DOE/EIA-O095(96)

Distribution Category UC-950

\title{
Inventory of Power Plants in the United States
}

\author{
As of January 1, 1996
}

December 1996

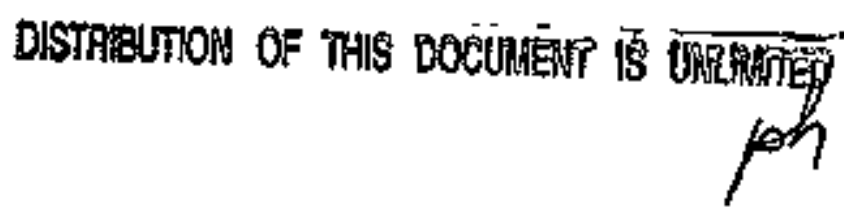

Energy Information Administration

Olfice of Coal, Nuclear, Electric

and Altemate Fuels

U.S. Department of Energy

Washington, DC 20585

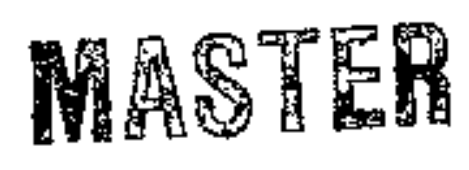

This report was prepared by the Energy Information Administration, the independent statistical and analytical agency within the Department of Energy. The trformsition contalned harein should not be construed as axtracaling of reflecting any polloy positton of the Department of Energy or any other arganization. 


\section{DISCLATMER}

Thls report was prepared as 2n acrount of work sponstred by an agency tof the Urited Stales Government. Neither the United 5tates Goverument nor any agency thereof, nor

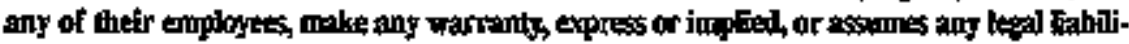

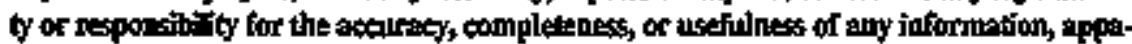
rates, product, or process itsolosed, or represents that its use would not infringe privately owned tights. Reference herin to any specific commercial product, process, or sertice by

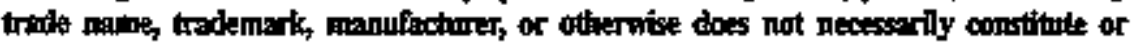
innply it esdorsenest, recommendation, or favoring by the Untted States Government or any agency theraut. The views and opinlons of authors expressed herels do not necesal: ily state or reflect those of the United States Gorernuent or any atsency thereof. 


\section{Contents}

Page

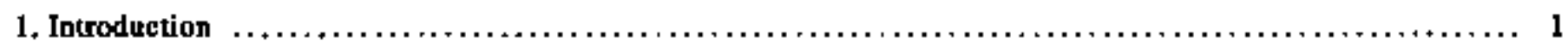

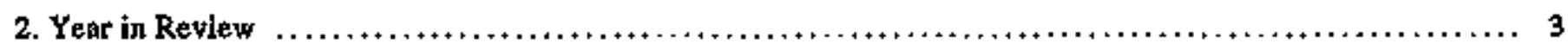

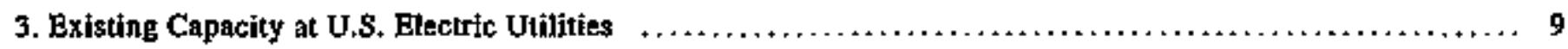

4. Planned Capacity Additions at $U . S$. Electric Utitities $\ldots \ldots \ldots \ldots \ldots \ldots \ldots \ldots \ldots \ldots \ldots \ldots \ldots \ldots \ldots \ldots$ Appendices

A. Technical Notes $\ldots \ldots \ldots \ldots \ldots+\ldots \ldots \ldots \ldots \ldots \ldots \ldots \ldots \ldots \ldots \ldots \ldots \ldots \ldots \ldots \ldots \ldots \ldots \ldots+\ldots \ldots \ldots, 259$

B. Table Coiles and References $\ldots \ldots \ldots \ldots \ldots \ldots \ldots \ldots \ldots \ldots \ldots \ldots \ldots \ldots \ldots \ldots \ldots \ldots \ldots \ldots \ldots, 267$

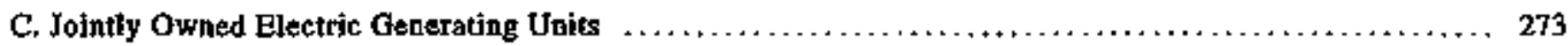

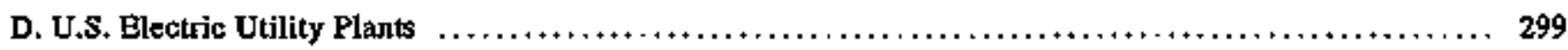

E. Plant-Level Statistics for U.S. Electric Utilitjes $\ldots \ldots \ldots \ldots \ldots \ldots \ldots \ldots \ldots \ldots \ldots \ldots \ldots \ldots \ldots \ldots, 393$

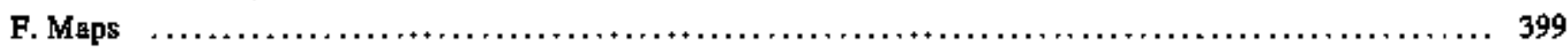

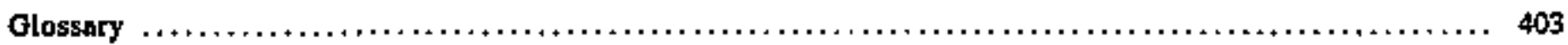


1. Existing Capacity and Planned Capacity Additions at U.S. Electric Utilittes by Energy Source, as of

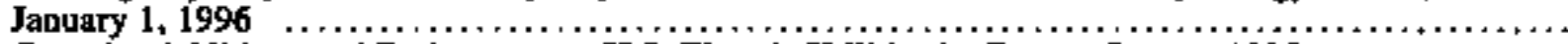

2. Capacity Additions and Retirements at U.S. Electric Utilities by Energy Source, $1995 \ldots \ldots \ldots \ldots \ldots$.

3. Combined Cycle Existing Capacity and Planned Capacity Additions at U.S. Electric Utilities by Prime Mover and Prjmary Energy Source, as of January 1, 1996

4. Fossil-Fueled Existing Capacity and Planned Capacity Additions at U.S. Electric Utilities by Prime Mover and Primary Energy Source, as of January 1, 1996

5. Fossil-Fueled and Nuclear Steam-Electric Existing Capacity and Planned Capacity Additioas at U.S. Electric Utilities, as of January 1, 1996

6. Existing Capacity at U.S. Electric Utilities by Prime Mover and Energy Source, as of January 1, 1996

7. Planned Capacity Additions at U.S. Electric Utilities, 1996 Through 2005 , as of Janutary 1, $1996 \ldots \ldots$

8. Planned Coal+ and Petroleum-Fired Capacity Additions at U.S. Electric Utilities, 1996 Through 2005, as of January 1,1996

9. Planned Gas-Fired and Hydroelectric Capacity Additions at U.S. Electric Utilities, 1996 Through 2005 , as of January 1, 1996

10. Planned Nuclear and Other Capacity Additions at U.s. Electric Ueilities, 1996 Through 2003 , as of January 1,1996

11. Planned Capacity Retrements at U.S. Electric Utilities, 1996 Through 2005 , as of January 1, $1996 \ldots$

12. Planned Coal- and Petroleum-Fired Capacity Retirements at U.S. Electric Utilities, 1996 Through 2005, as of Japuary 1,1996

Planned Gas-Fired and Hydroelectric Capacity Retirements at U.S. Ejectric Utilities, 1996 Through

2005, as of January 1, 1996

Existing Capacity and Plapned Capacity Additions at U.S. Electric Utilities by Energy Sonoce, North

American Electric Reliability Council Region, and Hawai, as of January $1,1996 \ldots \ldots \ldots \ldots \ldots \ldots$

15. Existing Capacity and Planned Capacity Additions at U.S. Electric Utilities by Energy Sonrce and

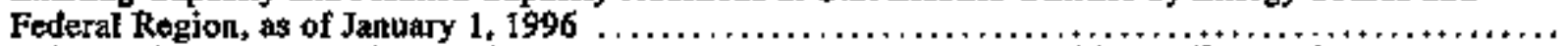

16. Existing Capacity and Planned Capacity Additions at U.S. Electric Utilities by Energy Source and

17. Existing Capacity and Planued Capacity Additions at U.S. Electric Ütilities by Energy Source and

State, as of January 1, 1996

18. Oenerating Units that Started Operation at U.S. Electric Utilities by State, Company, and Plant, 1995

19. Generating Units Retired from Service at U.S. Electric Utilities by State, Company, and Plant, 1995

20. Existing Generating Units at U.S. Electric Utilities by State, Company, and Plant, as of January 1, 1996

21. Existing Generating Units Powered by Renewable Energy Sources at U.S. Electje Utilities by State,

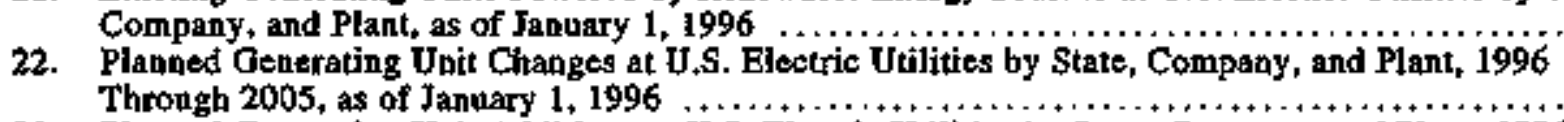

23. PJanned Genterating Unit Additions at U.S. Electric Utilities by State, Company, and Plant, 1996

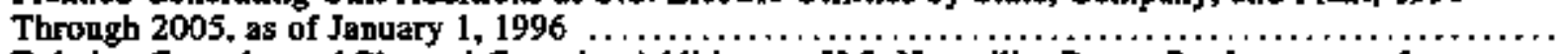

24. Existing Capacity and Planned Capacity Additions at U.S. Nonutility Power Producers, as of

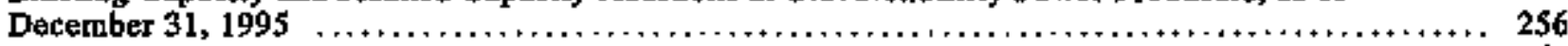

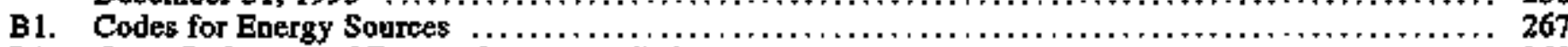

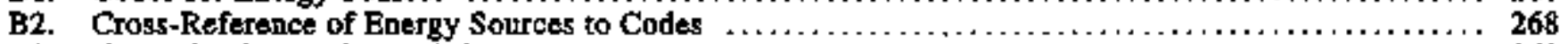

B3. Codes for Generating Unit Type $+\ldots, \ldots \ldots, \ldots, \ldots, \ldots \ldots \ldots, \ldots \ldots+\ldots+\ldots+\ldots \ldots \ldots \ldots+\ldots, 268$

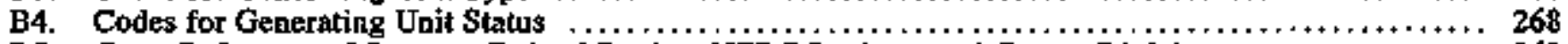

B5. Cross Reference of States to Federal Region, NERC Regions, and $C$ ensus Divisions .............. 269

C1. Jointly Owned Electric Generating Units by State, Company, and Plant, as of January 1, $1996 \ldots \ldots .273$

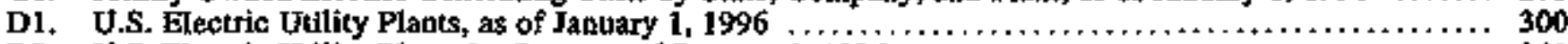

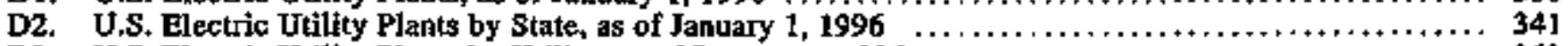

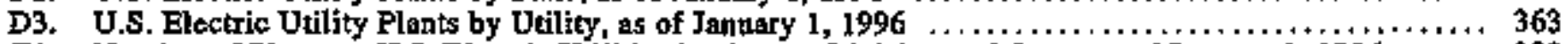

E1. Number of Plants at U.S. Electric Utilities by Census Division and State, as of Jaguary $1,1996 \ldots \ldots 39$

E2. Existing Capacity at U.\$. Electric Utilities by Census Division, State, and Prtme Mover, as of January

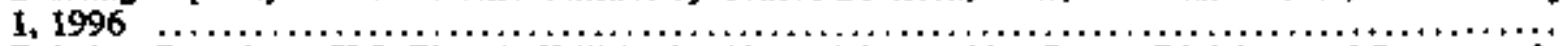

E3. Existing Capacity at U.S. Electric Uttities by Class of Ownership, Census Division, and State, as of Jantary 1, 1996 


\section{Illustrations}

1. Share of Capacity at U.S. Electric Utilities by Energy Source, as of January 1, $1996 \ldots \ldots \ldots \ldots \ldots .5$

2. Share of Capacity at U.S. Electric Utilities by Prime Mover, as of Jatuary $1,1996 \ldots \ldots \ldots \ldots \ldots \ldots, 5$

3. Coal-Fired Capacity at U.S. Electric Utilities by State, as of Japuary $1,1996 \ldots \ldots \ldots \ldots \ldots \ldots \ldots \ldots, 6$

4. Petroleum-Fired Capacity at U.S. Electric Utilities by State, as of January $1,1996 \ldots \ldots \ldots \ldots \ldots \ldots, 6$

5. Gas-Pired Capacity at U.S. Electric Utilities by State, as of January $1,1996 \ldots \ldots \ldots \ldots \ldots \ldots \ldots, 7$

6. Hydroelectric Capactty at U.S. Electric Utilitjes by State, as of Janvary $1,1996 \ldots \ldots \ldots \ldots \ldots \ldots \ldots 7$

7. Nuclear Capacity at U.S. Electric Utilities by $\$ t a t e$, as of Jabuary $1,1996 \ldots \ldots \ldots \ldots \ldots \ldots \ldots \ldots \ldots \ldots, 8$

8. Tatal Capacity at U.S. Electric Utilities by State, as of Jenuary 1, $1996 \ldots \ldots \ldots \ldots \ldots \ldots \ldots \ldots \ldots, 8$

9. Existing Capacity at U.S. Electric Utiljties by Prime Mover and Initial Year of Commercial Operation,

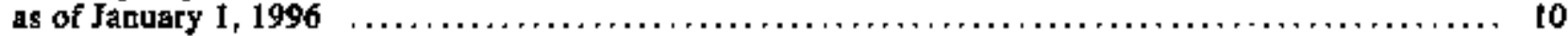

10. Capacity Additions at U.S. Electric Utilities by Energy Source, 1986 Through $1990 \ldots \ldots \ldots \ldots \ldots+\ldots \ldots$, II

11. Capacity Additions at U.S. Electric Utilities by Energy Source, 1991 Tbrough $1995 \ldots \ldots \ldots \ldots \ldots \ldots$. 11

12. Share of Planned Capacity Additions at U.S. Electric Utilitites by Energy Source, 1996 Througb $2005+13$

13. Planned Capacity Additions at U.S. Electric Utilities by Energy Source, 1996 Through $2000 \ldots \ldots . .16$

14. Planned Capacity Additions at U.S. Electric Utitities by Energy Source, 2001 Torough 2005 ....... 16

15. Planned Capacity Additions of More Than 1,000 Megawatts at U.S. Blectric Utiljies by Utility, 1996

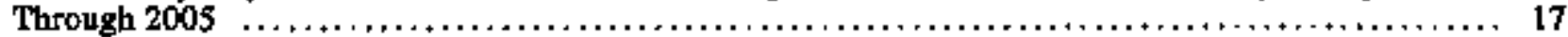

16. Planped Capacity Additions at U.S. Electric Utilities by State, 1996 Through $2005 \ldots \ldots \ldots \ldots \ldots \ldots . \ldots \ldots$

F1. North American Electric Reliability Council Regious for the Contiguous United States and Alaska ... 399

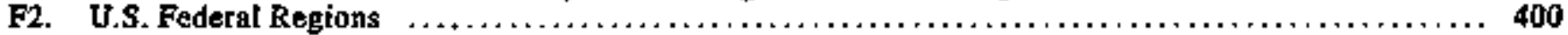

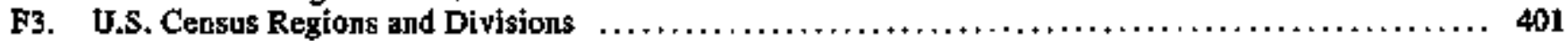




\section{Introduction}

The Inventory of Power Plants in the United States provides annual statistics on generating units operated by electric utilities in the United States (the $\$ 0$ States and the District of Columbia). Statisttes prosented in this report reflect the status of generating units as of January 1, 1996. The publication also provides a 10-year outlook for generating unit additions.

This report is prepared annually by the Coal and Electric Data and Renewables Division; Offjee of Coal, Nuclear, Electric and Alternate Fuels; Energy Information Adiministration (EIA); U.S. Depariment of Energy (DOE). Data summarized in this report are useful to a wide audience inciuding Congress; Federal and Stats agencjes; the electric utility industry; and the general public. Data presented in this report were assembled and published by the EIA to fulfill its data cojlection and dissemination responsibilities as specified in the Federal Energy Administration Act of 1974 (Public Law 93-275) as amended.

This report's primary data source, Form BIA-860. "Annual Electric Geperator Report," was revised in 1995 (see "Data Sources" below). One of the revisions is the collection of data as of January 1 of the reporting yearn-reporting year is the calcadar year in which the survey form is tequired to be filed with the BIA. Historically, the form has been used to coilect data as of the end of each calendar year. The calendar year as specified in the title of the historical series identified the year to which the data relate. For example, Inventory of Power Plants in the United States 1994, prepared from data reported on the form, reguesting data as of year-end 1994, reflects the status of clectric utilities' generators as of December 31 . 1994, and generator-related statisties for 1994. Starting with this issie, the title of the sertes is changed to reflect the revised reference point of data collection. This issue. Inventory of Power Plants in the United States, as of Januany J, 1996, contains data about the status of electrite utitjties' generators as of January 1, 1996, and essentially reflects generator-related statistics for 1995 . With the revised teference point, no continuity is lost in this historical annual pubijcation stejes. The title reflects a reporting yezr, rather than the year of the data. The next publication is expected to be published in October 1997, reflecting the status of electric utilities' generators as of January 1, 1997, and generator-related statistics for 1996 .

Chapter 2. "Year in Review" contains aggregate statistics on capacity at various regional levels and at the national level for existing and planned generating unit additions. Aggregate data on capacity at the national level are presented by energy source and prime mover. Aggregate data on capacity at various regiona? levels are presented by primary energy source. Planned capacity additions and retirements are sum. marized by year for 1996 through 2005.

Chapter 3. "Existing Capacity at U.S. Electric Utilities ${ }^{n}$ contains data on existing generating units as of January I, 1996 and generating units that were retired froen service during 1995. A summary of generating unit additions by energy source during 1995 is also included.

Chapter 4. "Planned Capacity Additions at U.S. Electric Utilities" contains information regarding gener. ating ugits scheduled to start commercial operation from 1996 through 2005. This chapter also contajns data about proposed changes (modifications and retirements) to existing and previously retired generating units.

This is a report of electric utility data; in cases where summary data of nonutility capacity are presented, it is specifrcally noted as such.

Generally, tables in this publication that contain electric utility capacity data present three measures of generator capacity --genesator nameplate capacity, net summer capability, and net winter capability. Howerer, the EIA uses net summer capability as its stattstic for analyzing electric utility cupacity. Therefore, all discussion of electric otility generating capacity in this publication relors to net summer eapability, unless otherwise stated. For an explanation of the three measures of generator capacity, see Appendix A, Technical Notes, "Explanacory Notes." Additionally, any discussion of generator capacity by energy source is based on the primary energy source used by the respective generating unit.

\section{Data Sources}

Data published in the Invensory of Power Plants in the Untied States were compiled from the Form EIA-860, "Annual Electric Generator Report," filed annually with the ELA, directly by tectric utitities, or through an agent of their choice. The 1996 submissions are the first to reflect the combined data collection efforts of the North American Electric Reliability Council (NERC) and the EIA. Effective with the 1996 reporting, respondents to Form EIA-860 are given the 
option to file directly with the ElA or through an agent of their choice, such as the respondent's regional electric reliability conncil. Since data requested in Form EIA-860 are also requested by the regional councils on Form EIA-411, "Coordinated Bulk Power Supply Program," Item 3, respendents who report data for Form EIA-4I1 can fulfill their reporting requirements for Form ELA-860 by reporting these data to their regional councils. The regional councils use these data for their planning process and regional analysis. The Form EIA-411 data are submitted annually to NERC by the regtonal councils. NERC, in tum, forwards these data ejectronically to the ELA. For 1996, 87 percent of responses were submitted directly to the EIA in hardcopy form bad 17 percent were submitted electronically by NERC.

The Form EIA 860 was revised in 1995, with changes effective with the 1996 reporting year, where reporting year is the calendar year in which the report is ffled with the EIA. Therefore, data in this publics- tion reflect the revised data collection. For mote information on this form, see the Technical Notes. Atso, Appendix B contains the tables of current codes and their definition.

Updates made during the past year for inclusion in this publication are as follows: (i) changes that reflect construction or modification within power plants or changes in power plant operations (includes the installation of new generators; the retirement of existing generators; the use of a primary energy saurce for dual-fired units different from that reported in the past; and the modification of generators, soch as the rewinding of stators or the retrofitting of associated generator equipment), (2) corrections to previously repotted data that were incorrect, (3) deletion of respondents that do not meet the reporing reguirements of Form EIA-860, (4) deletion of capacity when generators prevjously owned and operated by electric utilities are sold to nonutilities, and (5) the incluston of new respondents. 


\section{Year in Revlew}

As of Jannary 1, 1996, the existing capacity' of U.S. electric utilities totaled 706,111 megawatts (Tab]e 1). Based on primary energy source, coal-fired capacity represented 43 percent $(300,610$ megawatts) of the Nation's existing capacity (Figure 1). Gas-fired capacity accouated for 20 percent (142,536 megawatts); auclear, 14 percent (99,515 megawatts); renewable energy sources, ${ }^{2} 11$ percent $(77,600$ megawatts); petroleum, 9 percent (64,465 megawatts); and water (pumped storage hydroelectric), 3 percent (21,387 megawatts). The amount and geographical distribution of capacity by energy source is a function of availability and price of fuels and/or regulations. Capacity by energy source generally shows a geographical pattern such as, significant petroleunfired capacity in the East, hydroelectric in the West, and gas-fired capacity in the Coastal South (Figures 3-7).

Of the existing capacity, conventional steam-electric units accounted for 62 percent $(441,158$ megawatts). Nuclear units accounted for 14 percent; hydroelectric (conventional), 11 perceat; gas turbine, 7 percent; hydroolectric (pumped storage), 3 percent; combined cycle, 2 percent; interual combustion, geothermal, solar and wind combined, 1 percent (Figure 2).

In the revised Form EIA-860, respondents are requested to report up to 2 energy sources for each generator, os applicable, as opposed to the historical request for up to 3 energy sources, as applicable, on Form EIA-860. Historically, the amount of capacity in units that were capable of using solids, liquids, and gases averaged less than one percent of conventional steam-electric capacity. As of Janusry i, 1996, 190,604 mogawatts or 27 percent of capacity reported for thermal-electric units that had the capability to use more than one energy source. Of the 190,604 megawatts, 153,680 megawatts were in conventional stoam-electric units. The remaining 36,924 megawatts were in gas turbine, intersal combustion and combined cycle units.

In 1995, 5,752 megawatts in new units started commercial operation (Table 2). Gas-fired capacity accounted for $\mathbf{4 8}$ percent of this new capacity. Gas turbines and combined cycle units. accounted for 99 percent (2,741 megawatts) of gas-fireo capacity additions. The remainder of the pas-fired capacity added in 1995 included internal combustion unies totaling 6 megewatts; and a stean unit of 16 megawatts (Tablo 18).

No new nuclear units came on-line or retired during 1995. Currently, no new nuclear generating units are planned for installation over the next 10 years. As of January 1, 1996, construction of Tennessee Valley Anthority's Watts Bar, Unit 1, was conplete and in testing phase. It started commercial operation in May 1996.

In addition to adding new generating capscity, tectric atilities have engaged in other activjties to meet futore load requirements. These activities include retating, repowering, or life extension of existing nnits, purchases from nonutility power producers, and desuand-side managenent programs.

The amount of capacity planned to undergo changes during the pext 10 years totals 29,896 megawatts (Table 22). Of that total, 4,506 megawatts of capacity are proposed for retirement and 4,050 megawatts are proposed for repowering or life extension. This capacity planned for repawering or life extersision does not include the increase in capacity that usually results from repowering. For example, repowered combined cycle units usually restult in the addition of gas turbine units, which anay be added to the unit"s overall capacity. The 4,050 megawatts of capacity do not include any additional capacity supplemented by the added gas turbine units. Most of this capacity will be repowered to combined cycle or fluidized bed technology. The remaining approximate 21,340 megawatts include platned fuel changes, reratings of generating nnits, reactivation from retirement and deactivation to shutdown status.

Nonutilities are expected to supply a significant portion of the generating capacity needed to meet energy requirements of electric utilities (Table 24). The contribution of nonutility capacity to total elec. tricity supply has increased signifrcantly over the past several years and is expected to cantinue to iocreaso. Competitive bidding requirements by public utility commissions allow nonntilities to compete with elec. tric utilities for new capacity construction. This is expected to resust in an increasing share of nonutitity capacity in the electric power supply. Estiniated data for 1995 show that nonutility capacity totals 70 gigawatts for a gross generation of 375,901 gigawatthours with sales to electric utilitios of

I In all custo, capacity is nel summor capability, unless poled otherwist.

2 Renewable energy sources include water (conventional hydroelectric), geothermal, hionass, solar and wind.

3 Capacity for nonotility power producer facilities is seperator nameplate capacicy. For more information on nonufility eapocify, afece Electrie Power Antuai, Volvare 2, DOE/ELA-034B(95)/2 (Weshington, DC, December 1996). 
233,454 gigawatthours. Nonutility power producers project that nomutility capacity will be fueled mostly by gas and renewable energy sources. Nonutility capacity additions 4 planned for 1996 through 1998 total atore than 4 gigawatts, ${ }^{5}$ while electric utilities bave planned to add 11 gigavatts of new capacity (generator nameplate capacity) during this same period (Table 7).

Electric utilities are also engaged in demand-side management (DSM) programs aimed at reducing electricity use by implementing conservation and load management. The objective of most DSM programs is to provide cost-effective energy and capacity resources that postpone the need for construction of new power plants by nodifying the growth in demand and energy ase. Data collected by the EIA indicate that the number and scope of DSM programs in the United States are increasing. Estimated 1995 data show the total potential peakload reductions for DSM in 1995 was 47 gigawatts; 49 gigawatts and 58 gigawatts are projected for 1996 and 2000, respectively. ${ }^{\circ}$

1 These data represent planted capacily additions tor which a proposed date of operation in 1996, 1997, or 1998 was reported. Those dato moy differ frose oifur nonvitity planped capacity additions summarized in otber reports that additionally include planned capacity edditions for which a proposed date of operation was not feported.

S Soure: Energy Inforfagtion Administration, Porm ElA-867, "Anawal Nonoltitty Power Produces Report."

6 Soutes: Energy Informalkon Administration, Fotm EJA-861, “Annual Electric Uíliny Report." 
Figure 1. Share of Capacity at U.S. Electric Utlitles by Energy Source, as of January 1, 1996

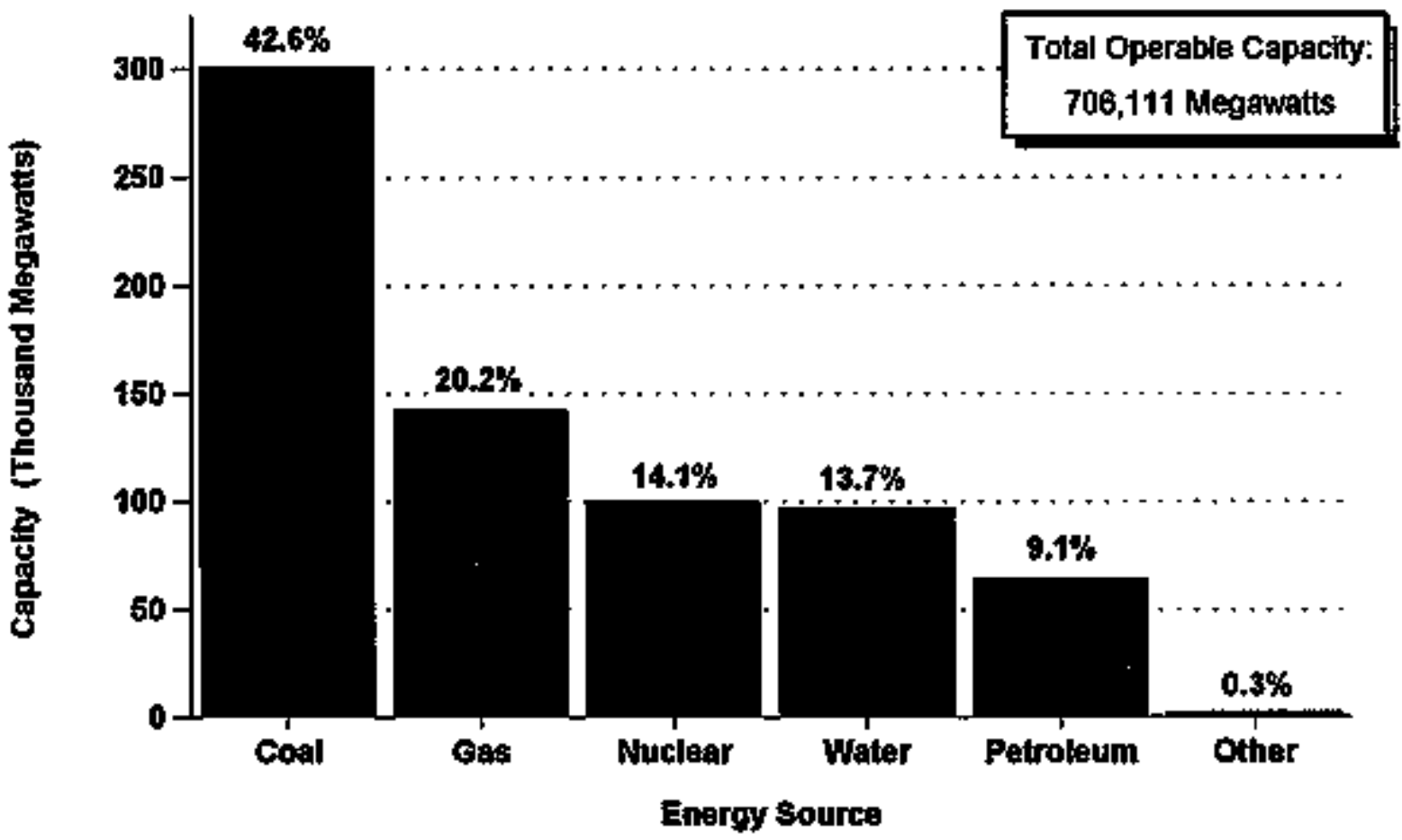

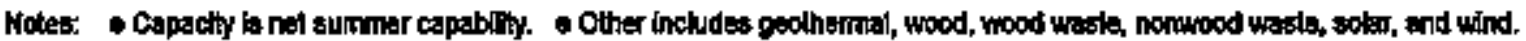

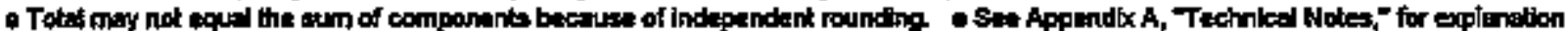
of reparting dicte.

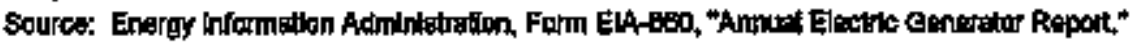

Figure 2. Share of Capacity at U.S. Electric Utilities by Prime Mover, as of January 1, 1996

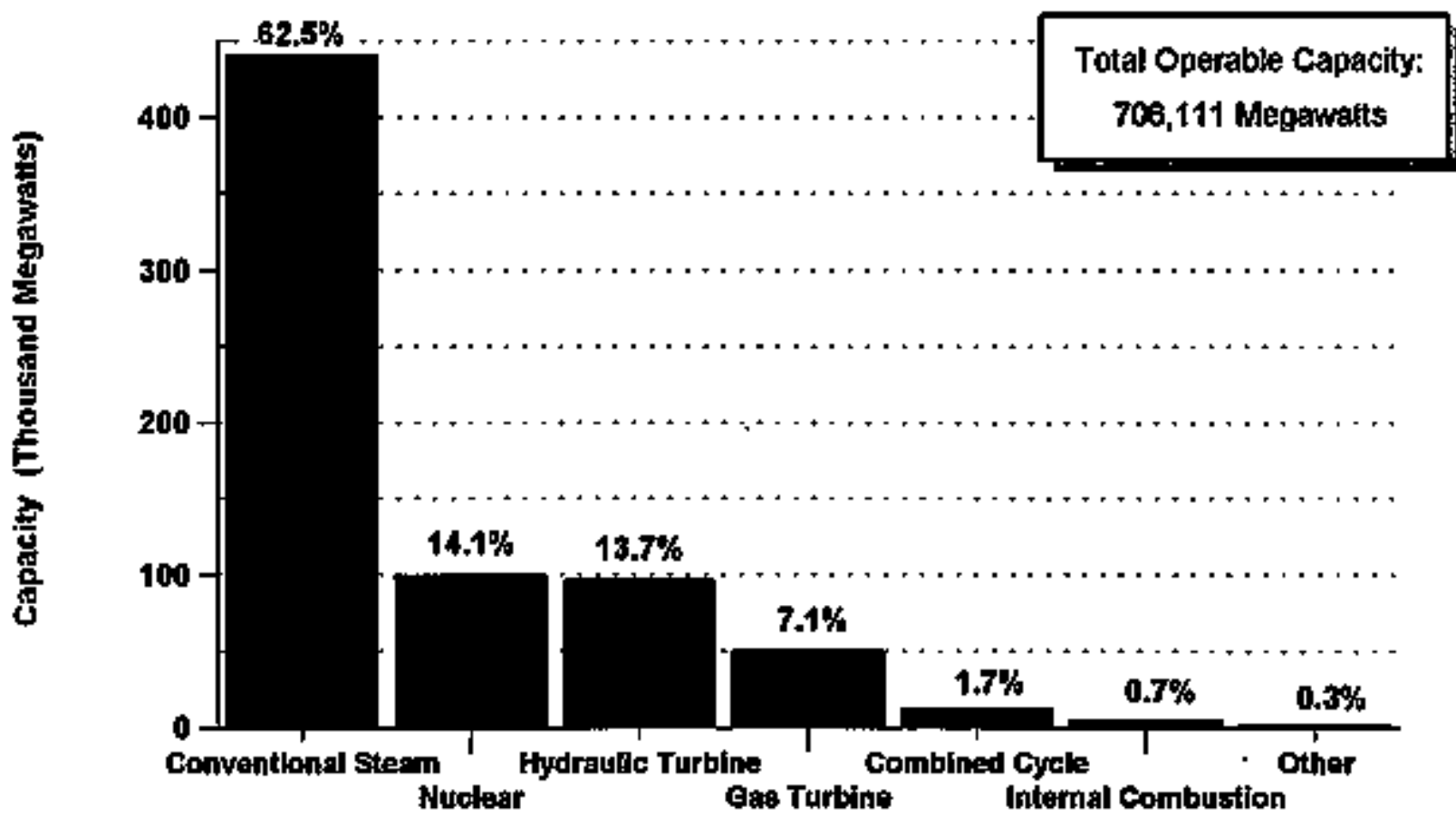

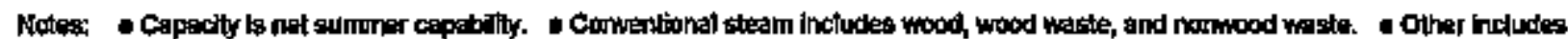

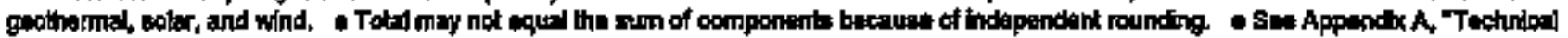
Notess," for explanation of reporting doth.

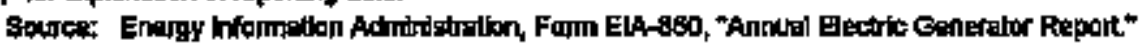

Energy Information Administration/Inventory of Power Plants in the United States as of Jantuary 1, 1996 


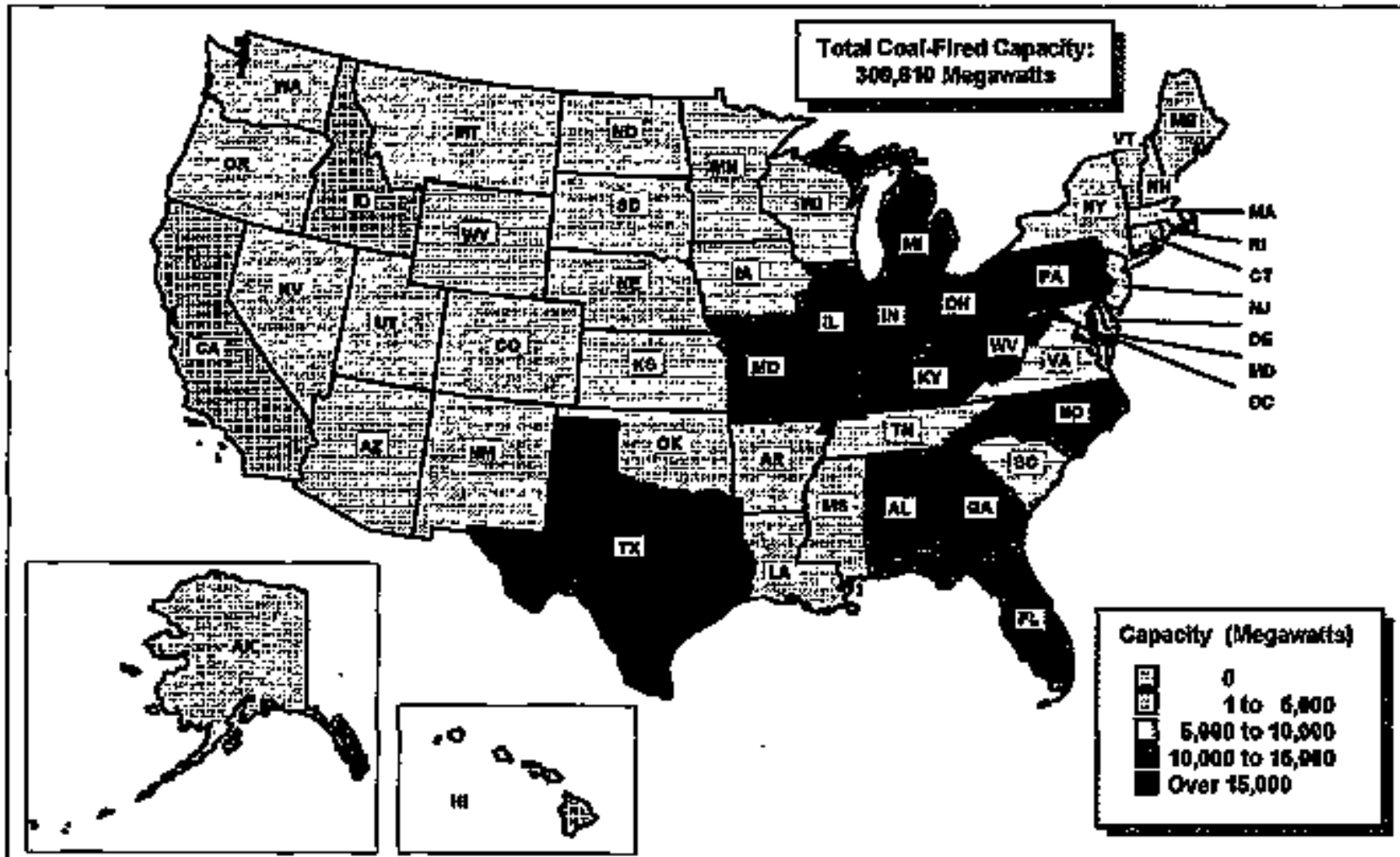

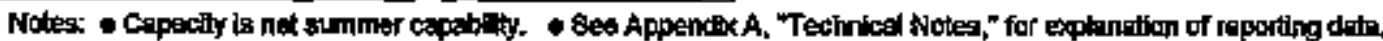

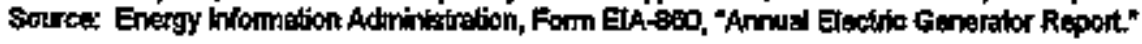

Figure 4. Petroleum-Fired Capacity at U.S. Electrie Utilities by State, a\& of January 1, 1996

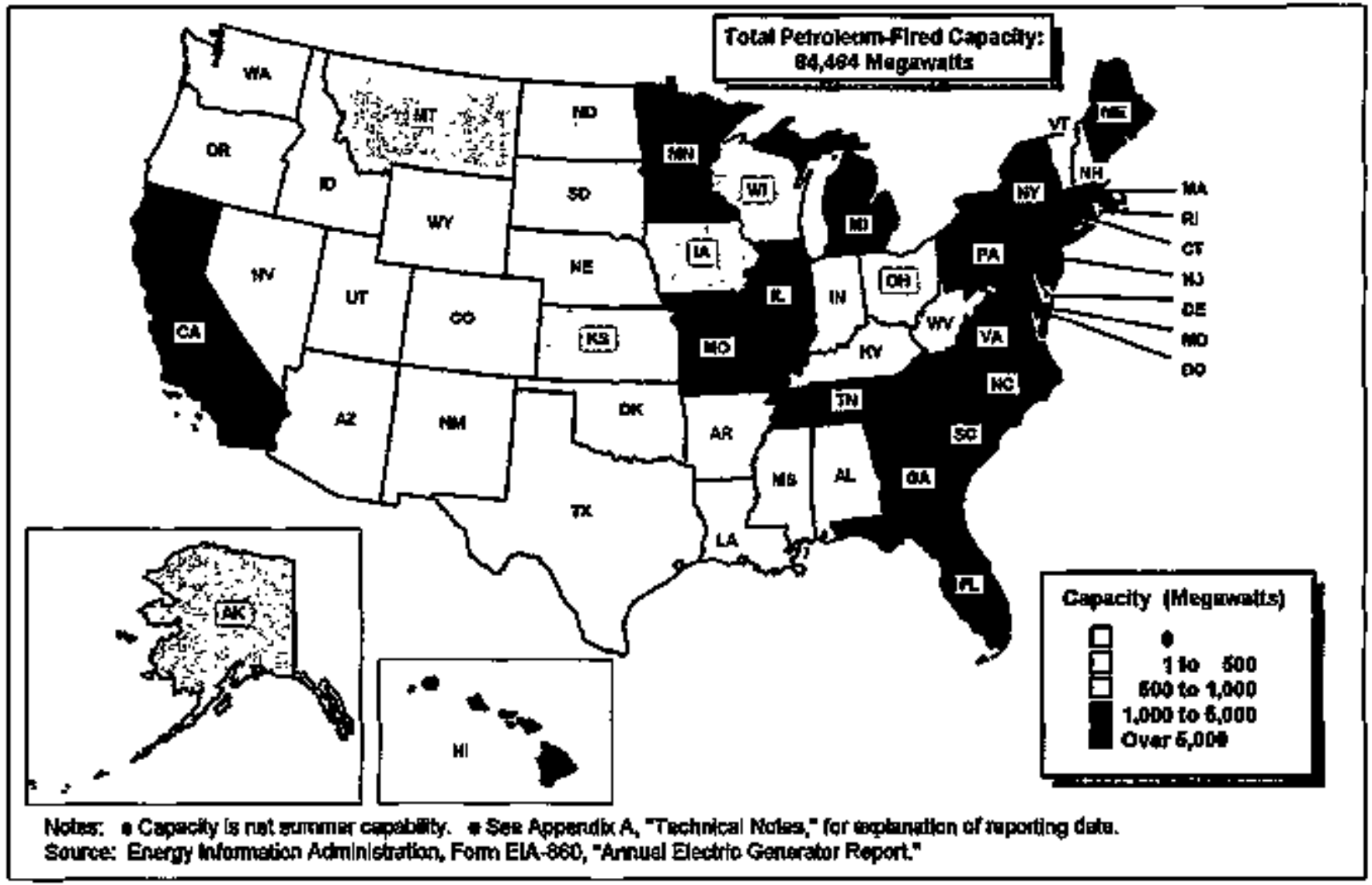

6 Energy Information Administration/Inventory of Power Plants in the United States as of January 1, 1996 
Figure 5. Gas-Fired Capaclty at U.S. Electric Utilities by State, as of January 1, 1996

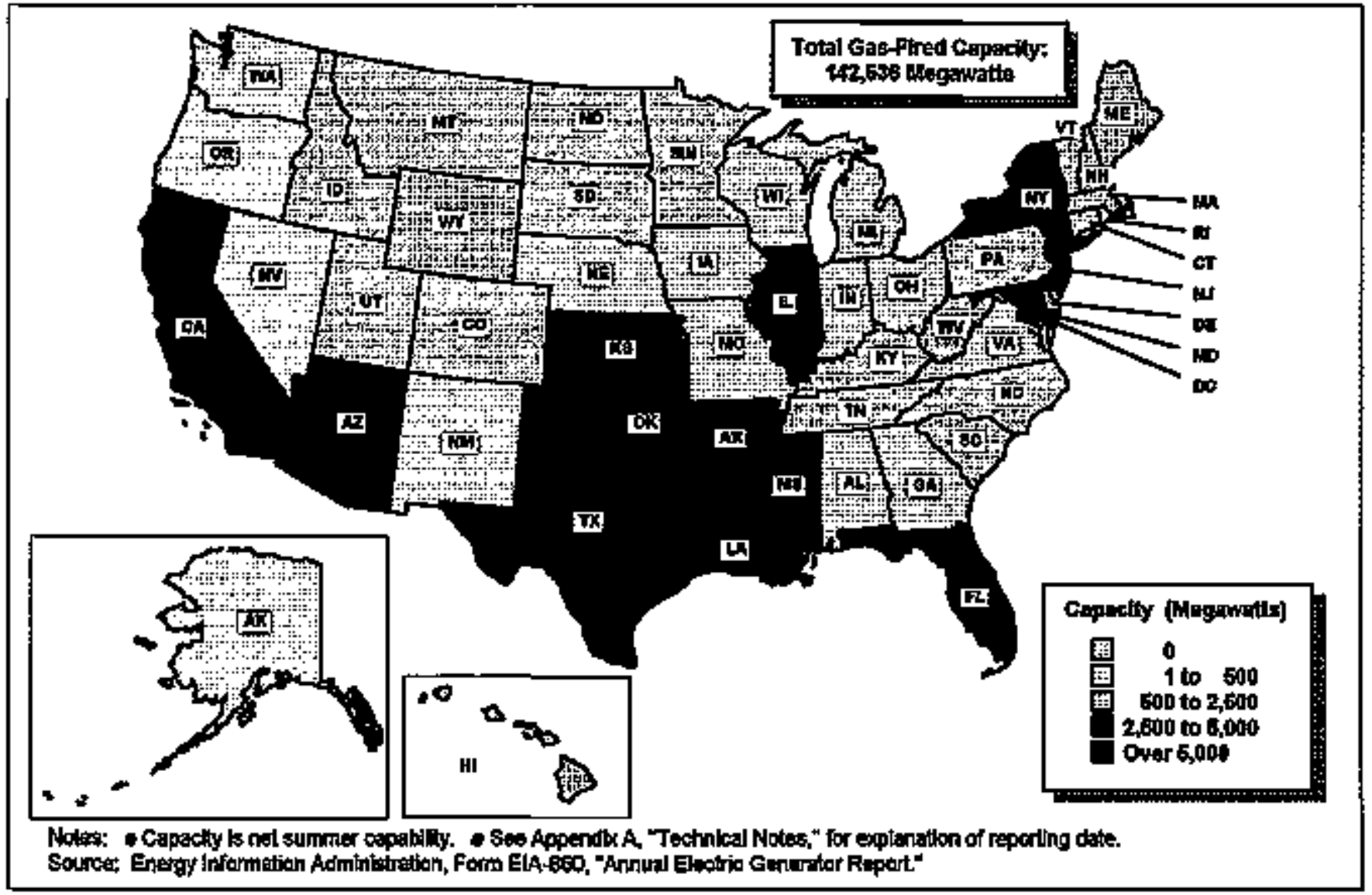

Figure 6. Hydroelectric Capacty at U.\$. Eleotric Utilties by State, as of January 1, 1996

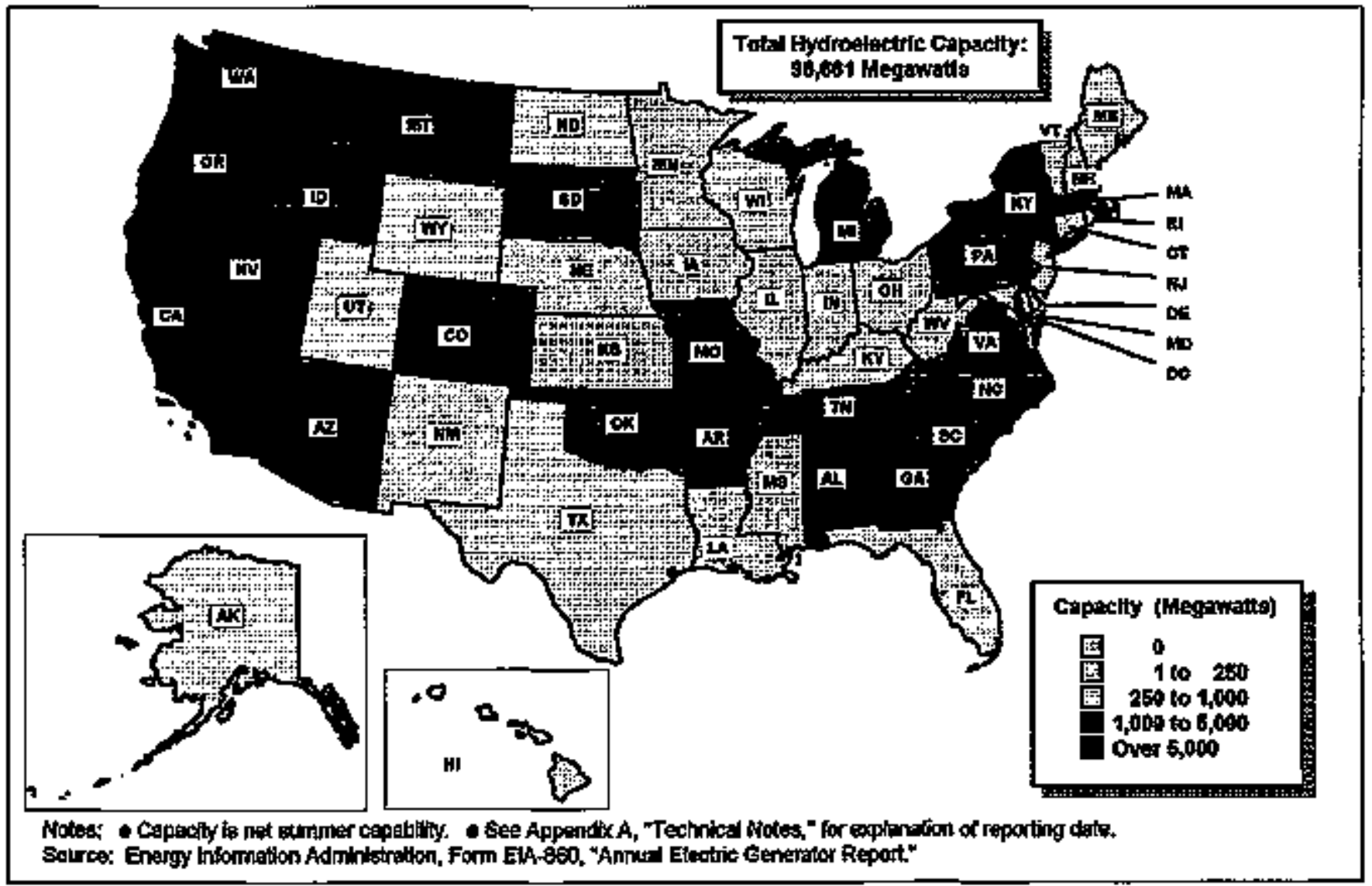

Energy Information Administration/Inventory of Power Plants in the Lnited States as of January 1, 19967 


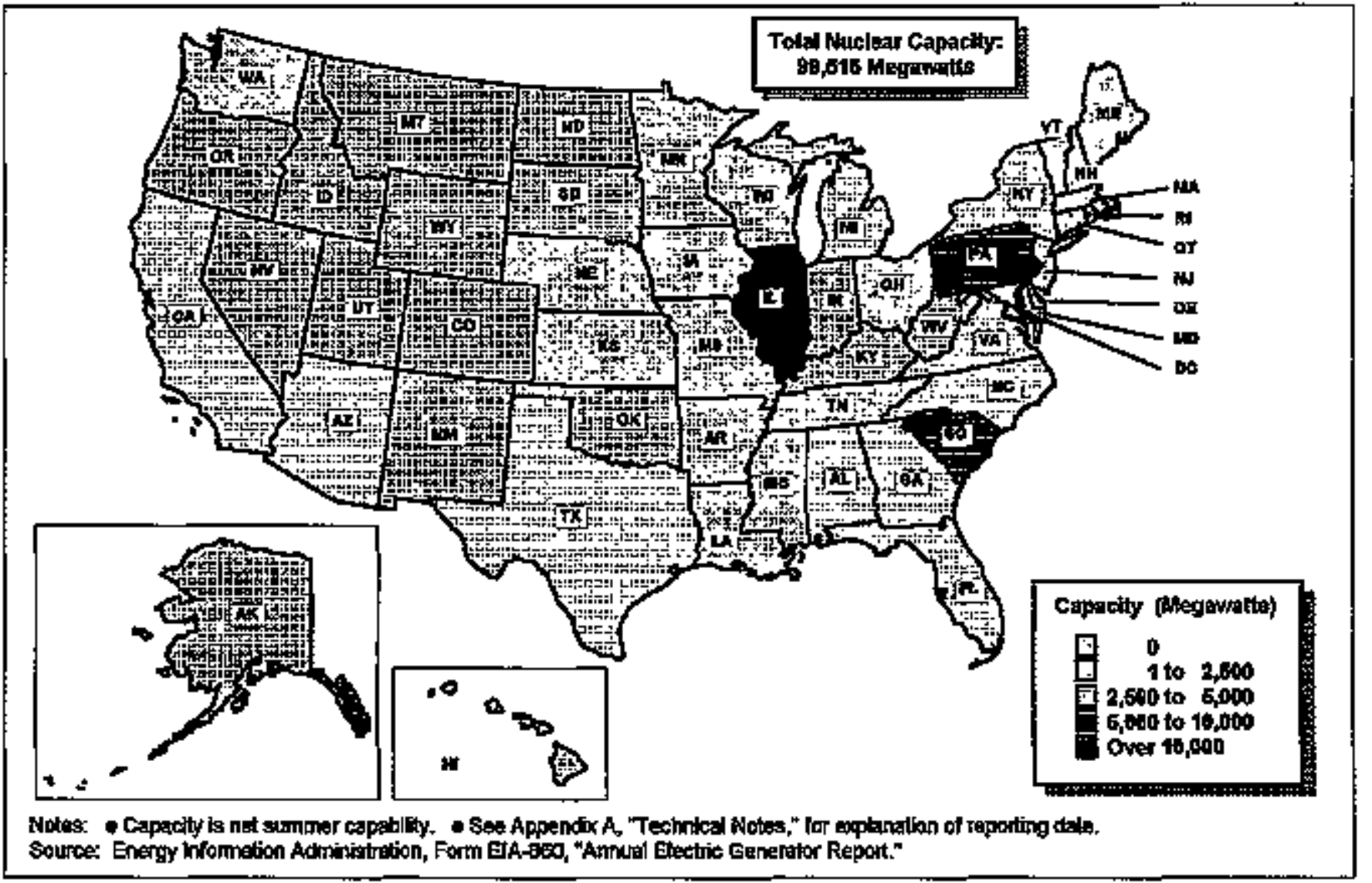

Figure 8. Total Capacly at U.S. Electric Utilities by State, as of January 1, 1996

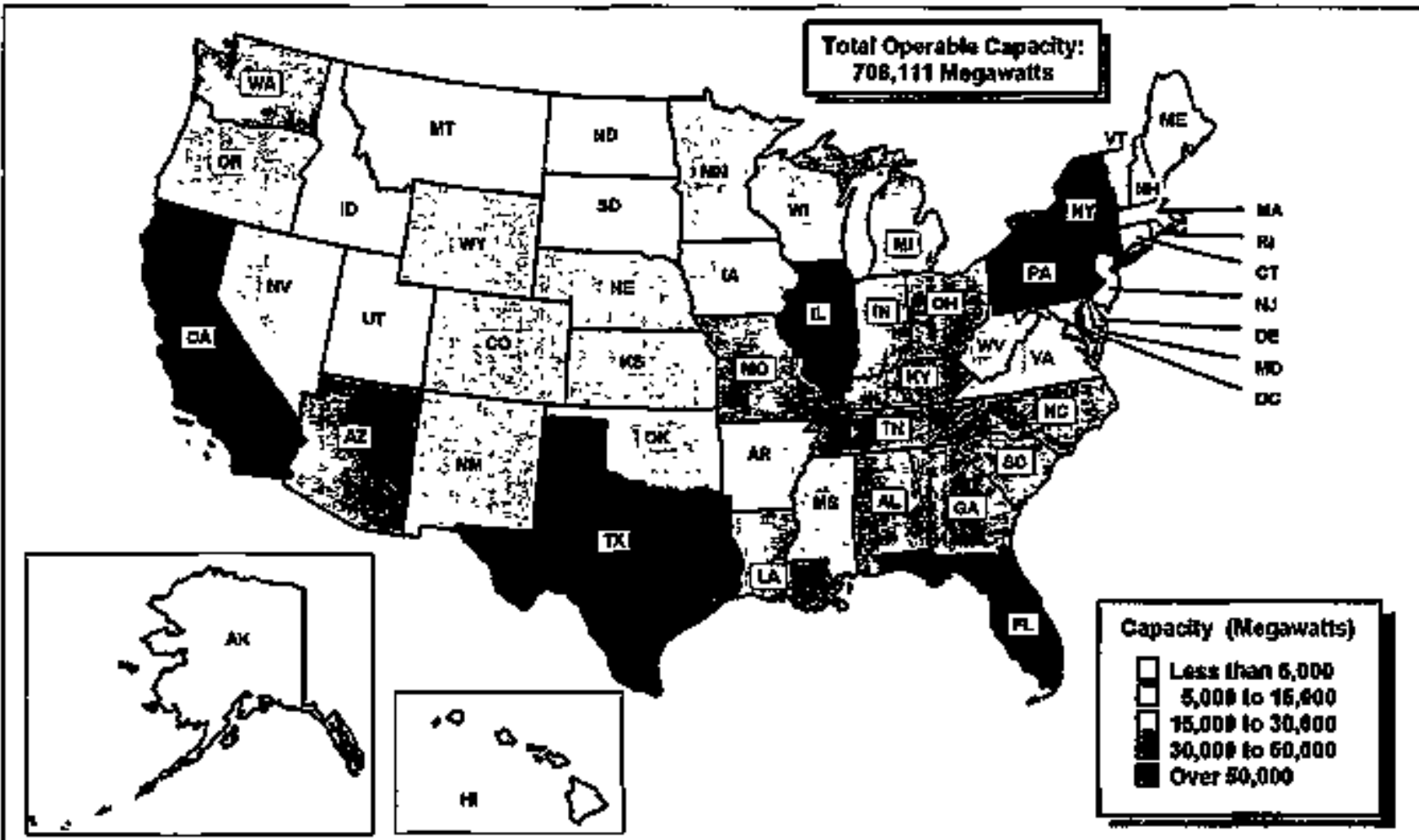

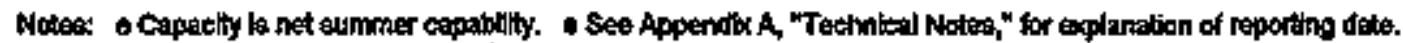

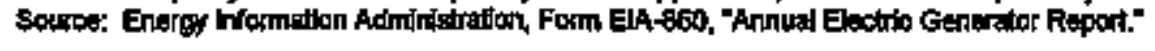

8 Energy Information Administration/Inventory of Powrer Plants in the United States as of Janusry 1, 1996 


\section{Existing Capacity at U.S. Electric Utilities}

As of January 1, 1996, the extsting capacity' of generating units operated by U.S. eleclute utilities totaled 706,111 megawatts (Table 1). Existing capacity can be divided into two categories: active and inactive. The active generating capacity totaled 694,201 megawatts. The inactive existing capacity $(11,910 \mathrm{mega}-$ watts) includied units that were in standby status or oot of service indefinitely. Existing electric generating capacity by prime mover and initial year of commercial aperation, is presented in Figure 9.

Conventiona! steam-electrtc cspacily, accounted for 62 percent (441,158 megawatts) of operable capacity; noclear, 14 percent $(99,515$ megawatts); hydroelectric (conventional), 11 percent $(75,274$ megawatts); gas urbine and internal combustion, 8 percent $(54,831$ megawatts); bydroelectric (pumped storage), 3 percent (21,387 megawatts); combined cycle, 2 percent (12,188 megawatts); and geatherntal, solar, and wind, less than 1 percent $(1,759$ megawatts) (Table 6 ).

The generating capacity of new units brought on line in 1995 totaled 5,752 megawats (Table 2); this additional capacity is in 88 generating units. The largest single unit (540 megawatts) to come on line was the bitominous-fired Cross, Unit 1, operated by South Carolina Public Servjec Authority. This unit tested for approximately 11 months before entering commercial operation this year."

Units powered by renewable energy sources that entered service is 1995 included only conventiopal hydroelectric additions. Conventional hydroelectric additions totaled 176 megawatts capacity in 10 nnits (Table 18), Other capacity included fossil-fueled steam, Enternal combustion (diesel), combined cycle, and gas turbine units. Electric ntility generating capacity additions by onergy source are presented for the 1986 through 1995 period in Figures 10 and 11.

Electric atilities reported 29,896 megawatts of existing capacity in generating units that will undergo changes duting the bext dectade (Table 22). Utilities have scheduled 4,506 unegawatts of capacity for retirement during the next decade (Table 11). A total of 4,050 megawats are proposed for repowering or life extension. The remaining changes proposed for existing units include fuel changes, reratings, retetivation, and deactivation.

Electric uttlities retited 954 megawatts of tapacity in 1995. Fossil-fueled steam-electric units, which bad an average size of 47 megawatcs accounted for almost 75 percent (705 megawatts) of the retired capacity. The Jargest fossil-fueled steam-electric unjt to retire in 1995 was the gas-fired 115-mogawatt Unit 3 at Cane Run, operated by Louisville Gas \& Electric Company. Niagara Mohawk Power Corporation retited 4 steanelectric units totaling 276 megawatts at its Oswego Plant. One of the 4 units was gas-fired while the remainder were petraleum-fired untits. Collectively, the 5 units retired at Louisville Gas \& Electric Corporation and Njagara Mobawk Power Corporation accounled for 55 percent of the fossil-fueled steamelectric capacity and 41 percent of the total U.S. retired capacity (Table 19). Niagara Mohawk Power Corporation also retired 4 gas-fired gas torbine units (156 megawatts) at its Albany Plant. The Oswego and Albany Plants of Niagara Mohawk Power Corporation collectively retired 8 units, totaling $\mathbf{5 3 2}$ megawatts in 1995 , 56 percent of the total U.S. retired capacity. The remaining 307 megawatts (32 percent) of retired capacity was in gas turbine, internal combustion, three snall wind units, and other stean units.

I [ ol] ooses, enpacity is net summer capability, unless noied otherwise.

- Apptared in Toblo [8 for 1994, as woll as 1995. Historical]y, first electefciay date represented the year that operation began. As of Janoary 1, J9\%, commercial operation date represents the year thal operatlon began. 
Figure 9. Existing Capacity at U.S. Electric Utllities by Prime Mover and Initial Year of Commerctal Operation, as of Jamuary 1, 1996

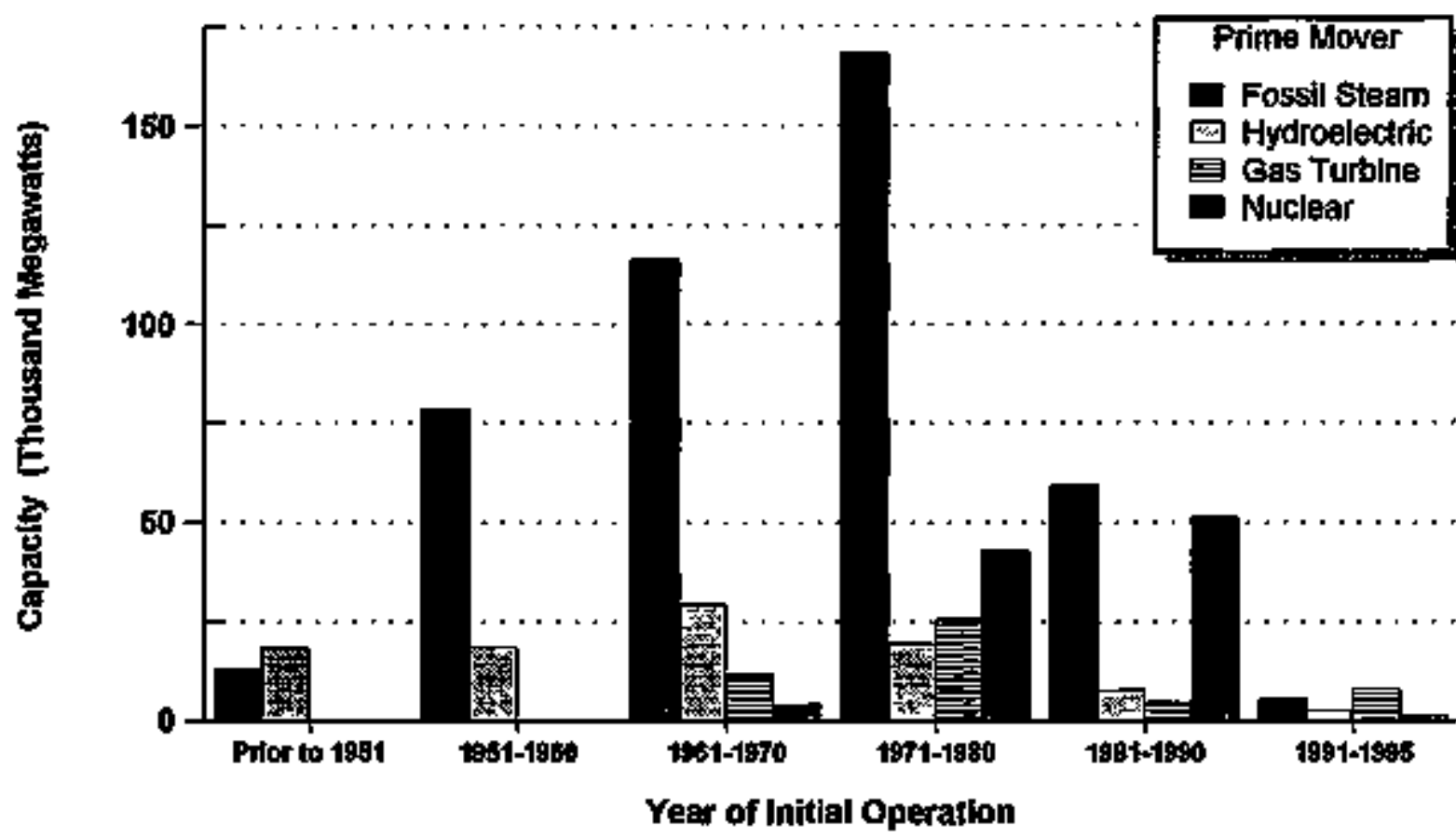

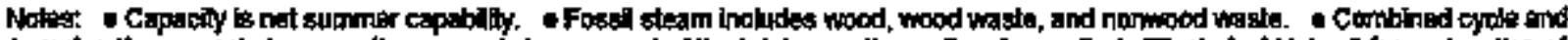

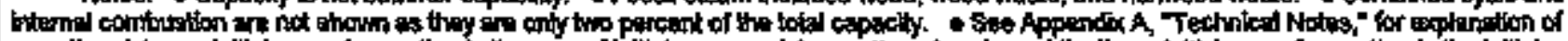

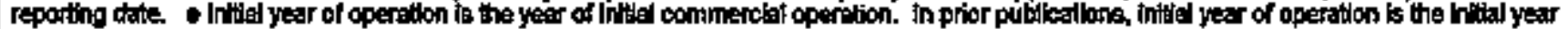

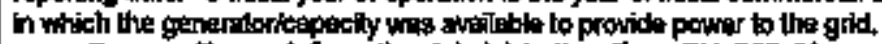

Sounce: Eneroy infometion Admintatration, Fonn ElA-s60, "Amnuel Electric Berterater Report" 


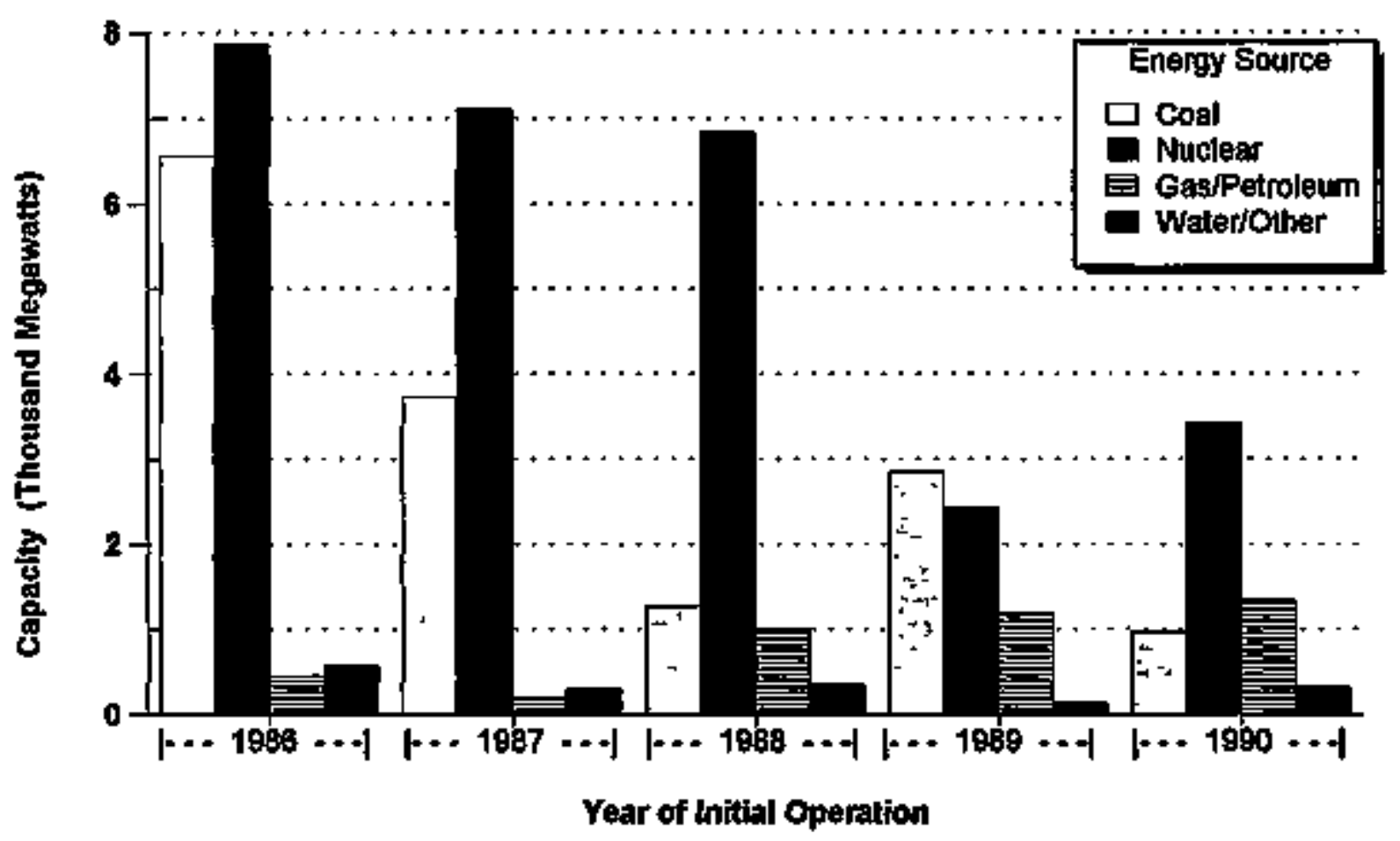

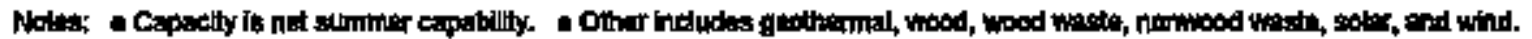

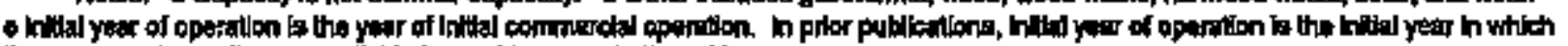

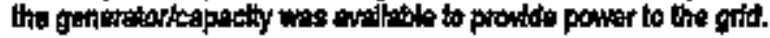

Source: Energy Intomation Adninietration, Fom ElA.690, "Anmual Electio Gernesator Report"

Figure 11. Capacity Additions at U.S. Electric Utiflites by Energy Source, 1991 Through 1995

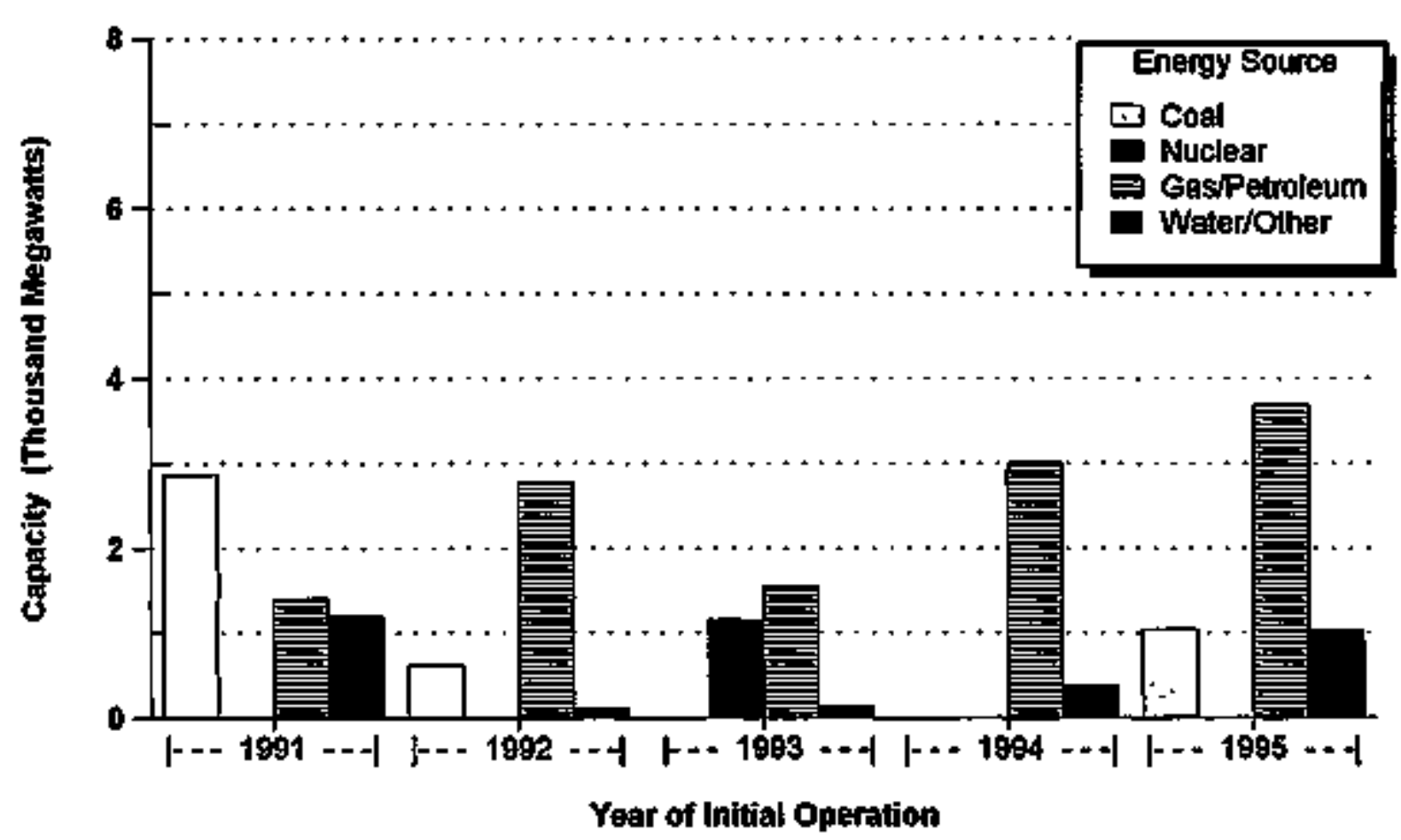

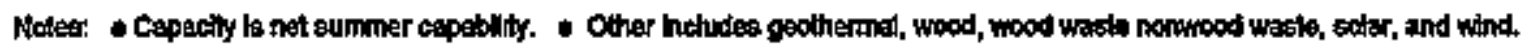

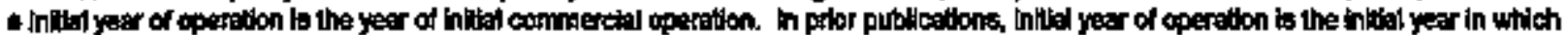

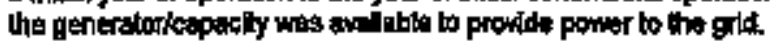

Spuce; Eneroy informetion Administralion, Fonm ElA-860, "Anntal Electrle Gernorator Report." 


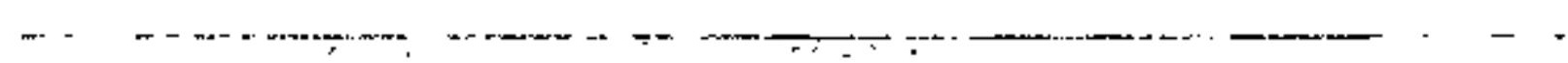




\section{Planned Capacity Additions at U.S. Electric Utilities}

Electric utilities have planned to Install 40,150 megawatts of capacity in their systemts during the next 10 years (Figures 15 and 16). This proposed new capacity includes generators that were in testing phase, in various stages of constroction, and in various stages of planning as of January 1, 1996.

Of the 40,150 megawatts of proposed tew capacity in new generating units, 4 percent $(1,806$ megawatts) had construction complete and was in the testing phase. Eleven percent $(4,475$ megawatts) was under construction and 84 percent $(33,870$ megawatts) was in various stages of planning. These proposed plans for 40,150 megawats of capacity are in $440 \mathrm{new}$ units. Additional capacity that electric utilities will realize through repowering or upgrading of their existing plants, or capacity that is owned and operated by nonutility generators is not included in these plans.

Gas accounts for the greatest share of additions in each of the next 10 years, with the exception of 1996 where coal accounts for the greatest share. Of the 440 generating units planned for installation in the next 10 years, more than balf are gas-fired units, totaling 27,371 megawatcs. Seventy-nine percent of new capacity will be in gas turbine and combined cycle units. Coal-fired unjts (including 2 integrated col gasification combined cycle unils) represent 12 percent (4,845 megawatts) of capacity additions (Figure 12). The remainder of additions witl be in nuclear stean-electric, other steam-electric, bydroelectric, internal combustion, conpressed ait energy storage, solar, and wind units (Table 23).

Figure 12. Share of Planned Capacity Additions at U.8. Electric Utilites by Energy Source, 1996 Through 2005

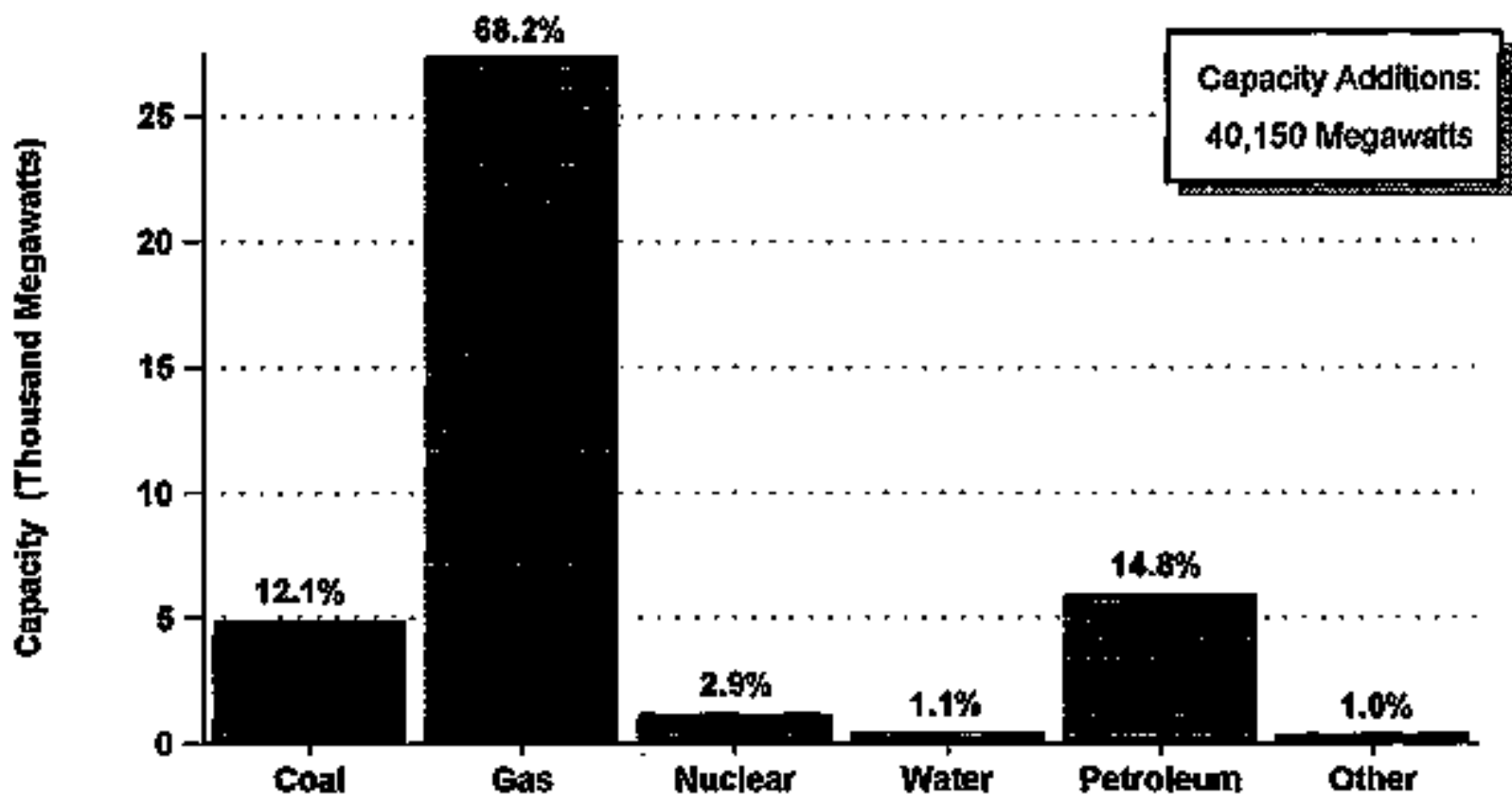

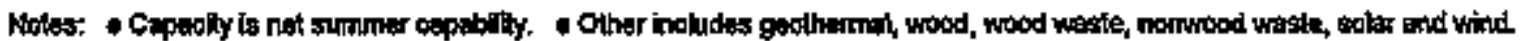

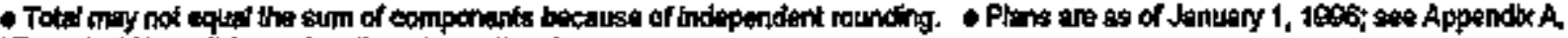
"Techntical Noles," for explanation of repoiting date.

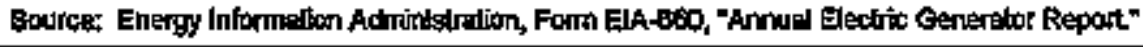


As of January 1, 1996, one nuclear unit (Tennessee Valley Authority's Watts Bar, Unit 1) was in testing phase. It began commercial operation in 1996.

During the next 10 years, electric utilities will continue to help fulfill their needs for new capacity by primarily adding new gas turbine and combined cycle units because these units haye lower installation costs and shorter lead-times for installation. At least 1.000 megawats of new gas turbite or combined cycle capacity are planned í each of 12 States--North Carolina, Ohio. Texas, New Jersey, Florida, Illinois, Missouri, Virginia, Marytand, Alabama, Kenturcky, and Georgita.

The most common type of repowering reported by electric utitities is reconfifuring an existing stealnelectric plant with a new combustion technology and adding a gas turbine. This type of repowering bas already beed completed by several electric utilities. Additional projects of this type are planned by other electric utilities over the next 10 years (Table 22). As of Janurary 1, I996, more than 4,000 megawatts of capacity have been reported for repowering or life extension during the next decade (Table 22).

Most clean coal projects plarned and undertaken by utilities were fiuidized bed combustion and integrated coal gasiffcation. These technologies improve powes plant effrciency, heip clean the air, and allow greater use of high-sulfur coal. Current 10-year plans inclade 340 megawatts of integrated coal gasification combined cycle capacity in Sierra Pacific Power Compagy's Pinon Pine, Unit 1 of Nevada and Tampa Electric Company's Polk, Untit 1 of Florida. Electric utilities have planned and are engaged in clean coal projects to comply with stricter environmental requirements proposed by the Clean Air Act Amendment. Other activjties planned and undertaken by electric utilities to provide an adequate and reliable supply of electricity include purchases from nonutility power producters and demand-side management programs designed to reduce overall demand and electricity use.

Several factors affect atility choices of tecbnology for new capacity, as well as their choices for future construction. Electric utilities select new generating units through a process knowa as capacity expansion planning. Typically, this process begins using sophisticated models to analyze potential options, based on tradeoffs among the technical characteristics of each option. As the capacity expansion planning process continues, these technical characteristics are evaluated in increasing detail. Among the characteristics considered are:

- Unit size capacity (megawalts) is the capability of the unit to generate power. Various measures are used. The nameplate capacity, for example, is determized by the manufacturer, and can be found attached to the unit.

- Capital cost is primarily, the initial construction cost of the generating unit. This represents most of the fixed-cost portion of the total utility investment.

- Operating and Maintenance (O\&M) costs are the annual costs associated with unit operation. These costs vary excording to the types of generating unit and fuel. The major component in the O\&M cost of a new coll-fired power plant, for example, is the cost of sorbent for flue gas desulfurization systems.

- Heat rate is the amount of fuel energy input required to generate one kilowatthour of electricity, expressed in Btu per kilowatthour. Units with heat rates are more efflctent. Efflciency becomes increasingly more inporant the greater the uncertainty in variable costs (for example, O\&M, fuel).

- Projected fuel prices are usualiy the largest component of the angoing annual costs of operating a generating unjt. Utilities use fuel price forecasts, developed either internally or by outside services, to analyze capacity addition decisions.

- Load-following capacity is the abjlity to change outpot as utility joad varies. This capability is important bocause utilities must often respond instantaneously to large variations in load.

- Reliabitity measures how available a generating unit is for operation in the likelihood of an outage, Utilities teed reliable equipment to ensuse that they can meet load as it occurs.

- Environmental performance is the relationship between generation and environmental residuals. This determines the ability and cost to meet environmenta' limitations. Environmental restrictions have grown considerably since the early 1970's.

Capacity-expansion planning models are needed to identify the least-cost additions needed to meet anticjpated load requirements, given various operational constraints. Modeling analyses are followed by detailed studies and compatisons of the specific options favored by the modeling, including assessments of site availability, permitting issues, and otber constraints. In recent years, the advent of integratedresource planging techntques has broadened the analytic framework to include demand-side management options as means for satisfying potential load growth.

Several new factors now also influence how utility capacity expansion techuology choices are made. These factors include the acid ratn provision (Titie IV) of the Clean Ajr Act Amendments of 1990, increasing use of prudency reviews by State utility regulatory commissions, mandatory incorporation of environmental externalities in utility capacity expanston plapning, in some States, increased availability of nonutsitity generation, and the diffictlty of siting and permitting new puclear plants. The exact nature of the impact of most of these factors has yet to be determised and is, in some cases, subject to considerable debate.

Even within this broader context, the basic factors considered by capacity expansion models continte to be major determinants of technology choice for new renerating units by electric utilities. Further, the technology choices of the independent power producter (IPP) segenent of the nonutility generation are often similar to the choices of atilitios. IPP choices are

14 Energy Information Administration/Inventory of Power Plants in the United States as of January 1, 1996 
driven by utility preferences, expressed through atility competitive bidding programs.

Electric ntilities require a mix of generatting units of different types to meet varying daily, weekly, and seasonal load requirements. Technologies with different cost and performance characteristics are of ten chosen to serve for different typos of duty:

- Baseload duty generaljing ugits are operated most of the time to meet loads that are always present. As a resuli, baseload units operate at constant output levels around the clock. The most impor* tant characteristics for a baseload generating unit are low operating costs and bigh avallability. Low-operating costs are a function of a high capacity factor and low-beat rates, low-fuel costs and low-OtM expenditures. Capital costs, which are spread over uany kilowatthours, are of somewhat lesser importance.

- Peakjng duty units are used only for very limited periods of time when the atitity's load is near its maximum. The most important characteristics of technologies used for peaking duty are a low capital cost since jt cannot be spread ovet many kilowatthours and the ability to provide variable capacity operation to meet changing loads. Because peaking units are used for a relatively small portion of the day, offtciency is less of a concern than is reliability.

- Intermediats duty units are operated less than baselcad units, but more than peaking units. Technologies are selected for intermediate duty that have a balapce of ralatively moderate capital and operating costs and have the ability to follow changing loads. Intermediate units generally have heat rates that fall between those of baseloat and peaking units.

Historically, different technologies typically have had characteristics that meet the requirements of each type of duty. Coal and nuclear units typically were selected for baseload duty because they had the lowest fuel and operating cost but could not change output easily to meat changing load. Their relatively high capital costs were not a barrier to baseload operation, because these units were operated most of the time. Natural gas and petrolenm-fired boilers have been used in intermediate load applications because they have cost characteristics between those of base and peaking duty units. Internal combustion engines and com* bustion turbines have very low capital costs, comparatjvely, and rapid start-up capabilities. The loads that hydroelectric units serve depend on water avajlabillty and cost of operation.

Since the early 1980 's, several factors bave changed the relative advantages of differeat types of generating units $\omega$ favor gas-fired conbustion turbines in botb simple and tombined cycle configurations.

- Lower gas costs. The price of natural gas to utilities bas declined sharply from the early 1980 's, spurred by structural changes in the gas industry. Lower gas prices have made gas economical to use in many intermediate and baseload applications where that fuel would not have been consid. ered before.

- Regulatory changes. The removal af most of the restrictions of the Power Plant and Industrial Fuel Use Act (PIFUA) in 1990 eliminated an important legal barrier to increased gas use by electric utilities and thus stimulated atility consideration of combustion turbines.

- Technological advances. Counbustion turbines are much more efficient and reliable than in the early 1980 's, and they are available in a wide range of capacities. Moreover, the devalopment of the phased-constraction concept has uade combustion turbines more attractive. At the same time, technologies for coal units (particularly new, morereliable and less-costly flue gas desulfurization systems and other clean coal technologies) have kept coal units competitive.

- Increased environmental regulations. Natural gas technologies cas generaily comply with most epvirontuental restrictions at a cost lower that other units. While the acid rain provisions of the Clean Air Act Amendments of 1990 apply primarily to existing coal-fired unjts, the New Sourcs Performance Standards (NSPS) still impose capital-intensive techpologicel control on now coal units. In addition, environmentally driven siting constraints also tend to favor small gasfired combustion turbines.

The factors that influence utility technology choices change over time and result in shifts in the mix of planned vtility (and nonutility) generating capacity. Moreover, the determinants of vtility decisjons for new capacity, notably fuel prices, are inherently uncertain. Utilities prefer a mix of onit types that enable them to diversify their technology and fuel choices and to meet different types of loads. Thus. although shifts occur, no one techoology or fuel completely dominates utility decisions for new capacity. 
Figure 13. Planned Capaclty Additions at U.S. Electric Utilities by Energy Source, 1996 Through 2000

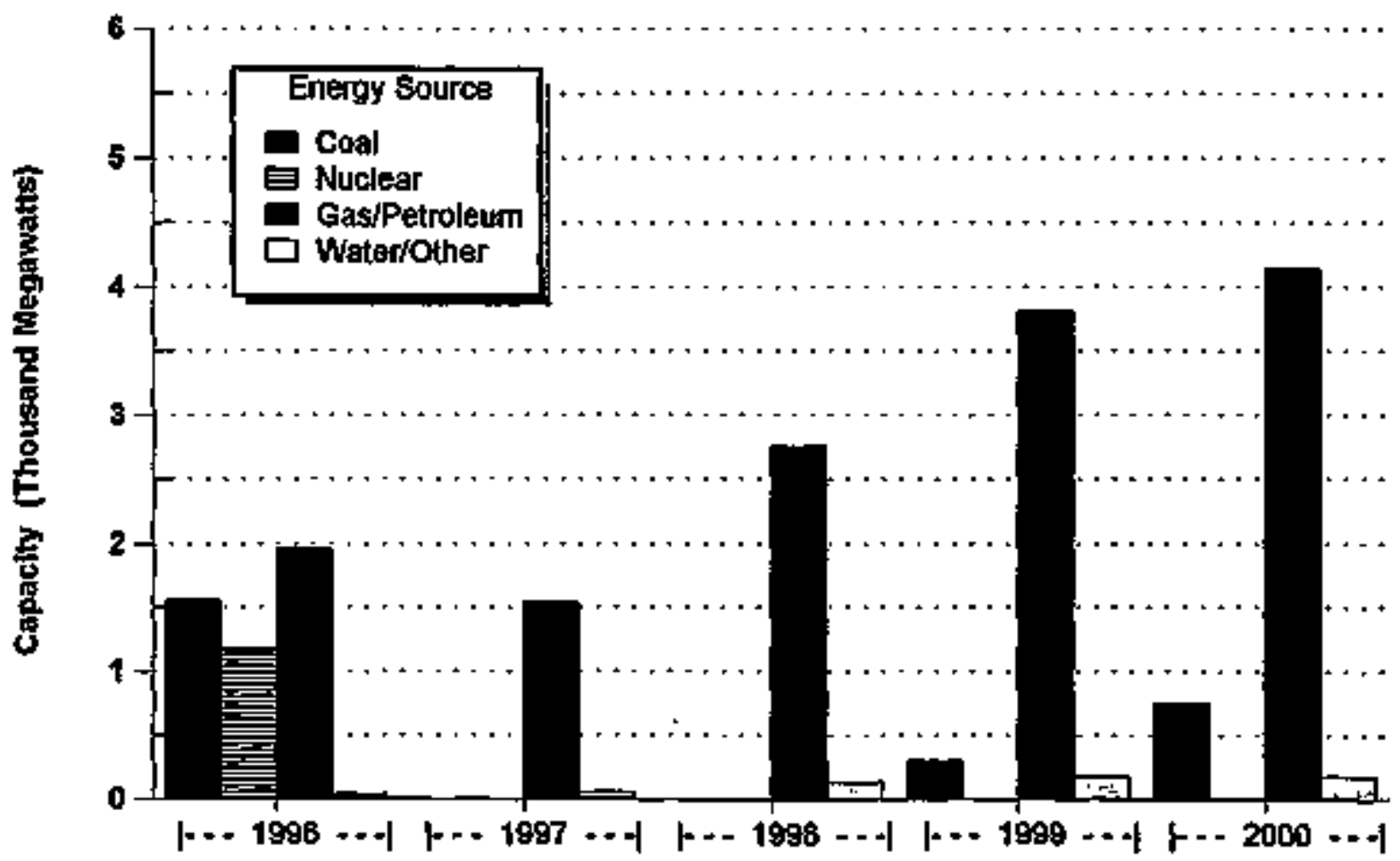

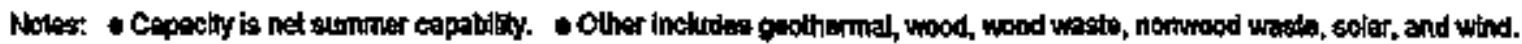

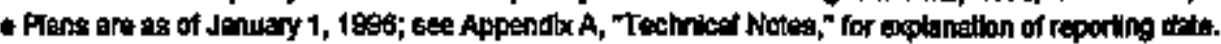

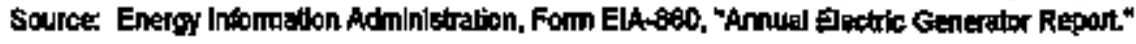

Figure 14. Planned Capacity Addlitions at U.S. Electric Utilltles by Energy Source, 2001 Through 2005

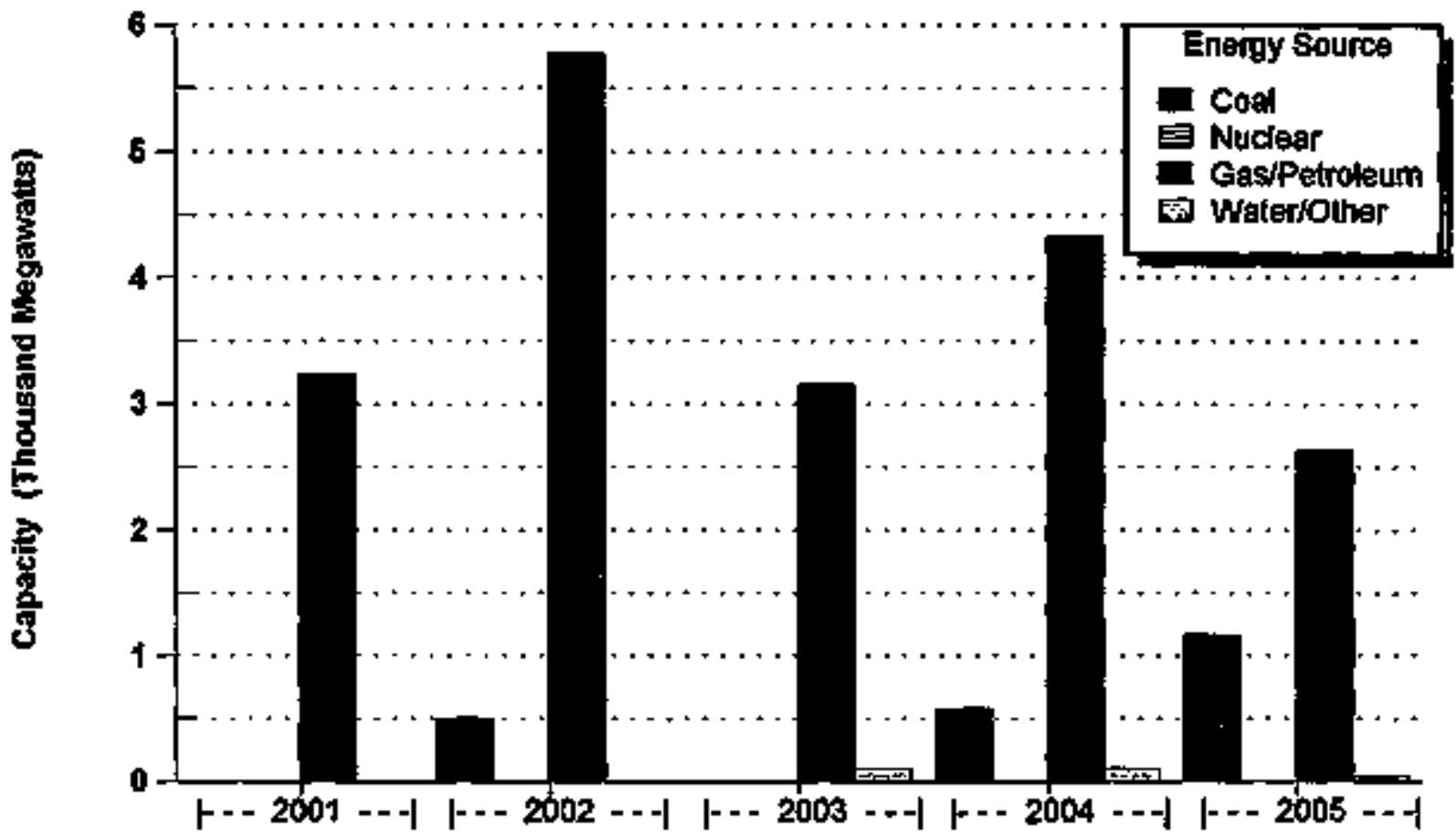

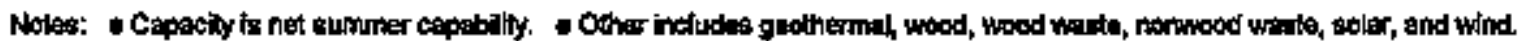

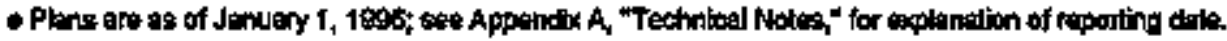

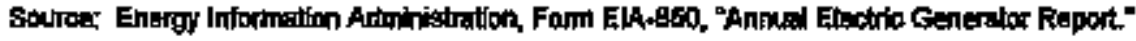

16 Energy Information Administration/Inventory of Power Plants in the United States as of January 1, 1996 
Figure 15. Planned Capacity Additions of More Than 1,000 Megawatts at U.S. Electrio Uttlities by Utilly, 1996 Through 2005

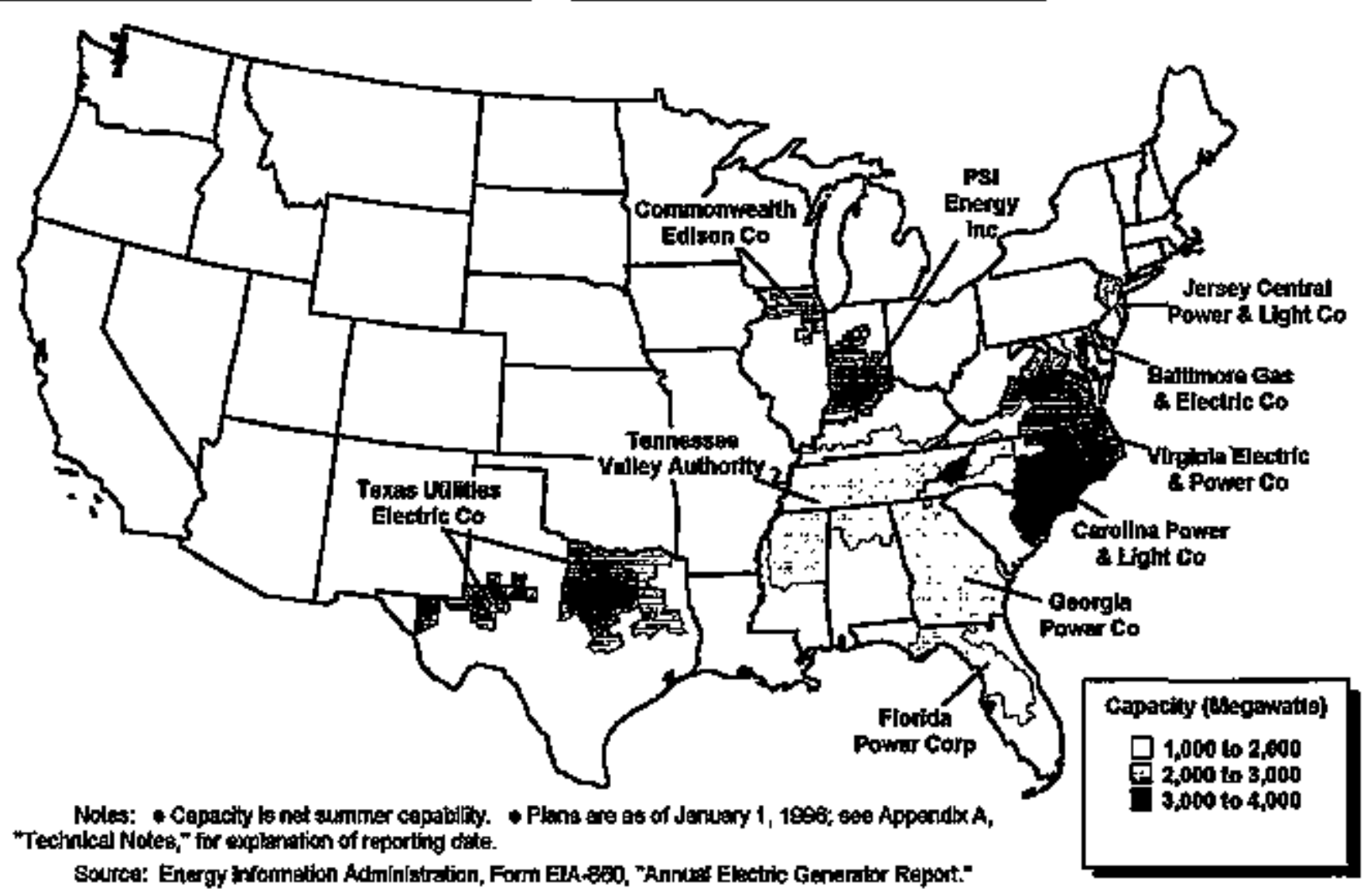




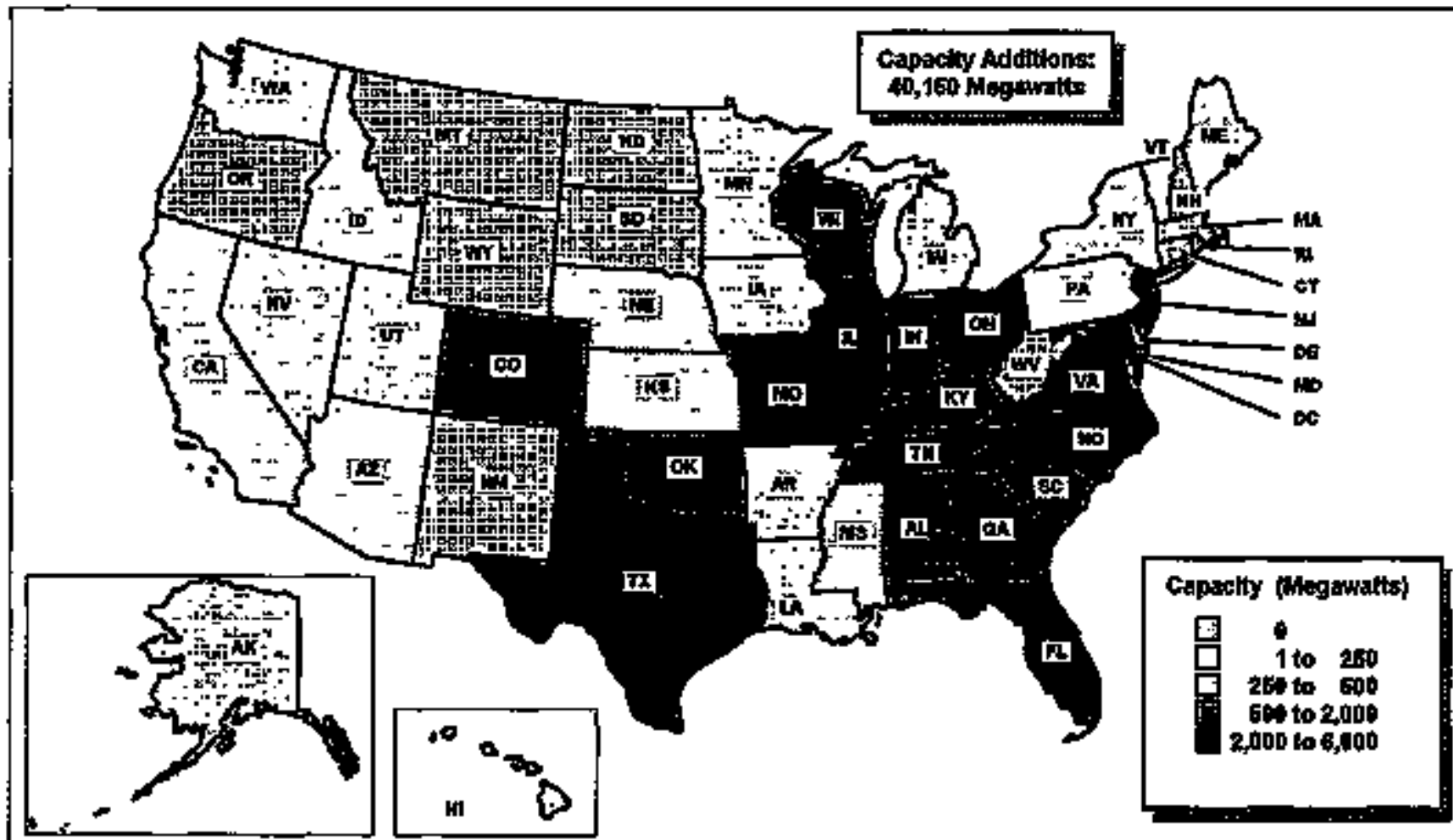

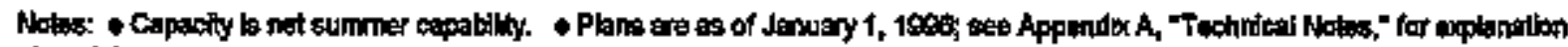
of reperting detp.

Source: Energy Information Admintstrution, Fomm ElA-660, "Annual Electro Generator Report" 
Table 1. Existing Capacity and Planned Capacity Additions at U.S. Electric Utilities by Fnergy Source, as of January 1, 1996

\begin{tabular}{|c|c|c|c|c|c|c|c|c|}
\hline \multirow[b]{2}{*}{ 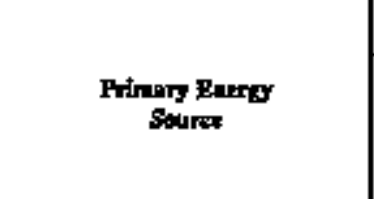 } & \multicolumn{4}{|c|}{ Boratipel } & \multicolumn{4}{|c|}{ 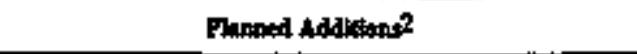 } \\
\hline & $\begin{array}{l}\text { Stumbtr } \\
\text { of Undt }\end{array}$ & 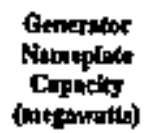 & 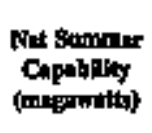 & 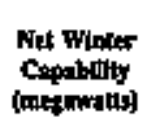 & 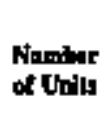 & 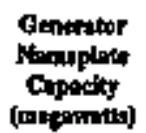 & 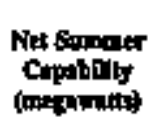 & 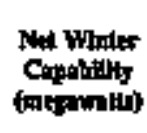 \\
\hline 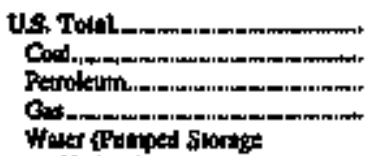 & $\begin{array}{r}10,346 \\
1,212 \\
3,277 \\
2,236\end{array}$ & $\begin{array}{l}75,40 t \\
324,400 \\
71,968 \\
153,668\end{array}$ & $\begin{array}{r}706,111 \\
300,610 \\
64,464 \\
142,536\end{array}$ & $\begin{array}{r}76,29 t \\
303,121 \\
70,195 \\
14,736\end{array}$ & $\begin{array}{r}13 \\
206\end{array}$ & $\begin{array}{r}44,003 \\
5,165 \\
6,984 \\
33,3,28\end{array}$ & $\begin{array}{r}4,150 \\
4,845 \\
5,451 \\
2,37\end{array}$ & $\begin{array}{r}44,515 \\
4,000 \\
6,802 \\
30,522\end{array}$ \\
\hline 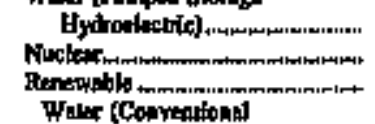 & $\begin{array}{r}140 \\
109 \\
3,420\end{array}$ & 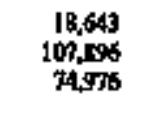 & $\begin{array}{l}21,357 \\
95,515 \\
T 7,500\end{array}$ & $\begin{array}{r}21,32 t \\
100,696 \\
76,828\end{array}$ & $\frac{-}{76}$ & $\begin{array}{r}1,276 \\
561\end{array}$ & 1,170 & 1,170 \\
\hline 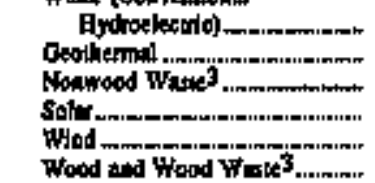 & $\begin{array}{r}3,37 \\
29 \\
14 \\
9 \\
18 \\
13\end{array}$ & $\begin{array}{r}72,41 \\
1,716 \\
316 \\
4 \\
3 \\
301\end{array}$ & $\begin{array}{r}75,274 \\
1.747 \\
260 \\
4 \\
8 \\
307\end{array}$ & $\begin{array}{r}74,513 \\
1,797 \\
267 \\
4 \\
8 \\
307\end{array}$ & $\begin{array}{l}6 \\
\frac{5}{2} \\
1 \\
-\end{array}$ & $\frac{474}{60}$ & $\begin{array}{r}431 \\
56 \\
126\end{array}$ & $\begin{array}{r}418 \\
\frac{16}{10} \\
-\end{array}$ \\
\hline
\end{tabular}

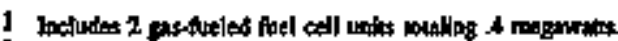

2 Planed addian ane for 1950 through 2005.

3 Blomas.

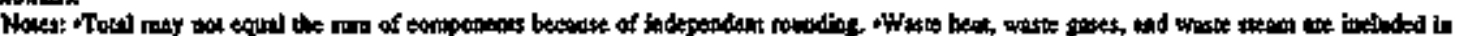

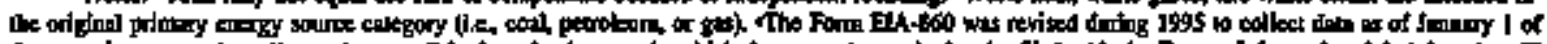

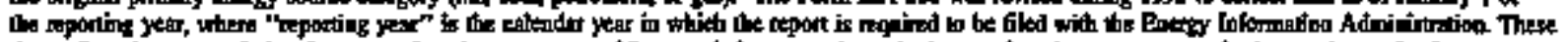

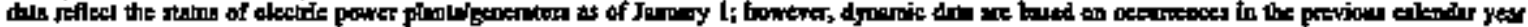

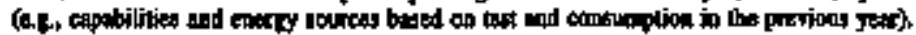

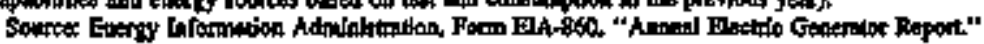

Table 2. Capacity Additions and Retirements at US. Electric Utilities by Energy Source, 1995

\begin{tabular}{|c|c|c|c|c|c|c|c|c|}
\hline \multirow[b]{2}{*}{$\begin{array}{c}\text { Protory thergy } \\
\text { Sauret }\end{array}$} & \multicolumn{4}{|c|}{ I } & \multicolumn{4}{|c|}{ Redirebutaitit } \\
\hline & $\begin{array}{l}\text { Noniber } \\
\text { of Dnils }\end{array}$ & 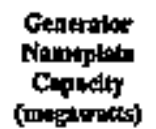 & Med Simmer & 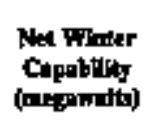 & 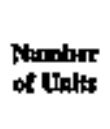 & 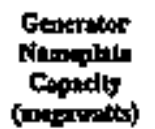 & Net sinmer & $\begin{array}{l}\text { Nat Whites } \\
\text { Capablly } \\
\text { (me-guralts) }\end{array}$ \\
\hline 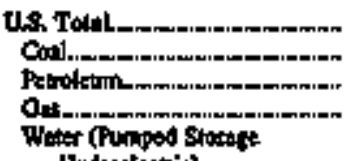 & $\begin{array}{r}4 \\
3 \\
34 \\
38\end{array}$ & $\begin{array}{l}6,603 \\
1,095 \\
1,191 \\
3,291\end{array}$ & $\begin{array}{r}5,752 \\
1,036 \\
\$, 929 \\
2,764\end{array}$ & $\begin{array}{l}6,479 \\
1,036 \\
1,217 \\
3,201\end{array}$ & $\begin{array}{r}1 \\
34 \\
17\end{array}$ & $\begin{array}{r}1,004 \\
2 \\
424\end{array}$ & $\begin{array}{r}94 \\
453 \\
495\end{array}$ & $\begin{array}{r}969 \\
2 \\
465 \\
509\end{array}$ \\
\hline 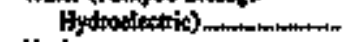 & 3 & 845 & 848 & 848 & - & - & 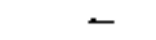 & - \\
\hline 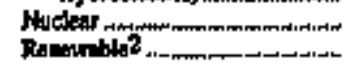 & $\overline{10}$ & $\overrightarrow{197}$ & $\overline{136}$ & $\overline{176}$ & $\overrightarrow{3}$ & $\overline{-}$ & - & 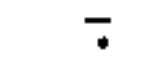 \\
\hline
\end{tabular}

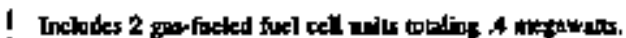

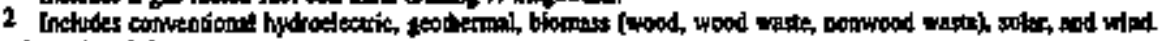

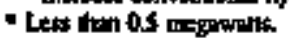

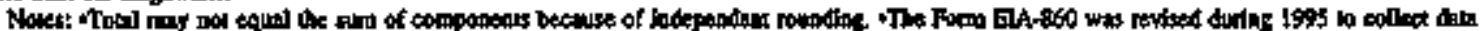

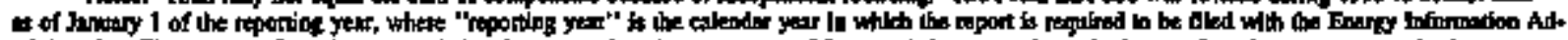

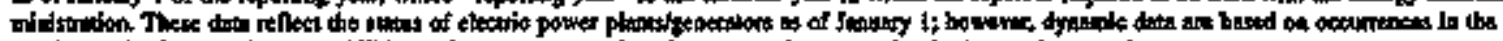

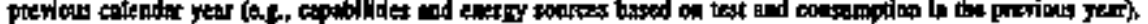

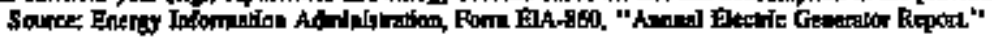


Table 3. Combmed Cycle Existing Capacity and Plamed Capacity Additions at U.S. Elecerric Utilities by Prime Mover and Primary Energy Source, as of January 1, 1996

\begin{tabular}{|c|c|c|c|c|c|c|c|c|}
\hline \multirow[b]{2}{*}{$\begin{array}{l}\text { Pring Moner } \\
\text { Pristar Souret }\end{array}$} & \multicolumn{4}{|c|}{ Existbig } & \multicolumn{4}{|c|}{ Finned Adidangl } \\
\hline & Nombar & 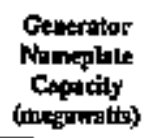 & 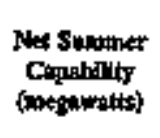 & 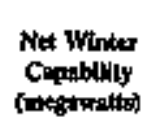 & $\begin{array}{l}\text { Nonbert } \\
\text { of Unites }\end{array}$ & $\begin{array}{l}\text { Conprolor } \\
\text { Napeptate } \\
\text { Copactily } \\
\text { (megroratts) }\end{array}$ & 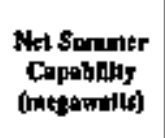 & 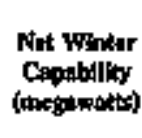 \\
\hline 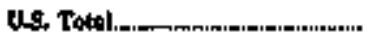 & 189 & 14,5n: & 12,189 & 13,169 & 64 & $9 ; 73$ & 7,45 & \$,Est \\
\hline 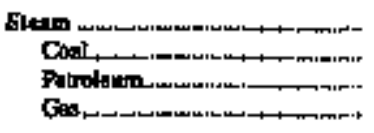 & $\frac{61}{7}$ & $\begin{array}{r}4,773 \\
286 \\
4,457\end{array}$ & $\underset{2,5,5}{5,523}$ & $\frac{5,758}{218}$ & $\begin{array}{r}27 \\
2 \\
3 \\
17\end{array}$ & $\begin{array}{r}3,620 \\
418 \\
280 \\
2,930\end{array}$ & $\begin{array}{r}2,773 \\
340 \\
2,98 \\
2,195\end{array}$ & $\begin{array}{r}2,995 \\
345 \\
264 \\
2,386\end{array}$ \\
\hline 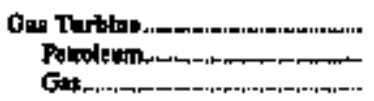 & $\begin{array}{r}124 \\
\text { 치 }\end{array}$ & $\begin{array}{r}9,804 \\
803 \\
8,942\end{array}$ & $\begin{array}{r}6,600 \\
675 \\
5,978\end{array}$ & $\begin{array}{r}3,417 \\
829 \\
6,583\end{array}$ & $\begin{array}{r}48 \\
4 \\
35\end{array}$ & $\begin{array}{r}6,744 \\
66 \\
6,358\end{array}$ & $\begin{array}{r}5,101 \\
74 \\
5,027\end{array}$ & $\begin{array}{r}5,586 \\
81 \\
5,506\end{array}$ \\
\hline
\end{tabular}

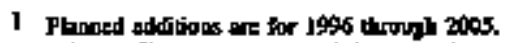

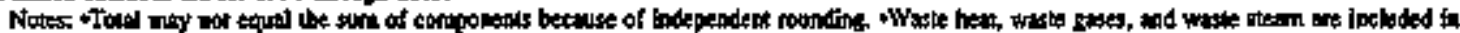

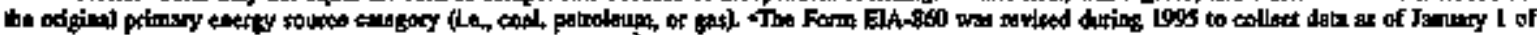

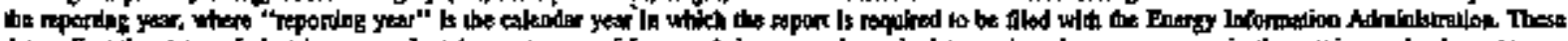

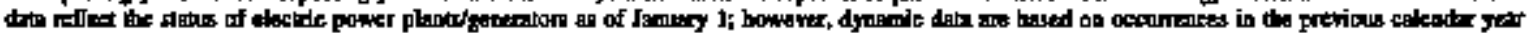

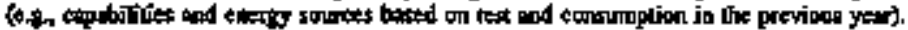

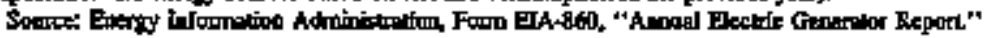

Table 4. Fossil-Faeled Existing Capacity and Plamed Capacity Additions at U.S. Flectric Ut:lities by Prime Mover and Primary Energy Source, as of January 1, 1996

\begin{tabular}{|c|c|c|c|c|c|c|c|c|}
\hline \multirow[b]{2}{*}{$\begin{array}{l}\text { Prime Mover } \\
\text { Enmisg Sotret }\end{array}$} & \multicolumn{4}{|c|}{ Exitetiogof } & \multicolumn{4}{|c|}{ Plenned didiliont } \\
\hline & $\begin{array}{l}\text { Nopmber } \\
\text { of Unt's }\end{array}$ & 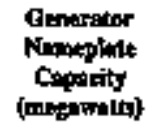 & $\begin{array}{l}\text { Net Summar } \\
\text { Capablity } \\
\text { (megtomatss) }\end{array}$ & 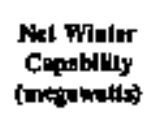 & $\begin{array}{l}\text { Nomber } \\
\text { of Untils }\end{array}$ & 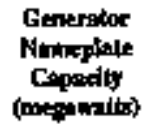 & 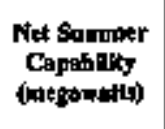 & $\begin{array}{l}\text { Nel Winter } \\
\text { Capabllity } \\
\text { (ntestumatis) }\end{array}$ \\
\hline 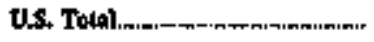 & 6,727 & 549,166 & sot,609 & set,ost & 363 & 45,471 & 38,166 & 4,613 \\
\hline 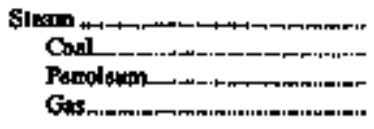 & $\begin{array}{r}2.191 \\
1.212 \\
229 \\
750\end{array}$ & $\begin{array}{r}415,860 \\
371,430 \\
99,526 \\
111,904\end{array}$ & $\begin{array}{r}416,976 \\
30,0,610 \\
36,669 \\
108,798\end{array}$ & $\begin{array}{r}40,444 \\
3013,121 \\
36,982 \\
109,342\end{array}$ & $\begin{array}{r}39 \\
13 \\
3 \\
23\end{array}$ & $\begin{array}{r}9,006 \\
5,165 \\
280 \\
1,360\end{array}$ & $\begin{array}{r}8,607 \\
4,845 \\
296 \\
3,525\end{array}$ & $\begin{array}{r}8,854 \\
4860 \\
264 \\
3,731\end{array}$ \\
\hline 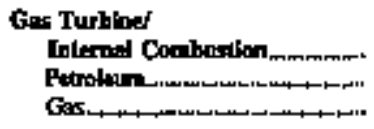 & $\begin{array}{l}4,566 \\
3,048 \\
1,488\end{array}$ & $\begin{array}{l}75,166 \\
32,3720 \\
40,755\end{array}$ & $\begin{array}{l}61,563 \\
27,795 \\
39,738\end{array}$ & $\begin{array}{l}71,608 \\
33,213 \\
38,395\end{array}$ & $\begin{array}{r}324 \\
84 \\
240\end{array}$ & $\begin{array}{r}35,665 \\
6,704 \\
29,051\end{array}$ & $\begin{array}{r}29,569 \\
5,712 \\
2,845\end{array}$ & $\begin{array}{r}33,759 \\
6,568 \\
27,191\end{array}$ \\
\hline 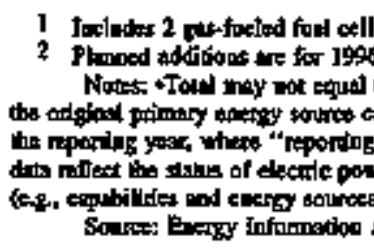 & unisterilir & consmptio & 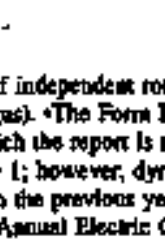 & $\begin{array}{l}\text { tic dath ate b } \\
\text { aerion Kepot }\end{array}$ & 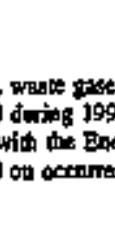 & Aformidon & 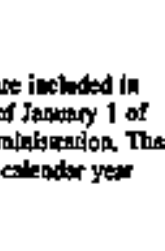 & \\
\hline
\end{tabular}


Table 5. Fossil-Fueled and Nuclear Steam-Electric Existing Capacity and Planned Capacity Additions at U.S. Electric Utilities, as of January 1,1996

\begin{tabular}{|c|c|c|c|c|c|c|c|c|}
\hline \multirow[b]{2}{*}{$\begin{array}{c}\text { Ptimary Batro } \\
\text { Sourch }\end{array}$} & \multicolumn{4}{|c|}{ Eutiting } & \multicolumn{4}{|c|}{ Planned Addifionsl } \\
\hline & $\begin{array}{l}\text { Number } \\
\text { of Inites }\end{array}$ & 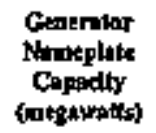 & 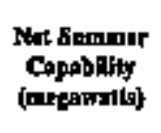 & 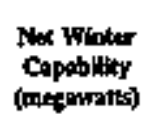 & $\begin{array}{l}\text { Nhiner } \\
\text { of linity }\end{array}$ & 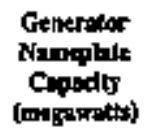 & 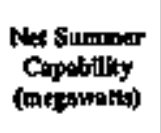 & 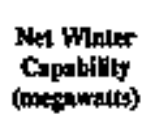 \\
\hline 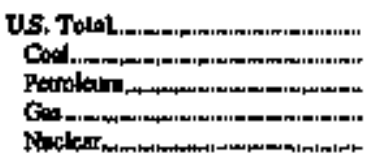 & $\begin{array}{r}2,310 \\
1,212 \\
2,9 \\
750 \\
109\end{array}$ & $\begin{array}{r}51.3,756 \\
324,430 \\
39,526 \\
111,904 \\
107,806\end{array}$ & $\begin{array}{r}545,592 \\
300,610 \\
36,669 \\
10,798 \\
99,515\end{array}$ & $\begin{array}{r}551,141 \\
300,121 \\
36,982 \\
109,342 \\
100,696\end{array}$ & $\begin{array}{r}4 \\
13 \\
3 \\
23 \\
1\end{array}$ & $\begin{array}{r}11,076 \\
5,165 \\
200 \\
4,360 \\
1,270\end{array}$ & $\begin{array}{r}9,577 \\
4,845 \\
238 \\
3,525 \\
1,170\end{array}$ & $\begin{array}{r}10,024 \\
4,860 \\
264 \\
3,791 \\
1,170\end{array}$ \\
\hline
\end{tabular}

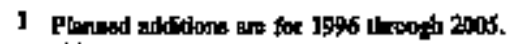

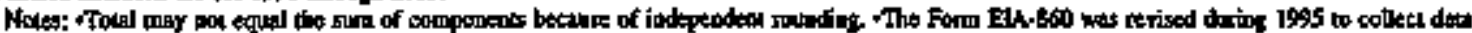

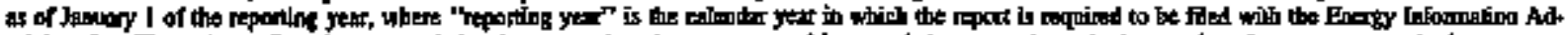

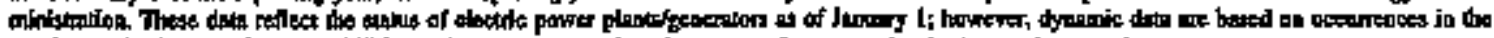

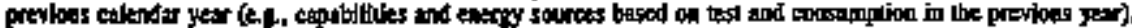

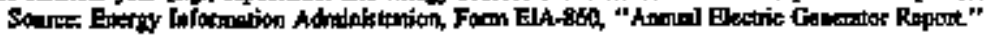


Table 6. Existing Capacity at U.S. Flectric Utilities by Prime Mover and Energy Source, as of January 1, 1996

\begin{tabular}{|c|c|c|c|c|}
\hline $\begin{array}{l}\text { Pribe Morat } \\
\text { Dento Sanrat }\end{array}$ & $\begin{array}{l}\text { Nonbar } \\
\text { of Doils }\end{array}$ & 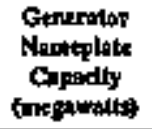 & $\begin{array}{l}\text { Net Summer } \\
\text { Coppollty } \\
\text { (megamots) }\end{array}$ & 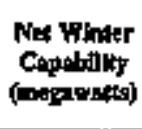 \\
\hline 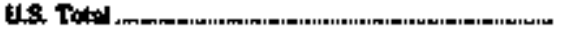 & 10,396 & 750,542 & 76, il1 & 79,59 \\
\hline 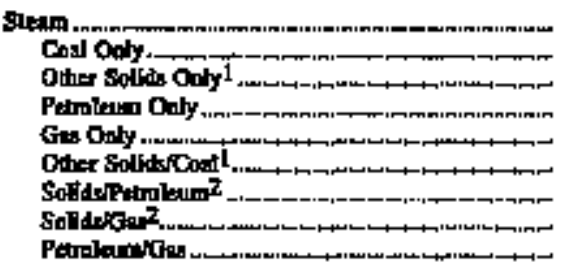 & $\begin{array}{r}2,157 \\
890 \\
8 \\
151 \\
96 \\
6 \\
83 \\
258 \\
645\end{array}$ & $\begin{array}{r}471,751 \\
274,459 \\
142 \\
25,435 \\
10,331 \\
182 \\
13,385 \\
37,434 \\
110,363\end{array}$ & $\begin{array}{r}441,15 \% \\
254,209 \\
148 \\
23,592 \\
9,759 \\
136 \\
12,624 \\
34,597 \\
106,303\end{array}$ & $\begin{array}{r}44,04 \\
256,659 \\
1 t 8 \\
23,641 \\
9,764 \\
155 \\
12,657 \\
34,621 \\
306,712\end{array}$ \\
\hline 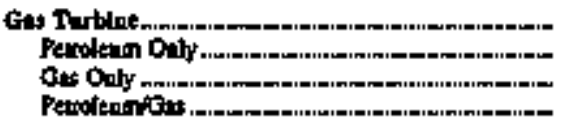 & $\begin{array}{r}1486 \\
620 \\
153 \\
713\end{array}$ & $\begin{array}{r}58,320 \\
2,001 \\
5,222 \\
30,107\end{array}$ & $\begin{array}{r}50,191 \\
19,662 \\
4,424 \\
26,105\end{array}$ & $\begin{array}{r}59,45 \\
24,005 \\
5,051 \\
30,399\end{array}$ \\
\hline 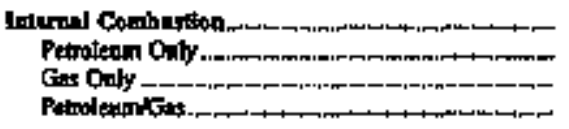 & $\begin{array}{r}2,956 \\
1,799 \\
35 \\
1,096\end{array}$ & $\begin{array}{r}4,455 \\
2,534 \\
35 \\
2,416\end{array}$ & $\begin{array}{r}1,640 \\
2,384 \\
32 \\
2,224\end{array}$ & $\begin{array}{r}4,701 \\
2,412 \\
32 \\
2,256\end{array}$ \\
\hline 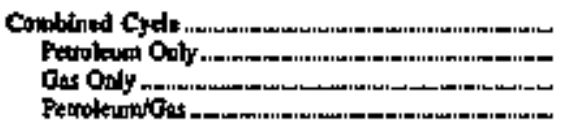 & $\begin{array}{r}189 \\
20 \\
121 \\
121\end{array}$ & $\begin{array}{r}14,5 \% \\
851 \\
3,375 \\
10,389\end{array}$ & $\begin{array}{r}12,188 \\
608 \\
2,486 \\
9,595\end{array}$ & $\begin{array}{r}13,169 \\
691 \\
3,123 \\
6,356\end{array}$ \\
\hline 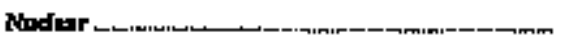 & 109 & $107,80 \times$ & 9,515 & 104,996 \\
\hline 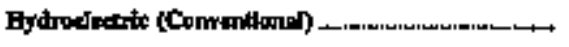 & 3,337 & $\pi, 471$ & 75,274 & 74,501 \\
\hline 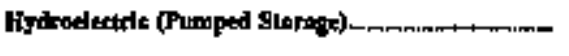 & 140 & $J: 640$ & 21,807 & $21,3: \mathbf{I}$ \\
\hline 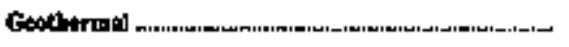 & 29 & 1,776 & 1,74 & $1, \pi \%$ \\
\hline Solpr & 9 & 4 & 4 & 4 \\
\hline Hitod.rminn & 18 & $\boldsymbol{t}$ & 8 & 8 \\
\hline
\end{tabular}

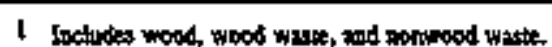

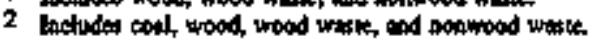

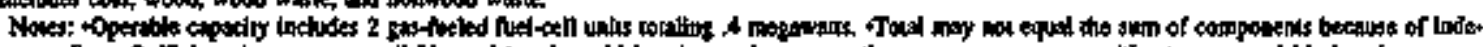

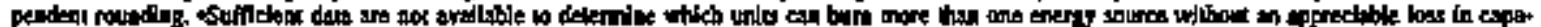

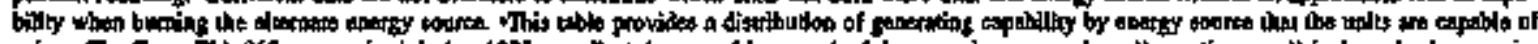

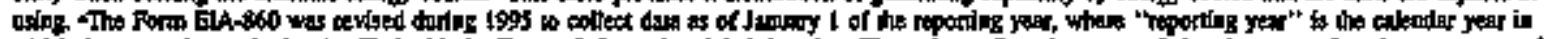

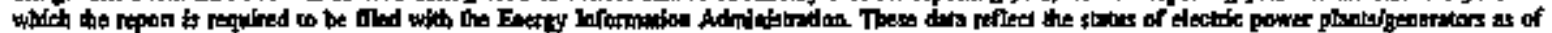

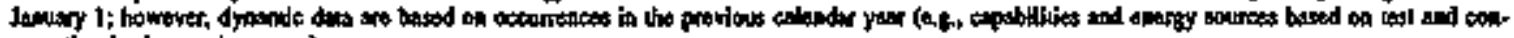
stription in ins perious year).

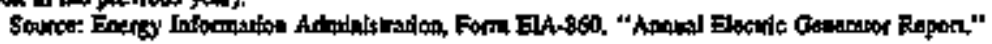


Table 7. Planned Capacity Additions at U.S. Electric Utilities, 1996 Through 2005, as of January 1, 1996

\begin{tabular}{|c|c|c|c|c|}
\hline Ytar & $\begin{array}{l}\text { Nomibet } \\
\text { of Utith }\end{array}$ & 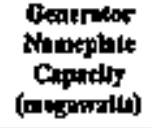 & 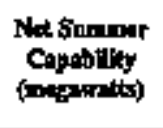 & 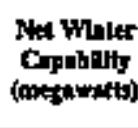 \\
\hline US, TolDl & 414 & 47600 & 10,150 & 44,585 \\
\hline 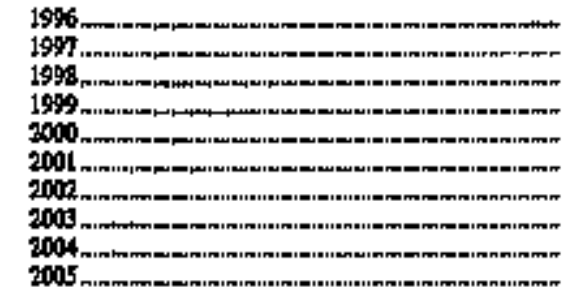 & $\begin{array}{l}78 \\
44 \\
48 \\
48 \\
29 \\
48 \\
30 \\
39 \\
24\end{array}$ & $\begin{array}{l}5,335 \\
1,917 \\
3,6017 \\
4,895 \\
5,876 \\
4,164 \\
7,424 \\
3,994 \\
5,994 \\
4,457\end{array}$ & $\begin{array}{l}4,726 \\
1,600 \\
2,886 \\
4,287 \\
5,068 \\
3,337 \\
6,274 \\
3,248 \\
5,006 \\
3,624\end{array}$ & $\begin{array}{l}5,021 \\
1,003 \\
3,268 \\
4,921 \\
5,561 \\
3,667 \\
7,062 \\
3,717 \\
5,599 \\
4,103\end{array}$ \\
\hline
\end{tabular}

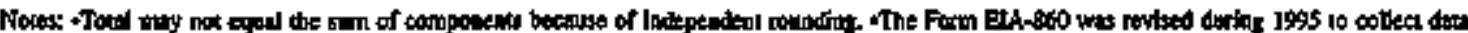

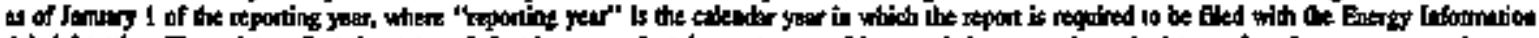

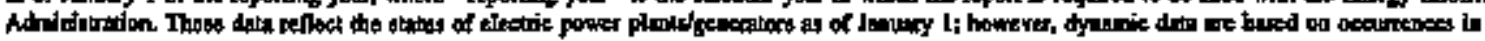

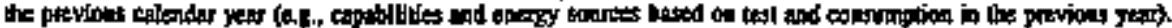

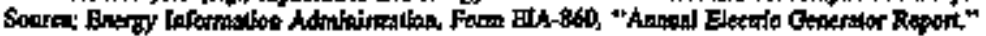

Tabłe 8. Plamed Coal- and Petroleum-Fired Capacity Additions at U.S. Electric Utilities, 1996 Through 2005, as of January 1, 1996

\begin{tabular}{|c|c|c|c|c|c|c|c|c|}
\hline \multirow[b]{2}{*}{ Yarr } & \multicolumn{4}{|c|}{ Cont } & \multicolumn{4}{|c|}{ Petrotinn } \\
\hline & $\begin{array}{l}\text { Nomber } \\
\text { of Utults }\end{array}$ & 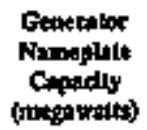 & 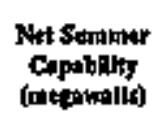 & 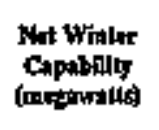 & $\begin{array}{l}\text { Number } \\
\text { of Utalts }\end{array}$ & 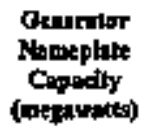 & 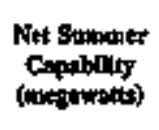 & 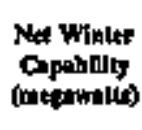 \\
\hline US. Todו & 13 & 5,166 & 4,1845 & 4060 & 87 & 6,$0 ; 4$ & sost & $6+3$ \\
\hline 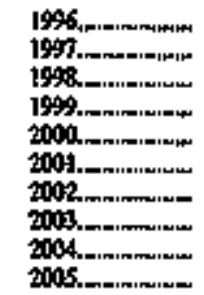 & $\begin{array}{l}\frac{6}{2} \\
\frac{1}{1} \\
\frac{1}{2} \\
2\end{array}$ & $\begin{array}{r}\frac{1,731}{7} \\
\frac{700}{800} \\
\frac{546}{580} \\
1,208\end{array}$ & $\begin{array}{r}1,558 \\
\overline{=} \\
300 \\
750 \\
500 \\
575 \\
1,162\end{array}$ & $\begin{array}{l}1,573 \\
\frac{-}{300} \\
750 \\
\frac{500}{5} \\
1,162\end{array}$ & $\begin{array}{l}34 \\
19 \\
1 \\
7 \\
6 \\
5 \\
6 \\
6 \\
2 \\
4\end{array}$ & $\begin{array}{r}618 \\
228 \\
3 \\
1,469 \\
845 \\
77 \\
1,152 \\
1,101 \\
315 \\
469\end{array}$ & $\begin{array}{r}531 \\
195 \\
3 \\
1,250 \\
722 \\
661 \\
979 \\
936 \\
271 \\
399\end{array}$ & $\begin{array}{r}605 \\
221 \\
3 \\
1,439 \\
831 \\
762 \\
1,125 \\
1,079 \\
313 \\
451\end{array}$ \\
\hline
\end{tabular}

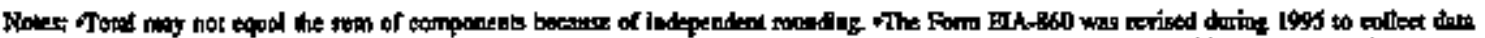

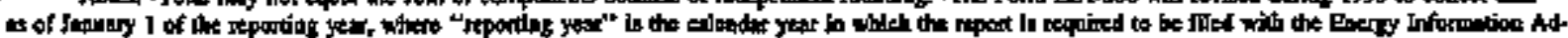

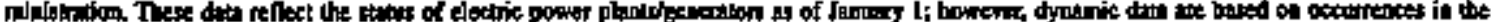

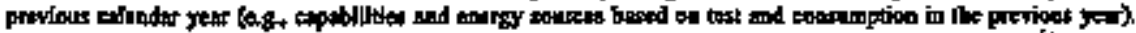

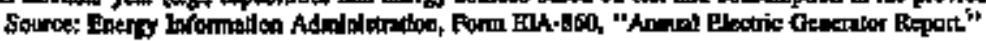


Table 9. Planned Gas-Fired and Hydroelectric Capacity Additlons at U.S. Electric Utilities, 1996 Throngh 2005, as of Jannary 1, 1996

\begin{tabular}{|c|c|c|c|c|c|c|c|c|}
\hline \multirow[b]{2}{*}{ Tar } & \multicolumn{4}{|c|}{ Ge: } & \multicolumn{4}{|c|}{ Bydroelectry' } \\
\hline & $\begin{array}{l}\text { Namber } \\
\text { of Units }\end{array}$ & 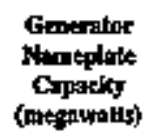 & $\begin{array}{l}\text { Net Summer } \\
\text { Capablit'y } \\
\text { (dhethwatts) }\end{array}$ & 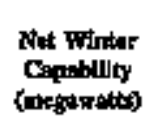 & $\begin{array}{l}\text { Mitaber } \\
\text { of Dalle }\end{array}$ & 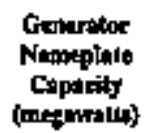 & 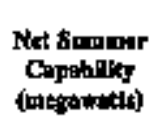 & 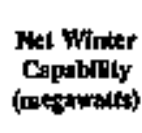 \\
\hline DS Totr.............. & 263 & 33,322 & $27,3 \pi 1$ & 30,922 & 65 & 474 & 431 & Alts \\
\hline 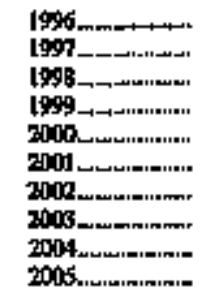 & $\begin{array}{l}30 \\
16 \\
30 \\
24 \\
28 \\
24 \\
39 \\
25 \\
34 \\
15\end{array}$ & $\begin{array}{l}1,681 \\
1,608 \\
3,461 \\
2,949 \\
4,099 \\
3,387 \\
5,724 \\
3,739 \\
4,995 \\
2,744\end{array}$ & $\begin{array}{l}1,4,34 \\
1,343 \\
2,748 \\
2,558 \\
3,418 \\
2,576 \\
4,792 \\
1,212 \\
4,060 \\
2,200\end{array}$ & $\begin{array}{l}1,640 \\
1,576 \\
3,134 \\
2,914 \\
3,806 \\
2,005 \\
3,435 \\
2,538 \\
4,571 \\
2,456\end{array}$ & $\begin{array}{r}9 \\
8 \\
13 \\
9 \\
24 \\
-2 \\
- \\
-\end{array}$ & $\begin{array}{r}29 \\
B I \\
147 \\
76 \\
143 \\
-3 \\
- \\
-\end{array}$ & $\begin{array}{r}28 \\
58 \\
135 \\
72 \\
136 \\
-2 \\
- \\
-\end{array}$ & $\begin{array}{r}27 \\
57 \\
137 \\
73 \\
131 \\
\frac{2}{-} \\
-\end{array}$ \\
\hline
\end{tabular}

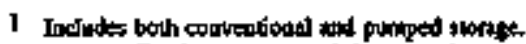

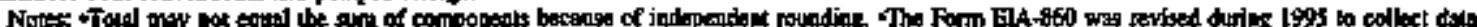

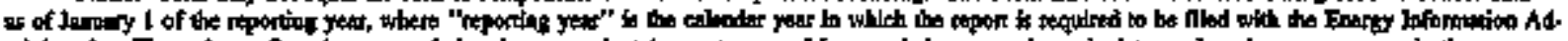

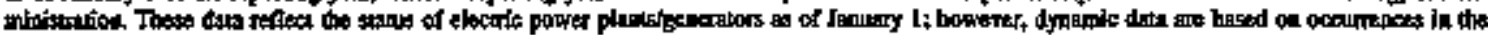

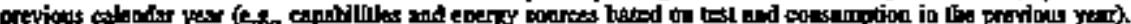

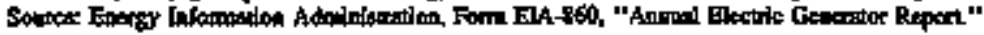

Table 10. Planned Nuclear and Other Capacity Additions at U.S. Electric Utiltites, 1996 Through 2005, as of January 1, 1996

\begin{tabular}{|c|c|c|c|c|c|c|c|c|}
\hline \multirow[b]{2}{*}{ Pear } & \multicolumn{4}{|c|}{ Nodear } & \multicolumn{4}{|c|}{ Other1 } \\
\hline & $\begin{array}{l}\text { Nonber } \\
\text { of Doises }\end{array}$ & 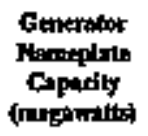 & 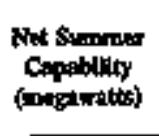 & $\begin{array}{l}\text { Nit Winter } \\
\text { Copability } \\
\text { (megroratts) }\end{array}$ & $\begin{array}{l}\text { Non ber } \\
\text { of Whits }\end{array}$ & 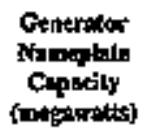 & $\begin{array}{l}\text { Ne! Snomor } \\
\text { Copobulity } \\
\text { (nrgawrits) }\end{array}$ & 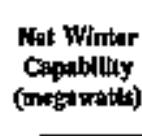 \\
\hline Dيs Told & t & 1,274 & $1,17 \%$ & 1,170 & t1 & $\mathbf{3}$ & 393 & 38 \\
\hline t996............ & $\mathbf{I}$ & 1,270 & 1,170 & 1.170 & 1 & 6 & 6 & 6 \\
\hline t & - & - & - & - & ] & - & $*$ & * \\
\hline מ & - & - & - & - & - & - & - & - \\
\hline [999.......... & - & - & - & - & $\mathbf{1}$ & too & 100 & 100 \\
\hline 2000 & - & - & - & - & 3 & 45 & 43 & $\mathbf{4 B}$ \\
\hline $2010 \mathrm{~L}$.............mmm & - & - & - & - & - & - & - & - \\
\hline 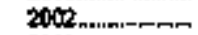 & - & - & - & - & - & - & - & - \\
\hline 2003 . & - & - & - & - & 1 & 100 & 100 & 100 \\
\hline 2004 m & - & - & $m$ & - & 1 & 100 & 100 & 100 \\
\hline 2005 & $\longrightarrow$ & - & $m$ & - & $\mathbf{j}$ & 36 & 34 & 34 \\
\hline
\end{tabular}

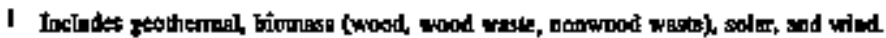

- Lase than ós meparith.

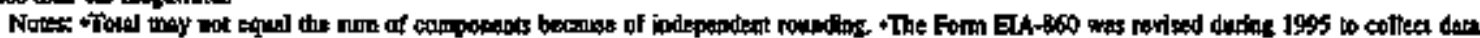

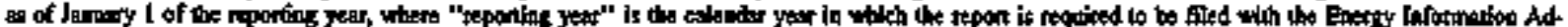

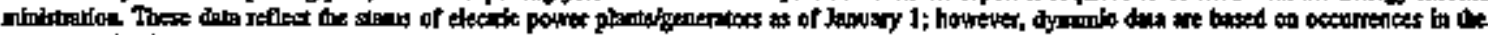

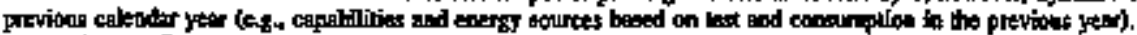

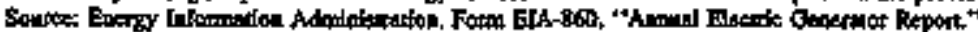


Table 11. Planned Capacity Retirements at U.S. Electric Utílities, 1996 Through 2005, as of January 1, 1996.

\begin{tabular}{|c|c|c|c|c|}
\hline Year & $\begin{array}{l}\text { Nurnber } \\
\text { of Units }\end{array}$ & 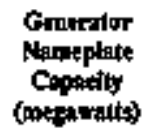 & 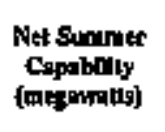 & $\begin{array}{l}\text { Net Finter } \\
\text { Capabrity } \\
\text { (mejontis) }\end{array}$ \\
\hline 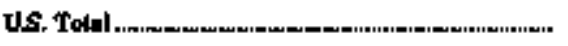 & 180 & 4,95 & 4,506 & 4,113 \\
\hline 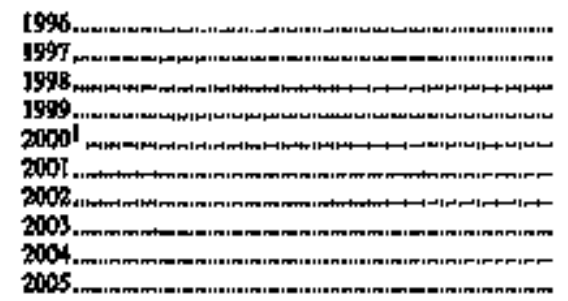 & $\begin{array}{r}6 \\
27 \\
11 \\
14 \\
9 \\
14 \\
44 \\
12 \\
23 \\
19\end{array}$ & $\begin{array}{r}132 \\
251 \\
470 \\
220 \\
236 \\
1,009 \\
147 \\
1,296 \\
851\end{array}$ & $\begin{array}{r}132 \\
240 \\
43 \\
428 \\
202 \\
195 \\
854 \\
1,248 \\
1,21 \\
803\end{array}$ & $\begin{array}{r}135 \\
241 \\
51 \\
204 \\
213 \\
213 \\
900 \\
1,27 \\
848\end{array}$ \\
\hline
\end{tabular}

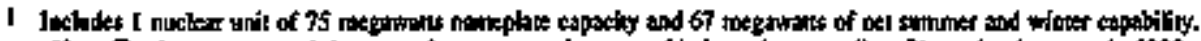

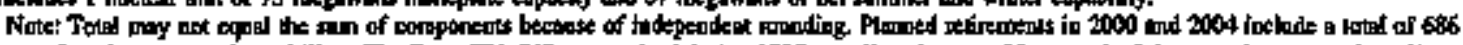

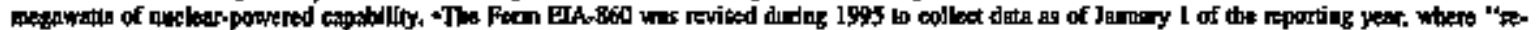

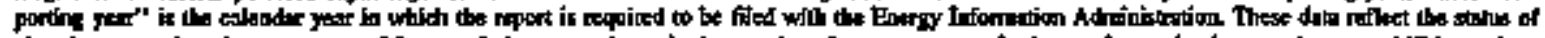

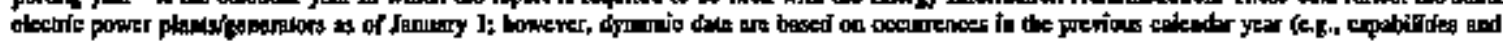

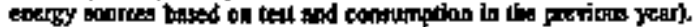

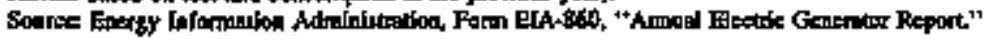

Table 12. Planned Coal- and Petroleum-Fired Capacity Retirements at U.S. Electric Utilities, 1996 Through 2005, as of January 1, 1996

\begin{tabular}{|c|c|c|c|c|c|c|c|c|}
\hline \multirow[b]{2}{*}{ Year } & \multicolumn{4}{|c|}{ Cool } & \multicolumn{4}{|c|}{ Petroleum } \\
\hline & $\begin{array}{l}\text { Nombar } \\
\text { of Uhits }\end{array}$ & 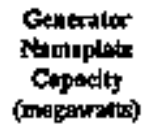 & 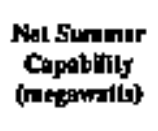 & $\begin{array}{l}\text { Nel White } \\
\text { Copabilly } \\
\text { (meperatils) }\end{array}$ & $\begin{array}{l}\text { Nurnber } \\
\text { of Units }\end{array}$ & 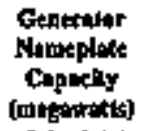 & $\begin{array}{l}\text { Net Sumnir } \\
\text { Copability } \\
\text { (megrasitus) }\end{array}$ & 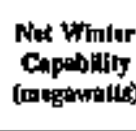 \\
\hline Uts. Twoll & 12 & 766 & 748 & $\pi 13$ & 80 & 1,500 & 1,317 & 1,463 \\
\hline 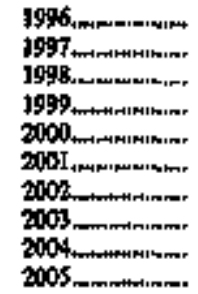 & $\begin{array}{l}\frac{7}{2} \\
\frac{4}{-} \\
\frac{-}{3} \\
\frac{3}{3}\end{array}$ & $\begin{array}{l}\frac{\overline{60}}{392} \\
\frac{-}{39} \\
\frac{-}{159} \\
155\end{array}$ & $\begin{array}{l}\overline{56} \\
\frac{355}{355} \\
= \\
= \\
158 \\
129\end{array}$ & $\begin{array}{l}\overline{56} \\
\overline{355} \\
= \\
- \\
= \\
158 \\
134\end{array}$ & $\begin{array}{r}5 \\
20 \\
1 \\
2 \\
6 \\
8 \\
14 \\
11 \\
11 \\
2\end{array}$ & $\begin{array}{r}130 \\
43 \\
3 \\
25 \\
20 \\
67 \\
232 \\
495 \\
472 \\
84\end{array}$ & $\begin{array}{r}131 \\
40 \\
3 \\
20 \\
17 \\
52 \\
191 \\
362 \\
416 \\
45\end{array}$ & $\begin{array}{r}134 \\
41 \\
3 \\
23 \\
18 \\
60 \\
272 \\
406 \\
472 \\
96\end{array}$ \\
\hline
\end{tabular}

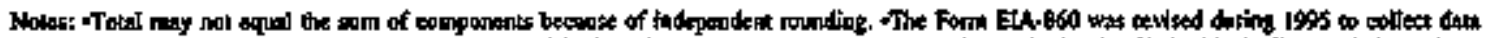

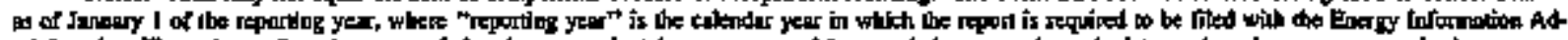

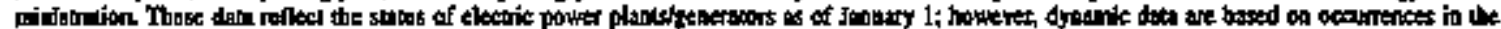

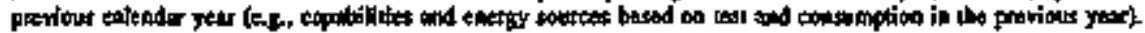

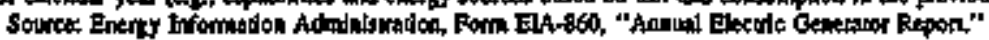


Table 13. Planned Gas-Fired and Hydroelectric Capacity Retirements at U.S. Electric Utilities, 1996 Through 2005, as of January 1, 1996

\begin{tabular}{|c|c|c|c|c|c|c|c|c|}
\hline \multirow[b]{2}{*}{ Yotr } & \multicolumn{4}{|c|}{ Ons } & \multicolumn{4}{|c|}{ Hytrofiludite } \\
\hline & 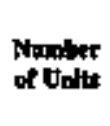 & 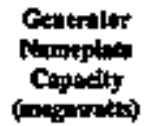 & 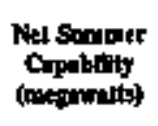 & 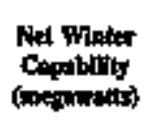 & $\begin{array}{l}\text { Nomiter } \\
\text { of Units }\end{array}$ & 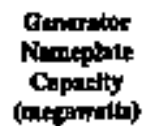 & 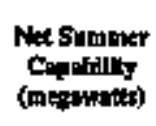 & 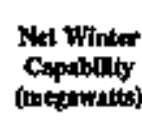 \\
\hline US. Totl & $\pi$ & 2,6001 & 2,45s & $\mathbf{2} ; \mathbf{2}$ & H & 17 & $\$$ & 12 \\
\hline 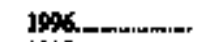 & - & - & - & - & 3 & $\mathbf{z}$ & I & I \\
\hline 1997. & $\boldsymbol{s}$ & 149 & 144 & 144 & - & - & - & - \\
\hline 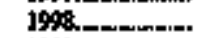 & 3 & 39 & 35 & 4 & 6 & 7 & 5 & 6 \\
\hline 199 & 2 & 49 & 51 & 51 & 6 & 4 & 2 & 3 \\
\hline 2000 . & 2 & 126 & 118 & 117 & - & عـ & - & - \\
\hline 2004. & 4 & 165 & 143 & [\$5! & 2 & 4 & I & 2 \\
\hline 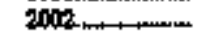 & 30 & $T H$ & 662 & 758 & - & $\rightarrow$ & - & - \\
\hline 2000. & $t$ & 23 & 26 & 26 & $\leftarrow$ & - & - & 一 \\
\hline 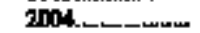 & 9 & 664 & EST & 637 & - & - & - & - \\
\hline -1905 2405 & 14 & 812 & 590 & 617 & 一 & - & 一 & - \\
\hline
\end{tabular}

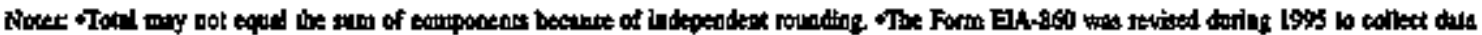

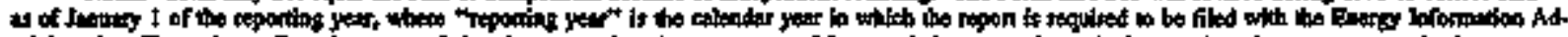

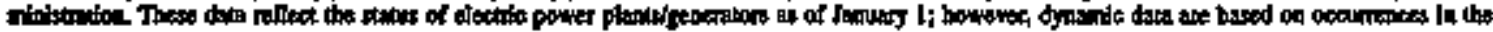

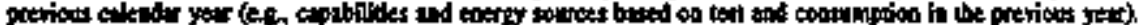

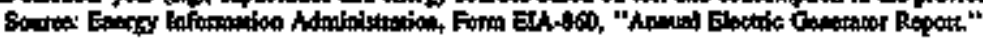


Table 14. Existing Capacity and Planned Capacify Additions at U.S. Electric Utilities by Enerzy Source, North American Electric Reliability Councll Region, and Hawaii, as of January 1, 1996

\begin{tabular}{|c|c|c|c|c|c|c|c|c|}
\hline \multirow{2}{*}{$\begin{array}{c}\text { MERc Region and Brwall } \\
\text { Priwewy Retris } \\
\text { Sourte }\end{array}$} & \multicolumn{4}{|c|}{ Extoling 1} & \multicolumn{4}{|c|}{ 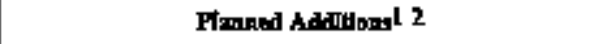 } \\
\hline & $\begin{array}{l}\text { Chumber } \\
\text { of thites }\end{array}$ & $\begin{array}{l}\text { Generator } \\
\text { Panpeplote } \\
\text { (megionets) }\end{array}$ & 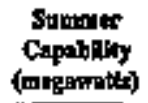 & $\begin{array}{c}\text { Winter } \\
\text { Crpablly } \\
\text { (mptomets) }\end{array}$ & Ninger & 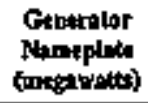 & 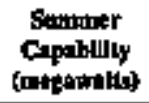 & 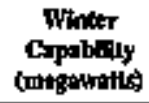 \\
\hline 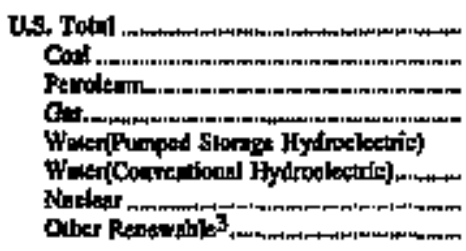 & $\begin{array}{r}10,396 \\
1,212 \\
3,277 \\
2,238 \\
140 \\
3,337 \\
109 \\
83\end{array}$ & $\begin{array}{r}79,562 \\
324,430 \\
71,908 \\
252,698 \\
18,643 \\
72,471 \\
197,896 \\
2,505\end{array}$ & $\begin{array}{r}70,131 \\
30,10,610 \\
64,464 \\
142,536 \\
21,387 \\
75,274 \\
99,515 \\
2,326\end{array}$ & $\begin{array}{r}78,097 \\
309,121 \\
70,195 \\
147,796 \\
21,321 \\
74,501 \\
100,696 \\
2,327\end{array}$ & $\begin{array}{r}44 \\
13 \\
87 \\
263 \\
\frac{6}{65} \\
1 \\
11\end{array}$ & $\begin{array}{r}7,600 \\
5,165 \\
6,984 \\
33,322 \\
\frac{474}{4,270} \\
387\end{array}$ & $\begin{array}{r}40,150 \\
4,845 \\
5,951 \\
27,371 \\
\frac{431}{1,170} \\
3 \$ 3\end{array}$ & $\begin{array}{r}4,565 \\
4,800 \\
6,832 \\
30,922 \\
-418 \\
1,130 \\
384\end{array}$ \\
\hline 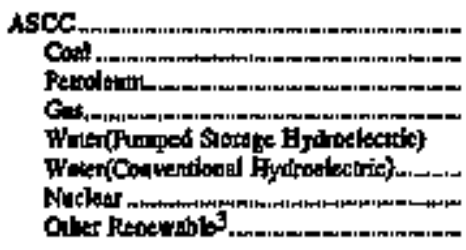 & $\begin{array}{r}595 \\
5 \\
464 \\
\frac{31}{54} \\
\overrightarrow{3}\end{array}$ & $\begin{array}{r}1,909 \\
54 \\
610 \\
90 \\
\frac{359}{-}\end{array}$ & $\begin{array}{r}1,732 \\
54 \\
572 \\
754 \\
\frac{159}{\square} \\
\because\end{array}$ & $\begin{array}{r}1,570 \\
54 \\
614 \\
843 \\
\frac{360}{2} \\
\end{array}$ & $\frac{\frac{6}{1}}{\frac{1}{2}}$ & $\begin{array}{l}\frac{5}{*} \\
\frac{5}{1} \\
\frac{1}{*}\end{array}$ & $\begin{array}{l}\frac{6}{*} \\
\frac{4}{1} \\
\frac{1}{7}\end{array}$ & $\frac{5}{4}$ \\
\hline 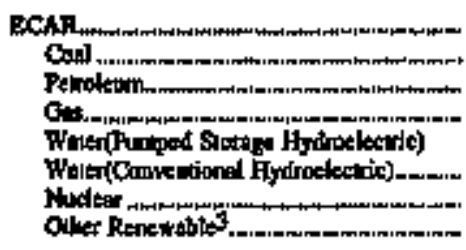 & $\begin{array}{r}3,125 \\
372 \\
300 \\
151 \\
17 \\
276 \\
9 \\
3\end{array}$ & $\begin{array}{r}11,4020 \\
90,594 \\
5,329 \\
4,983 \\
3,393 \\
1,150 \\
6,351 \\
90\end{array}$ & $\begin{array}{r}104,436 \\
83,356 \\
4,091 \\
4,321 \\
3,381 \\
1,017 \\
7,661 \\
90\end{array}$ & $\begin{array}{r}106,133 \\
84,220 \\
5,005 \\
4,397 \\
3,281 \\
1,054 \\
7,796 \\
90\end{array}$ & $\begin{array}{l}\frac{9}{4} \\
\frac{4}{6} \\
\frac{1}{-}\end{array}$ & $\begin{array}{r}5,500 \\
\frac{258}{2530} \\
\frac{115}{-}\end{array}$ & $\begin{array}{r}4,979 \\
2,609 \\
\frac{70}{2} \\
=\end{array}$ & $\begin{array}{r}5,76 \\
2 \pi 3 \\
5,375 \\
=\end{array}$ \\
\hline 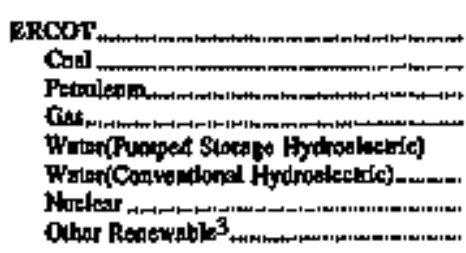 & $\begin{array}{r}360 \\
27 \\
32 \\
250 \\
\frac{46}{40} \\
1\end{array}$ & 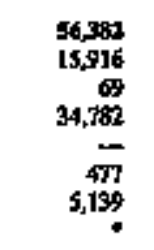 & $\begin{array}{r}53,400 \\
14,134 \\
58 \\
30,157 \\
5,519 \\
4,802\end{array}$ & $\begin{array}{r}53,597 \\
14,055 \\
60 \\
33,418 \\
\frac{4}{461} \\
4802 \\
-\end{array}$ & $\frac{28}{\frac{3}{22}}$ & $\begin{array}{r}5,900 \\
1,693 \\
3,208 \\
\bar{z}\end{array}$ & 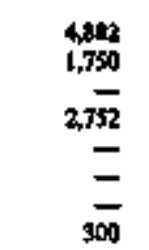 & $\begin{array}{r}5,097 \\
1,750 \\
3,047 \\
= \\
= \\
300\end{array}$ \\
\hline 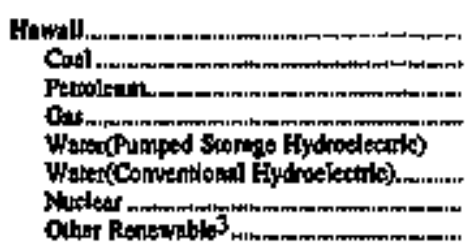 & $\begin{array}{l}\frac{97}{93} \\
\frac{7}{4} \\
=\end{array}$ & $\frac{1,659}{1,655}$ & $\begin{array}{r}1,600 \\
\frac{1}{3} \\
\frac{7}{-}\end{array}$ & $\begin{array}{r}1,600 \\
\frac{7}{3} \\
\frac{7}{-}\end{array}$ & $\begin{array}{l}\frac{14}{14} \\
\frac{}{\square} \\
= \\
=\end{array}$ & $\begin{array}{l}180 \\
\frac{183}{-} \\
= \\
=\end{array}$ & $\begin{array}{l}1510 \\
158 \\
= \\
= \\
=\end{array}$ & $\begin{array}{l}174 \\
\frac{174}{-} \\
= \\
= \\
=\end{array}$ \\
\hline 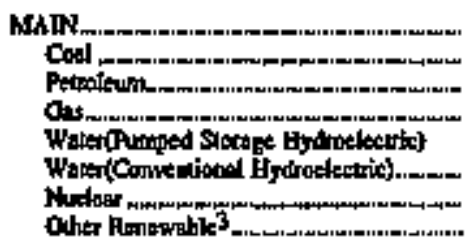 & $\begin{array}{r}755 \\
134 \\
297 \\
165 \\
2 \\
199 \\
17 \\
1\end{array}$ & $\begin{array}{r}56,366 \\
30,382 \\
4,045 \\
4,738 \\
408 \\
652 \\
56,139 \\
3\end{array}$ & $\begin{array}{r}51,430 \\
7,746 \\
3,588 \\
3,538 \\
350 \\
611 \\
14,902 \\
2\end{array}$ & $\begin{array}{r}52,657 \\
27,975 \\
3,960 \\
4,752 \\
275 \\
611 \\
15,063 \\
2\end{array}$ & 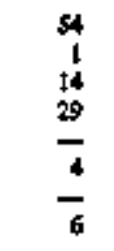 & 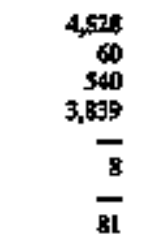 & $\begin{array}{r}3,879 \\
60 \\
461 \\
3,239 \\
\frac{7}{7}\end{array}$ & $\begin{array}{r}1,163 \\
60 \\
529 \\
3,729 \\
\frac{7}{7} \\
\frac{7}{77}\end{array}$ \\
\hline 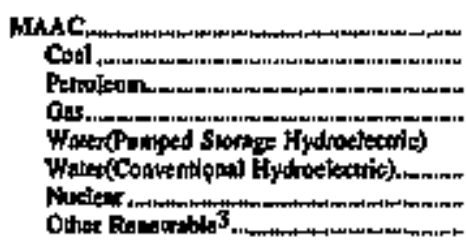 & $\begin{array}{r}49 \\
66 \\
219 \\
99 \\
13 \\
46 \\
13 \\
\end{array}$ & $\begin{array}{r}5,065 \\
59,279 \\
11,237 \\
9,389 \\
1,366 \\
1,135 \\
10,769 \\
-\end{array}$ & $\begin{array}{r}5,003 \\
17,814 \\
10,180 \\
8,732 \\
1,341 \\
1,1,53 \\
12,863 \\
-\end{array}$ & $\begin{array}{r}5,547 \\
18,062 \\
11,151 \\
9,683 \\
1,341 \\
1,169 \\
13,140 \\
=\end{array}$ & $\begin{array}{l}43 \\
1 \\
5 \\
37 \\
= \\
= \\
=\end{array}$ & $\begin{array}{r}5,4+4 \\
300 \\
2 \$ 1 \\
5,10 \% \\
= \\
= \\
=\end{array}$ & $\begin{array}{r}4,564 \\
300 \\
200 \\
4356 \\
= \\
= \\
=\end{array}$ & $\begin{array}{r}5,461 \\
300 \\
296 \\
4945 \\
= \\
= \\
=\end{array}$ \\
\hline
\end{tabular}

Sec frolnokes at and of thhle. 
Table 14. Existing Capacity and Planned Capacity Additions at U.S. Clectric Utilities by Energy Source, North American Electric Reliability Council Region, and Hawali, as of January 1, 1996 (Continued)

\begin{tabular}{|c|c|c|c|c|c|c|c|c|}
\hline \multirow{2}{*}{ 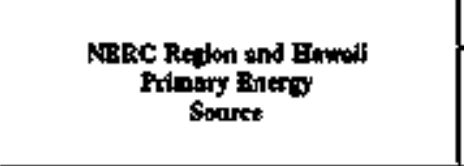 } & \multicolumn{4}{|c|}{ Extylingt } & \multicolumn{4}{|c|}{ Prened Adidond 2} \\
\hline & $\begin{array}{l}\text { Nomber } \\
\text { of Utits }\end{array}$ & 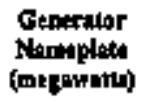 & $\underset{\text { Cupgiblity }}{\text { Sumejer }}$ & 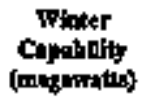 & $\begin{array}{l}\text { Ninber } \\
\text { of Dn'lls }\end{array}$ & 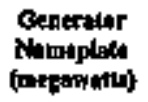 & 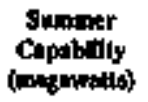 & 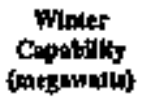 \\
\hline 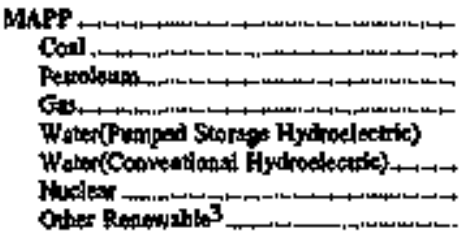 & $\begin{array}{r}1261 \\
131 \\
636 \\
290 \\
230 \\
8 \\
16\end{array}$ & $\begin{array}{r}32,57 \\
19,442 \\
3,134 \\
2,680 \\
3,179 \\
4,104 \\
189\end{array}$ & $\begin{array}{r}31,711 \\
\text { I8,908 } \\
2,186 \\
2,446 \\
3,168 \\
3,738 \\
176\end{array}$ & $\begin{array}{r}32,2,4 \\
18,866 \\
3,448 \\
2,795 \\
3,172 \\
3,817 \\
187\end{array}$ & $\begin{array}{r}21 \\
14 \\
1 \\
- \\
- \\
-\end{array}$ & $\begin{array}{r}79 \\
713 \\
40 \\
= \\
=\end{array}$ & $\begin{array}{r}40 \\
4 \\
99 \\
367 \\
= \\
= \\
-\end{array}$ & $\begin{array}{r}435 \\
111 \\
421 \\
= \\
=\end{array}$ \\
\hline 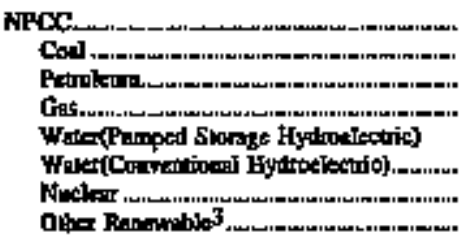 & $\begin{array}{r}1,21 \\
50 \\
366 \\
106 \\
24 \\
646 \\
14 \\
14\end{array}$ & $\begin{array}{r}36,808 \\
7,807 \\
17,710 \\
10,796 \\
2,693 \\
5,2060 \\
12,499 \\
173\end{array}$ & $\begin{array}{r}55,567 \\
7,16,2 \\
16,160 \\
10,006 \\
5,099 \\
3,375 \\
11,208 \\
107\end{array}$ & $\begin{array}{r}57,477 \\
7,468 \\
17,247 \\
10,693 \\
5,115 \\
5,425 \\
11,392 \\
147\end{array}$ & $\begin{array}{l}\frac{2}{2} \\
\frac{-}{2} \\
\frac{1}{1}\end{array}$ & $\frac{119}{\frac{7}{3}}$ & $\frac{114}{\frac{-1}{3}}$ & $\frac{-1}{\frac{1}{10}}$ \\
\hline 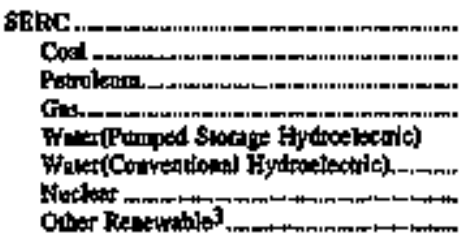 & $\begin{array}{r}1,400 \\
259 \\
364 \\
286 \\
31 \\
510 \\
33 \\
3\end{array}$ & $\begin{array}{r}165,690 \\
78,980 \\
71,876 \\
15,268 \\
6,074 \\
10,836 \\
37,686 \\
4\end{array}$ & $\begin{array}{r}13,04 \\
72,071 \\
20,053 \\
13,684 \\
6,105 \\
11,181 \\
30,942 \\
\end{array}$ & $\begin{array}{r}157,4 n \\
73,251 \\
2,057 \\
14,821 \\
6,109 \\
10,605 \\
30,541 \\
\end{array}$ & $\begin{array}{r}4 \\
49 \\
29 \\
\frac{1}{6} \\
-1 \\
-\end{array}$ & $\begin{array}{r}18,975 \\
1,619 \\
5,640 \\
10,44 \\
= \\
1,270 \\
-\end{array}$ & $\begin{array}{r}1,479 \\
1,464 \\
4,795 \\
5,050 \\
- \\
1,170 \\
-\end{array}$ & $\begin{array}{r}17,102 \\
1,474 \\
5,518 \\
8,940 \\
= \\
1,170 \\
-\end{array}$ \\
\hline 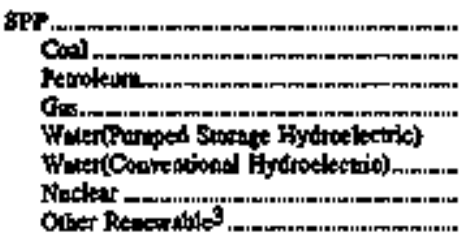 & $\begin{array}{r}1,136 \\
80 \\
40 t \\
546 \\
14 \\
90 \\
5 \\
-\end{array}$ & $\begin{array}{r}7,899 \\
23,274 \\
2,484 \\
36,942 \\
549 \\
2,375 \\
5,317 \\
-\end{array}$ & $\begin{array}{r}7,375 \\
26,503 \\
2,253 \\
34,7005 \\
505 \\
2,565 \\
4,572 \\
-\end{array}$ & $\begin{array}{r}71,456 \\
26,507 \\
2,342 \\
34,541 \\
505 \\
2,565 \\
4,896 \\
-\end{array}$ & $\begin{array}{r}40 \\
1 \\
3 \\
39 \\
\frac{9}{-} \\
-\end{array}$ & $\begin{array}{r}4,453 \\
652 \\
4 \\
3,6 \% 9 \\
\frac{-}{109} \\
-\end{array}$ & $\begin{array}{r}3,786 \\
662 \\
4 \\
3,018 \\
\frac{103}{103} \\
-\end{array}$ & $\begin{array}{r}4.98 \\
690 \\
4 \\
3,418 \\
-7 \\
-\end{array}$ \\
\hline 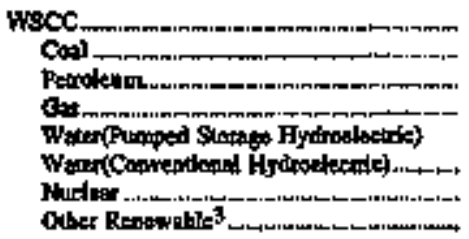 & $\begin{array}{r}1,970 \\
110 \\
165 \\
355 \\
17 \\
1,245 \\
8 \\
8\end{array}$ & $\begin{array}{r}132,354 \\
3,671 \\
2,759 \\
32,200 \\
4,310 \\
47,195 \\
9,564 \\
2,058\end{array}$ & $\begin{array}{r}12,751 \\
31,044 \\
2,469 \\
30,273 \\
4,706 \\
4,328 \\
9,227 \\
1,902\end{array}$ & $\begin{array}{r}130,316 \\
31,863 \\
2,613 \\
30,991 \\
4,707 \\
4,986 \\
9,259 \\
1,902\end{array}$ & $\begin{array}{r}42 \\
2 \\
1 \\
26 \\
\frac{1}{23} \\
- \\
-\end{array}$ & 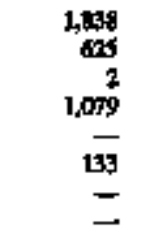 & $\begin{array}{r}1,654 \\
605 \\
2 \\
921 \\
-125 \\
- \\
-\end{array}$ & $\begin{array}{r}1776 \\
610 \\
2 \\
1,0 \times 2 \\
- \\
122 \\
- \\
-\end{array}$ \\
\hline
\end{tabular}

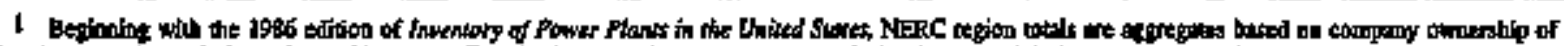

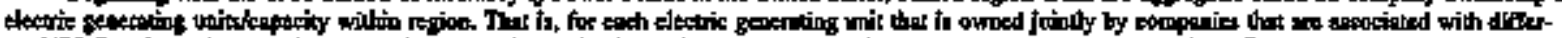

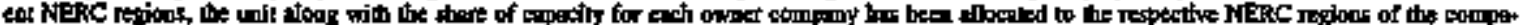

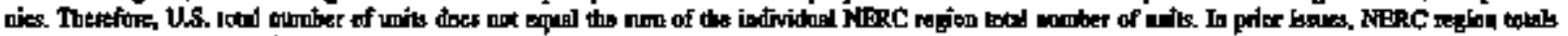

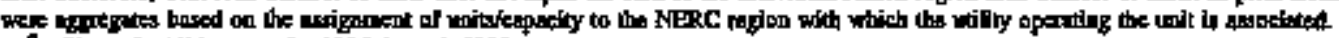

2 Pilned additions ate for 1996 throwh 2005 .

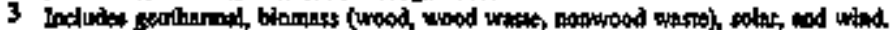

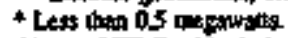

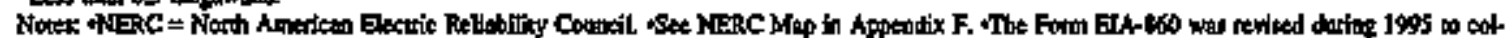

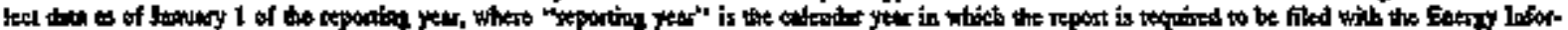

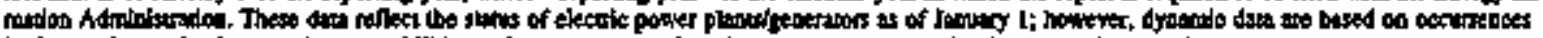

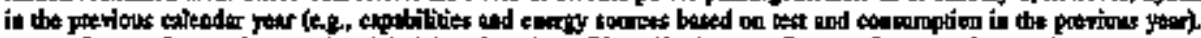

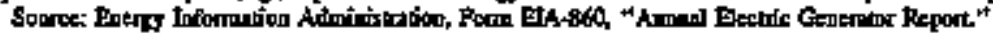


Table 15. Existing Capacity and Planned Capacity Additions at U.S. Electric Utilities by Energy Source and Federal Reglon, as of January 1, 1996

\begin{tabular}{|c|c|c|c|c|c|c|c|c|}
\hline \multirow[b]{2}{*}{$\begin{array}{c}\text { Federal Roghon } \\
\text { Ptimary Butroty } \\
\text { Soluroe }\end{array}$} & \multicolumn{4}{|c|}{ Entsting } & \multicolumn{4}{|c|}{ Fummet dtitulonal } \\
\hline & 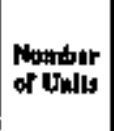 & 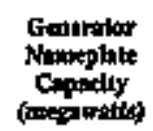 & 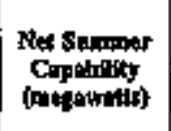 & 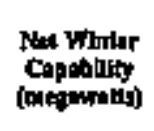 & $\begin{array}{l}\text { Nowber } \\
\text { of Undus }\end{array}$ & 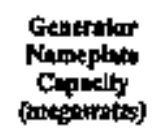 & 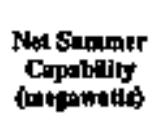 & 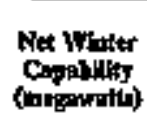 \\
\hline 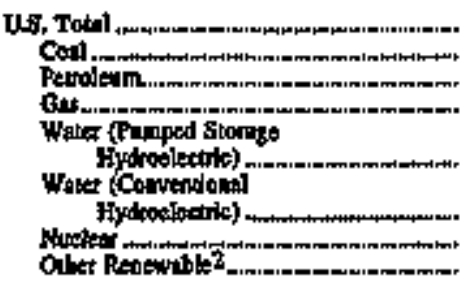 & $\begin{array}{r}10,396 \\
1,212 \\
3,277 \\
2,228 \\
140 \\
3,737 \\
108 \\
80\end{array}$ & $\begin{array}{r}180,542 \\
324,430 \\
71,908 \\
158,698 \\
18.643 \\
72,411 \\
107,846 \\
2,505\end{array}$ & $\begin{array}{r}706,111 \\
300,610 \\
64,464 \\
242,536 \\
21,387 \\
75,274 \\
90,543 \\
2336\end{array}$ & $\begin{array}{r}719,997 \\
303,121 \\
70,195 \\
147,736 \\
21,321 \\
71,501 \\
10,4096 \\
2,737\end{array}$ & $\begin{array}{r}40 \\
13 \\
87 \\
267 \\
-7 \\
65 \\
11\end{array}$ & $\begin{array}{r}47,643 \\
5,165 \\
6,934 \\
33,322 \\
= \\
4,74 \\
2,270 \\
357\end{array}$ & $\begin{array}{r}41,150 \\
4,154 \\
5,951 \\
27,371 \\
- \\
1,131 \\
1,170 \\
383\end{array}$ & $\begin{array}{r}44,565 \\
4,860 \\
6,832 \\
30,922 \\
- \\
418 \\
1,170 \\
334\end{array}$ \\
\hline 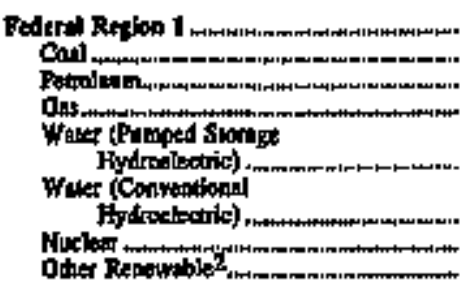 & $\begin{array}{r}63 \\
15 \\
231 \\
19 \\
8 \\
368 \\
8 \\
14\end{array}$ & $\begin{array}{l}2,101 \\
2,773 \\
9,152 \\
1,705 \\
1,453 \\
1,340 \\
6,846 \\
175\end{array}$ & $\begin{array}{r}22,480 \\
2,670 \\
9,529 \\
1,627 \\
1,659 \\
1,471 \\
6,389 \\
147\end{array}$ & $\begin{array}{r}13,704 \\
2,680 \\
6,964 \\
1,7 \pi 1 \\
1,675 \\
1,461 \\
6,400 \\
147\end{array}$ & $\begin{array}{l} \pm \\
z\end{array}$ & $\begin{array}{l}\frac{10}{-} \\
- \\
- \\
-6\end{array}$ & $\frac{45}{6}$ & $\begin{array}{l}\infty \\
= \\
- \\
- \\
\frac{44}{6}\end{array}$ \\
\hline 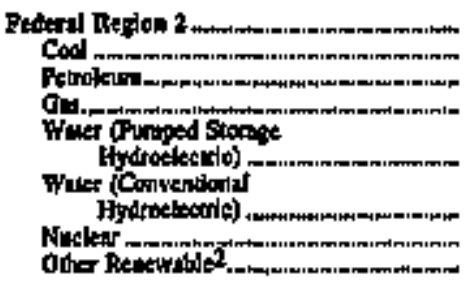 & $\begin{array}{r}668 \\
30 \\
176 \\
103 \\
10 \\
201 \\
10 \\
-10\end{array}$ & $\begin{array}{r}4,247 \\
5,736 \\
11,644 \\
14,528 \\
1,627 \\
3,922 \\
9,775 \\
-\end{array}$ & $\begin{array}{r}45,594 \\
5,499 \\
11,597 \\
13,525 \\
3,820 \\
3,906 \\
0,696 \\
-\end{array}$ & $\begin{array}{r}8,243 \\
5,497 \\
11,494 \\
14,634 \\
3,820 \\
3,945 \\
8,852 \\
-\end{array}$ & $\begin{array}{l}\frac{46}{2} \\
\frac{1}{2} \\
\frac{16}{-}\end{array}$ & $\begin{array}{r}\frac{3,173}{3} \\
3,107 \\
- \\
\frac{62}{-}\end{array}$ & $\begin{array}{r}\frac{2,243}{3} \\
2,641 \\
- \\
59 \\
-\end{array}$ & $\begin{array}{r}\frac{3,105}{3} \\
3,045 \\
- \\
\frac{5}{-}\end{array}$ \\
\hline 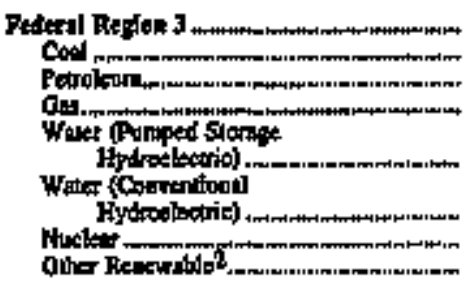 & $\begin{array}{r}607 \\
139 \\
293 \\
58 \\
19 \\
130 \\
15 \\
3\end{array}$ & $\begin{array}{r}12,360 \\
45,069 \\
10,47 \\
5,195 \\
3,544 \\
1,564 \\
15,419\end{array}$ & $\begin{array}{r}76,404 \\
42,851 \\
9,264 \\
4,675 \\
9,630 \\
2,0,000 \\
14,03\end{array}$ & $\begin{array}{r}78,724 \\
41,039 \\
10,075 \\
5,203 \\
3,600 \\
2,065 \\
14,212\end{array}$ & $\begin{array}{l}37 \\
2 \\
24 \\
12 \\
- \\
1 \\
= \\
-\end{array}$ & $\begin{array}{l}5,259 \\
724 \\
2,500 \\
2,000\end{array}$ & $\begin{array}{r}4,561 \\
691 \\
2,154 \\
1,715\end{array}$ & $\begin{array}{l}8,0101 \\
701 \\
2,400 \\
1,900\end{array}$ \\
\hline 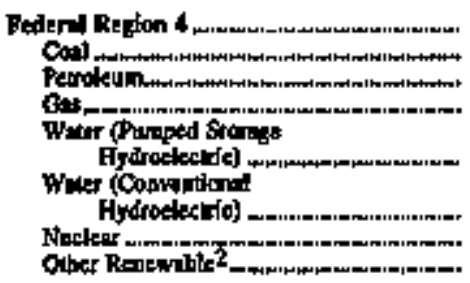 & $\begin{array}{r}1,47 \\
278 \\
328 \\
306 \\
25 \\
506 \\
29 \\
-\end{array}$ & $\begin{array}{r}161,206 \\
84,556 \\
21,592 \\
17,414 \\
4,1614 \\
10,718 \\
29,031 \\
-\end{array}$ & $\begin{array}{r}154,646 \\
76,962 \\
18906 \\
15.999 \\
4806 \\
10.986 \\
26,950 \\
-\end{array}$ & $\begin{array}{r}165,394 \\
78,104 \\
20,822 \\
16,972 \\
4,843 \\
10,3103 \\
27,149 \\
-\end{array}$ & $\begin{array}{r}101 \\
3 \\
14 \\
30 \\
- \\
3 \\
1 \\
-\end{array}$ & $\begin{array}{r}17,710 \\
1,195 \\
3,607 \\
11,567 \\
- \\
70 \\
1,270 \\
-\end{array}$ & $\begin{array}{r}14,389 \\
1,073 \\
3,060 \\
9,005 \\
- \\
1,67 \\
-170\end{array}$ & $\begin{array}{r}15,968 \\
1,075 \\
3,566 \\
10,005 \\
- \\
65 \\
1,17 \\
-\end{array}$ \\
\hline 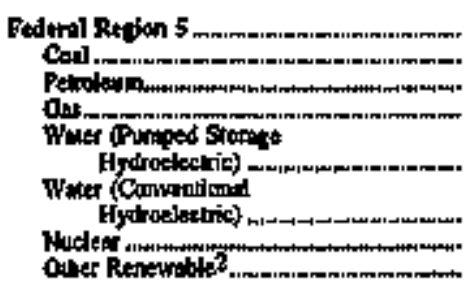 & $\begin{array}{r}2,401 \\
426 \\
603 \\
360 \\
6 \\
521 \\
26 \\
19\end{array}$ & $\begin{array}{r}134,563 \\
88,294 \\
9,564 \\
9,723 \\
1,979 \\
1,159 \\
2,576 \\
280\end{array}$ & $\begin{array}{r}125,656 \\
91,517 \\
8612 \\
5662 \\
1,872 \\
1,002 \\
21,664 \\
271\end{array}$ & $\begin{array}{r}126,711 \\
02,157 \\
9,662 \\
9,820 \\
1,872 \\
1,049 \\
22,077 \\
278\end{array}$ & $\begin{array}{l}95 \\
2 \\
13 \\
57\end{array}$ & $\begin{array}{r}8,47 \\
57 \\
26 \\
8,252\end{array}$ & $\begin{array}{r}1,148 \\
64 \\
25 \\
6,053\end{array}$ & $\begin{array}{r}0,211 \\
64 \\
26 \\
0,015\end{array}$ \\
\hline
\end{tabular}

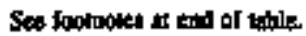


Table 15. Existing Capacity and Plamned Capacity Additions at U.S. Electric Utilities by Energy Sonrce and Federal Region, as of January 1, 1996 (Continued)

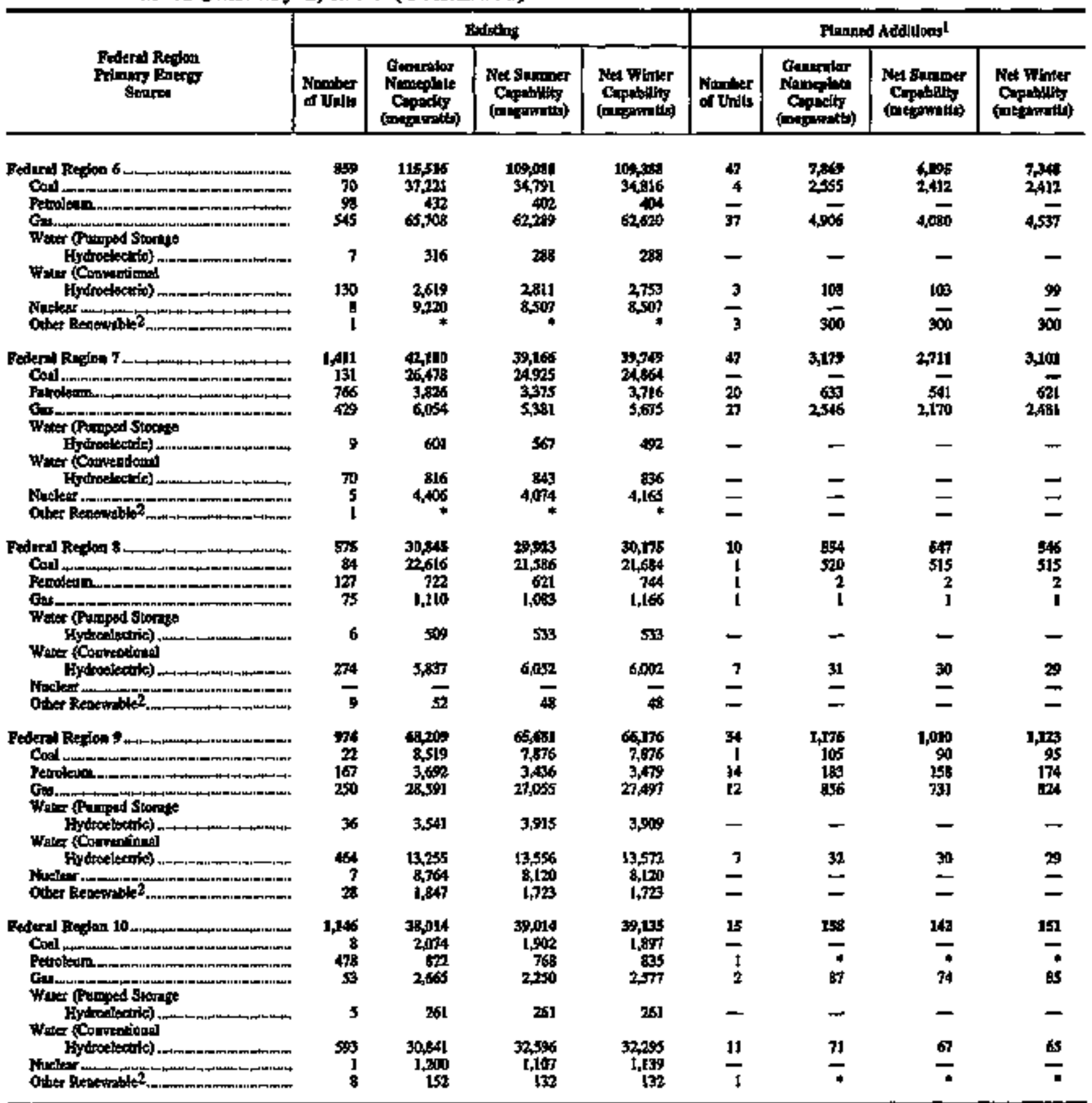

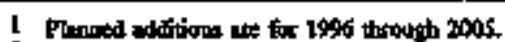

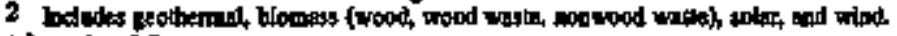

+ Lar than 0.5 miganitis.

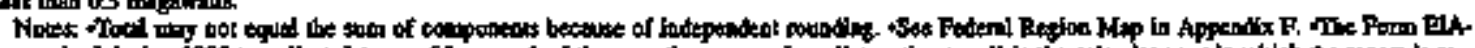

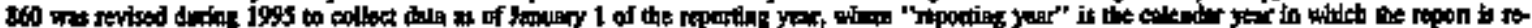

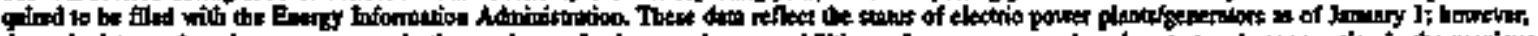

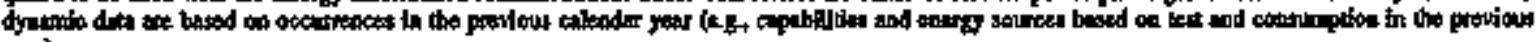
ye: $x$.

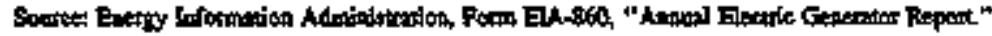


Table 16. Existing Capacity and Planmed Capacity Additions at US. Electric Utilities by Energy Source and Census Division, as of Jamuary 1, 1996

\begin{tabular}{|c|c|c|c|c|c|c|c|c|}
\hline \multirow[b]{2}{*}{ 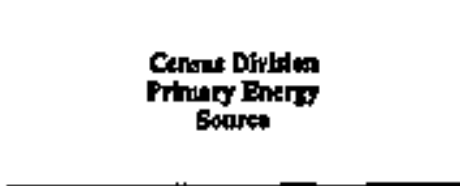 } & \multicolumn{4}{|c|}{ Exinting } & \multicolumn{4}{|c|}{ Planied Mddilont } \\
\hline & $\begin{array}{l}\text { stmerer } \\
\text { if Onils }\end{array}$ & 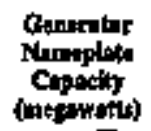 & 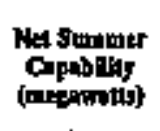 & 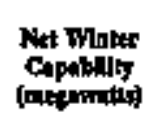 & $\begin{array}{l}\text { Nomber } \\
\text { ot Un'ts }\end{array}$ & 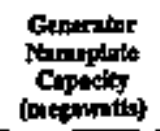 & 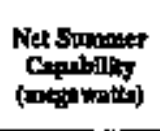 & 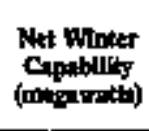 \\
\hline 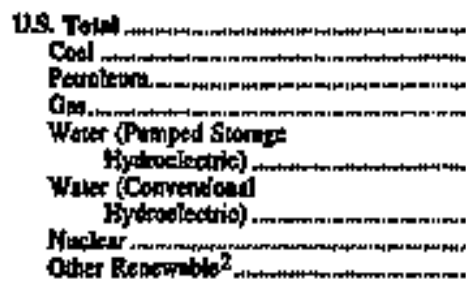 & $\begin{array}{r}10,395 \\
1,212 \\
3,277 \\
2,278 \\
140 \\
3,337 \\
109 \\
83\end{array}$ & 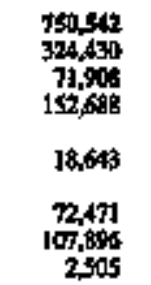 & $\begin{array}{r}706,111 \\
30,610 \\
61,464 \\
142,536 \\
21,387 \\
75,274 \\
99,545 \\
2 \$ 25\end{array}$ & $\begin{array}{r}35,107 \\
309,121 \\
70,195 \\
147,736 \\
21,321 \\
74,001 \\
100,406 \\
2,307\end{array}$ & $\begin{array}{r}416 \\
13 \\
87 \\
269 \\
- \\
65 \\
1 \\
11\end{array}$ & $\begin{array}{r}47,603 \\
5,165 \\
6,904 \\
13,3 \pm 2 \\
- \\
1,40 \\
307\end{array}$ & $\begin{array}{r}40,350 \\
4,845 \\
5,951 \\
27,37 \\
- \\
431 \\
1,17 \pi \\
383\end{array}$ & 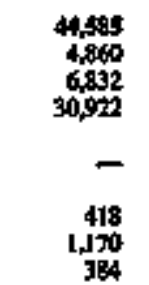 \\
\hline 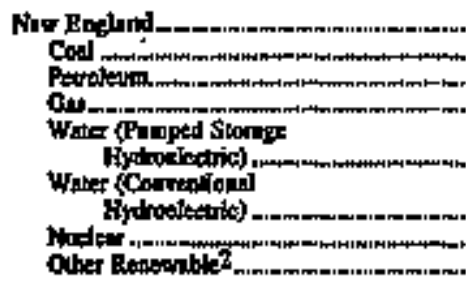 & $\begin{array}{r}6.63 \\
15 \\
235 \\
19 \\
8 \\
36 \\
4 \\
4\end{array}$ & $\begin{array}{l}2,101 \\
2,773 \\
9,1,53 \\
1,105 \\
1,453 \\
1,340 \\
6,866 \\
173\end{array}$ & $\begin{array}{r}2,4,40 \\
2,610 \\
8,503 \\
1,627 \\
1,69 \\
1,671 \\
6,383 \\
147\end{array}$ & $\begin{array}{r}21,204 \\
2,690 \\
8,964 \\
1,771 \\
1.675 \\
1,491 \\
6,490 \\
147\end{array}$ & $\frac{-}{3}$ & $\begin{array}{l}\frac{11}{-} \\
- \\
\frac{10}{6}\end{array}$ & $\frac{45}{6}$ & $\begin{array}{l}\frac{10}{-} \\
= \\
- \\
\frac{44}{6}\end{array}$ \\
\hline 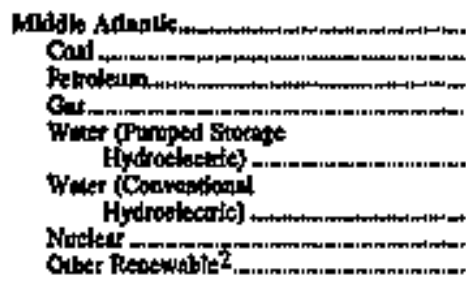 & $\begin{array}{r}900 \\
98 \\
203 \\
157 \\
29 \\
316 \\
19\end{array}$ & $\begin{array}{r}9,1,144 \\
25,005 \\
17,3,44 \\
15,018 \\
2,823 \\
4,513 \\
19,411 \\
-\end{array}$ & $\begin{array}{r}7,609 \\
2,002 \\
15,37 \\
13,972 \\
5,105 \\
4,553 \\
17,642 \\
-\end{array}$ & $\begin{array}{r}0,913 \\
2,357 \\
16,800 \\
15,172 \\
5,105 \\
4,608 \\
17,942 \\
-\end{array}$ & $\begin{array}{l}\frac{4}{2} \\
26 \\
- \\
\frac{18}{-}\end{array}$ & $\begin{array}{r}\frac{3,331}{3} \\
3,272 \\
- \\
\frac{62}{-}\end{array}$ & $\begin{array}{r}\frac{7}{3} \\
2,784 \\
- \\
\frac{5}{-}\end{array}$ & $\begin{array}{r}\frac{3,287}{3} \\
3,207 \\
- \\
= \\
-7\end{array}$ \\
\hline 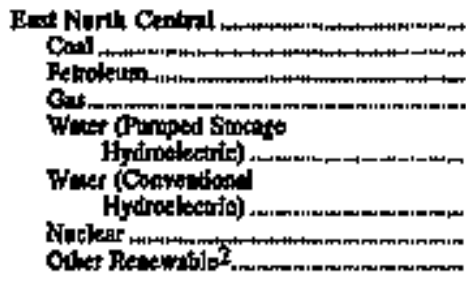 & $\begin{array}{r}1,685 \\
392 \\
496 \\
300 \\
6 \\
46 \\
73 \\
9\end{array}$ & $\begin{array}{r}125,320 \\
80,645 \\
8,433 \\
9,731 \\
1,979 \\
1,015 \\
21.821 \\
187\end{array}$ & $\begin{array}{r}1,4,735 \\
73,657 \\
7 \leq 97 \\
1,208 \\
1,672 \\
1080 \\
20,093 \\
196\end{array}$ & $\begin{array}{r}117,515 \\
76,537 \\
8,412 \\
9,138 \\
1,572 \\
906 \\
20,455 \\
196\end{array}$ & $\begin{array}{r}88 \\
\frac{7}{7} \\
-6 \\
\frac{8}{6}\end{array}$ & $\begin{array}{r}8,455 \\
60 \\
15 \\
8,246 \\
- \\
51 \\
\frac{51}{81}\end{array}$ & $\begin{array}{r}7,125 \\
60 \\
65 \\
6947 \\
- \\
30 \\
\frac{37}{77}\end{array}$ & $\begin{array}{r}8,191 \\
60 \\
6 \\
8,009 \\
- \\
30 \\
\frac{77}{77}\end{array}$ \\
\hline 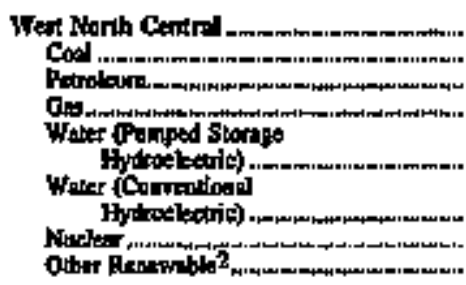 & $\begin{array}{r}1,108 \\
190 \\
903 \\
502 \\
9 \\
155 \\
8 \\
11\end{array}$ & $\begin{array}{r}59,001 \\
36,602 \\
5,405 \\
6,915 \\
601 \\
3,205 \\
6,161 \\
94\end{array}$ & $\begin{array}{r}55,504 \\
34,5,92 \\
4,775 \\
6,208 \\
5,77 \\
\\
3,351 \\
5,645 \\
\$ 12\end{array}$ & $\begin{array}{r}56,57 ! \\
34,866 \\
5,413 \\
6,571 \\
492 \\
3,349 \\
5,782 \\
83\end{array}$ & $\begin{array}{l}5 \\
1 \\
26 \\
28 \\
- \\
= \\
=\end{array}$ & $\begin{array}{r}3,202 \\
7 \\
644 \\
2,52 \\
- \\
= \\
=\end{array}$ & $\begin{array}{r}2,731 \\
4 \\
552 \\
2,175 \\
- \\
= \\
=\end{array}$ & $\begin{array}{r}3,121 \\
4 \\
632 \\
2,496 \\
- \\
= \\
-\end{array}$ \\
\hline 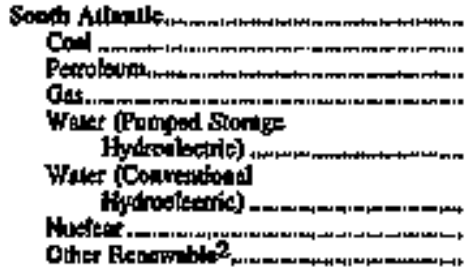 & 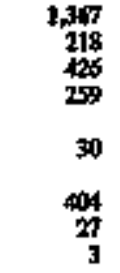 & $\begin{array}{r}148,996 \\
70,702 \\
24,691 \\
16,213 \\
5,612 \\
6,292 \\
25,468 \\
*\end{array}$ & $\begin{array}{r}136,336 \\
65,790 \\
21,979 \\
14,609 \\
5,656 \\
6,423 \\
23,792\end{array}$ & 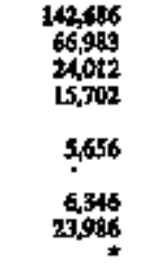 & $\begin{array}{l}94 \\
5 \\
34 \\
58 \\
- \\
1 \\
-\end{array}$ & $\begin{array}{r}18,004 \\
1,919 \\
5,891 \\
10,27 \\
- \\
1 \\
=\end{array}$ & $\begin{array}{r}14,6 \mathbf{5 3} \\
1,764 \\
5,005 \\
7,256 \\
- \\
=\end{array}$ & $\begin{array}{r}16,249 \\
1,770 \\
5,755 \\
8,690 \\
- \\
1 \\
=\end{array}$ \\
\hline
\end{tabular}

Sex hooluow at exc of colath 
Table 16. Existing Capacity and Planned Capacity Additions at U.S. Electric Utlities by Brergy Source and Census Division, as of January 1, 1996 (Continued)

\begin{tabular}{|c|c|c|c|c|c|c|c|c|}
\hline \multirow[b]{2}{*}{ 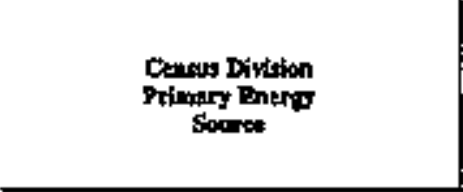 } & \multicolumn{4}{|c|}{ Extothe } & \multicolumn{4}{|c|}{ Finned Aditthml } \\
\hline & $\begin{array}{l}\text { khaner } \\
\text { ar Utelts }\end{array}$ & 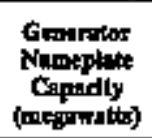 & 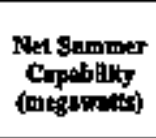 & 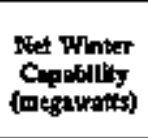 & $\begin{array}{l}\text { Nimber } \\
\text { of Unlta }\end{array}$ & 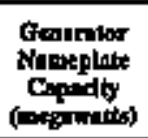 & 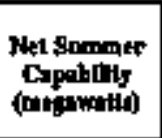 & 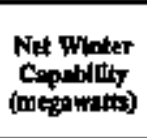 \\
\hline 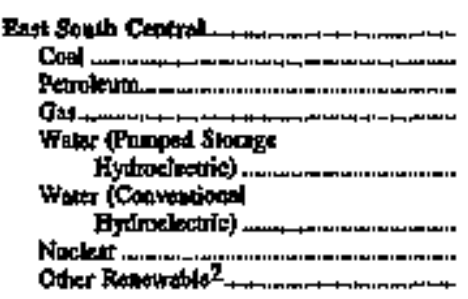 & $\begin{array}{r}478 \\
140 \\
38 \\
91 \\
4 \\
197 \\
8 \\
-\end{array}$ & $\begin{array}{r}64,651 \\
40,749 \\
1,693 \\
5,9000 \\
1,530 \\
5,732 \\
9,046 \\
7\end{array}$ & $\begin{array}{r}99,202 \\
36,550 \\
1,331 \\
5,808 \\
1,582 \\
5,956 \\
8,205 \\
\end{array}$ & $\begin{array}{r}59,658 \\
36,1000 \\
1,549 \\
5,933 \\
1,532 \\
5,560 \\
3,285 \\
-\end{array}$ & $\begin{array}{l}39 \\
\frac{3}{2} \\
33 \\
- \\
3 \\
-\end{array}$ & $\begin{array}{r}\frac{4,761}{256} \\
3,165 \\
- \\
1,270 \\
7\end{array}$ & $\begin{array}{r}\frac{4140}{258} \\
2,595 \\
- \\
1,170 \\
-\end{array}$ & $\begin{array}{r}\frac{4560}{251} \\
3,093 \\
- \\
1,170 \\
-\end{array}$ \\
\hline 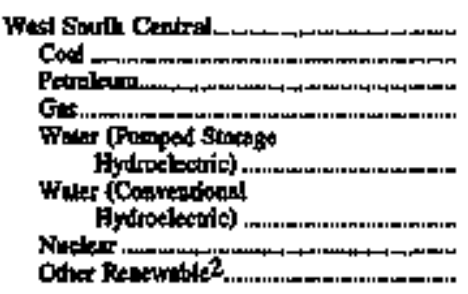 & $\begin{array}{r}648 \\
57 \\
91 \\
516 \\
7 \\
124 \\
8 \\
1\end{array}$ & $\begin{array}{r}10,996 \\
32,926 \\
3,4 \\
64,590 \\
316 \\
2,561 \\
9,204\end{array}$ & $\begin{array}{r}104010 \\
30,100 \\
359 \\
61,213 \\
288 \\
2753 \\
8,507 \\
7\end{array}$ & $\begin{array}{r}104,304 \\
30,915 \\
356 \\
61,540 \\
285 \\
2,695 \\
3,507\end{array}$ & $\begin{array}{r}47 \\
4 \\
37 \\
- \\
-3 \\
3\end{array}$ & $\begin{array}{r}7,860 \\
2,555 \\
4,906 \\
- \\
105 \\
300\end{array}$ & $\begin{array}{r}6,695 \\
2412 \\
\frac{-}{4,090} \\
- \\
103 \\
\frac{100}{300}\end{array}$ & $\begin{array}{r}7,368 \\
2,417 \\
\frac{-}{4,537} \\
- \\
\frac{99}{300}\end{array}$ \\
\hline 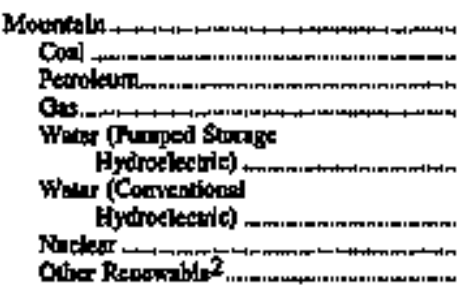 & $\begin{array}{r}849 \\
104 \\
112 \\
175 \\
12 \\
414 \\
3 \\
9\end{array}$ & $\begin{array}{r}3,641 \\
30,912 \\
499 \\
7,669 \\
697 \\
9,992 \\
4,210 \\
372\end{array}$ & $\begin{array}{r}50,503 \\
20,0026 \\
456 \\
6,537 \\
718 \\
9,908 \\
3,810 \\
49\end{array}$ & 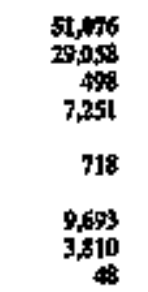 & $\begin{array}{r}16 \\
2 \\
1 \\
4 \\
- \\
= \\
-\end{array}$ & $\begin{array}{r}1,157 \\
625 \\
372 \\
- \\
51 \\
-\end{array}$ & $\begin{array}{r}972 \\
605 \\
2 \\
316 \\
- \\
-\end{array}$ & $\begin{array}{r}1,004 \\
610 \\
2 \\
36 \\
= \\
4 \\
=\end{array}$ \\
\hline 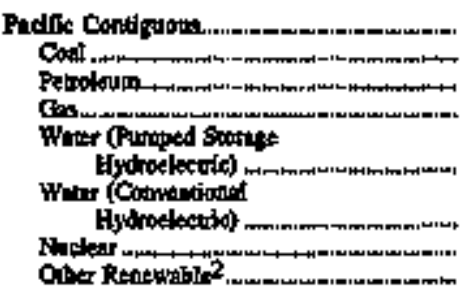 & $\begin{array}{r}1,154 \\
3 \\
54 \\
318 \\
35 \\
834 \\
5 \\
3\end{array}$ & $\begin{array}{r}17,790 \\
2,000 \\
2,064 \\
24,542 \\
3,6 \pm 2 \\
37,789 \\
5,755 \\
1,999\end{array}$ & $\begin{array}{r}7,025 \\
1,848 \\
1,803 \\
2,398 \\
3,991 \\
35,633 \\
5,417 \\
1,944\end{array}$ & $\begin{array}{r}70,396 \\
1,844 \\
1,546 \\
2,814 \\
3,955 \\
30,505 \\
5,409 \\
1,854\end{array}$ & $\begin{array}{l}24 \\
\frac{2}{10} \\
- \\
\frac{14}{-}\end{array}$ & $\begin{array}{l}\frac{44}{7} \\
567 \\
- \\
\frac{82}{-}\end{array}$ & $\begin{array}{l}86 \\
\frac{-}{465} \\
- \\
79 \\
-\end{array}$ & $\begin{array}{l}516 \\
\overline{5} \\
= \\
\overline{75} \\
\overline{-}\end{array}$ \\
\hline 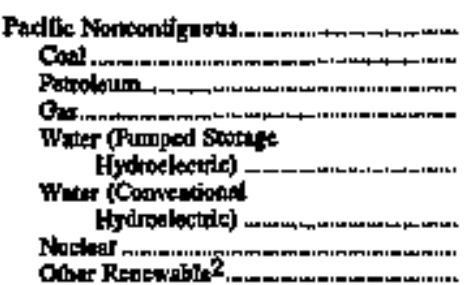 & $\begin{array}{r}655 \\
5 \\
559 \\
31\end{array}$ & $\begin{array}{r}3,588 \\
54 \\
2,266 \\
907 \\
-\end{array}$ & $\begin{array}{r}3,736 \\
54 \\
1,170 \\
754 \\
-\end{array}$ & $\begin{array}{r}3,472 \\
5,54 \\
2,212 \\
144\end{array}$ & $\begin{array}{r}\frac{19}{15} \\
- \\
\frac{1}{2}\end{array}$ & $\frac{180}{183}$ & $\begin{array}{r}164 \\
139 \\
- \\
\frac{1}{1}\end{array}$ & $\frac{110}{5}$ \\
\hline
\end{tabular}

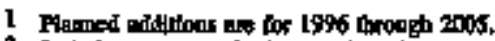

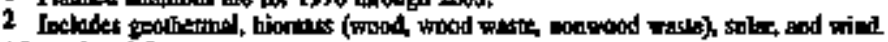

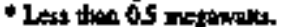

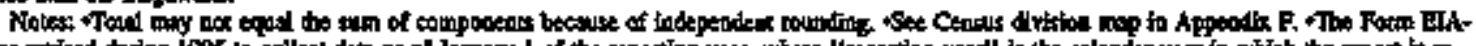

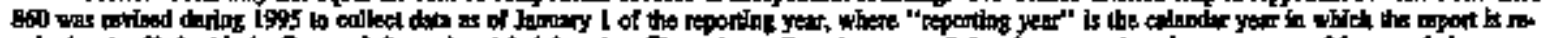

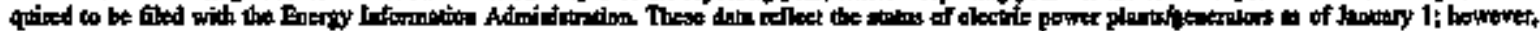

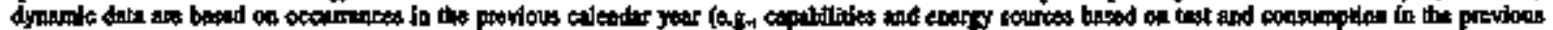
fear).

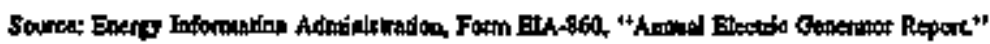


Table 17. Existing Capacity and Planned Capacity Additions at U.S. Electric Utilities by Energy Source and State, as of January 1, 1996

\begin{tabular}{|c|c|c|c|c|c|c|c|c|}
\hline \multirow[b]{2}{*}{$\begin{array}{c}\text { Stute } \\
\text { Pdinary Energy } \\
\text { Souret }\end{array}$} & \multicolumn{4}{|c|}{ Exwiting } & \multicolumn{4}{|c|}{ Puned Adations } \\
\hline & $\begin{array}{l}\text { Atoriber } \\
\text { of Unis }\end{array}$ & 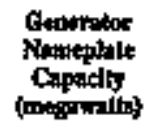 & 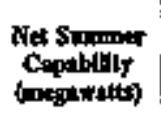 & 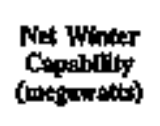 & $\begin{array}{l}\text { Number } \\
\text { of Ualins }\end{array}$ & 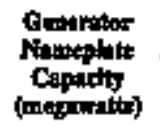 & 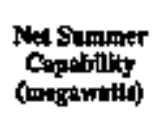 & 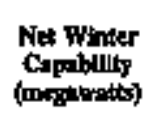 \\
\hline 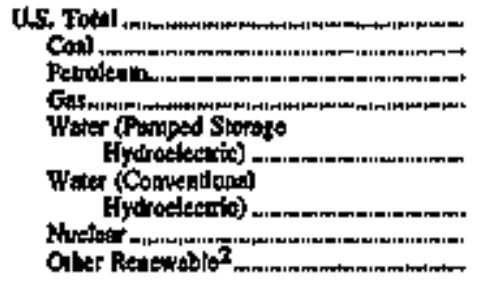 & $\begin{array}{r}20,396 \\
3,212 \\
3,277 \\
2,296 \\
140 \\
3,337 \\
109 \\
83\end{array}$ & 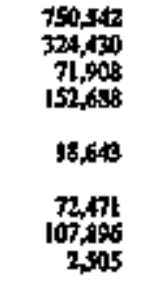 & $\begin{array}{r}706,111 \\
300,610 \\
64,464 \\
192,536 \\
21,387 \\
75,274 \\
99515 \\
2,326\end{array}$ & $\begin{array}{r}70,897 \\
303,121 \\
70,195 \\
147,796 \\
21,321 \\
74,901 \\
700,696 \\
2,327\end{array}$ & $\begin{array}{r}415 \\
13 \\
57 \\
253 \\
- \\
15 \\
11\end{array}$ & $\begin{array}{r}7,643 \\
5,165 \\
6,994 \\
38,328 \\
- \\
17270 \\
187\end{array}$ & 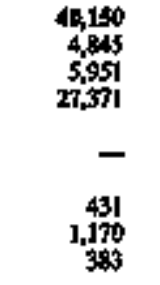 & $\begin{array}{r}4,595 \\
4,865 \\
6,802 \\
30,927 \\
- \\
416 \\
1,170 \\
384\end{array}$ \\
\hline 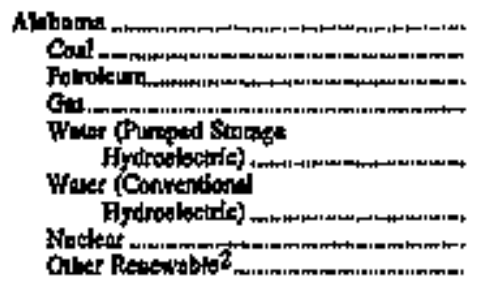 & $\begin{array}{r}158 \\
39 \\
1 \\
10\end{array}$ & $\begin{array}{r}21,79 \\
13,595 \\
21 \\
1,072 \\
- \\
2,858 \\
5,233 \\
-\end{array}$ & $\begin{array}{r}20,460 \\
11,667 \\
18 \\
978\end{array}$ & $\begin{array}{r}24,412 \\
1,57 \\
22 \\
1,113\end{array}$ & $\begin{array}{l}- \\
= \\
-\end{array}$ & $\begin{array}{r}1,69 \\
= \\
= \\
=\end{array}$ & $\frac{1,413}{1,413}$ & $\frac{1,616}{1,616}$ \\
\hline 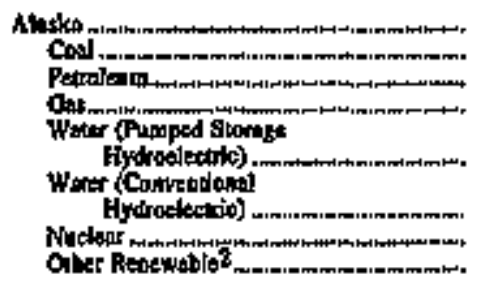 & $\begin{array}{l}55 \\
56 \\
31\end{array}$ & $\begin{array}{r}1,929 \\
54 \\
610 \\
907\end{array}$ & $\begin{array}{r}1,732 \\
54 \\
572 \\
754 \\
- \\
353 \\
-\end{array}$ & $\begin{array}{r}1,871 \\
54 \\
614 \\
843 \\
- \\
360 \\
-\end{array}$ & $\begin{array}{l}\frac{3}{1} \\
- \\
\frac{2}{1}\end{array}$ & $\begin{array}{l}\frac{6}{5} \\
5 \\
- \\
\frac{1}{4}\end{array}$ & $\begin{array}{l}\frac{6}{4} \\
- \\
\frac{1}{4}\end{array}$ & $\frac{5}{5}$ \\
\hline 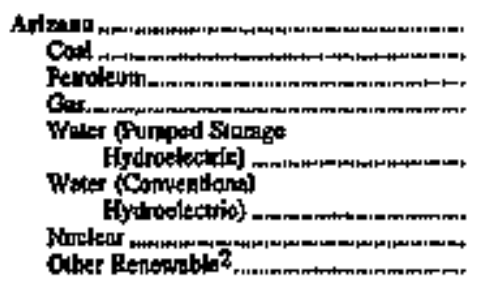 & $\begin{array}{r}129 \\
64 \\
7 \\
5 \\
6 \\
4 \\
3 \\
-\end{array}$ & $\begin{array}{r}16,667 \\
5,749 \\
121 \\
3,723 \\
109 \\
2,696 \\
4,210 \\
-\end{array}$ & $\begin{array}{r}15,231 \\
5,159 \\
95 \\
3,273 \\
185 \\
2,609 \\
3,810 \\
\end{array}$ & $\begin{array}{r}15,546 \\
5,159 \\
95 \\
3,397 \\
185 \\
2,699 \\
3,810 \\
\rightarrow\end{array}$ & $\begin{array}{l}\frac{3}{3} \\
- \\
= \\
=\end{array}$ & $\begin{array}{l}m \\
\overline{371} \\
- \\
=\end{array}$ & $\begin{array}{l}316 \\
\overline{316} \\
= \\
=\end{array}$ & $\frac{364}{364}$ \\
\hline 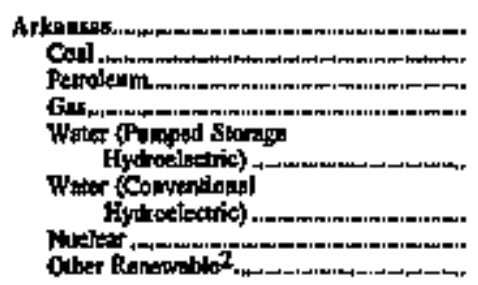 & $\begin{array}{r}105 \\
5 \\
31 \\
24 \\
1 \\
42 \\
2\end{array}$ & $\begin{array}{r}9,855 \\
3,958 \\
227 \\
2,628 \\
28 \\
1,168 \\
1,845 \\
-\end{array}$ & $\begin{array}{r}9,698 \\
3,817 \\
217 \\
2,585 \\
28 \\
1,297 \\
1,694 \\
-29\end{array}$ & $\begin{array}{r}9,899 \\
3,817 \\
217 \\
2,585 \\
28 \\
1,297 \\
1,694 \\
-\end{array}$ & $\begin{array}{l}\frac{3}{-} \\
- \\
\frac{3}{-}\end{array}$ & $\begin{array}{l}108 \\
= \\
- \\
= \\
=\end{array}$ & $\begin{array}{l}100 \\
= \\
- \\
\text { Ins } \\
-\end{array}$ & $\begin{array}{l}99 \\
= \\
- \\
- \\
9 \\
-\end{array}$ \\
\hline 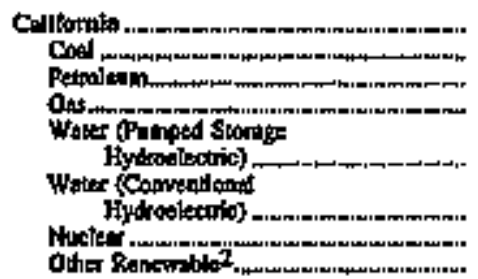 & $\begin{array}{r}178 \\
46 \\
168 \\
30 \\
402 \\
4 \\
28\end{array}$ & $\begin{array}{r}4,071 \\
1,857 \\
2,851 \\
3,352 \\
9,509 \\
4,555 \\
1,847\end{array}$ & $\begin{array}{r}0,3,02 \\
1,692 \\
22,040 \\
3,730 \\
9,800 \\
4,310 \\
1,723\end{array}$ & $\begin{array}{r}4,560 \\
1,796 \\
22,700 \\
3,724 \\
9,820 \\
4,360 \\
1,72\end{array}$ & $\begin{array}{l}\frac{16}{9} \\
- \\
\frac{7}{-}\end{array}$ & 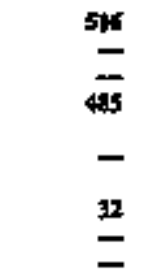 & $\begin{array}{l}446 \\
= \\
416 \\
- \\
= \\
=\end{array}$ & $\frac{7}{400}$ \\
\hline
\end{tabular}

Seo foothotios of end of ablith 
Table 17. Existing Capacify and Planned Capacity Additions at U.S. Mlectric Utilities by Energy Source and State, as of January 1, 1996 (Continued)

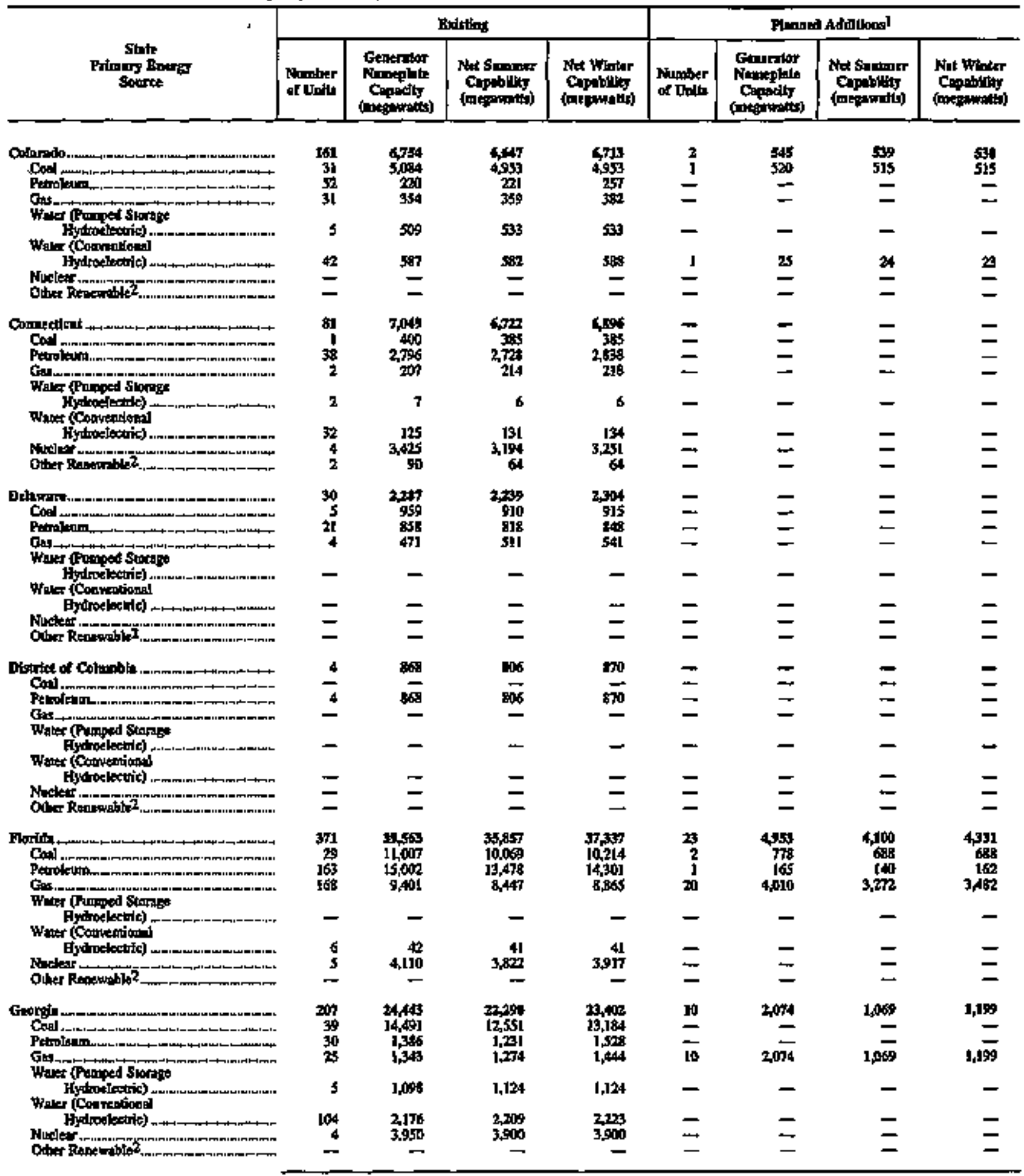

See footorots at end of urb. 
Table 17. Existing Capacity and Planned Capacity Additions at U.S. Electric Utllities by Energy Source and State, as of January 1 , 1996 (Contimued)

\begin{tabular}{|c|c|c|c|c|c|c|c|c|}
\hline \multirow[b]{2}{*}{ 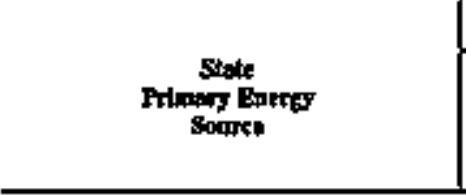 } & \multicolumn{4}{|c|}{ Erialleg } & \multicolumn{4}{|c|}{ Finnned Add'tzingl } \\
\hline & $\begin{array}{l}\text { Munber } \\
\text { s thets }\end{array}$ & 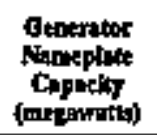 & 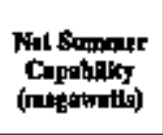 & 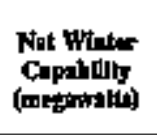 & $\begin{array}{l}\text { Nonbar } \\
\text { of Unils }\end{array}$ & 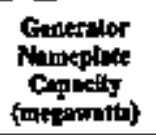 & 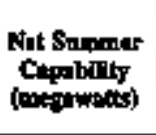 & 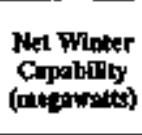 \\
\hline 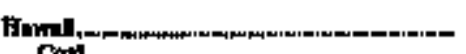 & 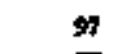 & 1,600 & 1,602 & $1, \infty 62$ & 14 & 183 & $1 \mathbf{s t}$ & 174 \\
\hline 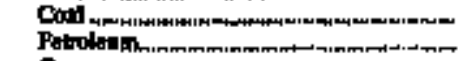 & $\overline{93}$ & $1, \overline{65 s}$ & 1,5958 & $\sqrt{5,98}$ & $\overline{14}$ & $\overline{189}$ & $\overline{1}$ & $\overline{174}$ \\
\hline 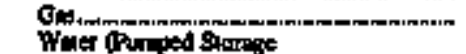 & - & - & - & - & - & - & - & - \\
\hline 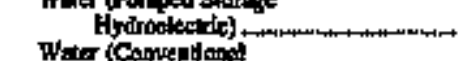 & - & - & - & - & $\rightarrow$ & - & - & + \\
\hline 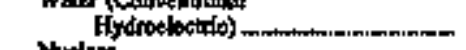 & 4 & 3 & 3 & 3 & - & - & - & - \\
\hline 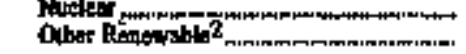 & $=$ & $\bar{z}$ & $\bar{z}$ & $z$ & $\bar{z}$ & 二 & $\bar{z}$ & 二 \\
\hline Lachon & $\mathbf{1 1 1}$ & 2,575 & 2,59 & 2,448 & 2 & 20 & $\mathbf{y}$ & $\mathbf{1 8}$ \\
\hline $\cos 1$. & - & - & - & - & - & - & - & - \\
\hline 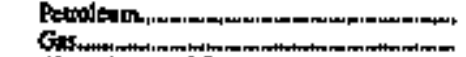 & $\begin{array}{l}2 \\
2\end{array}$ & 167 & $\begin{array}{l}6 \\
136\end{array}$ & 296 & $\bar{z}$ & $\Xi$ & $\bar{z}$ & $\Xi$ \\
\hline 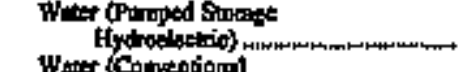 & - & - & - & - & - & 一 & - & - \\
\hline 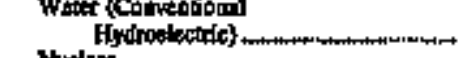 & 100 & 2203 & 2418 & 2,252 & 2 & 20 & 19 & 18 \\
\hline 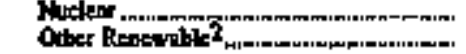 & $\bar{z}$ & $\bar{z}$ & $\bar{z}$ & $\bar{z}$ & $=$ & $\Xi$ & $=$ & $=$ \\
\hline Dilnobs. & 328 & 37,013 & 33,139 & 33,856 & 22 & 2,092 & 2,401 & 2,a10 \\
\hline Coll: & 56 & $\begin{array}{r}17,038 \\
3,015\end{array}$ & 14,916 & 15,005 & $\overline{1}$ & $\overline{5}$ & $\overline{5}$ & - \\
\hline Gan & 127 & 3,219 & 2904 & 3.135 & 17 & 2879 & 2418 & 2.783 \\
\hline 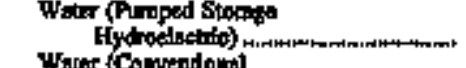 & - & - & - & - & - & - & - & - \\
\hline 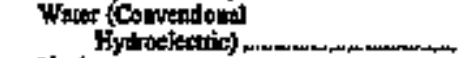 & 8 & & & & 4 & 8 & 7 & 7 \\
\hline 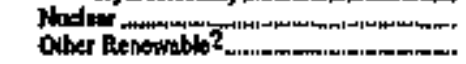 & $\underline{-3}$ & 93,734 & 12,609 & 12,296 & $\bar{z}$ & $=$ & $=$ & 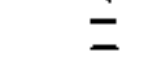 \\
\hline Ifallinitin & J6I & 25,1000 & 20,712 & 21,019 & 8 & 640 & 559 & 60 \\
\hline 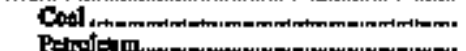 & 80 & 20967 & 18,850 & $\begin{array}{l}18990 \\
533\end{array}$ & $\bar{z}$ & $=$ & $=$ & $=$ \\
\hline Gon & 25 & 1,609 & 1.315 & 1,458 & 8 & 640 & $5 \$ 8$ & 643 \\
\hline 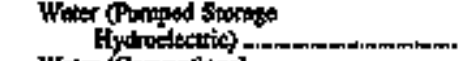 & - & - & - & - & - & - & - & - \\
\hline der (Compinitipial & $2 t$ & g & 68 & 68 & - & - & - & $\vec{a}$ \\
\hline 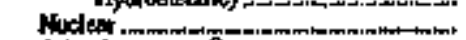 & 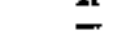 & - & - & 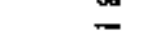 & - & - & - & $=$ \\
\hline 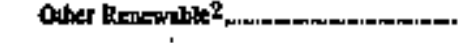 & - & - & - & - & - & - & - & - \\
\hline 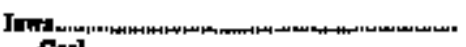 & and & 8,858 & 8,297 & 8 & 6 & 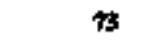 & $\omega$ & $\pi$ \\
\hline 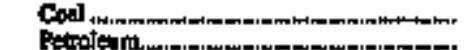 & 28 & $\frac{6298}{863}$ & $\begin{array}{l}5.995 \\
755\end{array}$ & $\begin{aligned} 6000 \\
8 \rightarrow 8\end{aligned}$ & 7 & $\overline{10}$ & $\overline{9}$ & $\bar{g}$ \\
\hline Gavo.: & 5 & $\stackrel{9}{9}$ & 835 & 1,020 & 2 & 63 & 54 & 62 \\
\hline 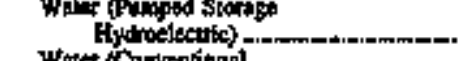 & - & - & - & - & - & $\rightarrow$ & $\rightarrow$ & - \\
\hline 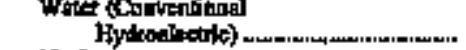 & 30 & 134 & 134 & 193 & - & - & - & - \\
\hline 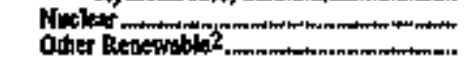 & i & 597 & 328 & 395 & $\bar{z}$ & $\ddot{z}$ & $=$ & $\bar{z}$ \\
\hline 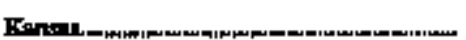 & $4: 18$ & 10,476 & 067 & 9754 & 10 & sat & 30 & 296 \\
\hline oit & 19 & 5,636 & 5,244 & $\$ 244$ & - & - & - & - \\
\hline 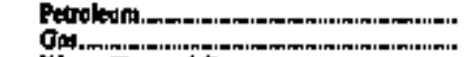 & 1212 & 2,935 & $\begin{array}{l}5,99 \\
3,695\end{array}$ & 2725 & $\stackrel{2}{8}$ & 301' & 251 & $\operatorname{los}$ \\
\hline 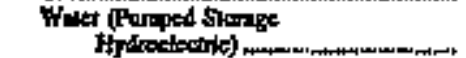 & - & - & - & - & - & - & - & - \\
\hline 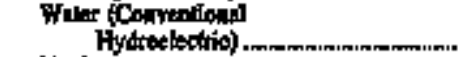 & & & & & - & - & - & $\rightarrow$ \\
\hline 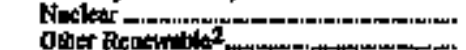 & $\underline{\mathbf{I}}$ & t,236 & 1,167 & 1,191 & $\bar{z}$ & $\bar{z}$ & $=$ & $z$ \\
\hline
\end{tabular}

See foolenest at end of ubte. 
Table 17. Existing Capacity and Planned Capacity Additions at US. Electric Utillties by Energy Source and State, as of January 1, 1996 (Continued)

\begin{tabular}{|c|c|c|c|c|c|c|c|c|}
\hline \multirow[b]{2}{*}{ 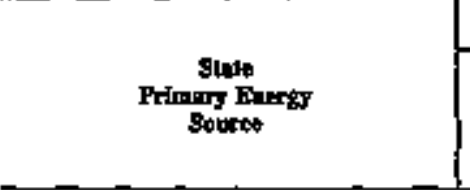 } & \multicolumn{4}{|c|}{ Balsting } & \multicolumn{4}{|c|}{ Planoed Adillkos I } \\
\hline & $\begin{array}{l}\text { Nonbs } \\
\text { of Lis's }\end{array}$ & 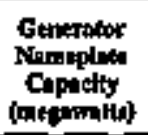 & 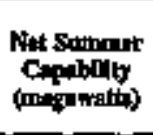 & 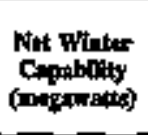 & $\begin{array}{l}\text { Nonber } \\
\text { of Untis }\end{array}$ & 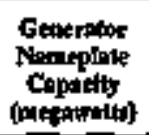 & 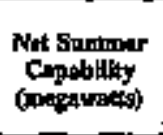 & 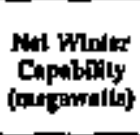 \\
\hline 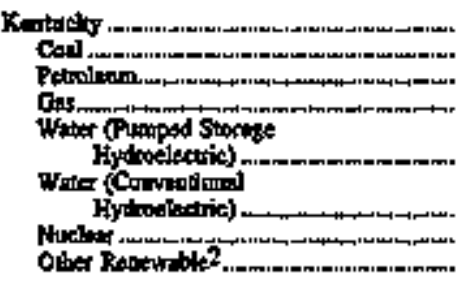 & $\begin{array}{r}12 \\
\text { 3: } \\
5 \\
9 \\
- \\
- \\
-\end{array}$ & $\begin{array}{r}17,579 \\
16,118 \\
278 \\
495 \\
- \\
748 \\
-\end{array}$ & $\begin{array}{r}15,425 \\
14,011 \\
\text { IE6 } \\
479 \\
- \\
789 \\
-\end{array}$ & $\begin{array}{r}15,612 \\
14, \frac{277}{200} \\
510 \\
- \\
\frac{675}{-}\end{array}$ & $\begin{array}{l}\frac{15}{2} \\
10 \\
- \\
\frac{3}{-}\end{array}$ & $\begin{array}{r}1,409 \\
\frac{146}{1,123} \\
- \\
\frac{70}{-}\end{array}$ & $\begin{array}{r}1,210 \\
\frac{10}{218} \\
9 \$ 56 \\
- \\
67 \\
- \\
-\end{array}$ & $\begin{array}{r}1,410 \\
\frac{251}{1,095} \\
- \\
\frac{6}{-}\end{array}$ \\
\hline 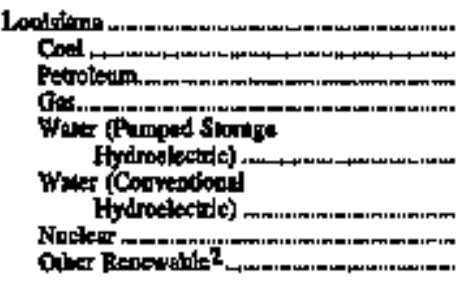 & $\begin{array}{r}109 \\
6 \\
2 \\
9 \\
-\end{array}$ & $\begin{array}{r}18,375 \\
3,000 \\
3,77 \\
13,061 \\
-\end{array}$ & $\begin{array}{r}17.019 \\
2,843 \\
35 \\
12.130 \\
-\end{array}$ & $\begin{array}{r}17,000 \\
2,343 \\
35 \\
12,131 \\
2.4 \\
2.11\end{array}$ & $\begin{array}{l}-2 \\
-2 \\
- \\
= \\
-\end{array}$ & $\begin{array}{l}249 \\
\frac{2}{249} \\
= \\
=\end{array}$ & $\begin{array}{r}212 \\
\frac{1}{212} \\
-\end{array}$ & $\frac{24}{244}$ \\
\hline 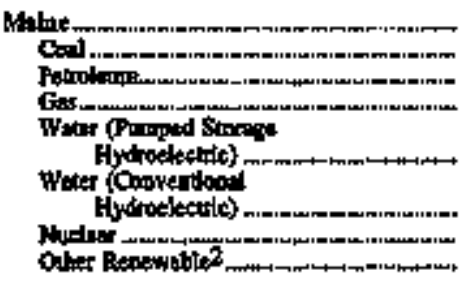 & $\begin{array}{r}- \\
141 \\
1\end{array}$ & $\frac{2,460}{1,130}$ & $\frac{2,452}{1,109}$ & $\frac{2,460}{1,127}$ & $\frac{5}{-}$ & $\begin{array}{l}- \\
\frac{45}{-}\end{array}$ & $\begin{array}{l}- \\
43 \\
-\end{array}$ & $\begin{array}{l}- \\
42 \\
-\end{array}$ \\
\hline 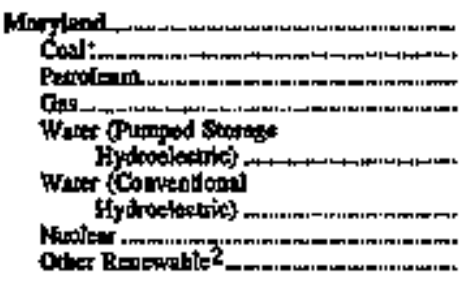 & $\begin{array}{r}104 \\
15 \\
4 \\
16\end{array}$ & $\begin{array}{r}11,762 \\
4,543 \\
1,501 \\
2,946 \\
- \\
1,894 \\
-\end{array}$ & 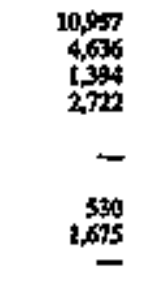 & $\begin{array}{r}1,3,311 \\
4,697 \\
1,516 \\
2,897\end{array}$ & $\begin{array}{r}17 \\
\vdots \\
5 \\
11 \\
- \\
- \\
-\end{array}$ & $\begin{array}{r}2736 \\
300 \\
24 t \\
t, 835 \\
= \\
= \\
=\end{array}$ & $\begin{array}{r}1,043 \\
300 \\
200 \\
1,575 \\
- \\
= \\
=\end{array}$ & $\begin{array}{r}2,274 \\
300 \\
2,76 \\
1,738 \\
= \\
=\end{array}$ \\
\hline 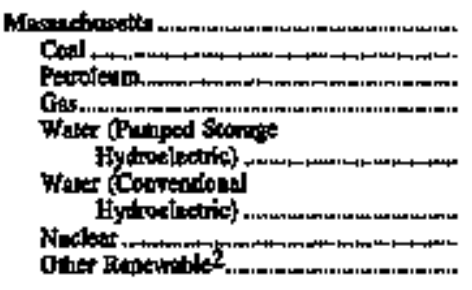 & $\begin{array}{r}206 \\
5 \\
101 \\
14 \\
5 \\
8 \\
8\end{array}$ & $\begin{array}{l}5,53 \\
1,764 \\
4,547 \\
1,005 \\
1,446 \\
201 \\
655 \\
4\end{array}$ & $\begin{array}{r}9,219 \\
1,707 \\
4,059 \\
993 \\
3,653 \\
207 \\
660 \\
4\end{array}$ & $\begin{array}{r}9,615 \\
1,724 \\
4,308 \\
1,040 \\
1,669 \\
208 \\
668 \\
1\end{array}$ & $\begin{array}{l}- \\
- \\
- \\
- \\
-\end{array}$ & $\begin{array}{l}- \\
- \\
- \\
- \\
-\end{array}$ & $\begin{array}{l}- \\
- \\
- \\
- \\
-\end{array}$ & $\begin{array}{l}- \\
\overrightarrow{-} \\
- \\
- \\
-\end{array}$ \\
\hline 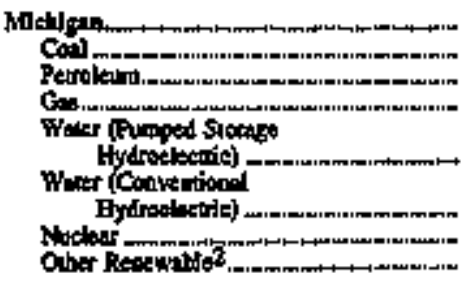 & $\begin{array}{r}557 \\
75 \\
167\end{array}$ & $\begin{array}{r}2,807 \\
12,727 \\
2,800 \\
1,567 \\
1,979\end{array}$ & $\begin{array}{r}21,5181 \\
11,794 \\
2,618 \\
1,444 \\
1,872 \\
263 \\
3,999 \\
-\end{array}$ & $\begin{array}{l}27,389 \\
11,400 \\
2,700 \\
1,596 \\
1,572 \\
27 ! \\
4,059 \\
-\end{array}$ & $\begin{array}{l}\frac{2}{2} \\
- \\
- \\
-\end{array}$ & $\begin{array}{l}\frac{3}{2} \\
- \\
- \\
-\end{array}$ & $\begin{array}{l}\frac{2}{2} \\
- \\
- \\
-\end{array}$ & $\begin{array}{l}\frac{2}{2} \\
- \\
- \\
- \\
-\end{array}$ \\
\hline
\end{tabular}

See footortes ar end of thble. 
Table 17. Existing Capacity and Planned Capacity Additions at U.S. Electric Utilities by Energy Source and State, as of January 1, 1996 (Continued)

\begin{tabular}{|c|c|c|c|c|c|c|c|c|}
\hline \multirow[b]{2}{*}{ 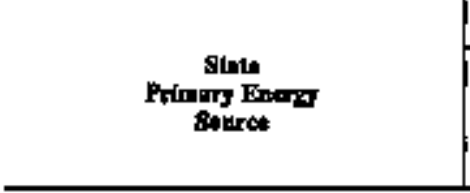 } & \multicolumn{4}{|c|}{ Entiding } & \multicolumn{4}{|c|}{ Planand Adtilloas I } \\
\hline & $\begin{array}{l}\text { Number } \\
\text { of Stintis }\end{array}$ & 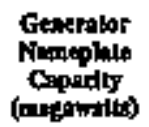 & $\begin{array}{l}\text { Nit Somner } \\
\text { Coqubility } \\
\text { (meganatits) }\end{array}$ & $\begin{array}{l}\text { Not Winter } \\
\text { Copobilly } \\
\text { fmegatity) }\end{array}$ & $\begin{array}{l}\text { Numbar } \\
\text { of Endls }\end{array}$ & 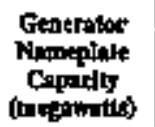 & 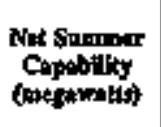 & 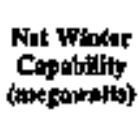 \\
\hline 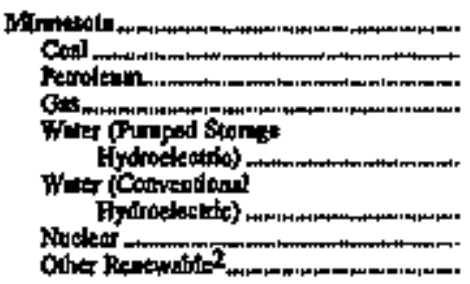 & $\begin{array}{r}376 \\
465 \\
96 \\
- \\
54 \\
30\end{array}$ & $\begin{array}{r}9,248 \\
5,638 \\
1,127 \\
493 \\
- \\
1,742 \\
94\end{array}$ & $\begin{array}{l}8,923 \\
5,600 \\
0,044\end{array}$ & $\begin{array}{r}9,196 \\
\text { s,619 } \\
1,251 \\
481 \\
- \\
1.42 \\
1.617 \\
83\end{array}$ & $\begin{array}{l}8 \\
\mathbf{1} \\
6 \\
\mathbf{1} \\
- \\
= \\
=\end{array}$ & $\begin{array}{r}24 \\
7 \\
11 \\
6 \\
- \\
= \\
=\end{array}$ & $\begin{array}{l}20 \\
11 \\
10 \\
= \\
=\end{array}$ & $\begin{array}{r}20 \\
11 \\
6 \\
= \\
= \\
=\end{array}$ \\
\hline 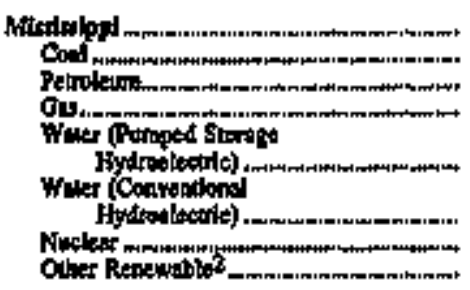 & $\begin{array}{l}5 \\
6 \\
2 \\
4 \\
- \\
-1 \\
-\end{array}$ & $\begin{array}{r}7,276 \\
2,150 \\
31 \\
3,723 \\
- \\
1,373 \\
-\end{array}$ & $\begin{array}{r}7,270 \\
2,255 \\
3,31 \\
3,711 \\
- \\
5,173 \\
-\end{array}$ & $\begin{array}{r}7,390 \\
2,208 \\
31 \\
3,758\end{array}$ & $\begin{array}{l}\frac{4}{4} \\
- \\
=\end{array}$ & $\begin{array}{l}\frac{3}{363} \\
- \\
= \\
=\end{array}$ & $\begin{array}{l}326 \\
= \\
325 \\
= \\
=\end{array}$ & $\begin{array}{l}372 \\
\overrightarrow{3 n} \\
= \\
=\end{array}$ \\
\hline 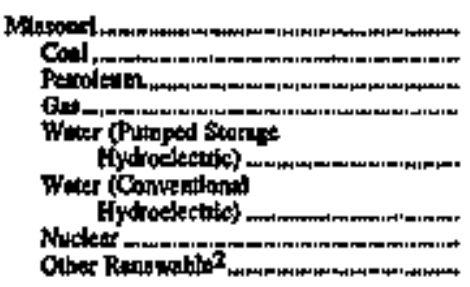 & $\begin{array}{r}39 \\
15 \\
195 \\
12 \\
9 \\
20 \\
1 \\
1\end{array}$ & $\begin{array}{r}37,014 \\
1,378 \\
1,909 \\
1,400 \\
601 \\
4,99 \\
1,296 \\
-\end{array}$ & $\begin{array}{r}15,74 \\
10,75 \\
1,710 \\
1,205 \\
565 \\
543 \\
2,125 \\
-\end{array}$ & $\begin{array}{r}15,904 \\
10,615 \\
1,866 \\
1,220 \\
492 \\
596 \\
1,169 \\
=\end{array}$ & $\begin{array}{l}24 \\
\frac{24}{10} \\
12 \\
- \\
= \\
-\end{array}$ & $\begin{array}{r}2,152 \\
\frac{531}{531} \\
= \\
= \\
=\end{array}$ & $\begin{array}{r}\frac{2,000}{452} \\
2,551 \\
= \\
= \\
=\end{array}$ & $\begin{array}{r}2,29 \\
520 \\
1,70 \\
= \\
=\end{array}$ \\
\hline 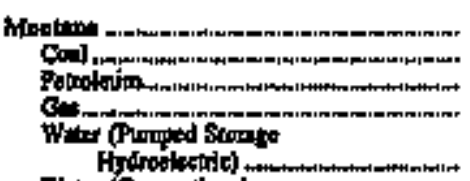 & $\frac{96}{3}$ & $\frac{1,596}{133}$ & $\frac{4043}{120}$ & $\frac{4,489}{141}$ & $\begin{array}{l}= \\
= \\
-\end{array}$ & $\begin{array}{l}\bar{z} \\
-\end{array}$ & $\begin{array}{l}\bar{z} \\
\bar{z}\end{array}$ & $\begin{array}{l}\bar{Z} \\
=\end{array}$ \\
\hline 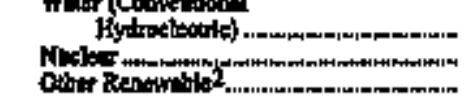 & $\frac{1}{2}$ & $\frac{2,487}{23}$ & $\frac{2,551}{13}$ & $\frac{2,52}{13}$ & $\vec{z}$ & $\bar{z}$ & $\underline{z}$ & $\bar{z}$ \\
\hline 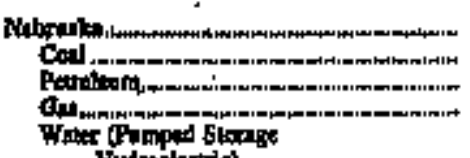 & 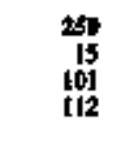 & $\begin{array}{r}5,77 \\
3,160 \\
388 \\
707\end{array}$ & $\begin{array}{c}\$ 5,59 \\
3,117 \\
331 \\
666\end{array}$ & 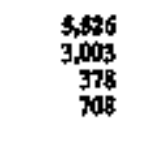 & $\frac{9}{4}$ & $\frac{15}{92}$ & $\frac{387}{79}$ & $\frac{644}{954}$ \\
\hline 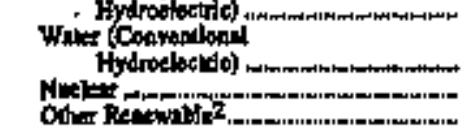 & $\begin{array}{r}2 \\
20 \\
2\end{array}$ & $\begin{array}{r}183 \\
1,396 \\
-\end{array}$ & $\begin{array}{r}167 \\
1,24 \\
-\end{array}$ & 1,157 & $\begin{array}{l}- \\
=\end{array}$ & $\begin{array}{l}- \\
\overline{-}\end{array}$ & $\begin{array}{l}= \\
\bar{z}\end{array}$ & $\begin{array}{l}- \\
=\end{array}$ \\
\hline 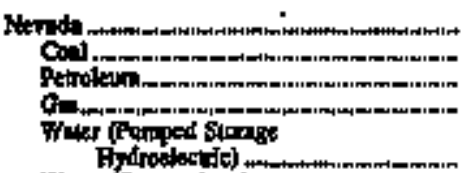 & $\begin{array}{r}70 \\
8 \\
21 \\
24 \\
-\end{array}$ & $\begin{array}{r}5,792 \\
2,769 \\
51, \\
1,918 \\
-\end{array}$ & $\begin{array}{r}5,566 \\
2,717 \\
50 \\
1,748 \\
-\end{array}$ & $\begin{array}{r}5,649 \\
2,717 \\
55 \\
1,890 \\
-\end{array}$ & $\begin{array}{l}1 \\
\frac{1}{2} \\
-\end{array}$ & $\begin{array}{l}105 \\
\frac{109}{-} \\
-\end{array}$ & $\begin{array}{l}90 \\
90 \\
= \\
-\end{array}$ & $\begin{array}{l}95 \\
95 \\
- \\
-\end{array}$ \\
\hline 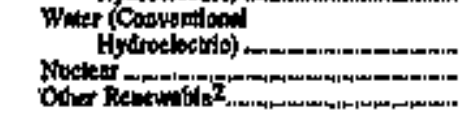 & $\frac{17}{-}$ & $\stackrel{1,046}{=}$ & $\underline{-1,046}$ & 1,047 & $\bar{z}$ & $\bar{z}$ & $\bar{z}$ & $\overline{-}$ \\
\hline
\end{tabular}

Sed fooknow at ond of twbe. 
Table 17. Existing Capacity and Plammed Capacity Additions at U.S. Electric Utitities by Energy Sonrce and State, as of January 1, 1996 (Continued)

\begin{tabular}{|c|c|c|c|c|c|c|c|c|}
\hline \multirow[b]{2}{*}{ 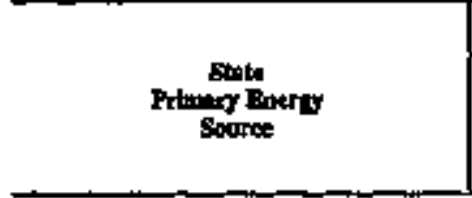 } & \multicolumn{4}{|c|}{ Ratifin: } & \multicolumn{4}{|c|}{ 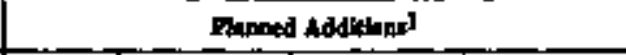 } \\
\hline & 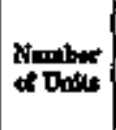 & 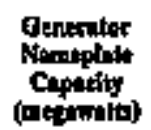 & 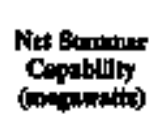 & 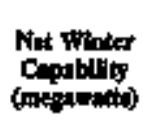 & $\begin{array}{l}\text { Nonber } \\
\text { if thitto }\end{array}$ & 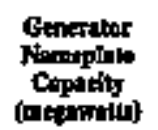 & 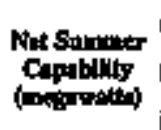 & 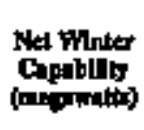 \\
\hline 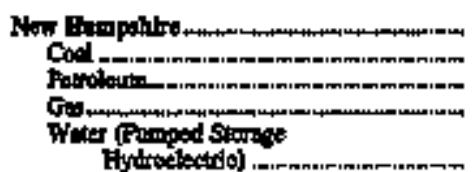 & $\begin{array}{r}44 \\
5 \\
-6\end{array}$ & $\begin{array}{r}2014 \\
509 \\
500 \\
-\end{array}$ & $\begin{array}{r}2846 \\
578 \\
409 \\
-\end{array}$ & $\begin{array}{r}2516 \\
796 \\
513 \\
- \\
-\end{array}$ & $\begin{array}{l}\bar{z} \\
=\end{array}$ & $\begin{array}{l}= \\
= \\
=\end{array}$ & $\begin{array}{l}\bar{z} \\
=\end{array}$ & $=$ \\
\hline 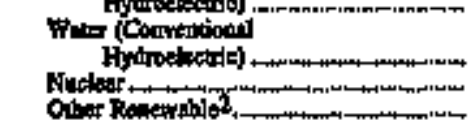 & $\begin{array}{l}- \\
12 \\
-\end{array}$ & $\underset{1,242}{2,24}$ & 1,135 & $\begin{array}{l}-\overline{156} \\
1,588 \\
-\end{array}$ & $\bar{z}$ & $\bar{z}$ & $=$ & $\begin{array}{l}= \\
z\end{array}$ \\
\hline 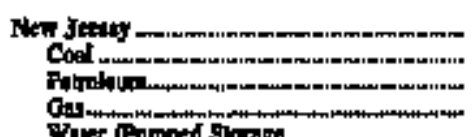 & $\begin{array}{r}117 \\
71 \\
37\end{array}$ & $\begin{array}{l}1,7,65 \\
1,710 \\
3,080 \\
5,031\end{array}$ & $\begin{array}{r}13,617 \\
1,629 \\
2,850 \\
3,056\end{array}$ & $\begin{array}{l}14,909 \\
1,658 \\
1,251 \\
5,70 \\
5,70\end{array}$ & $\frac{25}{25}$ & $\frac{3,107}{2,107}$ & $\frac{2,641}{2.041}$ & $\frac{3,045}{3,045}$ \\
\hline 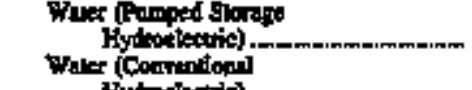 & 3 & 389 & 3900 & 390 & - & - & - & - \\
\hline 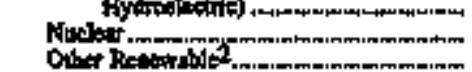 & -7 & $4 \sqrt{51}$ & $3, \overline{862}$ & 3,200 & $\Xi$ & $\bar{z}$ & $\Xi$ & こ \\
\hline 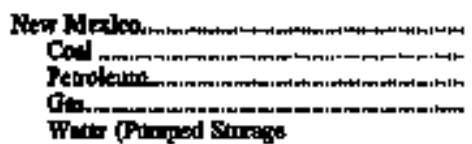 & $\begin{array}{r}55 \\
13 \\
7 \\
29\end{array}$ & $\begin{array}{l}5,199 \\
4,295 \\
1,49\end{array}$ & $\begin{array}{l}5,009 \\
3,909 \\
4,04\end{array}$ & $\begin{array}{l}5,095 \\
5,901 \\
1,060\end{array}$ & $\begin{array}{l}\bar{z} \\
\end{array}$ & $=$ & $=$ & $\bar{z}$ \\
\hline 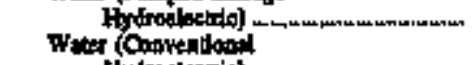 & - & - & - & - & - & - & - & - \\
\hline 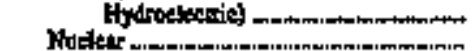 & 6 & -1 & 58 & SB & $=$ & $\bar{z}$ & $\vec{z}$ & $=$ \\
\hline 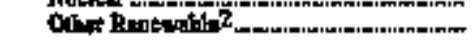 & - & - & - & - & - & - & - & ב \\
\hline 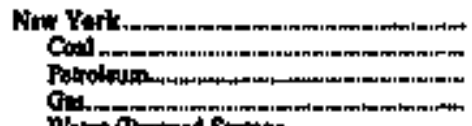 & $\begin{array}{c}56 \\
32 \\
135 \\
86\end{array}$ & $\begin{array}{l}32,460 \\
1,020 \\
8,558 \\
9,091\end{array}$ & $\begin{array}{l}3,1,16 \\
3,770 \\
7,610 \\
8,469\end{array}$ & $\begin{array}{r}33,302 \\
3,879 \\
8,263 \\
8,923\end{array}$ & $\frac{20}{2}$ & $\frac{66}{3}$ & $\frac{62}{3}$ & $\frac{6}{3}$ \\
\hline 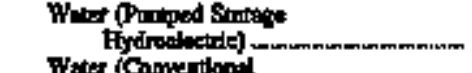 & 16 & 1,240 & 3,440 & 9,440 & - & - & - & - \\
\hline 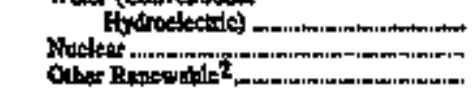 & $\frac{200}{6}$ & $\begin{array}{l}3,922 \\
5,624 \\
-\end{array}$ & $\begin{array}{l}3,906 \\
4,824 \\
-\end{array}$ & $\begin{array}{l}3,945 \\
4,902 \\
-\end{array}$ & $\frac{18}{2}$ & $\frac{62}{2}$ & $\frac{59}{=}$ & $\stackrel{s}{=}$ \\
\hline 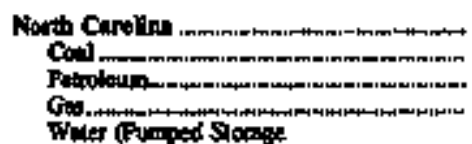 & $\begin{array}{l}195 \\
45 \\
41\end{array}$ & $\begin{array}{r}20,58 \\
2,994 \\
2,098 \\
333\end{array}$ & $\begin{array}{r}20,97 \\
12,440 \\
1,676 \\
314\end{array}$ & $\begin{array}{r}21991 \\
12513 \\
2037 \\
315\end{array}$ & $\frac{21}{11}$ & $\begin{array}{l}4,726 \\
3, \tilde{106} \\
1,520\end{array}$ & 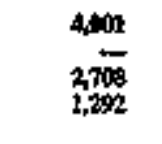 & $\stackrel{4,600}{3,463}$ \\
\hline 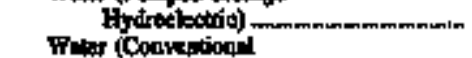 & - & - & - & - & - & - & - & - \\
\hline 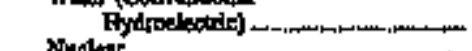 & B2 & 1,506 & 1,5038 & 1,418 & 一 & - & - & - \\
\hline 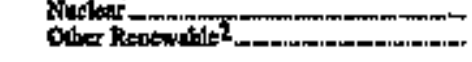 & -5 & 5,125 & 4069 & 4.039 & 二 & $\overline{-}$ & $\bar{z}$ & 二 \\
\hline 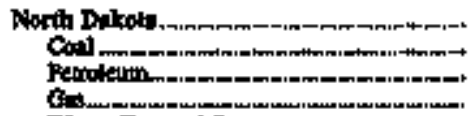 & $\begin{array}{l}46 \\
12 \\
27 \\
2\end{array}$ & $\begin{array}{r}4006 \\
700 \\
7 \\
8\end{array}$ & $\begin{array}{r}4.45 \\
3 \times 282 \\
69 \\
10\end{array}$ & $\begin{array}{r}4,56 \\
3,918 \\
83 \\
10\end{array}$ & $\begin{array}{l}= \\
z\end{array}$ & $\begin{array}{l}\bar{z} \\
=\end{array}$ & $\bar{z}$ & $\begin{array}{l}z \\
z\end{array}$ \\
\hline Wrer (Puppod Stongs" & - & - & - & - & - & - & $=$ & - \\
\hline Eiydrotestic) .... & 5 & 517 & sas & sts & - & - & - & - \\
\hline 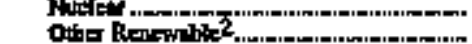 & $=$ & $=$ & 二 & 二 & $\vec{z}$ & $\Xi$ & 二 & 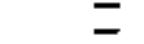 \\
\hline
\end{tabular}

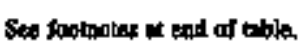


Table 17. Existing Capacity and Planned Capacity Additions at U.S. Blectric Utilities by Energy Source and State, as of January 1, 1996 (Continued)

\begin{tabular}{|c|c|c|c|c|c|c|c|c|}
\hline \multirow[b]{2}{*}{ 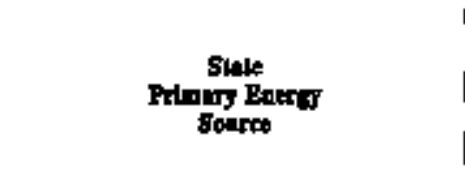 } & \multicolumn{4}{|c|}{ Edulne } & \multicolumn{4}{|c|}{ Fimaned Adelilond } \\
\hline & Nember & 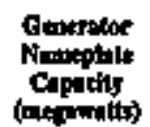 & 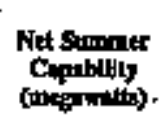 & 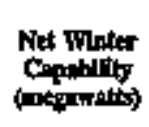 & $\begin{array}{l}\text { Nonber } \\
\text { of Uhifs }\end{array}$ & 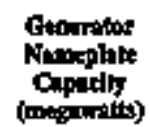 & Net Spaponer & 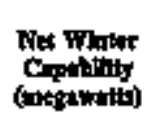 \\
\hline 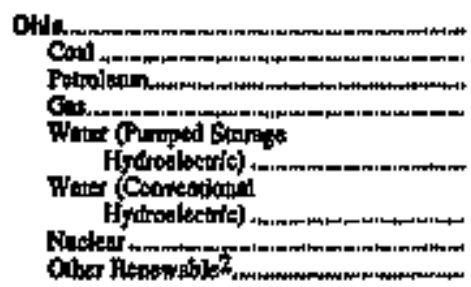 & $\begin{array}{r}245 \\
122 \\
72 \\
39 \\
- \\
? \\
2 \\
3\end{array}$ & 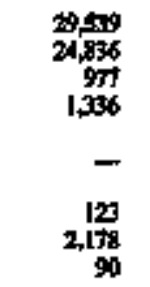 & 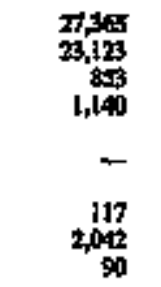 & $\begin{array}{r}29,004 \\
23,411 \\
1,046 \\
1,336 \\
= \\
2,24 \\
2,078 \\
90\end{array}$ & $\begin{array}{l}\frac{35}{29} \\
- \\
\frac{4}{-}\end{array}$ & $\begin{array}{r}3,810 \\
= \\
\frac{7}{4}, 767 \\
=\end{array}$ & $\begin{array}{r}3, \sqrt{58} \\
\frac{7}{3,155} \\
- \\
=\end{array}$ & $\begin{array}{r}3,600 \\
\frac{7}{3,037} \\
- \\
\frac{23}{-}\end{array}$ \\
\hline 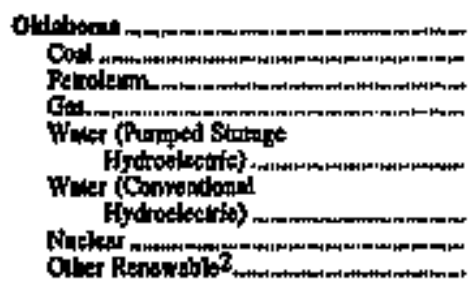 & $\begin{array}{r}155 \\
10 \\
27 \\
80 \\
6 \\
32 \\
=\end{array}$ & $\begin{array}{r}13,49 \\
5,206 \\
64 \\
745 \\
204 \\
=\end{array}$ & $\begin{array}{r}12,028 \\
4,031 \\
5,58 \\
7,004 \\
200 \\
776 \\
=\end{array}$ & $\begin{array}{r}12,0 \% 9 \\
4,835 \\
5,070 \\
200 \\
776 \\
=\end{array}$ & $\begin{array}{l}\frac{7}{7} \\
= \\
=\end{array}$ & $\begin{array}{l}9 \overline{7} \\
\frac{9}{7} \\
= \\
=\end{array}$ & $\begin{array}{l}703 \\
\frac{7}{703} \\
= \\
=\end{array}$ & $\begin{array}{l}\frac{787}{287} \\
- \\
=\end{array}$ \\
\hline 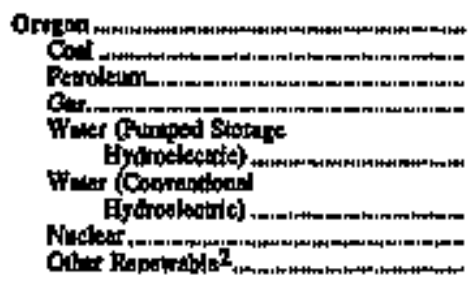 & $\begin{array}{r}16 \\
\frac{1}{2} \\
13 \\
- \\
\frac{17}{2}\end{array}$ & $\begin{array}{r}5,140 \\
561 \\
113 \\
935 \\
- \\
\frac{51}{52}\end{array}$ & $\begin{array}{r}16,406 \\
500 \\
100 \\
700 \\
- \\
\frac{9,0351}{35}\end{array}$ & $\begin{array}{r}10,556 \\
500 \\
116 \\
172 \\
- \\
\frac{9090}{35}\end{array}$ & $\begin{array}{l}= \\
= \\
= \\
=\end{array}$ & $\begin{array}{l}\bar{z} \\
= \\
=\end{array}$ & $\begin{array}{l}\bar{z} \\
= \\
= \\
=\end{array}$ & $\begin{array}{l}= \\
= \\
= \\
=\end{array}$ \\
\hline 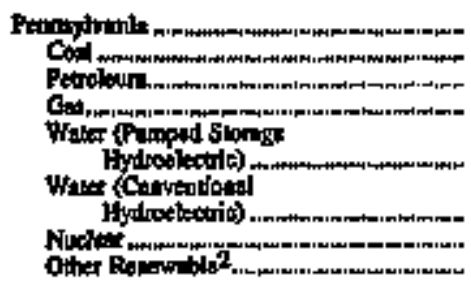 & $\begin{array}{r}234 \\
59 \\
107 \\
14 \\
10 \\
35 \\
9 \\
-\end{array}$ & $\begin{array}{r}36,937 \\
19,267 \\
5,606 \\
496 \\
1,196 \\
6,62 \\
9,036 \\
-\end{array}$ & 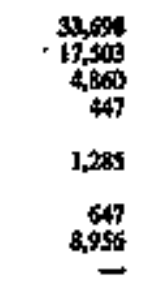 & 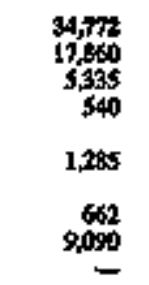 & $\begin{array}{l}\frac{1}{1} \\
- \\
=\end{array}$ & $\begin{array}{l}\frac{108}{165} \\
- \\
=\end{array}$ & $\begin{array}{l}140 \\
= \\
=\end{array}$ & $\begin{array}{l}16 \\
\frac{1}{162} \\
- \\
=\end{array}$ \\
\hline 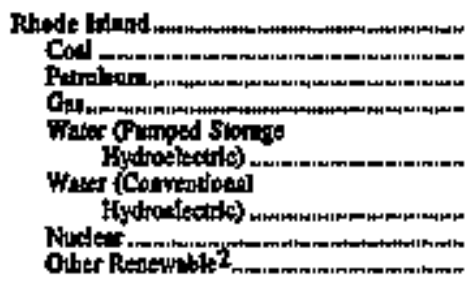 & $\begin{array}{l}\frac{18}{14} \\
\frac{3}{1} \\
\frac{1}{-}\end{array}$ & $\begin{array}{r}\frac{512}{2 I} \\
499 \\
- \\
\frac{2}{2}\end{array}$ & $\frac{42}{2 y 3}$ & $\begin{array}{l}\frac{505}{71} \\
513 \\
- \\
\frac{1}{-}\end{array}$ & $\begin{array}{l}= \\
= \\
= \\
=\end{array}$ & $\begin{array}{l}= \\
= \\
= \\
=\end{array}$ & $\begin{array}{l}= \\
= \\
= \\
=\end{array}$ & $\begin{array}{l}\bar{z} \\
= \\
=\end{array}$ \\
\hline 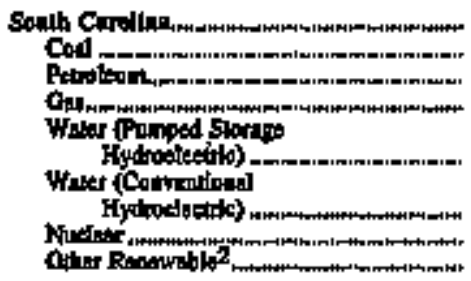 & $\begin{array}{r}216 \\
16 \\
11 \\
11 \\
11 \\
\frac{11}{7}\end{array}$ & $\begin{array}{r}18,013 \\
5,915 \\
1,413 \\
4,57 \\
2,186 \\
4,2,59 \\
6,799\end{array}$ & $\begin{array}{r}16,701 \\
5,352 \\
1,192 \\
, 345 \\
2,167 \\
1,762 \\
6,364 \\
-\end{array}$ & $\begin{array}{r}17,024 \\
5,394 \\
1,359 \\
416 \\
2,107 \\
1,262 \\
6,408 \\
-\end{array}$ & $\begin{array}{l}\mathbf{g} \\
\frac{1}{3} \\
- \\
= \\
-\end{array}$ & $\begin{array}{r}1,315 \\
-758 \\
- \\
=\end{array}$ & $\begin{array}{r}1,45 \\
\text { 135 } \\
-678 \\
- \\
=\end{array}$ & $\frac{1,167}{385}$ \\
\hline
\end{tabular}

See sootnots at eod of toble. 
Table 17. Existing Capacity and Planned Capacity Addittons at U.S. Electric. Utilities by Energy Source and State, as of January 1, 1996 (Continued)

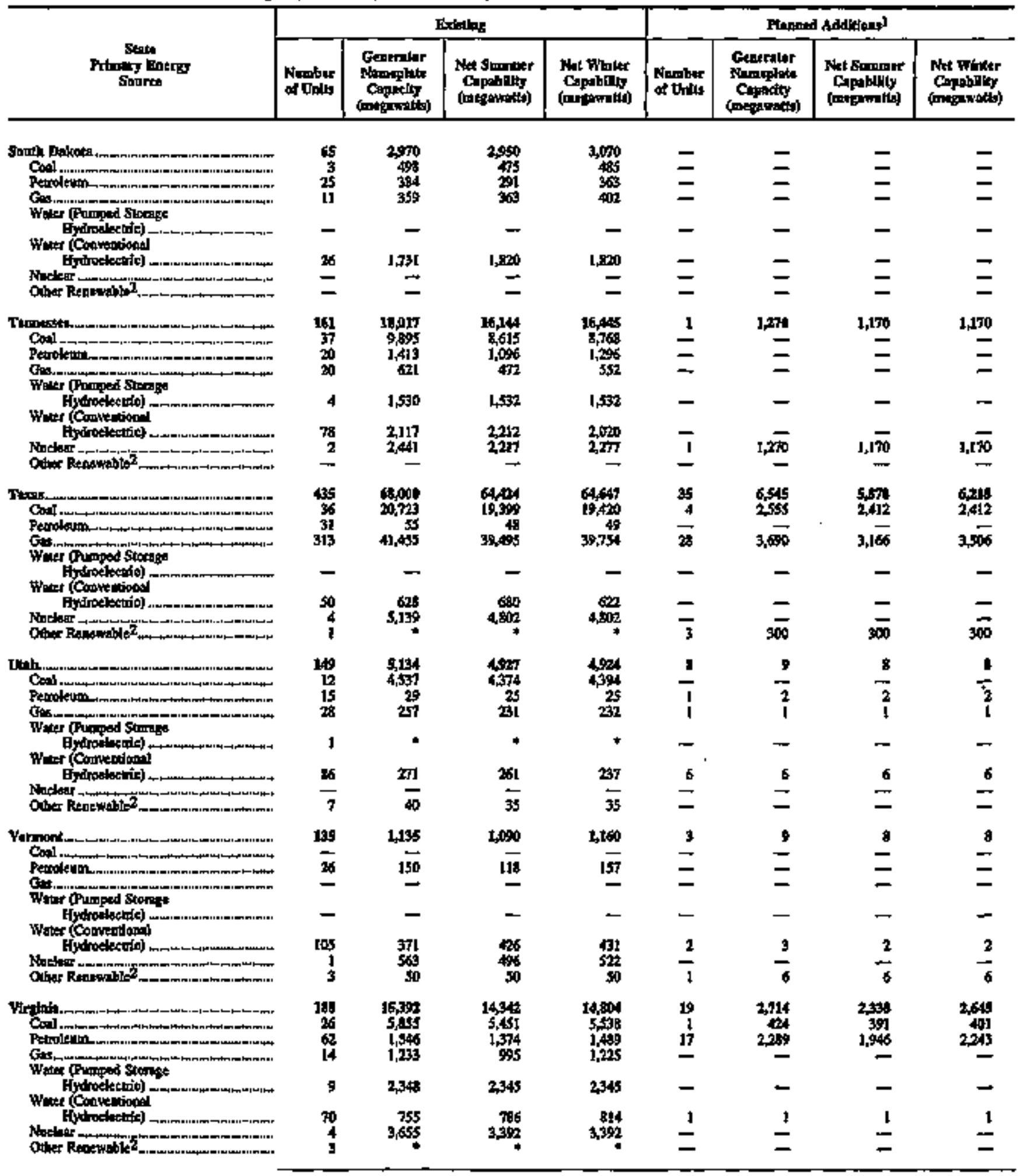

Sec toomotat at and of thble 
Table 17. Exdsting Capacity and Planned Capacity Additions at U.S. Electric Utilities by Energy Source and State, as of January 1, 1996 (Continued)

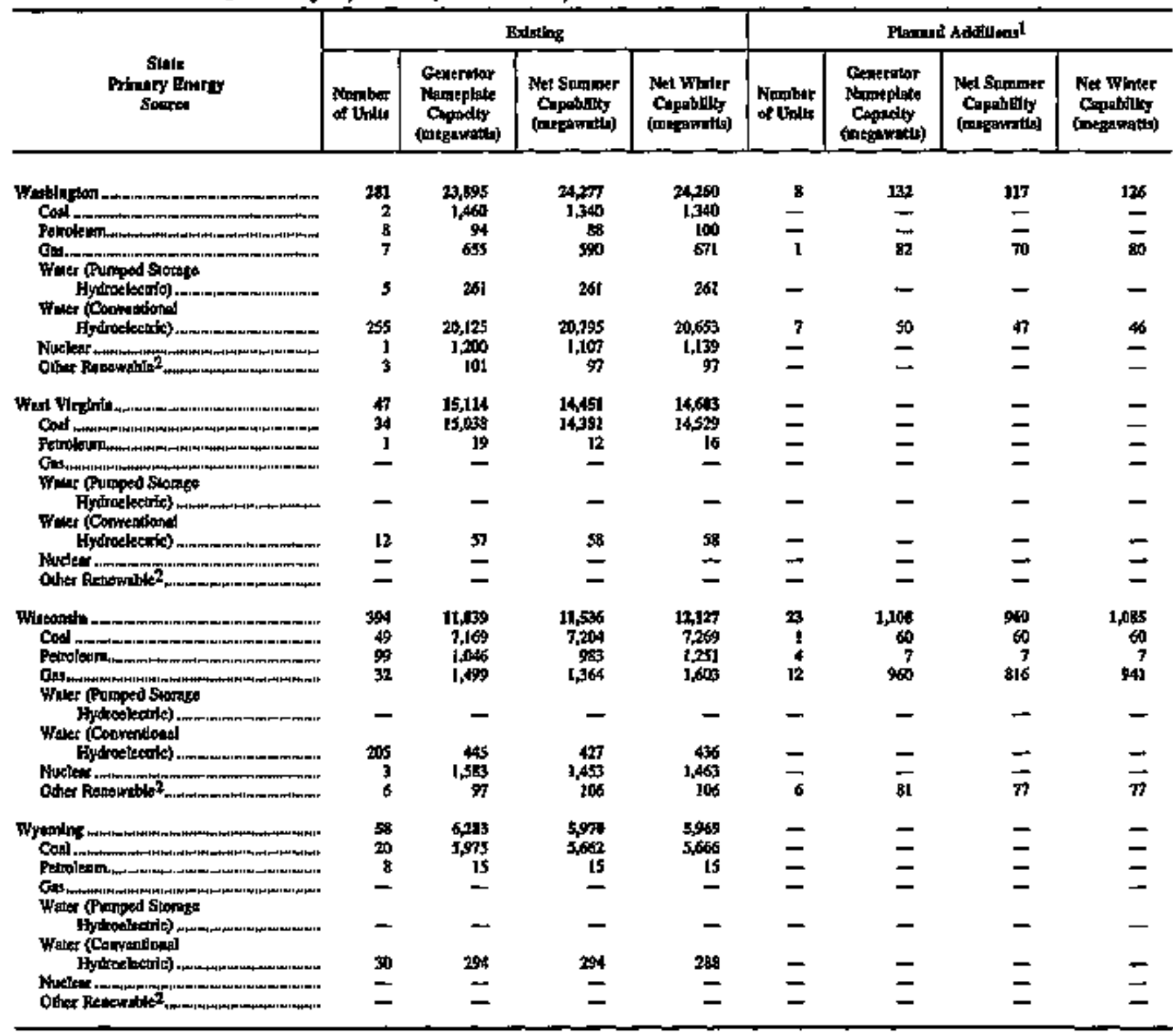

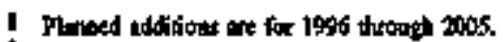

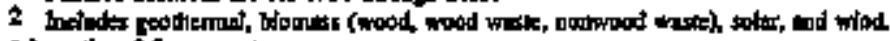

- Lete tha 0.5 metuwatis.

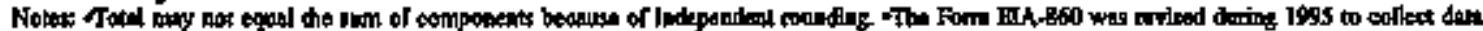

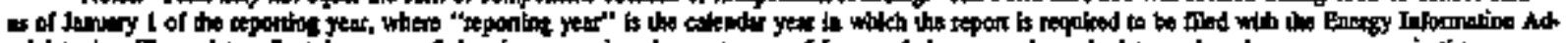

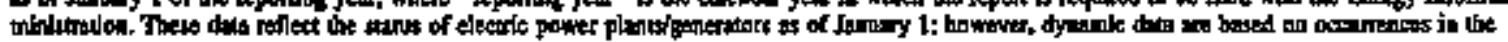

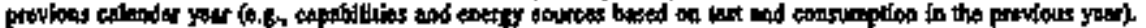

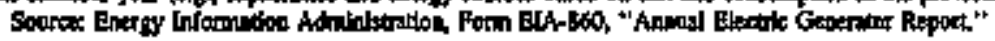


Table 18. Generating Units that Started Operation at US. Electric Utilities by State, Company, and Plant, 1995

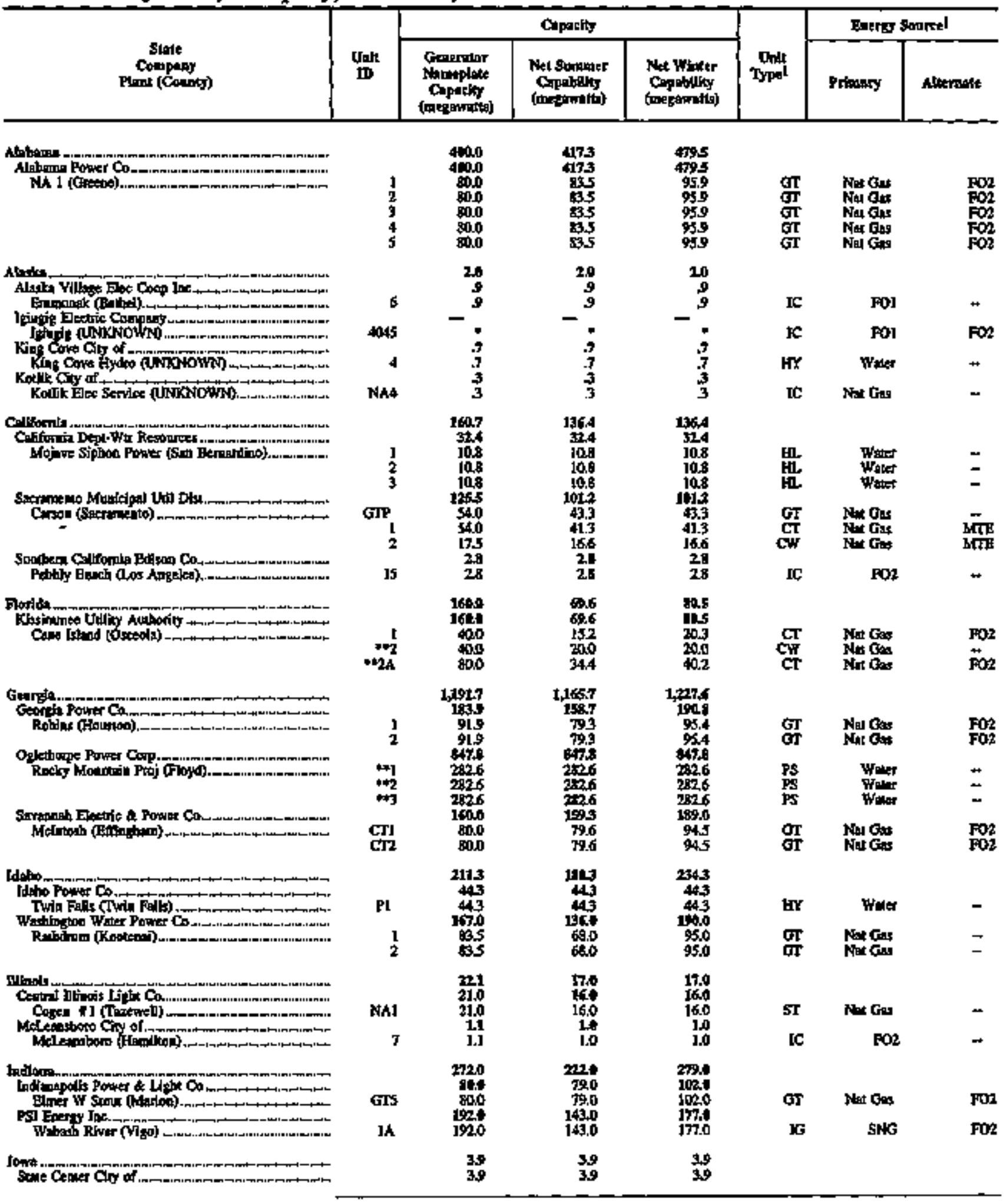

Ste foctistet at end of table.

42 Energy Information Administration/Inventory of Power Pleats in the United States as of January 1 , 1996 
Table 18. Generating Units that Started Operation at U.S. Electric Utilities by State, Company, and Plant, 1993 (Continued)

\begin{tabular}{|c|c|c|c|c|c|c|c|}
\hline \multirow[b]{2}{*}{ 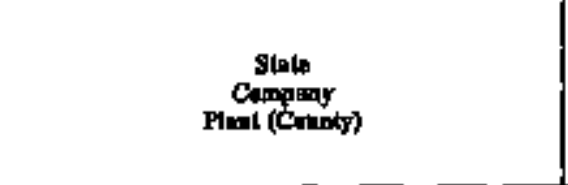 } & \multirow[b]{2}{*}{ to } & \multicolumn{3}{|c|}{ Capedity } & \multirow[b]{2}{*}{ Tyly } & \multicolumn{2}{|c|}{ Dneray Somex } \\
\hline & & 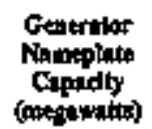 & 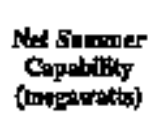 & 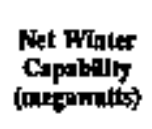 & & Prtingy & Alerpatta \\
\hline 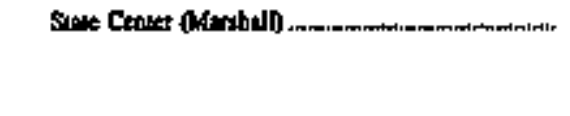 & $\begin{array}{l}1 \\
2 \\
3 \\
4\end{array}$ & $\begin{array}{r}0.6 \\
.6 \\
1.4 \\
1.4\end{array}$ & $\begin{array}{r}0.6 \\
.6 \\
1.4\end{array}$ & $\begin{array}{r}0.6 \\
1.4 \\
1.4\end{array}$ & $\begin{array}{l}\mathbf{K} \\
\mathbf{K C} \\
\mathbf{K C}\end{array}$ & $\begin{array}{l}\text { FOI } \\
\text { FOI } \\
\text { FOI } \\
\text { FOI }\end{array}$ & $\ddot{\ddot{z}} \bar{z}$ \\
\hline 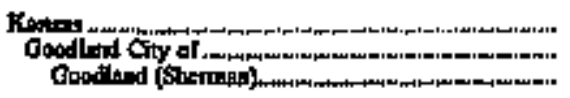 & 12 & $\begin{array}{l}1.0 \\
1.0 \\
1.0\end{array}$ & $\$$ & $\begin{array}{l}1.0 \\
1.0 \\
1.0\end{array}$ & $\mathbf{K}$ & Nat OH & $\mathrm{Fot}$ \\
\hline 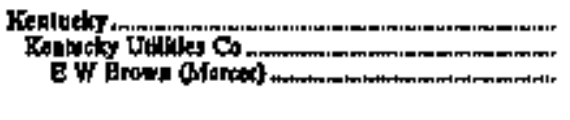 & 20 & $\begin{array}{l}2580 \\
2380 \\
1190 \\
1190\end{array}$ & $\begin{array}{l}219.0 \\
219.0 \\
110.0 \\
\text { t09.0 }\end{array}$ & $\begin{array}{l}245,0 \\
245.0 \\
110.0 \\
135,0\end{array}$ & GT & $\begin{array}{l}\text { Not fon } \\
\text { Wat Cox }\end{array}$ & $\begin{array}{l}\mathbf{F O 2} \\
\mathbf{E O Z}\end{array}$ \\
\hline 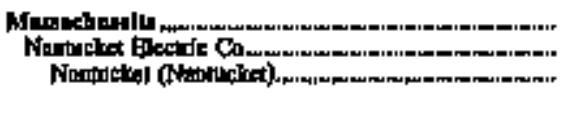 & 14 & $\begin{array}{l}5.0 \\
5.0 \\
2.5 \\
2.5\end{array}$ & $\begin{array}{l}60 \\
50 \\
25 \\
25\end{array}$ & $\begin{array}{l}\$ .0 \\
\$ .0 \\
2.5 \\
2.5\end{array}$ & $\underset{\mathbf{I C}}{\mathrm{IC}}$ & $\begin{array}{l}\text { FOZ } \\
\text { FOZ }\end{array}$ & $\stackrel{+}{-}$ \\
\hline 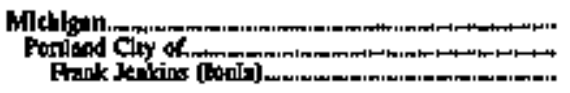 & $\mathbf{5}$ & $\begin{array}{l}20 \\
20 \\
20\end{array}$ & $\begin{array}{l}1.7 \\
1.7 \\
1.7\end{array}$ & $\begin{array}{l}1.7 \\
1.7 \\
1.7\end{array}$ & $\mathrm{k}$ & $\mathrm{FOP}$ & $\mathrm{Kax} G=$ \\
\hline 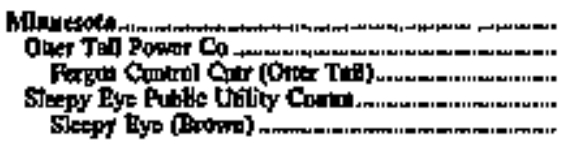 & I & $\begin{array}{l}3.8 \\
20 \\
2.0 \\
1.8 \\
1.8\end{array}$ & $\begin{array}{l}3.8 \\
\mathbf{2 0} \\
20 \\
\mathbf{1 . 0} \\
1.8\end{array}$ & $\begin{array}{l}3.8 \\
2.0 \\
2.0 \\
1.8 \\
1.8\end{array}$ & k & $\begin{array}{l}F O 2 \\
F O 2\end{array}$ & - \\
\hline 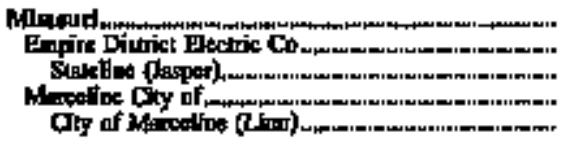 & $\begin{array}{l}1 \\
4\end{array}$ & $\begin{array}{r}98.4 \\
98.0 \\
98.0 \\
4 \\
.4\end{array}$ & $\begin{array}{r}38.4 \\
900 \\
940 \\
4\end{array}$ & $\begin{array}{r}98.4 \\
4.0 \\
4 \\
4 \\
40.0\end{array}$ & cr & $\begin{array}{l}\text { Nest Ches } \\
\text { FOC }\end{array}$ & $\begin{array}{l}\text { FOQ } \\
=\end{array}$ \\
\hline 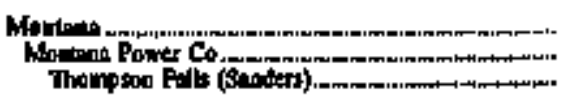 & 7 & $\begin{array}{l}516 \\
526 \\
516\end{array}$ & $\begin{array}{l}364 \\
364 \\
364\end{array}$ & $\begin{array}{l}364 \\
364 \\
364\end{array}$ & Hr & Witer & - \\
\hline 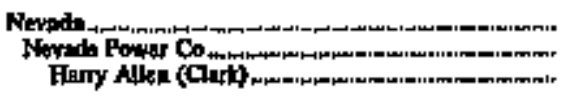 & GIJ & $\begin{array}{l}780 \\
780\end{array}$ & $\begin{array}{l}7.0 \\
7 \$ .0 \\
7 \% .0\end{array}$ & 70.0 & GI & Nel Gar & $\mathbf{F O}$ \\
\hline 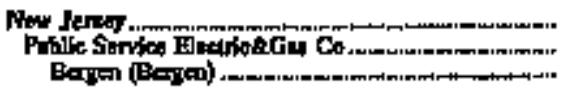 & $s$ & $\begin{array}{l}3257 \\
3252\end{array}$ & $\begin{array}{l}27.00 \\
20.0 \\
20.0\end{array}$ & $\begin{array}{l}2200 \\
2000\end{array}$ & CW & Nat Cux & - \\
\hline 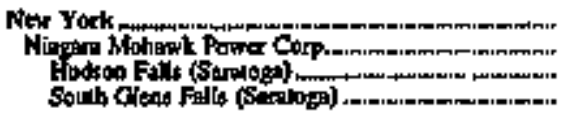 & $\hat{N B}$ & $\begin{array}{l}49.9 \\
49.9 \\
36.1 \\
19.8\end{array}$ & $\begin{array}{l}45,0 \\
45.0 \\
35.0 \\
10.0\end{array}$ & $\begin{array}{l}4.0 \\
450 \\
35.0 \\
10.0\end{array}$ & HY & What & $\ddot{n}$ \\
\hline 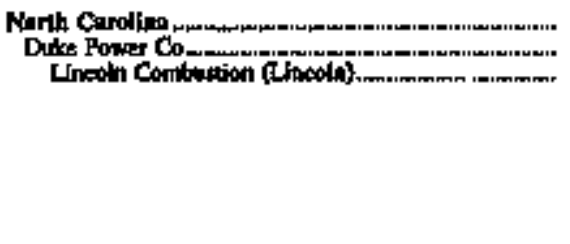 & $\begin{array}{r}1 \\
10 \\
11 \\
12 \\
2 \\
3 \\
4\end{array}$ & $\begin{array}{r}1,1616 \\
1,161.6 \\
96.8 \\
96.8 \\
96.8 \\
968 \\
968 \\
968 \\
968\end{array}$ & $\begin{array}{l}9010 \\
9040 \\
75.0 \\
75.0 \\
75.0 \\
75.0 \\
750 \\
750 \\
75.0\end{array}$ & $\begin{array}{r}\text { L.188.0 } \\
\text { Lt\$8.0 } \\
99.0 \\
99.0 \\
99.0 \\
99.0 \\
99.0 \\
99.0 \\
99.0\end{array}$ & 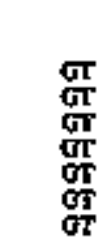 & $\begin{array}{l}\mathrm{FO2} \\
\mathrm{FO2} \\
\mathrm{FO2} \\
\mathrm{FO2} \\
\mathrm{EO2} \\
\mathrm{FO2} \\
\mathrm{FO2}\end{array}$ & 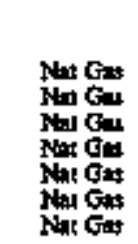 \\
\hline
\end{tabular}

Sec foutroks al and of tohice 
Table 18. Generating Units that Started Operation at U.S. Electric Utilities by State, Company, and Plant, 1995 (Continued)

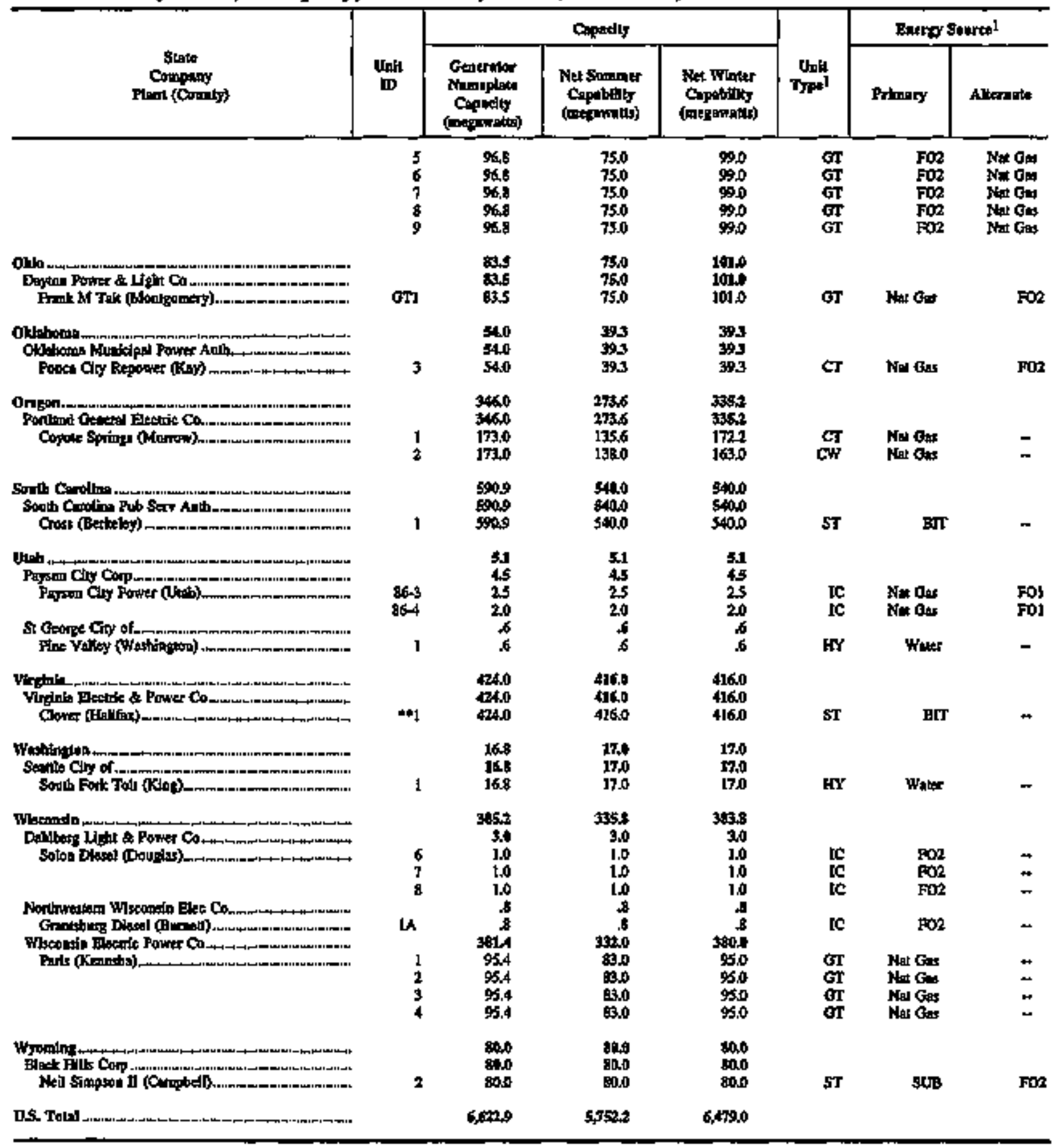

1 See Appendix B for codins

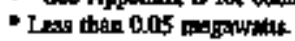

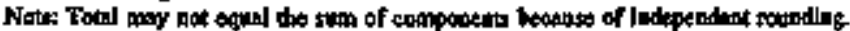

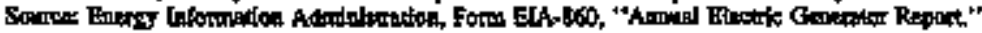


Table 19. Generating Units Retired from Service at US. Electric Utilities by State, Company, and Plant, 1995

\begin{tabular}{|c|c|c|c|c|c|c|c|c|}
\hline \multirow[b]{2}{*}{$\begin{array}{c}\text { Strite } \\
\text { Company } \\
\text { Flank (Conty) }\end{array}$} & \multirow[b]{2}{*}{$\underset{D}{\ln k}$} & \multicolumn{3}{|c|}{ Capoxiky } & \multirow[b]{2}{*}{ Throt } & \multicolumn{2}{|c|}{ Eneray Sinued } & \multirow[b]{2}{*}{$\begin{array}{c}\text { Year } \\
\text { Of } \\
\text { Opinirition }\end{array}$} \\
\hline & & 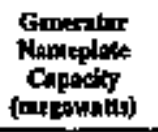 & 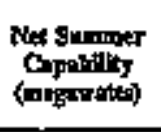 & 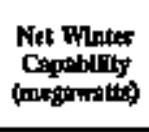 & & Primingy & Ahtrmate & \\
\hline 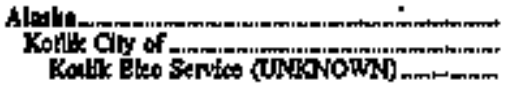 & NAT & $\frac{03}{2}$ & $\frac{21}{2}$ & $\frac{12}{7}$ & IC. & Nat Gy & $=$ & 1981 \\
\hline 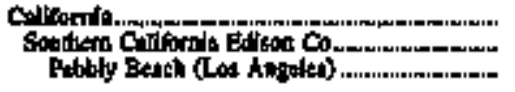 & 11 & $\begin{array}{l}10 \\
1.0 \\
1.0\end{array}$ & $\begin{array}{l}1.0 \\
100 \\
1.0\end{array}$ & $\begin{array}{l}1.4 \\
1.0\end{array}$ & $\mathbf{K}$ & $\mathrm{FO} 2$ & 4 & |QT⿰亻 \\
\hline 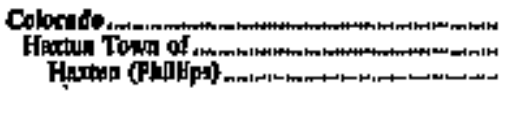 & I & 4 & $\hat{4}$ & $\begin{array}{l}4 \\
.4 \\
.1\end{array}$ & KC & $\begin{array}{l}\mathrm{FOO} \\
\mathrm{FO} 2\end{array}$ & $\ddot{z}$ & $\begin{array}{l}1944 \\
1919\end{array}$ \\
\hline 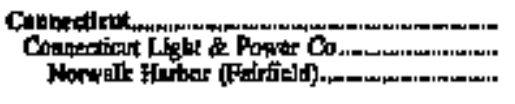 & 10 & $\begin{array}{l}163 \\
163 \\
163\end{array}$ & $\begin{array}{l}170 \\
170 \\
170\end{array}$ & $\begin{array}{l}\mathbf{1 2 3} \\
\mathbf{2 . 3} \\
\mathbf{1 2 3}\end{array}$ & $\mathbf{G r}$ & FO2 & $\hookrightarrow$ & 1966 \\
\hline 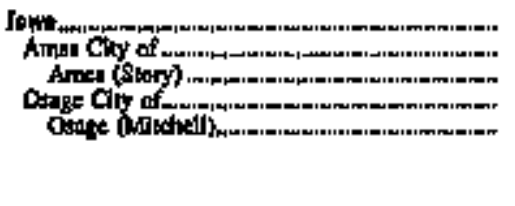 & $\begin{array}{l}4 \\
1 \\
2 \\
4\end{array}$ & $\begin{array}{r}3.3 \\
1.0 \\
1.0 \\
23 \\
5 \\
5 \\
1.3\end{array}$ & $\begin{array}{l}26 \\
1.0 \\
16 \\
4 \\
4 \\
A\end{array}$ & $\begin{array}{r}2.6 \\
1.0 \\
1.0 \\
1.6 \\
.4 \\
.4 \\
.8\end{array}$ & $\begin{array}{l}\mathbf{k} \\
\mathbf{k} \\
\mathbf{k}\end{array}$ & $\begin{array}{l}\mathrm{FOI} \\
\mathrm{PO2} \\
\mathrm{FO2}\end{array}$ & 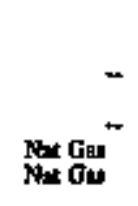 & $\begin{array}{l}1947 \\
1992 \\
1941 \\
1931\end{array}$ \\
\hline 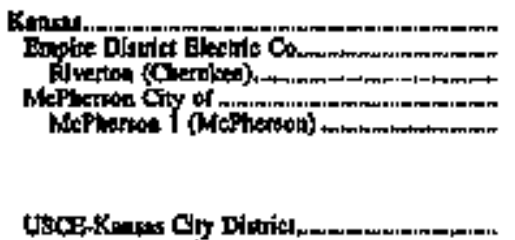 & $\begin{array}{r}\mathbf{6} \\
\mathfrak{3 y} \\
\mathbf{3 y} \\
3\end{array}$ & 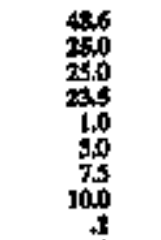 & $\begin{array}{r}55.6 \\
32.0 \\
320 \\
20.15 \\
1.0 \\
50 \\
75 \\
10.0 \\
11\end{array}$ & $\begin{array}{r}5.6 \\
\mathbf{3 2 . 0} \\
32.0 \\
23.5 \\
1.0 \\
50 \\
7.5 \\
10.0 \\
.1\end{array}$ & 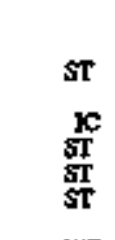 & 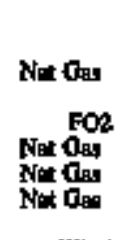 & $\begin{array}{l}\mathrm{FO2} \\
\mathrm{FO}_{2} \\
\mathrm{FO} 2 \\
\mathrm{FO} 2\end{array}$ & $\begin{array}{l}1939 \\
1949 \\
1948 \\
1952 \\
1958\end{array}$ \\
\hline 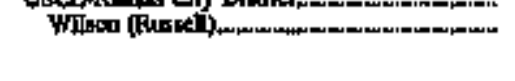 & $\frac{1}{2}$ & $*$ & & 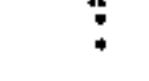 & HT & $\begin{array}{l}\text { Wind } \\
\text { Wind }\end{array}$ & $=$ & $\begin{array}{l}1948 \\
1984\end{array}$ \\
\hline 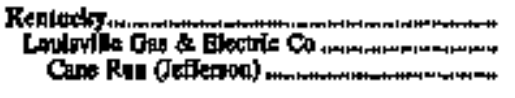 & 3 & $\begin{array}{l}147.1 \\
147.1 \\
147.1\end{array}$ & $\begin{array}{l}115.4 \\
118.10 \\
115.0\end{array}$ & $\begin{array}{l}1150 \\
1150 \\
115.0\end{array}$ & $\mathbf{S T}$ & Nor Oat & $\rightarrow$ & 1958 \\
\hline 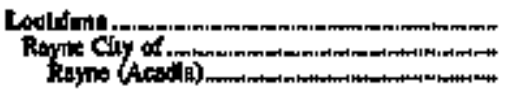 & 9 & $\begin{array}{l}4.1 \\
4.1 \\
4.1\end{array}$ & $\begin{array}{l}25 \\
2,5 \\
25\end{array}$ & $\begin{array}{l}25 \\
25 \\
25\end{array}$ & tc & Net Ou & FOS & 1969 \\
\hline 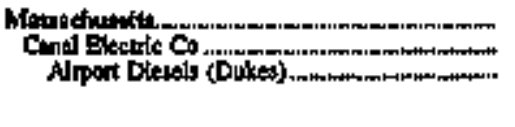 & $\frac{1}{2}$ & $\begin{array}{l}\frac{3.2}{3.2} \\
1.6 \\
1.6\end{array}$ & $\begin{array}{l}3.1 \\
3.1 \\
1.6 \\
1.6\end{array}$ & $\begin{array}{l}\text { 3.1 } \\
3.1 \\
1.6 \\
3.6\end{array}$ & 学 & $\begin{array}{l}\mathrm{Fo} 2 \\
\mathrm{FO} 2\end{array}$ & $=$ & $\begin{array}{l}1989 \\
1989\end{array}$ \\
\hline 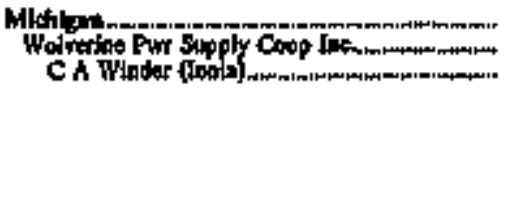 & $\begin{array}{l}1 \\
2 \\
3 \\
4 \\
5\end{array}$ & $\begin{array}{r}4,1 \\
41 \\
1.0 \\
1.1 \\
\text {. } \\
.5 \\
.5\end{array}$ & $\begin{array}{l}4 . \\
4.0 \\
1.0 \\
1.0 \\
1.0 \\
.5 \\
.5\end{array}$ & $\begin{array}{l}40 \\
40 \\
1.0 \\
1.0 \\
1.0 \\
5 \\
5\end{array}$ & 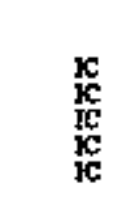 & $\begin{array}{l}\mathrm{FO} 2 \\
\mathrm{Foz} \\
\mathrm{Foz} \\
\mathrm{Fol}\end{array}$ & $\begin{array}{l}\bar{z} \\
\bar{z} \\
\bar{z}\end{array}$ & $\begin{array}{l}1990 \\
1945 \\
1946 \\
1941 \\
1941\end{array}$ \\
\hline 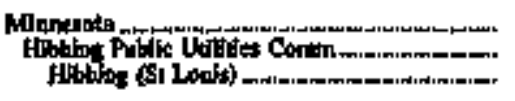 & 4 & 15 & $\begin{array}{l}1.5 \\
1.5 \\
1.5\end{array}$ & $\begin{array}{l}1.5 \\
1.5 \\
1.5\end{array}$ & ET & sup & Nut Gas & 19:1 \\
\hline 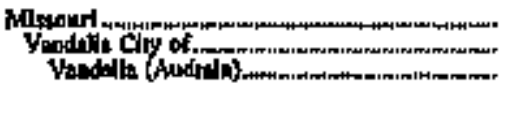 & 5 & $\begin{array}{l}2.0 \\
2.0 \\
1,0 \\
1,0\end{array}$ & $\begin{array}{r}1.6 \\
1.6 \\
.8\end{array}$ & $\begin{array}{r}1.6 \\
1.6 \\
8\end{array}$ & fe & $\mathbf{5 0 2}$ & 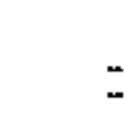 & $\begin{array}{l}1956 \\
1963\end{array}$ \\
\hline New Jeruy & & $\begin{aligned} 1003 \\
77.1\end{aligned}$ & $\mathbf{1 3 0 , 3}$ & 345,2 & & & & \\
\hline
\end{tabular}

Soe bootinated at ad of lable. 
Table 19. Generating Units Retired from Service at U.S. Electric Utilities by State, Company, and Plant, 1995 (Continued)

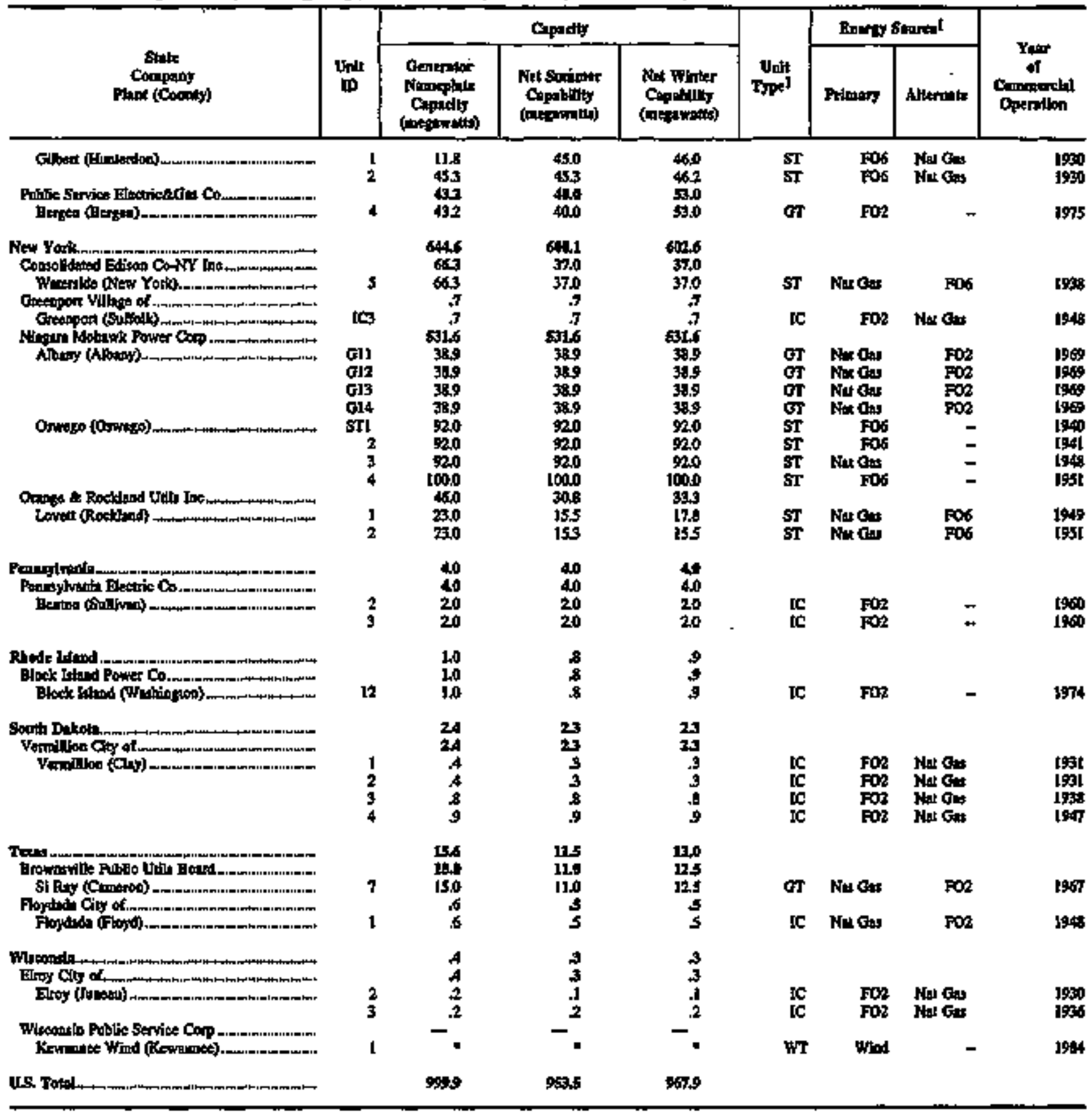

I Set Appeodla B for contis.

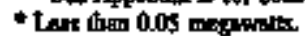

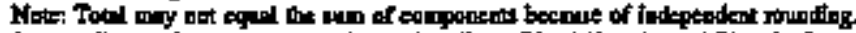

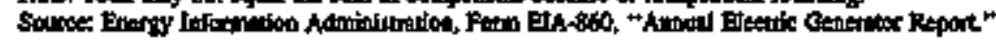


Table 20. Existing Generating Untts at U.S. Electric Utilities by State, Company, and Plant, as of January 1, 1996

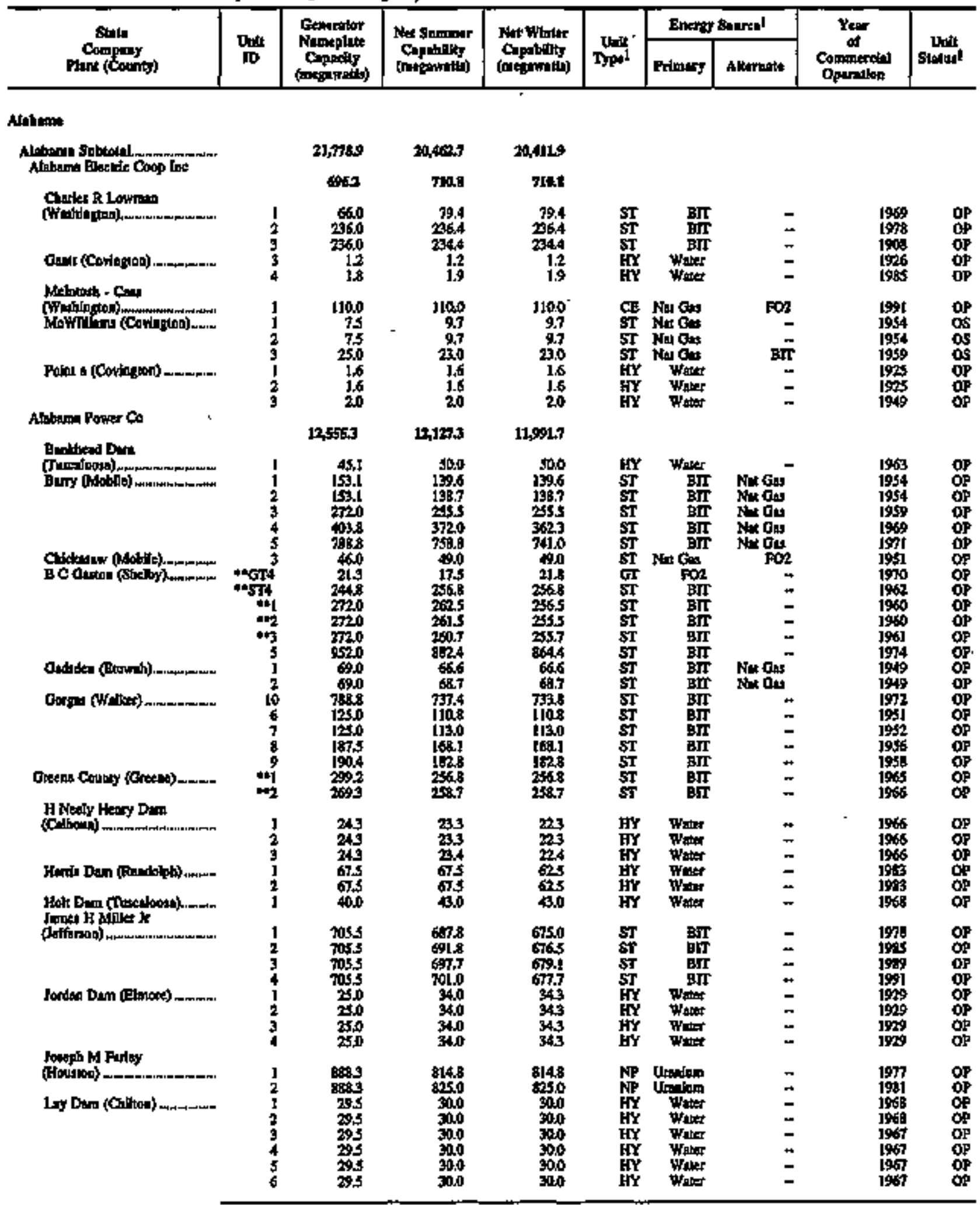

Seo lootnotes u end of toble. 
Table 20. Existing Generating Units at U.S. Flectric Utilities by State, Company, and Plant, as of January 1, 1996 (Continued)

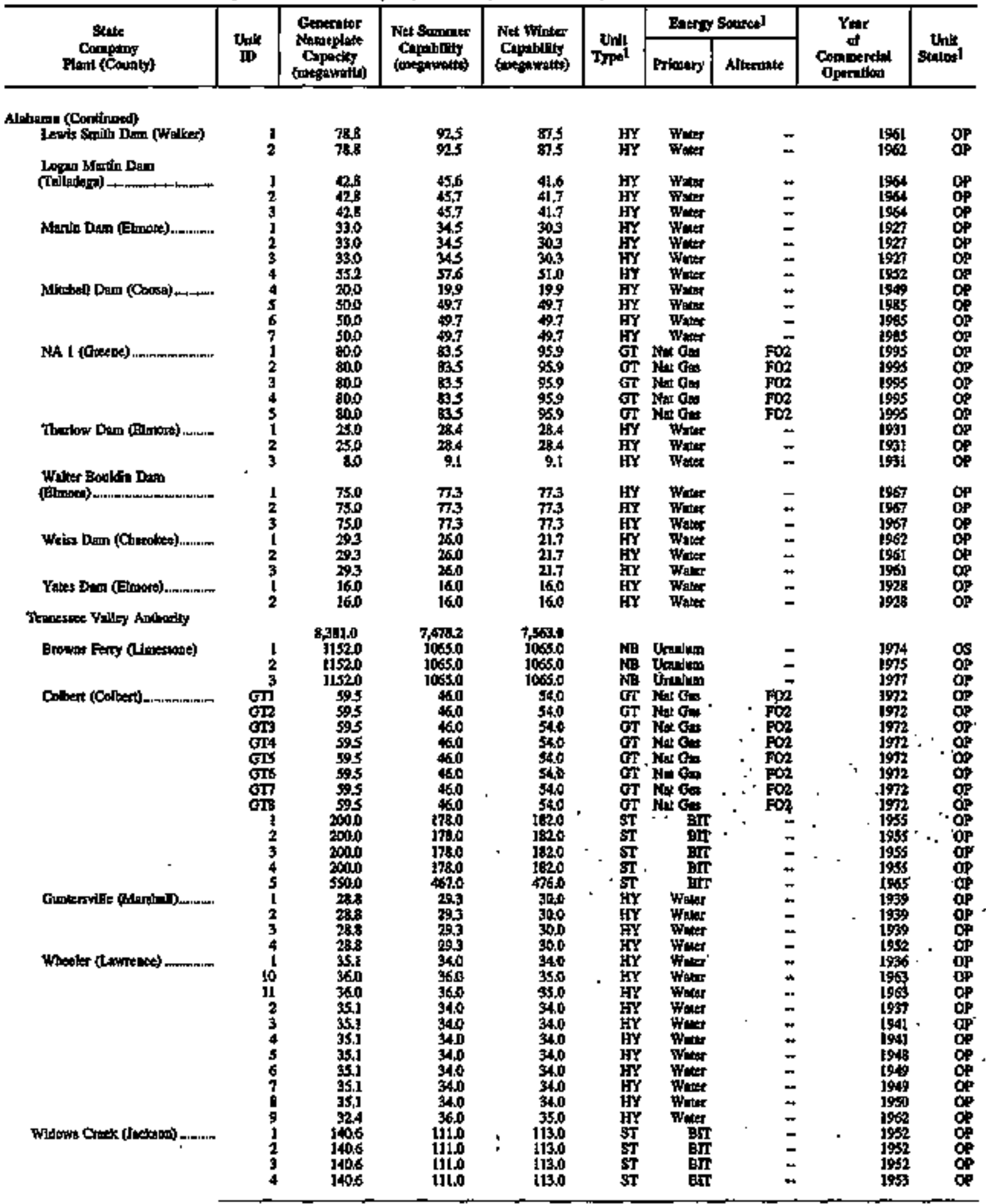

See footpotas at and of labke. 
Table 20. Existing Generating Units at U.S. Electric Utiltities by State, Company, and Plant, as of January 1, 1996 (Continued)

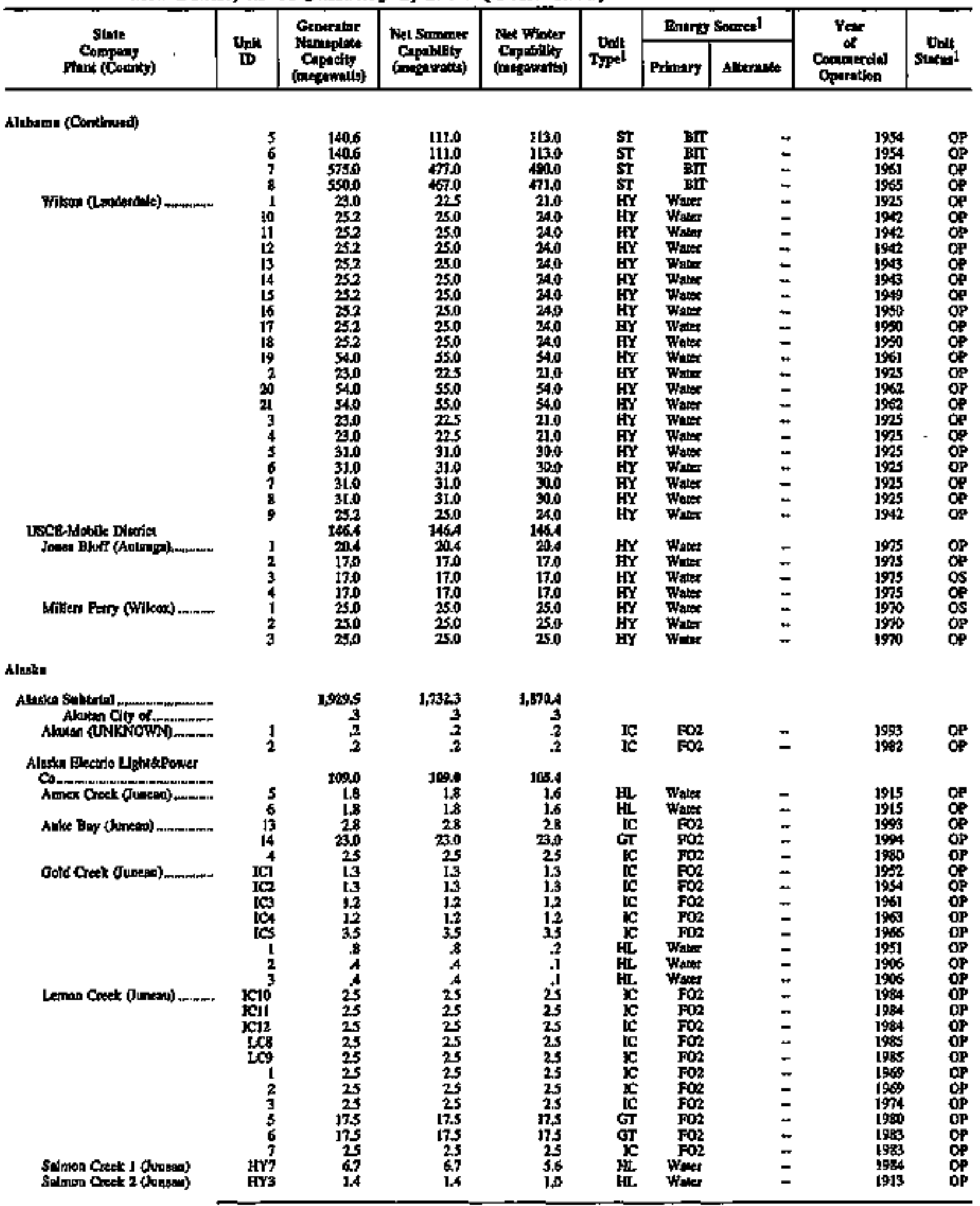

Sen foomones ut and of uble. 
Table 20. Existing Generating Units at U.S. Electric Utilities by.State, Company, and Plant, as of January 1, 1996 (Continued)

\begin{tabular}{|c|c|c|c|c|c|c|c|c|c|}
\hline \multirow{2}{*}{$\underset{\text { Coppongy }}{\text { Surat }(C \text { County) }}$} & \multirow{2}{*}{$\begin{array}{l}\text { Un't } \\
\text { D }\end{array}$} & \multirow{2}{*}{ 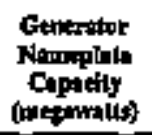 } & \multirow{2}{*}{ 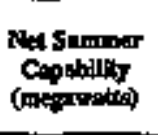 } & \multirow{2}{*}{ 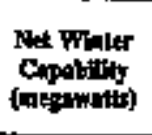 } & \multirow{2}{*}{$\begin{array}{l}\text { Unt: } \\
\text { Typal }\end{array}$} & \multicolumn{2}{|c|}{ Burpo soured } & \multirow{2}{*}{ Opention } & \multirow{2}{*}{ Untmin } \\
\hline & & & & & & Pranay & Alterante & & \\
\hline \multicolumn{10}{|l|}{ Alnska (Cophated) } \\
\hline & EY4 & $\mathrm{l.4}$ & 1.4 & 1,0 & EIL & Water & - & 1913 & OP \\
\hline \multicolumn{8}{|l|}{ Alpulat Furex \& Tokptrope } & & \\
\hline 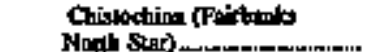 & $\mathbf{1}$ & . &. & .1 & IC & Fot & & & \\
\hline Minil soly & 2 & .I & $\overrightarrow{1}$ & II & IC & Fol & For & 1991 & OP \\
\hline & Coffimin Cove (Ptince of & 3 & 3 & 3 & IC & 702 & Fo1 & 1998 & $O^{P}$ \\
\hline & 3 & 2 & .2 & , . & ic & $\mathrm{FO} 2$ & Foj & 1992 & $O_{P}$ \\
\hline Oraile (Prince of Whlest:_._. & IC2 & $\frac{3}{7}$ & .3 & .3 & $\begin{array}{l}\text { IC } \\
\text { IC }\end{array}$ & $\mathbf{F 0 2}$ & 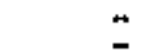 & 1976 & $\underset{O P}{O P}$ \\
\hline & $3 A$ & 1.5 & 1.6 & 16 & IC & Fol & $\bar{z}$ & 199 & op \\
\hline & 5 & t.I & 1,1 & 1.1 & IC & $=02$ & - & 193 & OP \\
\hline \multicolumn{10}{|l|}{ Don Lake Forithanks Nerth } \\
\hline \multirow{2}{*}{\multicolumn{10}{|c|}{ Eapte (Fodrants Nont }} \\
\hline & & & & & & & & & \\
\hline & 1 & $\frac{2}{2}$ & 2 & $\frac{2}{2}$ & ic & Fol & $\mathrm{FO}_{\mathrm{O}}$ & 1909 & $\stackrel{\text { OP }}{\mathrm{P}}$ \\
\hline \\
\hline Nomik Sur) & I & * & 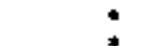 & 4 & $\mathbf{x}$ & Fo1 & FO2 & 1994 & OP \\
\hline \multirow{2}{*}{ Holls (Pinxe of Wols) } & & .1 & 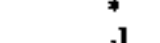 & * & $\mathbf{x}$ & Fo1 & FO2 & 1924 & OP \\
\hline & $\mathrm{IA}_{2}$ & I & .1 & 1 & (c) & Fo2 & $\bar{z}$ & 1993 & OP \\
\hline Hydiby-g (Proce of & & & & & & 106 & 7 & & \\
\hline Wales) & 3 & 3 & 9 & 3 & $\mathbf{x}$ & Fon 2 & - & 1980 & op \\
\hline & 4 & $\frac{1}{3}$ & 1 & +1 & ic & $F 00$ & $\overline{-}$ & 1979 & QP \\
\hline & $\frac{3}{6}$ & 4 & 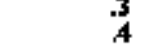 & 4 & Ic & $\mathrm{FO}_{2}$ & $\bar{z}$ & 1996 & OP \\
\hline 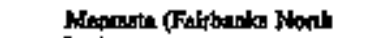 & & & & & & & & & \\
\hline SFM) & & 1 & 1 & + & $\mathbf{C}$ & $\mathrm{FOO}$ & FO1 & 193 & op \\
\hline & $\begin{array}{l}2 \\
3\end{array}$ & 4 & 4 & $\vdots$ & $\underset{\mathrm{K}}{\mathrm{IC}}$ & $\begin{array}{l}\mathrm{FO2} \\
\mathrm{FO2}\end{array}$ & $\begin{array}{l}\text { Fo1 } \\
\text { Fol }\end{array}$ & 1992 & $\underset{0 P}{O P}$ \\
\hline 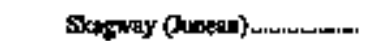 & i & .4 & .4 & 4 & HY & Wor & - & 1957 & of \\
\hline & {$[8$} & 13 & $\mathbf{L 3}$ & 1.3 & $\mathbf{x}$ & FQ2 & مـ & ]990 & OP \\
\hline & $\mathbf{2}$ & 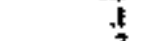 & .1 & .1 & सY & Walar & - & 1900 & OP \\
\hline & 3 & 3 & 3 & 3 & HY & Wor & 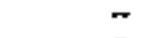 & 1981 & $\mathbf{0}$ \\
\hline & $6^{4}$ & $\frac{2}{9}$ & $\frac{2}{9}$ & $\frac{2}{9}$ & HY & Prar & & & op \\
\hline & $\$ A$ & 5 & 5 & 5 & $\mathbf{E}$ & $\mathbf{m o n}$ & - & 1996 & Pי \\
\hline & 9 & 13 & t3 & 5 & $\mathbf{k}$ & FO2 & & $1 \% 7$ & of \\
\hline Tealln fFourbember Nonlh & & & & & & & & & \\
\hline 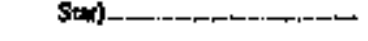 & Ih & 1 & $!$ & 4 & $\mathbf{c}$ & FO2 & Fol & 1999 & op \\
\hline & $\stackrel{2}{3}$ & J & E & .1 & IC & Fol & $\mathrm{FO2}$ & 199 & $\stackrel{0 P}{0}$ \\
\hline Tak Graibenks Nonth & & & & & & & & & \\
\hline $\operatorname{Sam})_{+}$ & 10 & 1.1 & 1.1 & $1, t$ & $\mathbf{C}$ & $\mathbf{F O 2}$ & EOI & 1989 & OP \\
\hline & 2 & 2 & $\frac{2}{3}$ & .2 & tc & 702 & Poi & [960) & $\mathbf{O P}$ \\
\hline & $\mathbf{s}$ & $\overrightarrow{3}$ & $\frac{3}{3}$ & .3 & IC & $p_{02}$ & Fol & $\begin{array}{l}1961 \\
\text { lost }\end{array}$ & op \\
\hline & 6 & $\mathbf{L}$ & 2.0 & 1.0 & IC & Fol & Fol & $19 m$ & DP \\
\hline & 7 & t3 & 13 & 1.3 & IC & $\mathrm{FOO}$ & Fo1 & 1904 & of \\
\hline & 8 & 4 & $\stackrel{a}{a}$ & $A$ & IC & $\mathrm{FO} 2$ & Fol & tgas & of \\
\hline Alukn Power Adminintertion & & & $y$ & & IC & & & L985 & \\
\hline & & 100.2 & toter & 1042 & & & & & \\
\hline Eldutat Maturusos & & & & & & & & & \\
\hline Swing & 1 & 150 & 15,0 & I5.0 & HYY & Wala & $\because$ & $\begin{array}{l}\text { toss } \\
\text { 595s }\end{array}$ & op \\
\hline SRewshinn (Jusesu) & & 23.6 & 23.6 & 236 & & Woler & $\therefore$ & 1973 & OP \\
\hline & 2 & 236 & 236 & 28.6 & 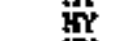 & Wur & - & 973 & op \\
\hline & 3 & $3 t . t$ & 31.] & 31.1 & $\boldsymbol{E} \boldsymbol{Y}$ & What & - & 1990 & $\phi P$ \\
\hline 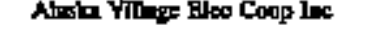 & & & & 73 & & & & & \\
\hline Alekonuk (Bobrel) & IA & $\frac{326}{3}$ & $\begin{array}{ll}33.1 \\
3\end{array}$ & $\frac{3}{3}$ & $\mathbf{K}$ & Fo1 & - & 1986 & \\
\hline & 2 & $\overrightarrow{3}$ & .2 & .2 & ce & Fol & $=$ & $\begin{array}{l}1970 \\
1970\end{array}$ & OF \\
\hline & & $\sqrt{3}$ & 3 & 3 & ic & FOI & - & 1974 & $\sigma_{P}$ \\
\hline Arablet (Kobal) & IC & $\$$ & .3 & .3 & $\mathbf{k}$ & Fol & - & 1985 & OP \\
\hline
\end{tabular}

Sec foolsoles at end of table. 
Table 20. Existing Generating Units at US. Electric Utilities by State, Company, and Plant, as of January 1, 1996 (Continued)

\begin{tabular}{|c|c|c|c|c|c|c|c|c|c|}
\hline \multirow{2}{*}{$\begin{array}{c}\text { Sate } \\
\text { Compiny } \\
\text { Plom (Coants) }\end{array}$} & \multirow{2}{*}{$\underset{\text { III }}{\text { Unk }}$} & \multirow{2}{*}{ 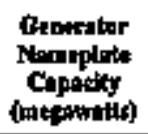 } & \multirow{2}{*}{ 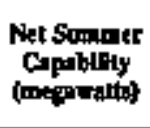 } & \multirow{2}{*}{$\begin{array}{l}\text { Net Winter } \\
\text { Capabilty } \\
\text { (medewatis }\end{array}$} & \multirow{2}{*}{$\underset{\text { Jynt }}{\text { Unt }}$} & \multicolumn{2}{|c|}{ Ronrey Souted } & \multirow{2}{*}{$\begin{array}{c}\text { Year } \\
\text { af } \\
\text { Commerital } \\
\text { Operition }\end{array}$} & \multirow{2}{*}{ Strank } \\
\hline & & & & & & Prinary & Alkermile & & \\
\hline \multicolumn{10}{|l|}{ Alalike (Continned) } \\
\hline & 1 & 0.2 & 0.2 & 0.2 & 1C. & Fol & - & 1984 & op \\
\hline & $3 \boldsymbol{k}$ & 3 & 3 & $\mathbf{3}$ & $\boldsymbol{x}$ & FOI & ـ & 2991 & $O P$ \\
\hline 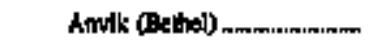 & 1 & $\vec{J}$ & 1 & I. & IC & Fol & - & 1971 & op \\
\hline & $\mathbf{3}^{2}$ & .1 & 1 & .1 & ic & Fo1 & $\bar{z}$ & 1969 & $\stackrel{p e}{o p}$ \\
\hline Brevilg Mftesion (Alome) & 1 & 2 & 2 & 2 & $\mathbf{k C}$ & Fol & 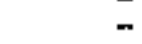 & 1996 & op \\
\hline & 2 & 3 & .2 & $\frac{2}{1}$ & $\underset{k}{c}$ & Fo1 & $=$ & 693 & $\stackrel{p P}{O P}$ \\
\hline Chevalk (Betit) & i & 3 & 3 & 3 & KC & Fol & - & 1977 & OP \\
\hline 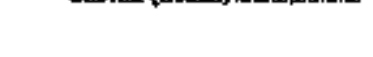 & $\begin{array}{l}2 \\
3\end{array}$ & $\frac{2}{4}$ & .2 & $\frac{7}{4}$ & ck & $\begin{array}{l}\mathrm{Foj} \\
\mathrm{Fo1}\end{array}$ & $\bar{z}$ & $\begin{array}{r}1976 \\
1979\end{array}$ & $\stackrel{\text { op }}{\text { op }}$ \\
\hline 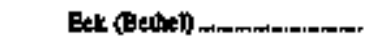 & i & 2 & 2 & 3 & $\mathbf{k}$ & Fol & n & 1977 & of \\
\hline - 8) & 24 & .2 & $\frac{1}{2}$ & $\frac{1}{2}$ & $\underset{\mathbf{c}}{\mathbf{c}}$ & $\begin{array}{l}\mathrm{Fol} \\
\mathrm{Fol}\end{array}$ & $\overline{-}$ & $\begin{array}{l}1998 \\
1998\end{array}$ & $\stackrel{\text { op }}{\text { OP }}$ \\
\hline 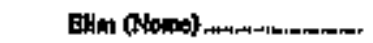 & i & 2 & .2 & 2 & $\underline{x}$ & Fo1 & $=$ & 1975 & OP \\
\hline (2) & 24 & .2 & 2 & $\frac{2}{3}$ & $\underset{\mathbf{c}}{\mathrm{c}}$ & $\begin{array}{l}\mathrm{Fo1} \\
\mathrm{Fol}\end{array}$ & $\ddot{-}$ & $\begin{array}{l}\text { t986 } \\
1991\end{array}$ & $\underset{\mathrm{dP}}{\mathrm{dP}}$ \\
\hline Bmponate (Betiol) & 2 & 3 & 3 & 3 & $\mathbf{x}$ & Fo1 & .. & 1977 & op \\
\hline & 5 & 8 & A & 6 & $\begin{array}{l}\mathbf{k} \\
\mathbf{k}\end{array}$ & $\begin{array}{l}\text { Fol } \\
\text { Fol }\end{array}$ & 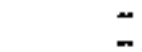 & $\begin{array}{l}\text { 19\$80 } \\
\text { j9g8 }\end{array}$ & $\begin{array}{l}\text { OP } \\
\text { OP }\end{array}$ \\
\hline & 6 & 9 & 9 & 9 & Ic & Fo1 & $=$ & 1995 & op \\
\hline 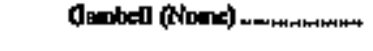 & $\operatorname{ICI}$ & 3 & .3 & 3 & jc & Fo1 & $\ddot{z}$ & t\$35 & op \\
\hline & $\frac{x_{2}}{1 C_{3}}$ & 3 & 3 & $\$$ & $\mathbf{c}$ & Fod & $=$ & $\begin{array}{l}1965 \\
1955\end{array}$ & op \\
\hline \multirow[t]{3}{*}{ 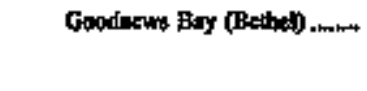 } & $\mathrm{IC2}$ & 2 & .2 & 2 & r & Fo1 & $=$ & t985s & QP \\
\hline & la & 2 & 2 & 7 & $\mathbf{l c}$ & Fol & $\leftrightarrow$ & {$[978$} & OP \\
\hline & $\mathbf{H A}$ & I & 1 & .1 & c & F01 & - & t99! & op \\
\hline \multirow[t]{2}{*}{ 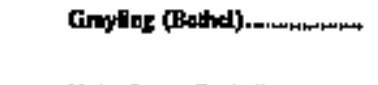 } & L & 2 & .2 & 2 & ch & poi & - & $98 ?$ & $\alpha P$ \\
\hline & 24 & .1 & $\frac{1}{2}$ & $\frac{1}{2}$ & c & Foil & $=$ & 991 & op \\
\hline \multirow{3}{*}{ Holy Cost (Betill) } & i & 2 & 2 & $\frac{1}{2}$ & 孯 & Fo1 & $\ddot{z}$ & 1960 & op \\
\hline & 2 & $\frac{2}{2}$ & 2 & 3 & kc & FOI & - & ini & op \\
\hline & I & 2 & .2 & $\frac{2}{2}$ & $\underset{\mathbf{c}}{\mathbf{r C}}$ & $\begin{array}{l}\text { FOl } \\
\text { Fol }\end{array}$ & $\ddot{z}$ & 1960 & of \\
\hline \multirow{3}{*}{ Hoopes Bay (Beuhel). } & 2 & 4 & $A$ & 4 & fic & Hol & 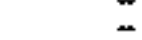 & $\begin{array}{l}1969 \\
1975\end{array}$ & $\stackrel{p P}{O P}$ \\
\hline & 4 & 4 & $\boldsymbol{A}$ & 4 & tc & Foi & - & $19 \%$ & op \\
\hline & & 6 & .6 & 6 & $\mathbf{c}$ & Fol & + & 1991 & OP \\
\hline \multirow[t]{3}{*}{ 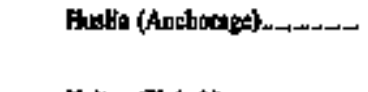 } & & 2 & .2 & 2 & $\mathbf{s}$ & Fol & - & 1969 & OP \\
\hline & $2 A$ & $\frac{5}{3}$ & .2 & 2 & sc & F01 & - & 1887? & $\mathrm{OP}$ \\
\hline & $\mathrm{A}^{3}$ & 3 & .2 & 2 & ce & Fol & - & 1994 & op \\
\hline 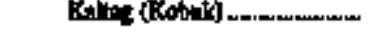 & ${ }_{2}$ & $\frac{1}{2}$ & .1 &.+1 & te & $\begin{array}{l}\text { Fol } \\
\text { Fo1 }\end{array}$ & $=$ & 1991 & OP \\
\hline & & 3 & 2 & .2 & ic & $\mathrm{Foj}$ & - & I & QP \\
\hline 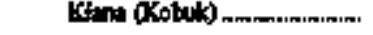 & IA & 3 & 3 & 3 & IC & Fol & + & 1090 & OP \\
\hline & 2 & 3 & 3 & 3 & IC & Fol & - & 197 & GP \\
\hline & & $\frac{3}{2}$ & .2 & .2 & IC & Fo1 & - & 1984 & op \\
\hline 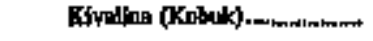 & I & 2 & 2 & 2 & IC & F01 & + & 1975 & OP \\
\hline & 2 & 3 & 3 & $\mathbf{3}$ & IC & Fol & - & (97) & QP \\
\hline & $4^{3}$ & $\frac{2}{3}$ & .2 & .2 & & Fo1 & 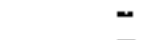 & test & OP \\
\hline 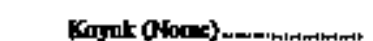 & ${ }_{1}^{4 A}$ & 3 & 2 & .3 & IC & $\begin{array}{l}\text { FoJ } \\
\text { Fol }\end{array}$ & $\overline{-}$ & $\begin{array}{l}1992 \\
1968\end{array}$ & OP \\
\hline & $\mathbf{2}$ & $\ddot{2}$ & 3 & 2 & Ic & Fol & 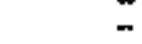 & 1970 & dP \\
\hline & 3 & 2 & 2 & 2 & IC & Fo1 & - & 9970 & OP \\
\hline 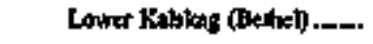 & i & $\overline{2}$ & .2 & .2 & ic & Foj & - & t 983 & OP \\
\hline & ZH & 3 & .2 & .2 & k & Fol & - & [986 & op \\
\hline & 3 & 2 & .2 & 3 & 1c & Fol & $\ddot{z}$ & 1977 & OP \\
\hline 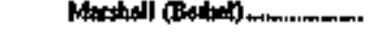 & 1 & $\frac{3}{3}$ & 2 & $\frac{3}{3}$ & F & Fo1 & - & 1970 & op \\
\hline & $\mathbf{2 k}_{3}$ & $\frac{2}{2}$ & 2 & 3 & $\underset{c}{c}$ & Fo1 & $=$ & $\begin{array}{l}1987 \\
1970\end{array}$ & op \\
\hline Makreryuk (Bdbel) & $\vec{i}$ & 2 & 2 & $\overrightarrow{3}$ & 1c & Fol & - & 1969 & of \\
\hline & 2 & 2 & $\mathbf{z}$ & 2 & $\mathbf{c}$ & FOI & - & 1971 & $\mathbf{Q P}$ \\
\hline & 3 & 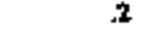 & 2 & 2 & 16. & FOI & 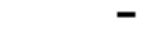 & 1970 & OP \\
\hline 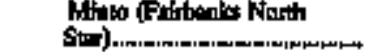 & & 2 & .2 & 2 & $\mathrm{rc}$ & Forl & - & J985 & \\
\hline & 103 & 2 & 2 & 2 & $\mathbf{K}$ & Fol & 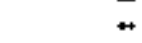 & 1985 & op \\
\hline & ik & J & .1 & $+\mathbf{l}$ & $\mathbf{K}$ & FOI & - & 1992 & OP \\
\hline Moortsin Villoge (Redtel) & I & 4 & 4 & 4 & $\mathbf{C}$ & Eol & - & 1984 & OP \\
\hline & $\mathbf{3}$ & $\mathbf{3}$ & 3 & 3 & $\mathbf{K C}$ & Fol & 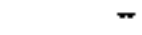 & 1982 & $\boldsymbol{O P}$ \\
\hline
\end{tabular}

Sec foornows to end of table. 
Table 20. Existing Generating Units at U.S. Electric Utilities by State, Company, and Plant, as of January 1, 1996 (Continued)

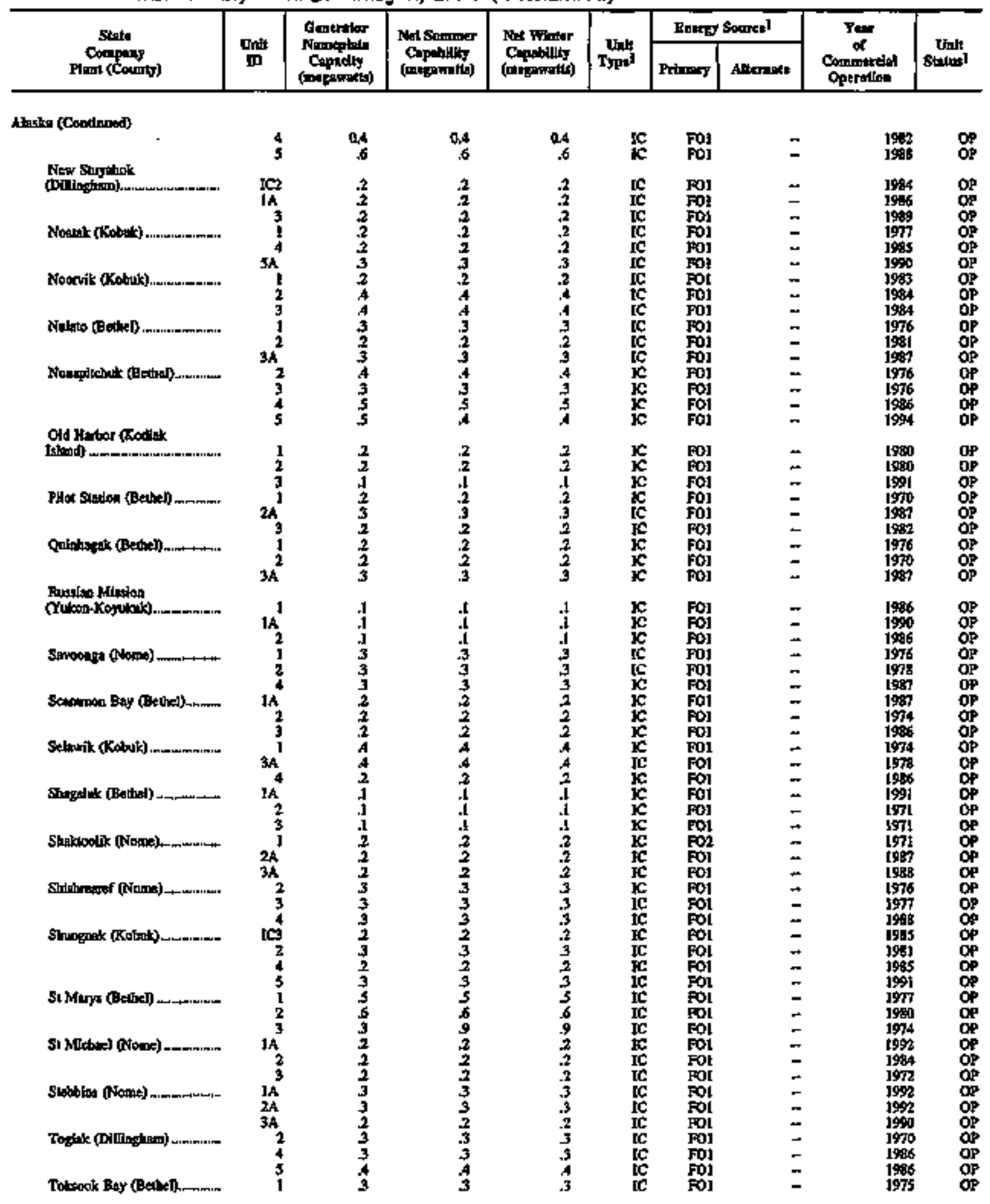

Sed Jounogtes at end of tible. 
Table 20. Existing Generating Units at U.S. Electric Utilities by State, Company, and Plant, as of January 1, 1996 (Continued)

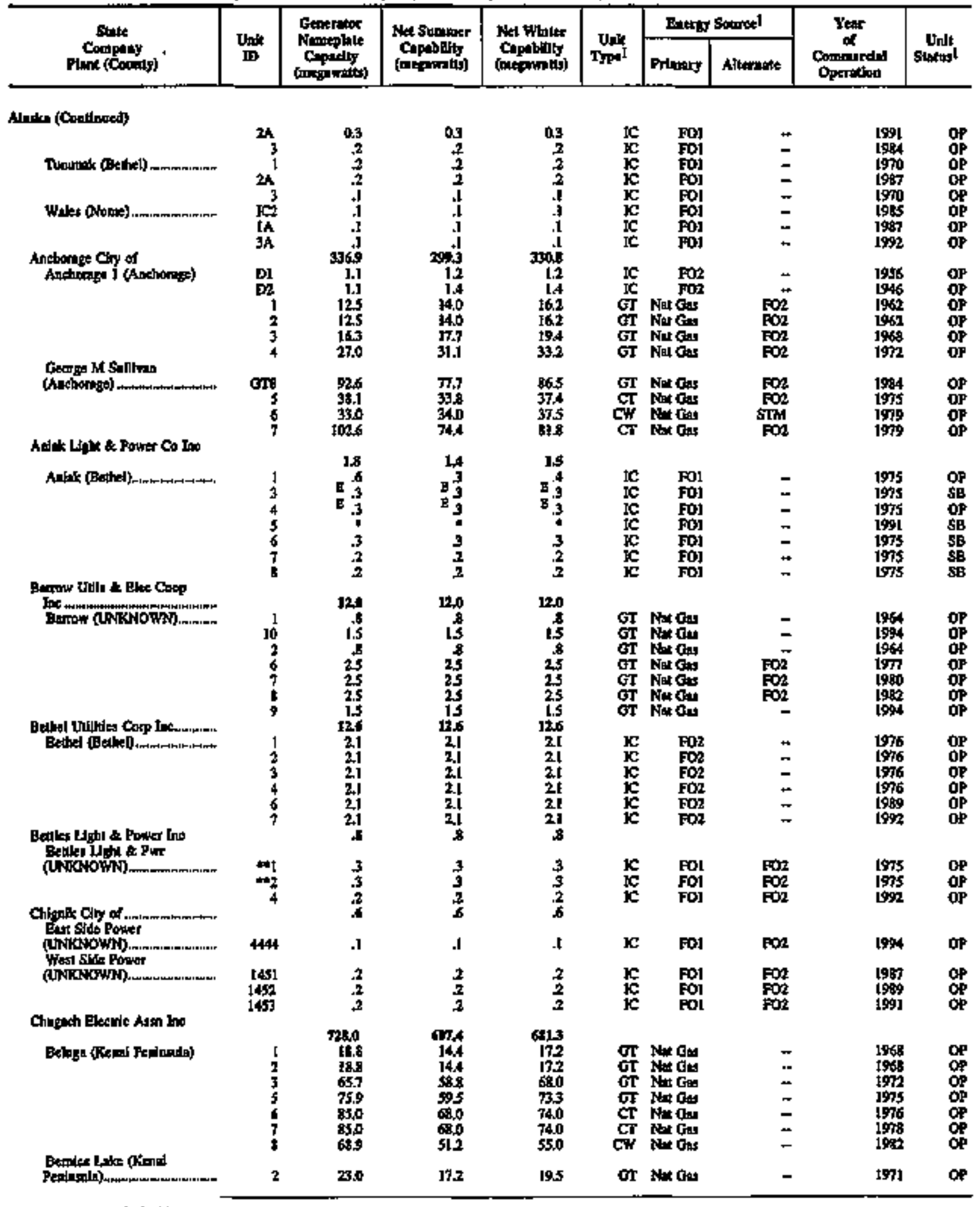

Ses footended al ced of Itable. 
Table 20. Existing Generating Units at U.S. Electric Utilities by State, Company, and Plant, as of January 1, 1996 (Continued)

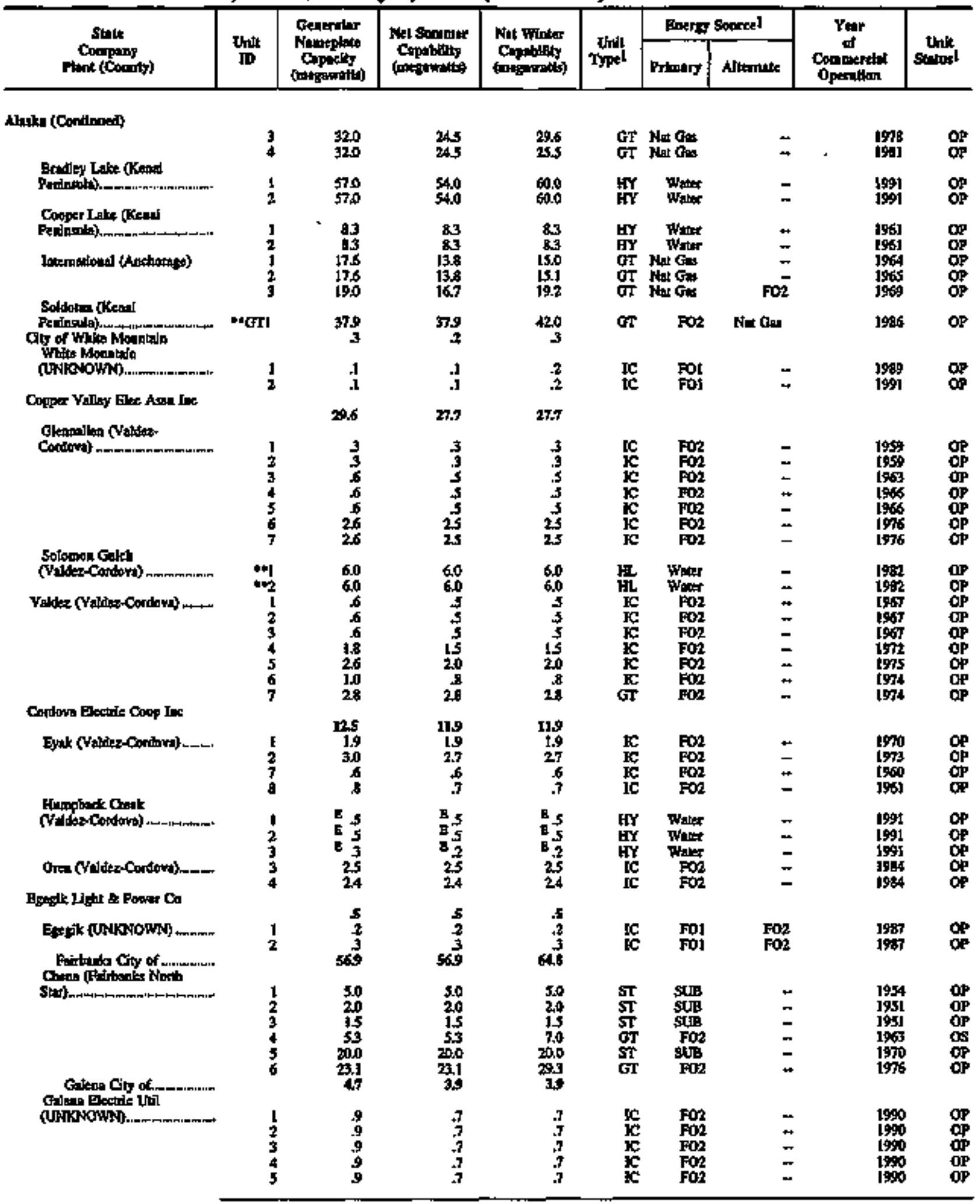

Sen footholes it end of tabili. 
Table 20. Existing Generating Units at U.S. Electric Utilities by State, Company, and Plant, as of January 1, 1996 (Continued)

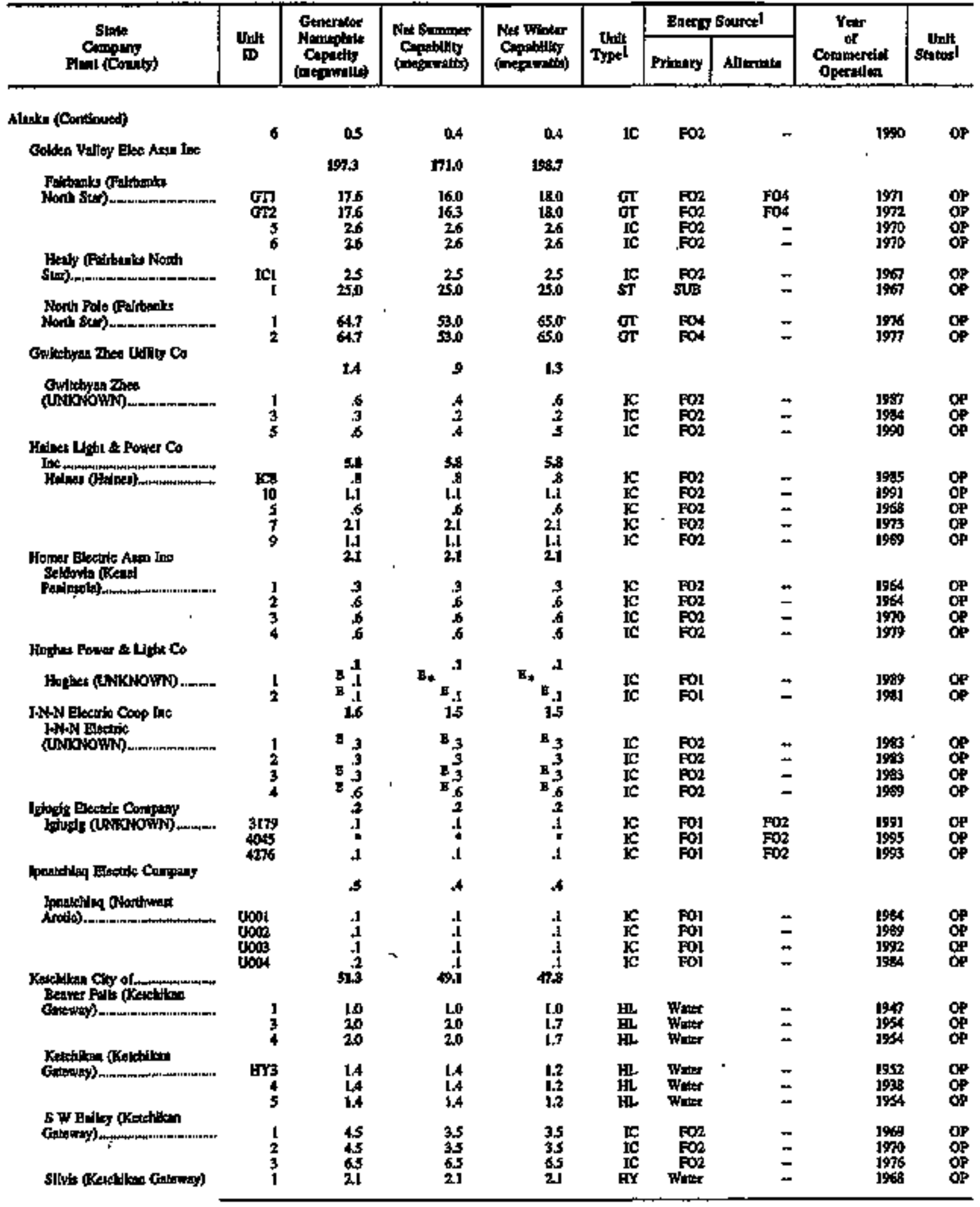

Ses footrolses it end of thlto. 
Table 20. Existing Generating Units at U.S. Electric Utilties by State, Company, and Plant, as of January 1, 1996 (Continued)

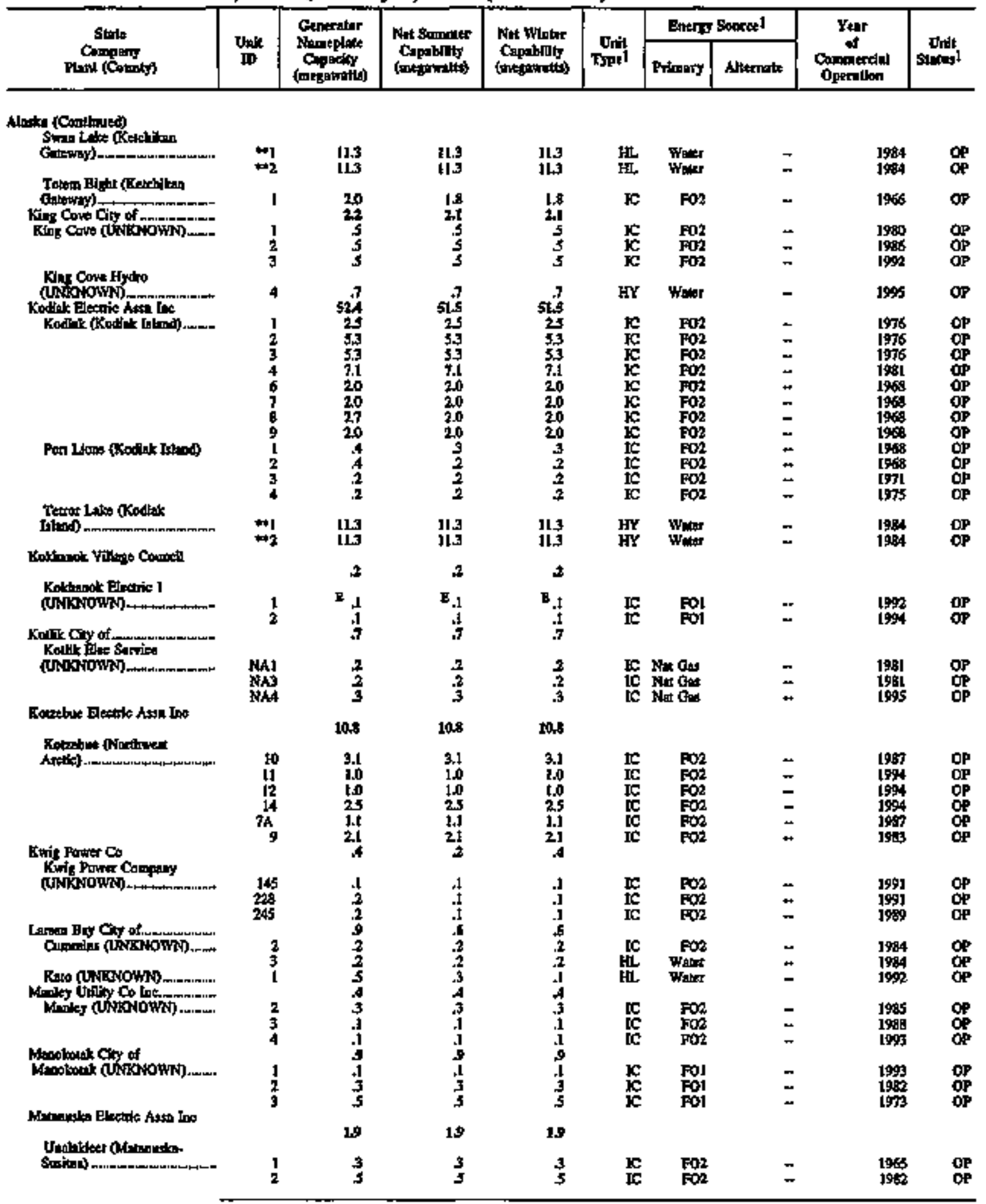

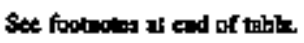


Tahle 20. Existing Generating Units at U.S. Ellectric Utillities by State, Company, and Plant, as of January 1, 1996 (Continued)

\begin{tabular}{|c|c|c|c|c|c|c|c|c|c|}
\hline \multirow{2}{*}{$\begin{array}{c}\text { Slote } \\
\text { Conpong } \\
\text { Plemt }\{\text { Coonty\} }\end{array}$} & \multirow{2}{*}{$\begin{array}{l}\text { Un't } \\
\text { 血 }\end{array}$} & \multirow{2}{*}{ 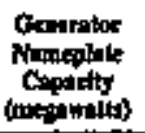 } & \multirow{2}{*}{ 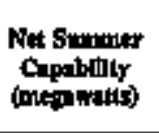 } & \multirow{2}{*}{ 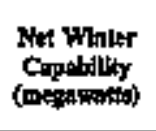 } & \multirow{2}{*}{ Unit } & \multicolumn{2}{|c|}{ Burrgy Sourel } & \multirow{2}{*}{$\begin{array}{c}\text { Year } \\
\text { of } \\
\text { Comonindal } \\
\text { Operiftor }\end{array}$} & \multirow{2}{*}{ Until } \\
\hline & & & & & & Primarg & Alermate & & \\
\hline \multicolumn{10}{|l|}{ Alakin (Contitined) } \\
\hline & 3 & 05 & 0.5 & 0.5 & $\begin{array}{l}\text { tc } \\
\text { IC }\end{array}$ & FOn & $\ddot{-}$ & 19,3 & of \\
\hline Uaplabtets-Wind & & & & & & & & & \\
\hline 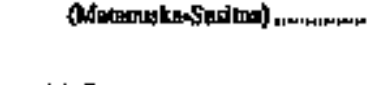 & $\begin{array}{l}1 \\
2 \\
3\end{array}$ & $\ddot{*}$ & 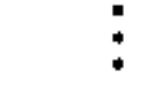 & 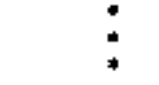 & $\begin{array}{l}\text { WT } \\
\text { wT } \\
\text { wT }\end{array}$ & $\begin{array}{l}\text { Wind } \\
\text { Whind } \\
\text { Whad }\end{array}$ & $\ddot{\Xi}$ & $\begin{array}{l}1902 \\
1902 \\
1942\end{array}$ & $\begin{array}{l}\text { op } \\
\text { of } \\
\text { op }\end{array}$ \\
\hline \multicolumn{10}{|l|}{ MeGrup Wjat a Power Co } \\
\hline MeOtint (Yukso+Kopulatit) & $\begin{array}{l}3 \\
4 \\
5 \\
6 \\
7\end{array}$ & $\begin{array}{r}21 \\
3 \\
2 \\
6 \\
.6 \\
.4 \\
4\end{array}$ & $\begin{array}{r}2.0 \\
.2 \\
2 \\
6 \\
4 \\
4\end{array}$ & $\begin{array}{r}2.1 \\
.2 \\
.2 \\
.6 \\
.7 \\
.4\end{array}$ & IC & $\begin{array}{l}\text { Fol } \\
\text { Fol } \\
\text { Fol } \\
\text { Fo1 } \\
\text { Fol }\end{array}$ & $\begin{array}{l}\mathrm{FO} 2 \\
\mathrm{Fo2} \\
\mathrm{FO2} \\
\mathrm{FO2} \\
\mathrm{FO} 2\end{array}$ & $\begin{array}{l}1919 \\
1979 \\
1997 \\
1998 \\
1993\end{array}$ & $\begin{array}{l}o p \\
\alpha p \\
\phi p \\
\alpha p \\
\phi p\end{array}$ \\
\hline \multicolumn{10}{|l|}{ Mkltalla Powr \& Lighl } \\
\hline 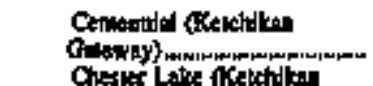 & 106 & 3.3 & 3.3 & 33 & $\mathbf{K}$ & FO2 & - & 5 & op \\
\hline Gereway & ! & 1.0 & 2.0 & 1.0 & HY & Water & - & 1908 & op \\
\hline 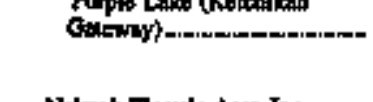 & $\begin{array}{l}1 \\
2 \\
3\end{array}$ & $\begin{array}{l}1.3 \\
13 \\
13\end{array}$ & $\begin{array}{l}1.3 \\
1.3 \\
1.3\end{array}$ & $\begin{array}{l}1.3 \\
1.3 \\
1.3\end{array}$ & $\begin{array}{l}\mathrm{HY} \\
\mathrm{HY} \\
\mathrm{HY}\end{array}$ & Water & $\ddot{z}$ & $\begin{array}{l}1986 \\
1986 \\
1962\end{array}$ & $\begin{array}{l}\text { OP } \\
\text { OP }\end{array}$ \\
\hline \multicolumn{10}{|l|}{ Nalpek Ilectik Assn InC } \\
\hline Natint (Eristol Boy).......... & $\begin{array}{r}N A 1 \\
N A 2 \\
N A 3 \\
N A 4 \\
N A S \\
4 \\
5 \\
6 \\
7 \\
8\end{array}$ & $\begin{array}{r}1.1 \\
1.1 \\
9 \\
9 \\
9 \\
5 \\
4 \\
4 \\
4 \\
1.0\end{array}$ & 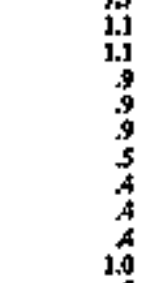 & 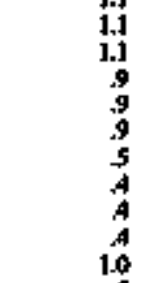 & $\begin{array}{l}\text { IC } \\
\text { IC } \\
\text { IC } \\
\text { IC } \\
\text { IC } \\
\text { IC } \\
\text { IC } \\
\text { IC } \\
\text { IC } \\
\text { IC }\end{array}$ & 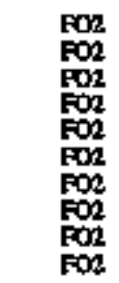 & $\begin{array}{l}\bar{z} \\
= \\
\bar{z} \\
\bar{z} \\
=\end{array}$ & $\begin{array}{l}1988 \\
1988 \\
1991 \\
1998 \\
1993 \\
1965 \\
1977 \\
1977 \\
1977 \\
1977\end{array}$ & 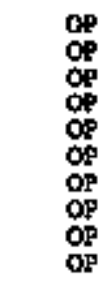 \\
\hline 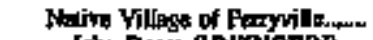 & & s & $s$ & 5 & & & & & \\
\hline Joht Deere (UNONOWM) & $\begin{array}{l}l \\
2 \\
3\end{array}$ & $\begin{array}{l}3 \\
3 \\
1\end{array}$ & $\begin{array}{l}.2 \\
.2 \\
.1\end{array}$ & $\begin{array}{l}2 \\
2 \\
1\end{array}$ & $\begin{array}{l}\mathbf{K} \\
\mathbf{K C} \\
\mathbf{K C}\end{array}$ & $\begin{array}{l}\text { FOI } \\
\text { ROI } \\
\text { ROI }\end{array}$ & $\begin{array}{l}\mathrm{Fos} \\
\mathrm{Fog} 2\end{array}$ & $\begin{array}{l}1992 \\
19982 \\
1992\end{array}$ & $\stackrel{o P}{o p}$ \\
\hline \multicolumn{10}{|l|}{ Nams loint Unaly Syateng } \\
\hline 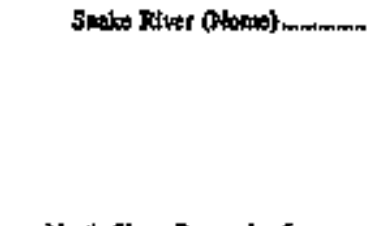 & $\begin{array}{r}1 \\
10 \\
11 \\
12 \\
2 \\
5 \\
6 \\
9\end{array}$ & $\begin{array}{r}6 \\
6 \\
1.5 \\
3.8 \\
6 \\
1.2 \\
1.0 \\
29\end{array}$ & $\begin{array}{r}.6 \\
6 \\
\mathbf{1 . 5} \\
3.3 \\
\mathbf{4} \\
\mathbf{1 . 0} \\
\mathbf{1 . 9}\end{array}$ & $\begin{array}{r}.6 \\
.6 \\
1.5 \\
3.6 \\
1.2 \\
1.0 \\
2.9\end{array}$ & $\begin{array}{l}\text { Ic } \\
\text { ic } \\
\text { IC } \\
\text { IC } \\
\text { IC } \\
\text { IC } \\
\text { IC } \\
\text { IC }\end{array}$ & $\begin{array}{l}\mathrm{FO2} \\
\mathrm{FO2} \\
\mathrm{FO2} \\
\mathrm{FO2} \\
\mathrm{FO2} \\
\mathrm{FO2} \\
\mathrm{FO2} \\
\mathrm{FO2}\end{array}$ & $\begin{array}{l}= \\
= \\
\ddot{ } \\
= \\
=\end{array}$ & $\begin{array}{l}1963 \\
1978 \\
19488 \\
1991 \\
1963 \\
1994 \\
1972 \\
1968\end{array}$ & 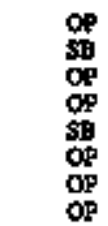 \\
\hline \multicolumn{10}{|l|}{ Nonth slope Borowgh of } \\
\hline $\begin{array}{l}\text { NSB Annltovik Pass } \\
\text { North Stopt\}... }\end{array}$ & $\begin{array}{l}1 \\
2 \\
3 \\
4 \\
5\end{array}$ & $\begin{array}{l}\text { Y. } \\
\mathrm{E} \\
\mathrm{E} .3 \\
\mathrm{E} .3 \\
\mathrm{E} .3 \\
\mathrm{E} .2 \\
.2\end{array}$ & 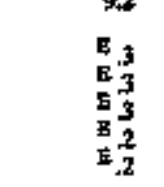 & 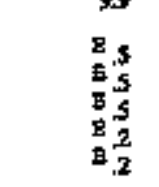 & $\begin{array}{l}\text { IC } \\
\text { IC } \\
\text { IC } \\
\text { IC } \\
\text { tC }\end{array}$ & $\begin{array}{l}\text { FoI } \\
\text { Fol } \\
\text { FO1 } \\
\text { Fol } \\
\text { Fol }\end{array}$ & $\begin{array}{l}\bar{z} \\
\bar{z} \\
\pm\end{array}$ & $\begin{array}{l}1994 \\
1994 \\
1994 \\
1994 \\
1994\end{array}$ & $\begin{array}{l}\text { OP } \\
\text { of } \\
\text { OP } \\
\text { OP } \\
\text { OP }\end{array}$ \\
\hline 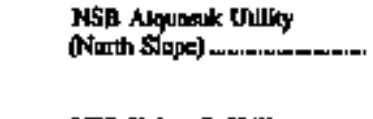 & $\begin{array}{l}P 01 \\
P G 6\end{array}$ & $\begin{array}{l}\text { E } .3 \\
\text { E } .4 \\
\text { E. } .7\end{array}$ & $\begin{array}{l}E .3 \\
E .4 \\
E .6\end{array}$ & $\begin{array}{l}\mathrm{E}, 3 \\
\mathrm{E}^{, 3} \\
\mathrm{E}\end{array}$ & $\begin{array}{l}\text { IC } \\
\text { IC }\end{array}$ & $\begin{array}{l}\text { FOI } \\
\text { FOI } \\
\text { Fol }\end{array}$ & $\because$ & $\begin{array}{l}1986 \\
1985 \\
1986\end{array}$ & $\begin{array}{l}\text { of } \\
\text { of }\end{array}$ \\
\hline 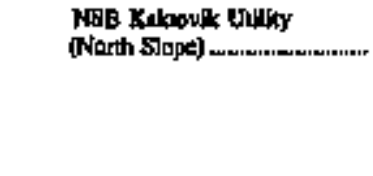 & $\begin{array}{l}\text { POJ } \\
\text { PGL } \\
\text { PO3 } \\
\text { PG4 } \\
\text { POS }\end{array}$ & $\begin{array}{l}\mathrm{E} .3 \\
\mathrm{E} .3 \\
\mathrm{E} .3 \\
\mathrm{E} .3 \\
\mathrm{E} .2 \\
.2\end{array}$ & 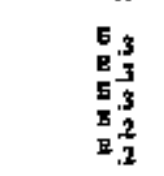 & $\begin{array}{l}\text { E. } \\
\mathrm{E}: 3 \\
\mathrm{E} .3 \\
\mathrm{~g} \cdot 3 \\
\mathrm{H} .2\end{array}$ & $\begin{array}{l}\text { IC } \\
\text { IC } \\
\text { IC } \\
\text { IC } \\
\text { IC }\end{array}$ & $\begin{array}{l}\text { Fol } \\
\text { Fol } \\
\text { FO1 } \\
\text { FOd } \\
\text { FOl }\end{array}$ & $\begin{array}{l}\bar{z} \\
\bar{z}\end{array}$ & $\begin{array}{l}1990 \\
1990 \\
1990 \\
1951 \\
1951\end{array}$ & $\begin{array}{l}\text { op } \\
\text { op } \\
\text { op } \\
\text { of } \\
\text { oP }\end{array}$ \\
\hline
\end{tabular}

Sec roolmont at end of ubte. 
Table 20. Existing Generating Units at U.S. Electric Utilities by State, Company, and Plant, as of January 1, 1996 (Continued)

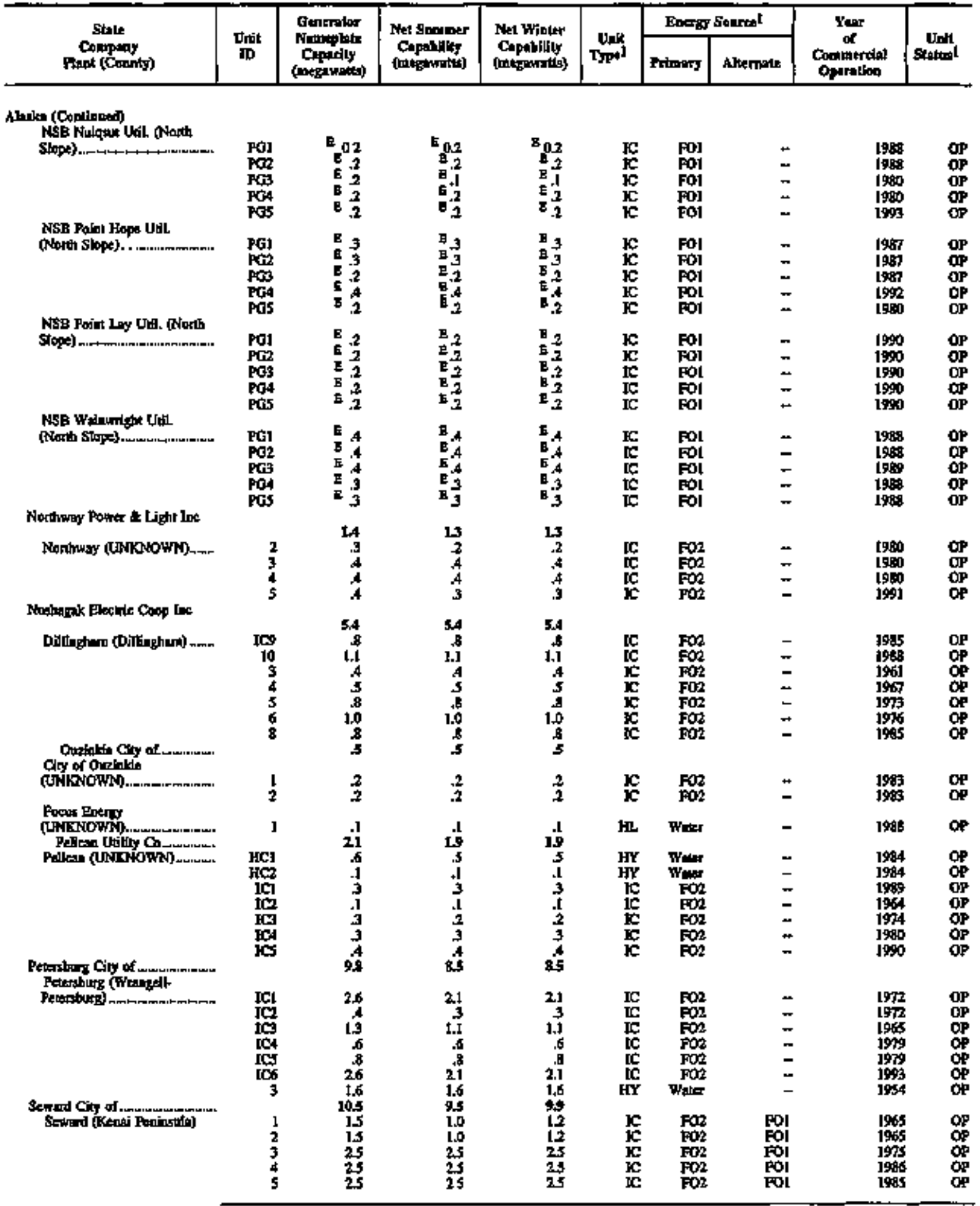

Ser taounowe * and of thite 
Tahle 20. Existing Generating Units at U.S. Electric Utilities by State, Company, and Plant, as of January 1, 1996 (Continued)

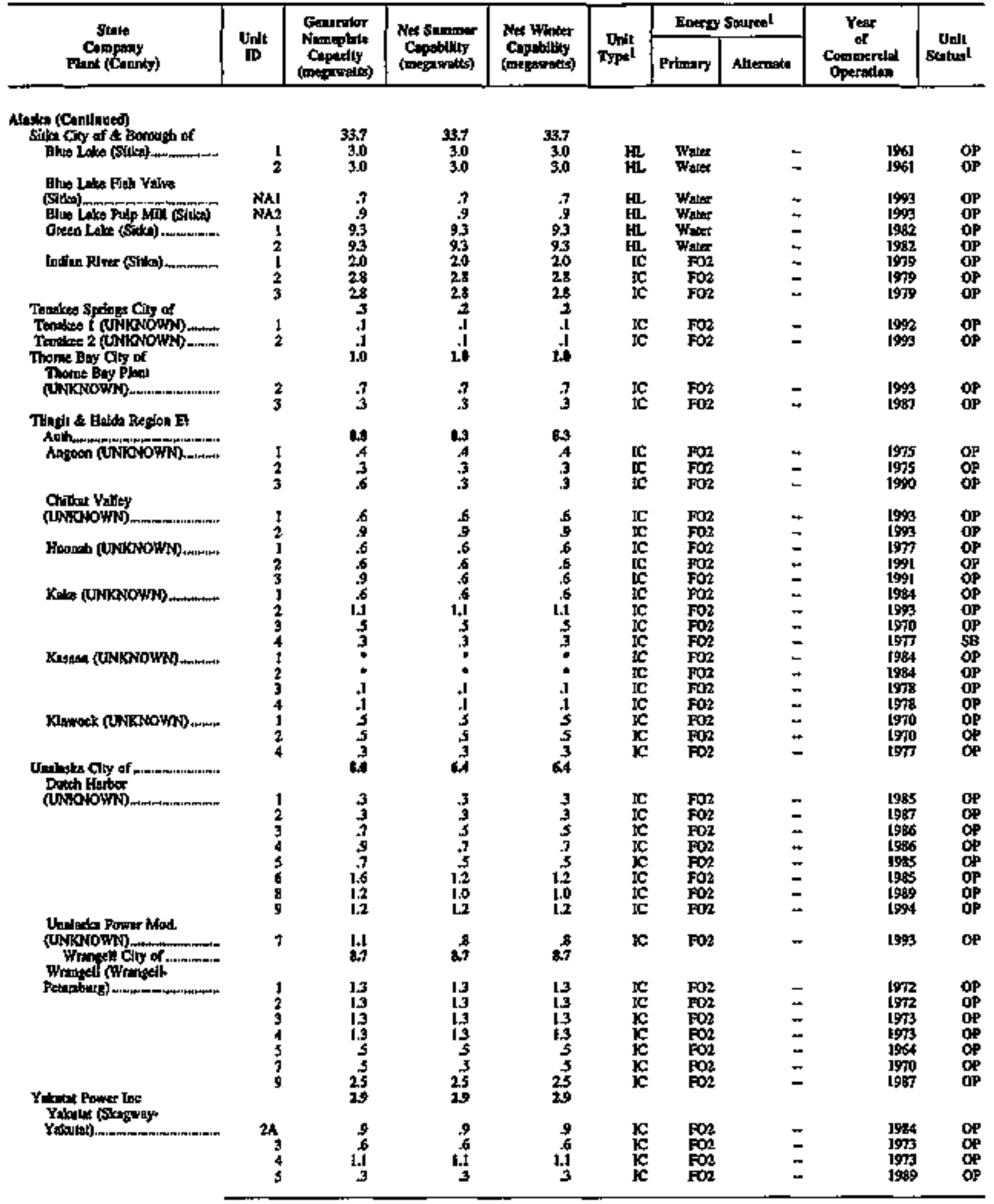

Ses foothoted at end of table. 
Table 20. Existing Generating Units at U.S. Electric Utilities by S'tate, Company, and Plant, as of January 1, 1996 (Continued)

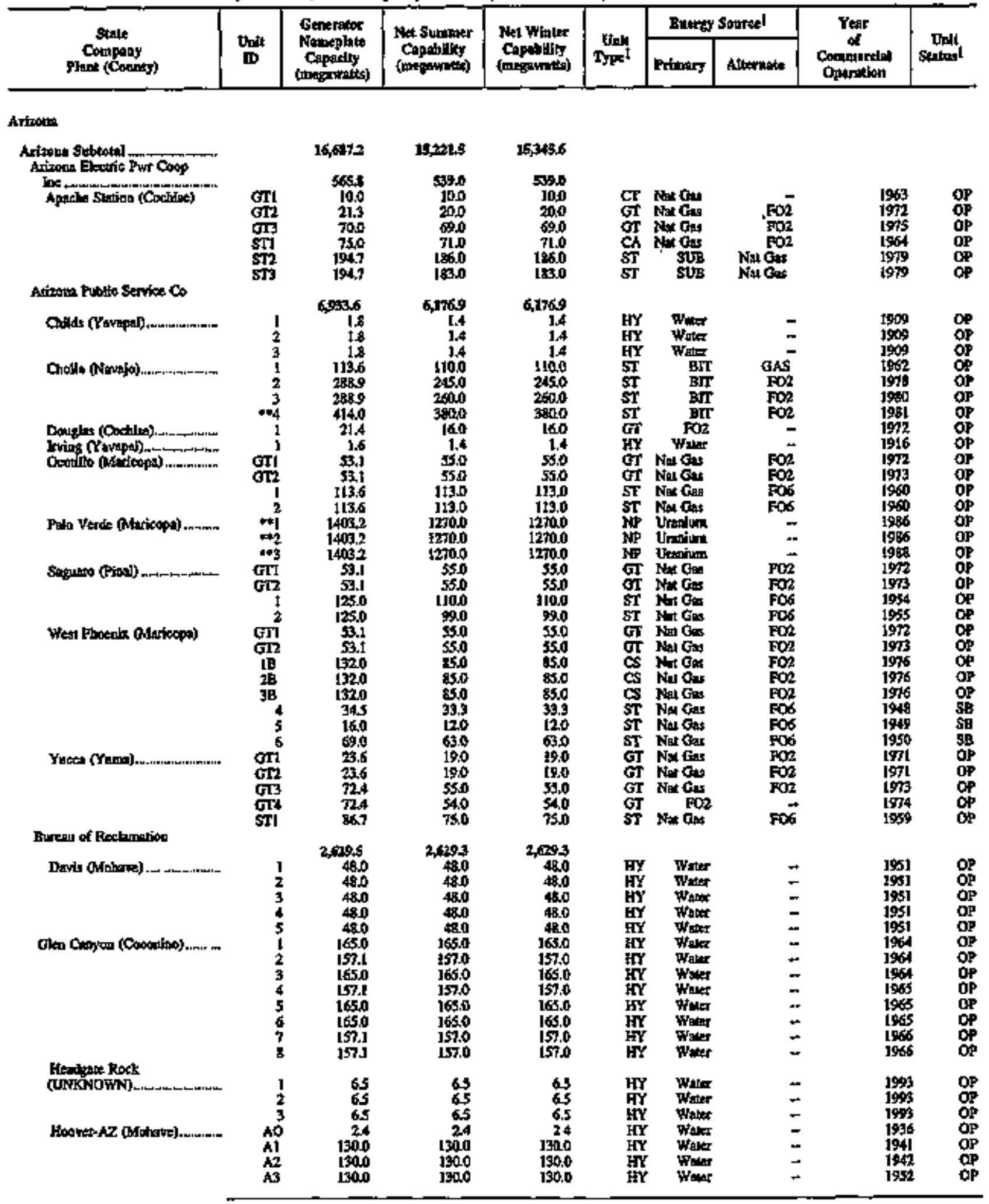

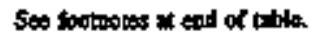


Table 20. Existing Generating Units at U.S. Flectric Utilities by State, Company, and Plant, as of January 1, 1996 (Continued)

\begin{tabular}{|c|c|c|c|c|c|c|c|c|c|c|}
\hline \multirow{2}{*}{$\begin{array}{c}\text { Sirts } \\
\text { Conpany } \\
\text { Phand (Counly) }\end{array}$} & \multirow{2}{*}{$\underset{\mathbf{b}}{\mathbf{U}}$} & \multirow{2}{*}{ 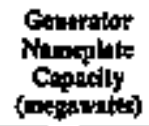 } & \multirow{2}{*}{ 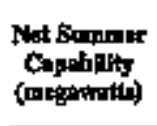 } & \multirow{2}{*}{ 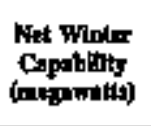 } & \multirow{2}{*}{ That } & \multicolumn{2}{|c|}{ Eneray Soured } & \multirow{2}{*}{\multicolumn{2}{|c|}{$\begin{array}{c}\text { Year } \\
\text { ot } \\
\text { Commerchol } \\
\text { Optenilon }\end{array}$}} & \multirow{2}{*}{ thit } \\
\hline & & & & & & Printry & Aktrnole & & & \\
\hline \multicolumn{11}{|l|}{ Artanu (Comlaned) } \\
\hline & A4 & 1900 & 130,0 & 130.0 & HY & Wur & - & & 1958 & \\
\hline & AS & $\$ 27,0$ & 1270 & 177.0 & EY & Wuter & 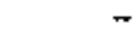 & & 1943 & 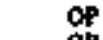 \\
\hline & A6 & $\begin{array}{l}5300 \\
130,0\end{array}$ & $\begin{array}{l}1900 \\
1900\end{array}$ & $\begin{array}{l}150.0 \\
130.0\end{array}$ & $\begin{array}{l}\text { hy } \\
\text { HY }\end{array}$ & $\begin{array}{l}\text { War } \\
\text { Wur }\end{array}$ & $\overline{-}$ & & $\begin{array}{l}19399 \\
1939\end{array}$ & $\stackrel{\mathrm{gP}}{\mathrm{OP}}$ \\
\hline & A8 & 61.5 & 61.5 & fil.s & HY & Finter & - & & 1987 & \\
\hline & 19 & 68.5 & 685 & 685 & fYY & Wmer & - & & 1902 & \\
\hline & No & 24 & 24 & 24 & KY $\mathbf{Y}$ & Wha & ـ & & 1936 & op \\
\hline 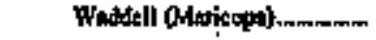 & RGS & 10.0 & 100 & 20.0 & fs & Honst & - & & 1993 & OP \\
\hline & PES & 10,0 & $\begin{array}{l}10.0 \\
100\end{array}$ & $\begin{array}{l}10,0 \\
100\end{array}$ & PS & Wwr & $\bar{z}$ & I & 1993 & GP \\
\hline & Es. & I0.0 & 100 & Hot & RS & Whar & + & & 1999 & $\begin{array}{l}\text { EP } \\
\text { OP }\end{array}$ \\
\hline \multirow{6}{*}{ 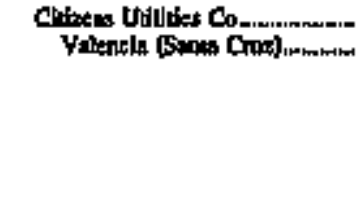 } & & EAA & 43. & 510 & & & & & & \\
\hline & GII & I6.8 & 193 & 15.8 & $\mathbf{G}$ & Non ons & $\mathrm{FO} 2$ & & 1989 & 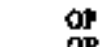 \\
\hline & GI2 & $\begin{array}{l}\text { IEB } \\
\text { las }\end{array}$ & $\begin{array}{l}13.5 \\
{[3.5}\end{array}$ & $\begin{array}{l}15.8 \\
160\end{array}$ & $\begin{array}{l}\text { GT } \\
\text { GT }\end{array}$ & $\operatorname{Nin}_{n=1}$ & FO2 & & 1989 & OP \\
\hline & 1 & 1.0 & .9 & 9 & IC & $\mathrm{FO}_{\mathrm{O} 2}$ & $\operatorname{sex} G_{t s}$ & & 1949 & \\
\hline & 2 & 10 & 9 & 9 & IC & F02 & rot Gas & & 1949 & \\
\hline & 3 & $\begin{array}{l}1.0 \\
1.0\end{array}$ & 9 & 9 & KC & $\mathrm{Fon}$ & Net Gou & & 1949 & op \\
\hline \multirow{7}{*}{ 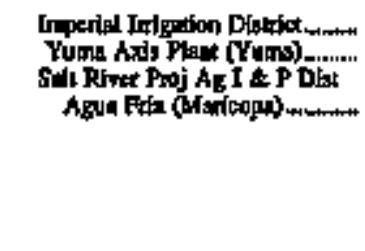 } & & 234 & 22 & $2 \geq 0$ & & & & & & \\
\hline & 1 & 23.4 & 220 & 220 & GT & $\mathbf{P O Z}$ & - & & 1978 & OP \\
\hline & & & & 4,60034 & & & & & & \\
\hline & $\mathrm{AF}_{\mathrm{A}}$ & $\begin{array}{l}113.6 \\
113.6\end{array}$ & $\begin{array}{l}3630 \\
1330\end{array}$ & $\begin{array}{l}1140 \\
1140\end{array}$ & $\underset{S T}{S T}$ & Non Gas & $\mathrm{FOZ}$ & & 1954 & op \\
\hline & A & 163.2 & 181.0 & 1840 & st & Nol Gess & FO2 & & $\begin{array}{l}1396 \\
1961\end{array}$ & ar \\
\hline & $A \sqrt{4}$ & 80,6 & 720 & 87,0 & GT & Nar Gas & EON & & 1975 & OP \\
\hline & APF & $\frac{71.2}{71.2}$ & 70.0 & 75.0 & 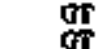 & fal $\mathrm{O}_{\mathrm{t}}$ & FO2 & & 1974 & of \\
\hline Corondso (Apatres) & ACOI & 410.9 & 36.0 & 365.0 & $\mathbf{s T}$ & BIT & sut & & 1919 & 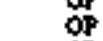 \\
\hline (1) & $\cos 2$ & 410.9 & 350.0 & 350.0 & $\mathbf{S T}$ & BIT & suB & & I960 & ap \\
\hline \multirow[t]{4}{*}{ Gossol (Minflopa) } & C)। & 7.5 & git & 40 & $\mathbf{S I}$ & Mal Gas & For & & 1942 & \\
\hline & $\infty_{0}$ & 75 & 8.0 & $\begin{array}{l}8.0 \\
80\end{array}$ & STI & Nun $G$ & Fod & & 1942 & 8 \\
\hline & ct & is & 8.0 & 8.0 & ST & Nol Car & pos & & 1948 & $\begin{array}{ll}\mathrm{sB} \\
\mathrm{s}\end{array}$ \\
\hline & $\alpha$ & $\mathbf{3 0}$ & 3,0 & 3.0 & HY & Water & 4 & & 1939 & ap \\
\hline \multirow{4}{*}{ 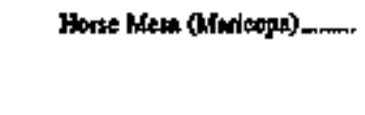 } & Ho1 & 9.9 & 10,0 & 100 & $\mathrm{kY}$ & Waler & - & & 1927 & $\mathbf{P}$ \\
\hline & Bhi2 & 9.9 & 100 & 100 & $\mathbf{H Y}$ & Waler & - & & 1927 & P \\
\hline & FMa3 & 99 & 100 & I0.0 & ตY & Walor & 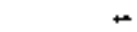 & & 1927 & Ur \\
\hline & FA & 99.9 & 98,0 & 980 & ps & Water & - & & $19 \pi$ & is \\
\hline Kynens (Hadscopa) & $\mathbf{K Y} \mathbf{I}$ & 345 & 340 & 340 & $\mathbf{s T}$ & Nad $\mathrm{Gar}$ & FOr & & 1952 & OP \\
\hline & $\mathbf{K Y 2}$ & 73.5 & 720 & 72.0 & $\boldsymbol{\Sigma} \mathbf{T}$ & Nur Gar & FOE & & 1954 & OP \\
\hline & $\underset{K Y Y_{4}}{K Y 3}$ & 53.1 & \$70 & $\begin{array}{l}30,0 \\
690\end{array}$ & GT & Nat Gas & FO2 & & I972 & $\underset{\text { OP }}{\mathrm{P}}$ \\
\hline & KYy & 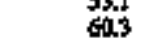 & 310 & 61,0 & a & $\begin{array}{l}\text { Nar Gas } \\
\text { Nim ots }\end{array}$ & $\mathrm{FO} 2$ & & $\begin{array}{l}19 \pi 1 \\
1973\end{array}$ & op \\
\hline & KY 6 & s03 & 500 & $\$ 0$ & GT & Wo Gess & Fon & & 19T3 & $\mathrm{QP}$ \\
\hline 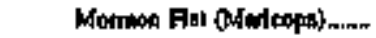 & MFt & 9.2 & 110 & 31.0 & $\mathbf{H Y}$ & Waer & $=$ & & 1926 & op \\
\hline & MIP2 & 48.6 & 470 & 47,0 & PS & Wous & - & & 1971 & op \\
\hline 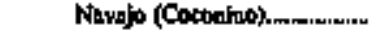 & mikYl & 803.2 & 750.0 & 750,0 & sT & stise & - & & 1974 & F \\
\hline & aNAY2 & 803.2 & 7500 & 7500 & $\mathbf{s t}$ & stos & - & & 1975 & $\mathbf{P}$ \\
\hline & & 803 & 750 & 75 & sT & so & - & & 1976 & S \\
\hline 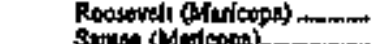 & Roos & 360 & 960 & 360 & $\mathbf{E Y}$ & What & - & & 1973 & PP \\
\hline & $\begin{array}{l}\text { STH } \\
\text { ST2 }\end{array}$ & $\begin{array}{l}10.5 \\
103.5\end{array}$ & $\begin{array}{l}76,0 \\
74.0\end{array}$ & $\begin{array}{l}87.0 \\
85.0\end{array}$ & cs & Nat Gas & $\mathrm{FO2}$ & & $\begin{array}{l}1974 \\
1974\end{array}$ & op \\
\hline & $\mathrm{SZ3}$ & 1035 & 800 & 91.0 & cs & $N \neq G_{\Delta x}$ & Fot & & 1974 & OP \\
\hline & ST4 & 103.5 & $\pi 0$ & 3890 & es & $\operatorname{Nan} G u$ & Fot & & ins & OP \\
\hline Soeth Consolidaled & sci & 1* & (d & 4 & $17 y$ & Won: & 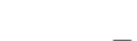 & & 1081 & De \\
\hline Stewot Notonoin & scl & $1 A$ & 1.4 & 3.4 & HY & Wots & - & & $19 \% 1$ & De \\
\hline (Mindoppe) & SM & lof & 1310 & 19.0 & HY & Wher & - & & 1950 & of \\
\hline Lewon Electic Pow & & $1,604,6$ & 13150 & 13150 & & & & & & \\
\hline DE Mour Petrie (Pinno) & $\underset{G T I}{\text { GTI }}$ & 65 & 470 & m.0 & $\mathbf{G T}$ & Nex Clas & $\operatorname{Fon}$ & & 1973 & $\stackrel{p P}{O P}$ \\
\hline п) & GI & 270 & $\underset{250}{240}$ & $\begin{array}{l}24,0 \\
\mathbf{2 5 0}\end{array}$ & GI & $\begin{array}{l}\text { Nat Gas } \\
\text { Nat Gas }\end{array}$ & $\mathrm{Foz}_{02}$ & & $\begin{array}{l}1972 \\
19 m 2\end{array}$ & $\stackrel{O P}{O P}$ \\
\hline & ors & 27,0 & 250 & 250 & or & $N=d_{a}$ & $\mathrm{FO2}$ & & 1974 & of \\
\hline & STI & 1085 & 8.0 & 51.0 & ST & Nax de. & $F 06$ & & 1998 & pe \\
\hline & $\frac{\sin }{s+3}$ & $\begin{array}{l}\mathrm{I0s.8} \\
113.6\end{array}$ & $\begin{aligned} 8 \mathrm{t} .0 \\
1040\end{aligned}$ & $\begin{array}{r}85.0 \\
104.0\end{array}$ & $\begin{array}{l}\mathbf{S T} \\
\mathrm{ST}\end{array}$ & Nax Gas & 506 & & $\begin{array}{l}1960 \\
1960\end{array}$ & $O P$ \\
\hline & & & & & & & & & & \\
\hline
\end{tabular}

Ses frodeolen at and of whing. 
Tabie 20. Existing Generating Units at U.S. Electric Utlities by State, Company, and Plant, as of Janurary 1, 1996 (Continted)

\begin{tabular}{|c|c|c|c|c|c|c|c|c|c|}
\hline \multirow{2}{*}{$\begin{array}{c}\text { Btata } \\
\text { Company } \\
\text { Plom (Count) }\end{array}$} & \multirow{2}{*}{ Ins' } & \multirow{2}{*}{ 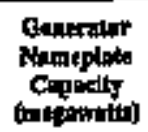 } & \multirow{2}{*}{ 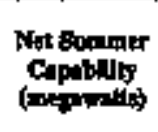 } & \multirow{2}{*}{ 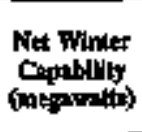 } & \multirow{2}{*}{ Jalt } & \multicolumn{2}{|c|}{ Enengt sourcel } & \multirow{2}{*}{$\begin{array}{c}\text { Yerr } \\
\text { of } \\
\text { Opantinition }\end{array}$} & \multirow{2}{*}{ Dinlint } \\
\hline & & & & & & 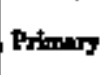 & Alitmate & & \\
\hline
\end{tabular}

Artzen (Conthioed)

Noth 1 pop (Pran)

Springaville (Aperde)

U S Brtedn of Iadien Affits

Cootidas (Gili)

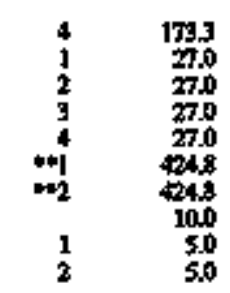

1100
250
250
230
250
3500
3600
140
50
50

Artionsos

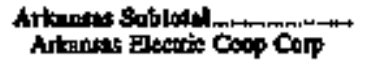

Carl Billay (Woodent)

Dam 9 (Coming) -

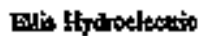

(C)

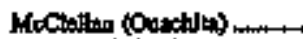

Thooles Bribotion

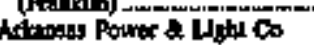

Atroust: Whatear Oen

(Pape)

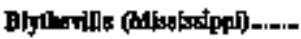

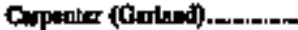

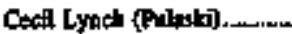

Etrolion Nosed st

Erincis)

Anrey Cooch (Loflytu)

Indkpentiedect

(independienc:)

Lon Culvetion aros

Spring)

Mabelprale (Punnci).

Renpel (Hins Spring)

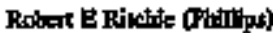

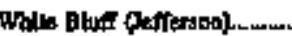

intorion $\mathrm{C}^{2} \mathrm{r}$ of

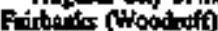

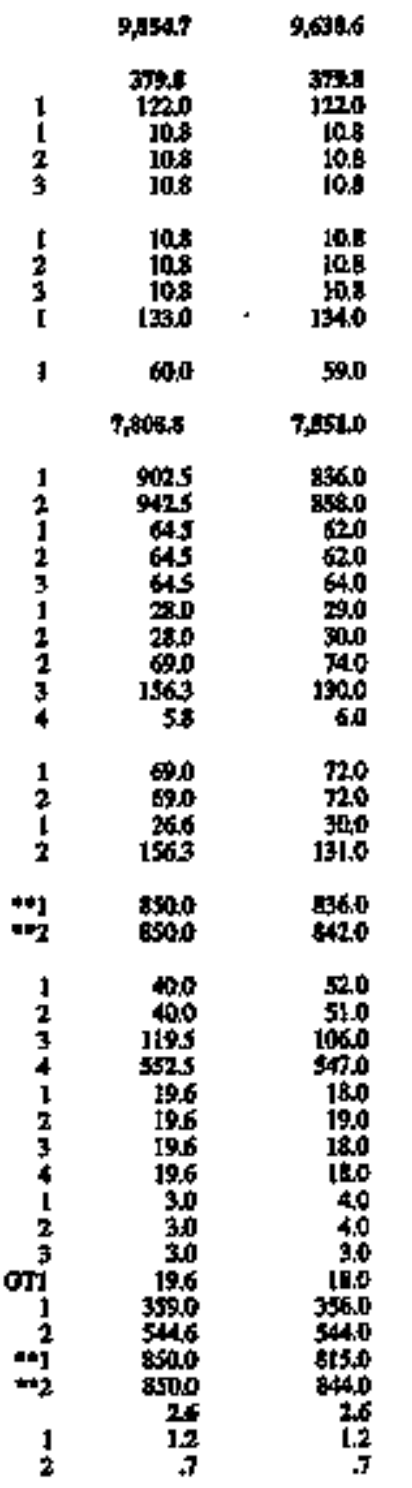

1906

3rs:

ja.t

10.8 3 WY Ware

jo.8 fr war

to.8

108

10.8

108

1340

59.0

T도에.

6350

85.0

620

S20

64.40

29.0

300

1900
600

20

300

30,0
131.0

fr Wrar

HY Wher

HY Wer

LY Wros

ST NN OhA

ST Nat Ges

Fos

$\begin{array}{ll}- & 1995 \\ = & 1999 \\ \Rightarrow & 1925\end{array}$

1967

1972

1972

1974

$19 \% 0$

-

$19 \%$

1929

OP
DP
OP
OP
OP
OP
OP
OS
OS

$\begin{array}{lllll}5160 & 50 B & - & 1983 & O P\end{array}$

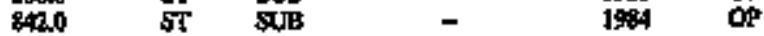

S20 ST Nates For 1950 s:

\$t, 0 ST Ne h.

1060 ST yert Ga

ST ST No

IBO GI Not

I9.4 GT Hat Go

130 Or ve कि

190 GT Kas o.

40 HY Whet

4.0 HY Wr Wrot

190

3560

544.0

244.0

CI Nar Gat

ST N* Dat

ST Niv Gu

BT SUB

$\begin{array}{cc}- & 1989 \\ \overline{-} & 1998 \\ \mathrm{~F} & 1972\end{array}$

Fos

1063

OP
Op
OP
OP
OP
op
OP
OP
OP

2.6

$\frac{1}{7}$

IC FOn Nat Grs

Nol Ges

Fo6

FOS

Fo2

Fod

Fo?

Foy

$=$

Fon

FOS

EOS

-

1950

1953

1970

1970

1970

tomo

1925

ighs

1925

$19 \pi$

1961

196

$19 \%$

1957

1948

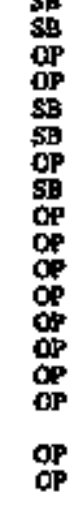

Ses roomoned an and of rablo.

62 Fnergy Information Admioistration/tnyentory of Power Plants in the United States as of Japuary 1,1996 
Table 20. Existing Generating Units at U.S. Flectric Utillties by State, Company, and Plant, as of January 1, 1996 (Continned)

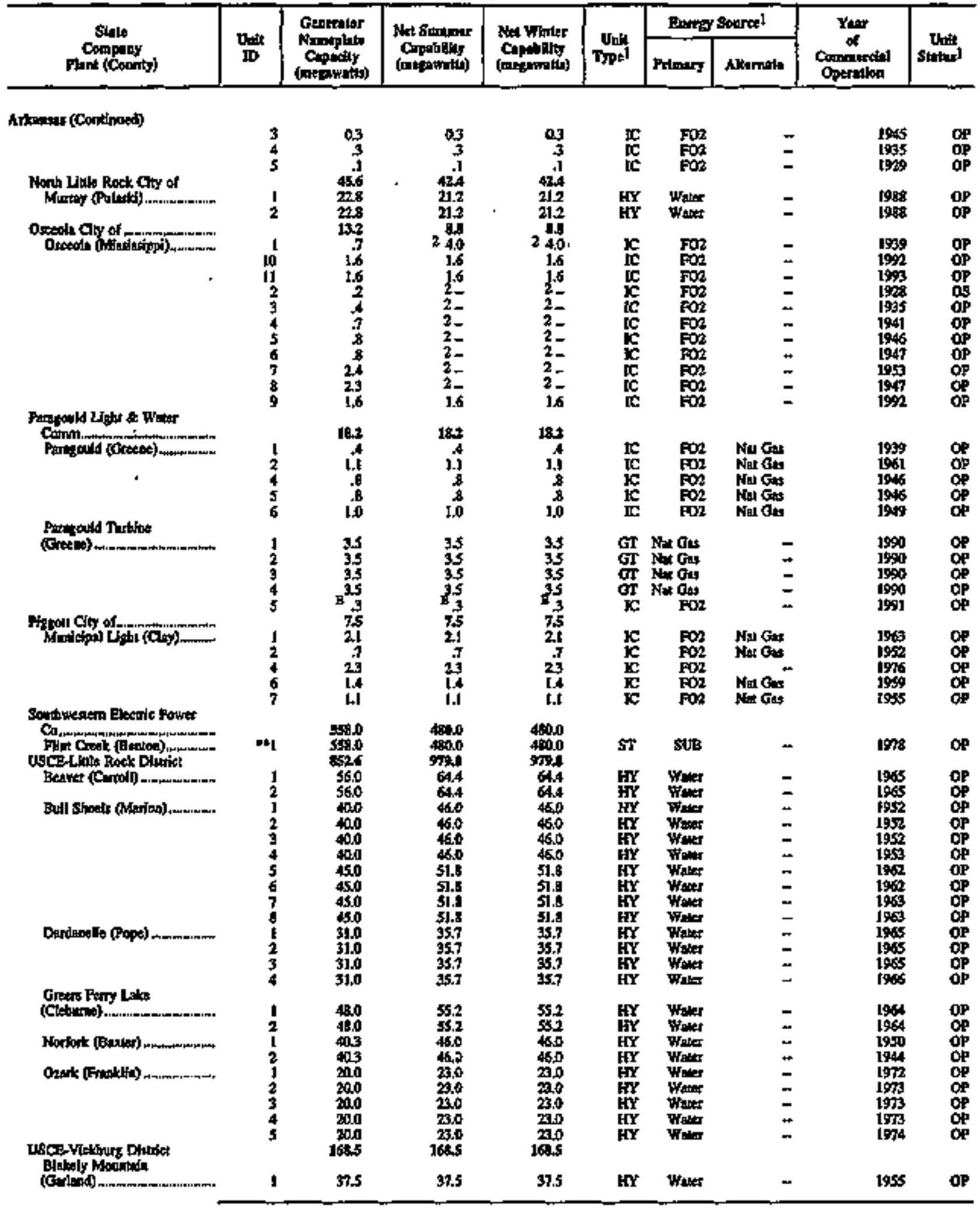

See foothoinar at end of table 
Table 20. Existing Generating Units at U.S. Electric Utilities by State, Company, and Plant, as of January 1, 1996 (Continued)

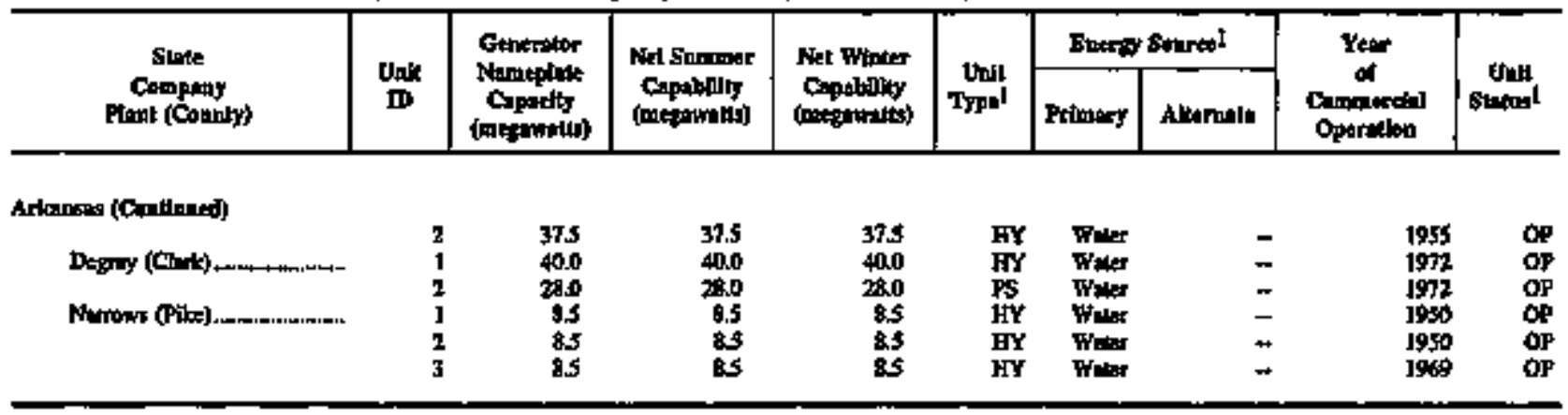

Sec foctolot al ted of thith

64 Energy Information Administration/Inventory of Power Plants in the United States as of January 1, 1996 
Table 20. Existing Generating Units at U.S. Electric Utilities by State, Company, and Plant, as of January 1, 1996 (Continued)

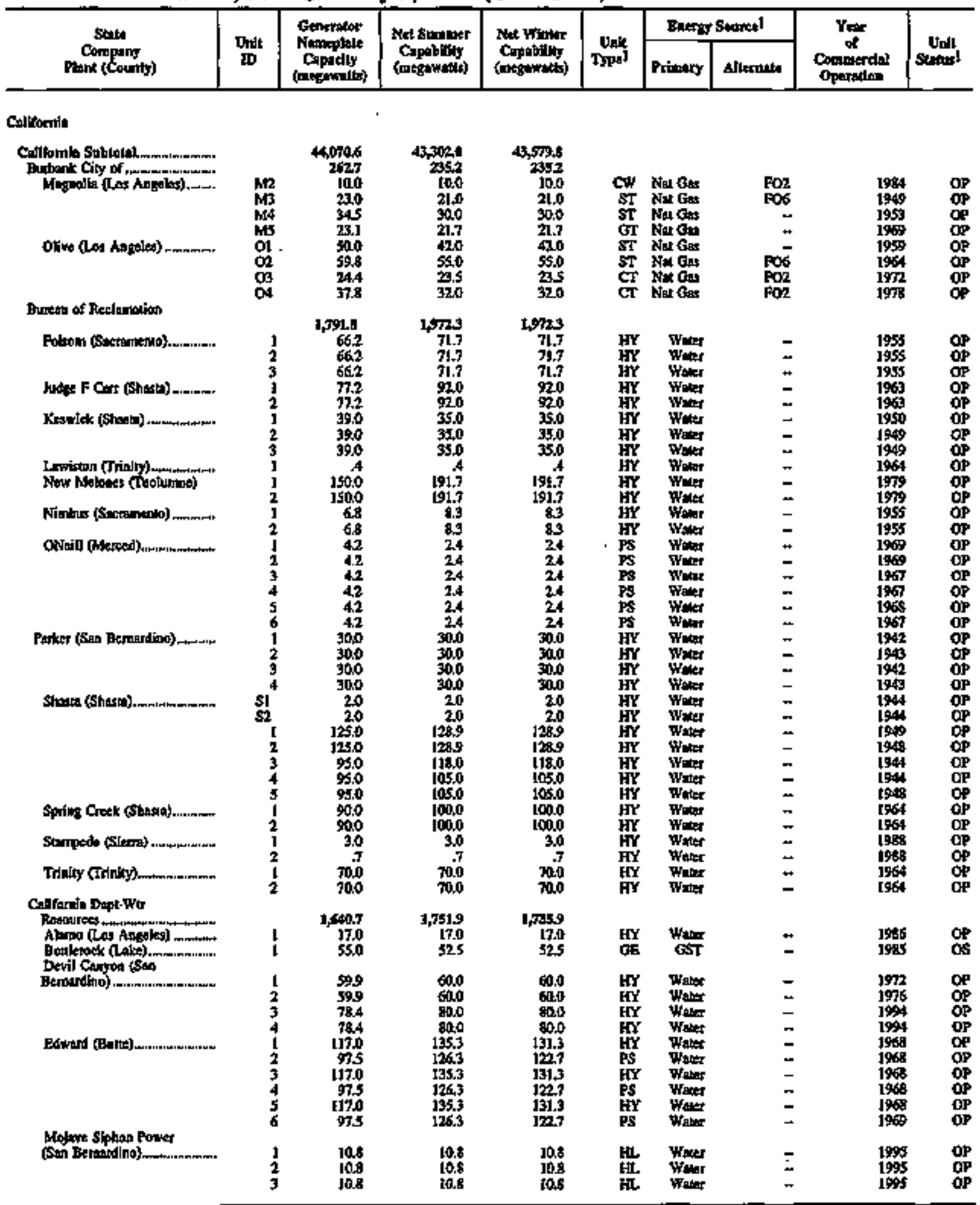

Sect loodmotes at enot of wble. 
Table 20. Existing Generating Units at U.S. Electric Utilities by State, Company, and Plant, as of January 1, 1996 (Continued)

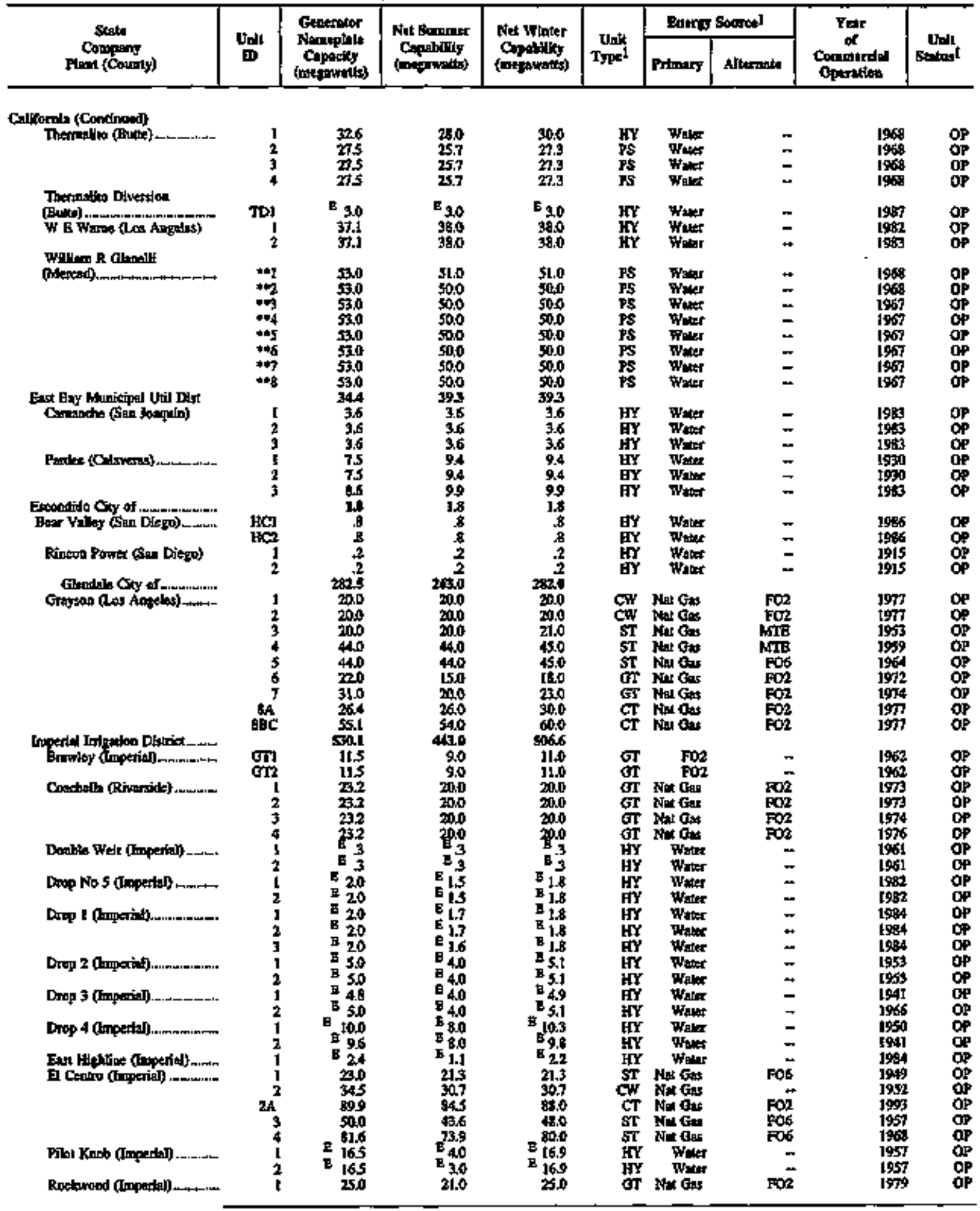

Ses sootions at end of tabls. 
Table 20. Existing Generating Units at U.S. Electric Utilities by State, Company, and Plant, a of January 1, 1996 (Continned)

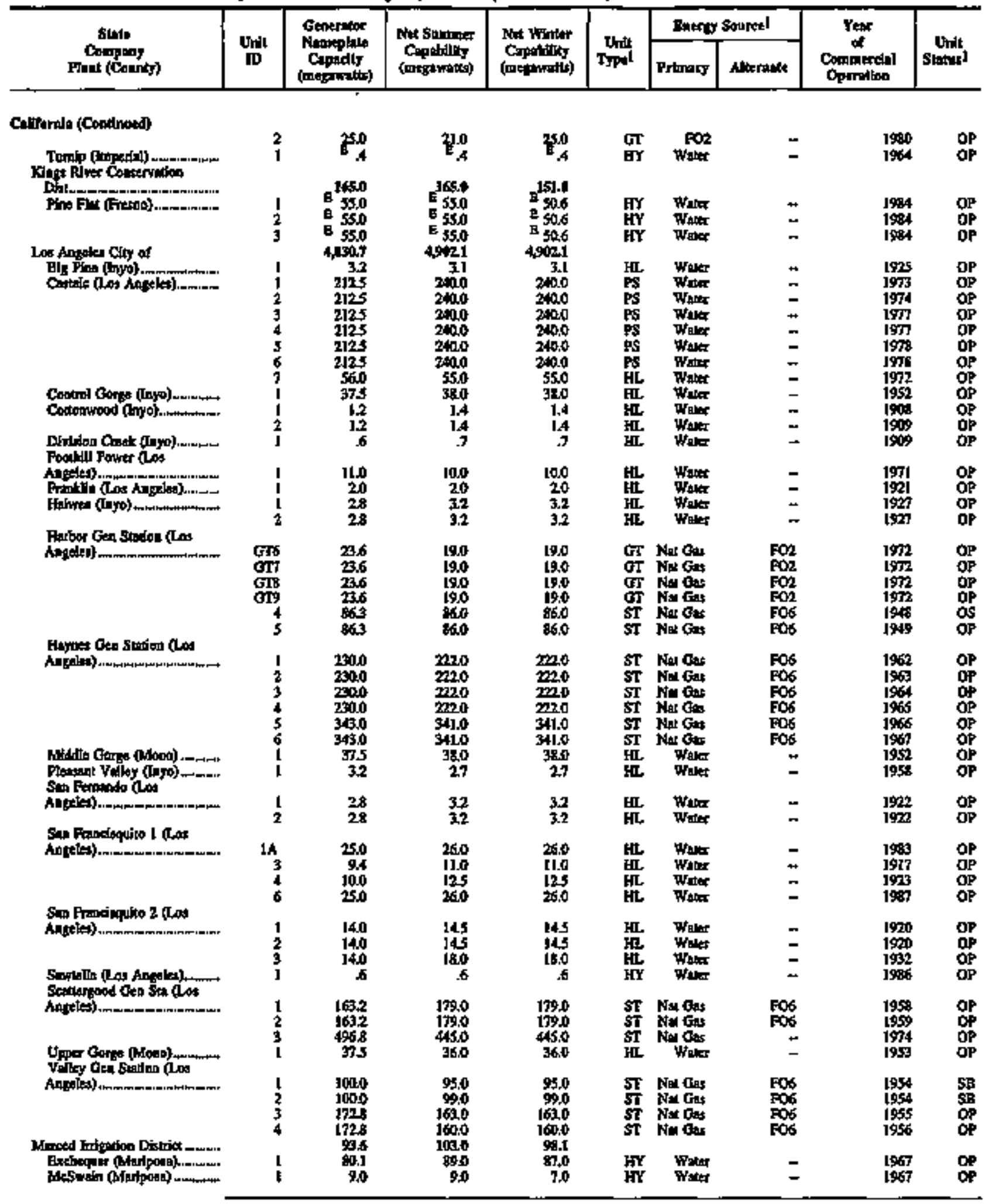

Sect founowy at ent of eabto. 
Table 20. Existing Generating Units at U.S. Electric Utilities by State, Company, and Plant, as of Jamuary 1, 1996 (Continned)

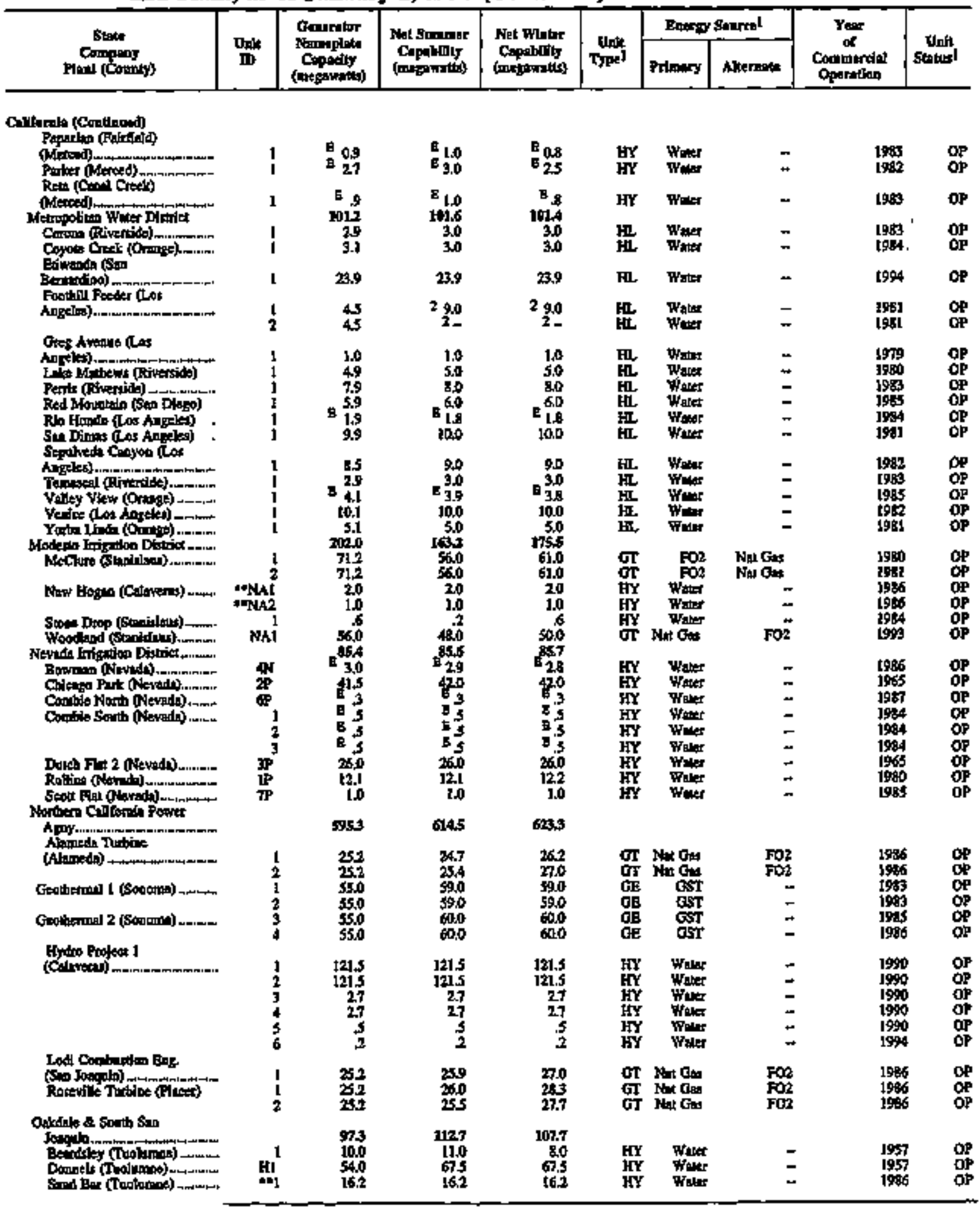

Sec frowiond at ode af tabte. 
Table 20. Existing Generating Units at U.S. Electric Utilities by State, Company, and Plant, as of Jaauary 1, $19 \%$ (Continued)

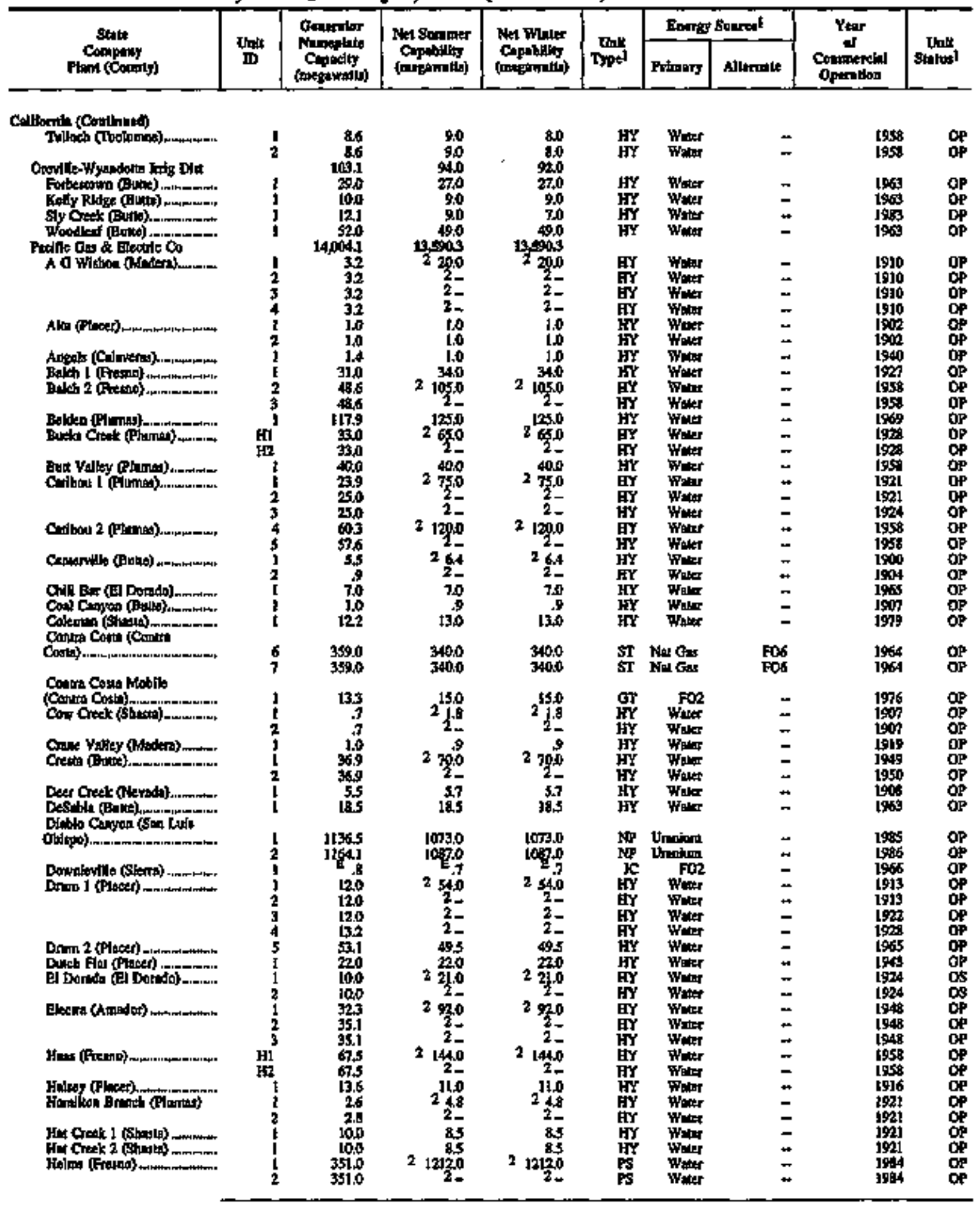

Sow fooleoke at end of tabte. 
Table 20. Existing Generating Units at U.S. Ełectric Utilities by State, Company, and Plant, as of January 1, 1996 (Continued)

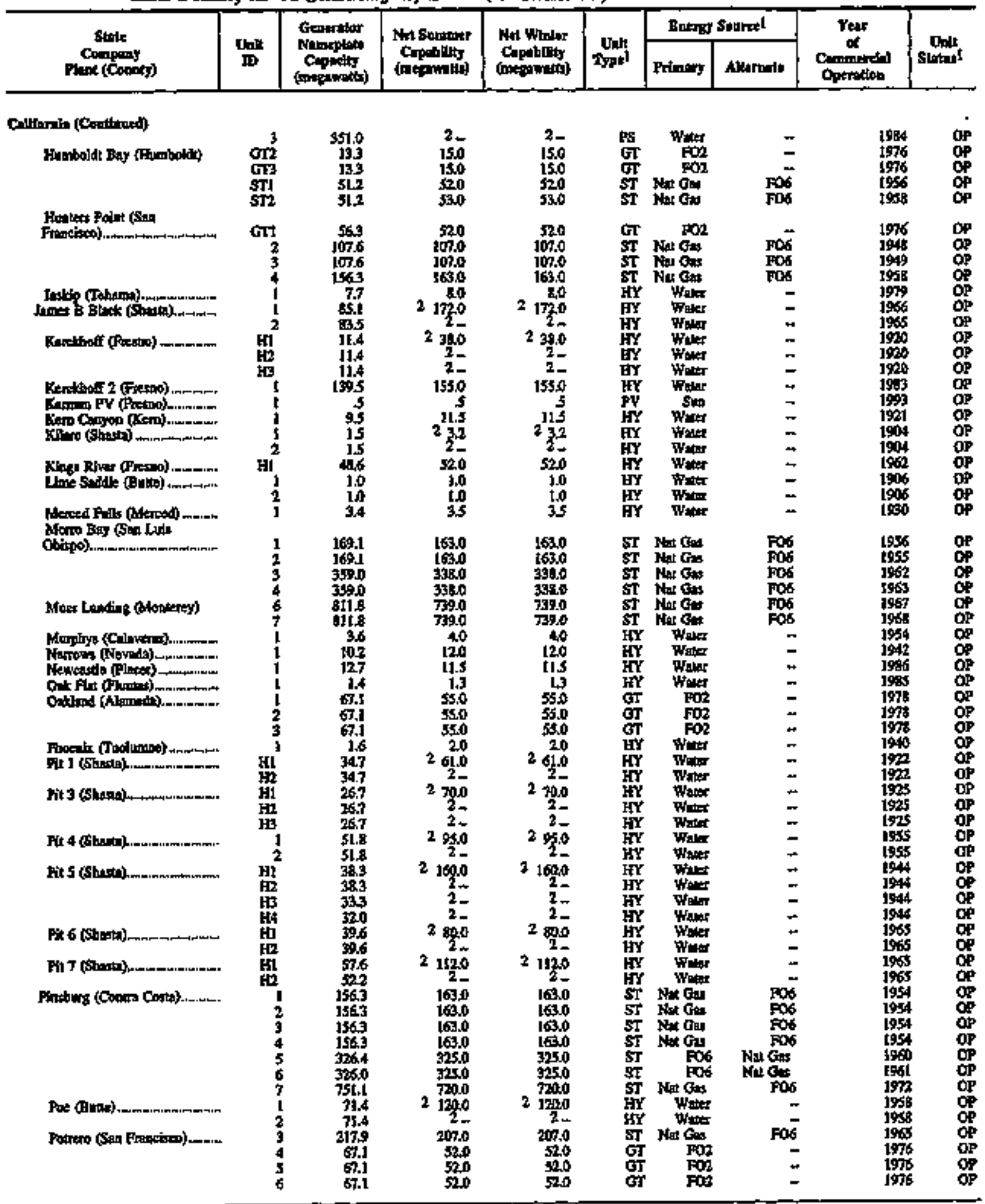

Soc toonoces an and of tible. 
Table 20. Existing Generating Ünits at U.S. Electric Utilities by State, Company, and Plant, as of January 1, 1996 (Continued)

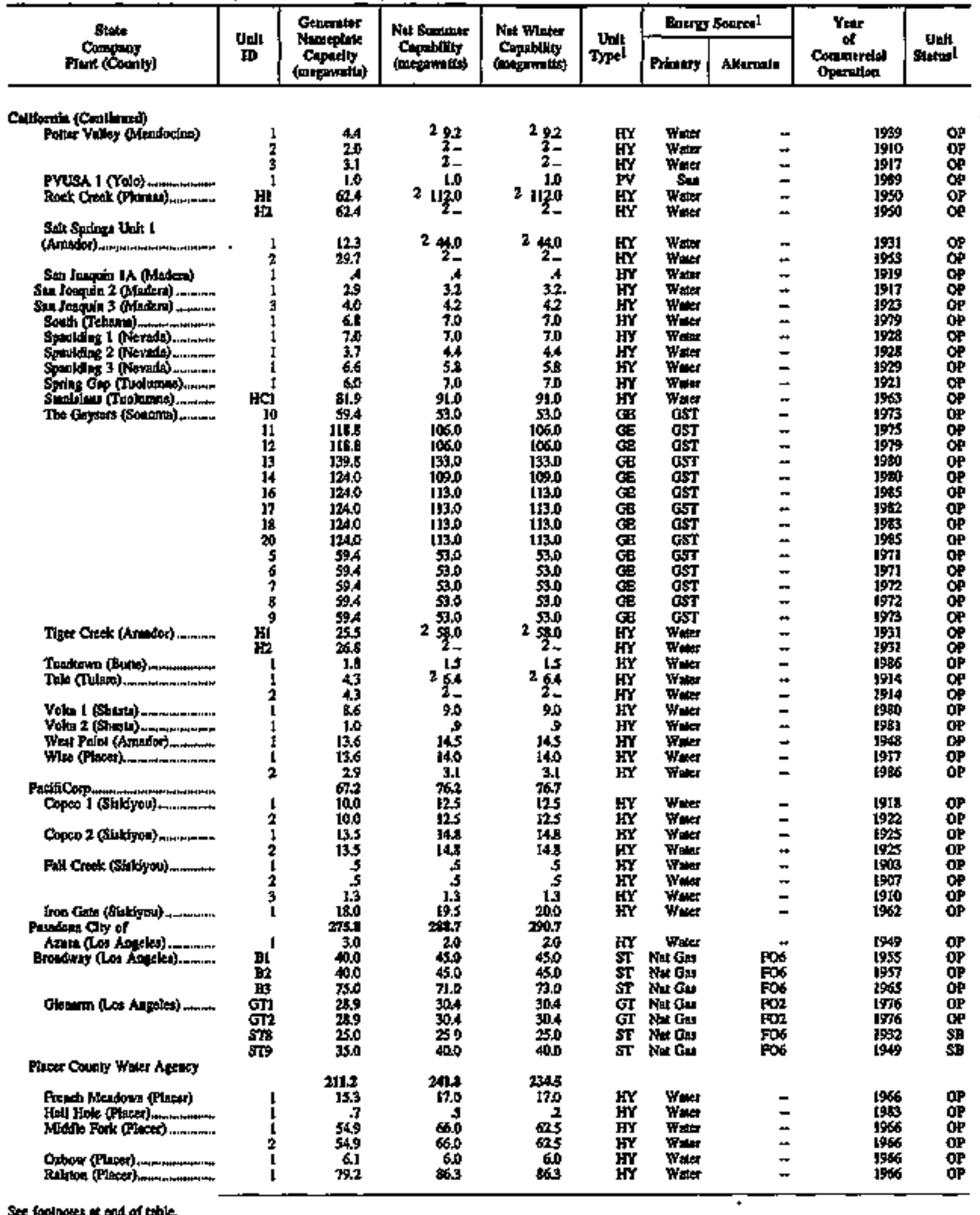


Table 20. Existing Generating Units at U.S. Electric Utilities by State, Company, and Plant, as of January 1, 1996 (Continued)

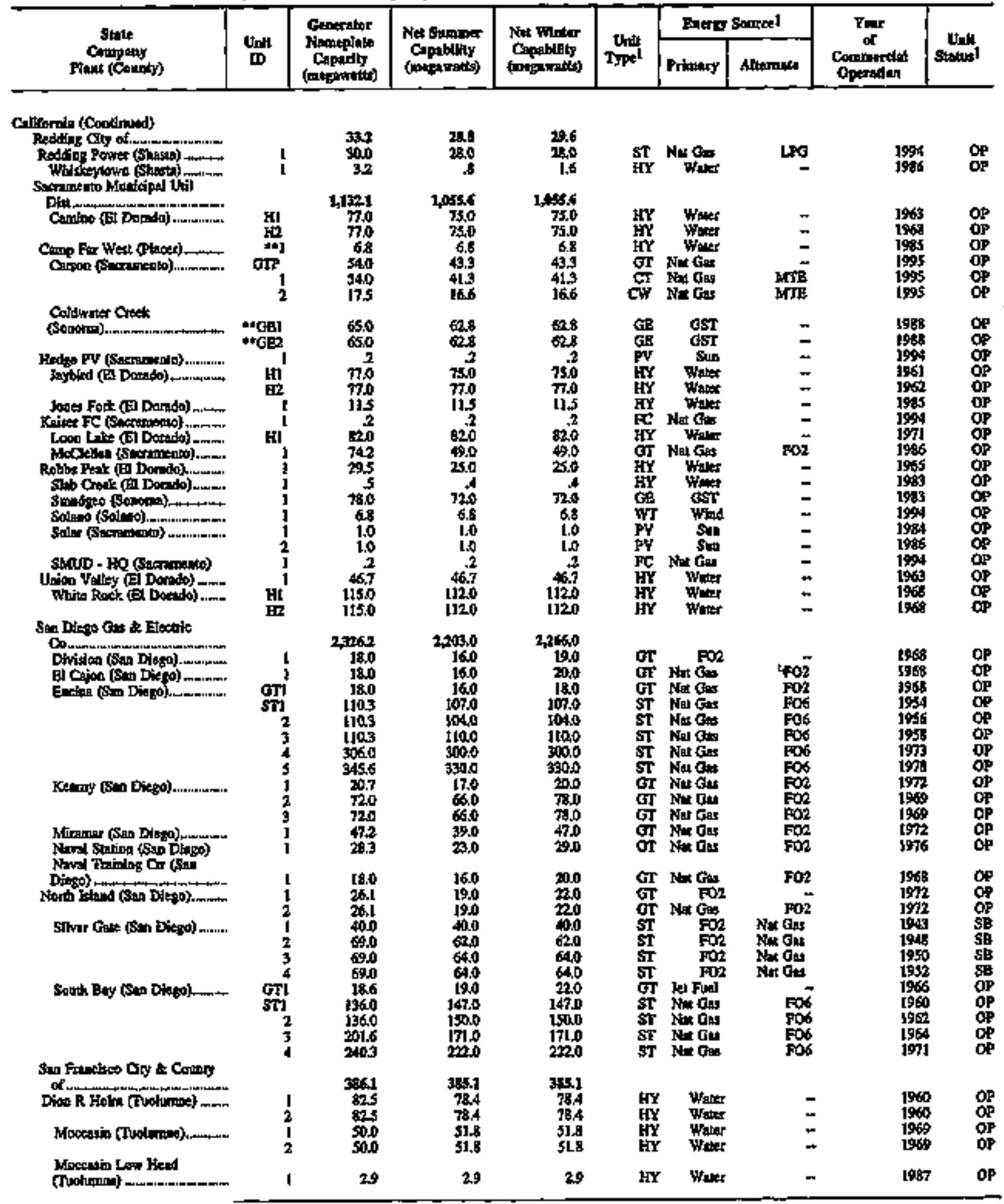

Sec footions at ado of thith

72 Energy Information Administration/Inventory of Power Plants in the United States as of January 1,1996 
Table 20. Existing Generating Units at U.S. Electric Utillties by State, Company, and Plant, as of January 1, 1996 (Continued)

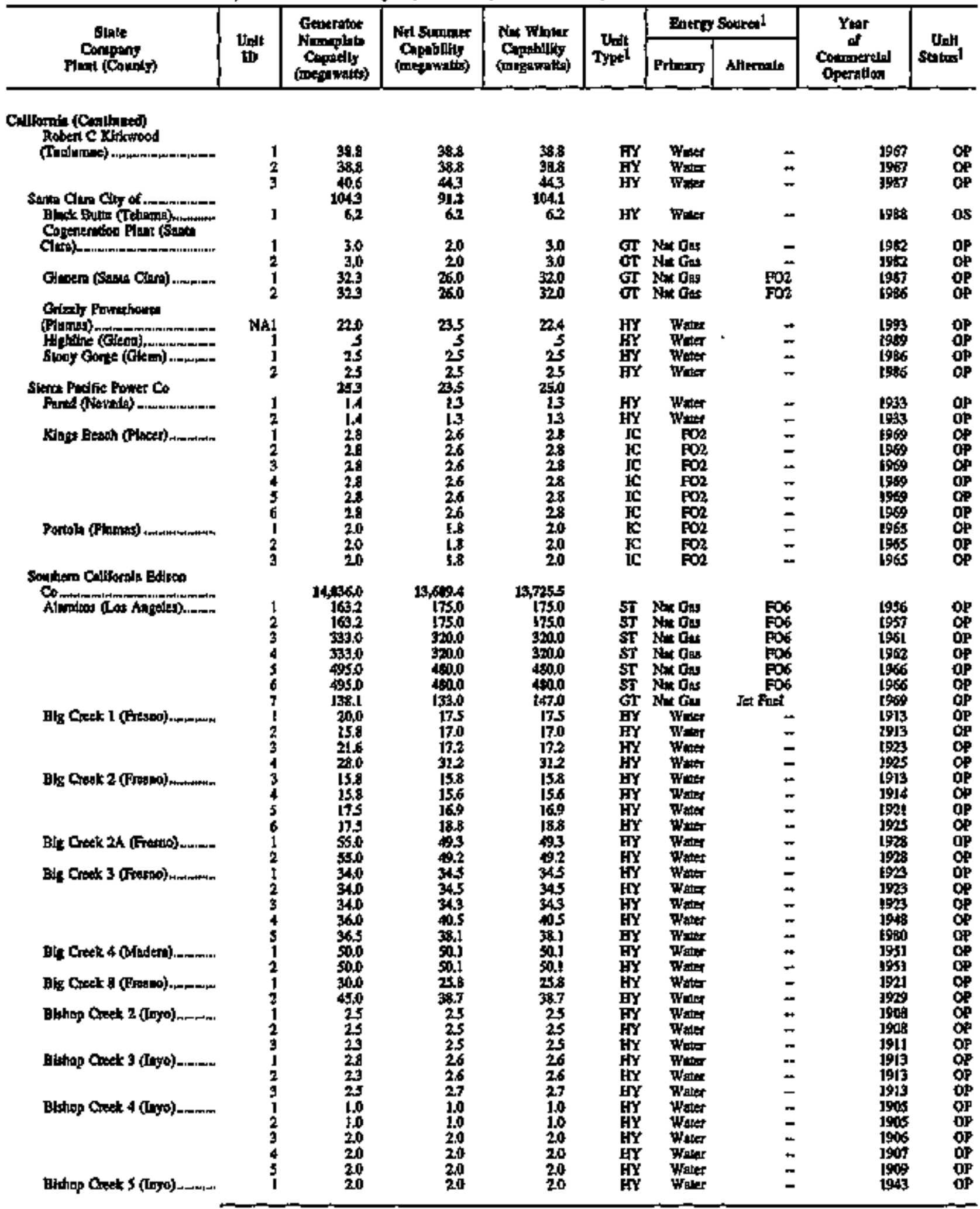

See foowetes it and of chls. 
Table 20. Existing Generating Units at U.S. Electric Utilities by State, Company, and Plant, as of January 1, 1996 (Conthued)

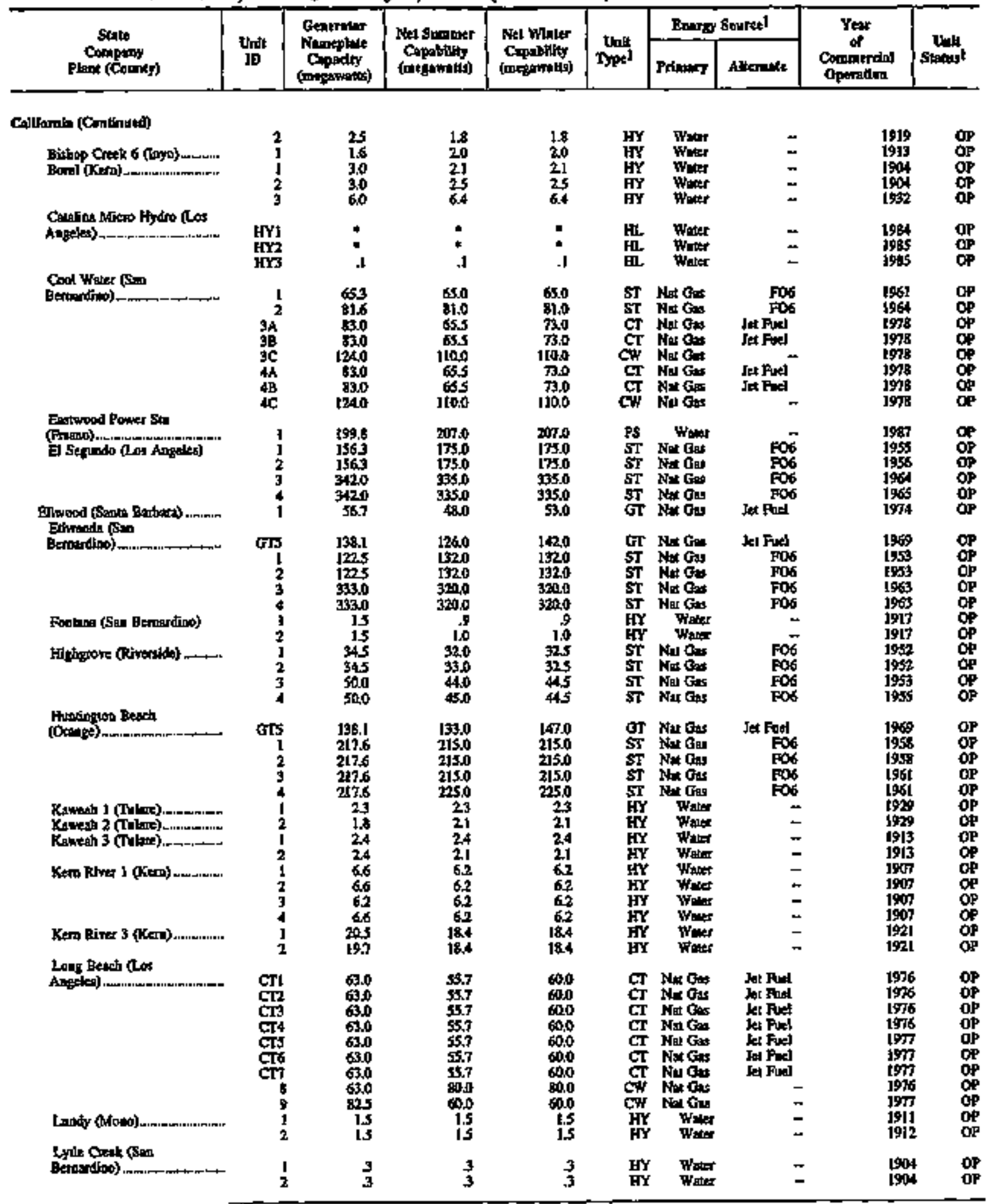

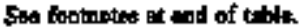


Table 20. Existing Generating Units at U.S. Electric Utilities by State, Company, and Plant, as of January 1, 1996 (Continued)

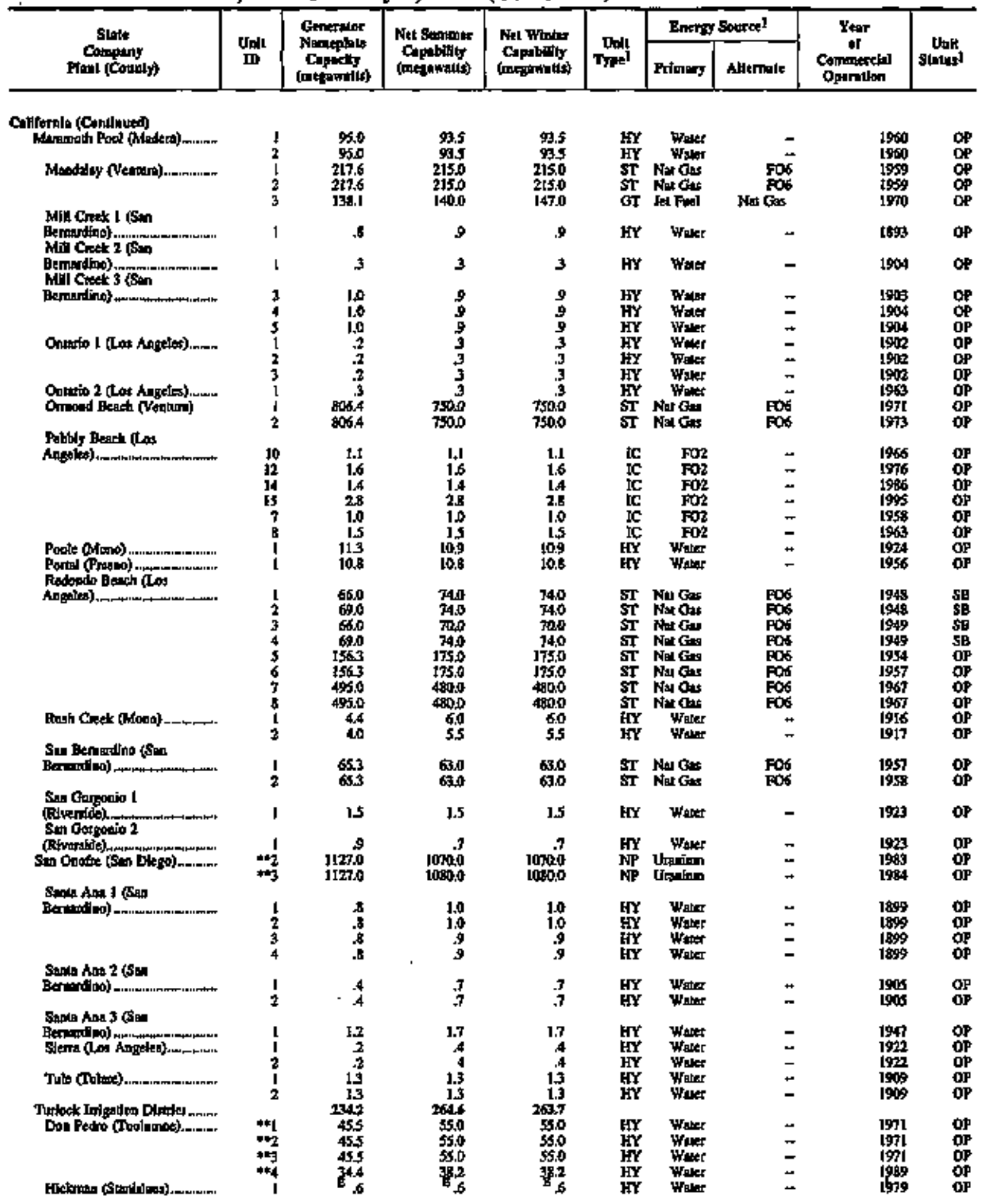

Seo soonoles ax end of nable. 
Table 20. Existing Generating Units at U.S. Electric Utilities by State, Company, and Plant, as of Jamuary 1, 1996 (Continued)

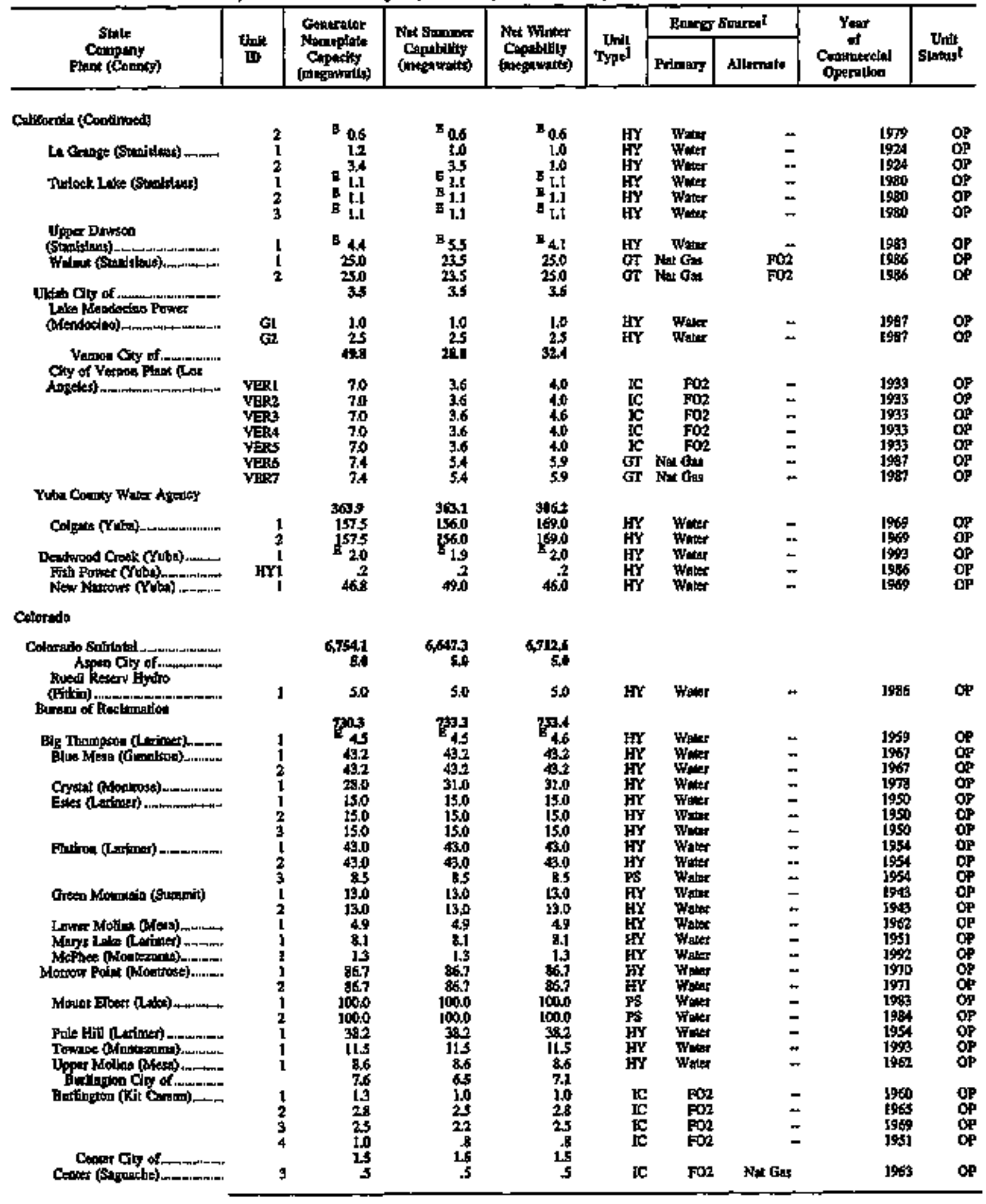

Sect tooboust in etid of cable. 
Table 20. Existing Generating Units at U.S. Electric Utilities by State, Company, and Plant, as of January 1, 1996 (Continued)

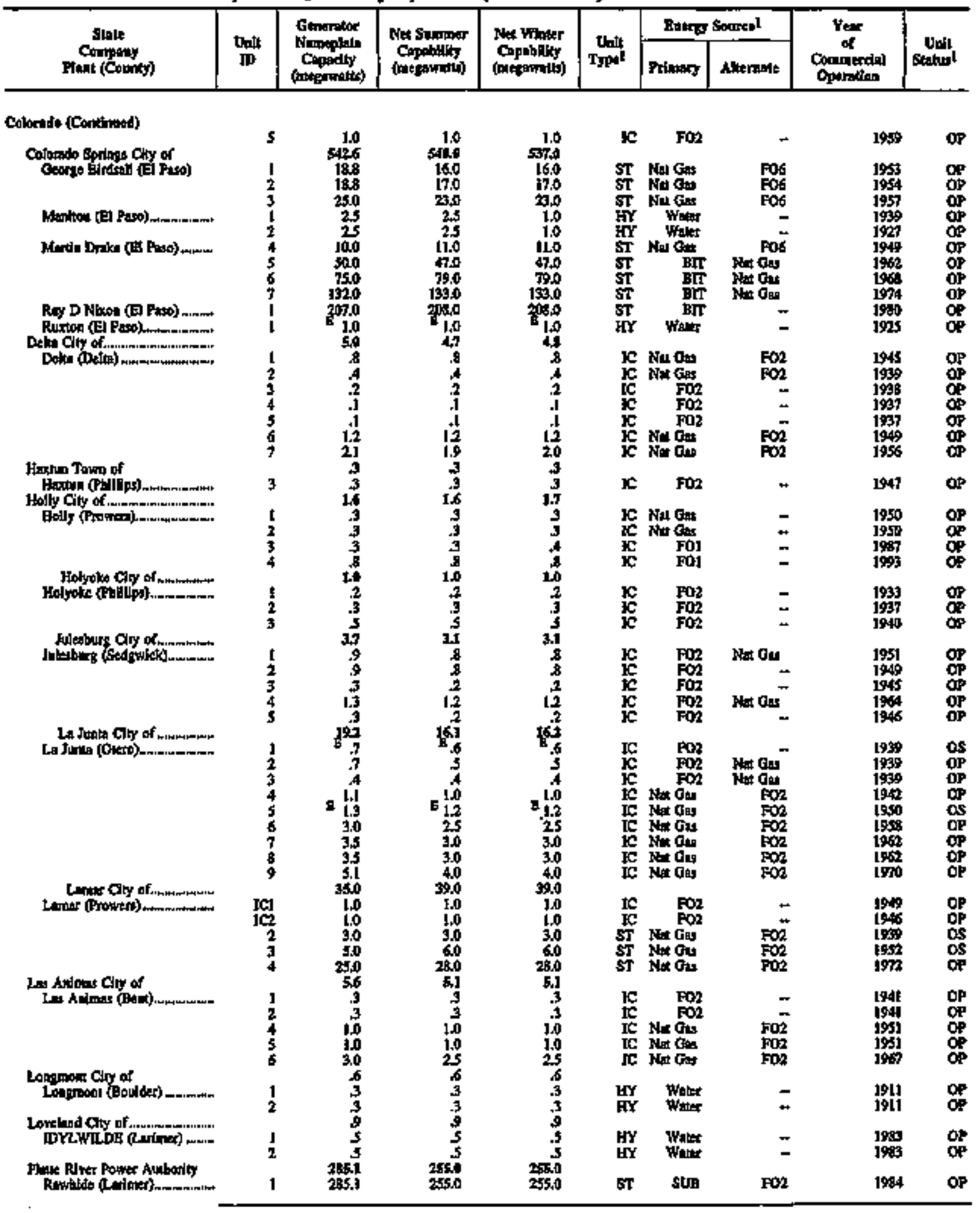

Ses foowoles at end or table. 
Table 20. Existing Generating Units at U.S. Electric Utilities by State, Company, and Plant, as of January 1, 1996 (Continued)

\begin{tabular}{|c|c|c|c|c|c|c|c|c|c|}
\hline 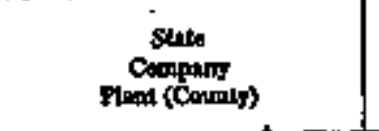 & $\begin{array}{l}\text { Untit } \\
\text { D }\end{array}$ & 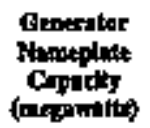 & 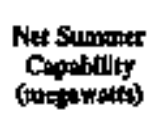 & 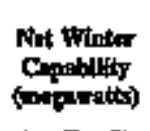 & Trot & Batror & Allesilute & 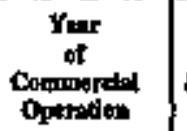 & tonth \\
\hline 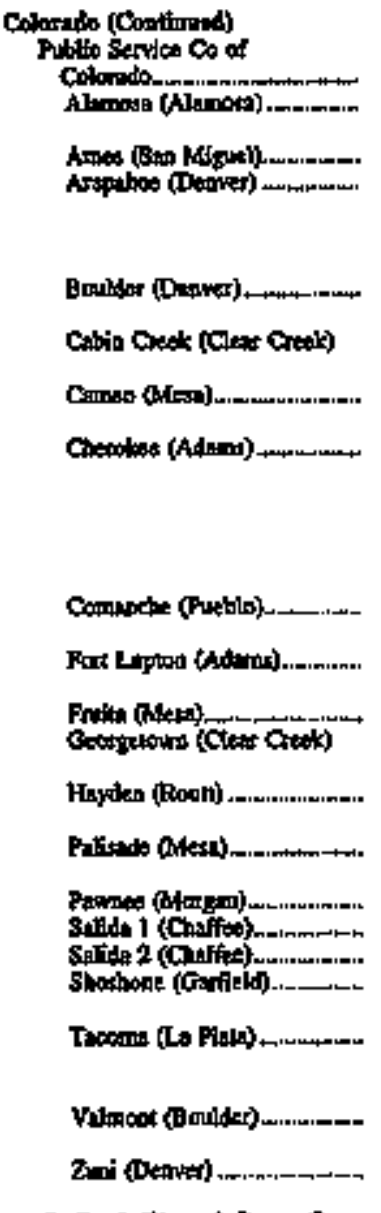 & 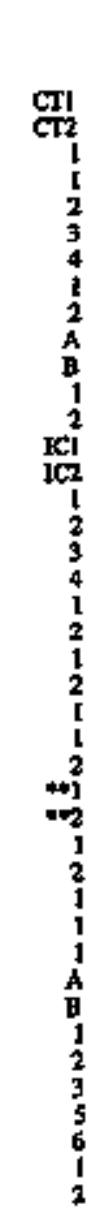 & 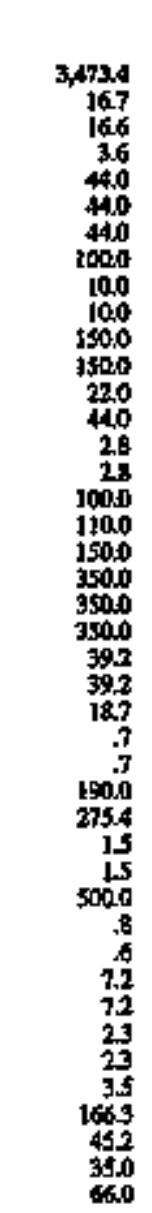 & 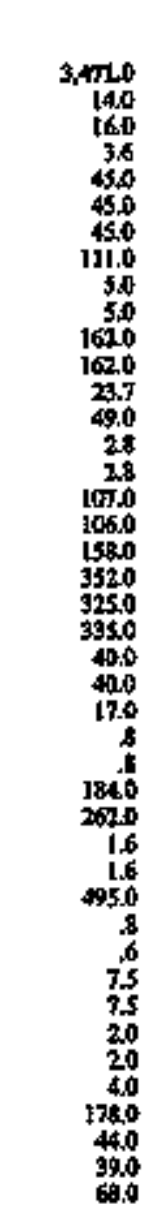 & 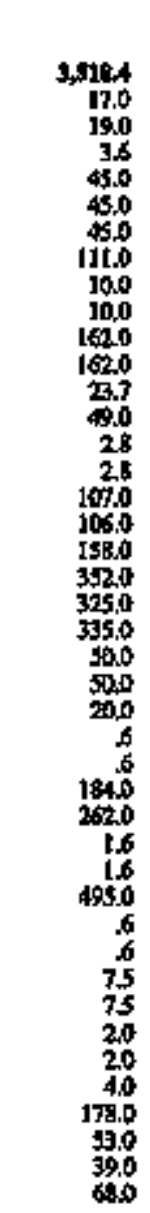 & 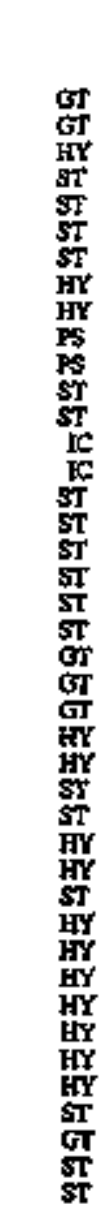 & 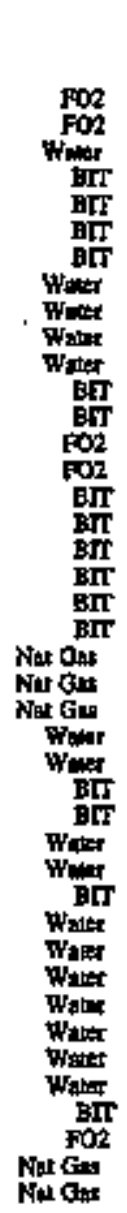 & 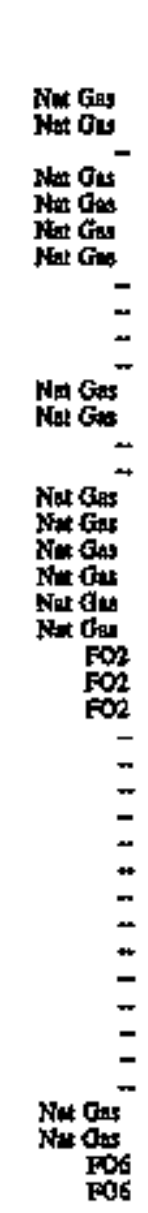 & 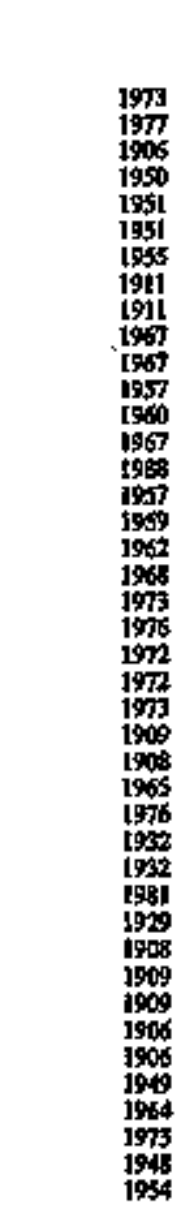 & 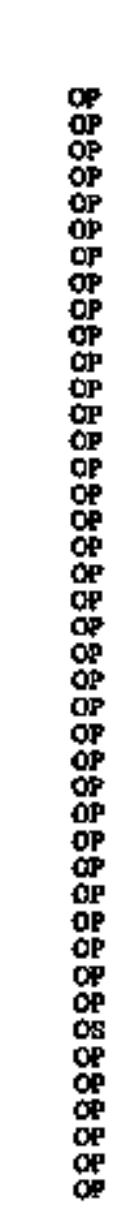 \\
\hline 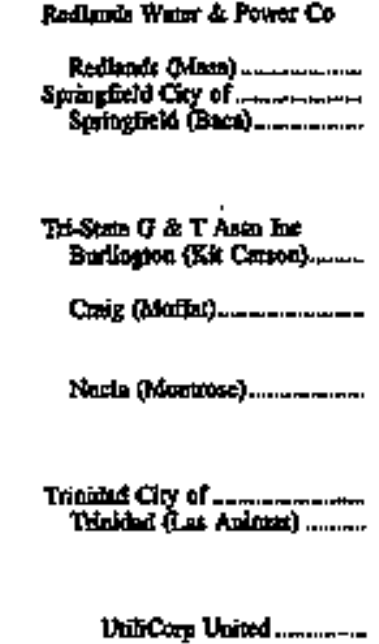 & 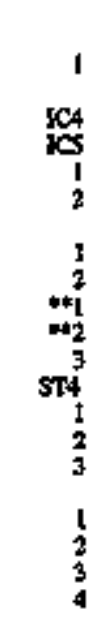 & $\begin{array}{r}1.4 \\
1.4 \\
2.8 \\
.6 \\
1.3 \\
1.3 \\
1.25 \\
46.3 \\
46.3 \\
446.4 \\
446.4 \\
4 \$ 6.4 \\
79.4 \\
11.5 \\
11.5 \\
11.5 \\
11.3 \\
3.8 \\
3.5 \\
1.9 \\
1.9 \\
7.5\end{array}$ & $\begin{array}{r}1.4 \\
1.4 \\
2.4 \\
.6 \\
1.9 \\
.2 \\
1.454 .0 \\
50.0 \\
50.0 \\
428.0 \\
42.0 \\
408.0 \\
65.1 \\
11.3 \\
11.3 \\
11.3 \\
11.4 \\
3.3 \\
3.9 \\
1.9 \\
1.9 \\
12.0\end{array}$ & $\begin{array}{r}1.4 \\
1.4 \\
2.6 \\
5 \\
8 \\
13 \\
2 \\
1.40 \\
60.0 \\
60.0 \\
428.0 \\
4280 \\
40.0 \\
66.1 \\
11.3 \\
11.3 \\
11.3 \\
11.4 \\
3.8 \\
3.8 \\
1.9 \\
19 \\
7.0\end{array}$ & 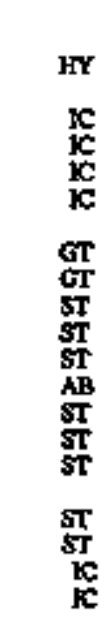 & 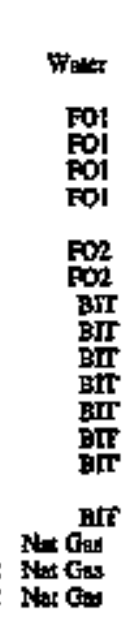 & $\begin{array}{l}\text { Nat Gas } \\
\text { Nat Gas } \\
\text { Nal Got } \\
\text { Nat Gat }\end{array}$ & $\begin{array}{l}1992 \\
1950 \\
1960 \\
1965 \\
1950 \\
1977 \\
1977 \\
1990 \\
1979 \\
1994 \\
1991 \\
1959 \\
1959 \\
1959 \\
1950 \\
1960 \\
1966 \\
1966\end{array}$ & 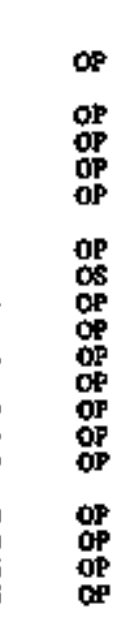 \\
\hline
\end{tabular}

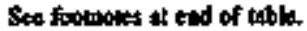


Table 20. Existing Generating Units at U.S. Electric Utilities by State, Company, and Plant, as of January 1, 1996 (Continued)

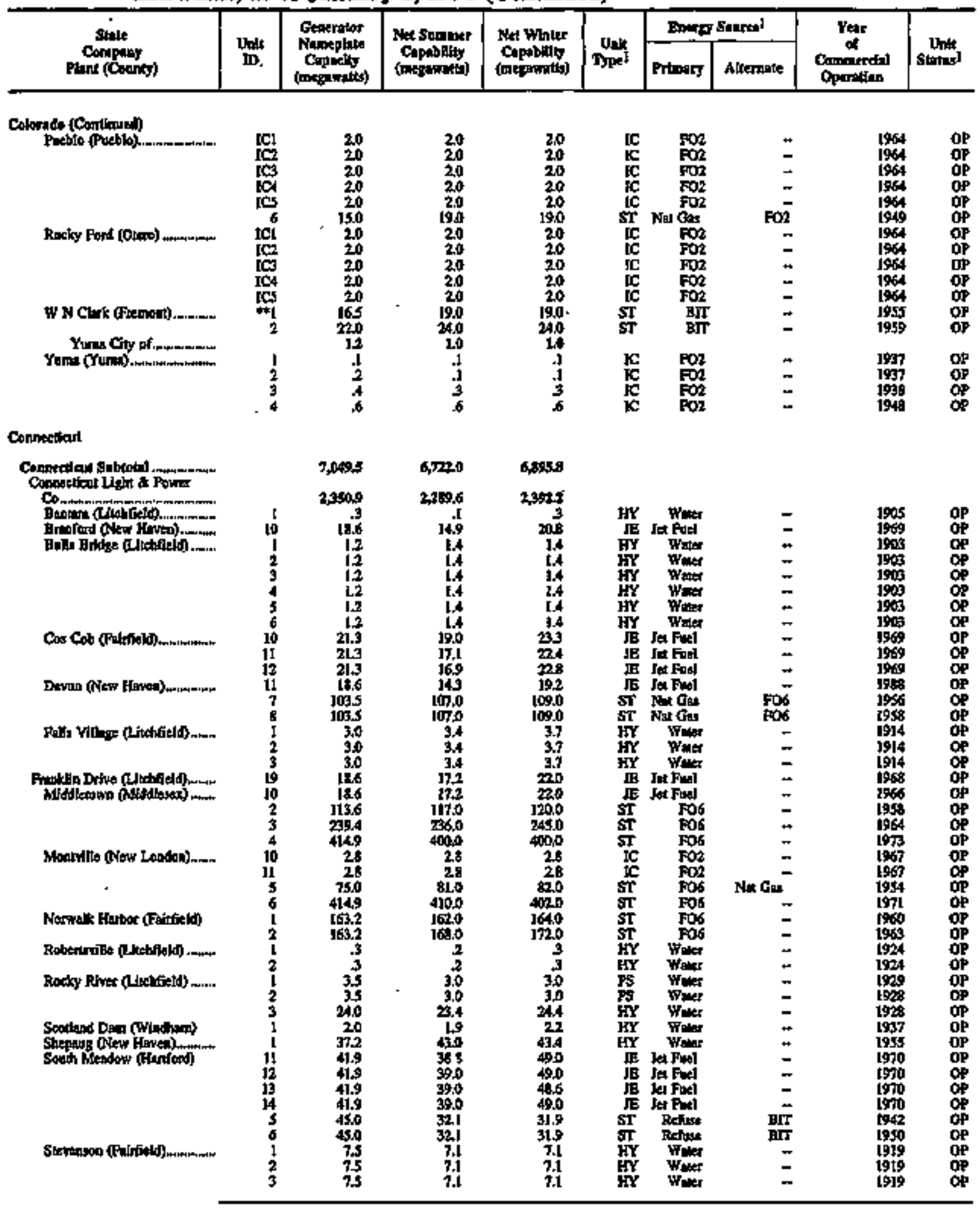

Sex footnoled at end of ubita. 
Table 20. Existing Generating Units at U.S. Electric Utilities by State, Company, and Plant, as of January 1, 1996 (Continued)

\begin{tabular}{|c|c|c|c|c|c|c|c|c|c|}
\hline \multirow{2}{*}{$\begin{array}{c}\text { Suts } \\
\text { Compiny } \\
\text { Plane (Corndy) }\end{array}$} & \multirow{2}{*}{$\begin{array}{l}\text { Un't } \\
\text { ID }\end{array}$} & \multirow{2}{*}{ 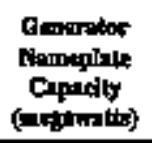 } & \multirow{2}{*}{ 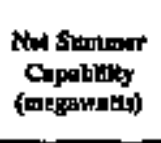 } & \multirow{2}{*}{ 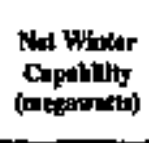 } & \multirow{2}{*}{ Type!t } & \multicolumn{2}{|c|}{ Bontw Soureal } & \multirow{2}{*}{$\begin{array}{l}\text { Pear } \\
\text { of } \\
\text { Conumbrden } \\
\text { Oparadun }\end{array}$} & \multirow{2}{*}{ Vonk } \\
\hline & & & & & & Prtming & Altermito & & \\
\hline \multirow{2}{*}{\multicolumn{10}{|c|}{ Comeedlat (Corlhaned) }} \\
\hline & 4 & 80 & 7.6 & 7.6 & HY & Wher & + & 1936 & OP \\
\hline \multirow[t]{5}{*}{ Totrillo (Nerr Londm) } & I & 4 & A & $A$ & $\mathbf{H Y}$ & Water & 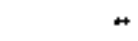 & [926 & OP \\
\hline & 2 & 3 & .4 & 4 & $\mathbf{F Y}$ & Water & $\omega$ & IS06 & OP \\
\hline & 3 & 4 & 4 & 4 & $\mathbf{H Y}$ & What & r & 1906 & OP \\
\hline & 4 & 4 & A & 4 & $\mathbf{E Y}$ & Water & + & 1949 & OP \\
\hline & 5 & 4 & 4 & 4 & FY & Wher & $\leftrightarrow$ & 1949 & OP \\
\hline \multirow{4}{*}{ 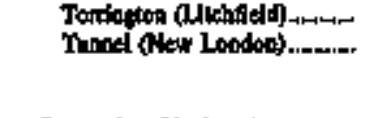 } & 10 & 186 & 17.2 & 218 & B & let Fuel & . & $16 t$ & OP \\
\hline & I & 1.0 & 8 & 1.1 & $\underset{\mathbf{E Y}}{\mathbf{E Y}}$ & Water & + & 1919 & OP \\
\hline & 20 & 186 & 16.9 & 20.8 & Jis & Jat Bof & - & [190 & op \\
\hline & & 1.0 & 8 & 2.1 & HT & Wats & $*$ & 1948 & DP \\
\hline \multicolumn{10}{|l|}{ Conecticer Yenke Acors } \\
\hline \multirow{3}{*}{ 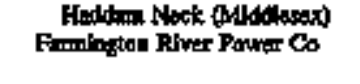 } & $+\infty$ & 600.3 & 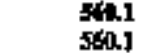 & $\begin{array}{l}5 \sin 2 \\
583,2\end{array}$ & NPP & D-milum & $\leadsto$ & 1058 & of \\
\hline & 7 & & & & Mr & Urill & 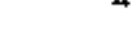 & $1 \times 0.8$ & Wr \\
\hline & & $\mathbf{8 0} 0$ & at & \$4 & & & & & \\
\hline 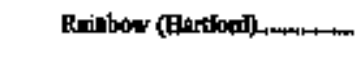 & I & 4.0 & 40 & 4.0 & EYY & Whest & - & 1925 & op \\
\hline \multicolumn{9}{|l|}{ Nopthest Nirclest Exary } & op \\
\hline \multirow{3}{*}{ Co } & & 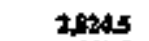 & $2,603.7$ & 4,6679 & & & & & \\
\hline & $\rightarrow$ & $\begin{array}{l}6615 \\
9099\end{array}$ & $\begin{array}{l}641.0 \\
471.1\end{array}$ & $\begin{array}{l}617.7 \\
874.5\end{array}$ & $\begin{array}{l}\text { NB } \\
\text { NP }\end{array}$ & $\begin{array}{l}\text { Unotum } \\
\text { Unowium }\end{array}$ & $\bar{z}$ & t9mo & op \\
\hline & $+\infty \frac{1}{3}$ & 1253.1 & 1119.6 & 1145.7 & $\mathbf{N P}$ & Lraphm & $=$ & Igxo & op \\
\hline \multirow{2}{*}{ 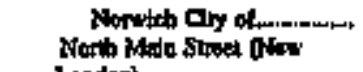 } & & 188 & 103 & 214 & & & & & \\
\hline & & & & & & & & & \\
\hline \multirow{4}{*}{ 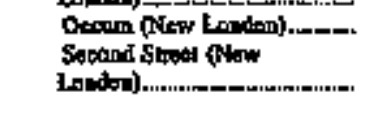 } & 3 & 168 & 153 & 18.4 & GT & $\mathrm{FO} 2$ & $*$ & Ish & OP \\
\hline & 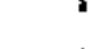 & 8 & .8 & .9 & GYY & Wuter & - & 1936 & $\mathbf{O P}$ \\
\hline & $\mathbf{I}$ & $A$ & 4 & $A$ & HY & Wret & - & [927? & op \\
\hline & 2 & $A$ & 4 & $A$ & $\mathbf{E Y}$ & Watex & - & 1927 & OP \\
\hline \multicolumn{10}{|l|}{ Tenbh Steter MeH } \\
\hline \multirow{6}{*}{ 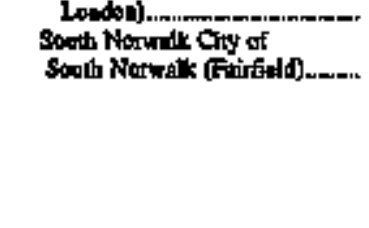 } & ! & $\begin{array}{r}1,4 \\
173\end{array}$ & $\begin{array}{r}1.4 \\
t 6.1\end{array}$ & 1.4 & EY & Wator & - & t9on & DP \\
\hline & $\mathbf{1}$ & 5.0 & 50 & 5.1 & IC & $F O 2$ & - & 1972 & OP \\
\hline & 2 & 20 & 1.0 & 1.9 & IC & $F=0$ & - & t940 & OP \\
\hline & 3 & 20 & 1.6 & 19 & IC & $\mathrm{FO2}$ & - & $\lg 2$ & DP \\
\hline & $\begin{array}{l}4 \\
5\end{array}$ & $\begin{array}{l}3.3 \\
40\end{array}$ & $\begin{array}{l}3.1 \\
3.4\end{array}$ & $\begin{array}{l}3.2 \\
1.5\end{array}$ & IC & $F_{002}$ & $\bar{z}$ & $\begin{array}{l}\text { to5i } \\
\text { to60 }\end{array}$ & $\begin{array}{l}D P \\
D P\end{array}$ \\
\hline & 6 & 1.0 & 1.1 & 1.1 & 政 & $\mathrm{FO}$ & - & 1990 & OP \\
\hline & & 1,2061 & $1,173,7$ & 1,1840 & & & & & \\
\hline $\begin{array}{l}\text { Bidgapont Harbor } \\
\text { (Pairfizeld) }\end{array}$ & & & & & & & & & \\
\hline 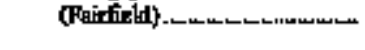 & 1 & 15 & 920 & 850 & ST & Fo6 & - & 1957 & $\mathrm{sg}$ \\
\hline & 2 & $\begin{array}{l}179.5 \\
399.5\end{array}$ & 170.0 & $\begin{array}{l}1700 \\
3850\end{array}$ & $\mathbf{S T}$ & $\begin{array}{l}F 06 \\
8 \pi\end{array}$ & $50 \overline{6}$ & 1961 & $\underset{\rho p}{o p}$ \\
\hline & 4 & 18.6 & 17.1 & 220 & $\mathbb{I E}$ & Je: Funt & - & 1969 & op \\
\hline Bngtid (Nest Horeal) & 7 & sol 0 & 34,1 & 35,0 & & & - & j948 & si \\
\hline & 8 & 30.8 & 38.5 & +400 & si & 906 & - & 1953 & si \\
\hline New Heven Habor ONow & & & & & & & & & \\
\hline 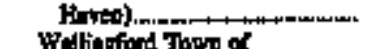 & $=1$ & 4603 & 217.9 & 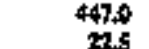 & $\boldsymbol{s T}$ & Fos & $\operatorname{Sim~Oas}$ & 1975 & OP \\
\hline 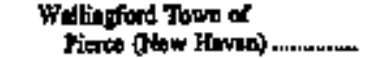 & $\mathbf{l}$ & 223 & $\frac{2245}{75}$ & 72,5 & & & & & \\
\hline 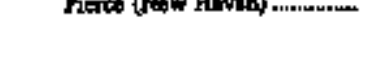 & 2 & 75 & 7,3 & 7.5 & $\frac{51}{5 \pi}$ & Fod & $\bar{z}$ & 1959 & $\underset{G P}{\operatorname{dos}}$ \\
\hline
\end{tabular}

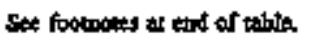


Table 20. Existing Generating Units at US. Flectric Utilities by State, Company, and Plant, as of Jamuary 1, 1996 (Continued)

\begin{tabular}{|c|c|c|c|c|c|c|c|c|c|}
\hline \multirow{2}{*}{$\begin{array}{c}\text { Btale } \\
\text { Conpenyy } \\
\text { Flant (County) }\end{array}$} & \multirow{2}{*}{ thatk } & \multirow{2}{*}{ 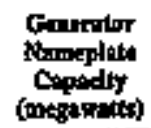 } & \multirow{2}{*}{ 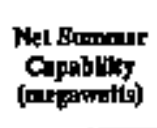 } & \multirow{2}{*}{$\begin{array}{l}\text { Net Whater } \\
\text { Capublity } \\
\text { (atopintis) }\end{array}$} & \multirow{2}{*}{$\begin{array}{r}\text { Dnh } \\
\text { Typol }\end{array}$} & \multicolumn{2}{|c|}{ Fintrs Solaxpi I } & \multirow{2}{*}{$\begin{array}{c}\text { Yenr } \\
\text { of } \\
\text { Comprerdial } \\
\text { Optitilon }\end{array}$} & \multirow{2}{*}{ Sinting } \\
\hline & & & & & & Primarp & Ahernats & & \\
\hline
\end{tabular}

Duhware

\begin{tabular}{|c|c|c|c|c|c|c|c|c|c|}
\hline Delmare Sobtodal. & & $2,28,44$ & $2,25,4$ & 2,3506 & & & & & \\
\hline Culutana (Now Crote) & 11 & $\begin{array}{r}20811.6 \\
26.6 \\
26.6\end{array}$ & $\begin{array}{r}2,0540 \\
275 \\
225\end{array}$ & $\begin{array}{r}2,112,0 \\
25,0 \\
250\end{array}$ & $\mathbf{G T}$ & $\mathrm{FO} 2$ & $\overline{-}$ & $\begin{array}{l}1973 \\
1973\end{array}$ & $\begin{array}{l}\text { OP } \\
\text { OP }\end{array}$ \\
\hline 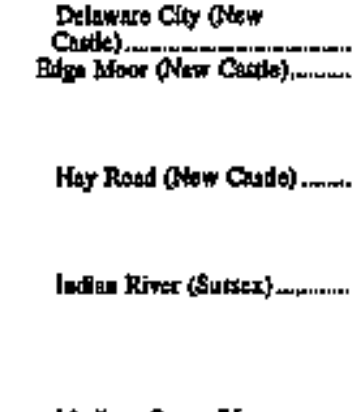 & $\begin{array}{r}10 \\
10 \\
3 \\
4 \\
5 \\
5 \\
2 \\
3 \\
4 \\
1 \\
10 \\
2 \\
3 \\
4\end{array}$ & $\begin{array}{r}18.6 \\
12.5 \\
75.0 \\
176.5 \\
446.0 \\
103.5 \\
109.5 \\
109.5 \\
160.0 \\
01.6 \\
18.6 \\
18.6 \\
176.8 \\
442.4\end{array}$ & $\begin{array}{r}140 \\
13.0 \\
84.0 \\
167.0 \\
\$ 35.0 \\
112.0 \\
1120 \\
112.0 \\
175.0 \\
290 \\
170 \\
\$ 90 \\
162.0 \\
403.0\end{array}$ & $\begin{array}{r}180 \\
15.0 \\
84.0 \\
167.0 \\
435.0 \\
1220 \\
120 \\
1200 \\
175.0 \\
90.0 \\
21.0 \\
900 \\
1650 \\
403.0\end{array}$ & 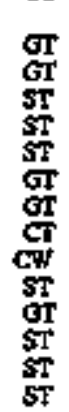 & 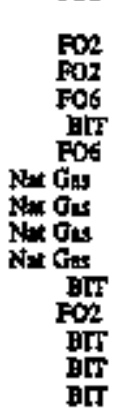 & Nat Gas & $\begin{array}{l}1968 \\
1963 \\
1954 \\
1966 \\
1973 \\
1998 \\
1989 \\
1995 \\
1999 \\
1957 \\
1967 \\
1959 \\
1950 \\
1980\end{array}$ & $\begin{array}{l}O P \\
O P \\
O P \\
O P \\
O P \\
O P \\
O P \\
O P \\
O P \\
O P \\
O P \\
O P \\
O P \\
O P\end{array}$ \\
\hline 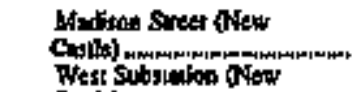 & I & 113 & 11.0 & 14.0 & GT & $\mathrm{PO} 2$ & $x^{2}$ & 1962 & OP \\
\hline Catly. & $\mathbf{t}$ & $\begin{array}{r}16.2 \\
196.3\end{array}$ & $\begin{aligned} 14,0 \\
175,0\end{aligned}$ & $\begin{array}{r}19.0 \\
1820\end{array}$ & $\boldsymbol{G T}$ & Fon & - & 1964 & $\alpha P$ \\
\hline 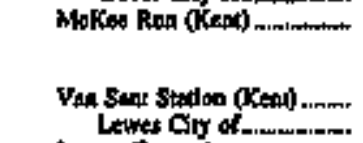 & $\begin{array}{l}1 \\
\frac{2}{3} \\
1\end{array}$ & $\begin{array}{r}18.8 \\
18.8 \\
213.6 \\
45.1 \\
20\end{array}$ & $\begin{array}{r}17,0 \\
170 \\
100 \\
390 \\
1.6\end{array}$ & $\begin{array}{r}17.0 \\
170 \\
1080 \\
450 \\
2.1\end{array}$ & $\begin{array}{l}s \tau \\
s \tau \\
s T \\
g r\end{array}$ & $\begin{array}{l}\text { Fo6 } \\
\text { For } \\
\text { Fo6 } \\
\text { Fo6 }\end{array}$ & $\begin{array}{l}\text { Nht Gus } \\
\text { Nat Gas } \\
\text { Nat Gans } \\
\text { Nut Gou }\end{array}$ & $\begin{array}{l}1962 \\
1962 \\
1975 \\
1991\end{array}$ & $\begin{array}{l}o p \\
o p \\
o p \\
o p\end{array}$ \\
\hline 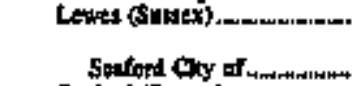 & $\frac{7}{8}$ & $\begin{array}{l}\mathbf{3 . 0} \\
\mathbf{1 . 0} \\
\mathbf{7 . 0}\end{array}$ & 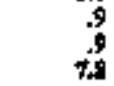 & $\begin{array}{l}1.0 \\
1.0 \\
t\end{array}$ & 倠 & $\begin{array}{l}\mathrm{Fot} \\
\mathrm{Fon} 2\end{array}$ & $\bar{z}$ & 1990 & $\underset{\text { of }}{\mathrm{OP}}$ \\
\hline 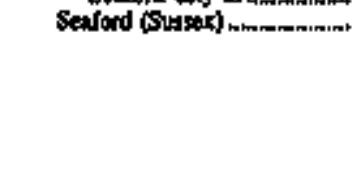 & $\begin{array}{l}1 \\
2 \\
3 \\
5 \\
6 \\
7\end{array}$ & $\begin{array}{r}1.4 \\
1.4 \\
1.1 \\
8 \\
20 \\
1.1\end{array}$ & $\begin{array}{l}1.4 \\
1.4 \\
1.5 \\
2.0 \\
1.1\end{array}$ & $\begin{array}{l}1.4 \\
1.4 \\
1.1 \\
.8 \\
2,0 \\
1.1\end{array}$ & $\begin{array}{l}\text { 琶 } \\
\text { IC } \\
\text { IC } \\
\text { IC } \\
\text { IC }\end{array}$ & $\begin{array}{l}\mathrm{FO2} \\
\mathrm{FO2} \\
\mathrm{FO2} \\
\mathrm{FO2} \\
\mathrm{FO2} \\
\mathrm{FO2}\end{array}$ & $\begin{array}{l}+ \\
\ddot{-} \\
\ddot{-} \\
\ddot{-}\end{array}$ & $\begin{array}{l}1998 \\
1954 \\
1950 \\
1947 \\
1962 \\
1989\end{array}$ & $\begin{array}{l}\not p \\
\not p \\
o p \\
o p \\
o p \\
O P\end{array}$ \\
\hline Dtarka of Columbla & & & & & & & & & \\
\hline 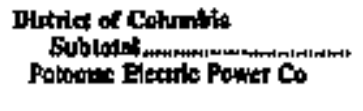 & & 668.0 & s060 & Gate & & & & & \\
\hline 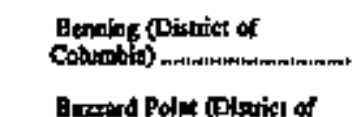 & 15 & $\begin{array}{l}2800 \\
290.0 \\
290.0\end{array}$ & $\begin{array}{l}2750.0 \\
275.0\end{array}$ & $\begin{array}{l}5700 \\
275,11 \\
275.0\end{array}$ & $\begin{array}{l}\$ \mathbf{T} \\
\mathbf{S T}\end{array}$ & $\begin{array}{l}\mathrm{Fp4} \\
\mathrm{FO4}\end{array}$ & $\begin{array}{l}\text { Fo2 } \\
\text { FO2 }\end{array}$ & $\begin{array}{l}1968 \\
1972\end{array}$ & $\stackrel{O P}{O P}$ \\
\hline Columatda) & WAS & $\begin{array}{l}144.0 \\
1440\end{array}$ & $\begin{array}{l}129.0 \\
128,0\end{array}$ & 1600 & GT & $\begin{array}{l}\mathrm{FO2} \\
\mathrm{Foz}\end{array}$ & $\overline{-}$ & 1968 & op \\
\hline Farda & & & & & & & & & \\
\hline 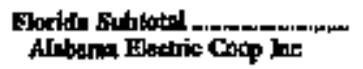 & & 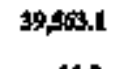 & $35,356,5$ & 37,1374 & & & & & \\
\hline 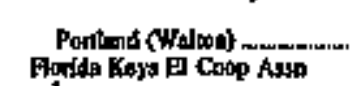 & I & 110 & $\lim _{100}$ & 100 & $\boldsymbol{\sigma T}$ & $\mathbf{F} 02$ & - & 1964 & or \\
\hline 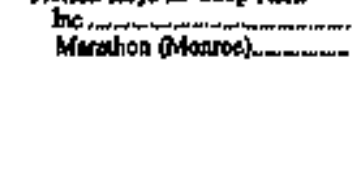 & $\begin{array}{l}3 \\
4 \\
5 \\
6 \\
7\end{array}$ & $\begin{array}{r}18.4 \\
3.0 \\
3.0 \\
3.0 \\
2.5 \\
25\end{array}$ & $\begin{array}{l}16.5 \\
2.5 \\
2.5 \\
2.5 \\
2.5 \\
2.5\end{array}$ & $\begin{array}{l}16.5 \\
2.5 \\
2.5 \\
2.5 \\
2.5 \\
2.5\end{array}$ & $\begin{array}{l}\text { IC } \\
\text { IC } \\
\text { Ix } \\
\text { xc }\end{array}$ & $\begin{array}{l}\mathrm{FO2} \\
\mathrm{Fo2} \\
\mathrm{FO2} \\
\mathrm{Fon} \\
\mathrm{Fo2}\end{array}$ & $\begin{array}{l}\bar{z} \\
\bar{z} \\
z\end{array}$ & $\begin{array}{l}1998 \\
1959 \\
1959 \\
1973 \\
1973\end{array}$ & $\begin{array}{l}O P \\
O P \\
O P \\
O P \\
O P\end{array}$ \\
\hline
\end{tabular}

Sec fortories al end of ILbla, 
Table 20. Extsting Generating Units at U.S. Electric Utlities by State, Company, and Plant, as of January 1, 1996 (Continuedi)

\begin{tabular}{|c|c|c|c|c|c|c|c|c|c|}
\hline \multirow{2}{*}{$\begin{array}{c}\text { State } \\
\text { Company } \\
\text { Plant \{Couply\} }\end{array}$} & \multirow{2}{*}{$\begin{array}{c}\text { Untk } \\
\text { m }\end{array}$} & \multirow{2}{*}{ 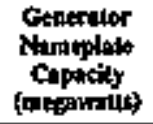 } & \multirow{2}{*}{ 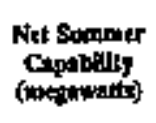 } & \multirow{2}{*}{$\begin{array}{l}\text { Net Pinter } \\
\text { Capibifity } \\
\text { (meigeratis) }\end{array}$} & \multirow{2}{*}{$\begin{array}{l}\text { Uny } \\
\text { Type1 }\end{array}$} & \multicolumn{2}{|c|}{ Batro Soocel } & \multirow{2}{*}{$\begin{array}{c}\text { Year } \\
\text { of } \\
\text { Cominarelal } \\
\text { Operollon }\end{array}$} & \multirow{2}{*}{ Dn:t } \\
\hline & & & & & & Primary & Altentald & & \\
\hline Copltureil) & 8 & $\begin{array}{l}20 \\
20\end{array}$ & $\begin{array}{l}2.0 \\
2.0\end{array}$ & $\begin{array}{l}20 \\
20\end{array}$ & 丞 & $\begin{array}{l}\text { Fon } \\
\text { Fot }\end{array}$ & $\vec{m}$ & $\begin{array}{l}1969 \\
1999\end{array}$ & $\begin{array}{l}\text { OP } \\
\text { OP }\end{array}$ \\
\hline 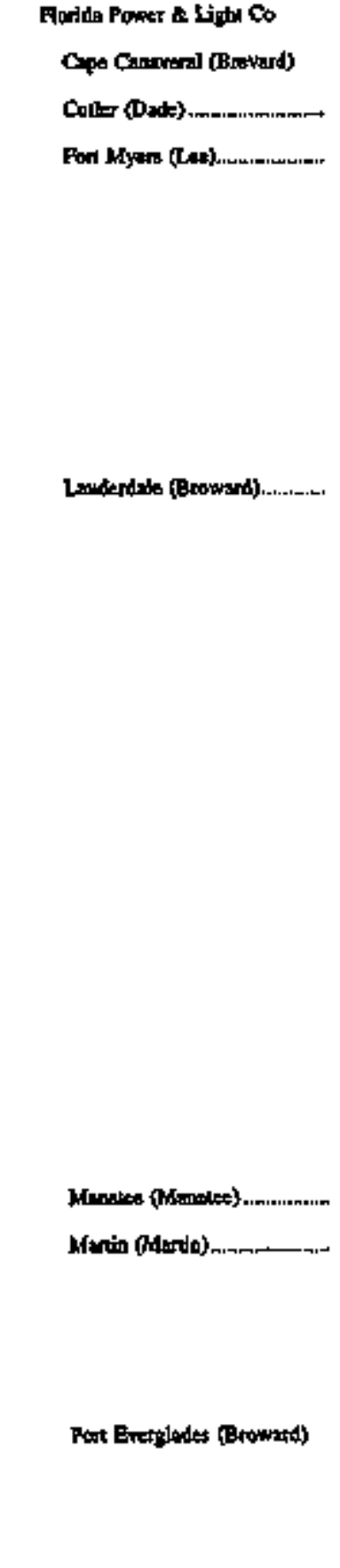 & 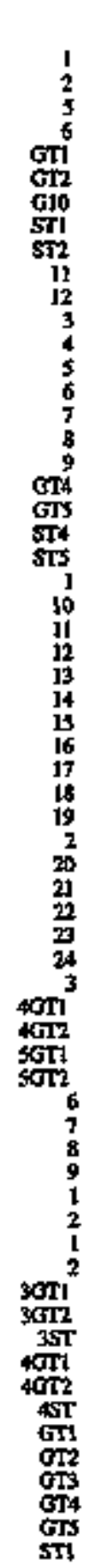 & 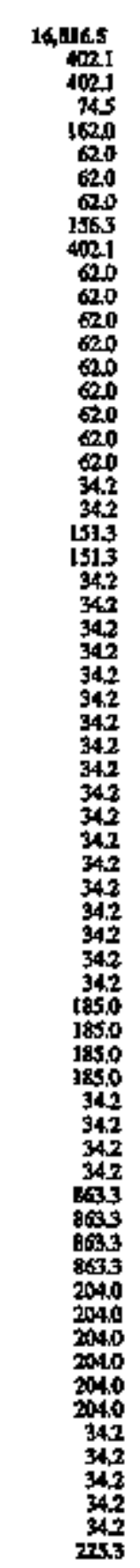 & 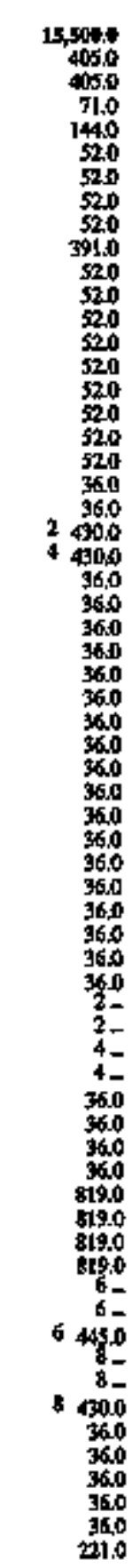 & 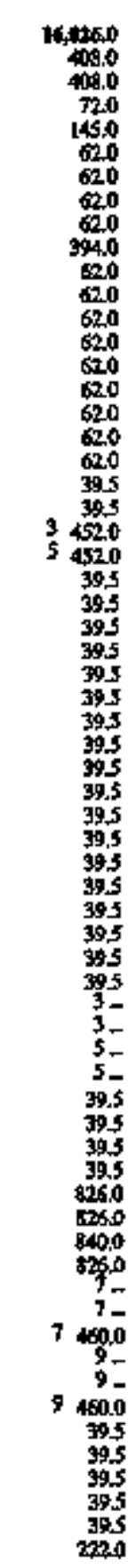 & 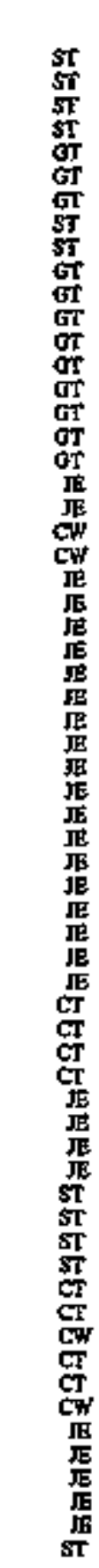 & 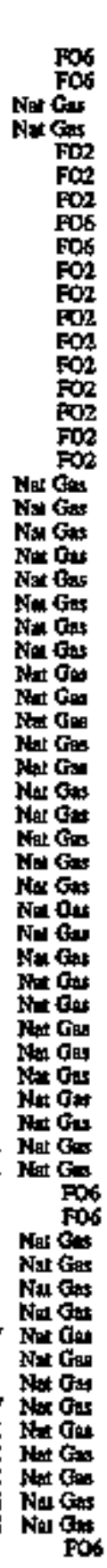 & 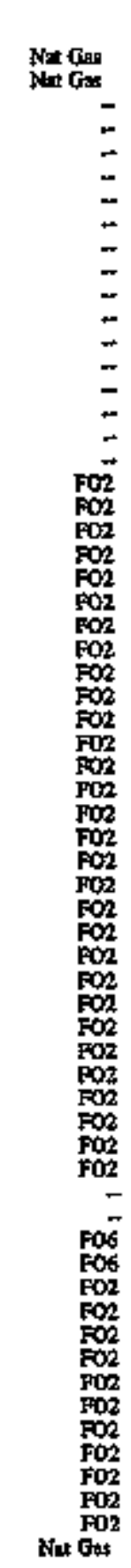 & 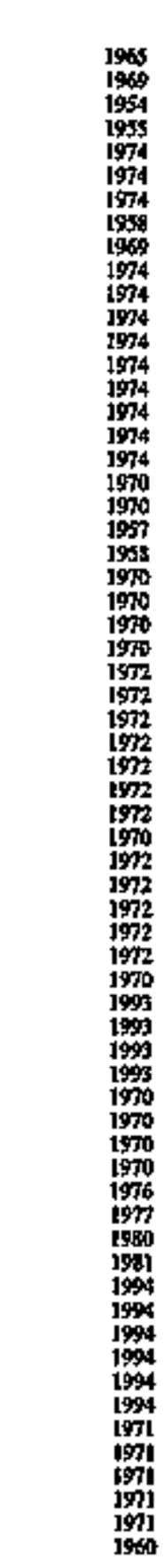 & 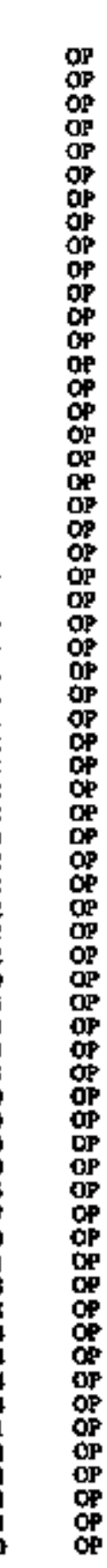 \\
\hline
\end{tabular}

Set fostrotea a end of thile. 
Table 20. Existing Generating Units at U.S. Electric Utilities by State, Company, and Plant, as of January 1, 1996 (Continued)

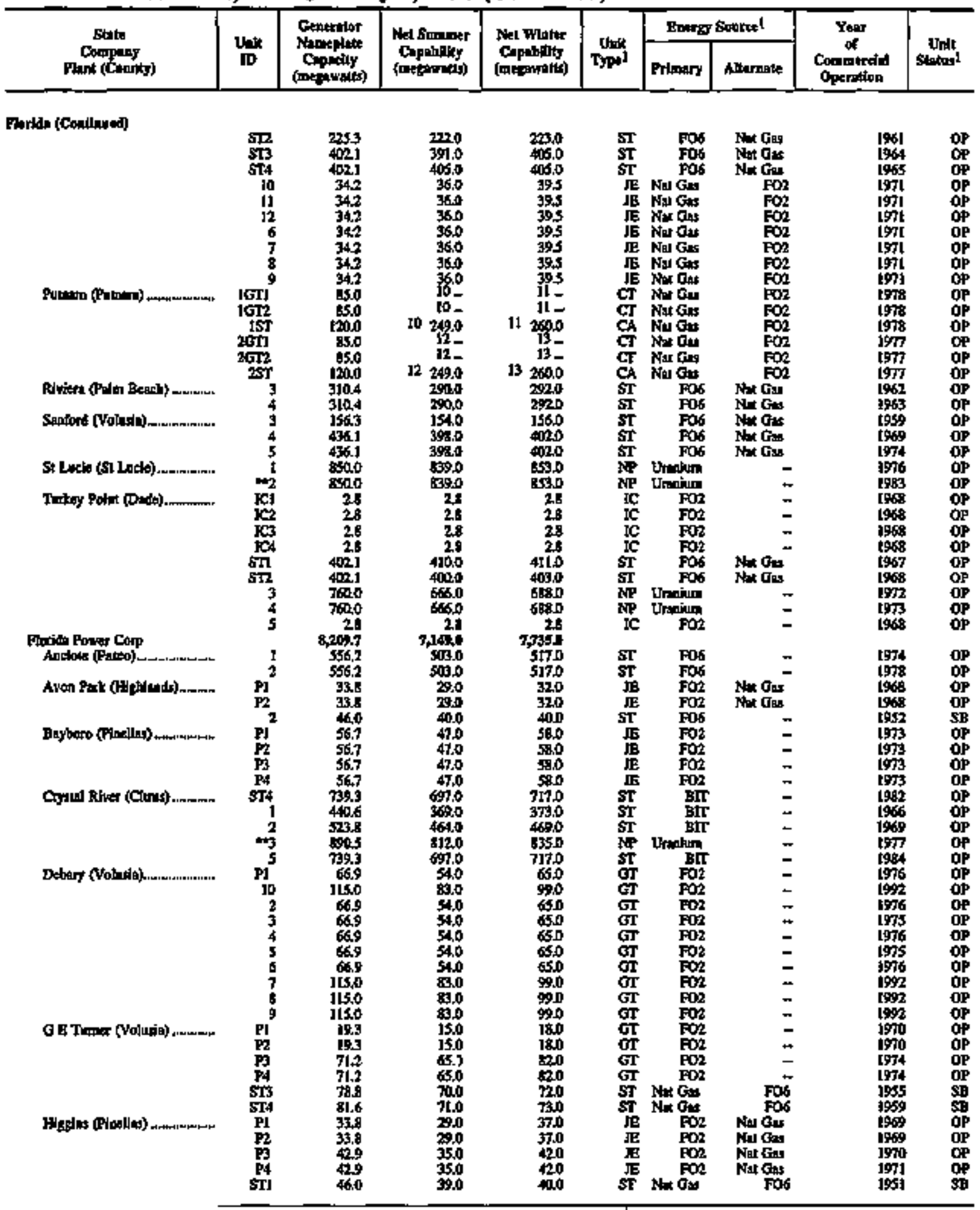

Sec foomotis at ead of uble. 
Table 20. Existing Generating Units at U.S. Electric Utilities by State, Company, and Plant, as of January 1, 1996 (Conthued)

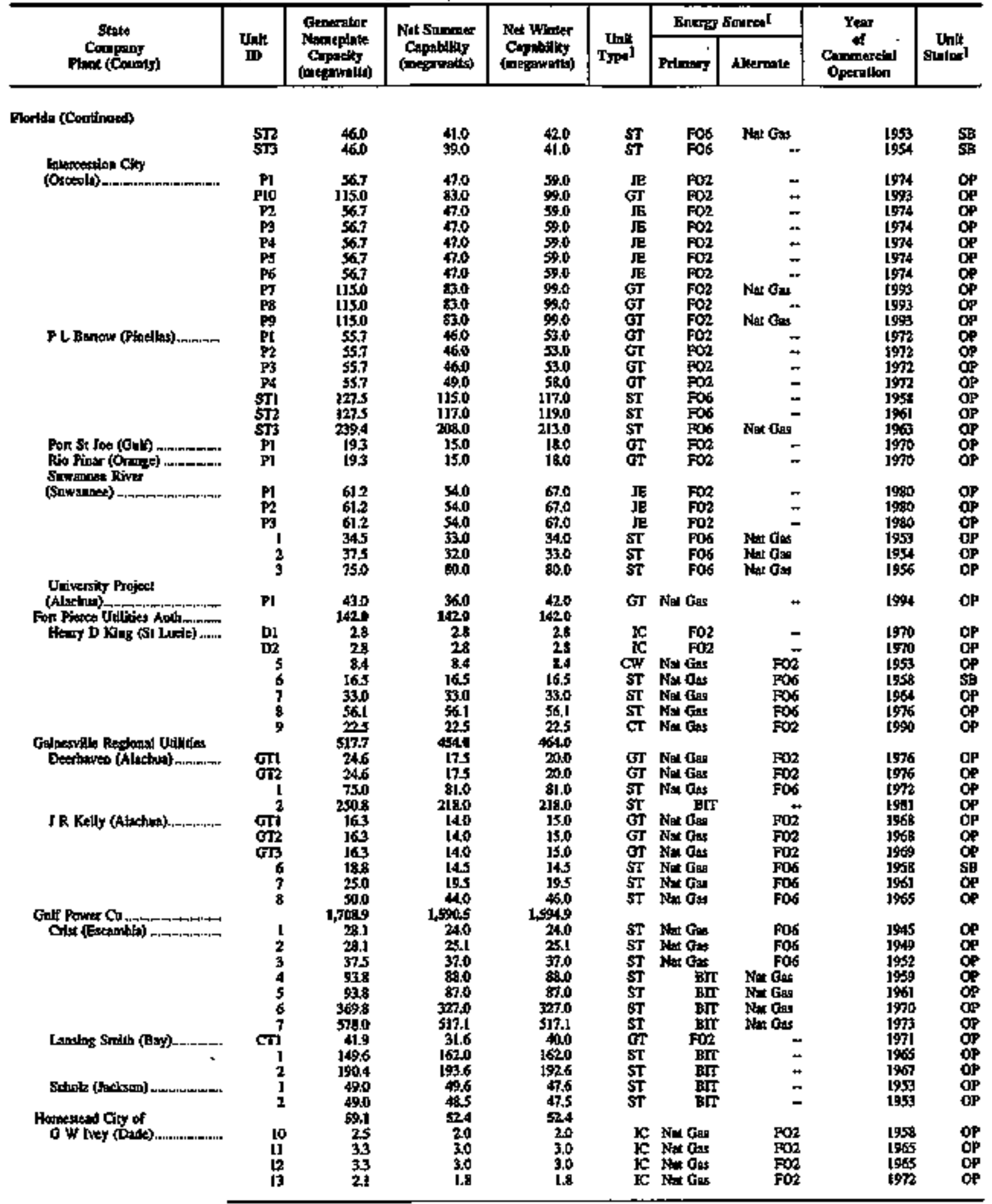

Sec tootootat at end of table. 
Table 20. Existing Generating Units at U.S. Electric Utilities by State, Company, and Plant, as of January 1, 1996 (Continued)

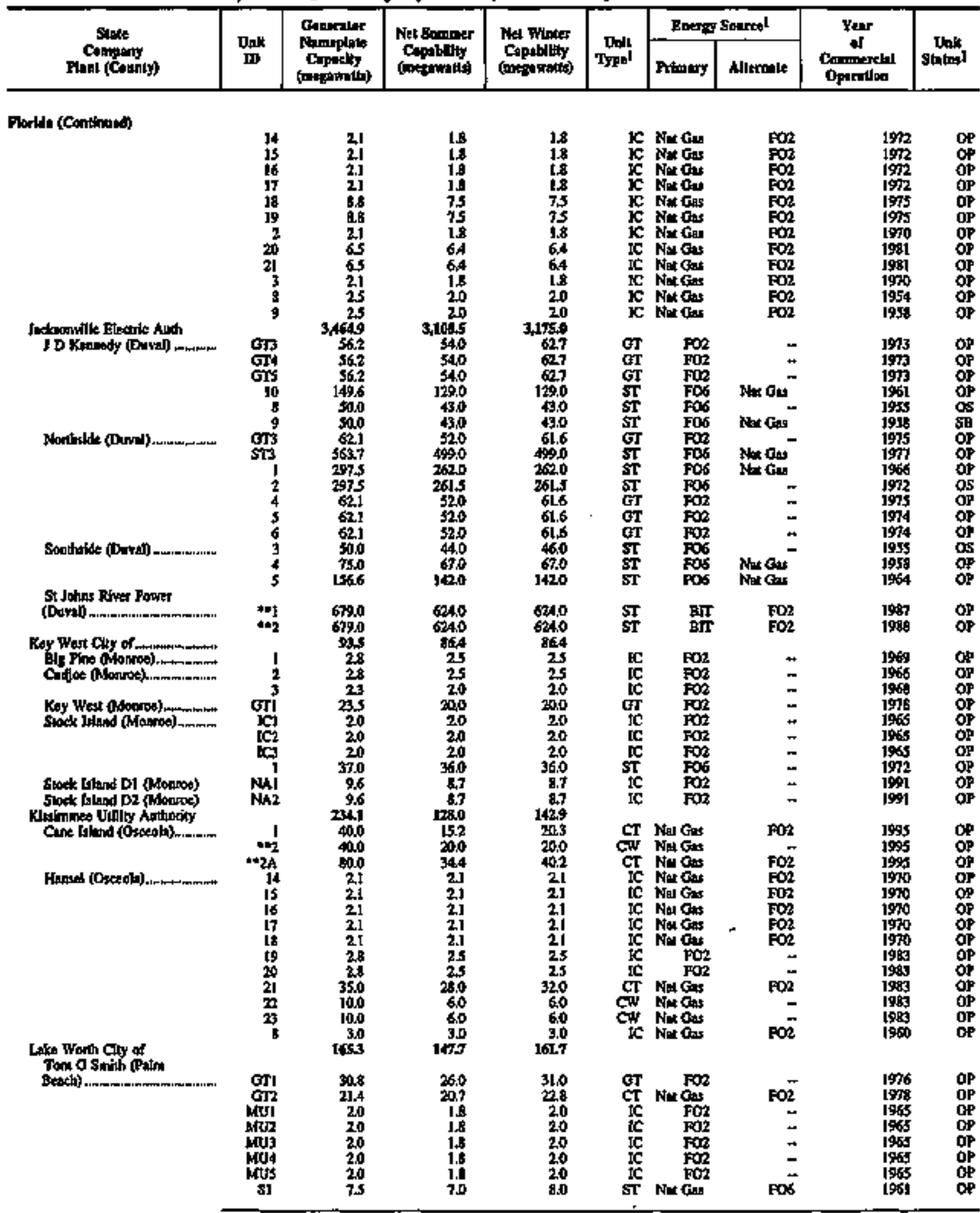

Ser forbotos at end of catle. 
Tahle 2A. Existing Generating Units at U.S. Mectric Utilities by State, Company,. and Plant, as of January 1, 1996 (Continued)

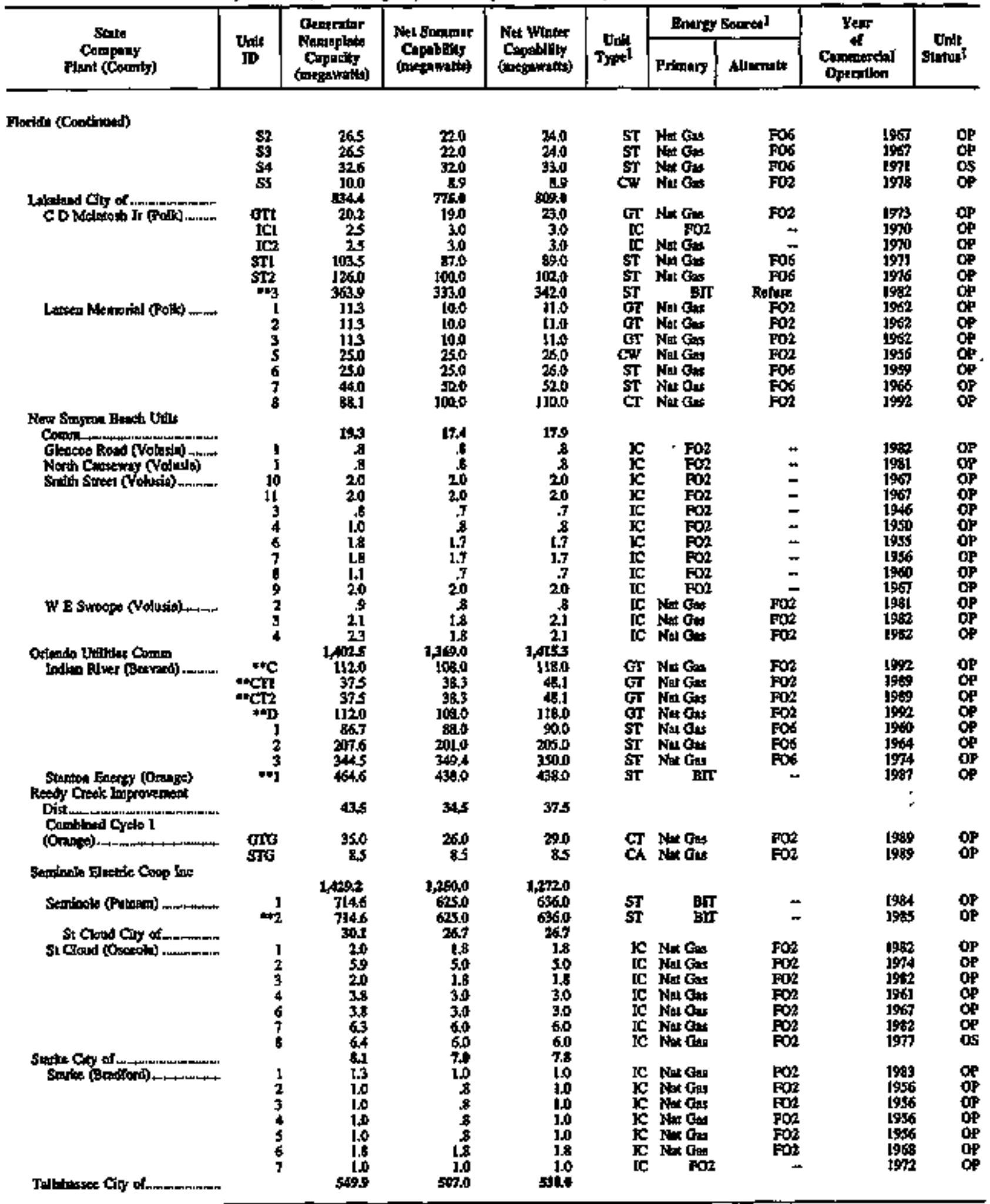

See foatnotas al eod of tribl. 
Table 20. Existing Generating Units at U.S. Electric Utilities by State, Company, and Plant, as of Jamuary 1, 1996 (Continued)

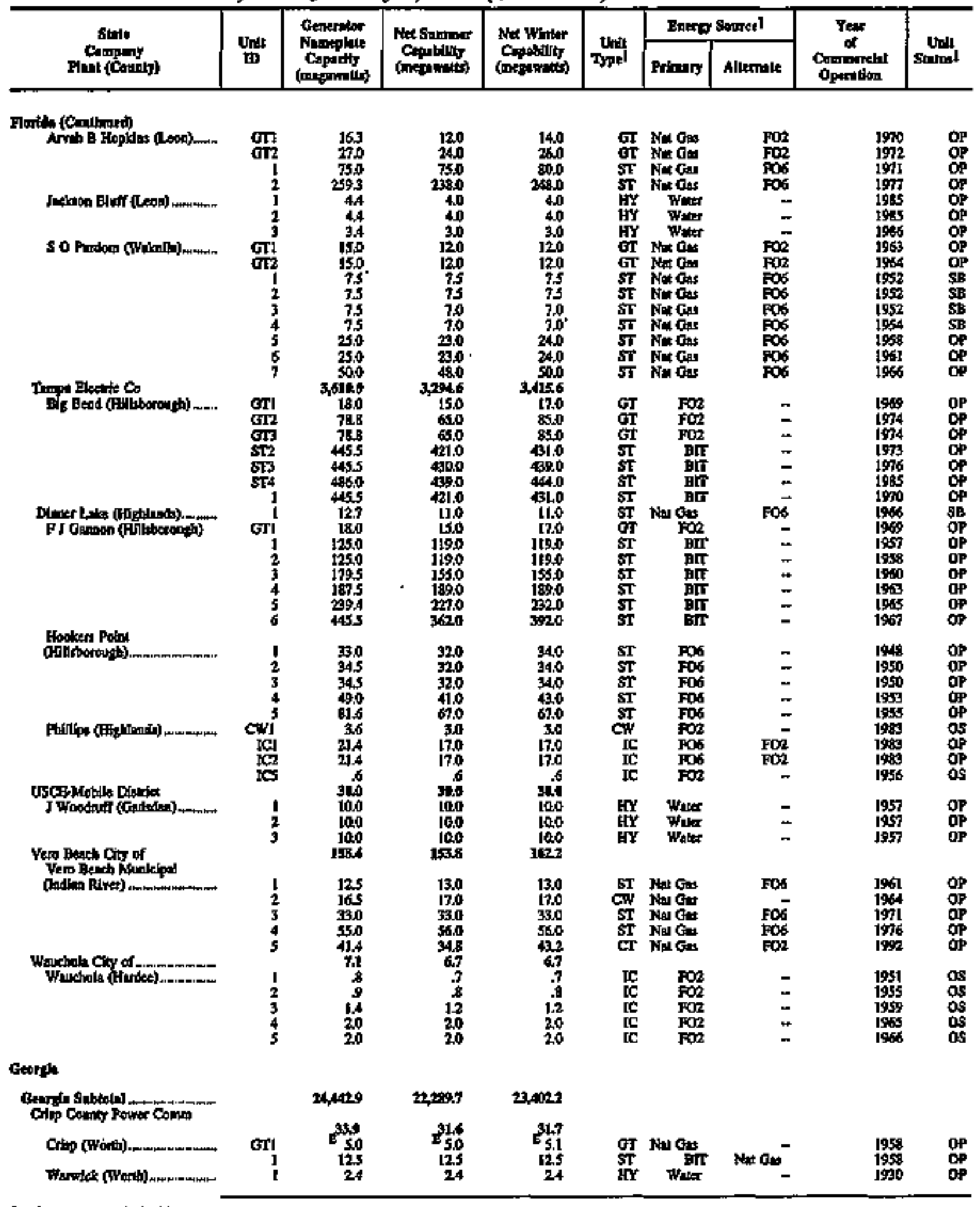

Sect foomides at end of able. 
Table 20. Existing Generating Units at U.S. Electric Utilities by State, Company, and Plant, as of January 1, 1996 (Continned)

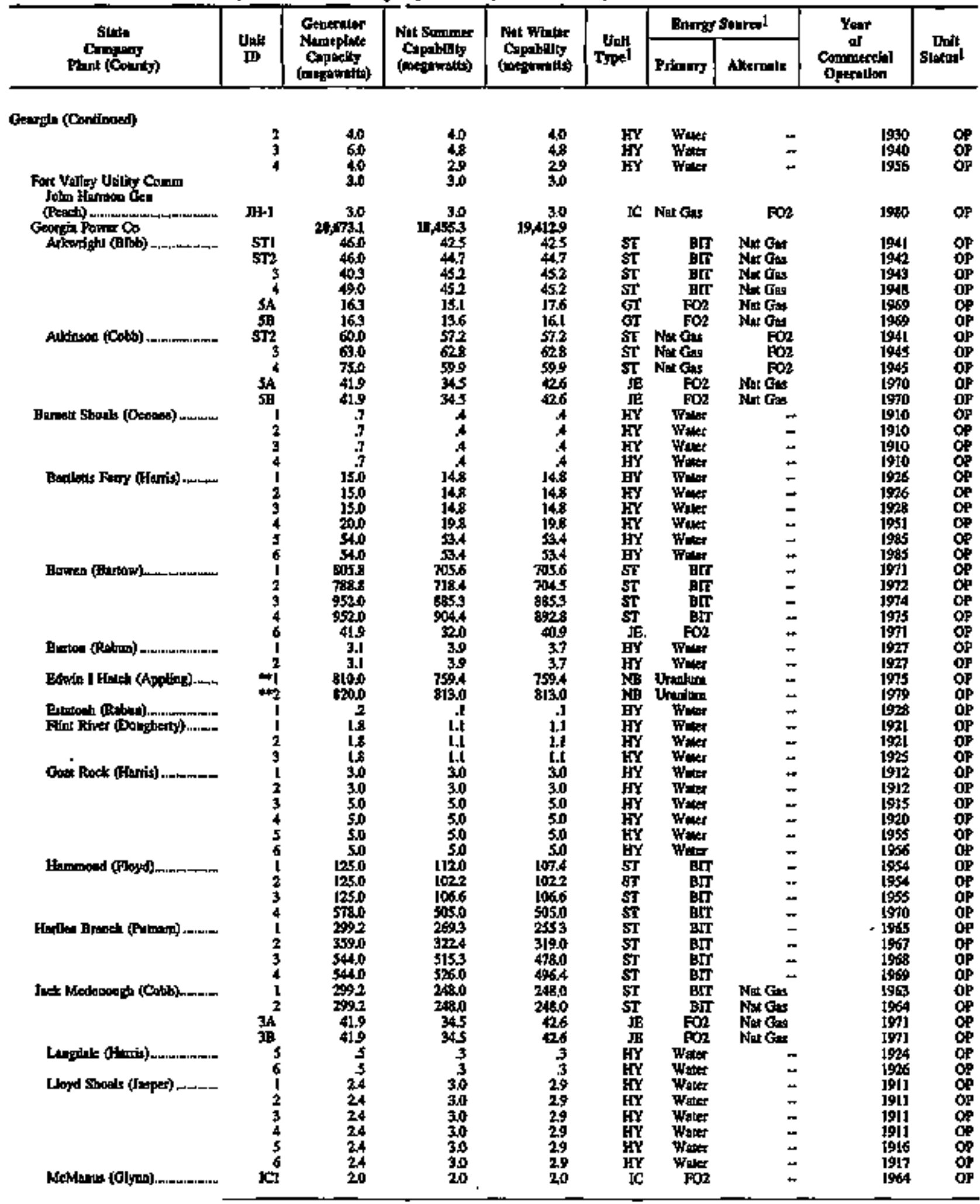

Sea foomotess at end of table. 
Table 20. Existing Generating Units at U.S. Electric Utilities by State, Company, and Plant, as of January 1, 1996 (Continued)

\begin{tabular}{|c|c|c|c|c|c|c|c|c|c|}
\hline \multirow{2}{*}{$\begin{array}{c}\text { Siete } \\
\text { Combenting } \\
\text { Flath (Cosinty) }\end{array}$} & \multirow{2}{*}{ Intit } & \multirow{2}{*}{ 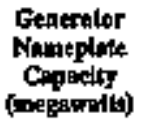 } & \multirow{2}{*}{ 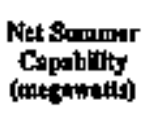 } & \multirow{2}{*}{ 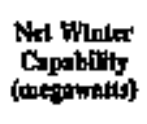 } & \multirow{2}{*}{ That } & \multicolumn{2}{|c|}{ Energy Souret } & \multirow{2}{*}{$\begin{array}{c}\text { Year } \\
\text { Co } \\
\text { Comercind } \\
\text { Operalion }\end{array}$} & \multirow{2}{*}{ Stont } \\
\hline & & & & & & Pratatery & Alternate & & \\
\hline \multicolumn{10}{|l|}{ Gearyle (Corllnoed) } \\
\hline & & 500 & 493 & 43.3 & $\mathbf{S T}$ & FOS & - & 1997 & OP \\
\hline & 2 & 98,8 & 787 & 78.7 & $\underline{B}$ & Fos & - & 1958 & QP \\
\hline & $3 \mathrm{k}$ & 35.4 & 50: & 68 & Gr & $\mathrm{FO2}$ & - & $1 \% 12$ & $0 P$ \\
\hline & 30 & $\mathbf{5 5 A}$ & 508 & 63.8 & GT & $\mathrm{Fon}$ & 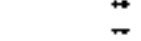 & $\begin{array}{l}1912 \\
1972\end{array}$ & OP \\
\hline & $4 A$ & 55,4 & 90,8 . & 63,8 & 臭 & $\mathrm{FO} 2$ & - & 1972 & OP \\
\hline & $A B$ & 554 & 508 & 解 & GT & FO2 & + & 1972 & DP \\
\hline & $\begin{array}{l}4 C \\
4 D\end{array}$ & $\begin{array}{l}55.4 \\
55.4\end{array}$ & $\begin{array}{l}50.8 \\
50.8\end{array}$ & $\begin{array}{l}618 \\
698\end{array}$ & $\begin{array}{l}\mathbf{G T} \\
\mathbf{G T}\end{array}$ & $\begin{array}{l}\mathrm{FO2} 2 \\
\mathrm{FO} 2\end{array}$ & $=$ & $\begin{array}{l}1952 \\
1972\end{array}$ & OP \\
\hline & $4 \mathrm{E}$ & $\begin{array}{l}554 \\
554\end{array}$ & $\begin{array}{l}508 \\
508\end{array}$ & 68 & Gr & $\mathrm{FOz}$ & $\approx$ & $16 / 2$ & ${ }_{P}$ \\
\hline \multirow{5}{*}{ Mdictiell (Dougherty) } & 1 & 27.6 & 20.1 & 20.1 & ST & BUT & $\bar{z}$ & $\begin{array}{l}1972 \\
1948\end{array}$ & $\stackrel{\phi P}{O P}$ \\
\hline & 2 & 27,6 & 185 & 185 & ș & Htr & + & |셩 & op \\
\hline & 3 & 163.2 & 159,4 & 59,4 & $\mathbf{I T}$ & HIT & - & 1945 & OP \\
\hline & $4 A$ & $\begin{array}{l}419 \\
419\end{array}$ & $\begin{array}{l}39.1 \\
39.1\end{array}$ & $\begin{array}{l}41.9 \\
41.9\end{array}$ & $\sqrt{\mathbb{E}}$ & $\begin{array}{l}\mathrm{FO} 2 \\
\mathrm{FO} 2\end{array}$ & 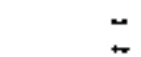 & $\begin{array}{l}1971 \\
1971\end{array}$ & $\stackrel{P}{O P}$ \\
\hline & $4 C$ & 41.9 & 33.1 & 41.9 & J & $\mathrm{FO2}$ & - & 1971 & $\Delta P$ \\
\hline \multirow[t]{5}{*}{ Morgen Fills (Fultcon) } & 1 & 24 & $1 / 4$ & 1.3 & $\begin{array}{l}\mathbf{H Y} \\
\mathbf{H Y}\end{array}$ & Wmo & 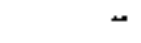 & 1903 & op \\
\hline & $\mathbf{2}$ & 24 & {$[.4$} & $\begin{array}{l}1.3 \\
13\end{array}$ & $\begin{array}{l}\text { HY } \\
\text { HY }\end{array}$ & $\begin{array}{l}\text { Woter } \\
\text { Water }\end{array}$ & $\bar{z}$ & $\begin{array}{l}1909 \\
1903\end{array}$ & op \\
\hline & 4 & 24 & 14 & 13 & HY & Wwer & - & 1900 & op \\
\hline & $\begin{array}{l}5 \\
6\end{array}$ & $\begin{array}{l}24 \\
24\end{array}$ & 1.4 & $\frac{13}{1.3}$ & $\begin{array}{l}\text { HY } \\
\text { HY }\end{array}$ & Woter & $\bar{z}$ & 1903 & OP \\
\hline & 7 & 24 & 1.4 & 13 & HY & What & - & 1903 & OP \\
\hline \multirow{2}{*}{ 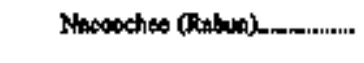 } & 1 & 24 & 28 & 28 & HY & Wutr & . & 1926 & $a p$ \\
\hline & 3 & 24 & 28 & 28 & $\mathbf{H Y}$ & Weter & 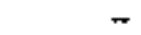 & 1926 & OP \\
\hline \multirow[t]{3}{*}{ 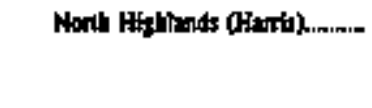 } & 1 & 9,2 & 9.7 & 9.6 & $\mathbf{H Y}$ & Water & - & 1963 & OP \\
\hline & $\frac{2}{3}$ & 9.2 & $\begin{array}{l}9.7 \\
9.7\end{array}$ & 9.6 & hy & Wor & $\ddot{2}$ & 1960 & op \\
\hline & 4 & 25 & 21 & 21 & HY & Wettr & $\pi$ & [9967 & OP \\
\hline 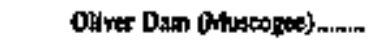 & 1 & 180 & 16.0 & 15.5 & HY & Water & - & 1959 & $\mathrm{PP}$ \\
\hline & 2 & 160 & 160 & 155 & HY & Wats & - & 1959 & OP \\
\hline & 3 & 180 & 160 & 155 & HY & Woter & - & 1959 & OP \\
\hline & 4 & 60 & 53 & 5.2 & HY & Wetrs & - & 1959 & OP \\
\hline 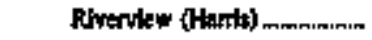 & 1 & $\frac{7}{2}$ & 1 & .1 & HY & Wayr & - & 1918 & OP \\
\hline & 2 & 2 &. & .1 & $\mathbf{H Y}$ & Wotr & - & 1918 & OP \\
\hline Foblas (Housson) & $\begin{array}{l}1 \\
2\end{array}$ & 919 & $\begin{array}{l}793 \\
793\end{array}$ & $\begin{array}{l}95.4 \\
95.4\end{array}$ & GT & Not Gas & $\mathrm{FO} 2$ & 199 & pp \\
\hline Scleter (Mbortoe) & $* * 1$ & 890 & 8327 & 9322 & sT & BrT & - & 198 & aP \\
\hline & $\cdots_{2}$ & B325 & 8325 & 8321 & ș & EIT & - & 1984 & OP \\
\hline & -3 & 3910 & 844.0 & 844.0 & $S T$ & BIT & $n$ & 1987 & op \\
\hline & +4 & 5910 & 8440 & 844.0 & sT & E[T & - & 1989 & of \\
\hline Shedolr Dan (Batutwin) & 1 & 225 & 20.0 & 20.1 & HY & Wwar & - & 1958 & OP \\
\hline & $\mathbf{z}$ & 225 & 200 & 20.5 & HY & Wat & - & 1959 & OP \\
\hline 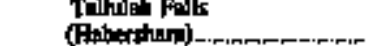 & & & & & HY & Wher & - & 1913 & \\
\hline & 2 & 120 & 108 & 10.8 & $\mathbf{H Y}$ & Water & $=$ & {$[913$} & op \\
\hline & 3 & 120 & 10.8 & 108 & HYY & Water & - & 1914 & of \\
\hline & 4 & 120 & 108 & 10.8 & HY & Water & ـ & [9]3 & OP \\
\hline & 5 & I20 & 108 & 10.8 & HY & Whar & - & [19]3 & op \\
\hline & 6 & 120 & 108 & 108 & $\mathbf{H Y}^{\mathbf{Y}}$ & Weter & - & 1920 & OP \\
\hline Tutara (Renon) & i & 80 & 73 & 73 & HYY & Wort & - & 1925 & $\mathbf{Q P}$ \\
\hline & $i$ & 80 & 73 & 7.3 & ḦY & Wher & - & 1925 & OP \\
\hline - Tugno (trphersham) & J & I13 & $11 . \overline{2}$ & 11.2 & HY & Watter & - & t923 & OP \\
\hline & 2 & 113 & 112 & 11.2 & BY & Water & $\leftrightarrow$ & {$[928$} & p \\
\hline & 3 & 113 & 11.2 & 11.2 & HY & Water & - & 1924 & $\mathbf{o p}$ \\
\hline & & It 3 & 11.2 & 11.2 & HYे & Water & . & 1924 & op \\
\hline 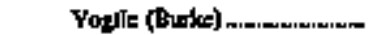 & 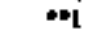 & 21600 & 1164.0 & 1164.0 & $\mathrm{NP}$ & tharian & 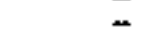 & 1967 & $O P$ \\
\hline & $m$ & 11000 & J164.0 & JTS4.0 & & Utaninm & ـ & 1989 & OP \\
\hline 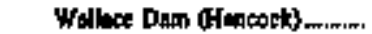 & $\mathbf{I}$ & 522 & 51.5 & 51,5 & HY & Waber & - & 1980 & $\phi P$ \\
\hline & 2 & 52.2 & 515 & $\$ 15$ & $\mathbf{H Y}$ & Walue & - & 1980 & $\alpha p$ \\
\hline & 3 & 563 & $\begin{array}{l}55.5 \\
55.5\end{array}$ & $\begin{array}{l}55.5 \\
55.5\end{array}$ & HYY & Wabre & $\overline{-}$ & 1980 & ${ }_{0 p}^{0 p}$ \\
\hline & 3 & S22 & 515 & G.5 & $\mathbf{H Y}$ & $\begin{array}{l}\text { Warta } \\
\text { Water }\end{array}$ & $=$ & 1940 & هా \\
\hline & & 52.2 & 515 & $\$ 1.5$ & EY & Walus & - & 1979 & $\alpha$ \\
\hline Wansley (Feard) & t & 9520 & 86.4 & $8640^{\circ}$ & $\mathbf{s T}$ & $B R$ & - & 1976 & $\mathrm{OP}$ \\
\hline & 4 & 9620 & 868.1 & 868.1 & $\mathbf{s T}$ & BIT & - & 197 & QP \\
\hline & SA & 528 & SAd & 66.1 & GT & Fo2 & - & 1980 & P \\
\hline
\end{tabular}

Bet foutrobes at end of thits. 
Table 20. Existing Generating Units at U.S. Electric Utilities by State, Company, and Plant, as of January 1, 1996 (Conthned)

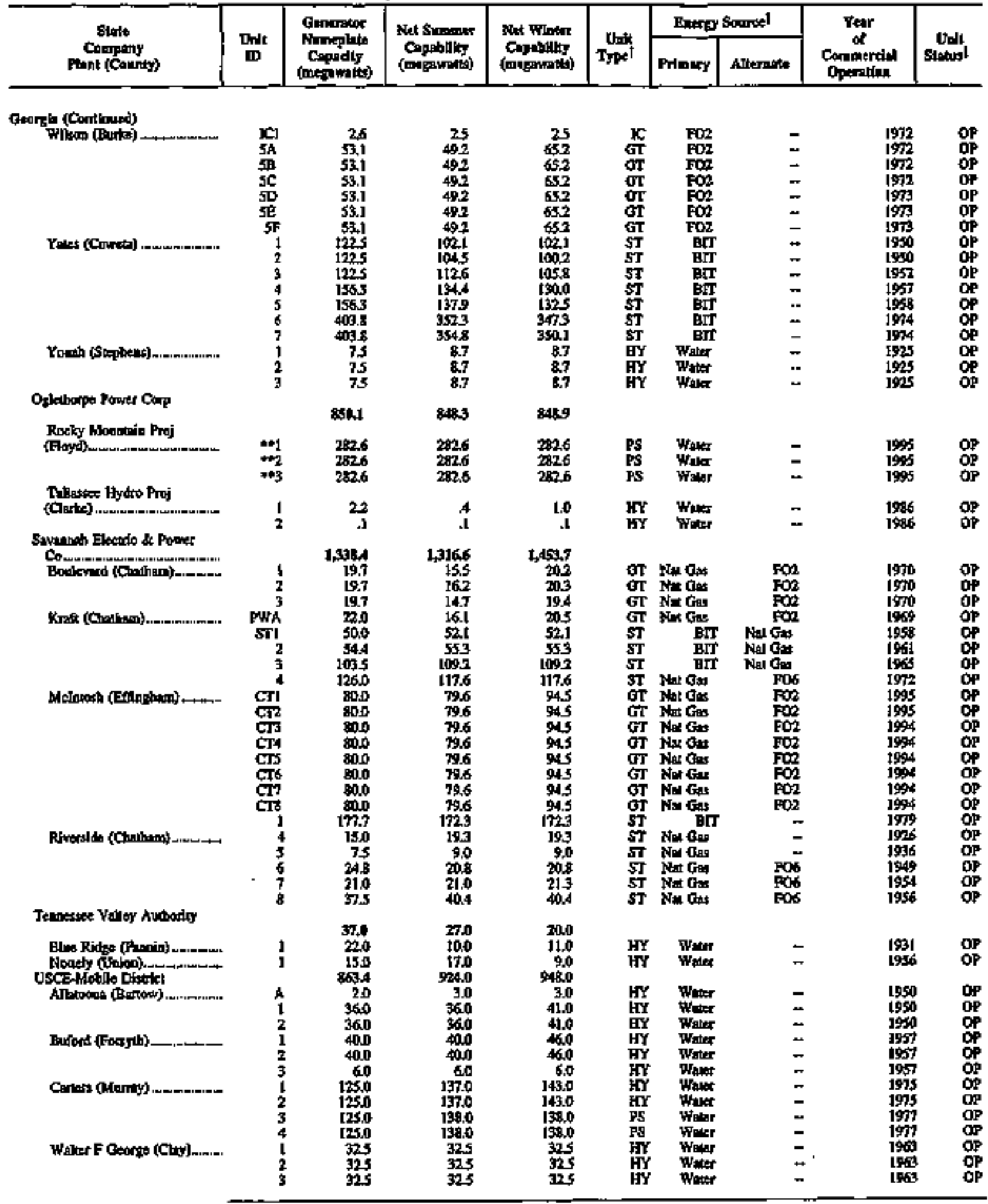

Sol footones at and of toble. 
Tahle 20. Existing Generating Units at U.S. Electric Utilities by State, Company, and Plant, as of January 1, 1996 (Continued)

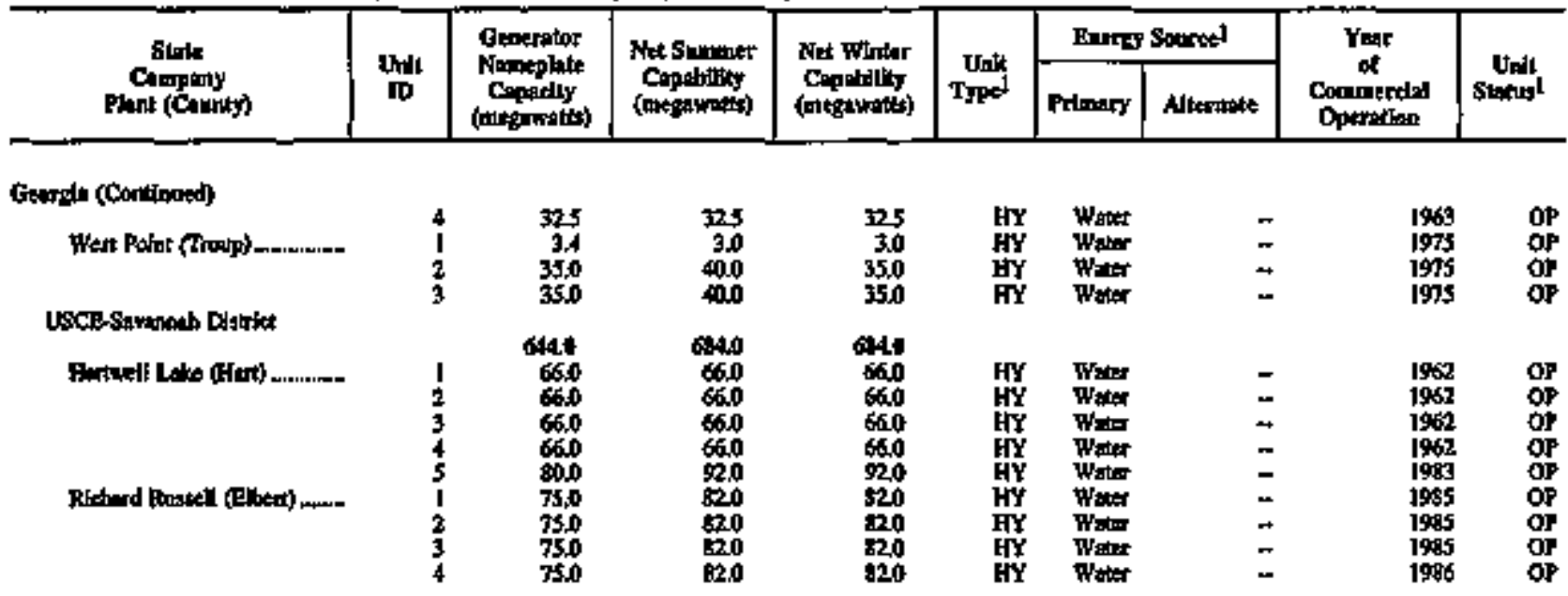

Empan

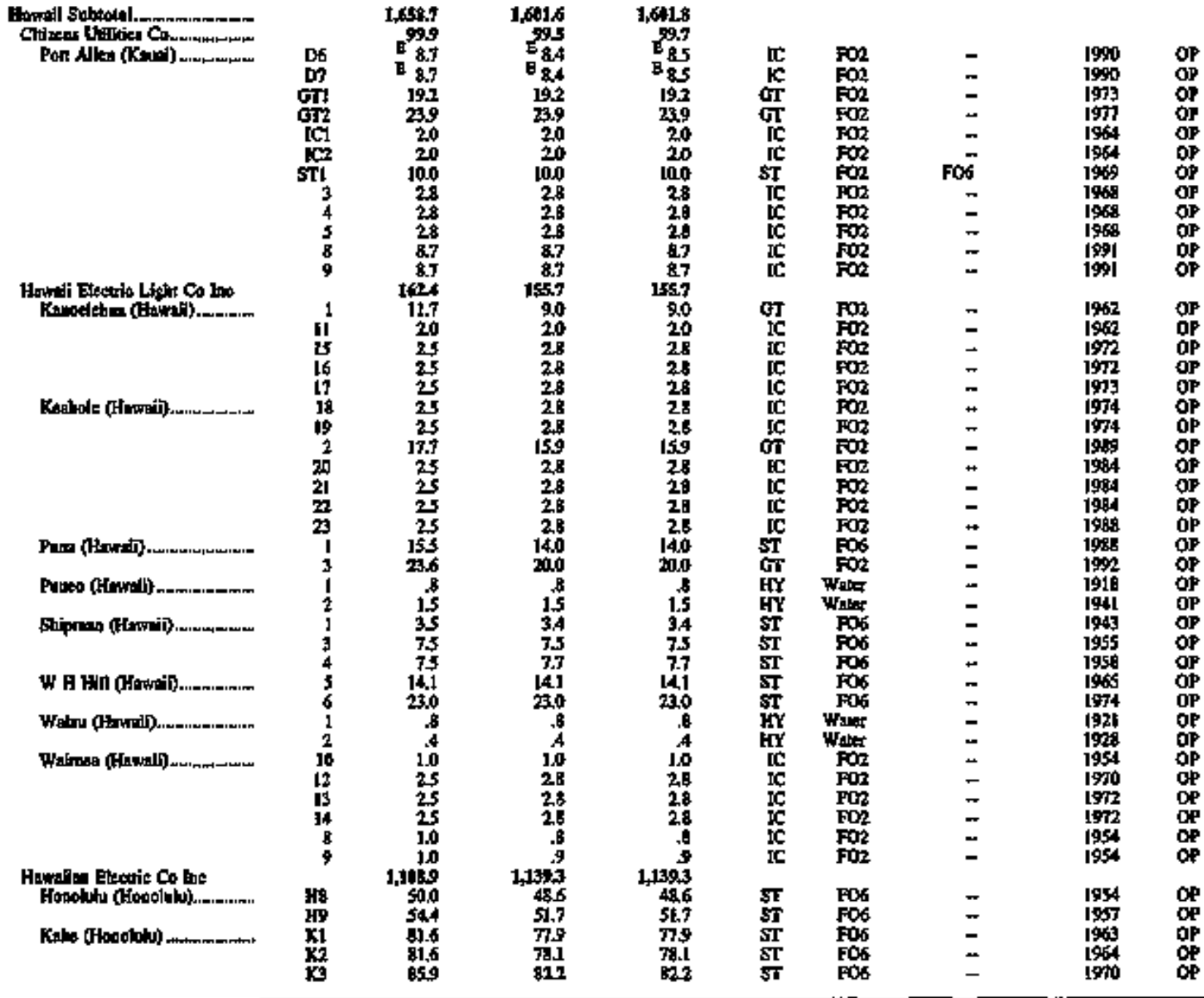

Bes loomptos an end of thile. 
Table 20. Existing Generating Units at U.S. Electric Utilities by State, Company, and Plant, as of January 1, 1996 (Continued)

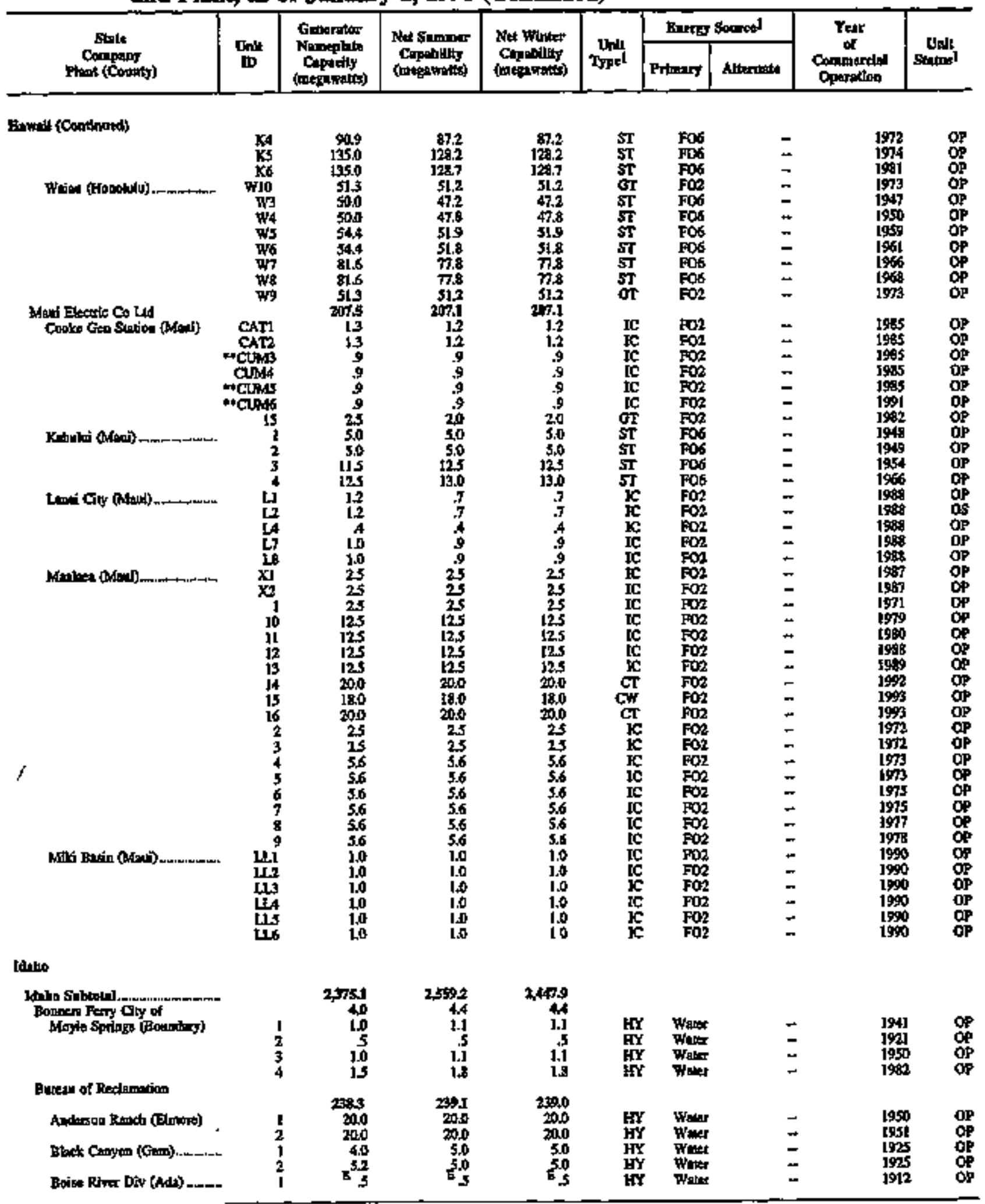

Sec footsons an and of toble.

92 Energy Information Administration/Inventory of Power Plants in the United States as of January 1, 1996 
Table 20. Exlsting Generating Units at U.S. Electric Utilities by State, Company, and Plant, as of Jamuary 1, 1996 (Continned)

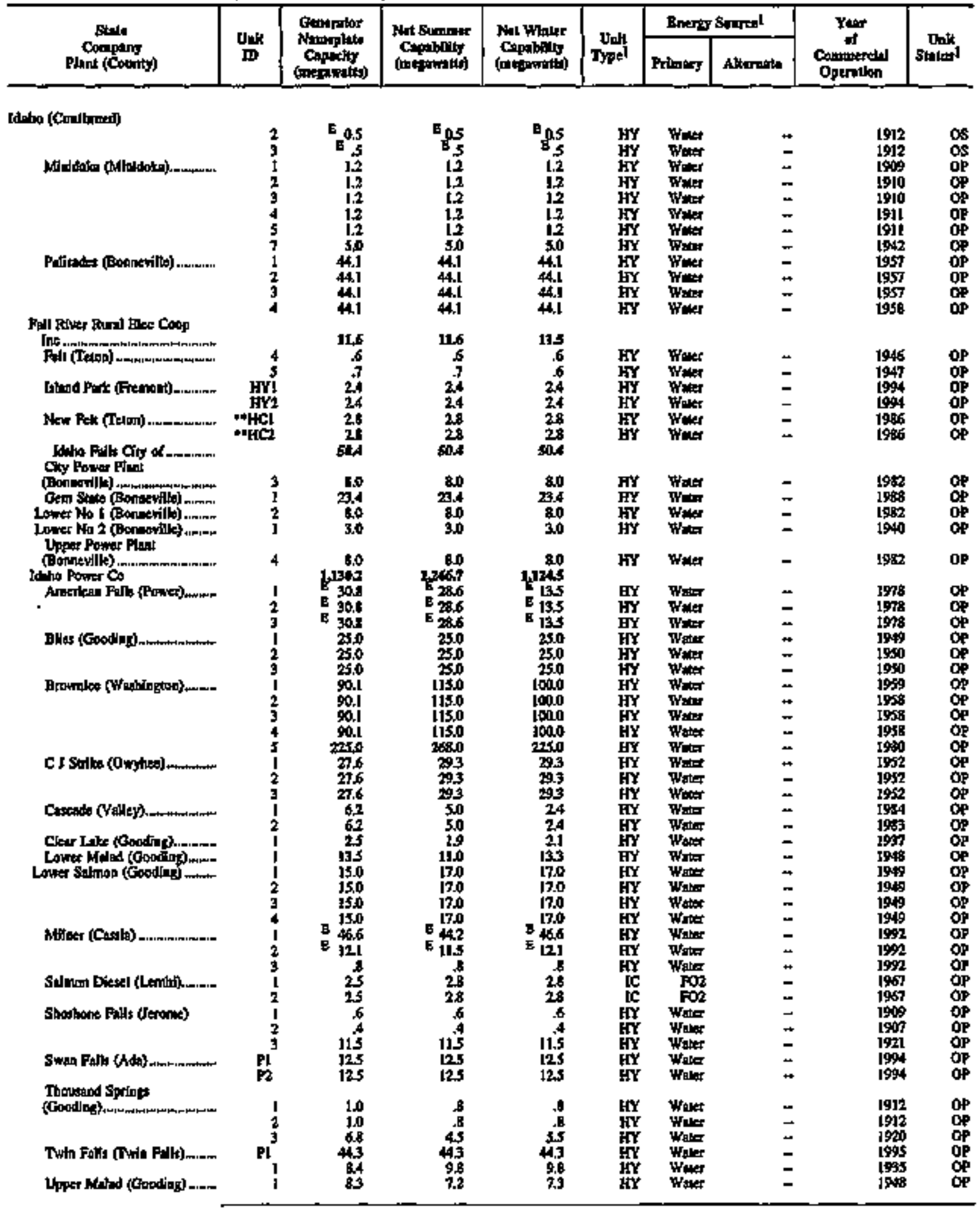

See toonores ex end of able. 
Table 20. Existing Generating Unlts at U.S. Electric Utilities by State, Coimpany, and Plant, as of January 1, 1996 (Continnued)

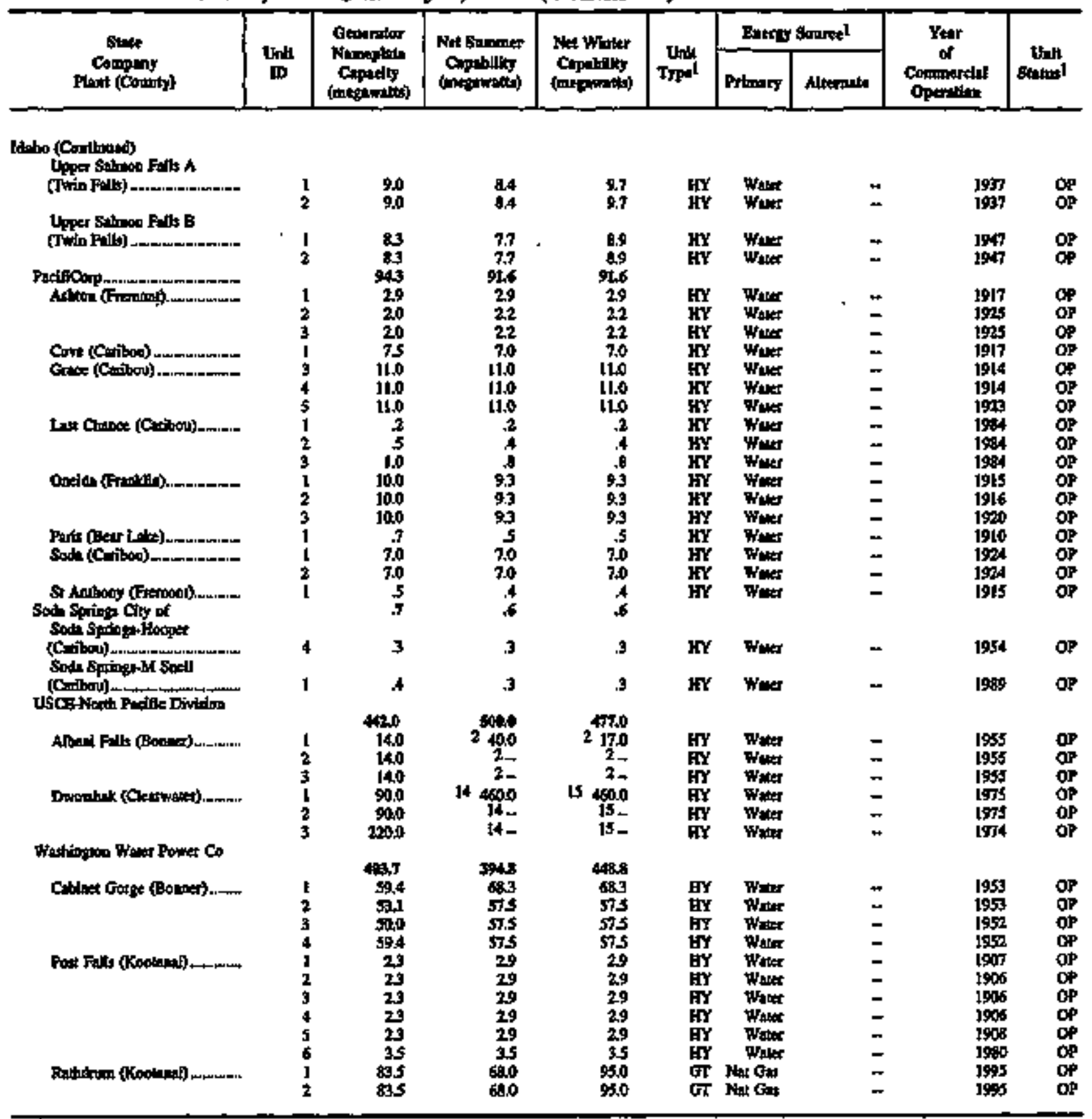

sas foothotes al ead of talle. 
Table 20. Existing Generating Units at U.S. Electric Utilities by State, Company, and Plant, as of January 1, 1996 (Continued)

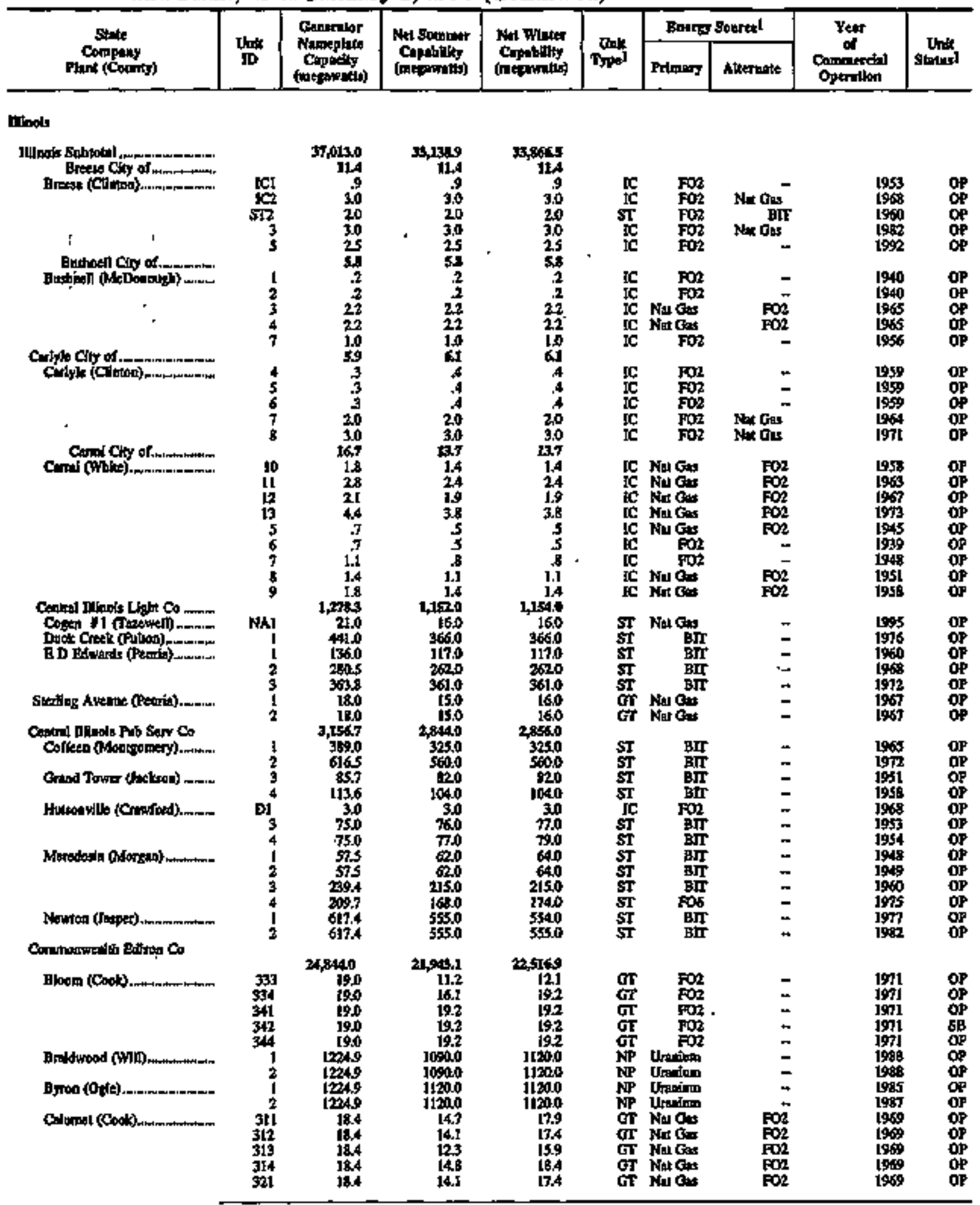

Ses foutonger at of of thble 
Tahle 20. Existimg Generating Units at U.S. Electric Utilities by State, Company, and Plant, as of January 1, 1996 (Continued)

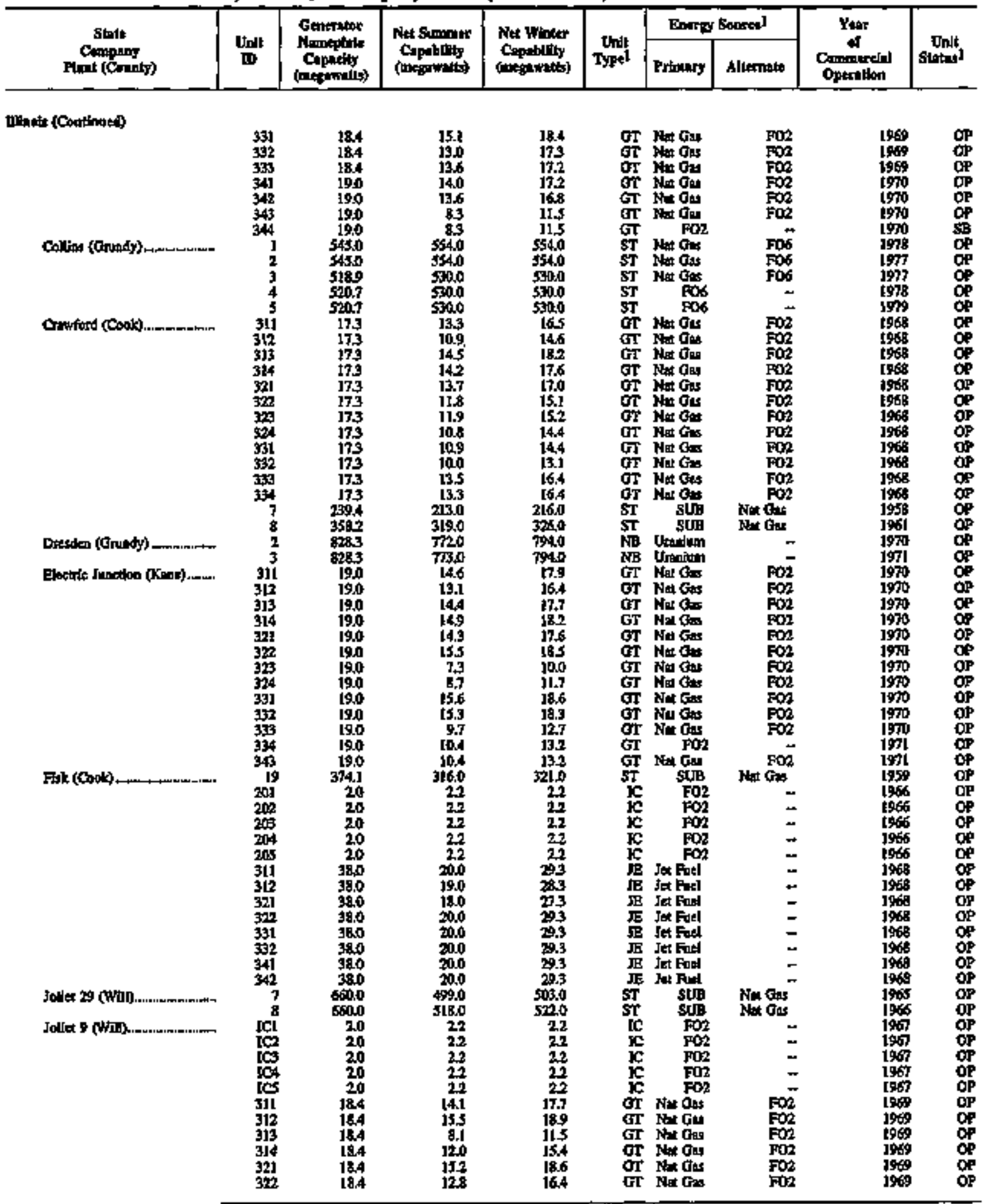

Seo footpoks at and of loble 
Table 20. Existing Generating Units at U.S. Electric Utillities by State, Company, and Plant, as of January 1, 1996 (Continued)

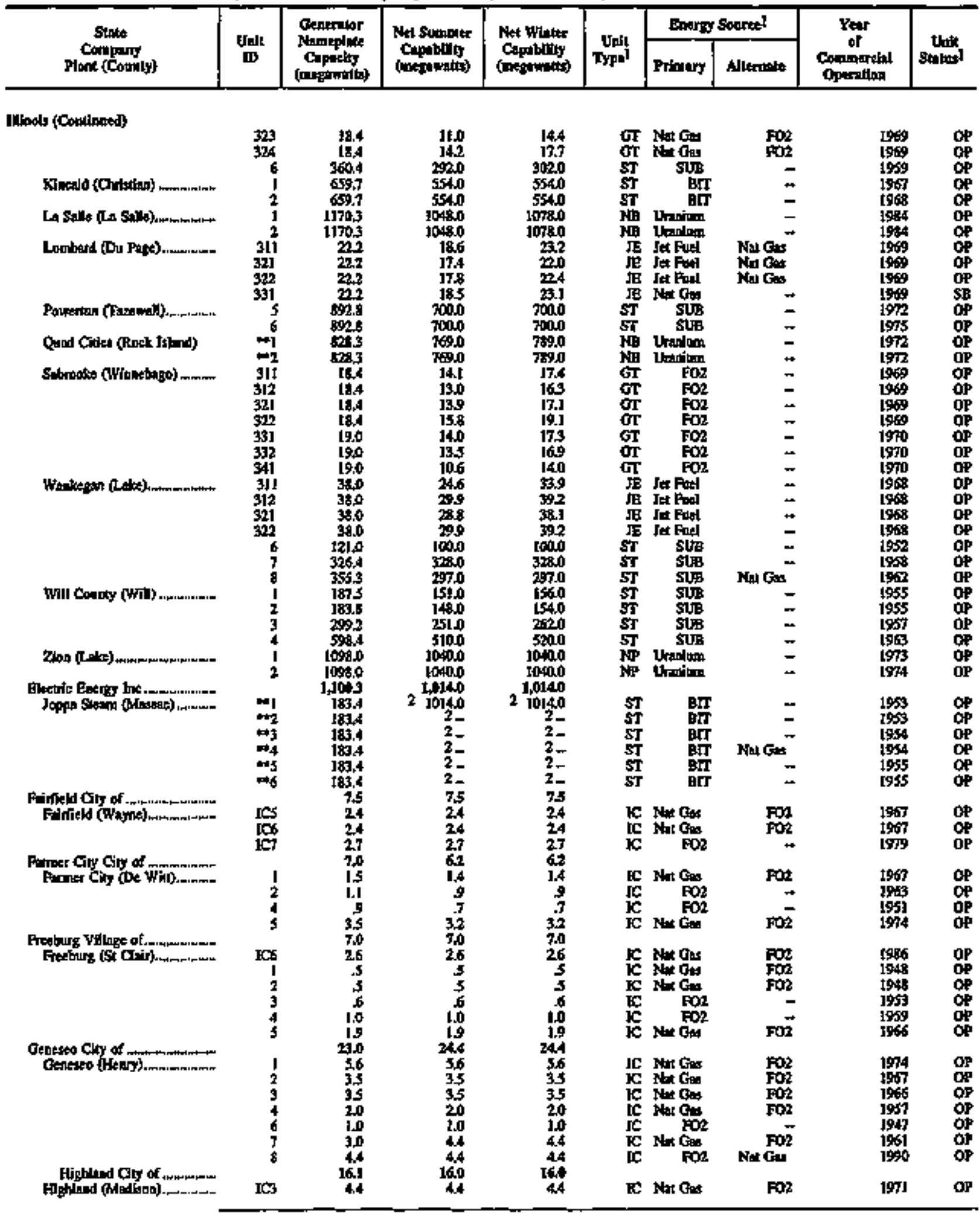

Sos footootes to eod of trible. 
Table 20. Existing Gererating Units at U.S. Electric Utillties by State, Company, and Plant, as of January 1, 1996 (Conthued)

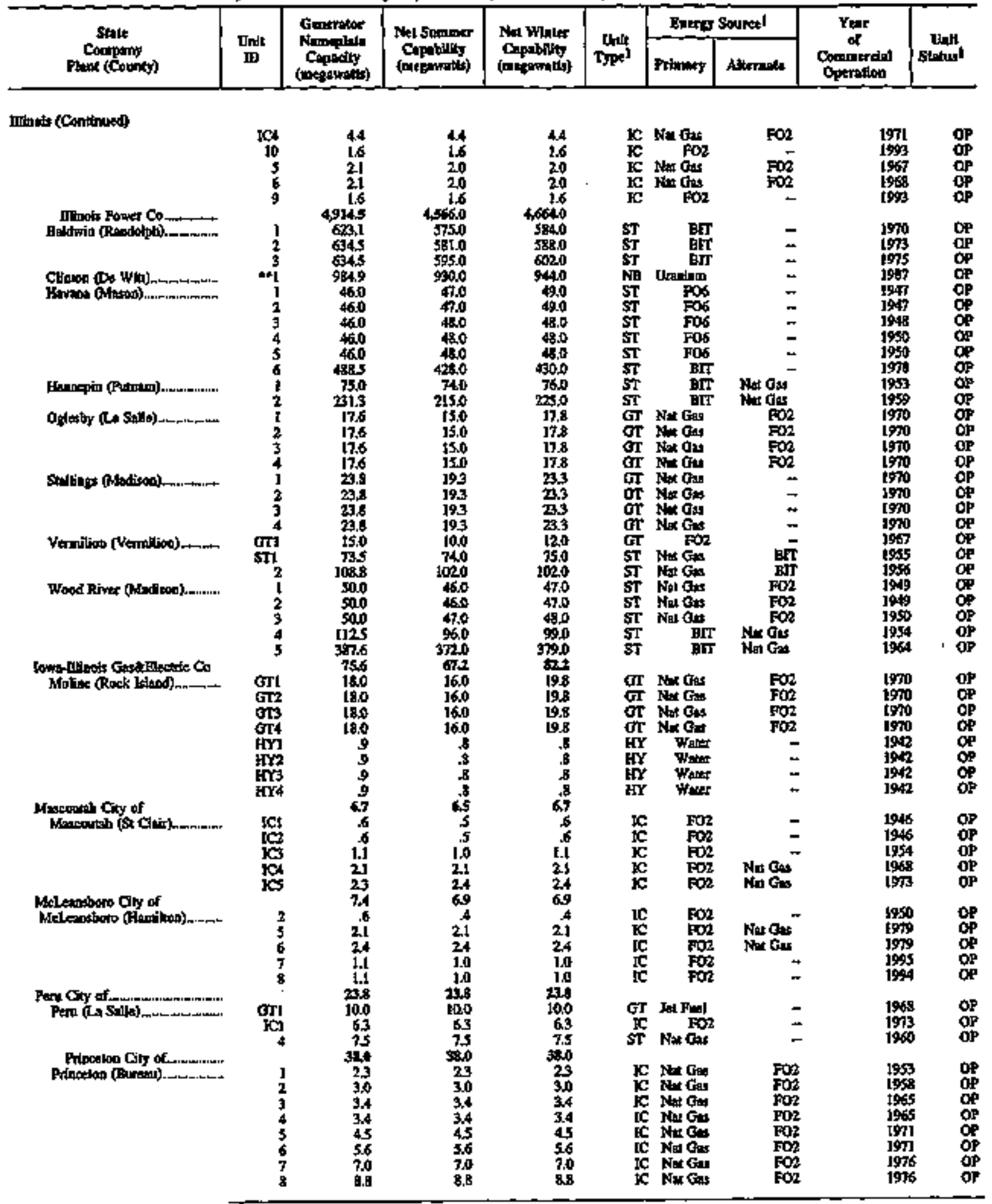

See papanotes at end of thle. 
Table 20. Existing Generating Units at U.S. Electric Utilities by State, Company, and Plant, as of January 1, 1996 (Continued)

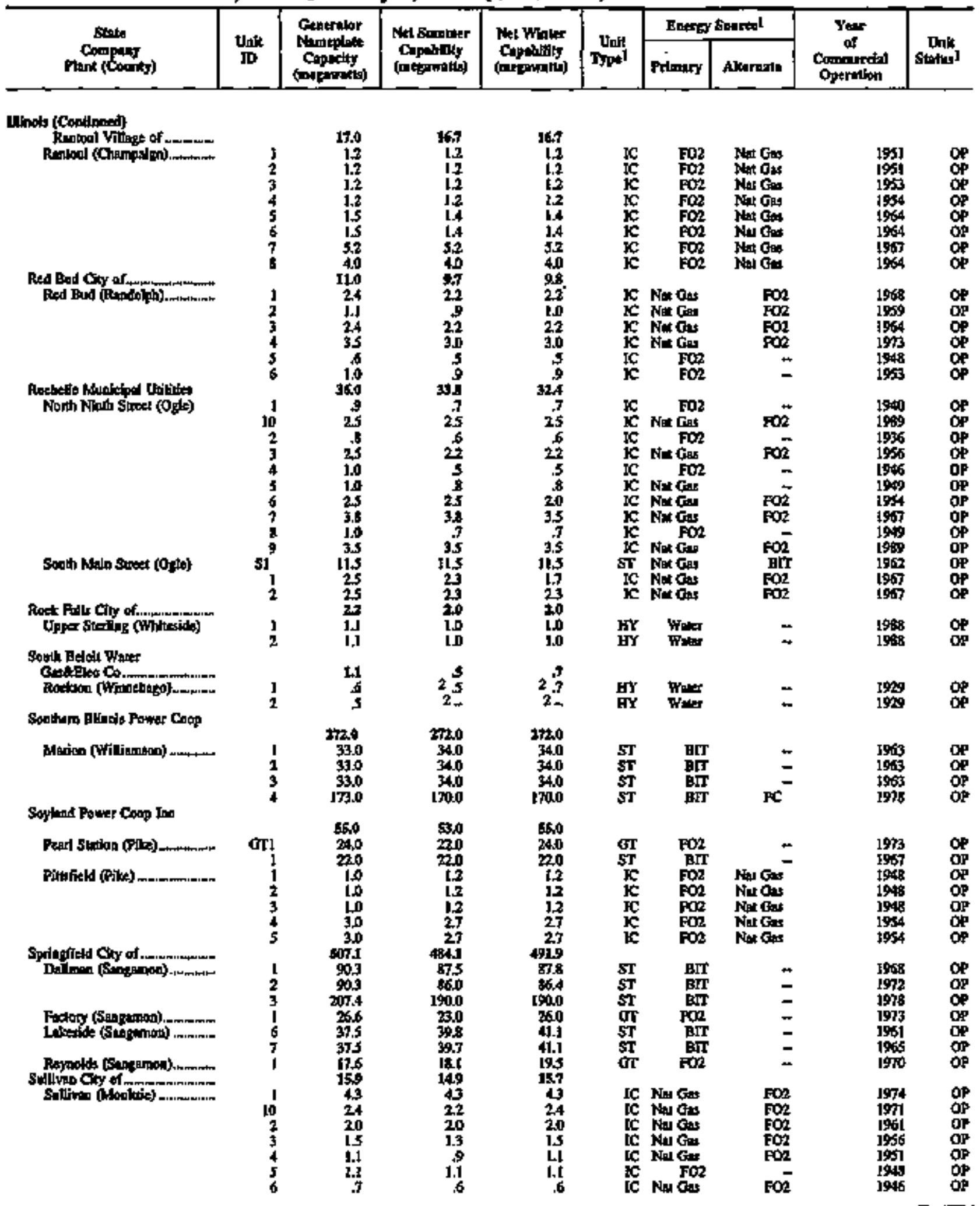

Ses foomotes at exd of teble. 
Table 20. Existing Generating Units at U.S. Electric Utilities by State, Company, and Plant, as of January 1, 1996 (Continued)

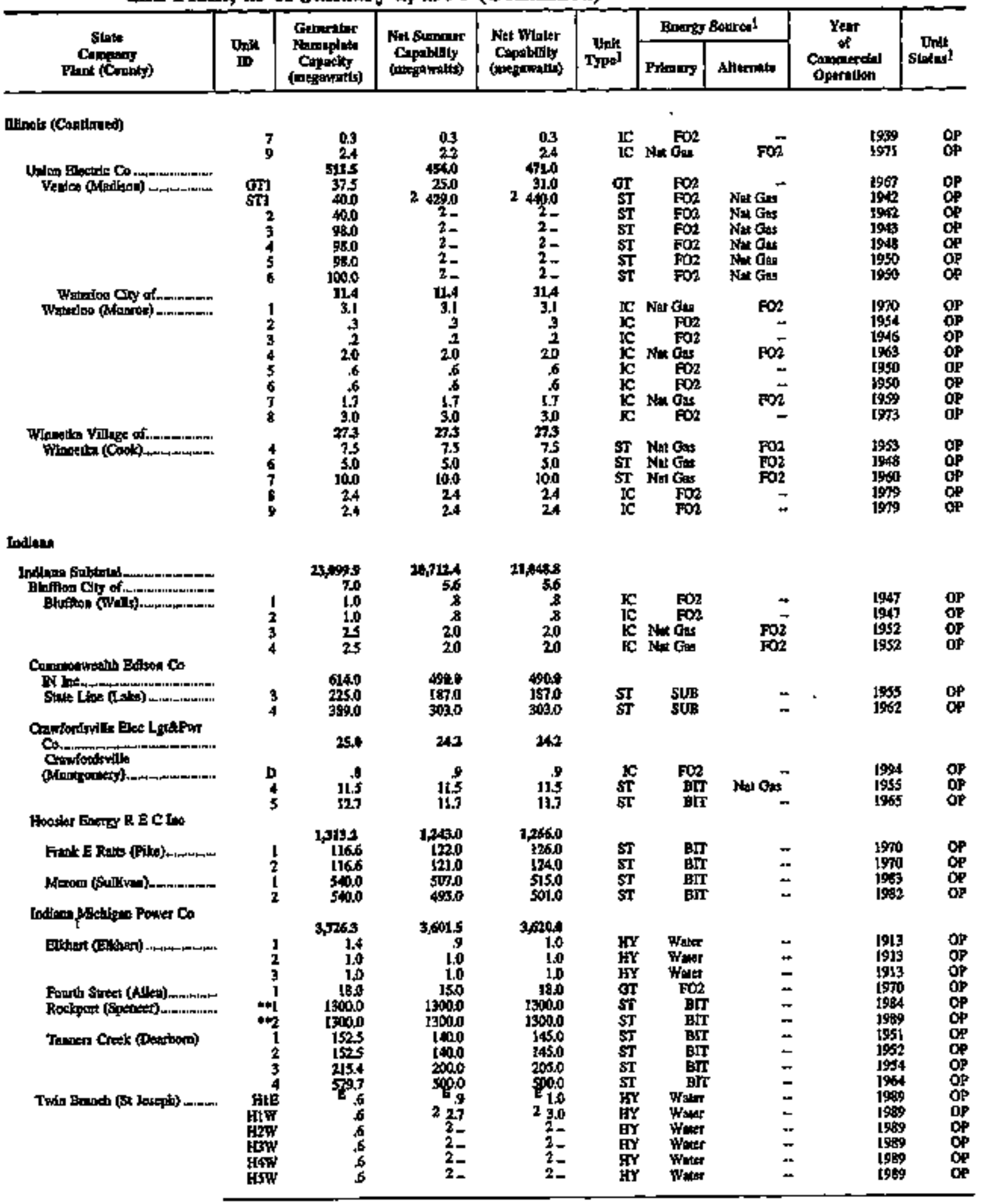

Sec tootingtes it end of thill 
Table 20. Existing Generating Units at U.S. Electric Utilities by State, Company, and Plant, as of January 1, 1996 (Continued)

\begin{tabular}{|c|c|c|c|c|c|c|c|c|c|}
\hline \multirow{2}{*}{$\begin{array}{c}\text { State } \\
\text { Companny } \\
\text { Ploni (Coaurts) }\end{array}$} & \multirow{2}{*}{$\begin{array}{l}\text { Dntt } \\
\text { ID }\end{array}$} & \multirow{2}{*}{ 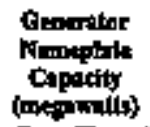 } & \multirow{2}{*}{$\begin{array}{l}\text { Net Bumoner } \\
\text { Cappabilly } \\
\text { (mejprotts) }\end{array}$} & \multirow{2}{*}{ 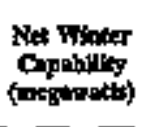 } & \multirow{2}{*}{$\begin{array}{c}\text { thalt } \\
\text { Typet }\end{array}$} & \multicolumn{2}{|c|}{ Binergy Sonree] } & \multirow{2}{*}{$\begin{array}{c}\text { Yaur } \\
\text { of } \\
\text { Commerelol } \\
\text { Operation }\end{array}$} & \multirow{2}{*}{$\begin{array}{c}\text { Unil } \\
\text { Stalles }\end{array}$} \\
\hline & & & & & & Primury & Allerrinta & & \\
\hline \multicolumn{10}{|l|}{ Incilana (Cootinowed) } \\
\hline & 衉 & 0.6 & $\frac{2}{2}-$ & $2=$ & $\boldsymbol{H} \boldsymbol{Y}$ & Whater & $\ddot{z}$ & 199 & op \\
\hline \multirow{2}{*}{\multicolumn{10}{|c|}{ Indlang hounkfipil Power }} \\
\hline & & 165.5 & 1400 & 164,0 & & & & & \\
\hline 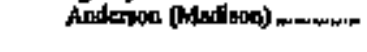 & ACTI & 41.5 & $\begin{array}{l}36.0 \\
360\end{array}$ & 41.0 & Gr & Nat Gas & F02 & 1992 & op \\
\hline 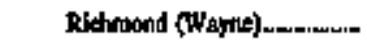 & RCTH & 41.3 & 300 & $\mathbf{4 1 . 0}$ & 故 & Het & Fon & 1992 & \\
\hline \multicolumn{10}{|l|}{ 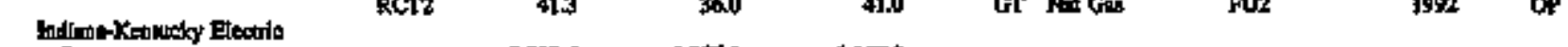 } \\
\hline \multirow{6}{*}{ 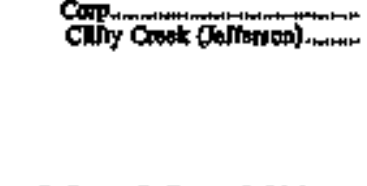 } & & 133036 & 1,2170 & $1,253$. & & & & & \\
\hline & 1 & 2173 & wis.0 & 2150 & ST & EIT & - & 1955 & of \\
\hline & 2 & 2173 & 201.0 & 209,0 & $\underset{S T}{S T}$ & BIT & $=$ & 1955 & $\mathbf{m}$ \\
\hline & $\frac{3}{4}$ & $\begin{array}{l}2173 \\
2173\end{array}$ & 191.0 & 199.0 & ST & BrT & 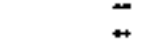 & 1965 & $\stackrel{\mathrm{OP}}{\mathrm{OP}}$ \\
\hline & 5 & 217.3 & 2070 & 225,0 & ST & हा & - & 1955 & F \\
\hline & 6 & 2173 & 2030 & 211.0 & sT & EIT & - & 1965 & op \\
\hline \multicolumn{10}{|l|}{ Jnofanapoli Power a Lijat } \\
\hline 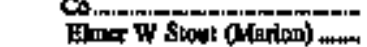 & & $\begin{aligned} 3,3146 \\
25,4\end{aligned}$ & 2006 & $3,0,0$ & GF & & & 1973 & \\
\hline & Gr: & 31.4 & $\mathbf{2 0 0}$ & $\mathbf{3 0 4}$ & GT & Fon & $=$ & 1973 & $\alpha$ \\
\hline & GT3 & 2.4 & 200 & 250 & $G$ & $P D 2$ & - & 1973 & $\phi_{p P}$ \\
\hline & GI5 & 8000 & 79.0 & 102-8 & Gr & Flat Ge & $\begin{array}{ll}\mathrm{FO2} \\
\mathrm{FO2}\end{array}$ & 1998 & $\stackrel{\alpha p}{\alpha p}$ \\
\hline & ICI & 28 & 3,0 & 30 & IC & F02 & - & 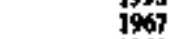 & \\
\hline & 3 & 375 & 350 & 40,0 & ST & 802 & - & j9:41 & 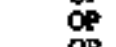 \\
\hline & 4 & 375 & 350 & 40,0 & SI & $\mathrm{F} 02$ & + & 1947 & OP \\
\hline & $\frac{5}{6}$ & 1136 & 1050 & 109.0 & ST & $B \pi$ & 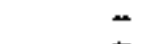 & 1956 & $\stackrel{Q P}{\alpha}$ \\
\hline & j & 430.9 & 4220 & 2000 & sT & BII & $=$ & 1973 & of \\
\hline \multirow[t]{6}{*}{ 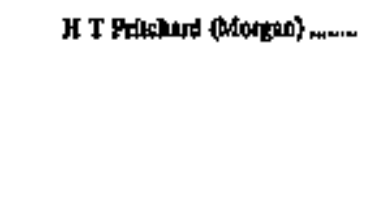 } & ICI & 28 & 30 & 3.0 & ic & $\mathrm{Fot}$ & - & 1967 & er \\
\hline & sti & 460 & 390 & 39.0 & ST & $\mathrm{Fo2}$ & $*$ & 1949 & $\underset{g P}{\operatorname{Pr}}$ \\
\hline & 2 & $\begin{array}{l}46,0 \\
500\end{array}$ & 43.0 & $\begin{array}{l}39,0 \\
490\end{array}$ & $\begin{array}{l}\$ \mathbf{T} \\
\mathbf{S T}\end{array}$ & $\mathrm{FO}$ & $=$ & $\begin{array}{l}1951 \\
1951\end{array}$ & $\stackrel{\alpha P}{O P}$ \\
\hline & 4 & 290 & 550 & 57.0 & $\mathbf{s} \mathbf{T}$ & BII & 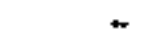 & 1953 & $\alpha$ \\
\hline & 3 & 69,0 & 620 & 63.0 & $\mathbf{S T}$ & BII & - & 1953 & 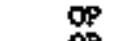 \\
\hline & 6 & 1136 & 99.0 & 1000 & $\mathbf{S T}$ & BT & - & & or \\
\hline \multirow{2}{*}{ 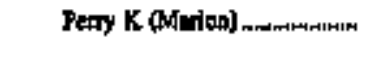 } & es & 50 & 300 & 3.0 & ST & Bri & - & 1938 & $\underset{\mathbf{O P}}{\mathbf{O P}}$ \\
\hline & 4 & $E_{50}^{150}$ & 845 & $E_{d d}^{370}$ & ST & BrT & $=$ & 1925 & 90 \\
\hline Pemy W ONaIOA) & 7 & 11.6 & 120 & 500 & $\mathbf{s t}$ & BII & $\mathbf{F O} 2$ & 196 & $\alpha$ \\
\hline Peketibere $($ Piko) & (C) & 28 & 3,0 & 3.0 & $\mathbf{s}$ & FO2 & + & 1967 & $O P$ \\
\hline & $\sqrt{102}$ & 28 & 3.0 & 3.0 & $\mathbf{s t}$ & FOS & 2 & 1967 & OP \\
\hline & $\sin$ & 253,4 & 200 & 290 & 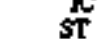 & But & $\bar{z}$ & 1969 & of \\
\hline & $5 T 2$ & 471.0 & 4180 & 4180 & $\mathbf{s}$ & $\mathrm{BrT}$ & 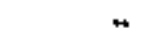 & 190 & op \\
\hline & STY & 5744 & 5100 & 510,0 & SI & BII & - & $19 m$ & OP \\
\hline & 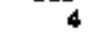 & 574.2 & 515.0 & 515.0 & $s t$ & BTI & - & 1986 & $\mathbf{O P}$ \\
\hline Jasper Ofy of m & & 14.5 & 135 & 13.5 & & & & & \\
\hline Jatper 2 (Dobolo) & I & 145 & {$[3.5$} & 13.3 & $\mathbf{s t}$ & Btr & Mat & 1968 & OP \\
\hline Log & 4 & 100 & 26.5 & & & BतF & $=$ & 2058 & OP \\
\hline & $\mathbf{s}$ & 25.0 & 220 & $n_{0}$ & st & Bत & 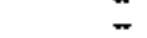 & 198 & OP \\
\hline & 6 & ISd & 1f:0 & 17.0 & का & $N e x$ & $F 02$ & 1969 & OP \\
\hline Nonbeta ladias Pot Sery & & & & & & & & & \\
\hline - & & 4097 & $3,390,4$ & $3,398,4$ & & & & & \\
\hline Hedily (Fonlest) & $\begin{array}{l}10 \\
7\end{array}$ & $\begin{array}{r}37.5 \\
1940\end{array}$ & $\begin{array}{r}31,0 \\
1600\end{array}$ & $\begin{array}{r}31,0 \\
160.0\end{array}$ & $\begin{array}{l}9 \pi \\
s !\end{array}$ & Nat G & Nar Go- & 3968 & OP \\
\hline & 8 & 421.6 & 320.0 & 3200 & $\$ T$ & BIT & Nat $\mathrm{k}$. & 1968 & OP \\
\hline 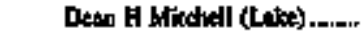 & it & j15.1 & 1100 & 100 & $S !$ & ȘIB & BIT & 1970 & 邑 \\
\hline & 4 & 139.1 & 18.0 & 125.0 & ST & Nex Gou & sup & 1956 & OP \\
\hline & 5 & 138, & 125,0 & $\begin{array}{l}1250 \\
1250\end{array}$ & $\begin{array}{l}S \mathrm{ST} \\
\mathbf{S T}\end{array}$ & stb & E[T & 1959 & OP \\
\hline & 9 & 374 & 17.0 & 170 & 亩 & Net Ge & & 1966 & \\
\hline MIdtgan Clyy (Ln Parte) & 12 & 5400 & 400 & 499.0 & 57 & BT & sup & 1974 & op \\
\hline & 2 & 700 & 600 & $\$ 0$ & ST & Nat ot & - & 190 & OP \\
\hline Non & 3 & 700 & 60,0 & 600 & ST & Nat Ges & $\approx$ & 1951 & $O P$ \\
\hline 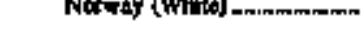 & 2 & $\begin{array}{l}2.0 \\
2.0\end{array}$ & 200 & 200 & $\begin{array}{l}\mathrm{HY} \\
\mathrm{HY}\end{array}$ & $\begin{array}{l}\text { Wath } \\
\text { Wanr }\end{array}$ & 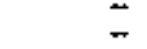 & 1923 & op \\
\hline
\end{tabular}

Sec foothes at and of ctible 
Table 20. Existing Generating Units at .U.S. Electric Utilities by State, Conapany, and Plant, as of Jamuary 1, 1996 (Continued)

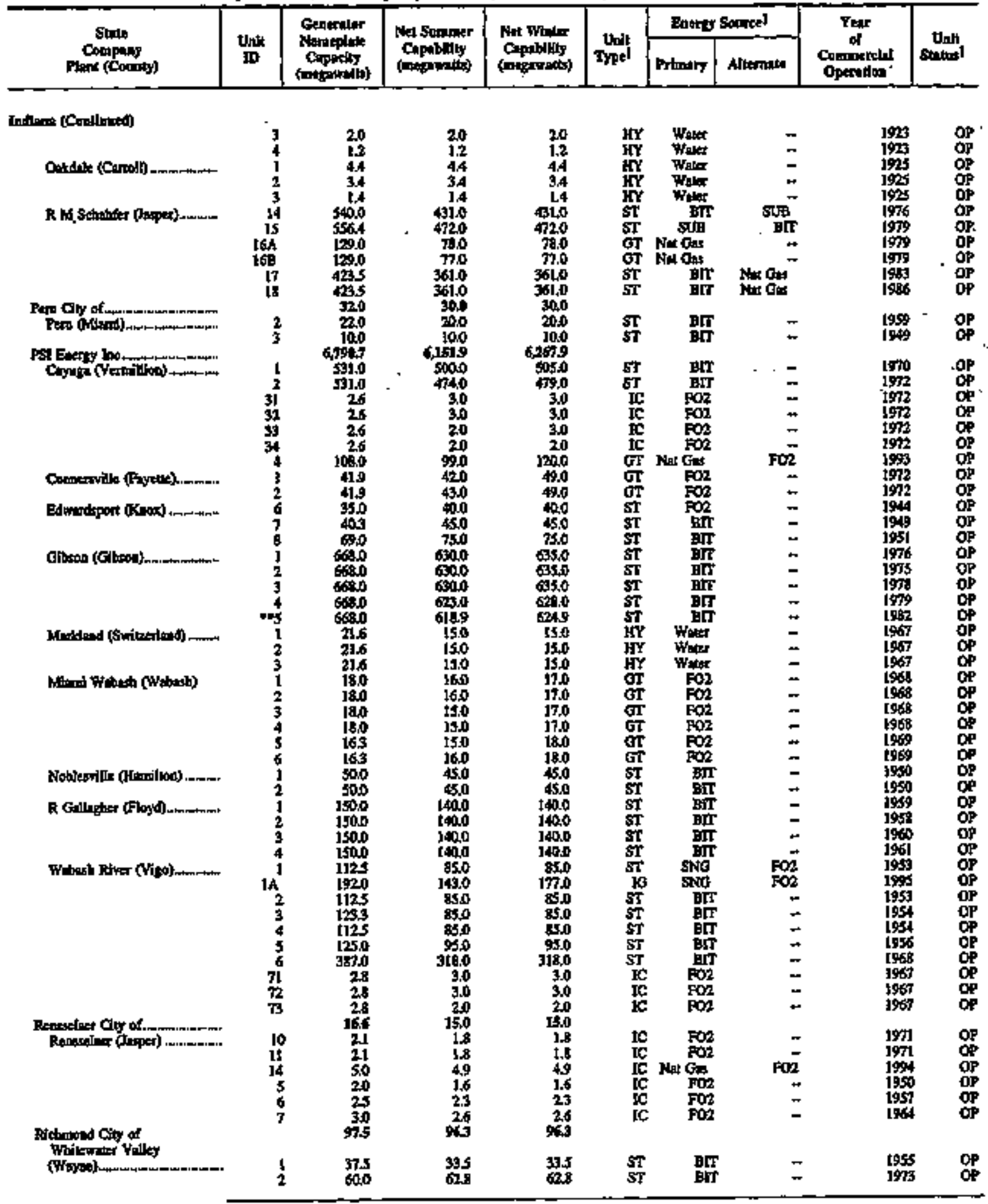

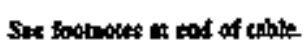


Table 20. Existing Generating Units at US. Electric Utilities by State, Company, and Plant, as of January 1, 1996 (Continued)

\begin{tabular}{|c|c|c|c|c|c|c|c|c|c|}
\hline \multirow{2}{*}{ 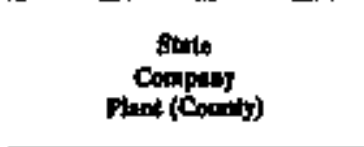 } & \multirow{2}{*}{ Int: } & \multirow{2}{*}{ 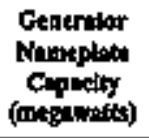 } & \multirow{2}{*}{ 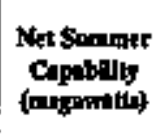 } & \multirow{2}{*}{ 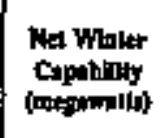 } & \multirow{2}{*}{$\underset{\text { Typt }}{\text { Uth }}$} & \multicolumn{2}{|c|}{ Emarg Snarm } & \multirow{2}{*}{ 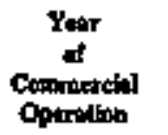 } & \multirow{2}{*}{ Sistuts } \\
\hline & & & & & & Prtang & Ahermati & & \\
\hline 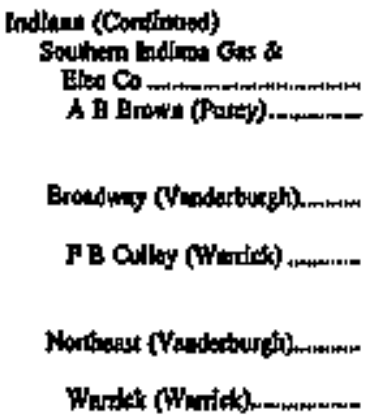 & $\begin{array}{r}1 \\
2 \\
4 \\
1 \\
2 \\
1 \\
2 \\
3 \\
1 \\
2 \\
+4\end{array}$ & $\begin{array}{r}1,570.8 \\
265.2 \\
265.2 \\
08.2 \\
53.1 \\
88.9 \\
46.0 \\
103.7 \\
265.2 \\
10.7 \\
11.5 \\
32.0\end{array}$ & $\begin{array}{r}1,220,0 \\
2500 \\
250,0 \\
90,0 \\
500 \\
650 \\
46,0 \\
920 \\
2500 \\
10.0 \\
100 \\
1350\end{array}$ & $\begin{array}{r}1,260.0 \\
250.0 \\
260.0 \\
87.0 \\
60.0 \\
750 \\
160 \\
920 \\
2100 \\
120 \\
13.0 \\
1350\end{array}$ & $\begin{array}{l}S T \\
S T \\
\text { GT } \\
G T \\
\text { GT } \\
\text { ST } \\
\text { ST } \\
\text { ST } \\
\text { GT } \\
\text { GT } \\
\text { ST }\end{array}$ & 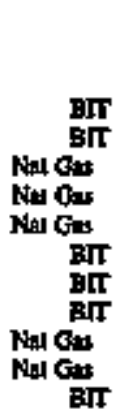 & $\begin{array}{r}\overline{-} \\
\mathrm{FO}_{2} \\
\mathrm{FO} \\
\mathrm{FO} 2 \\
\overline{-} \\
\overline{-} \\
\overline{-} \\
-\end{array}$ & $\begin{array}{l}1979 \\
1986 \\
1991 \\
1971 \\
1981 \\
1955 \\
1966 \\
1973 \\
1953 \\
1964 \\
1950\end{array}$ & $\begin{array}{l}O P \\
O P \\
O P \\
O P \\
O P \\
O P \\
O P \\
O P \\
O P \\
O P \\
O P\end{array}$ \\
\hline
\end{tabular}

Sut tootnotat al ead of uble 
Table 20. Existing Generating Units at US. Electric Utilities by State, Company, and Plant, as of January 1, 1996 (Continued)

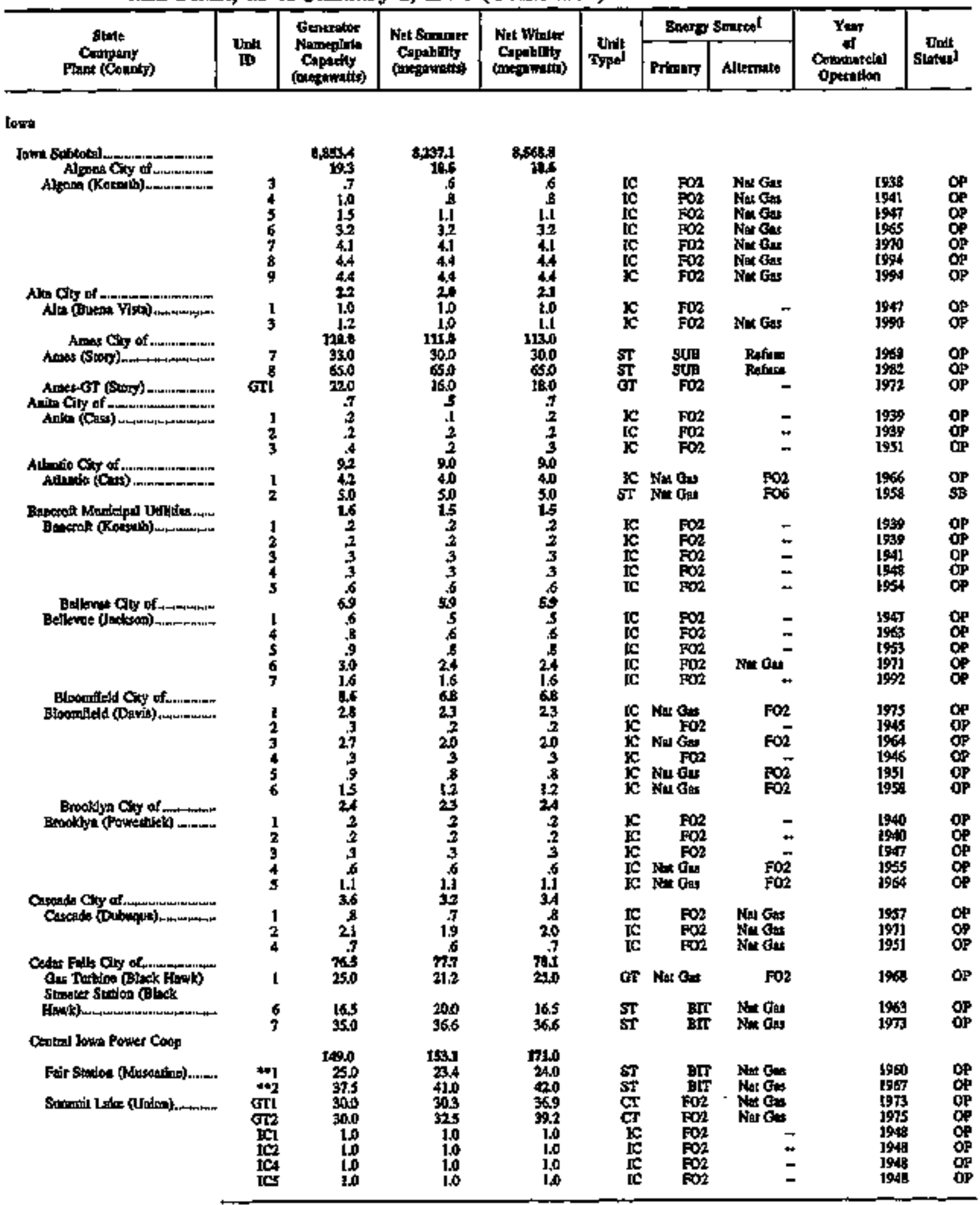

bee footnotes al and of uble. 
Table 20. Existing Generating Units at U.S. Electric Utiltties by State, Company, and Plant, as of January 1, 1996 (Continued)

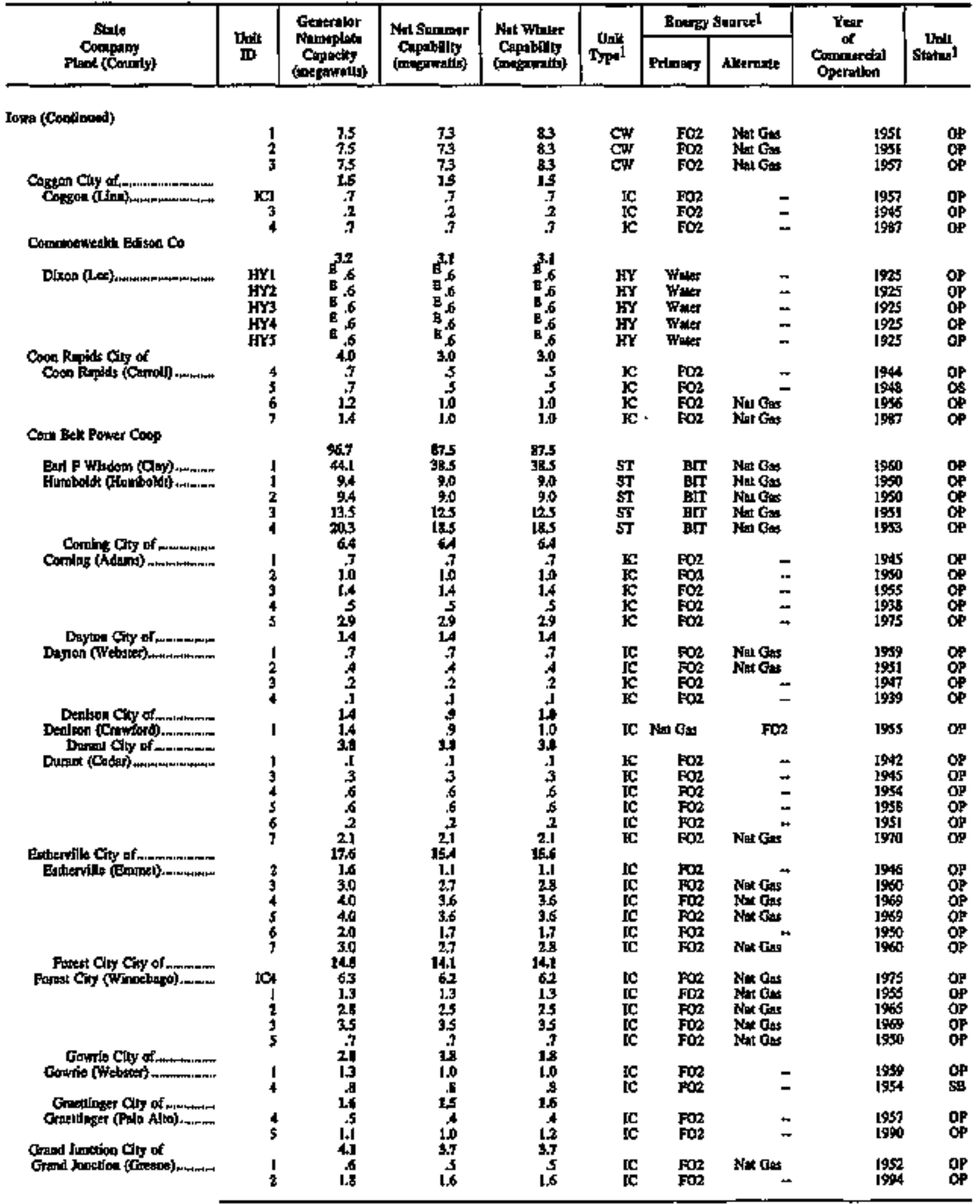

Sea fookneks ax and af toble. 
Tahle 20. Existing Generating Units at U.S. Electric Uttlities by State, Company, and Plant, as of January 1, 1996 (Continued)

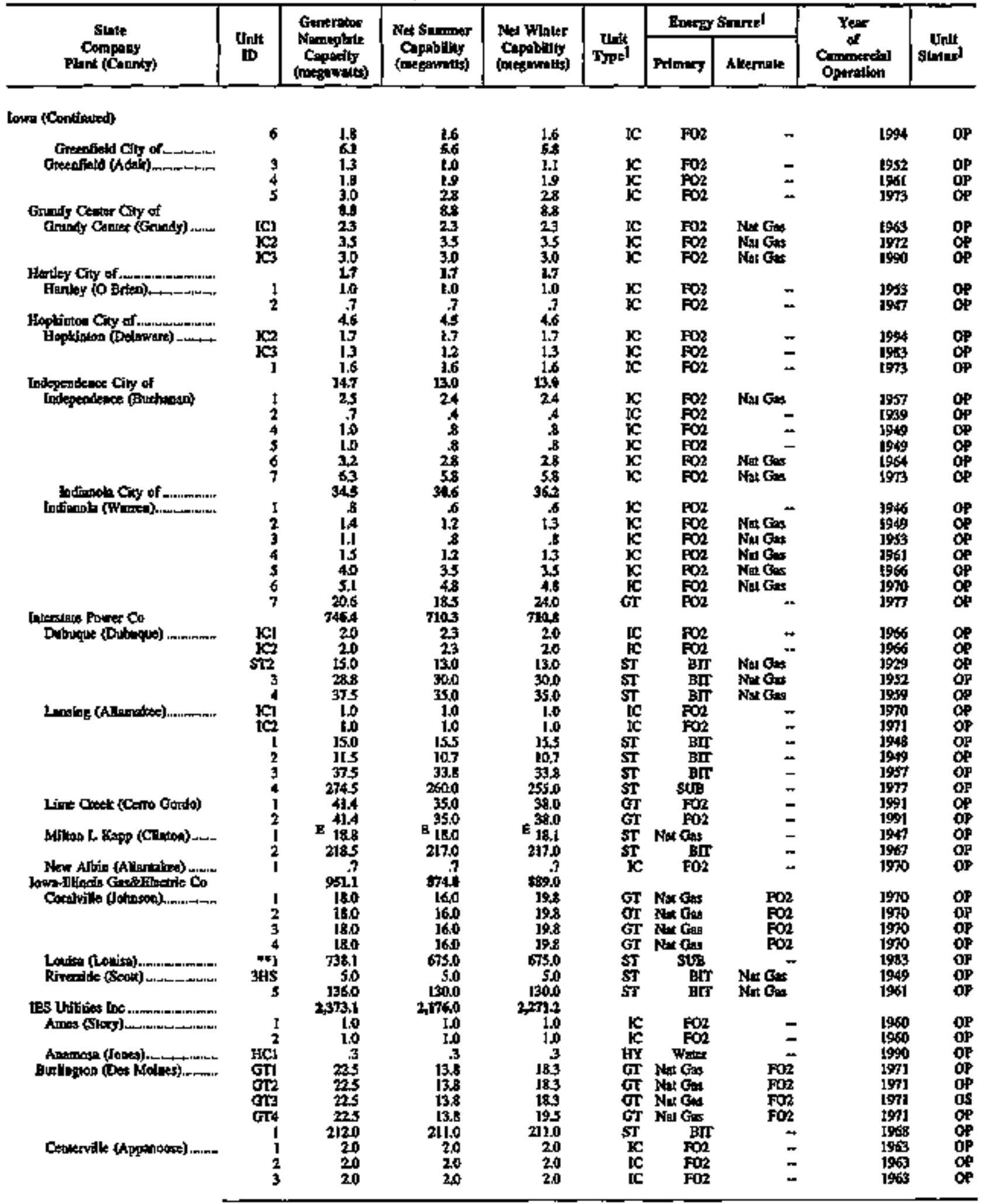

Sectoonous al edd of table 
Table 20. Existing Generating Units at U.S. Electric Utilities by State, Company, and Plant, as of Jamuary 1, 1996 (Continued)

\begin{tabular}{|c|c|c|c|c|c|c|c|c|c|}
\hline \multirow{2}{*}{$\underset{\text { Cimpoint }}{\text { Stitt }}$} & \multirow{2}{*}{ Dinh } & \multirow{2}{*}{ 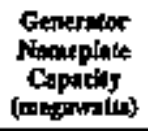 } & \multirow{2}{*}{$\begin{array}{l}\text { Net \$rminer } \\
\text { Capablity } \\
\text { (neperwats) }\end{array}$} & \multirow{2}{*}{ 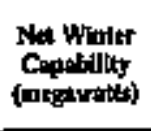 } & \multirow{2}{*}{ Theit } & \multicolumn{2}{|c|}{ Bnerg Sorat } & \multirow{2}{*}{$\begin{array}{c}\text { Yor } \\
\text { of } \\
\text { Combertily } \\
\text { Operation }\end{array}$} & \multirow{2}{*}{ Stint } \\
\hline & & & & & & Prhary & Alliningta & & \\
\hline \multicolumn{10}{|l|}{ fom (Cothmed) } \\
\hline - Deans Anold fling & $m \mathbf{t}$ & 597.2 & 578.0 & 5050 & N日 & Urandan & - & 1975 & OP \\
\hline 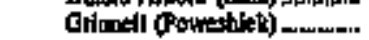 & j & 223 & 243 & 235 & GT & Net Ces & - & 1990 & $O P$ \\
\hline & 2 & ns & 229 & $\overline{372}$ & GT & Nat $0=$ & $=$ & j991 & OP \\
\hline Towa Fals (buedla) & j & 5 & 5 & 5 & 荘 & Water & - & 1926 & OP \\
\hline 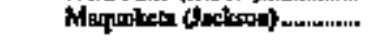 & 1 & .6 & $\frac{6}{6}$ & .6 & $\begin{array}{l}\text { HY } \\
\text { HY }\end{array}$ & $\begin{array}{l}\text { Wata } \\
\text { watax }\end{array}$ & $\ddot{+}$ & 1924 & op \\
\hline Mathatlotin Gimabdl)...n. & Ic| & 25 & 20 & 20 & IC & $\mathbf{F 0 2}$ & - & 1941 & op \\
\hline & $I C$ & 25 & 15 & 19 & IC & $\mathrm{FO}_{2}$ & - & 1942 & OP \\
\hline & 1 & 8.4 & 50,0 & 703 & aT & $\mathrm{FO} 2$ & $=$ & 1979 & OP \\
\hline & $\frac{2}{3}$ & 67.4 & $\begin{array}{l}3048 \\
504\end{array}$ & 703 & GT & Fod & $\bar{z}$ & $\begin{array}{l}1986 \\
1978\end{array}$ & OP \\
\hline Oynnm (Whalla) & $\cdots$ & 2260 & 74,0 & 7140 & sT & sivg & $\mathrm{Nat}-$ & I981 & $\mathrm{OP}$ \\
\hline Privite Cresk (umo). & $\mathbf{i}$ & $\begin{array}{l}23.0 \\
230\end{array}$ & 200 & $\frac{220}{220}$ & $\begin{array}{l}\mathbf{S T} \\
\mathbf{S T}\end{array}$ & $\begin{array}{l}\text { BIT } \\
\text { BIF }\end{array}$ & $\begin{array}{l}N * G_{n} \\
N_{n}\end{array}$ & $\begin{array}{l}1950 \\
1951\end{array}$ & $\underset{0 P}{05}$ \\
\hline & 3 & 50,0 & 490 & 495 & ST & BIt & Nat Gas & 1958 & of \\
\hline & 4 & [ 4838 & 142, & 1420 & ST & BIT & Nox & 1967 & op \\
\hline 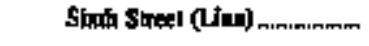 & 1 & 10.0 & 3,0 & 60 & sT & Bत & Refiese & 1921 & $\rho p$ \\
\hline & 2 & 60 & 3,0 & 60 & sT & bit & Refole & 1930 & op \\
\hline & 4 & 150 & 18.0 & $\begin{array}{r}17.0 \\
\mathbf{3 0}\end{array}$ & ST & ATr & Refose & 1942 & OP \\
\hline & $\frac{6}{7}$ & 150 & 180 & $\begin{array}{l}300 \\
170\end{array}$ & ST & $\begin{array}{l}\text { 最 } \\
\text { BIT }\end{array}$ & Refinse & $\begin{array}{l}1925 \\
19455\end{array}$ & DP \\
\hline & a & 288 & 30.0 & 270 & sT & Bit & Refilse & 1950 & âp \\
\hline Swtherland (Manhalli)...--- & 1 & 375 & 31.0 & 320 & 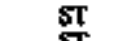 & BT: & $\mathrm{N}=\mathrm{G}$ 的 & ISSS & QP \\
\hline & $\begin{array}{l}2 \\
3\end{array}$ & $\begin{array}{l}37.5 \\
61.6\end{array}$ & $\begin{array}{l}31.0 \\
B 000\end{array}$ & $\begin{array}{l}320 \\
815\end{array}$ & $\begin{array}{l}\text { ST } \\
\text { ST }\end{array}$ & Bir & $\begin{array}{l}\text { Not Gex } \\
\text { Nan Gex }\end{array}$ & $\begin{array}{l}1953 \\
1961\end{array}$ & $\begin{array}{l}\text { GP } \\
\text { OP }\end{array}$ \\
\hline Binbatton Chy of & & 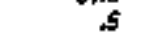 & 4 & A & & & & & 政 \\
\hline \multirow{5}{*}{ 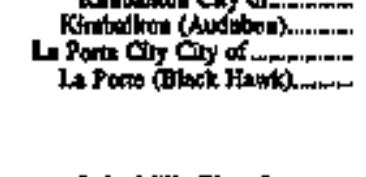 } & $\mathbf{5}$ & 5 & 4) & 4 & $\mathbf{K}$ & $\mathbf{F O 2}$ & $=$ & I970 & $\mathbf{O P}$ \\
\hline & 2 & 1.J & 1.1 & i.t & Ic & F02 & NNot & 1963 & op \\
\hline & 3 & .3 & 3 & 3 & Ic & $\mathrm{FO2}$ & + & [940 & OP \\
\hline & 4 & 6 & .6 & 6 & IC & FO2 & $=$ & 1950 & OP \\
\hline & $\mathbf{s}$ & 11.6 & 114 & 11.4 & & $\mathbf{F O 2}$ & Wat & 1956 & OP \\
\hline \multirow{5}{*}{ 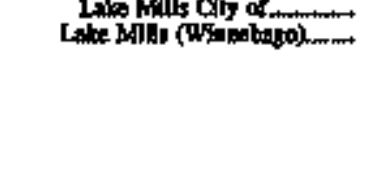 } & I & 2 & ב & 3 & $\mathbf{K}$ & FO2 & + & I931 & DP \\
\hline & $i$ & 3 & 3 & 3 & IC & Fol & - & 1937 & OP \\
\hline & 3 & 9 & .3 & 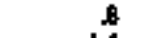 & ic & $\mathbf{F O 2}$ & Nat $O x$ & 1956 & of \\
\hline & 4 & 1.4 & 1,4 & 1.4 & IC. & $\mathrm{Fo2}$ & Nuto & 1962 & OP \\
\hline & 5 & 3.0 & 3,0 & 3.0 & ic & Fo2 & Neat Gex & 1969 & DP \\
\hline \multirow{3}{*}{ 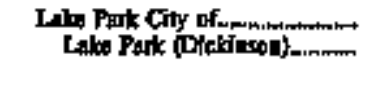 } & & 5.8 & 5.8 & 58 & $\mathrm{IC}$ & $\mathrm{FO} 2$ & - & 1979 & \\
\hline & I & $\log _{7}$ & 5 & 3 & $\boldsymbol{x}$ & $\mathbf{F O Z}$ & + & $\operatorname{tg} 0$ & \\
\hline & $i$ & 10 & $x$ & 8 & [c & Fo2 & $=$ & 1058 & OP \\
\hline \multirow{6}{*}{ 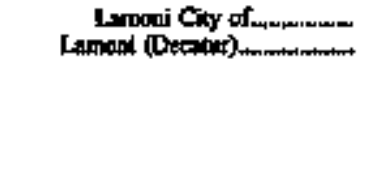 } & & 5.7 & 5.3 & $5 \mathbf{5}$ & & & & & \\
\hline & I & 28 & 28 & 28 & Ic & $\mathrm{FOC2}$ & Lat $6 x$ & 1973 & CP \\
\hline & $i$ & .2 & 2 & $\frac{2}{7}$ & $\mathbf{c}$ & FG2 & & $19+0$ & OP \\
\hline & 3 & $\stackrel{3}{7}$ & $\frac{2}{6}$ & $\frac{2}{6}$ & ch & $\mathrm{FO2}$ & $\bar{z}$ & I941 & OP \\
\hline & 5 & 1.2 & .1 .1 & 1.1 & IC & $\mathrm{FOA}$ & Nat $\mathrm{Otan}^{-}$ & 1995 & oq \\
\hline & 6 & 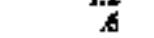 & 6 & .6 & IC & FO2 & Sher & L9s & op \\
\hline Lalneas Cly of & & 1.6 & 15 & 15 & & & & & \\
\hline 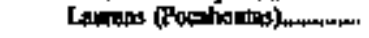 & 3 & 8 & 8 & 8 & $\mathbf{x}$ & FOO & - & 1952 & OP \\
\hline Leagr Ciry of & 4 & 2.8 & $\underset{23}{83}$ & $\underset{23}{83}$ & & $F O 2$ & 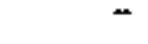 & 1951 & $O P$ \\
\hline Lepor (Toylon) & $\boldsymbol{l}$ & 3 & 3 & 3 & $\mathbf{K}$ & FO2 & $\leftrightarrow$ & 1945 & op \\
\hline & 2 & I.I & I. & l. & $\mathbf{c}$ & Fon & - & 1965 & OP \\
\hline & $\mathbf{3}$ & 9 & 9 & 9 & If & $\mathrm{FO2}$ & - & 1966 & $\mathbf{O P}$ \\
\hline Mesilyta Towp of & & & 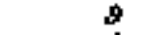 & 1.1 & & & & & \\
\hline 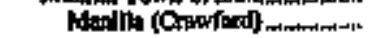 & COI & 3 & 4 & 3 & 1c & $\mathrm{FO2}$ & - & 199 & op \\
\hline & ICO & .6 & 3 & .6 & $\mathbf{R}$ & $\mathbf{F O}$ & 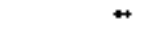 & 1965 & OP \\
\hline 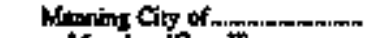 & & & 1.1 & $\mathbf{1 1}$ & & & & & \\
\hline Manning $(C+n d t)$ & $\boldsymbol{I}$ & 5 & 3 & 3 & IC & EO6 & $=$ & t925: & os \\
\hline & 2 & .3 & 3 & 3 & 㹂 & Fos & $\overline{-}$ & 1928 & $\phi s$ \\
\hline Mologited Cuy of & & 16.6 & $\mathbf{t 5 0}$ & 15.4 & & & & & \\
\hline 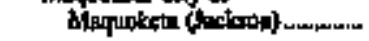 & 1 & 1.4 & 1.0 & 10 & & Nat Gas & FOI & 1947 & $O P$ \\
\hline & 2 &. & 5 & 5 & ic & $\therefore 502$ & - & 1998 & OP \\
\hline & $\begin{array}{l}3 \\
4\end{array}$ & $\begin{array}{l}2 ! \\
1.6\end{array}$ & 20 & $\begin{array}{l}2.1 \\
12\end{array}$ & $\begin{array}{l}\text { IC } \\
\text { IC }\end{array}$ & Nim $\operatorname{Fas}_{\mathrm{F} 02}$ & PO2 & $\begin{array}{l}19696 \\
1941\end{array}$ & क्p \\
\hline
\end{tabular}

Soe foolmoled at ead of hable 
Table 20. Existing Generating Units at U.S. Electric Utilities by State, Company, and Plant, as of Jamuary 1, 1996 (Contioned)

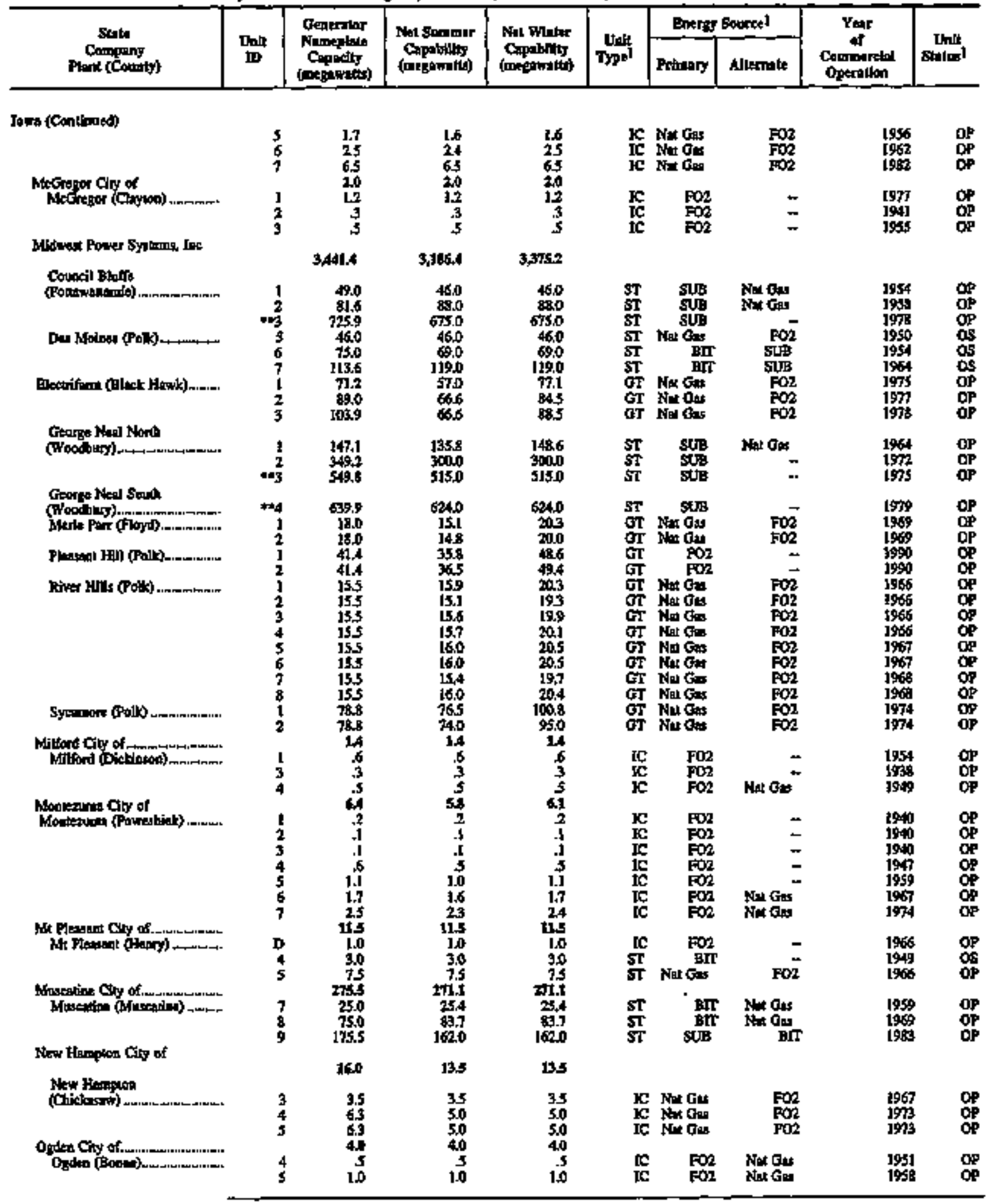

Sow Footholss at apd of lable. 
Table 20. Existing Generating Unlts at U.S. Electric Utilities by State, Company, and Ptant, as of January 1, 1996 (Continued)

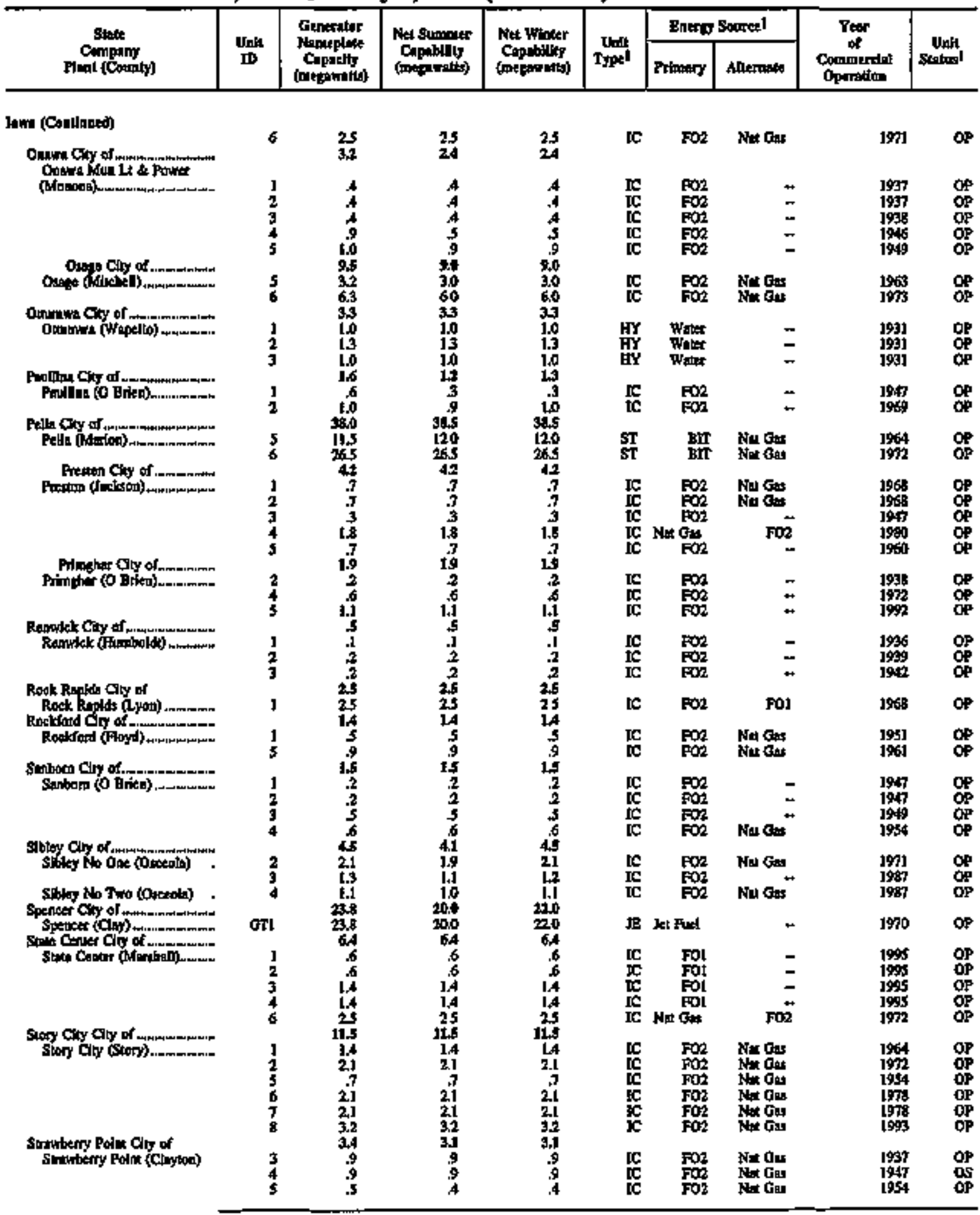

Set foothoute al ead of lobk. 
Table 20. Fxisting Generating Units at U.S. Electric Utilities by State, Company, and Plant, as of Jamuary 1, 1996 (Continued)

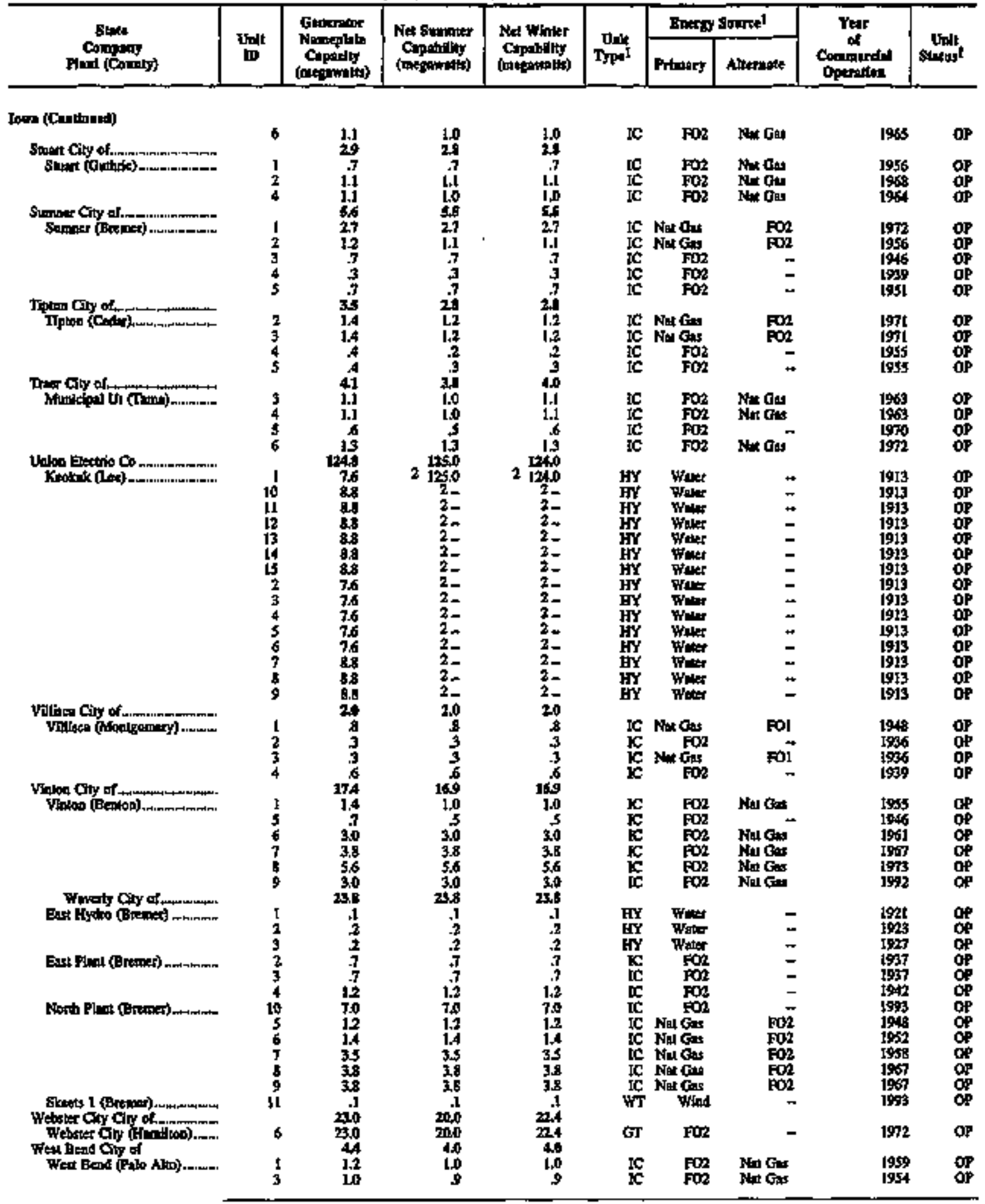

See foocnowa at end of able. 
Table 20. Existlag Generating Units at U.S. Electric Utilities by State, Company, and Plant, as of January 1, 1996 (Contimued)

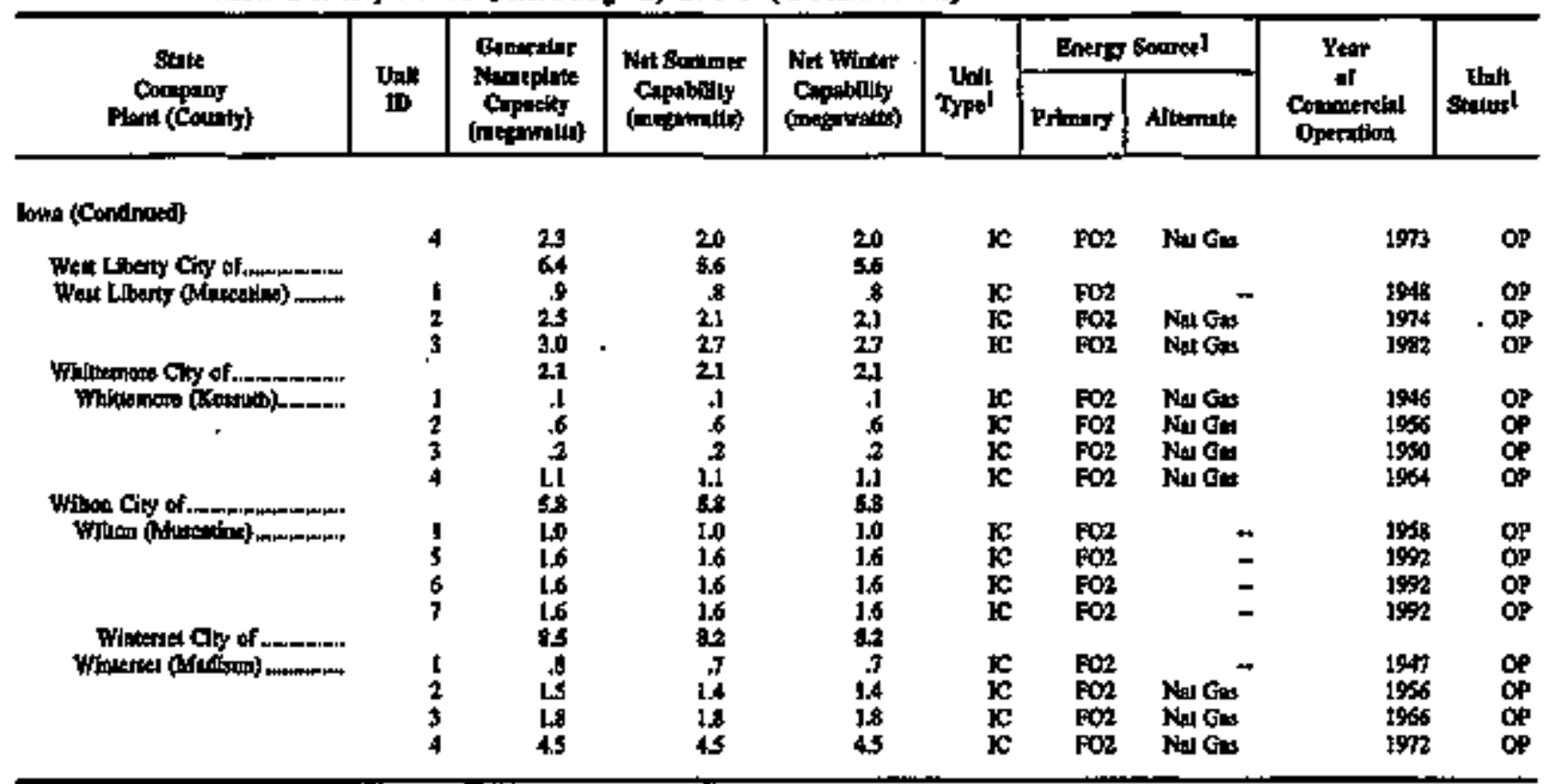

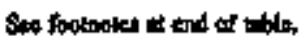


Table 20. Existing Generating Units at U.S. Electric Utilities by State, Company, and Plant, as of January 1, 1996 (Continued)

\begin{tabular}{|c|c|c|c|c|c|c|c|c|c|}
\hline \multirow{2}{*}{$\underset{\text { Comping }}{\text { Stant }}$} & \multirow{2}{*}{$\frac{\text { Dht }}{\text { ID }}$} & \multirow{2}{*}{ 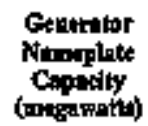 } & \multirow{2}{*}{ 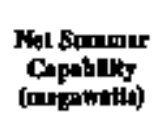 } & \multirow{2}{*}{ 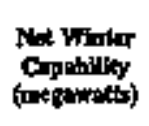 } & \multirow{2}{*}{$\operatorname{thot}_{\text {Type }}^{2}$} & \multicolumn{2}{|c|}{ Buthty Sourt? } & \multirow{2}{*}{$\begin{array}{c}\text { Yart } \\
\text { of } \\
\text { Comintroind } \\
\text { Opterallon }\end{array}$} & \multirow{2}{*}{$\begin{array}{c}\text { Opli } \\
\text { sutetest }\end{array}$} \\
\hline & & & & & & Putmaty & Aturate & & \\
\hline \multicolumn{10}{|l|}{ Kant: } \\
\hline \multirow{3}{*}{ 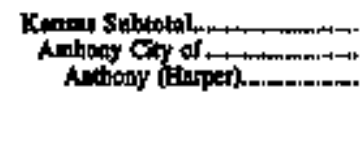 } & \multicolumn{2}{|r|}{$10,475,9$} & \multirow{6}{*}{ 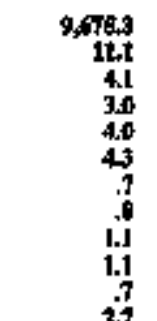 } & \multirow{2}{*}{$9,760,8$} & & \multirow{4}{*}{$\begin{array}{l}\text { Net Ges } \\
\text { Nat Gas } \\
\text { Nar Gst }\end{array}$} & \multirow{4}{*}{$\begin{array}{l}\mathrm{FO} 2 \\
\mathrm{FO}\end{array}$} & \multirow{4}{*}{$\begin{array}{l}1972 \\
1976 \\
1981\end{array}$} & \multirow{4}{*}{$\underset{O P}{O P}$} \\
\hline & & & & & & & & & \\
\hline & [c] & $\begin{array}{l}4.1 \\
3.0 \\
4.0\end{array}$ & & $\begin{array}{l}4.1 \\
30 \\
4,0\end{array}$ & $\begin{array}{l}\text { 致 } \\
\text { IC }\end{array}$ & & & & \\
\hline Astland City of & & $\mathbf{5 0}$ & & 4,4 & & & & & \\
\hline Asilend (Clmb)............m. & $\begin{array}{l}1 \\
2 \\
3 \\
4 \\
5\end{array}$ & $\begin{array}{r}7 \\
13 \\
13 \\
9\end{array}$ & & $\begin{array}{r}.7 \\
8 \\
1.1 \\
1.1 \\
.7\end{array}$ & 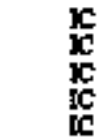 & $\begin{array}{l}\text { Nar Ga: } \\
\text { Nu Ge: } \\
\text { Nou Gas } \\
\text { Nu Ges } \\
\text { Fo? }\end{array}$ & $\begin{array}{l}\mathrm{FO2} \\
\mathrm{FO} \\
\mathrm{FO} 2 \\
\mathrm{FO2}\end{array}$ & $\begin{array}{l}1900 \\
1974 \\
1969 \\
1950 \\
197 \pi\end{array}$ & $\begin{array}{l}\text { OP } \\
\text { OP } \\
\text { OP } \\
O P \\
O P\end{array}$ \\
\hline \multirow{2}{*}{ 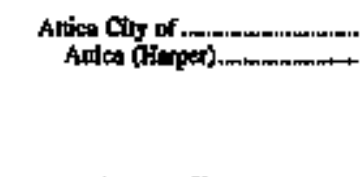 } & & $\mathrm{E}$ & & $\mathrm{E}_{1.0}^{30}$ & & & & & \\
\hline & $\begin{array}{r}165 \\
1 \\
2 \\
4 \\
5\end{array}$ & $\begin{array}{r}E .1 .1 \\
\text { E. } 5 \\
.9 \\
3 \\
.3 \\
23.7\end{array}$ & 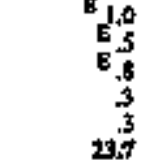 & 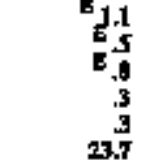 & $\begin{array}{l}\boldsymbol{c} \\
\mathbf{x} \\
\mathbf{x} \\
x \\
\mathbf{x}\end{array}$ & $\begin{array}{l}\mathrm{Faz} \\
\mathrm{FO2} \\
\mathrm{FO2} \\
\mathrm{FO2} \\
\mathrm{FO2}\end{array}$ & 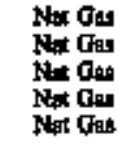 & $\begin{array}{l}1984 \\
1954 \\
1950 \\
1961 \\
1961\end{array}$ & $\begin{array}{l}\text { OP } \\
\text { OP } \\
\text { OP } \\
\text { OP } \\
\text { OP }\end{array}$ \\
\hline 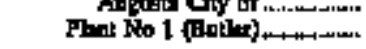 & 1 & 1.1 & 1.1 & $1+1$ & $\boldsymbol{x}$ & & Fon & 1984 & op \\
\hline & $\frac{2}{3}$ & $\stackrel{4}{10}$ & 1.4 & 10 & ic & Nel $\begin{array}{c}F_{02} \\
\text { Oes }\end{array}$ & $\mathrm{FO}^{-}$ & $\begin{array}{l}1915 \\
1949\end{array}$ & OP \\
\hline & 4 & 7 & .3 & .7 & $\mathbf{x}$ & FO2 & 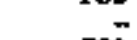 & 1939 & op \\
\hline & 5 & $\begin{array}{l}23 \\
23\end{array}$ & $\begin{array}{l}2.3 \\
2.3\end{array}$ & $\begin{array}{l}2.3 \\
23\end{array}$ & $\underset{x}{x}$ & $\begin{array}{l}\text { Net Gas } \\
\text { Not Get }\end{array}$ & $\begin{array}{l}\mathrm{FO} 2 \\
\mathrm{PO} 2\end{array}$ & $\begin{array}{l}1956 \\
19556\end{array}$ & of \\
\hline & 7 & 20 & 20 & 20 & $\mathbf{c}$ & Nal Gas & Fon & 1964 & op \\
\hline Plan $\mathrm{Xl}_{2} 2$ (Bullox) & $i$ & $\begin{array}{l}40 \\
10\end{array}$ & $\begin{array}{l}40 \\
40\end{array}$ & $\begin{array}{l}40 \\
40\end{array}$ & 究 & Nol Gas & $\mathrm{FOO}_{\mathrm{FO}}$ & $\begin{array}{l}1969 \\
1960\end{array}$ & op \\
\hline & 3 & 60 & 60 & 60 & $\pi$ & Nate & $\mathrm{FO2}$ & 1981 & $O P$ \\
\hline Balawis Giy Cly of & & $\mathbf{L 1}$ & 4.6 & 5.2 & & & & & \\
\hline 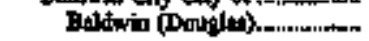 & $\mathbf{J}$ & .6 & $A$ & 4 & $\mathbf{c}$ & $\mathrm{Fot}$ & Nax Gos & 1950 & $g 9$ \\
\hline & 3 & 1.1 & 10 & 1.0 & 突 & $\mathrm{Faz}$ & fing & 1956 & $\frac{\alpha}{\mathrm{p}}$ \\
\hline & 3 & l. & .2 & $\begin{array}{l}1.8 \\
1.0\end{array}$ & $\mathbf{k}$ & Fon & Nat Gis & 1964 & OP \\
\hline & 6 & 1.1 & $\ddot{z}$ & 1.0 & $\mathbf{c}$ & FO2 & Nat Gas & 1904 & \\
\hline Belleville ciny of & & 13.1 & 13.1 & 13.1. & & & & & \\
\hline Beterile (Repubitic)........ & 1 &.$\hat{6}$ & .6 & .6 & $\boldsymbol{x}$ & $\mathbf{F O z}$ & Nax Ois & 1946 & op \\
\hline & 2 & .6 & 3 & 6 & re & Fot & Wh & 1946 & क्中 \\
\hline & 4 & $\overrightarrow{10}$ & 1.0 & $1 \overrightarrow{0}$ & $\mathbf{K}$ & FO2 & Nat Ges & $\begin{array}{l}15909 \\
1955\end{array}$ & OP \\
\hline & $\mathbf{s}$ & 1.8 & 1.8 & 1,8 & $\mathbf{x}$ & Fo? & Now Onis & 1961 & op \\
\hline & 6 & 38 & 3,8 & 3.8 & $\mathbf{x}$ & Fo2 & Netos & 1966 & op \\
\hline Befon $\mathrm{Or}$ & 7 & $\begin{aligned} 5.1 \\
104\end{aligned}$ & 5.1 & 5.1 & $\boldsymbol{c}$ & & & 157 & \\
\hline 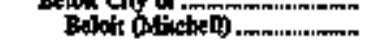 & $\mathbf{J}$ & 1.5 & 10 & 1.0 & ce & Fo: & Net Gn & & \\
\hline & 2 & 15 & 1.0 & 1.0 & $\mathbf{k}$ & FQ2 & Nat Gas & t995: & op \\
\hline & 3 & 20 & 20 & 20 & $\mathbf{c}$ & $\mathrm{FQ}$ & Nat Ga & t96t & OP \\
\hline & $\frac{4}{5}$ & 9 & $\frac{3}{.7}$ & $\frac{33}{7}$ & je & $\mathrm{FO} 2$ & Non Giat & $\begin{array}{l}994 \\
1990\end{array}$ & dp \\
\hline & 6 & 4.1 & 38 & $\ddot{3.8}$ & IL & $\mathbf{F}$ & Nel $\mathrm{Oas}$ & 1971 & oe \\
\hline & 7 & 60 & 60 & 6.0 & II & $\mathbf{F O 2}$ & Nar Ges & 190 & 9 \\
\hline Earillpgane Chy of & & & 4.3 & 4t & & & & & \\
\hline 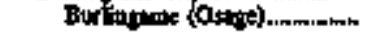 & 1 & 1.1 & $i, 4$ & I.t & KC & Fon & Nar Gat & Ims & op \\
\hline & $\frac{2}{3}$ & 8 & $\stackrel{4}{8}$ & $\vec{g}$ & Ic & $\mathrm{FO2}$ & Nat Ga & 1991 & $\frac{\alpha}{\alpha p}$ \\
\hline & 4 & l.t. & 1.1 & $\vec{j}$ & II & $\mathbf{F} \mathbf{w}_{2}$ & Nu G & 1969 & op \\
\hline & 5 & 9 & 8 & 9 & $\overline{\mathrm{JC}}$ & 102 & Nu Gas & 1900 & 8 \\
\hline Buctuptom $\left(C_{0}\left(f_{y}\right)\right.$........ & IC6 & 48 & $\begin{array}{l}84 \\
4.8\end{array}$ & 8,8 & Ic. & Net Cer & Fos & 1093 & \\
\hline & 1 & 3 & 3 & 3 & IC & Fot & & 1935 & $O P$ \\
\hline & 2 & 13 & 1.3 & 1.3 & ic & Nin G*t & Fo2 & 1962 & op \\
\hline & 3 & 8 & 8 & .8 & ic & Nat Ge & Fo2 & 1954 & $o p$ \\
\hline & 5 & \pm .0 & 10 & $\stackrel{3}{1.0}$ & IC & Nat Gas & FÖ & $\begin{array}{l}1945 \\
1955\end{array}$ & op \\
\hline Chanoke Ofty of & & $\sin 6$ & 51.5 & $\mathbf{0 . 1}$ & & & & & \\
\hline Gutus i (kotho) & 1 & 40 & 40 & 4.2 & $\mathbf{s T}$ & Nat Gat & For & 1949 & op \\
\hline & 5 & $\begin{array}{r}1.7 \\
10,0\end{array}$ & $\frac{15}{98}$ & $\begin{array}{r}\mathbf{1 . 3} \\
\mathbf{1 0 . 0}\end{array}$ & $\begin{array}{l}\text { IC } \\
\mathbf{E T}\end{array}$ & $\begin{array}{l}\text { Nat Gs } \\
\text { Nat Gut }\end{array}$ & $\begin{array}{l}\text { FO2 } \\
\text { Fos }\end{array}$ & $\begin{array}{l}1955 \\
1957\end{array}$ & op \\
\hline
\end{tabular}

Sies focturtes in end of uble. 
Table 20. Existing Generating Units at U.S. Electric Utulities by State, Company, and Plant, as of January 1, 1996 (Continued)

\begin{tabular}{|c|c|c|c|c|c|c|c|c|c|}
\hline \multirow{2}{*}{ 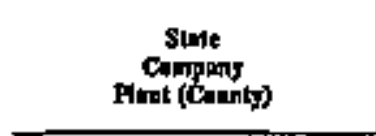 } & \multirow{2}{*}{ Dit } & \multirow{2}{*}{ 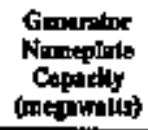 } & \multirow{2}{*}{ 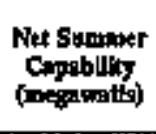 } & \multirow{2}{*}{ 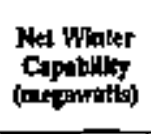 } & \multirow{2}{*}{ Type } & \multicolumn{2}{|c|}{ Energ Sompe? } & \multirow{2}{*}{$\begin{array}{c}\text { Year } \\
\text { of } \\
\text { Conmertiol } \\
\text { Operation }\end{array}$} & \multirow{2}{*}{ Stothos } \\
\hline & & & & & & Primary & Altermate & & \\
\hline \multicolumn{10}{|l|}{ Kuntas (Condinued) } \\
\hline Cherese 2 (Neosita). & 7 & 20 & 20 & 20 & EC & Nat Gy & FO2 & 1965 & $O P$ \\
\hline & $j$ & 20 & 20 & 20 & IC & The & $\mathrm{FO2}$ & 1960 & op \\
\hline 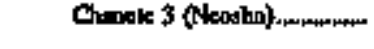 & Fit & 70 & 69 & 6.9 & IC & $\mathrm{FOC}$ & Nat Gas & 1986 & $\phi_{P}$ \\
\hline & 11 & 60 & 5.9 & 5 & 党 & $\begin{array}{l}\mathrm{PO2} \\
\mathrm{FO}\end{array}$ & Nax Cos & $19 \%$ & OP \\
\hline & 13 & 60 & 60 & 60 & IC & Fon & & 1991 & $\mathrm{OP}$ \\
\hline & 4 & 7.0 & 69 & 69 & ic & $F 02$ & Nax Oas & 198 & of \\
\hline \multirow{6}{*}{ 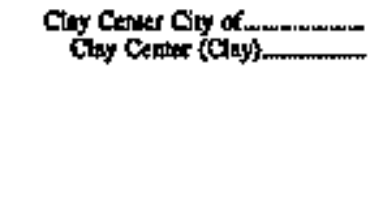 } & & 17.6 & 17.5 & 17.5 & & & & & \\
\hline & ICI & 9 & 9 & 5 & IC & Nat Gas & FO2 & 195 & OP \\
\hline & IC & 2.1 & $\begin{array}{l}2.1 \\
50\end{array}$ & 2.1 & IC & Ner Oes & $\mathrm{FOz}$ & 1966 & op \\
\hline & 4 & 1.5 & 1.5 & 1.5 & sT & Ner Gas & Fos & $19 / 2$ & op \\
\hline & 5 & 30 & 3.0 & 3.0 & $s \mathbf{s}$ & Nar car & FOS & 194 & op \\
\hline & 6 & 50 & $\$ 0$ & s.to & $\$ \mathbf{T}$ & Nat Gas & Fos & I961 & OP \\
\hline \multirow{2}{*}{ 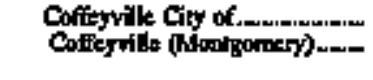 } & & 5B.S & 59.5 & 50,5 & & & & & \\
\hline & $\frac{6}{7}$ & $\begin{array}{l}18.5 \\
40,0\end{array}$ & $\begin{array}{l}17.5 \\
30.0\end{array}$ & $\begin{array}{l}18,5 \\
40,0\end{array}$ & $\begin{array}{l}\mathbf{S T} \\
\mathbf{S T}\end{array}$ & $\begin{array}{l}\text { Ner Oay } \\
\text { Net Gas }\end{array}$ & I & $\begin{array}{l}1956 \\
1973\end{array}$ & ${ }_{O P}$ \\
\hline \multirow{6}{*}{ 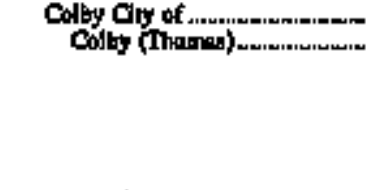 } & & $17 A$ & 136 & 13.6 & & & & & \\
\hline & 3 & 25 & 3.8 & 1.6 & IC & For & $\mathrm{N} \in \mathrm{Gm}$ & 196 & $O P$ \\
\hline & 4 & 1.8 & 13 & 10 & [C & $\mathrm{FO2}$ & Nat Gas & 1958 & $O P$ \\
\hline & $\frac{5}{6}$ & 1.4 & $\begin{array}{l}100 \\
3.5\end{array}$ & 3.5 & IC & $\begin{array}{l}\text { fue } \\
\text { futs }\end{array}$ & Noth & $\begin{array}{l}1958 \\
197 \mathrm{~L}\end{array}$ & of \\
\hline & 7 & 45 & $\mathbf{3}, 5$ & 3.5 & [C & Fos & $\mathrm{Na}$ Ga & 1971 & $O P$ \\
\hline & 8 & $\begin{array}{l}2.8 \\
8.5\end{array}$ & 25 & 25 & IC & Fó & Nate & ighi & of \\
\hline \multirow{3}{*}{ Ellinirood City of } & I & 2.1 & III & 19 & $\mathbf{K C}$ & 502 & $\operatorname{Stat} \sigma_{m}$ & I965 & op \\
\hline & 3 & 1.4 & 13 & 13 & $\mathrm{k}$ & $\mathrm{FO} 2$ & Nat ons & 1957 & OP \\
\hline & 3 & .6 & 5 & 5 & c & $\mathrm{FOZ}^{2}$ & 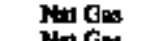 & 1948 & 㻃 \\
\hline \multirow{3}{*}{ 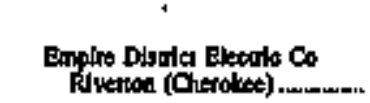 } & 5 & 1.3 & $\mathbf{1 0}$ & $\begin{array}{l}1.0 \\
3.0\end{array}$ & C & $\begin{array}{l}\mathrm{FO} 2 \\
\mathrm{FO} 2\end{array}$ & For Ges & 1968 & DP \\
\hline & & 132.6 & 1375 & 1575 & N & & & $1 \times / 1$ & DP \\
\hline & 10 & 16.3 & 165 & 163 & Or & Non Out & For & L9B8 & op \\
\hline & II & 163 & 165 & 165 & GI & Nut Gar & $\mathbf{F O}$ & 1960 & op \\
\hline & $?$ & 37.5 & 389 & 38.0 & $\boldsymbol{s T}$ & stop & 㗐 & 1950 & op \\
\hline & $\begin{array}{l}3 \\
0 \\
0\end{array}$ & 30.0 & 340 & 940 & $\mathbf{S T}$ & SUR & 照 & 1954 & 郘 \\
\hline 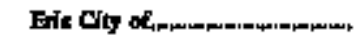 & & 4.8 & 4.4 & 4.4 & $\mathbf{G I}$ & Al wit & & 19604 & OP \\
\hline 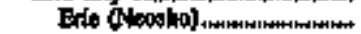 & I & & & .6 & sc & FO2 & 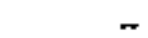 & 195 & OP \\
\hline & $\mathbf{3}$ & 13 & 1.0 & L0 & $\mathbf{k}$ & $\mathrm{EOS}$ & - & 1958 & op \\
\hline & 4 & 15 & 15 & 15 & $\mathbf{c}$ & $\mathrm{FO2}$ & - & 1964 & op \\
\hline & $\frac{3}{x}$ & 1.0 & 1.0 & 1.0 & $\mathbf{K}$ & 502 & $\rightarrow$ & 1992 & OP \\
\hline Fitdonia Cirs of ............ & 0 & 74 & 7,0 & $\begin{array}{r}3 \\
7.0\end{array}$ & IC & FOI & - & 1992 & OP \\
\hline Frodente (WWilron) & cs & 9 & 9 & 9 & $\mathbf{K C}$ & FO2 & Nat Gon & $19 n 8$ & OP \\
\hline & $10 x$ & 3 & 9 & 9 & FC & $\mathrm{FO2}$ & Nil Gos & 1978 & OP \\
\hline & +6 & 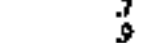 & $\frac{7}{9}$ & .7 & IC & 502 & Nol Ger & 1978 & of \\
\hline & 10 & 9 & 8 & $\hat{8}$ & te & $\mathrm{FO}$ & Nar Ges & $19 \%$ & op \\
\hline & 1 & 9 & 8 & 8 & IC & FO2 & Nat Gess & 1948 & op \\
\hline & 2 & 13 & 13 & 1.3 & IC & $\mathrm{FO}$ & Nar Gat & 1953 & OP \\
\hline & 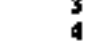 & $\stackrel{4}{6}$ & 3 & $\sqrt[3]{5}$ & $\underset{\text { fic }}{\text { IC }}$ & $\mathrm{FO2}$ & $\begin{array}{l}\text { Nat Gas } \\
\text { Nat Git }\end{array}$ & $\begin{array}{l}1927 \\
1991\end{array}$ & $\stackrel{O P}{O P}$ \\
\hline Ganderer Gity of & & 392 & 31.0 & 31.0 & & & & & \\
\hline Gendbar (Darnsoon) & $\mathrm{Crl}$ & 19.5 & 15.0 & 150 & $\mathbf{G r}$ & $\mathrm{FO} 2$ & Nar $\mathrm{Oar}$ & 1990 & op \\
\hline Grnet Gny of. & $\cos$ & $\begin{array}{r}19,6 \\
9,3\end{array}$ & $\begin{array}{r}16.0 \\
84\end{array}$ & $\begin{array}{r}16,0 \\
8,4\end{array}$ & GT & FO2 & Not Ger & 1990 & of \\
\hline Gemerl bhunispal & & & & & & & & & \\
\hline (Andenton) & $\mathbf{K S}$ & 24 & 22 & 22 & k & Nor Gus & $\mathbf{F O}$ & 1951 & op \\
\hline & IOS & 25 & 23 & 23 & Ic & $\mathrm{F02}$ & 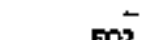 & 1976 & OP \\
\hline & I & 4 & 1.4 & 1.4 & IC & Nipt Gas & 102 & $\begin{array}{l}1961 \\
199010\end{array}$ & OP \\
\hline & 3 & 5 & $\hat{\mathrm{IA}}$ & 1.4 & Ic & Nort $\mathrm{Cos}$ & $\mathrm{FO}_{2}$ & 1955 & OP \\
\hline & & 50 & .9 & 9 & IC & Npp Gas & $\mathrm{EO}$ & J94: & OP \\
\hline Grtatd Cry of & & 3.7 & 29 & $\mathbf{3 . 3}$ & & & & & \\
\hline Gined (Crapulond) & 1 & i.4 & 1.J & 1.3 & IC & Not Gat & $\mathbf{F O} 2$ & 1955 & as \\
\hline & 1 & 23 & 18 & 20 & IC & Nar Ges & Fo2 2 & $1 \% 2$ & os \\
\hline Coodland Giry of & & 189 & 169 & 164. & & & & & \\
\hline Goodlend (Stwnen) & 30 & 2.1 & 1.8 & 21 & IC & Nar Gat & FO2 & 1971 & OP \\
\hline
\end{tabular}

See foonalut at end of table. 
Table 20. Existing Generating Units at U.S. Electric Utillties by State, Company, and Plant, as of January 1, 1996 (Continued)

\begin{tabular}{|c|c|c|c|c|c|c|c|c|c|}
\hline \multirow{2}{*}{$\begin{array}{c}\text { Seato } \\
\text { Conpany } \\
\text { Flent (Courty) }\end{array}$} & \multirow{2}{*}{ thit } & \multirow{2}{*}{ 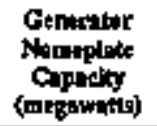 } & \multirow{2}{*}{ 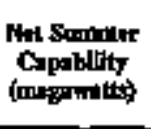 } & \multirow{2}{*}{$\begin{array}{l}\text { Net Winter } \\
\text { Copiniliky } \\
\text { (oneganatis) }\end{array}$} & \multirow{2}{*}{ This } & \multicolumn{2}{|c|}{ Brergy Sourwa2 } & \multirow{2}{*}{$\begin{array}{c}\text { Yoar } \\
\text { of } \\
\text { Commeredel } \\
\text { Operalles }\end{array}$} & \multirow{2}{*}{ that } \\
\hline & & & & & & Printiry & Alemente & & \\
\hline \multicolumn{10}{|l|}{ 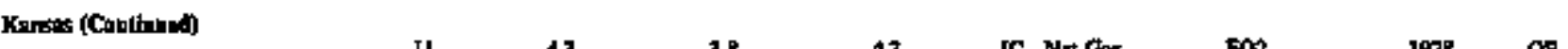 } \\
\hline Ginenaburg Ciry of & $\begin{array}{r}\text { Il } \\
12 \\
3 \\
5 \\
6 \\
7 \\
8\end{array}$ & $\begin{array}{l}1.3 \\
1.3 \\
18 \\
1.3 \\
2.3 \\
2.3 \\
3.0 \\
3.8\end{array}$ & $\begin{array}{r}3.8 \\
9 \\
.5 \\
.9 \\
2.0 \\
20 \\
4.8 \\
3.4\end{array}$ & $\begin{array}{l}4.3 \\
1.0 \\
1.0 \\
2.3 \\
23 \\
5.0 \\
7.4\end{array}$ & $\begin{array}{l}\text { IC } \\
\text { IC } \\
\text { IC } \\
\text { IC } \\
\text { IC } \\
\text { IC } \\
\text { IC }\end{array}$ & 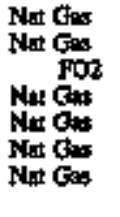 & $\begin{array}{l}\mathrm{FO}_{2} \\
\mathrm{FO2} \\
-2 \\
\mathrm{FO} 2 \\
\mathrm{FO}_{2} \\
\mathrm{FO2} \\
\mathrm{FO} 2\end{array}$ & $\begin{array}{l}1978 \\
1995 \\
1995 \\
1990 \\
1962 \\
1965 \\
1975\end{array}$ & $\begin{array}{l}\phi p \\
o p \\
o p \\
o p \\
o p \\
o p \\
O P\end{array}$ \\
\hline 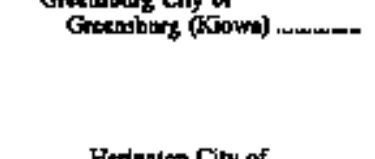 & $\begin{array}{l}1 \\
3 \\
4 \\
5 \\
6\end{array}$ & $\begin{array}{l}2.1 \\
1.1 \\
1.1 \\
2.1 \\
1.4\end{array}$ & $\begin{array}{l}20 \\
1.3 \\
1.1 \\
1.9 \\
1.3\end{array}$ & $\begin{array}{l}2.0 \\
1.1 \\
1.1 \\
1.9 \\
1.7\end{array}$ & $\begin{array}{l}\text { IC } \\
\text { IC } \\
\text { IC } \\
\text { IC } \\
\text { IC }\end{array}$ & $\begin{array}{l}\text { Nal Gus } \\
\text { Nal Ges } \\
\text { Nol Gas } \\
\text { Nor Ges } \\
\text { Nat das }\end{array}$ & $\begin{array}{l}\mathrm{FO2} \\
\mathrm{FO2} \\
\mathrm{FO2} \\
\mathrm{FO2} \\
\mathrm{FO} 2\end{array}$ & $\begin{array}{l}1965 \\
1969 \\
1956 \\
1972 \\
1983\end{array}$ & $\begin{array}{l}O P \\
O P \\
O P \\
O P \\
O P\end{array}$ \\
\hline 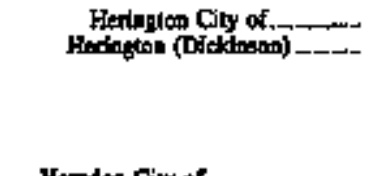 & $\begin{array}{l}1 \\
2 \\
3 \\
4 \\
5\end{array}$ & $\begin{array}{l}9.7 \\
2.1 \\
1.4 \\
4.3 \\
.8 \\
1.1\end{array}$ & $\begin{array}{l}7.0 \\
1.6 \\
1.0 \\
3.1 \\
.3 \\
1.0\end{array}$ & $\begin{array}{l}7.7 \\
1.6 \\
1.1 \\
3.5 \\
3 \\
1.0\end{array}$ & $\begin{array}{l}x \\
x \\
x \\
x \\
x \\
x\end{array}$ & $\begin{array}{l}\text { Nus Gos } \\
\text { Nus Gs } \\
\text { Na Ges } \\
\text { FO2 } \\
\text { Nat Gou }\end{array}$ & $\begin{array}{l}\mathrm{FO} \\
\mathrm{FO} 2 \\
\mathrm{FO} 2 \\
\mathrm{FO}\end{array}$ & $\begin{array}{l}1968 \\
1962 \\
1973 \\
1947 \\
1951\end{array}$ & $\begin{array}{l}\text { OP } \\
\text { OP } \\
O P \\
\text { sp } \\
\text { OP }\end{array}$ \\
\hline 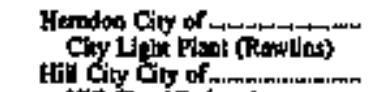 & I & $\begin{array}{r}3 \\
3 \\
73\end{array}$ & 6 & $\frac{3}{65}$ & c & For & - & 1950 & OP \\
\hline 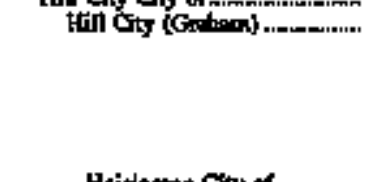 & $\begin{array}{l}1 \\
2 \\
3 \\
4 \\
5 \\
6\end{array}$ & $\begin{array}{r}1.4 \\
1.4 \\
.7 \\
1.4 \\
1.4 \\
1.4\end{array}$ & $\begin{array}{l}1.2 \\
1.2 \\
1.5 \\
1.3 \\
1.3\end{array}$ & $\begin{array}{r}1.2 \\
1.2 \\
6 \\
1.0 \\
13 \\
13\end{array}$ & $\begin{array}{l}n \\
1 c \\
1 c \\
k \\
k \\
c\end{array}$ & 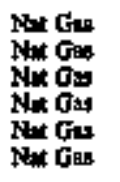 & $\begin{array}{l}\mathrm{FO2} \\
\mathrm{PO2} \\
\mathrm{FOZ} \\
\mathrm{FOOZ} \\
\mathrm{FO2} \\
\mathrm{FO2}\end{array}$ & $\begin{array}{l}1962 \\
1962 \\
1952 \\
1967 \\
1934 \\
1974\end{array}$ & $\begin{array}{l}O P \\
O P \\
O P \\
O P \\
\text { OP } \\
\text { OP }\end{array}$ \\
\hline 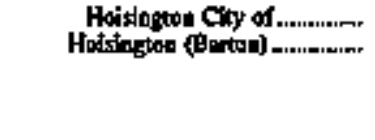 & $\begin{array}{l}1 \\
6 \\
7 \\
8\end{array}$ & $\begin{array}{r}13.2 \\
.2 \\
2.0 \\
4.0 \\
7.0\end{array}$ & $\begin{array}{r}13.2 \\
2.2 \\
4.0 \\
7.0\end{array}$ & $\begin{array}{r}13.2 \\
2 \\
20 \\
4.0 \\
70\end{array}$ & $\begin{array}{l}\mathrm{k} \\
\mathrm{k} \\
\mathrm{k}\end{array}$ & 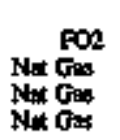 & $\underset{\mathrm{Fon}}{\mathrm{Fon}}$ & $\begin{array}{l}1940 \\
1961 \\
1966 \\
1961\end{array}$ & $\begin{array}{l}o p \\
\text { oP } \\
\text { OP } \\
\text { OP }\end{array}$ \\
\hline $\begin{array}{l}\text { Holton Ciky of } \\
\text { Fiotion (Jectson). }\end{array}$ & $\begin{array}{r}10 \\
11 \\
5 \\
6 \\
7 \\
8 \\
9\end{array}$ & $\begin{array}{l}163 \\
20 \\
2.5 \\
.9 \\
1.8 \\
2.8 \\
4.3 \\
20\end{array}$ & $\begin{array}{r}14.5 \\
1.8 \\
2.3 \\
.7 \\
1.4 \\
2.4 \\
3.9 \\
1.8\end{array}$ & $\begin{array}{r}157 \\
20 \\
24 \\
9 \\
18 \\
27 \\
40 \\
20\end{array}$ & $\begin{array}{l}\text { EC } \\
\text { KC } \\
\text { Kc } \\
\text { 1C } \\
\text { IC } \\
\text { IC } \\
\text { IC }\end{array}$ & 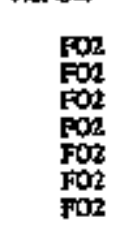 & 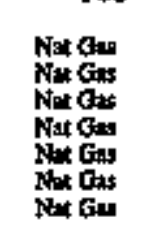 & $\begin{array}{l}1978 \\
1994 \\
1951 \\
1958 \\
1969 \\
1969 \\
1998\end{array}$ & $\begin{array}{l}\not P \\
O P \\
O P \\
o p \\
o p \\
o P \\
O P\end{array}$ \\
\hline 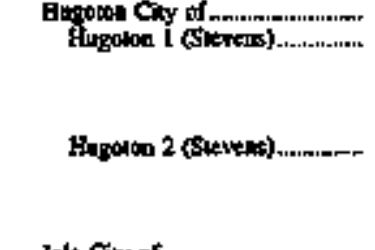 & $\begin{array}{r}1 \\
2 \\
4 \\
6 \\
10 \\
7 \\
8 \\
94\end{array}$ & $\begin{array}{r}15.4 \\
.8 \\
2 \\
4 \\
1.4 \\
43 \\
23 \\
21 \\
43\end{array}$ & $\begin{array}{r}14.1 \\
.5 \\
.1 \\
.4 \\
1.2 \\
4.0 \\
2.1 \\
1.5 \\
4.0\end{array}$ & $\begin{array}{r}14.1 \\
.6 \\
.1 \\
1.2 \\
4.0 \\
2.1 \\
1.5 \\
4.0\end{array}$ & $\begin{array}{l}\text { IC } \\
\text { IC } \\
\text { IC } \\
\text { IC } \\
\text { IC } \\
\text { IC } \\
\text { IC } \\
\text { IC }\end{array}$ & 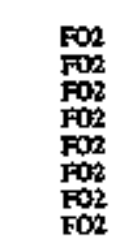 & 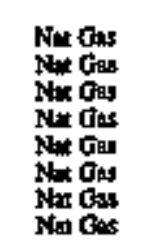 & $\begin{array}{l}1949 \\
1924 \\
1940 \\
1959 \\
1983 \\
1964 \\
1971 \\
1994\end{array}$ & $\begin{array}{l}\text { Qp } \\
\text { op } \\
o p \\
\text { op } \\
\text { op } \\
\text { op } \\
\text { op } \\
\text { op }\end{array}$ \\
\hline 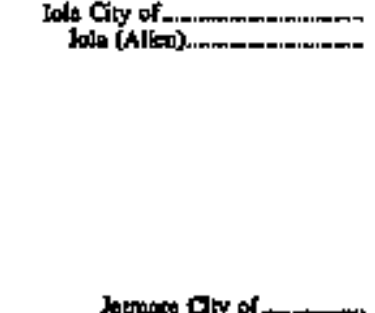 & $\begin{array}{r}10 \\
11 \\
12 \\
13 \\
4 \\
5 \\
6 \\
7 \\
5 \\
9\end{array}$ & $\begin{array}{l}28.5 \\
2.8 \\
21 \\
21 \\
2.1 \\
3.5 \\
5.0 \\
2.8 \\
2.7 \\
2.8 \\
2.8\end{array}$ & $\begin{array}{l}34.7 \\
2.9 \\
2.2 \\
2.0 \\
2.1 \\
4.4 \\
5.4 \\
3.0 \\
2.9 \\
3.0 \\
3.0 \\
6.0\end{array}$ & $\begin{array}{l}30.7 \\
2.9 \\
2.2 \\
20 \\
2.1 \\
4.4 \\
5.4 \\
3.0 \\
2.9 \\
3.0 \\
3.0 \\
6.0\end{array}$ & $\begin{array}{l}\text { IC } \\
\text { IC } \\
\text { IC } \\
1 C \\
5 T \\
5 T \\
1 C \\
16 \\
k \\
K \\
K\end{array}$ & 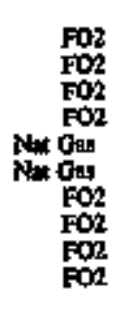 & 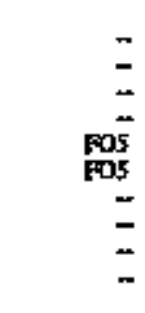 & $\begin{array}{l}1981 \\
1988 \\
1989 \\
1988 \\
1949 \\
1997 \\
1969 \\
1971 \\
1976 \\
1977\end{array}$ & $\begin{array}{l}\text { OP } \\
\text { op } \\
\text { op } \\
\text { OP } \\
\text { op } \\
\text { OP } \\
\text { OP } \\
\text { OP } \\
\text { OP } \\
\text { OP }\end{array}$ \\
\hline 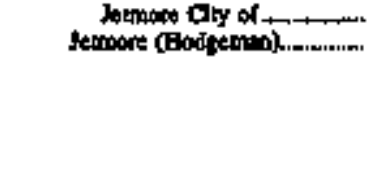 & $\begin{array}{l}1 \\
2 \\
3 \\
4 \\
5 \\
6\end{array}$ & $\begin{array}{r}6 \\
1.0 \\
4 \\
3 \\
5 \\
15 \\
13\end{array}$ & $\begin{array}{r}6.0 \\
1.0 \\
.4 \\
.2 \\
.8 \\
1.5 \\
1.2\end{array}$ & $\begin{array}{r}6.0 \\
1.0 \\
4 \\
.2 \\
.8 \\
1.5 \\
1.2\end{array}$ & $\begin{array}{l}\mathbf{K} \\
\mathrm{KC} \\
\mathrm{CC} \\
\mathrm{LC} \\
\mathrm{IC} \\
\mathrm{ICC}\end{array}$ & $\begin{array}{l}\mathbf{F O 2} \\
\mathrm{FO2} \\
\mathrm{FO2} \\
\mathrm{FO2} \\
\mathrm{FO2} \\
\mathrm{FO2}\end{array}$ & 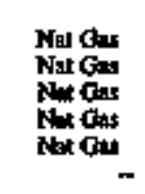 & $\begin{array}{l}1960 \\
\text { I951 } \\
1946 \\
1954 \\
1966 \\
1966\end{array}$ & $\begin{array}{l}\text { op } \\
\text { op } \\
\text { op } \\
\text { op } \\
\text { op } \\
\text { or }\end{array}$ \\
\hline
\end{tabular}

See foction al exd of table. 
Table 20. Existing Generating Units at US. Electric Utilities by State, Company, and Plant, as of Janwary 1, 1996 (Continued)

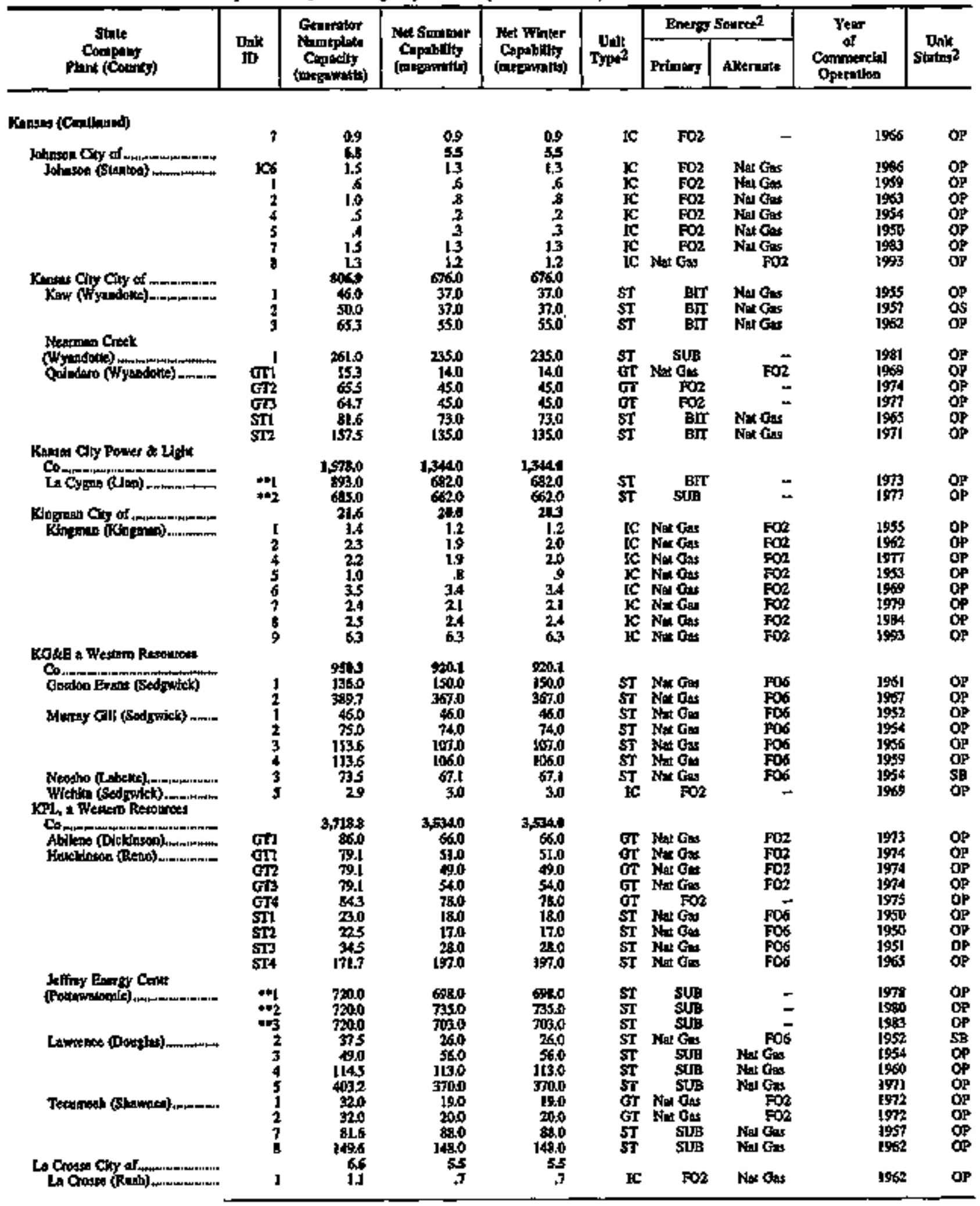

Sec foolnotes at the of lablo. 
Table 20. Existing Generating Units at U.S. Electric Utilities by State, Company, and Plant, as of Jamuary 1, 1996 (Conthued)

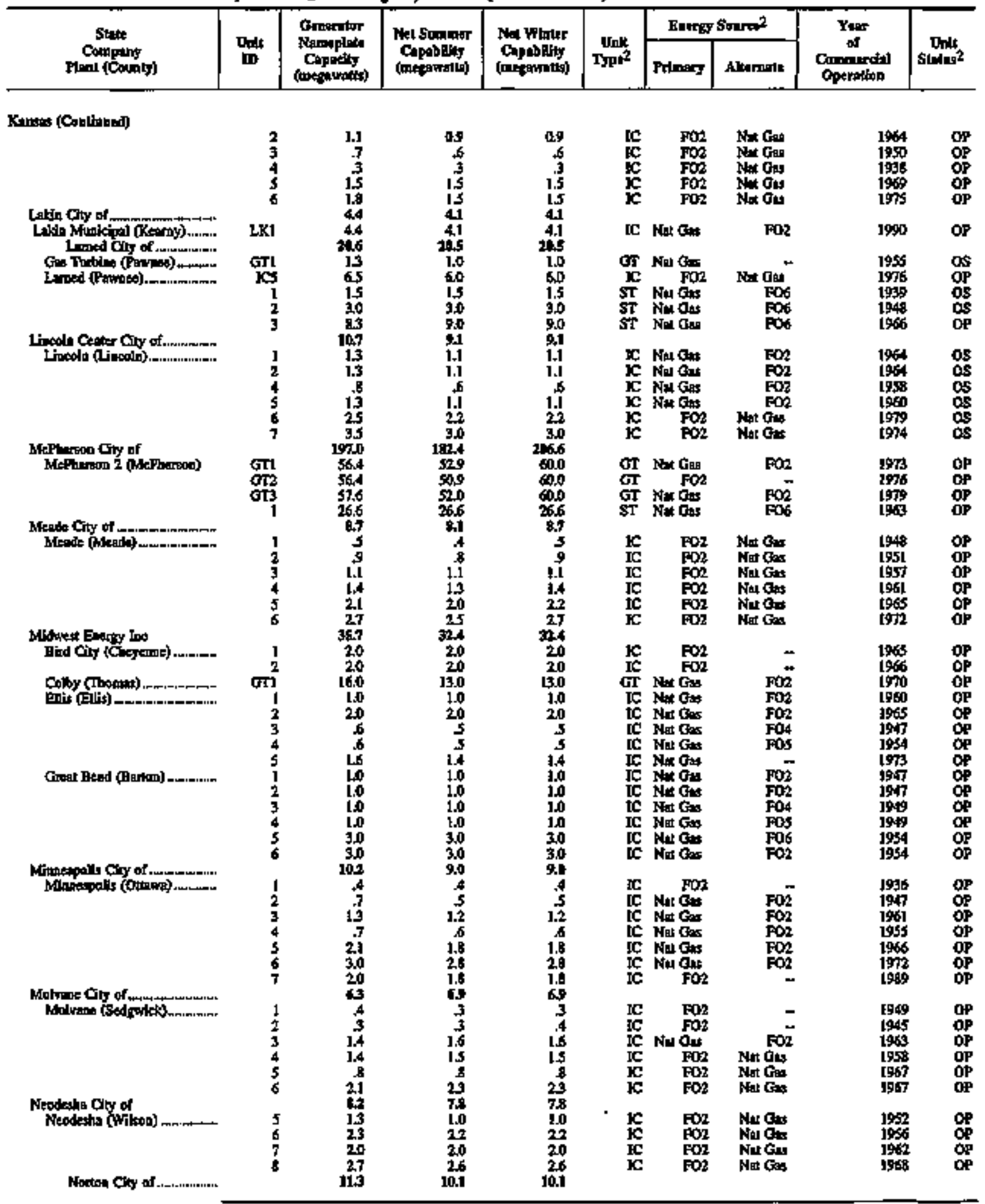

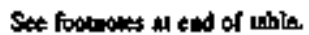


Table 20. Existing Generating Units at U.S. Electric Utillties by State, Company, and Plant, as of January 1, 1996 (Continued)

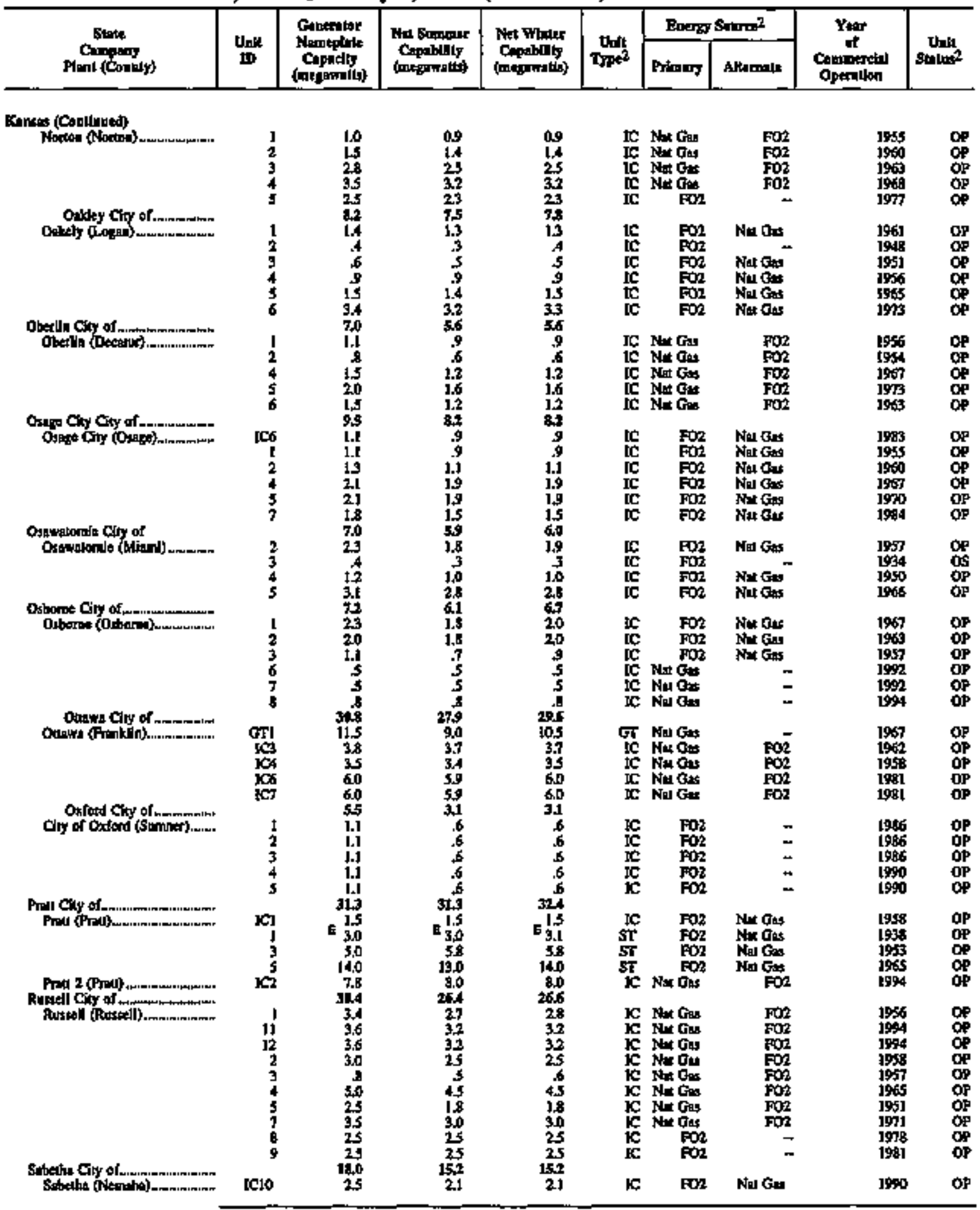

Sec frooleokes nt cad of uble. 
Table 20. Existing Generating Units at U.S. Electric Utilities by State, Company, and Plant, as of January 1, 1996 (Continued)

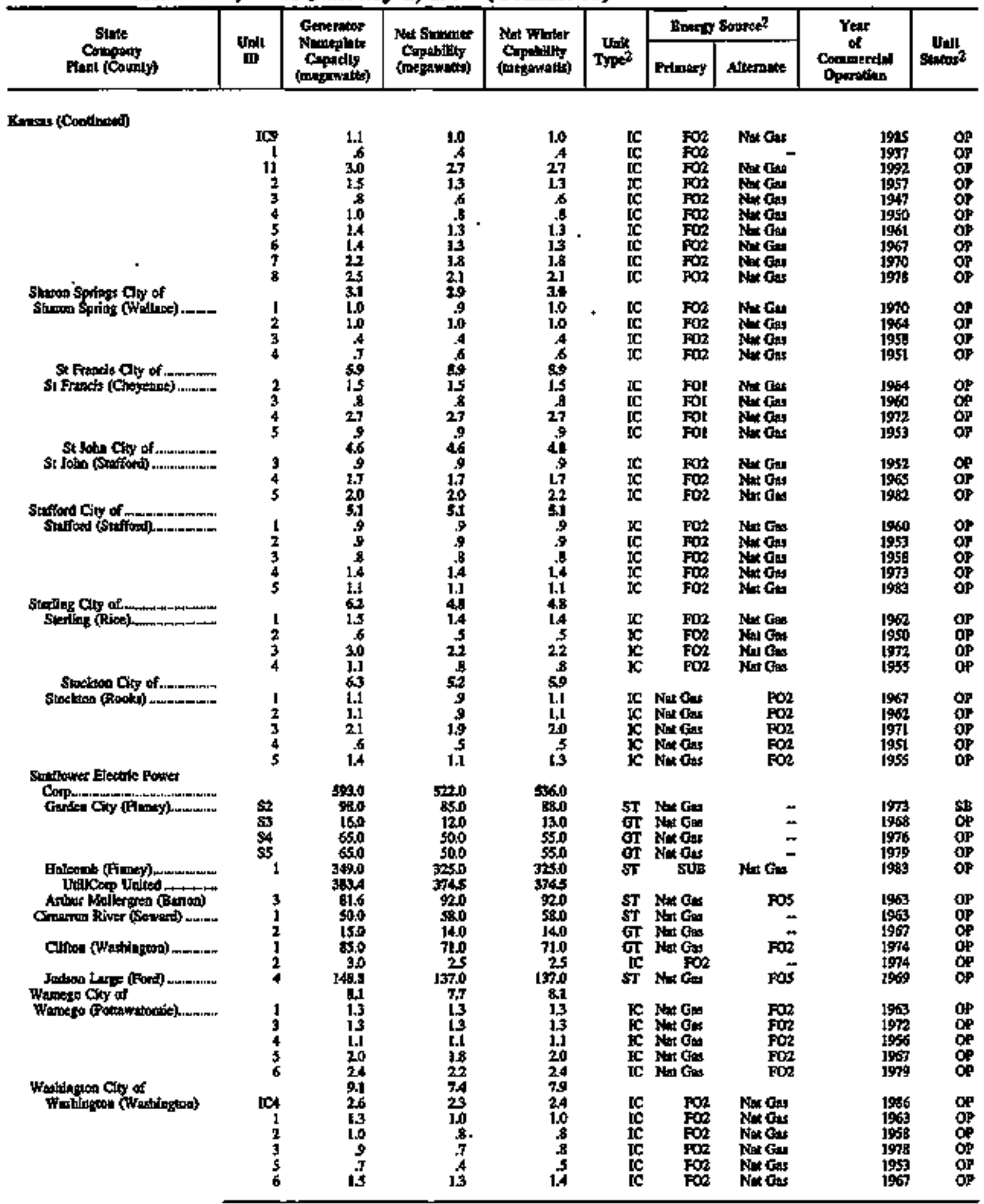

Sist footnotes ax end of thit. 
Table 20. Existing Generating Units at U.S. Electric Utilities by State, Company, and Plant, as of January 1, 1996 (Continned)

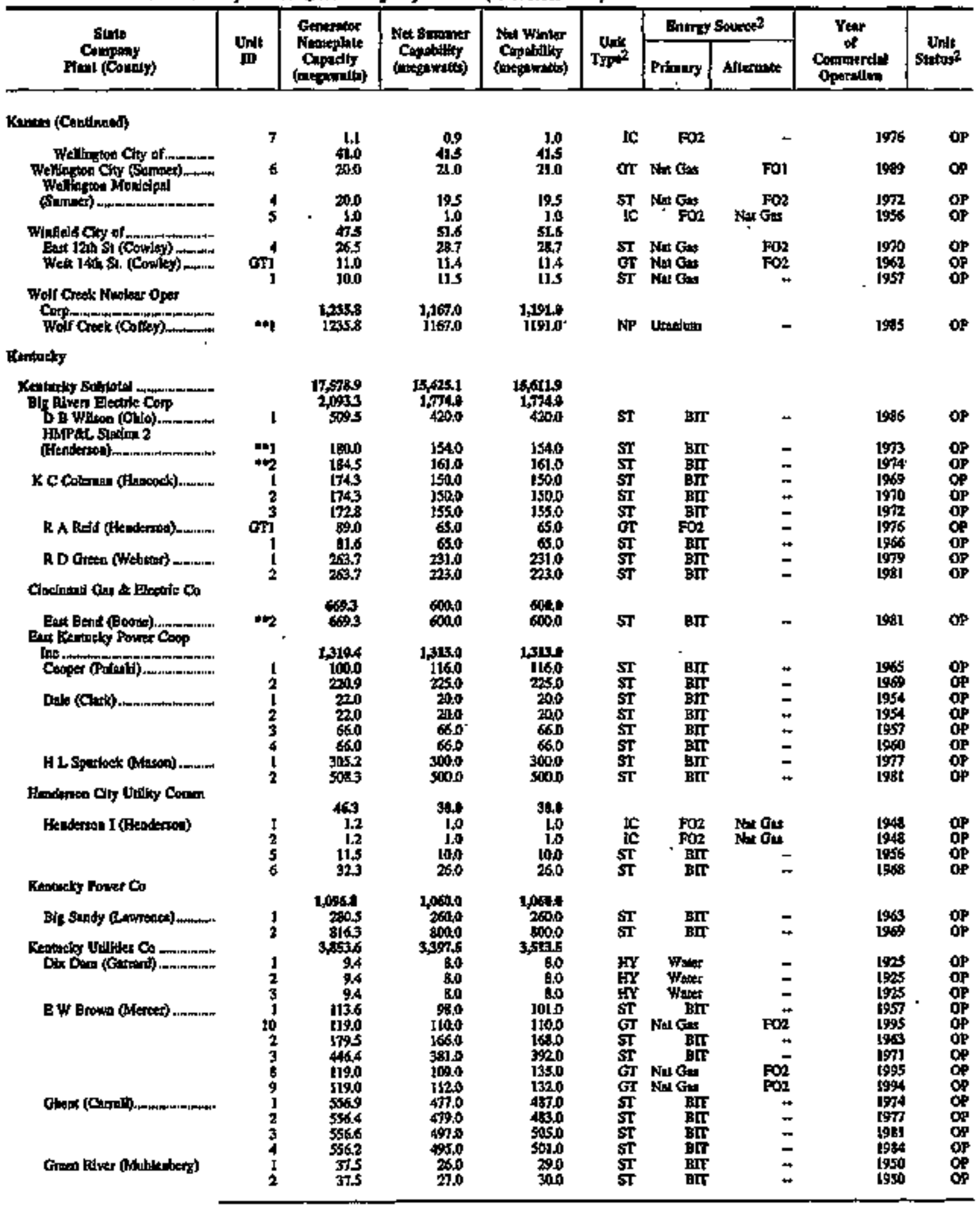

See footholta a and of thite. 
Table 20. Existing Generating Units at U.S. Electric Utilities by State, Company, and Plant, as of January 1, 1996 (Continued)

\begin{tabular}{|c|c|c|c|c|c|c|c|c|c|}
\hline \multirow{2}{*}{ 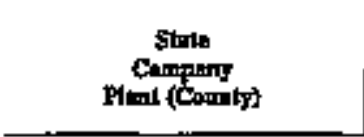 } & \multirow{2}{*}{$\begin{array}{l}\text { In't } \\
\text { ID }\end{array}$} & \multirow{2}{*}{ 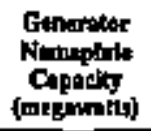 } & \multirow{2}{*}{ 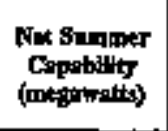 } & \multirow{2}{*}{ 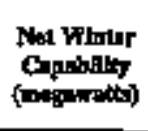 } & \multirow{2}{*}{$\begin{array}{l}\text { Unity } \\
\text { Typet }\end{array}$} & \multicolumn{2}{|c|}{ Eners Souret? } & \multirow{2}{*}{$\begin{array}{c}\text { Yaar } \\
\text { of } \\
\text { Commerctal } \\
\text { Openilon }\end{array}$} & \multirow{2}{*}{$\operatorname{Unan}_{\text {sients }}$} \\
\hline & & & & & & Prtmary & Alarmbe & & \\
\hline \multicolumn{10}{|l|}{ Kentody (Continged) } \\
\hline & 3 & 75.0 & 71.0 & $n 20$ & sT & 时 & - & 1954 & op \\
\hline & 4 & 113.6 & losso & \pm 11.0 & ST & BII & _- & 1959 & of \\
\hline 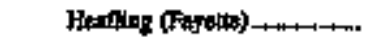 & j & 20.7 & 1700 & 200 & GI & 102 & Nat Ges & 2970 & OP \\
\hline (2) - & 2 & 20.7 & 160 & 19.0 & GI & Fo2 & Mot $6 *$ & 1970 & \\
\hline Lock (Bhrocr) & 3 & 20.7 & 170 & 200 & $\mathbf{r r}$ & $\mathbf{F O 2}$ & Non B० & 1900 & op \\
\hline & $\mathbf{3}$ & 7 & 15 & $\begin{array}{l}1.5 \\
2.5\end{array}$ & $\begin{array}{l}\text { HY } \\
\text { HY }\end{array}$ & What & 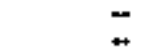 & 1929 & OP \\
\hline & 3 & 7 & is & 15 & HY & Waler & - & [9LI & op \\
\hline Pineville (Bell) & 3 & 37.5 & 330 & 34.0 & sT & BrT & $=$ & [\$5] & op \\
\hline Tyou (Woodford).........- & 1 & 31.3 & 27.0 & 300 & $\underline{s T}$ & 502 & - & lap? & of \\
\hline & $\frac{2}{3}$ & $3 \mathrm{L3}$ & $\begin{array}{l}35.0 \\
720\end{array}$ & $\frac{330}{30}$ & $\begin{array}{ll}S T \\
\xi T T\end{array}$ & $\mathbf{F D 2}$ & $\ddot{z}$ & $19+8$ & oP \\
\hline \multirow{4}{*}{ 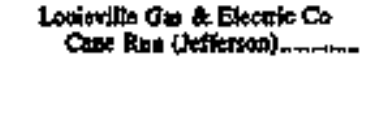 } & & 3,1356 & $2,621$. & 2,8500 & & $\mathbf{m}$ & 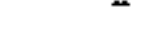 & & \\
\hline & I1 & 163 & 16,0 & 19.0 & Gr & Nhe Ony & $\mathbf{F m 2}$ & 1968 & op \\
\hline & 4 & 1692 & 135,0 & 1550 & \$T & & - & [962 & op \\
\hline & $\begin{array}{l}5 \\
6\end{array}$ & $\begin{array}{l}309.4 \\
2020\end{array}$ & $\begin{array}{l}160.0 \\
240.0\end{array}$ & $\begin{array}{l}16 \% 0 \\
240.0\end{array}$ & $\begin{array}{l}s T \\
s T\end{array}$ & Bys & $\ddot{z}$ & $\begin{array}{l}2966 \\
t 906\end{array}$ & op \\
\hline \multirow[t]{4}{*}{ 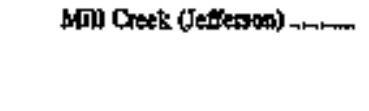 } & I & 3.55 .5 & 309.0 & $\sin 0$ & sT & BटT & - & 1972 & $\mathbf{O P}$ \\
\hline & 2 & 355.5 & 301.0 & 301.0 & 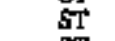 & $\mathrm{BIT}$ & - & 1504 & OP \\
\hline & 3 & 4026 & 386.0 & 3860 & ET & BT & - & 1978 & OP \\
\hline & 4 & 54.6 & 400 & $\$ 90.0$ & ST. & Ex & - & 198 & op \\
\hline \multirow{6}{*}{ 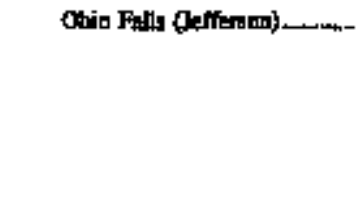 } & $\frac{1}{2}$ & $\begin{array}{l}100 \\
100\end{array}$ & 60 & $\begin{array}{l}5.1 \\
5.1\end{array}$ & $\begin{array}{l}\text { HY } \\
\text { HY }\end{array}$ & Woter & 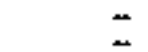 & $\begin{array}{l}1928 \\
1928\end{array}$ & op \\
\hline & 3 & 100 & 60 & $\$$ & HY & Wast & $=$ & 1928 & of \\
\hline & 4 & 100 & 60 & 5.1 & EY & What & - & 1928 & op \\
\hline & $\begin{array}{l}5 \\
6\end{array}$ & 20.0 & 6.0 & $\begin{array}{l}5.1 \\
5.1\end{array}$ & FY & Wh & 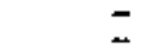 & 1928 & op \\
\hline & 7 & 100 & 60 & 5.1 & HY & Water & $*$ & 1928 & of \\
\hline & 8 & 5100 & 6.0 & 5.1 & HY & Water & - & 1928 & of \\
\hline 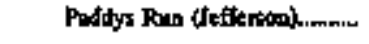 & 11 & 5160 & E 150 & 164 & $\sigma$ & Nat Gos & - & 1968 & of \\
\hline & $\mid 2$ & 326 & ${ }^{E} 26,0$ & E35.4 & ar & Fat 0 & - & 1968 & op \\
\hline 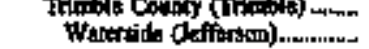 & 1 & $E^{5060.1}$ & E. 17.0 & $e^{40.3}$ & GT & Nax the & $\ddot{4}$ & 1960 & op \\
\hline & 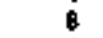 & E 250 & E 160 & 25.6 & or & $\mathrm{Not}$ & $=$ & & op \\
\hline Zusn (Jaffertm) & 1 & E 120 & $E=16.0$ & 18.4 & Gi & 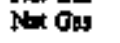 & - & 1909 & OP \\
\hline Orenstore Cyy of & & 4160 & 390.3 & 390,3 & & & & & \\
\hline Eftur Serith (Derien) .....- & $\mathbf{l}$ & 151.0 & 141.I & 141.1 & ST & E[T & - & 1964 & OP \\
\hline Phis Cty of & $\mathbf{z}$ & 2650 & 2493 & 249.3 & ST & BI & - & 1974 & OP \\
\hline 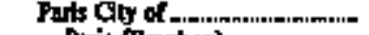 & & 11.8 & 11.1 & 11.1 & & & & & \\
\hline Pirit (Babon).................... & $\mathbf{I}$ & 1.4 & 1.3 & 1.3 & IS & Bot & - & 1952 & oP \\
\hline & 3 & 1.4 & 13 & 13 & IE & Fon & - & 1954 & OP \\
\hline & 4 & 10 & .78 & 1.7 & Fc & $\mathrm{FO}_{2}$ & $\ddot{z}$ & $\begin{array}{l}\$ 934 \\
1947\end{array}$ & of \\
\hline & $\xi$ & i.t & 1.1 & t..1 & 距 & $\mathrm{PO} 2$ & - & $19 \% 9$ & $\mathbf{Q P}$ \\
\hline & 6 & 3.1 & 29 & 29 & te & $\mathbf{F o n}$ & - & 1974 & of \\
\hline & 7 & 3.1 & 29 & 29 & IC & $\mathrm{PO} 2$ & - & 1974 & $O P$ \\
\hline Tenostite Valky hutherity & & 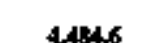 & & $3, \pi 0,0$ & & & & & \\
\hline 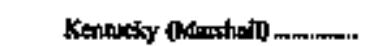 & 」 & tho & 37,0 & 15.0 & HY & Water & - & 1945 & OP \\
\hline & 2 & 320 & 310 & 15.0 & HY & Water & - & 1944 & QP \\
\hline & 3 & 320 & 32.0 & 15.0 & $\mathbf{E Y}$ & Watre & - & 1944 & $\mathbf{O P}$ \\
\hline & 4 & 38.4 & 37.0 & 150 & $\mathbf{E Y}$ & Water & - & 1945 & of \\
\hline & $\$$ & 370 & 44.4 & $\mathbf{L S . 0}$ & EY & Whaper & - & 1946 & OP \\
\hline Paradise (Blubluberg)_-Cm & 1 & 7040 & 391.0 & 6L5;0 & ST & BतT & - & 1963 & op \\
\hline & 2 & 7040 & 593.0 & 6150 & $\mathbf{s T}$ & EnT & - & 1963 & OP \\
\hline & 3 & 11502 & 977.0 & 10360 & $\mathbf{s T}$ & EIT & - & $19 \pi 0$ & OP \\
\hline 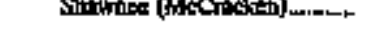 & 10 & 173,9 & 134,0 & 138.0 & ST & BST & $=$ & 1953 & op \\
\hline & $\begin{array}{r}10 \\
2\end{array}$ & $\begin{array}{l}1750 \\
1750\end{array}$ & $\begin{array}{l}1400 \\
1340\end{array}$ & $\begin{array}{l}167,0 \\
138.0\end{array}$ & $\begin{array}{l}s T \\
s T\end{array}$ & 然 & $\bar{z}$ & 1956 & $\sigma_{0 P}$ \\
\hline & 3 & $\begin{array}{l}1750 \\
1750\end{array}$ & $\begin{array}{l}+34,0 \\
134,0\end{array}$ & $\begin{array}{l}130.60 \\
130.0\end{array}$ & $\begin{array}{l}\text { sI } \\
\mathbf{s T}\end{array}$ & BIT & $\bar{z}$ & $\begin{array}{l}1093 \\
1959\end{array}$ & op \\
\hline & 4 & 1750 & 1340 & 139.0 & ST & BI & ـ & 1954 & OP \\
\hline & $\mathbf{s}$ & 1750 & 1340 & 1390 & $\mathbf{s T}$ & BतT & - & I954 & OP \\
\hline & 6 & 1750 & 1340 & 1380 & ST & ㅍ. & 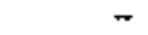 & 1954 & OP \\
\hline & 7 & [750 & 1340 & 138.0 & ST & Bris & - & 1994 & OP \\
\hline & 8 & 1750 & 1340 & 138.0 & $\mathbf{s T}$ & BIT & - & 1955 & OP \\
\hline & $\mathbf{9}$ & 1750 & 1340 & 130.0 & รT & ВतT & - & 1955 & OP \\
\hline 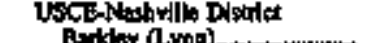 & & 461,0 & 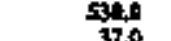 & 5364 & & & & & $\mathbf{A P}$ \\
\hline 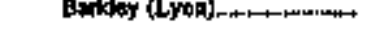 & I & 32.5 & 37. & 370 & HI & fros & * & 1966 & OP \\
\hline
\end{tabular}

Ses footunies at end of toblt. 
Table 20. Existhg Generating Units at U.S. Electric Utilities by State, Company, and Plant, as of January 1,1996 (Contimued)

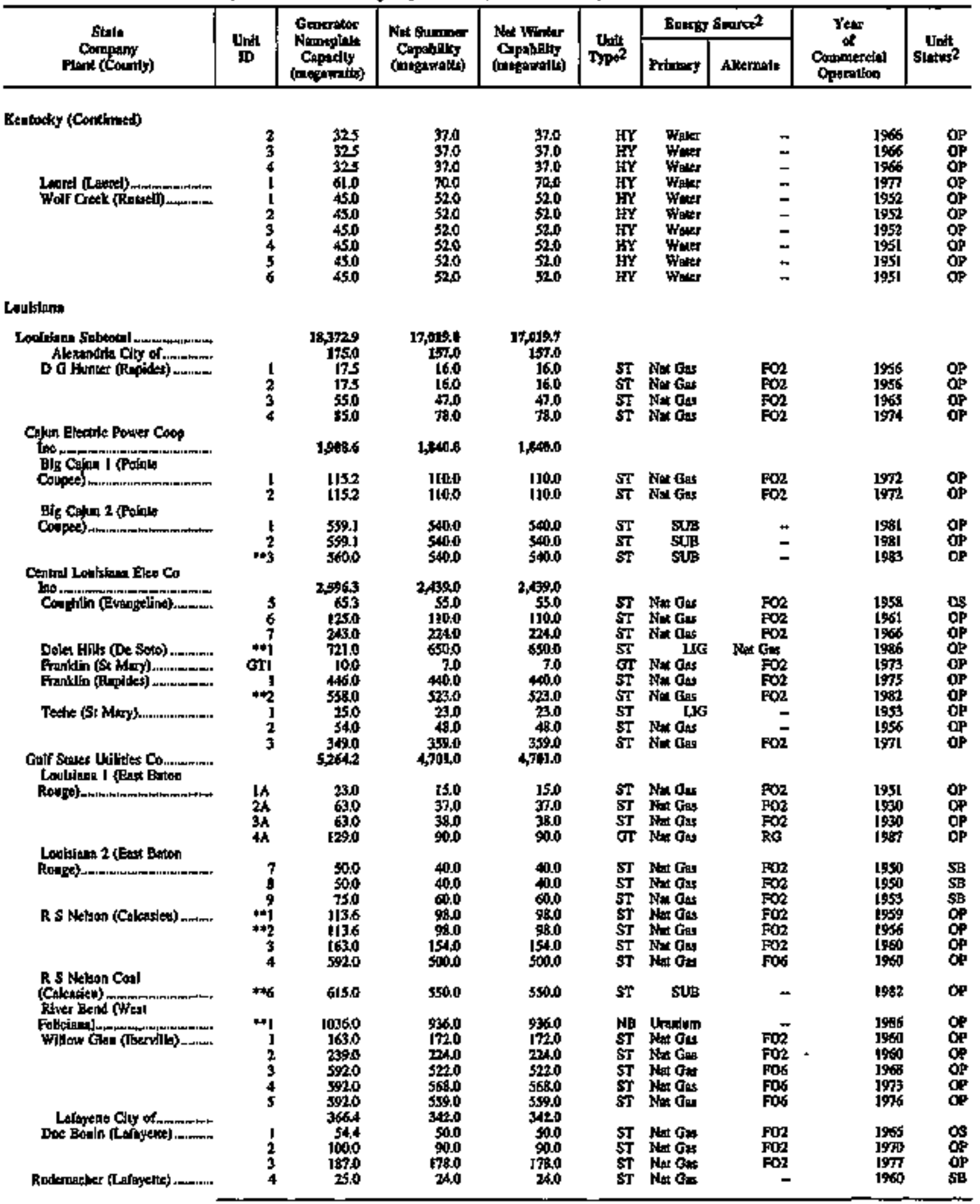

See focennous at end of follo. 
Table 20. Existing Generating Units at U.S. Electric Utilities by State, Company, and Plant, as of January 1, 1996 (Continued)

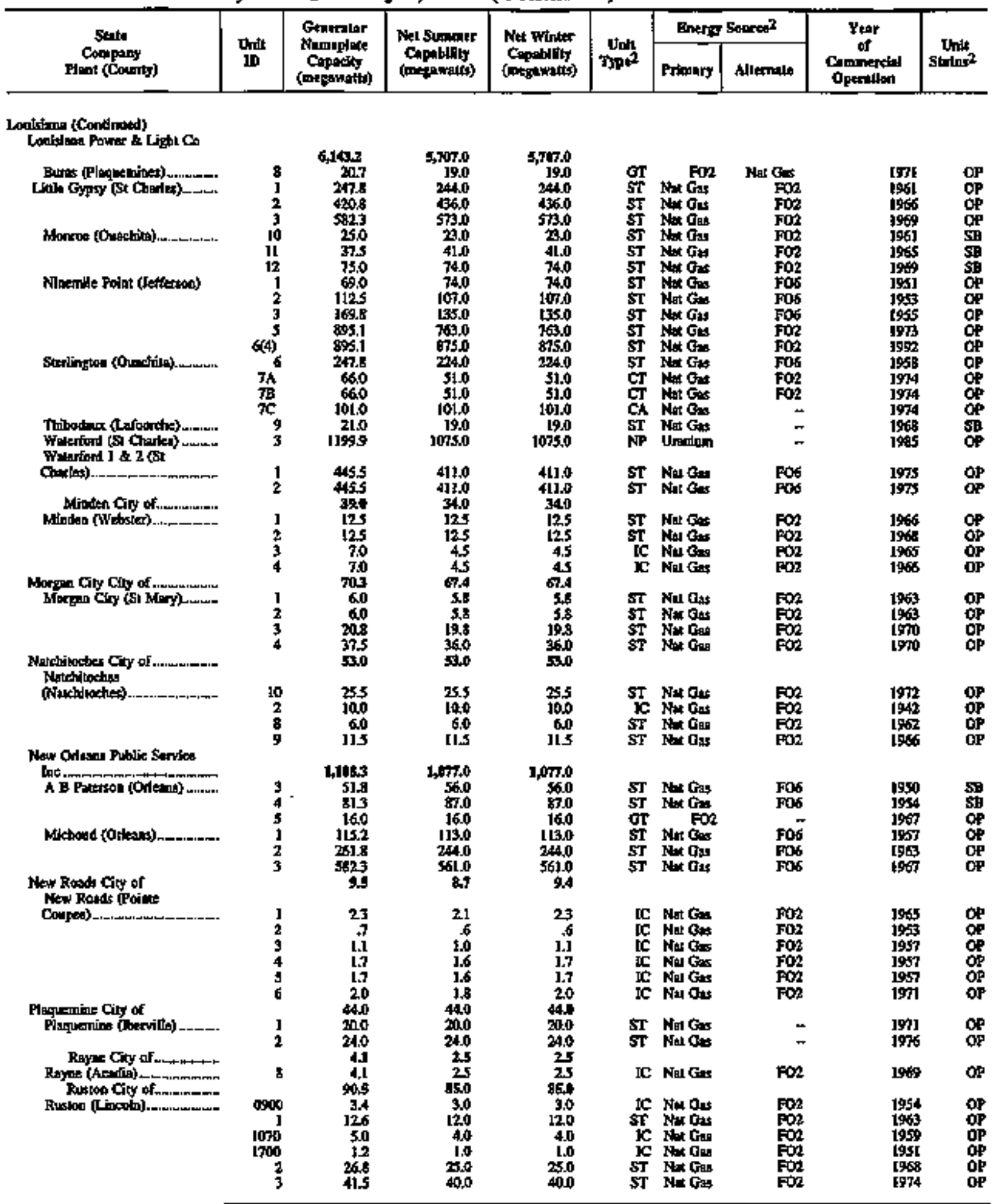

Sas foctunctes ot end of reble. 
Table 20. Existing Generating Units at U.S. Electric Utilities by State, Company, and Plant, as of January 1, 1996 (Continued)

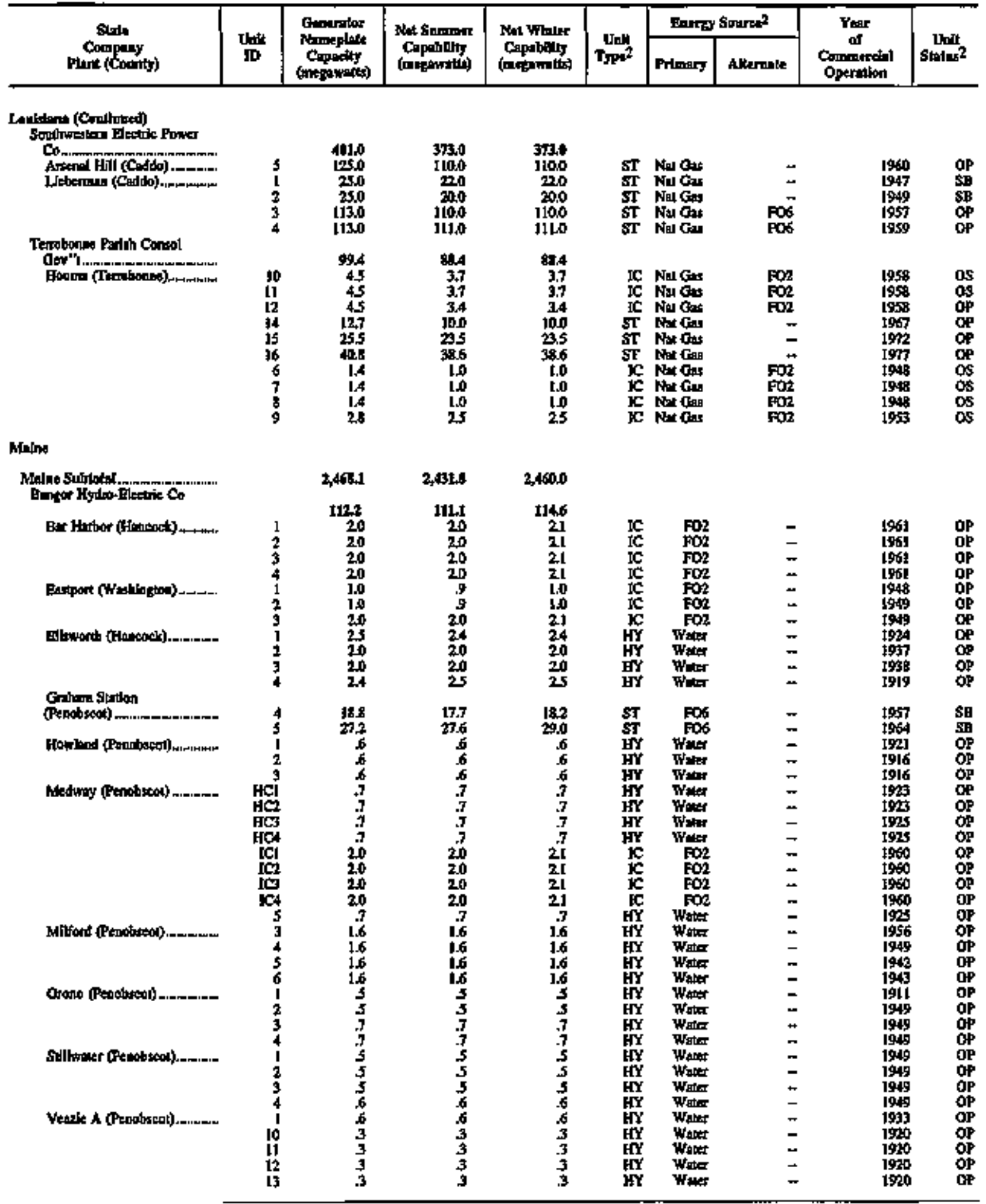

Sect boonotes we end of table. 
Table 20. Existing Generating Units at U.S. Electric Utiltites by State, Company, and Plant, as of January 1, 1996 (Continued)

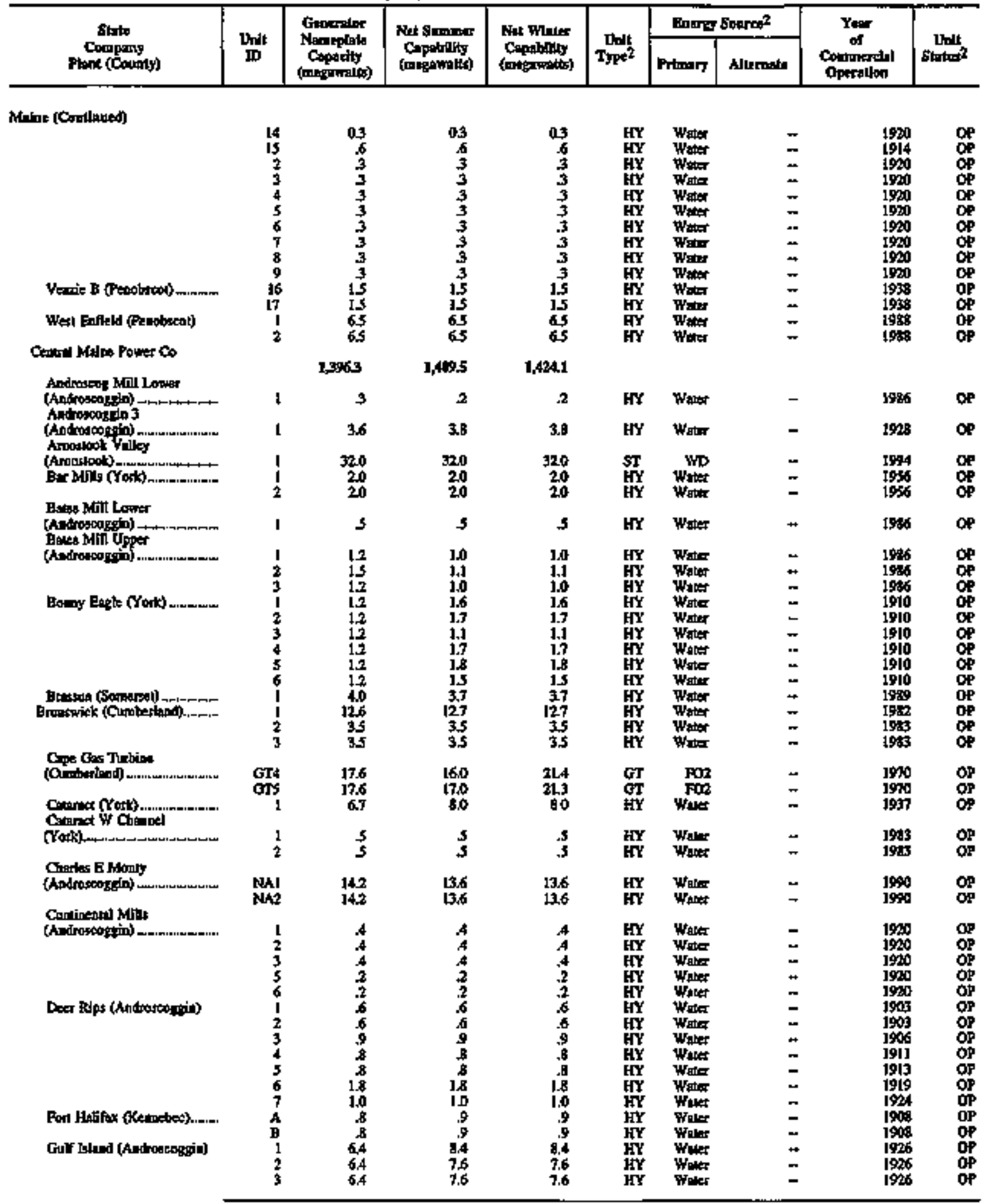

Sex foomon at end of toble. 
Table 20. Existing Generating Units at U,S. Electric Utilities by State, Company, and Plant, as of January 1, 1996 (Continued)

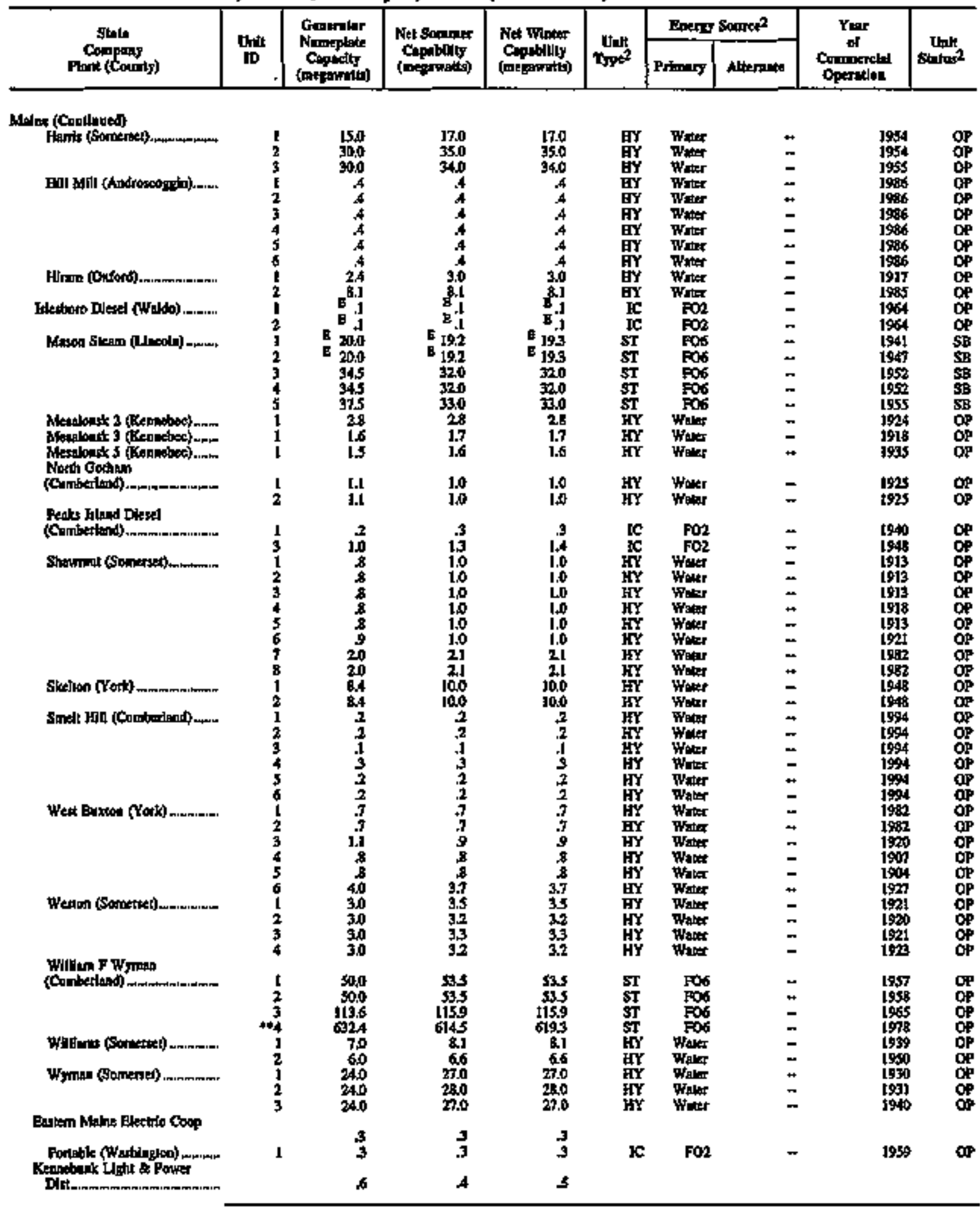

Sec foolnowe at and of ublan, 
Table 20. Existing Generating Units at U.S. Electric Utilities by State, Company, and Plant, as of January 1, 1996 (Continued)

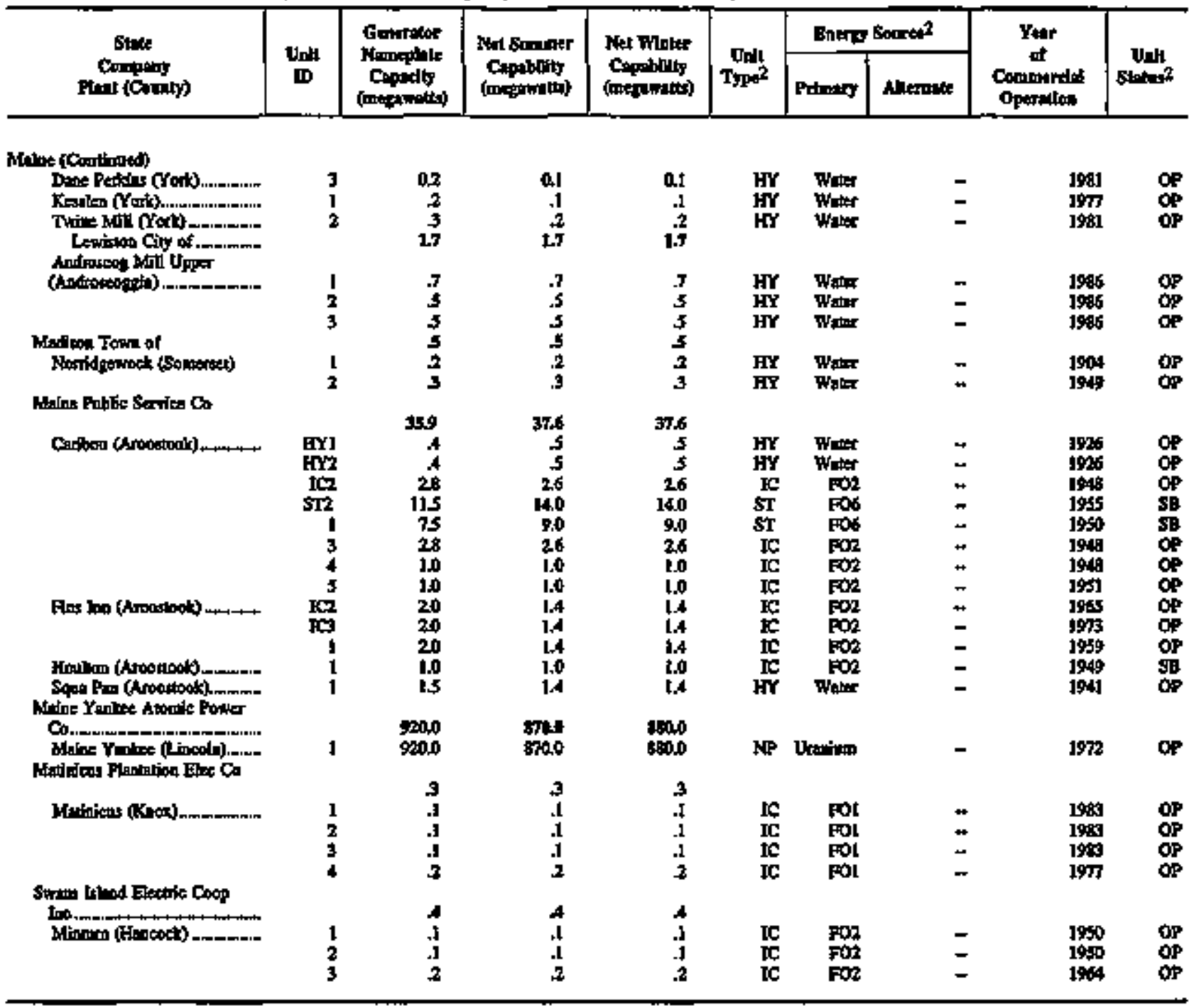

Sect footions at tad of able. 
Table 20. Existing Generattng Units at U.S. Electric Utilities by State, Company, and Plant, as of January 1, 1996 (Continned)



Sec soomowa at end of tabta. 
Table 20. Exxisting Generating Units at U.S. Electric Utiltties by State, Company, and Plant, as of Jamuary 1, 1996 (Continued)

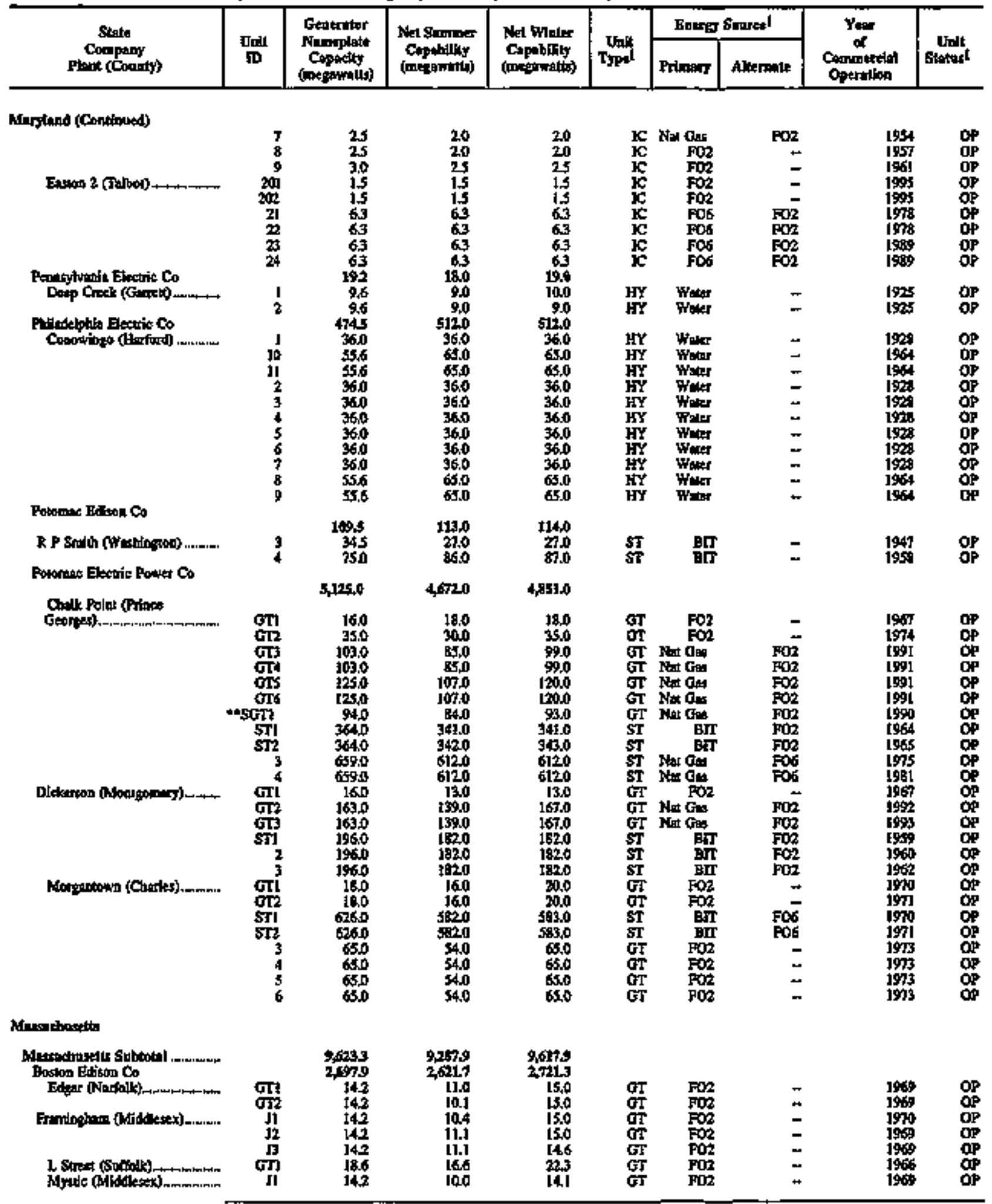

Ses footontes al and of isble. 
Table 20. Existing Generating Units at U.S. Electric Utilities by State, Company, and Plant, as of Jamuary 1, 1996 (Contimued)

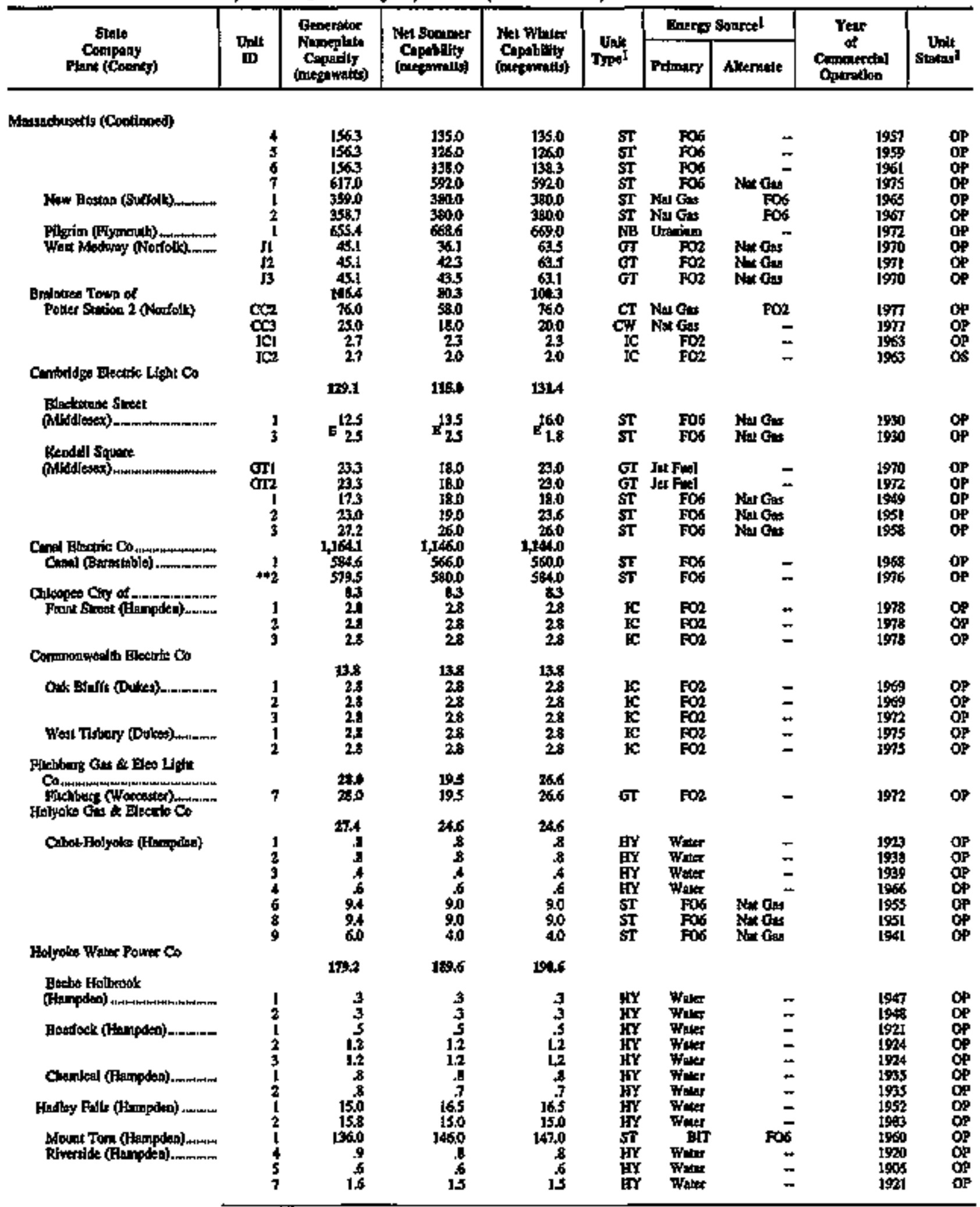

Ses foolnoke to end of abto. 
Table 20. Existing Generating Units at US.S. Electric Utilities by State, Company, and Plant, as of January 1, 1996 (Continued)

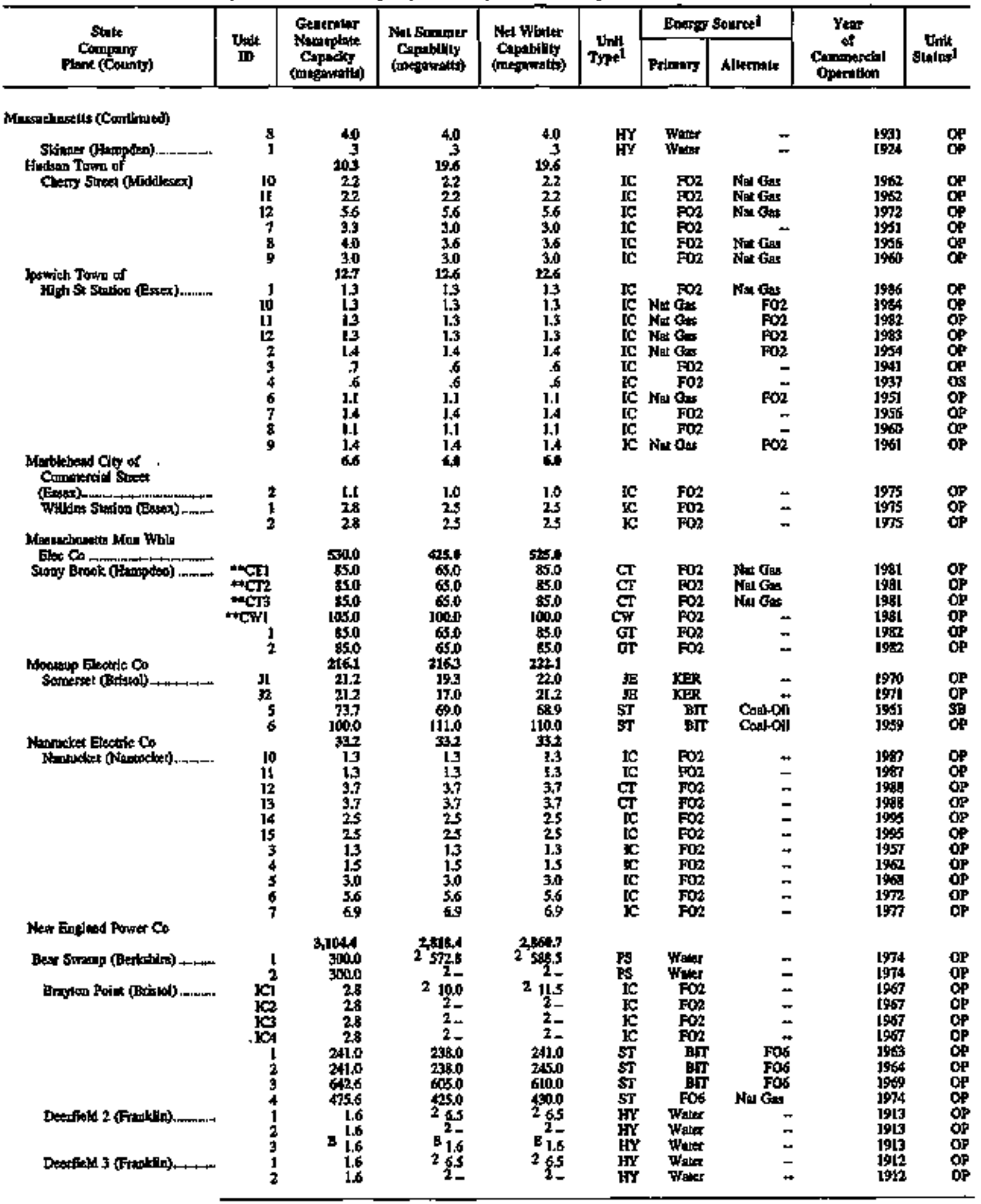

See rootoros at ead of table. 
Table 20. Existing Generating Units at U.S. Electric Utilities by State, Company, and Plant, as of January 1, 1996 (Continued)

\begin{tabular}{|c|c|c|c|c|c|c|c|c|c|}
\hline \multirow{2}{*}{ 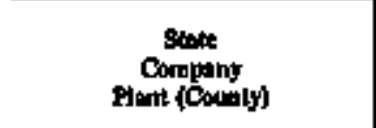 } & \multirow{2}{*}{ Inn } & \multirow{2}{*}{ 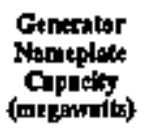 } & \multirow{2}{*}{ 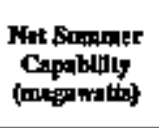 } & \multirow{2}{*}{$\begin{array}{l}\text { Nat Wintar } \\
\text { Coppobillity } \\
\text { (inopowats) }\end{array}$} & \multirow{2}{*}{ Thin } & \multicolumn{2}{|c|}{ Eneryy Soutreel } & \multirow{2}{*}{$\begin{array}{c}\text { Year } \\
\text { of } \\
\text { Comberdiel } \\
\text { Operallon }\end{array}$} & \multirow{2}{*}{$\begin{array}{c}\text { Unh } \\
\text { statest }\end{array}$} \\
\hline & & & & & & Prinurg & Aliernilo & & \\
\hline \multirow{2}{*}{\multicolumn{10}{|c|}{ Mnswedrustits (C) mlinsted) }} \\
\hline & & & 2_ & 2 - & $\mathbf{H Y}$ & Wuser & - & 1912 & op \\
\hline Deafiedd \& (Fimkln).mm & 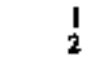 & 1.6 & 25.7 & $\begin{array}{l}25.7 \\
2=\end{array}$ & $\begin{array}{l}\mathbf{E Y} \\
\mathbf{H Y}\end{array}$ & $\begin{array}{l}\text { Water } \\
\text { Water }\end{array}$ & $=$ & $\begin{array}{l}1912 \\
1912\end{array}$ & of \\
\hline & $\overline{3}$ & If & 2- & $2-$ & HY & Water & . & 1913 & OP \\
\hline 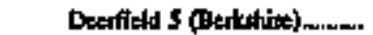 & 1 & 17,6 & 140 & IA,0 & HY & Water & - & 1974 & $O P$ \\
\hline 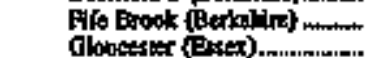 & $\mathbf{j}$ & $\begin{array}{l}11.3 \\
20\end{array}$ & 219.9 & 29.9 & $\underset{\text { IC }}{\text { HY }}$ & $\begin{array}{l}\text { Wract } \\
\text { FO2 }\end{array}$ & $\ddot{m}$ & $\begin{array}{l}1974 \\
1963\end{array}$ & OP \\
\hline & 10 & $\begin{array}{l}20 \\
2.6\end{array}$ & $2=$ & $2-$ & Ic & $\mathrm{FO2}$ & $\bar{z}$ & $\begin{array}{l}1971 \\
1971\end{array}$ & OP \\
\hline & 2 & 20 & $2-$ & $2=$ & 政 & $\mathbb{N O}_{12}$ & $\ddot{z}$ & 1963 & op \\
\hline & 4 & 2.0 & $2=$ & $\frac{2}{y}=$ & ch & $\cos$ & $=$ & 1964 & op \\
\hline & 5 & $\begin{array}{l}20 \\
28\end{array}$ & $2-$ & $\begin{array}{l}2= \\
2=\end{array}$ & $\underset{x}{x}$ & $\mathrm{Fon}_{2}$ & $\vec{z}$ & $\begin{array}{l}1964 \\
1967\end{array}$ & $\underset{O P}{O P}$ \\
\hline & $\begin{array}{l}7 \\
8\end{array}$ & 26 & $2=$ & & 茂 & $\mathbf{F O 2}$ & 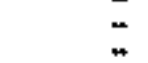 & 1967 & $\underset{O P}{O P}$ \\
\hline & 9 & 28 & $2 \ln ^{2}$ & $2=$ & ic & $\mathrm{FO} 2$ & - & 1967 & $O P$ \\
\hline 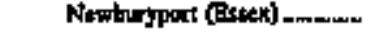 & $\begin{array}{l}1 \\
2\end{array}$ & $\begin{array}{l}2.8 \\
2.8\end{array}$ & $2 \log$ & $2 \frac{11.5}{25}$ & IC & $\mathrm{FO2}$ & $\ddot{-}$ & $\begin{array}{l}1970 \\
1970\end{array}$ & $\stackrel{p P}{O P}$ \\
\hline & 3 & 28 & $\frac{2-}{2}$ & $2-$ & rc & $\mathrm{Fon}$ & 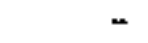 & 190 & $\mathrm{OP}^{+}$ \\
\hline & 4 & 28 & ${ }^{2}-0$ & $2-$ & Ic & Fon & $=$ & 1970 & $\mathrm{op}$ \\
\hline Salem Harbor (Esaex) & I & 819 & 79.0 & 81.0 & ST & BI & For & 1952 & OP \\
\hline & $\mathbf{2}$ & $\begin{array}{r}820 \\
1608\end{array}$ & $\begin{array}{r}78,0 \\
143,0\end{array}$ & $\begin{array}{r}780 \\
1430\end{array}$ & $\underset{\mathbf{S T}}{\mathbf{S T}}$ & $\begin{array}{l}\text { BIT } \\
\text { BIT }\end{array}$ & $\begin{array}{l}\text { FOS } \\
\text { FOS }\end{array}$ & $\begin{array}{l}1952 \\
1958\end{array}$ & OP \\
\hline & 4 & 475.6 & 3500 & 350.0 & ST & For & - & 1,973 & OP \\
\hline \multirow{3}{*}{ 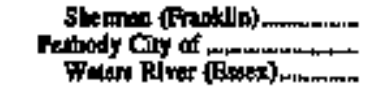 } & $\mathbf{i}$ & 7.2 & 65 & 6.5 & EY & Water & $\leftarrow$ & 1926 & OP \\
\hline & & 649 & 4.6 & 695 & & & & & \\
\hline & 1 & 21.3 & 140 & 200 & $\mathbf{r}$ & Nax Ox & FO2 & 1971 & $O P$ \\
\hline \multirow{8}{*}{ 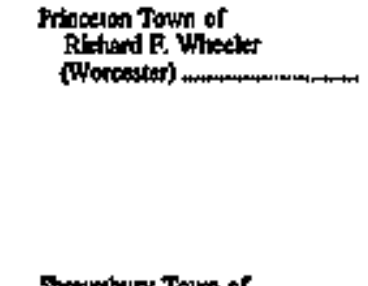 } & & 43.6 & $\begin{array}{r}30.6 \\
5\end{array}$ & $\begin{array}{r}45.9 \\
49\end{array}$ & Gr & Nan Gen & $\mathrm{FO} 2$ & 1991 & $\mathrm{OP}_{\mathrm{P}}$ \\
\hline & $\mathbf{I}$ & - & .1 & .] & WT & Wind & - & 1984 & op \\
\hline & 2 & • & .1 & .1 & $\mathbf{Y T}$ & Wind & - & t984 & OP \\
\hline & 3 & * & 1 & 1 & HT & Wind & + & Is84 & OP \\
\hline & 5 & 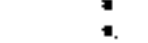 & 1 & 1 & HT & Whad & $\bar{z}$ & $\begin{array}{l}1594 \\
1984\end{array}$ & $\underset{\mathrm{op}}{\mathrm{pP}}$ \\
\hline & 6 & * & إ & .j & FT & Wind & + & Ists & OP \\
\hline & 3 & * & I & آ. & WT & WInd & - & I9134 & op \\
\hline & 8 & - & .1 & .1 & WT & Wiad & - & 1984 & OP \\
\hline \multicolumn{10}{|l|}{ Showbury Tows of } \\
\hline Skrowabngy (Wonstulur) & ! & $\begin{aligned} 140 \\
28\end{aligned}$ & 14.8 & 2.8 & ac & $\mathrm{FO}_{2}$ & - & 1969 & \\
\hline & 3 & 28 & 2.3 & 2,8 & Ic & rog & - & I\$69 & OP \\
\hline & 3 & 28 & 2.8 & 2.8 & ic & Fod & - & 1975 & op \\
\hline & 4 & 28 & 20 & 2.8 & Ic & $\mathrm{FO2}$ & - & 1975 & oP \\
\hline & 5 & 28 & 2,8 & 28 & IC & FO2 & $r$ & 1978 & OP \\
\hline Oeny Flaod (Brintol) & CA9 & $\$ 5.0$ & 810 & 870 & & & Fos & 1975 & \\
\hline & 8 & 203 & $\mathbf{2 5 0}$ & 260 & $\mathbf{S T}$ & Fos & po4 & 1968 & of \\
\hline & $9 h$ & 230 & 16.8 & 230 & CT & Nal Gad & $\mathbf{m o n}$ & 3976 & $\mathbf{O P}$ \\
\hline \multicolumn{10}{|l|}{ 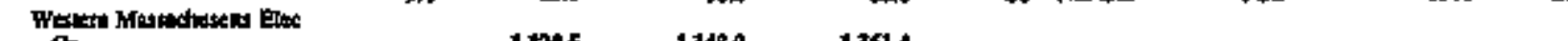 } \\
\hline 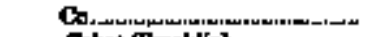 & & $1,+2,2,5$ & $1,348,0$ & $1,361.4$ & & & & & \\
\hline 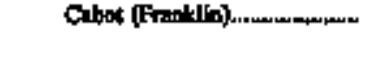 & $\begin{array}{l}1 \\
2 \\
3\end{array}$ & $\begin{array}{l}8.5 \\
85\end{array}$ & $\begin{array}{l}\mathbf{R} . \mathbf{B} \\
\mathbf{B . B} \\
\mathbf{B . 8}\end{array}$ & $\begin{array}{l}8.8 \\
8.8 \\
8.8\end{array}$ & HYY & $\begin{array}{l}\text { What } \\
\text { Wout }\end{array}$ & $=$ & $\begin{array}{l}1915 \\
1915\end{array}$ & of \\
\hline & 3 & 85 & $\begin{array}{l}8.8 \\
8.8\end{array}$ & 悬, & $\begin{array}{l}\mathbf{H Y} \\
\mathbf{H Y}\end{array}$ & Waur & 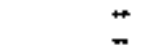 & $\begin{array}{l}1 \$ 16 \\
1916\end{array}$ & op \\
\hline & 5 & 8.5 & 8.8 & 8. & $\mathbf{H Y}$ & Whit & $=$ & 1917 & or \\
\hline & 5 & 5.5 & BE & 8.5 & FY & Wuer & - & 9917 & op \\
\hline \multirow{4}{*}{ 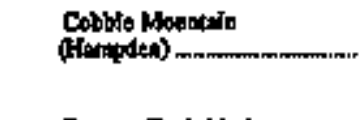 } & & & & & & & & & \\
\hline & 1 & 13.6 & 14,0 & 140 & HY & Wath & - & 1930 & OF \\
\hline & 2 & 5.8 & 6.0 & 60 & HY & Water & - & 1930 & OP \\
\hline & & 13.6 & 14,0 & 140 & $\mathbf{H Y}$ & Waver & - & 1930 & OP \\
\hline 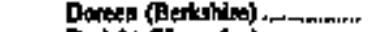 & 10 & 18.6 & i6.6 & 21.I & 氜 & Jat Fot & $\leftrightarrow$ & 196 & op \\
\hline 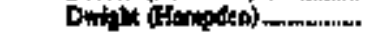 & 2 & $s$ & .6 & 6 & FY & Waller & - & 1920 & oP \\
\hline & 3 & 5 & 6 & .6 & HY & Want & $=$ & 1920 & $O P$ \\
\hline & 4 & 3 & 6 & .6 & EY & Wor & - & 1920 & ot \\
\hline 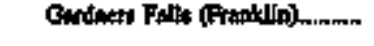 & $\frac{1}{3}$ & औ & 5 & 5 & HY & Wum & - & 1904 & OP \\
\hline & 3 & 9 & 1.0 & 1, & HI & Whoter & $\sigma$ & & DP \\
\hline
\end{tabular}

Ses rootingen all and of lablat 
Table 20. Existing Generating Units at U.S. Electric Utilities by State, Company, and Plant, as of January 1, 1996 (Continued)

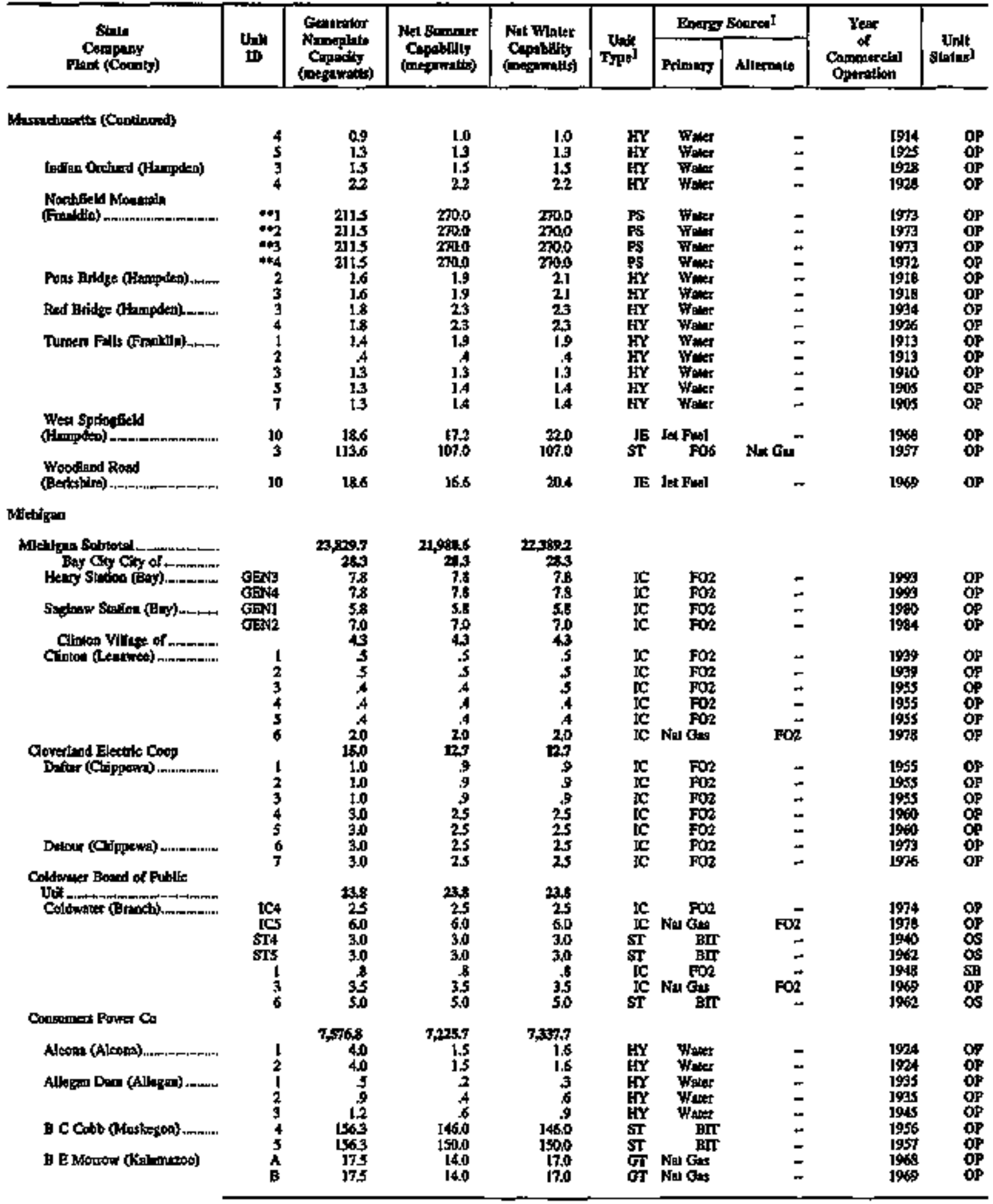

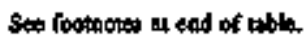


Table 20. Existing Generating Units at U.S. Electric Utilities by State, Company, and Plant, as of January 1, 1996 (Continued)

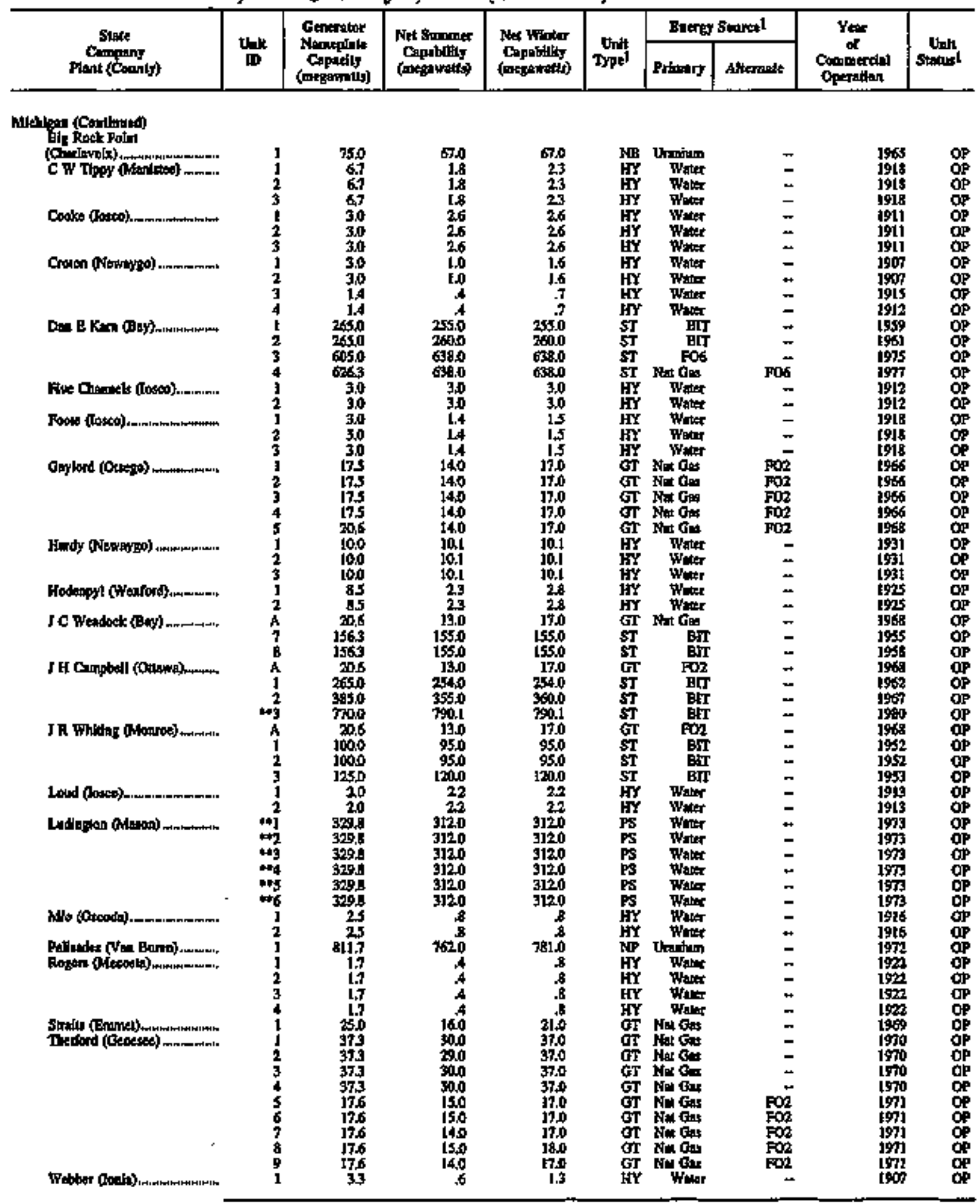

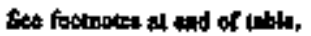


Table 20. Existing Generating Units at U.S. Electric Utilities by State; Company, and Piant, as of January 1, 1996 (Continued)

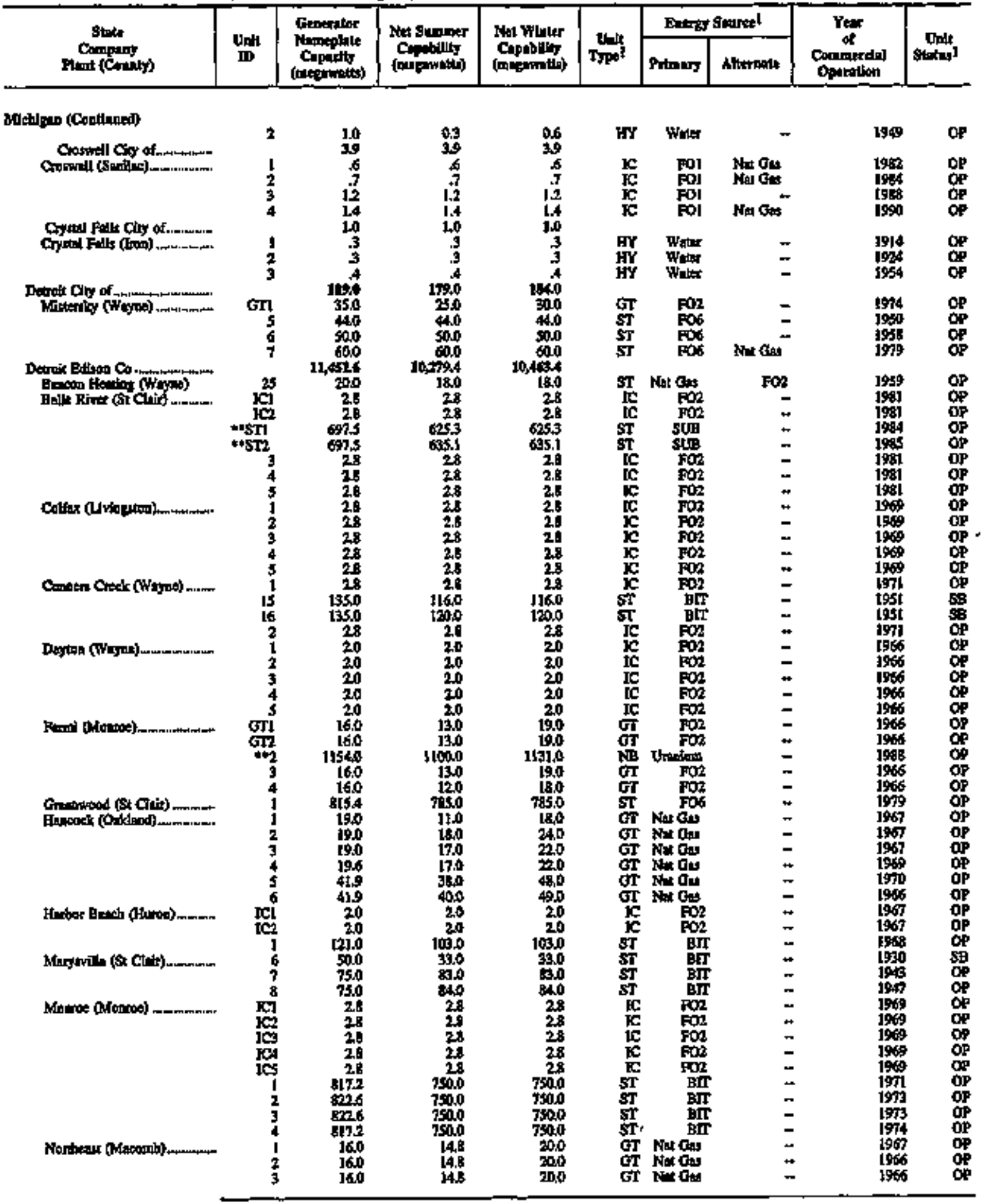

Sop foctinotis at wod of toble.

134 Bnergy Information Administration/Ioventory of Power Plants in the United States as of January 1, 1996 
Table 20. Existing Generating Units at U.S. Electric Utilities by State, Company, and Ptant, as of January 1, 1996 (Continued)

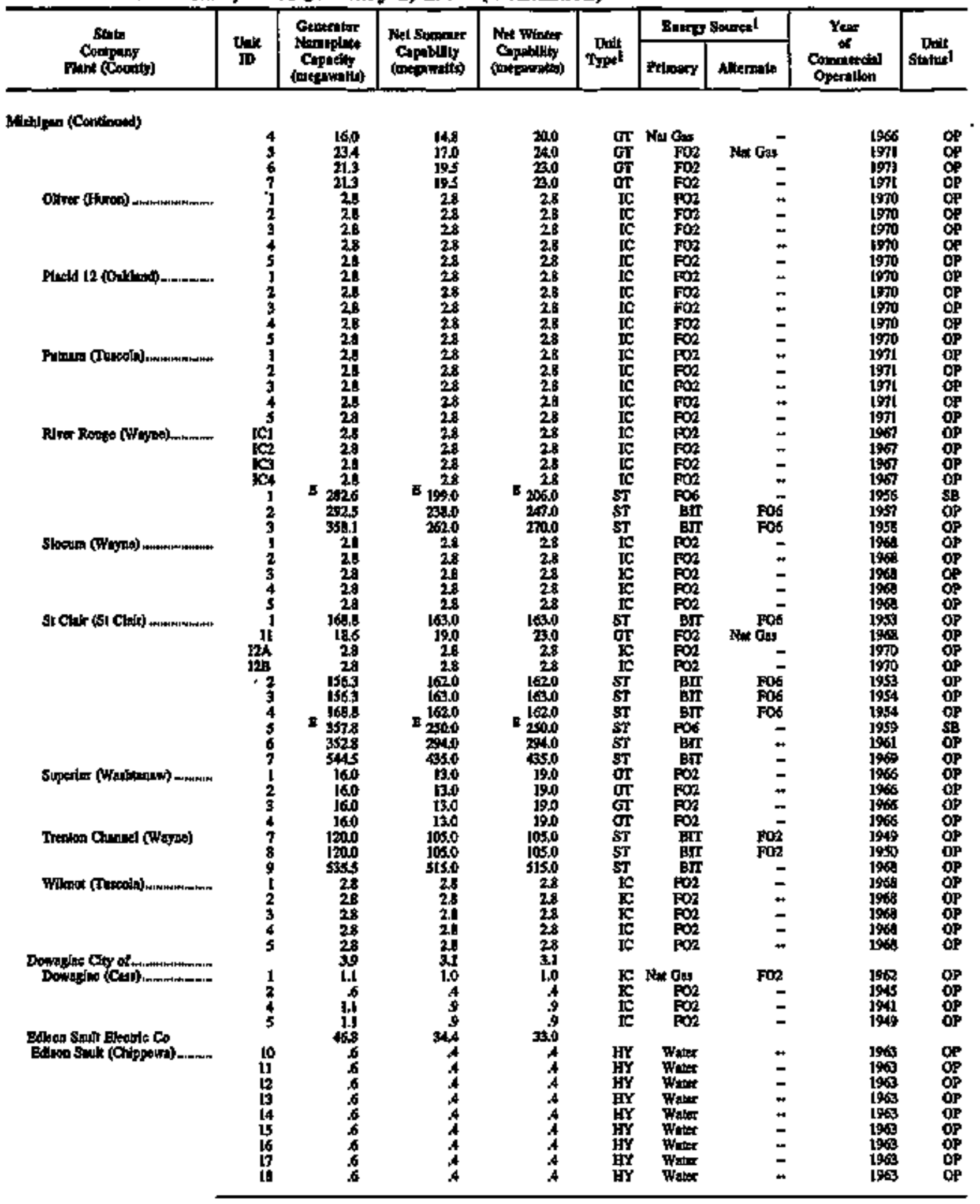

See foonoted at etd of uble. 
Table 20. Existing Generating Units at US. Electric Utilities by State, Company, and Plant, as of Jamuary 1, 1996 (Continued)

\begin{tabular}{|c|c|c|c|c|c|c|c|c|c|}
\hline \multirow{2}{*}{$\begin{array}{c}\text { Siste } \\
\text { Comping } \\
\text { Pland (Corloly) }\end{array}$} & \multirow{2}{*}{ Utit } & \multirow{2}{*}{ 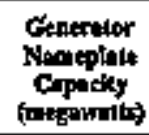 } & \multirow{2}{*}{ 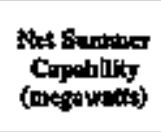 } & \multirow{2}{*}{ 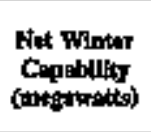 } & \multirow{2}{*}{$\underset{\text { Type }}{\text { Unt }}$} & \multicolumn{2}{|c|}{ Energy Sourcel } & \multirow{2}{*}{$\begin{array}{c}\text { Teur } \\
\text { of } \\
\text { Cominutectiol } \\
\text { Oparacion }\end{array}$} & \multirow{2}{*}{ Shots } \\
\hline & & & & & & Prtinicy & Atterrtote & & \\
\hline 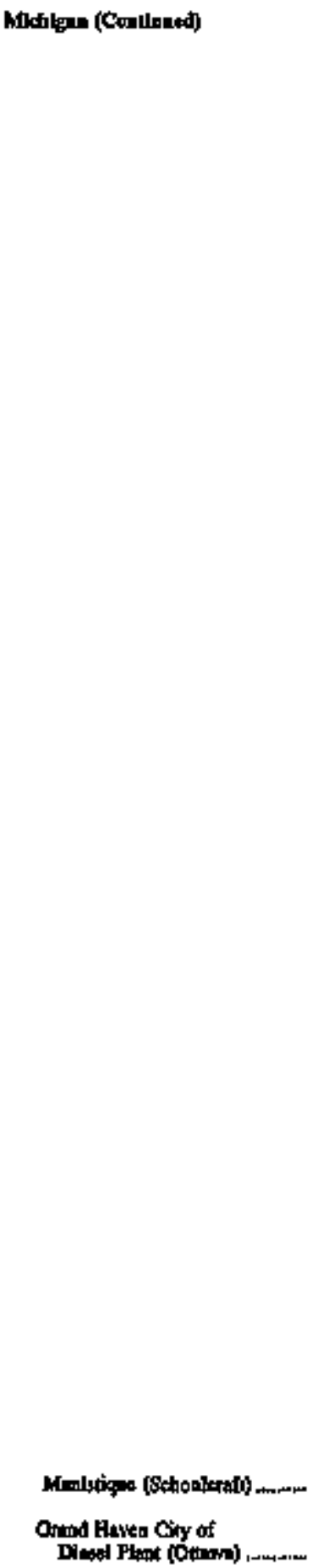 & 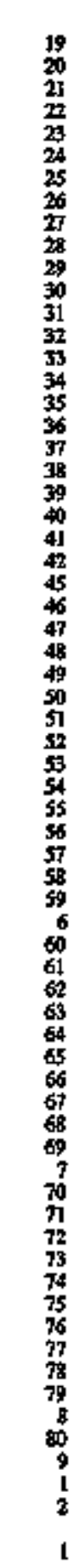 & 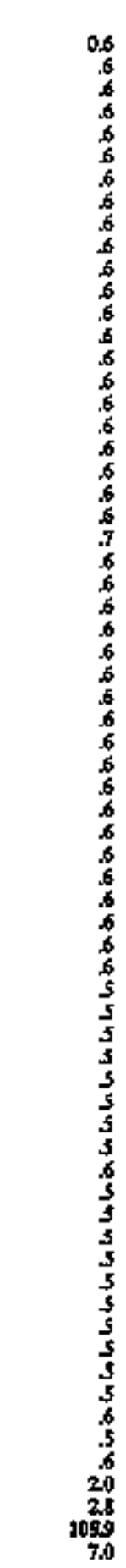 & 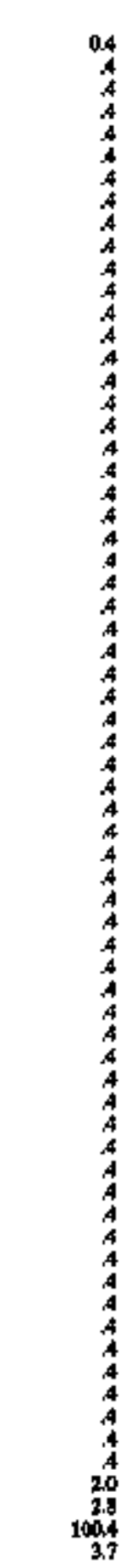 & 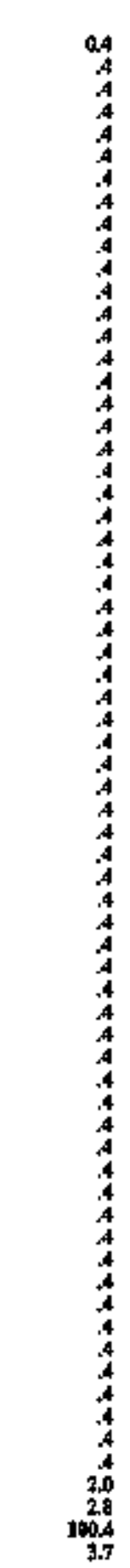 & 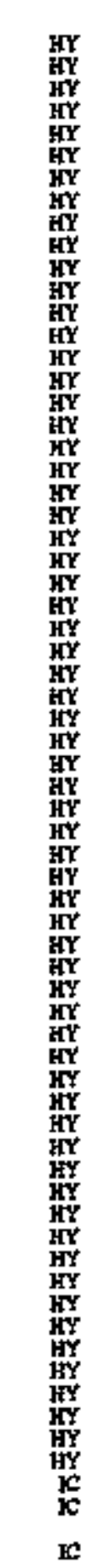 & 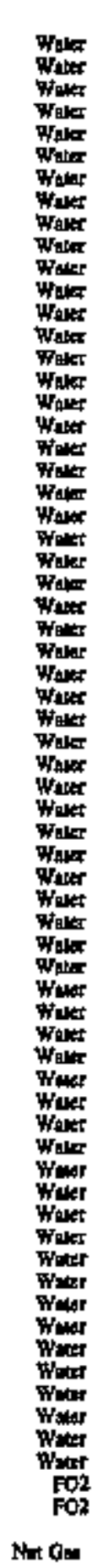 & 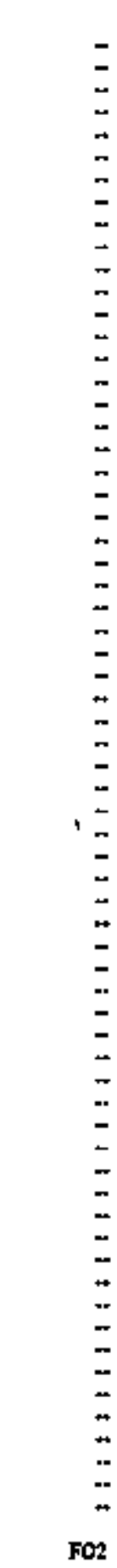 & 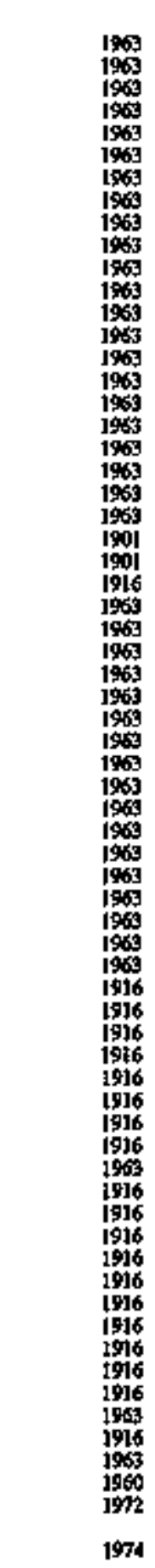 & 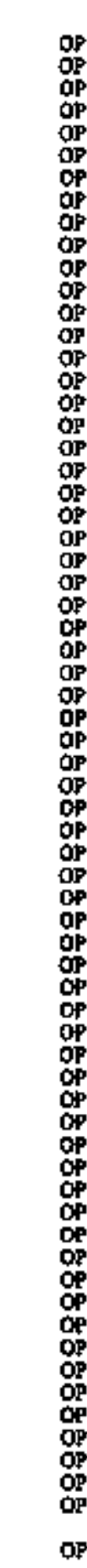 \\
\hline
\end{tabular}

soe footingtat al end of lable. 
Table 20. Existing. Generating Units at U.S. Electric Utilities by State, Company, and Plank, as of January 1, 1996 (Continued)

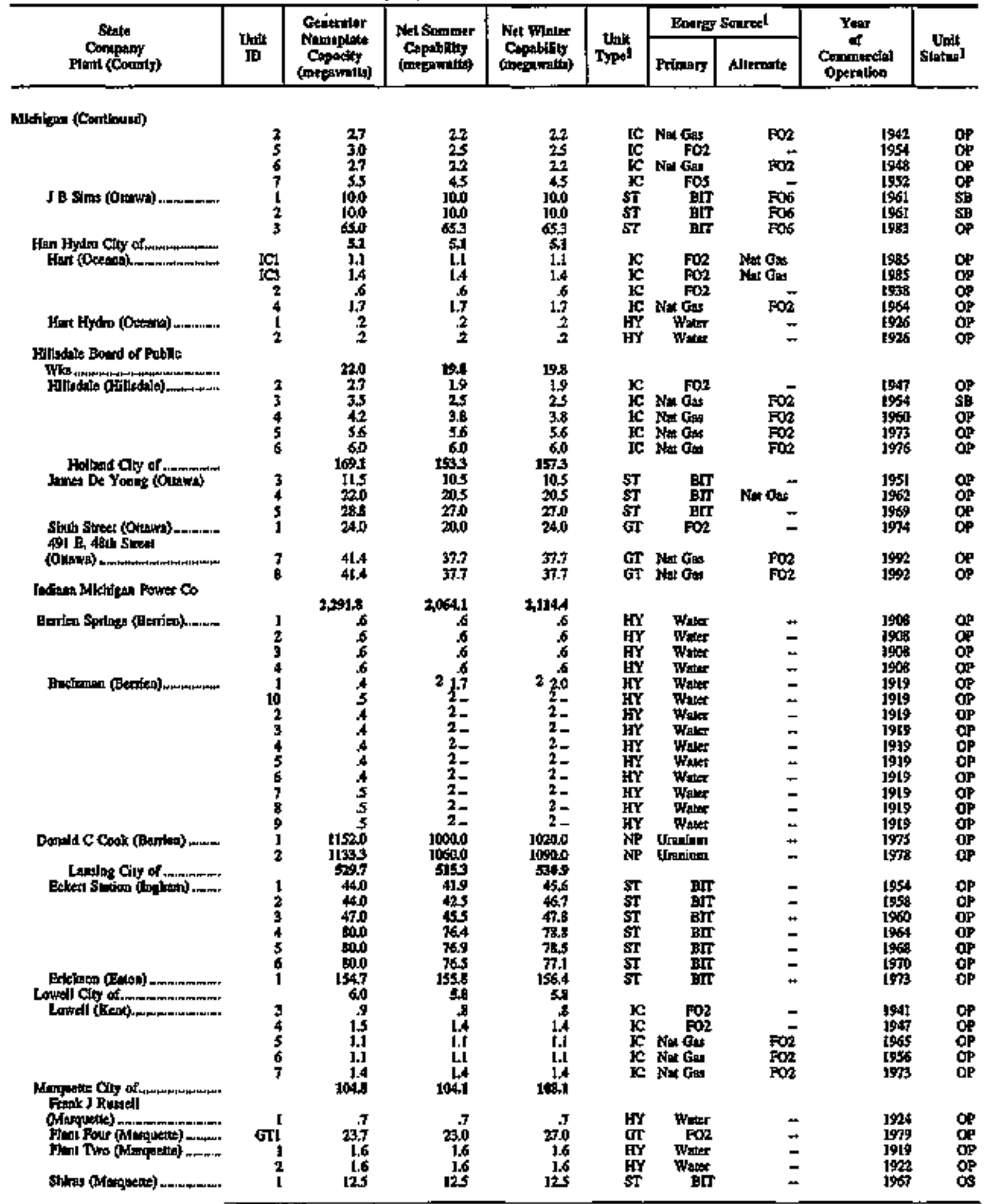

sod footinowe ar ted of thble 
Table 20. Existing Generating Units at U.S. Electric Utillties by State, Company, and Plant, as of January 1, 1996 (Continued)

\begin{tabular}{|c|c|c|c|c|c|c|c|c|c|}
\hline \multirow{2}{*}{$\begin{array}{c}\text { Sosle } \\
\text { Conpony } \\
\text { Plown (Compty) }\end{array}$} & \multirow{2}{*}{ thit } & \multirow{2}{*}{ 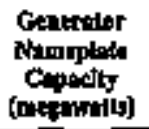 } & \multirow{2}{*}{ 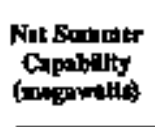 } & \multirow{2}{*}{ 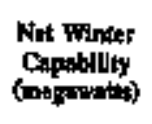 } & \multirow{2}{*}{$\begin{array}{c}\text { Thil } \\
\text { Typel }\end{array}$} & \multicolumn{2}{|c|}{ Earigs Santod } & \multirow{2}{*}{$\begin{array}{c}\text { Year } \\
\text { of } \\
\text { Cammercial } \\
\text { Operation }\end{array}$} & \multirow{2}{*}{ Thing } \\
\hline & & & & & & Priming & Ahemple & & \\
\hline \multicolumn{10}{|l|}{ Midligan (Corllaned) } \\
\hline & $\mathbf{2}$ & 21,5 & $\begin{array}{l}21.0 \\
437\end{array}$ & $\begin{array}{r}21,0 \\
43.7\end{array}$ & $\begin{array}{l}\text { ST } \\
\text { ST }\end{array}$ & $\begin{array}{c}\text { BTT } \\
\text { surs }\end{array}$ & $\bar{z}$ & 192 & OP \\
\hline & & 119 & 10.8 & 10.0 & & & & & \\
\hline Worthell (Cediowo) & 19 & $\begin{array}{l}\mathbf{l}, \mathbf{I} \\
\mathbf{2}\end{array}$ & 19 & .9 & IC & $\underset{\mathrm{EO} 2}{\mathrm{EO} 2}$ & $\begin{array}{l}\text { Nal Gas } \\
\text { Nut Gos }\end{array}$ & 1973 & $\begin{array}{l}\mathrm{OP} \\
\mathrm{OP}\end{array}$ \\
\hline & IC4 & 10 & 3 & .7 & ic & $\mathrm{PO} 2$ & & 3942 & $\stackrel{p p}{o p}$ \\
\hline & ICS & $\begin{array}{l}1.7 \\
5.7\end{array}$ & 5.4 & $\begin{array}{l}1.4 \\
5.6\end{array}$ & $\begin{array}{l}\mathbf{F C} \\
\text { IC }\end{array}$ & $\mathrm{FO2}$ & $\begin{array}{l}\text { Nex Gas } \\
\text { Nat Gay }\end{array}$ & $\begin{array}{l}1948 \\
1978\end{array}$ & $\stackrel{O P}{O P}$ \\
\hline & I & 2 & 3 & .2 & EYY & Wher & - & 1978 & oP \\
\hline & 3 & I & 13 & $\therefore$ & $\mathbf{H Y}$ & Wast & - & 1929 & $\phi p$ \\
\hline 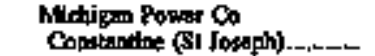 & & 25 & 2.7 & 210 & $\mathbf{H Y}$ & Wher & & & \\
\hline 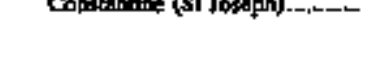 & 2 & $\frac{3}{3}$ & $\begin{array}{l}2= \\
2=\end{array}$ & $\begin{array}{l}21,0 \\
2= \\
2=\end{array}$ & my & Wale & $=$ & 1923 & $\stackrel{O P}{O P}$ \\
\hline & 4 & $\frac{3}{3}$ & $2=$ & $2=$ & $\begin{array}{l}\mathbf{H Y} \\
\mathbf{H Y}\end{array}$ & $\begin{array}{l}\text { Wolter } \\
\text { Wakt }\end{array}$ & $\overline{-}$ & $\begin{array}{l}1999 \\
1993\end{array}$ & $\frac{O P}{O P}$ \\
\hline Motwille (51 Joteph) _...- & i & 4 & 6.9 & 6 & EY & Wayr & - & 1923 & op \\
\hline & 2 & 4 & $8=$ & $6=$ & HY & Wow & $=$ & 1923 & op \\
\hline & & 4 & 6 & $6-$ & EY & War & $\bar{z}$ & 1923 & op \\
\hline \\
\hline 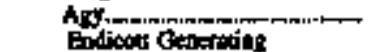 & & 55.6 & 500 & $\mathbf{5 . 0}$ & & & & & \\
\hline 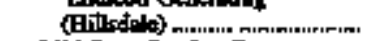 & $t$ & 55,0 & 500 & 35.0 & $\mathbf{S T}$ & BIT & $\mathrm{FO} 2$ & 1982 & DP \\
\hline Mid-Stil Servies Co & & .6 & 5 & 5 & & & & & \\
\hline 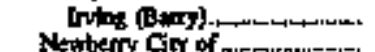 & I & 5.6 & 45 & $\frac{5}{45}$ & HY & Wats & $\omega$ & [9\$0 & DP \\
\hline Newbery (Lues). & 1 & $\mathbf{1 1}$ & 25 & 25 & tc & $\mathrm{FO}$ & - & 1974 & OP \\
\hline & 2 & 7 & 5 & $\frac{5}{4}$ & IC & $\mathrm{FO}$ & 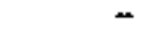 & 1948 & op \\
\hline \multirow{3}{*}{ 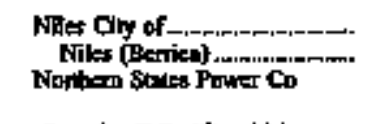 } & & 1.8 & 15 & 15 & IC & $\mathbf{P O O}$ & - & t9:8s & of \\
\hline & $\mathbf{1}$ & 5 & 5 & 5 & HYY & Witer & - & 1928 & OP \\
\hline & & & 19 & 14 & & & & & \\
\hline Superier Fulls (Godetic) _.mm. & 1 & 7 & 1.0 & 10 & HY & & & 1917 & \\
\hline & 2 & .7 & 1.0 & i. 0 & HY & Water & $\overline{-}$ & 1907 & OP \\
\hline \multirow{4}{*}{ 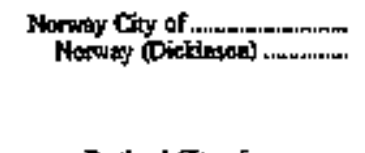 } & & 56 & 47 & 4.7 & & & & & \\
\hline & 1 & 20 & 1,5 & 15 & HYY & Wake & $\ddot{-}$ & 1905 & $\underset{O P}{O P}$ \\
\hline & $\mathbf{2}$ & E 1.2 & 8.1 .2 & $E_{1.1}^{1.2}$ & HY & Wart & $=$ & $\begin{array}{l}1905 \\
198 \%\end{array}$ & $\stackrel{O P}{O P}$ \\
\hline & 4 & B 1.2 & & 8.9 & HY & Witer & - & 1985 & OP \\
\hline \multirow{3}{*}{ 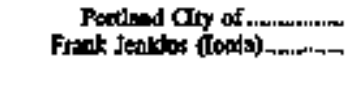 } & & $3 \mathbf{s}$ & 32 & $3 \mathbf{3}$ & & & & & \\
\hline & 3 & 3 & .3 & 3 & $\mathrm{k}$ & Fon & \pm & 1938 & sB \\
\hline & $\begin{array}{l}4 \\
5\end{array}$ & 20 & 1.7 & 1.7 & IE & $\mathrm{FO}_{2}^{+02}$ & Now Gas & 1505 & 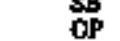 \\
\hline \multirow{2}{*}{ 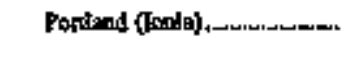 } & 5 & .1 & .1 & .1 & $\mathbf{H Y}$ & water & 2 & 1930 & OP \\
\hline & 2 & $\stackrel{3}{7}$ & 9 & .3 & HYY & Waver & $=$ & [9\$0 & $P$ \\
\hline \multirow{5}{*}{ 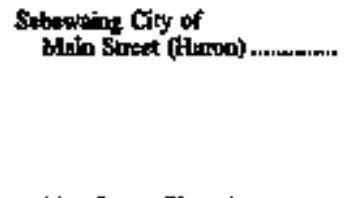 } & $\mathbf{1}$ & $\begin{array}{r}1.7 \\
1.0\end{array}$ & $\begin{aligned} 99 \\
.9\end{aligned}$ & 107 & IC & Nhat Gas & $\mathrm{FO} 2$ & [965] & \\
\hline & 2 & .9 & 8 & .9 & 政 & Fo2 & - & $19 \%$ & op \\
\hline & 3 & 1.1 & $\begin{array}{l}1.1 \\
1.3\end{array}$ & 1.13 & If & Nat Ge & $\mathrm{Fog}_{2}$ & 1966 & op \\
\hline & 5 & t.. & $\begin{array}{l}1.3 \\
1.1\end{array}$ & $\begin{array}{l}1.3 \\
1+1\end{array}$ & IC & Not Gts & $\mathrm{FO2}$ & $\begin{array}{l}1968 \\
1979\end{array}$ & $\underset{\alpha P}{\alpha}$ \\
\hline & 6 & .7 & 6 & .7 & IC & Nar Gut & P02 & 196 & OP \\
\hline \multirow[t]{3}{*}{ 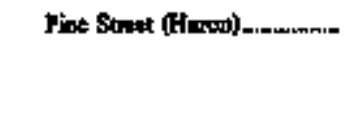 } & i & 1.1 & 1.1 & 1.1 & c & No Ots & FO2 & 1969 & $\alpha p$ \\
\hline & 2 & I.I & $\begin{array}{l}1.1 \\
1.1\end{array}$ & $\mathbf{I + 1}$ & IC & Nol Gas & FO2 & 1969 & $O P$ \\
\hline & 3 & & $\begin{array}{l}1.1 \\
1.1\end{array}$ & I.I & 喿 & $\operatorname{Fon}$ & - & 1984 & Op \\
\hline St Loull Gy of & & 39 & $\begin{array}{l}1.1 \\
3.3\end{array}$ & 3.1 & K & $\mathbf{F O} 2$ & - & 1988 & GP \\
\hline St Lowts (Gondo $)$ & 1 & 1.4 & 1.4 & I. & $\mathbf{k C}$ & FO2 & Nat $G=$ & 1958 & OP \\
\hline & 2 & 7 & 7 & $\ddot{7}$ & 器 & $\mathrm{EO} 2$ & 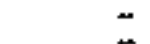 & 1945 & $Q P$ \\
\hline & $\begin{array}{l}3 \\
4\end{array}$ & $\begin{aligned} 1.0 \\
.5\end{aligned}$ & $\begin{array}{r}1,0 \\
5\end{array}$ & 1.0 & 点 & $F_{\mathrm{FO} 2}$ & \pm & $\begin{array}{l}1951 \\
1936\end{array}$ & op \\
\hline & 5 & 2 & 2 & 3 & $\mathbf{H Y}$ & witer & $=$ & 1919 & of \\
\hline Shroit $\mathrm{Ory}$ & 6 & 2 & .2 & .2 & $\mathbf{H Y}$ & Wheth & - & I919 & OP \\
\hline 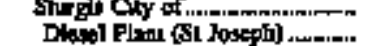 & & 124 & 112 & 11.2 & to & 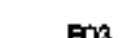 & - & 100 & OP \\
\hline & $\mathbf{z}$ & 3.0 & .8 & .8 & IC & 80 & 5 & 5948 & op \\
\hline & 4 & 1.0 & .6 & 6 & IC & $\mathrm{FO} 2$ & - & 1947 & OP \\
\hline
\end{tabular}

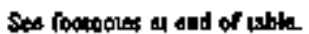


Table 20. Existing Generating Units at U.S. Electric Utilities by State, Company, and Plant, as of January 1, 1996 (Continued)

\begin{tabular}{|c|c|c|c|c|c|c|c|c|c|}
\hline \multirow{2}{*}{$\begin{array}{c}\text { Stato } \\
\text { Conpany } \\
\text { Plont (Contrily) }\end{array}$} & \multirow{2}{*}{ Jutit } & \multirow{2}{*}{ 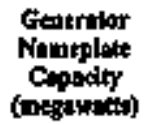 } & \multirow{2}{*}{ 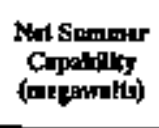 } & \multirow{2}{*}{$\begin{array}{l}\text { Net Whater } \\
\text { Coppotiky } \\
\text { (megenetis) }\end{array}$} & \multirow{2}{*}{ Thalt } & \multicolumn{2}{|c|}{ Bnerga Soureal } & \multirow{2}{*}{$\begin{array}{c}\text { Year } \\
\cdot \text { of } \\
\text { Commeretal } \\
\text { Oparnition }\end{array}$} & \multirow{2}{*}{ Sintt } \\
\hline & & & & & & Prturagy & Alkersale & & \\
\hline \multicolumn{10}{|l|}{ Ittetlagen (Continued) } \\
\hline & 3 & 10 & 0.6 & 0.6 & IC & $F 02$ & & 1947 & op \\
\hline & 6 & 60 & 60 & 60 & Ic & Nat G. & FO2 & 1981 & of \\
\hline \multirow[t]{3}{*}{ Hyato Flan (Sc Jaseph) } & I & 4 & $A$ & 4 & HY & Walat & $=$ & $19 ! 1$ & op \\
\hline & 3 & 8 & . & .8 & भrr & $\begin{array}{l}\text { Want } \\
\text { Wakt }\end{array}$ & $\bar{z}$ & 1998 & op \\
\hline & 4 & $\boldsymbol{8}$ & . & $\$$ & HY $\mathbf{Y}$ & Wow & - & 1983 & QP \\
\hline \multirow{5}{*}{ 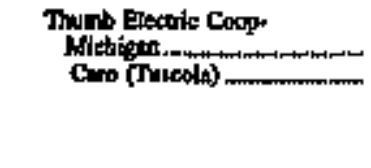 } & & & & & & & & & \\
\hline & & 11.6 & 10.5 & 10.5 & & & & & \\
\hline & $\begin{array}{l}1 \\
2\end{array}$ & $\begin{array}{l}13 \\
13\end{array}$ & $\begin{array}{l}1.0 \\
1.0\end{array}$ & 10 & IC & $\begin{array}{l}\mathrm{FO}_{2} \\
\mathrm{~F} 02\end{array}$ & $\bar{z}$ & 1949 & $\underset{Q P}{Q P}$ \\
\hline & 3 & 13 & 1.0 & is & ic & Fon & $\overline{-}$ & 1952 & oP \\
\hline & 4 & 15 & 1.5 & 1.5 & IC & $\mathbf{F O 2}$ & - & 1984 & OP \\
\hline \multirow[t]{5}{*}{ 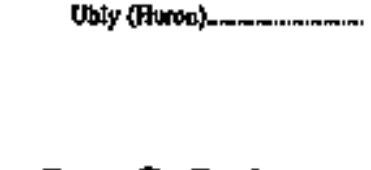 } & $\mathbf{I}$ & 6 & 6 & 8 & IC & 702 & - & 1935 & OP \\
\hline & 3 & .7 & .7 & $\frac{5}{7}$ & IC & $\begin{array}{l}F 02 \\
F 02\end{array}$ & $\bar{z}$ & $\begin{array}{l}1983 \\
1938\end{array}$ & $\stackrel{O P}{Q P}$ \\
\hline & 4 & $=10$ & 10 & 1.0 & ic & For & - & 1947 & 䒠 \\
\hline & $\mathbf{5}$ & E 26 & E 15 & $E_{15}$ & IC & FO2 & $=$ & 1987 & op \\
\hline & 6 & $\begin{array}{l}15 \\
320\end{array}$ & 13 & $\begin{array}{l}15 \\
363\end{array}$ & IC & Nat Gas & $\mathbf{F 0 2}$ & 1993 & op \\
\hline \multirow{4}{*}{ 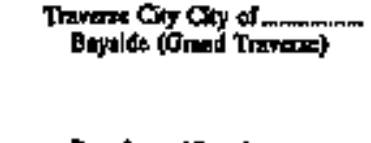 } & 1 & 25 & 3.j & 3.1 & ST & BRT & $=$ & 1946 & $O P$ \\
\hline & $i$ & 50 & 6.] & 6.1 & $\mathbf{s T}$ & BIT & - & joss & op \\
\hline & 3 & 73 & 96 & 9.6 & ST & Net Gis & $=$ & 1954 & $\mathrm{OP}$ \\
\hline & 4 & 140 & 15.] & IS.1 & ST & BT & $r$ & 1968 & OP \\
\hline \multirow{3}{*}{ 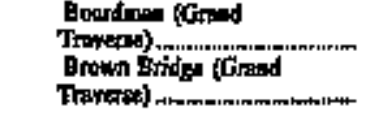 } & HC1 & [.0 & $\boldsymbol{g}$ & .9 & HYY & Water & - & 1985 & op \\
\hline & & & & & & & & & \\
\hline & 3 & 4 & 3 & 4 & HY & Water & - & 1921 & OP \\
\hline 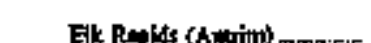 & 2 & 3 & 3 & -4 & $\begin{array}{l}\mathrm{BY} \\
\mathbf{H Y}\end{array}$ & Whiter & $\ddot{z}$ & 1921 & op \\
\hline 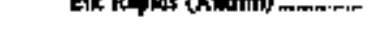 & $\rightarrow+4$ & 4 & 2 & 2 & HY & Woter & - & 1984 & or \\
\hline 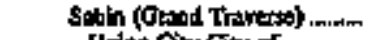 & HCI & 5 & 4 & 3 & HY & Water & - & 1985 & OP \\
\hline 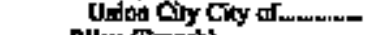 & & 13 & 13 & 13 & & & & & \\
\hline 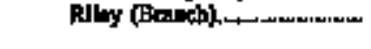 & 1 & $\frac{3}{3}$ & 3 & .3 & HY & Winter & $\overline{-}$ & 10,22 & $\stackrel{o p}{o p}$ \\
\hline Uston Chy (Brueh)...minmm & 1 & 3 & 3 & .3 & IC & $\mathrm{FO2}$ & + & 1941 & OP \\
\hline & 2 & 3 & 3 & 3 & c & FÖ & - & 1941 & OP \\
\hline 1 牧 & 3 & $\mathbf{3}$ & 3 & 3 & tc & FO2 & - & 1941 & OP \\
\hline 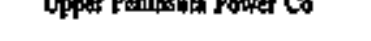 & & 116.5 & 127.6 & 1278. & & & & & \\
\hline MuTria (AI & 1 & & 5 & .5 & HY & Whar & - & 1968 & op \\
\hline 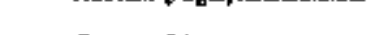 & $i$ & 5 & .6 & $\mathscr{E}$ & EY & Water & . & 1988 & op \\
\hline 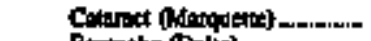 & $\mathbf{1}$ & 20 & 15 & 1.5 & HY & Water & - & 1958 & OP \\
\hline 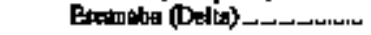 & *11 & IIs & 13.] & I3.1 & ST & EIT & - & $195 \%$ & $O P$ \\
\hline & +2 & ] 5 & 13.2 & 13.2 & $\mathbf{s} \mathbf{r}$ & BIT & - & 1965 & OP \\
\hline Gtadthone (Delan) & I & 22.6 & 20.8 & 27.5 & GI & $\mathrm{FO2}$ & - & 1975 & op \\
\hline 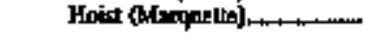 & ! & 10 & 10 & 1.0 & HY & Woter & - & 1958 & OP \\
\hline & $\begin{array}{l}2 \\
3\end{array}$ & 20 & $\begin{array}{l}3.5 \\
1.8\end{array}$ & $\begin{array}{l}15 \\
1.8\end{array}$ & $\begin{array}{l}\text { HY } \\
\text { HY }\end{array}$ & $\begin{array}{l}\text { Watur } \\
\text { Water }\end{array}$ & $\ddot{-}$ & 1998 & op \\
\hline 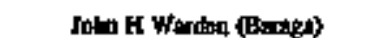 & $\mathbf{j}$ & 18,8 & 17.7 & 17.7 & $S T$ & Sit tes & BII & 1969 & $\mathbf{O P}$ \\
\hline 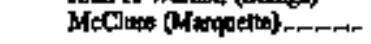 & j & 4.0 & 43 & 43 & HY & Wxts & - & jgts & $O P$ \\
\hline & 2 & 40 & 4.4 & 44 & HY & Water & - & J98s & op \\
\hline Ponts: Gifoughtor) & ] & 226 & 235 & 27.5 & GI & Fon & - & 1973 & op \\
\hline Pricked (Bardpa\}) & $\mathbf{]}$ & t.1 & 1.1 & I.I & HY & Water & $=$ & 1991 & OP \\
\hline & 2 & 1.1 & I.I & I.I & HY & Wath & - & 991 & of \\
\hline 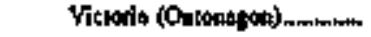 & 1 & 60 & 6.2 & 6.2 & HY & Wotur & - & J931 & op \\
\hline & 2 & 60 & 6.2 & 62 & HY & Water & - & 1931 & $\mathbf{O P}$ \\
\hline 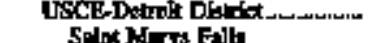 & & & 200 & & & & & & \\
\hline (Chlppowh)._. & & 48 & 53 & 53 & HY & W-ier & - & 1951 & $O P$ \\
\hline & 10 & 20 & 20 & 20 & $\mathbf{H Y}$ & Water & - & 1932 & OP \\
\hline & 2 & 48 & 5.3 & 5.3 & HYY & Water & 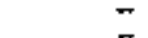 & 1931 & OP \\
\hline & $\$ A$ & 20 & 20 & 20 & $\mathbf{H Y}$ & Witer & $=$ & 1954 & op \\
\hline Wroconin Elachte Power Co & & & & & & & & & \\
\hline & & FIS.8 & 6824 & 6.4.2 & & & & & \\
\hline 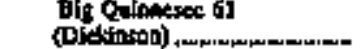 & 4 & 1.8 & ?- & $7-$ & НYY & Watex & • & 1914 & OP \\
\hline & 5 & 1.6 & 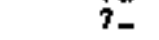 & 7 & KY & Wan & + & 1914 & OP \\
\hline
\end{tabular}

Sec focthotes al and of Ioble. 
Table 20. Exdsting Generating Units at U.S. Electric UTtilities by State, Company, and Plant, as of Jamuary 1, 1996 (Continued)

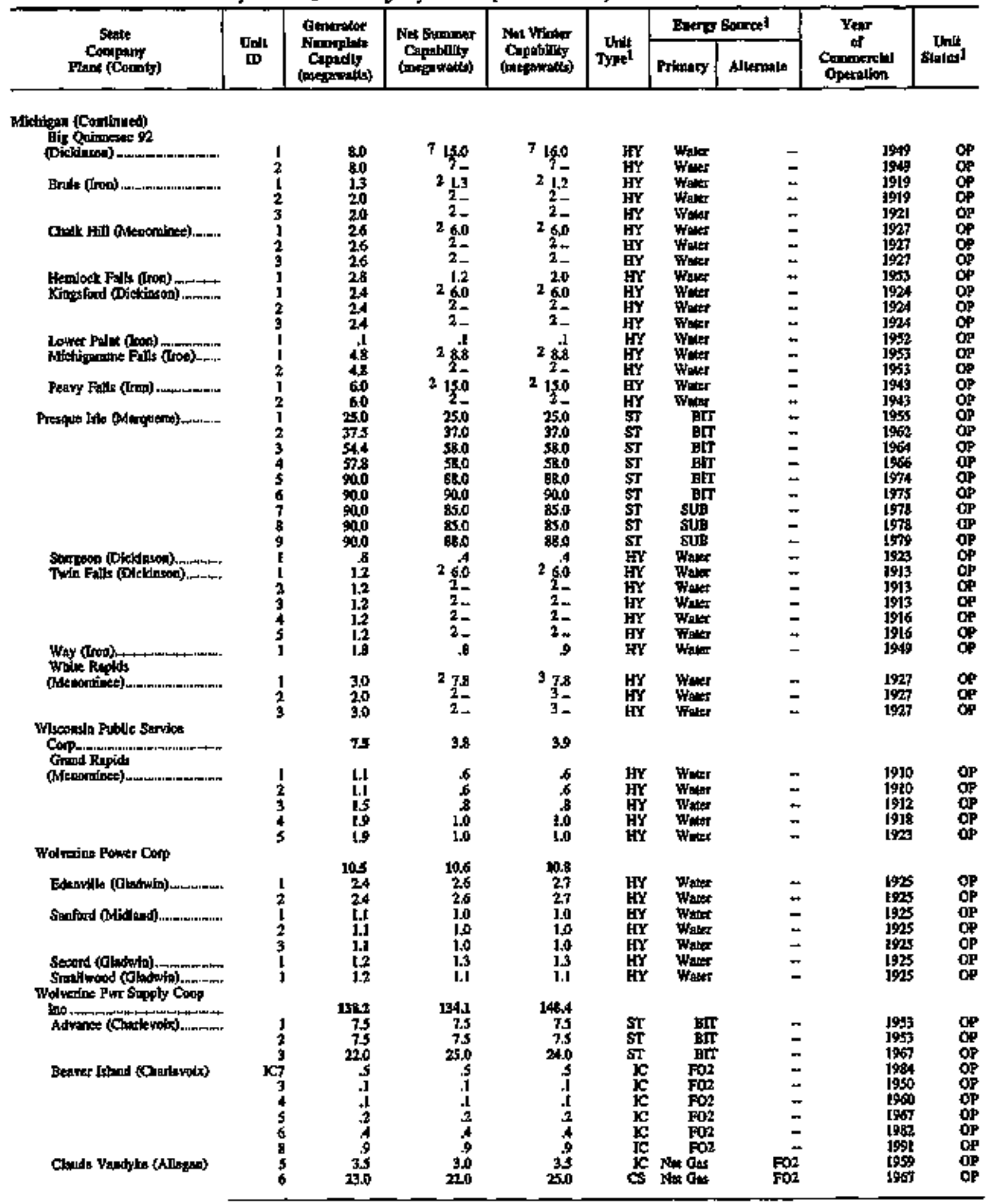

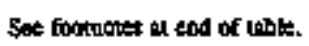


Table 20. Existing Generating Units at U.S. Electric Utillites by State, Company, and Plant, as of January 1, 1996 (Continued)

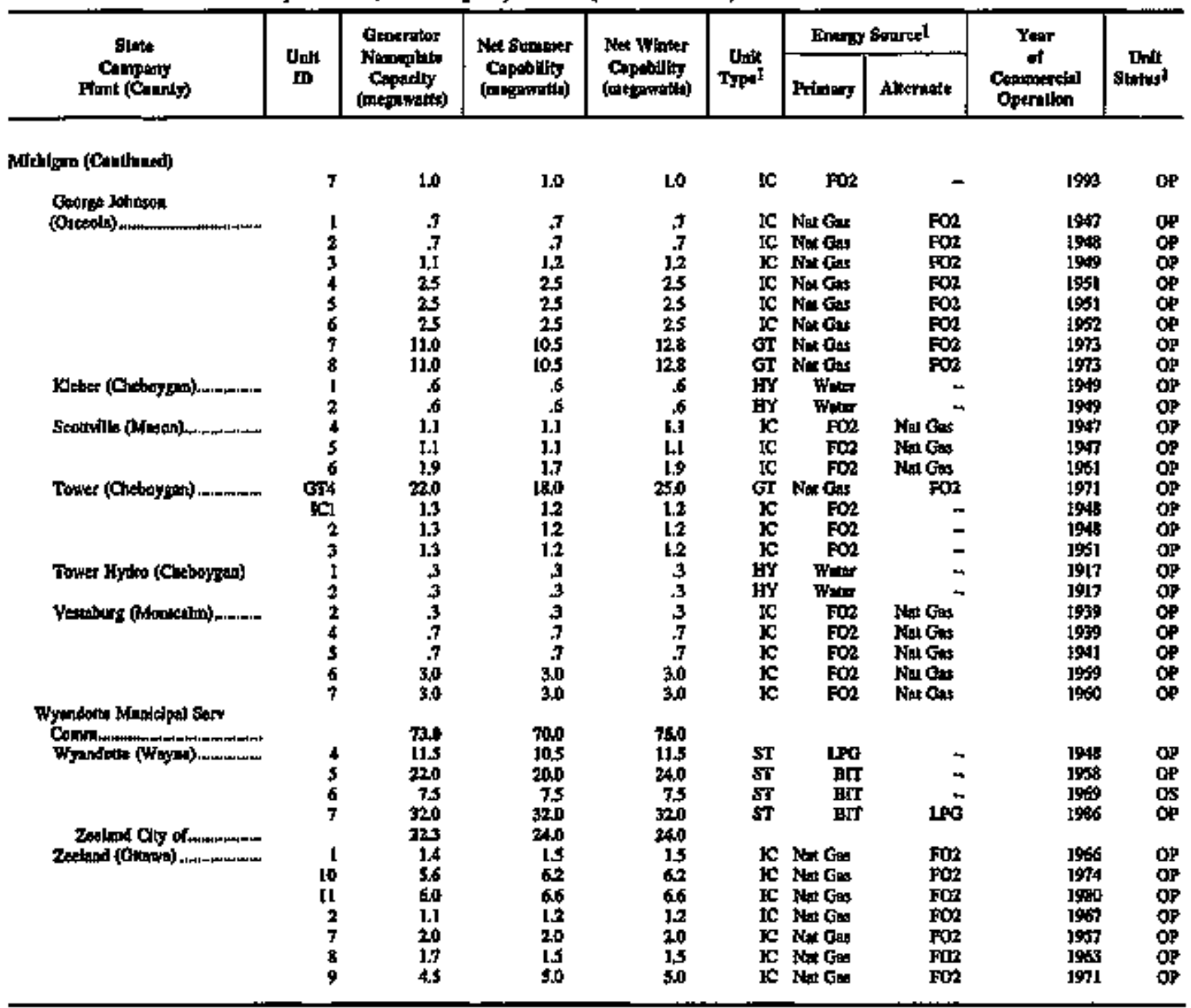

Sect toonotes ate end of ablo. 
Table 20. Existing Generating Units at U.S. Electric Utilties by State, Company, and Plant, as of January 1, 1996 (Continued)

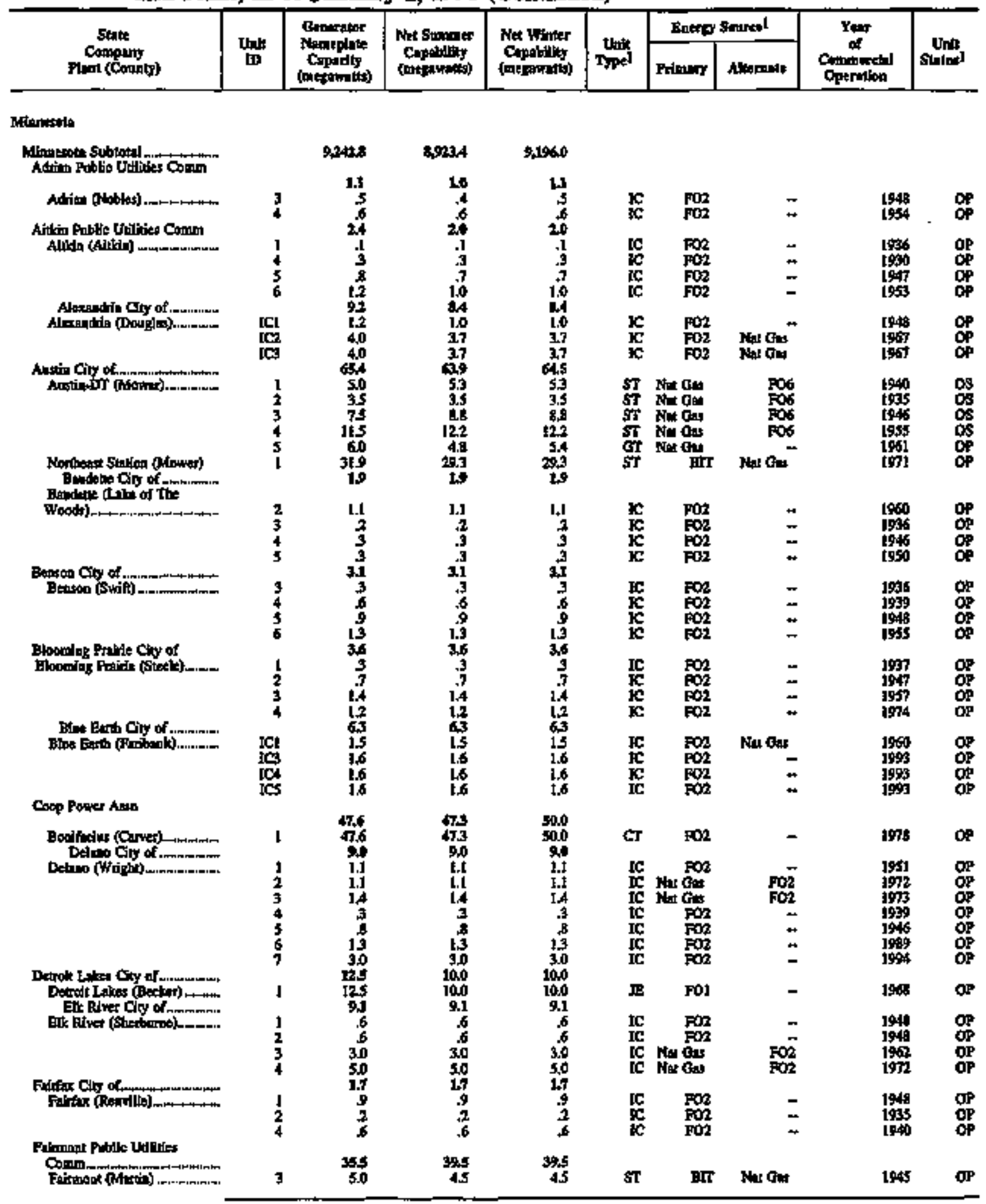

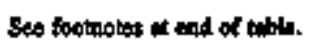


Table 20. Existing Generating Units at U.S. Electric Utilities by State, Company, and Plant, as of January 1, 1996 (Continued)

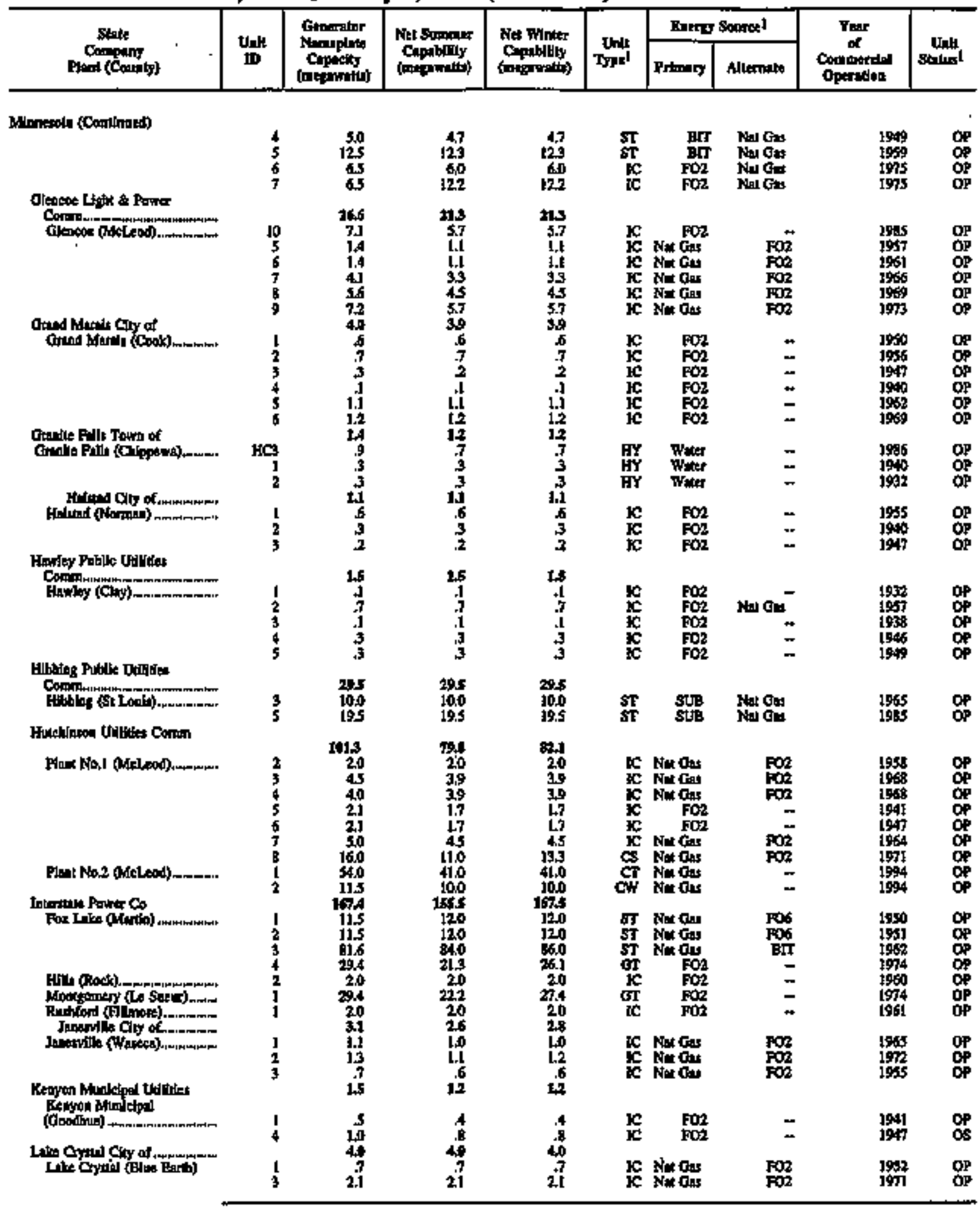

Seo soomolis at end of oble. 
Table 20. Existing Generating Units at U.S. Electric Utilities by State, Company, and Plant, as of January 1, 1996 (Continued)

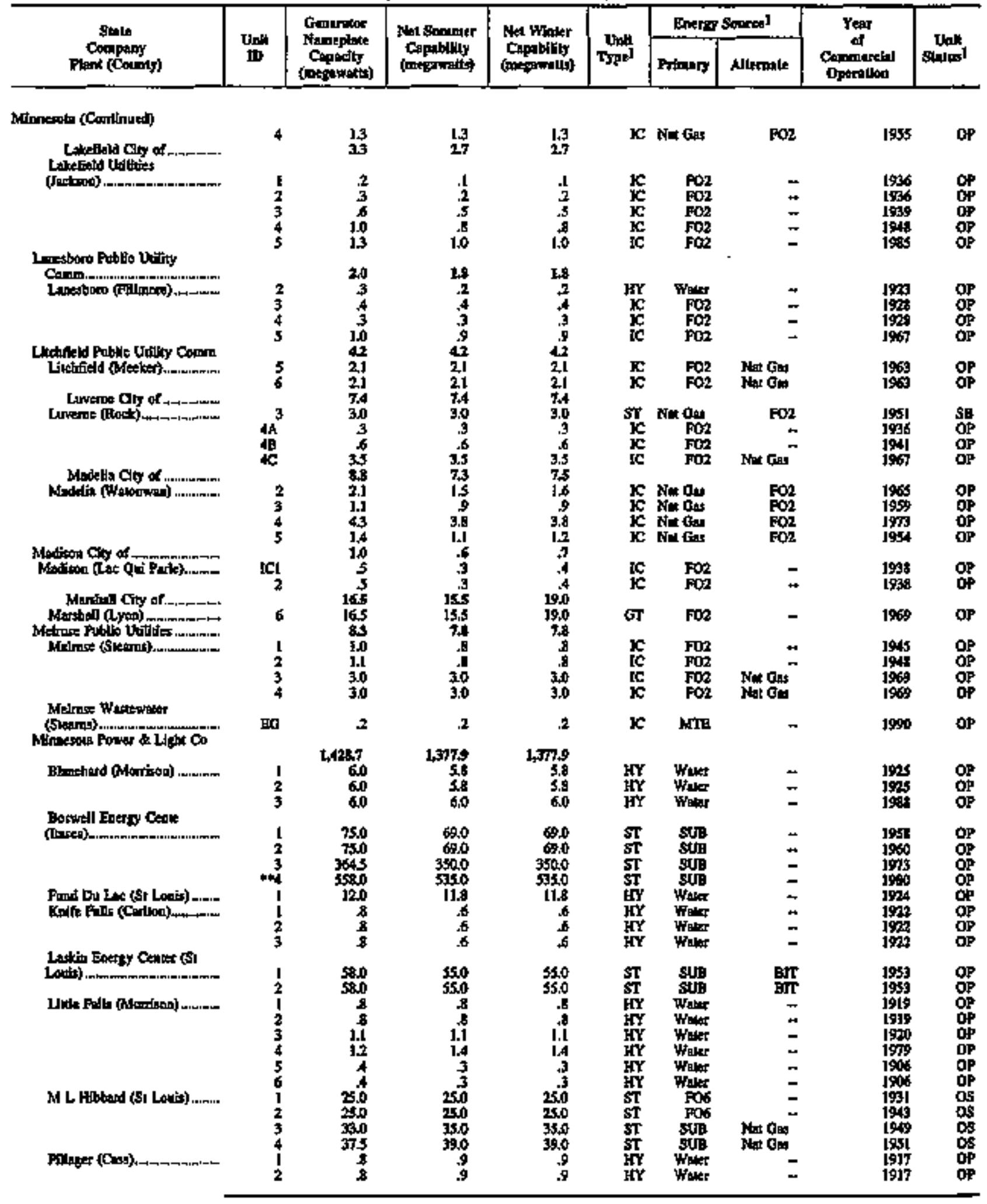

Sec footnotes at end of lable 
Table 20. Existing Generating Units at U.S. Electric Utilities by State, Company, and Plant, as of Jamuary 1, 1996 (Continned)

\begin{tabular}{|c|c|c|c|c|c|c|c|c|c|}
\hline \multirow{2}{*}{$\underset{\text { Sonte }}{\text { Congy }}$} & \multirow{2}{*}{$\begin{array}{l}\text { thit } \\
\text { JD }\end{array}$} & \multirow{2}{*}{ 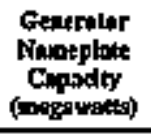 } & \multirow{2}{*}{ 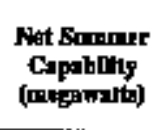 } & \multirow{2}{*}{ 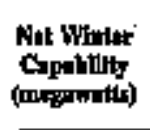 } & \multirow{2}{*}{$\underset{\text { Typt }}{\text { Und }}$} & \multicolumn{2}{|c|}{ Bntry Sorropl } & \multirow{2}{*}{$\begin{array}{c}\text { Year } \\
\text { of } \\
\text { Commitrial } \\
\text { Operation }\end{array}$} & \multirow{2}{*}{ 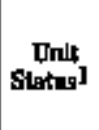 } \\
\hline & & & & & & Primong & Alementh & & \\
\hline \multicolumn{10}{|l|}{ Minmespola (Coptitmued) } \\
\hline 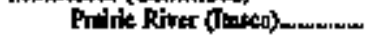 & 1 & 0.7 & 0.5 & os & frY & Yrater & - & 12020 & op \\
\hline & $\mathbf{z}$ & 4 & $A$ & 4 & HY & Wave & - & 1920 & OP \\
\hline 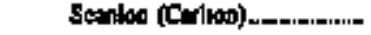 & 1 & 4 & 4 & $A$ & HY & Waks & + & 1923 & OP \\
\hline & 2 & 4 &.$A$ & .4 & $\begin{array}{l}\text { HYY } \\
\boldsymbol{H Y \gamma}\end{array}$ & $\begin{array}{l}\text { Wart } \\
\text { Waver }\end{array}$ & - & $\begin{array}{l}1923 \\
1923\end{array}$ & $\begin{array}{l}\text { OP } \\
\text { OP }\end{array}$ \\
\hline & 4 & 4 & .4 & .4 & $\mathbf{H Y}$ & What & ـ & 1923 & OP \\
\hline 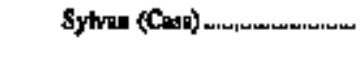 & $\mathbf{3}$ & 6 & 6 & 6 & $\begin{array}{l}\text { fYY } \\
\mathrm{FY}\end{array}$ & War & = & $\begin{array}{l}1913 \\
1913\end{array}$ & op \\
\hline & 3 & .6 & .6 & .6 & 湈Y & Went & 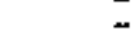 & [915 & OP \\
\hline Thomian (Chyllon) & 1 & $\begin{array}{l}13.0 \\
13.0\end{array}$ & 125 & 125 & KY & Warer & $\overline{-}$ & 1907 & op \\
\hline & 3 & 150 & 12.5 & 12.5 & $\mathrm{HY}$ & Wiler & - & I907 & OP \\
\hline & 4 & 108 & 125 & I): & HY & Woler & - & 1914 & OP \\
\hline & 5 & $\begin{array}{l}108 \\
120\end{array}$ & $\begin{array}{l}12.5 \\
12.5\end{array}$ & 125 & $\begin{array}{l}\text { HYर } \\
\boldsymbol{H Y}\end{array}$ & $\begin{array}{l}\text { Wokr } \\
\text { Walur }\end{array}$ & 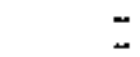 & 1919 & $\underset{\text { OP }}{\text { OP }}$ \\
\hline 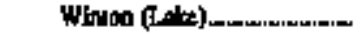 & 2 & 20 & 20 & 20 & HY & Waver & - & 152 & op \\
\hline Hontert City of & 3 & $\frac{30}{350}$ & 20 & 20 & HY & Wakr & - & 1523 & OP \\
\hline Moothesd (Chy).- & 6 & 100 & 63 & Lat & GT & $\mathrm{FO}$ & - & 1961 & OP \\
\hline & 7 & 25.0 & 23.0 & 23.0 & sT & Uo & - & $15 n 0$ & $\mathbf{S B}$ \\
\hline \multicolumn{10}{|l|}{ 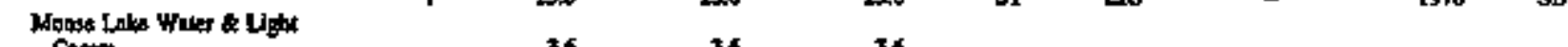 } \\
\hline 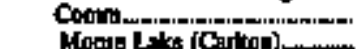 & & 36 & $\begin{array}{l}\mathbf{3 . 6} \\
\mathbf{1 3}\end{array}$ & 3.4 & & & & & \\
\hline 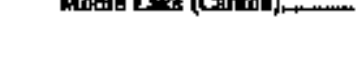 & $\begin{array}{l}2 \\
4\end{array}$ & $\begin{array}{l}1.3 \\
1.0 \\
13\end{array}$ & $\begin{array}{l}1.3 \\
1.0 \\
1.3\end{array}$ & 1.0 & 皆 & Nim & $\mathrm{FO}_{2}$ & 1958 & of \\
\hline Mort City ol _........ & & 139 & 12,4 & 13,1.1 & & & & & \\
\hline Morn (k nabos) & 2 & 1.1 & 9 & 9 & $\mathbf{c}$ & Now Gas & $\mathrm{FO} 2$ & t957 & OP \\
\hline & 5 & 58 & 5.7 & 5,7 & x & Nod Ga & $\mathrm{FO} 2$ & $\mathrm{~km}$ & OP \\
\hline \multirow{2}{*}{\multicolumn{9}{|c|}{ Moundein Lntas Cly of }} & OP \\
\hline & & & & & & & & & \\
\hline (Contorroesd) - - & 1 & .7 & $A$ & 5 & IC & FO2 & - & 1946 & OP \\
\hline & 2 & 1.1 & 10 & $\begin{array}{l}1.1 \\
3\end{array}$ & tc & F02 & 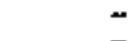 & 1954 & $\mathrm{QP}$ \\
\hline & 3 & 2 & 2 & 2 & [C & F02 & - & 1935 & OP \\
\hline & 4 & $2 t$ & 1.8 & 19 & IC & Fos & - & 1968 & OP \\
\hline & & 1.4 & 1.3 & 1.3 & tc & Fo2 & - & 1959 & OP \\
\hline \multicolumn{10}{|l|}{ Now Frapo Min Chity } \\
\hline New Pronot (te Setol...... & $\mathbf{1}$ & 18.3 & 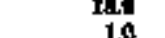 & 1.00 & $\mathbf{C}_{-}+2+2$ & Nonot & EOS & & \\
\hline & 2 & $4, A$ & 44 & 44 & $\mathrm{k}$ & $x+0$ & $\mathrm{Fos}$ & 1978 & OP \\
\hline . & $\overline{\mathbf{3}}$ & 24 & 25 & 25 & $\mathbf{k C}$ & Nat Gar & $\mathrm{FO} 2$ & 1962 & op \\
\hline & 4 & 3.5 & 3.6 & 3.6 & $\mathbf{K C}$ & Nax Gar & Fon & 1968 & OP \\
\hline & $s$ & .6 & .6 &, 6 & 1C. & Nan ats & - & 1944 & oP \\
\hline & 6 & 60 & 5.9 & 5.9 & $\mathrm{k}$ & the out & $\mathrm{FO2}$ & t9e? & OP \\
\hline \multirow{2}{*}{\multicolumn{10}{|c|}{ New Un Publife Utiliten }} \\
\hline 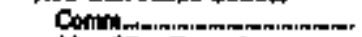 & & \$5.0 & 36.3 & 40 & & & & & \\
\hline 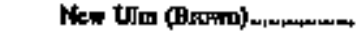 & 3 & 600 & s.1 & 15 & $\boldsymbol{r T}$ & $\operatorname{tint} \mathrm{Gs}$ & ElT & 1967 & OP \\
\hline & 4 & 150 & [3.1 & J3.5 & $\mathbf{s T}$ & Nat Gas & ETT & 1964 & op \\
\hline \multirow{2}{*}{\multicolumn{10}{|c|}{ 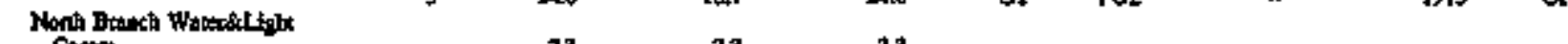 }} \\
\hline comm_- & & & & & & & & & \\
\hline Horlh Brinch (Chisege) $->-$ & $\mathbf{1}$ & .9 & .9 & 5 & $\mathbf{x}$ & $\mathrm{FO} 2$ & Fat $\mathrm{G}_{\mathrm{s}}$ & 1960 & OP \\
\hline & 4 & 1.4 & 1.4 & I.A & $\mathbf{K}$ & FO2 & Nar Ge: & 191 & of \\
\hline 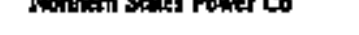 & & $6,521.3$ & 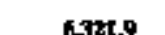 & $6 \mathbf{- 7}, \mathbf{2}$ & & & & & \\
\hline Alim \$ Xlog (Thelpington) & & & & 5ु: 0 & ST & BRT & WD & 1968 & \\
\hline Alline Tech (teanepith.m. & $i$ & 1.6 & 1.6 & 1.6 & It & Fot & Fo2 & 19963 & op \\
\hline Bick Dos (Dikota) & i & 810 & 360 & 640 & sT & EIT & Nox Gass & 1952 & 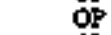 \\
\hline & 2 & 1970 & 100.0 & 101.0 & A & Bis & & 1954 & $\alpha$ \\
\hline & $\mathbf{3}$ & 1140 & 100.0 & 920 & $\mathbf{s T}$ & EIr & Nat Bas & 1955 & $O P$ \\
\hline & 4 & 1900 & 175.0 & 170.0 & \$T & BIT & Nol Gu & 1960 & $\alpha$ \\
\hline 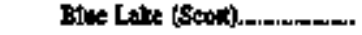 & I & 36.7 & 40 & 60.0 & GT & FO2 & $\rightarrow$ & 1974 & $\alpha$ \\
\hline & 2 & 56.7 & 47.0 & 6000 & GI & $\mathrm{FO}_{\mathrm{O}}$ & $\bar{z}$ & 1974 & OP \\
\hline & 5 & 367 & $\begin{array}{l}47.0 \\
4.0\end{array}$ & $\Leftrightarrow$ & GT & $\mathrm{NO}_{2}$ & $\bar{z}$ & 1979 & ه্p \\
\hline Ornite Cly (Bentoo).......... & t & 18,0 & 15.0 & 20.0 & GT & $\mathbf{F o n}$ & Not Gas & 1969 & OP \\
\hline & $\frac{2}{3}$ & $\begin{array}{l}180 \\
180\end{array}$ & $\begin{array}{l}15.0 \\
15.0\end{array}$ & $\begin{array}{l}20.0 \\
20.0\end{array}$ & $\frac{G T}{\sigma T}$ & $\mathrm{FO}_{2}$ & $\begin{array}{l}\text { Nat Gas } \\
\text { Nax Gay }\end{array}$ & $\begin{array}{l}1969 \\
1969\end{array}$ & op \\
\hline
\end{tabular}

See foolnotes al enó of lable 
Table 20. Existing Generating Units at U.S. Electric Utilities by State, Company, and Plant, as of January 1, 1996 (Continued)

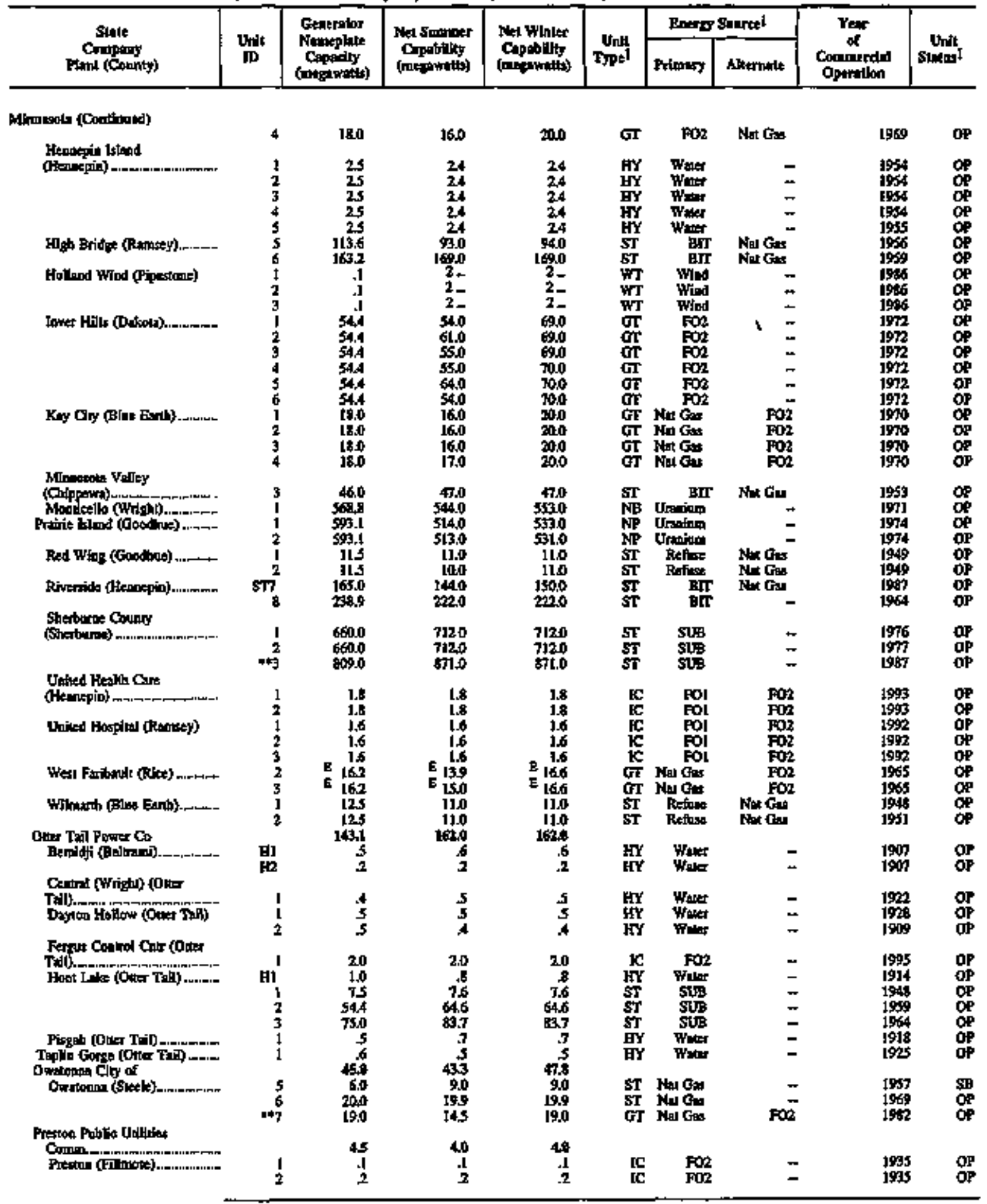

Ses footnotas at end of toble 
Table 20. Existing Generating Units at U.S. Flectric Utilities by State, Company, and Plant, as of January 1, 1996 (Continued)

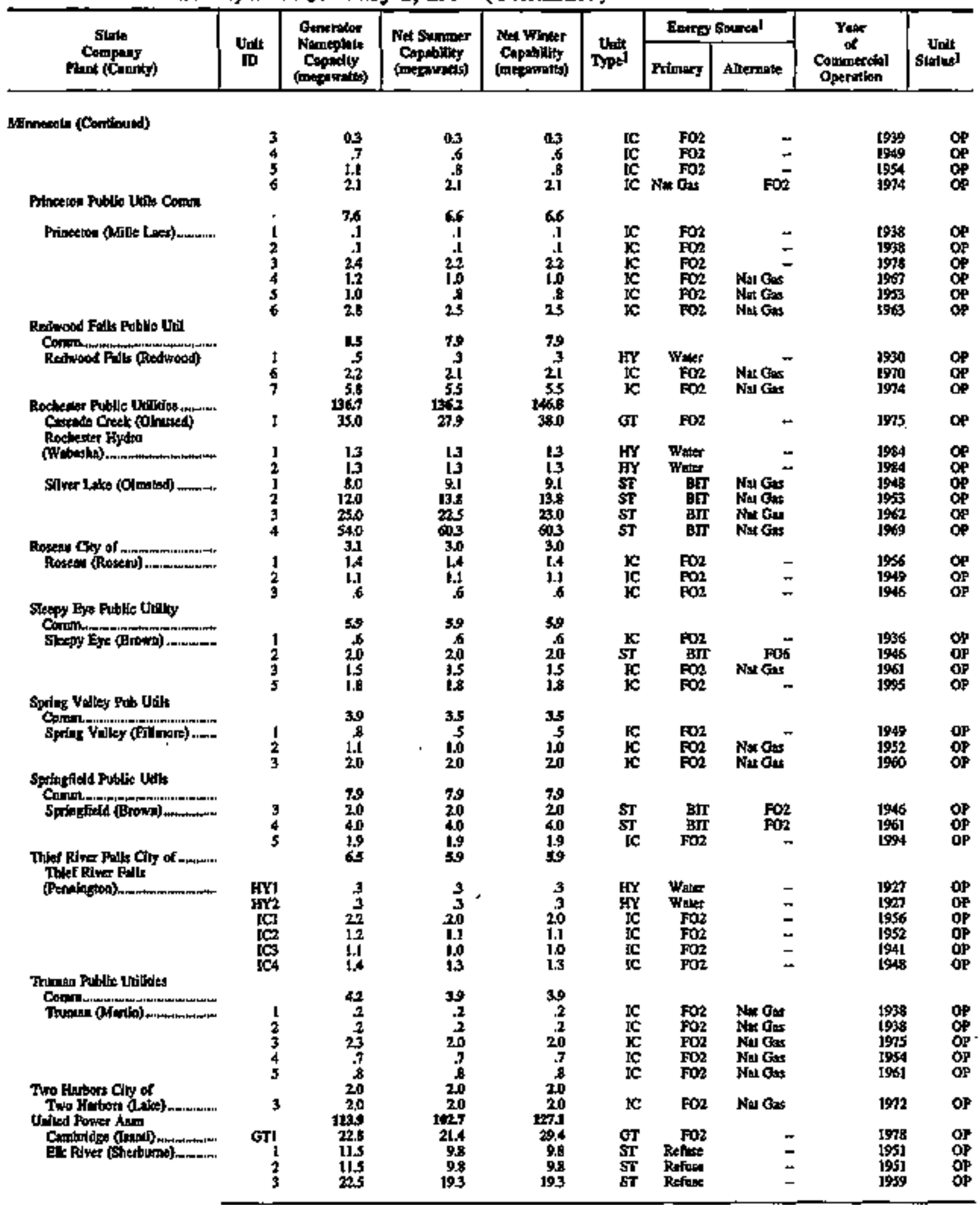

Set fortaoles at and of bite. 
Table 20. Existing Generating Units at U.S. Electric Utillties by State, Company, and Plant, as of January 1, 1996 (Continized)

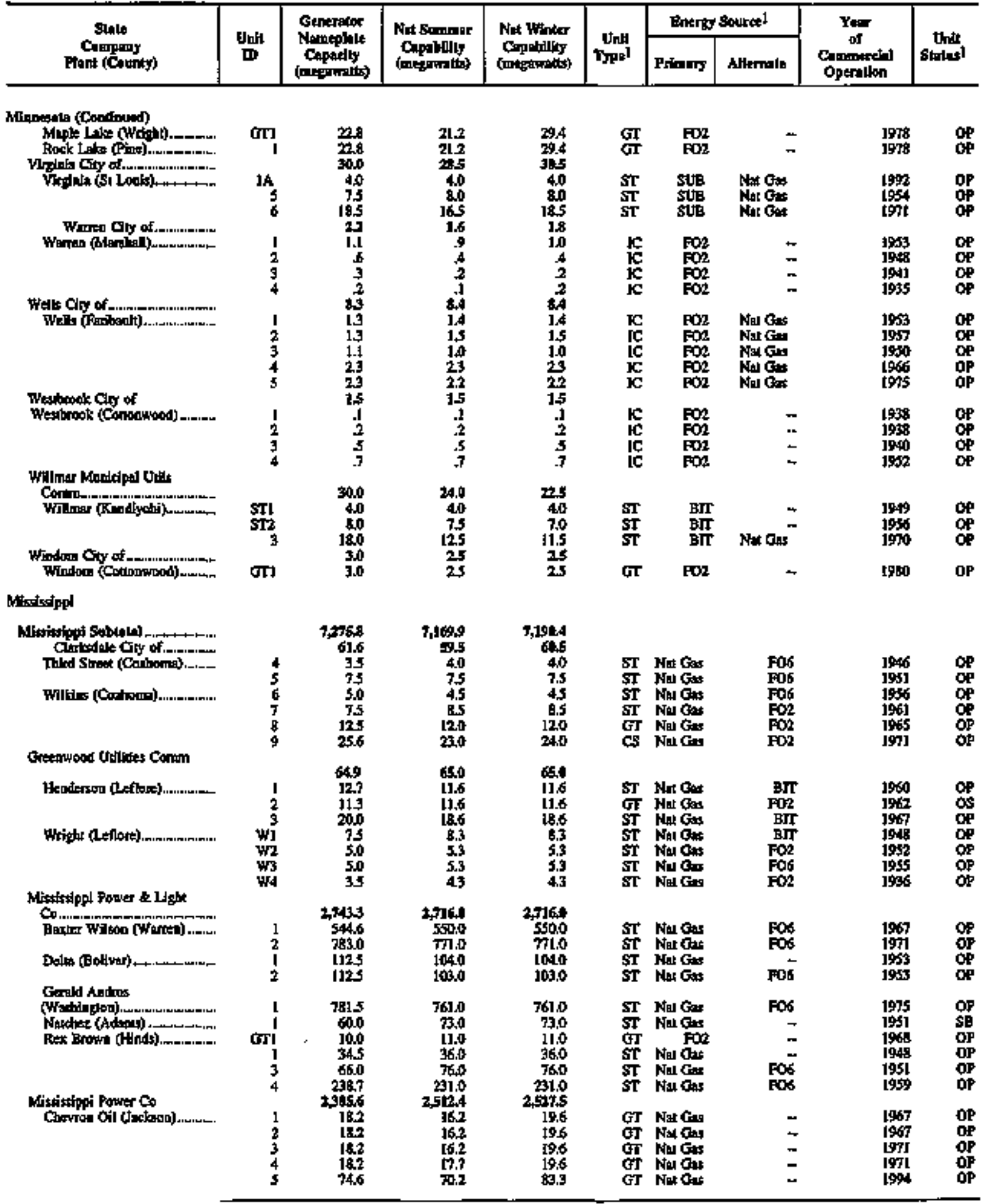

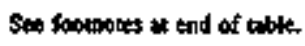


Table 20. Existing Generating Units at U.S. Electric Utilities by State, Company, and Plant, as of January 1, 1996 (Conthmed)

\begin{tabular}{|c|c|c|c|c|c|c|c|c|c|}
\hline \multirow{2}{*}{ 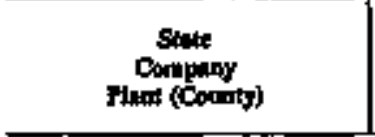 } & \multirow{2}{*}{$\frac{D_{-i *}}{\pi}$} & \multirow{2}{*}{ 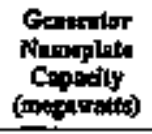 } & \multirow{2}{*}{ 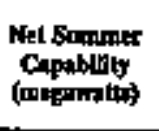 } & \multirow{2}{*}{ 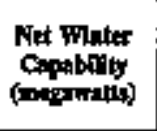 } & \multirow{2}{*}{ Tint } & \multicolumn{2}{|c|}{ Bourb Soores! } & \multirow{2}{*}{$\begin{array}{c}\text { Tear } \\
\text { of } \\
\text { Cominidald } \\
\text { Operallon }\end{array}$} & \multirow{2}{*}{ 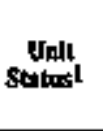 } \\
\hline & & & & & & Ptimary & Ahirmale & & \\
\hline \multicolumn{10}{|l|}{ 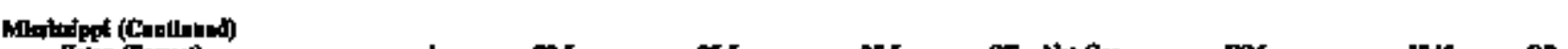 } \\
\hline 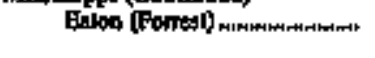 & $\frac{1}{2}$ & 225 & $\begin{array}{l}25,5 \\
25.5\end{array}$ & $\begin{array}{l}255 \\
255\end{array}$ & ST & Not Gas & FO6 & joxs & of \\
\hline & 3 & 225 & 25.3 & 283 & si & Natat & Fos & 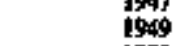 & of \\
\hline 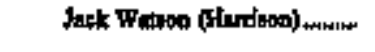 & $A$ & 39.4 & 35.2. & 43,5 & $\underset{S T}{\mathrm{~J}}$ & Nol Ge & $\mathrm{Fon}$ & 1970 & of \\
\hline & $\begin{array}{l}1 \\
2\end{array}$ & 75.00 & 81.7 & $\begin{array}{l}81.7 \\
873\end{array}$ & $\begin{array}{l}S T \\
S T\end{array}$ & 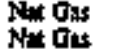 & $\begin{array}{ll}\mathrm{Fot}_{2} \\
\text { Fol }\end{array}$ & $\begin{array}{l}1998 \\
1950\end{array}$ & $\stackrel{O P}{O P}$ \\
\hline & 3 & E120 & 131.6 & 11.6 & sT & Stet Gen & Fos & 192 & $\alpha$ \\
\hline & 4 & 250.0 & 261.3 & 261.8 & $\stackrel{s T}{s T}$ & 照 & Nar Gs: & 1968 & OP \\
\hline 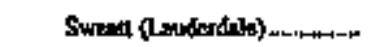 & $\vec{A}$ & 39.4 & 35.0 & 435 & Je & $\sin 0$ & Nod & $\begin{array}{l}1973 \\
6971\end{array}$ & $\frac{O P}{\alpha p}$ \\
\hline & i & 400 & 468 & 468 & $\mathbf{s T}$ & Net Goss & Fos & 1951 & OP \\
\hline Victor J Dared 1 r & 2 & $40: 0$ & 46. & 46.8 & $\mathbf{5 T}$ & Ne Gas & FO6 & 1953 & OP \\
\hline 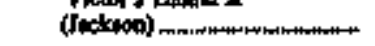 & 1 & 5000 & \$35.5 & $\operatorname{sing}$ & sT & BLF & Fos & $19 m$ & OP \\
\hline & +2 & 300,0 & $\$ 45,4$ & 550,4 & ST & BतT & F06 & 1981 & or \\
\hline \multicolumn{10}{|l|}{ Puote Sen Condm of Yexoo } \\
\hline 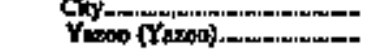 & ori & 3.6 .6 & 14,0 & $\begin{array}{l}386 \\
16,6\end{array}$ & $\boldsymbol{\sigma}$ & Nex Gas & $\mathrm{FO} 2$ & 1968 & of \\
\hline & 2 & 30 & 35 & 62 & $s t$ & $\mathbf{N u n} \tilde{\sigma} \mathbf{s}$ & $\mathrm{FO} 6$ & 1945 & os \\
\hline & & 12.7 & 115 & 12.7 & ST & Ner Gas & Fo6 6 & 1954 & os \\
\hline \multicolumn{10}{|l|}{ South Mindaippi Fl Pwr } \\
\hline & & 6138 & 6130 & 6130 & & & & & \\
\hline 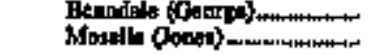 & I & $\begin{array}{l}162 \\
59.0\end{array}$ & $\frac{160}{500}$ & $\begin{array}{l}160 \\
590\end{array}$ & or & Sat $G:$ & $\mathrm{FO}_{\overline{6}}$ & 1959 & $\stackrel{a p}{O P}$ \\
\hline & 2 & 590 & $\$ 00$ & 590 & ST & $\mathrm{Nr} \mathrm{Gon}$ & Fos & Gro & op \\
\hline & 3 & 590 & 99.0 & 590 & $\mathbf{s} \mathbf{T}$ & Nat Gas & FOS & 1910 & of \\
\hline 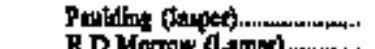 & $\mathbf{l}$ & 20.6 & 200 & 200 & $\mathbf{T}$ & $\mathbf{7 0 2}$ & - & 1972 & gP \\
\hline 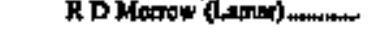 & I & 200.0 & 20060 & 2000 & $\mathbf{S T}$ & $\mathrm{Br}$ & - & 1978 & OP \\
\hline \multicolumn{10}{|l|}{ Sylkm Eneryy Rotomed } \\
\hline 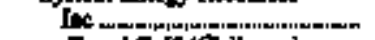 & & $1,372.5$ & 1,1720 & 1,173a & & & & & \\
\hline Grand Gưff (Clalberst) & $\boldsymbol{\bullet + 1}$ & [372, & 11730 & 1173.0 & NB & Utaraffen & - & 19:5 & $\mathbf{O P}$ \\
\hline \multicolumn{10}{|l|}{ Minood } \\
\hline 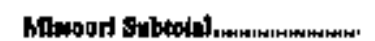 & & $17,053,7$ & 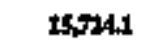 & Is,904as & & & & & \\
\hline Alany Oby of & & 83 & 60 & & & & & & \\
\hline 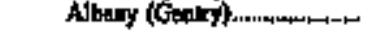 & ICS & 1.2 & 1.2 & 1,2 & IC & $\mathrm{FOZ}$ & 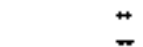 & 1983 & op \\
\hline & 1 & 2.1 & 2.12 & $\begin{array}{l}1.2 \\
2.1\end{array}$ & IC & Fon & $\bar{z}$ & 1969 & $O P$ \\
\hline & 2 & 1.0 & 9,0 & 10 & IC & $\mathbf{F O}$ & $\leftrightarrow$ & 1978 & op \\
\hline & $\overline{3}$ & 8 & & 7 & IC & FO2 & - & 19\$4 & $\mathbf{D P}$ \\
\hline \multicolumn{10}{|l|}{ 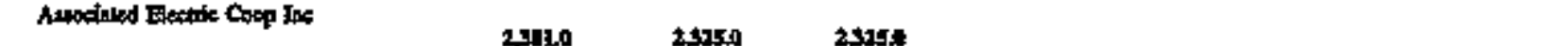 } \\
\hline Now Muddd Nerr Kaddd & 1 & 6000 & 58020 & 5800 & $\mathbf{s t}$ & sur & $*$ & 1972 & OP \\
\hline & 2 & 600,0 & 5800 & 5000 & $S T$ & SUL & - & 197 & of \\
\hline 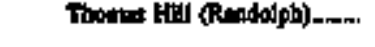 & 1 & & 775.0 & 175.0 & $\mathbf{s T}$ & sile & $\bar{z}$ & 1946 & of \\
\hline & $\frac{2}{3}$ & $\begin{array}{l}285.00 \\
6700\end{array}$ & 273,0 & 275,0 & $\begin{array}{l}\mathbf{s T} \\
\mathbf{s T}\end{array}$ & stis & $\overline{-}$ & $\begin{array}{l}1999 \\
19 \$ 2\end{array}$ & $\stackrel{0 P}{O P}$ \\
\hline Uplarvills (Pabn-m) & 1 & & 25 & 22 & Gr & $\mathrm{PO2}$ & 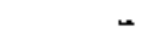 & 1976 & op \\
\hline & $i$ & 23,0 & 225 & 225 & G & FOI & $*$ & 1976 & or \\
\hline Bethoy Cly of-- & 1 & I0.1 & .23 & 98 & c c & $\mathbf{F O} 2$ & - & 19여 & OP \\
\hline & 2 & $\therefore$ & $\$$ & 9 & ch & Fon & 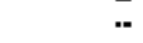 & 1949 & or \\
\hline & 3 & 1.5 & 1.5 & 1.5 & IC & $\mathbf{F O}$ & Nhetsit & 1950 & op \\
\hline & s & 1.8 & 1.6 & 1.3 & 岕 & 002 & Nat Gas & 1981 & OP \\
\hline & 6 & 9 & 9 & $\therefore$ & Ic & Fot & Wat Gat & 1981 & pp \\
\hline & 7 & 1.2 & $\frac{1.2}{12}$ & 1.2 & IC & Foc & 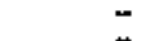 & I9893 & ap \\
\hline 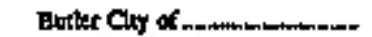 & & 6.t & $\$$ & 44 & & & 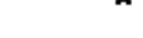 & & \\
\hline Butker $(B=0)$ & IC6 & 1,4 & 1.0 & & & & - & & OP \\
\hline & 1 & 4 & 3 & 3 & If & Fon & - & 1929 & OP \\
\hline & 2 & 7 & $\begin{array}{l}5 \\
\mathbf{6}\end{array}$ & 5 & IC & Fon & $N \in \mathrm{G}_{01}^{-}$ & 1938 & $\alpha$ \\
\hline & 4 & 1.4 & 1.0 & 1.0 & IC & Fo? & tos & 1952 & OP \\
\hline & 5 & 1.4 & 1.0 & 1.0 & X & Fo? & Jan Gs & 1959 & OP \\
\hline Cinplat Oly of.. & & 6.7 & 4 & 62 & & & & & \\
\hline
\end{tabular}

See tootolita at end of toblen. 
Table 20. Existing Generating Units at U.S. Electric Utilities by State, Company, and Plant, as of January 1, 1996 (Continued)

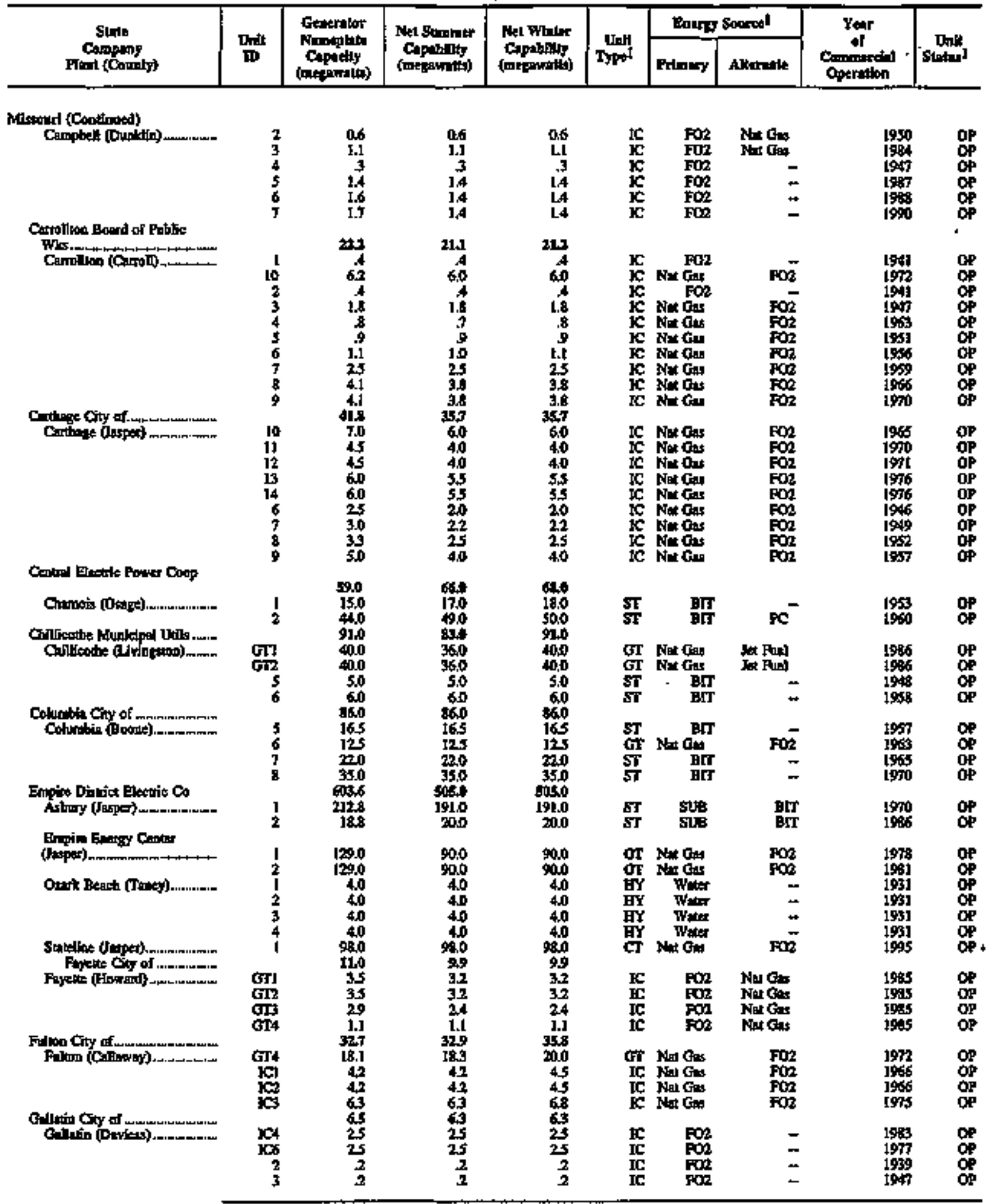

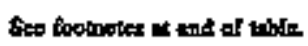


Tahle 20. Existing Generating Units at U.S. Electric Utilities by State, Company, and Plant, as of January 1, 1996 (Continued)

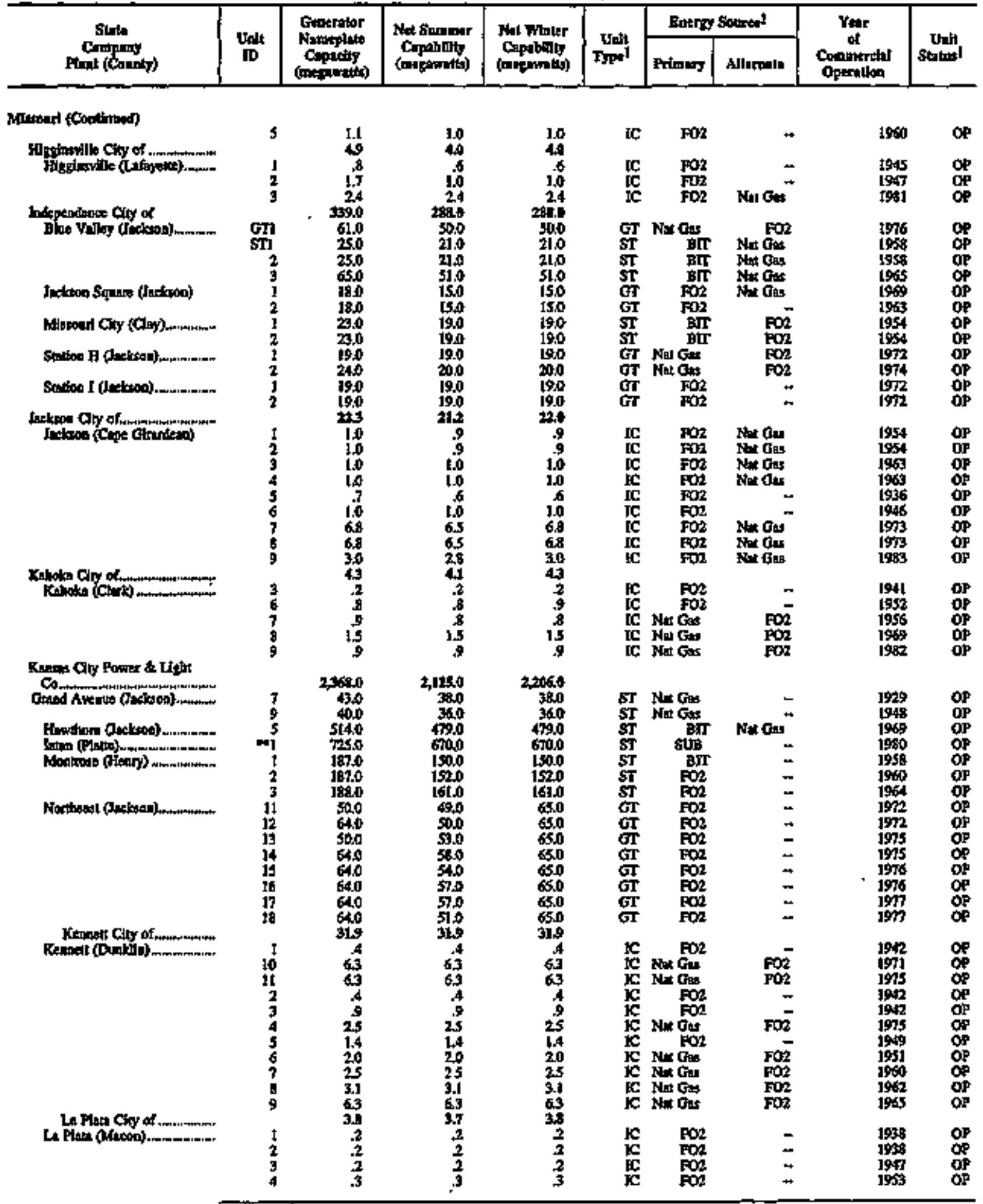

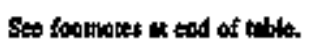


Table 20. Existing Generating Units at U.S. Flectric Utilities by State, Company, and Plant, as of January 1, 1996 (Continued)

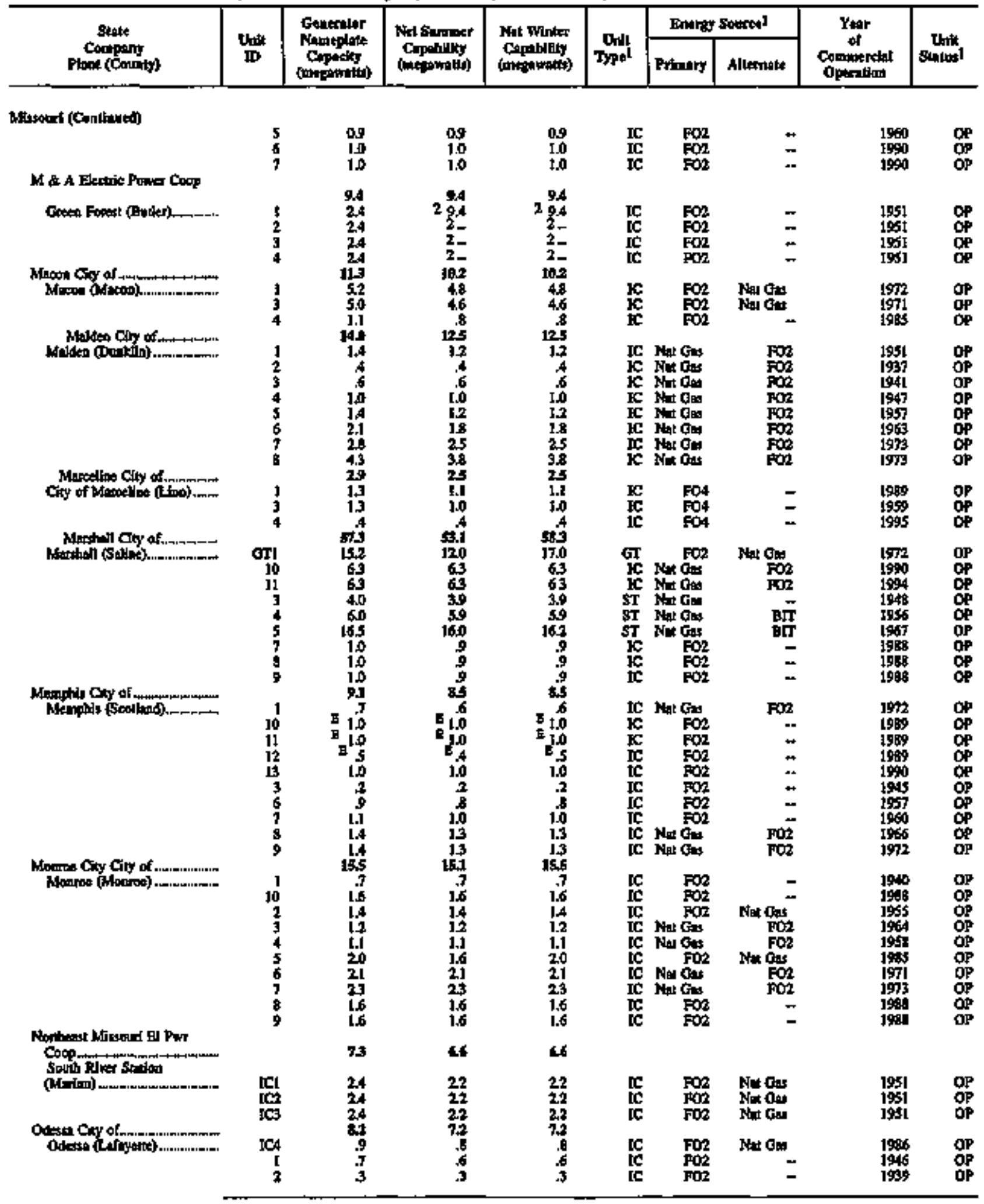

Sec fontoros at end at tible. 
Table 20. Existing Generating Units at U.S. Electric Utilities by State, Company, and Plant, as of January 1, 1996 (Continned)

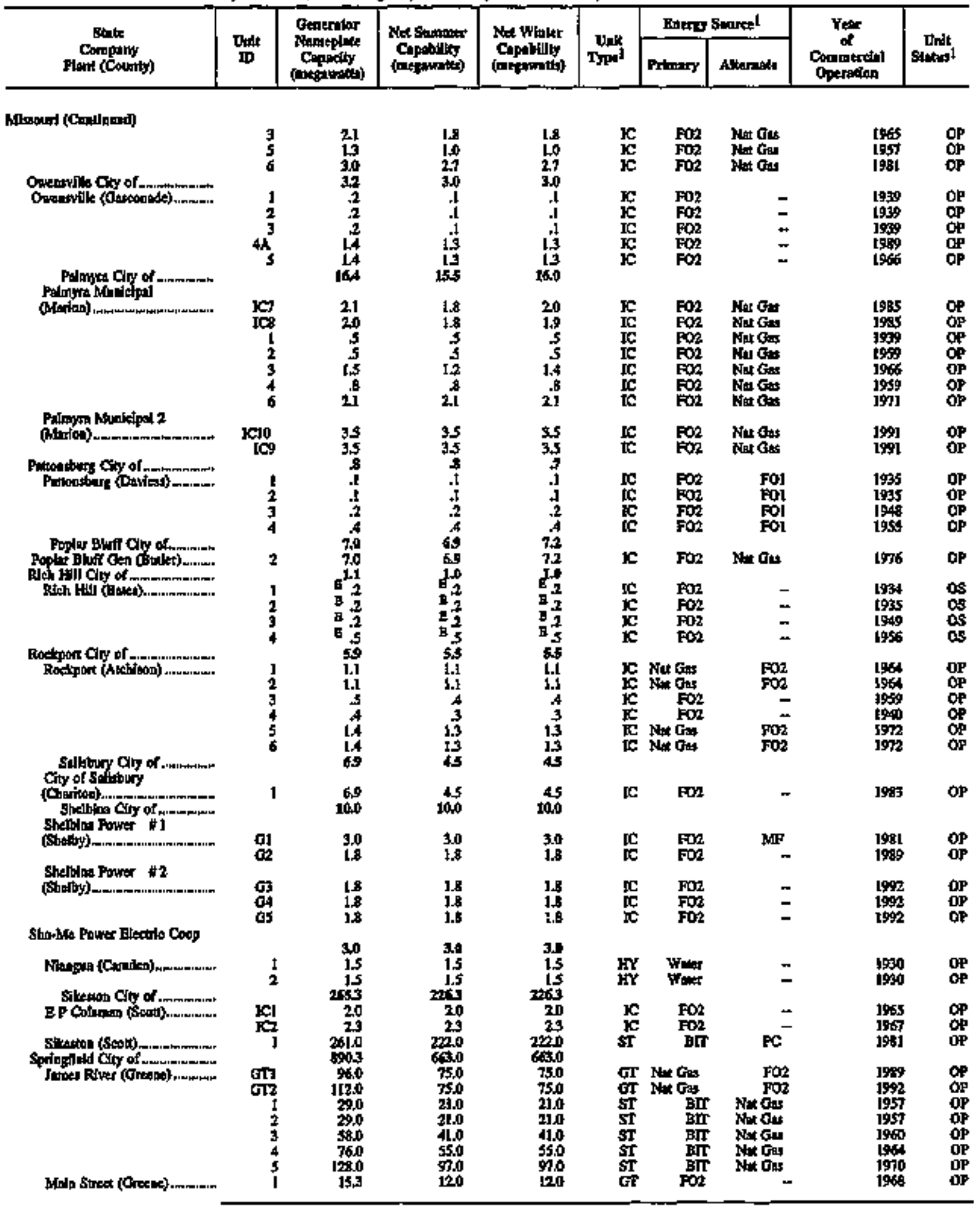

Sers tootinotes at end of tible, 
Table 20. Existing Generating Untits at U.S. Electric Utilities by State, Company, and Plant, as of January 1, 1996 (Continued)

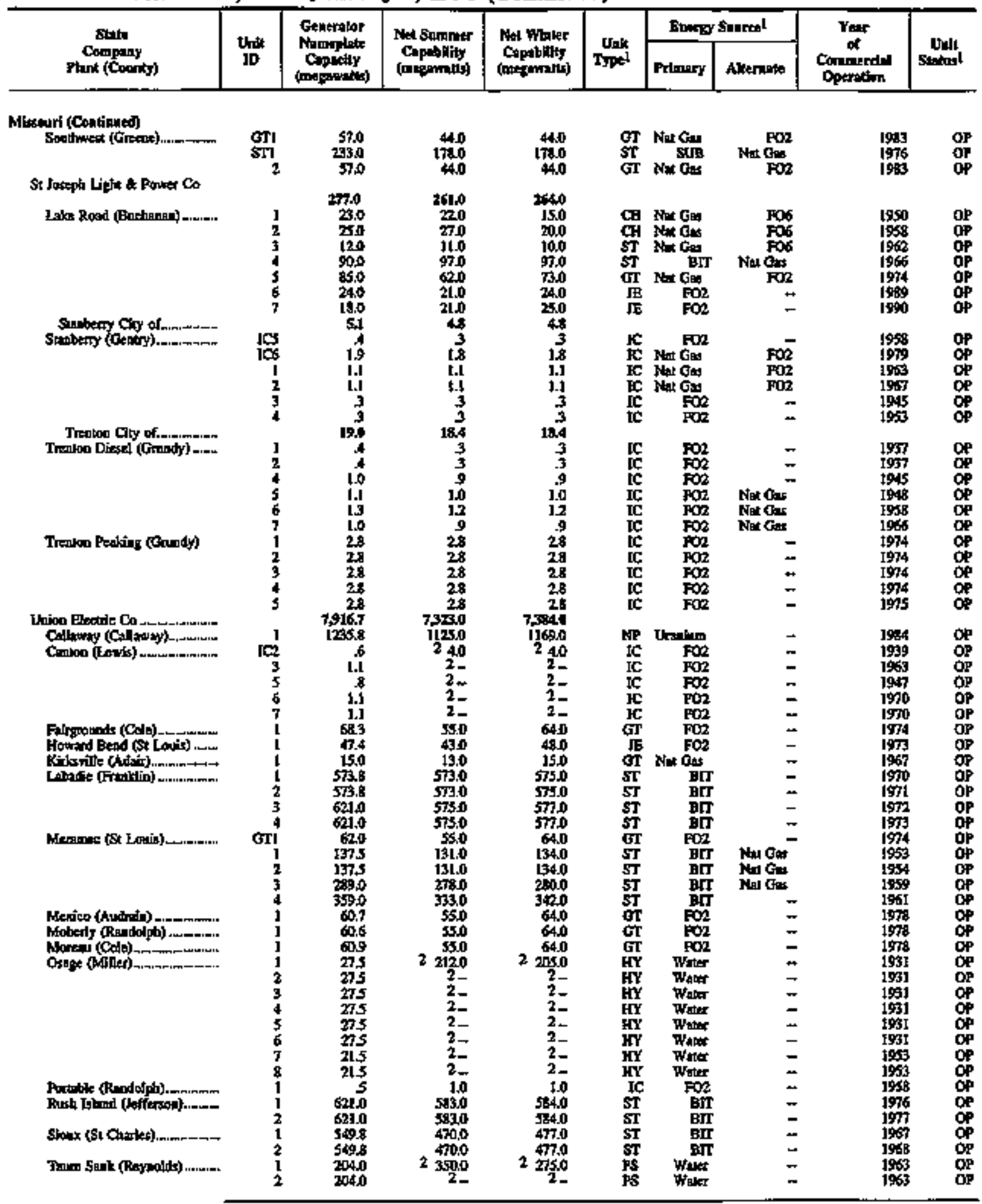

Sec fortores is ed of abje 
Table 20. Existing Generating Units at U.S. Electric Utilities by State, Company, and Plant, as of January 1,1996 (Continued)

\begin{tabular}{|c|c|c|c|c|c|c|c|c|c|}
\hline \multirow{2}{*}{$\begin{array}{c}\text { Stalo } \\
\text { Conpapy } \\
\text { Pant (County) }\end{array}$} & \multirow{2}{*}{ thitt } & \multirow{2}{*}{ 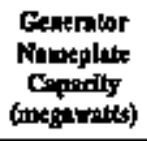 } & \multirow{2}{*}{$\begin{array}{l}\text { Nat Sumper } \\
\text { Copabliky } \\
\text { (nwegmonts) }\end{array}$} & \multirow{2}{*}{$\begin{array}{l}\text { Stel Whater } \\
\text { Crpublity } \\
\text { (nimequntis) }\end{array}$} & \multirow{2}{*}{ Thek } & \multicolumn{2}{|c|}{ Eneroy Sourcel } & \multirow{2}{*}{ 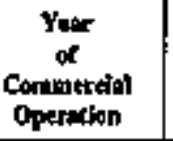 } & \multirow{2}{*}{$\begin{array}{l}\text { Undt } \\
\text { Sistust }\end{array}$} \\
\hline & & & & & & Petmary & Akernots & & \\
\hline \multicolumn{10}{|l|}{ 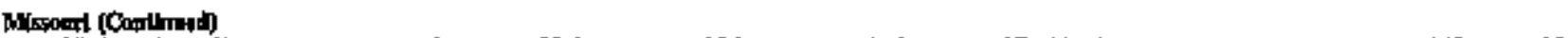 } \\
\hline Wrowec1 (Cape Girndesu) & I & 30.6 & 25,0 & 31.0 & GT & Nel Gas & $\leftrightarrow$ & I967 & OP \\
\hline Uhloomilk CYy of & & 9.1 & 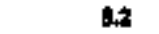 & 12 & & & & & \\
\hline 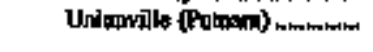 & 1 & 8 & 6 & 6 & IC & $\mathrm{FQP}$ & $\stackrel{*}{*}$ & 1970 & op \\
\hline & 3 & 1.8 & $\begin{array}{l}1.8 \\
3\end{array}$ & $\begin{array}{l}1.8 \\
.3\end{array}$ & IC & FU2 & Netudi. & $\begin{array}{l}1975 \\
1935\end{array}$ & OP \\
\hline & 4 & 10 & 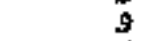 & 9 & IC & Fan & - & $19 \pi 0$ & $\mathbf{O P}$ \\
\hline & $\mathbf{s}$ & $A$ & 4 & 4 & $\mathbf{K C}$ & $\mathrm{FO} 2$ & - & I955 & op \\
\hline & 6 & $A$ & 4 & 4 & $\mathbf{K C}$ & 1002 & - & t95s & oP \\
\hline & ? & 1.1 & 9 & 9 & $\mathbf{~}$ & FOP & - & 1962 & OP \\
\hline & $\mathbf{z}$ & 14 & l.I & 1.1 & $\mathbf{K}$ & EOQ & Nal Gas & 1967 & OP \\
\hline & 9 & 20 & 2.0 & 2.0 & $\mathbf{c}$ & FOR & - & 1994 & OP \\
\hline \multirow{5}{*}{ 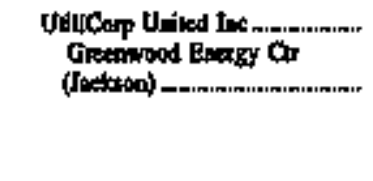 } & & 4920 & 1450 & 6450 & & & & & \\
\hline & 1 & 61,0 & (ब, 0 & 600 & GT & FO2 & - & 1975 & OP \\
\hline & 2 & 610 & 580 & $\$ 8.0$ & GT & FOA & - & $\$ 975$ & OP \\
\hline & 3 & 610 & 600 & 600 & $\mathbf{G T}$ & $\mathrm{FO} 2$ & - & sin? & OP \\
\hline & 4 & 61.0 & $\infty 0$ & 500 & or & $\mathrm{EO} 2$ & ـ & $\operatorname{sen}$ & $O P$ \\
\hline Kanapl Cly Inal (Platta) & ] & L8.0 & 13.0 & 13.0 & IE & Wax Gas & Ant & $19 m$ & OP \\
\hline & 2 & 19.0 & 13.0 & 13.0 & JE & Not Ous & 1et ful & $19 m$ & OP \\
\hline Nevidn (Vetaon). & 了 & 23.0 & 200 & 200 & Gr & $\mathrm{FO} 2$ & $=$ & 1974 & OP \\
\hline Rulph Gran (Gis) & GTI & 660 & 65 & 650 & Gr & Nat das & 的 & 195] & $\mathbf{Q P}$ \\
\hline Slbloy (1nowon) & $\mathbf{l}$ & 55.0 & 530 & $\$ 3.0$ & $\mathbf{s T}$ & BlT & - & 1960 & OP \\
\hline & 2 & 500 & 53.0 & $5 \mathbf{5 0}$ & $\mathbf{5 T}$ & BIT & - & IST2 & OP \\
\hline & 3 & 4180 & 3\%0 & 3900 & $5 T$ & BrT & 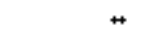 & 1969 & OP \\
\hline \multicolumn{10}{|l|}{ Usce-kings City Diduict } \\
\hline & & 20.0 & $240 . T$ & 240.7 & & & & & \\
\hline \multirow[t]{6}{*}{ Elany Truman (Bendan)...ninn } & 1 & 270 & $3 t .0$ & 31.0 & Ps & Water & + & 1982 & os \\
\hline & 2 & 270 & 31.0 & 31.0 & PS & Wues & - & 1982 & $\alpha s$ \\
\hline & 3 & $27 \pi$ & 31.0 & 31.0 & PS & Water & + & 1982 & OP \\
\hline & 4 & 27.0 & 31.0 & 31.0 & FS & Wyar & - & 1982 & os \\
\hline & 5 & 27,0 & 3110 & 31.0 & Fs & Water & - & 1961 & $\mathbf{O P}$ \\
\hline & 6 & $2 \pi 0$ & 35.0 & 31.0 & ps & Water & $=$ & 1979 & oP \\
\hline \multirow{5}{*}{ 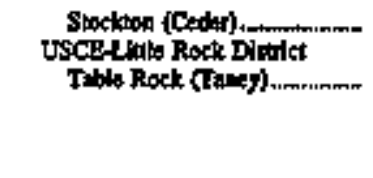 } & I & $\begin{array}{r}45,3 \\
200,0\end{array}$ & $\begin{array}{r}54.7 \\
23000\end{array}$ & $\begin{array}{r}54.7 \\
230,040\end{array}$ & HY & Waler & - & 1973 & op \\
\hline & I & 50,0 & 575 & $n 5$ & HY & Water & - & 1959 & DP \\
\hline & 2 & 50.0 & 57 & 575 & HY & Hat & - & 1959 & of \\
\hline & 3 & 500 & 575 & 575 & HY & Wuter & - & 1961 & of \\
\hline & 4 & 50.0 & 575 & $\$ 75$ & HY & Wats & - & 1961 & QP \\
\hline \multirow{3}{*}{ 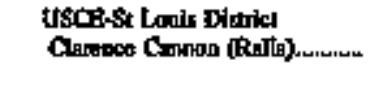 } & & 今.0 & 50 & $\mathbf{5 8 . 0}$ & & & & & \\
\hline & 1 & 27.0 & 27.0 & 20.0 & $\mathbf{H Y}$ & Wour & - & 1944 & OP \\
\hline & 2 & 31.0 & 31.0 & $3 ! .0$ & PS & Wer & - & 1984 & $O P$ \\
\hline \multirow{7}{*}{ 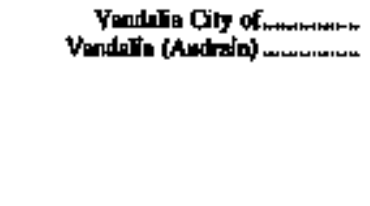 } & & 74 & 6.] & 6.1 & & & & & \\
\hline & ] & 1.3 & 10 & 10 & k & $\mathbf{F O} 2$ & - & 1967 & OP \\
\hline & 10 & IA & 1.1 & 1.J & k & $\mathrm{FO} 2$ & - & 1984 & of \\
\hline & 11 & Lo & 1.0 & 10 & K & $\mathrm{FO} 2$ & - & 1993 & of \\
\hline & 12 & 1.0 & 1.0 & 1.0 & $\mathbf{k}$ & FO2 & - & 1993 & $O P$ \\
\hline & B & 10 & 8 & 8 & c & $\mathrm{FO} 2$ & - & 195 & of \\
\hline & 9 & 1.4 & 1.2 & 1.2 & IC & $\mathrm{FO2}$ & - & $19 n$ & OP \\
\hline
\end{tabular}

Sec fornots at cod of table

Energy Information Administration/Inventory of Power Plants in the United States as of January I, 1996 Iss 
Table 20. Existing Generating Units at U.S. Electric Utilities by State, Company, and Plant, as of January 1, 1996 (Continued)

\begin{tabular}{|c|c|c|c|c|c|c|c|c|c|}
\hline \multirow{2}{*}{$\begin{array}{c}\text { State } \\
\text { Conprang } \\
\text { Flem (Coonty) }\end{array}$} & \multirow{2}{*}{ that } & \multirow{2}{*}{ 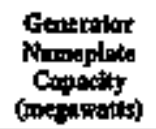 } & \multirow{2}{*}{ 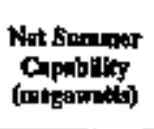 } & \multirow{2}{*}{$\begin{array}{c}\text { Med Plater } \\
\text { Copbits } \\
\text { (mejomitis) }\end{array}$} & \multirow{2}{*}{ Typt } & \multicolumn{2}{|c|}{ Bxtry Sumpl } & \multirow{2}{*}{$\begin{array}{c}\text { Yaar } \\
\text { of } \\
\text { Commured } \\
\text { Operetito }\end{array}$} & \multirow{2}{*}{ Standt } \\
\hline & & & & & & PrImaxy & Alternite & & \\
\hline
\end{tabular}

Montana

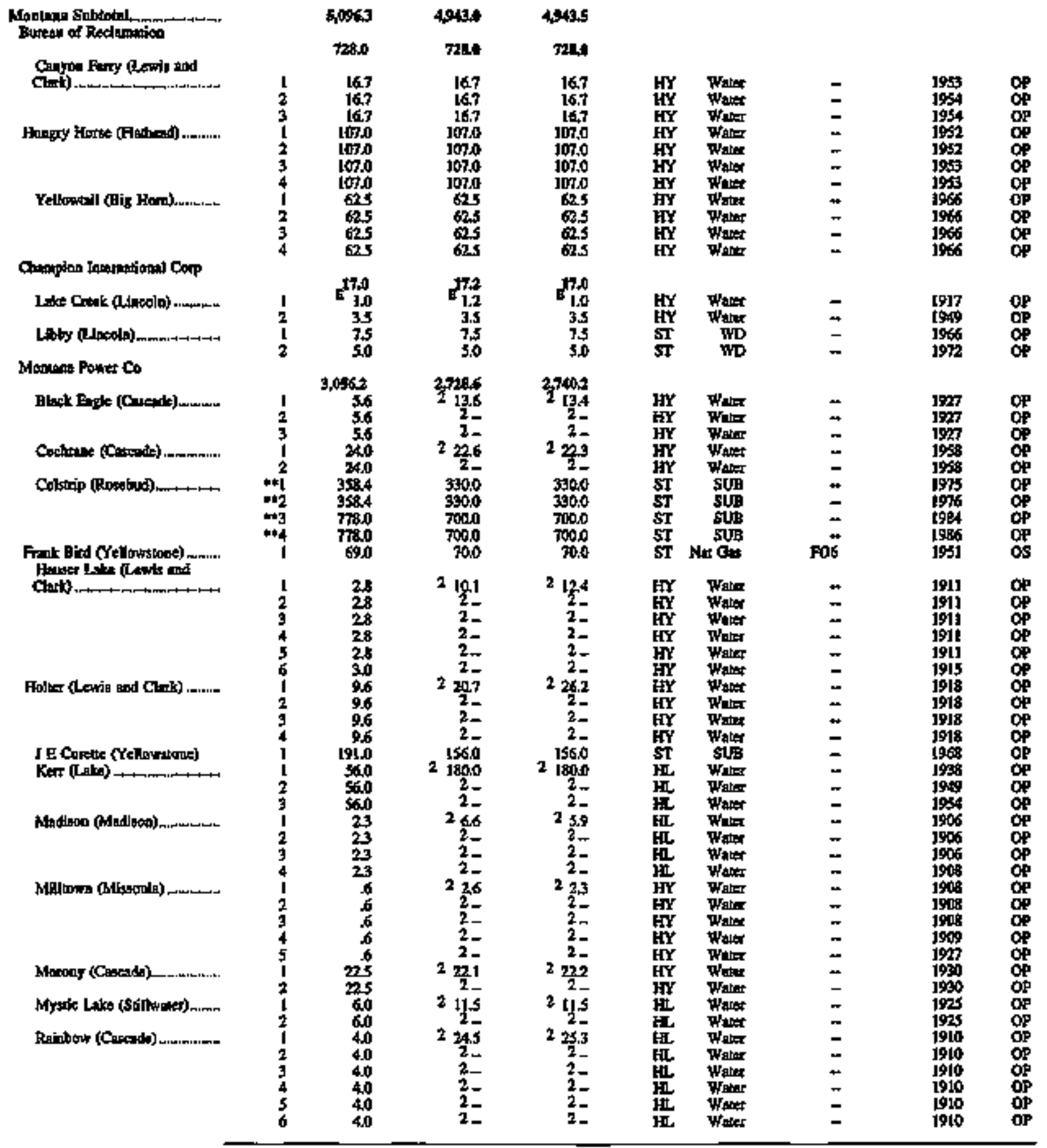

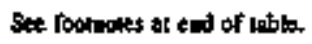


Table 20. Exdsting Generating Uinits at U.S. Blectric Utillties by State, Company, and Plant, as of January 1, 1996 (Continued)

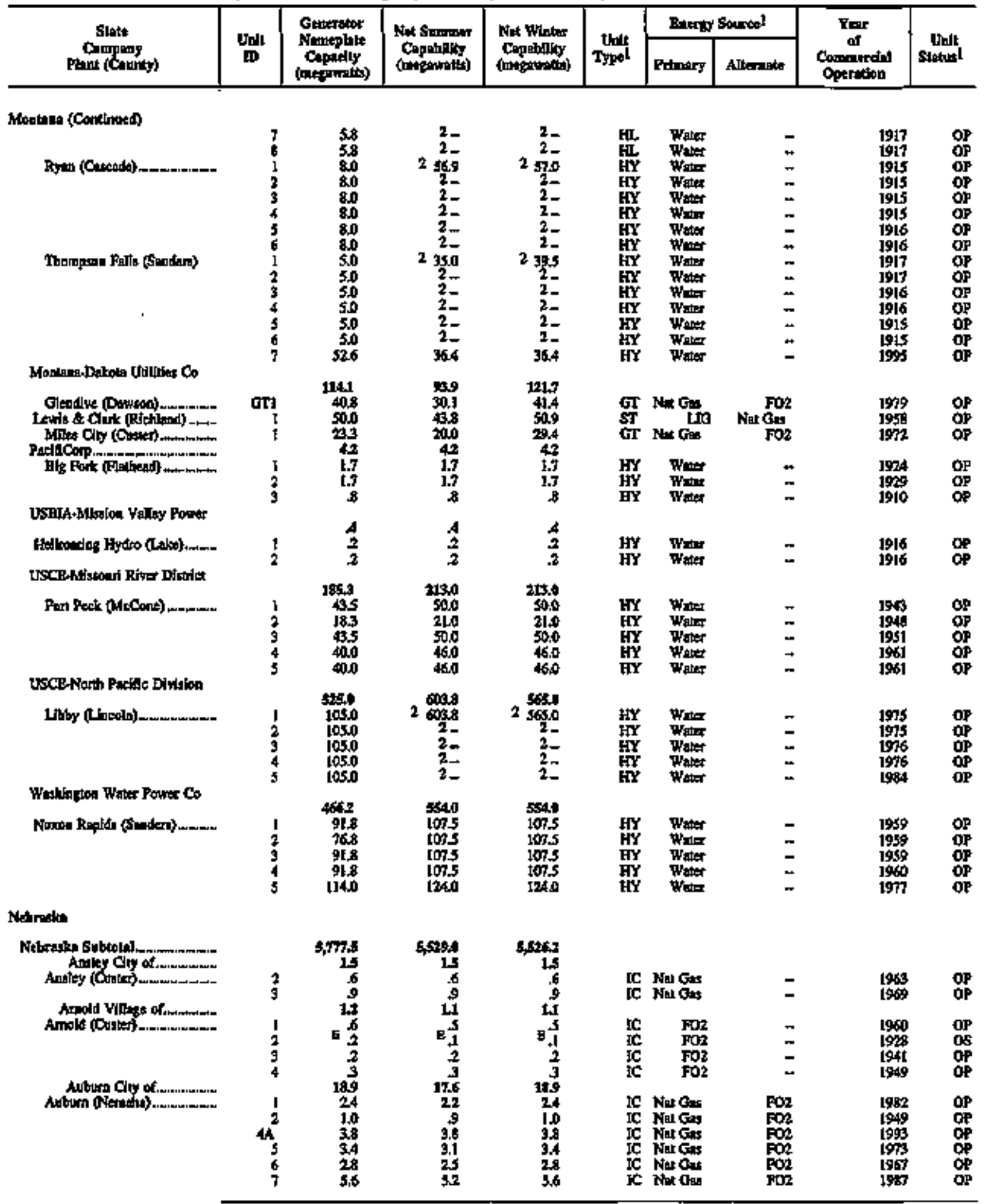

Ses footrutios at end of tables. 
Talule 20. Existing Generating Units at U,S. Electric Uttlitles by State, Company, and Plant, as of Jamuary 1, 1996 (Continued)

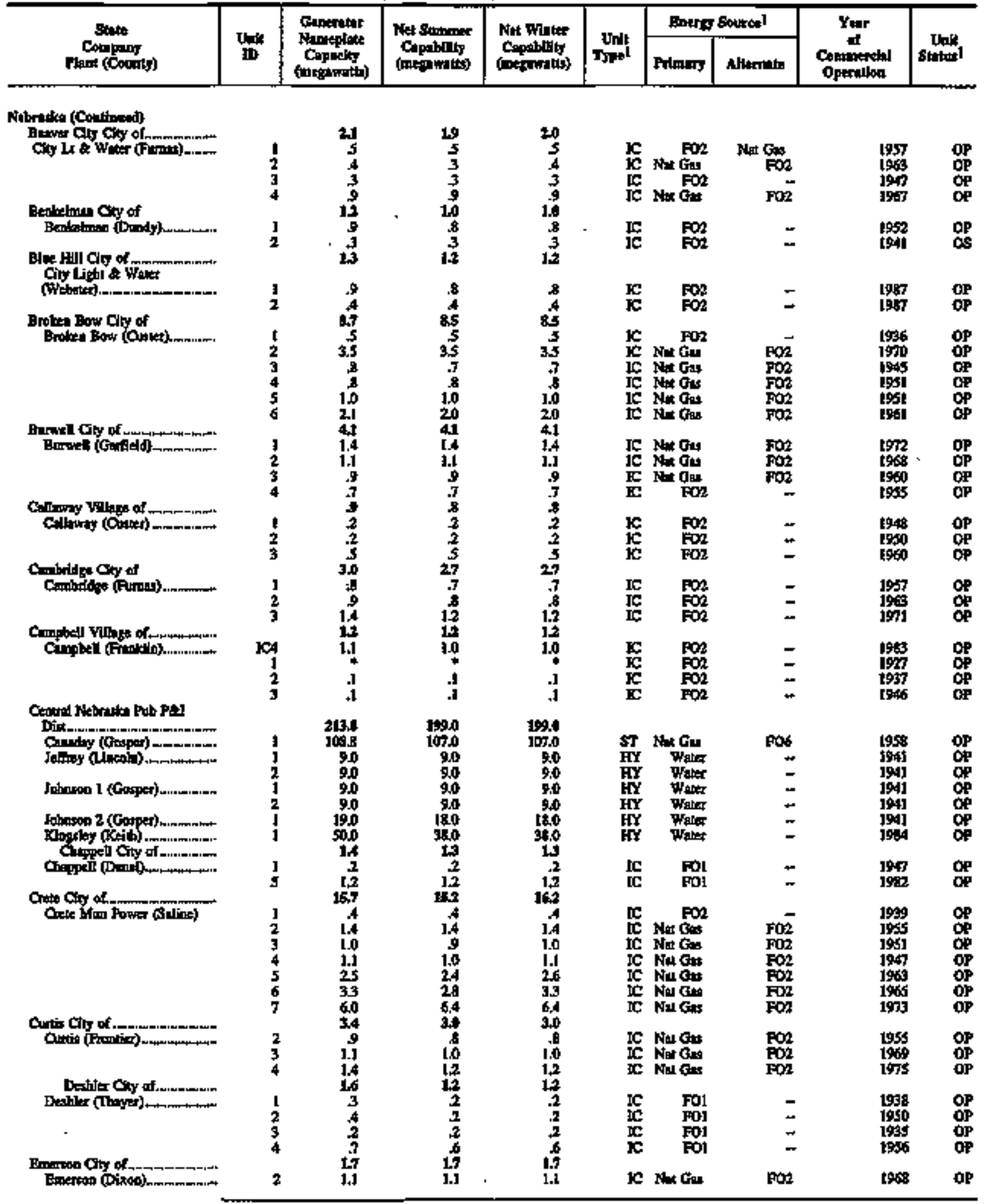

Eeo foctuoles un end of toble. 
Table 20. Existing Generating Units at U,S. Electric Utilities by State, Company, and Plant, as of January 1, 1996 (Continued)

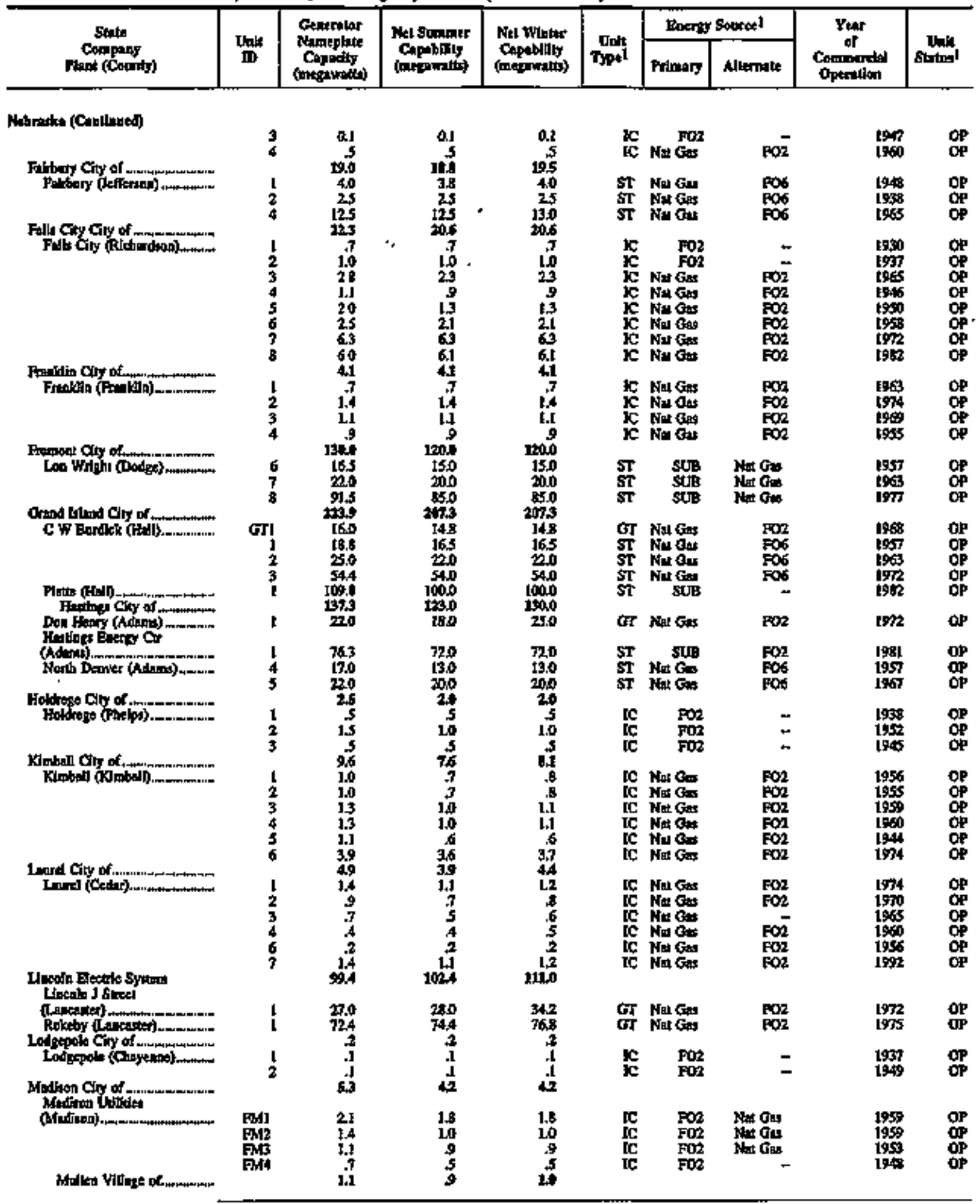

San foolmown at apd of uble. 
Table 20. Existing Generating Units at U.S. Electric Utullties by State, Company, and Plant, as of January 1, 1996 (Contimued)

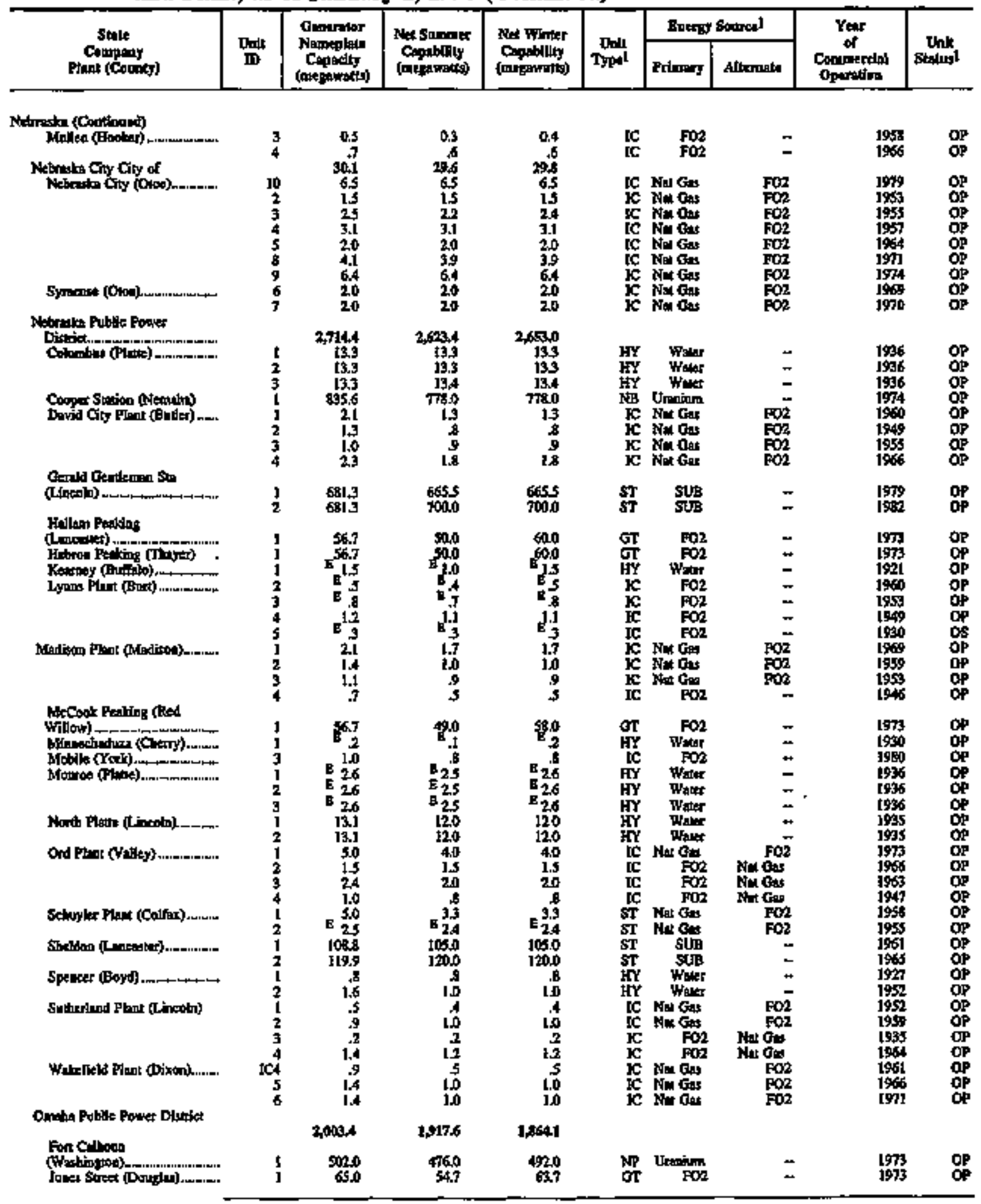

Sec footnokd at and of tabje. 
Table 20. Existing Generating Units at U.S. Electric Utilities by State, Company, and Plant, as of Jamuary 1, 1996 (Continued)

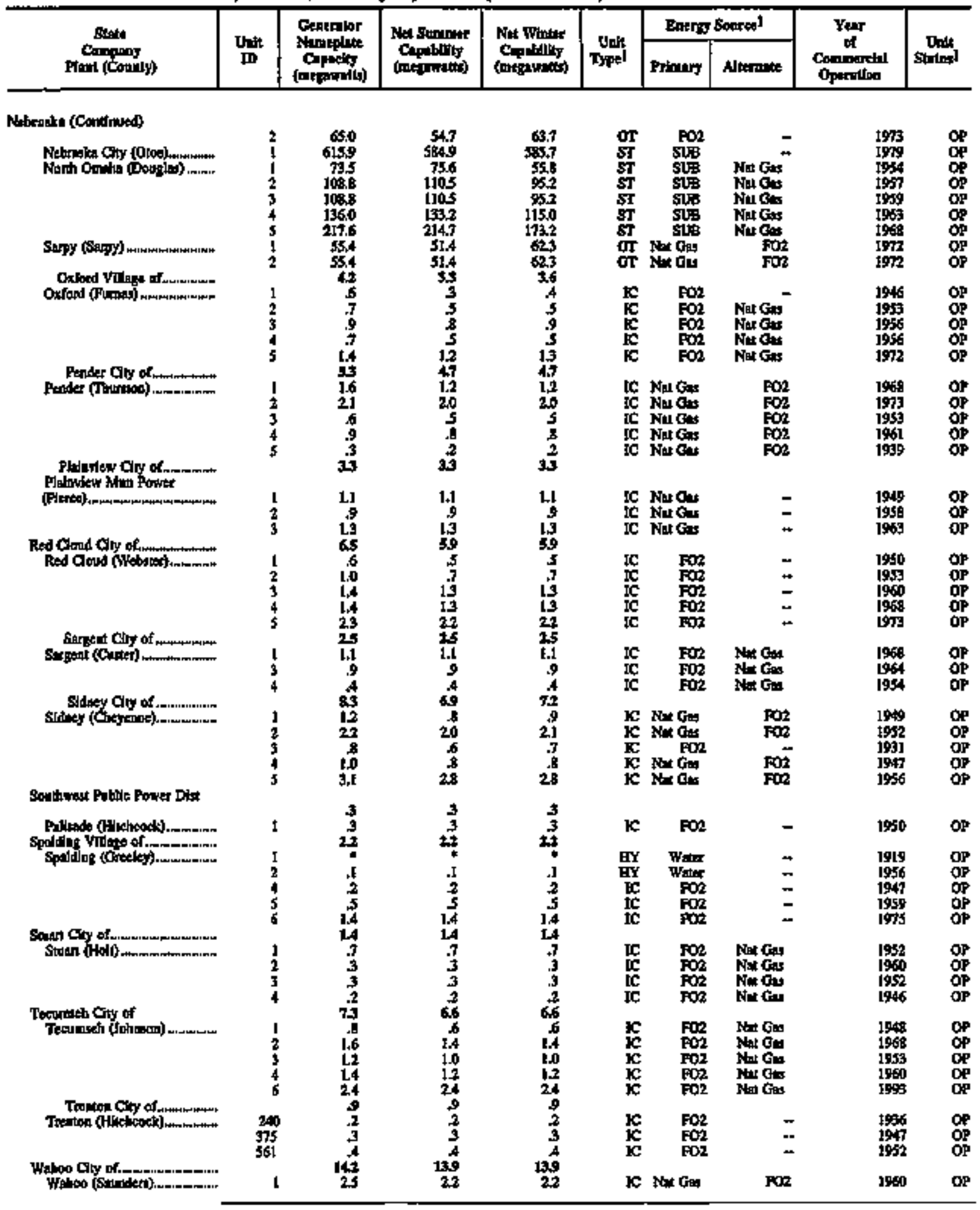

Sec footalas at end of bitho 
Table 20. Existing Generating Units at U.S. Electric Utilities by State, Company, and Plant, as of January 1, 1996 (Continued)

\begin{tabular}{|c|c|c|c|c|c|c|c|c|c|}
\hline \multirow{2}{*}{$\begin{array}{c}\text { Stante } \\
\text { Condent } \\
\text { Filund (Coundy) }\end{array}$} & \multirow{2}{*}{ Ud } & \multirow{2}{*}{ 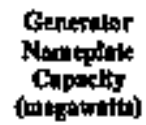 } & \multirow{2}{*}{$\begin{array}{l}\text { Net Summet } \\
\text { Copobitity } \\
\text { (metowatts) }\end{array}$} & \multirow{2}{*}{ 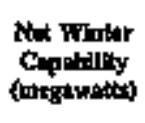 } & \multirow{2}{*}{$\begin{array}{c}\text { Jelf } \\
\text { Typel }\end{array}$} & \multicolumn{2}{|c|}{ Bteng Sourcel } & \multirow{2}{*}{ 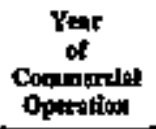 } & \multirow{2}{*}{ 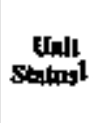 } \\
\hline & & & & & & Prtuang & Allernatio & & \\
\hline
\end{tabular}

Nobrisiog (Contimuted) Clly of Waknfield (Dirot)..... Wave Giy of

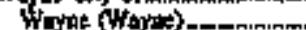

West Poda: Cry of Weal Pedrot Wumicipail (Ouming)

wilber Cis of Willier (Sallot).

Where Cily of Wister (Clinini)

$\begin{array}{rr}2 & 0 \\ 3 & 4 \\ 4 & 3 \\ 5 & 2 \\ 5 & 3 \\ 2456 & 3 \\ 1 & 15 \\ 7 & 1 \\ 3 & 2 \\ 4 & 2 \\ 6 & 5\end{array}$

0.5
1.5
1.2
23
3.4
3.3
3.2
13.4
5.8
1.8
1.9
3.3
4.9
1.5

4
1.2
23
4.1
1.6
1.0
6
10
6
5
6

Neveda

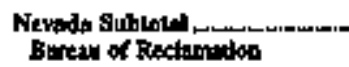

Heover Dam Prxt Pbad (Clak).

Nowada Foner Co

Gut (Clant)

Inry Allen (Clok)

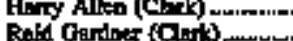
Rolit Ganderer (Clait)

Son $P=x(C l . j)$

Surrixe (C)

Stierst Parite Power Co Batdo Monntin (Landec)

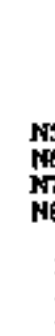

S.rta1

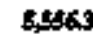

$1,007,0$

1,374

1300

L00

1270

1300

L300

1300

1300

1300

17260

3,017, t

2.4

869

86.9

60

869

500

90.0

65.0

75.0

900

1140

1140

I1400

2700

究0

9010

900

90.0
820

s50.

1,201.1.

20

20

1,7256

50

700

70.0

200

420

900

660

67.0

70

I 100

I Lat

1100

700

700

7006

800

t>113

18

1.8

0.5
4.5
1.2
2.3
3.4
5.9
3.9
13.4
6.9
1.9
1.9
3.3
4.9
1.5

thet:6

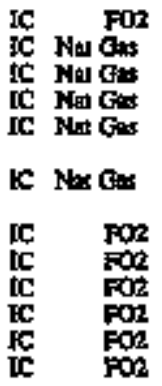

$\underset{T \rightarrow 2}{\operatorname{Fon} 2}$

ros

Fol

$\begin{array}{ll}- & 1952 \\ - & 1945\end{array}$

$-$

$\pi$

$-$

IC Mal Ges

IC Nat Gas

IC Nal Cos

IC $\quad$ FO?

ic

$\begin{array}{ll}\text { IC } & \mathbf{F O 2} \\ \text { IC } & \mathbf{F i 2} \\ \text { IC } & \text { FO2 }\end{array}$

1,배삿

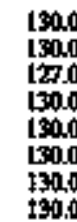

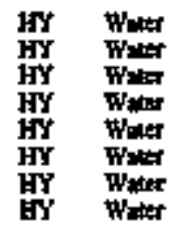

FO2

Fon

bht Ge

Nate Tou

$\begin{array}{ll} & 1960 \\ + & 1954 \\ - & 1947 \\ - & 1965\end{array}$

1936

1971

1947

190

t915

1946

1956

1960

1964
1968

op
op
op
op
op
OP
OP
OP
OP
OP
OP
OP

Op
Op
Op
Op
Op

$\begin{array}{ll}1947 & \text { OP } \\ 1959 & \text { OP } \\ 1965 & \text { OP } \\ 1911 & \text { OP } \\ 1960 & \text { OP } \\ 1960 & \text { OP } \\ 1954 & \text { OP } \\ 1947 & \text { OP } \\ 1965 & \text { OP }\end{array}$

Sect foomotes at and of table. 
Table 29. Existing Generating Units at U.S. Electric Utilities by State, Company, and Plant, as of January 1, 1996 (Continued)

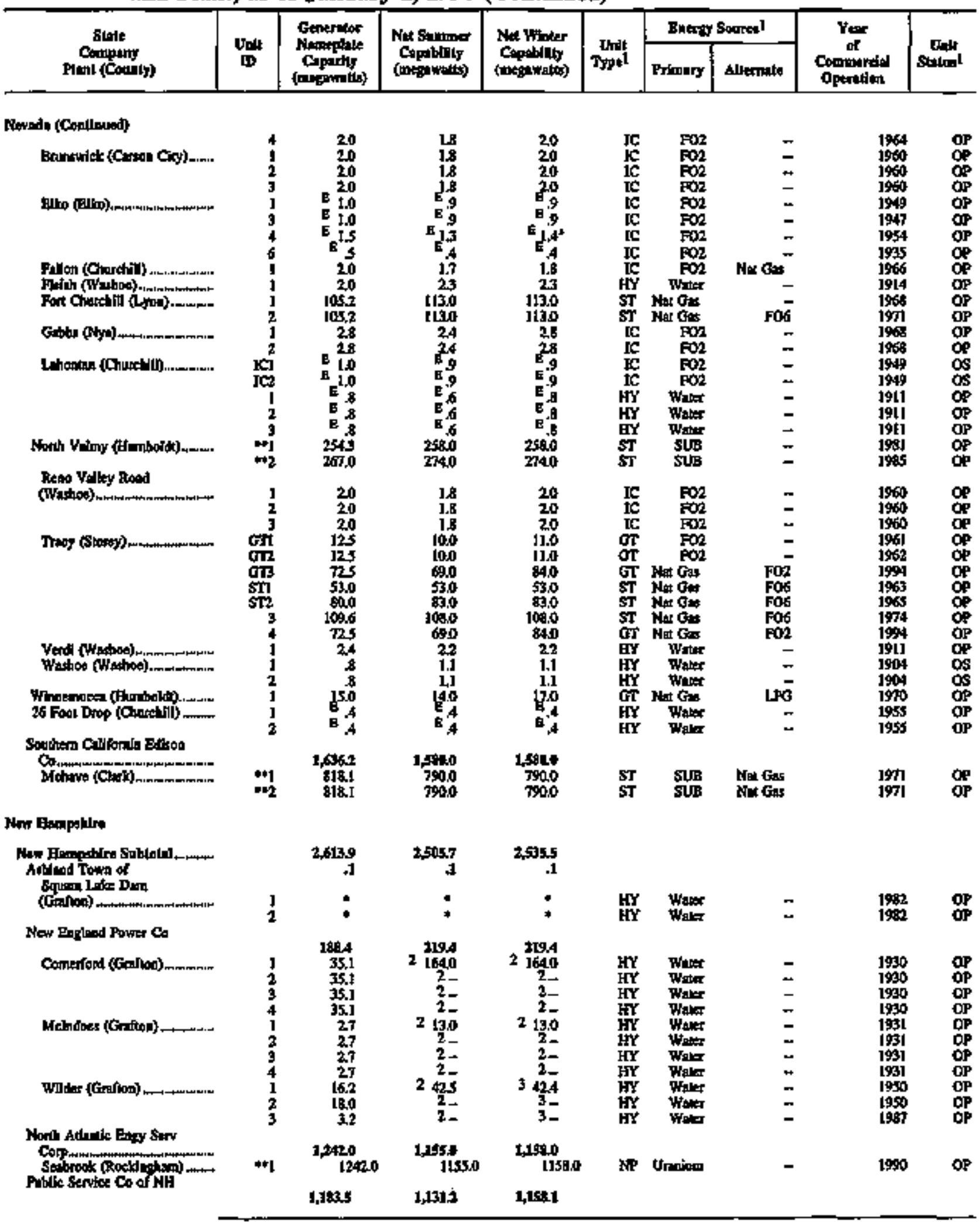

Seo footionet in end of cables. 
Table 20. Existing Generating Units at U.S. Electrfe Utilities by State, Company, and Plant, as of Janwary 1, 1996 (Continued)

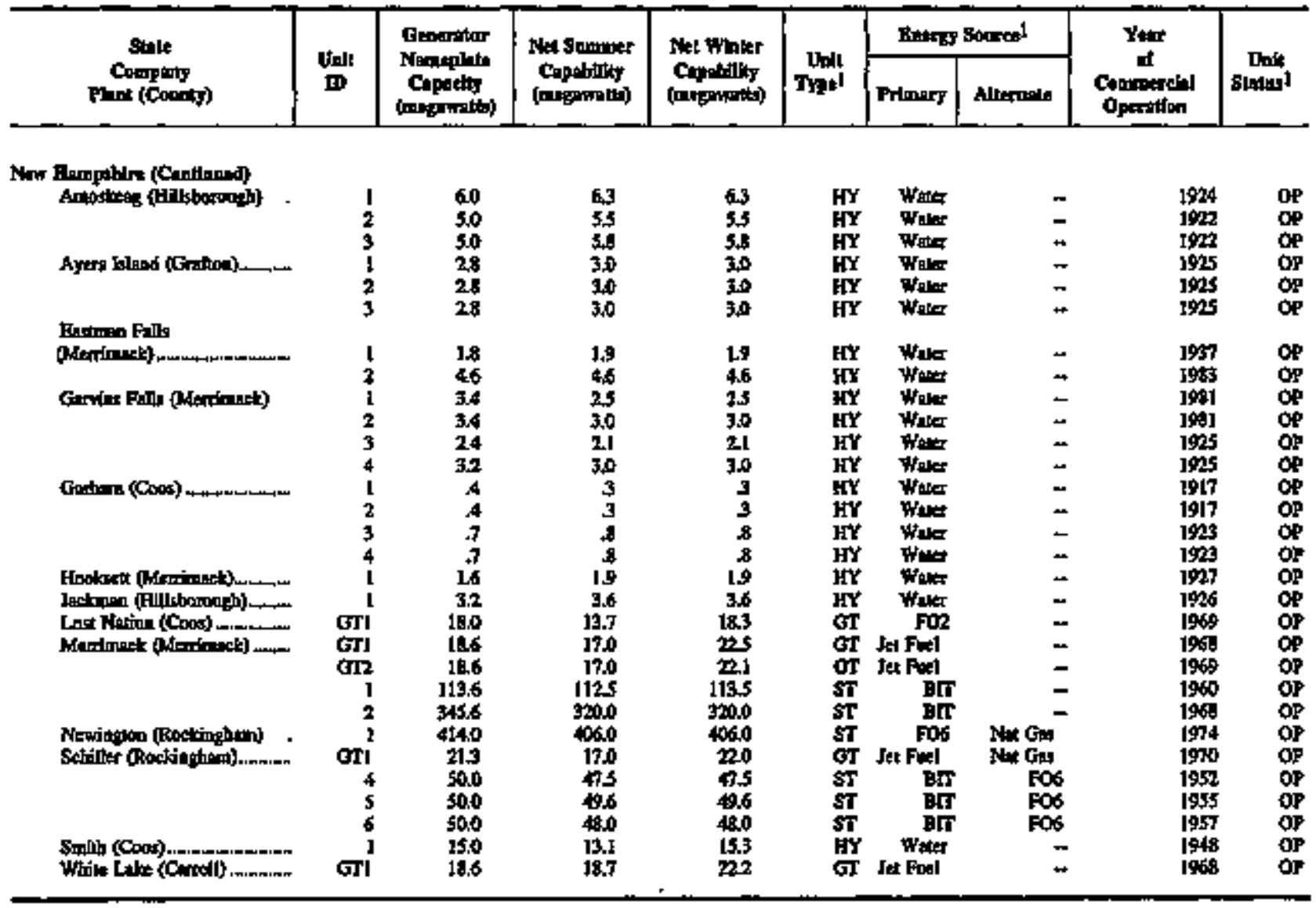

Sac foothow an and of babje. 
Table 20. Existing Generating Units at U.S. Electric Utilities by State, Company, and Plant, as of Jamuary 1,1996 (Continued)

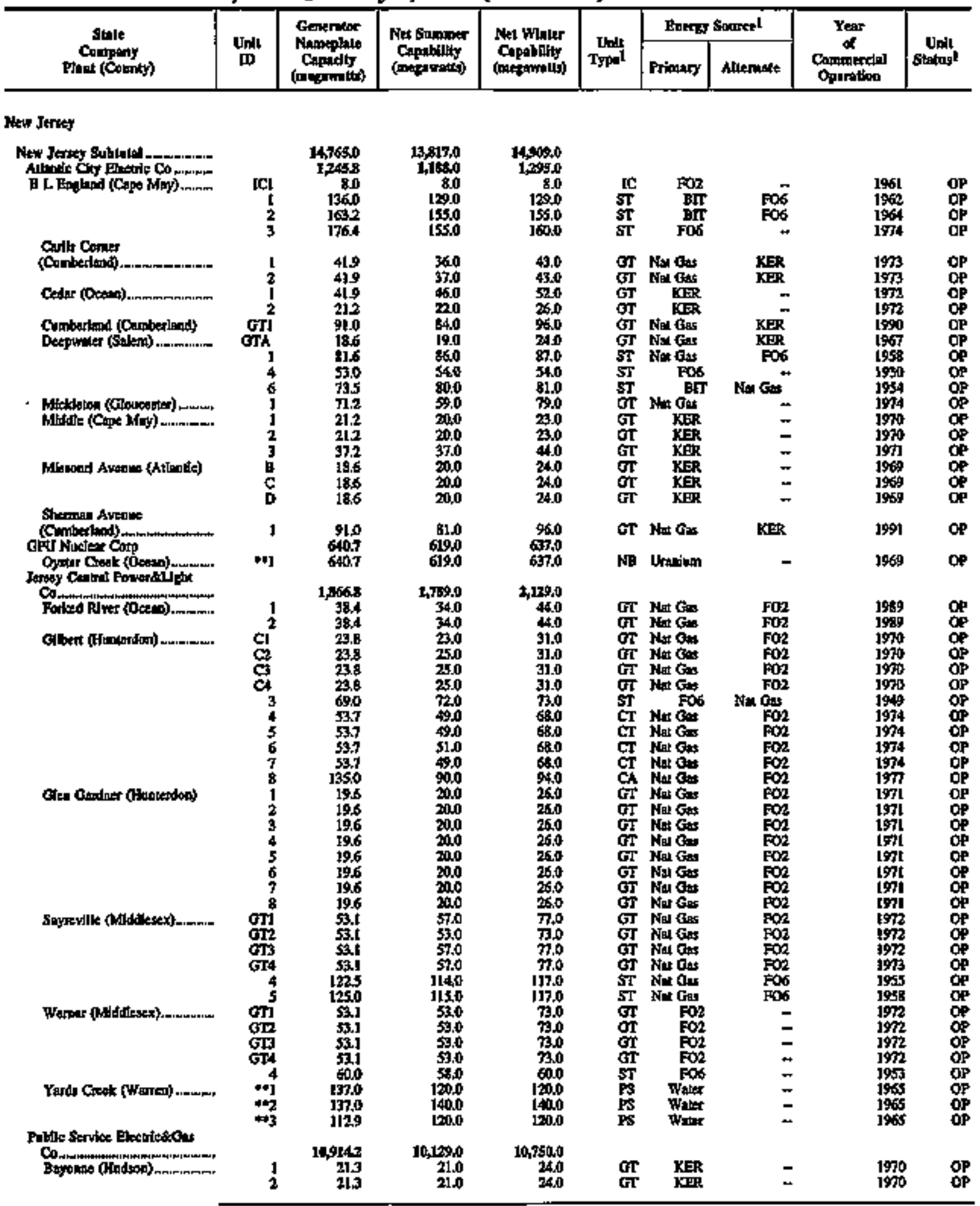

Sto fooknow at and of enble. 
Table 20. Existing Generating Units at U.S. Flectric Utllities by State, Company, and Plant, as of January 1, 1996 (Continued)

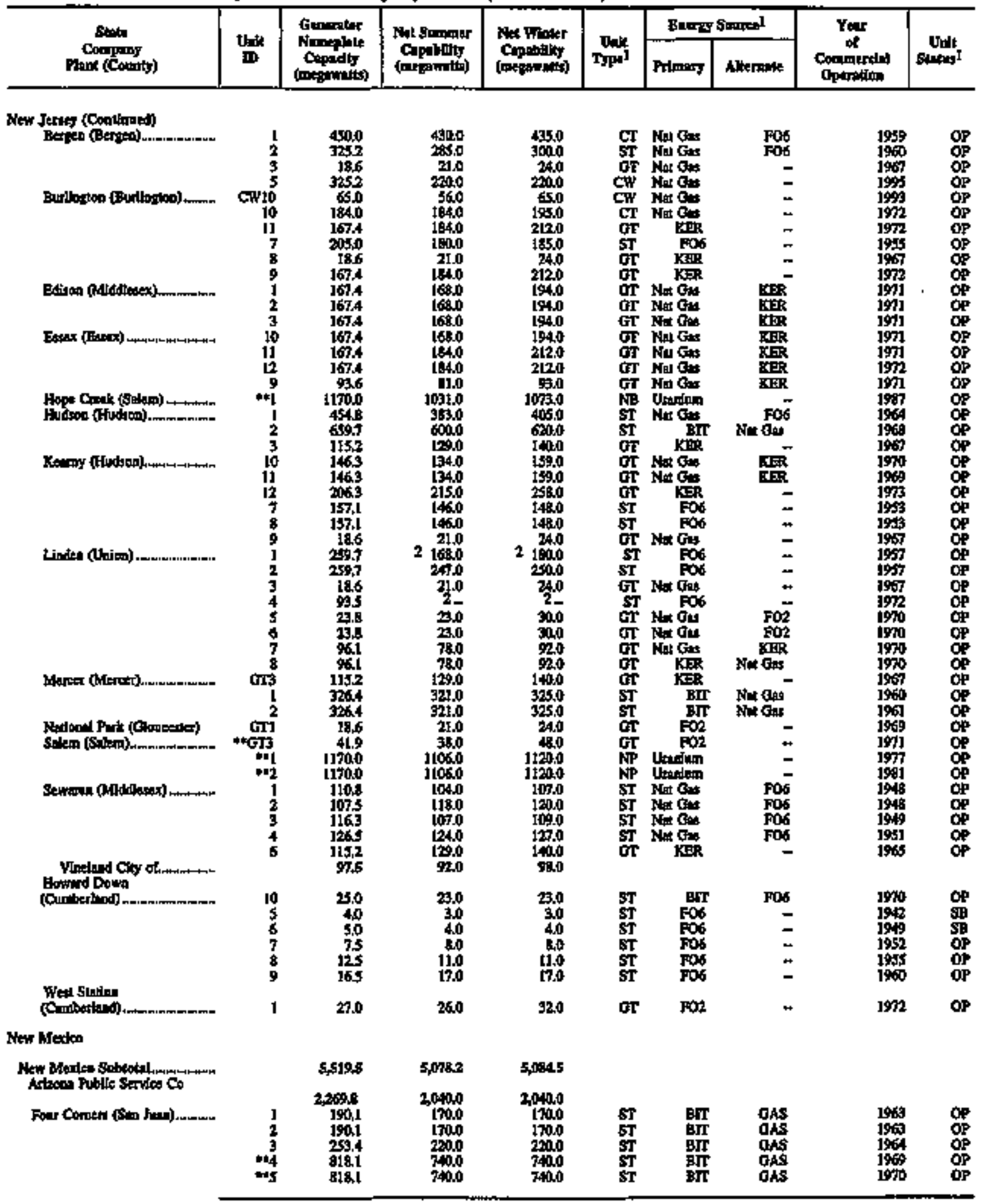

Sec footonots at etd of uble. 
Table 20. Existing Generating Units at U.S. Electric Utillities by State, Company, and Ptant, as of January 1, 1996.(Continued)

\begin{tabular}{|c|c|c|c|c|c|c|c|c|c|}
\hline \multirow{2}{*}{$\begin{array}{c}\text { Shalo } \\
\text { Conpasy } \\
\text { Funt (County) }\end{array}$} & \multirow{2}{*}{$\underset{\mathrm{D}}{\mathrm{DW}}$} & \multirow{2}{*}{ 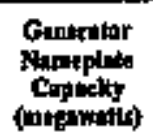 } & \multirow{2}{*}{$\begin{array}{l}\text { Mel Sommet } \\
\text { Copoblity } \\
\text { (orejowats) }\end{array}$} & \multirow{2}{*}{ 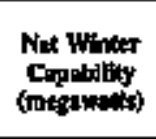 } & \multirow{2}{*}{$\underset{\text { TrFat }}{\text { Dont }}$} & \multicolumn{2}{|c|}{ Eors Souret 1} & \multirow{2}{*}{$\begin{array}{c}\text { Yers } \\
\text { of } \\
\text { Cocontrelin } \\
\text { Operetion }\end{array}$} & \multirow{2}{*}{ Undt } \\
\hline & & & & & & Primary & Alternote & & \\
\hline \multicolumn{10}{|l|}{ 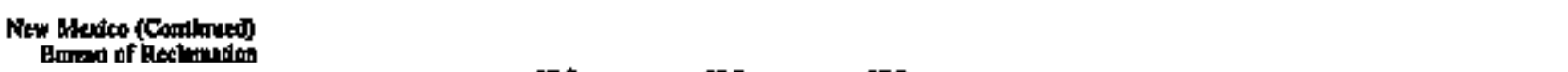 } \\
\hline & & \multirow{12}{*}{ 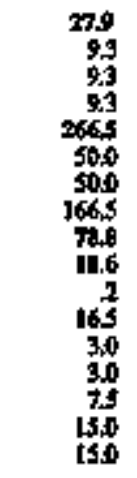 } & \multirow{12}{*}{$\begin{array}{r}23.5 \\
9.3 \\
9.3 \\
9.3 \\
246.5 \\
480 \\
480 \\
150.2 \\
78.8 \\
18.6 \\
.2 \\
16.5 \\
3.0 \\
3.0 \\
75 \\
15.0 \\
150\end{array}$} & \multirow{12}{*}{ 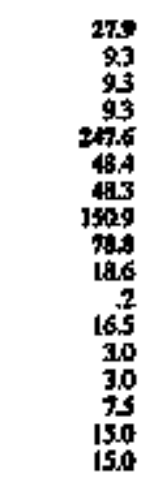 } & & \multirow{3}{*}{$\begin{array}{l}\text { Water } \\
\text { Wrat } \\
\text { Water }\end{array}$} & \multirow{3}{*}{$\overline{-}$} & \multirow{3}{*}{$\begin{array}{l}1940 \\
1940 \\
1940\end{array}$} & \multirow{3}{*}{$\begin{array}{l}\text { of } \\
\text { of } \\
\text { OP }\end{array}$} \\
\hline 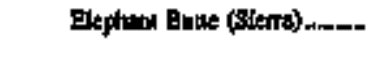 & 1 & & & & $\underset{\mathbf{H Y}}{\mathbf{E Y}}$ & & & & \\
\hline EPato Elosito $C_{0}$ & 3 & & & & $\mathbf{H Y}$ & & & & \\
\hline 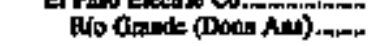 & 6 & & & & $\mathbf{S T}$ & Nay O: & $\mathbf{F O n}$ & 195t & of \\
\hline & 7 & & & & SI & Non Gas & $\begin{array}{l}\mathrm{FO} 2 \\
\mathrm{FO} 2\end{array}$ & 1968 & OP \\
\hline \multirow{5}{*}{ 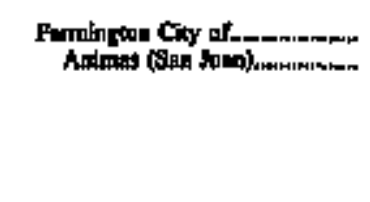 } & & & & & & & & & \\
\hline & GI & & & & $\mathbf{C T}$ & Ner Gas & 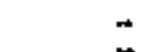 & 1094 & op \\
\hline & SI4 & & & & $\stackrel{\mathbf{m r}}{\mathbf{s r}}$ & Nit Gu & $\ddot{z}$ & 1959 & oP \\
\hline & 1 & & & & $C W$ & Net Gns & - & 195 & OP \\
\hline & 3 & & & & $\underset{\text { ST }}{\mathrm{CW}}$ & Nat Gat & $\stackrel{+}{*}$ & 1055 & op \\
\hline Narefo (Ftan Juan) & $\mathbf{j}$ & & & & KY & Waber & $\overline{-}$ & 1909 & or \\
\hline & & & & & $\mathbf{H Y}$ & Walut & $\leftrightarrow$ & 1989 & OP \\
\hline \multicolumn{10}{|l|}{ Los Counk flucte Coop } \\
\hline 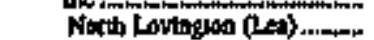 & \$] & 1600 & 160 & 160 & $\mathbf{s T}$ & NuG & $\mathbf{F O 2}$ & I\$62 & se \\
\hline 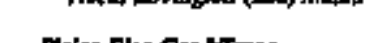 & 52 & $3 \mathbf{3 0}$ & 30 & 330 & ST & Nat Ga & Fon & 196 & SB \\
\hline \multicolumn{10}{|l|}{ Plalan Blec GapdTans } \\
\hline 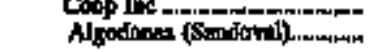 & $\mathbf{I}$ & $\begin{array}{l}270,0 \\
15.0\end{array}$ & 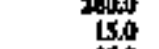 & $\begin{array}{l}20,150 \\
150\end{array}$ & $\mathbf{S T}$ & Net Cat & FOS & 1904 & SE \\
\hline & 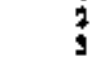 & $\begin{array}{l}15.0 \\
15.0\end{array}$ & ${ }_{\mathbf{5} 5.0}^{5.0}$ & $\begin{array}{l}15.0 \\
15.0\end{array}$ & ST & Net Gan & $\begin{array}{l}\mathbf{F O 6} \\
\mathbf{1 0 6}\end{array}$ & ${ }_{1954}$ & sip \\
\hline 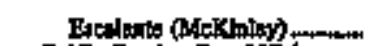 & 1 & 200 & $2,50.0$ & 2350 & $\mathbf{g r}$ & suts & 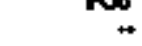 & 1984 & De \\
\hline Pable Serves Co of $\mathrm{NA}$ & & 1,450 & 1,T:8 & 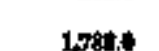 & & & & & \\
\hline 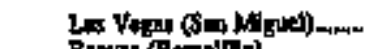 & 1 & 200 & 20,0 & 200 & $\boldsymbol{G r}$ & $\mathrm{FO}_{2}$ & $\mathrm{Stat} \mathrm{OH}$ & 1973 & op \\
\hline Revel (Berpillio) & $\begin{array}{l}1 \\
2\end{array}$ & $\begin{array}{l}4.0 \\
44.0\end{array}$ & 440 & $\begin{array}{r}4,0 \\
440\end{array}$ & $\mathbf{S T}$ & Sorten & $\begin{array}{l}106 \\
\mathbf{1 0 6}\end{array}$ & 1960 & $\begin{array}{l}\text { OP } \\
\text { OP }\end{array}$ \\
\hline & 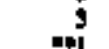 & 60 & $\begin{array}{r}66,0 \\
3160\end{array}$ & $\begin{array}{r}660 \\
3160\end{array}$ & $\stackrel{s t}{s T}$ & Mex Oas & Fo6 & 1962 & op \\
\hline $\operatorname{Sen}$ Jun (Sar Jas) & 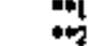 & $\begin{array}{l}361.0 \\
150.0\end{array}$ & 3160 & $\begin{array}{l}3160 \\
3120\end{array}$ & $\frac{5 T}{S T}$ & $\begin{array}{l}\text { sute } \\
\text { șters }\end{array}$ & $\ddot{-}$ & 596 & of \\
\hline & if & 534.0 & 480 & 4800 & $s T$ & surs & 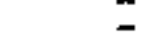 & 1979 & PP \\
\hline & 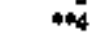 & $\$ 3140$ & 4980 & 4980 & $\boldsymbol{E} \mathbf{T}$ & 518 & $*$ & 19 & OP \\
\hline Rutum Rublo Servise Co & & $2:$ & & 110 & & & & & \\
\hline Raton (Colfax) & $\mathbf{3}$ & ists & $\begin{array}{r}11,9 \\
1,8\end{array}$ & $\begin{array}{l}17.9 \\
1.8\end{array}$ & $\boldsymbol{S T}$ & Brt & $*$ & 3935 & 80 \\
\hline & 4 & 38 & 3,2 & 3.2 & ST & 些 & $=$ & $195]$ & of \\
\hline 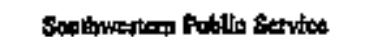 & & & & & & & & & \\
\hline C. C. & & 5014 & 421 & 492 & & & & & \\
\hline Culdod (Eddy) & 5 & 163 & 160 & 160 & 罙 & Hot Gas & $=$ & $\operatorname{in}$ & op \\
\hline 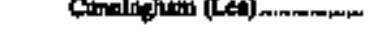 & 2 & $\begin{array}{l}75.0 \\
151.4\end{array}$ & 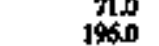 & 1960 & $\begin{array}{l}S T \\
S I\end{array}$ & $X_{0}$ & Fo: & 1965 & $o p$ \\
\hline Maddax (Led) (1) & $j$ & I 1346 & $1] 80$ & 1480 & $\mathbf{S T}$ & 整 & $*$ & 199 & og \\
\hline & $\frac{1}{3}$ & 16.9 & 600 & 100 & $\underset{\mathbf{\sigma r}}{\mathbf{\sigma r}}$ & Hat & $\bar{z}$ & $\begin{array}{l}1976 \\
1963\end{array}$ & \\
\hline Duconaced (Qump). & 3 & 1,0 & 1.0 & 1.0 & IC & $\mathbf{P O}$ & $\Rightarrow$ & 1975 & of \\
\hline & 4 & $E \frac{23}{13}$ & $=20$ & $\mathbf{8}_{12}^{20}$ & IC & Nats & FOت & 1959 & op \\
\hline & 6 & A.] & 30 & 30 & k & Ning & rot & 1960 & op \\
\hline & 8 & 3,0 & 30 & 50 & $\mathbf{r C}$ & pos & $\leadsto$ & 194 & of \\
\hline Tritalter Mestoo Powe & & & & & & & 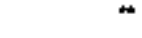 & $19 \pi$ & \\
\hline 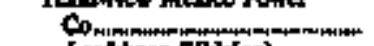 & & s50 & 443 & & & & & & \\
\hline Loddibors (EldaIpo) & I & 13.3 & 96 & 11.3 & CT & 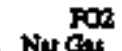 & $=$ & 1964 & si \\
\hline & 3 & 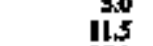 & $\begin{array}{c}47 \\
1099\end{array}$ & 110 & SA & Nor Ea & FO4 & 1949 & 58 \\
\hline & 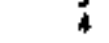 & 250 & 19.0 & 220 & sT & Not 4 4 & $\mathrm{PO4}$ & 1968 & $\mathrm{SB}$ \\
\hline US Doplon Alnmes An: & & & & & & & & & \\
\hline 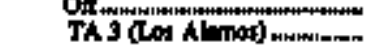 & $5 T 2$ & 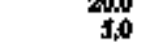 & 20 & 2010 & $\mathbf{S T}$ & Nar Ots & FOR & ספ\$\$ן & of \\
\hline
\end{tabular}

Sed footinow at end of ualle 
Table 20. Existing Generating Units at U.S. Electric Utillttes by State, Company, and Plant, as of January 1, 1996 (Continued)

\begin{tabular}{|c|c|c|c|c|c|c|c|c|c|}
\hline \multirow{2}{*}{$\begin{array}{l}\text { Sinte } \\
\text { Coupoang } \\
\text { Pined (Conterly) }\end{array}$} & \multirow{2}{*}{ Und } & \multirow{2}{*}{ 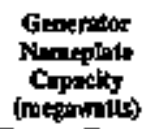 } & \multirow{2}{*}{ 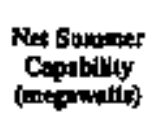 } & \multirow{2}{*}{$\begin{array}{l}\text { Net Wintar } \\
\text { Cepofillity } \\
\text { (mesamitis) }\end{array}$} & \multirow{2}{*}{$\underset{\text { Typt }}{\text { Uhit }}$} & \multicolumn{2}{|c|}{ Entros Saural } & \multirow{2}{*}{$\begin{array}{c}\text { Year } \\
\text { of } \\
\text { Cominarlel } \\
\text { Dperitinte }\end{array}$} & \multirow{2}{*}{ Stativil } \\
\hline & & & & & & Primary & Alterriate & & \\
\hline
\end{tabular}

New Madm (Coodimeth)

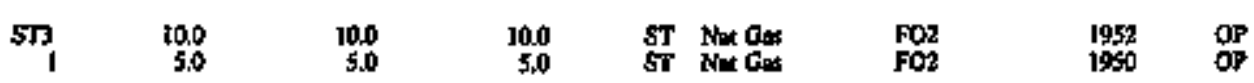

Not Yotk

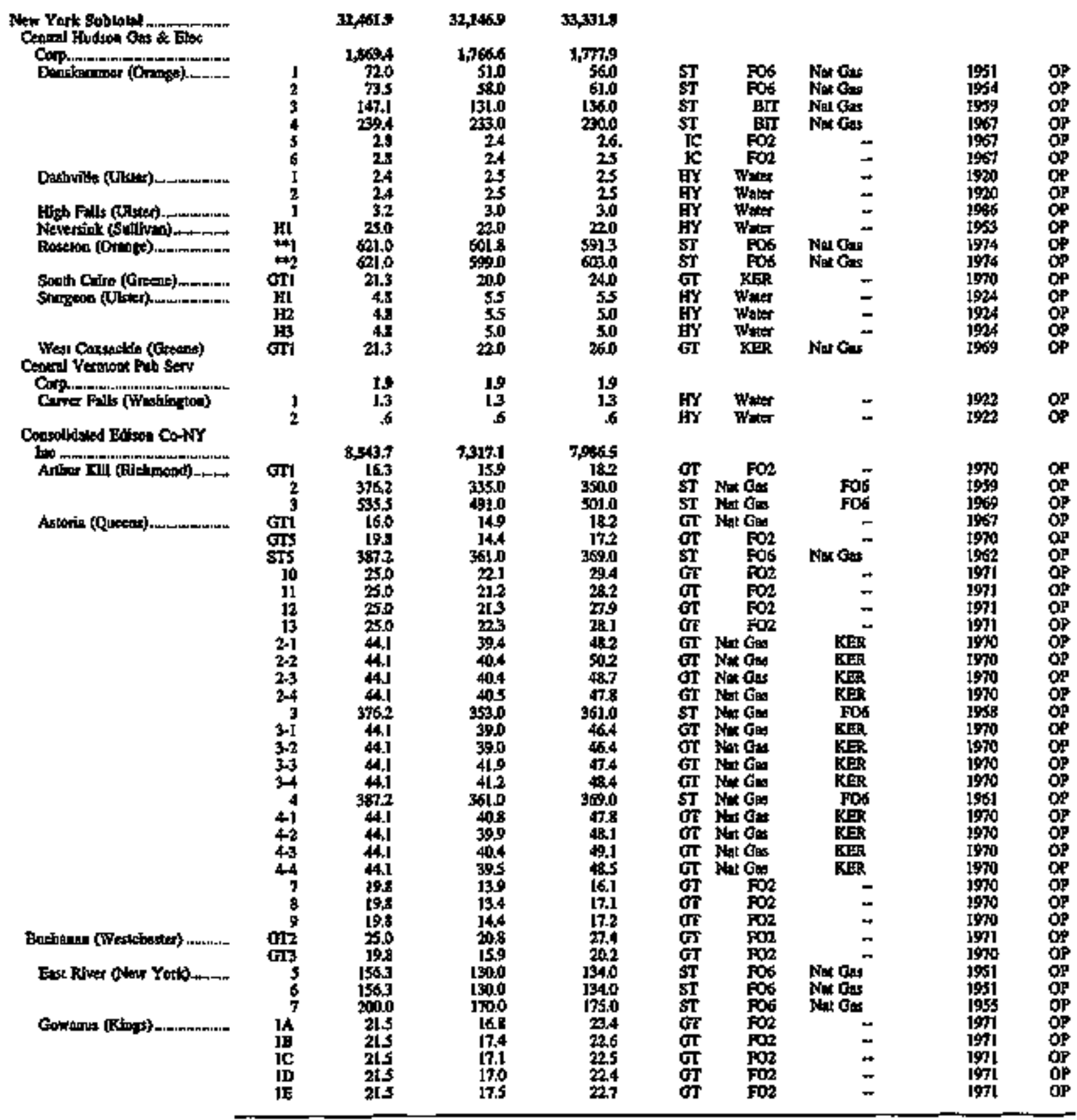

Ser foomotes at end of trible.

168 Energy Information Administration/Inventory of Power Piants in the United States as of January 1, 1996 
Table 20. Existing Generating Units at U.S. Electric Utultitles by State, Company, and Plant, as of January 1, 1996 (Continued)

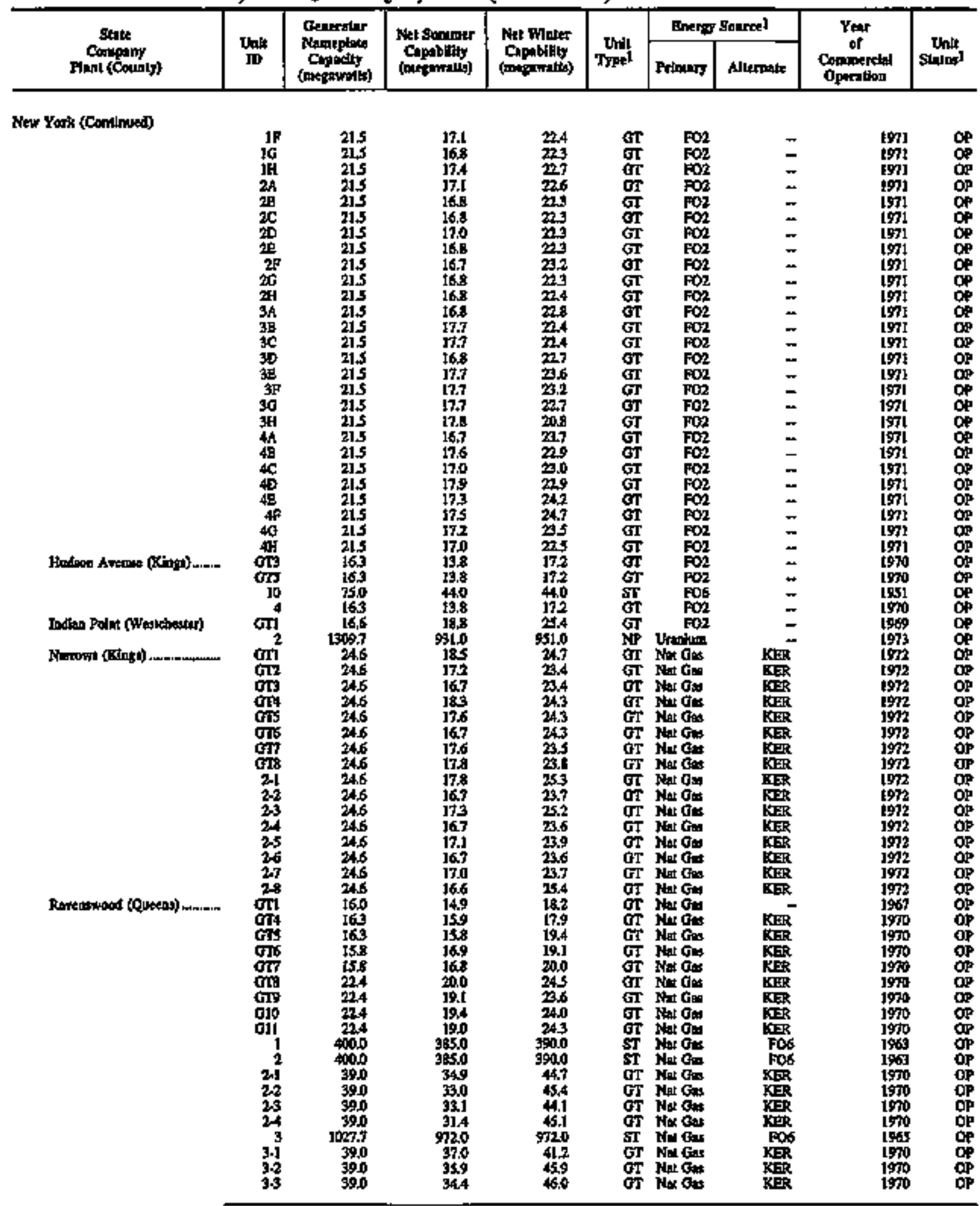

Ses foctiotios al and of able. 
Table 20. Existing Generating Units at U.S. Electric Utilities by State, Compary, and Plant, as of January 1, 1996 (Continused)

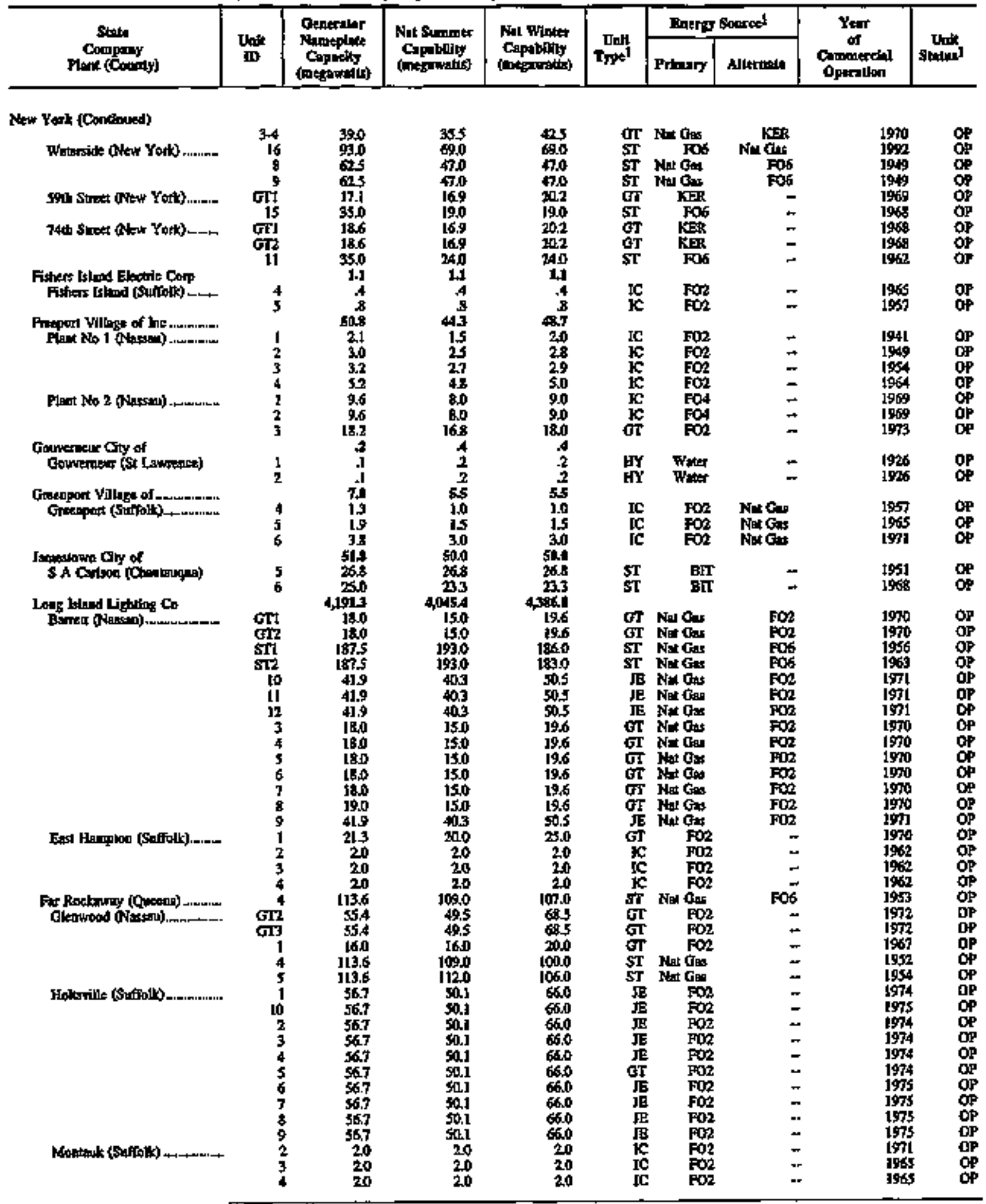

Sec footodes ar end of teibla.

170 Energy Information Adminfstration/Inventory of Power Plants in the United States as of January 1, 1996 
Table 20. Existing Generating Units at U.S. Electric Utilities by State, Company, and Plant, as of January 1, 1996 (Continued)

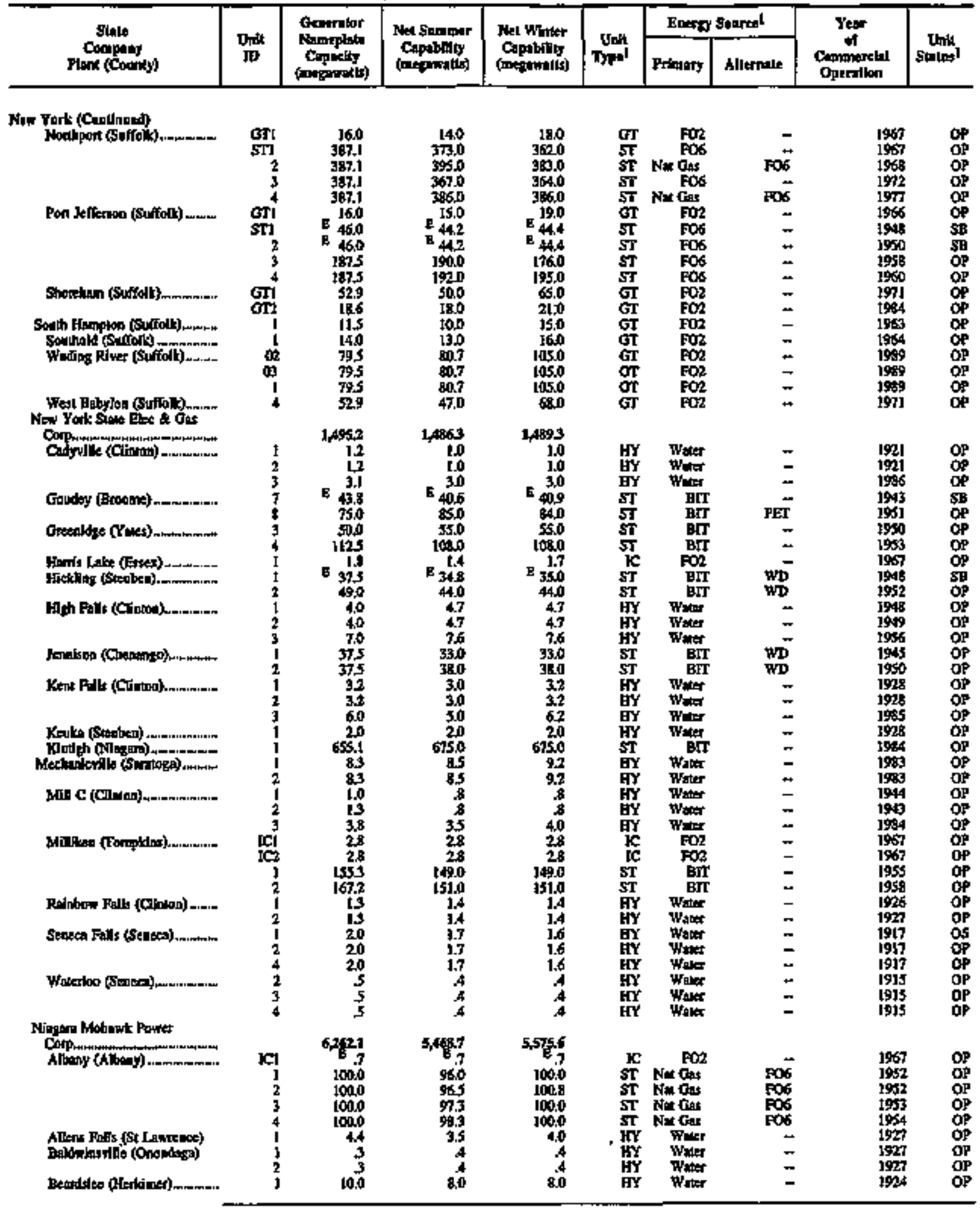

Seo foodmonas at ond of tabte 
Table 20. Existing Generating Units at U.S. Electric Utllities by State, Company, and Plant, as of January 1, 1996 (Continued)

\begin{tabular}{|c|c|c|c|c|c|c|c|c|c|}
\hline \multirow{2}{*}{$\begin{array}{c}\text { Stato } \\
\text { Coraptiny } \\
\text { Ptank (Cormity) }\end{array}$} & \multirow{2}{*}{$\underset{\text { Jphik }}{\text { Up }}$} & \multirow{2}{*}{ 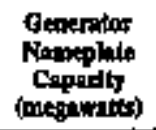 } & \multirow{2}{*}{ 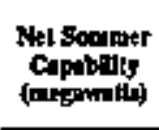 } & \multirow{2}{*}{$\begin{array}{l}\text { Het Winter } \\
\text { Copebllity } \\
\text { (miganotsis) }\end{array}$} & \multirow{2}{*}{ 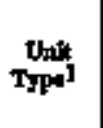 } & \multicolumn{2}{|c|}{ Eneng Soorced } & \multirow{2}{*}{$\begin{array}{c}\text { Year } \\
\text { of } \\
\text { Commanclal } \\
\text { Optritlon }\end{array}$} & \multirow{2}{*}{$\begin{array}{l}\text { Inik } \\
\text { Shtow } 1\end{array}$} \\
\hline & & & & & & Primat & Alernate & & \\
\hline \multicolumn{10}{|l|}{ New Yark (Canulanet) } \\
\hline & 2 & 10.0 & $\mathbf{8 0 0}$ & 80 & $\mathbf{B Y}$ & Water & - & 1924 & $\mathbf{O P}$ \\
\hline 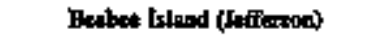 & $m^{-1}$ & 4.0 & $\mathbf{3 . 3}$ & 33 & $\mathbf{E Y}$ & Water & - & 1968 & OP \\
\hline & $\omega_{2}$ & 40 & 3.3 & 3.3 & HY & Water & - & 1960 & OP \\
\hline 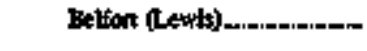 & i & 4 & 4 & 4 & $\mathbf{H Y Y}$ & Wh & $\overrightarrow{4}$ & 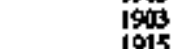 & ap \\
\hline & $\begin{array}{l}2 \\
3\end{array}$ & $\begin{array}{r}6 \\
1.0\end{array}$ & $\stackrel{A}{a}$ & $\stackrel{4}{1.0}$ & $\begin{array}{l}\mathbf{H Y} \\
\mathbf{H Y}\end{array}$ & $\begin{array}{l}\text { What } \\
\text { Witer }\end{array}$ & $\because$ & $\begin{array}{l}1915 \\
1918\end{array}$ & $\stackrel{O P}{O P}$ \\
\hline Bemens Bedoge (Orwego) & I & 64 & $\mathbf{6 3}$ & 6.2 & $\mathbf{E Y}$ & Water & $=$ & 1970 & OP \\
\hline & 2 & 64 & 65 & 62 & हYY & Water & $\ddot{-}$ & 1970 & $\mathbf{O P}$ \\
\hline & 4 & $\begin{array}{l}7.00 \\
7.0\end{array}$ & $\begin{array}{l}7.0 \\
7.0\end{array}$ & 7.0 & thy & $\begin{array}{l}\text { Whet } \\
\text { Water }\end{array}$ & $\bar{z}$ & $\begin{array}{l}1900 \\
1964\end{array}$ & GP \\
\hline 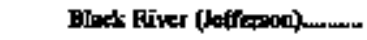 & i & 200 & 1.7 & 19 & $\mathbf{H Y}$ & Wntra & ـ & 1920 & op \\
\hline & $\mathbf{2}$ & 20 & 1.7 & 1.9 & $\mathbf{H Y}$ & Water & $=$ & 1920 & op \\
\hline & 3 & 20 & 17 & 1.9 & Нy & Walet & .. & 1920 & of \\
\hline stost & I & 144 & 140 & 14.9 & HY & Wobr & - & 1957 & $\mathbf{a P}$ \\
\hline 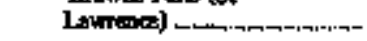 & I & 75 & 3.4 & 74 & $\mathbf{H Y}$ & Water & - & I923 & OP \\
\hline \multirow{6}{*}{ CR Horikg (teis) } & 2 & 85 & $7 A$ & 7.4 & EX & Wan & - & 1923 & op \\
\hline & $\begin{array}{l}\text { ICI } \\
\text { S6I }\end{array}$ & $\begin{array}{r}E .7 \\
217.6\end{array}$ & $\begin{array}{r}67 \\
1900\end{array}$ & $\begin{array}{l}\text { B.7 } \\
1900\end{array}$ & $\underset{\mathbf{S T}}{\mathbf{L C}}$ & $\underset{B T T}{F O 2}$ & 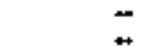 & $\begin{array}{l}1997 \\
1958\end{array}$ & $\begin{array}{l}\text { OP } \\
\mathbf{G P}\end{array}$ \\
\hline & 63 & 920 & 850 & $\mathbf{8 5 0}$ & $\mathbf{S T}$ & BII & - & 1942 & OP \\
\hline & 64 & 100.0 & geon & 90,0 & $\mathbf{S T}$ & BaT & - & 1948 & OP \\
\hline & 65 & $\operatorname{logh}$ & 90,0 & 900 & $\underset{\mathbf{S T}}{\mathbf{S T}}$ & Br & - & 1053 & OP \\
\hline & 67 & 217.6 & 19000 & $\begin{array}{l}9000 \\
1850\end{array}$ & $\begin{array}{l}\mathbf{S T} \\
\mathbf{S T}\end{array}$ & $\begin{array}{l}\text { Bir } \\
\text { BIT }\end{array}$ & $\ddot{2}$ & $\begin{array}{l}1954 \\
1957\end{array}$ & $\begin{array}{l}\text { OP } \\
\text { OP }\end{array}$ \\
\hline 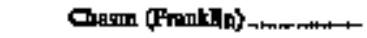 & 1 & 1.0 & 10 & $\mathrm{LS}$ & EY & Wate & $\leftrightarrow$ & 1913 & of \\
\hline & 2 & 1.0 & 1,0 & 1,3 & Ex & Waber & - & 1913 & op \\
\hline 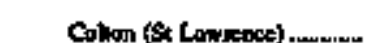 & 3 & 100 & 1.2 & 1,3 & EY & Waver & - & 1926 & op \\
\hline & $\begin{array}{l}1 \\
3\end{array}$ & 300 & $\begin{array}{l}95 \\
95\end{array}$ & 95 & HY & Wurs & $\ddot{+}$ & 1962 & OP \\
\hline & 3 & 300 & 90 & 90 & HY & Water & - & 1908 & op \\
\hline Deferiel (Jeffetion) & 1 & 3.6 & 29 & 34 & $\mathbf{H Y}$ & Water & ـ & by & OP \\
\hline & 2 & 3.6 & $\frac{29}{29}$ & 3.4 & BY & Whit & $*$ & 192 & op \\
\hline \multirow{5}{*}{ Durkitk (Chontrugyas) } & 3 & $\mathrm{H}^{3.5}$ & 29 & 3.4 & HY & Water & - & 1925 & op \\
\hline & IG & 5 & 03 & Bs 5 & ic & FO2 & - & 1990 & OP \\
\hline & \$TS & 2180 & IS50 & 195.0 & sT & BIT & $\leftarrow$ & 1960 & $\mathbf{o p}$ \\
\hline & 1 & 960 & 910 & 910 & $\$ \mathbf{T}$ & BIT & - & 1990 & op \\
\hline & $\frac{1}{3}$ & $\begin{array}{r}950 \\
2180\end{array}$ & 2000 & $\begin{array}{r}920 \\
1980\end{array}$ & $\stackrel{S T}{\mathbf{S T}}$ & $\begin{array}{l}\text { BiT } \\
\text { BIT }\end{array}$ & 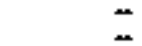 & $\begin{array}{l}1950 \\
1998\end{array}$ & $\underset{O P}{O P}$ \\
\hline 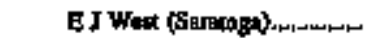 & 1 & 100 & 3.7 & 7.3 & BY & Wate & $\leftrightarrow$ & 1930 & oP \\
\hline & 2 & 30.0 & 7.7 & 7.7 & HY & Water & - & [1990 & OP \\
\hline \multirow{3}{*}{ 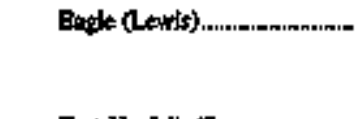 } & 1 & 13 & 10 & 1.0 & EY & Wur & - & 1914 & op \\
\hline & 2 & 14 & 1.0 & 1.0 & $\begin{array}{l}\text { GY } \\
\text { HY }\end{array}$ & Wht & $\approx$ & 1915 & op \\
\hline & 4 & 21 & 20 & 20 & $\begin{array}{l}\mathbf{H Y} \\
\mathbf{H Y}\end{array}$ & Water & $\ddot{*}$ & isig & GP \\
\hline \multicolumn{10}{|l|}{ Estrt Nottolt (St } \\
\hline Leturetes) & & 3.0 & 3.6 & 3.6 & EY & Wats & - & 1928 & of \\
\hline Eel Weir (si Laracos) & 1 & $s$ & 3 & 3 & HY & Wuter & - & 198 & op \\
\hline & 2 & 1.1 & 5 & 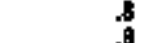 & HY & Whet & - & 1938 & OP \\
\hline & 3 & & & & EY & Water & - & 1938 & op \\
\hline 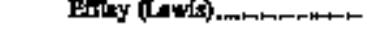 & 1 & 4 & 4 & $A$ & EY & Water & 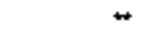 & 1902 & of \\
\hline & 3 & 4 & $\stackrel{4}{6}$ & 6 & हY & Water & $\bar{z}$ & 1909 & OP \\
\hline & 4 & $t .6$ & 13 & 13 & HY & Wor & $\bar{z}$ & 1923 & of \\
\hline Elner (Lewis)............-. & i & 8 & 8 & 8 & HY & Wuter & ـ & 1916 & op \\
\hline & $i$ & 8 & $\mathbf{8}$ & .8 & HYY & Whate & - & 1916 & $\mathbf{p P}$ \\
\hline Fphraltb (Fulkon) & I & I.4 & 3 & 6 & $\mathbf{E Y}$ & Water & - & 1520 & OP \\
\hline & 2 & 12 & 5 & 6 & 奛 & Water & $=$ & 191t & pp \\
\hline & 3 & 1.3 & $\frac{3}{5}$ & 6 & ㅂy & Water & $\bar{z}$ & 1911 & os \\
\hline 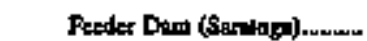 & j & 12 & $\vec{g}$ & $g$ & BY & What & $=$ & 1911 & OP \\
\hline & 2 & 12 & .9 & 9 & ey & Water & $=$ & $\begin{array}{l}1924 \\
1924\end{array}$ & OP \\
\hline & 3 & $i .2$ & 9 & 9 & EY & $W_{n=r}$ & $=$ & 1924 & of \\
\hline & 4 & 12 & 9 & 9 & $\mathbf{H Y}$ & Wher & - & 1924 & $O P$ \\
\hline & 5 & 12 & 9 & 9 & & Water & 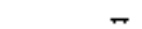 & 1924 & OP \\
\hline Five Fills (St lapuenes) & J & 224 & 23.9 & 23.9 & HY & water & - & 1955 & op \\
\hline 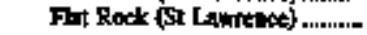 & i & 3.0 & 25 & 25 & EYY & Wats & - & 1924 & OP \\
\hline & 2 & 3.0 & 25 & 25 & $\mathbf{H Y}$ & Witer & - & 1924 & OP \\
\hline Finflitin (Fraridiln) & I & L.I & 1.0 & 1.1 & HY & Wuter & - & $\$ 911$ & OP \\
\hline
\end{tabular}

Seo toonetes all and of whe. 
Table 20. Exdstlug Generating Units at U.S. Blectric Utifities by State, Company, and Plant, as of January 1, 1996 (Continured)

\begin{tabular}{|c|c|c|c|c|c|c|c|c|c|}
\hline \multirow{2}{*}{$\underset{\text { Contesty }}{\text { Sind }(\operatorname{Coc} 1 y)}$} & \multirow{2}{*}{ Uh:k } & \multirow{2}{*}{ 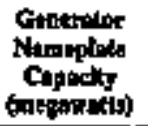 } & \multirow{2}{*}{ 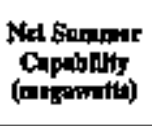 } & \multirow{2}{*}{ 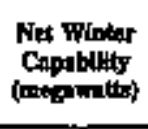 } & \multirow{2}{*}{$\underset{T_{T E}}{\text { Dolt }}$} & \multicolumn{2}{|c|}{ Bnerey Sourded } & \multirow{2}{*}{ 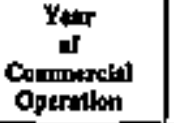 } & \multirow{2}{*}{ 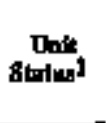 } \\
\hline & & & & & & Prtway & Akimalo & & \\
\hline \multicolumn{10}{|l|}{ Ney Yock (Contowed) } \\
\hline & 2 & I.1 & 10 & I.I & HY & Wrater & - & 1926 & OP \\
\hline Aulon (Otwoga) & 1 & .8 & 5 & 5 & 榃 & Wust & . & 1924 & OP \\
\hline Geprood (Oteane) & $\begin{array}{l}2 \\
1\end{array}$ & 5 & $\frac{5}{3}$ & .5 & Hy & Whar & 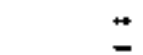 & 108 & op \\
\hline 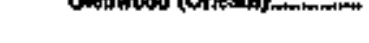 & $i$ & 5 & 2 & II & 慰Y & Wuts & 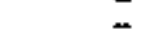 & $\begin{array}{l}1900 \\
1950\end{array}$ & op \\
\hline & 3 & 5 & 2 & 1 & $\overrightarrow{\mathbf{H}} \dot{\mathbf{Y}}$ & Witer & . & 1980 & $\mathbf{O P}$ \\
\hline 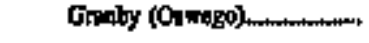 & 1 & 5.0 & $3 \overline{5}$ & 35 & HY & $W_{n=1}$ & - & 1953 & op \\
\hline Green fyland (Abany)....... & $\begin{array}{l}2 \\
1\end{array}$ & 5.0 & 3.5 & 3.4 & fry & Wits & $\overline{-}$ & $\begin{array}{l}1963 \\
19 n 1\end{array}$ & op \\
\hline & 3 & 15 & 1.1 & 14 & $\underset{\mathrm{WY}}{\mathrm{FY}}$ & Wuter & $=$ & 197 & 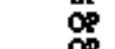 \\
\hline & 3 & 1.5 & l.1 & 1.4 & HYY & Wher & $\bar{z}$ & 1971 & OP \\
\hline 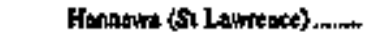 & 1 & 3.6 & 3.7 & 37 & $\mathbf{H Y}$ & Ww & - & 1914 & $\mathrm{CP}^{\mathrm{P}}$ \\
\hline Herings (Jefferood) & 2 & 3.6 & $\begin{array}{l}3.7 \\
1.1\end{array}$ & 3.7 & IY & Wes: & $=$ & 1920 & Op \\
\hline & 2 & $1+6$ & I.I & 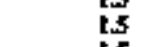 & HY & Wuter & $\bar{m}$ & t924 & op \\
\hline & 3 & 1.5 & i.1 & t.5 & $\mathbf{F Y}$ & How & - & 1924 & op \\
\hline 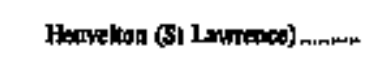 & 1 & 5 & 4 & .4 & $\underset{\mathbf{H Y}}{\mathbf{H Y}}$ & Wom & $\stackrel{+}{-}$ & 194 & OP \\
\hline \multirow{3}{*}{ Heb Don (Oswego).......... } & i & 8 & 1.0 & 13 & HY & Water & $\bar{z}$ & 1923 & OP \\
\hline & $\frac{1}{3}$ & 1.8 & $\begin{array}{l}10 \\
10\end{array}$ & is & HY & $\begin{array}{l}\text { Water } \\
\text { Wutor }\end{array}$ & 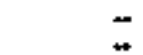 & In:5 & $\underset{\phi}{\phi}$ \\
\hline & 4 & 2,2 & 1.0 & 20 & 些Y & What & 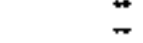 & 1900 & OF \\
\hline \multirow[t]{2}{*}{ 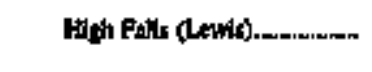 } & i & 1.6 & 1.6 & 5.6 & FY & Witer & - & 102 & pP \\
\hline & 3 & 1.6 & 1.6 & $\begin{array}{l}1.6 \\
1.6\end{array}$ & $\begin{array}{l}\text { EY } \\
\text { HY }\end{array}$ & Wor & $\ddot{z}$ & $\begin{array}{l}1925 \\
1925\end{array}$ & op \\
\hline \multirow{3}{*}{ 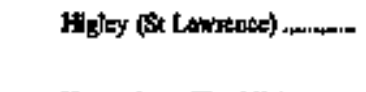 } & i & 12 & t.t & 12 & KY & Water & ـ & 1913 & OP \\
\hline & 3 & 1.2 & t,I & 1.2 & $\mathbf{H Y}$ & Wait & - & 1913 & $\mathbf{Q P}$ \\
\hline & 3 & 21 & i... & 1.7 & HY & Wotes & - & 1943 & OP \\
\hline \multirow{4}{*}{ 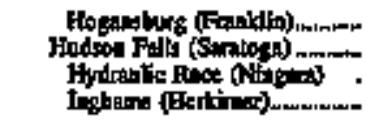 } & $i$ & 7 & 4 & 4 & HY & Water & - & 1930 & op \\
\hline & A & 36.1 & 35.0 & 15,0 & EY & Wator & - & 1995 & Op \\
\hline & I & 4.7 & 20 & 48 & $\mathbf{F Y}$ & $W_{n}=$ & 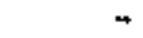 & 19012 & $\alpha^{\prime}$ \\
\hline & $\frac{1}{2}$ & 32 & 25 & 25 & HY & Watr & $\ddot{z}$ & 3912 & op \\
\hline \multirow[t]{2}{*}{ Jolnsoorile (Renspetion) } & 1 & 24 & is & 15 & HY & Water & $\bar{z}$ & 1909 & op \\
\hline & 2 & 24 & IS & 1.5 & tiY & Wrax & $\leftrightarrow$ & 1909 & OP \\
\hline \multirow[t]{2}{*}{ 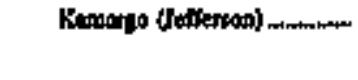 } & 1 & 18 & 1.6 & $1 \mathrm{~A}$ & EY & Watur & - & 1921 & OP \\
\hline & $\frac{2}{3}$ & 1.8 & 1.6 & 1.6 & $\underset{\mathbf{H Y}}{\mathbf{E Y}}$ & Water & $=$ & 1921 & op \\
\hline 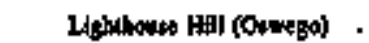 & & 3.9 & 38 & 38 & $\mathbf{H Y}$ & Witer & $=$ & 1930 & or \\
\hline & 2 & $\mathbf{1 . 8}$ & 38 & 38 & $\mathbf{H Y}$ & Whax & - & 1930 & OP \\
\hline 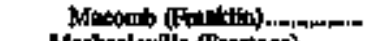 & I & 10 & 9 & 1.0 & EY & Water & - & 1940 & $\mathrm{OP}$ \\
\hline 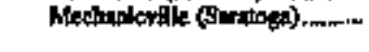 & 1 & 8 & 4 & $\underset{s}{5}$ & $\begin{array}{l}\mathbf{H Y} \\
\mathbf{H Y}\end{array}$ & $W_{m=x}$ & $\overline{-}$ & 1890 & OP \\
\hline & 3 & 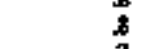 & 4 & 3 & HY & Water & $=$ & $1 \%$ & os \\
\hline & & 7 & 4 & 5 & HY & Wes & 4 & 189: & os \\
\hline & $\frac{5}{7}$ & 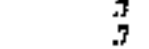 & $\stackrel{4}{4}$ & $\begin{array}{l}5 \\
s\end{array}$ & $\begin{array}{l}\mathbf{H Y} \\
\mathbf{H Y}\end{array}$ & Wothe & $\bar{z}$ & $18 \%$ & of \\
\hline Minewo (Ouncto)............. & HYi & 1.6 & 13 & 1.5 & HY & Water & - & L15 & OP \\
\hline & $\begin{array}{l}\mathrm{HY}_{2} \\
\mathrm{HY3}\end{array}$ & 1.6 & 6.3 & $\begin{array}{l}1.5 \\
1.5\end{array}$ & $\mathbf{H Y}$ & $W_{\text {intr }}$ & + & 1915 & $O P$ \\
\hline & HY4 & 1.6 & 1.3 & 13 & $\begin{array}{l}\mathbf{H Y} \\
\mathbf{H Y}\end{array}$ & Water & $\bar{z}$ & $\begin{array}{l}1915 \\
1975\end{array}$ & op \\
\hline & EYS & 1.6 & 13 & 15 & $\mathbf{y}$ & Waret & - & I915 & op \\
\hline Modhler (thatinet) & I & 40 & 43 & 43 & Eे & Wote & $\rightarrow$ & 690 & op \\
\hline 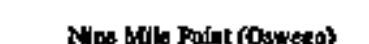 & i & $\begin{array}{r}40 \\
6418\end{array}$ & $\begin{array}{r}4.3 \\
617.0\end{array}$ & 648 & HY & Witr & $\bar{z}$ & 1929 & op \\
\hline & +2 & 1259.3 & 10063 & 10563 & N⿴囗十 & Upandes & $\bar{z}$ & 1908 & ap \\
\hline Norfouk (st Satritencos) & i & 45 & 38 & 93 & फY & Whet & - & 192 & op \\
\hline 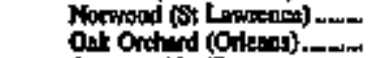 & 1 & $\mathbf{x}_{4}^{20}$ & 20 & $\frac{22}{64}$ & $\underset{H Y Y}{H Y}$ & $\begin{array}{l}\text { Wovt } \\
\text { Warf }\end{array}$ & $\overline{+}$ & $\begin{array}{l}1923 \\
1941\end{array}$ & $\begin{array}{l}\text { OP } \\
\text { OP }\end{array}$ \\
\hline 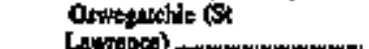 & N & $E_{2}$ & $\mathbf{E}_{\mathbf{2}}$ & $\mathbf{B}_{2}$ & & Wout & & 1988 & \\
\hline [1, & ${ }_{1}$ & -2 & -4 & .2 & $\underset{H Y Y}{\mathbf{H Y}}$ & $\begin{array}{l}\text { Wakt } \\
\text { Wuler }\end{array}$ & $\overline{-}$ & $\begin{array}{l}1988 \\
1913\end{array}$ & op \\
\hline 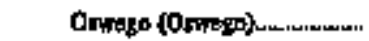 & ci & E 7 & 9 & .7 & & FO2 & $=$ & 1957 & $O P$ \\
\hline & $\mathbf{S O}$ & E. & $\stackrel{B}{E}$ & 8 & c & $P_{2}^{2}$ & 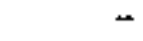 & $19 n 6$ & 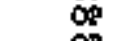 \\
\hline & $\mathbf{S T 3}$ & 902.8 & 9020 & 9020 & $\underset{\boldsymbol{E}}{\mathbf{K}}$ & FOA & $=$ & $\begin{array}{l}19 \% 0 \\
1976\end{array}$ & $\stackrel{\mathscr{P}}{\mathrm{s}}$ \\
\hline & " & $\$ 020$ & 598,0 & 636.1 & ST & FDS & - & $19 \% 0$ & op \\
\hline
\end{tabular}

Set footinoke to ond af uble 
Table 20. Existing Generating Units at U.S. Flectric Utilities by State, Company, and Plant, as of January 1, 1996 (Continued)

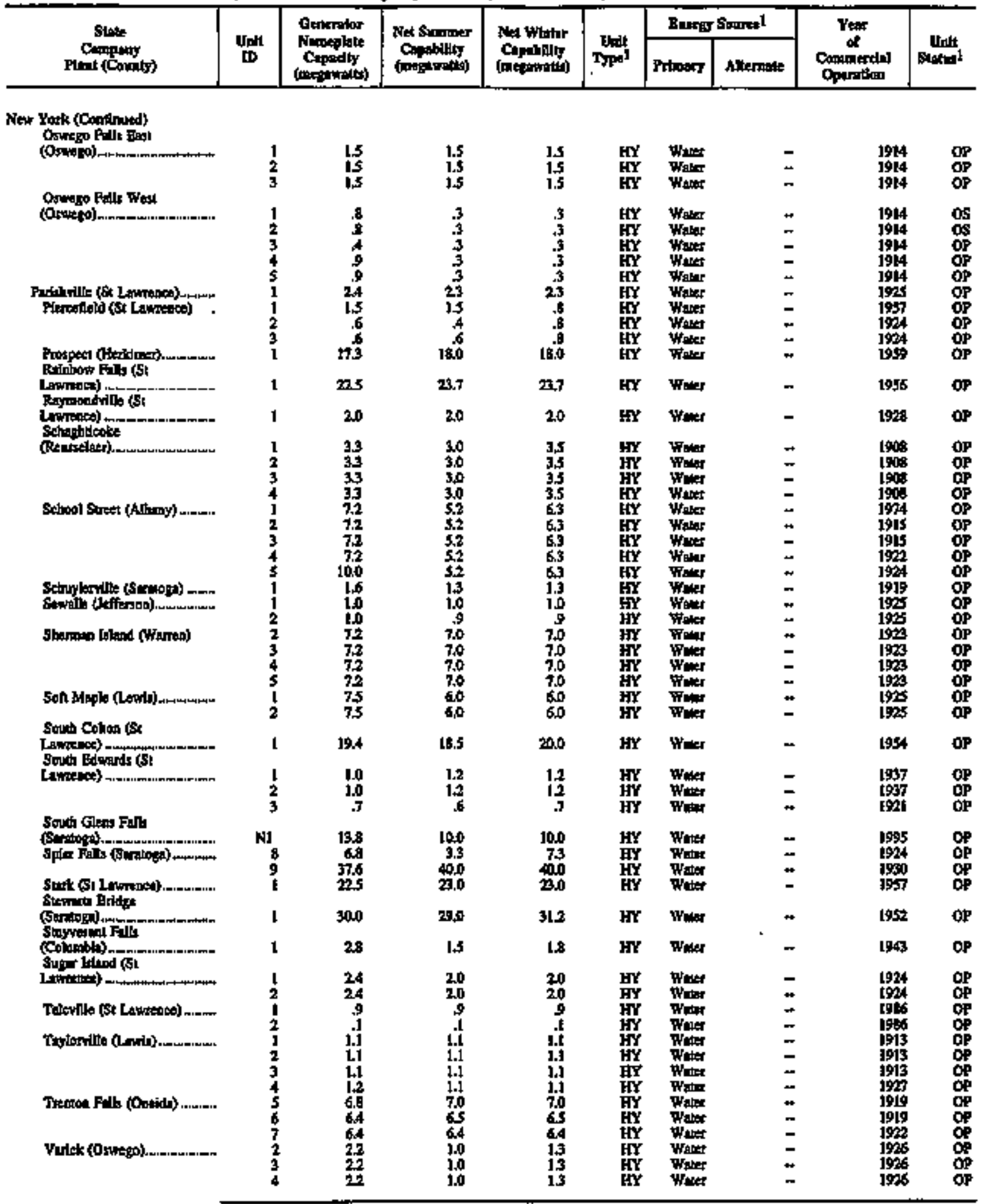

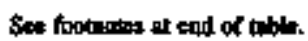


Table 20. Existing Generating Unfts at U.S. Electric Utilities by State, Company, and Plant, as of January 1, 1996 (Continued)



Sos focingers al opd of table. 
Table 20. Existing Generating Units at U.S. Electric Utilittes by State, Company, and Plant, as of January 1, 1996 (Continued)

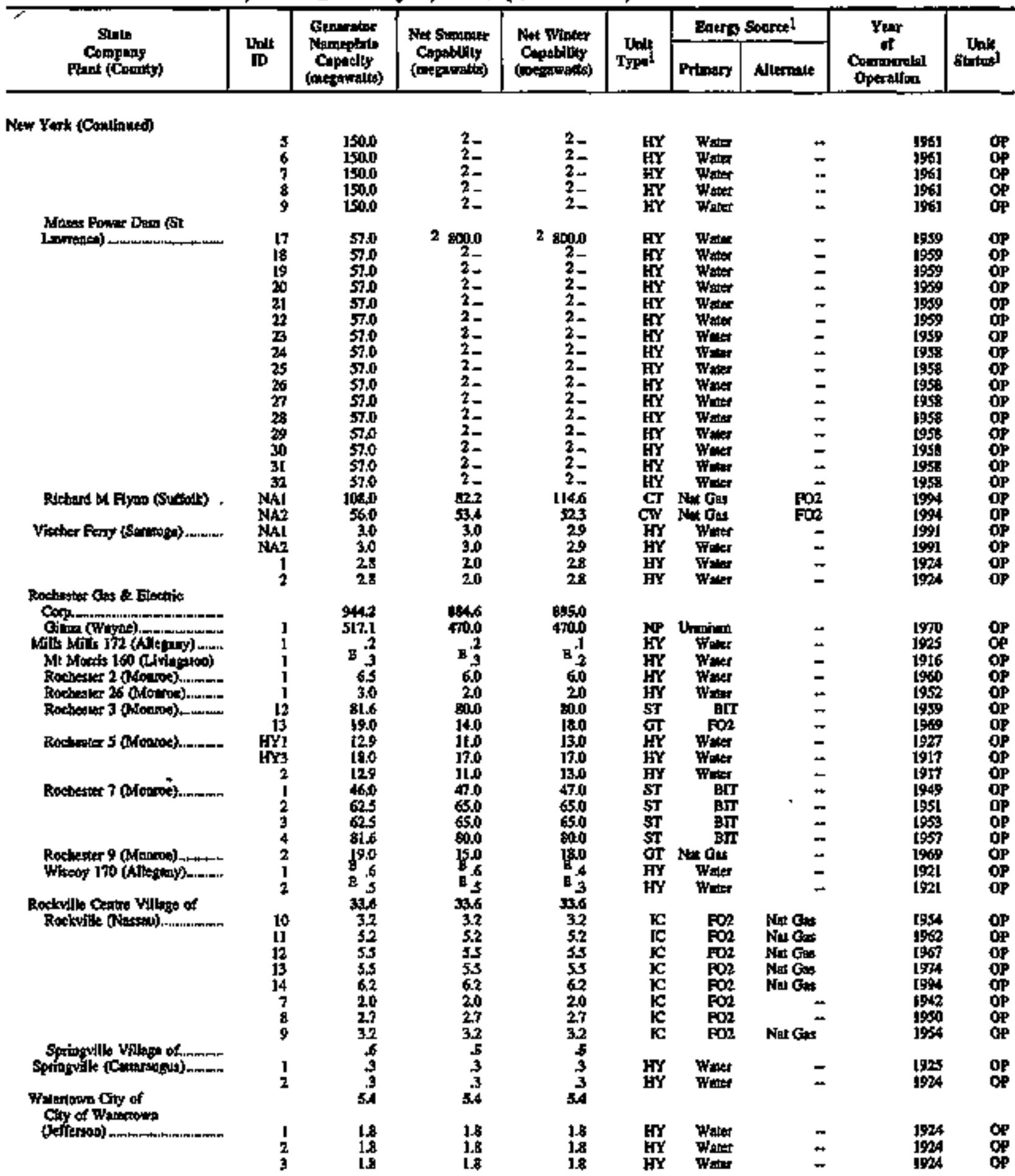

Narth 6umlng

North Combin Stbrolal .......

21, 556.1

$20,596.5$

209909

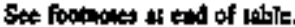

I76 Energy Information Administration/Apventory of Power Plants in the United States as of January I, 1996 
Table 20. Existing Generating Units at U.S. Electric Utilities by State, Company, and Plant, as of January 1, 1996 (Continued)

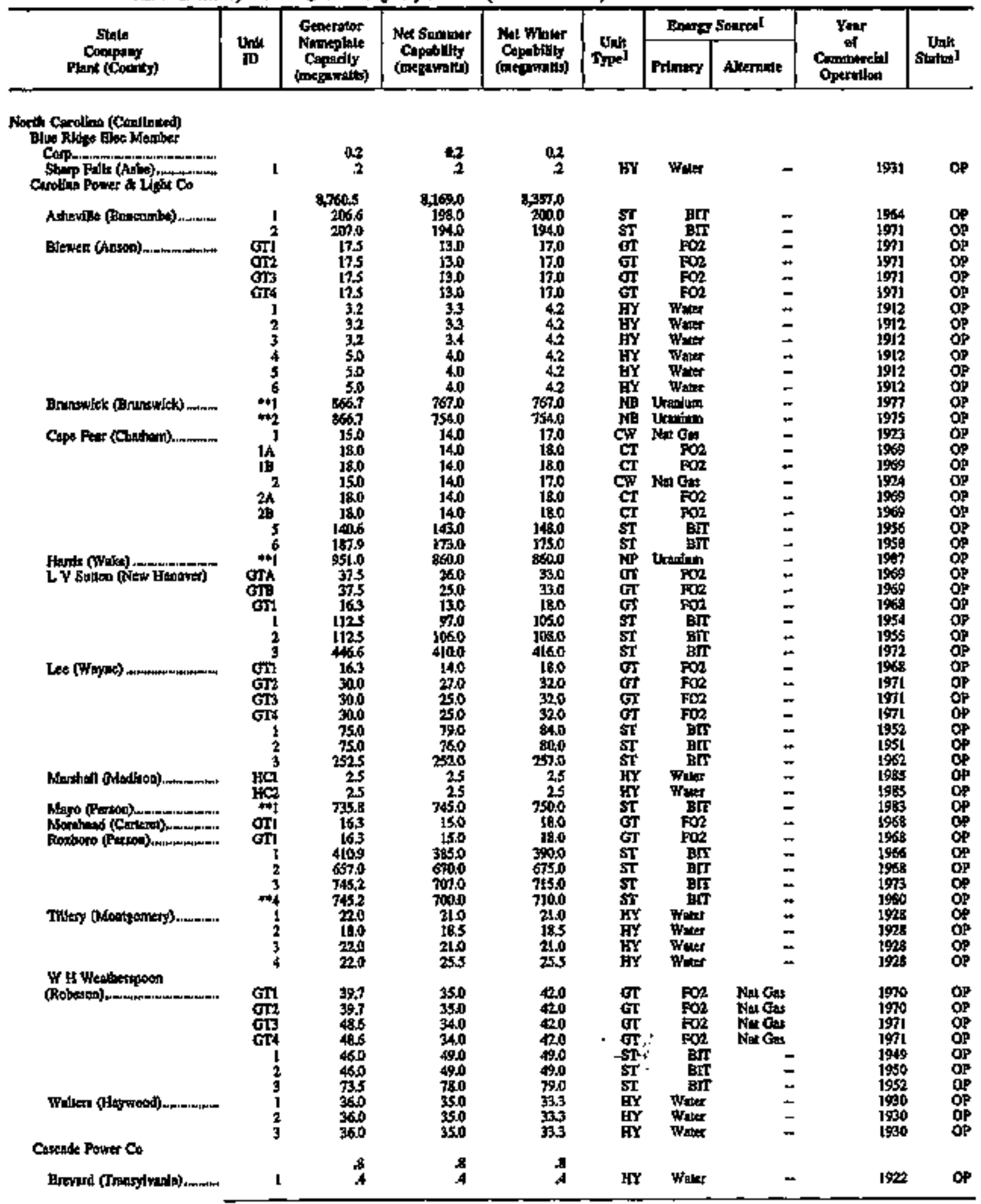

Sect footnotes at end of thbla 
Table 20. Existing Generating Units at U.S. Electric Utilities by State, Company, and Plant, as of January 1, 1996 (Continued)

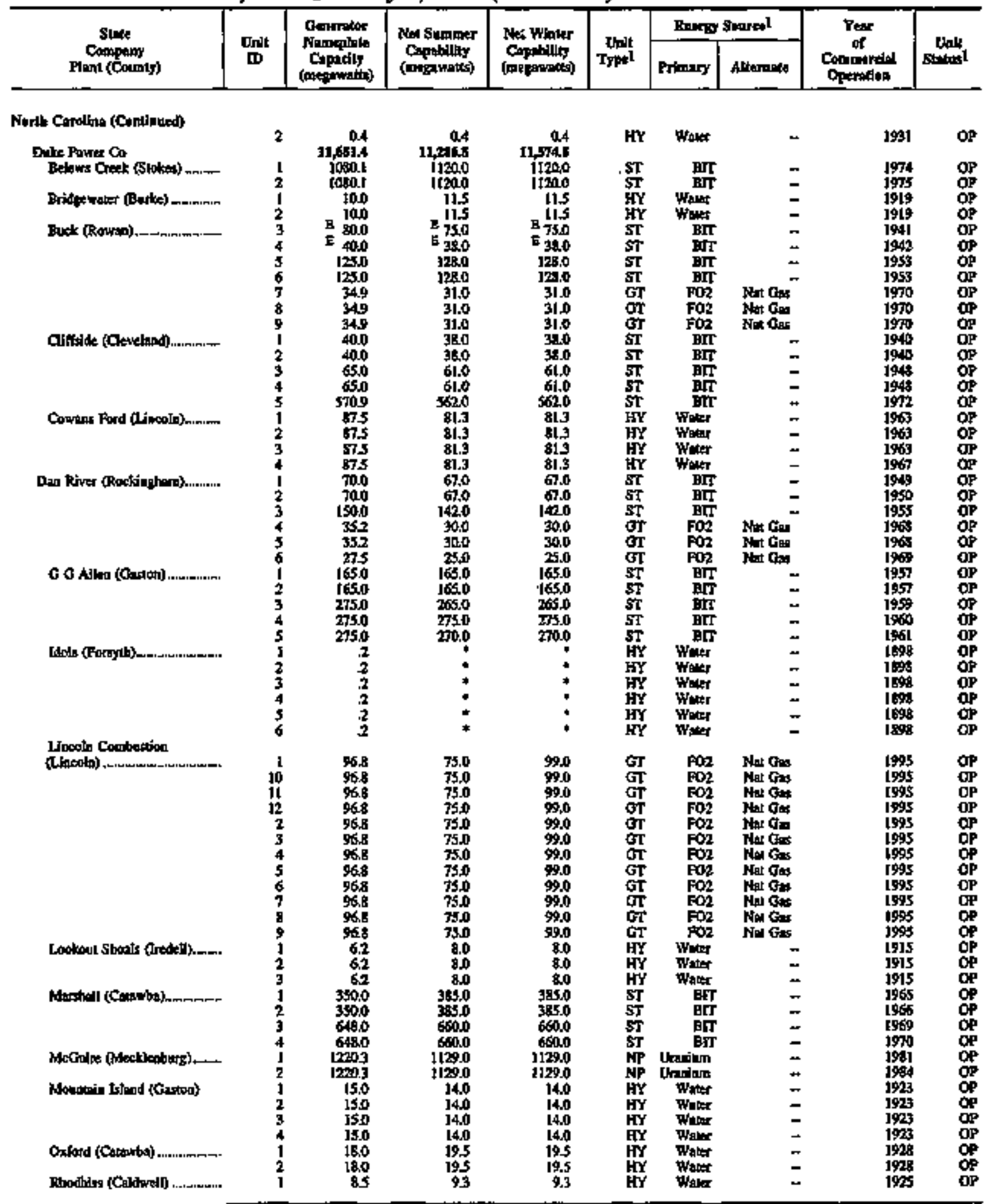

Sea foothowa st end of labk. 
Table 20. Existing Generating Units at U.S. Electric Utalities by State, Company, and Plant, as of January 1, 1996 (Continued)

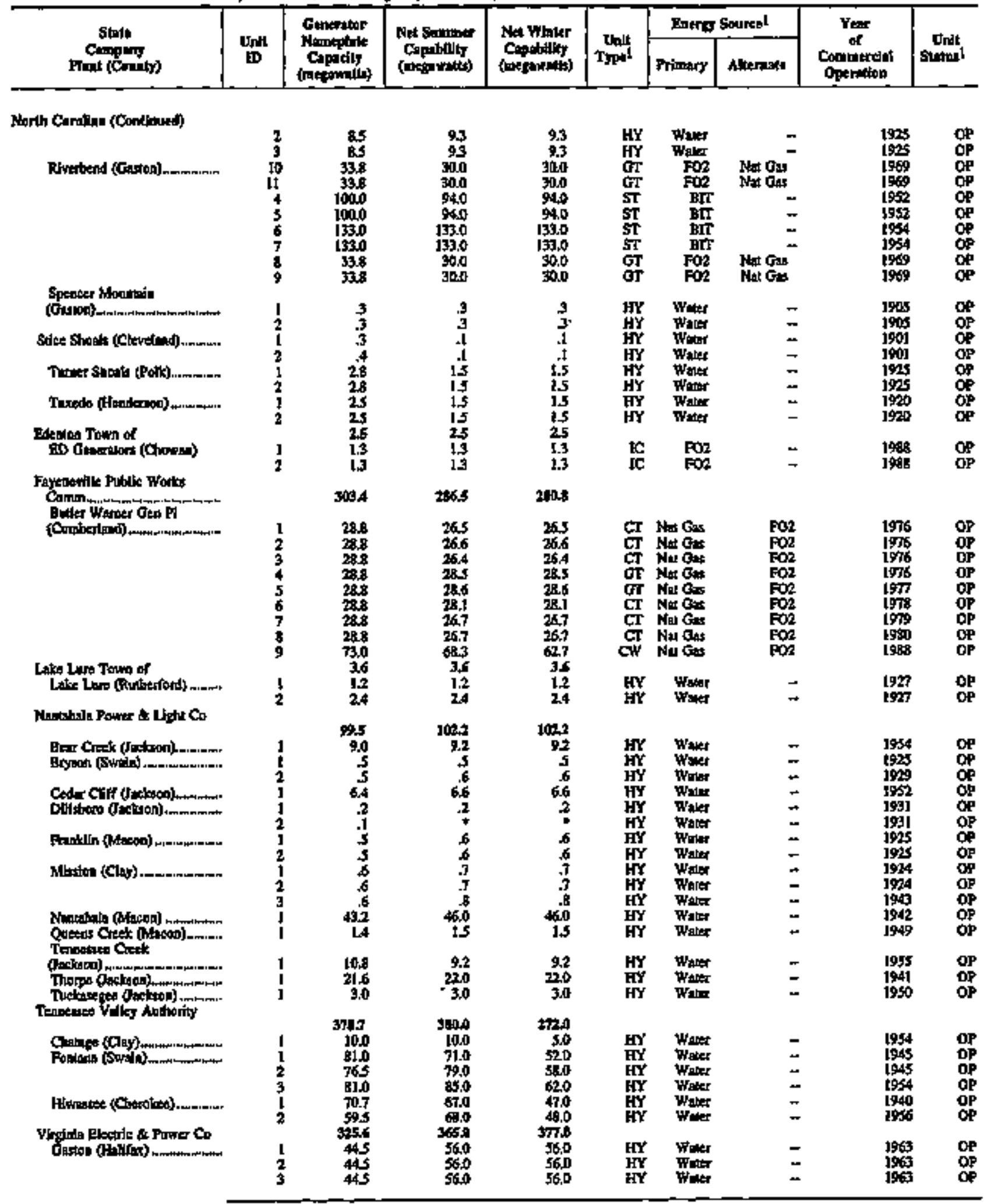

Seo roolpolut at end al temble. 
Table 20. Exdsting Generating Units at U.S. Electric Ut3lities by State, Company, and Plant, as of January 1, 1996 (Continued)

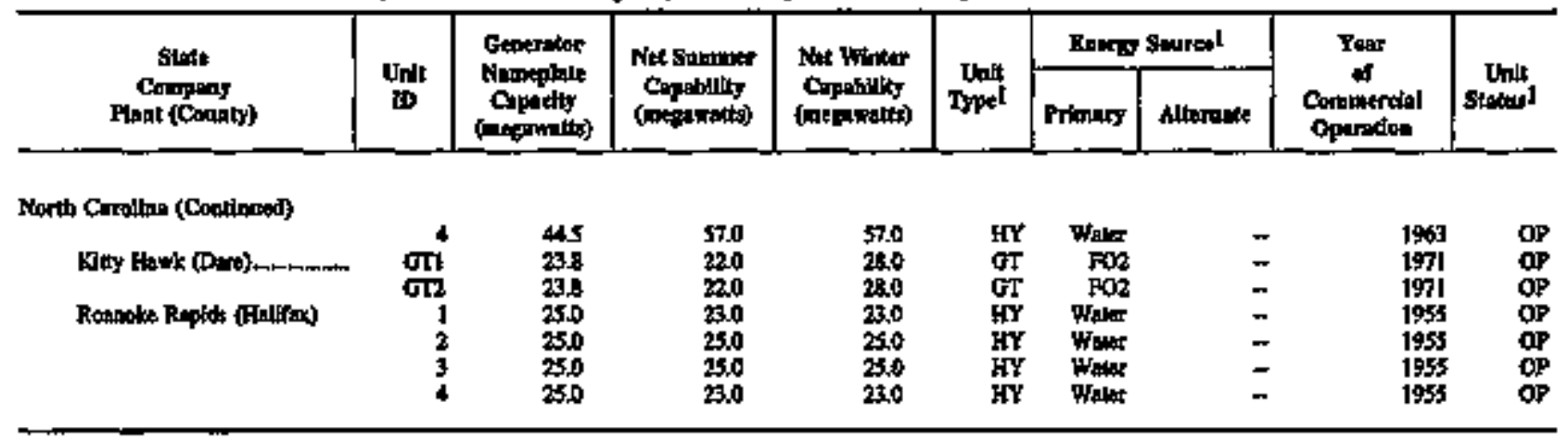

Sec focmotes ar ead of uble.

180 Energy Information Adrinistration/Inventory of Power Plants in the United States as of Janusry 1, 1996 
Table 20. Existing Generating Units at U.S. Electric Utaltties by State, Company, and Plant, as of January 1, 1996 (Continued)

\begin{tabular}{|c|c|c|c|c|c|c|c|c|c|}
\hline \multirow{2}{*}{$\begin{array}{c}\text { Slope } \\
\text { Couppany } \\
\text { Fisal (County) }\end{array}$} & \multirow{2}{*}{$\begin{array}{c}\text { Unlt } \\
\text { ID }\end{array}$} & \multirow{2}{*}{ 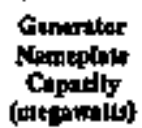 } & \multirow{2}{*}{$\begin{array}{l}\text { Net Solomer } \\
\text { Capabllity } \\
\text { (negerwats) }\end{array}$} & \multirow{2}{*}{ 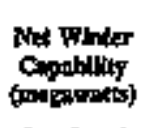 } & \multirow{2}{*}{ 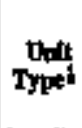 } & \multicolumn{2}{|c|}{ Bourt Aburts } & \multirow{2}{*}{$\begin{array}{c}\text { Varr } \\
\text { of } \\
\text { Commind } \\
\text { Operitim }\end{array}$} & \multirow{2}{*}{ Sula } \\
\hline & & & & & & Prtanary & Alleranive & & \\
\hline
\end{tabular}

Noril Donotim

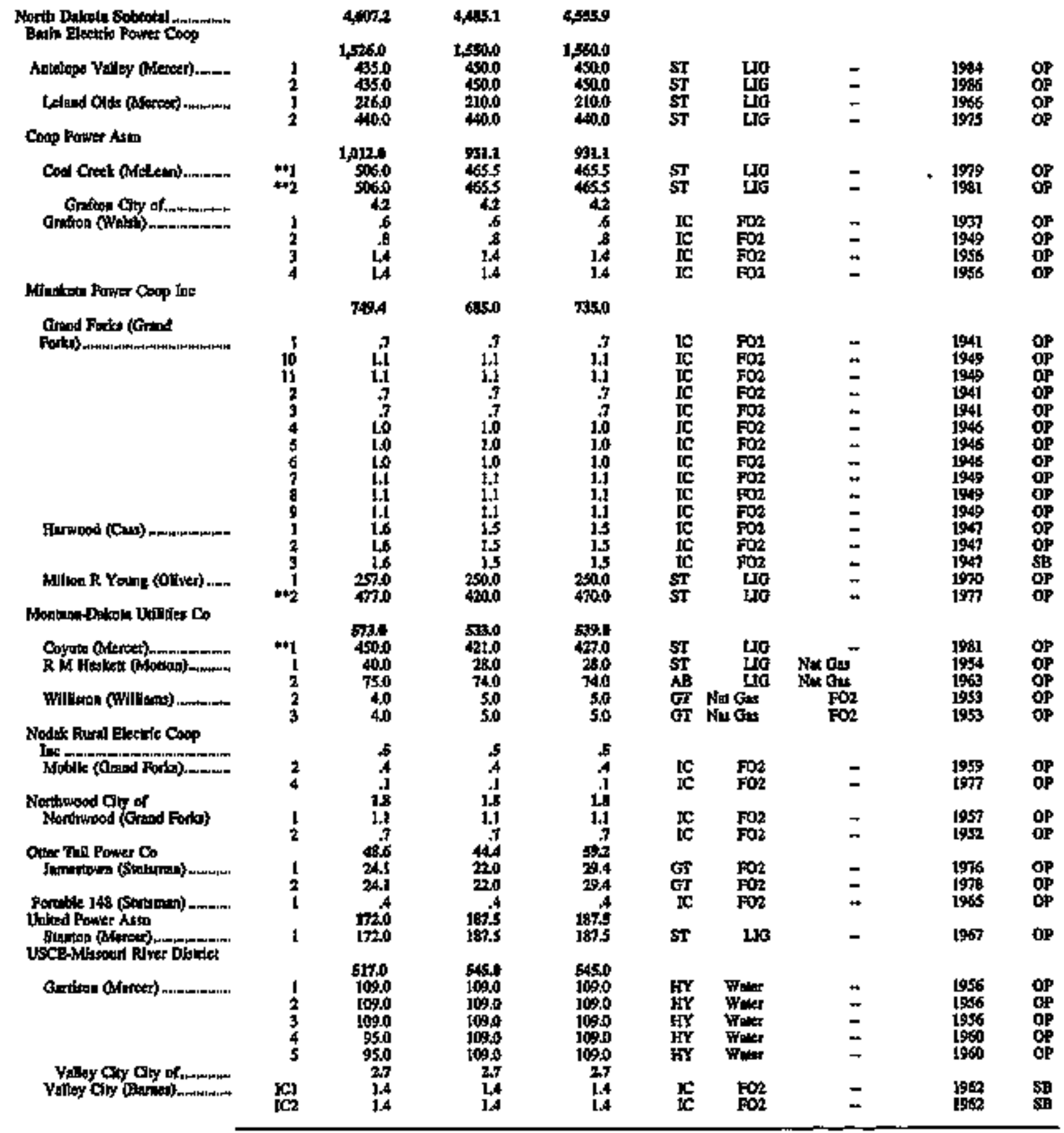

Het tootwotes at tend of abs. 
Table 20. Existing Generating Units at U.S. Electric Utilities by State, Company, and Plant, as of January 1, 1996 (Continued)

\begin{tabular}{|c|c|c|c|c|c|c|c|c|c|}
\hline \multirow{2}{*}{$\begin{array}{c}\text { Stste } \\
\text { Compoing } \\
\text { Plomt (County) }\end{array}$} & \multirow{2}{*}{$\underset{\mathbf{m}}{\mathbf{U}}$} & \multirow{2}{*}{ 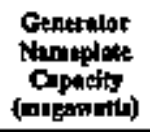 } & \multirow{2}{*}{ 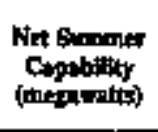 } & \multirow{2}{*}{ 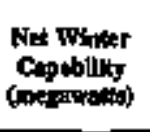 } & \multirow{2}{*}{ Unit } & \multicolumn{2}{|c|}{ Barryy Soomed } & \multirow{2}{*}{ 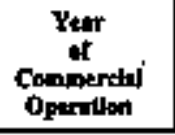 } & \multirow{2}{*}{ Solitid } \\
\hline & & & & & & Printry & Alemollo & & \\
\hline \multicolumn{10}{|l|}{ Ohis } \\
\hline 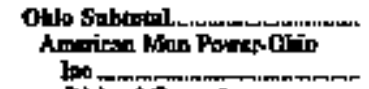 & & $\begin{array}{r}39,5392 \\
2009\end{array}$ & $\begin{array}{r}m, 355,0 \\
212.3\end{array}$ & $\begin{array}{r}200016 \\
2123\end{array}$ & & & & & \\
\hline $\begin{array}{l}\text { Richud Dorrowh } \\
\text { (Washington) }\end{array}$ & $\begin{array}{l}+1 \\
+1 \\
+4\end{array}$ & $\begin{array}{l}53.3 \\
593 \\
53.3 \\
500\end{array}$ & $\begin{array}{l}5.0 \\
\$ 30 \\
59.0 \\
53.3\end{array}$ & $\begin{array}{l}53.0 \\
53.0 \\
53.0 \\
53.3\end{array}$ & $\begin{array}{l}S T \\
S T \\
S T \\
S T\end{array}$ & $\begin{array}{l}\text { BIT } \\
\text { BIr } \\
\text { BIr } \\
\text { BIr }\end{array}$ & $\begin{array}{l}\ddot{+} \\
\ddot{m}\end{array}$ & $\begin{array}{l}1968 \\
1985 \\
1918 \\
1908\end{array}$ & $\begin{array}{l}\text { of } \\
\text { of } \\
\text { of } \\
\text { of }\end{array}$ \\
\hline 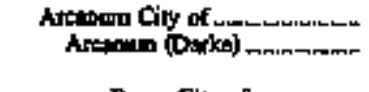 & $\mathbf{1}$ & $\frac{13}{5.6}$ & $\begin{array}{l}1.2 \\
5.7 \\
5\end{array}$ & 1.7 & IC & PO2 & $\bar{x}$ & $\begin{array}{l}1951 \\
1946\end{array}$ & $\begin{array}{l}\text { op } \\
\text { of }\end{array}$ \\
\hline 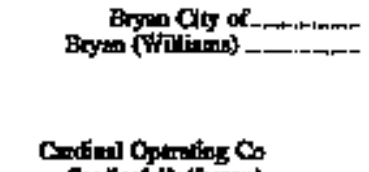 & $\begin{array}{l}1 \\
2 \\
5 \\
6\end{array}$ & $\begin{array}{r}39.3 \\
15.8 \\
16,0 \\
25 \\
5.0 \\
1,8005\end{array}$ & $\begin{array}{r}40,0 \\
160 \\
160 \\
200 \\
6.0 \\
1,800\end{array}$ & $\begin{array}{r}4000 \\
160 \\
16.0 \\
20 \\
60 \\
1,890\end{array}$ & $\begin{array}{l}\text { or } \\
\text { ơ } \\
\text { 宁 }\end{array}$ & 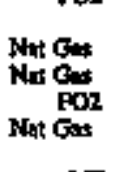 & $\begin{array}{l}\mathrm{Fan}_{\mathrm{FO}} \\
\mathrm{FO2}\end{array}$ & $\begin{array}{l}1970 \\
1988 \\
1948 \\
1963\end{array}$ & $\begin{array}{l}\text { OP } \\
\text { OP } \\
\text { OP } \\
\text { OP }\end{array}$ \\
\hline 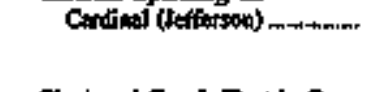 & $\begin{array}{l}* 1 \\
* 2\end{array}$ & $\begin{array}{l}615.2 \\
615.2 \\
650.0\end{array}$ & $\begin{array}{l}585.0 \\
585.0 \\
630.0\end{array}$ & $\begin{array}{l}6000 \\
6000 \\
63010\end{array}$ & $\begin{array}{l}\text { ST } \\
\text { ST } \\
\text { STT }\end{array}$ & $\begin{array}{l}\text { BrT } \\
\text { BII } \\
\text { BII }\end{array}$ & $\ddot{-}$ & $\begin{array}{l}1967 \\
1967 \\
1977\end{array}$ & of \\
\hline 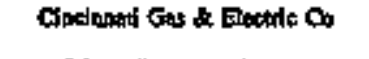 & & $\$, 132.6$ & & & & & $\cdots$ & & \\
\hline 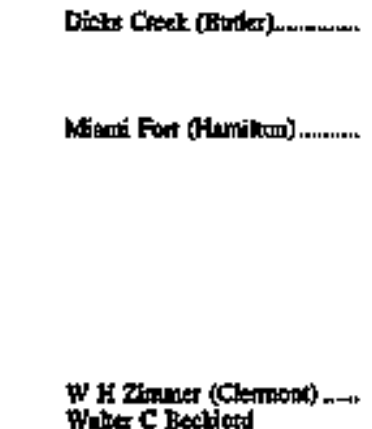 & 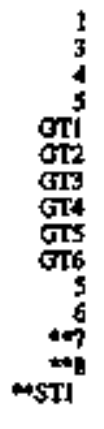 & $\begin{array}{r}120.3 \\
15.3 \\
20.4 \\
20.0 \\
53.1 \\
53.1 \\
15.3 \\
15.3 \\
15.3 \\
15.3 \\
100.0 \\
169.2 \\
551.1 \\
551.7 \\
1425.6\end{array}$ & $\begin{array}{l}\$ 20 \\
140 \\
15.0 \\
15.0 \\
48.0 \\
480 \\
140 \\
140 \\
140 \\
140 \\
80.0 \\
1630 \\
900.0 \\
500.0 \\
1360.0\end{array}$ & $\begin{array}{r}1100 \\
200 \\
21.0 \\
220 \\
640 \\
650 \\
200 \\
19.0 \\
200 \\
1900 \\
800 \\
2610 \\
5010 \\
5000 \\
1300.0\end{array}$ & $\begin{array}{l}J E \\
\text { Gr } \\
\text { Gr } \\
\text { Ur } \\
\text { Gr } \\
\text { GT } \\
\text { GT } \\
\text { Gr } \\
\text { GT } \\
\text { Or } \\
\text { ST } \\
\text { ST } \\
\text { ST } \\
\text { ST } \\
\text { ST }\end{array}$ & 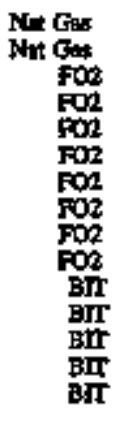 & $\begin{array}{r}F 02 \\
F 02 \\
= \\
= \\
= \\
= \\
= \\
= \\
= \\
= \\
=\end{array}$ & $\begin{array}{l}1965 \\
1969 \\
1969 \\
1969 \\
1991 \\
1971 \\
1911 \\
1971 \\
1911 \\
1971 \\
1949 \\
1960 \\
1915 \\
1978 \\
1991\end{array}$ & $\begin{array}{l}o p \\
o p \\
o p \\
\phi p \\
o p \\
o p \\
o p \\
o p \\
o p \\
o p \\
o p \\
o p \\
o p \\
o p \\
o p\end{array}$ \\
\hline 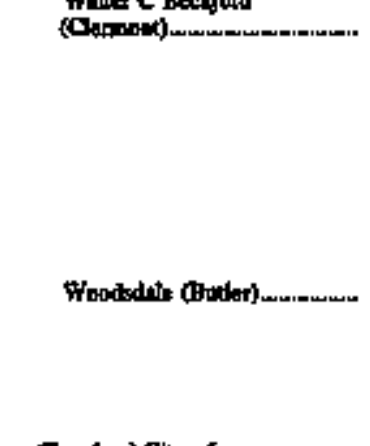 & 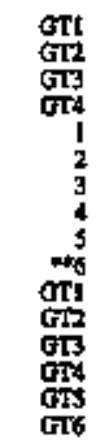 & $\begin{array}{l}49.6 \\
49.6 \\
48.6 \\
486 \\
115.0 \\
152.5 \\
125.0 \\
169.2 \\
244.8 \\
460.8 \\
96.4 \\
95.4 \\
95.4 \\
95.4 \\
94.4 \\
94.4\end{array}$ & $\begin{array}{l}46.0 \\
7.0 \\
47.0 \\
45.0 \\
94.0 \\
94.0 \\
198.0 \\
150.0 \\
298.0 \\
414.0 \\
77.0 \\
77.0 \\
77.0 \\
77.0 \\
77.0 \\
77.0\end{array}$ & $\begin{array}{l}610 \\
610 \\
61.0 \\
610 \\
940 \\
940 \\
1290 \\
1500 \\
2000 \\
4210 \\
940 \\
940 \\
940 \\
940 \\
940 \\
940\end{array}$ & $\begin{array}{l}G T \\
G T \\
\text { GT } \\
\text { GT } \\
\text { ST } \\
\text { ST } \\
\text { SI } \\
\text { ST } \\
\text { ST } \\
\text { ST } \\
\text { Gr } \\
\text { Gr } \\
\text { GT } \\
\text { GT } \\
\text { GT } \\
\text { Gr }\end{array}$ & 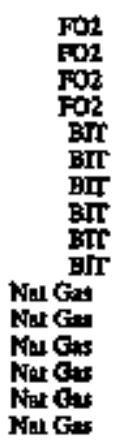 & 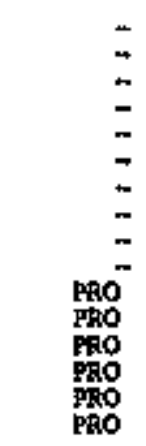 & $\begin{array}{l}1972 \\
1972 \\
1972 \\
1992 \\
1902 \\
1953 \\
1954 \\
1958 \\
1962 \\
1959 \\
1993 \\
1992 \\
1992 \\
1992 \\
1992 \\
1992\end{array}$ & 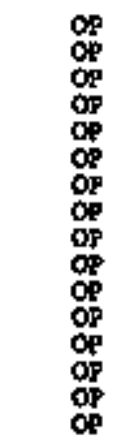 \\
\hline 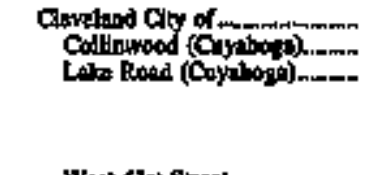 & $\begin{array}{r}3 \\
10 \\
11 \\
8 \\
9\end{array}$ & $\begin{array}{r}20000 \\
16.0 \\
25.0 \\
85.0 \\
25.0 \\
25.0\end{array}$ & $\begin{array}{l}205.0 \\
16.0 \\
25.0 \\
95,0 \\
25.0 \\
25.0\end{array}$ & $\begin{array}{r}212.4 \\
180 \\
25.0 \\
850 \\
25.0 \\
250\end{array}$ & $\begin{array}{l}G T \\
\text { ST } \\
\text { sT } \\
\text { ST }\end{array}$ & $\begin{array}{c}\text { Not Cas } \\
\text { BII } \\
\text { BIr } \\
\text { BIr } \\
\text { BIT }\end{array}$ & $\begin{array}{r}\text { Fon } \\
= \\
= \\
=\end{array}$ & $\begin{array}{l}1919 \\
1953 \\
1961 \\
1941 \\
1959\end{array}$ & $\begin{array}{l}\text { of } \\
\text { os } \\
\text { os } \\
\text { os } \\
\alpha\end{array}$ \\
\hline $\begin{array}{l}\text { Wat 4lal Streel } \\
\text { (Oryphog) }\end{array}$ & ! & $\begin{array}{l}16.0 \\
160\end{array}$ & 160 & 180 & Gr & Ner Gax & $\begin{array}{l}\mathrm{FO} 2 \\
\mathrm{FO} 2\end{array}$ & 1970 & op \\
\hline 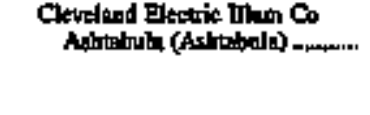 & $\begin{array}{l}5 \\
6 \\
7\end{array}$ & $\begin{array}{r}4,126,6 \\
2600 \\
460 \\
46,0\end{array}$ & $\begin{array}{r}3.50 .4 \\
243.0 \\
43.4 \\
43.0\end{array}$ & $\begin{array}{r}7,400 \\
2440 \\
440 \\
44.0\end{array}$ & $\begin{array}{l}\mathbf{S T} \\
\mathbf{S T}\end{array}$ & $\begin{array}{l}\text { grr } \\
\text { exr } \\
\text { Brr }\end{array}$ & $\ddot{z}$ & $\begin{array}{l}1958 \\
19972 \\
1972\end{array}$ & $\begin{array}{l}\text { OP } \\
\text { OP } \\
\text { OP }\end{array}$ \\
\hline
\end{tabular}

Ses footonot we tod of thite 
Table 20. Existing Generating Units at US. Electric Utillities by State, Company, and Ptant, as of January 1, 1996 (Continued)

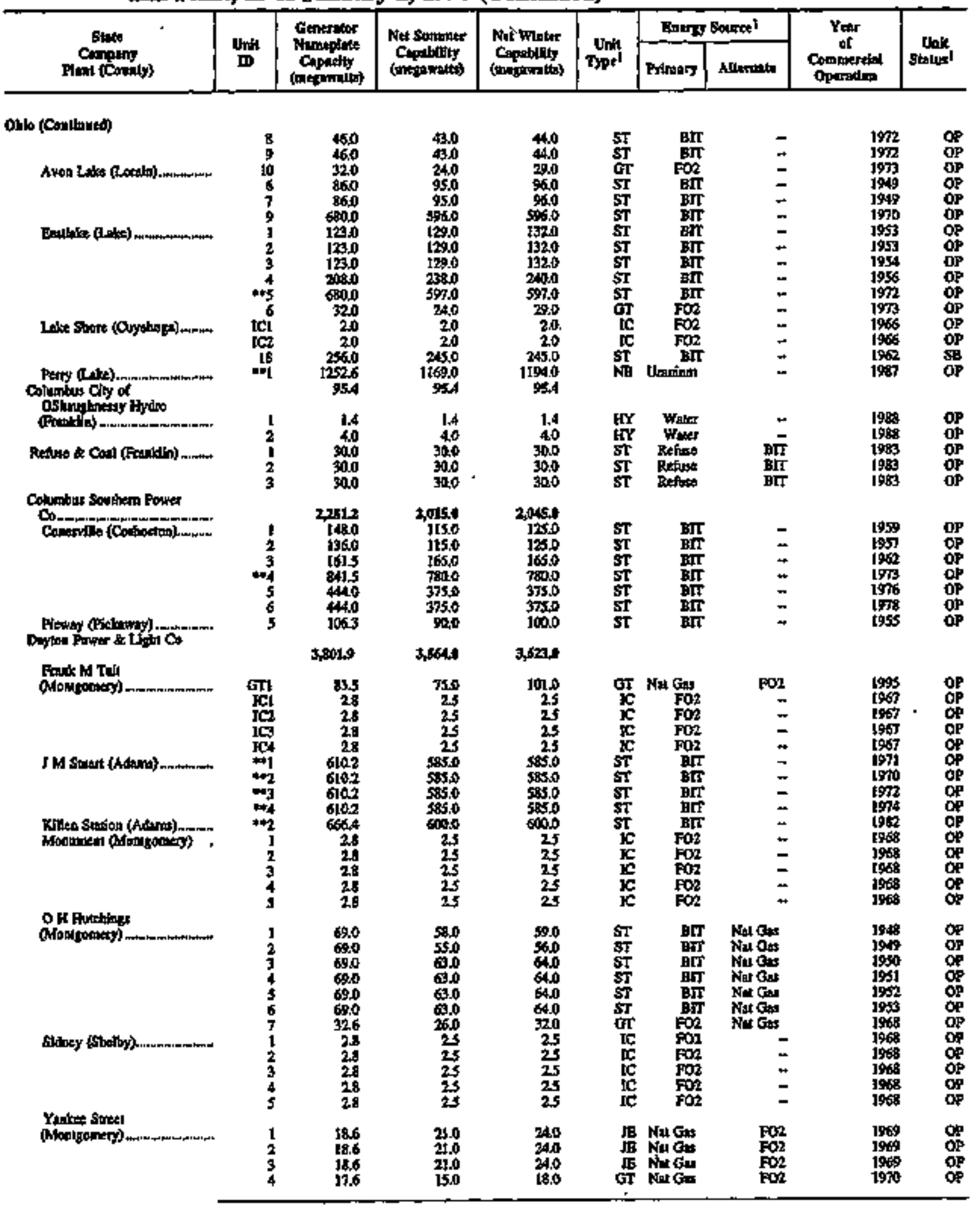

Sect foobinotes and of thiblo. 
Table 20. Existing Generating Units at U.S. Electric Utiltiles by State, Company, and Plant, as of Jenuary 1, 1996 (Continued)

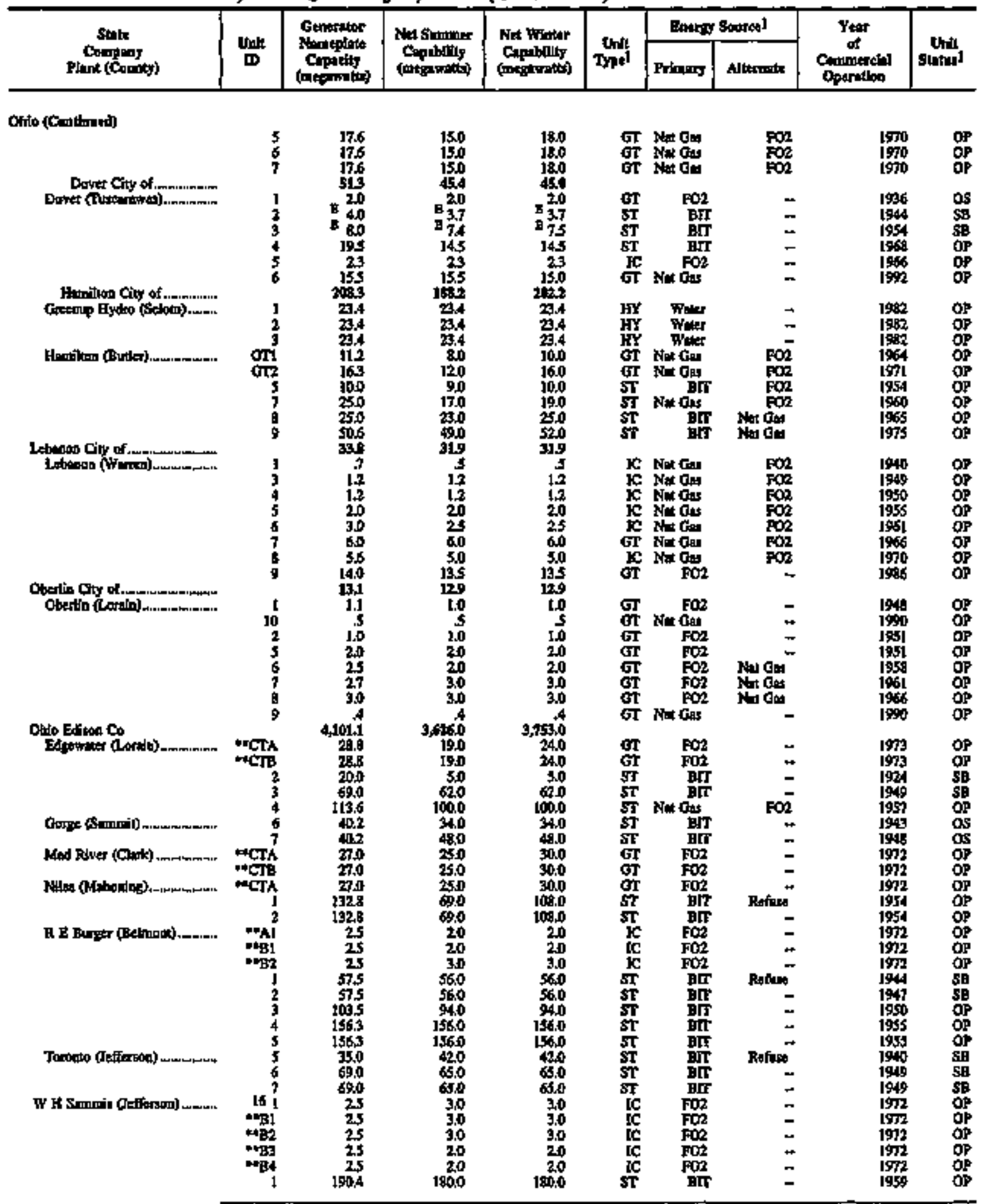

Sec foolionst ot end of tats. 
Table 20. Existing Generating Units at U.S. Electric Utilities by State, Company, and Plant, as of January 1, 1996 (Continued)

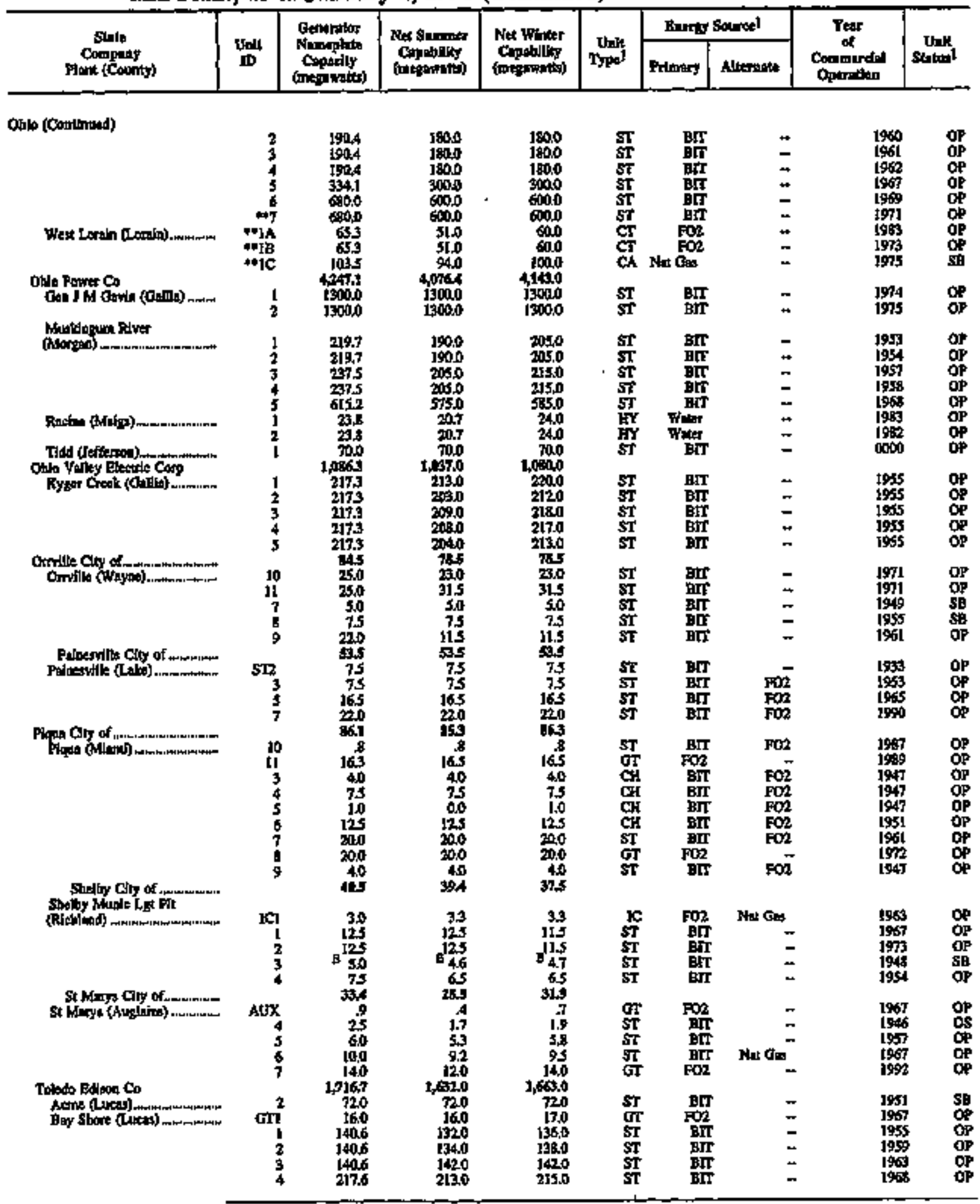

Ses tootions it and of toble. 
Table 20. Existing Generating Units at U.S. Electric Utillities by State, Company, and Plant, as of January 1, 1996 (Continued)

\begin{tabular}{|c|c|c|c|c|c|c|c|c|c|}
\hline \multirow{2}{*}{$\begin{array}{c}\text { Stat } \\
\text { Cocopany } \\
\text { Plont (County) }\end{array}$} & \multirow{2}{*}{$\frac{\text { tht }}{\text { Int }}$} & \multirow{2}{*}{ 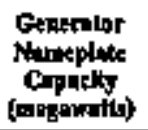 } & \multirow{2}{*}{ 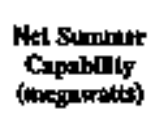 } & \multirow{2}{*}{ 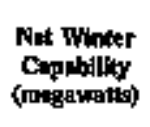 } & \multirow{2}{*}{$\begin{array}{c}\text { Undt } \\
\text { Typel }\end{array}$} & \multicolumn{2}{|c|}{ Energy Sobeed } & \multirow{2}{*}{ 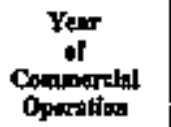 } & \multirow{2}{*}{ 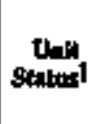 } \\
\hline & & & & & & Prtery & Altarinte & & \\
\hline 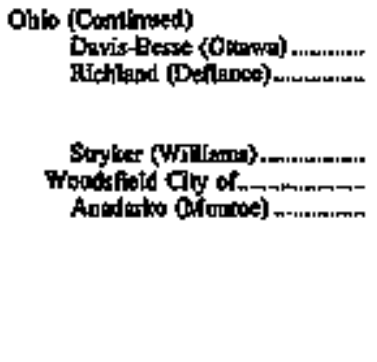 & $\begin{array}{r}-1 \\
1 \\
2 \\
3 \\
1 \\
10 \\
11 \\
6 \\
7 \\
8 \\
9\end{array}$ & $\begin{array}{r}95.2 \\
15.0 \\
150 \\
150 \\
19.4 \\
9.0 \\
1.2 \\
1.2 \\
.6 \\
13 \\
15 \\
2.2\end{array}$ & $\begin{array}{r}873.0 \\
11.0 \\
11.0 \\
11.0 \\
17.0 \\
8.0 \\
1.2 \\
1.2 \\
.6 \\
1.3 \\
1.5 \\
2.2\end{array}$ & $\begin{array}{r}883.0 \\
140 \\
140 \\
140 \\
18.0 \\
1.0 \\
12 \\
1.2 \\
6 \\
1.3 \\
1.5 \\
2.2\end{array}$ & $\begin{array}{l}\text { NP } \\
\text { GT } \\
\text { GT } \\
\text { GT } \\
\text { Gr } \\
\text { GT } \\
\text { GT } \\
\text { GT } \\
\text { GT } \\
\text { GT } \\
\text { GT }\end{array}$ & 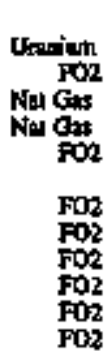 & 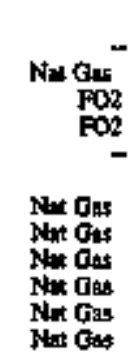 & $\begin{array}{l}1977 \\
1965 \\
1966 \\
1966 \\
1969 \\
1983 \\
1989 \\
1945 \\
1957 \\
1965 \\
1971\end{array}$ & 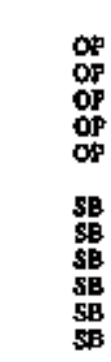 \\
\hline
\end{tabular}

Olakionas

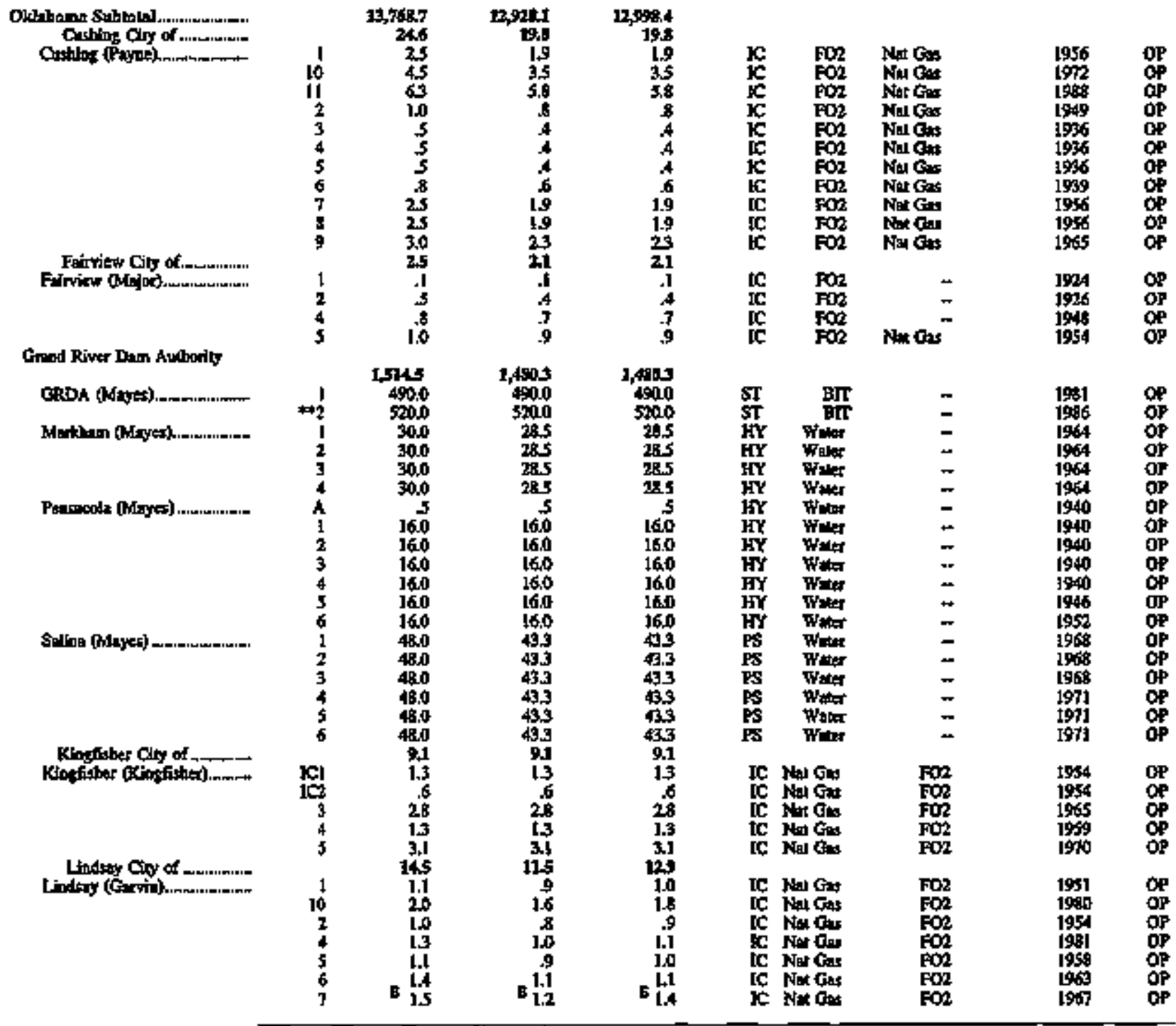

Set frotuntas at ead of thile. 
Table 20. Existing Generating Units at U.S. Electric Utilities by State, Company, and Plant, as of January 1, 1996 (Continued)

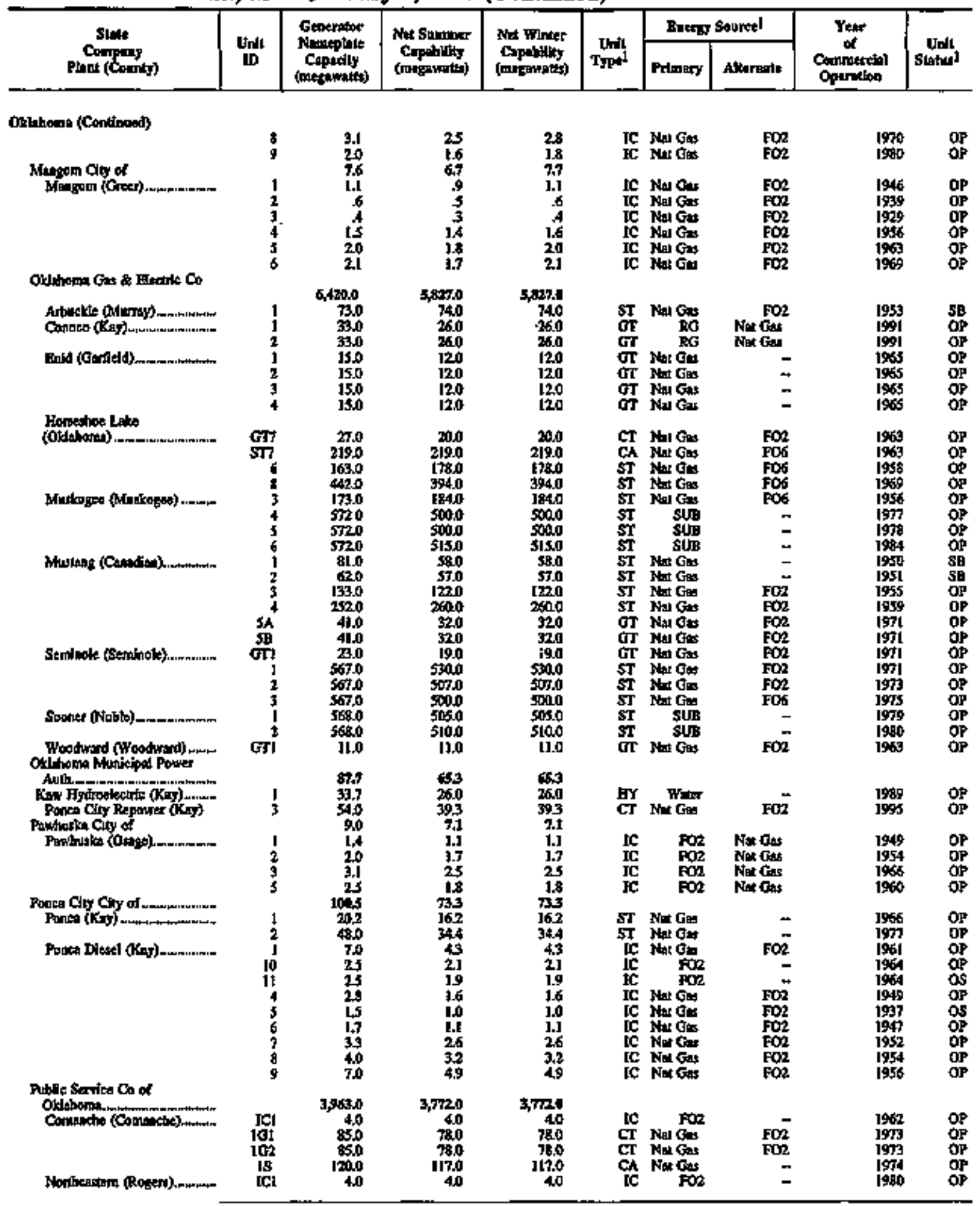

Ste fortinotet at ead of tahla. 
Tahle 20. Existing Generating Units at U.S. Electric Utilities by State, Company, and Plant, as of January 1, 1996 (Continued)

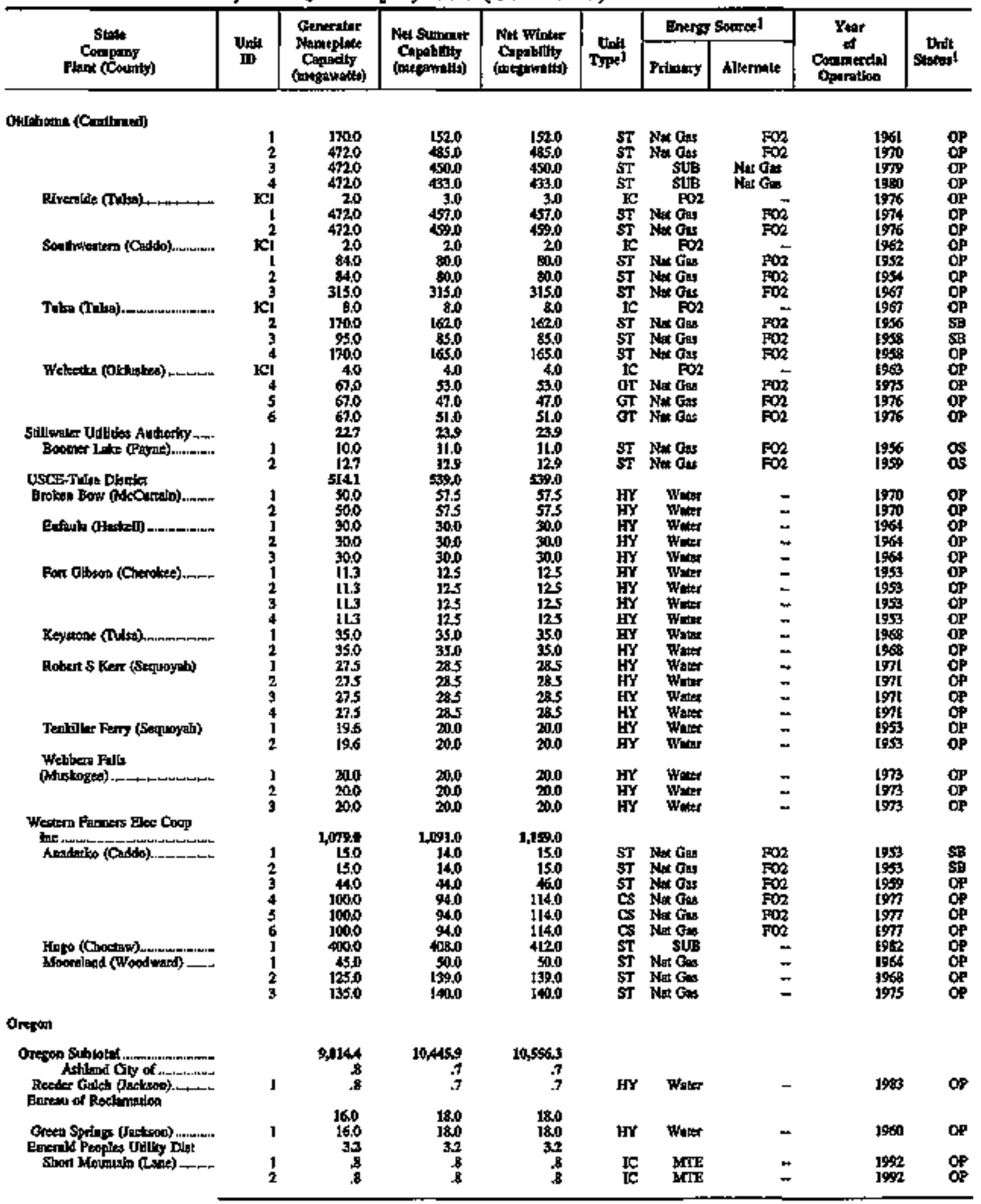

Soe frotorons at end of tabla. 
Table 20. Existing Generating Units at US. Electric Utilities by State, Company, and Plant, as of January 1, 1996 (Continued)

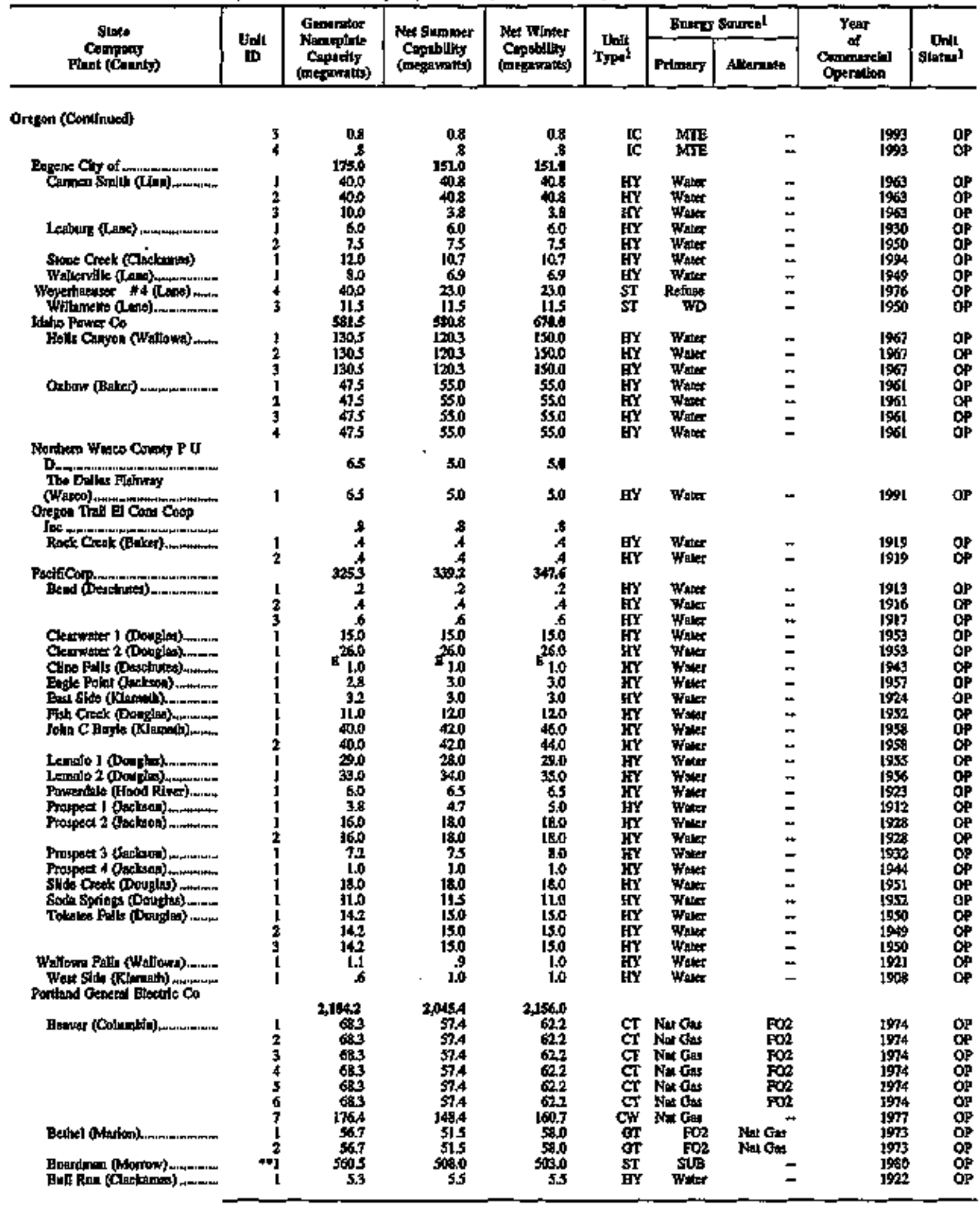

Sea tooinoles at end of able. 
Table 20. Existing Generating Units at U.S. Electric Utilities by State, Company, and Plant, as of Jamuary 1, 1996 (Continued)

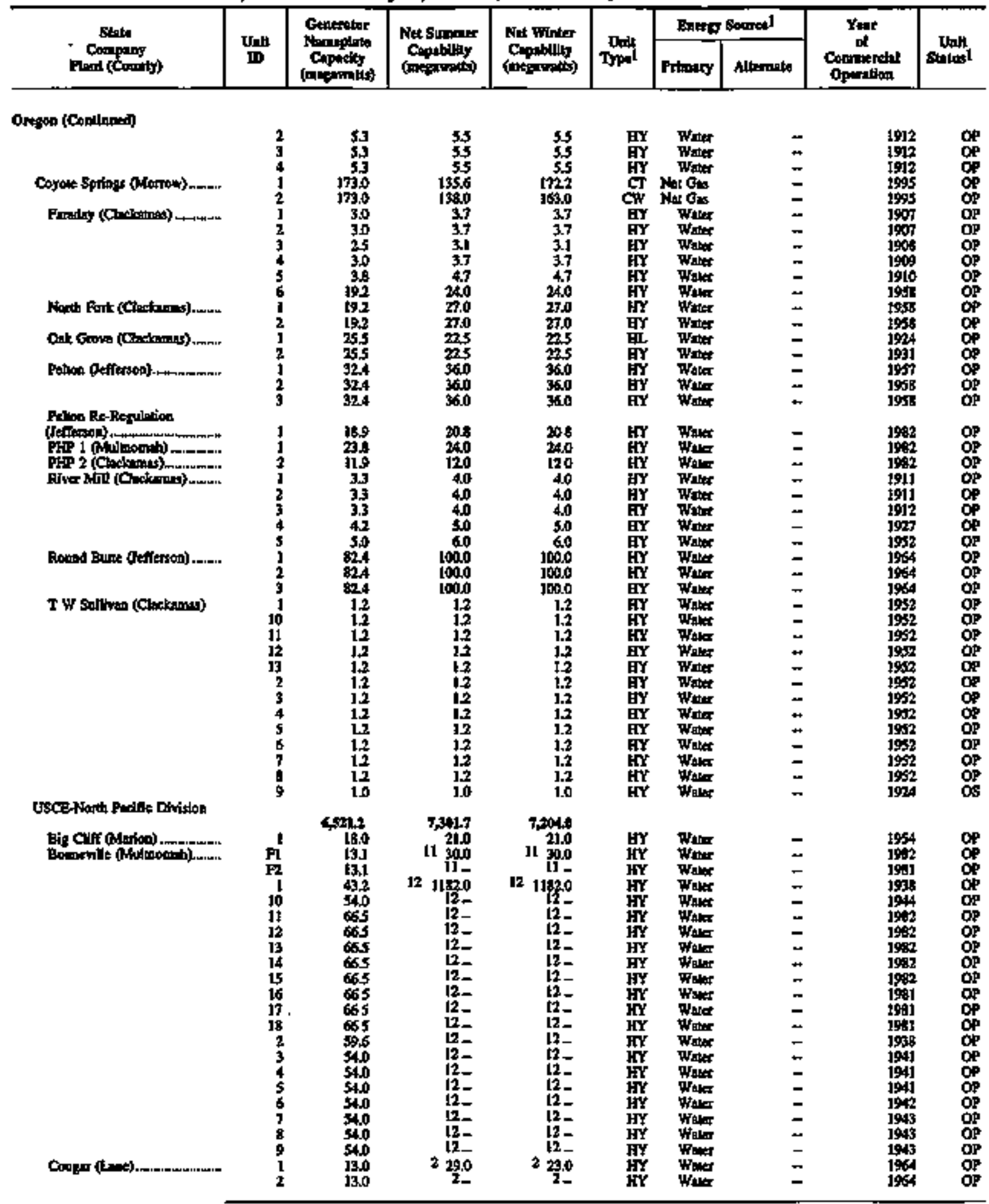

Ste footaoks al ead of table. 
Tabłe 20. Existing Generating Units at U.S. Electric Utiltiles by State, Company, and Plant, as of January 1,1996 (Continued)

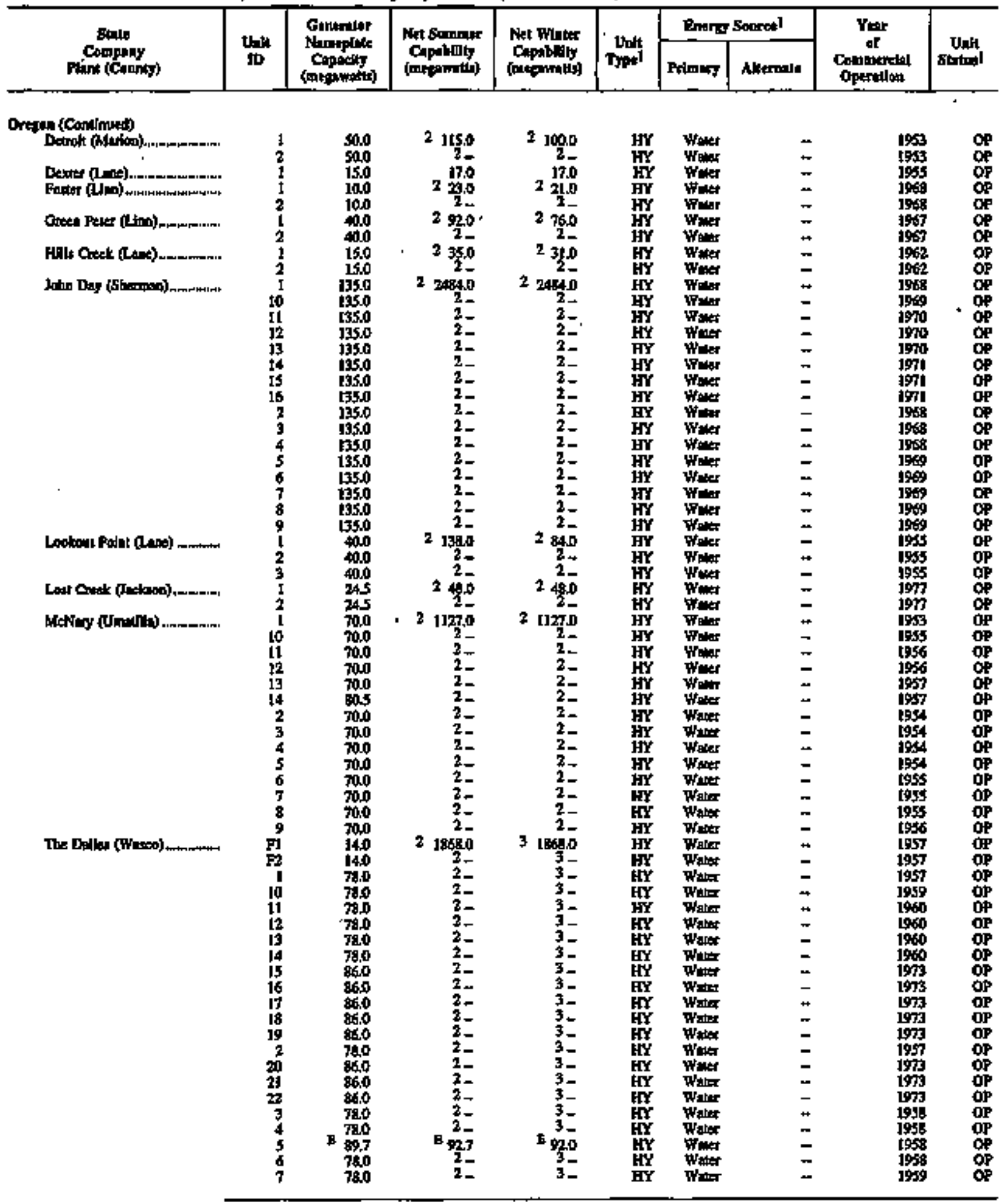

Sas foctingter it end of table. 
Table 20. Existing Generating Units at U.S. Electric Utilities by State, Company, and Plant, as of January 1, 1996 (Continued)

\begin{tabular}{|c|c|c|c|c|c|c|c|c|c|}
\hline \multirow{2}{*}{$\begin{array}{c}\text { Shlo } \\
\text { Company } \\
\text { Phant (Cowiky) }\end{array}$} & \multirow{2}{*}{$\begin{array}{l}\text { Drtit } \\
\text { W }\end{array}$} & \multirow{2}{*}{ 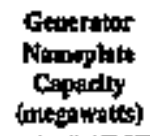 } & \multirow{2}{*}{ 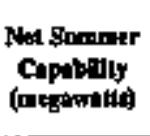 } & \multirow{2}{*}{ 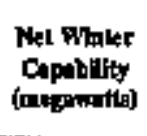 } & \multirow{2}{*}{$\begin{array}{l}\text { Dat: } \\
\text { Type1 }\end{array}$} & \multicolumn{2}{|c|}{ Entry Sourtel } & \multirow{2}{*}{ 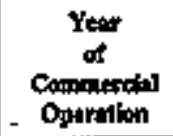 } & \multirow{2}{*}{$\begin{array}{l}\text { Untt } \\
\text { slathey }\end{array}$} \\
\hline & & & & & & Prtarary & Aflmante & & \\
\hline \multicolumn{10}{|l|}{ 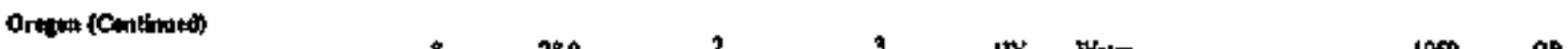 } \\
\hline & & $\begin{array}{l}78.0 \\
78.0\end{array}$ & $\begin{array}{l}2= \\
2=\end{array}$ & $3-$ & $\begin{array}{l}\mathbf{H Y} \\
\mathbf{H Y}\end{array}$ & $\begin{array}{l}\text { Water } \\
\text { Wots }\end{array}$ & & $\begin{array}{l}195 \\
195\end{array}$ & $\underset{O P}{O P}$ \\
\hline
\end{tabular}

$\sec$ footols at ond of able. 
Tahle 20. Exlsting Generating Units at U.S. Electric Utilities by State, Company, and Plant, as of January 1, 1996 (Continued)

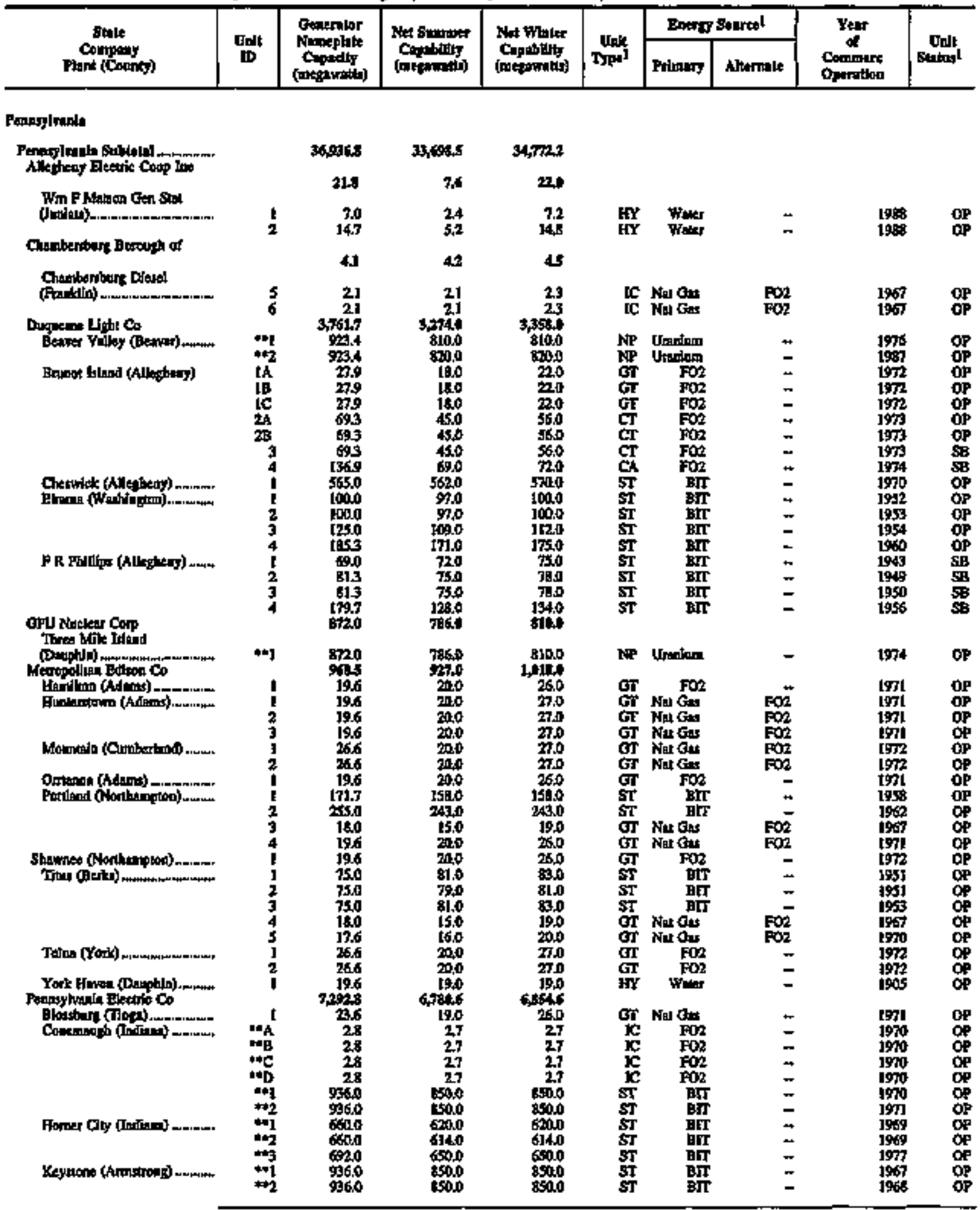

Sec foovinites at tod of tiblt. 
Table 20. Existing Generating Units at U.S. Electric Utillties by State, Company, and Plant, as of January 1, 1996 (Continued)

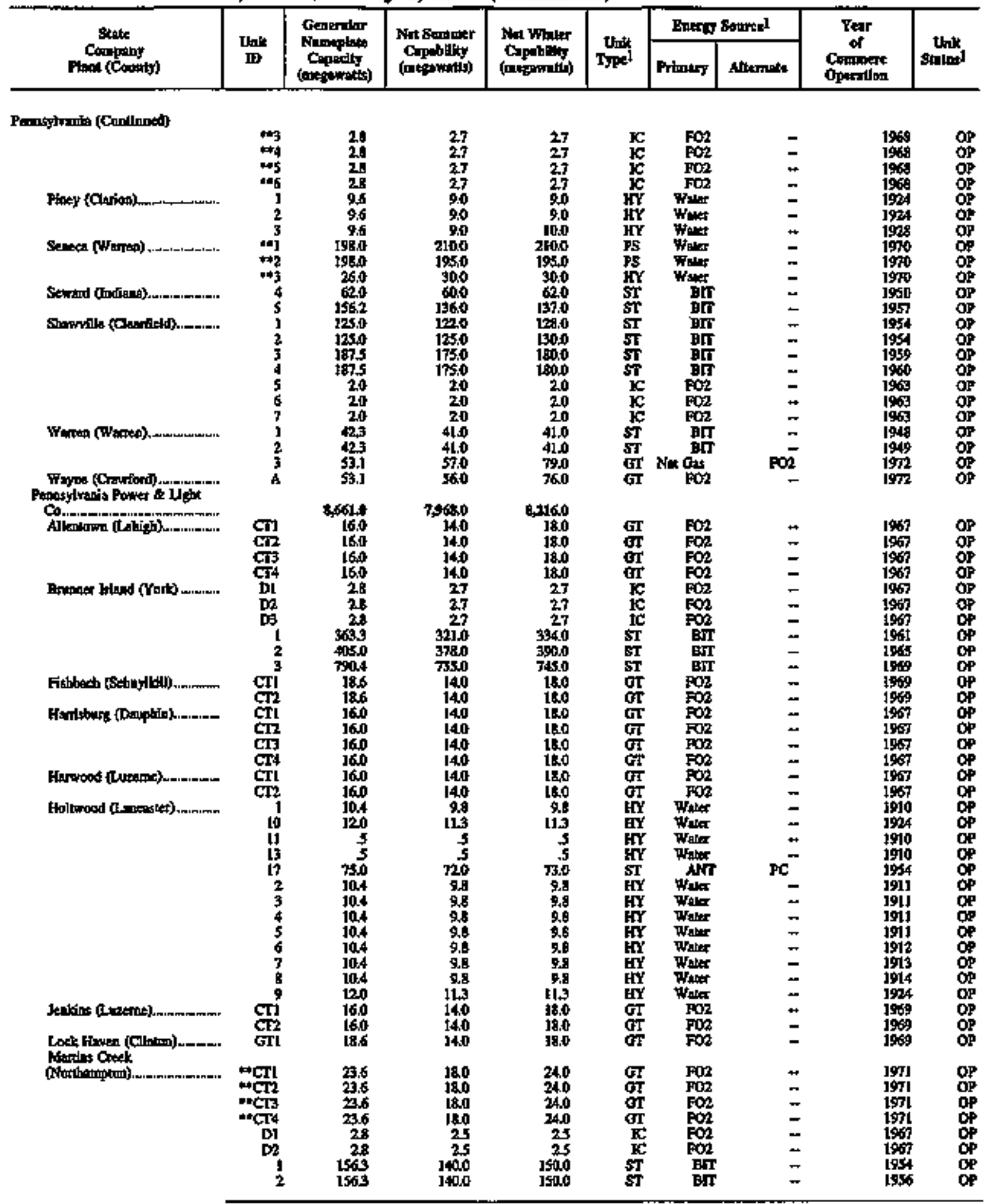

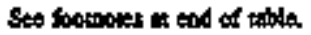


Table 20. Existing Generating Units at U.S. Electric Utilities by State, Company, and Plant, as of January 1, 1996 (Continued)

\begin{tabular}{|c|c|c|c|c|c|c|c|c|c|}
\hline \multirow{2}{*}{$\begin{array}{c}\text { Simte } \\
\text { Congeng }\end{array}$} & \multirow{2}{*}{$\begin{array}{l}\text { Unh } \\
\text { II }\end{array}$} & \multirow{2}{*}{ 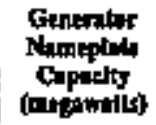 } & \multirow{2}{*}{ 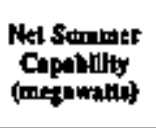 } & \multirow{2}{*}{ 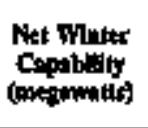 } & \multirow{2}{*}{ Tink } & \multicolumn{2}{|c|}{ Einerizy Sanded } & \multirow{2}{*}{$\begin{array}{c}\text { Yent } \\
\text { of } \\
\text { Compinate } \\
\text { Oparition }\end{array}$} & \multirow{2}{*}{$\begin{array}{c}\text { Whit } \\
\text { slofus) }\end{array}$} \\
\hline & & & & & & Pribugary & Alemple & & \\
\hline \multicolumn{10}{|l|}{ Penriplinula (Continoed) } \\
\hline & 3 & 805 & $\sin 0$ & 807.0 & $\mathbf{S T}$ & For & - & 1975 & OP \\
\hline Montour Glochowr) & 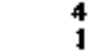 & $\begin{array}{l}850,5 \\
805.5\end{array}$ & 785.0 & 755.0 & ST & FOS & $\dot{+}$ & 195 & $\begin{array}{l}\text { OP } \\
\text { op }\end{array}$ \\
\hline & 13 & 17.2 & 15.0 & 15.0 & $\mathrm{st}$ & $\mathrm{FO} 2$ & вत्र & Ins & op \\
\hline & 2 & BIS4 & 7450 & 7550 & st & Bir & - & 1973 & op \\
\hline Sunbury (Spydet) & ${ }^{* * C T I}$ & 236 & 18.0 & 240 & कr & $\mathbf{E O}$ & - & 1971 & OP \\
\hline & ACIZ & 200 & 180 & 240 & or & $\mathrm{FO}$ & - & 1971 & of \\
\hline & Di & 2.8 & $\begin{array}{l}3.0 \\
3.0\end{array}$ & $\begin{array}{l}3.0 \\
3.0\end{array}$ & k & $\mathrm{FO2}$ & $\bar{z}$ & 1967 & op \\
\hline & 1 & 75.0 & 700 & 76,0 & ST & ANI & $P C$ & $19+9$ & OP \\
\hline & 2 & 75.0 & 700 & 160 & $\stackrel{5 T}{s T}$ & AN & $\mathbf{C C}$ & iges & OP \\
\hline & 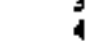 & 1563 & 1280 & [340' & ST & 最 & $\bar{c}$ & 1951 & OP \\
\hline 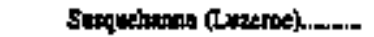 & $\cdot * \mathbf{i}$ & 1161.2 & 1090.0 & 1070 & NB & Uranlorm & - & 1983 & OP \\
\hline & $\mathbf{m j}_{\mathbf{j}}$ & 1168.2 & 10940 & jiod & m & Uhting & - & 1965 & $\mathrm{OP}$ \\
\hline Wallenpmupack (Pik)...-.-. & 1 & 200 & mo & 200 & EY & $\mathbf{W}$ atter & - & 1926 & op \\
\hline 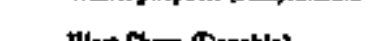 & 2 & 200 & 20 & 20 & $\mathbf{H Y}$ & Water & - & 1926 & OP \\
\hline 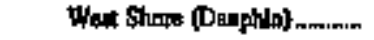 & CTi & 386 & 14.0 & 180 & $\mathbf{T r}$ & $\mathrm{FO} 2$ & - & 1969 & PP \\
\hline 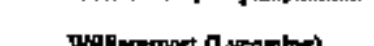 & CT2 & 18.6 & 140 & 18:0 & 6r & EOR & - & 1969 & op \\
\hline 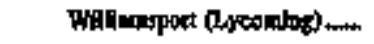 & CrI & 160 & 140 & 180 & or & FO2 & - & 1967 & of \\
\hline & Caz & 160 & 14.0 & 180 & or & $\mathbf{F O 2}$ & - & 1967 & $\boldsymbol{\phi P}$ \\
\hline \multicolumn{10}{|l|}{ Peanglvalu Poster Co } \\
\hline \multirow[t]{3}{*}{ Bnuce Kanafiold (Beava) } & $m$ & 913.8 & 781.0 & 7600 & str & BUT & - & 1976 & OP \\
\hline & $* 2$ & 9138 & 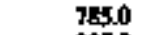 & 7800 & ST & BIT & - & $19 m$ & OP \\
\hline & $+\infty$ & 9138 & 805.0 & 8000 & sT & BT & - & 196 & $\phi$ \\
\hline 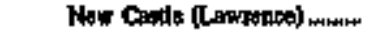 & $\mathscr{M A}_{\mathbf{A}}$ & 28 & $\mathbf{3 0}$ & 30 & $\mathbf{1 C}$ & $\mathbf{F O 2}$ & - & 1968 & Op \\
\hline & $=\oplus \mathrm{g}$ & 28 & 3,0 & $\mathbf{3 0}$ & $\mathbf{k}$ & FO2 & - & 19648 & op \\
\hline & 1 & 37.5 & 35.0 & 250 & sT & BT & - & 1939 & $\mathrm{Sg}$ \\
\hline & 2 & 40 & 48,0 & 48.0 & ST & BIT & - & 1997 & si \\
\hline & 4 & 978 & $\begin{array}{l}96.0 \\
98.0\end{array}$ & 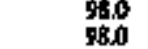 & st & 然 & $=$ & 1932 & op \\
\hline & 5 & 1560 & imf.0 & 1370 & st & BIT & - & 1964 & op \\
\hline 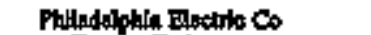 & & a90, & 8,2006 & 8,596 & & & & & \\
\hline Chester (Detmumb)...-..... & $?$ & 186 & 13.0 & 180 & $\mathbf{g}$ & FO2 & + & 1969 & op \\
\hline & 8 & 18.6 & 130 & IA.0 & ơr & Fon & - & 1969 & op \\
\hline & $\$$ & 18.6 & $\mathbf{1 3 0}, \mathbf{0}$ & Loto & 6T & Fol & - & 196 & or \\
\hline Crobity (Chester) , & CI & 28 & 27 & 27 & IC & $\mathrm{Fo2}$ & - & 1967 & OP \\
\hline & 1 & 187.5 & 144.0 & 1470 & sT & BII & & 1954 & of \\
\hline & 2 & 2300 & 200911 & 2110 & $\mathbf{s T}$ & Nar Gas & FO6 & igss & of \\
\hline 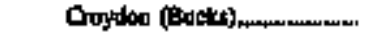 & I1 & 66.3 & 90 & 600 & $\mathbf{G P}^{2}$ & Foe & 4 & 1974 & OP \\
\hline & $\mathbf{2}$ & 683 & 47.0 & 600 & or & $\mathrm{EOZ}$ & $\overline{-}$ & $\begin{array}{l}1974 \\
1974\end{array}$ & OP \\
\hline & 21 & 68 & $\begin{array}{l}6.0 \\
\$ .0\end{array}$ & $\begin{array}{l}390 \\
60.0\end{array}$ & wr & Fon & $\ddot{-}$ & $\begin{array}{l}19944 \\
1994\end{array}$ & op \\
\hline & 31 & 68.3 & m.0 & G12 & ư & $\mathbf{E g}$ & - & 1974 & or \\
\hline & 32 & 66.3 & 45,0 & 590 & GT & FQ2 & - & 1974 & OF \\
\hline & 41 & 683 & 4.0 & 600 & $\mathbf{\sigma}$ & $\mathrm{Foz}$ & $=$ & 1974 & OP \\
\hline & 42 & 65:3 & 45.0 & 500 & 宁 & $\mathbf{F} \mathbf{2} \mathbf{z}$ & - & 1974 & DP \\
\hline Delaware (Phludadphis)......... & 1 & 2.8 & 27 & 27 & IC & $\mathbf{F O l}$ & + & 1969 & OP \\
\hline & 10 & 196 & 15,0 & 10.0 & GT & 502 & - & 1969 & OP \\
\hline & 11 & 116 & 15.0 & 180 & $G \mathbf{T}$ & Fo2 2 & - & 1960 & OP \\
\hline & 32 & 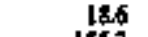 & 15.0 & 180 & G & F02 & - & 1969 & $\mathbf{O P}$ \\
\hline & 7 & $\mathbf{L S 6 3}$ & $12 \mathbf{x}_{0} 0$ & 1280 & 5T & EOS & $\rightarrow$ & 1953 & op \\
\hline & 6 & 156.3 & 1240 & 1280 & $\mathbf{5 T}$ & FO6 & - & 1953 & of \\
\hline & 9 & 21.3 & $\mathbf{t 5 . 0}$ & 200 & $\boldsymbol{\sigma r}$ & Fon & - & 1970 & op \\
\hline 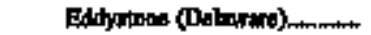 & 1 & 3536 & 2793 & 2800 & $\mathbf{S T}$ & BII & - & 1960 & OP \\
\hline & 10 & 18,6 & & 180 & & FO2 & - & $19 \%$ & DP \\
\hline & 1 & 359.6 & 3000 & 3100 & $\mathbf{s}$ & Bतr & - & LeO & OP \\
\hline & $\mathbf{2 0}$ & $1 \pm .6$ & 140 & 180 & GI & EOS & $\omega$ & 199 & of \\
\hline & 3 & 3914 & 3300 & 3300 & sT & FO6 & - & 1974 & OP \\
\hline & 30 & 24,3 & 170 & 2000 & $\mathbf{G r}$ & FO2 & 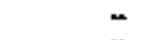 & 1910 & of \\
\hline & 4 & 3910 & $\begin{array}{l}35000 \\
0\end{array}$ & 3900 & ST & $\mathbf{E O S}$ & 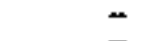 & 1976 & of \\
\hline Foll (Buct) & $\begin{array}{l}40 \\
\text { I }\end{array}$ & 21.3 & $\underset{170}{100}$ & 20.0 & GI & $\mathrm{FO}$ & $=$ & 1970 & op \\
\hline Fub towat.... & 3 & $\begin{array}{l}21.3 \\
21,3\end{array}$ & 150 & 2000 & OT & $\begin{array}{ll}502 \\
\mathrm{EOO} 2\end{array}$ & $\bar{z}$ & $\begin{array}{l}\text { Liflu } \\
\text { tofto }\end{array}$ & 罗 \\
\hline & 3 & 213 & 160 & 200 & GT & $\mathrm{PO} 2$ & 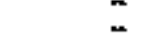 & 1970 & oe \\
\hline 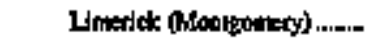 & 1 & 11395 & 10550 & 10620 & NB & Uranitur & - & 1986 & OP \\
\hline & $i$ & 11385 & J1150 & $\mathrm{jt} 3 \mathbf{3 0}$ & $\mathbf{M g}$ & Drapium & - & 1990 & of \\
\hline
\end{tabular}

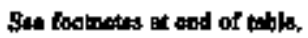


Tahle 20. Existing Generating Units at U.S. Electric Utilities by State, Company, and Plant, as of January 1, 1996 (Continued)

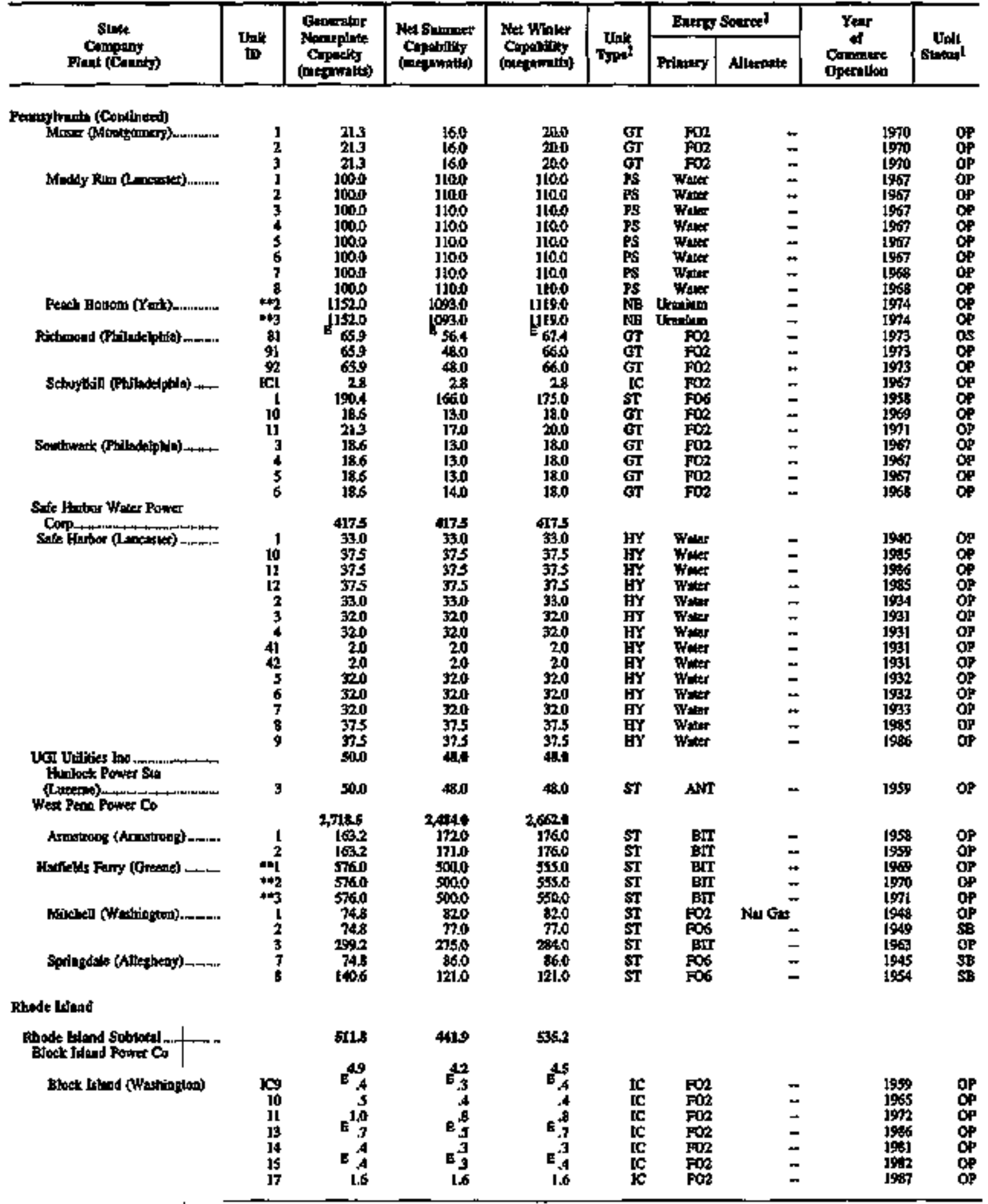

set foctones and of ublo! 
Table 20. Existing Generating Units at U.S. Electric Utilities by State, Company, and Plant, as of January 1, 1996 (Continued)

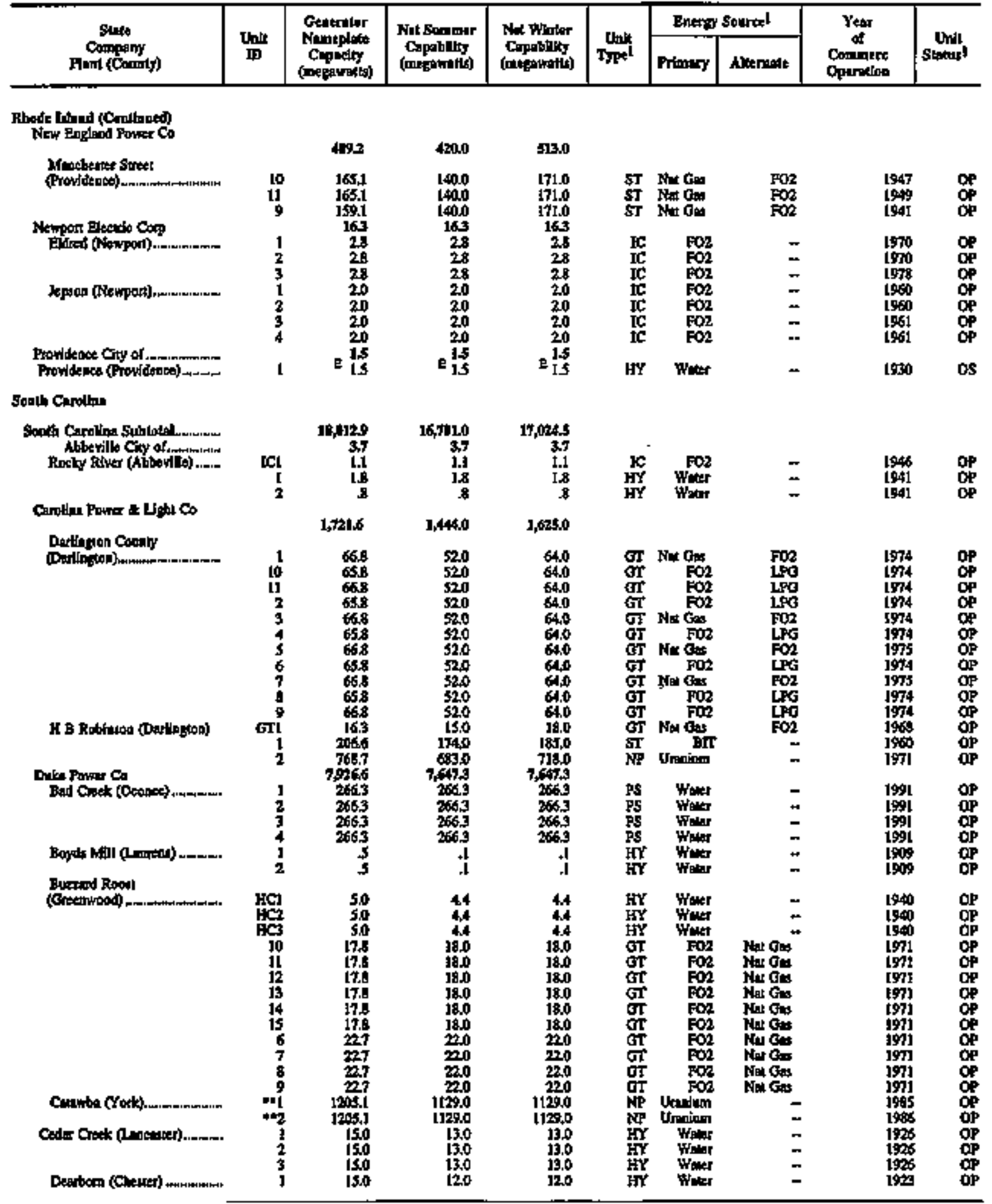

Sec footuoles at ind af cabte. 
Table 20. Existing Generating Units at U.S. Electric Utilities by State, Company, and Piant, as of January 1, 1996 (Continted)

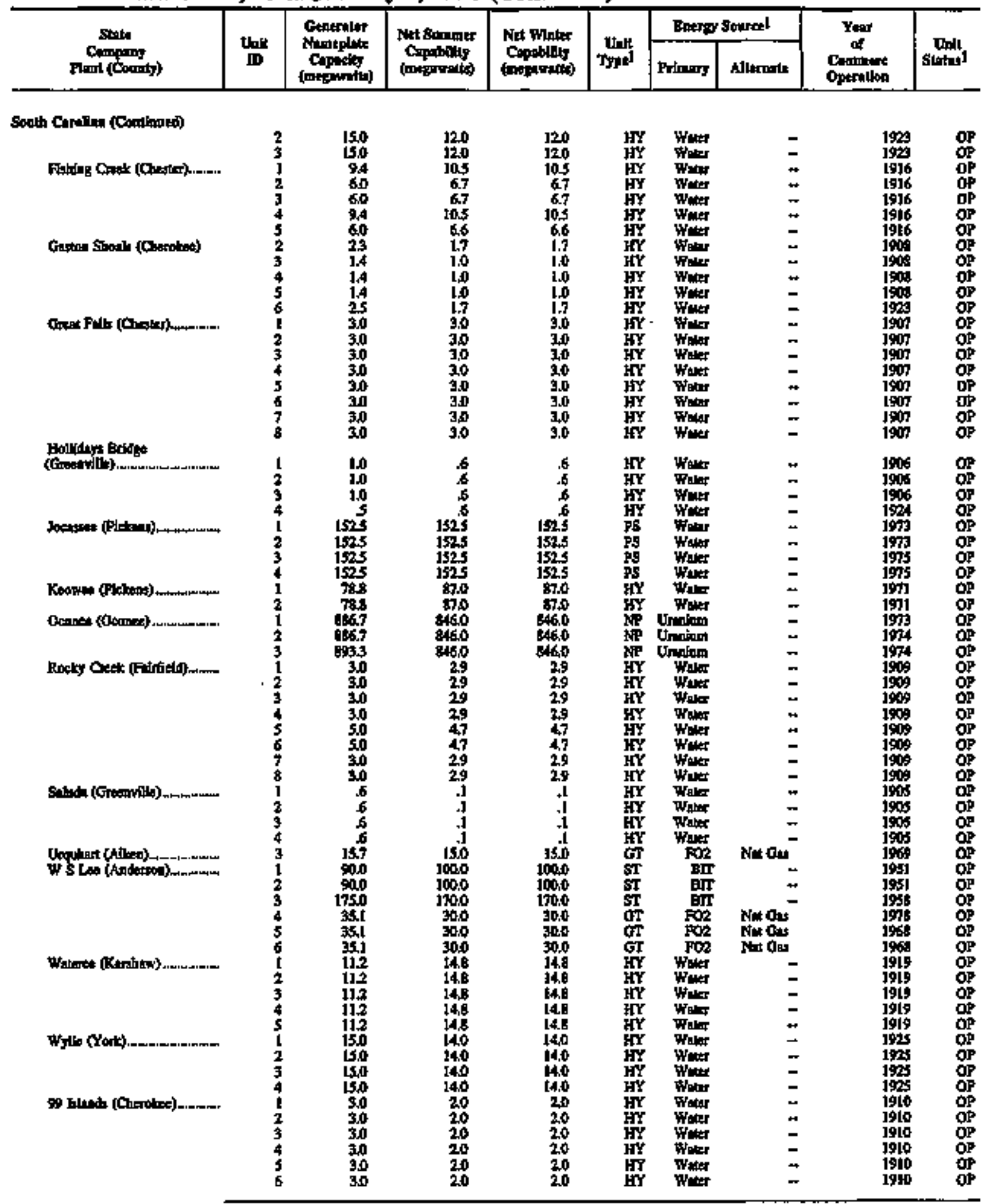

Sat forteolnt if end of tatis. 
Table 20. Existing Generating Units at U.S. Electric Utilities by State, Company, and Plant, as of January 1, 1996 (Contimued)

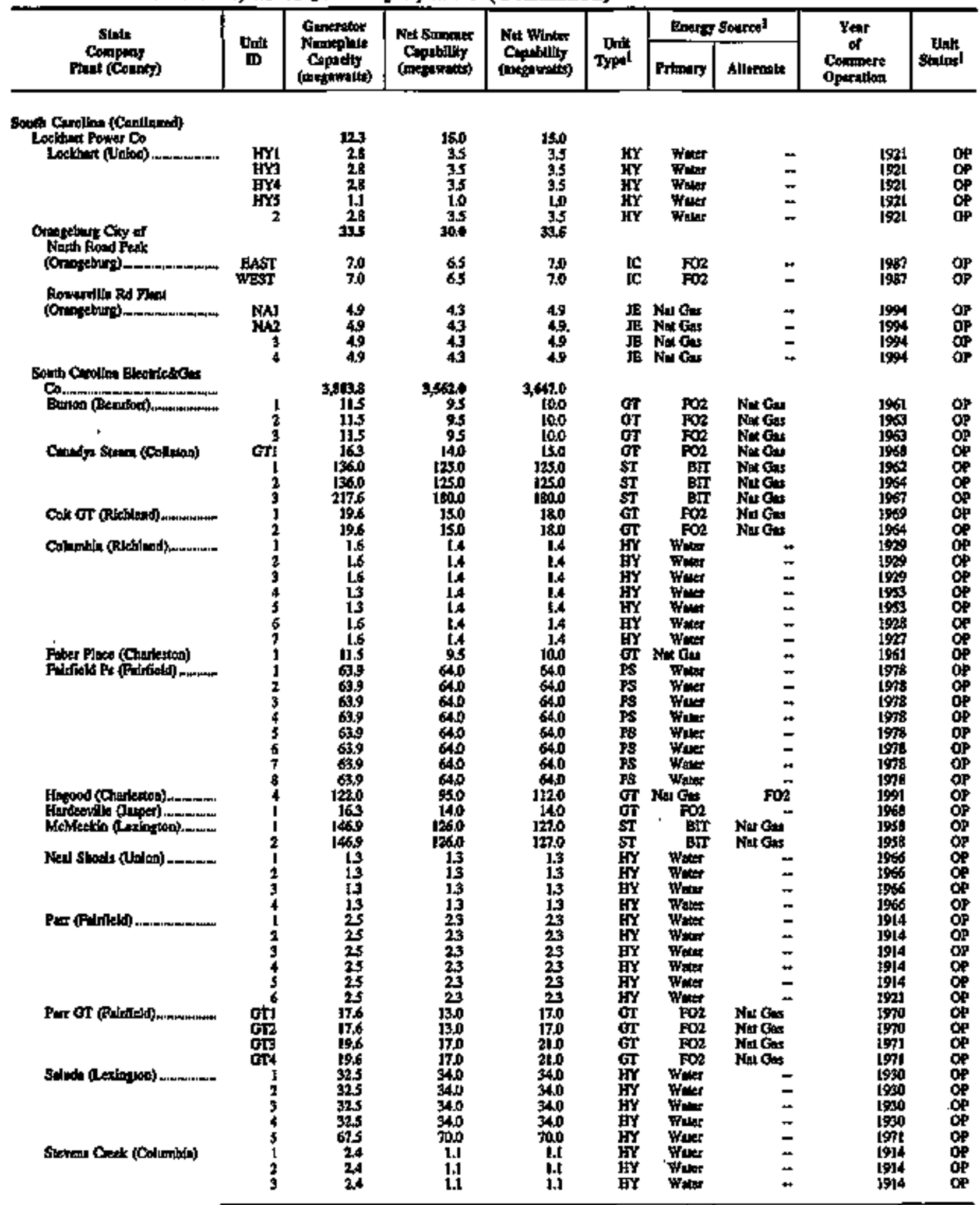

Ses boolnotes in end of table. 
Table 20. Existing Generating Units at U.S. Electric Uttittes by State, Company, and Plant, as of January 1, 1996 (Conthued)

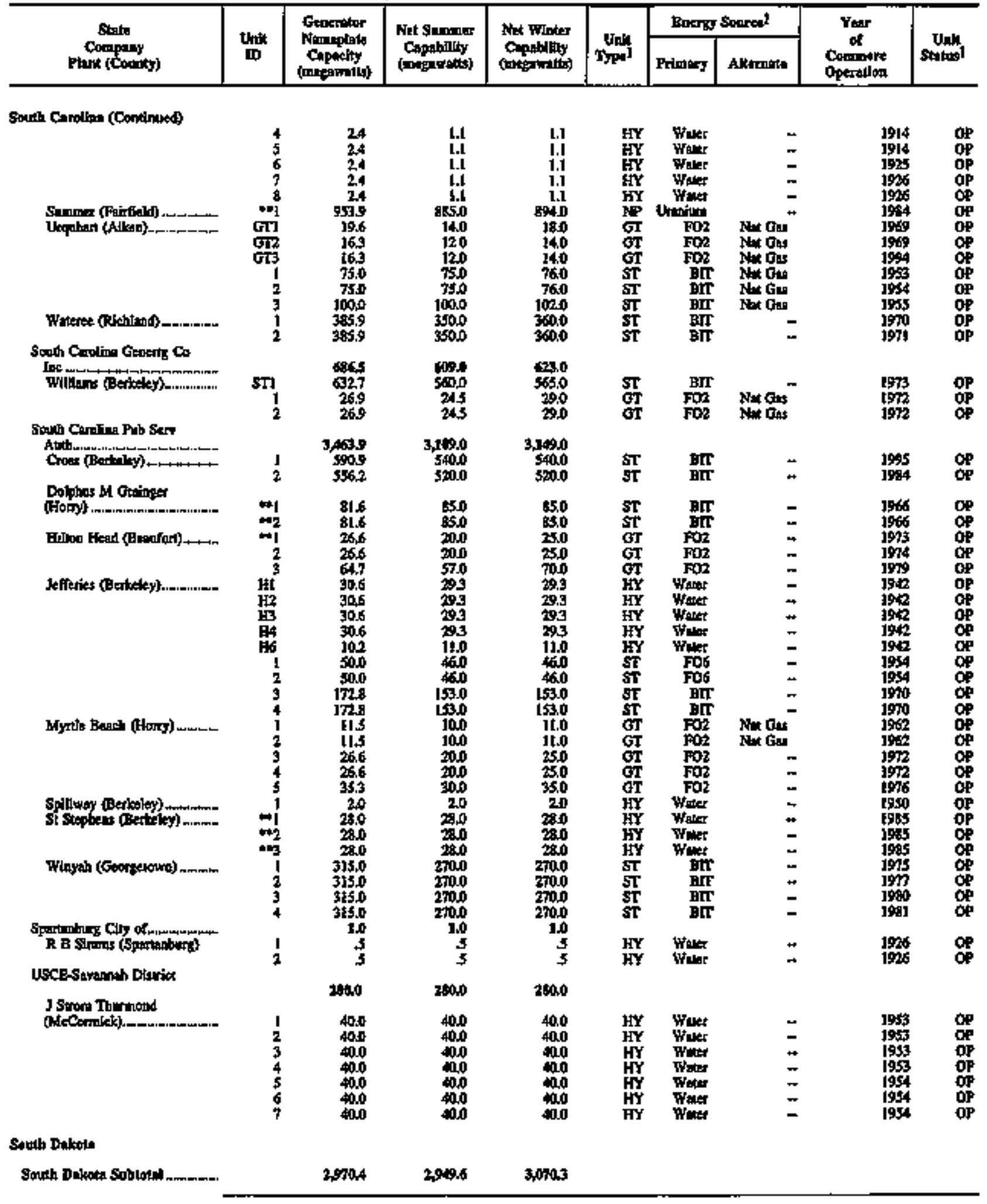

Sten Folnotite at enit of thble. 
Table 20. Existing Generating Units at U.S. Electric Utilities by State, Company, and Plant, as of January 1, 1996 (Continued)

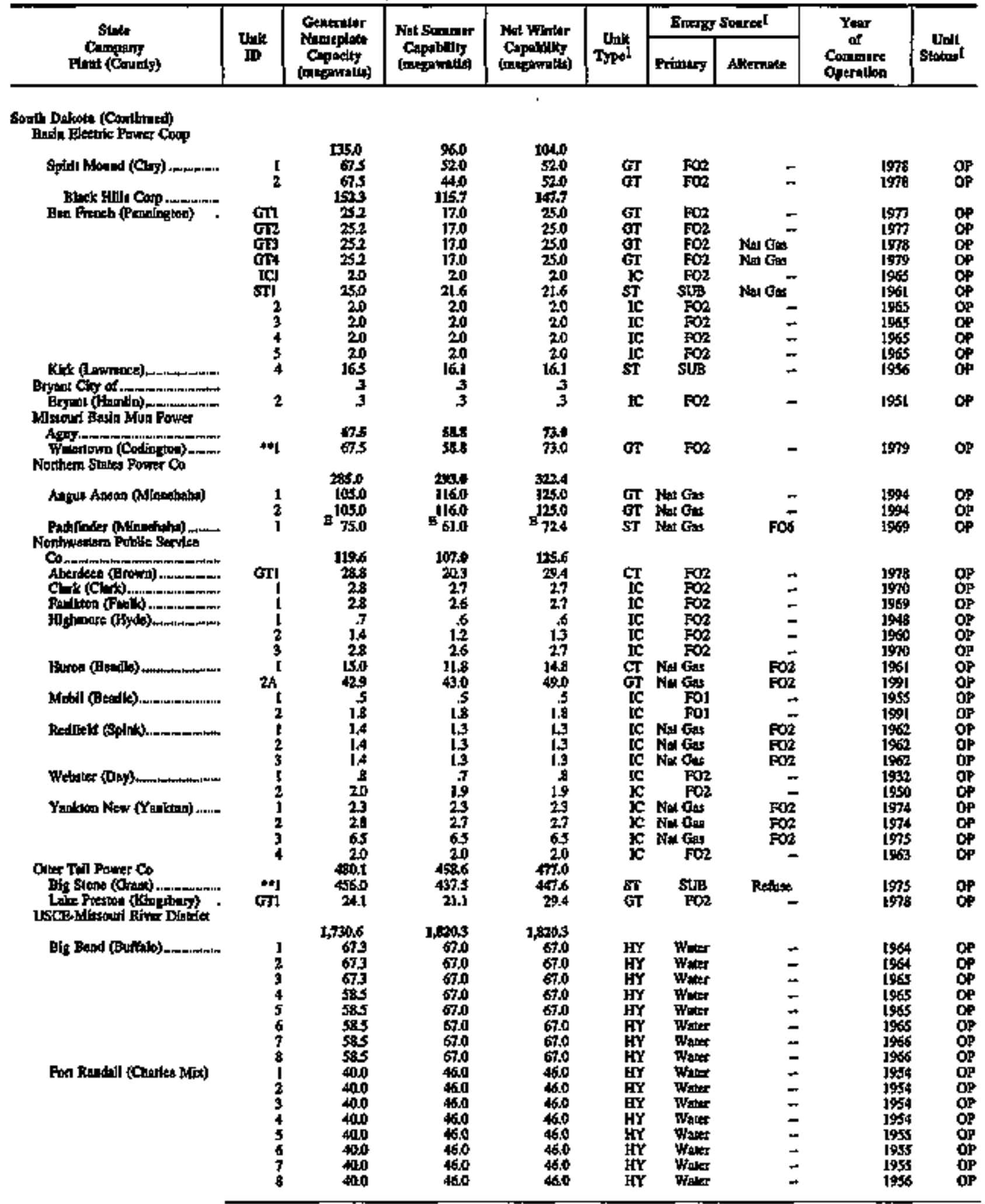

Sed foovines at end of tabte. 
Table 20. Existing Generating Units at U.S. Electric Utilities by State, Company, and Plant, as of January 1, 1996 (Continued)

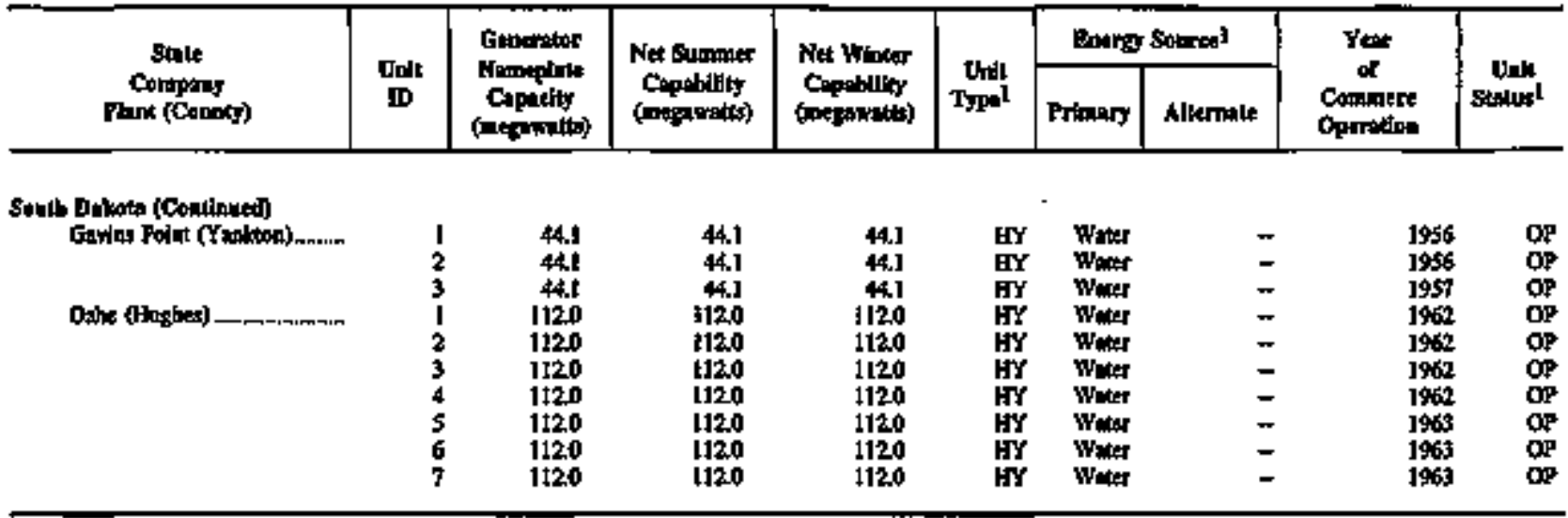

Sec poouroes it und of able. 
Table 20. Existing Generating Units at US. Electric Utilities by State, Company, and Plant, as of January 1, 1996 (Continued)

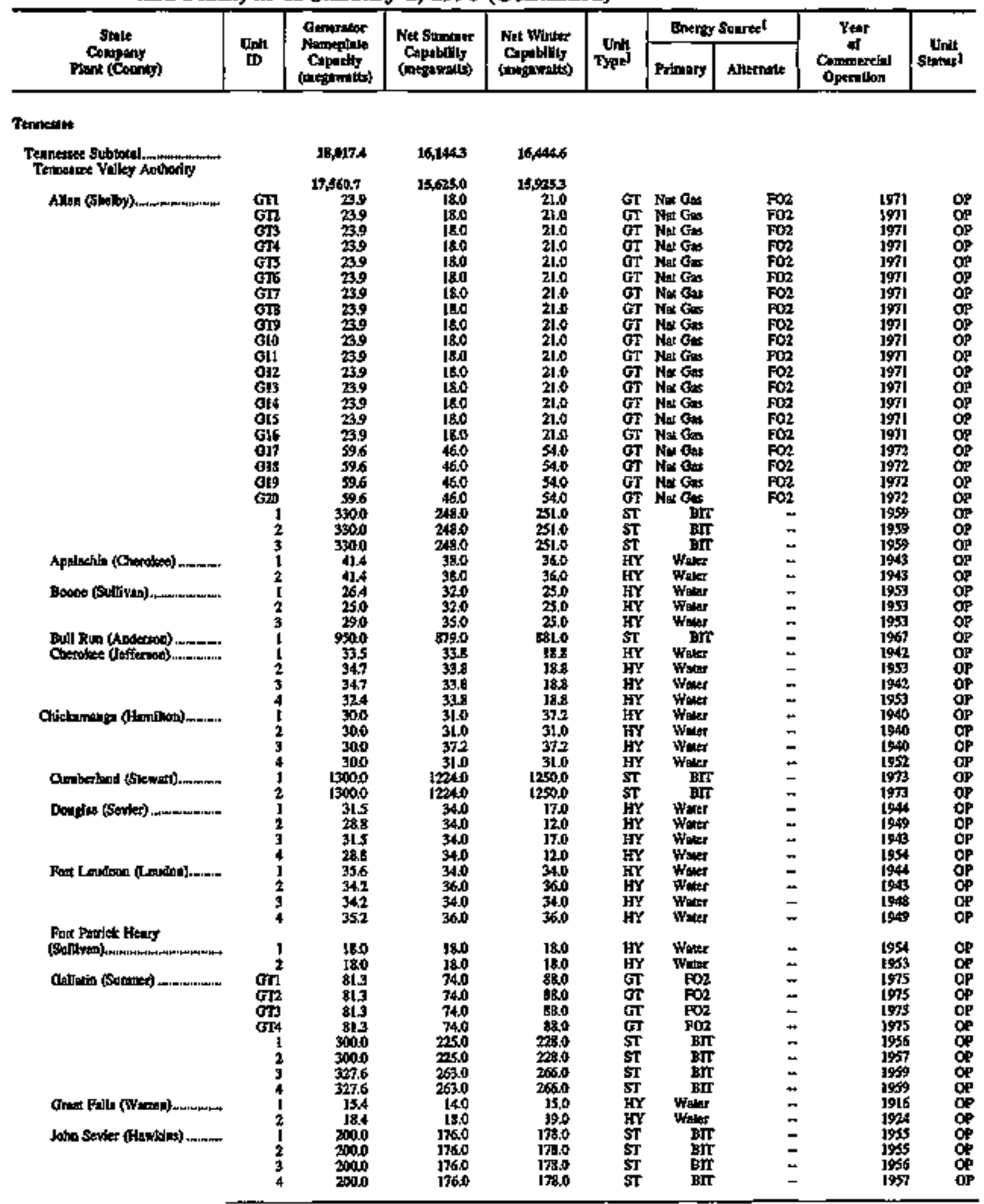

Sec footrolep It and af tabte. 
Table 20. Existing Generating Units at U.S. Electric Utilitles by State, Company, and Plant, as of January 1, 1996 (Continuted)

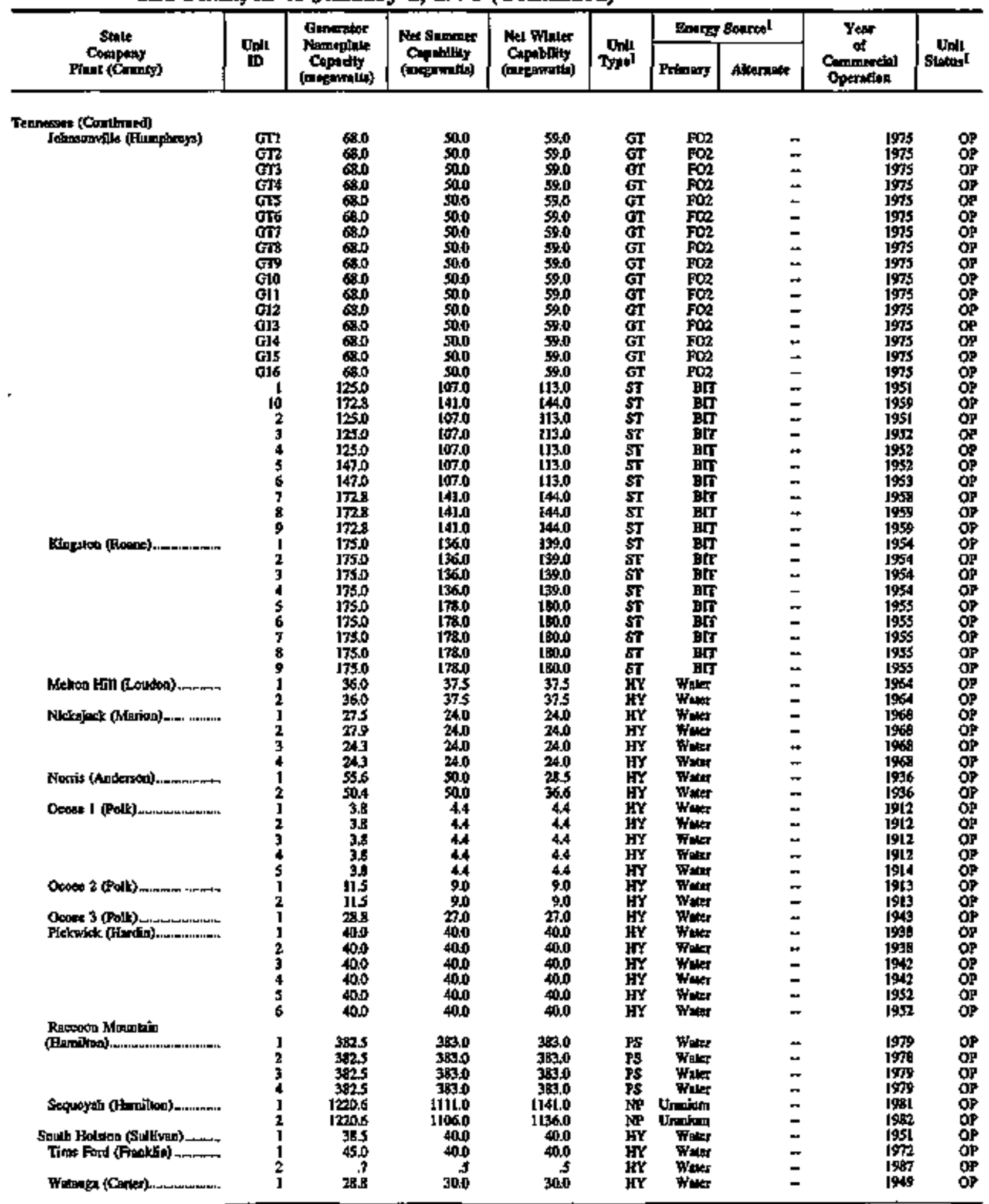

Sec fochoores tal tad of table. 
Table 20. Existing Generating Units at U.S. Flectric Utilities by State, Company, and Plant, as of January 1, 1996 (Continued)

\begin{tabular}{|c|c|c|c|c|c|c|c|c|c|}
\hline \multirow{2}{*}{ 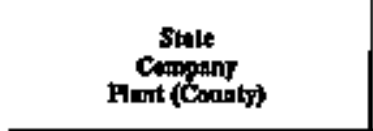 } & \multirow{2}{*}{ IIII } & \multirow{2}{*}{ 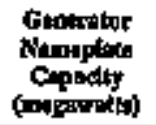 } & \multirow{2}{*}{ 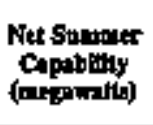 } & \multirow{2}{*}{ 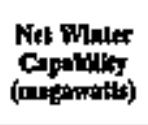 } & \multirow{2}{*}{ Typel } & \multicolumn{2}{|c|}{ Eneraty Soored] } & \multirow{2}{*}{$\begin{array}{c}\text { Year } \\
\text { of } \\
\text { Comintretal } \\
\text { Operation }\end{array}$} & \multirow{2}{*}{$\begin{array}{l}\text { That } \\
\text { Stateos 1 }\end{array}$} \\
\hline & & & & & & Prtmary & Atkenole & & \\
\hline \multicolumn{10}{|l|}{ Tennetere (Conitiond) } \\
\hline & 2 & 288 & 300 & 300 & $\mathbf{E Y}$ & Wale & 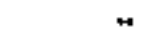 & 1949 & op \\
\hline Woll Bar (Rkeg) & $\mathrm{sTn}_{\mathrm{sT2}}^{\mathrm{sT3}}$ & $\begin{array}{l}600 \\
6000 \\
600\end{array}$ & $\begin{array}{l}5600 \\
560 \\
\$ 60\end{array}$ & $\begin{array}{r}564 \\
56,0 \\
560\end{array}$ & $\underset{S T}{S T}$ & $\begin{array}{l}\text { BIT } \\
\text { BIT } \\
\text { BIT }\end{array}$ & $\bar{z}$ & $\begin{array}{l}1942 \\
1942 \\
1943\end{array}$ & $\begin{array}{l}\text { SB } \\
\text { sis } \\
\text { sB }\end{array}$ \\
\hline & $\mathbf{s t}$ & 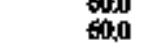 & 3600 & 560.0 & $\begin{array}{l}\text { SI } \\
\mathbf{S T}\end{array}$ & EII & $\ddot{z}$ & 1943 & sa \\
\hline What Bar Eydro (Rhat mem. & FY1 & 335 & 35.5 & 35.5 & EY & Waver & 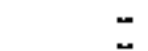 & 1942 & op \\
\hline & $\begin{array}{l}\text { Pys } \\
\text { Fys } \\
\text { pys } \\
\text { Hys }\end{array}$ & 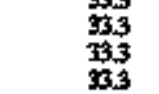 & $\begin{array}{l}37.0 \\
35.5\end{array}$ & $\begin{array}{l}3.9 \\
37.0 \\
35.5 \\
35.5\end{array}$ & thy & Whar & $\ddot{z}$ & $\begin{array}{l}1942 \\
1942 \\
1944 \\
1944\end{array}$ & 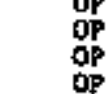 \\
\hline Wilour (C-ntar) & 1 & 1.3 & 1.3 & 1.3 & $\mathbf{H}$ & Wale & $\leftrightarrow$ & $19+2$ & op \\
\hline & $\begin{array}{l}2 \\
3 \\
4\end{array}$ & $\begin{array}{l}1.3 \\
1.3 \\
7.0\end{array}$ & $\begin{array}{l}1.9 \\
1.3 \\
7.2\end{array}$ & $\begin{array}{l}1.3 \\
1.3 \\
7.2\end{array}$ & $\begin{array}{l}\text { HY } \\
\text { țY } \\
\text { Erर }\end{array}$ & $\begin{array}{l}\text { Walex } \\
\text { Whakx } \\
\text { Whax }\end{array}$ & $\begin{array}{l}- \\
\ddot{z}\end{array}$ & $\begin{array}{l}1912 \\
1926 \\
1910\end{array}$ & $\underset{\partial P}{O P}$ \\
\hline CSCE-Natwils Dignda & & 456.7 & 5193 & 5193 & & & & & \\
\hline 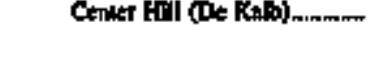 & $\begin{array}{l}1 \\
2 \\
3\end{array}$ & $\begin{array}{l}45,0 \\
45.0\end{array}$ & 520 & 5200 & 昂 & $\begin{array}{l}W_{w+r} \\
W_{a t a r}\end{array}$ & $\overline{-}$ & $\begin{array}{l}1950 \\
1951\end{array}$ & op \\
\hline 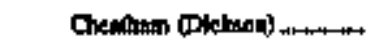 & 3 & $\begin{array}{l}45.0 \\
120\end{array}$ & 320 & $\begin{array}{l}52.0 \\
13.8\end{array}$ & $\begin{array}{l}\text { HY } \\
\mathbf{H Y}\end{array}$ & Witex & 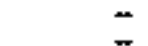 & 190 & op \\
\hline 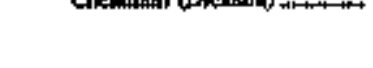 & 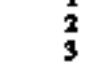 & $\begin{array}{l}120 \\
120\end{array}$ & $\begin{array}{l}13.8 \\
13.8\end{array}$ & $\begin{array}{l}13.8 \\
13.8\end{array}$ & 旦Y & $W_{w+a r}$ & $\overline{-}$ & 19958 & op \\
\hline Condill Eivll (SThllib) & j & $\frac{333}{313}$ & 380 & 380 & 昏 & Water & 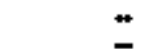 & 1973 & op \\
\hline & 3 & 333 & 38.0 & 38.0 & $\begin{array}{l}\text { HI } \\
\text { HY }\end{array}$ & Wint & $\bar{z}$ & 1974 & op \\
\hline Dele Hollow $(\mathrm{Cwy})$ & 1 & 18.0 & 20.7 & 207 & EY & Water & - & 1948: & OP \\
\hline & 3 & $\begin{array}{l}180 \\
180\end{array}$ & 20.7 & 20.7 & HY & $\begin{array}{l}\text { Water } \\
\text { Whter }\end{array}$ & $\overline{-}$ & 1949 & OP \\
\hline J P PAleal (Dondson) & 1 & 280 & 300 & $\mathbf{3 0 , 0}$ & HY & Water & 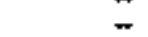 & 1970 & op \\
\hline 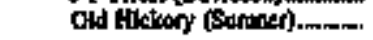 & j & 384 & 238 & 28.8 & EY & Water & - & 1957 & op \\
\hline & 2 & 350 & 29.0 & 29.0 & EY & Wret & - & 196 & OP \\
\hline & 3 & 250 & 29.0 & 29.0 & EY & Water & - & 1957 & $\boldsymbol{O Q}$ \\
\hline & & 250 & 290 & 2900 & BY & Wating & $*$ & 1969 & OP \\
\hline \multicolumn{10}{|l|}{ Tas: } \\
\hline \multirow{7}{*}{ 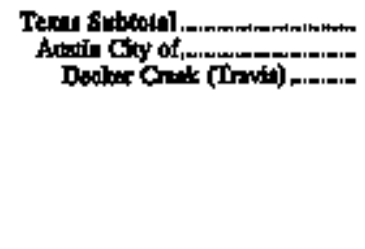 } & & $0,000,1$ & 644242 & 64,6469 & & & & & \\
\hline & & 1,000 & 1,403 & 1,443 & & & & & \\
\hline & ort & $\$ 1.6$ & sol. & 500 & GT & Nat Gan & $\mathbf{E O}_{2}$ & 198 & op \\
\hline & 西 & 31.6 & 50.0 & 500 & G & Nix the & Fug & 1988 & $\underset{\mathrm{OP}}{\mathrm{OP}}$ \\
\hline & Gry & 51.5 & so.0 & 500 & $\overrightarrow{\mathbf{G}}$ & Net Gas & 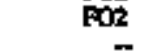 & 1968 & OP \\
\hline & 1 & 320 & 321.0 & 3210 & $5 \mathbf{T}$ & Notots & $\mathrm{FO}$ & 197 & op \\
\hline & 2 & 405 & 405.0 & 405.0 & $\mathbf{s i}$ & Note tin & 502 & 1978 & OP \\
\hline Holly Street (Thowis) & 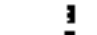 & 100,0 & 1000 & 1000 & $\mathbf{T T}$ & Nut Gen & Fo5 & 1960 & OP \\
\hline & 2 & 1000 & 1000 & 1000 & ET & Now On & P05 & 1964 & \\
\hline & 3 & 1650 & 165.0 & 16500 & 52 & Now Gas & $\mathbf{F}$ & 1967 & op \\
\hline \multirow{2}{*}{\multicolumn{10}{|c|}{ 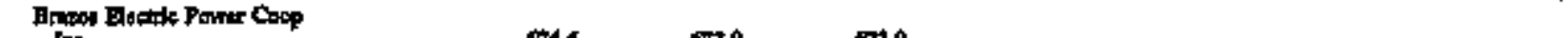 }} \\
\hline & & 874 & का:0 & 672.0 & & & & & \\
\hline 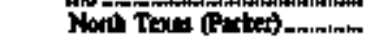 & $\mathbf{3}$ & 165 & 170 & 17,0 & $5 T$ & Mux Gas & FOS & 1958 & OP \\
\hline & $\mathbf{2}$ & 365 & 170 & 35.0 & $5 T$ & Now & $\mathbf{F o 6}$ & 1058 & op \\
\hline 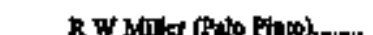 & 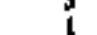 & 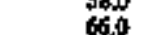 & $\$ 50$ & 390 & ST & Nite Das & 506 & 1963 & 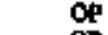 \\
\hline & 2 & 1000 & 1160 & 1960 & st & Nerdat & $\mathrm{FO2}$ & 1968 & op \\
\hline & $\overline{3}$ & 2000 & 2000 & 2000 & $\mathbf{s t}$ & Net Gu & For & 1975 & op \\
\hline & $\$$ & $\mathrm{J1}, \mathrm{s}$ & 104,0 & 104,0 & GI & Non Gas & - & 1994 & of \\
\hline \multirow{3}{*}{ Brome Bhiver Authodity } & 5 & ${ }_{21}^{118}$ & 1040 & 1040 & $\boldsymbol{\sigma}$ & Net Oas & - & 1994 & OP \\
\hline & & & & 23 & & & & & \\
\hline & I & & $\mathrm{ll}, \mathrm{s}$ & 11,5 & fFY & Water & $\sigma$ & 1948 & OP \\
\hline 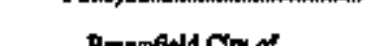 & 2 & 25 & 11.5 & 115 & $\ddot{\mathbf{Y Y}}$ & Wuter & - & 1942 & De \\
\hline Brownilold (Tenty) & OTI & $\begin{array}{ll}24,35 \\
6,5\end{array}$ & $\begin{array}{l}1+3 \\
5.5\end{array}$ & 58 & ET & Nut Gu & $\mathrm{POz}$ & 1953 & OP \\
\hline & 1 & 20 & :0 & 1.0 & $\mathrm{~K}$ & Now Gat & $\mathrm{pop}$ & 1951 & OP \\
\hline & 3 & 3.1 & 1.8 & 20 & 点 & Now Gas & $\mathrm{FOZ}$ & 1984 & \\
\hline & 4 & $\begin{array}{l}27 \\
3,6\end{array}$ & $\begin{array}{l}1.8 \\
20\end{array}$ & $\begin{array}{l}1.8 \\
24\end{array}$ & k & $N_{n=} \frac{d t}{d t}$ & ${ }_{P \infty 2}^{P 02}$ & $\begin{array}{l}1954 \\
1057\end{array}$ & of \\
\hline
\end{tabular}

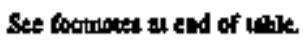


Table 20. Existing Generating Units at US. Electric Utilifies by State, Company, and Plant, as of January 1, 1996 (Continued)

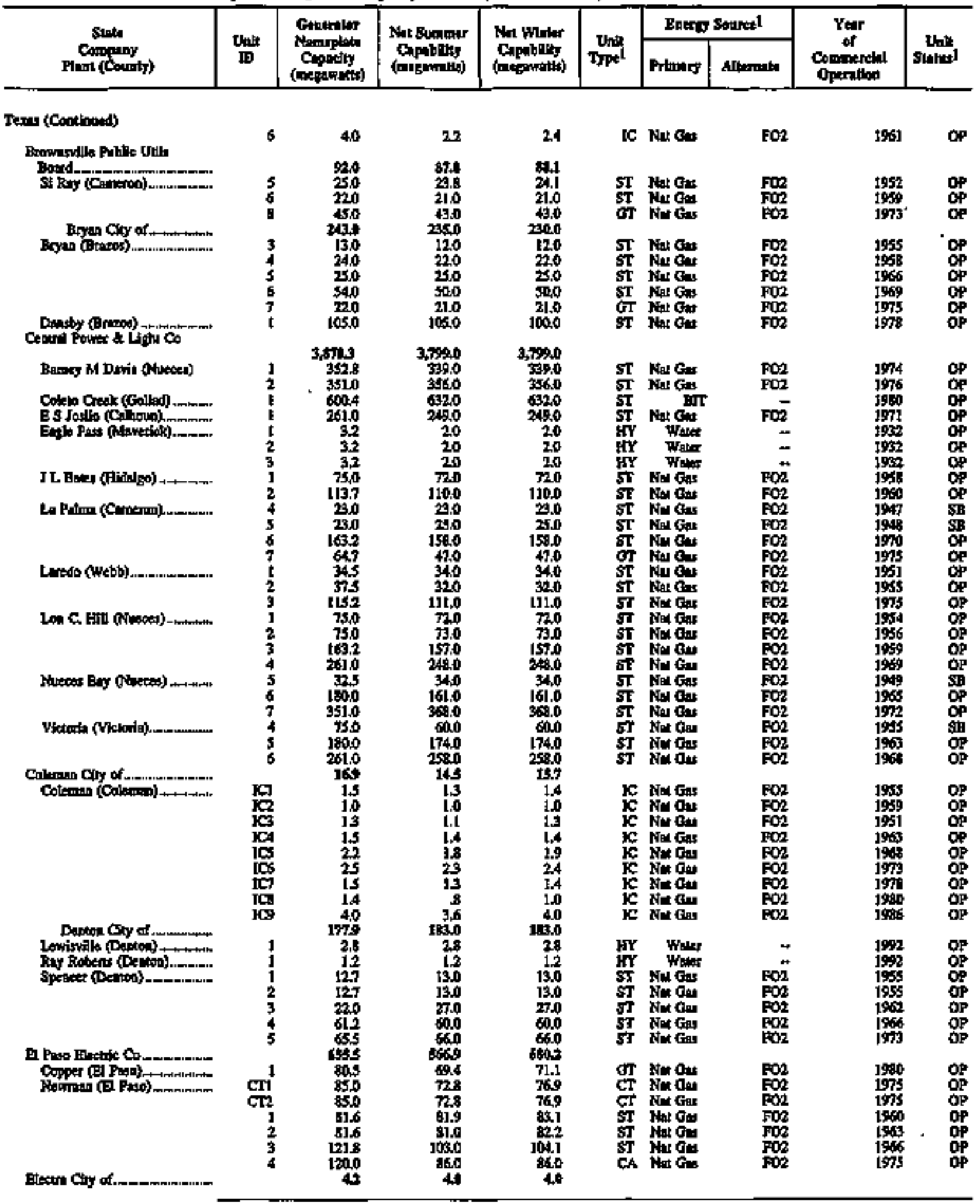

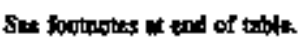


Table 20. Exdsting Generating Unuts at U.S. Electric Utilities by State, Company, and Plant, as of Jamaary 1, 1996 (Continued)

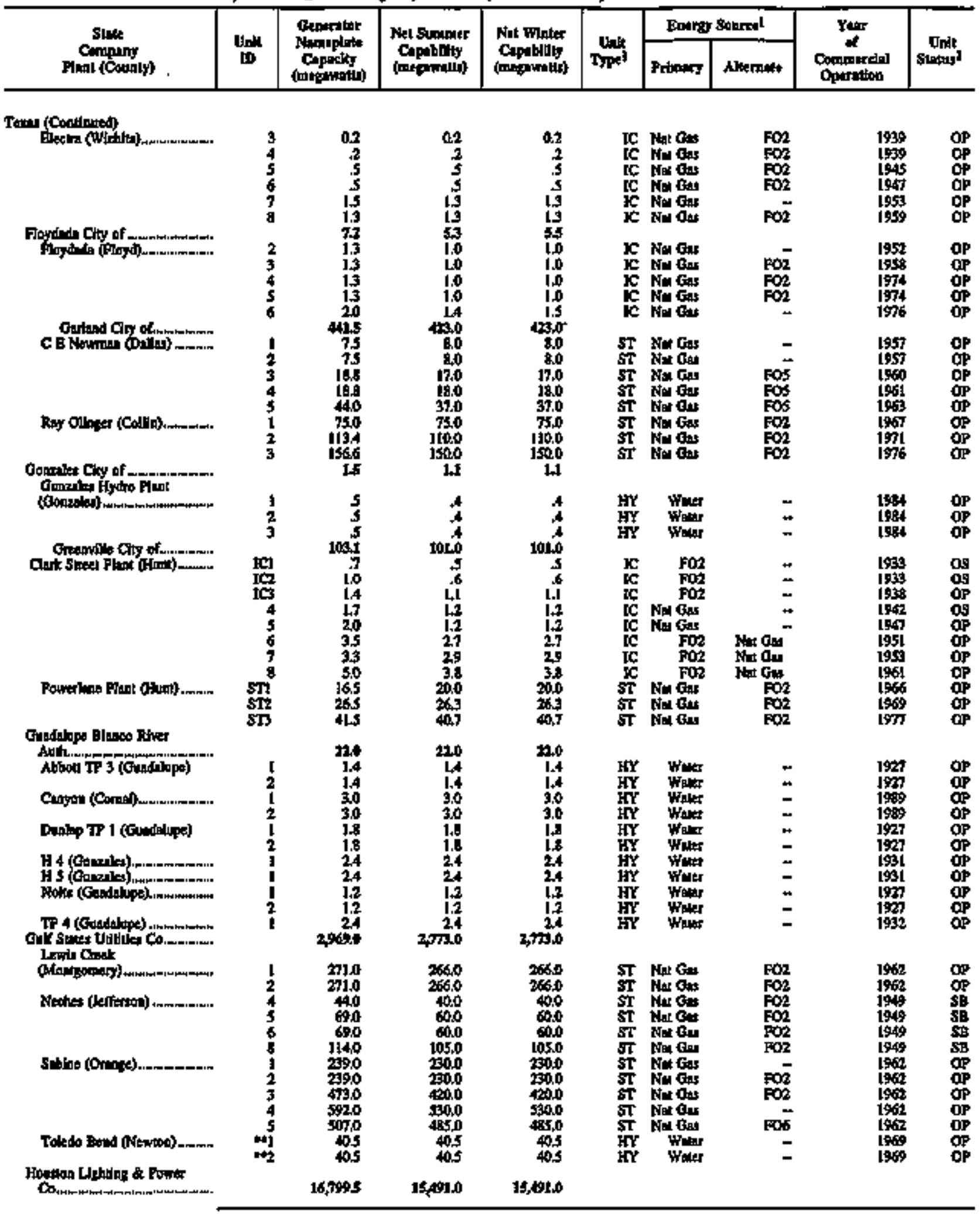

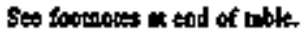


Table 20. Existing Generating Units at U.S. Flectric Utilities by State, Company, and Plant, as of January 1, 1996 (Continued)

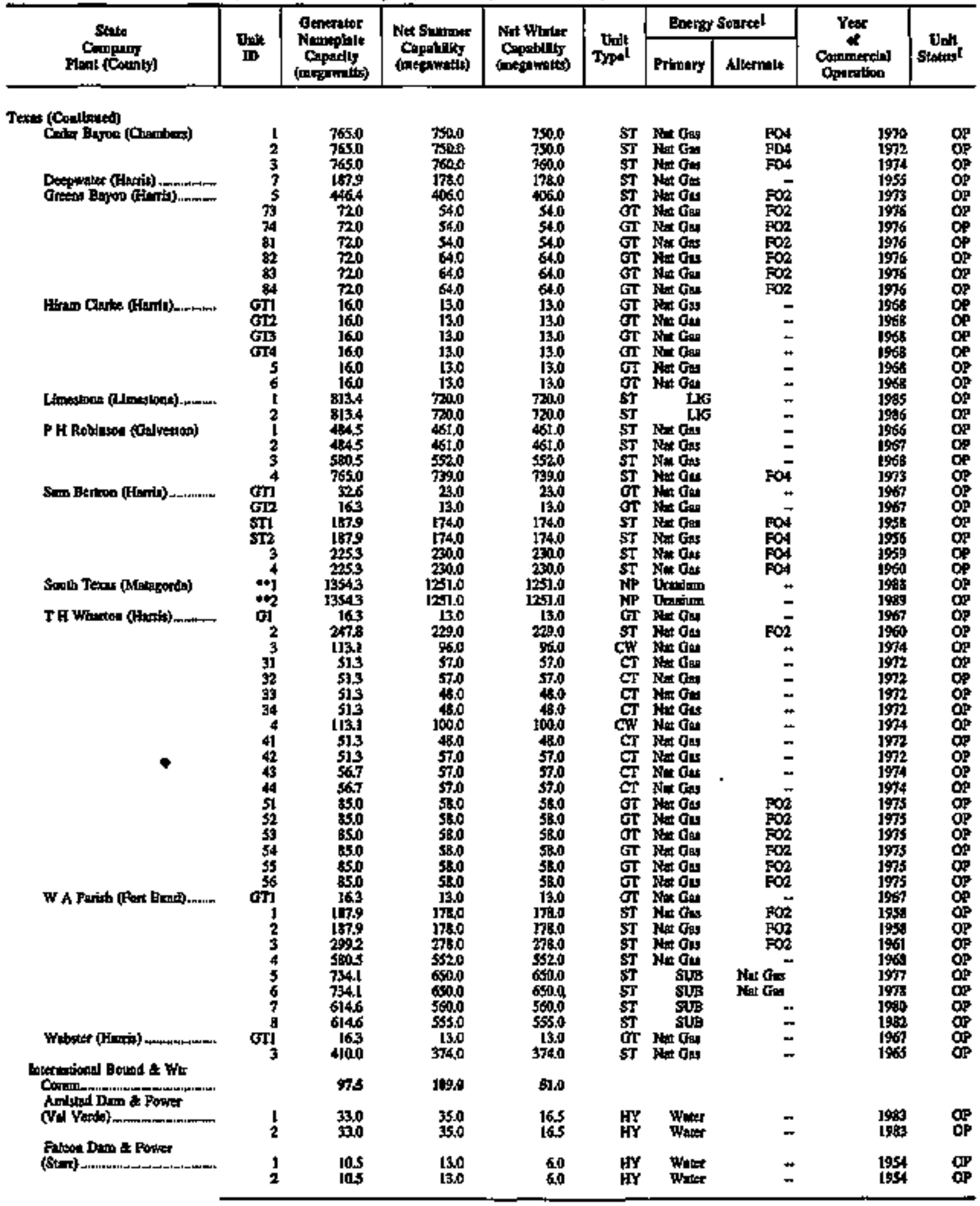

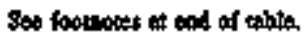


Table 20. Existing Generating Units at U.S. Electric Utillties by State, Company, and Plant, as of January 1, 1996 (Continued)

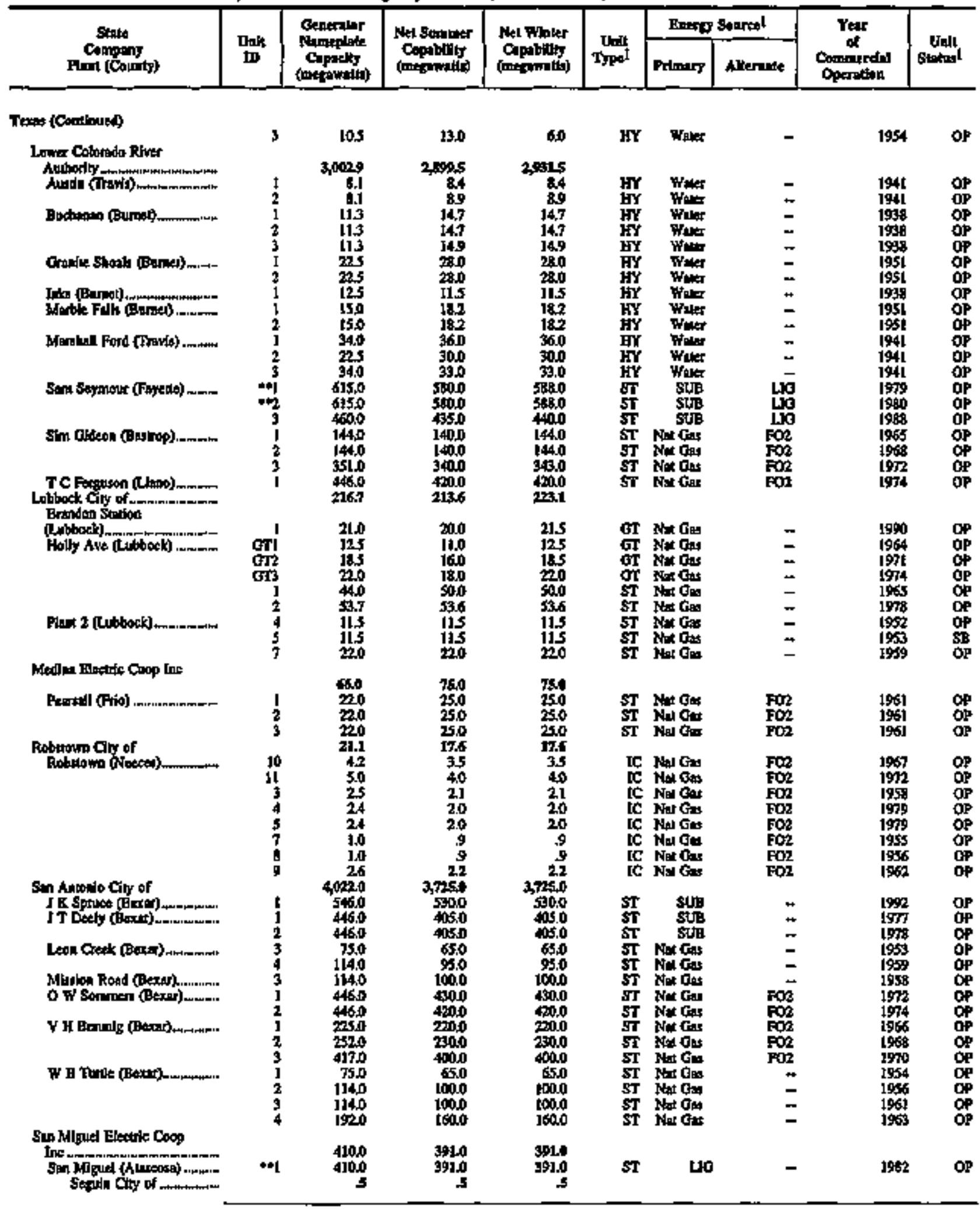

See toomoles at and of whle. 
Table 20. Existing Generating Units at U.S. Flectric Utilities by Stafe, Company, and Plant, as of January 1, 1996 (Continued)

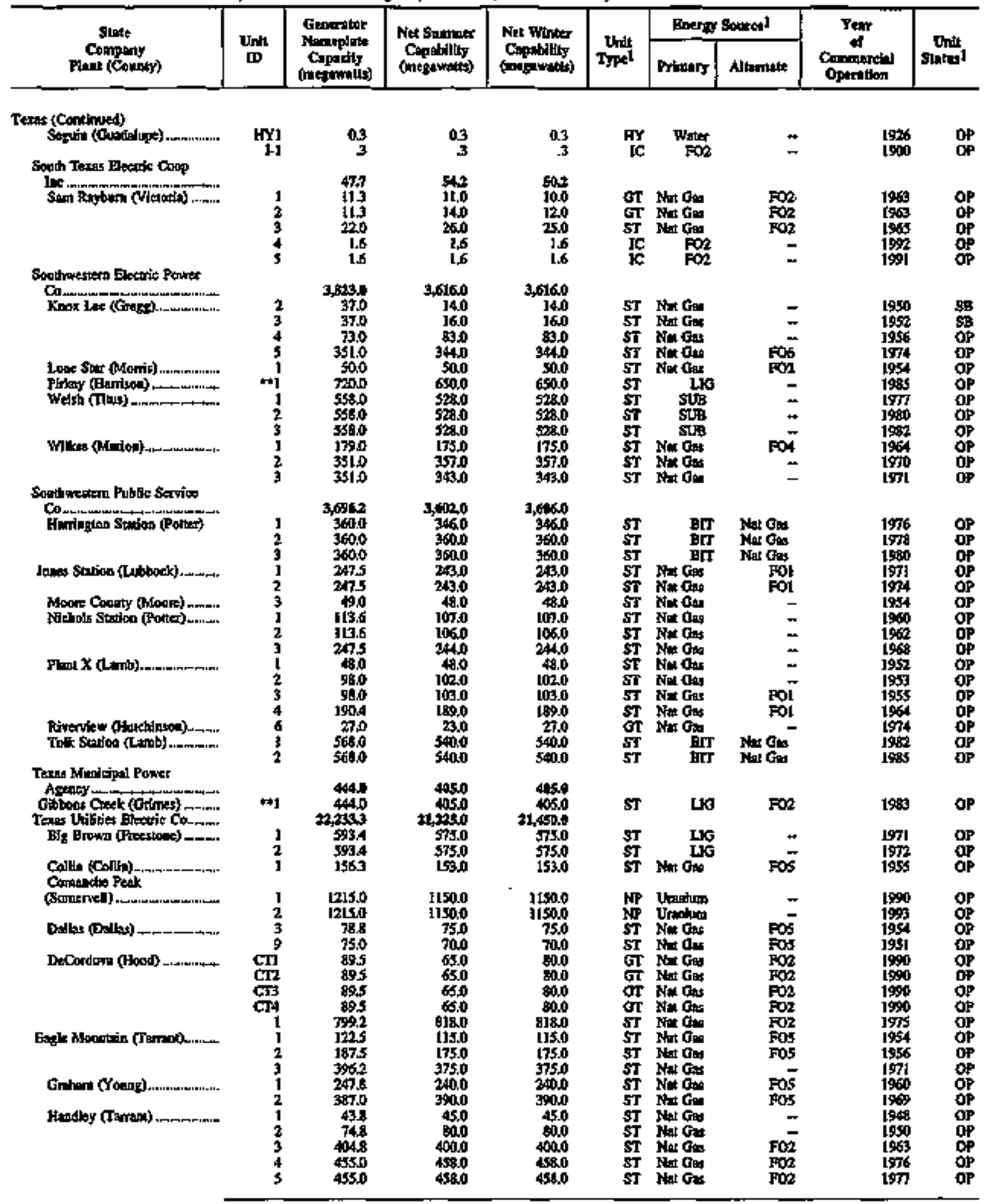

Sec footmotes al end of thbls. 
Table 20. ExIsting Generating Units at U.S. Electric Utilities by State, Company, and Plant, as of January 1, 1996 (Continued)

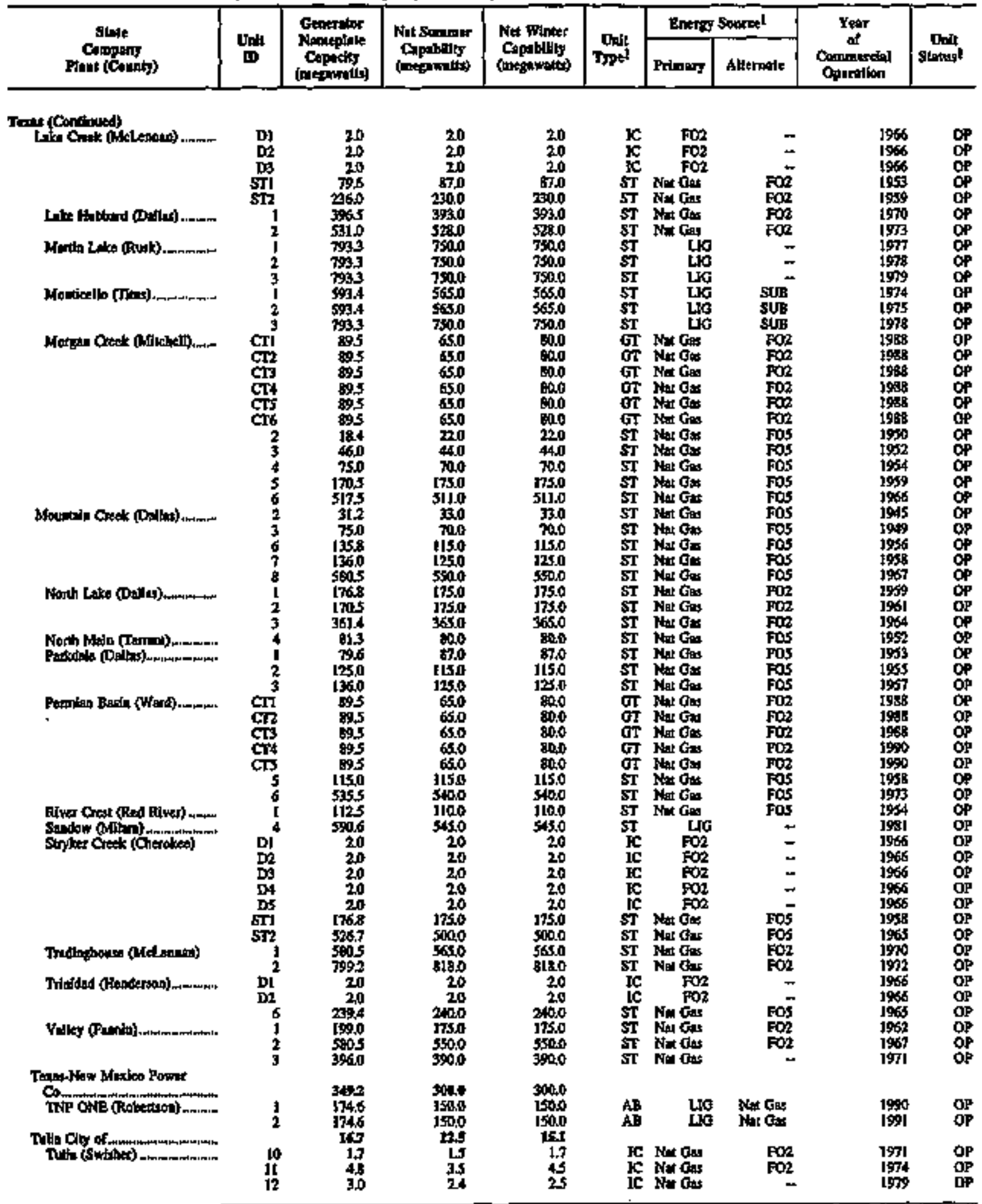

Sex tootneks it end of uble. 
Table 20. Existing Generating Units at U.S. Electric Utiltites by State, Company, and Ptant, as of Jamuary 1, 1996 (Continued)

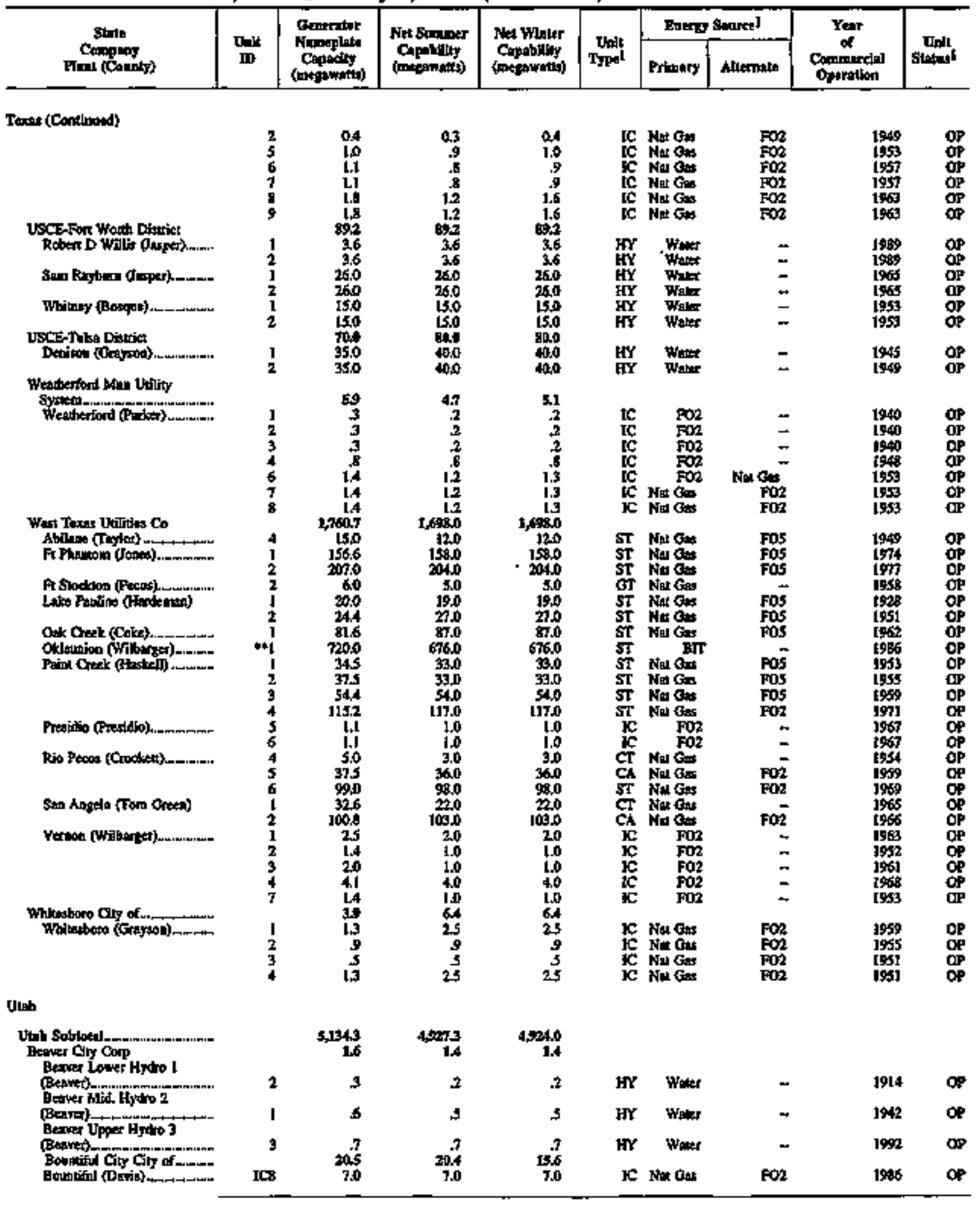

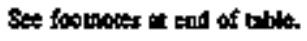


Table 20. Existing Generating Units at U.S. Electric Utilittes by State, Company, and Plant, as of January 1, 1996 (Continued)

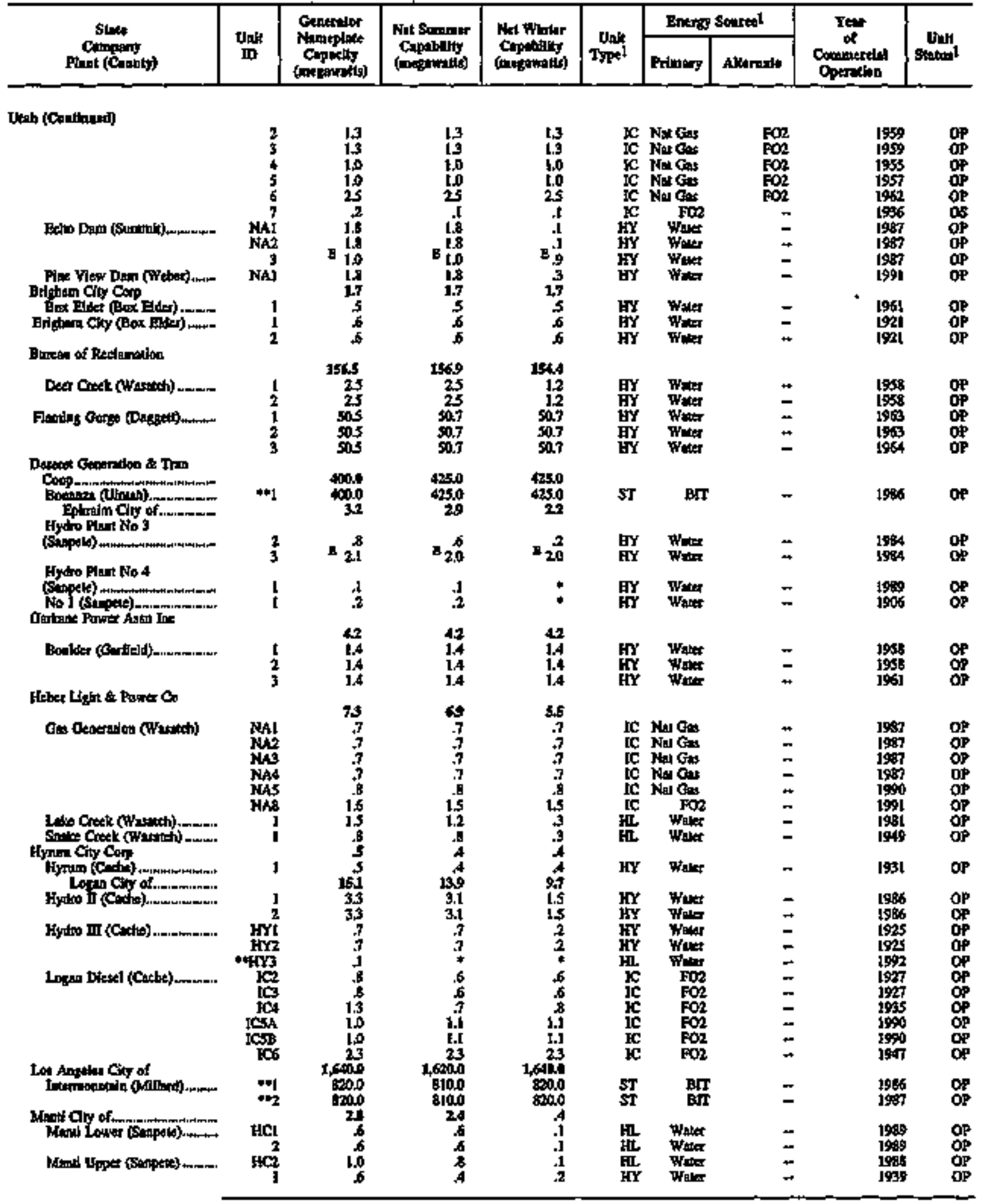

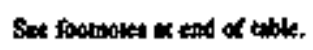


Table 20. Existing Generating Units at U.S. Electric Utilities by State, Company, and Plant, as of January 1, 1996 (Continued)

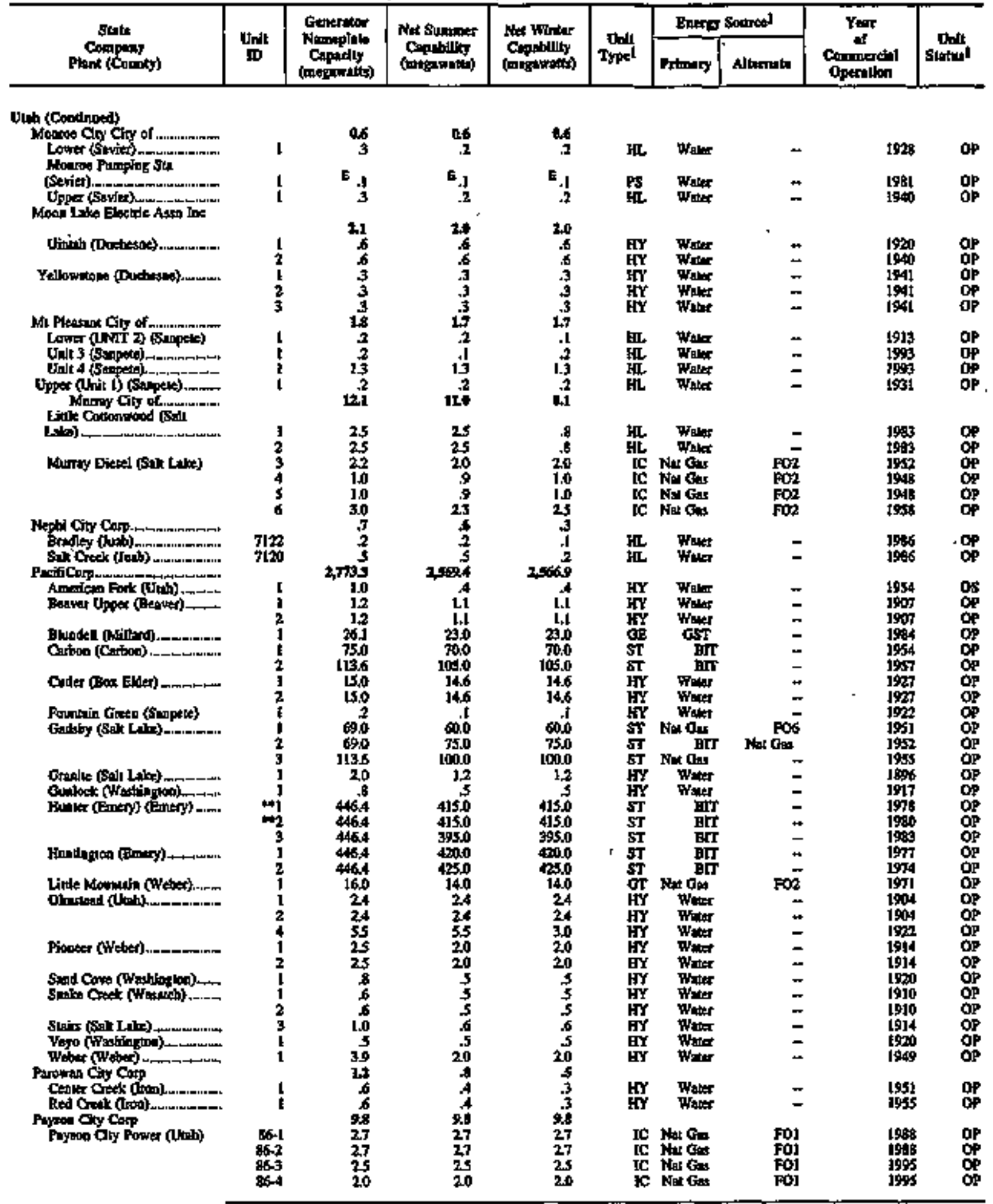

sen fooboligy at end of whik. 
Table 20. Existing Generating Units at U.S. Electric Utilitles by State, Company, and Plant, as of January 1, 1996 (Continued)

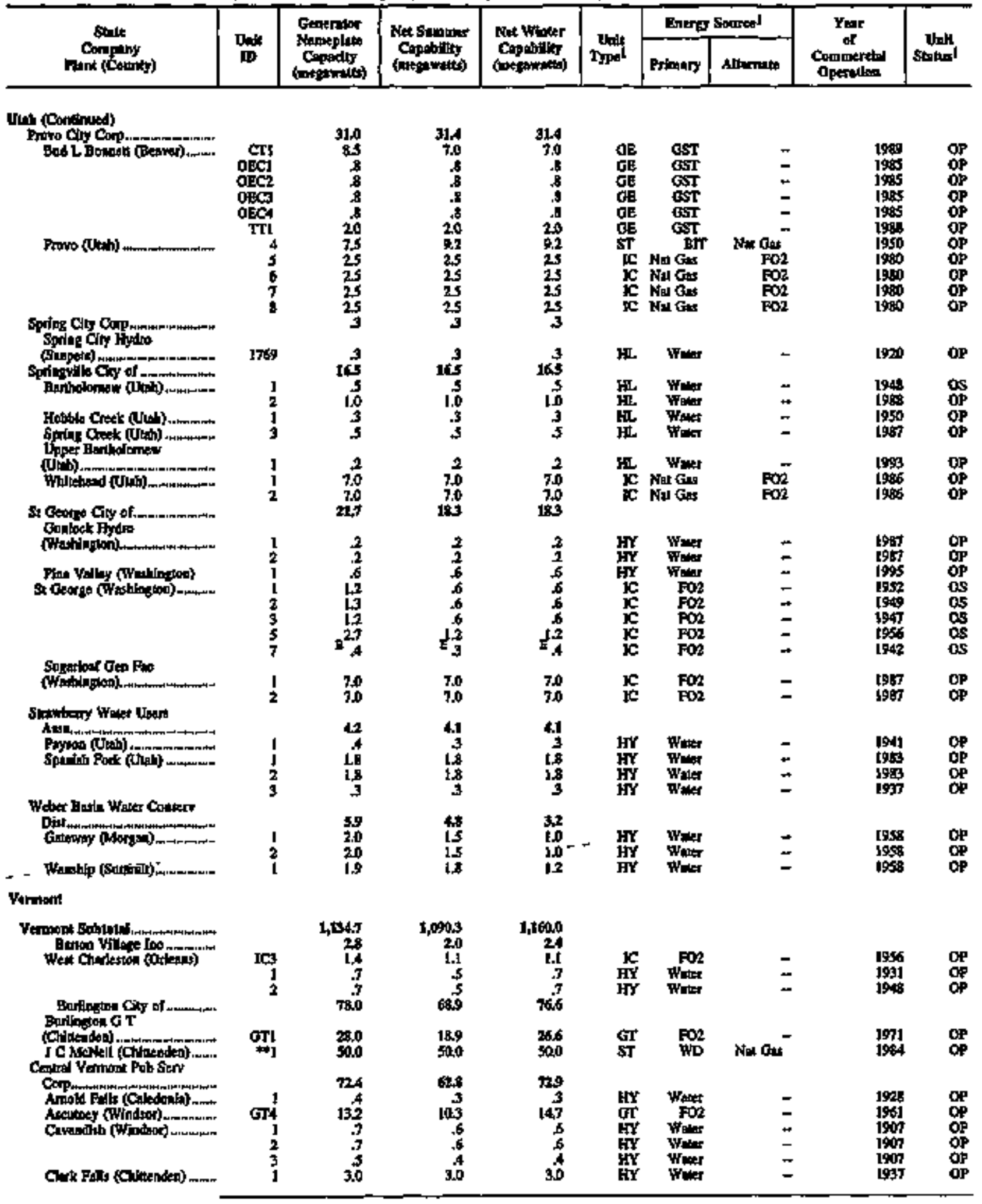

Sea foowates nil end of table 
Table 20. Existing Generating Units at U.S. Electric Utilities by State, Company, and Plant, as of January 1, 1996 (Continued)

\begin{tabular}{|c|c|c|c|c|c|c|c|c|c|}
\hline \multirow{2}{*}{ 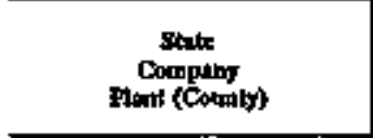 } & \multirow{2}{*}{$\underset{\text { In }}{\text { In }}$} & \multirow{2}{*}{ 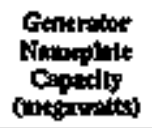 } & \multirow{2}{*}{ 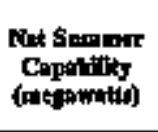 } & \multirow{2}{*}{ 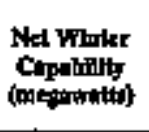 } & \multirow{2}{*}{ Thall } & \multicolumn{2}{|c|}{ Batry Som } & \multirow{2}{*}{ 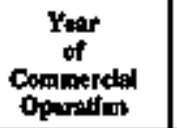 } & \multirow{2}{*}{$\begin{array}{l}\text { thit } \\
\text { sintuss }\end{array}$} \\
\hline & & & & & & Prituary & Dllerato & & \\
\hline \multicolumn{10}{|l|}{ Yeruon (Comtanued) } \\
\hline East Banet (C.ledonim) & I & 23 & 13 & 19 & HYY & Waks & $*$ & 1994 & OP \\
\hline Falfa Fall (Fraklia) & 3 & 14 & i.t. & 1.6 & $\mathbf{H Y}$ & Waks & - & 1919 & OP \\
\hline Onpe (Celedacio). & 2 & 22 & 2,1 & 21 & $\underset{\mathbf{H Y}}{\mathbf{H Y}}$ & $\begin{array}{l}\text { Waitr } \\
\text { Wasex }\end{array}$ & $\bar{z}$ & 1919 & $\begin{array}{l}\text { OP } \\
\text { OP }\end{array}$ \\
\hline Oage (ciledonis) & 2 & $\overrightarrow{4}$ & $\ddot{5}$ & .5 & HY & Wart & 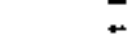 & 1921 & op \\
\hline 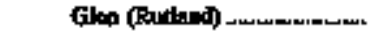 & 1 & 10 & L. & 1.0 & $\mathbf{t} \boldsymbol{Y}$ & Waker & - & 3920 & \\
\hline \multirow{2}{*}{\multicolumn{9}{|c|}{ Lower Hetodetaty }} & $\mathbf{Q P}$ \\
\hline & & & & & & & & & $\mathbf{O P}$ \\
\hline & 2 & $\ddot{s}$ & 6 & .6 & $\mathbf{E x}$ & Wast & $=$ & 3917 & OP \\
\hline & 3 & 8 & $A$ & .6 & EY' & What & ـ & [9]7 & OP \\
\hline MTlisa (Chitienden), & I & 38 & 33 & 3.3 & EYY & Water & - & 1929 & $\mathbf{a P}$ \\
\hline & 2 & 3.8 & 3.6 & 3,6 & $\overline{\mathbf{H Y}}$ & Warre & - & 1929 & aP \\
\hline Pastompsic (Caledonis) & I & .7 & .7 & 7 & IT & Want & - & 1929 & OP \\
\hline Poveh (Rothiol) & i & 4 & .3 & .3 & HY & Waver & - & $192 t$ & op \\
\hline Pelesson (Coivenden) & i & 6.4 & 58 & 6.4 & HYY & What & $*$ & $19+8$ & OP \\
\hline 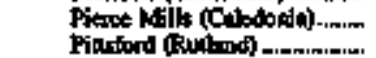 & I & 13 & .2 & 15 & $\underset{\boldsymbol{H Y}}{\mathbf{H Y}}$ & $\begin{array}{l}\text { Waver } \\
\text { Whaver }\end{array}$ & $\ddot{z}$ & 1928 & OP \\
\hline 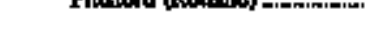 & 2 & 3 & l.1. & 1. & HY & Water & $=$ & t914 & of \\
\hline & 3 & 1.0 & 10 & 1.0 & HYY & Whet & ـ & [\$14 & OP \\
\hline Ruettand (Ruthod) & का5 & 133 & 104 & 141 & $\mathbf{G r}$ & $\mathrm{FO} 2$ & - & 1962 & $\mathbf{O P}$ \\
\hline 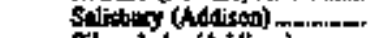 & I & 13 & 1.2 & $1+2$ & $\mathbf{H Y}$ & Walex & - & 1917 & OP \\
\hline & i & 32 & 22 & 2.2 & मY & Water & - & 1917 & OP \\
\hline 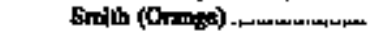 & HC2 & 5 & .2 & 4 & HYY & Wut & - & 1980 & pp \\
\hline & $\mathbf{c i}^{1}$ & 1.0 & 3 & 1.0 & HY & Whas & $=$ & I984 & OP \\
\hline 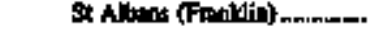 & IC1 & 13 & 1.1 & 1.2 & IC & $\mathrm{FO}$ & $\overline{-}$ & 1950 & OP \\
\hline 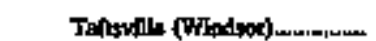 & $\vec{J}$ & 3 & $A$ & A & HY & $\underset{w+1}{F 02}$ & $\check{-}$ & $\begin{array}{l}t 950 \\
1948\end{array}$ & op \\
\hline Weybadded (Addlson) & i & 30 & 3.4 & 3.4 & EY & Whatr & $=$ & 1951 & AP \\
\hline 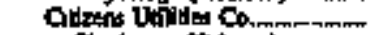 & & 143 & 15.3 & 123 & & & 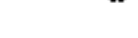 & & \\
\hline Chodestoe (0,ketit) & 1 & 8 &, 8 & .6 & EY & 마an & . & 1923 & op \\
\hline Hewpont (OAkenn) -.......... & 1 & 1.7 & 1.6 & 1.6 & HY & Wat & - & 1940 & 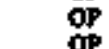 \\
\hline & 11 & 19 & 16 & 1.6 & $\mathbf{H Y}$ & Wher & $\ddot{z}$ & Its? & OPp \\
\hline & 3 & .6 & 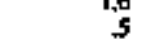 & $\begin{array}{l}1.8 \\
5\end{array}$ & $\mathbf{H Y}$ & $\begin{array}{l}\text { Wuter } \\
\text { Whater }\end{array}$ & $\overline{.}$ & 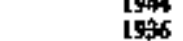 & GP \\
\hline \multirow{6}{*}{ Rerpor DAlesel (Ontests) } & 10 & L.I & 1.0 & 1.0 & ic & $\mathrm{FO} 2$ & - & 199 & OP \\
\hline & 4 & 9 & 9 & .9 & ic & $\mathrm{FO}$ & + & 1948 & OP \\
\hline & 6 & 9 & 9 & 9 & $\underset{\text { IC }}{\text { IC }}$ & $\begin{array}{l}\mathrm{Fon} \\
\mathrm{FO}\end{array}$ & $\ddot{\ddot{z}}$ & 1988 & OP \\
\hline & to & $\overrightarrow{\boldsymbol{y}}$ & $\overrightarrow{\boldsymbol{g}}$ & .9 & Ic & $\mathrm{OO} 2$ & - & tods & OP \\
\hline & i & 3.1 & 1.0 & 1.0 & tc & $\infty 2$ & - & 1954 & OP \\
\hline & 9 & 1.1 & 1,0 & 10 & IC & FO2 & - & 1954 & OP \\
\hline \multirow{4}{*}{ 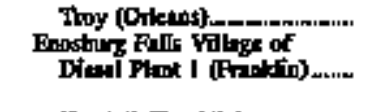 } & 1 & 6 & .6 & .6 & $\mathbf{E Y}$ & Water & - & 1925 & OP \\
\hline & & 19 & $\mathbf{j}$ & 1.8 & & & & & \\
\hline & ICI & .7 & .6 & .6 & te & $\mathbf{F O} 2$ & - & 1949 & oP \\
\hline & [C2 & 2 & 3 & .3 & tc & $\mathrm{FO} 2$ & - & 1938 & OP \\
\hline Kendstl (Fraklln) & EY2 & 4 & .3 & 3 & $\mathbf{E Y}$ & Watrax & - & 1992 & $\mathbf{O P}$ \\
\hline Whage Fhe (Frindlo)...... & सY1 & .6 & .6 & .6 & HY & Waler & - & I944 & OP \\
\hline & & 1089 & 93.5 & 1169 & & & & & \\
\hline Berila $\$$ (Woulington) & GN & 48.6 & 412 & 57.1 & $\mathbf{G T}$ & XER & - & 1972 & OP \\
\hline 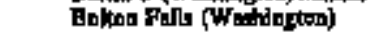 & & 4.4 & 27 & 3,9 & HY & Wabr & - & 1985 & $\mathrm{OP}$ \\
\hline & 2 & 4.4 & 27 & $\mathbf{3 . 9}$ & HY & Wuter & - & 1986 & $\mathbf{O P}$ \\
\hline Cribution (Bemiogron) & i & .1 & .1 & .1 & WT & Wod & - & 190 & op \\
\hline & 2 &.$t$ & .1 & .1 & WT & Wied & - & 1989 & OP \\
\hline Colanitur $1 \delta$ & OTI & 1700 & 11.7 & 15.7 & GT & Fo2 & - & 1965 & OP \\
\hline Estex Jetétion 19 & & & & & & & & & \\
\hline (Chloteden) ...-LC & En & 1.8 & 20 & 20 & $\mathbf{H Y}$ & Wator & + & 1917 & OP \\
\hline & 留 & 18 & 20 & 200 & $\underset{\forall Y Y}{\mathbf{H Y}}$ & $\begin{array}{l}\text { Waler } \\
\text { Warer }\end{array}$ & $=$ & t\$17 & op \\
\hline & $\mathbf{H}$ & 18 & 20 & 20 & $\begin{array}{l}\text { HY } \\
\text { HYY }\end{array}$ & Whet & $\bar{z}$ & [917 & QP \\
\hline & ICS & 3.0 & \pm .1 & 1.1 & IC & $\mathrm{FO}$ & - & 194 & DP \\
\hline & 106 & 10 & 1.1 & j.j & Ic & $\mathrm{FO}$ & - & {$[9.9]$} & op \\
\hline & ICI & [.0 & I.j & I.I & $\mathbf{K C}$ & $\mathbf{F} 2$ & - & 194 & Op \\
\hline & ICs & E.0 & 1.1 & 1.1 & $\mathbf{K C}$ & FO2 & * & [94] & OP \\
\hline 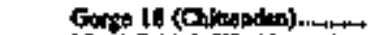 & 1 & 300 & 33 & 3.3 & HYY & Winter & - & 1928 & op \\
\hline Mrilieto $6 C$ & 1 & 5.0 & 4.9 & 4.9 & t & Waver & - & 5927 & $=$ \\
\hline
\end{tabular}

Sog foctingtes al end of toble. 
Table 20. Existing Generating Units at U.S. Electric Utilities by State, Company, and Plant, as of January 1, 1996 (Continuted)

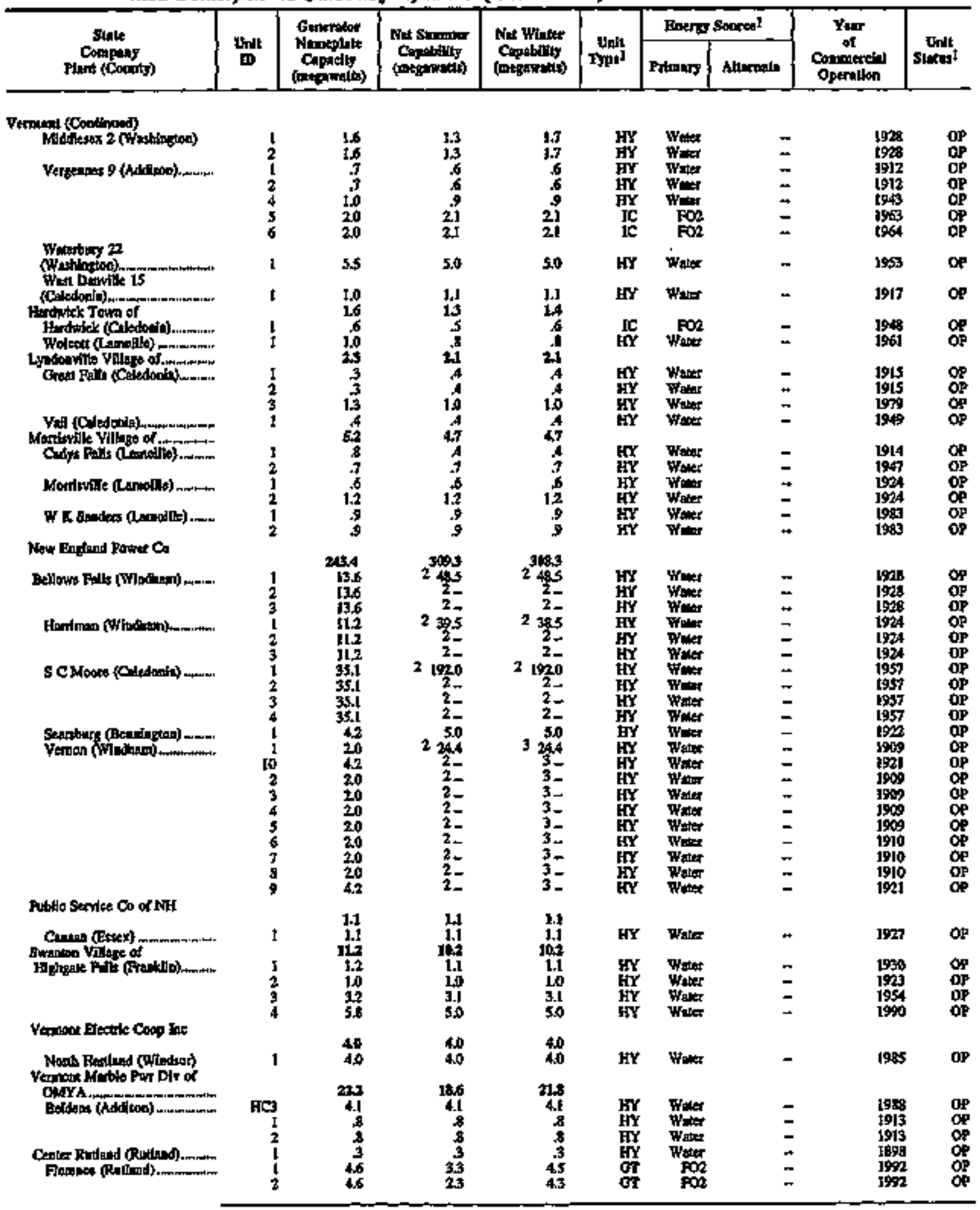

5et foctencel at end of whic. 
Table 20. Exdsting Generating Units at U.S. Electric Utilities by State, Company, and Plant, as of January 1, 1996 (Conthued)

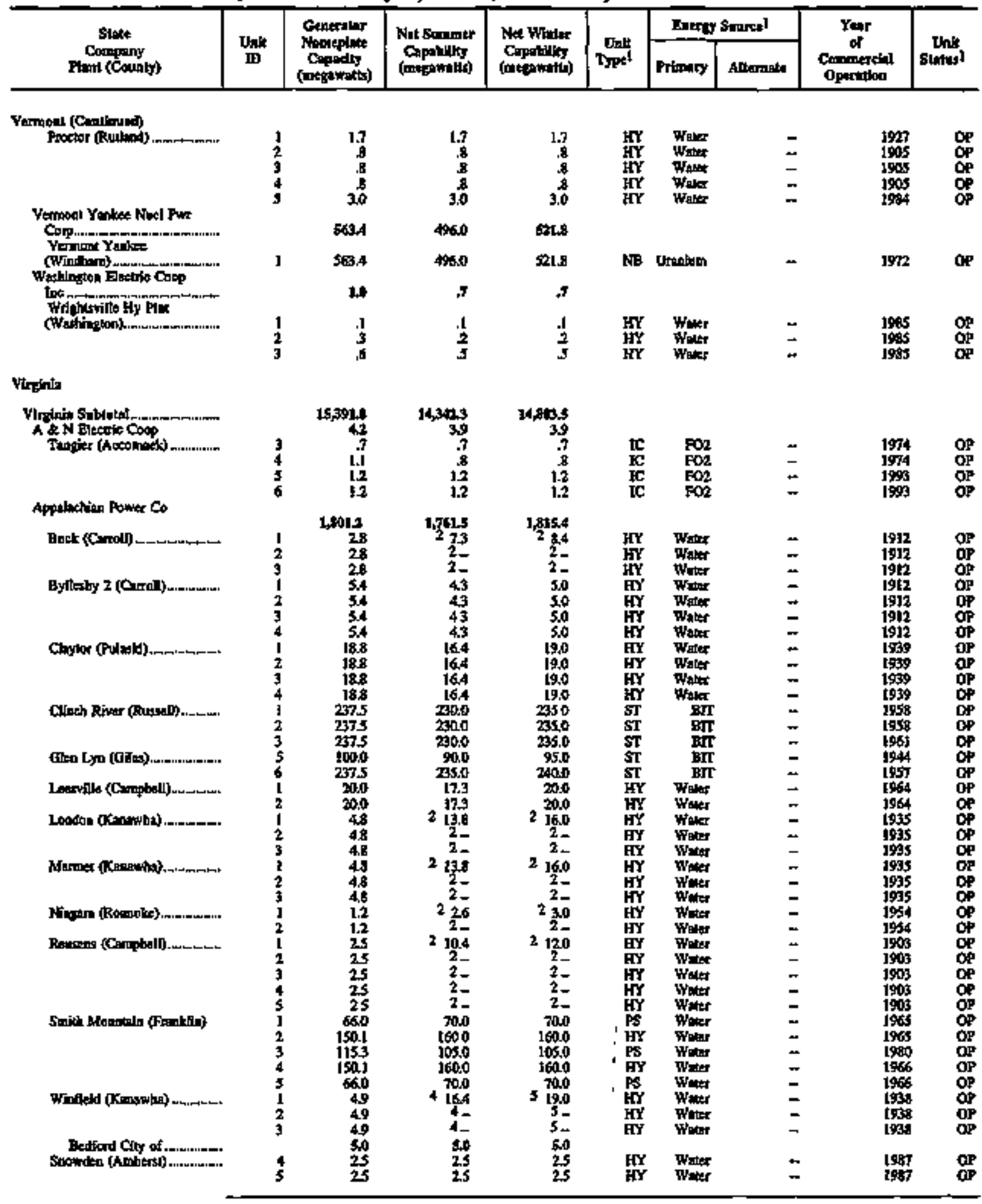

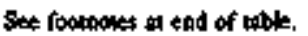


Table 20. Existing Generating Units at U.S. Electric Utilities by State, Company, and Plant, as of January 1, 1996 (Continuted)

\begin{tabular}{|c|c|c|c|c|c|c|c|c|c|}
\hline \multirow{2}{*}{ 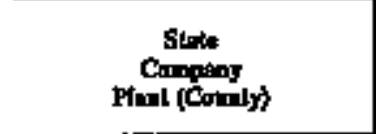 } & \multirow{2}{*}{ Unit } & \multirow{2}{*}{ 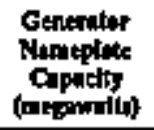 } & \multirow{2}{*}{ 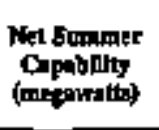 } & \multirow{2}{*}{ 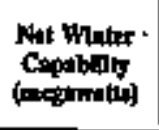 } & \multirow{2}{*}{$\operatorname{limin}_{\text {Typat }}$} & \multicolumn{2}{|c|}{ Enery Sourel } & \multirow{2}{*}{$\begin{array}{c}\text { Year } \\
\text { ot } \\
\text { Conmerded } \\
\text { Operimion }\end{array}$} & \multirow{2}{*}{$\begin{array}{l}\text { Undt } \\
\text { Stetest }\end{array}$} \\
\hline & & & & & & PAmary & Altemente & & \\
\hline \multicolumn{10}{|l|}{ Virinin \{Continod) } \\
\hline Cotig-golebont Elatic & & a. & 0,3 & $0 \mathbf{3}$ & & & & & \\
\hline $\begin{array}{l}\text { Alesdon Creek (Cribi) nmm- } \\
\text { Culpeper Towa of }\end{array}$ & $\mathbf{J}$ & 7.3 & 6 & $\frac{3}{65}$ & ผY & Whator & $=$ & 1938 & OP \\
\hline $\begin{array}{l}\text { Wet Sping Sireel } \\
\text { (Olpepex) }\end{array}$ & IT & (8) & $\boldsymbol{7}$ & 8 & GT & $\mathrm{FO2}$ & - & 1974 & op \\
\hline & 24 & 20 & 20 & 20 & IC & $\mathrm{Fol}_{202}$ & $=$ & 1969 & oP \\
\hline & $2 \pi$ & 15 & $i 2$ & t. 3 & $\mathbf{G r}$ & $\mathrm{N} \times \mathrm{DAO}_{\mathrm{H}}$ & $F O \overline{2}$ & $\begin{array}{l}1914 \\
1962\end{array}$ & of \\
\hline & 5 & 4 & 8 & 9 & 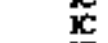 & Not 0 & Fon & t959 & OP \\
\hline & & 9 & .7 & 8 & $\mathbf{K C}$ & Nen Gas & F02 & E97 & op \\
\hline Dawille Cry of & & 113 & 10.5 & 10.5 & & & & & \\
\hline 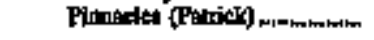 & I & 38 & 35 & 35 & $\mathbf{H Y}$ & Wuts & $*$ & 19383 & op \\
\hline & 2 & 3,8 & 35 & 33 & FY & Wirar & $\overline{-}$ & 1938 & OP \\
\hline \multicolumn{10}{|l|}{ 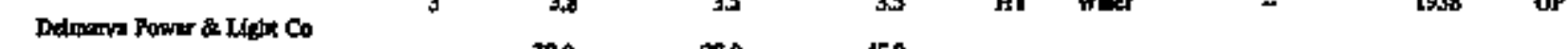 } \\
\hline 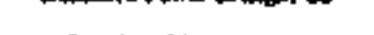 & & 390 & 380 & 450 & & & & & \\
\hline 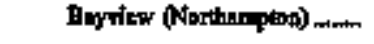 & 1 & 2.4 & 20 & 20 & c c & Fon & ـ & 5963 & OP \\
\hline & 2 & 20 & 20 & 20 & ç & FO2 & $\approx$ & 1963 & op \\
\hline & 4 & 2.0 & 20 & 20 & c & $\mathrm{FO2}$ & $\bar{z}$ & 1963 & OP \\
\hline & 3 & 20 & 20 & 20 & $\mathbf{k}$ & $\mathrm{FO} 2$ & 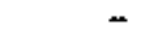 & 1963 & $\mathrm{OP}$ \\
\hline & 6 & 20 & 20 & 20 & C: & $\mathrm{BO}$ & $*$ & 1963 & op \\
\hline \multirow{3}{*}{ 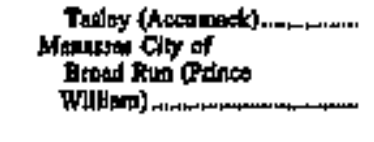 } & 20 & $\frac{27.0}{3 \pm 0}$ & $\begin{array}{l}260 \\
3 \neq 3\end{array}$ & $\begin{array}{l}3300 \\
3300\end{array}$ & $\boldsymbol{\sigma} \mathbf{T}$ & FO2 & - & 1972 & DP \\
\hline & H1 & $\mathrm{B}_{5}$ & & & & Weer & - & 1958 & op \\
\hline & H2 & $E$ & 8.9 & 8 & HY & Wher & - & 1967 & $\mathrm{OP}$ \\
\hline \multirow{5}{*}{$\begin{array}{l}\text { Oruch Strex Plonl } \\
\text { (Fince Wiltem) }\end{array}$} & & & & & & & & & \\
\hline & 点 & $\begin{array}{l}1.0 \\
1.0\end{array}$ & $\begin{array}{l}1.0 \\
1.0\end{array}$ & 100 & 犖 & FOR & $\ddot{z}$ & 1979 & $\stackrel{O P}{O P}$ \\
\hline & c3 & 1.0 & 3.0 & 10 & $\mathbf{c}$ & poi & - & 1979 & op \\
\hline & C4 & $\begin{array}{l}1.0 \\
17\end{array}$ & 1.0 & 1.0 & $\mathbf{c}$ & $\mathbf{B O 2}$ & $\vec{E}$ & 1979 & of \\
\hline & $\stackrel{\mathrm{C}}{\mathrm{C}}$ & 1.7 & 1.6 & 18 & tc & $\mathrm{Non}$ & $\overline{-}$ & 190 & of \\
\hline \multirow{4}{*}{$\begin{array}{l}\text { Gastria Ditre Plas } \\
\text { (Princs Willmin) }\end{array}$} & & & & & & & & & \\
\hline & ch & 1.7 & 1.6 & 1.06 & k & Fon & $=$ & 190 & op \\
\hline & $\mathbf{c s}$ & 1.7 & 3.6 & 1.6 & א & Fó & - & $19 \%$ & op \\
\hline & C & 1.7 & 1.6 & 1.6 & $\mathbf{K}$ & $\mathbf{F o}$ & 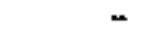 & 1992 & op \\
\hline \multirow{5}{*}{ 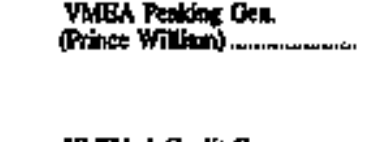 } & & & & & & & & & \\
\hline & vin & 1.7 & 1.6 & 1.6 & $\mathbf{K}$ & $\mathrm{FO2}$ & - & 1992 & $Q p$ \\
\hline & YL1 & 1.7 & 1.6 & 1.4 & 䆓 & Fon & - & 1993 & of \\
\hline & $v_{12}$ & 1.7 & 1.6 & 16 & 点 & $\mathrm{mog}^{2}$ & $\ddot{z}$ & 1903 & OP \\
\hline & & & & & & & & & \\
\hline \multirow{7}{*}{ 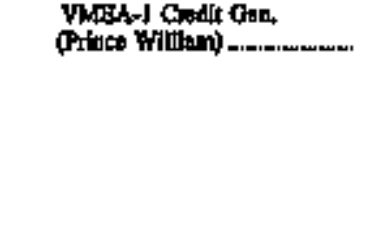 } & vio & 1.7 & 1.6 & 16 & 阷 & $\mathbf{F o z}$ & - & 1990 & 암 \\
\hline & v3 & 1.7 & 1.6 & 1.6 & 空 & 502 & - & 1990 & op \\
\hline & $\begin{array}{l}\text { vat } \\
\text { vis }\end{array}$ & 1.7 & 1.6 & 16 & c & Fon & $\approx$ & 1990 & of \\
\hline & $v_{6}$ & 1.7 & 3.6 & 1.0 & ç & & $=$ & 1990 & $O P$ \\
\hline & $\eta$ & 1.7 & 1.6 & 1.6 & c & $\mathrm{FO2}$ & - & 1990 & OP \\
\hline & ve & 1.7 & 18 & 1.6 & F & Po2 & - & Jor & op \\
\hline & vo & 1.7 & 1.6 & 0.6 & EC & Poi & + & $19 \%$ & $\sigma$ \\
\hline \multirow{3}{*}{ 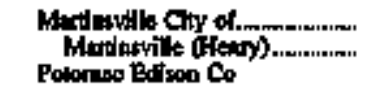 } & & 15 & 1. & 13 & & & & & \\
\hline & 1 & 5.3 & 1 & 1. & EY & Winer & - & 1924 & OP \\
\hline & & 46 & 46 & 46 & & & & & \\
\hline Inoy (Puge) " & 1 & .6 & 21.6 & 218 & $\mathbf{H Y}$ & Wor & - & 1927 & op \\
\hline & 3 & $\frac{4}{6}$ & ${ }_{2}^{2}-$ & $2_{2}^{2+}$ & $\begin{array}{l}\mathbf{H Y} \\
\mathbf{H Y}\end{array}$ & $\begin{array}{l}\text { Wolter } \\
\text { Witer }\end{array}$ & 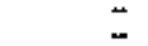 & $\begin{array}{l}1927 \\
1927\end{array}$ & op \\
\hline Netwpon (Pte) & j & .4 & 21.4 & 214 & & & $=$ & 1945 & or \\
\hline & $i$ & 4 & $2-$ & $2=$ & HY & Whler & - & 1923 & $O P$ \\
\hline & 3 & 6 & ${ }^{2}=$ & ${ }^{2}=$ & HY & Wur & . & 1925 & OP \\
\hline 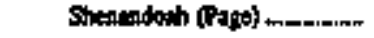 & 1 & .3 & 2,8 & 2.8 & FY' & Her & - & 1925 & or \\
\hline & $\frac{2}{3}$ & .3 & ${ }_{2}^{2}-$ & $\begin{array}{l}2- \\
2-\end{array}$ & $\begin{array}{l}\mathbf{H Y} \\
\mathbf{H Y}\end{array}$ & 'Wurer & $\rightleftarrows$ & 1925 & OP \\
\hline & 3 & آ. & & & KY & Water & $=$ & 100 & OP \\
\hline
\end{tabular}

Ser footingted al end of tobk. 
Table 20. Existing Generating Units at US. Electric Uttlities by State, Company, and Plant, as of January 1, 1996 (Continued)

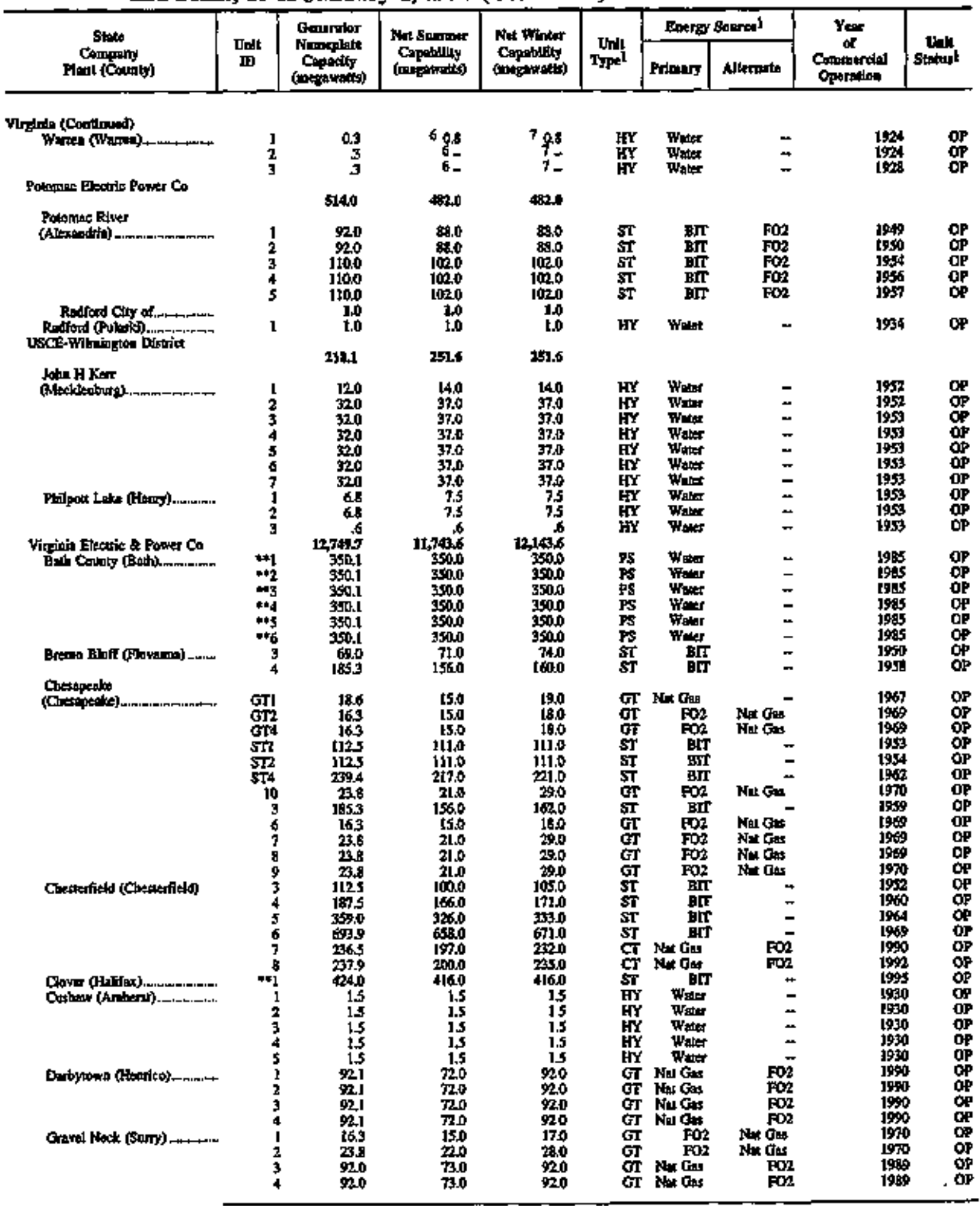

Sectoonobs at end af able. 
Table 20. Existing Generating Units at U.S. Electric Utalities by State, Company, and Plant, as of January 1, 1996 (Continued)

\begin{tabular}{|c|c|c|c|c|c|c|c|c|c|}
\hline \multirow{2}{*}{$\begin{array}{c}\text { Stank } \\
\text { Conpping } \\
\text { Plant (County) }\end{array}$} & \multirow{2}{*}{$\begin{array}{l}\text { Dndt } \\
\text { ID }\end{array}$} & \multirow{2}{*}{ 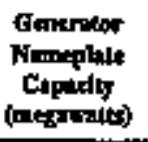 } & \multirow{2}{*}{ 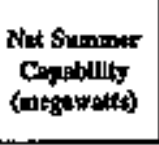 } & \multirow{2}{*}{ 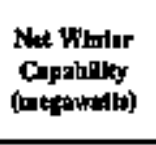 } & \multirow{2}{*}{$\begin{array}{l}\text { Init } \\
\text { Typel }\end{array}$} & \multicolumn{2}{|c|}{ Bnerip Soureel } & \multirow{2}{*}{$\begin{array}{c}\text { Year } \\
\text { of } \\
\text { Cominarcel } \\
\text { Operailen }\end{array}$} & \multirow{2}{*}{$\begin{array}{c}\text { thak } \\
\text { Stalus } 1\end{array}$} \\
\hline & & & & & & Primary & Alitentse & & \\
\hline \multicolumn{10}{|l|}{ 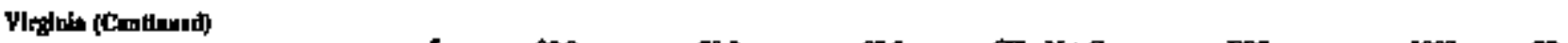 } \\
\hline & 5 & 920 & 73.0 & 92.0 & GT & Net Ges & F02 & 1989 & OP \\
\hline & 6 & 920 & 730 & 920 & GT & Net Gax & F02 & 1989 & $\mathbf{O P}$ \\
\hline \multirow[t]{4}{*}{ Low Moor (Aloghany) } & GII & 20.3 & I5.0 & 18,0 & GI & FO2 & $=$ & 1971 & OP \\
\hline & GIR & 20.7 & IS.0 & 180 & GT & For & - & 1971 & of \\
\hline & $\mathbf{G I J}$ & 207 & 15,4 & 180 & GT & $\mathbf{F O} 2$ & - & 1971 & op \\
\hline & ort & 20.7 & IS.0 & 18.0 & Gr & $\mathrm{PO} 2$ & - & 1971 & OP \\
\hline \multirow{6}{*}{ 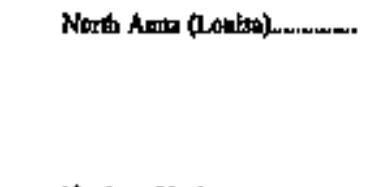 } & FCd & 10 & 10 & 1.0 & $\mathbf{H Y}$ & What & . & 1981 & OP \\
\hline & sPI & $\bullet$ & $\bullet$ & 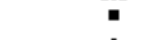 & PV & Son & - & 1968 & QP \\
\hline & $\mathbf{s P 2}$ & 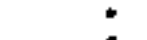 & 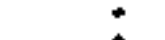 & $\because$ & PV & Son & * & 1985 & OP \\
\hline & $\mathrm{SP3}$ & - & • & - & PV & San & + & L\$\$85 & QP \\
\hline & •1 & 9797 & 853.0 & 591,0 & NP & Jrandum & - & 1978 & $O P$ \\
\hline & -2 & 979.7 & $\$ 97.0$ & $\$ 970$ & NP & Unativan & - & $19 \%$ & OP \\
\hline \multirow{5}{*}{$\begin{array}{l}\text { Nortbern Keck } \\
\text { (Ridtumowd) }\end{array}$} & & & & & & & & & \\
\hline & GI & 20.7 & 16.0 & 19.0 & Gr & Fo? & - & 19 & $\operatorname{og}$ \\
\hline & OT2 & 203 & 16.0 & 19.0 & or & F02 & 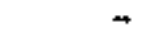 & $19 n 1$ & op \\
\hline & GI3 & 207 & 16,4 & 19.0 & GI & F02 & - & 197 & $\mathrm{OP}$ \\
\hline & GT4 & 30.7 & (6,4,4 & 19.0 & GT & $-\mathrm{FO} 2$ & - & 197 & OP \\
\hline \multicolumn{10}{|l|}{ Powm Point (Pincs } \\
\hline \multirow{11}{*}{ Whallo) } & GTI & 16,0 & 130 & 16.5 & G. & $\mathrm{FOZ}$ & $=$ & 190 & $O P$ \\
\hline & GI2 & 160 & 33.0 & 16.0 & GT & FO2 & - & 196 & $O P$ \\
\hline & GI3 & 16.0 & 13,0 & 16.0 & GI & F02 & - & 1900 & op \\
\hline & GT4 & 16.0 & 13.0 & 16.0 & GT & $\mathrm{FO2}$ & - & 196 & OP \\
\hline & GTS & 16.0 & 190 & 160 & GT & Fo2 & - & 1968 & op \\
\hline & GT6 & 16.0 & 19.0 & 16.0 & GT & $\mathrm{Foz}$ & - & 196 & op \\
\hline & 1 & 690 & 34.0 & 740 & $\mathbf{S T}$ & Fos & $*$ & 1948 & OP \\
\hline & 1 & 69.0 & 69.0 & 71.0 & $\mathbf{S T}$ & Fos & - & 1551 & op \\
\hline & 3 & 113.6 & 101.0 & $\cos 0$ & $S T$ & BतT & - & [955: & OP \\
\hline & 4 & 209.4 & 2210 & 2200 & ST & EIT & - & 1962 & OP \\
\hline & $\mathbf{s}$ & 8920 & 786.0 & $\mathbf{z 0 1 . 0}$ & $\mathbf{s t}$ & Fod & - & 1975 & OP \\
\hline Surty (Surcy)...-. & $\mathbf{1}$ & 897.5 & 201.0 & $\$ 0 \mathrm{~L} .0$ & NP & Un:alom & - & 1972 & OP \\
\hline \multirow{4}{*}{ 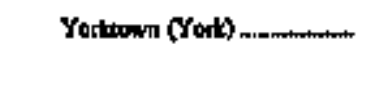 } & 2 & 897.5 & 801,0 & sollo & $\mathrm{MP}$ & Uramong & - & 197 & QP \\
\hline & $\mathbf{]}$ & 187.5 & 159.0 & 1610 & $s t$ & BIT & - & 1957 & OP \\
\hline & 2 & 187.5 & 161,0 & 1720 & $\mathbf{S T}$ & HIT & - & 1959 & OP \\
\hline & 3 & $\$ 20$ & 8180 & 920.0 & $s T$ & BET & - & 1974 & CP \\
\hline
\end{tabular}

Set foolbolea al end of labk 
Table 20. Existing Generating Uhits at U.S. Electric Utillties by State, Company, and Pkant, as of January 1, 1996 (Continued)

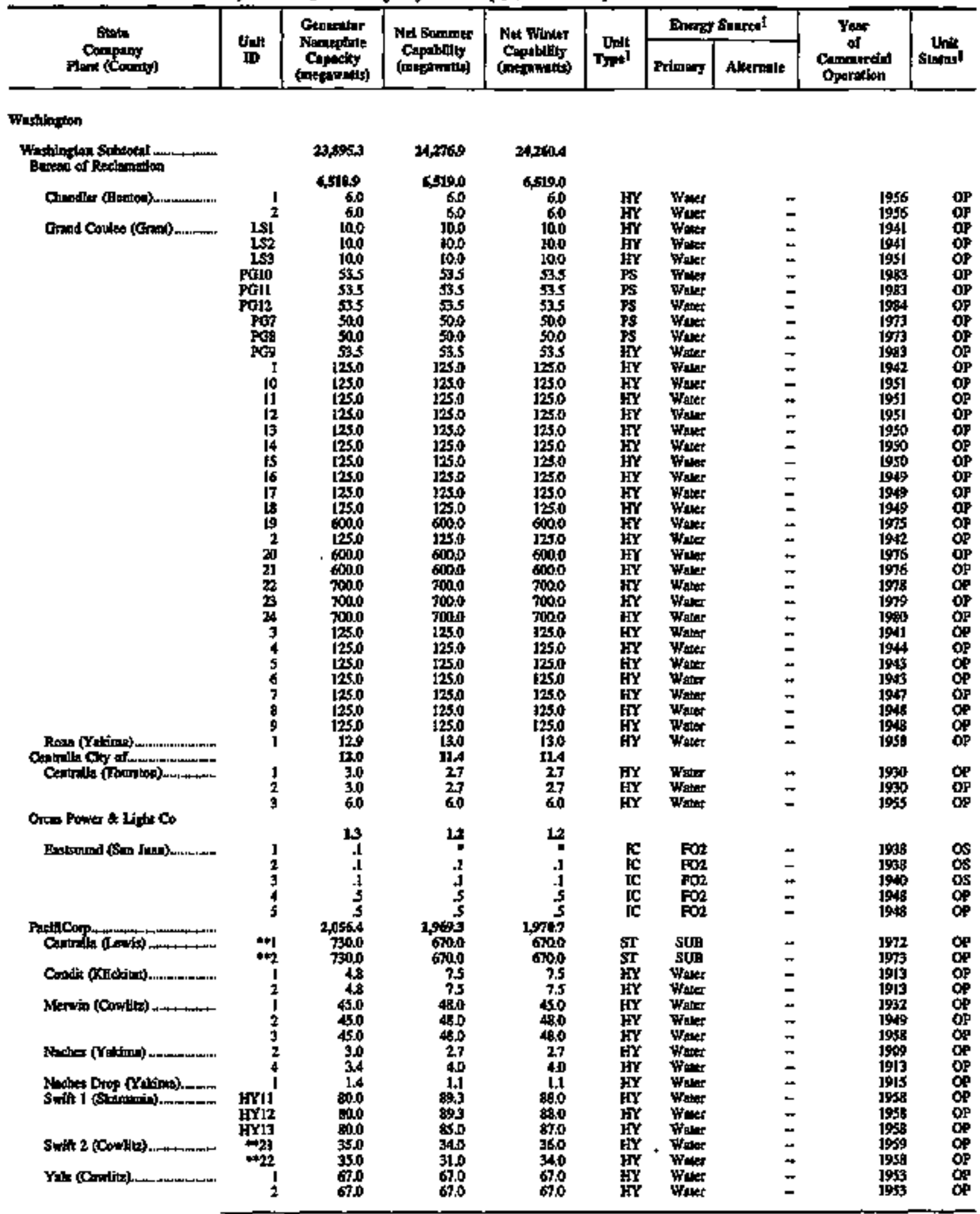

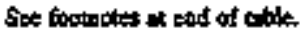


Table 20. Existing Generating Units at U.S. Electric Utilities by State, Company, and Plant, as of January 1, 1996 (Continued)

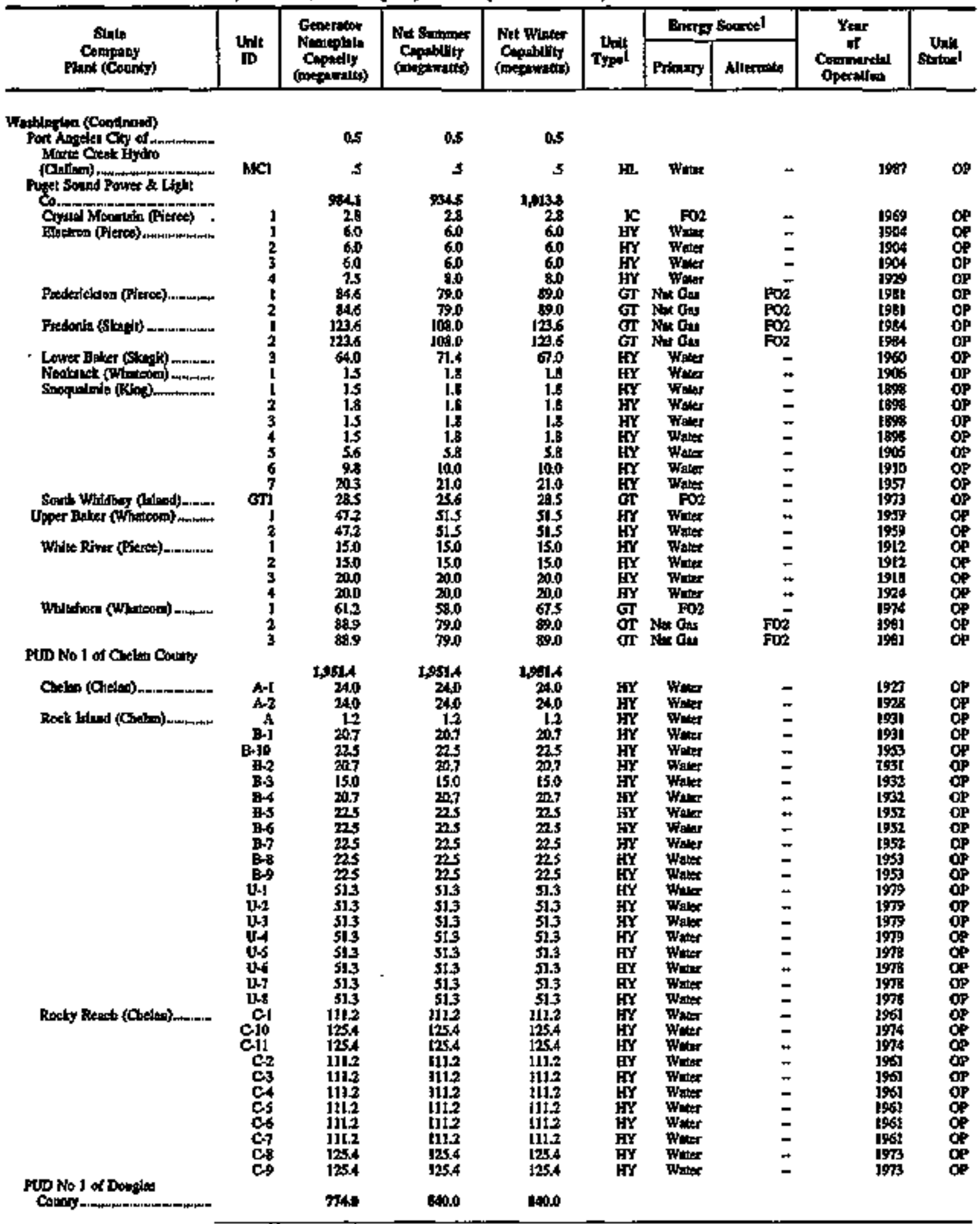

Sue foctotes al ead of tible. 
Table 20. Existing Generating Units at U.S. Electric Utilities by State, Company, and Plant, as of January 1, 1996 (Continued)

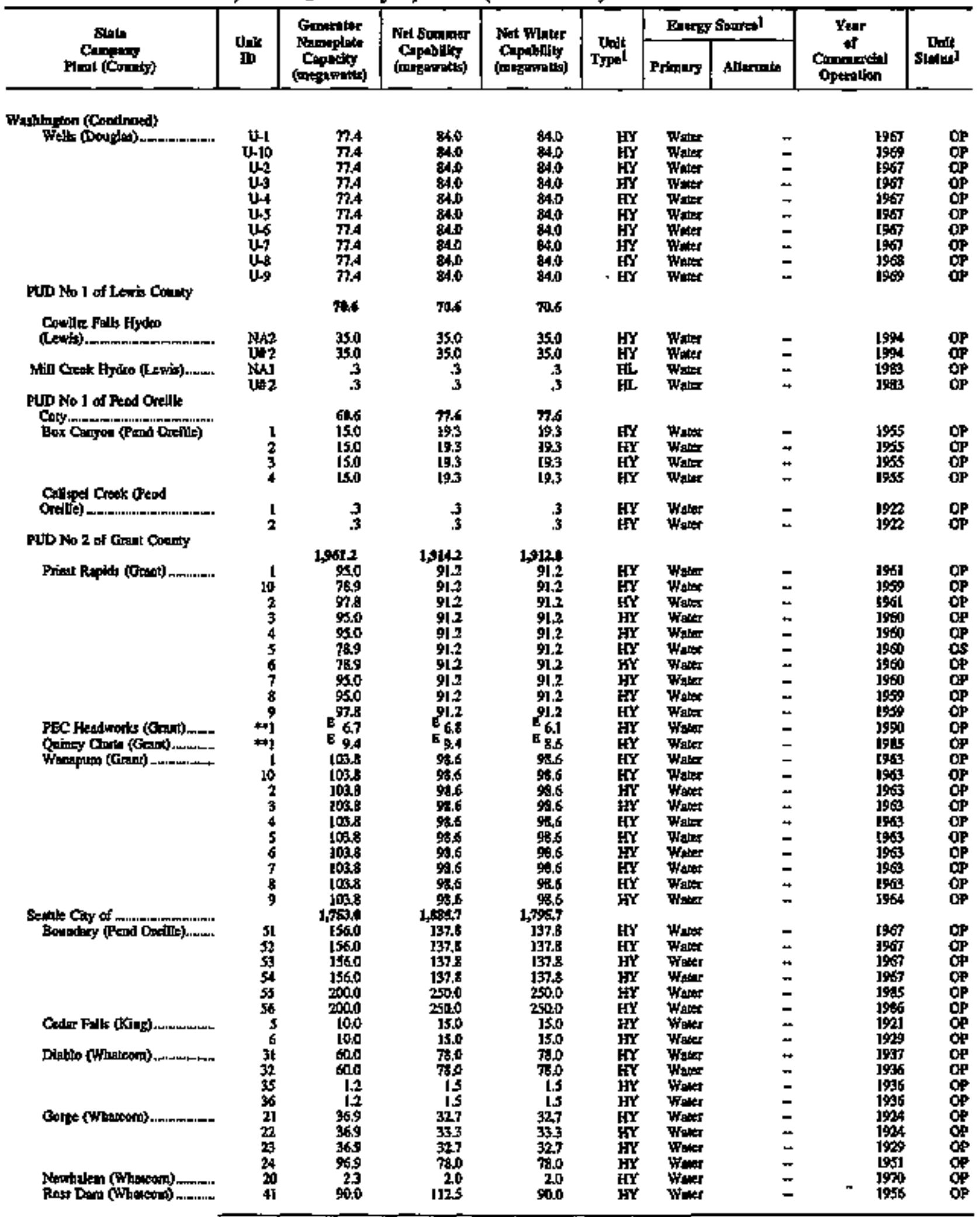

Sect foothots at and of Iable 
Table 20. Exdsting Generating Units at U.S. Electric Utilities by State, Company, and Plant, as of Jamuary 1, 1996 (Continned)

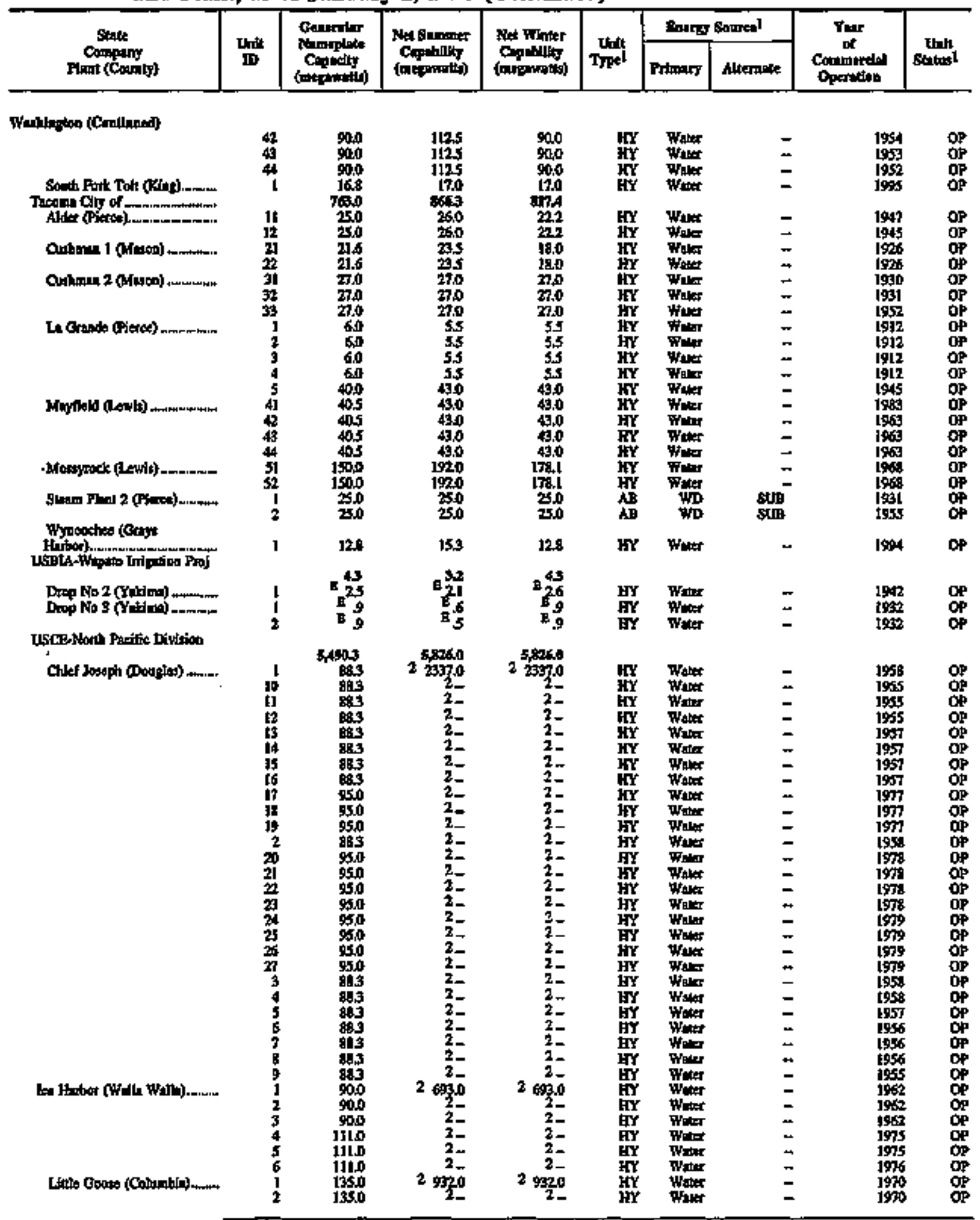

Sec boenots it end of thibl. 
Table 20. Existing Generating Units at U.S. Electric Utilities by State, Company, and Plant, as of January 1, 1996 (Continued)

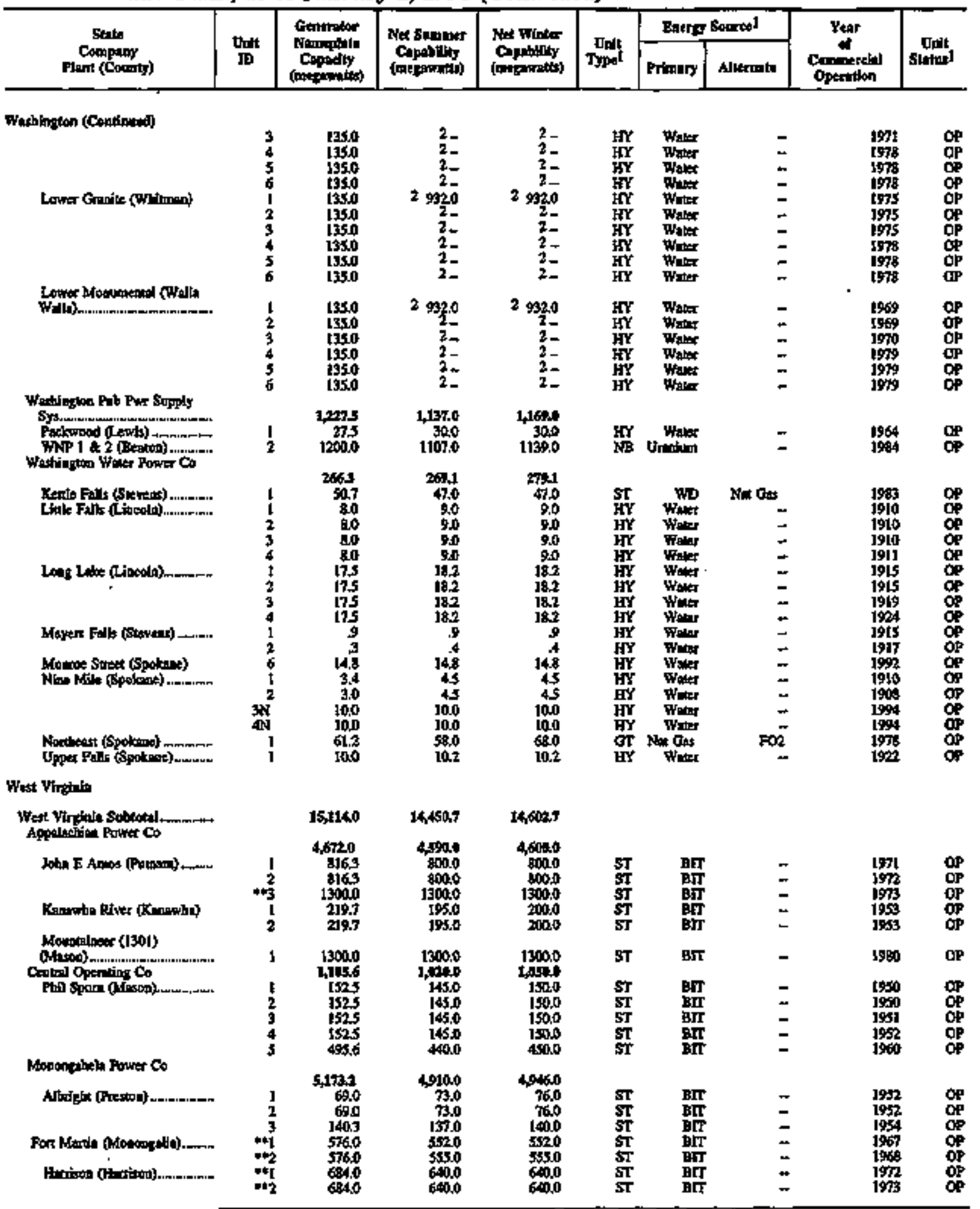

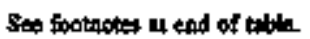


Table 20. Existing Generating Units at U.S. Electric Utilities by State, Company, and Plant, as of January 1, 1996 (Continued)

\begin{tabular}{|c|c|c|c|c|c|c|c|c|c|}
\hline \multirow{2}{*}{$\underset{\text { Conits }}{\text { Pont (domity) }}$} & \multirow{2}{*}{$\underset{\text { ID }}{\text { Unt }}$} & \multirow{2}{*}{ 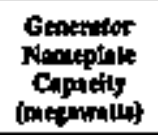 } & \multirow{2}{*}{ 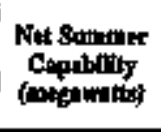 } & \multirow{2}{*}{ 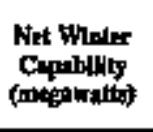 } & \multirow{2}{*}{ Uopel } & \multicolumn{2}{|c|}{ Bnery Sontcel } & \multirow{2}{*}{$\begin{array}{c}\text { Year } \\
\text { of } \\
\text { Commental } \\
\text { Optnilon }\end{array}$} & \multirow{2}{*}{ thin: } \\
\hline & & & & & & Primary & Alturtest: & & \\
\hline \multicolumn{10}{|l|}{ 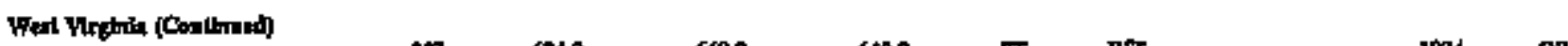 } \\
\hline & $\bullet$ & 6940 & 6400 & 640.0 & $5 T$ & EfT & م & 1974 & OP \\
\hline מ................ & $* * 1$ & 6840 & 6140 & 621.0 & si & 焉 & - & 1979 & op \\
\hline Ruswlak (Obrion) & 5 & $\begin{array}{r}68.0 \\
\$ 5.0\end{array}$ & $\begin{array}{r}6140 \\
\mathbf{4 6 0}\end{array}$ & $\begin{array}{r}61,0 \\
\$ 8.0\end{array}$ & $\begin{array}{l}\mathbf{S T} \\
\mathbf{S T}\end{array}$ & $\begin{array}{l}\text { EHT } \\
\text { BTT }\end{array}$ & $\ddot{-}$ & $\begin{array}{l}1980 \\
1943\end{array}$ & $\underset{\mathrm{OP}}{\mathrm{OP}}$ \\
\hline (1) & 6 & 348 & 91.0 & 94.0 & sT & BI & - & 1951 & $O P$ \\
\hline 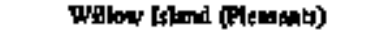 & 1 & 500 & $\begin{aligned} 540 \\
t=10\end{aligned}$ & $\mathbf{5 s 0}$ & ST & BTt & $\bar{z}$ & 1949 & $\partial P$ \\
\hline Oho Postor Ca & & $2,1,451$ & 2,20100 & $2+21060$ & $\mathbf{S T}$ & BII & - & 1950 & OP \\
\hline Kunner (Wwashob)............. & 1 & 2375 & 2000 & 2100 & ST & BI & - & 1958 & OP \\
\hline & 2 & 2375 & 2000 & 3100 & $\mathbf{S T}$ & BAT & $\overrightarrow{+}$ & 198 & $\mathrm{OP}_{\mathrm{OP}}$ \\
\hline Mikhell (Morstall) & $\hat{1}$ & 8163 & 8000 & $\operatorname{sen} 0$ & SI & BIT & $\underline{-}$ & 1971 & OP \\
\hline$x^{2}$ & 2 & 8163 & 8000 & 8000 & $s T$ & BAT & . & $19 i$ & op \\
\hline \multicolumn{10}{|l|}{ Potomen pdinot Cs } \\
\hline Das 4 (Jefforend) & 1 & 5 & 25 & 25 & КY & Water & $\leftarrow$ & $190 \%$ & op \\
\hline & 2 & $\stackrel{5}{9}$ & $\begin{array}{l}2- \\
2-\end{array}$ & $2=$ & $\underset{\mathbf{H Y}}{\mathbf{Y Y}}$ & $\begin{array}{l}\text { Wala } \\
\text { Waser }\end{array}$ & $\bar{z}$ & 1909 & $\stackrel{p P}{O P}$ \\
\hline Dan 5 (Bextuty) & 1 & 6 & 21,0 & 21,0 & KY & Wifter & $=$ & 1919 & op \\
\hline (2) & 2 & 6 & $2-$ & $2=$ & $\underline{\underline{Y}}$ & Water & - & igts & op \\
\hline 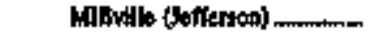 & 1 & 8 & 228 & 228 & YY & Wuth & - & 1913 & OP \\
\hline & 2 & 1.0 & ${ }^{2}-$ & $2_{-}^{2+}$ & $\frac{K Y}{f \mathbf{Y}}$ & Whatior & $\ddot{z}$ & 1939 & $\stackrel{o p}{O P}$ \\
\hline Yirgials Eetric \& Potror Co & & 1,76h1 & $1,6+x$, & $1,7+9$. & & Wares & $=$ & 19916 & OP \\
\hline 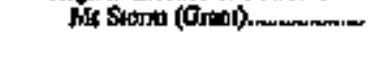 & Fi: & 596.2 & $\begin{array}{r}120 \\
5330\end{array}$ & 5450 & $\underset{\mathbf{S T}}{\mathbf{g r}}$ & let Fonfl & $\bar{z}$ & $\begin{array}{l}1967 \\
1965\end{array}$ & $\stackrel{o p}{o p}$ \\
\hline & 2 & 570.2 & 5310 & 545.0 & ST & BII & $=$ & 1965 & op \\
\hline & 3 & 520 & $\$ 21,0$ & 5360 & $s T$ & BTT & $=$ & 1973 & op \\
\hline \multirow{2}{*}{ 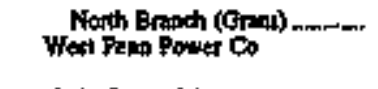 } & i & 800 & 740 & $\pi .0$ & $A$ & $W C$ & BपT & 1992 & $O P$ \\
\hline & & s1.2 & $\mathbf{2 0}$ & Bat & & & & & \\
\hline \multirow{4}{*}{ 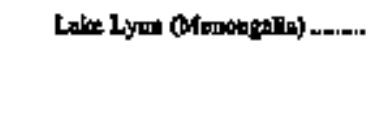 } & $\mathbf{I}$ & 128 & 130 & 13.0 & BY & Wuts & - & 1926 & op \\
\hline & 2 & I2.3 & IRT & E.t & द्रY & Wrar & $\omega$ & 1906 & DP \\
\hline & 3 & 128 & 130 & 13,0 & GY & Wabe & $=$ & 1923 & op \\
\hline & 4 & 12.8 & 13.0 & 13.0 & HY & Wave & - & 1926 & op \\
\hline \multicolumn{10}{|l|}{ Wheonds } \\
\hline \multirow{7}{*}{ 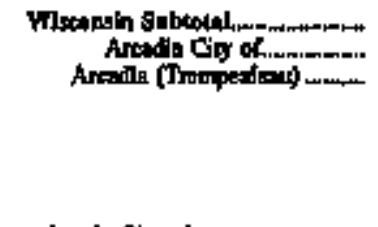 } & & 11,8086 & 11,035 & $12,126,7$ & & & & & \\
\hline & & $\mathbf{9 1}$ & & s.t & & & & & \\
\hline & t & 14 & & & IC & $\mathrm{FO}$ & - & 1956 & ap \\
\hline & 2 & 10 & 20 & 1.0 & IC & $\begin{array}{l}\mathrm{F02} \\
\mathrm{FOn}\end{array}$ & $\overrightarrow{+}$ & 1948 & OP \\
\hline & 4 & .2 & .2 & 2 & $x$ & Fon & $=$ & 1930 & op \\
\hline & 5 & 3.1 & 30 & 3.0 & c & Non Gar & $\mathrm{FO} 2$ & 1971 & op \\
\hline & 6 & 70 & 3,2 & 32 & k & Nut Gu & 502 & 1986 & OP \\
\hline \multirow{3}{*}{ 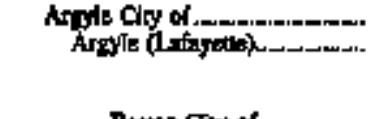 } & t & 23 & 24 & 24 & & & & & \\
\hline & 3 & 1.] & L2 & 1.2 & $\underset{\mathrm{K}}{\mathrm{H}}$ & wotr & 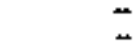 & 1929 & os \\
\hline & 4 & 1.1 & 1,2 & $\frac{1,2}{11}$ & $\mathrm{x}$ & $\mathbf{F O 2}$ & 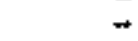 & 1989 & OP \\
\hline Butan Chy of & & 42 & 4,2 & 4,7 & & & & & \\
\hline 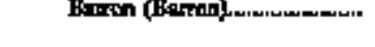 & $\frac{\mathrm{H} 2}{3}$ & 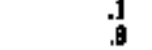 & .8 & 8 & 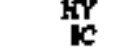 & Fon & 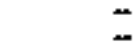 & 1923 & $\stackrel{\text { op }}{\text { op }}$ \\
\hline & i & 13 & 1,3 & 13 & $\ddot{\mathbf{c}}$ & FO2 & $\rightarrow$ & IS54 & OP \\
\hline & $\mathbf{9}$ & 20 & 20 & 20 & IC & $\mathrm{FOP}$ & - & 1960 & $\mathbf{D P}$ \\
\hline 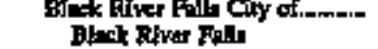 & & & & 40 & & & & & \\
\hline 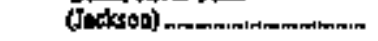 & & 5 & 6 & .6 & hY & Waler & - & [94] & \\
\hline & KY2 & 3 & 3 & 3 & HY & Waler & - & I5]9 & OP \\
\hline & I & 3 & 3 & 3 & tc & For & - & 1945 & $\mathrm{SB}$ \\
\hline & 3 & $\frac{3}{9}$ & $\overrightarrow{9}$ & 9 & c & Fon & 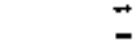 & $\begin{array}{l}\text { 1941 } \\
\text { l9499 }\end{array}$ & S: \\
\hline & 4 & 1.4 & t.4 & 1.4 & $\mathbf{x}$ & Fo2 & $=$ & I955 & $\mathbf{S B}$ \\
\hline Cadkon YIlot & & 19 & $\mathbf{1 2 7}$ & $\mathbf{1 . 8}$ & & & & & \\
\hline Cublon (Mforoo)......... & 3 & $\mathbf{3}$ & 3 & 3 & $\mathbf{K}$ & $\mathrm{FO} 2$ & + & 1932 & oP \\
\hline+ & 4 & 5 & $\therefore$ & 4 & le & $\mathrm{FO}$ & & 1962 & 5 \\
\hline 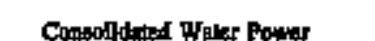 & 5 & I.I & 100 & 1.2 & IC & EO2 & Net Gats & 1969 & OP \\
\hline (Co & & $2 \mathbf{L A}$ & $\mathbf{2 H . 1}$ & 21.1 & & & & & \\
\hline
\end{tabular}

Sen toomotes of end of trble. 
Table 20. Existing Generating Units at U.S. Electric Utilities by State, Company, and Flant, as of January 1, 1996 (Continued)

\begin{tabular}{|c|c|c|c|c|c|c|c|c|c|}
\hline \multirow{2}{*}{ 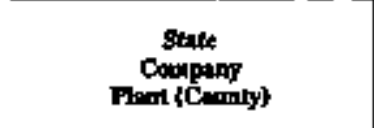 } & \multirow{2}{*}{ Un } & \multirow{2}{*}{ 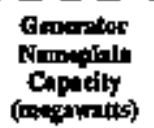 } & \multirow{2}{*}{ 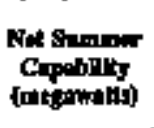 } & \multirow{2}{*}{ 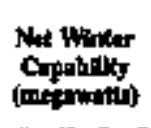 } & \multirow{2}{*}{ Thut } & \multicolumn{2}{|c|}{ Ewerisy Soundel } & \multirow{2}{*}{ 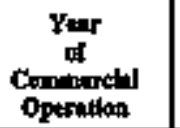 } & \multirow{2}{*}{ Dinit } \\
\hline & & & & & & Phtancy & Allemanto & & \\
\hline \multicolumn{10}{|l|}{ Wheoption (Copliqued) } \\
\hline Biron (Nood) & $\frac{1}{2}$ & 15 & $\mathbf{2 3}$ & $\begin{array}{l}1.3 \\
1.3\end{array}$ & $\begin{array}{l}\text { sy } \\
\text { HYY }\end{array}$ & $\begin{array}{l}\text { Water } \\
\text { Waler }\end{array}$ & $\overline{-}$ & 5916 & $\stackrel{\circ P}{O P}$ \\
\hline & 3 & & 4 & 4 & $\mathbf{H Y}$ & Whater & + & 192] & op \\
\hline Du Bay (Pantoges) & $\mathbf{z}$ & $\begin{array}{l}1,2 \\
2.0\end{array}$ & $\mathbf{2 2}$ & 1.2 & $\underset{\mathbf{H Y}}{\mathbf{H Y}}$ & Wow & $=$ & $\sin _{19012}$ & op \\
\hline & 4 & 20 & 20 & $\frac{2.0}{20}$ & $\underset{\mathbf{H Y}}{\mathbf{H Y}}$ & Wala & $\overline{-}$ & $\begin{array}{l}19+2 \\
19+2\end{array}$ & $\stackrel{p}{o p}$ \\
\hline Steved Polne (Pondes) & i & 8 & 8 &. & in & Prover & - & 1918 & op \\
\hline & 3 & $\begin{array}{l}\not{8} \\
8 \\
8\end{array}$ & 8 & .8 & 敵 & Whor & $\bar{z}$ & $\begin{array}{l}1915 \\
1918\end{array}$ & of \\
\hline & 4 & 8 & , & 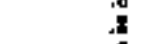 & fir & Wakr & 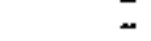 & 1918 & op \\
\hline & 8 & $\underset{8}{8}$ & 8 & 8 & $\underset{\mathbf{f r}}{\mathbf{f r}}$ & $\begin{array}{l}\text { Waler } \\
\text { Fist }\end{array}$ & 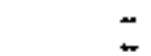 & 1918 & of \\
\hline Whesestix Reputsts (Wood) & 1 & 23 & 23 & 2.3 & HY & What & - & 1900 & op \\
\hline \multirow{7}{*}{ 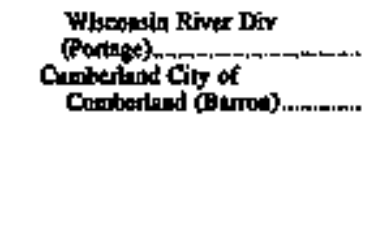 } & & & $\mathbf{2 , 3}$ & 2.3 & $\mathbf{H} \mathbf{Y}$ & Water & - & 1920 & OP \\
\hline & $\mathbf{t}$ & 15 & 1.5 & 1.5 & НY & Waks & + & 1955 & OP \\
\hline & & 112 & Its & its & & & & & \\
\hline & 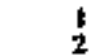 & +7 & $+\frac{7}{2}$ & 3 & $\underset{c c}{x}$ & $\begin{array}{ll}\mathrm{FO}_{2} \\
\mathrm{FO2}\end{array}$ & $\ddot{z}$ & 1945 & $\stackrel{O P}{O P}$ \\
\hline & 3 & 3 & 2 & .2 & ce & $\mathrm{FO2}$ & - & 1939 & $o p$ \\
\hline & 4 & 14 & 1.4 & i.4 & je & & $=$ & 1094 & $\alpha$ \\
\hline & $s$ & 21 & 20 & 20 & k & $N=0$ & $\mathbf{F O 2}$ & 1966 & or \\
\hline \multirow{2}{*}{\multicolumn{10}{|c|}{ 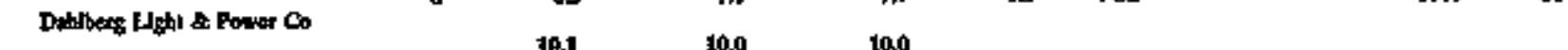 }} \\
\hline & & & 10.0 & & & & & & \\
\hline 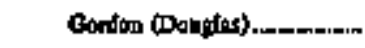 & $\mathbf{a}$ & .1 & 1 & .1 & HY & Whater & - & 1934 & $Q P$ \\
\hline & 2 & $\frac{1}{2}$ & .7 & $\frac{1}{7}$ & thy & For & 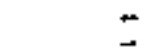 & 1945 & $\underset{\mathbb{p}}{\mathbb{p}}$ \\
\hline & 8 & .7 & .7 & .7 & IC & 1002 & $\vec{r}$ & 1948 & OP \\
\hline Nancy (Wothbom) & $\mathbf{j}$ & 3 & 3 & .3 & HY & Weter & $\bar{m}$ & 1953 & OP \\
\hline & $i$ & 3 & 2 & .2 & $\mathbf{H y}$ & Wut & - & 1953 & op \\
\hline Solon Dítes (Dodgles)......... & ] & 1.0 & 1.0 & 2.0 & ic & $\mathrm{FO2}$ & ـ & 1984 & $\mathbf{0}$ \\
\hline & $\mathbf{2}$ & 10 & 1.0 & 1.0 & IC & FO2 & $=$ & 1989 & $\underset{O P}{O P}$ \\
\hline & 4 & $\begin{array}{l}1.0 \\
t .0\end{array}$ & 2.0 & 1.0 & IC & $\operatorname{Fon}$ & $\bar{z}$ & 198 & $\stackrel{Q P P}{O P}$ \\
\hline & 5 & in & 1.0 & 1.0 & tc & Poi & - & [sos & op \\
\hline & 6 & 10 & 50 & 1.0 & IC & $\mathbf{F O n}$ & 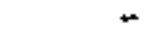 & 1995 & GP \\
\hline & $\mathrm{T}$ & 1.0 & 1.0 & 1.0 & IC & $\mathbf{F O 2}$ & $=$ & issos & OP \\
\hline & 8 & 1.0 & 1.0 & 1.0 & IC. & $\mathrm{EO} 2$ & - & 1995 & OP \\
\hline \multicolumn{10}{|l|}{ Daryitus Power Coop } \\
\hline 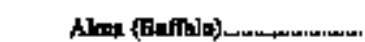 & $\mathbf{I}$ & & 213 & 243 & sT & EIr & stut & $\tan$ & DP \\
\hline & 2 & 150 & 21.9 & 21.9 & ST & BIT & sus & 54 & OP \\
\hline & 3 & 150 & 20.1 & 20.1 & $\mathbf{s T}$ & ET & sug & 1951 & $\mathrm{OP}$ \\
\hline & 4 & 34.4 & $\begin{array}{l}7.2 \\
6.5\end{array}$ & $\begin{array}{l}3.2 \\
B .5\end{array}$ & st & BST & sug & $\begin{array}{l}15 a 3 \\
1960\end{array}$ & OP \\
\hline Flamlieal (Rosk) & i & 50 & 7.0 & 70 & FY & Water & + & 1951 & OP \\
\hline & 2 & 50 & 7.0 & 7,00 & HY & Walat & $\ddot{-}$ & 1951 & op \\
\hline Genog (Vtemon) .......... & 3 & 345.6 & 37.2 & 37.2 & ST & What & $\operatorname{sun}^{-}$ & $\begin{array}{l}1551 \\
1969\end{array}$ & of \\
\hline 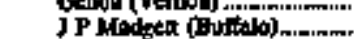 & & 3970 & 3723 & 37.3 & si & stip & $\mathrm{F02}$ & 1979 & $o$ \\
\hline Groy $\mathrm{G}$ \% of & & 24 & 23 & 25 & & & & & \\
\hline 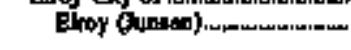 & 4 & 3 & .25 & 23 & IC & $\mathrm{BO} 2$ & Net Ge & 1945 & $\underset{\alpha P}{\alpha}$ \\
\hline Exmolinat of of & & & 20 & 21 & & & & & \\
\hline 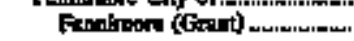 & 4 & 12 & 1.1 & 1,1 & tc & Fol & $\vec{r}$ & 1962 & $\mathbf{Q P}$ \\
\hline \multirow{3}{*}{$\begin{array}{l}\text { Crestum Vilhog of } \\
\text { Cower Wod (Strwano)._.. }\end{array}$} & $=$ & 1.0 & 1.0 & 1.0 & $\mathbf{x}$ & Fo1 & - & 1959 & o \\
\hline & 1 & 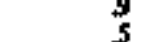 & 7 & $\frac{7}{3}$ & EY & War. & $\rightarrow$ & 1967 & op \\
\hline & 2 & $\overrightarrow{3}$ & لّ & إ. & 证 & Wats & - & 1967 & $\phi$ \\
\hline ذpper Weed (Shaqudo) & 1 & j & 1 & 1 & FY & Waler & - & 1946 & Pr \\
\hline Kombuget cily of & 7 & $\stackrel{2}{463}$ & $\begin{array}{r}.2 \\
42.6\end{array}$ & 2 & HY & Waler & - & J96!] & QP \\
\hline Conbined Locts & & & 31 & 31 & HY & $\mathbf{W r}$ & $\ldots$ & 1988 & op \\
\hline 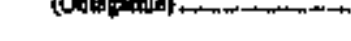 & BC2 & $3 \mathbf{3}$ & 3.1 & 3.1 & 踣 & Wher & 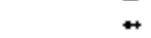 & 198 & ap \\
\hline 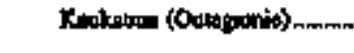 & 1 & 24 & 24 & 24 & 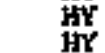 & Wut & $\ddot{z}$ & 1940 & $\underset{\mathbf{O P}}{\mathbf{P}}$ \\
\hline
\end{tabular}

See roopowe at end of uble. 
Table 20. Existing Generating Units at U.S. Electric Utilities by State, Company, and Plant, as of Jannary 1, 1996 (Continued)

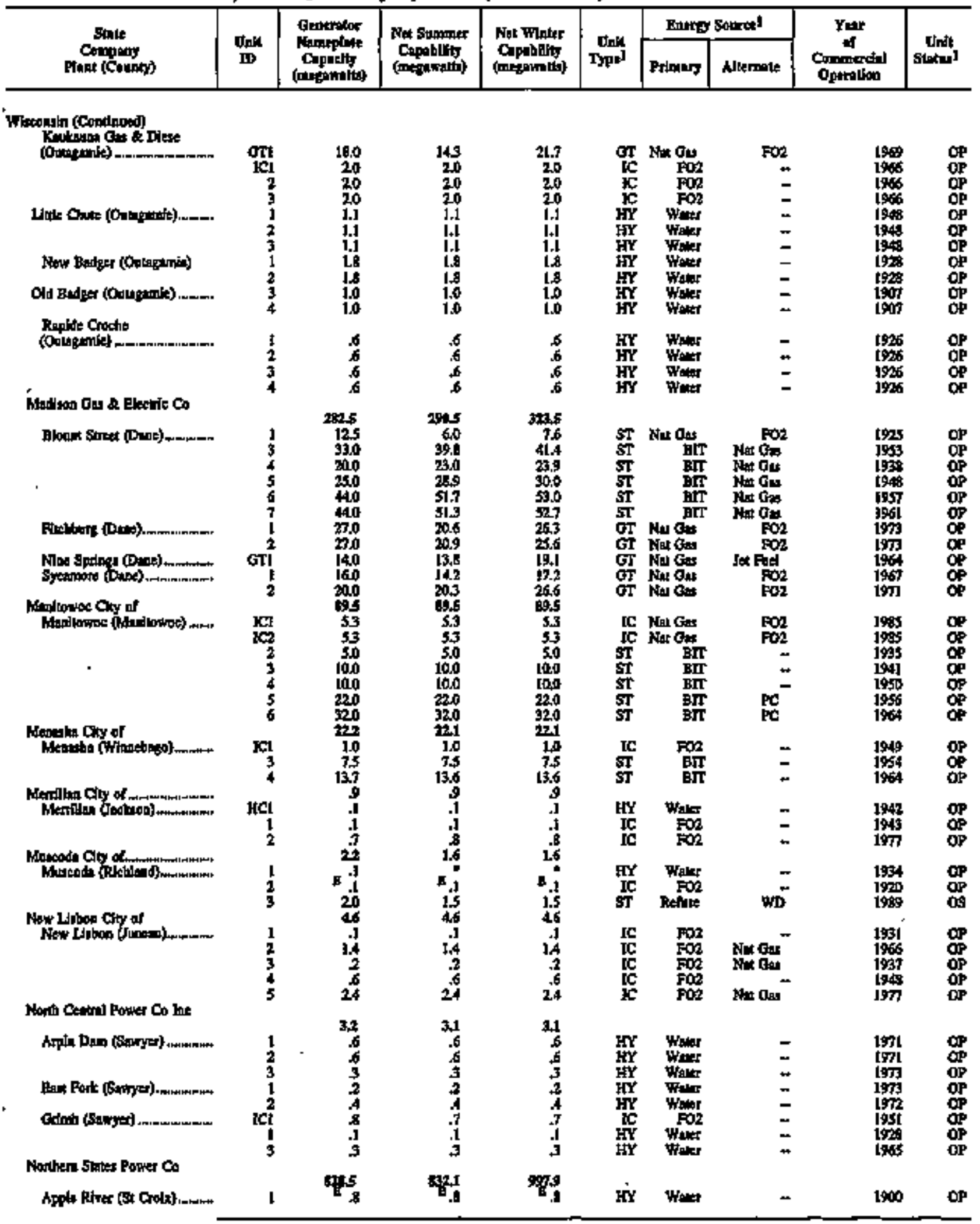

Son foolnotol at and af tabit. 
Table 20. Existing Generating Units at U.S. Electric Utilities by State, Company, and Plant, as of January 1, 1996 (Confinued)

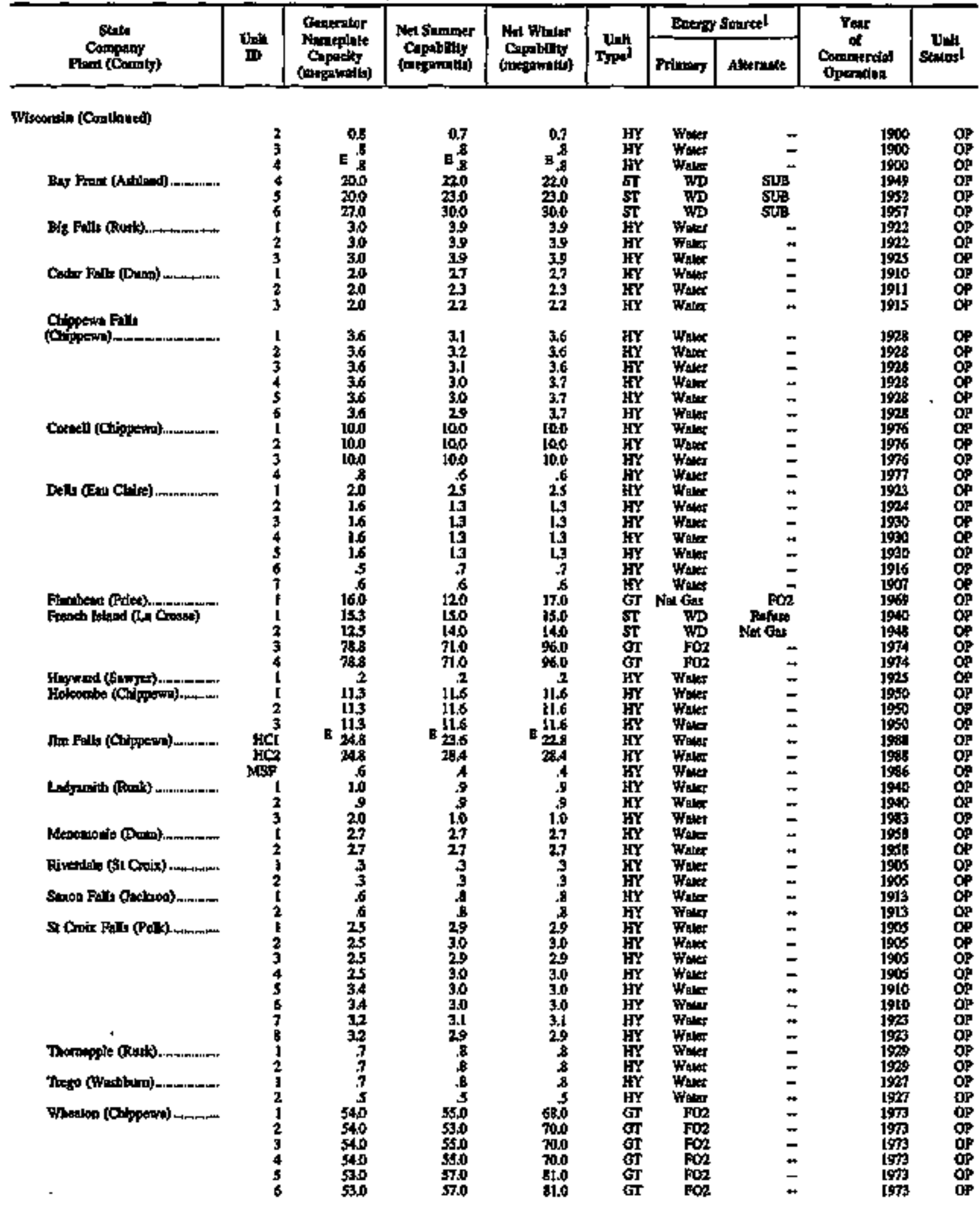

Ses tookotise aㅏ end of ublo. 
Table 20. Txisting Generating Units at U.S. Electric Utilities by State, Company, and Plant, as of January 1, 1996 (Continued)

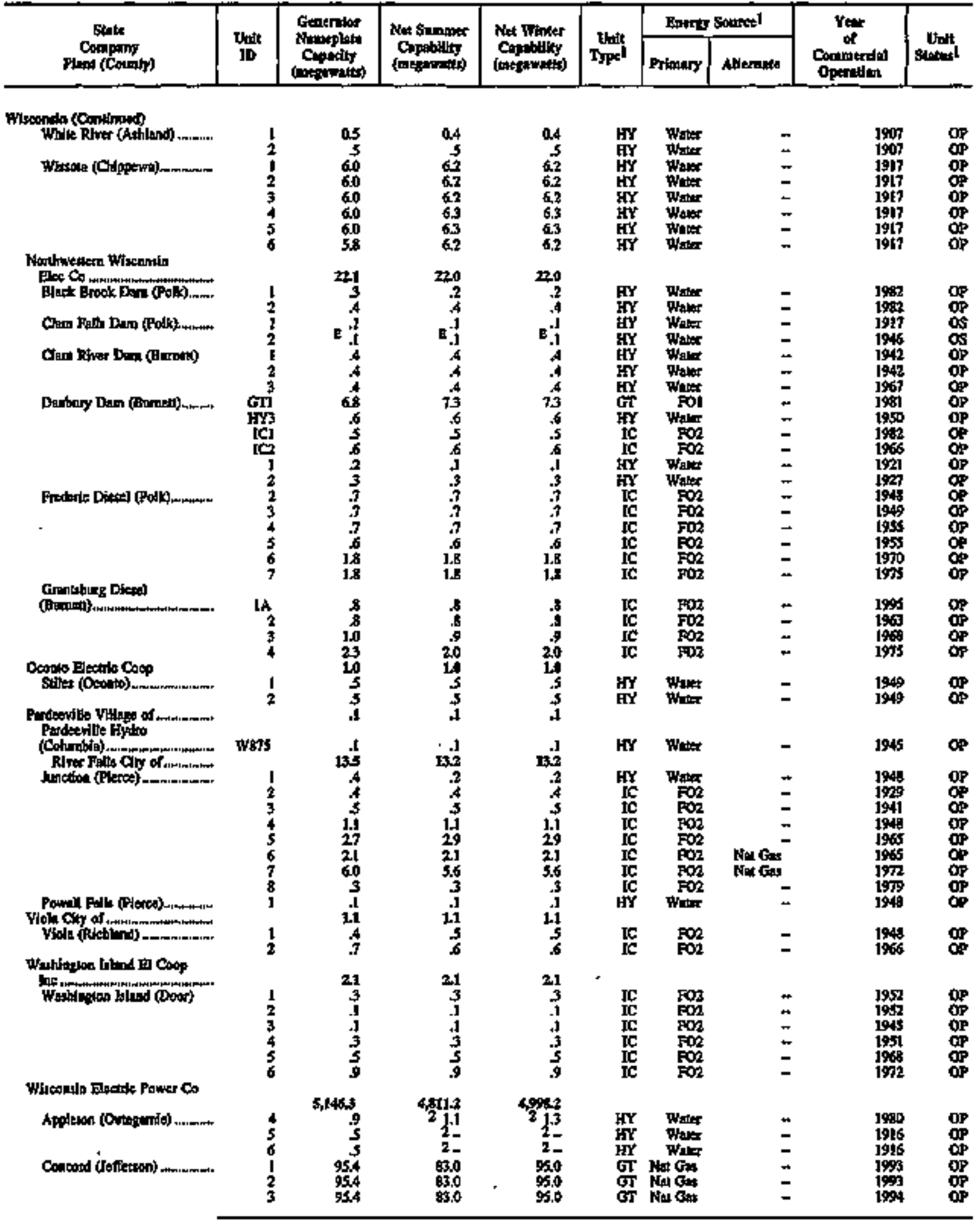

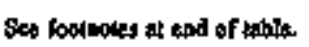


Table 20. Existing Generating Units at U.S. Electric Utilities by State, Company, and Plant, as of January 1, 1996 (Continued)

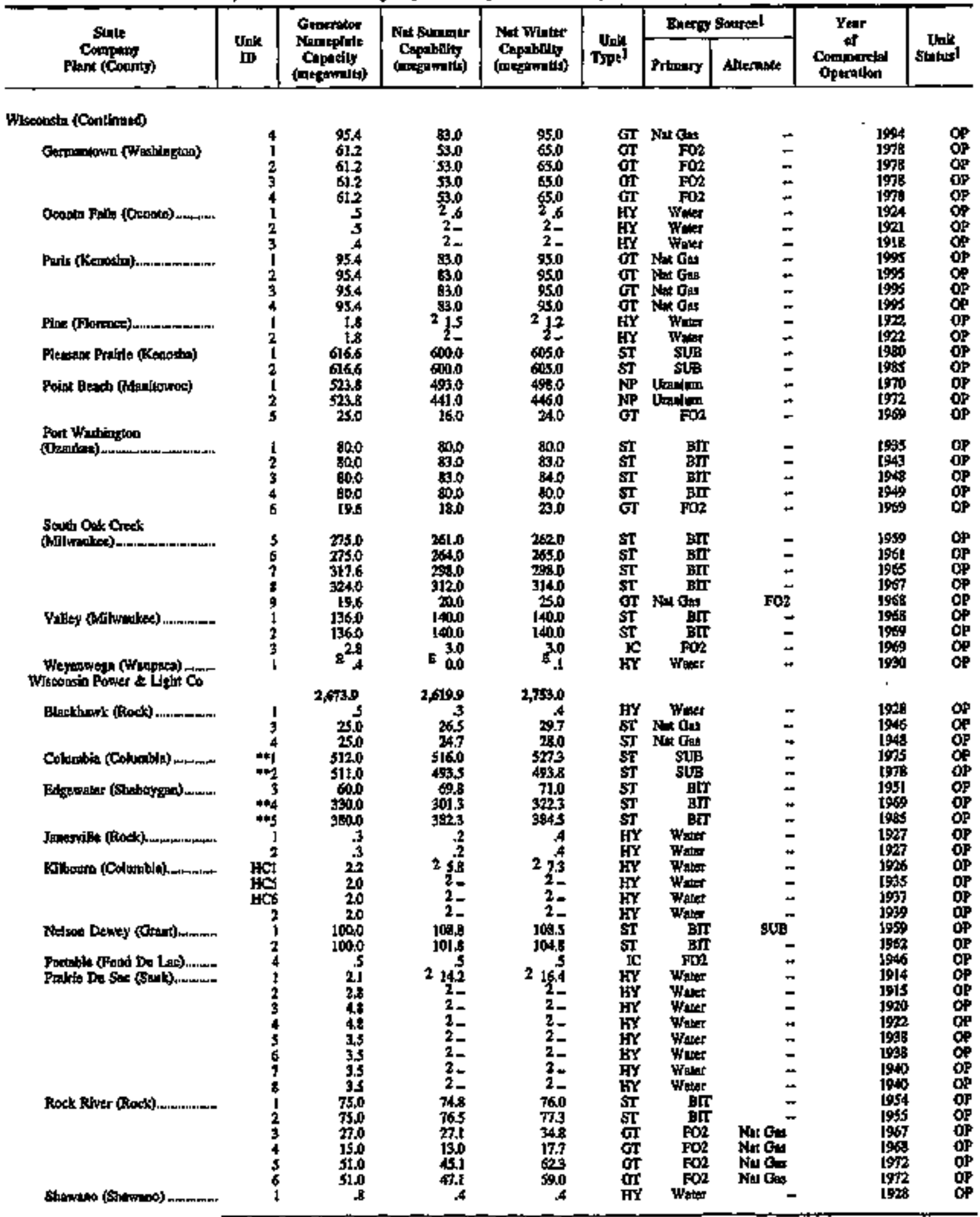

Son foospous at end of table 
Tahle 20. Existing Generating Units at U.S. Electric Utilities by State, Company, and Plant, as of January 1, 1996 (Continned)

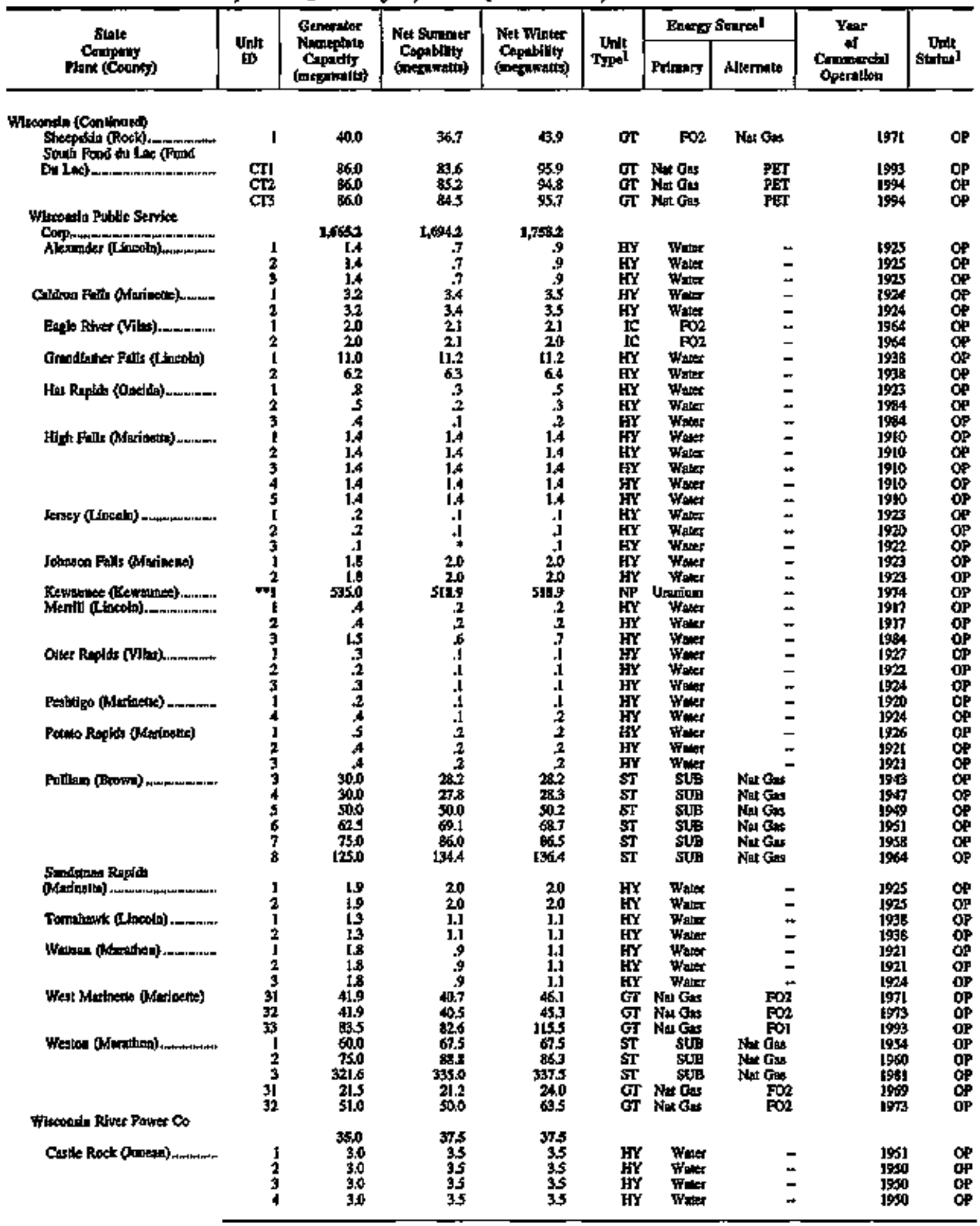

See foosectes at edd of table 
Table 20. Existing Generathing Units at U.S. Electric Utilities by State, Company, and Plant, as of January 1, 1996 (Continued)

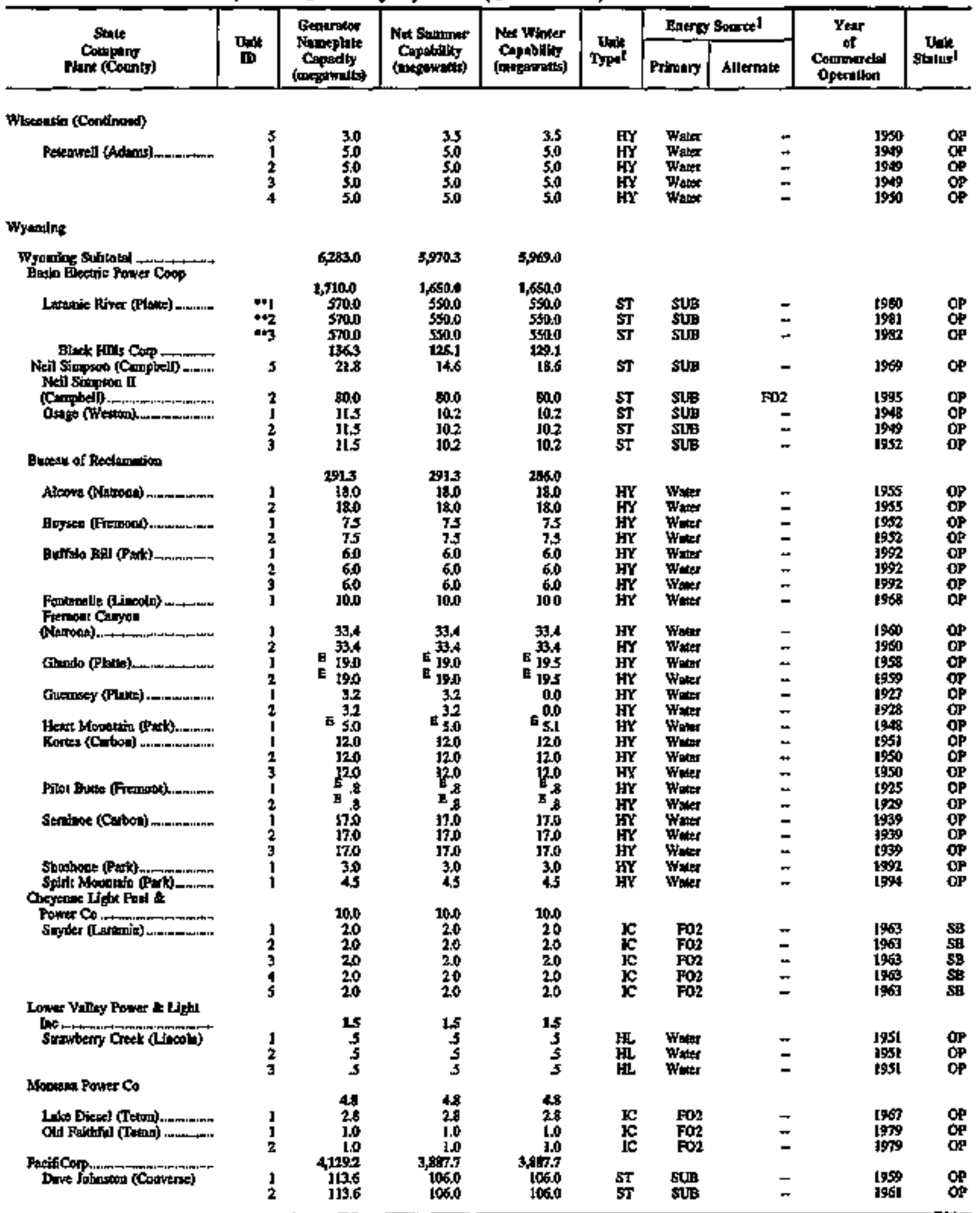

Set footnilits al etd of pole. 
Table 20. Existing Generating Units at U.S. Electric Utllities by State, Company, and Plant, as of January 1, 1996 (Conthued)

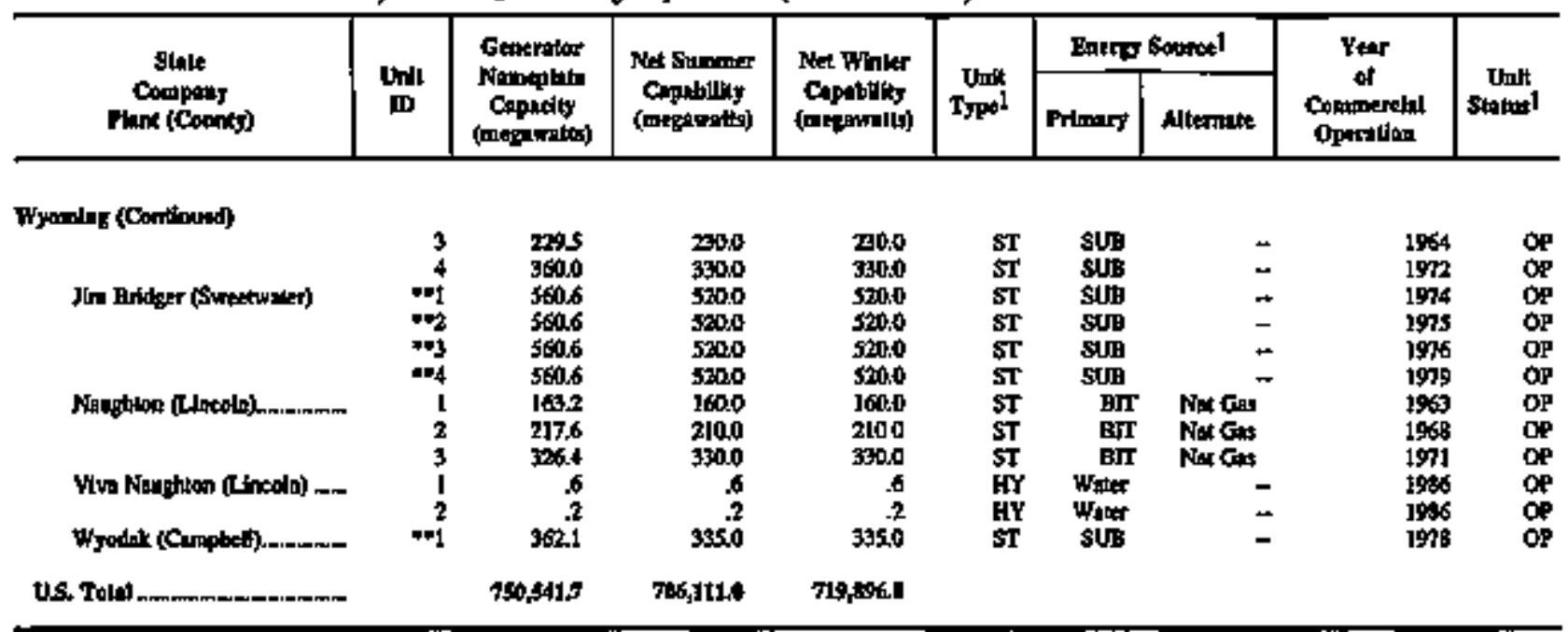

I Ste Appendix B tor codet.

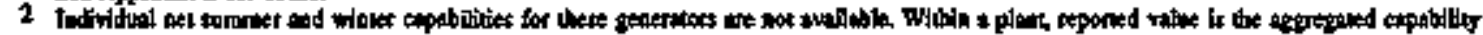
of all those gacrablon.

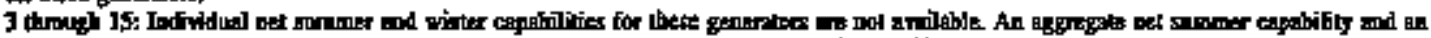

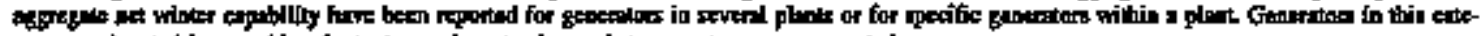

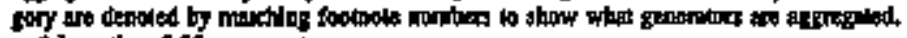

- Lert lian 0.0S metewats.

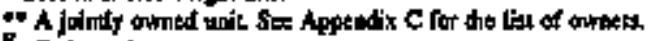

B Eifituted.

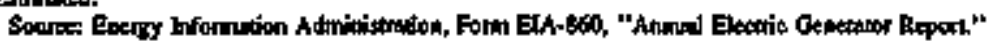


Table 21. Existing Generating Units Powered by Renewable Energy Sottrces at U.S. Electric Utilities by State, Company, and Plant, as of January 1, 1996

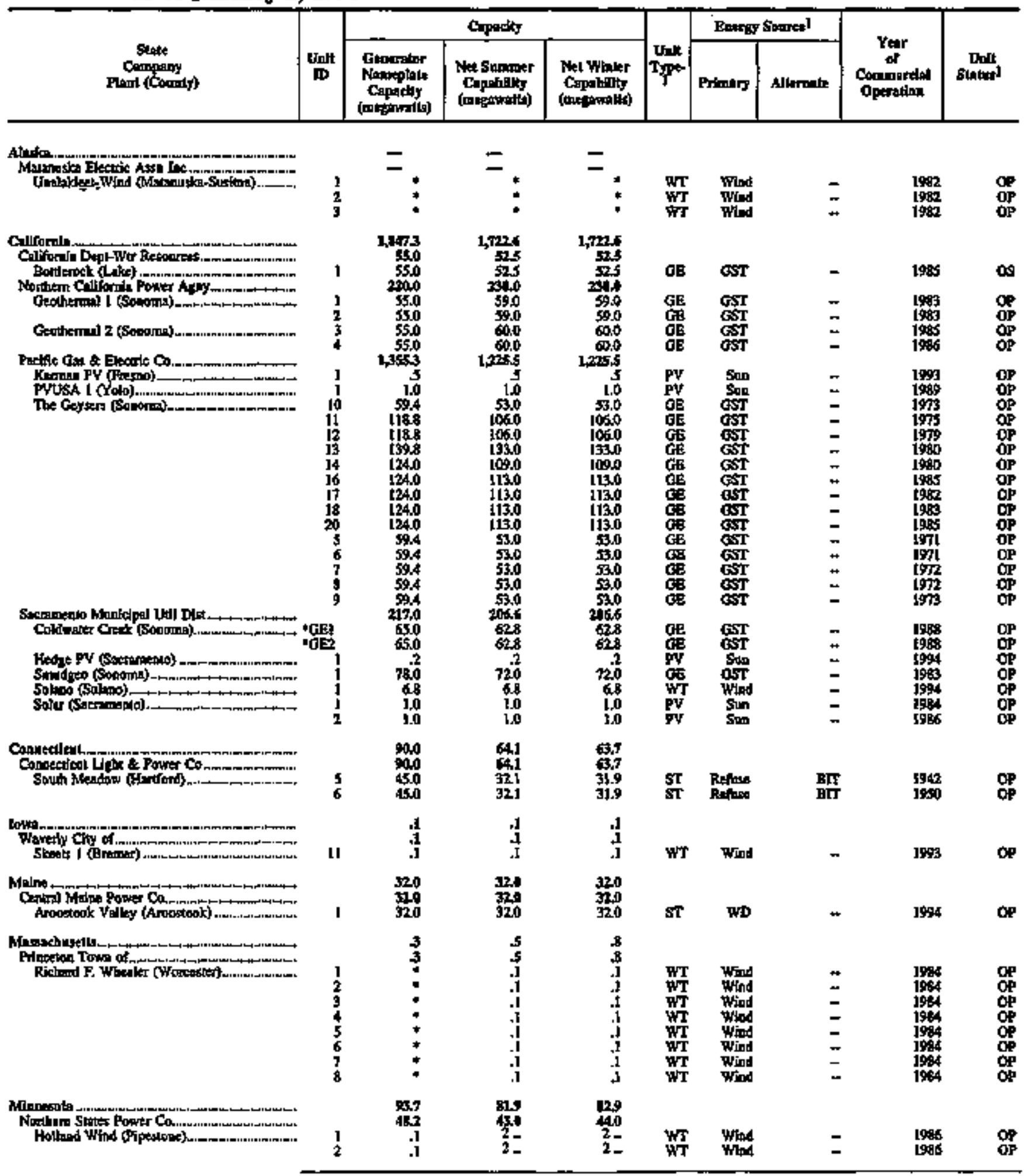

See frotenols at ead af bols. 
Table 21. Existing Generating Units Powered by Renewable Energy Sources at U.S. Electrlc Utillties by State, Company, and Ptant, as of January 1, 1996 (Continued)

\begin{tabular}{|c|c|c|c|c|c|c|c|c|c|}
\hline \multirow[b]{2}{*}{$\underset{\text { Fimal (Cotanty) }}{\text { Stritp }}$} & \multirow[b]{2}{*}{$\begin{array}{c}\text { Unit } \\
\text { m }\end{array}$} & \multicolumn{3}{|c|}{ Cupariky } & \multirow[b]{2}{*}{ Tpit } & \multicolumn{2}{|c|}{ Eodry SureaI } & \multirow[b]{2}{*}{$\begin{array}{c}\text { Your } \\
\text { of } \\
\text { Coninimital } \\
\text { Optrition }\end{array}$} & \multirow[b]{2}{*}{$\begin{array}{l}\text { thit: } \\
\text { sifuns: }\end{array}$} \\
\hline & & 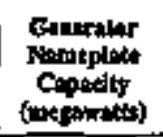 & 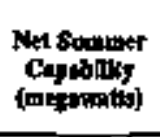 & 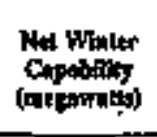 & & Promary & Alfermisto & & \\
\hline 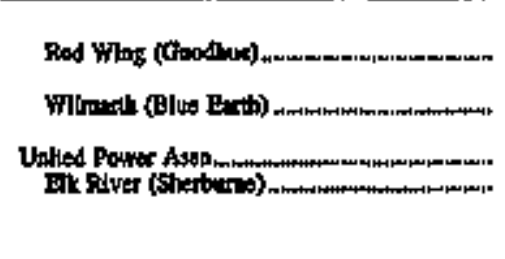 & $\begin{array}{l}3 \\
1 \\
2 \\
1 \\
2 \\
1 \\
2 \\
3\end{array}$ & $\begin{array}{l}01 \\
10.5 \\
11.5 \\
12.5 \\
12.5 \\
45.5 \\
11.5 \\
1.5 \\
22.5\end{array}$ & $\begin{array}{l}2 . \\
11.0 \\
200 \\
11.4 \\
11.0 \\
3.5 \\
9,8 \\
9.8 \\
15.3\end{array}$ & $\begin{array}{r}2 . \\
110 \\
11.0 \\
11.19 \\
11.0 \\
38.9 \\
9.8 \\
9.8 \\
193\end{array}$ & $\begin{array}{l}\text { WT } \\
\text { ST } \\
\text { ST } \\
\text { ST } \\
\text { ST } \\
\text { ST } \\
\text { SI } \\
\text { ST }\end{array}$ & 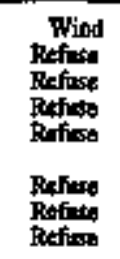 & $\begin{array}{l}\text { Kat Oat } \\
\text { Nat Ges } \\
\text { Nut Gat } \\
\text { Nat Gas } \\
= \\
=\end{array}$ & $\begin{array}{l}1996 \\
1949 \\
19959 \\
1948 \\
1951 \\
1951 \\
1951 \\
1959\end{array}$ & $\begin{array}{l}\text { OP } \\
O P \\
O P \\
O P \\
O P \\
O P \\
O P \\
O P\end{array}$ \\
\hline 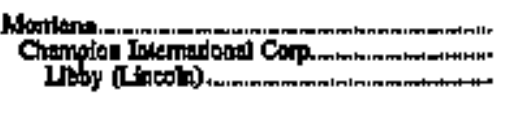 & I & $\begin{array}{l}12.5 \\
12,5 \\
7.5 \\
5,0\end{array}$ & $\begin{array}{l}125 \\
125 \\
7.0\end{array}$ & $\begin{array}{r}125 \\
12.5 \\
7.5 \\
5.0\end{array}$ & $\begin{array}{l}\mathbf{S T} \\
\mathbf{S T}\end{array}$ & WD & $=$ & $\begin{array}{l}1966 \\
19912\end{array}$ & of \\
\hline 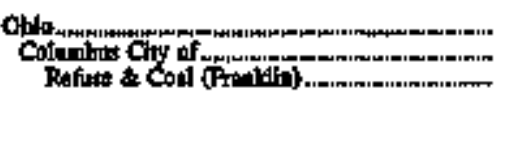 & j & $\begin{array}{l}90,0 \\
90,0 \\
30,0 \\
30,0 \\
30.0\end{array}$ & $\begin{array}{l}900 \\
900 \\
300 \\
300 \\
30,0\end{array}$ & $\begin{array}{l}900 \\
90,0 \\
300 \\
300 \\
300\end{array}$ & $\begin{array}{l}\text { ST } \\
\text { SI }\end{array}$ & $\begin{array}{l}\text { Refind } \\
\text { Refinat }\end{array}$ & 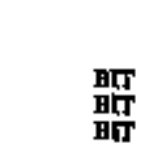 & $\begin{array}{l}1963 \\
19635 \\
19635\end{array}$ & op \\
\hline 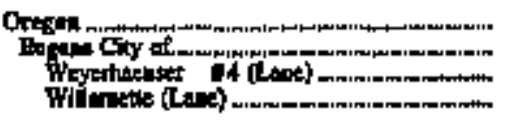 & 4 & $\begin{array}{l}\text { st.s } \\
\$ 1.5 \\
40.0 \\
11.5\end{array}$ & $\begin{array}{l}345 \\
345 \\
24.0 \\
115\end{array}$ & $\begin{array}{l}345 \\
34.5 \\
23.0 \\
11.5\end{array}$ & $\begin{array}{l}\text { ST } \\
\text { ST }\end{array}$ & RAntod & $\bar{z}$ & 1996 & $\stackrel{O P}{O P}$ \\
\hline 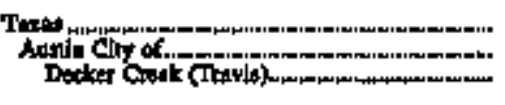 & prs & $\begin{array}{l}3 \\
3 \\
3\end{array}$ & $\begin{array}{l}3 \\
3 \\
3\end{array}$ & $\begin{array}{l}\frac{3}{3} \\
3\end{array}$ & PV & \$up & 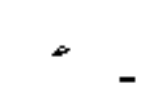 & 1987 & OP \\
\hline 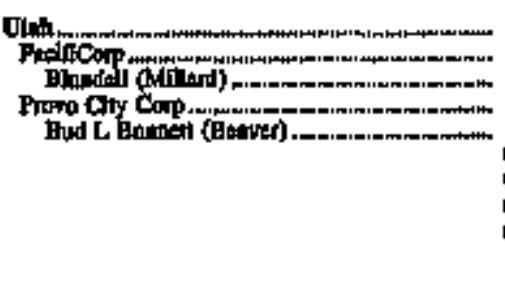 & 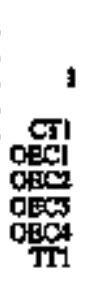 & $\begin{array}{r}39.6 \\
36.1 \\
26.1 \\
13.5 \\
85 \\
8 \\
8 \\
8 \\
8 \\
20\end{array}$ & $\begin{array}{r}35.2 \\
2.0 \\
21.0 \\
1.4 \\
7.0 \\
8 \\
8 \\
8 \\
8 \\
20\end{array}$ & $\begin{array}{r}252 \\
230 \\
200 \\
1 \pm 4 \\
70 \\
8 \\
8 \\
8 \\
8 \\
8 \\
20\end{array}$ & 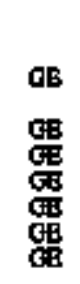 & $\begin{array}{l}\text { CST } \\
\text { GST } \\
\text { GST } \\
\text { gST } \\
\text { dST } \\
\text { dST }\end{array}$ & 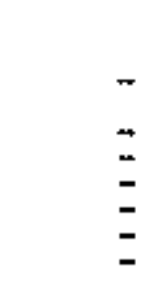 & $\begin{array}{l}1904 \\
1989 \\
1995 \\
1985 \\
1955 \\
1985 \\
1988\end{array}$ & $\begin{array}{l}\text { of } \\
\text { op } \\
\text { op } \\
\text { Op } \\
\text { op } \\
\text { op } \\
\text { op }\end{array}$ \\
\hline 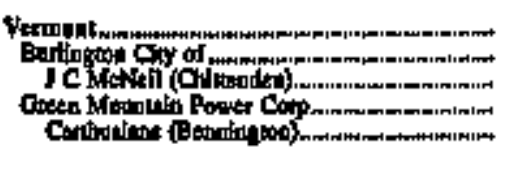 & $\begin{array}{r}* 1 \\
3 \\
3\end{array}$ & $\begin{array}{r}50.2 \\
50.0 \\
50.0 \\
.7 \\
.1\end{array}$ & 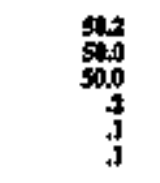 & $\begin{array}{r}50.3 \\
500 \\
500 \\
.7 \\
.1\end{array}$ & $\begin{array}{l}\text { ST } \\
\text { wT }\end{array}$ & $\begin{array}{l}\text { WD } \\
\text { whod } \\
\text { Whod }\end{array}$ & $\begin{array}{r}\text { Nat Out } \\
- \\
-\end{array}$ & $\begin{array}{l}1984 \\
1989 \\
1959\end{array}$ & $\begin{array}{l}\text { OP } \\
\text { OP } \\
\text { OP }\end{array}$ \\
\hline 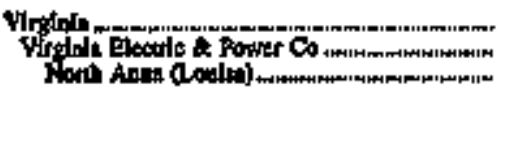 & SPP & 4 & 4 & $\begin{array}{l}4 \\
: \\
:\end{array}$ & $\underset{\mathbf{P W}}{\mathbf{P W}}$ & Sin & $\approx$ & $\begin{array}{l}1955 \\
19 \% 5 \\
19 \% 5\end{array}$ & $\stackrel{o p}{\phi p}$ \\
\hline 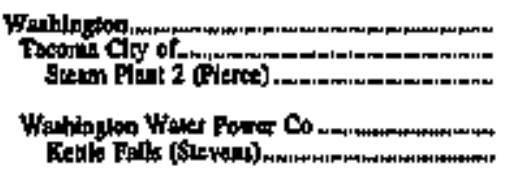 & $\begin{array}{l}1 \\
2 \\
1\end{array}$ & $\begin{array}{l}100.7 \\
5100 \\
25.0 \\
25.0 \\
60.7 \\
50.7\end{array}$ & $\begin{array}{l}97 \% \\
500 \\
25,0 \\
25,0 \\
47,0\end{array}$ & $\begin{array}{l}95 \\
5 \mathrm{gid} \\
25,0 \\
25,0 \\
47.5\end{array}$ & $\begin{array}{l}\text { AR } \\
\text { AB } \\
5 T\end{array}$ & $\begin{array}{l}\text { WD } \\
\text { WD } \\
\text { WD }\end{array}$ & $\begin{array}{l}\text { sth } \\
\text { sub } \\
\text { Nat Ges }\end{array}$ & $\begin{array}{l}1931 \\
1955 \\
1993\end{array}$ & $\begin{array}{l}o p \\
\text { op } \\
\text { op }\end{array}$ \\
\hline 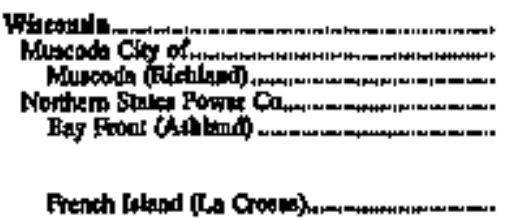 & $\begin{array}{l}3 \\
4 \\
5 \\
3\end{array}$ & 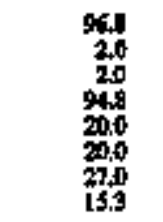 & $\begin{array}{r}105.5 \\
15 \\
15 \\
1040 \\
20.0 \\
230 \\
30.0 \\
150\end{array}$ & $\begin{array}{r}1055 \\
15 \\
15 \\
1+40 \\
20.0 \\
200 \\
300 \\
150\end{array}$ & $\begin{array}{l}S T \\
S T \\
S T \\
S T \\
S T\end{array}$ & $\begin{array}{r}\text { Refue } \\
\text { WD } \\
\text { WD } \\
\text { WD } \\
\text { WD }\end{array}$ & $\begin{array}{l}\text { WD } \\
\text { sws } \\
\text { sust } \\
\text { sus } \\
\text { Refuste }\end{array}$ & $\begin{array}{l}1999 \\
1999 \\
1959 \\
1957 \\
1940\end{array}$ & $\begin{array}{l}\text { OS } \\
\text { of } \\
\text { op } \\
\text { of } \\
\text { of }\end{array}$ \\
\hline
\end{tabular}

Seo boonotes at end o lablo. 
Table 21. Existing Generating Units Powered by Renewable Energy Sources at U.S. Electric Utilities by State, Company, and Plant. as of January 1, 1996 (Contjnued)

\begin{tabular}{|c|c|c|c|c|c|c|c|c|c|}
\hline \multirow[b]{2}{*}{$\begin{array}{c}\text { Strit } \\
\text { Compayy } \\
\text { Pant (Connty) }\end{array}$} & \multirow[b]{2}{*}{$\begin{array}{l}\text { Ink } \\
\text { in }\end{array}$} & \multicolumn{3}{|c|}{ Crparity } & \multirow[b]{2}{*}{ Typs } & \multicolumn{2}{|c|}{ Entry Sonreal } & \multirow[b]{2}{*}{$\begin{array}{c}\text { Yas } \\
\text { ot } \\
\text { Comaraid } \\
\text { Operatiod }\end{array}$} & \multirow[b]{2}{*}{$\begin{array}{c}\text { JdI! } \\
\text { Sthtosl }\end{array}$} \\
\hline & & 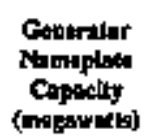 & 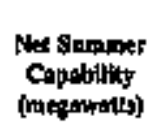 & 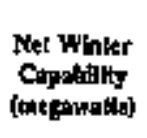 & & Primary & Alitinata & & \\
\hline & 2 & 125 & 140 & 140 & $\mathbf{S T}$ & MD & Nal Gat & t\$48 & $\mathrm{QP}$ \\
\hline US. Todel & & 2,605.1 & $2,3 \pm 6,4$ & 2,3273 & & & & & \\
\hline
\end{tabular}

1 Soa Appeodix $\mathbf{B}$ tor codiss.

- less then ous megniritr.

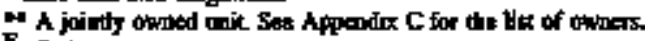

E Brianaled.

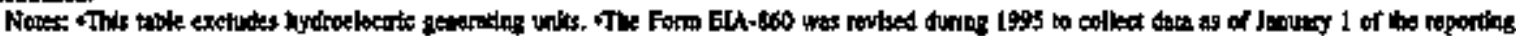

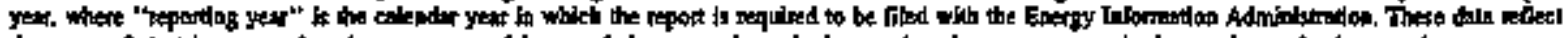

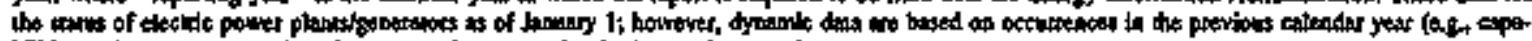

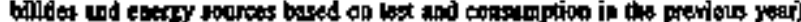

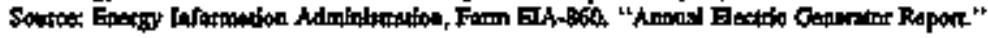


Table 22. Plamned Generating Unit Changes at U.S. Electric Utilities by State, Company, and Plant, 1996 Through 2005 as of January 1, 1996

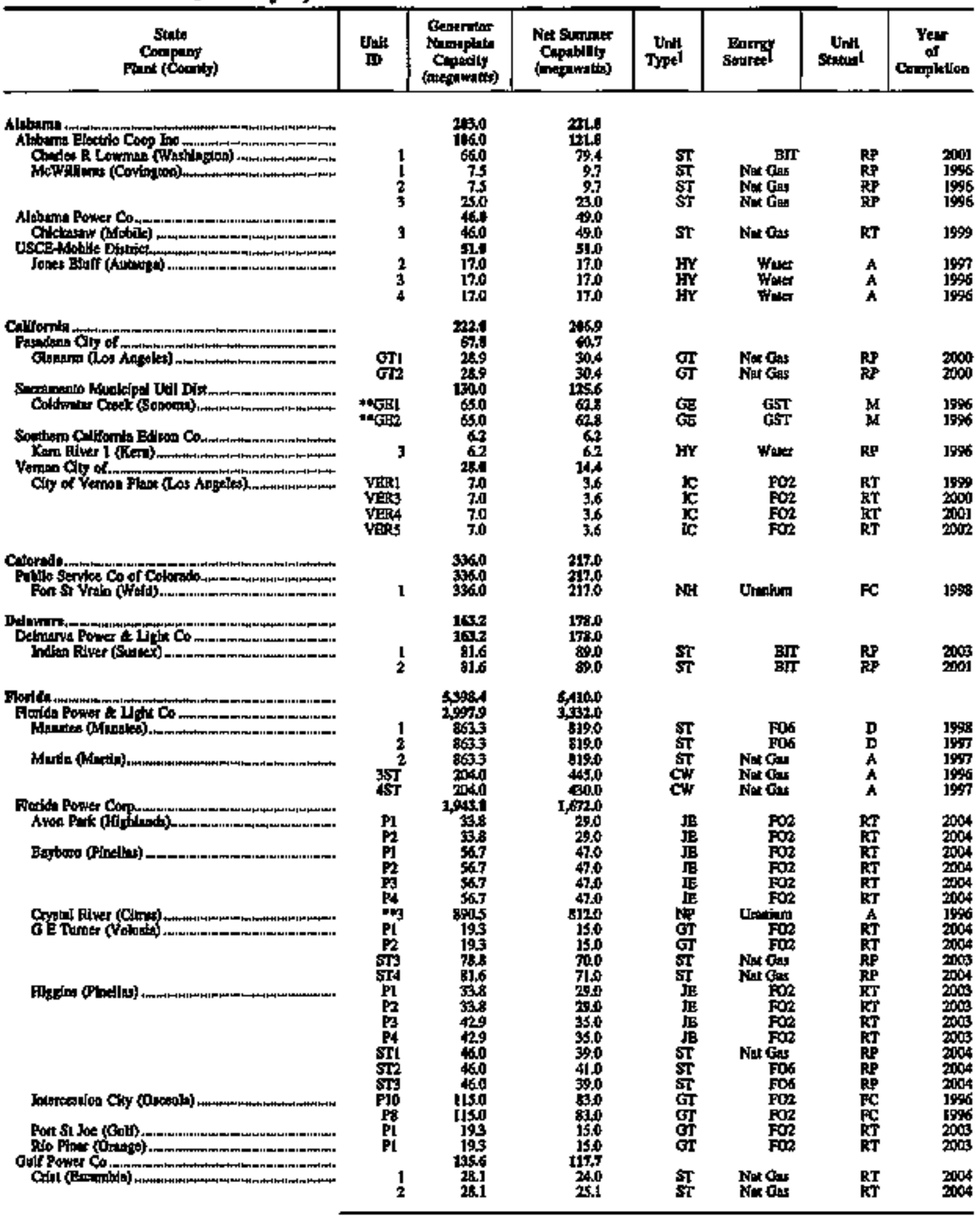

Ses boptoutex an end of tabla. 
Table 22. Planned Generating Unit Changes at U.S. Electric Utilities by State, Company, and Plant, 1996 Through 2005 as of January 1, 1996 (Contlnued)

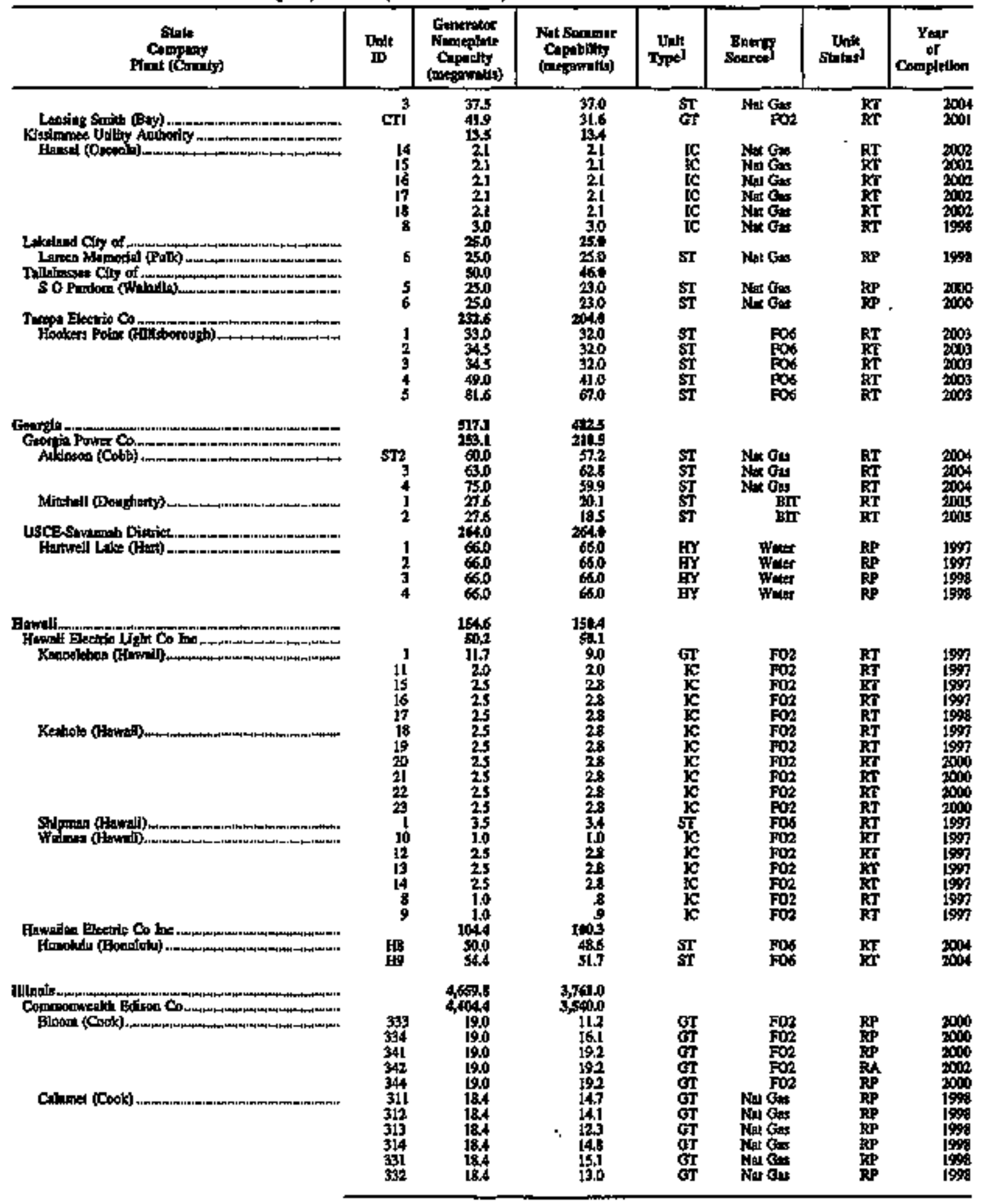

Sea sootale: at and of tite. 
Table 22. Planned Generating Unit Changes at U.S. Electric Utilittes by State, Company, and Plant, 1996 Through 2005 as of January 1, 1996 (Continued)

\begin{tabular}{|c|c|c|c|c|c|c|c|}
\hline 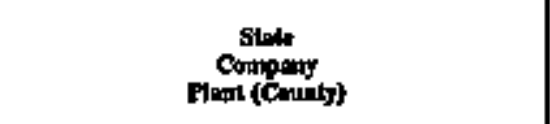 & $\begin{array}{l}\text { DhIt } \\
\text { if }\end{array}$ & 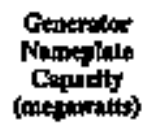 & 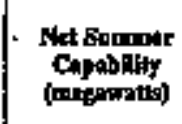 & Typal & $\begin{array}{l}\text { Entardy } \\
\text { Soureal }\end{array}$ & Uate: & $\begin{array}{c}\text { Tear } \\
\text { of } \\
\text { Conplistion }\end{array}$ \\
\hline 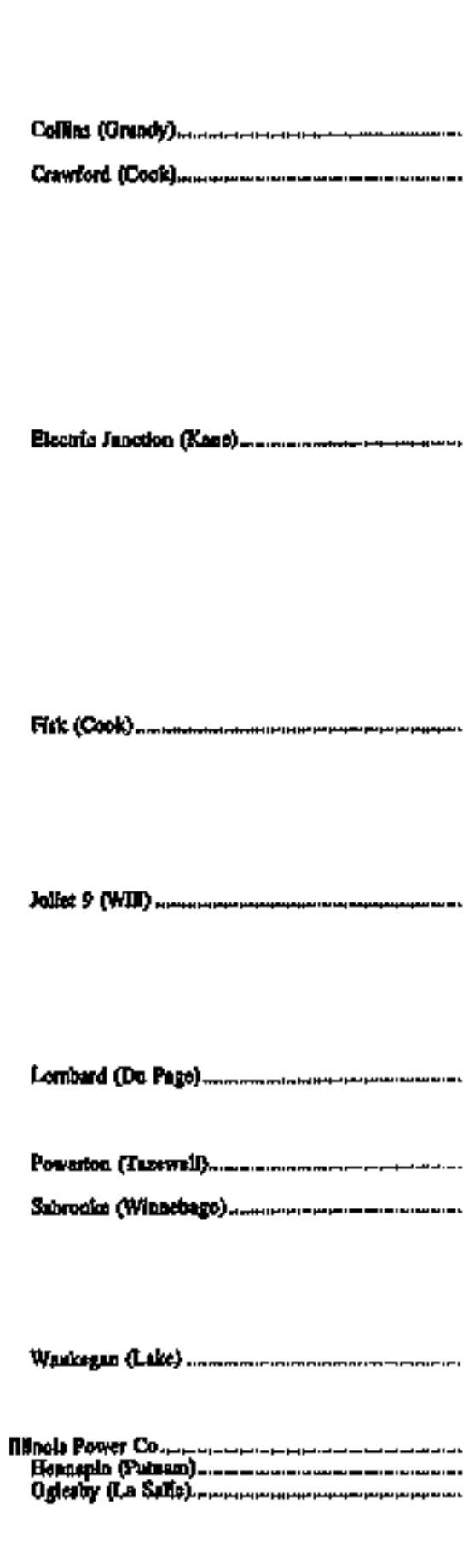 & 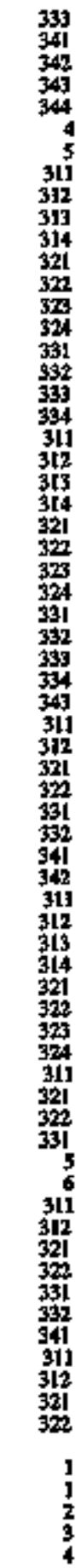 & 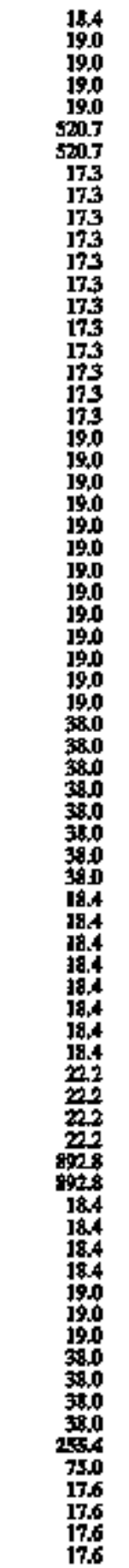 & 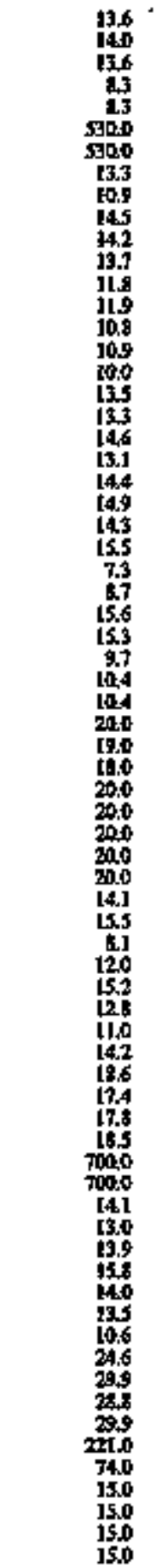 & 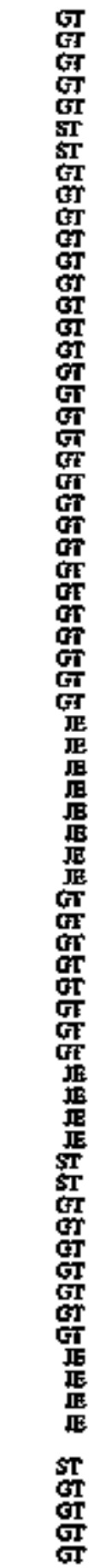 & 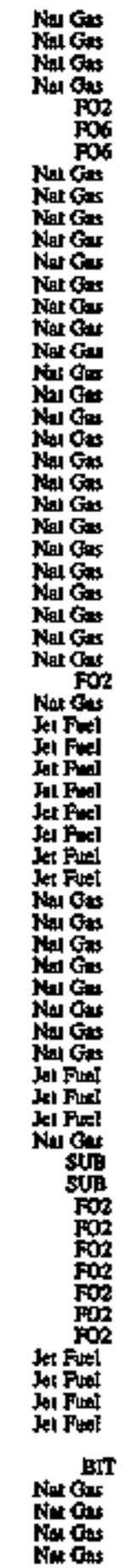 & 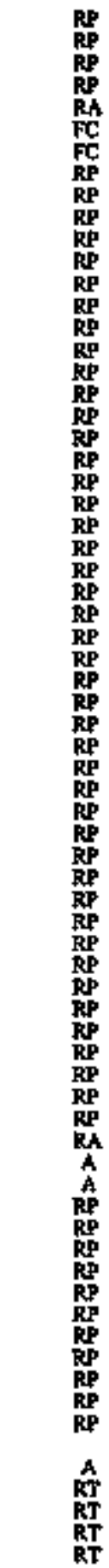 & 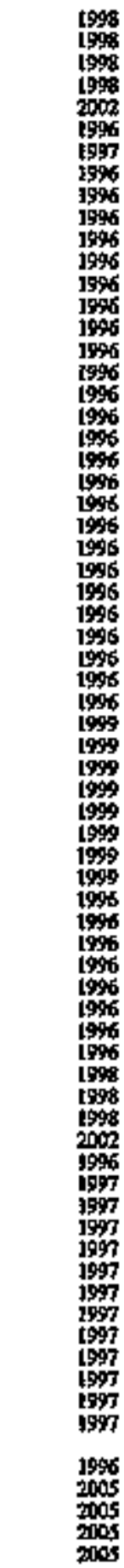 \\
\hline
\end{tabular}

Sol taokingen at and of tablo. 
Table 22. Planned Generating Unit Changes at U.S. Flectric Utilities by State, Company, and Plant, 1996 Through 2005 as of January 1, 1996 (Continued)

\begin{tabular}{|c|c|c|c|c|c|c|c|}
\hline $\begin{array}{l}\text { State } \\
\text { Company } \\
\text { Fine (Connty) }\end{array}$ & UfPH & 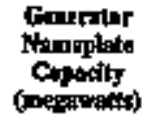 & 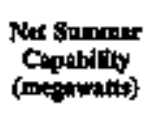 & Un't & $\begin{array}{l}\text { Dinerest } \\
\text { Sanrtal }\end{array}$ & $\underset{\text { stutosi }}{\operatorname{Un}}$ & $\begin{array}{l}\text { Yer } \\
\text { of } \\
\text { Compdetion }\end{array}$ \\
\hline 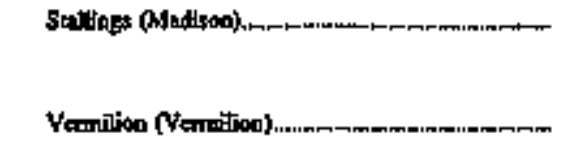 & $\begin{array}{r}1 \\
\frac{2}{3} \\
4 \\
\text { OTI }\end{array}$ & $\begin{array}{l}\mathbf{2 3 , 8} \\
\mathbf{2 3 , 8} \\
\mathbf{2 3 , 8} \\
\mathbf{2 3 . 8} \\
\mathbf{1 5 . 0}\end{array}$ & $\begin{array}{l}19.3 \\
19.3 \\
19.3 \\
19.3 \\
10.0\end{array}$ & $\begin{array}{l}\text { GI } \\
\text { GI } \\
\text { GT } \\
\text { Gr }\end{array}$ & 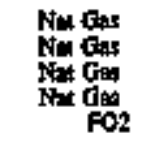 & $\begin{array}{l}\mathbf{R T} \\
\mathbf{R T} \\
\mathbf{R T} \\
\mathbf{R T}\end{array}$ & $\begin{array}{l}2005 \\
2005 \\
2005 \\
2005 \\
2002\end{array}$ \\
\hline 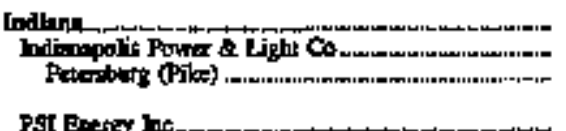 & $x_{101}$ & $\begin{array}{r}94.3 \\
5.5 \\
2.5 \\
2.5\end{array}$ & $\begin{array}{r}912.0 \\
6.0 \\
3.0 \\
3.0\end{array}$ & $\begin{array}{l}S T \\
S T\end{array}$ & $\mathrm{FO2}_{\mathrm{FO2}}$ & $\underset{\mathbf{D}}{\mathbf{D}}$ & 1996 \\
\hline 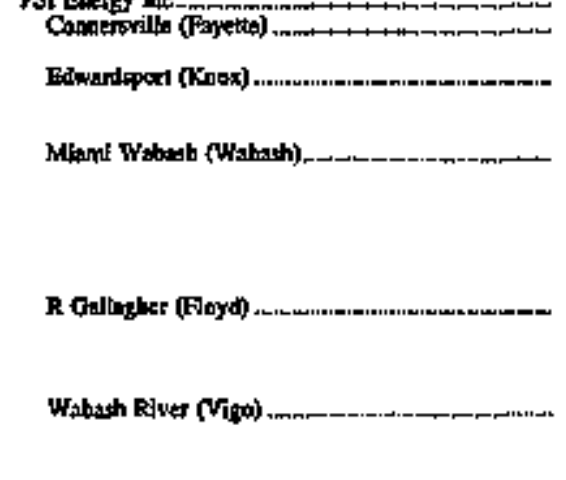 & $\begin{array}{l}1 \\
2 \\
6 \\
7 \\
6 \\
1 \\
7 \\
3 \\
4 \\
5 \\
6 \\
1 \\
2 \\
3 \\
4 \\
71 \\
72 \\
73\end{array}$ & $\begin{array}{r}41.9 \\
\$ 1.9 \\
38.0 \\
40.9 \\
69.0 \\
18.0 \\
18.0 \\
18.0 \\
18.0 \\
16.3 \\
163 \\
150.0 \\
150.0 \\
1500 \\
150.0 \\
28 \\
2.8 \\
2.8\end{array}$ & 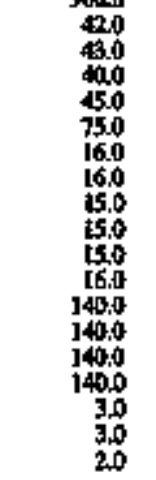 & 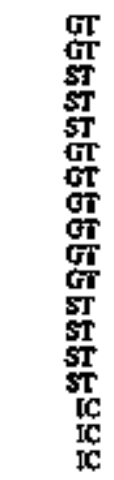 & 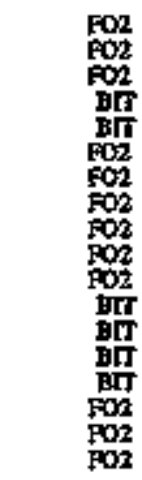 & $\begin{array}{l}R T \\
R T \\
R T \\
R T \\
R T \\
R T \\
R T \\
R T \\
R T \\
R T \\
\mathbf{R T} \\
\mathbf{E T} \\
\mathbf{R T} \\
\mathbf{D} \\
\mathbf{D} \\
\mathbf{D} \\
\mathbf{D} \\
\mathbf{E T} \\
\mathbf{R T} \\
\mathbf{R T}\end{array}$ & $\begin{array}{l}2005 \\
2005 \\
2004 \\
2004 \\
2004 \\
2002 \\
2002 \\
2002 \\
2002 \\
2002 \\
2002 \\
2005 \\
2005 \\
2005 \\
2005 \\
2004 \\
2002 \\
2002\end{array}$ \\
\hline 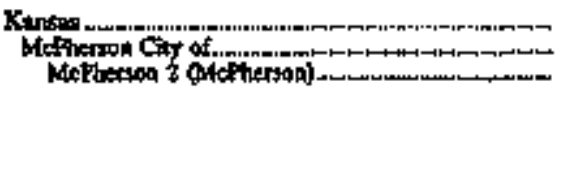 & 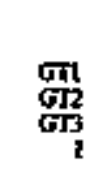 & $\begin{array}{l}\mathbf{t} 5,0 \\
+\$ .0 \\
5.4 \\
56.4 \\
57.6 \\
26.6\end{array}$ & $\begin{array}{l}180.4 \\
12.4 \\
32.9 \\
50.9 \\
5.0 \\
25.6\end{array}$ & $\begin{array}{l}\mathbf{G T} \\
\mathbf{G T} \\
\mathbf{G T}\end{array}$ & 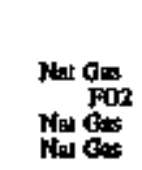 & $\underset{\mathbf{R T}}{\mathbf{R T}}$ & $\begin{array}{l}2002 \\
2002 \\
2002 \\
2005\end{array}$ \\
\hline 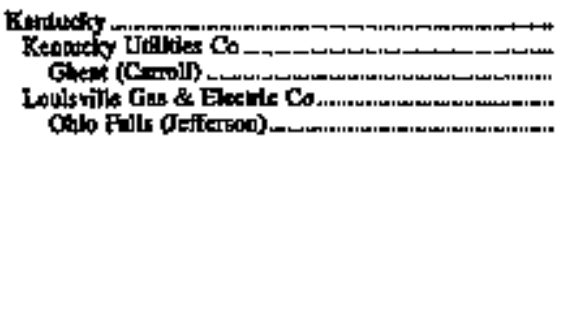 & $\begin{array}{l}1 \\
2 \\
3 \\
4 \\
5 \\
6 \\
7 \\
5\end{array}$ & 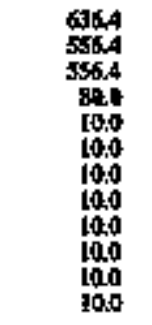 & $\begin{array}{r}57.0 \\
4500 \\
470 \\
400 \\
60 \\
60 \\
60 \\
60 \\
60 \\
60 \\
60 \\
60\end{array}$ & $\begin{array}{l}\mathbf{S T} \\
\mathbf{H Y} \\
\mathbf{H Y} \\
\mathbf{H Y} \\
\mathbf{H Y} \\
\mathbf{W Y} \\
\mathbf{W Y} \\
\mathbf{H Y} \\
\mathbf{H Y}\end{array}$ & 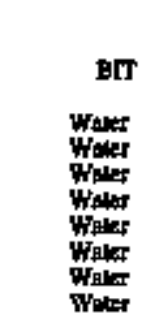 & $\begin{array}{l}\mathbf{D} \\
\mathbf{A} \\
\mathbf{D} \\
\mathbf{A} \\
\hat{A} \\
A \\
\mathbf{A} \\
\mathbf{D}\end{array}$ & $\begin{array}{l}2000 \\
2009 \\
1986 \\
1996 \\
2003 \\
2000 \\
2009 \\
2003 \\
1996\end{array}$ \\
\hline 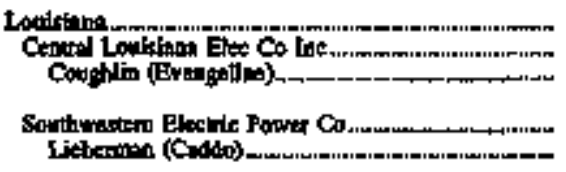 & $\$$ & $\begin{array}{l}115,3 \\
94,3 \\
25,0 \\
653 \\
254 \\
250\end{array}$ & $\begin{array}{l}\mathbf{2 0 . 0} \\
24.0 \\
5 \times 10 \\
200 \\
200\end{array}$ & $\begin{array}{l}\text { ST } \\
\mathbf{S T} \\
\mathbf{S T}\end{array}$ & 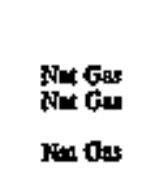 & $\begin{array}{l}\mathbf{R A} \\
\mathbf{R A} \\
\mathbf{R T}\end{array}$ & $\begin{array}{l}2000 \\
1999 \\
2005\end{array}$ \\
\hline 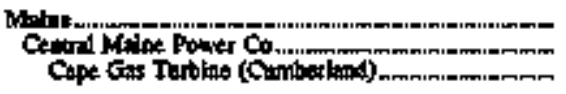 & ot4 & $\begin{array}{l}17.6 \\
17.6\end{array}$ & $\begin{array}{l}160 \\
16040 \\
160\end{array}$ & GT & $\mathrm{POZ}$ & $\mathbf{R T}$ & 1999 \\
\hline 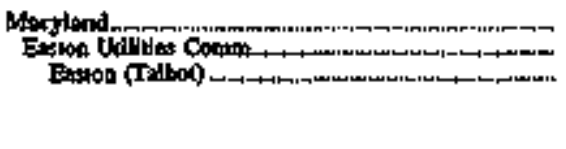 & $\begin{array}{l}7 \\
8 \\
9\end{array}$ & $\begin{array}{l}2.1 \\
21.5 \\
2.5\end{array}$ & $\begin{array}{l}6.5 \\
6.5 \\
2.0 \\
2.0 \\
2.5\end{array}$ & $\begin{array}{l}\mathrm{Ic} \\
\mathrm{dc}\end{array}$ & 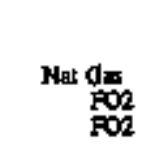 & $\begin{array}{l}\mathbf{E T} \\
\mathbf{R T} \\
\mathbf{R T}\end{array}$ & $\begin{array}{l}1999 \\
2000 \\
2001\end{array}$ \\
\hline 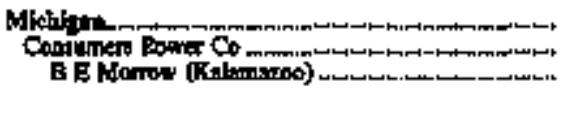 & $\mathbf{B}$ & $\begin{array}{r}3,31.9 \\
378.8 \\
175 \\
175\end{array}$ & $\begin{array}{r}3,105 \\
4129 \\
I 40 \\
140\end{array}$ & GT & $\begin{array}{l}\text { Nat Ġt } \\
\text { Nat Cat }\end{array}$ & $\begin{array}{l}\text { RT } \\
\text { RT }\end{array}$ & $\begin{array}{l}2002 \\
2002\end{array}$ \\
\hline
\end{tabular}

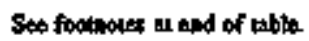


Table 22. Planned Generating Unit Changes at U.S. Electric Utilities by State, Company, and Plant, 1996 Through 2005 as of January 1, 1996 (Continued)

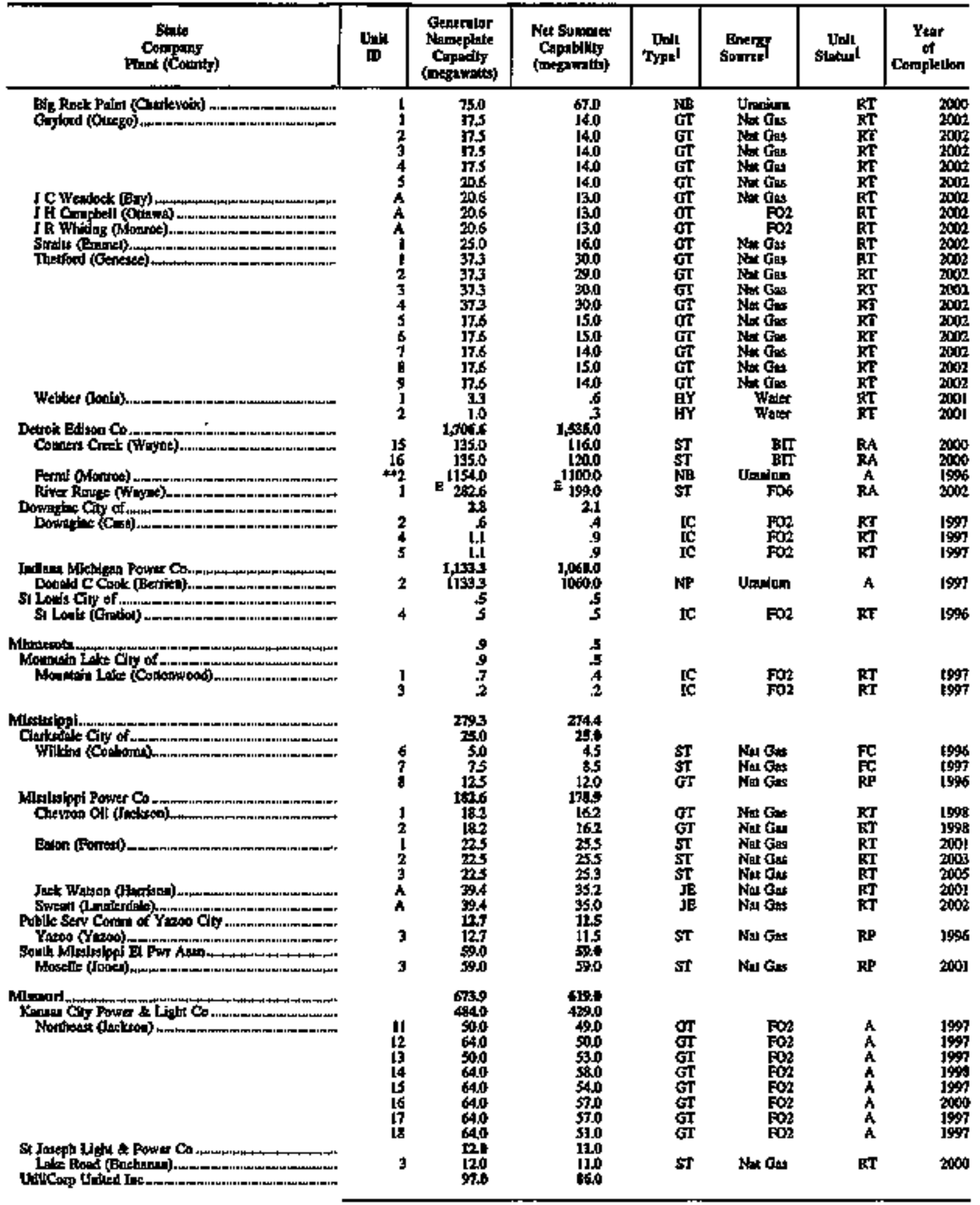

Set footmotrs at ead of whk. 
Table 22. Planned Generating Unit Changes at U.S. Electric Utiltites by State, Company, and Plant, 1996 Through 2005 as of January 1, 1996 (Continued)

\begin{tabular}{|c|c|c|c|c|c|c|c|}
\hline 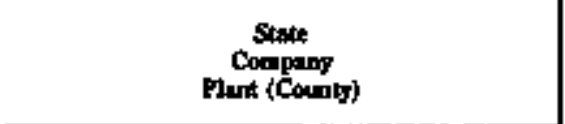 & II & 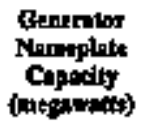 & $\begin{array}{l}\text { Nel Sumiter } \\
\text { Capabitly } \\
\text { (mequarats) }\end{array}$ & Typll & Bowrit & ornt & Caniditin \\
\hline 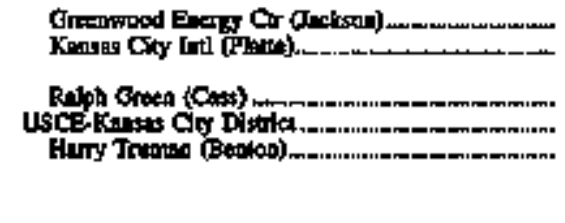 & $\mathrm{cTI}^{\frac{1}{1}}$ & $\begin{array}{l}61,0 \\
15,0 \\
180 \\
0.0 \\
098 \\
27.0 \\
27,0 \\
27,0\end{array}$ & $\begin{array}{l}600 \\
130 \\
130 \\
00 \\
0.00 \\
310 \\
310 \\
31.0\end{array}$ & $\begin{array}{l}\text { GT } \\
\mathbb{I R} \\
\text { GT } \\
\text { Fs } \\
\text { PS }\end{array}$ & $\begin{array}{c}\text { Foz } \\
\text { Nat Ges } \\
\text { Nel Ges } \\
\text { Wave } \\
\text { Wuer } \\
\text { Wuer }\end{array}$ & $\begin{array}{l}\mathbf{A} \\
\mathbf{A} \\
\mathbf{A} \\
\mathbf{A} \\
\mathbf{R P} \\
\mathbf{R P}\end{array}$ & $\begin{array}{l}1996 \\
1996 \\
1996 \\
1996 \\
1997 \\
1996 \\
1996\end{array}$ \\
\hline 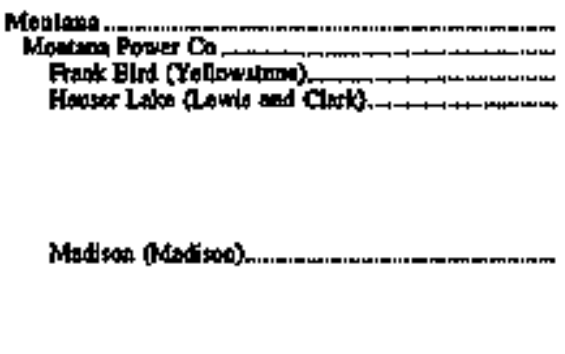 & $\begin{array}{l}5 \\
5 \\
2 \\
3 \\
4 \\
5 \\
6 \\
1 \\
2 \\
3 \\
4\end{array}$ & 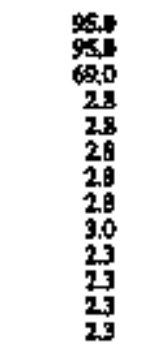 & $\begin{array}{r}16.7 \\
15.7 \\
700 \\
101 \\
= \\
= \\
= \\
= \\
65 \\
= \\
=\end{array}$ & 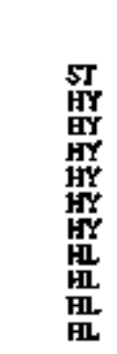 & Wht Gy & $\begin{array}{l}\text { RT } \\
\text { RPP } \\
\text { RPP } \\
\text { RPP } \\
\text { RPP } \\
\text { RPP } \\
\text { RPP } \\
\text { RPP } \\
\text { RPP } \\
\text { RPP }\end{array}$ & $\begin{array}{l}1997 \\
2002 \\
2002 \\
2002 \\
2002 \\
2002 \\
2000 \\
2002 \\
2002 \\
2002 \\
2002\end{array}$ \\
\hline 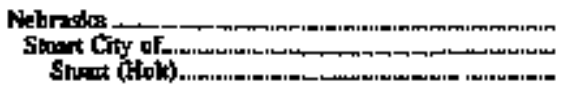 & 4 & $\frac{2}{2}$ & $\frac{2}{2}$ & tc & $\mathrm{EO} 2$ & RT & 1996 \\
\hline 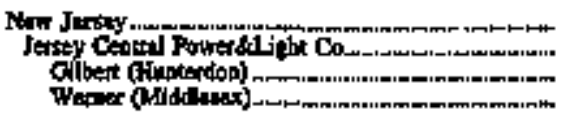 & $\begin{array}{l}3 \\
4\end{array}$ & $\begin{array}{c}125 \\
600 \\
600\end{array}$ & 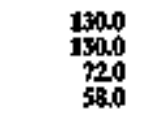 & ST & $\begin{array}{l}\text { E06 } \\
\text { EO6 }\end{array}$ & RT & $\begin{array}{l}1996 \\
1996\end{array}$ \\
\hline 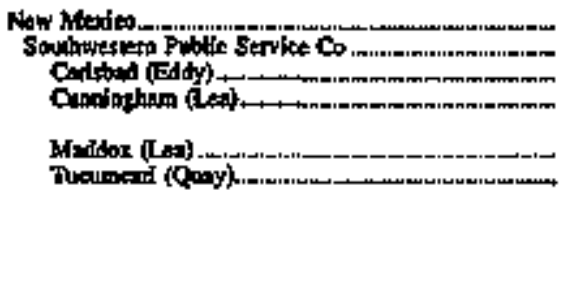 & $\begin{array}{l}5 \\
1 \\
2 \\
2 \\
3 \\
4 \\
6 \\
8 \\
9\end{array}$ & $\begin{array}{r}363.7 \\
383.7 \\
16.3 \\
75.0 \\
190.4 \\
86.9 \\
1.0 \\
2.3 \\
4.1 \\
3.0 \\
4.6\end{array}$ & $\begin{array}{r}34.4 \\
364 \\
160 \\
700 \\
196.0 \\
660 \\
10 \\
20 \\
3.0 \\
30 \\
50\end{array}$ & $\begin{array}{l}\text { GI } \\
\text { sT } \\
\text { sit } \\
\text { ic } \\
\text { ic } \\
\text { ic } \\
\text { ic } \\
\text { ic }\end{array}$ & 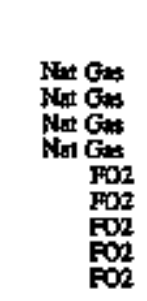 & $\begin{array}{l}\text { ET } \\
\text { RT } \\
\mathbf{R T} \\
\text { AT } \\
\text { RT } \\
\mathbf{R T} \\
\mathbf{R T} \\
\mathbf{R T} \\
\text { RT }\end{array}$ & $\begin{array}{l}2001 \\
1927 \\
2005 \\
2001 \\
2001 \\
2001 \\
2001 \\
2001 \\
2001\end{array}$ \\
\hline 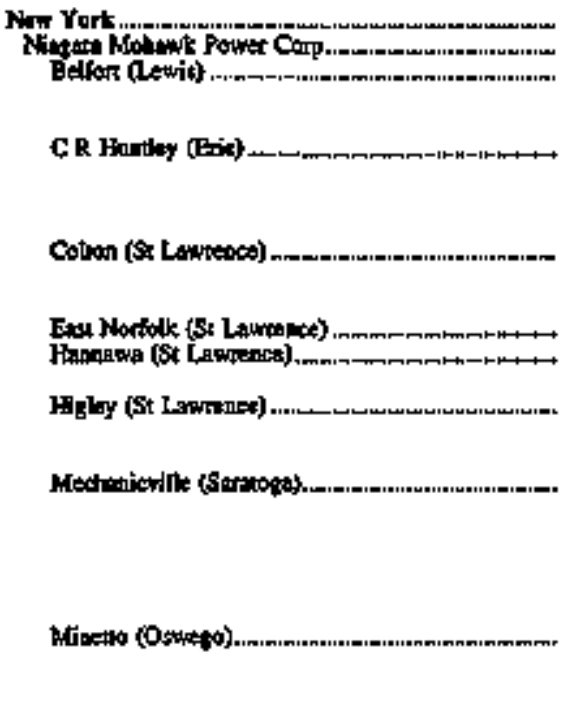 & $\begin{array}{r}1 \\
2 \\
3 \\
63 \\
64 \\
65 \\
66 \\
3 \\
2 \\
3 \\
1 \\
1 \\
2 \\
1 \\
2 \\
3 \\
1 \\
2 \\
3 \\
4 \\
5 \\
7 \\
\text { tYy } \\
\text { HY2 } \\
\text { HY }\end{array}$ & 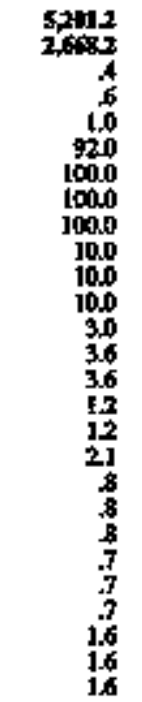 & 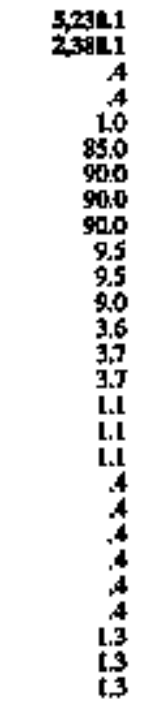 & 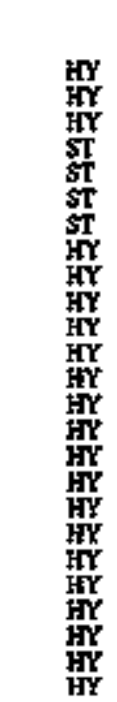 & 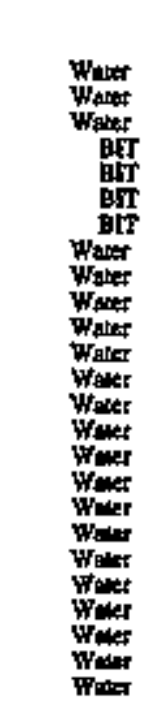 & $\begin{array}{l}\mathbf{R T} \\
\mathbf{R T} \\
\mathbf{R T} \\
\mathbf{R T} \\
\mathbf{R T} \\
\mathbf{R T} \\
\mathbf{R T} \\
\mathbf{R P} \\
\mathbf{R P} \\
\mathbf{R P} \\
\mathbf{R P} \\
\mathbf{R P} \\
\mathbf{R P} \\
\mathbf{R T} \\
\mathbf{R T} \\
\mathbf{R T} \\
\mathbf{R T} \\
\mathbf{R T} \\
\mathbf{R T} \\
\mathbf{R T} \\
\mathbf{R T} \\
\mathbf{R T} \\
\mathbf{R P} \\
\mathbf{R P} \\
\mathbf{R P}\end{array}$ & $\begin{array}{l}1990 \\
1998 \\
1990 \\
1909 \\
1999 \\
1999 \\
1998 \\
2000 \\
2000 \\
2000 \\
2000 \\
2000 \\
2000 \\
1998 \\
1998 \\
1998 \\
1999 \\
1999 \\
1999 \\
1999 \\
1999 \\
1999 \\
2000 \\
2000 \\
2000\end{array}$ \\
\hline
\end{tabular}

Ses fooponas at and of talbie. 
Table 22. Planned Generating Unit Changes at U.S. Electric Utilities by State, Company, and Plant, 1996 Through 2005 as of January 1, 1996 (Conthued)

\begin{tabular}{|c|c|c|c|c|c|c|c|}
\hline 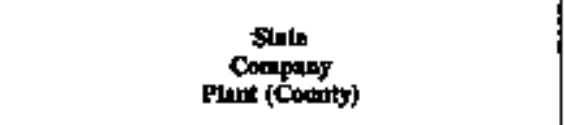 & $\begin{array}{l}\text { Unith } \\
\text { I }\end{array}$ & 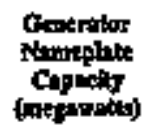 & $\begin{array}{l}\text { Net Sumpiner } \\
\text { Copphillyy } \\
\text { (mrogencts) }\end{array}$ & Typall & $\begin{array}{l}\text { Entry } \\
\text { Sourtal }\end{array}$ & Uint & $\begin{array}{c}\text { Year } \\
\text { of } \\
\text { Completion }\end{array}$ \\
\hline 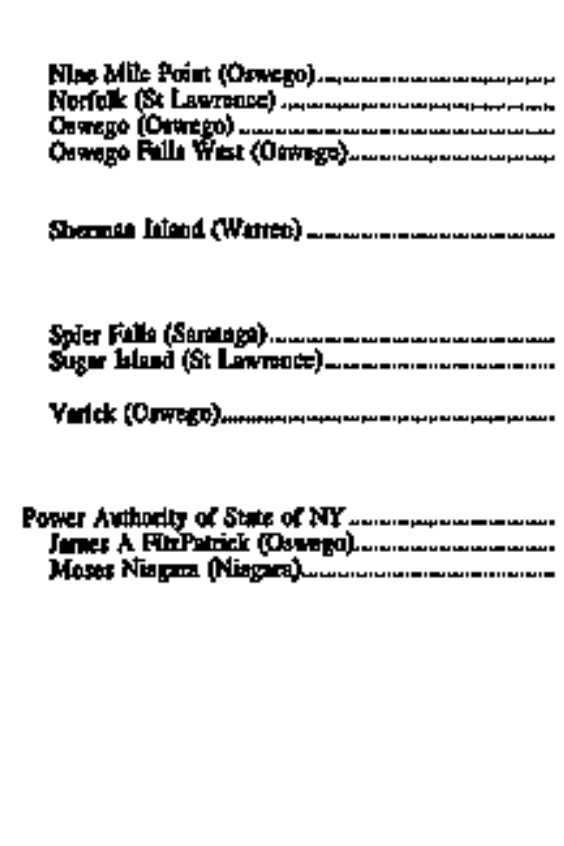 & $\begin{array}{c}\mathbf{H Y 4} \\
\mathbf{H Y 5} \\
+42 \\
1 \\
513 \\
513 \\
1 \\
2 \\
3 \\
2 \\
2 \\
3 \\
4 \\
5 \\
8 \\
1 \\
2 \\
2 \\
3 \\
4 \\
5 \\
1 \\
1 \\
1 \\
\text { t0 } \\
11 \\
12 \\
2 \\
3 \\
5 \\
6 \\
7 \\
8 \\
9\end{array}$ & 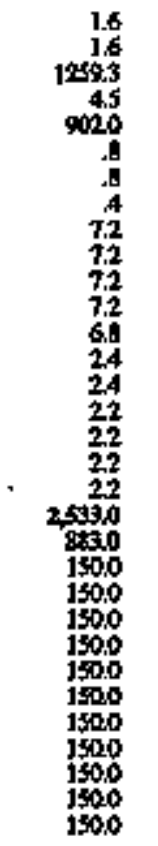 & $\begin{array}{r}1.3 \\
1.3 \\
5036.3 \\
3.8 \\
9020 \\
3 \\
3 \\
3 \\
7.0 \\
7.0 \\
7.0 \\
3.0 \\
3.3 \\
20 \\
20 \\
1.0 \\
1.0 \\
1.0 \\
1.0 \\
2,850.0 \\
60000 \\
2050.0 \\
= \\
= \\
= \\
= \\
= \\
= \\
= \\
= \\
\end{array}$ & 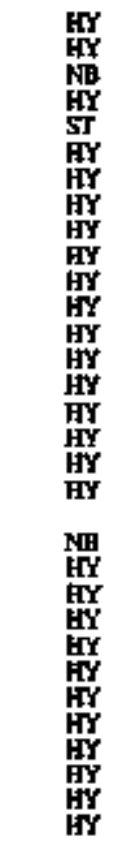 & 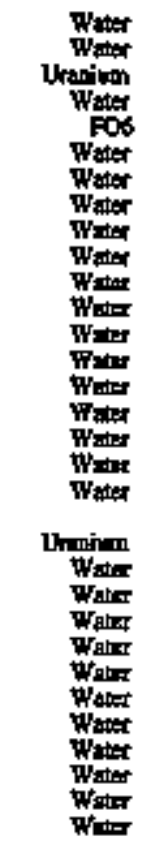 & $\begin{array}{l}\mathbf{R P} \\
\mathbf{R P} \\
\mathbf{A} \\
\mathbf{R P} \\
\mathbf{R A} \\
\mathbf{R T} \\
\mathbf{R T} \\
\mathbf{R P} \\
\mathbf{R P} \\
\mathbf{R P} \\
\mathbf{R P} \\
\mathbf{R P} \\
\mathbf{R P} \\
\mathbf{R P} \\
\mathbf{R P} \\
\mathbf{a P} \\
\mathbf{R P} \\
\hat{\mathbf{A}} \\
\hat{\mathbf{A}} \\
\hat{\mathbf{A}} \\
\hat{\mathbf{A}} \\
\hat{\mathbf{A}} \\
\hat{\mathbf{A}} \\
\hat{\mathbf{A}} \\
\hat{\mathbf{A}} \\
\hat{\mathbf{A}}\end{array}$ & 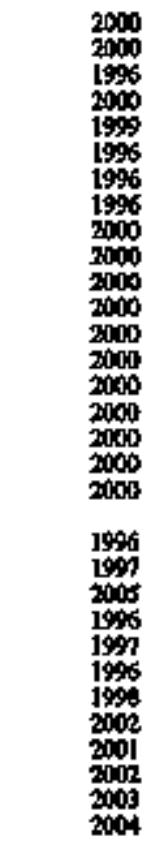 \\
\hline 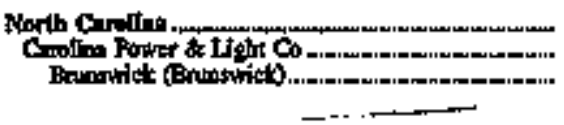 & $\omega_{2}$ & $\begin{array}{r}1,793,4 \\
1,73,4 \\
866,7 \\
866,7\end{array}$ & $\begin{array}{r}1,2.1 .0 \\
1,521.0 \\
75.0 \\
754.0\end{array}$ & N⿴囗十 & $\begin{array}{l}\text { Uhrim } \\
\text { Whetmm }\end{array}$ & $\hat{A}$ & 1999 \\
\hline 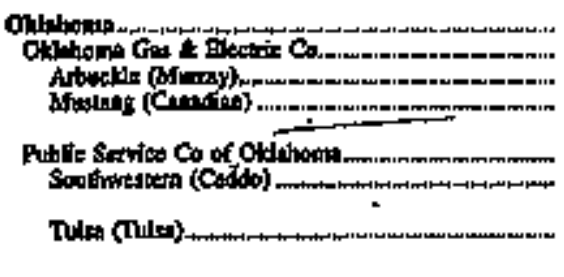 & $\begin{array}{l}1 \\
1 \\
3 \\
1 \\
2 \\
2 \\
3\end{array}$ & 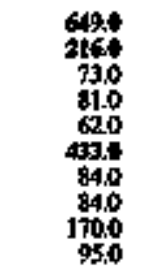 & 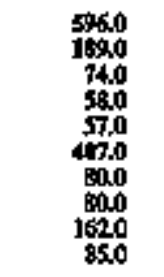 & $\begin{array}{l}S T \\
S T \\
S T \\
S T \\
S T \\
S T \\
S T\end{array}$ & 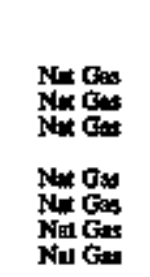 & $\begin{array}{l}\mathbf{R P} \\
\mathbf{R P} \\
\mathbf{R P} \\
\mathbf{R T} \\
\mathbf{R F} \\
\mathbf{R} \\
\mathbf{R h}\end{array}$ & $\begin{array}{l}2001 \\
2001 \\
2000 \\
2004 \\
2004 \\
3905 \\
1998 \\
1998\end{array}$ \\
\hline 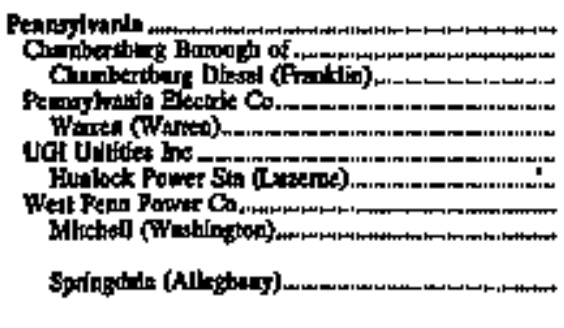 & $\begin{array}{l}5 \\
2 \\
3 \\
1 \\
2 \\
7 \\
8\end{array}$ & $\begin{array}{r}452 \\
211 \\
21 \\
423 \\
423 \\
50.0 \\
50.0 \\
3640 \\
74.8 \\
748 \\
748 \\
1406\end{array}$ & 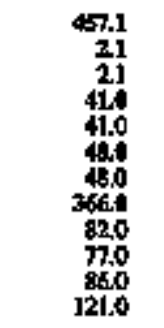 & $\begin{array}{l}\text { IC } \\
\mathbf{S T} \\
\mathbf{S T} \\
\mathbf{S T} \\
\underset{\mathbf{S T}}{\mathbf{S T}}\end{array}$ & $\begin{array}{l}\text { Nar Gas } \\
\text { BIT } \\
\text { ANT } \\
\text { FO2 } \\
\text { Fo5 } \\
\text { FO6 } \\
\text { FO6 }\end{array}$ & $\begin{array}{l}\text { RP } \\
\mathbf{R P} \\
\text { RT } \\
\text { A } \\
\text { RA }\end{array}$ & $\begin{array}{l}1996 \\
1997 \\
2904 \\
1997 \\
1997 \\
2000 \\
2000\end{array}$ \\
\hline 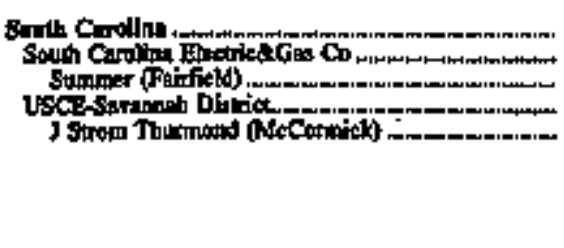 & $\begin{array}{r}-1 \\
1 \\
2 \\
3 \\
4 \\
5\end{array}$ & 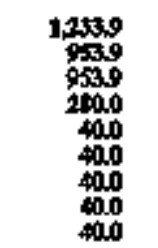 & $\begin{array}{r}1,165.0 \\
885,0 \\
8850 \\
2800 \\
40.0 \\
40.0 \\
40.0 \\
40.0 \\
40.0\end{array}$ & 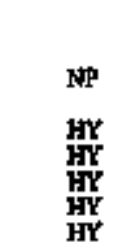 & 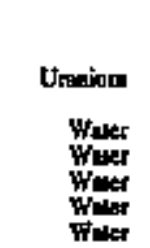 & 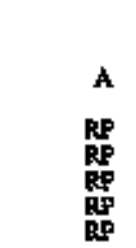 & $\begin{array}{l}1996 \\
2000 \\
2001 \\
2000 \\
2002 \\
2002\end{array}$ \\
\hline
\end{tabular}

Ser footontet at end of mallo. 
Table 22. Planned Generating Unit Changes at U.S. Electric Utilities by State, Company, and Plant, 1996 Through 2005 as of January 1, 1996 (Continued)

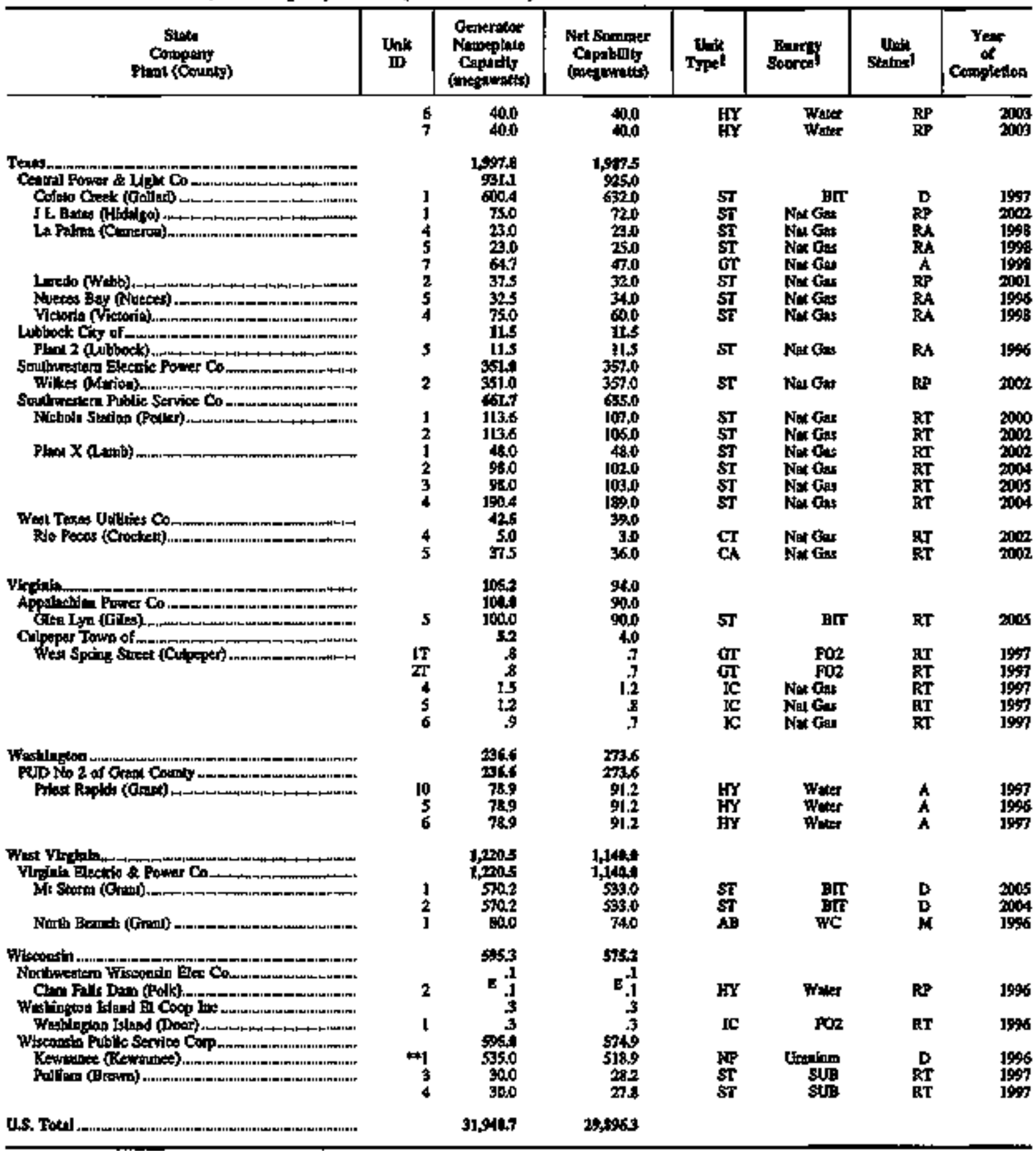

\footnotetext{
1 Ses Appendix $B$ for coder

- less then 0,05 mogrwath.

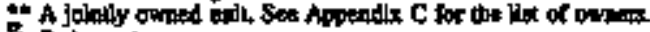

Estimilied.

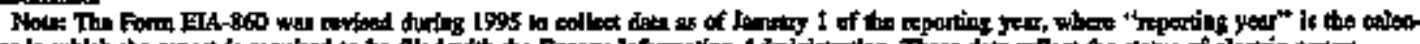

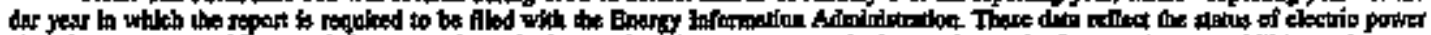

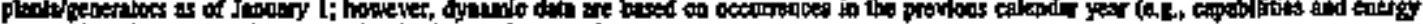

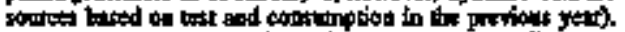

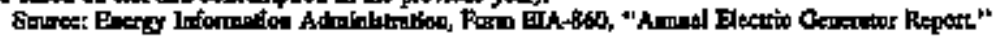


Table 23. Planned Generating Unit Additions at U.S. Electric Utilities by State, Company, and Plant, 1996 Through 2005 as of Jamuary i, 1996

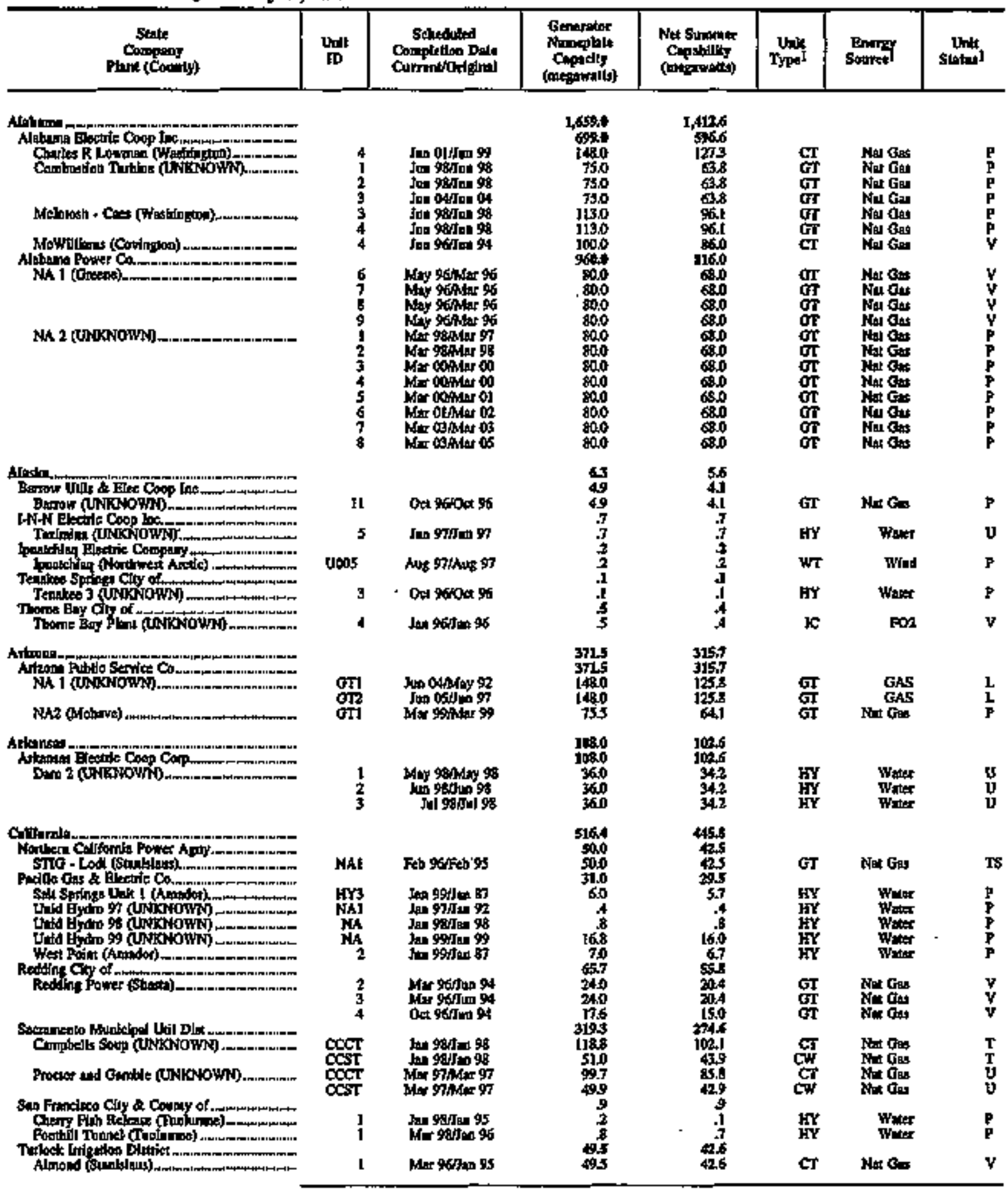

Sos rooknowe at tad of uabto. 
Table 23. Planned Generating Unit Additions at U.S. Electric Utilities by State, Company, and Plant, 1996 Through 2005 as of Jantsary 1, 1996 (Continued)

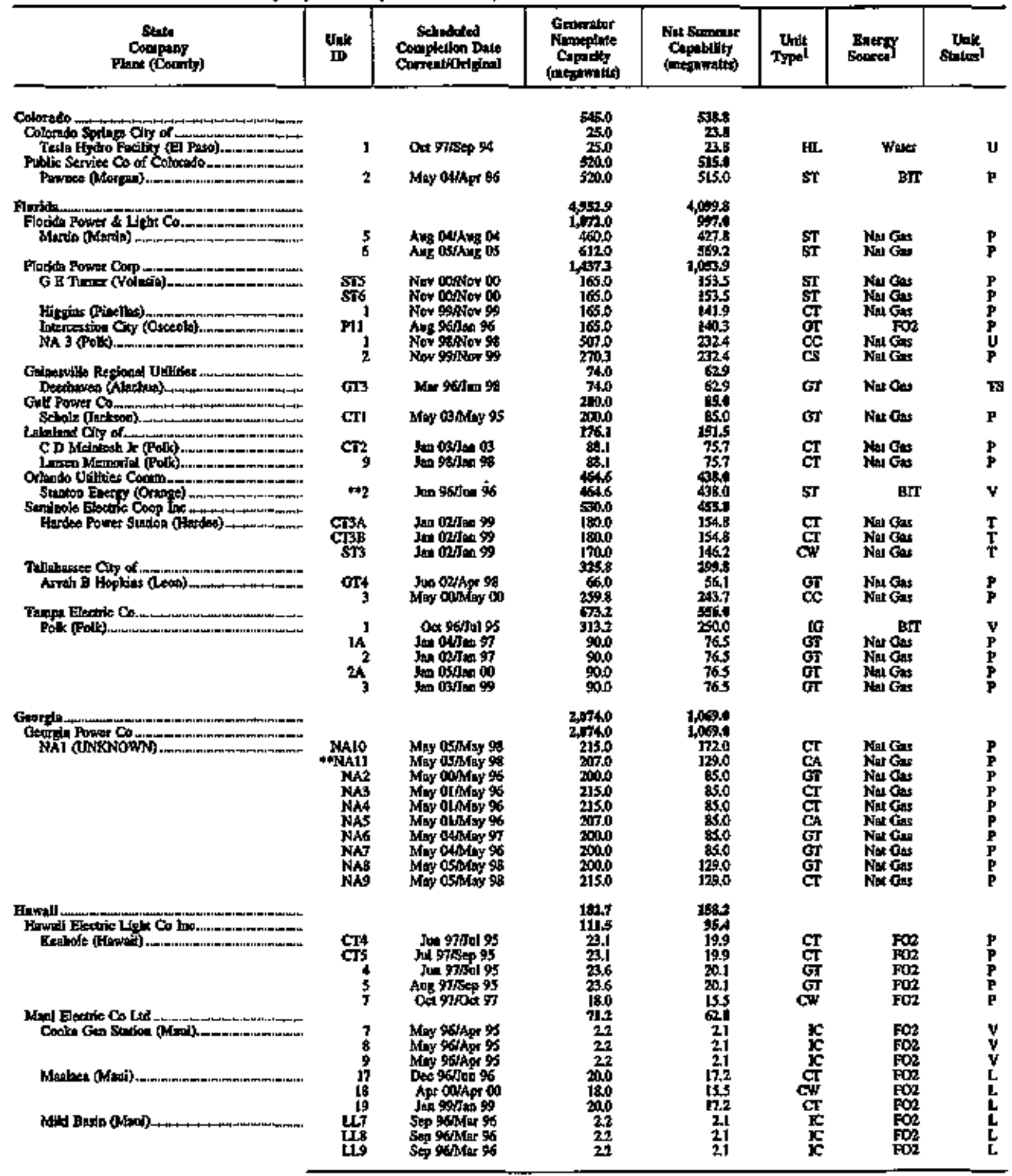

Set foomold at and of bitte. 
Table 23. Plamned Generating Unilt Additions at U.S. Electric Utilities by State, Company, and Plant, 1996 Through 2005 as of January 1, 1996 (Continued)

\begin{tabular}{|c|c|c|c|c|c|c|c|}
\hline Plant (Coututy) & IInt & 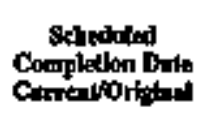 & 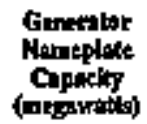 & $\begin{array}{l}\text { Net Sowner } \\
\text { Crpabtily } \\
\text { (dieprintis) }\end{array}$ & $\underset{\text { thpet }}{\text { thet }}$ & $\begin{array}{l}\text { Futerity } \\
\text { soorte }\end{array}$ & statul \\
\hline 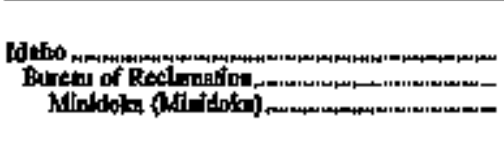 & 9 & 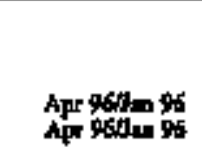 & $\begin{array}{l}2.6 \\
200 \\
100 \\
100\end{array}$ & $\begin{array}{r}19.0 \\
15.5 \\
9.5\end{array}$ & $\begin{array}{l}\mathbf{H Y} \\
\mathbf{H Y}\end{array}$ & Water & $\stackrel{P}{\mathbf{P}}$ \\
\hline 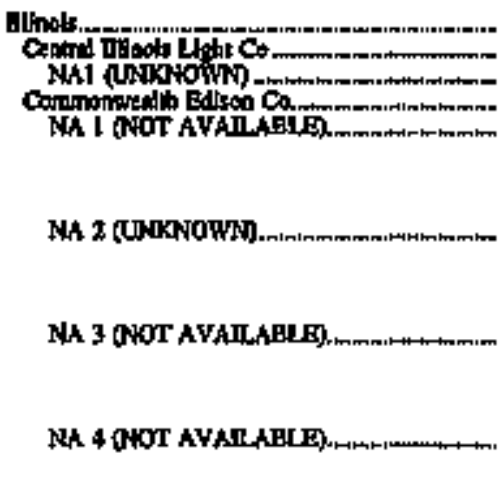 & 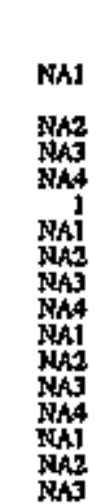 & 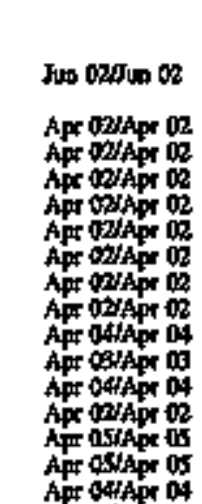 & $\begin{array}{r}28919 \\
1000 \\
1000 \\
26150 \\
1750 \\
1750 \\
1750 \\
1750 \\
1750 \\
1750 \\
1750 \\
1750 \\
3750 \\
1750 \\
1750 \\
1750 \\
175.0 \\
1750 \\
1750\end{array}$ & 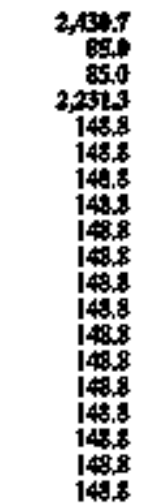 & $\begin{array}{l}\text { GI } \\
\text { GI } \\
\text { GI } \\
\text { GI } \\
\text { GT } \\
\text { GI } \\
\text { GI } \\
\text { GI } \\
\text { GI } \\
\text { GI } \\
\text { GI } \\
\text { GI } \\
\text { GI } \\
\text { GI } \\
\text { GI } \\
\text { GI }\end{array}$ & 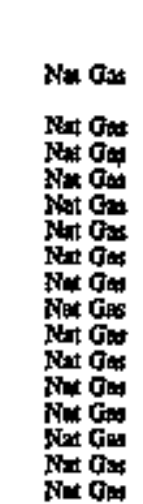 & $\begin{array}{l}p \\
p \\
p \\
p \\
p \\
p \\
p \\
p \\
p \\
p \\
p \\
p \\
p \\
P \\
P \\
P\end{array}$ \\
\hline 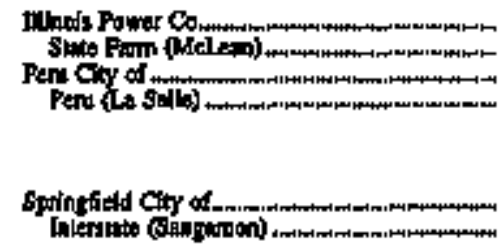 & $\begin{array}{r}1 \\
\operatorname{HCt} \\
\operatorname{Hct} \\
\operatorname{HC} \\
1\end{array}$ & 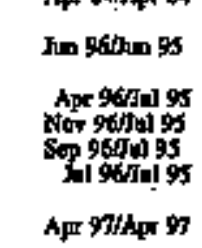 & $\begin{array}{r}53 \\
53 \\
7.6 \\
1.9 \\
19 \\
19 \\
19 \\
1540 \\
{[540}\end{array}$ & $\begin{array}{r}5.7 \\
5.3 \\
7.2 \\
1.8 \\
1.5 \\
1.8 \\
10.8 \\
102.0\end{array}$ & $\begin{array}{l}\text { IC } \\
\text { FY } \\
\mathbf{H Y} \\
\mathbf{H Y} \\
\mathbf{H Y} \\
\text { Or }\end{array}$ & $\begin{array}{l}\text { Foz } \\
\text { Water } \\
\text { Water } \\
\text { Water } \\
\text { Whet Oas }\end{array}$ & $\begin{array}{l}\mathbf{T S} \\
\mathbf{v} \\
\mathbf{u} \\
\mathbf{u} \\
\mathbf{v}\end{array}$ \\
\hline Indiars & $\begin{array}{r}\text { NA6 } \\
\text { NA7 } \\
\text { NAS } \\
! \\
2 \\
3 \\
4 \\
5\end{array}$ & 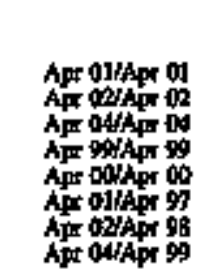 & $\begin{array}{l}6400 \\
6400 \\
80.0 \\
80.0 \\
80.0 \\
000 \\
80.0 \\
80.0 \\
80.0 \\
80.0\end{array}$ & 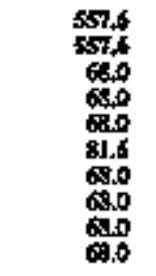 & $\begin{array}{l}\text { GT } \\
\text { GT } \\
\text { GT } \\
G T \\
\text { or } \\
\text { Gr } \\
\text { GT } \\
\text { GT }\end{array}$ & 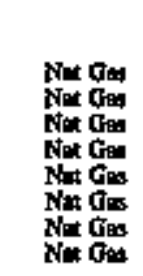 & $\begin{array}{l}\mathbf{p} \\
\mathbf{p} \\
\mathbf{p} \\
\mathbf{p} \\
\mathbf{P} \\
\mathbf{P} \\
\mathbf{P} \\
\mathbf{P}\end{array}$ \\
\hline 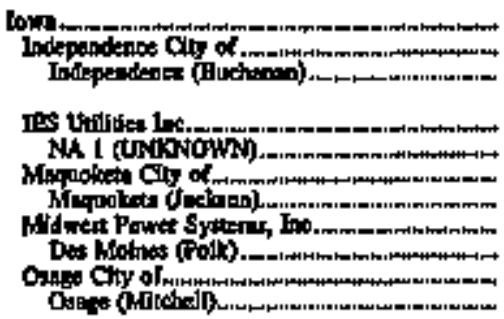 & $\begin{array}{r}6 \\
9 \\
1 \\
6 \\
6 A \\
7\end{array}$ & 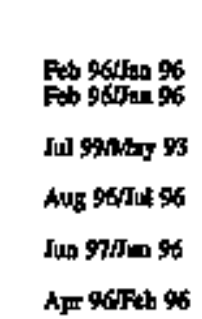 & $\begin{array}{r}7.5 \\
3.7 \\
19 \\
19 \\
38.0 \\
380 \\
15 \\
18 \\
250 \\
250 \\
40 \\
40\end{array}$ & 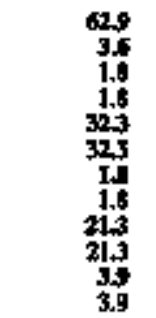 & 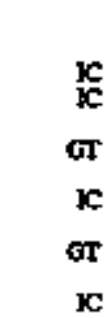 & $\begin{array}{r}\text { OOz } \\
\text { FOt } \\
\text { Star Go: } \\
\text { FOz } \\
\text { Jat Os: } \\
\text { FOn }\end{array}$ & $\begin{array}{l}\mathbf{v} \\
v \\
p \\
v \\
p \\
y\end{array}$ \\
\hline 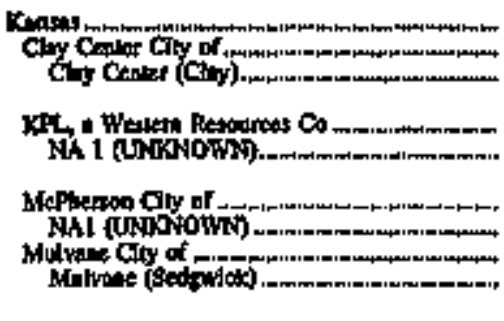 & 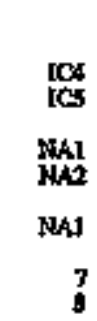 & 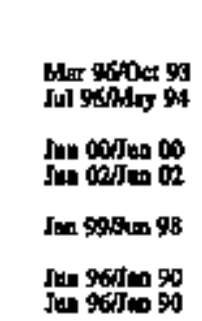 & $\begin{array}{r}301.9 \\
7.5 \\
3.5 \\
3.5 \\
174 . \\
87.0 \\
87.6 \\
135.5 \\
315.6 \\
1.2 \\
.6 \\
.6\end{array}$ & $\begin{array}{r}25,2 \\
6.5 \\
3.4 \\
3.4 \\
147.9 \\
74.0 \\
74.0 \\
983 \\
98.3 \\
1.2 \\
.6 \\
.6\end{array}$ & $\begin{array}{l}\mathbf{K} \\
\mathbf{K} \\
\mathbf{o r} \\
\mathbf{\sigma r} \\
\mathbf{G} \\
\mathrm{TC}\end{array}$ & 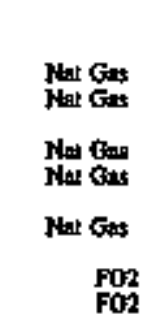 & $\begin{array}{l}\mathbf{Y} \\
\mathbf{Y} \\
\mathbf{P} \\
\mathbf{P} \\
\mathbf{P} \\
\mathbf{Y} \\
\mathbf{V}\end{array}$ \\
\hline
\end{tabular}

Sep foototies al ead of thble 
Table 23. Planned Generating Unit Additions at US. Electric Utilities by State, Company, and Plant, 1996 Through 2005 as of January 1, 1996 (Continued)

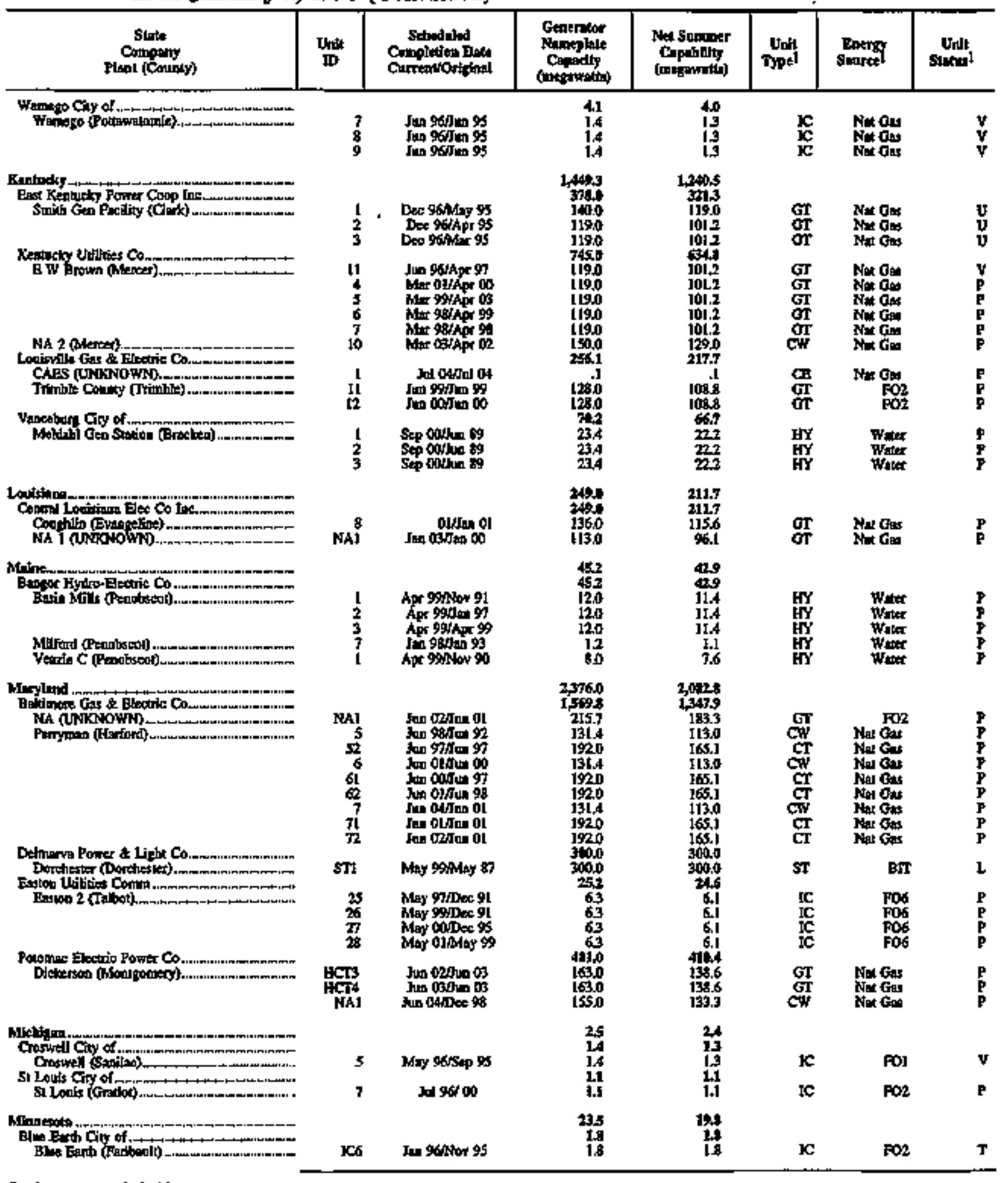

Sos foomoled an opd of ulipla. 
Table 23. Planned Generafing Unit Additions at U.S. Electric Utilities by State, Company, and Plant, 1996 'Through 2005

as of January 1, 1996 (Continued)

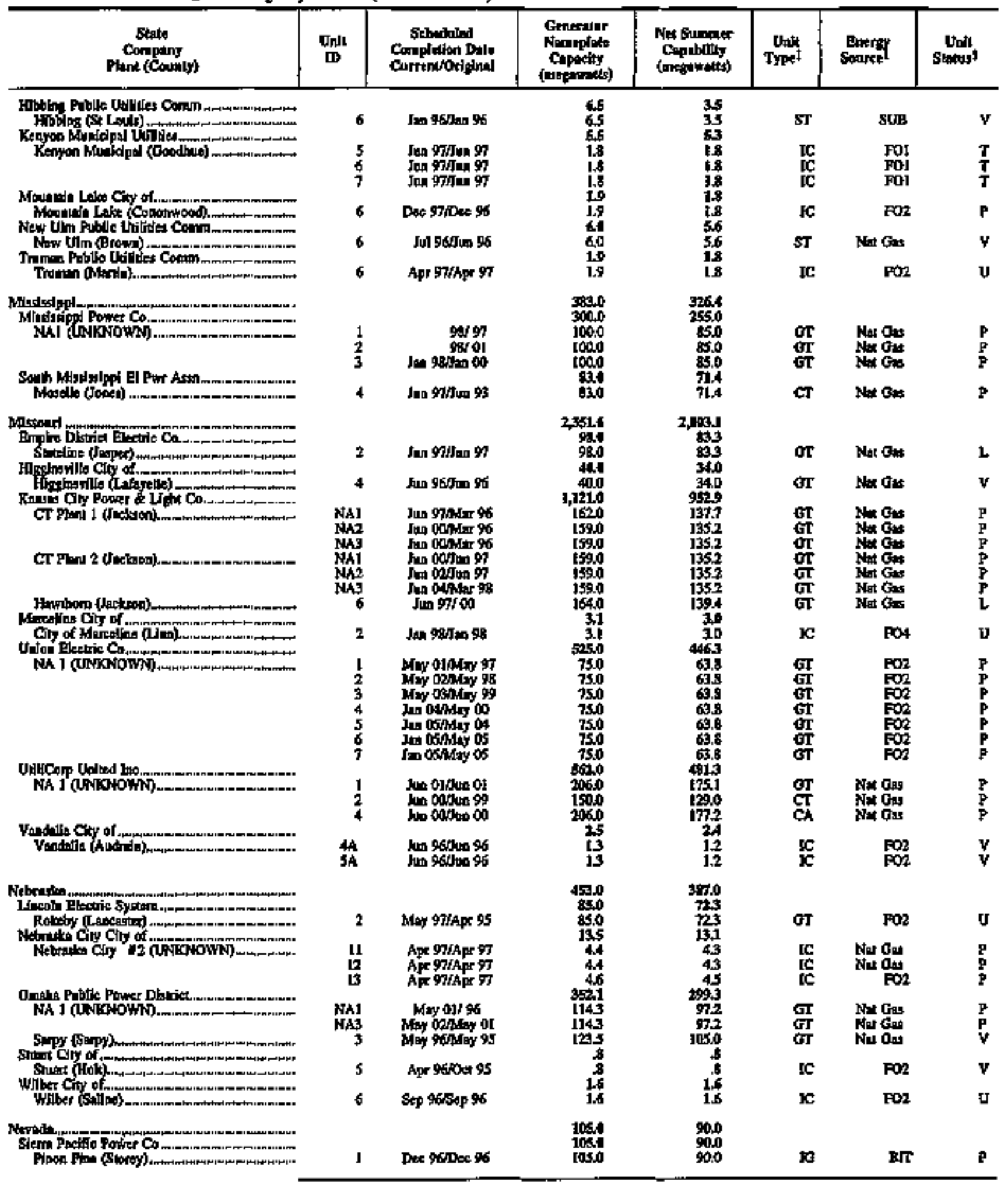

Sect footions ax ent of utile. 
Table 23. Plamed Generating Unit Additions at U.S. Electric Uilities by State, Company, and Plant, 1996 Through 2005 as of Jamuary 1, 1996 (Conthmed)

\begin{tabular}{|c|c|c|c|c|c|c|c|}
\hline 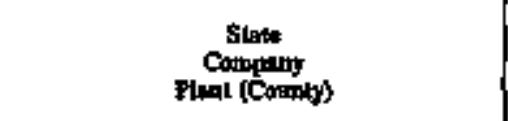 & $\begin{array}{l}\text { D'll } \\
\text { D }\end{array}$ & 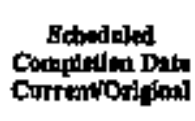 & 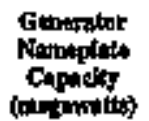 & 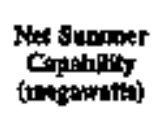 & Iypot? & Sotercet & Drolut \\
\hline 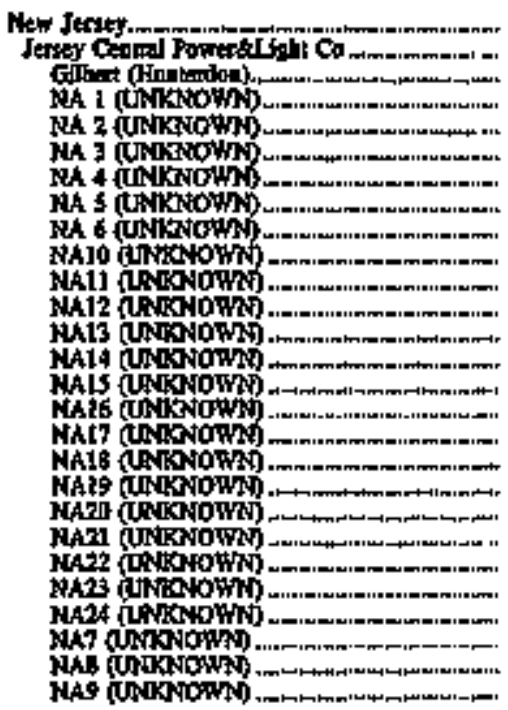 & $\begin{array}{r}\mathbf{1 0} \\
\mathbf{1} \\
\mathbf{1} \\
\mathbf{1} \\
\mathbf{j} \\
\mathbf{j} \\
\mathbf{j} \\
\mathbf{j} \\
\mathbf{1} \\
\mathbf{j} \\
\mathbf{j} \\
\mathbf{j} \\
\mathbf{j} \\
\mathbf{j} \\
\mathbf{j} \\
\mathbf{j} \\
\mathbf{j} \\
\mathbf{1} \\
\mathbf{1} \\
\mathbf{j} \\
\mathbf{j} \\
\mathbf{j} \\
\mathbf{j} \\
\mathbf{j} \\
\mathbf{1}\end{array}$ & 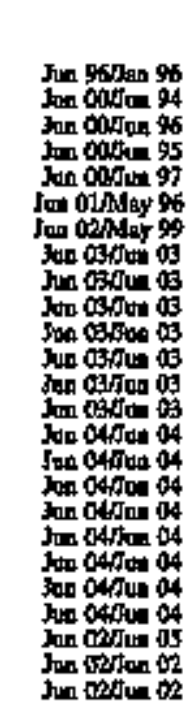 & $\begin{array}{l}3, t+0 \\
3,1+0 \\
16[0 \\
161.0 \\
161.0 \\
161.0 \\
161.0 \\
161.0 \\
161.0 \\
1100 \\
1100 \\
1100 \\
1100 \\
1100 \\
1200 \\
1100 \\
1100 \\
1 \neq 00 \\
1100 \\
1100 \\
1100 \\
1100 \\
1100 \\
1100 \\
1100 \\
1100 \\
1100\end{array}$ & 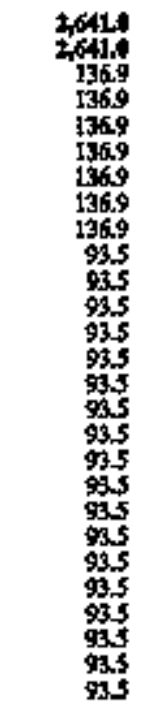 & 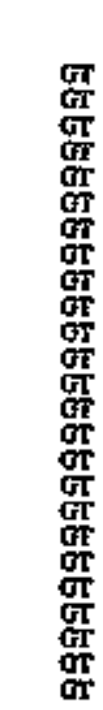 & 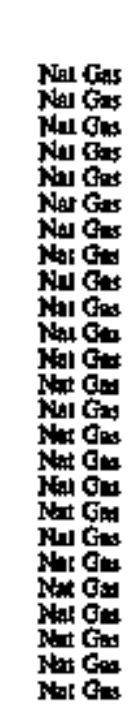 & 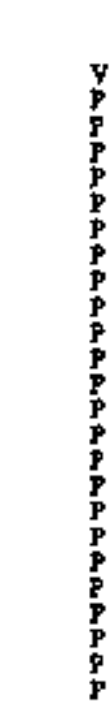 \\
\hline 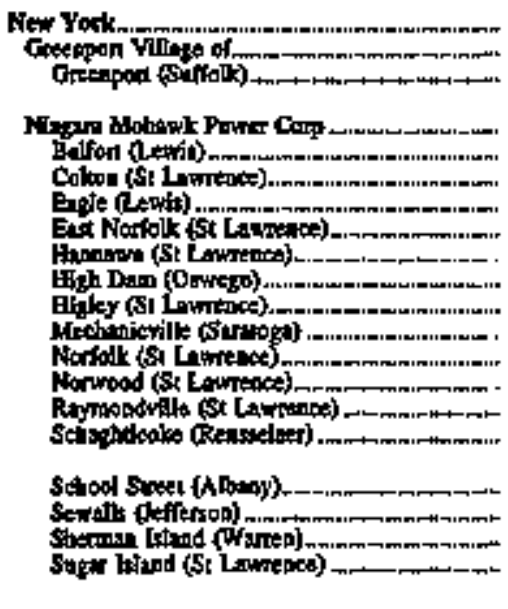 & $\begin{array}{r}2 \\
7 \\
4 \\
4 \\
5 \\
2 \\
3 \\
5 \\
4 \\
N 1 \\
2 \\
2 \\
2 \\
5 \\
6 \\
6 \\
3 \\
6 \\
3 \\
4\end{array}$ & 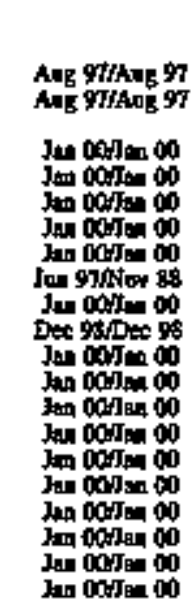 & 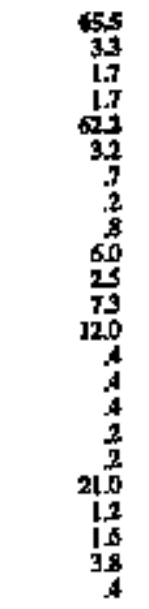 & $\begin{array}{r}6.3 \\
3.7 \\
1.6 \\
1.6 \\
59.1 \\
3.0 \\
.7 \\
.2 \\
.7 \\
5.7 \\
2.4 \\
6.9 \\
11.4 \\
.4 \\
.4 \\
.4 \\
.1 \\
.1 \\
20.0 \\
1.1 \\
1.5 \\
3.6 \\
.4\end{array}$ & $\begin{array}{l}\text { IC } \\
\text { IC } \\
\text { HYY } \\
\text { EYY } \\
\mathbf{H Y Y} \\
\mathbf{H Y} \\
\mathbf{H Y} \\
\mathbf{H Y} \\
\mathbf{H Y} \\
\mathbf{H Y} \\
\mathbf{H Y} \\
\mathbf{H Y} \\
\mathbf{H Y} \\
\mathbf{H Y} \\
\mathbf{H Y} \\
\mathbf{H Y} \\
\mathbf{H Y} \\
\mathbf{H Y} \\
\mathbf{H Y}\end{array}$ & 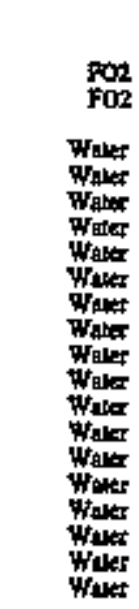 & $\begin{array}{l}\mathbf{U} \\
\mathbf{U} \\
\mathbf{P} \\
\mathbf{p} \\
\mathbf{p} \\
\mathbf{P} \\
\mathbf{p} \\
\mathbf{p} \\
\mathbf{p} \\
\mathbf{p} \\
\mathbf{p} \\
\mathbf{p} \\
\mathbf{p} \\
\mathbf{p} \\
\mathbf{p} \\
\mathbf{P} \\
\mathbf{p} \\
\mathbf{p} \\
\mathbf{p} \\
\mathbf{p}\end{array}$ \\
\hline 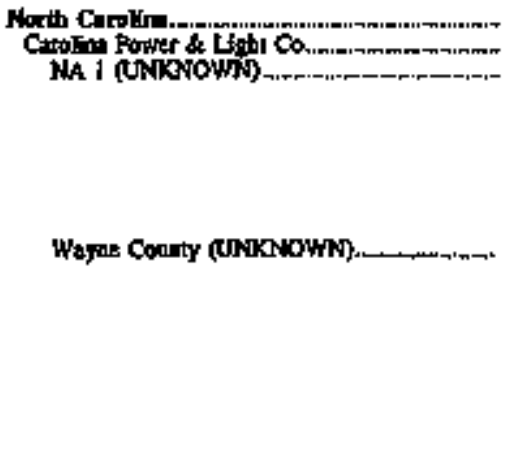 & $\begin{array}{r}1 \\
2 \\
3 \\
4 \\
5 \\
6 \\
7 \\
1 \\
10 \\
2 \\
3 \\
4 \\
5 \\
6 \\
7 \\
8\end{array}$ & 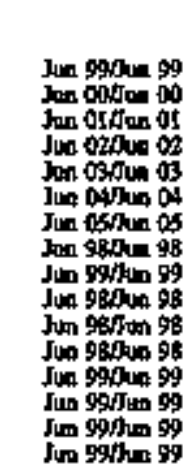 & $\begin{array}{r}4,745 \\
4,319,3 \\
8470 \\
3660 \\
3650 \\
3650 \\
3660 \\
2440 \\
2440 \\
211.4 \\
1250 \\
2118 \\
2158 \\
1350 \\
1250 \\
1250 \\
1250 \\
1250\end{array}$ & $\begin{array}{r}4,000.5 \\
3,671.4 \\
720.0 \\
311.1 \\
311.4 \\
311.1 \\
311.3 \\
207.4 \\
207.4 \\
180.0 \\
106.3 \\
180.0 \\
180.0 \\
114.8 \\
106.3 \\
106.3 \\
10.3 \\
105.3\end{array}$ & 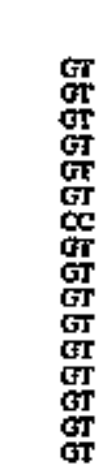 & 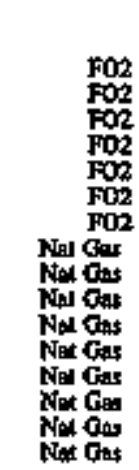 & $\begin{array}{l}\mathbf{p} \\
\mathbf{p} \\
\mathbf{p} \\
\mathbf{p} \\
\mathbf{p} \\
\mathbf{p} \\
\mathbf{p} \\
\mathbf{L} \\
\mathbf{p} \\
\mathbf{L} \\
\mathbf{L} \\
\mathbf{p} \\
\mathbf{p} \\
\mathbf{p} \\
\mathbf{p} \\
\mathbf{p}\end{array}$ \\
\hline
\end{tabular}

Sec foopones at ond of cable 
Table 23. Planned Generating Unit Additions at U.S. Electric Utilities by State, Company, and Plant, 1996 Through 2005 as of January 1, 1996 (Continued)

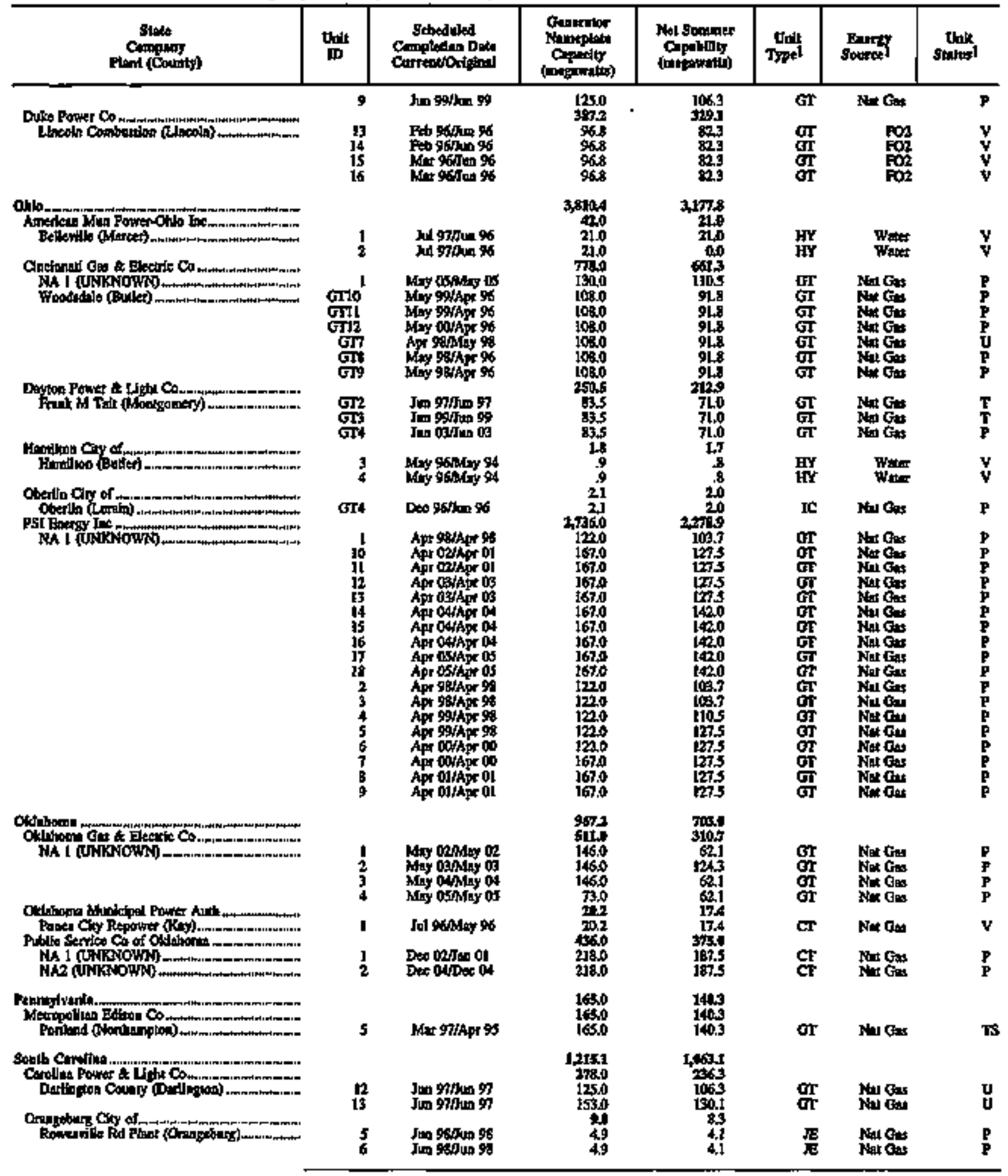

ses footrokes al and of bibit. 
Table 23. Planned Generating Unit Additions at U.S. Electric Utilities by State, Company, and Plant, 1996 Through 2005 as of January 1, 1996 (Continuzeii)

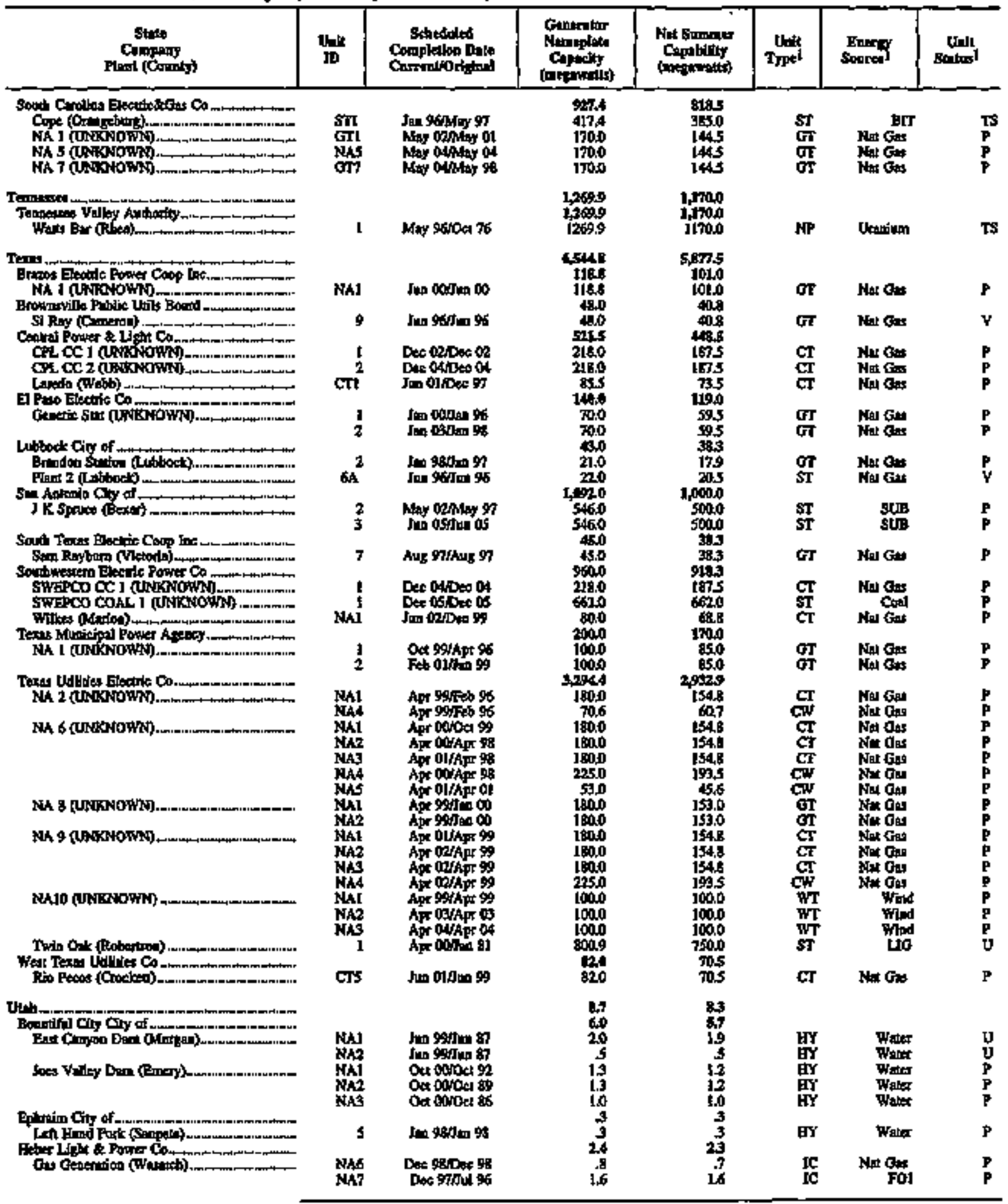

Set footions at end of tablo 
Table 23. Planned Generating Unit Additions at U.S. Ejectric Utilities by State, Company, and Plant, 1996 Throwgh 2005

as of January 1, 1996 (Continued)

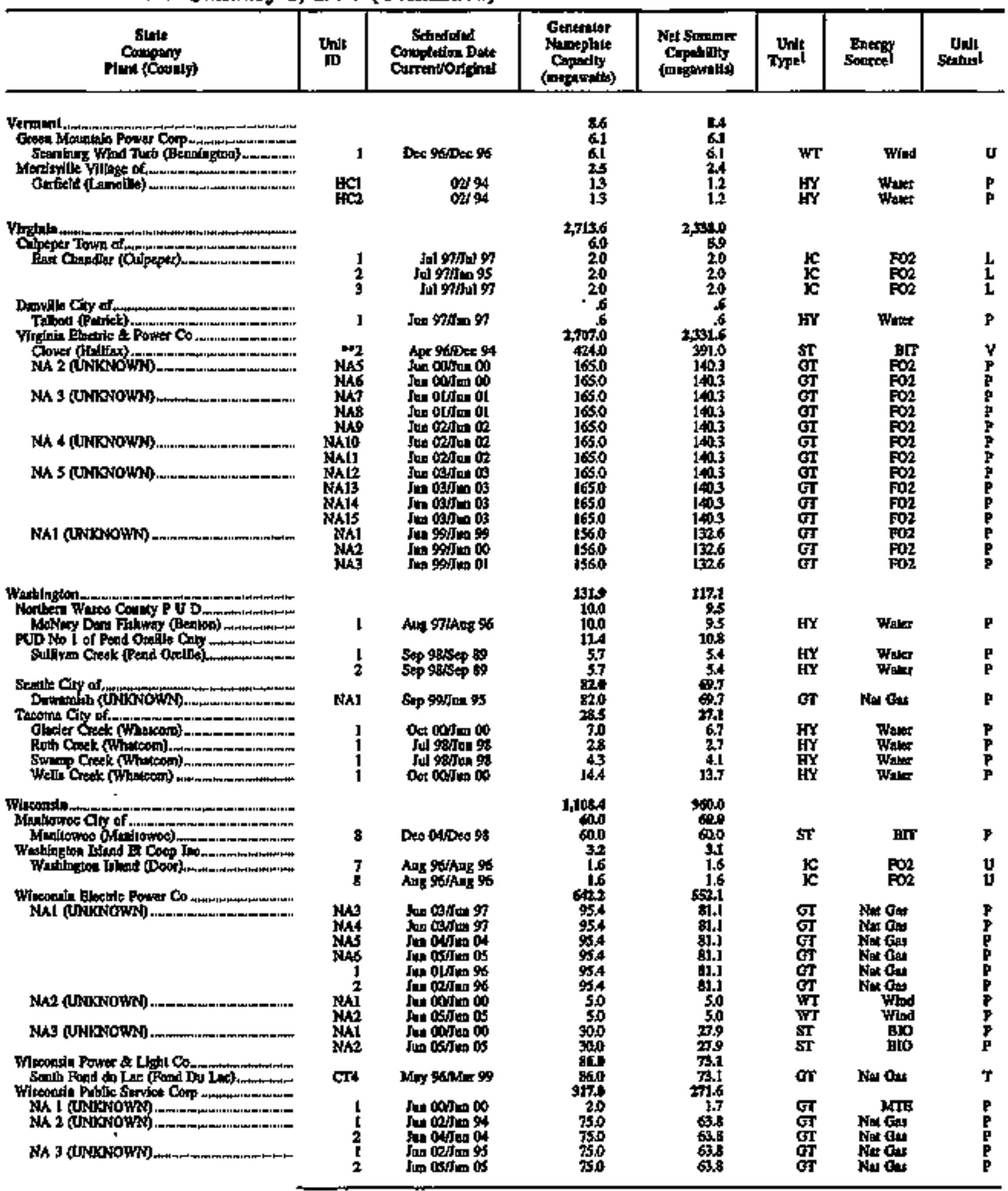

Sec lootholig at end of ublo. 
Table 23. Planned Generating Unit Additions at U.S. Electric Utflities by State, Company, and Plant, 1996 Through 2005 as of January 1, 1996 (Continued)

\begin{tabular}{|c|c|c|c|c|c|c|c|}
\hline 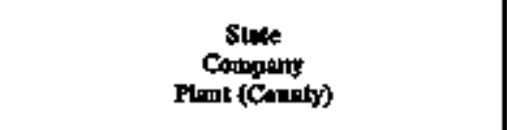 & II & 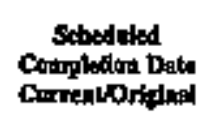 & 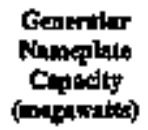 & 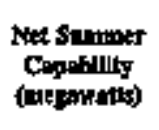 & لt!l & Baproy & Itatul \\
\hline 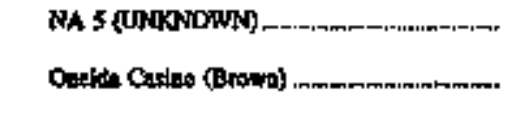 & & 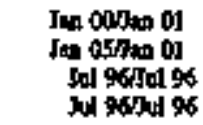 & $\begin{array}{r}100 \\
1.0 \\
20 \\
20\end{array}$ & $\begin{array}{r}10.0 \\
1.0 \\
20 \\
20\end{array}$ & pW & w & \\
\hline 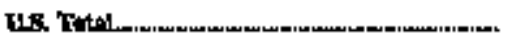 & & & 4,60025 & $40,15: 2$ & & & \\
\hline
\end{tabular}

1 Sect Appentix $\mathrm{B}$ ti codet.

- Less bin dos morrastit.

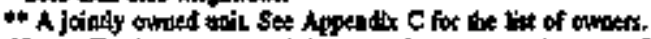

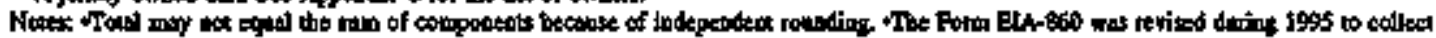

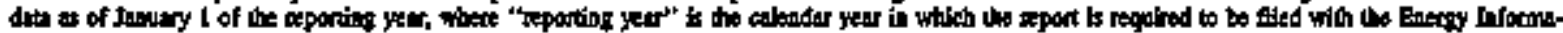

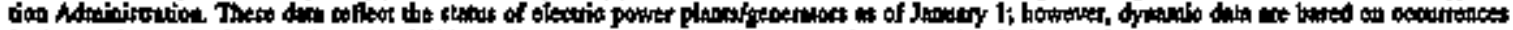

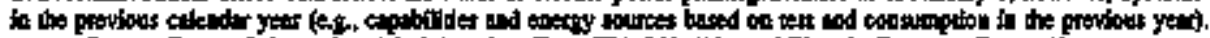

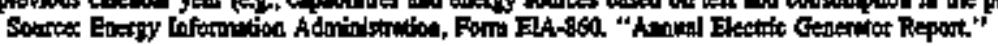

Table 24. Existing Capacity and Planned Capacity Additions at U.S. Nonutility Power Producers, as of December 31, 1995 (megawatts)

\begin{tabular}{|c|c|c|}
\hline Enerty Starte & $\begin{array}{l}\text { Botiting } \\
\text { 160s }\end{array}$ & 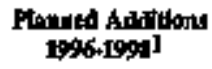 \\
\hline 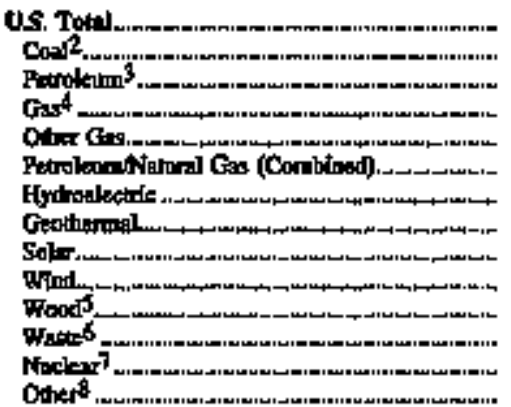 & $\begin{array}{r}70,754 \\
10,877 \\
2,116 \\
27,506 \\
1,217 \\
10,479 \\
3,799 \\
1,295 \\
354 \\
1,7239 \\
6,855 \\
3,430 \\
- \\
574\end{array}$ & $\begin{array}{l}4,004 \\
348 \\
21 \\
1,046 \\
\frac{1,144}{54} \\
- \\
-70 \\
264 \\
-\end{array}$ \\
\hline
\end{tabular}

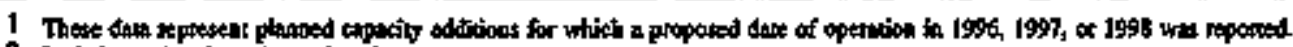

2 lucludes andhacite cutm and oos watte

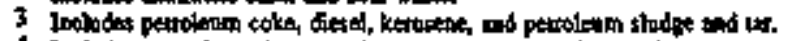

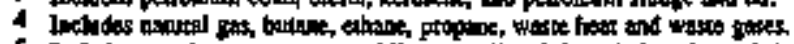

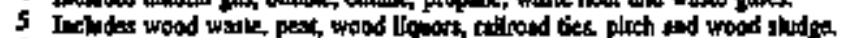

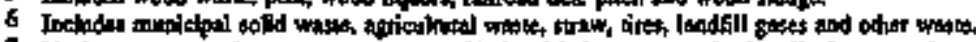

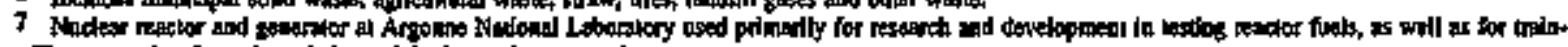

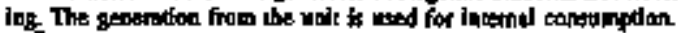

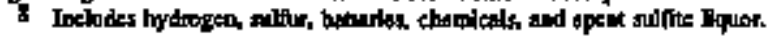

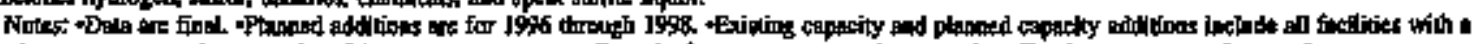

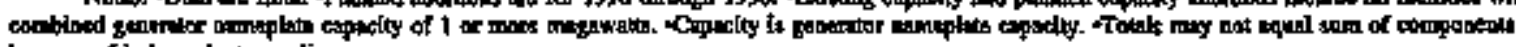
beentere of indepeodent mundin:

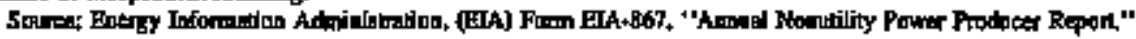


Appendix A

Technical

Notes 


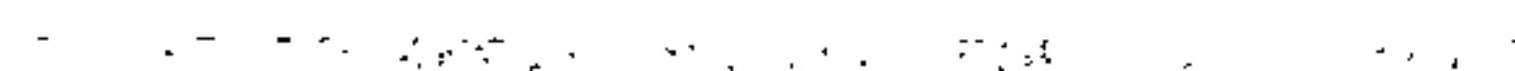




\section{Technical Notes}

\section{Sources of Data}

A synopsis of the data collection system used to prepare the Inventory of Power Plants in the United States is presented below. This synopsis reflects the Form EIA-860 data collection system, as of January 1, 1996.

The following are the primary changes that were made to the Form EIA-860 data collection during 1995, which are effective with the January 1 , 1996 reporting. (For an update on the definitions of various codes, see Appendix B.)

- Datz are reported as of January 1 of the reporting year, where reporting year is the calendar year in which the report is filed with the Energy Informa. tion Adminisiration.

- Respondents were given the option to file directly with EIA or file through an agent of their choice.

- Scbedule II - Power Plant Site Information: The requirements for elevation and type of cooling wore eliminated.

- Schedule III - Generator Inforuatod: Tho requirements for service type, first olectricity date, start-up fuels, third entergy source, date of cancellation, and reasons for delay/cancellation wers eliminated. The requirements for "mode of transportation of fizel" and "ownership type" were added.

\section{Form EIA-B60, "Annual Electric Cenerator Report"}

The Form EIA-860 provides for the annual data collection of information pertaining to powar plants owned and operated by electric utilities. The survey includes information on existing power plants and the 10-year plans for new plants, generating unit additons, modifications, and retirements. Data on Form EIA-860 are collected from all electric utitities in the United States that operate powter plants ot plan to operate a power plant within 10 years of the reporting year.

Instrument and Desige History. The Form ELA-860 was implemented in January 1985 to collect data as of year-end 1984. The Federal Energy Administration Act of 1974 (Public Law 93-275) defines the legislative authority to collect these data.

Data Processing. Data on Form ELA-860 are collected from approximately 900 respondents. The forms are mailed to the respondents in November or Decenber to collect data as of January 1 of the reporting year, where the reporting year is the celender year in which the report is frled. Effective with the 1996 reparting, respondents have the option of filing Form EIA-860 directly with the EIA or through an agent-such as the respondent's regional electric reliability council. For the 1996 reporting, 775 respondents filed directly with the EIA and 117 respondents filed through their regional electric reljability coureil. Data reported through the regional olectric reliability coutucils are submitted to the BLA electronically from the North American Electric Reljability Council (NERC). Forms filed directly with the EIA are due February 15 of the reporting calendar year. The submittal date of Form EIA-860 by respondents who file throngb their agent is determined by the agent. Extensions for filing may be granted the EIA, upon request.

Data for each respondent are preprinted from the applicable data base. Respondenis are instructed to verify all preprinted data and to supply missiag data, Processing of the data on Form BIA-860 is the responsibility of the Blectric Oenerating and Fuels Data Branch, CoaI and Electric Data and Renewables Division of the Office of Coal, Nuclear, Electric and Alternate Fuels. The system used to process data reported on Form EIA-860 was designed by this office. The data are manually edited before being keyed fot automatic data processing. Computer pro- 
grams containing additional edit checks are run. Respondents are contacted if necessary, to obtain correction or clarification of teported data, and to obtain missing data as a result of the manoul and automated editing process.

Presentation. Data from Form EIA-860 are summarized in the Inventory of Power Plants in the United States. This report presents eggregate totals for electric utilities in the United Stares, by Federal region, NERC region, Cenzus division, and State. The data are also used as input to publications and studites by other offices in the Department of Enercy.

Information Callected. A summary of the four schedules contained in Form EIA-860 is presented below.

1. Schedule I - Identification and Certification: Respondent's mailing address; namo and telephone number of contact person; and name and title of certifying official.

2. Schedule II - Power Plant Site Information: For each reported power plant, the following are specified: plant name; county location; State location; zipcode; name of cooling water source or source of water for hydroelectric power; and indicator of plant's cogeneration function.

3. Schedule III - Generator Information

a. For obch existing gentrator (active and inactive), the following are specified: plant name; generator identification; prime mover; nameplate rating; date of initial commercial opsration; energy sources used duting the repotting year for the production of electricity; heat rate; net summer capability; and net winter capability; ownership; identification; modes of transportation of fuet.

b. For each generator scheduled for initjal commercial operation within 10 years, the following are specified: plant name; generator identification; prime mover; nameplate rating; dates scheduled for initial commercial operation; proposed energy sources; and proposed net summer and net winter capabilities; ownership identification; modes of transportation of fuel.

c. Previously reported proposed generators that have been casceled or indefinitely postponed since the last reporting period are reported.

d. Ten-year plans for changes to existing generators are reportec. These proposed changes include change in Exel, life extension, or repowering, and rerating. Additionally, proposed changes in the status of existing geberators during the next ten years, incinding deactivation, change in ownership, retirement, and reactivation are reported.

e. Generators that have been retired during the reporting period and their date of retirement are reposted.

4. Schedule IV - OwTership of Generators Jointly Owned or Exclusively Ownad by Others: For operable generators and proposed new generators that are jointly owned, or for any generator that the respondent operates, but bas 100 percent own. ership outside the operating company, the following are reported: plant name, generator identification, prime mover, each owner's name, and thesir perceot ownership.

\section{Qually of Data}

The Office of Coal, Nuclear, Electric and Alternate Futels (CNEAF) is responsible for routine data improvenient and quality assurance activities. All operations of CNEAF are done in accordance with formal standards established by the Energy Information Admixistration (EIA). These standards are the guidelines for ensuring quality statistics. Data improventent efforts include verffication of datakeyed input by automated computerized methods, editing by subject matter specialists, and foltowep on submissions by noorespondents. The CNEAF supports the quality assurance offorts of the data collectors by providing advisory roviews of information requirements, and of proposed designs for new and revised data collection forms and systems. The actual performance of working data collection systems is validated once they are implemented. Respondents' computerized data files ate checked to identify those who fail to respond to the sarvey. By law, nonte. spondents may be fined or otherwise penalized for not filing at EIA data form as prescribed in the instructions. Before invoking the law, the EIA tries to obtain the required taformation by encouraging cooperation of nonrespondents.

\section{Updating and Editing of Data}

Autonated systems used to edit data include both deterministic checks, in which records are checked for the presence of data in required filelds, and statistical checks, in which the data are checked against a range of values besed on bistorical data values and for logical or mathematical consistency with data eloments reported in the source documents.

\section{CNEAF Data Revision Policy}

The Otfice of Coal, Nuclear, Electric and Aiternate Fuels has adopted the following policy with respect to the revision and correction of recurregt data in energy publications:

1. Annual survey data collected by this office are published eitber as preliminary or final when first appearing in a data report. Data initially relsased as preliminary witl be so noted in the report. These data wilt be revised, if necessary, ond declared final in the nexi publication of the data.

2. All montbly and quarterly survey data collected by this office are published as preliminary. These 
data are tevised only after the completion of the 12-month cycle of the data. No revisions are made to the published data before this.

3. The magnituse of charges due to revisions experianced in the past will be included in the data reports, so that the reader can assess the accuracy of the data.

4. After data are published as final, corrections will be made only in the event of a greater than one percent difference at the national lovel. Corrections for differences that are less than the before-mentioned threshold are left to the discretion of the Office Director.

\section{Confldentiallty of the Data}

Data coliected on Form EIA-860 are not confidential.

\section{Obtaining Copies of Data}

Upon EIA approval of the Inventory of Power Plants in the United States, the data become available for public use on a cost-recovery basis. Computer listings are obtained by submitting a written request to:

Energy Information Administration, BI-524

Fortestal Building

U.S. Department of Energy

Washington, DC 20585

These data are also available on machine-readable tapes. Tapes may be purchased by using Visa, MasterCard, or Anerican Express cards, as well as money orders or checks payable to the National Technical Infotmation Servjes (NTIS). Purchasers may also use NTIS and Government Printing Office depos* itory accounts. To place an order, contact:

National Technical Information Service (NTIS)

Offtce of Data Base Services

U.S. Department of Cotnmerke

5285 Port Royal Road

Springfield, Virginta 22,161

(703) $487-4650$

\section{Explanatory Notes}

\section{U.S. Aggregater}

Data from Form EIA-860 are submitted at the generator tevel. These data are then aggregated to provide totals by energy source (cosl, petroleum, gas, water, nuclear, other) and geographic ares (State, NERC region, Federal region, Census division). Addjtionally, at the national ievel data are aggregated to provide totals by prime mover.

\section{NERC Aggregates}

Beginning with the 1986 edition of Inventory of Power Plants th the United States, NERC tegton totals are aggregates based on company ownership of electric generating unit/capacity within region. That is, for eacb electric genteratiog unit that is owned jointly by companies that are associated with different NERC regions, the unit along with the share of capacity for each owner company has been allocated to the companies' respective NERC regions. In issues prior to 1986, NERC region totals were aggregates based on the assignment of units/capacity to the NERC region with which the utility operating the unit is associated.

\section{Generator Nameplate Capacity Versus Generator Capabillty}

Generator nameplate capacity is determined by the generator manufacturer under specified test conditions normally conducted at the faciory. The tnanufacturer starnss the achioved test capacity on the metal nameplate attached to the generator. Oenerator capability, on the other hand, is determined by the utility operating the generator, and is based on historical per. formance of the generator and associated equipinent. Generator nameplate capacity and generator capability generally differ from each other because the test conditlons used to establish the nameplate rating difier from those normally encountered in daily power plast operations. Different steam working pressures and temperatures, capacity limitations of boilers, cooling systems, turbines, and anvironmental control equipment, different hydrogen pressures osed to cool the generator, and reliability considerations cause dis. crepancies between bameplate and operating capacity.

Generator nameplate capacity reflects the capability of the generator to generate electrictity without regard to electrical loads from associated equipment such as boilers, particulate collectors, flue gas desulfurization units, and plant lighting. Generator nameplate capacity is therefore the gross capacity of the equipment. Net capability refers to the ability of the generator to generate electric power, taking into consideration the electrical requirements of associated plant equipment. For example, the electricity to run flue gas desulfurization equipment comes from electricity generated at the plant. Net, thenefore, refers to the electricity available to be sent offsite (for con. stemption) after plant eloctrical loads have been satisfied.

Net summer and net winter capability (the capacity of the generator that is generally achievable during the sumber and winter months, respectively, after plant electrical reguirements have been satisfied) is deter. mined by the atility operating the generator on the basis of bistorical performance of the gecerator and associated equipment. The summer and winter figures are usually not the same because of the differences in ambient temperatures during each season. Power plant cooling capacity, an essential part of electric power generation, decreases as air and water temperatures 
increase. Summer capability is therefore generally lower than winter capabitity, because high summer temperatures can strain power plant cooling capacity to the extent that maximam olectric power generation cannot be achieved. The statistics cited in the narrative in this publication are based on net summer capability, unless spectfied otherwise.

\section{Net Summar Capablity and Net Winter Capabllity Estimates}

Estimated values for net summer capability and net winter capability for nonpuctear electric generating units were doveloped by use of a rogression formula, using year-end 1992 data on net summer capability, net winter capability, and generator nameplate capacity of units in commercial operation during three intervals of time: 1940 or eatlier, 1941 through 1980 . and 1981 to present. A zero-intercept linear regression model with generator aameplate capacity as the regressor data was used since examination of the data shows that the intercepts are generally near zero. In all formulas,

the symbol, *, is an operator meaning multiplied by.

For nonmuclear units,

Net Summar/Winter Capability $=\mathrm{b} *$ (Nameplate Capacity),

where

b, represents the slope or factor by which name. plate eapacity has to be moltiplied to obtain a capability estimate, using this modet,

C. represents the standard error for $b$,

Generator Nameplate Capacity is expressed in kilowatts.

Net Summer Capability

$b=90, d=.04,1940$ or eartier, $b=.927, \sigma=$ $.002,1941-1980 ; b=.937, \sigma=.004,1981$ through present, for cosl steam units (Unis Types, ST, AB. CH)

$b=1,00, a=.03,1940$ or earlier; $b=.961, \sigma=$ $.002,1941-1980 ; b=.93, \sigma=.01,1981$ through present, for noncoal stean vnits (Unit Types, ST, $\mathrm{AB}, \mathrm{CH}$ )

$b=.856, \sigma=.003,1980$ or earlier; $b=.85, \sigma=$ .01, 1981 through present, for gas-turtitue units (Unit Types, GT, JE) $\mathrm{b}=.94, \sigma=, 01,1940$ or earlier; $\mathrm{b}=.84, \sigma=.01$, $1941-1980 ; \mathrm{b}=.86, \mathrm{a}=02$, 1981 through present, for combined-cycle units (Unit Types, CA, CS, CW, CT, 1G)

$\mathrm{b}$ w $.884, \sigma=.009,1940$ or earlier; $\mathrm{b}=.925, \mathrm{~b}=$ $.002,1941-1980 ; \mathrm{b}=.976, \sigma=.003,1981$ through present, for intersal combustion units (Untt Type, IC)

$b=.975, a=, 005,1940$ or earlier; $b=1.034, \sigma=$ $.004,1941-1980 ; b=.950, \sigma=.008,1981$ through present, for conventional and pipeline hydroelectric anits (Unit Types, HY, HL)

$b=.93, \sigma=.03,1940$ or earlier; $b=1.03, \sigma=$ $.01,1941-1980 ; b=1.01, \sigma=.006,1981$ through present, for pumaped-storage bydroelectric units (Unit Type, PS)

b = 3, for all other units (Unit Types, $C E, F C$, GE, $O C$, PV, SS, WTy, where limited data are available.

\section{Net Winter Capability}

$b=.88, \sigma=.05,1940$ or earlier; $b=.934, \sigma=$ $.002,1941 \cdot 1980 ; \mathrm{b}=.940$, व $=.004,1981$ through present, for coal steam units (Unit Types, ST, AB, PB)

$b=1.02, \sigma=.03,1940$ or earlies; $b=.965, \sigma=$ $.002,1941-1980 ; b=.94, d=, 01,1981$ through present, for noncoal steam units (Unit Types, ST, $\mathrm{AB}, \mathrm{PB}$ )

$b=1.023, \sigma=.004,1980$ or earlier; $b=.98, \sigma=$ .01, 1981 througb present, for gas-turbine units (Unit Types, GT, JE)

$\mathrm{b}=1.02, \sigma=, 03,5940$ or earljer; $\mathrm{b}=.96, \mathrm{a}=$ $.01,1941-1980 ; \mathrm{b}=.94, \sigma=.02,1981$ through present, for combined-cycle units (Unit Types, $\mathrm{CA}, \mathrm{CS}, \mathrm{CW}, \mathrm{CT}, \mathrm{IG})$

$b=.893, \sigma=.008,1940$ or earlier; $b=940, \sigma=$ $.002,1941-1980 ; b=.987, \sigma=.002,1981$ through present, for internal combustion unlts (Unit Type, IC)

$b=979, \sigma=, 005,1940$ or earlier; $b=1,026, a=$ $.004,1941-1980 ; b=.92, \sigma=.01,1981$ through present, for conventional and pipeline hydroelectric units (Unit Types, HY, HL)

$b=96, \sigma=.05,1940$ or earlier; $b=1.02, \sigma=$ $.01,1941-1980 ; b=1.03, \sigma=.01,1981$ through present, for pumped-storage hydroelectric units (Unit Type, PS)

$b=1$, for all other units (Unit Types, FC, GE, OC, PV, SS, WT, CE), where limited data are available.

9 Respondents report summer and winter eapebiltity and nowsplate for all neclear anito. 


\section{Generator Nameplate Capaclty Estimates}

Estimated values for genterator nameplate capacity for projected new generators were obtained by osing the average ratio that existed between the nameplate capacity and summer capability of specific types of operable generators as of year-end 1988. Proposed new gentrators with no reported nameplate capacity were limited to simple cycle gas turbine, combined cycle gas turbine and combjined cycle steam generators. In the formulas that follow,

the symbol, *, is an operator meaning multiplied by.

Generator Nameplate Capacity-Summer
Capability*1.20009, for gas nurbine generators(Unit Type=GT)

Generator Nameplate Capacity=Summer Capability*1.18918, for combine cycle gas turbine generators(Unit Type- CT)

Generator Nameplate Capacity=Summer Capability*1.14827, for combine cycle steam generators(Unit Type=CW)

\section{Definitions of Terms}

\section{Exlsting CapacityfExlsting Units}

Capacity/units that are existing, including those that are on standby and those that ase out of service for an indefinite period of tome.

\section{Planned Additlons/Additionel Units}

Capacity/units scheduled for initial commercial operation within 10 years of the reporting pertod of the poblication, uniess otherwise specified.

\section{Scheduled Completion}

Current/Original: For projected generating unit additions, the estimated date the unit is scheduled to start commercial operation, both the cutrent date and the original soheduled date.

\section{Rounding Autes for Data}

Given an n digit number with $r$ digits to the left of the decimal and $d+t$ digits in the fraction part, with $d$ being the place to which the number is to be rounded and $t$ being the remaining digits which will be truncated, this number is rounded to $r+d$ digits by adding 5 to the $(r+d+1)$ th digit when the number is positive or by subtracting 5 when the number is negative. The $t$ digits are then truncated at the $(x+\mathrm{d}+1)$ th digit. The symbol for a rounded number truncated to zero is $\left({ }^{*}\right)$.

\section{Use of the Glossary}

The terms in the Clossary have been defined for general use. Restrictions an the definitions as used in these data collection systems are included in each definition when necessary to define the terms as they are used in this report. 
1. 


\section{Appendix B}

Table Codes and

References 


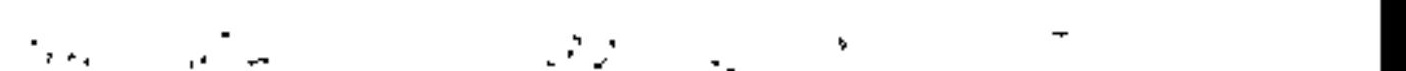




\section{Appendix B}

\section{Table Codes and References}

Table B1. Codes for Energy Sources

\begin{tabular}{|c|c|}
\hline Codk & Bntagy Sturet \\
\hline ANT & 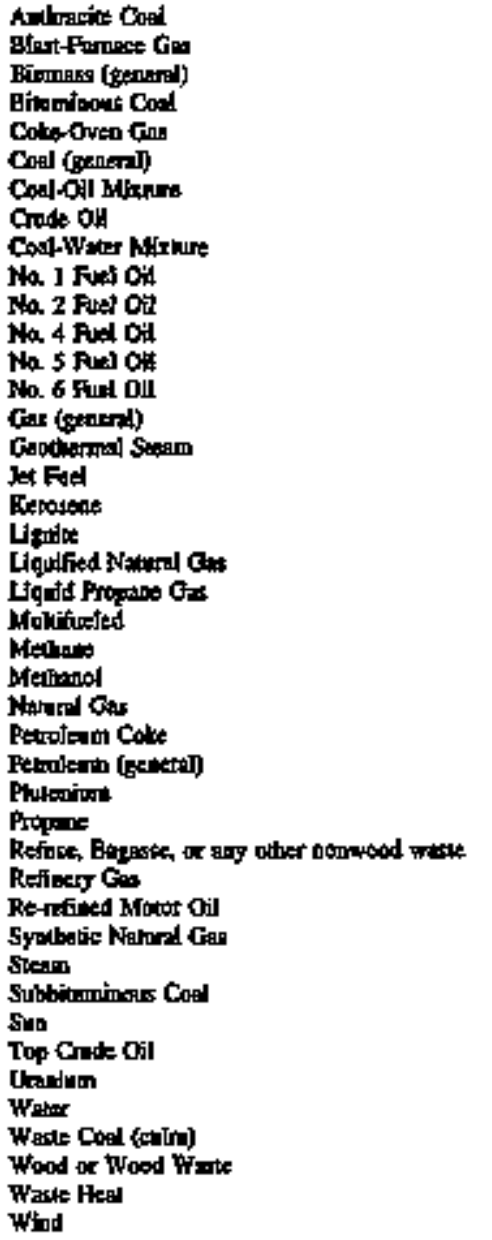 \\
\hline
\end{tabular}

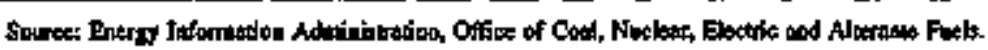




\begin{tabular}{|c|c|}
\hline Hett's Sortrat & Condo \\
\hline 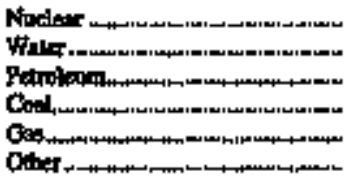 & 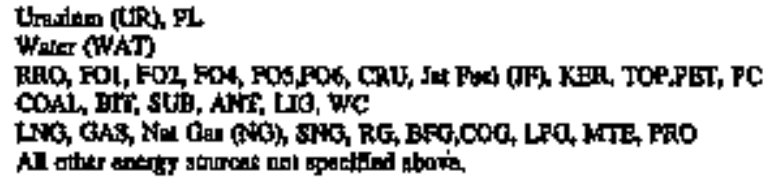 \\
\hline
\end{tabular}

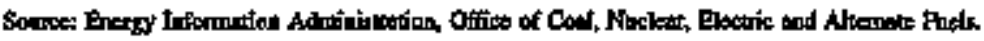

Table B3. Codes for Generating Unit Type

\begin{tabular}{|c|c|}
\hline Codo & Gentratiag Doll Type \\
\hline 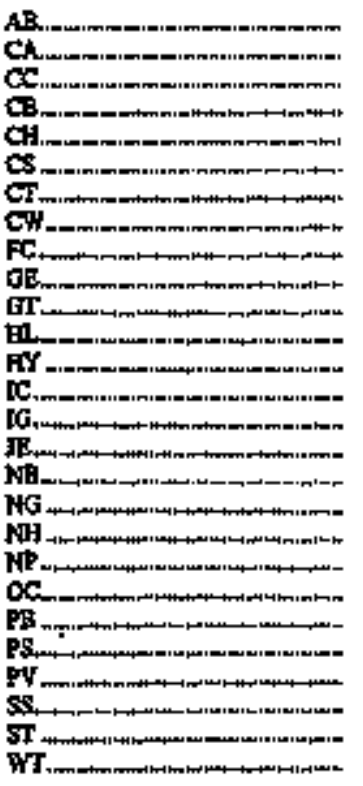 & 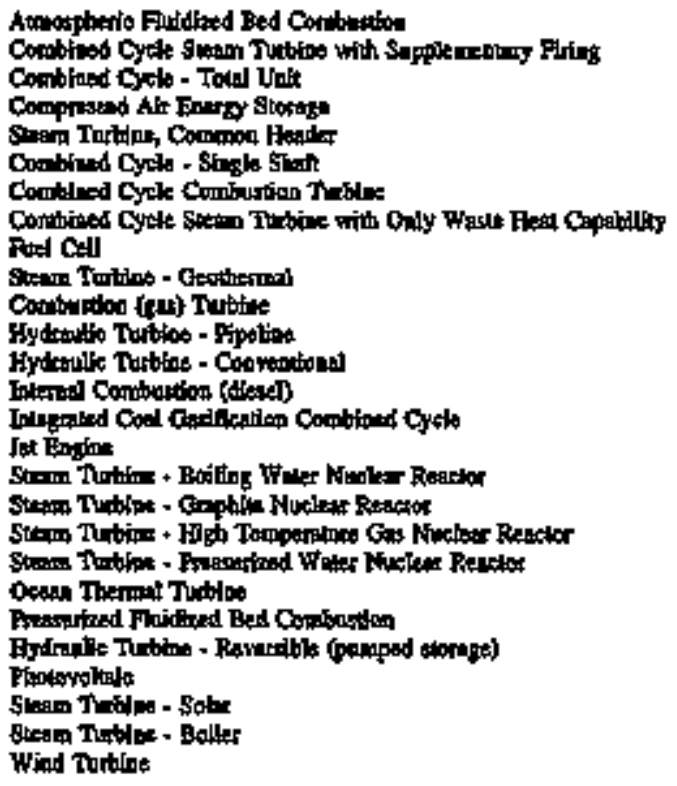 \\
\hline
\end{tabular}

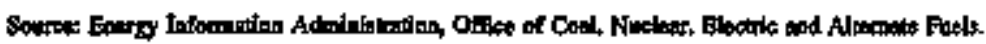

Table B4. Codes for Generating Unit Status

\begin{tabular}{|c|c|}
\hline Code & 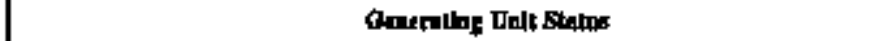 \\
\hline 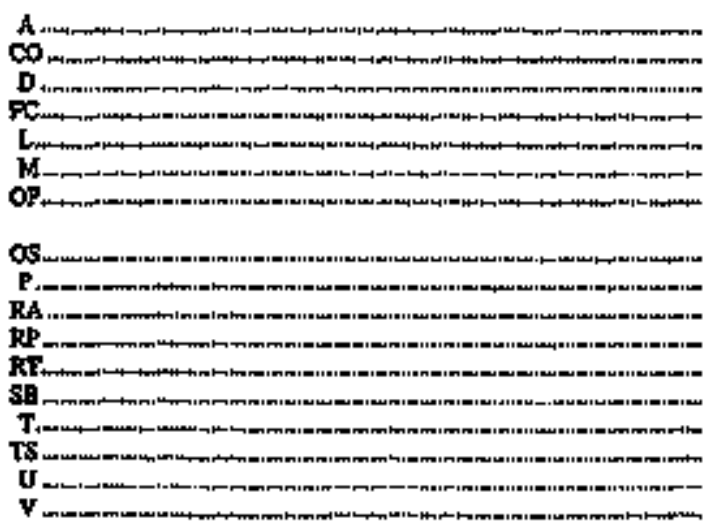 & 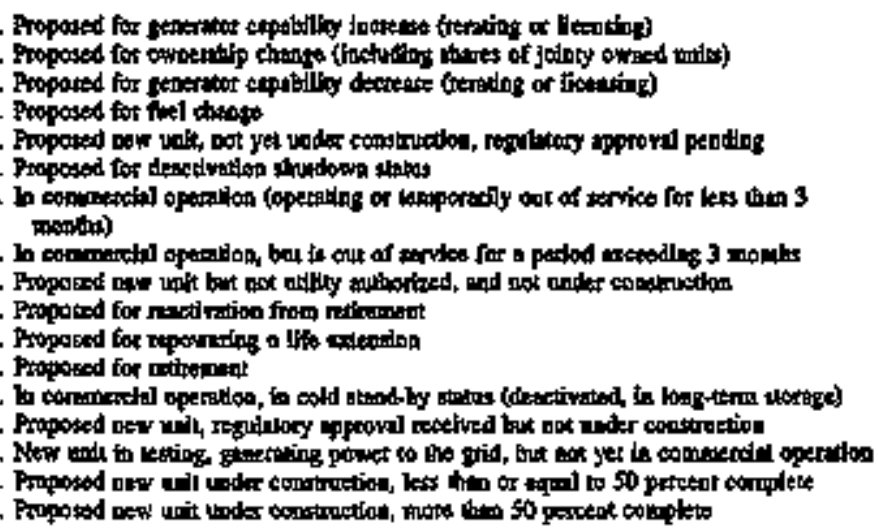 \\
\hline
\end{tabular}

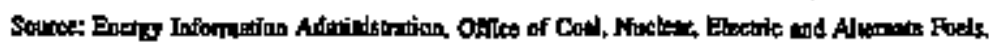


Table B5. Cross Reference of States to Federal Regions, NERC Regions, and Census Divisions

\begin{tabular}{|c|c|c|c|}
\hline Stalo & Federal Rejion & NGRC Acolon & Censos Dhoften \\
\hline 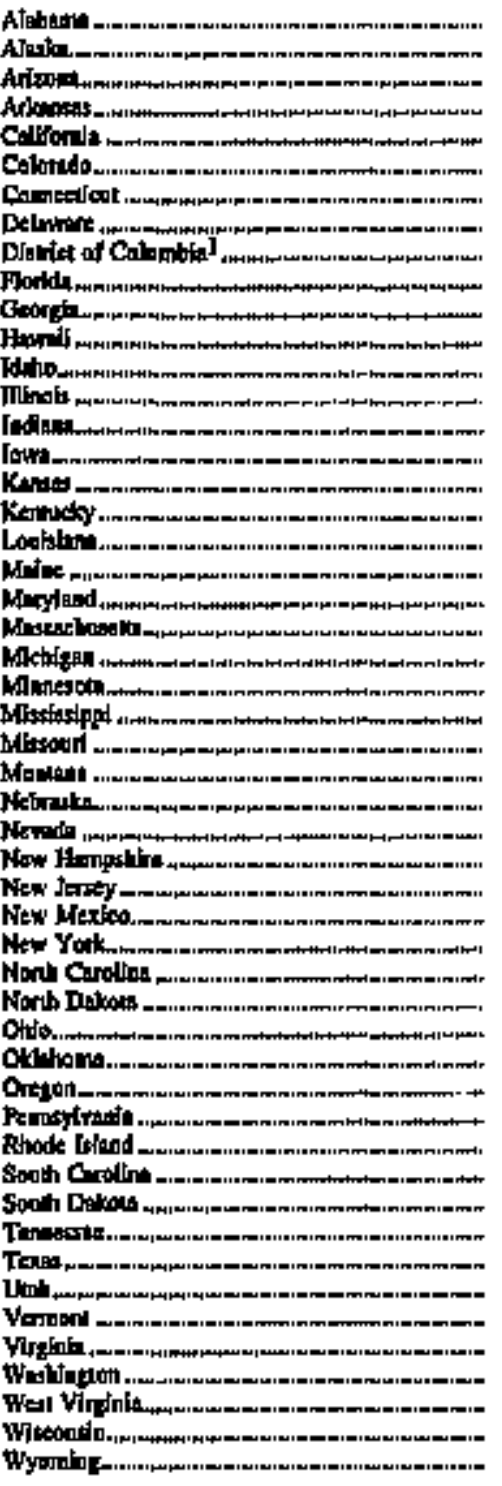 & 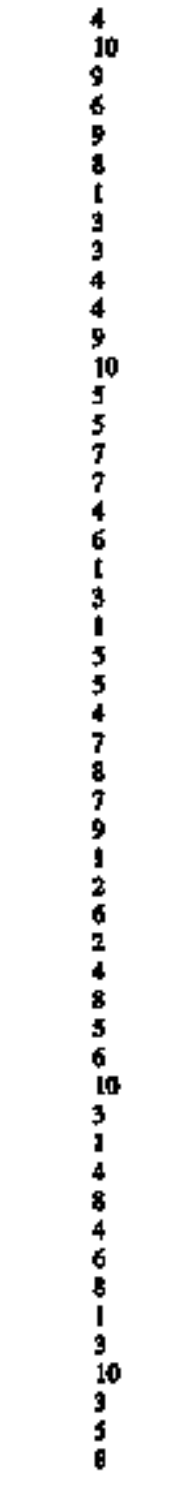 & 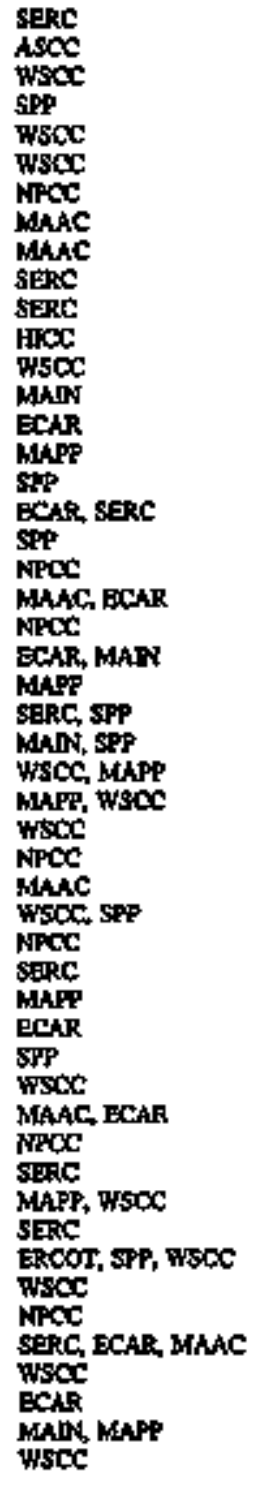 & 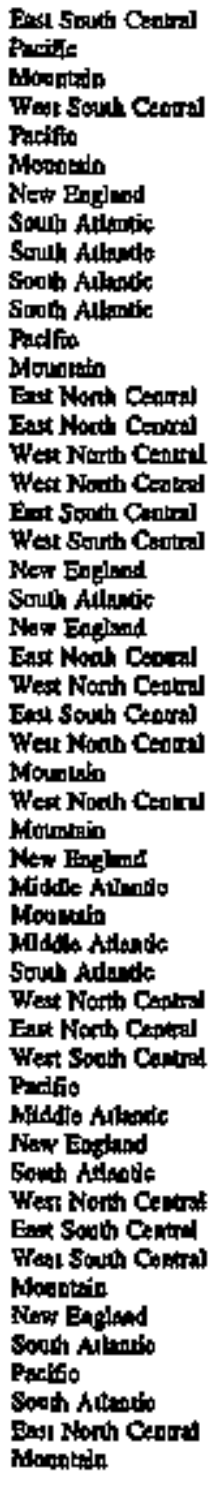 \\
\hline
\end{tabular}

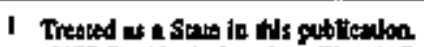

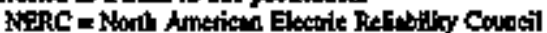

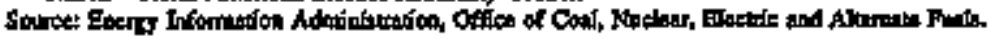





\section{Appendix C}

\section{Jointly Owned Electric \\ Generating Units}





\section{Jointly Owned Electric Generating Units}

Table C1. Jointiy Owned Electric Generating Units by State, Company, and Plant, as of January 1, 1996

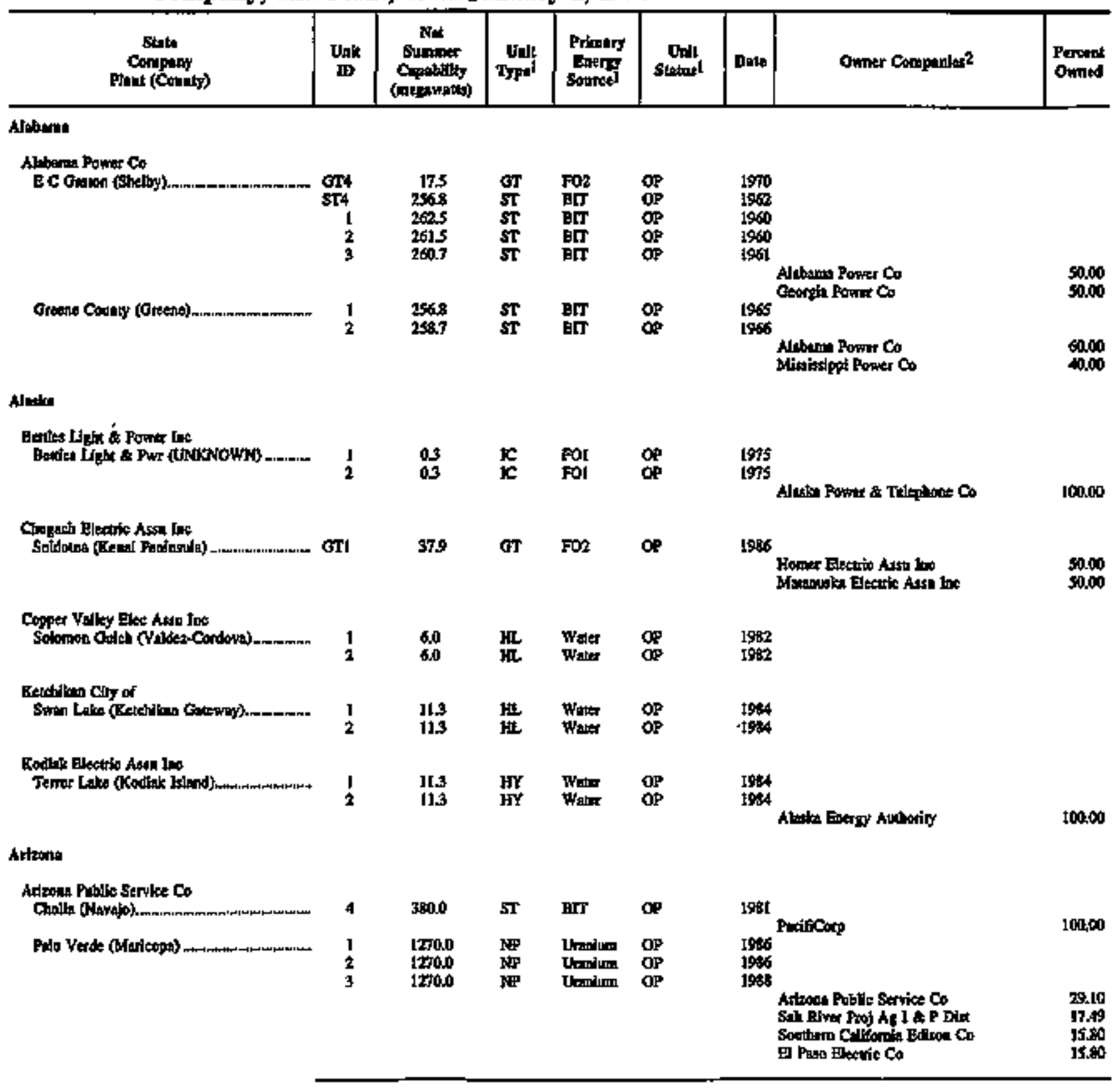

Sec foounove at and of uable. 
Table C1. Jointly Owned Electric Generating Units by State, Company, and Plant, as of January 1, 1996 (Continued)

\begin{tabular}{|c|c|c|c|c|c|c|c|c|}
\hline $\begin{array}{c}\text { State } \\
\text { Componyt } \\
\text { Font (Conty) }\end{array}$ & $\begin{array}{l}\text { Un't } \\
\text { ID }\end{array}$ & 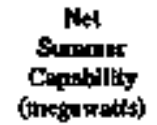 & $\begin{array}{c}\text { Un't } \\
\text { TYPस! }\end{array}$ & $\begin{array}{l}\text { Potpory } \\
\text { Bactiy } \\
\text { Soerenl }\end{array}$ & thit & Dote & Opmar Corpandet? & $\begin{array}{l}\text { Parreat } \\
\text { Ophutd }\end{array}$ \\
\hline
\end{tabular}

Artzona

Arizonin Pablie Service Co

\begin{tabular}{|c|c|c|c|c|}
\hline 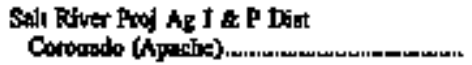 & Col & $35 \times 50$ & ST & HIT \\
\hline Nopto (Cophrino) & $\begin{array}{l}\text { NAVI } \\
\text { NAV2 } \\
\text { NAVZ }\end{array}$ & $\begin{array}{l}7500 \\
75000 \\
7500\end{array}$ & $\begin{array}{l}\mathbf{S T} \\
\mathbf{S T} \\
\mathbf{S T}\end{array}$ & $\begin{array}{l}\text { SUR } \\
\text { suB } \\
\text { suR }\end{array}$ \\
\hline
\end{tabular}

Patic servios Co of MMC

Sowber Cingen P A

Los Anseles Cor of

Sall River Proj Ag I \& P Diot

Cotorodo (Apache)

$\infty 1$

OP

or

OP

I979

1974

Selt Riwer Prod Ag 1 \& P Dist

top,os

1975

I976

Selt gexwer Prod Ag 1 \& P Dlt

Atone Polplit Service Co

Tusser Eloctrir Ponter Co

Los Angelea Ghy of

Merila Forer Co

Burav of Bech mation

Tucose Bectic Powar Ce

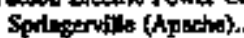

$\begin{array}{lllll}3600 & 5 T & \text { SUB } & O P & 1985 \\ 3600 & 5 T & \text { sUB } & O P & 1990\end{array}$

Tucape Electilo Powner Co

10000

Arronsis

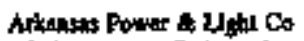

lodependioce (lodepepdencos.

8360

$5 T$ 5Ua

DP

1955

Attanst: Etectric Coop Corp

Altantes Poner \& Liph Co

Mitsassippi Pow or \& Lipt: Co

Jansuboco Cly of

Convay Corp

Wetl Merophts Dity or

IN84

Oupeola chity of

$21+70$

1400

750

4,20

11,30

24,30

$2 \quad 8420 \quad$ ST 5 STB OP

Arkanes Bectid Coop Corp

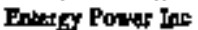

Mlagligipol Power \& Uph o

Janstrece Clity of

Conway Coms

Wes Meriphts Cly of

Optople City of

Who Blatf (Jemonon)

BLS.0 ST STP OP 1990

1981

Arktomas Ponke \& Litit Co

Aflensts Ilectric Coop Car

Ioneduto City of

Cornmy Con

Weat Rerojphis Clyoy of

35, 00

31.50

25.00

5.00

200

1.00

000

35,00

31,50

25.00

5.00

2.00

1,00

0.50

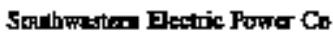

Fint Cresk (Bentron)

1400 ST SUI 0 \%

1978

Soinhweslam Etectid Power Co Arikansas Elestric Coop Corp

5000 \$2:100

Colltornda

Cillomin DeptWr Resoumes:

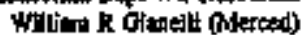

$\begin{array}{llllll}1 & 510 & \text { PS } & \text { Water } & \text { OP } & 1968 \\ 2 & 500 & \text { PS } & \text { Waber } & \text { DP } & 1958 \\ 3 & 50.0 & \text { PS } & \text { Wakt } & \text { OP } & 1957\end{array}$

Ste focloores at and of bitis.

274 Energy Information Adurinistration/Inventory of Power Plants in the United States as of January 1, 1996 
Table C1. Jointly Owned Electric Generating Units by State, Company, and Plant, as of January 1, 1996 (Continued)

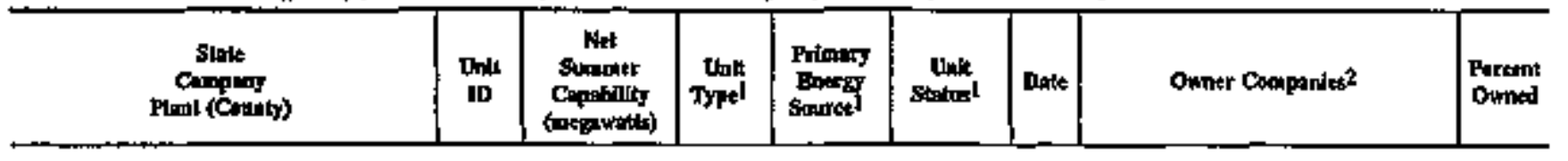

Californla

Olitionil Dept-Wtr Rescorses

Modespo Inlrallon Distipat

New Hoge (Coldeverit)

NAI 20 HY Water

$\begin{array}{llll}\text { NA2 } & \text { 1.0 } & \text { HY War }\end{array}$

CP $\quad 1986$

OP $\quad 1986$

Culyert Conty Wober Dis

10000

Oindele of Soult San Joagutio

Sort Bor (Tooluting)

HY Waver

OP

1986

Til-Dan Power Mubodly

10000

Secturasto Mondipd Uail Dit

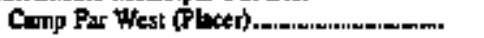

Coldwiter Oreak (Sveonn).

62. GE GST OP

Igs

Sonth Sortex Water District

เ90.06]

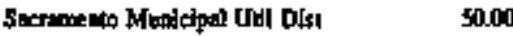

Madesto rrigailoo Didites

sime cher Gy of

1000

Sonherm Coliformia Edigen Co

San Ooote (Sin Dieto)

$3 \quad 1070$

10800

NP Urmivar OP

1983

Souben Oalitordia EAbon Ca

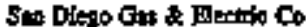

Ansein Gity of

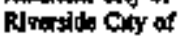

75,05

20100

3.16

1.79

Turlock Imlgatlap Didricl

Don Pecto (Tlobmens)

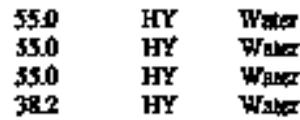

OP
OP
OP
OP

1971

197]

1971

1989

Teloct hrigation Distriat

Modedo Irigrion Distlitat

69.46

Cotorido

Puble Servite Co of Colormo

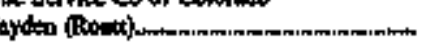

$\begin{array}{lllll}1 & \text { t\$40 } & 5 T & \text { BT } & \text { OP } \\ 2 & 2520 & 5 T & \text { BT } & \text { OP }\end{array}$

1955

Pubbie Service Co of Colorads 75.50 1976 Parfilloos

2450

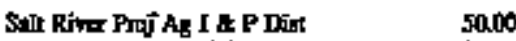

Poblic Serviee Co of Cotoredo

Postificor

12.64

TNESte 6 \& T AUID be

Orais (Mlaffet).

$\begin{array}{lllll}1 & 426.0 & \text { ST } & \text { BT } & O P \\ 2 & 4280 & \text { ST } & \text { BTT } & \text { OP }\end{array}$

1980

1975

Bive fosturotes at end of able 
Table C1. Jointly Owned Electric Generating Units by State, Company, and Plant, as of January 1, 1996 (Continued)

\begin{tabular}{|c|c|c|c|c|c|c|c|c|}
\hline 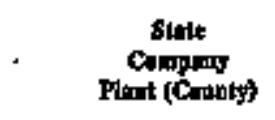 & $\underset{10}{\text { Dn't }}$ & 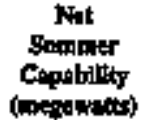 & Lntt & 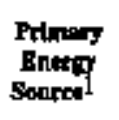 & $\begin{array}{c}\text { Uat } \\
\text { Etutas l }\end{array}$ & Dest & Owmer Coosparles & $\begin{array}{l}\text { Pertart } \\
\text { Dirned }\end{array}$ \\
\hline
\end{tabular}

Coternata

Th-sing G A T Asto to

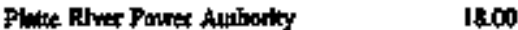
Paiflcor

La'icoip Uained

W $N$ Cladk (Frthot)

ST

BUr

OP

1955

Liticop Utiled

topoo

Conpuetient

Consectiar Yandes Alom Pror Co

Hadain Nock OMlianlesex)

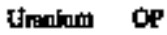

1960

Conoedtol lijht \& Potwer Co

Porite Seritue Co of NA

Nisw England Ponar Co

Hosloos Edtan Co

Whltex Ilominting Co

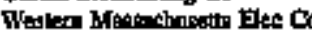

Cuptril MFias Powra Co

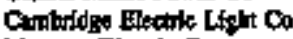

Monbop Electrit Co

Captral Yemat Fut Serv Corp

Northesst Nuder Elourg Co

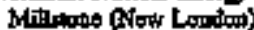

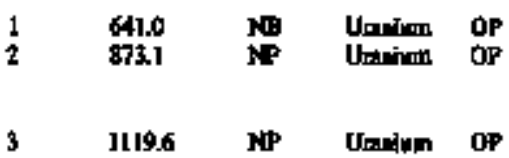

Vhiled IItumineting $\mathrm{Cs}$

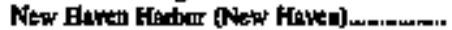

F06

OP

1975

Utived Iluminadog Co

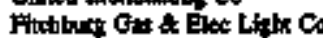

Hoblate Cor of

North Atalborough Toty of

Livtielon Totwa of 1986

Connecticat Light \& Perer Do

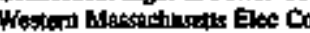

sings

Endend Ponwar Co

Sman Mon of Coop

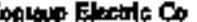

Inined Illaninating Co

Protic Service $C$ of $N$.

Cenotel Maine Porter Co

Contral Verpont Poll Sorv Capp

Foridip

Flocith Formes \& lidet Co

St Lwoik (Sil Ledo)

2 850.0 NP Ura:4:m OP

1963

Forjos Powar \& Ujbi Ca 
Table C1. Jointly Owned Electric Generating Units by State, Company, and Plant, as of January 1, 1996 (Continued)

\begin{tabular}{|c|c|c|c|c|c|c|c|c|}
\hline $\begin{array}{c}\text { Stok } \\
\text { Compang } \\
\text { Plant (Cowaty) }\end{array}$ & Utaik & 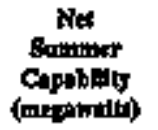 & 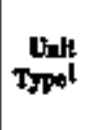 & 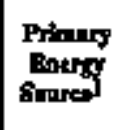 & Unk & Dote & Onder Compentalstz & $\begin{array}{l}\text { Peremp } \\
\text { Omued }\end{array}$ \\
\hline \multicolumn{9}{|l|}{ Blontidn } \\
\hline 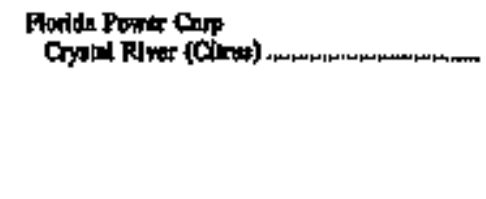 & 3 & 8120 & NF & Uningan & $\mathbf{Q P}$ & $19 \pi$ & 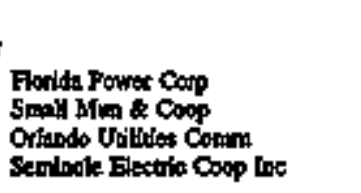 & $\begin{array}{l}\$ 0.45 \\
6.25 \\
1.60 \\
1.30\end{array}$ \\
\hline \multirow[t]{2}{*}{ 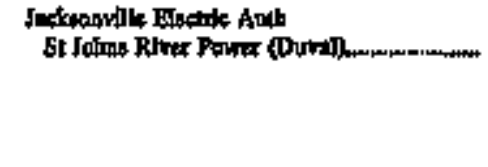 } & 1 & $\begin{array}{l}6240 \\
6240\end{array}$ & $\begin{array}{l}\mathbf{S T} \\
\mathbf{S T}\end{array}$ & $\begin{array}{l}\text { BnT } \\
\text { BIr }\end{array}$ & $\begin{array}{l}\text { OP } \\
\text { of }\end{array}$ & $\begin{array}{l}1987 \\
19898\end{array}$ & & \\
\hline & & & & & & & 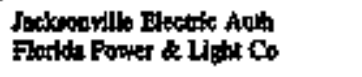 & $\begin{array}{l}50.00 \\
50.00\end{array}$ \\
\hline \multirow[t]{2}{*}{ 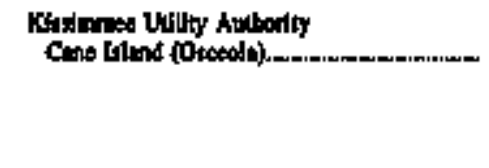 } & $2^{2}$ & $\begin{array}{l}200 \\
344\end{array}$ & $\stackrel{c r}{c r}$ & Mis Gas & $\begin{array}{l}\text { OP } \\
\text { op }\end{array}$ & $\begin{array}{l}19 \% 5 \\
19 \% 5\end{array}$ & & \\
\hline & & & & & & & 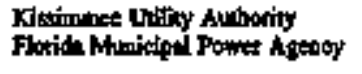 & 50,00 \\
\hline 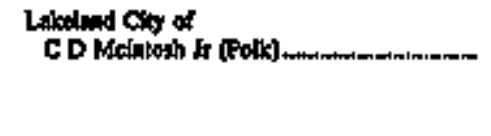 & 3 & 333.0 & $\mathbf{S T}$ & BIT & $Q P$ & 1982. & 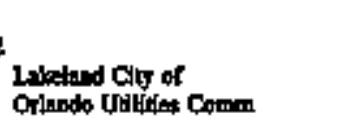 & $\infty_{400}^{\infty}$ \\
\hline \multirow[t]{3}{*}{ 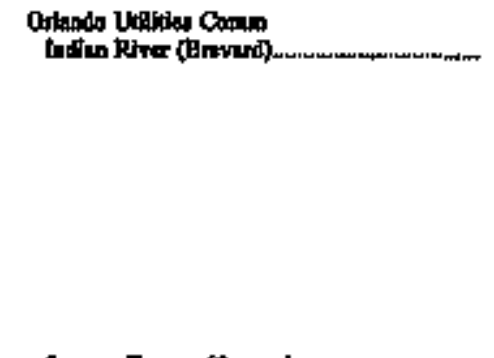 } & c & 1080 & $G$ & Nut Ge: & op & 1992 & Orlando thatilits Cann & $\begin{array}{l}79.00 \\
21.00\end{array}$ \\
\hline & $\begin{array}{l}\text { CTI } \\
\text { CTt }\end{array}$ & $\begin{array}{l}38.3 \\
38.3\end{array}$ & $\vec{\sigma}$ & $\begin{array}{l}\text { Nut Gar } \\
\text { Nut Gas }\end{array}$ & of & $\begin{array}{l}1998 \\
1998\end{array}$ & 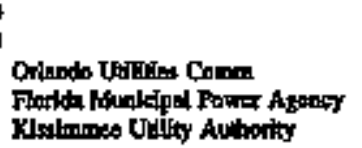 & $\begin{array}{l}49.60 \\
39.00 \\
12.20\end{array}$ \\
\hline & $\mathbf{b}$ & 108:0 & Gr & Now ous & OP & 1992 & Griando Utrides Compan & 29.00 \\
\hline \multirow[t]{2}{*}{ 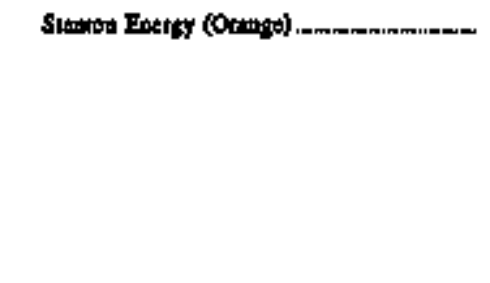 } & $\mathbf{J}$ & 400 & $\mathbf{S T}$ & BMT & op & 1987 & 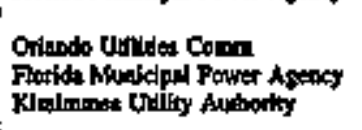 & $\begin{array}{l}60.55 \\
2563 \\
482\end{array}$ \\
\hline & 2 & 4880 & ST & ETr & $\mathbf{v}$ & 1996 & 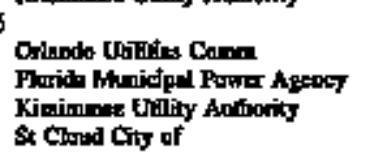 & $\begin{array}{l}7 ., 59 \\
24.17 \\
3.83 \\
3.41\end{array}$ \\
\hline 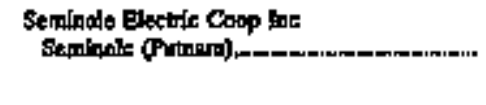 & 2 & 6250 & $\mathbf{\$ T}$ & EtT & OP & 1985 & Frty Flonin Bmk & 100.00 \\
\hline \multicolumn{9}{|l|}{ Georglat } \\
\hline \multirow[t]{2}{*}{ 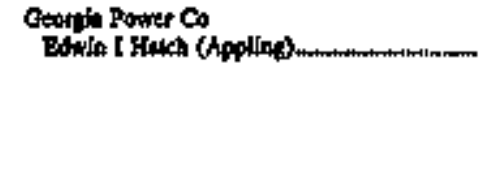 } & t & 899.4 & NB & $\begin{array}{l}\text { Utation } \\
\text { Utantum }\end{array}$ & $\underset{\mathrm{OP}}{\mathrm{OP}}$ & $\begin{array}{l}1975 \\
1979\end{array}$ & & \\
\hline & & & & & & & 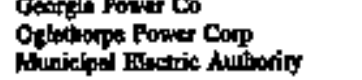 & $\begin{array}{l}30.00 \\
31 . x 0\end{array}$ \\
\hline
\end{tabular}

Ser foelootes at end of toble. 
Table C1. Jointly Owned Electric Generating Units by State, Company, and Plant, as of January 1, 1996 (Contimned)

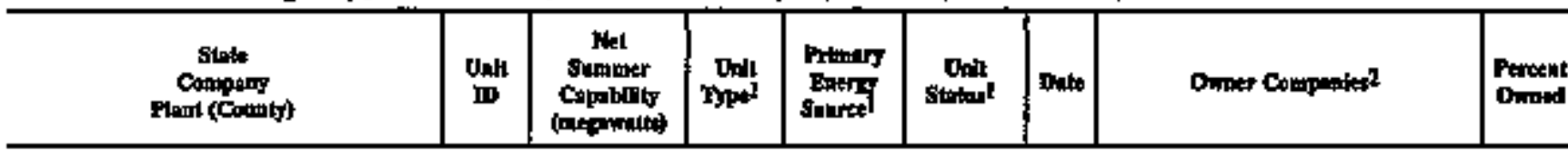

Georth

Geargia Porver Co

NA] (UNKNOWN)

NAII

1290

C Nat Ge

Solater (atomax)

\begin{tabular}{lllll}
\hline 192.2 & $5 T$ & $B T$ & $O P$
\end{tabular}

\begin{tabular}{|c|c|c|c|c|}
\hline & 4 & $\begin{array}{l}84400 \\
8440\end{array}$ & $\begin{array}{l}\mathbf{S T} \\
\mathbf{S T}\end{array}$ & $\begin{array}{l}\text { BrT } \\
\text { HEr }\end{array}$ \\
\hline Vo & $\mathbf{1}$ & $\begin{array}{l}1164.0 \\
I 1640\end{array}$ & $\begin{array}{l}\mathbf{N P} \\
\mathbf{N P}\end{array}$ & 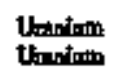 \\
\hline
\end{tabular}

Wmakg atsard

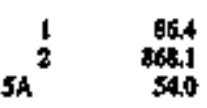

$\begin{array}{ll}\text { sT } & \text { BLT } \\ \text { हT } & \text { BUT } \\ \text { GT } & \text { FO2 }\end{array}$

$\underset{\text { Op }}{\operatorname{Op}}$

0yletborpe Power CocT

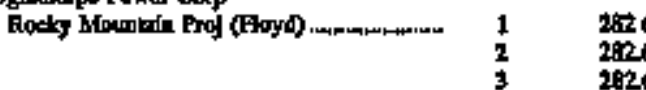

Whis

Wher

$\underset{\text { Op }}{\operatorname{Op}}$

2005

Dalion Gidy af

2.20

Coroplant Po

Allbera Powe Co

1992

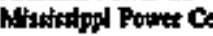

60,00

20.00

$19+4$

Georgla Foner Co

Ojectorpe Powar Con

Mualcipal Buatic Anoboriky

Dathog Chy of

IFT

1909

Cangala torer co

$19 \mathrm{ses}$

Guif pown co

Jys

Ceociga Pow Co

Ortiline Punt Carp

Aluritalat Elecitio Anthotity

Datho Oy of

1976

1978

$19 \%$

Coortis Power Co

Odeuborpo Power Com

Modocipal electio loborky

Dation Cos of

20.00

8.40

60.00

30.20

(4)

Ts.00

$\mathbf{6}, \mathbf{0}$

45.70

3000

2270

160

53.50

3000

15.10

140

glathere Poner Corp

Oection Ponte Co

Frant?

Mod Eatric Co Lta

Cootte Gen Strition (Menl)

cthrs

$\begin{array}{ll}\mathbf{a g} & \mathrm{k} \\ \mathbf{a g} & \mathrm{K} \\ \mathbf{a g} & \mathrm{k}\end{array}$

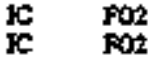

Fot

$\underset{\text { op }}{\text { op }}$

1905
joiks

196:

Palm Cosp

100.00

Tdabo

Fdll Riva Rurali Elus Coep Ine Now

$\begin{array}{llll}\text { HCl } & 28 & \text { HY } \\ \text { HC2 } & 28 & \text { Writs }\end{array}$

$\underset{\text { op }}{\operatorname{mp}}$

1936

1966

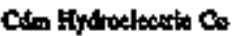

100.00

Winols

Commontream Exidia co

Quad Cites (thoxk iflund)

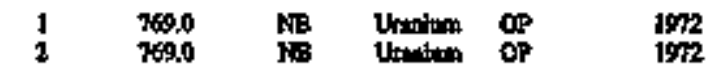

Commocreallb Efleos Co

7500

Sal botwoted at ad of trible

278 Energy Information Administration/Inventory of Power Plants in the United States as of January 1, 1996 
Table C1. Jointly Owned Electric Generating Units hy State, Company, and Plant, as of January 1, 1996 (Contimued)

\begin{tabular}{|c|c|c|c|c|c|c|c|c|}
\hline $\begin{array}{c}\text { State } \\
\text { Comping } \\
\text { Plane (Coint) }\end{array}$ & Utolt & 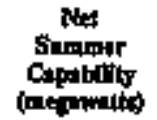 & Dat: & 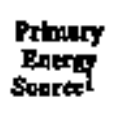 & Jinkt & Deto & 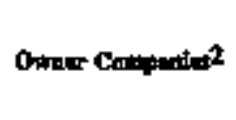 & $\begin{array}{l}\text { Pureynt } \\
\text { Dinfut }\end{array}$ \\
\hline
\end{tabular}

1.17ok

Commonaralih Edrom Co

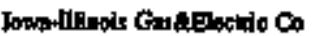

20,00

Boptalc Enoraty Ic

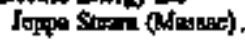

$\begin{array}{ccc}10140 & \text { ST } & \text { BIT } \\ 00 & \text { ST } & \text { BIT } \\ 0.0 & \text { ST } & \text { BIT } \\ 0.0 & \text { ST } & \text { IIT } \\ 00 & \text { ST } & \text { EIT } \\ 0.0 & \text { ST } & \text { BIT }\end{array}$

$\begin{array}{ll}O P & 1953 \\ O P & {[958} \\ O P & 1954 \\ O P & 1954 \\ O P & 1955 \\ O P & 1955\end{array}$

Uingon Eecrio co

Irinais Powre co

Kencuchy Litides

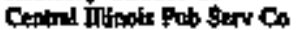

$\sin$

20.00

2000

Diterto Porra

Cilento (De Hit)

930

Na

tration

op

1967

Itinols Powit Co

Soptind Ponst Goop int

8679

Indlank

Indion Mindigen Pows Co

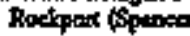

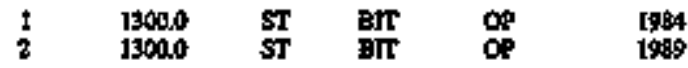

AEP Geantion Co

Kenanty Power Co

Inilime Mighign Fower on

3000

PSt Enatgr the

O'thon (O)

op

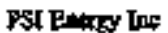

Wath Vatley Powt Asm inc

Inditin Mualifipeil Powa Agtory

sols

20,00

Soulks Indin Gat \& Elec Oo

Warick (Wurifly)

135,0 ST BIT

$\mathbf{\phi}$

$19 \times 0$

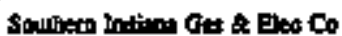

Alcos Centarting Corp

30.00

stodo

turt

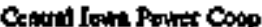

Evir Sladon (Mumatio)

$\begin{array}{llll}23.4 & \text { ST } & \text { BrT } & \text { OP } \\ 41.0 & \text { ST } & \text { BrT } & \text { OP }\end{array}$

3900

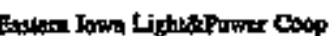

100.00

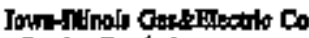

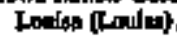

675

ST SUR:

op

1903

Ispm Iningly duspiptric Co

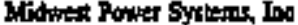

Cobtal Iow4 Pewer Coop

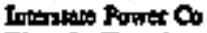

Waredy Ciy of

Cexpect Ciny of

Hartan City of

Ifpiod ثty of

Elifiding ofly of

40.00

45,00

460

400

1.10

050

0.80

0,0

050

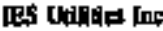

Dume Arolu atho)

3280 Ni Utitam OP

1975

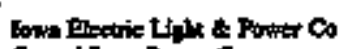

Cetrifl Jors Pouar Cocp

Com Beb Foner Coo;

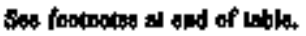

Energy Information Administration/Inventory of Power Plants ig the United States as of January 1, 1996279 
Table C1. Jointly Owned Electric Generating Units by State, Company, and Plant, as of Jantary 1, 1996 (Continued)

\begin{tabular}{|c|c|c|c|c|c|c|c|c|}
\hline 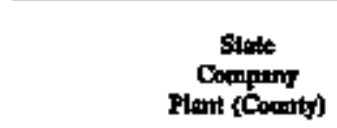 & $\begin{array}{c}\text { Unl! } \\
\text { tit }\end{array}$ & $\begin{array}{c}\text { Net } \\
\text { Cummer } \\
\text { Capobty }\end{array}$ & Tynl| & $\begin{array}{l}\text { Pringy } \\
\text { Satiry } \\
\text { Stareal }\end{array}$ & $\begin{array}{c}\text { Untit } \\
\text { Sinqay }\end{array}$ & Dele & Onater Conmanim? & $\begin{array}{l}\text { Ptrcent } \\
\text { Proud }\end{array}$ \\
\hline
\end{tabular}

Como

\begin{tabular}{|c|c|c|c|c|}
\hline Orainesa (Wopello) ....... & $\mathbf{I}$ & 7140 & $\mathbf{s t}$ & sUl: \\
\hline 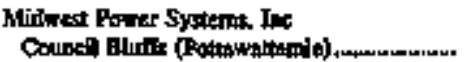 & 3 & 675.0 & st & stJ! \\
\hline 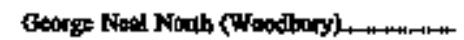 & 3 & 515.0 & $5 T$ & EH: \\
\hline George Noal Sarlh (Woodbary) & 4 & 6240 & $\mathbf{s T}$ & SU! \\
\hline
\end{tabular}

Loma Soulued Uillities C.

lows Patio Service Co

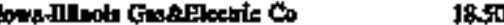

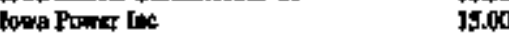

lows Electrle Light \& Pawer Co

Millinest Power Systernt. In

Midweit Pornes Sybem, lat 46.70

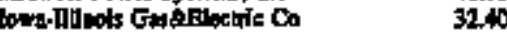

Conited Jown Power Coop 11.50

Com Belt Power Coop 3.80

Coter Fall City of 3.10

Aluatic Coty of 2.50

1975

lowe IIItoin Geshiflectrie Co 29,00

Jown Sowthon Lilintes Co 20.00

1979

Hidfrat Powar Symens, be $\quad 43.00$

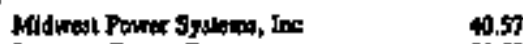

Iotwatals Powe Co

Monthwet Iowa Powner Coop. \$.03

Nouthowation Pubtic Strvice Co

Com Beft lownt Coop

Argon CK of 2,54

Webat Cly Ory of 2 .6

Code Fits Cin of 2.50

Soencer Cly of

Sitit Mot \& Coop

[.9]

xars

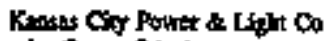

La Gres (tin)

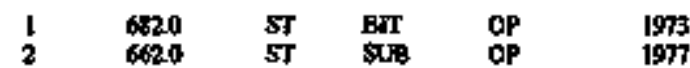

Knity Ging Power \& 1/ht Co

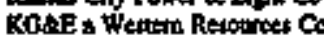

50.00

50.10

KPl, a Wexin Resources Co

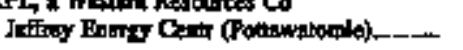

$2 \quad 698$

68.0

700

$\begin{array}{ll}\text { ST } & \text { SUB } \\ \text { ST } & \text { STI } \\ \text { ST } & \text { S्रU⿴囗 }\end{array}$

1978

op 1900

1903

KPL, : Watem Thoursea 6

RGere a Wealin Resparea Co

UillCort Lnited Ins

UiliCenp United

64.00

20.010

g.10

8.10

WoI Cust Nucletr Oper Corp

Wolf Crest (Cofficy)........................

1 t167.0 Nis Unme op

19 ist

KG8E a Wentrin Resouret Co 77,0

Fansa City Powet \& lith co 47,00

Sn Mon exp

Keatingy

Hig Binter Dethit Corp

Futpl stotion 2 (trandaroen)

L540

$\begin{array}{lll}\text { ST } & \text { EIT } & \text { OP } \\ \text { ST } & \text { EET } & \text { OP }\end{array}$

$\pm 973$

1974

Sen footingtes at end of table. 
Table C1. Jointly Omed Electric Generating Units by State, Company, and Plant, as of January 1, 1996 (Contlmued)

\begin{tabular}{|c|c|c|c|c|c|c|c|c|}
\hline 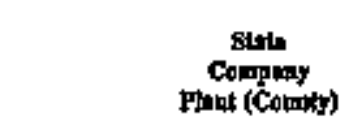 & $\underset{\text { Unt }}{\text { Unt }}$ & 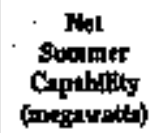 & $\underset{\text { Typh }}{\text { Xns }}$ & Pelnoty & $\begin{array}{c}\text { Uall } \\
\text { Staltonl }\end{array}$ & Dove & Dwrer Componites? & $\begin{array}{l}\text { Perwent } \\
\text { Owned }\end{array}$ \\
\hline
\end{tabular}

Kentodis

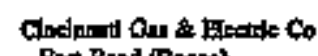

Bux Bood (Boon)?

Loulmils Gan \& Electio Co

Trinble Conos (Jimble)

Lealedare

Ciun Btectio Pewer Cosp las

Bif Cojun 2 pointo Con

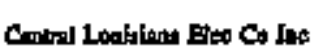

Dolat thll (De Soto).

Funtion (Ruplida)

(1)

Gut Staten Utillies Co

B S Alekan (Calcarla)

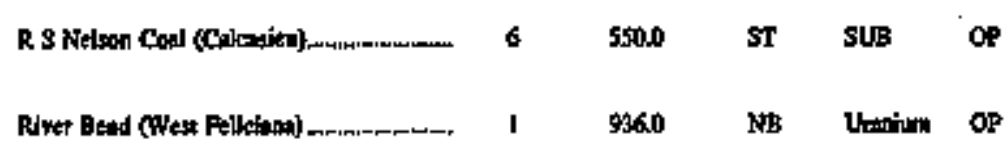

Malue

Contad Maim Ponte co

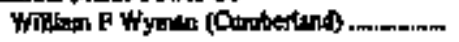

4
1981

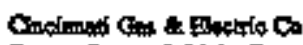

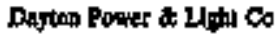

69.00 31.00

1900

Lonitrile Gu Besta Co 75.00

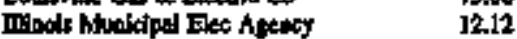

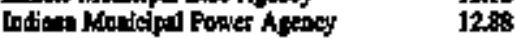

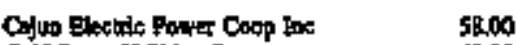

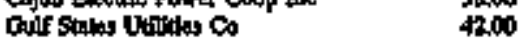

1986

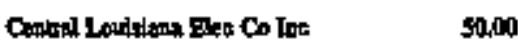

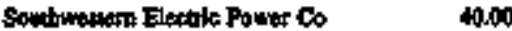

Nothes, Tex: Eto Coop los 600

$19: 2$

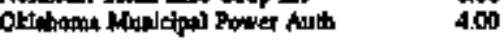

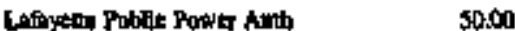

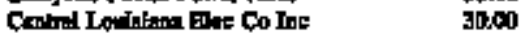

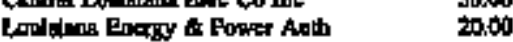

1959

195

Citgo Putrolnun Gow

Comoco in: 36,10

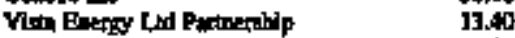

1982

Goif Sutho Uuififis Co

2.00

$19: 6$

Onif Stant Utibles Co

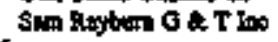

7000

30.00

Durf stans cintitios Co

Colvo Elecerle Pawas coop ins 30.00

918

\begin{tabular}{|c|c|}
\hline 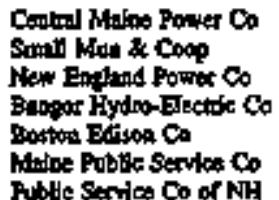 & $\begin{array}{l}39.15 \\
10.87 \\
9.27 \\
8.73 \\
5.59 \\
3.35 \\
3.24\end{array}$ \\
\hline
\end{tabular}

Sed foolugit a the of coble. 
Table C1. Jointly Owned Electric Generating Units by State, Company, and Plant, as of January 1, 1996 (Continued)

\begin{tabular}{|c|c|c|c|c|c|c|c|c|}
\hline 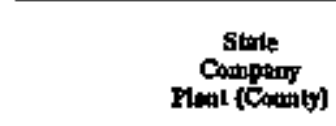 & $\begin{array}{c}\text { Lhot } \\
\text { ID }\end{array}$ & Natur & Typet & 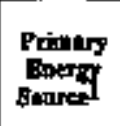 & $\underset{\text { Staksol }}{\text { Conk }}$ & Dascos & Omper Conpanile? & $\begin{array}{l}\text { Peroent } \\
\text { Chmed }\end{array}$ \\
\hline
\end{tabular}

\section{Mnnglead}

Polome Decto Poner Co

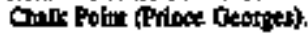
soTJ

240 GT Nat Ga OP

1930

Sotuket Marytand E Coop Iac

10000

MFosichinusetis

Cagl Electis $C_{0}$

ST FO6 OP

1976

Compl Elestic Co

Moutanp Elextric Co

$\$ 000$

Mussaturets Mun Whe Elec Co Slooy, Brook (Bhappdin)

OP
OP
OP

199]
$19 \% 1$
$19 \% 1$

$19 \% 1$

Mesuchusers Mon Whl Elec Co Gn:an Mountein Powur CoTp Cyndinvillo YHate of

Waster Mareschnseltr Fes Co Nonbafold Mounatio (Frapldio)

$\begin{array}{lll}2700 & \text { PS } & \text { Wata } \\ 2700 & \text { PS } & \text { Warar } \\ 2700 & \text { PS } & \text { Water } \\ 2700 & \text { PS } & \text { Water }\end{array}$

OP
OP
$O P$

1973
1973
1973
1972

Counatiant Light \& Porrex $C_{0}$

81.00 Westem Itasongunets Blac Co

\section{Nositger}

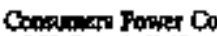 \\ If Camphell (O) \\ Lutionton (Mtest)}

Detroit Batsoo Co

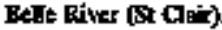

Ferd (Moproe)

Terterse Giry Ciry of

Flt Rapits (Antrim)

Uapor Paningule Power Co

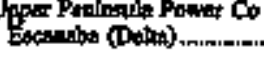

Mitimente

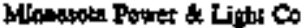

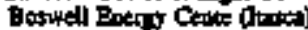

sed pootnotes at end of table.

\begin{tabular}{|c|c|c|}
\hline $7 \$ 0.1$ & $\mathbf{s t}$ & BIT \\
\hline $\begin{array}{r}3120 \\
3120 \\
3120 \\
3120 \\
3120 \\
3120\end{array}$ & $\begin{array}{l}\text { PS } \\
\mathbf{P S} \\
\mathbf{P S} \\
\mathbf{P S} \\
\mathbf{P S} \\
\mathbf{P S}\end{array}$ & 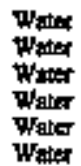 \\
\hline
\end{tabular}

$\begin{array}{llll}6253 & 5 T & \text { SUR } & \text { OP } \\ 6251 & 5 T & \text { SUt } & \text { OP }\end{array}$

I 100.0 NB Uhanm op

$\begin{array}{llll}0.2 & \text { HY } & \text { What } & \text { OP } \\ 0.2 & \text { HY } & \text { Wata } & \text { OP }\end{array}$

13.1 5 EIT OP

I3.2 ST BT OP
1990

Conaranes Penter Co

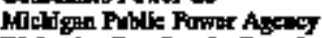

1973

Wolvetipo Fur Supply Cocp Int

1973

I97

1973

1973

1979

Corsintars Pontr Co

Derroit ardiesa Co

1984

Deroil Earou Co Mictuopon Poblic Power Agzary I988

Datroll Efinga Co

I984

1984

Animin Cowny

100.00

1958

1958

Entaliba Oy of
5100

49.00

81.39

10000

93.31

4.0

1.99

10000

595.0 ST SUB OP $\quad$ OP\$O 
Table C1. Jointly Owned Electric Generating Units by State, Company, and Plant, as of January 1, 1996 (Contimued)

\begin{tabular}{|c|c|c|c|c|c|c|c|c|}
\hline $\begin{array}{c}\text { Scote } \\
\text { Componty } \\
\text { Flent (Cornty) }\end{array}$ & Inlt & $\begin{array}{c}\text { Net } \\
\text { sommer } \\
\text { Copablly } \\
\text { (ouegyitits) }\end{array}$ & Dnd! & $\begin{array}{c}\text { Primary } \\
\text { Bustify } \\
\text { Sosred }\end{array}$ & thif & Date & Down Cmopentest2 & $\begin{array}{l}\text { Percent } \\
\text { Onned }\end{array}$ \\
\hline
\end{tabular}

Mbnotsole

Mlnosoda Powar \& 1 Jit Co

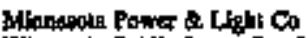

socto

Wisconato Publls Porwer Ine Sys

20.00

Yonlom Stos Power Ca

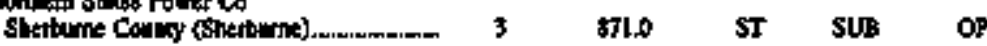

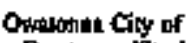

Orpasones (Stotala).

$7 \quad 145$ GT Nater or

1987

Nontem Stues Portit Co

59.00

Soolhem Minecoti Mun P Agry $\quad 41.00$

Mlarespel

Mlansadppt Power to

Vickor I Danlol Ir (Jacksom)

$\begin{array}{llll}535.9 & \text { ST } & \text { RT } & \text { OP } \\ 545.4 & \text { ST } & \text { BIT } & \text { OP }\end{array}$

top

Mrisissipgi Power Co

Golf Powar Co

5000

Syalom Anergy Reagureap Ioc

Grand Gut? (Clelbomo)

11720 NB Unathon OP

19.5s

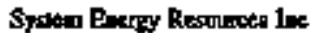

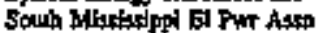

$\operatorname{sins}$

1001

Mlowert

Kandas Coty Powet a Light to

Igل的 (P)alls)

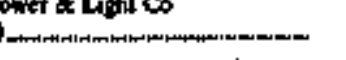

650 ST SUB oP

Mioniena

Montan Popur Co

Colstitg (Roseburi)

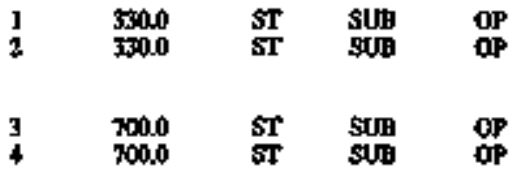

197

1976 Mownes Power os s0.00

1904

Patst Soind Powt a Eigh co

Krasas Cy Power of Light Co

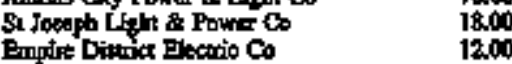

960

Monena Poner Co

Puget Somd Power is Ligh Co

Porrind Gesteral Eloctric Co 20.00

Wathingua Warer Power co

Pavificitp

Neredt

Nerate Fown $\mathrm{Co}$

Fiofd Gundar (4)

2750 ST $\quad$ BTT

DP

19 is

Cr.Fomin Dept-tit Resourses

67.80

Nevadt Ponst Ca

3220

Slam Paskite Fowre $C$

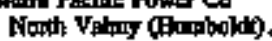

$\begin{array}{lll}2213 & \text { ST } & \text { stg } \\ 2740 & \text { ST }\end{array}$

op

1981

19:5

Ithe Pown Co

Sowthem Culkorala EAson Co

Nohere (Cletks

Ses foothobes at end of tabita. 
Table C1. Jointly Owned Electric Generating Units by State, Company, and Plant, as of January 1, 1996 (Continued)

\begin{tabular}{|c|c|c|c|c|c|c|c|c|}
\hline $\begin{array}{c}\text { Slats } \\
\text { Componf } \\
\text { Flam (Countor) }\end{array}$ & Un't & 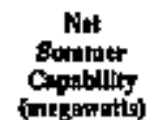 & $\underset{\text { Typel }}{\text { tylt }}$ & $\begin{array}{l}\text { Priniogy } \\
\text { Enterty } \\
\text { Soorcel }\end{array}$ & stak & Dan & Ownar Corvpante? & $\begin{array}{l}\text { Percind } \\
\text { Deried }\end{array}$ \\
\hline
\end{tabular}

\section{Nerade}

Sorubar Coilutorin Ediso Co

Sowhen Callionta Ealson co Los Ariptes toy af

Monde Foprar $C_{0}$

Bedr Rher Fo엉 AE ] \& P Dit

5600

2000

$[0,06$

New Eanpstire

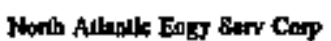

Sabrook (Rockinghan).m.
1155.0
Nip Uiming of
6190

tary Cend Fowteditat Co

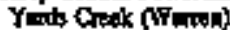

fiope Creet (Stitom)

Solan (tsinien)
Oruter Orets

990

\begin{tabular}{|c|}
\hline 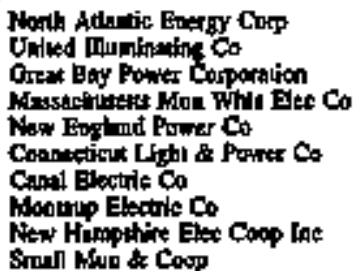 \\
\hline
\end{tabular}

Sinl Mur \& Coop

1969

Jersey Genual Poweribupi Co

10000

tano PS Water OP

$\begin{array}{lllll}1400 & P S & \text { Wowr } & \text { OP } & 1965 \\ \text { t200 } & \text { PS } & \text { Wokt } & \text { OP } & \end{array}$

Iersey Central Power\&lith Co

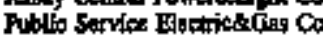

5000

$\$ 0,00$

I9:47

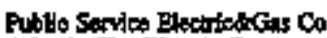

1971

Altutic aly Eletric Co

95.00

5,00

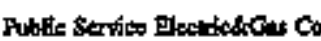

Philadolphala Biecade Co

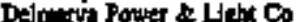

Allmic City Etectuic bo

42.59

4259

7.41

7.41

Neor phads

Arizonat Polio Strulos Co

Four Corrers (San Junn)

$\begin{array}{lllll}4 & 7400 & \text { ST } & \text { BTT } & \text { OP } \\ 5 & 7400 & \text { ST } & \text { BIT } & \text { OP }\end{array}$

1960

Soupera Calfonda Edisas Co Artronh Poblic Șervec Co

Public service Co of NMI

Selt Buret Ftod A I \& P Dit

E1 Paso Electric ह

Tuesop Platerie Fower Co

48.00

15.00

13,00

2000

706

7.6

Pabic Service Co of Nht

S.n hun (S月n Inan).

\begin{tabular}{|c|c|c|c|}
\hline 1 & $\begin{array}{l}316.0 \\
3120\end{array}$ & $\begin{array}{l}\mathbf{S T} \\
\mathbf{S T}\end{array}$ & $\begin{array}{l}\text { SUUB } \\
\text { SUR }\end{array}$ \\
\hline 3 & 48R난 & $\mathbf{5 T}$ & stto \\
\hline$d$ & 48.0 & $s t$ & st/ \\
\hline
\end{tabular}

1916

1973

Publle Service co of MLI 1979

5000

50.00

$\begin{array}{ll}\text { Publle Servike Co of NM } & 5000 \\ \text { Century Power Corp } & 5004\end{array}$

$\begin{array}{ll}\text { Publle Serviles Co of N1M } & 5000 \\ \text { Cedtury Power Con } & 5004\end{array}$ 1992

Puble Sarice Do of NMC 55\$7 thenction

Set foostints at and of table. 
Table C1. Jointly Owned Electric Generating Uniks by State, Company, and Plant, as of January 1, 1996 (Continued)

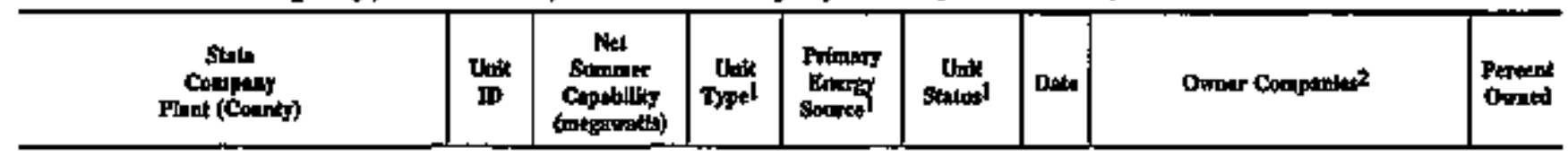

New Merko

Pable Service Co of NM

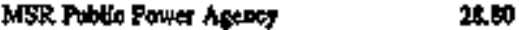

Farmingon Cly of

New Yorte

\begin{abstract}
Caphal Hiadson Gas a Rlac Corp
'Roselon (Dtaspet)
\end{abstract}

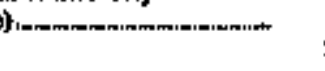

$\begin{array}{lllll}\mathbf{2} & 6018 & \mathrm{ST} & \mathrm{FO6} & \mathrm{OP} \\ 599.0 & \mathrm{ST} & \text { FO6 } & \text { OP }\end{array}$

Ningapd Molumuk Power Corp

Betbee hitud (kefterrou)

Nins Nallo Point (Onwego)

Onweso (Oswaso) ST6

Onipo a Rocklend Udis ho

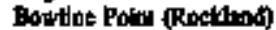

\section{Noril Cartilina}

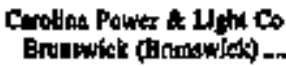

Hanis (Watral.

Mryo (Persan)

Rorbero (Rersad

(1)

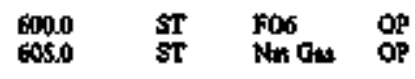

Contal Hudeon Gas \& Elex Can

Comsollated Exion Co-NY tho

Nopara Noturats Power Oorp

35.00

$\$ 0.00$

27.00

1908

1963

It)

Hytom Eovepian Lo

500.60

Nhepu Mobust Porser Corp

Centud Hindso Ges a Eles Cots

Long buand Lighting co

18000

Mew Yot Store finc a cos Com 19:00

Rochoster Oa \& Electic Corp

Ningrom Mohemic Powet Cop

Roxtestar Gas os Electric Comp

76.00

24.00

1572

1074

Comatidat Bditoo Co-NY IDe

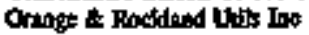

67.00 33.00

(97)

1975

Croltad Pown $*$ Lid $C_{0}$ Norb Carolin Eastet M P A

B1.67 1987

Caroting Powt to Loft $C$

Norih Corotion Finster M P A

I8.33

t5es

Canolin Potre of Lidex Co 19.80

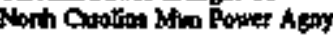

16.17

63.83

16.17

Norlb Cymlen Fiblem M P A

87,06

1294

Ses foomotes an end of table.

Energy Information Addinistration/Inventory of Power Plants in the United States as of Janvary 1, 1996 28S 
Table C1. Jointly Owned Electric Generating Units by State, Company, and Plant, as of January 1, 1996 (Contimned)

\begin{tabular}{|c|c|c|c|c|c|c|c|c|}
\hline $\begin{array}{l}\text { sento } \\
\text { Conpont } \\
\text { Fund (County) }\end{array}$ & $\begin{array}{l}\text { Uay: } \\
\text { ID }\end{array}$ & Sol & Typell & 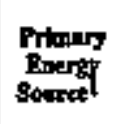 & $\begin{array}{l}\text { Unit } \\
\text { Statiosl }\end{array}$ & Dalt & Omare Compintar & Percted \\
\hline \multicolumn{9}{|l|}{ Morth ther } \\
\hline 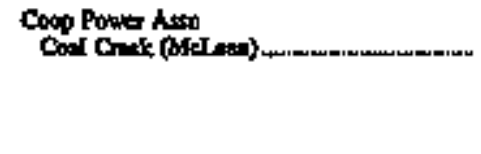 & 1 & 4655 & $\mathbf{s T}$ & $\underset{\mathbf{L O O}}{\mathbf{L}}$ & $\underset{0 P}{0 P}$ & 1979 & $\begin{array}{l}\text { Lolied Piater Atst } \\
\text { Coop Powar Asal }\end{array}$ & $\begin{array}{l}\operatorname{4x00} \\
5600\end{array}$ \\
\hline 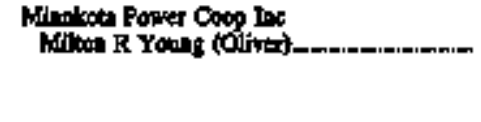 & 2 & $\$ 200$ & $\mathbf{S T}$ & LTt & $\mathbf{O P}$ & $19 \% 7$ & 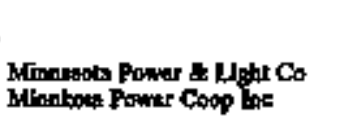 & $\begin{array}{l}70,00 \\
30,00\end{array}$ \\
\hline 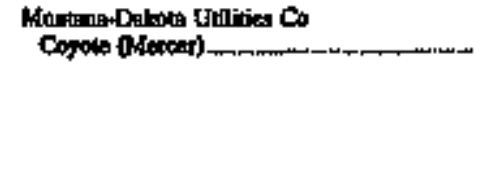 & 1 & $\mathbf{2 1 . 0}$ & $\mathbf{S T}$ & LIG & $\mathbf{0 p}$ & tpol & 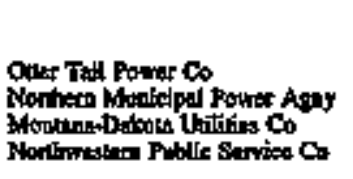 & $\begin{array}{l}35.00 \\
30.00 \\
25.00 \\
10.00\end{array}$ \\
\hline \multicolumn{9}{|l|}{ Mino } \\
\hline 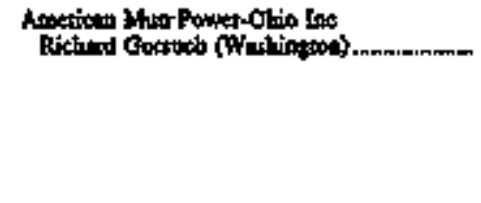 & $\begin{array}{l}1 \\
2 \\
3 \\
4\end{array}$ & $\begin{array}{l}51.0 \\
53.0 \\
53.0 \\
53.3\end{array}$ & $\begin{array}{l}\mathbf{S T} \\
\mathbf{S T} \\
\mathbf{S I}\end{array}$ & $\begin{array}{l}\text { Err } \\
\text { BIr } \\
\text { Brr } \\
\text { BrT }\end{array}$ & $\begin{array}{l}O P \\
O P \\
O P \\
O P\end{array}$ & 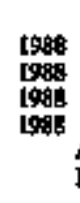 & 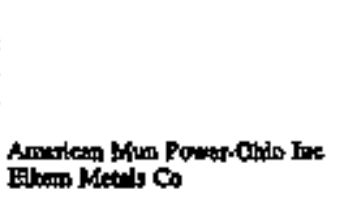 & $\begin{array}{l}79.15 \\
20.85\end{array}$ \\
\hline 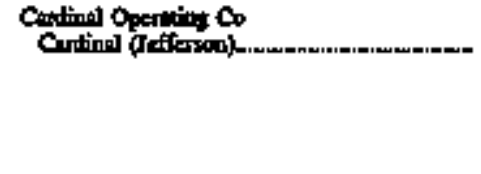 & $\begin{array}{l}1 \\
2 \\
3\end{array}$ & $\begin{array}{l}\operatorname{sis} 0 \\
\operatorname{sos} 0 \\
6 \sin 0\end{array}$ & $\begin{array}{l}\mathbf{S T} \\
\mathbf{S T} \\
\mathbf{S T}\end{array}$ & $\begin{array}{l}\text { झाt } \\
\text { BrT } \\
\text { BIr }\end{array}$ & $\begin{array}{l}\text { OP } \\
\text { OP } \\
O P\end{array}$ & $\begin{array}{l}1967 \\
1987 \\
1977\end{array}$ & $\begin{array}{l}\text { Ohis Pown Co } \\
\text { Bratago Powe Ing }\end{array}$ & $\begin{array}{l}100.00 \\
100000\end{array}$ \\
\hline 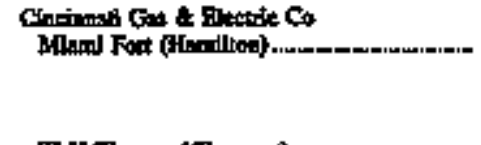 & 7 & $\begin{array}{l}5000 \\
500,0\end{array}$ & $\begin{array}{l}\mathbf{S T} \\
\mathbf{S T}\end{array}$ & BIT & OP & $\begin{array}{l}1975 \\
1975\end{array}$ & 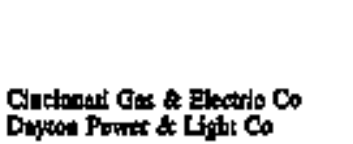 & $\begin{array}{l}6400 \\
3600\end{array}$ \\
\hline 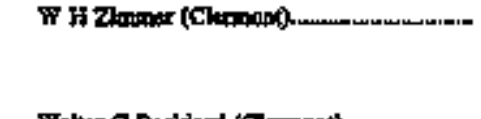 & $\mathbf{5 T}$ & 13000 & $\mathbf{S T}$ & EIT & $\mathbf{O P}$ & 1991 & 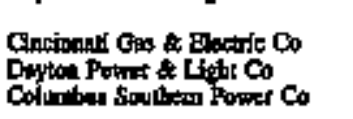 & $\begin{array}{l}46.50 \\
28.10 \\
28.40\end{array}$ \\
\hline 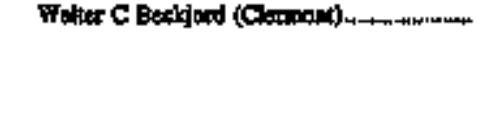 & 6 & 4140 & $\mathbf{S T}$ & EET & OP & 1969 & 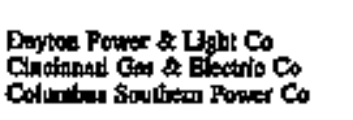 & $\begin{array}{l}50.00 \\
37.50 \\
12.50\end{array}$ \\
\hline 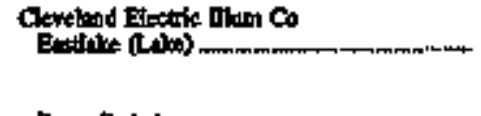 & 5 & 597.0 & $\mathbf{s T}$ & BII & OP & 1972 & 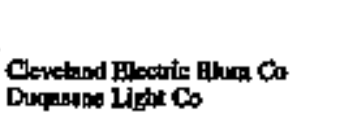 & 68.80 \\
\hline 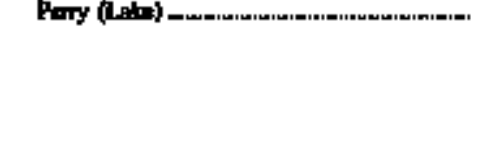 & $\mathbf{1}$ & 11690 & NB & Uhingn & OP & 1987 & 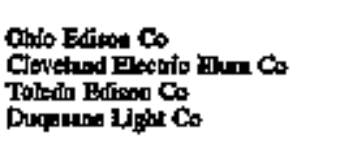 & $\begin{array}{l}35.24 \\
31.51 \\
19.91 \\
13.74\end{array}$ \\
\hline 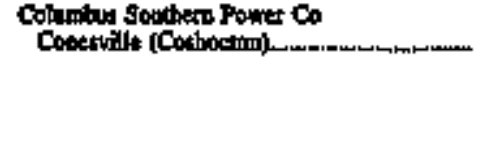 & 4 & 7800 & $\mathbf{S T}$ & EMT & $\mathbf{C P}$ & 1973 & 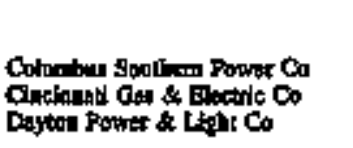 & $\begin{array}{l}40.50 \\
40,00 \\
1650\end{array}$ \\
\hline 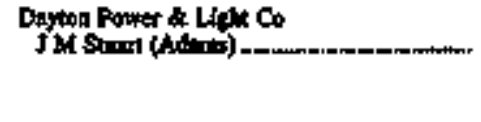 & $\begin{array}{l}1 \\
7 \\
3\end{array}$ & $\begin{array}{l}5.5 .0 \\
5.050 \\
585.0\end{array}$ & $\begin{array}{l}S \mathrm{Sr} \\
\mathbf{S T}\end{array}$ & $\begin{array}{l}\text { BUT } \\
\text { BIT } \\
\text { BIT }\end{array}$ & $\begin{array}{l}\text { OP } \\
\text { OPP } \\
\text { dP }\end{array}$ & $\begin{array}{l}1971 \\
1970 \\
1972\end{array}$ & & \\
\hline
\end{tabular}

Sect fotootas al ted of this. 
Table C1. Jointly Owned Electric Generating Units by State, Company, and Plant, as of January 1, 1996 (Continued)

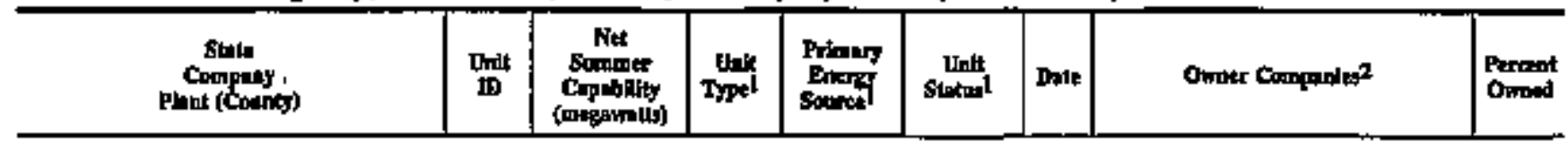

OHW

Dopton Powner L Ligh Co

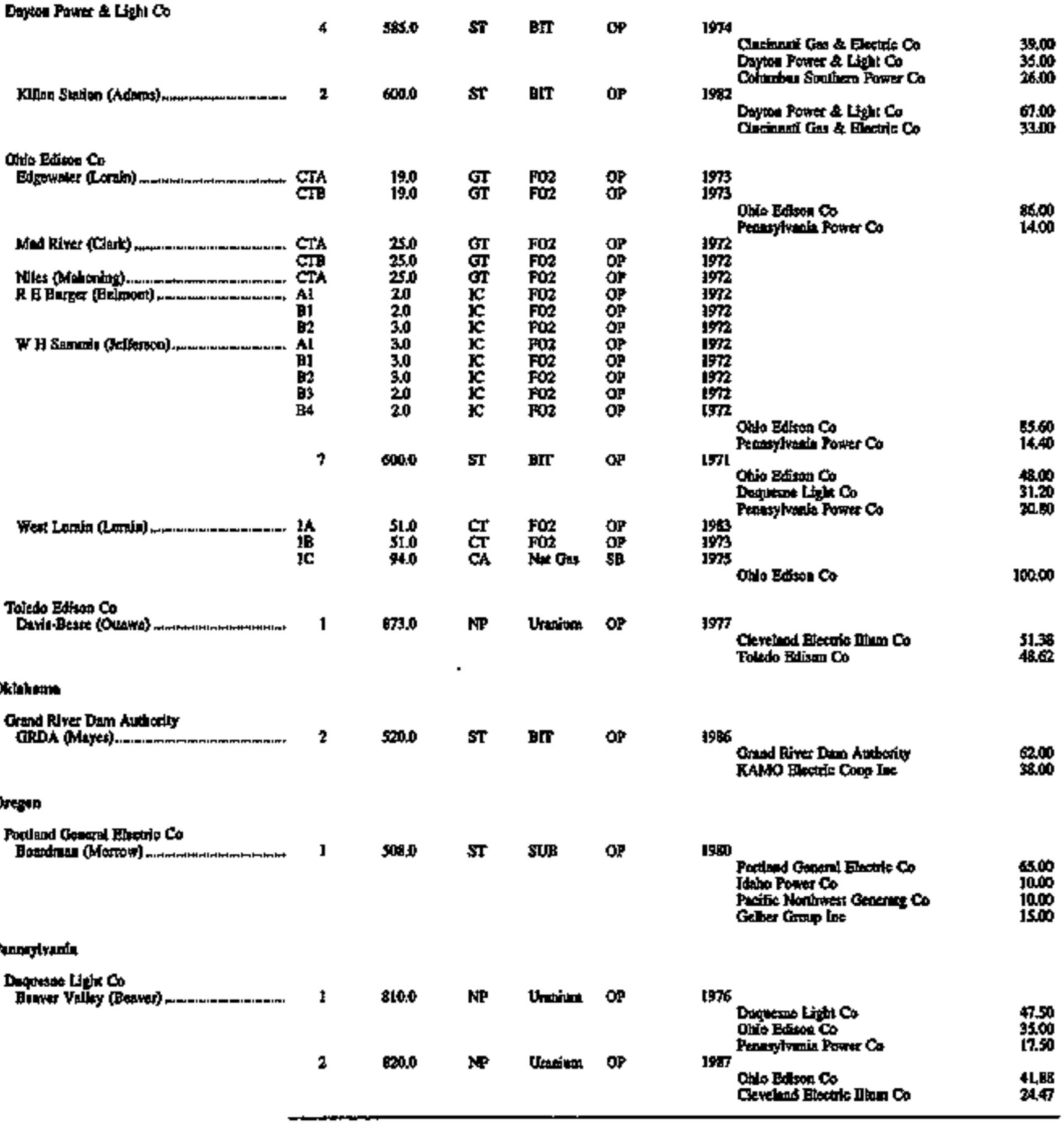

San foolnows al end of takle 
Tahle C1. Jointly Owned Electric Generating Units by State, Company, and Ptant, as of January 1, 1996 (Continued)

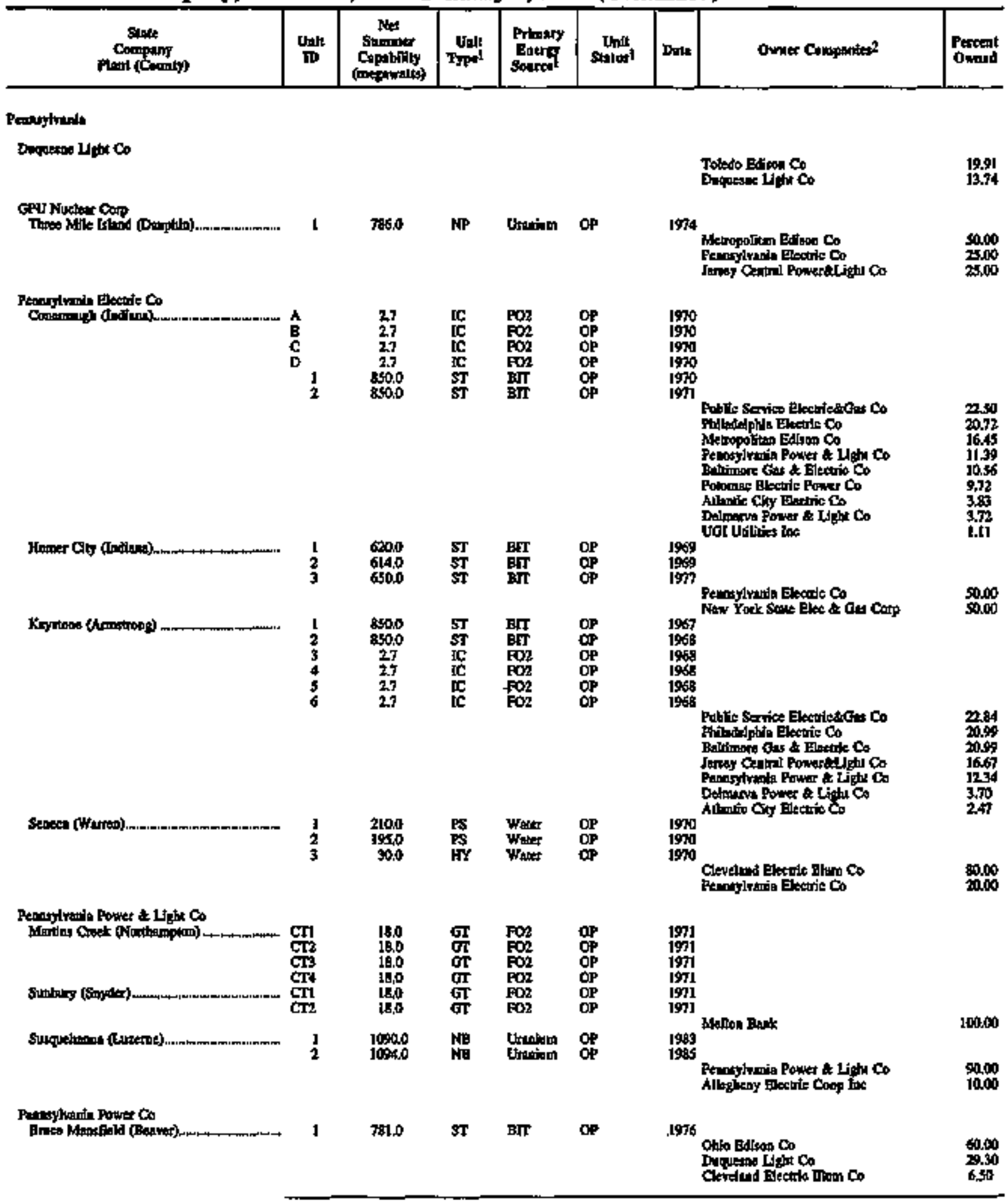

Set footacket at ed of thile. 
Table C1. Jointly Owned Electric Generating Units by State, Company, and Plant, as of January 1, 1996 (Continued)

\begin{tabular}{|c|c|c|c|c|c|c|c|c|}
\hline 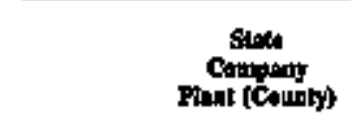 & $\begin{array}{l}\text { Unht } \\
\text { II }\end{array}$ & 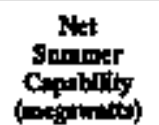 & Dat & 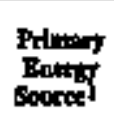 & Stunk & Duta & Orane Coupualest & $\begin{array}{l}\text { Puncant } \\
\text { Omand }\end{array}$ \\
\hline
\end{tabular}

\section{Pataglymit:}

Pennolvasia Powet Co

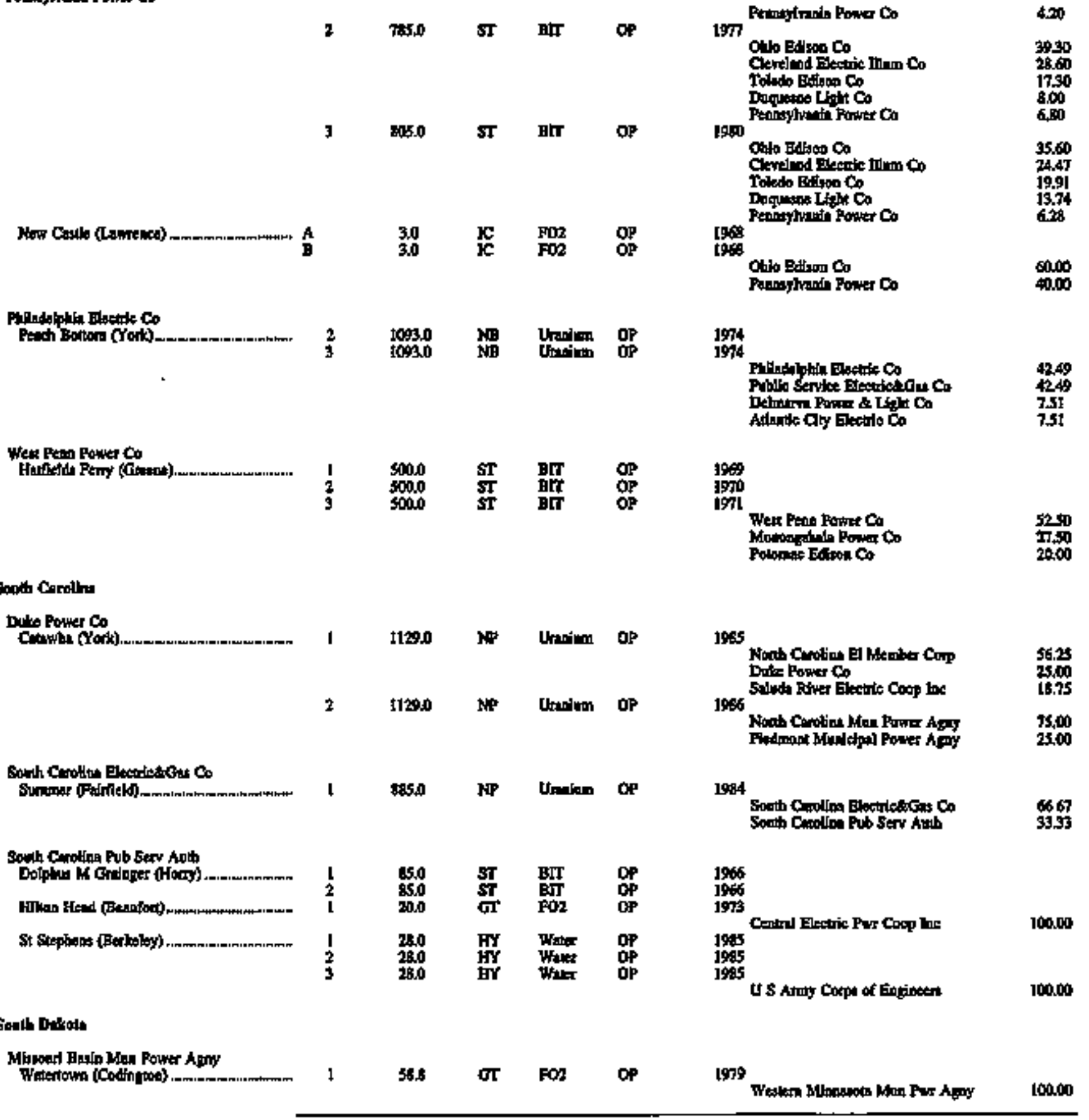

Ste Sochons te ead of lable 
Table C1. Jointly Owned Electric Generating Units by Stato, Company, and Plant, as of January 1, 1996 (Conthued)

\begin{tabular}{|c|c|c|c|c|c|c|c|c|}
\hline 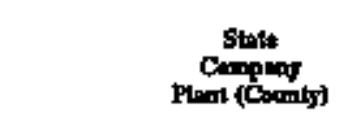 & $\begin{array}{c}U_{\text {ditl }} \\
\text { ib }\end{array}$ & 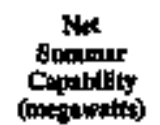 & Ing & 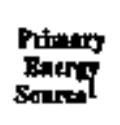 & $\begin{array}{c}\text { Jalk } \\
\text { Stritul }\end{array}$ & Doter & Otwer Conpunted2 & $\begin{array}{l}\text { Poreent } \\
\text { Omint }\end{array}$ \\
\hline
\end{tabular}

Pennsylranti

Peansyhrita Poner Co

$\begin{array}{lllll}2 & 7850 & \text { ST } & \text { ET } & \text { OP } \\ 3 & 2050 & \text { ST } & \text { BT } & \text { OP }\end{array}$

Nerr Cotsk (Lowrence)

B

$\begin{array}{llll}3.0 & \mathrm{E} & \mathrm{FO} 2 & \mathrm{OP} \\ 3.0 & \mathrm{FC} & \mathrm{FO}\end{array}$

Palbedelpbia Eloctric Co

Rench Boltom (Yotk)

1093.0 NR Ukánim OP

$19 m$

Ponoplramia Powar Co

4.7n

Gito BCisol Co.

Clerelond Ek=xtic IInon Co

Toloda EAlipa $C_{0}$

Dunament Uith $\mathrm{C}_{0}$

1900

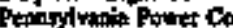

OHo Betiton Co

Clevelund Electric thom $C_{0}$

Trakdo Eílina Co

Dugname 1] th $C_{0}$

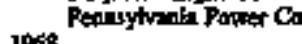

1968

OHín Bdison Co

Pannirytranta Poure Co

38.30

296

17,30

800

68

35.60

24.4

1951

6.73

60.06

4000

What Fean forver $\mathrm{Co}_{0}$

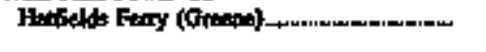

$\begin{array}{lllll}3 & 5000 & \text { ST } & \text { BIT } & \text { OP } \\ 2 & 5000 & \text { ST } & \text { BI } & \text { OP } \\ 3 & 5000 & \text { ST } & \text { BT } & \text { OP }\end{array}$

[974

1974

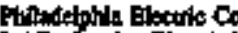

Putlit Service Electictios Co

Deltrente Powre \& Light C.

Atertis Cly Eloctats Co

4249

4249

7.51

1969
1971

West Fem Ponke Co

Mowongatich Fower Co

Pokomenc Bdipon Co

50

27.50

21000

Soub Caroina

Dole Purrer $C_{0}$

Catants (Yrat)

1985

Wath Curotena E1 Nember Corp

Doves Porver $\mathrm{C}_{0}$

56,25

2 11290 NQP Unanimor OP

1906

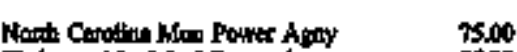

Sorth Corolina Eloctick ots $\mathrm{C}_{0}$

Somine (Fotrifiekt).

845.0 NP Utadium of

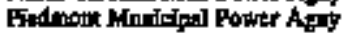

200

Soth Colitin Pob Sar Ant

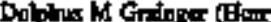

trich tiend (Becoution)

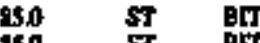

55 Bt

200 GI FOD

St Stapkent (Bertintery)

$28.0 \quad$ HY Wout

250 HY Wupt

OP 1994

Sorth Curoting Electictoses Co

South Croding Pin Berv A th 32.33

19es

1966

1973

Centel Electric Pwr Coop loc

10000

19:

u s Army Corp of Endines: 10000

South bosocta

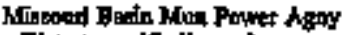

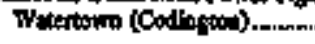

sti or for op

1979

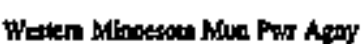

10000

Sea floomotes at end of oble.

290 Energy Information Admtaistration/Inventory of Power Plants in the United States as of January 1, 1996 
Table C1. Jointly Owned Electric Generating Units by State, Company, and Plant, as of January 1, 1996 (Continued)

\begin{tabular}{|c|c|c|c|c|c|c|c|c|}
\hline 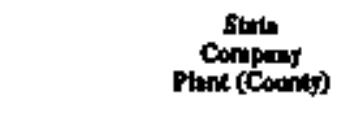 & Dnit & 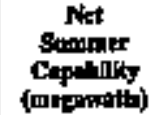 & 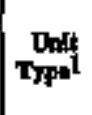 & 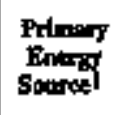 & $\underset{\text { Datak }}{\text { Datos }}$ & Dout & 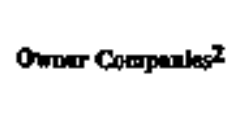 & $\begin{array}{l}\text { Pintet } \\
\text { Oinath }\end{array}$ \\
\hline
\end{tabular}

\section{Penanginanta}

Fannoyivala Powar Co

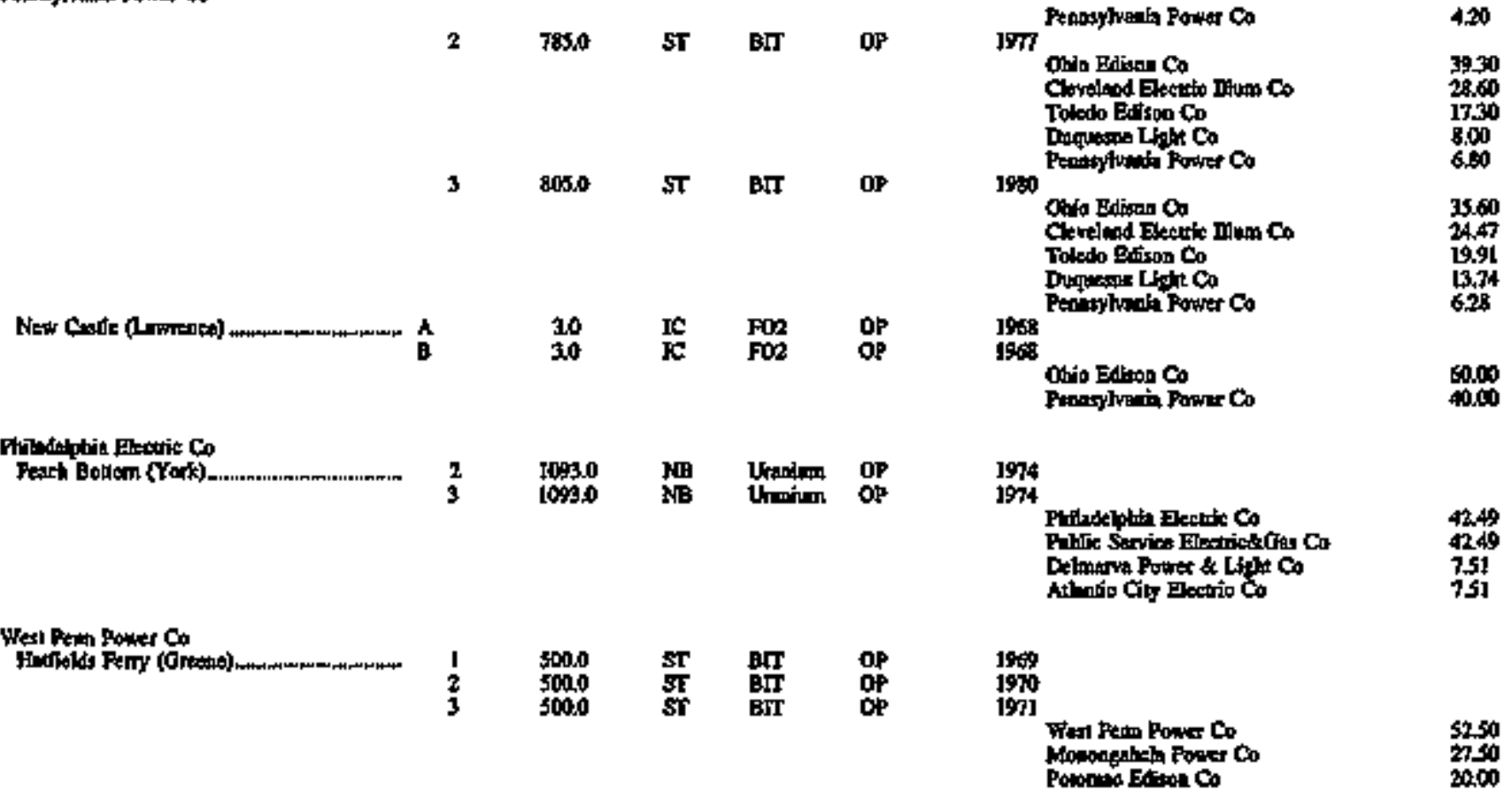

\section{Sodth Cirmtinth}

Dube Power Co

I II29,0 NP Utaniwa OP 1985

211290 NiP Unnion of

Nurth Choilno 自 Member Corp Dulk Porier Co

56.25

Lo:85

Thoo

Pledmox Marid pal Forver hapy $\quad$ XS.00

Soroth Caroling Blectriesclas Co

Sunmer (Finfiledd)

NP Urniom OP

1984

Soulh Caroint plectidestast Co Souli Caratin Pab Sery Anb

Sopih Canolian Fub Serv suth

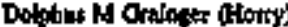

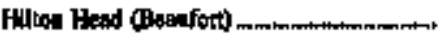

SI Steptenu (Betkeky)

1973

Cestrit Blectric Pur Coop Ins $\quad 100.00$

25, सY W

U S Aroy Capt of Eopionses

100.00

Sodth Dalrete

Milpouti Bavin Mua Power Asay

Wateriotila (Codfagion).

1979

Ses frolnower at end of table. 
Table C1. Jointly Owned Electric Generating Units by State, Company, and Plant, as of January 1, 1996 (Contimured)

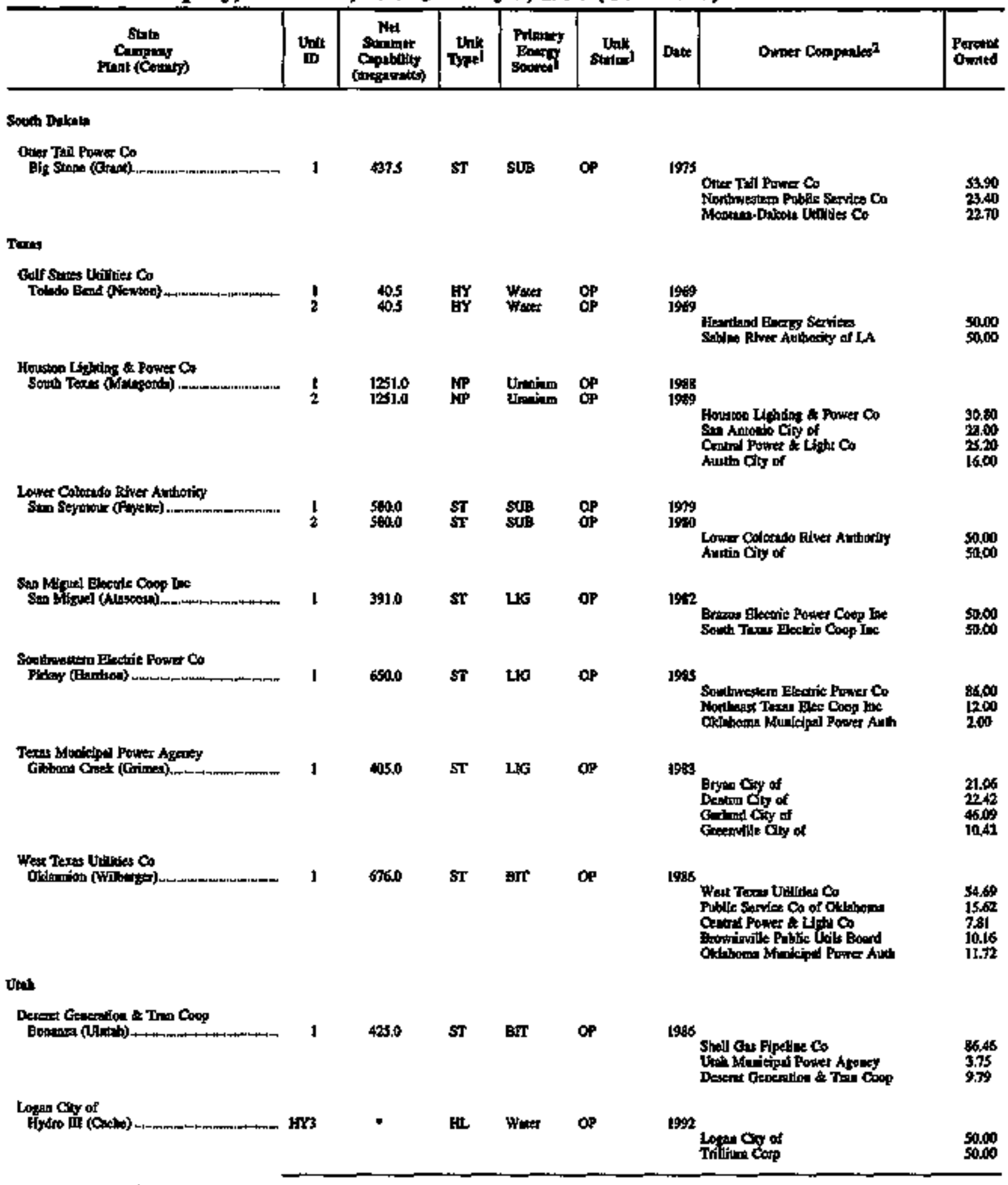

Ses footocke git ent of table.

292 Energy Information Administration/Inventory of Power Plants in the United States as of January 1, 1996 
Table C1. Jointly Owned Electric Generating Units by State, Company, and Plant, as of January 1, 1996 (Contimued)

\begin{tabular}{|c|c|c|c|c|c|c|c|c|}
\hline $\begin{array}{c}\text { Statu } \\
\text { Company } \\
\text { Ptenn (Connty) }\end{array}$ & $\begin{array}{c}\text { Ondt } \\
\text { In }\end{array}$ & 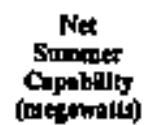 & Dntt & 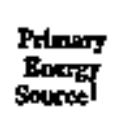 & $\begin{array}{c}\text { Unk } \\
\text { Stutogl }\end{array}$ & Dots & Owner Chipenies & $\begin{array}{l}\text { Pereest } \\
\text { Owath }\end{array}$ \\
\hline
\end{tabular}

Uah

Los Angeles City of

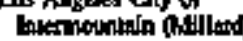

Padncorp

Huter (Enery) (Emary).

..m.n.

$\begin{array}{ll}1 & 8100 \\ 2 & 810.0\end{array}$

$\begin{array}{lll}\text { ST } & \mathrm{BN} & \mathrm{OP} \\ \text { SI } & \mathrm{BIT} & \mathrm{OP}\end{array}$

115.0 ST BT

1150 ST BIT

oP

$3 \quad 4150$

Verment

Burlingan Cliy of

J C kew ell (Owilenden)

Brifination of of

Oenin Yerourt Pat Sorv Corp

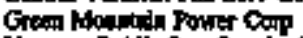

Vermoes Public Put Supply Auth

solo

20,00.

11.00

Vrointa

Virdn'a Electic a Ponn Ca

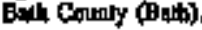

350,0
350.0
350.0
35000
350,0
350.0

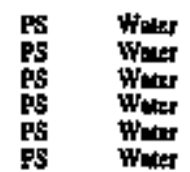

$o p$
$o p$
$o p$
$o p$
$o p$
$o p$

Clower (t)

456

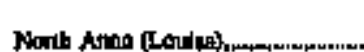

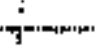

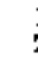

ต9.0

ST BNT

ST BIT

$\underset{\mathbf{v}}{\mathbf{p}}$

No traium

NP

op

Wastingtom

PatfllCon

Gorailh (Lowis)

1670

670

$\mathbf{S T}$

suts

$\underset{\text { op }}{\text { op }}$

$19 \mathrm{~m}$

Patacom

Whaintod Waer Foner Co

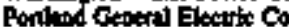

Sertilk Oty of

PUD No I of Sontoon'th Conty

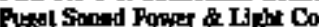

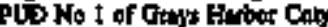

SwA 2 (Cow 15$)$

$21 \quad 34$

EY

Wour

OP

Tecoma Chy of

60.00

40.00

1996

Viridin Blection \& Pount Co

50.0.

50,00

Vhinin Flectite \& Parrer Co

혀,40

11,60

PUD No 2 \& Gran Cowny

PEC Headtwotks (Garic)

Oudpy Chate (GKant)

$\begin{array}{llll}6.8 & \text { HY } & \text { Wokr } & \text { OP } \\ 9.4 & \text { HY } & \text { Waver } & \text { OP }\end{array}$

PUD No I of Cowtin Couny

Soath Colnntia Basio he Dtt

Eetr Calambit Basin Ir Dist

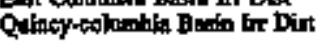

100.00

3933

34.39

4750

t5.00

250

800

700

800

32.39

Sect footpoket at and of tablot.

Energy Information Administration/Inventory of Power Plants in the United States as of January 1, 1996293 
Table C1. Jointly Owned Electric Generating Units by State, Company, and Plant, as of Janmary 1, 1996 (Continued)

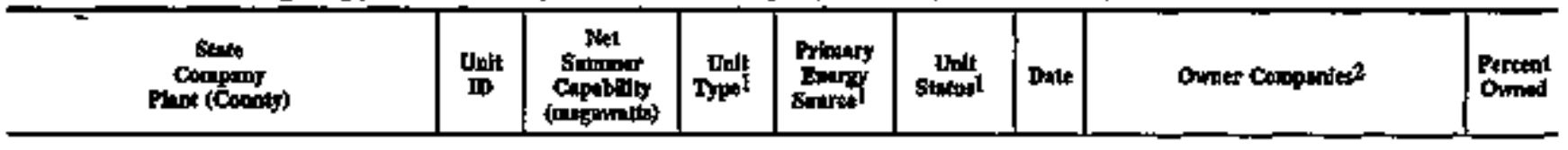

Weat Vinghen

Appilation towet Co
Jola $\mathrm{B}$ Amos (Putans)

Moconowita Pourer co

Font Mitio (botronglin).

$\begin{array}{llllll}3 & 19000 & \text { ST } & \text { BT } & \text { OP } & 1973\end{array}$

Alido Powec Co

Appolarhin Power Co

66.70

7

Duquane Light Co \$0,00

Mononertiot Powter Co

Potomed Edison Co

$2 \quad 55 s 0 \quad$ sr $\quad$ BIT OP

Hition (1)

6400

600

$\begin{array}{ll}\mathbf{S T} & \mathbf{E T} \\ \mathbf{S T} & \mathbf{B T} \\ \mathbf{S T} & \mathbf{B E}\end{array}$

West fenn Power Co

Folon:or Edison Co 30.00

20.00

1972

1994

Want Pean Power Co

Powoulc Editon Ca

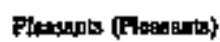

6140

ST 79

Moantyolo Porter Co

42.24

3276

1979

West Fan Powur Co

Polomec Edivon Co

Moosepileta Poytu Co

2000

Fineorita

Dainglund Powre Coosp

Geara (Vetion).

377.2 ST BiT

196

Daligind Powter Coop

Copp Pous Ass

5000

solo

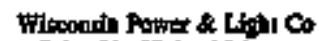

Columbin (Cohtrohisi)

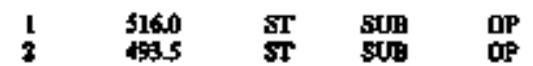

Edforwing (Steboryan).

3013 红

of

$5 \quad 3923 \quad$ ST $\mathrm{BH} \quad \mathrm{OP}$

Wipeosinin Publie Service Cep

Koripulas (Kewanned) -

\$189

Nop Upaim op

1975

Fimeondu Fumer o Ust Co

46,50

3L80

1949

Mralpon Gar \& Electio Co

2200

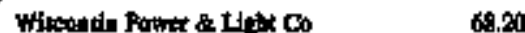

195

Thantin Fublio Service Corp 31.80

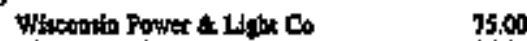

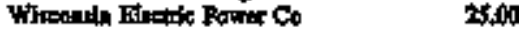

1974

Timenuln Publle Sorvie Corp 4L20

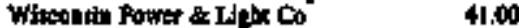

Mrilion ons \& Bectio Co

[7, 80

Wyoulas

Buin Eoculs Powar Coop

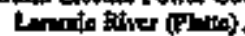

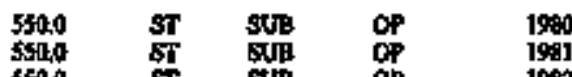

s500 \&5 stot

Badin Elecoic Powat Coop

This:un G t T Am lie

4277

16.4

5 incola Eexte Sywan 1276

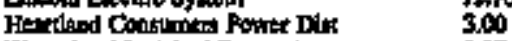

Wyomint Hodedpal Fourer Asoury $\quad \mathbf{1 3 7}$

Sex foothots at end of bible.

294 Bnergy Information Administrationfinventovy of Power Plants in the United States as of JanuEry 1,1996 
Table C1. Jointly Owned Electric Generating Units by State, Company, and Plant, as of Janiary 1, 1996 (Continued)

\begin{tabular}{|c|c|c|c|c|c|c|c|c|}
\hline $\begin{array}{c}\text { Cotspanty } \\
\text { Fins (Cominty) }\end{array}$ & Un'ti & 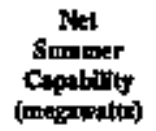 & Typt & $\begin{array}{l}\text { Podmang } \\
\text { Butrat } \\
\text { Soneref }\end{array}$ & $\begin{array}{l}\text { Untit } \\
\text { Etainst }\end{array}$ & Date & Omper Componate & $\begin{array}{l}\text { Pereent } \\
\text { Orred }\end{array}$ \\
\hline \multicolumn{9}{|l|}{ Wyonint } \\
\hline 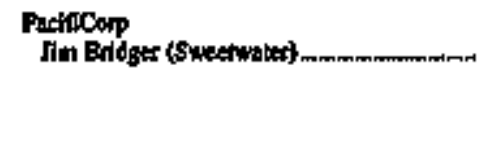 & $\begin{array}{l}1 \\
2 \\
3 \\
4\end{array}$ & $\begin{array}{l}5200 \\
520,0 \\
520,0 \\
520.0\end{array}$ & $\begin{array}{l}\mathbf{S T} \\
\mathbf{S T} \\
\mathbf{S T} \\
\mathbf{S T}\end{array}$ & $\begin{array}{l}\text { sug } \\
\text { suB } \\
\text { sUB } \\
\text { suB }\end{array}$ & $\begin{array}{l}Q P \\
Q P \\
Q P \\
O P\end{array}$ & $\begin{array}{l}1974 \\
1975 \\
\text { l976 } \\
\text { I979 }\end{array}$ & 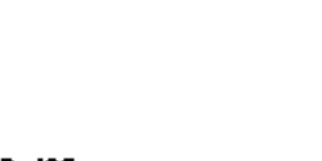 & \\
\hline Wyodel (Canpbol) & $\mathbf{]}$ & 3050 & $\mathbf{s T}$ & gut & op & 3978 & 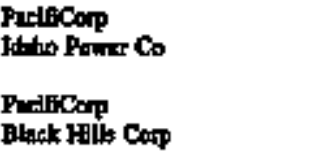 & $\begin{array}{l}56,67 \\
35.30 \\
89,010 \\
20,00\end{array}$ \\
\hline
\end{tabular}

I Sex Appeadx B for todin,

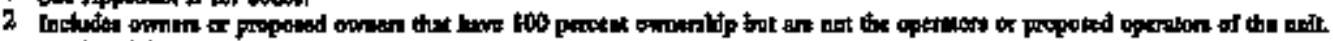

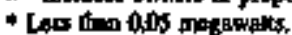

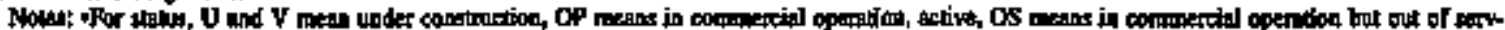

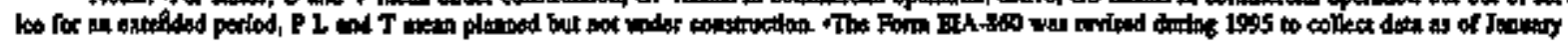

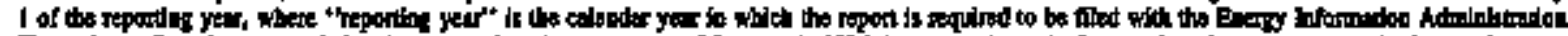

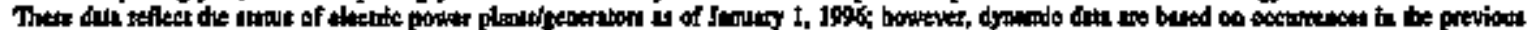

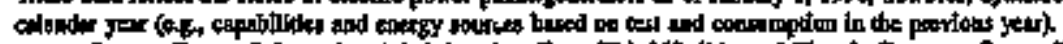

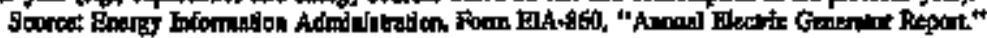





\title{
Appendix D
}

\author{
U.S. Electric \\ Utility Plants
}





\section{Appendix D}

\section{U.S. Electric Utility Plants}


Table D1. U.S. Electric Utillty Plants, as of Janmary 1,199

\begin{tabular}{|c|c|c|}
\hline Plont Xinme & Uldity than & silada \\
\hline 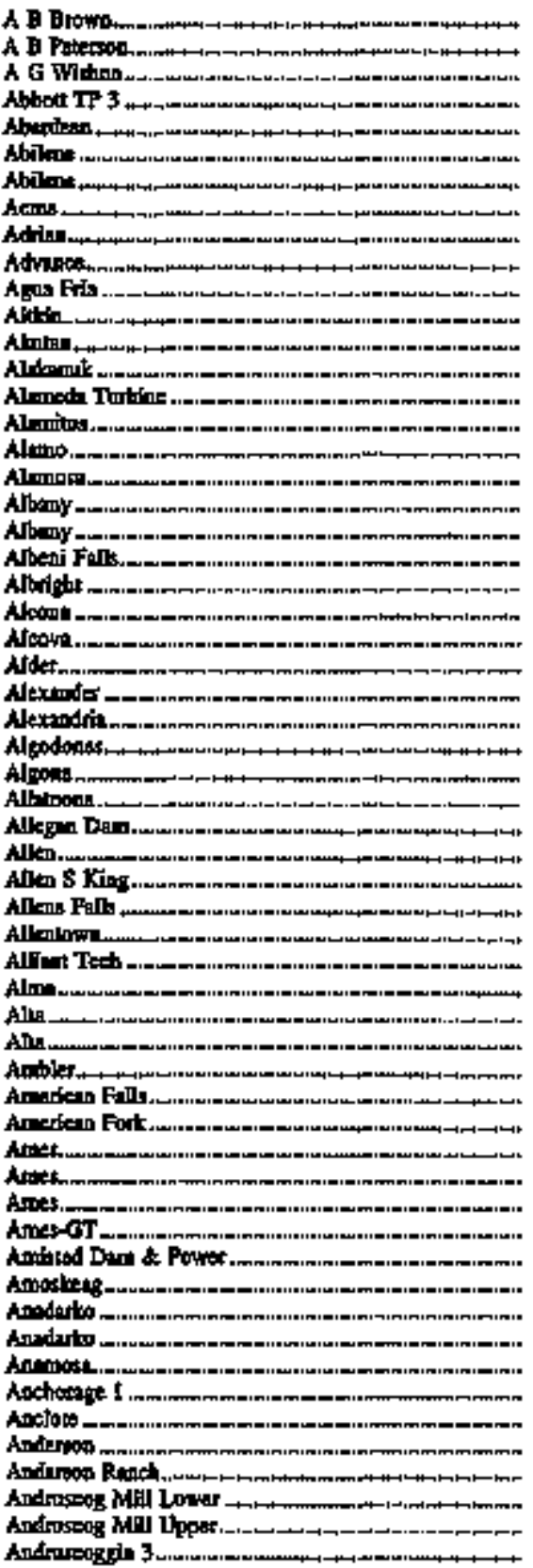 & 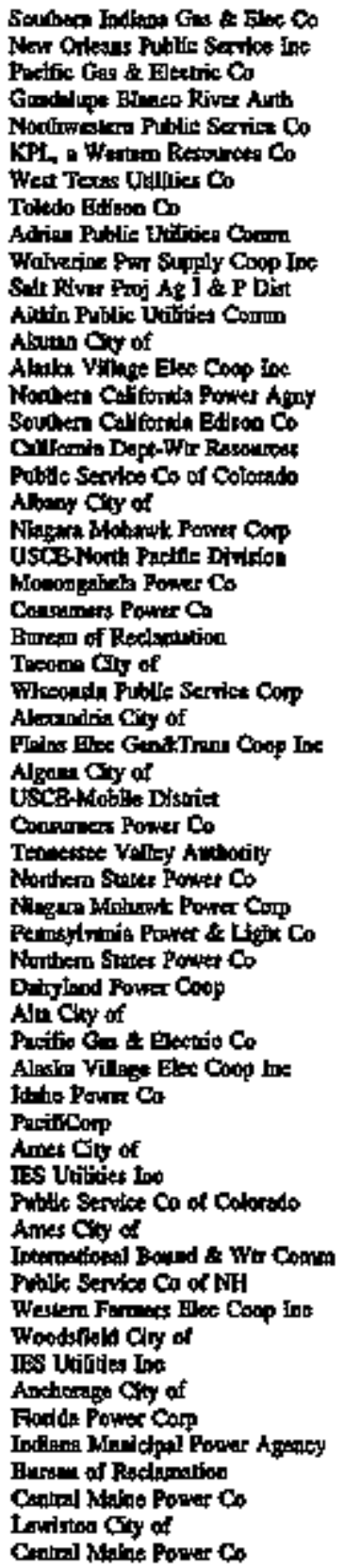 & 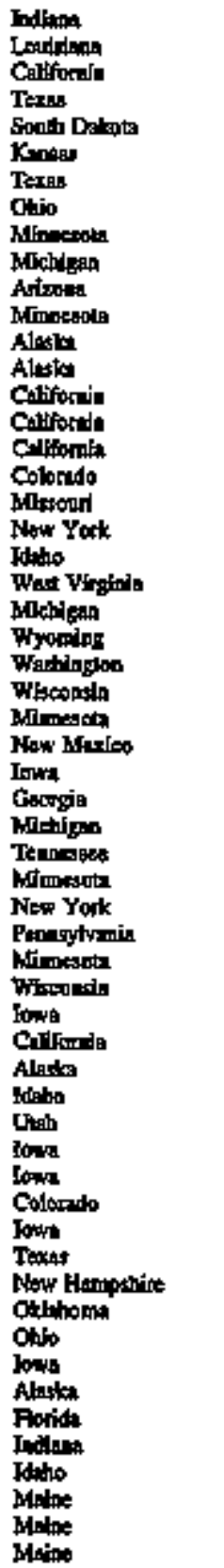 \\
\hline
\end{tabular}

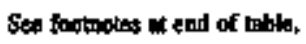


Table D1. U.S. Electric Utilly Plants, as of January 1, 1995 (Conthrued)

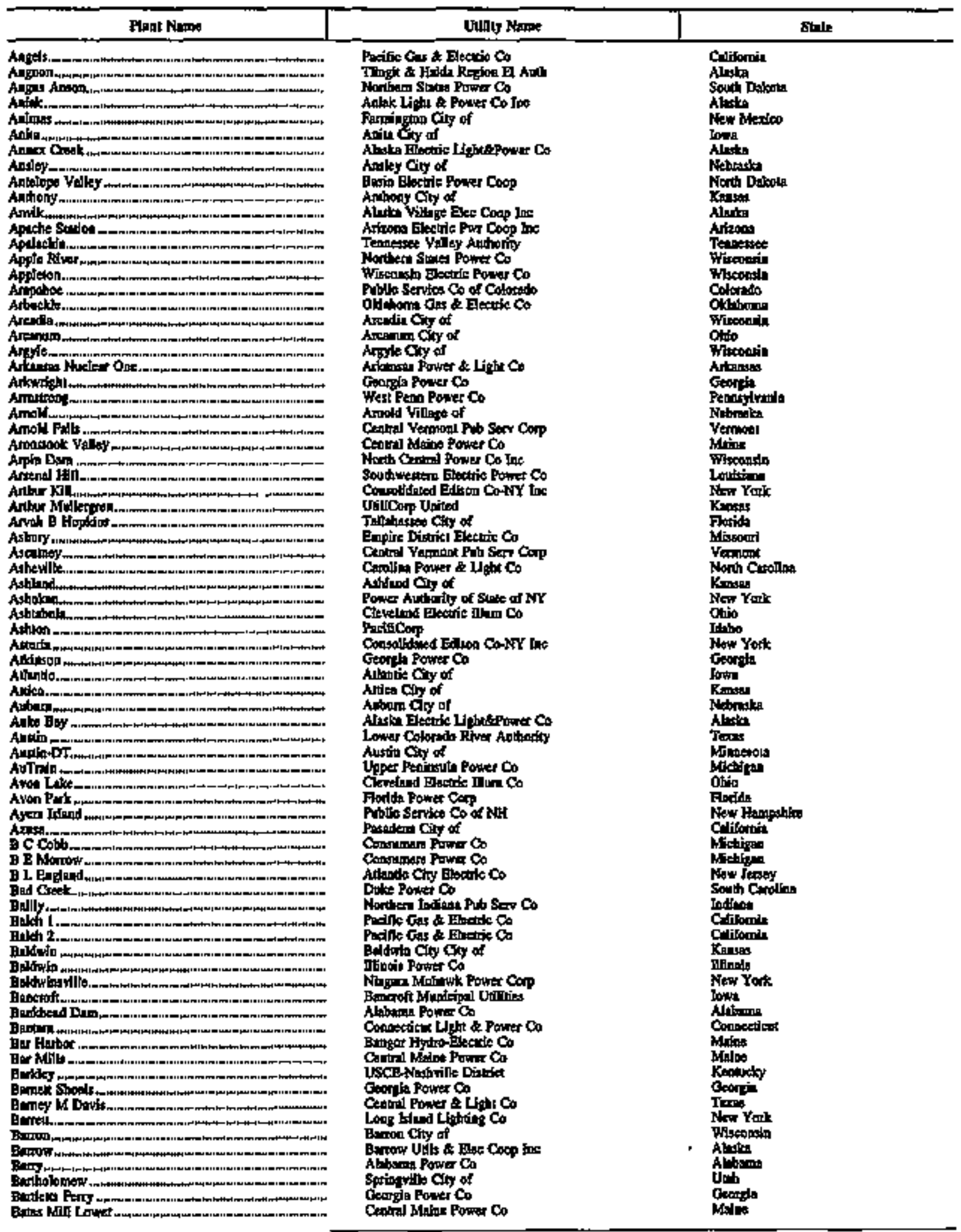

see foomates at end of toble. 
Tabłe DL. U.S. Flectric Utility Plants, as of Jannary 1, 1995 (Continued)

\begin{tabular}{|c|c|c|}
\hline Plont Nhand & Uully hat & senton \\
\hline 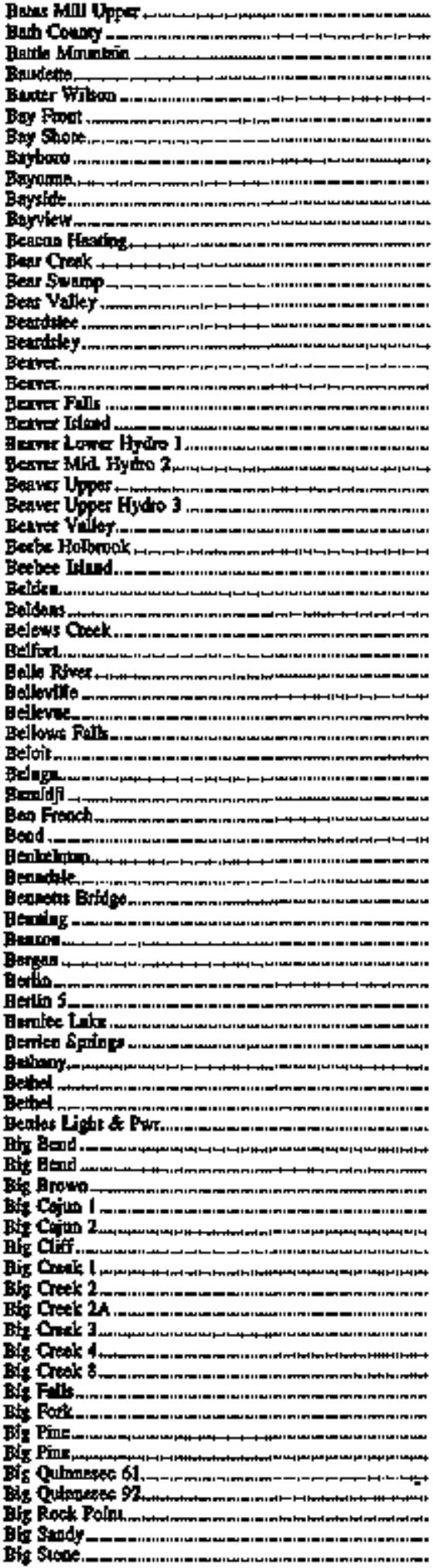 & 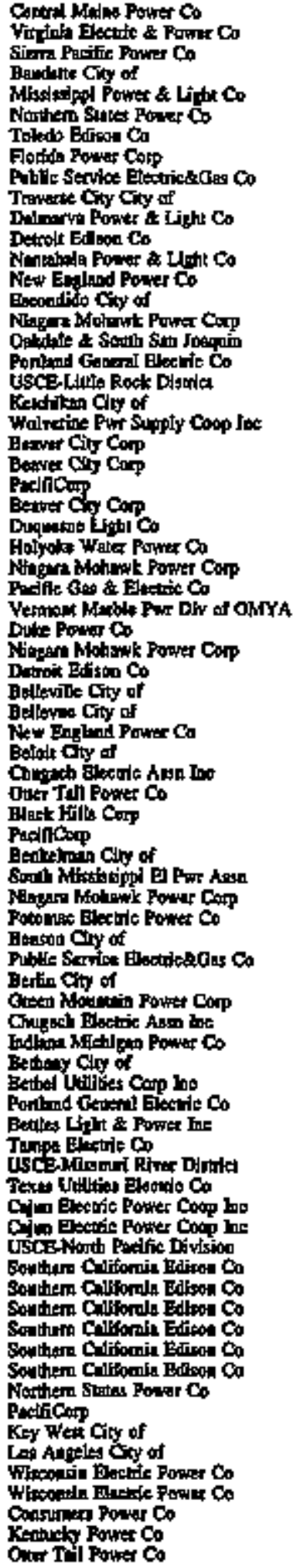 & 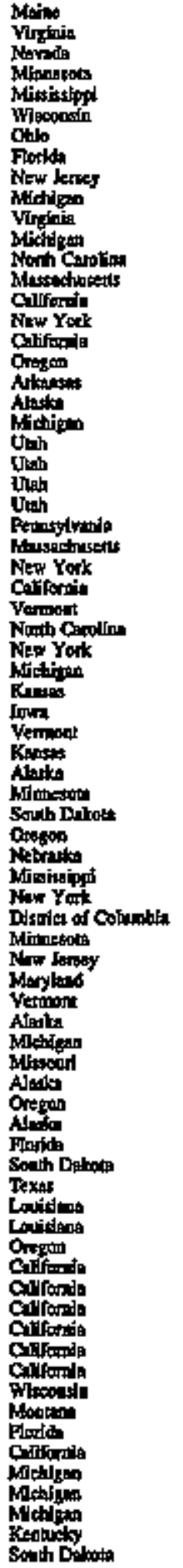 \\
\hline
\end{tabular}

Sor bookotes at end of ubte. 
Tabje D1. U.S. Electric Utility Plants, as of January 1, 1995 (Continued)

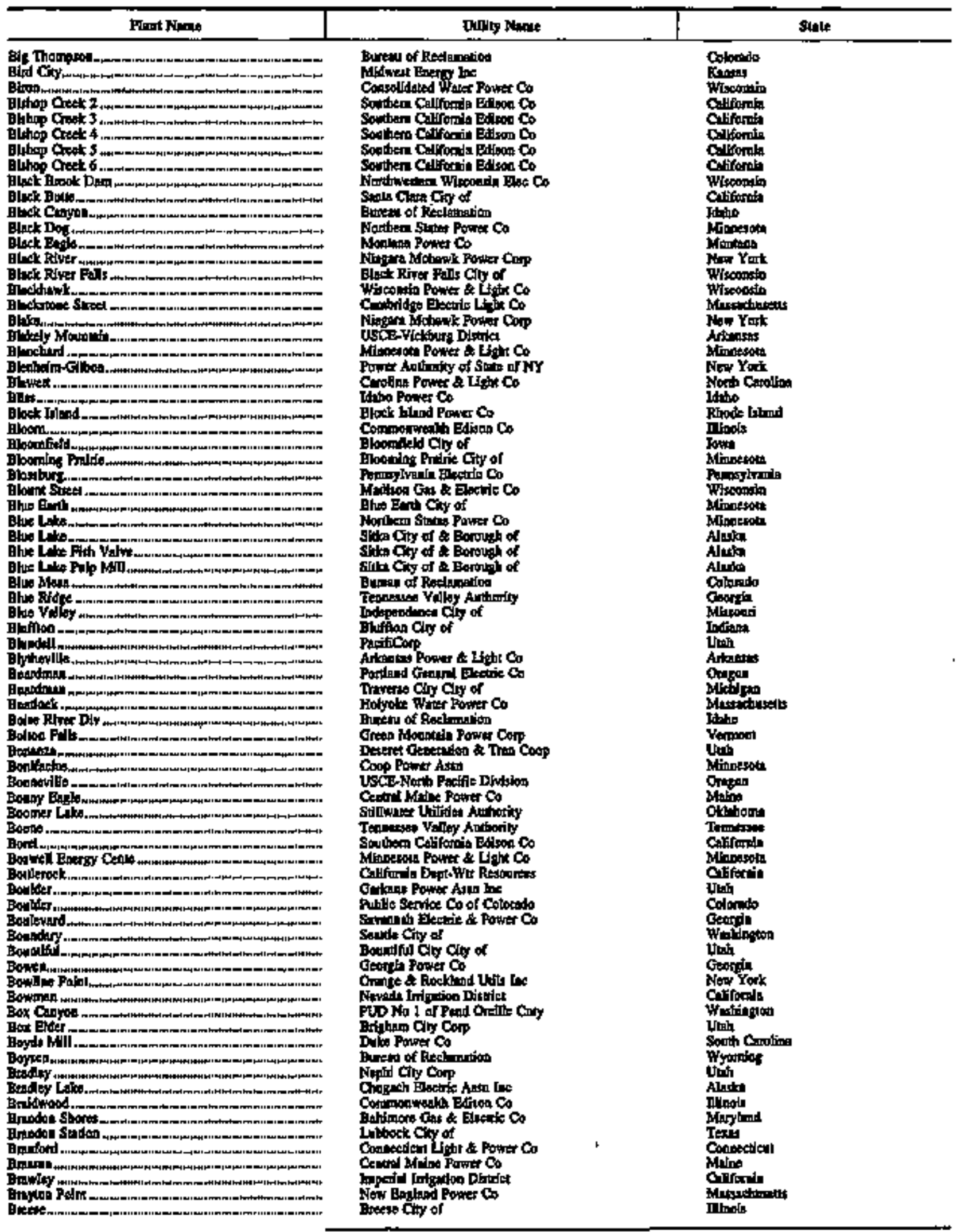

Sad fooknow at and of table 
Table D1. U.S. Electric Utility Plants, as of Jancary 1, 1995 (Contionued)

\begin{tabular}{|c|c|c|c|}
\hline Plant Nabut & Utilty Xtank & Stats & : \\
\hline 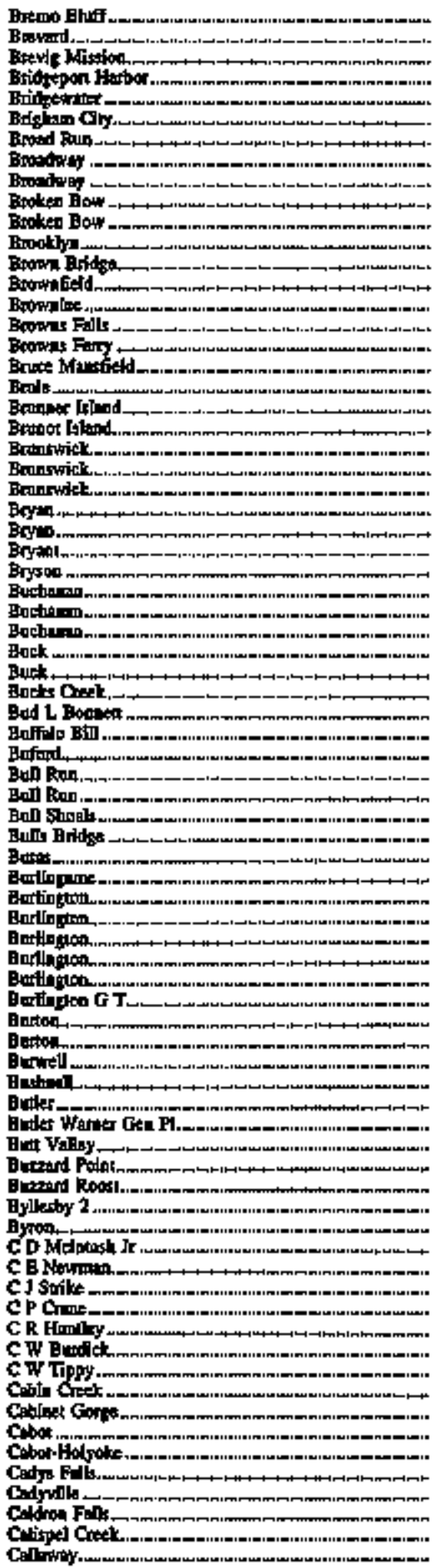 & 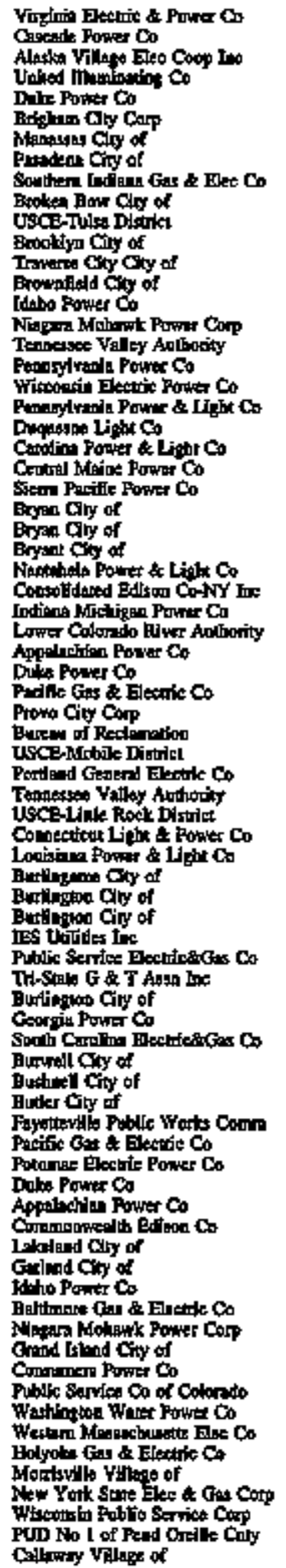 & 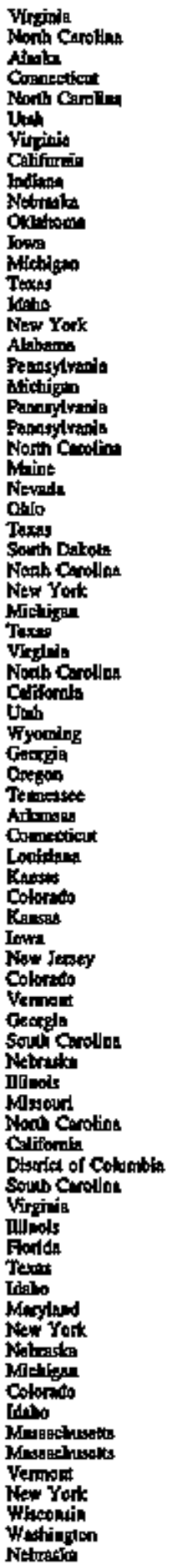 & \\
\hline
\end{tabular}

Ses toconols an ead of thik. 
Teble D1. U.S. Electric Utility Plents, as of January 1, 1995 (Continued)

\begin{tabular}{|c|c|c|c|}
\hline Fint Nrma & Ulilfy Nome & & State \\
\hline 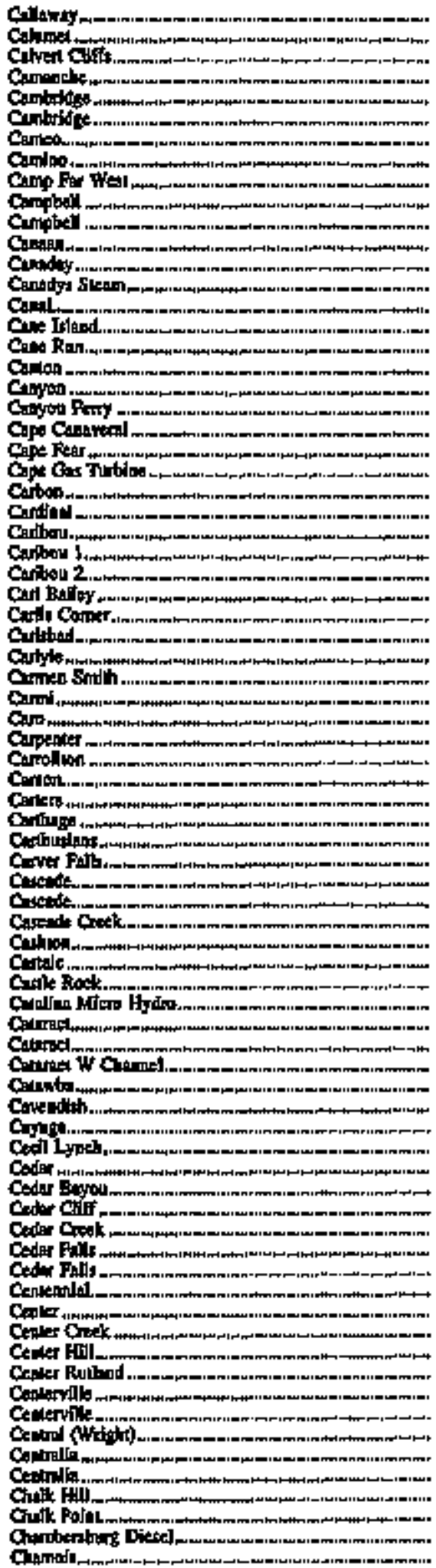 & 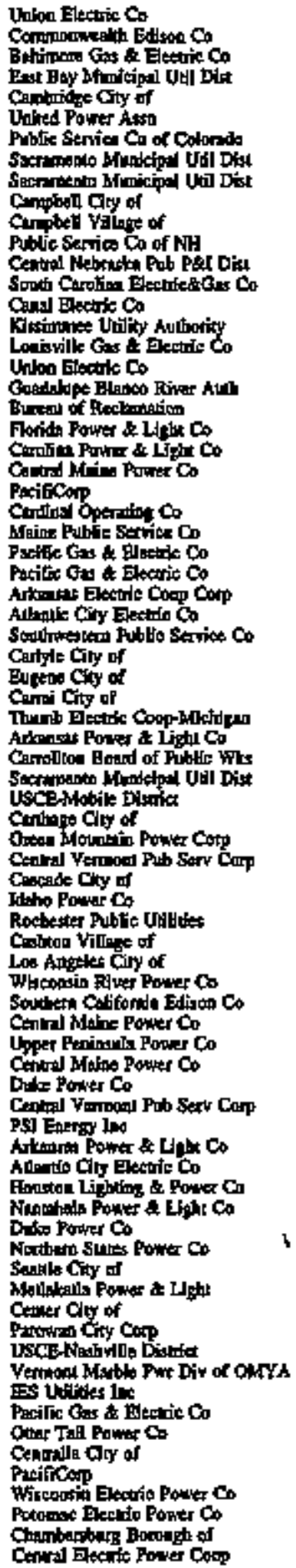 & 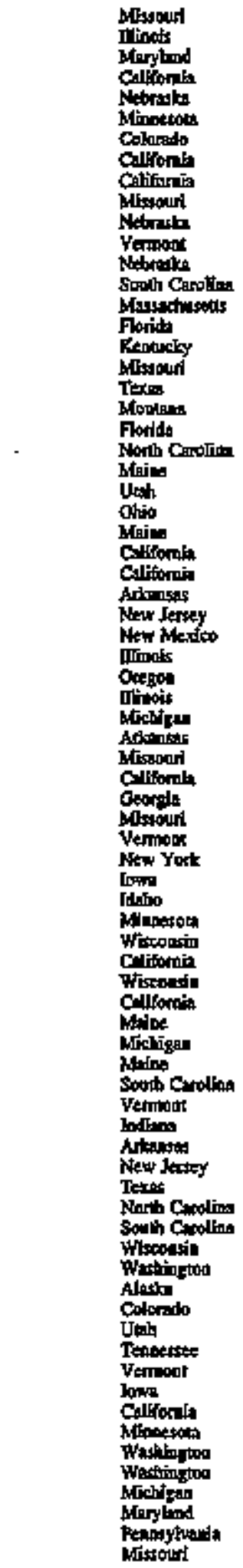 & \\
\hline
\end{tabular}

Set focknowe a and of lable. 
Tabje D1. U.S. Electric Utitity Plants, as of January 1, 1995 (Continued)

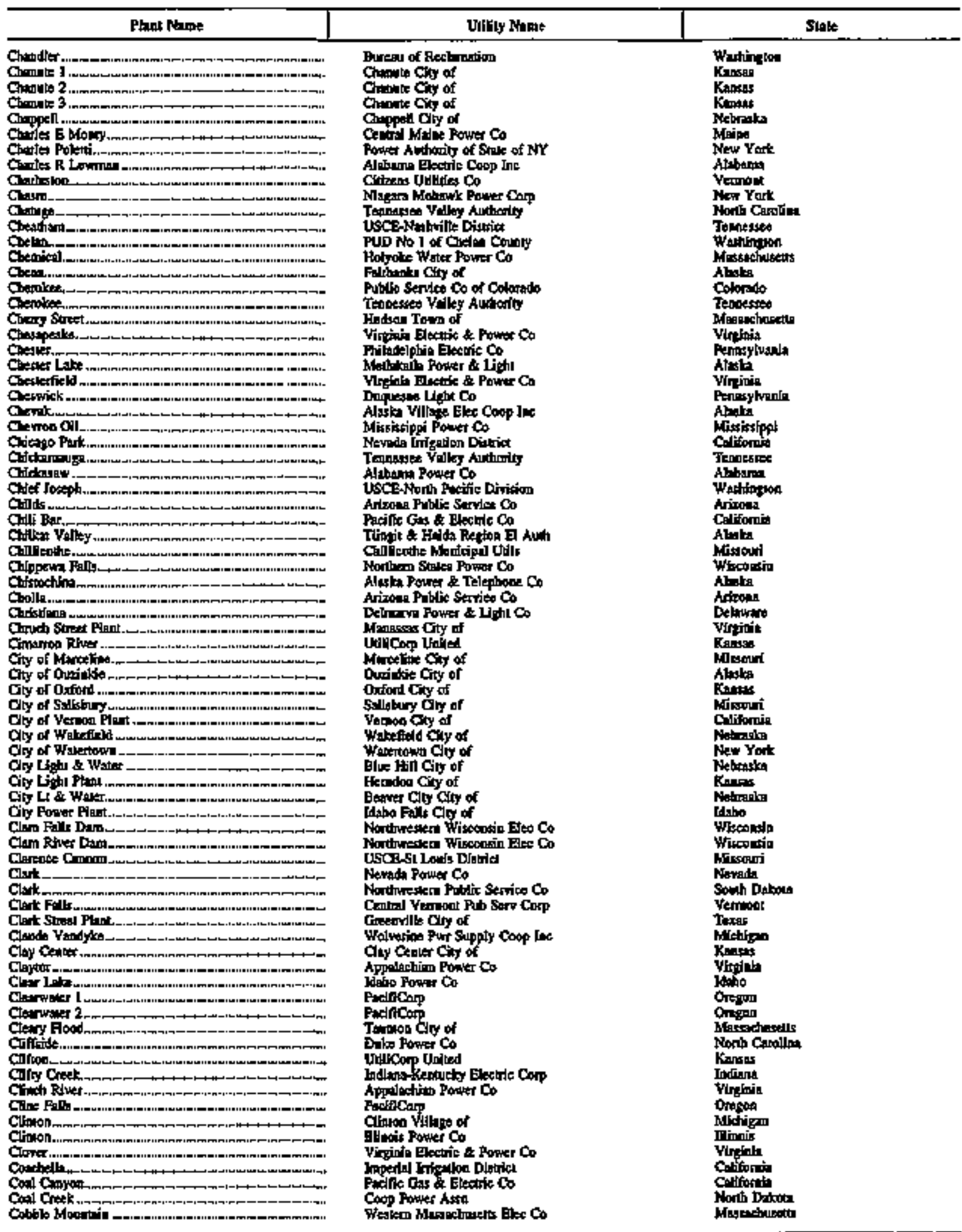

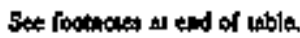


Table D1. U.S. Etectric Utilty Plants, as of Jamuary 1, 1995 (Continuted)

\begin{tabular}{|c|c|c|}
\hline Pient Napie & Wolity Nothe & State \\
\hline 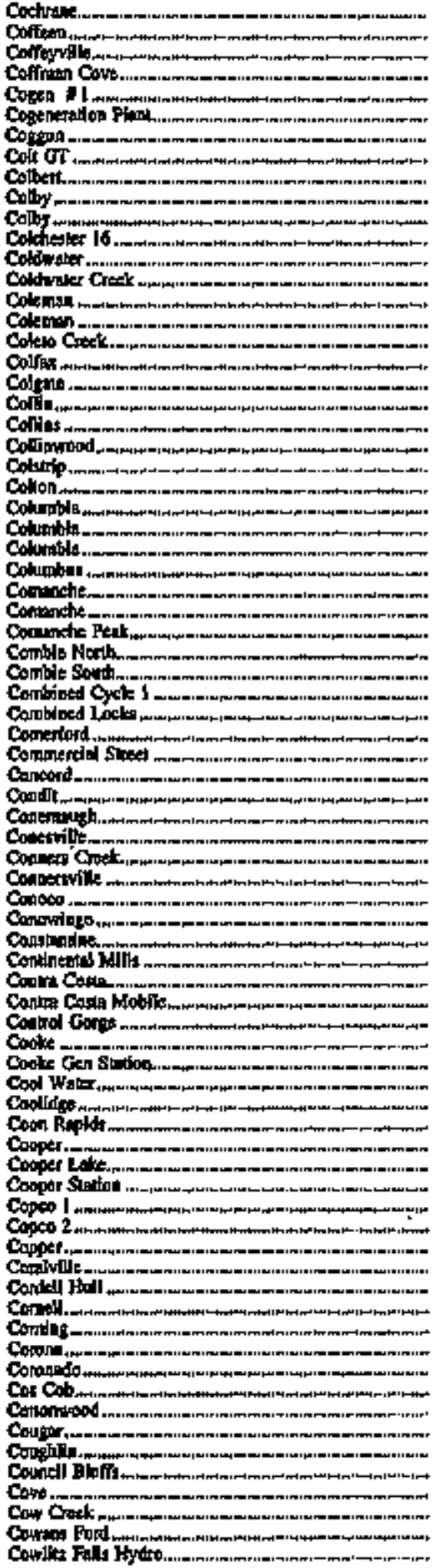 & 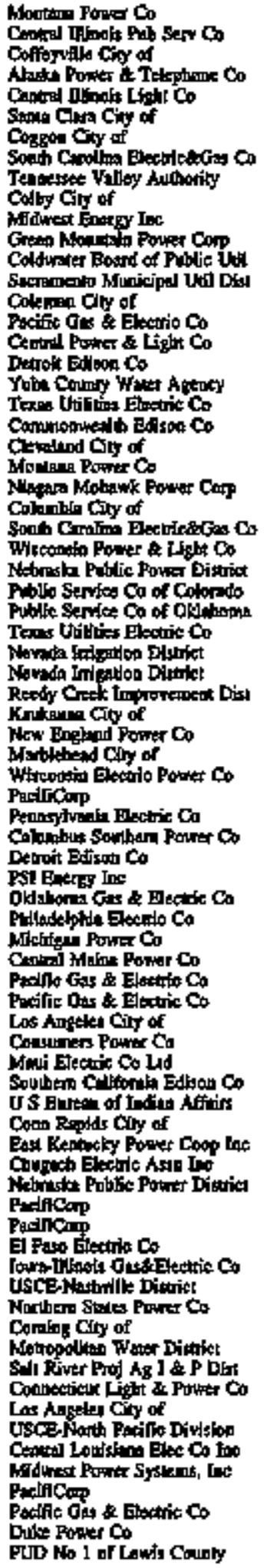 & 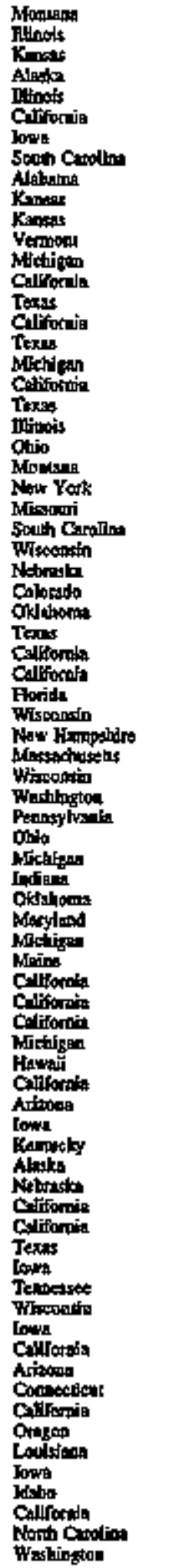 \\
\hline
\end{tabular}

Slat foctorstes it and of trblt. 
Table D1. U.S. Electrie Uthity Plants, as of Jannary L, 1995 (Comtinued)

\begin{tabular}{|c|c|c|}
\hline Plant Xoe & Julisy Nome & Itale \\
\hline 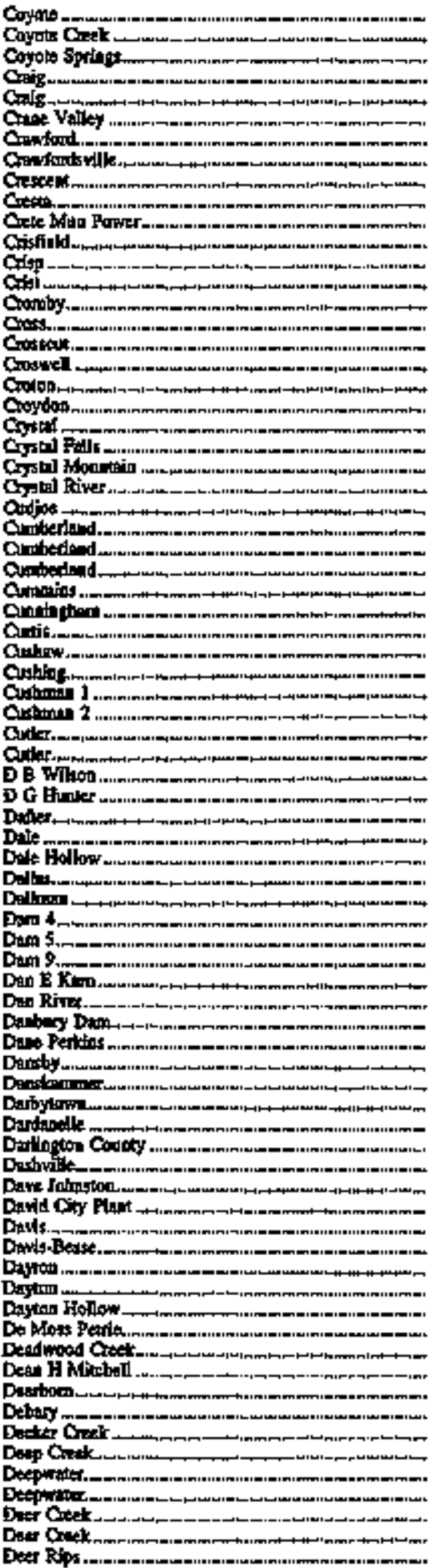 & 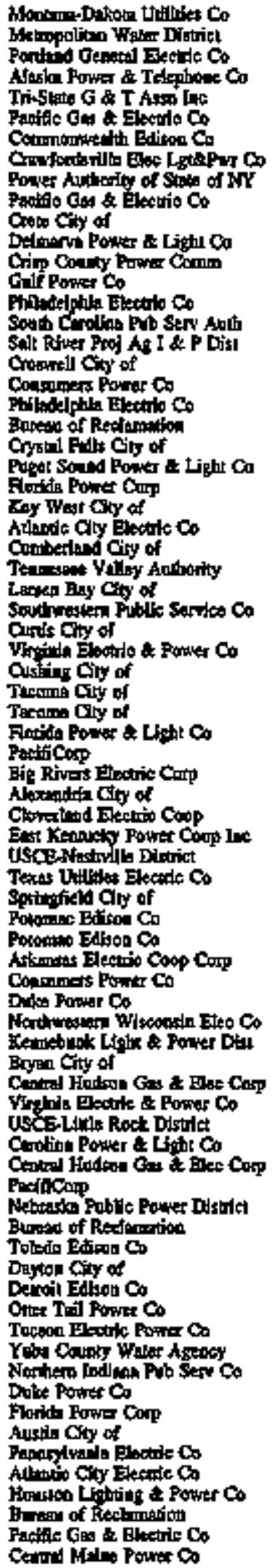 & 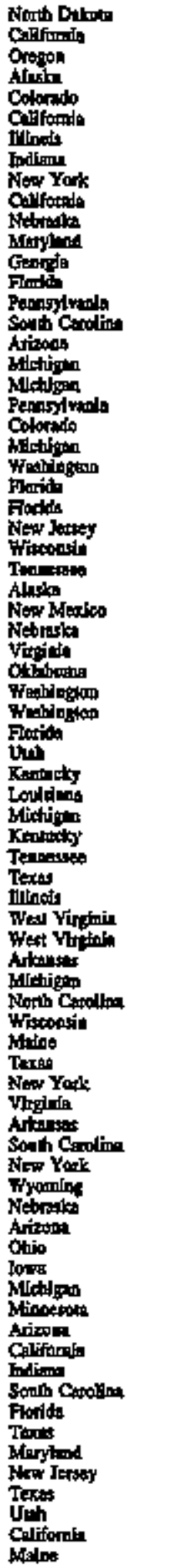 \\
\hline
\end{tabular}

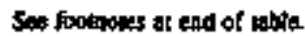


Table D1. U.S. Electric Utillity P1pnts, as of January 1, 1995 (Continued)

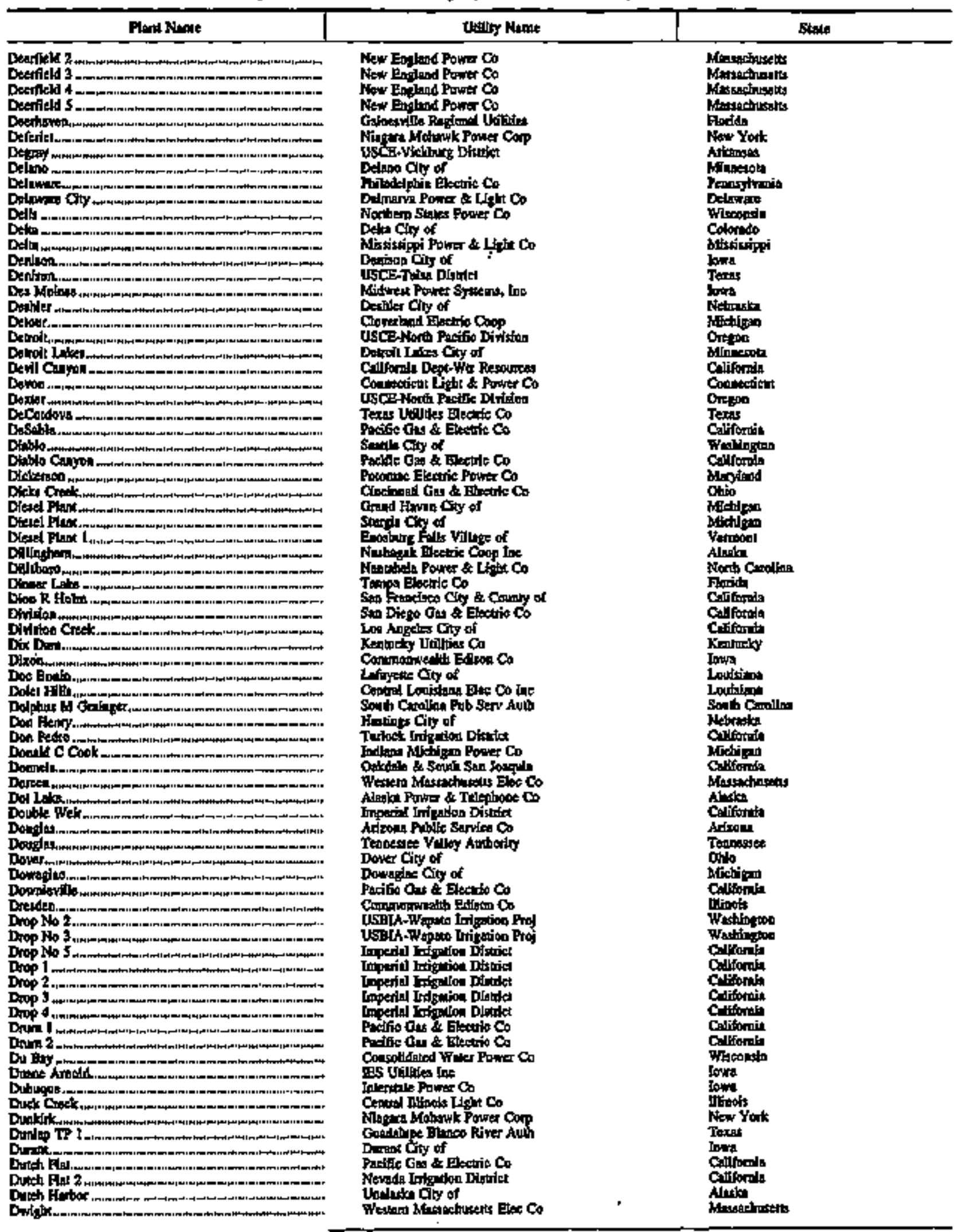

Seo foolnowis al and of thile. 
Tahle D1. U.S. ERlectric Utillty Plants, as of Jamuary I, 1995 (Contimued)

\begin{tabular}{|c|c|c|}
\hline Plank Nanat & Dullty Nens. & stato \\
\hline 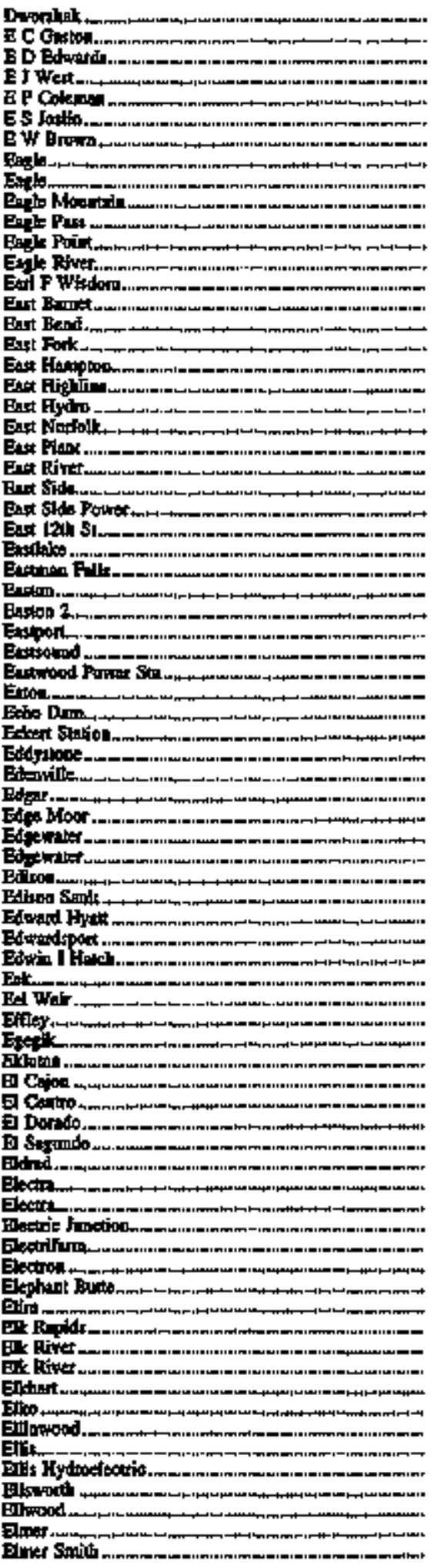 & 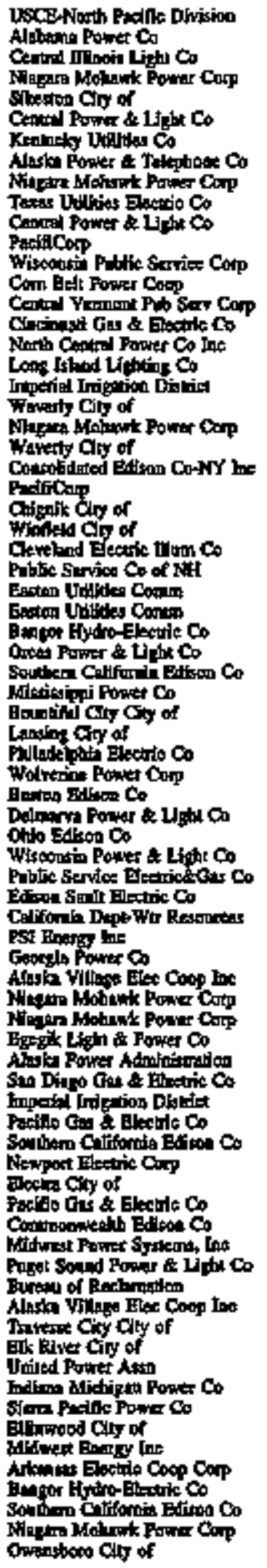 & 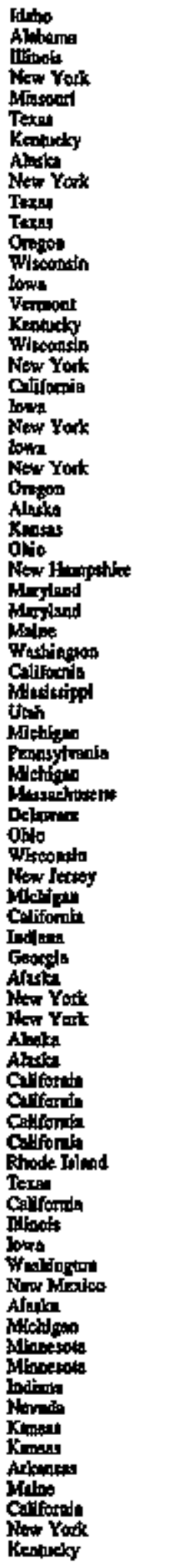 \\
\hline
\end{tabular}

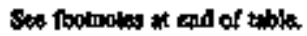


Table D1. U.S. Eleetret Uitulity Plants, as of Jaruary 1, 1995 (Continued)

\begin{tabular}{|c|c|c|c|}
\hline Plant Nowe & Uulty htom: & & State \\
\hline 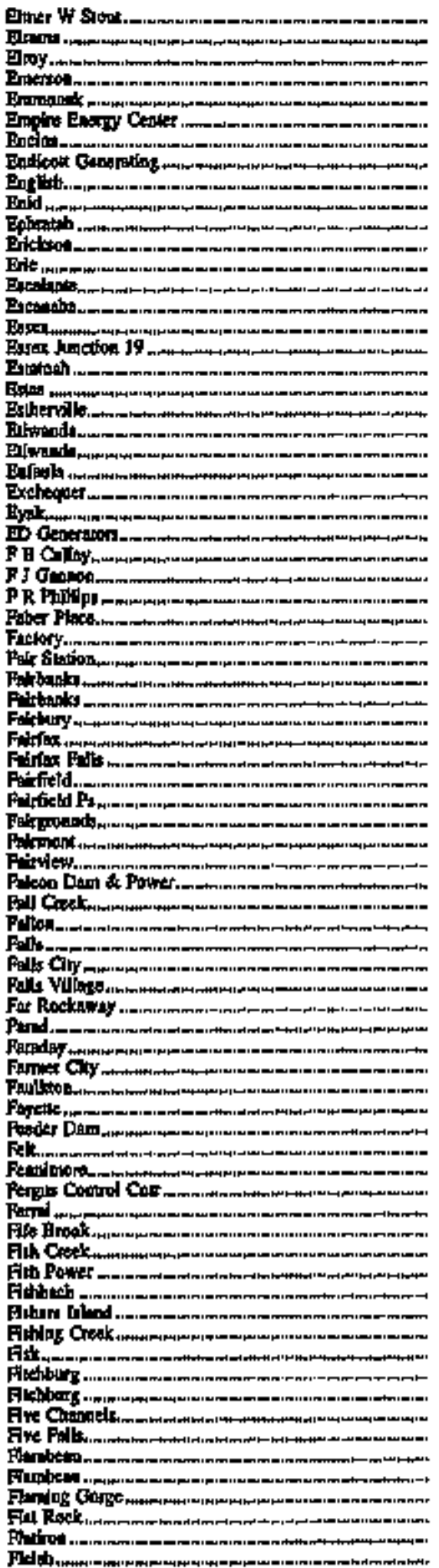 & 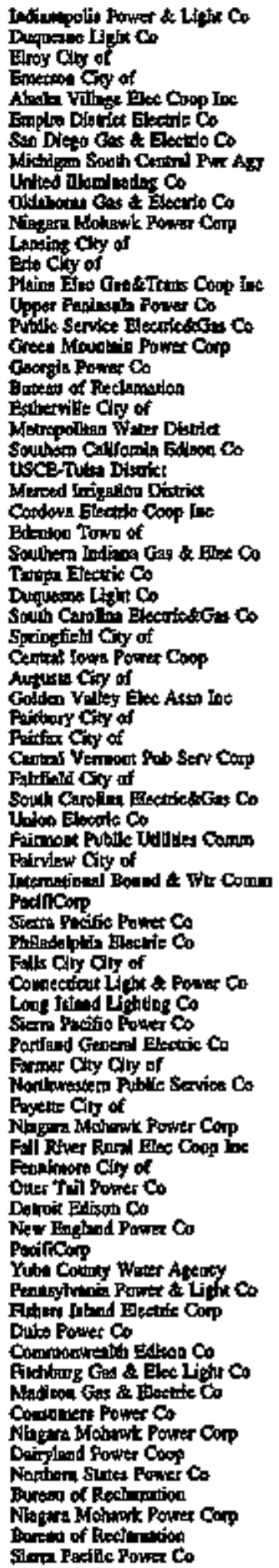 & 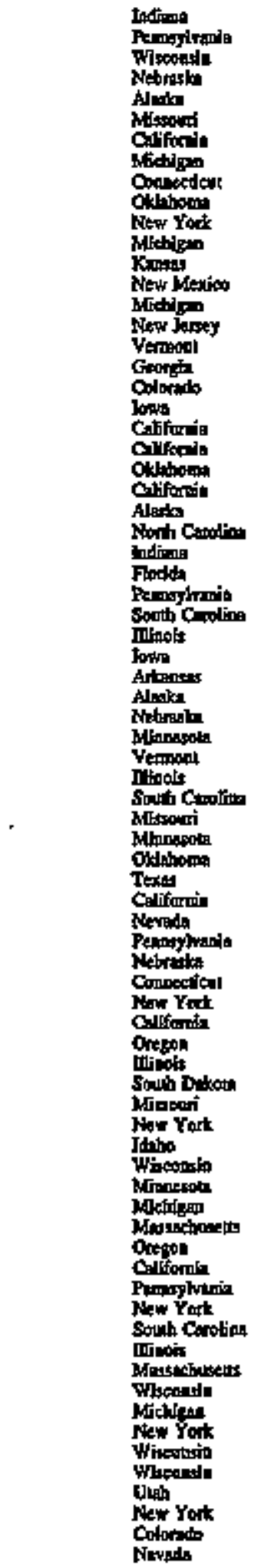 & \\
\hline
\end{tabular}

Sex toothoses ot terd of tobla. 
Table D1. G.S. Electric Utitity Flants, as of Jamuary 1, 1995 (Contimued)

\begin{tabular}{|c|c|c|}
\hline Plant Nape & Denlly Nate & Stinte \\
\hline 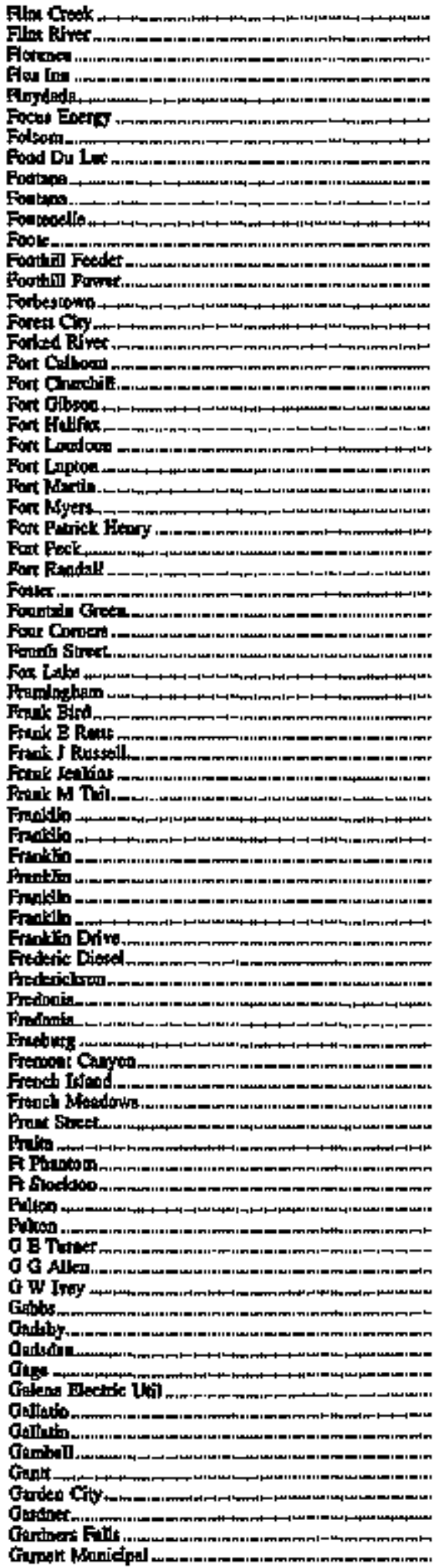 & 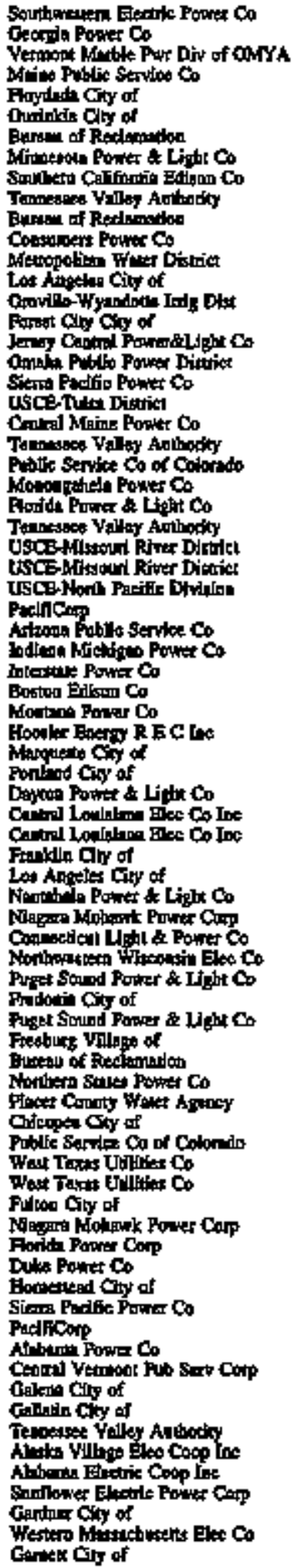 & 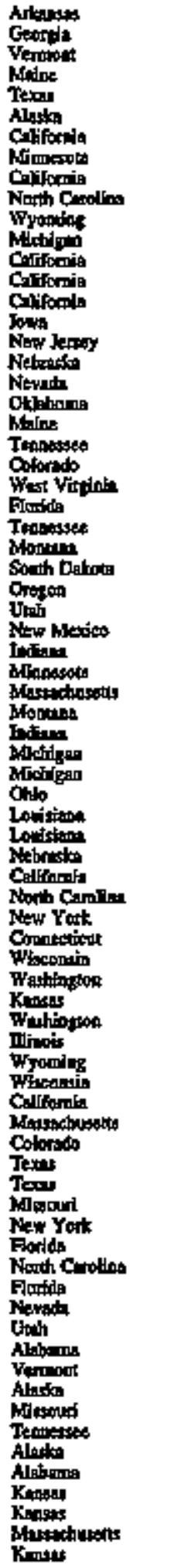 \\
\hline
\end{tabular}

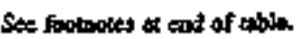


Table D1. U.S. Electric Utility Plants, as of January 1, 1995 (Continued)

\begin{tabular}{|c|c|c|}
\hline Fland Nanp & Dhilly lavere & Stale \\
\hline 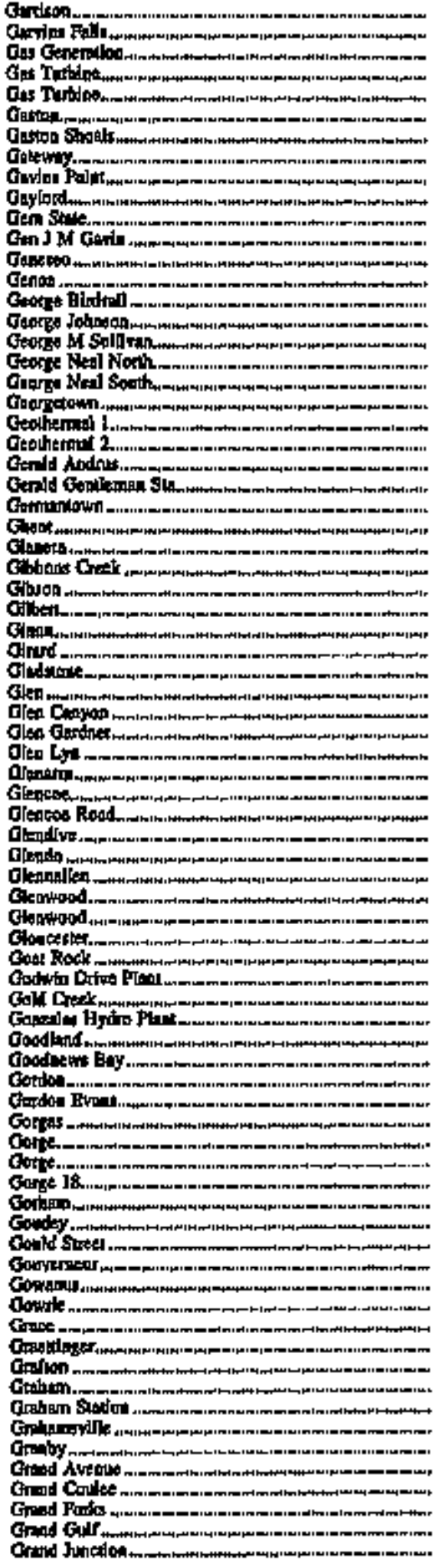 & 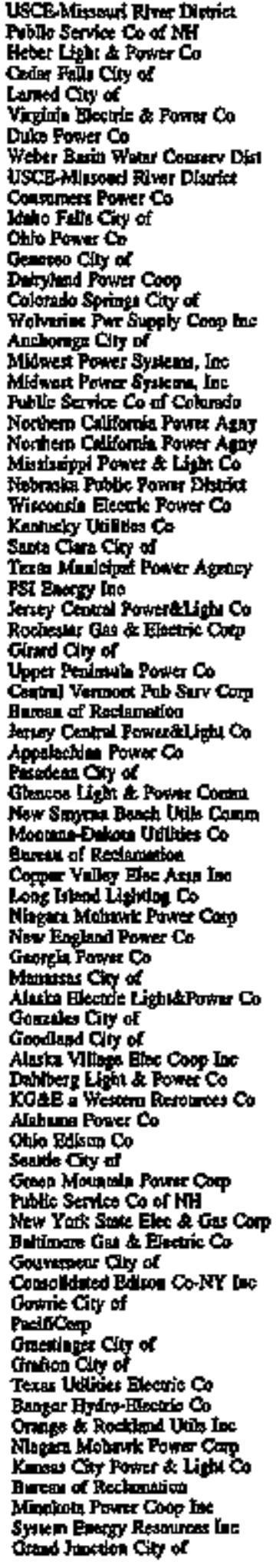 & 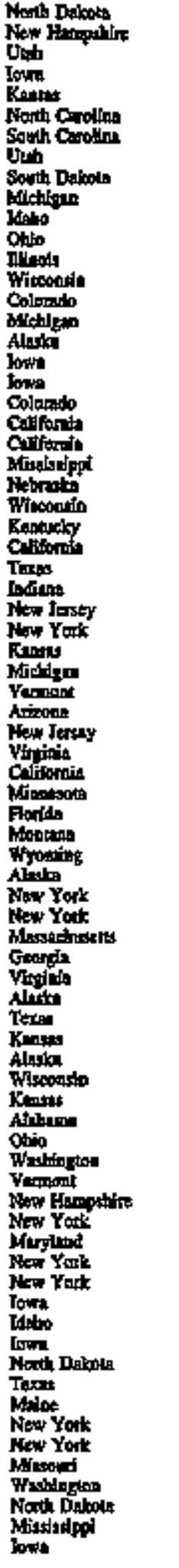 \\
\hline
\end{tabular}

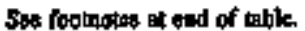


Tabje D1. U.S. Blectric Utility Plants, as of January 1, 1995 (Continued)

\begin{tabular}{|c|c|c|}
\hline Planc Ftamb & Udititg Rtine & shane \\
\hline 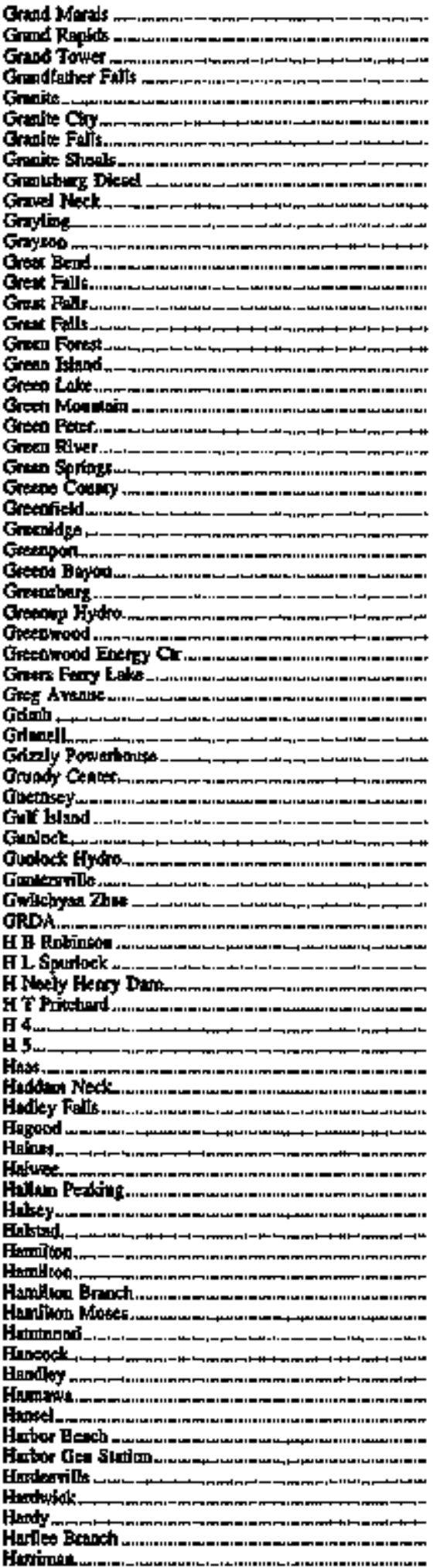 & 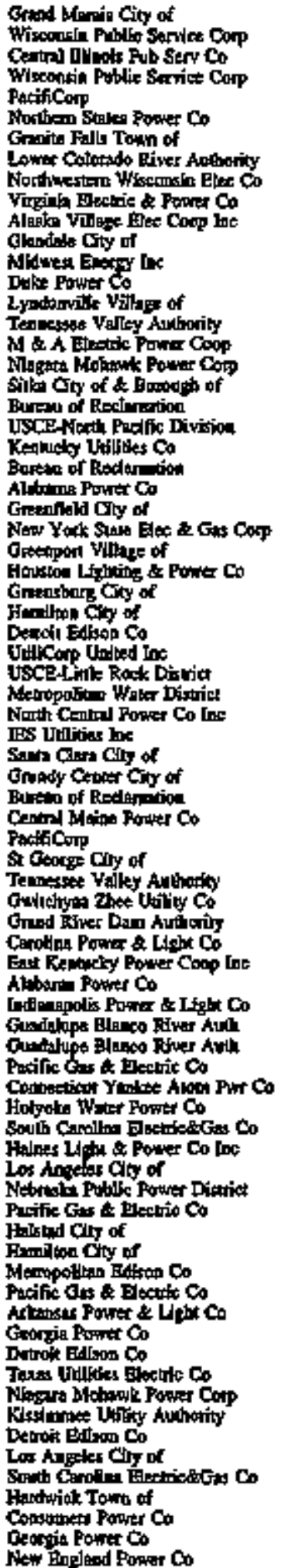 & 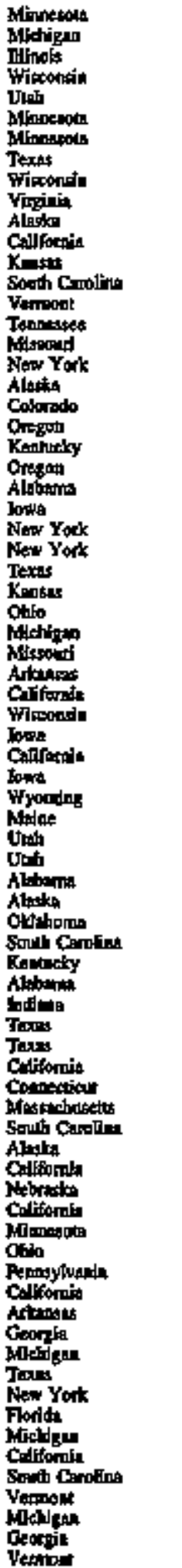 \\
\hline
\end{tabular}

Sea foolooted it end of nbte. 
Table DL. U.S. Electric Utilly Plants, as of January 1, 1995 (Continued)

\begin{tabular}{|c|c|c|}
\hline Piant Nerie & Difl'sty Netnt & Stale \\
\hline 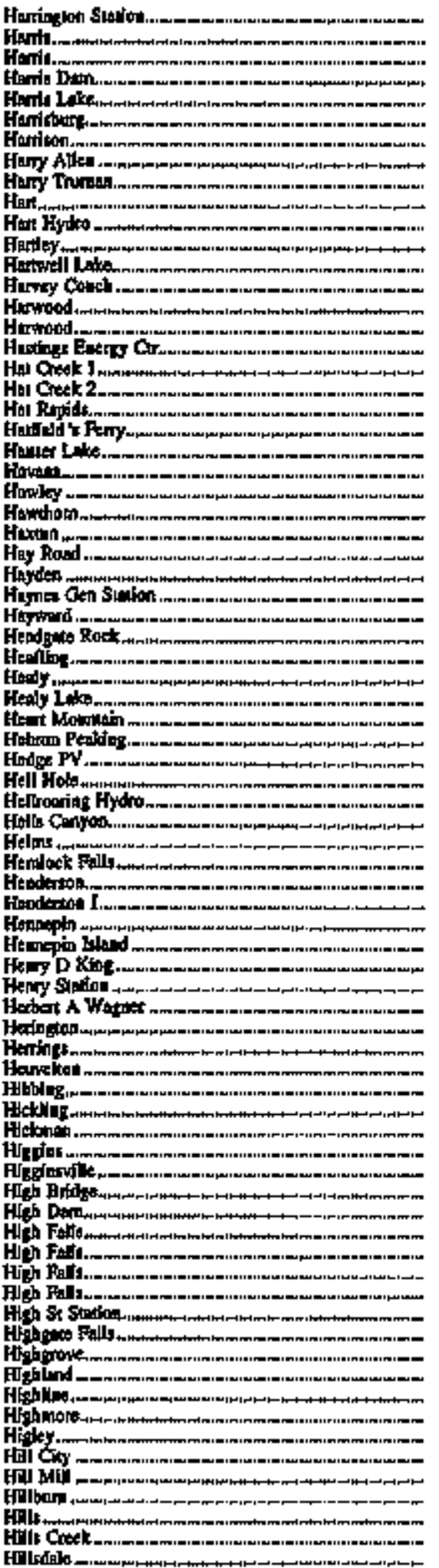 & 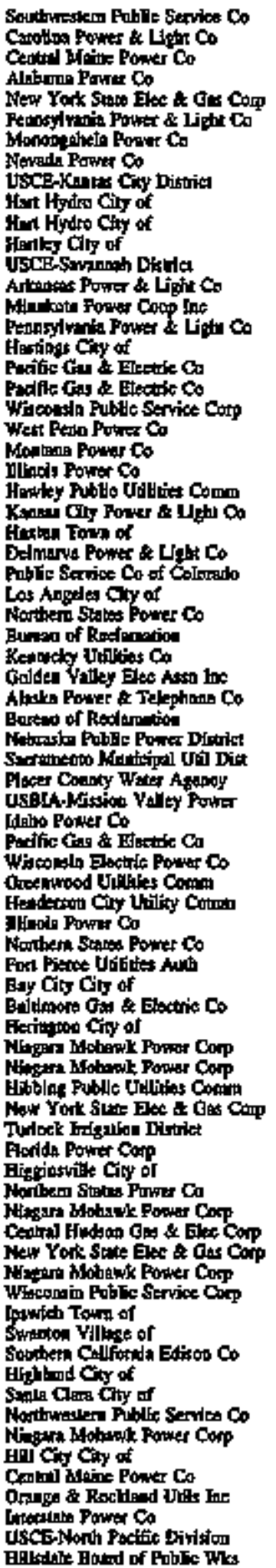 & 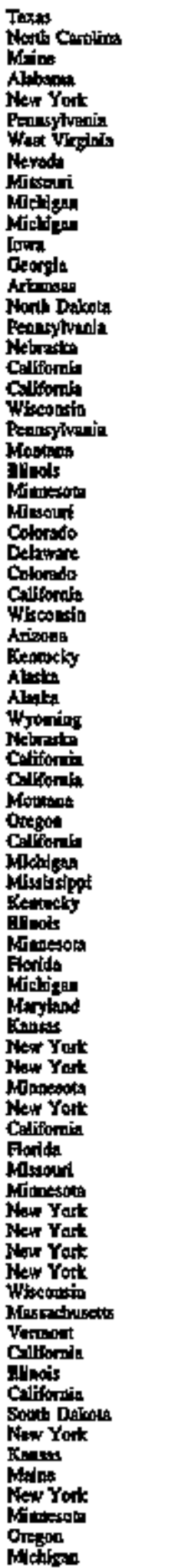 \\
\hline
\end{tabular}

Seo bootinotes at end of table. 
Tahle D1. US. Electric Uthilty Plants, of of Jannary 1, 1995 (Continued)

\begin{tabular}{|c|c|c|}
\hline Phnt Rane & Ditily Nump & stalt \\
\hline 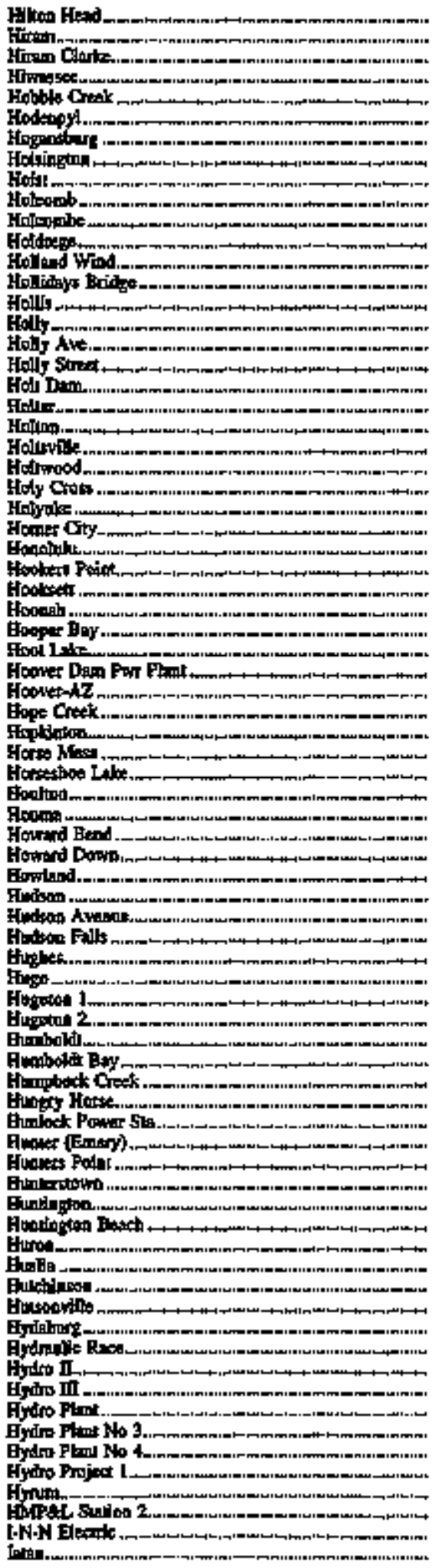 & 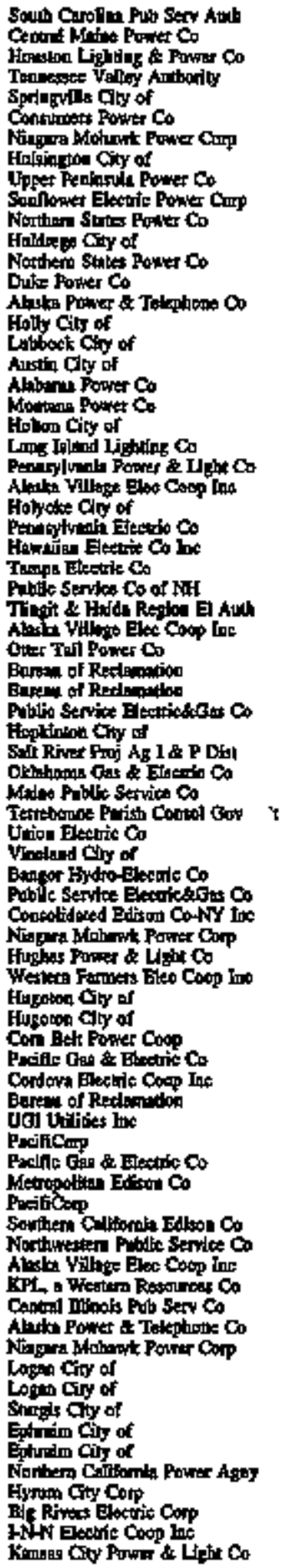 & 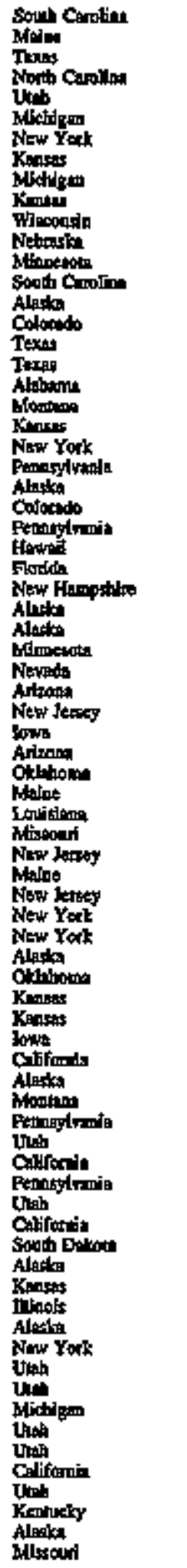 \\
\hline
\end{tabular}

Sen footorgtes al ead of tabls. 
Table D1. U.S. Electric Utility Plants, as of January 1, 1995 (Continued)

\begin{tabular}{|c|c|c|}
\hline Plant Nhat & twell' Nen: & State \\
\hline 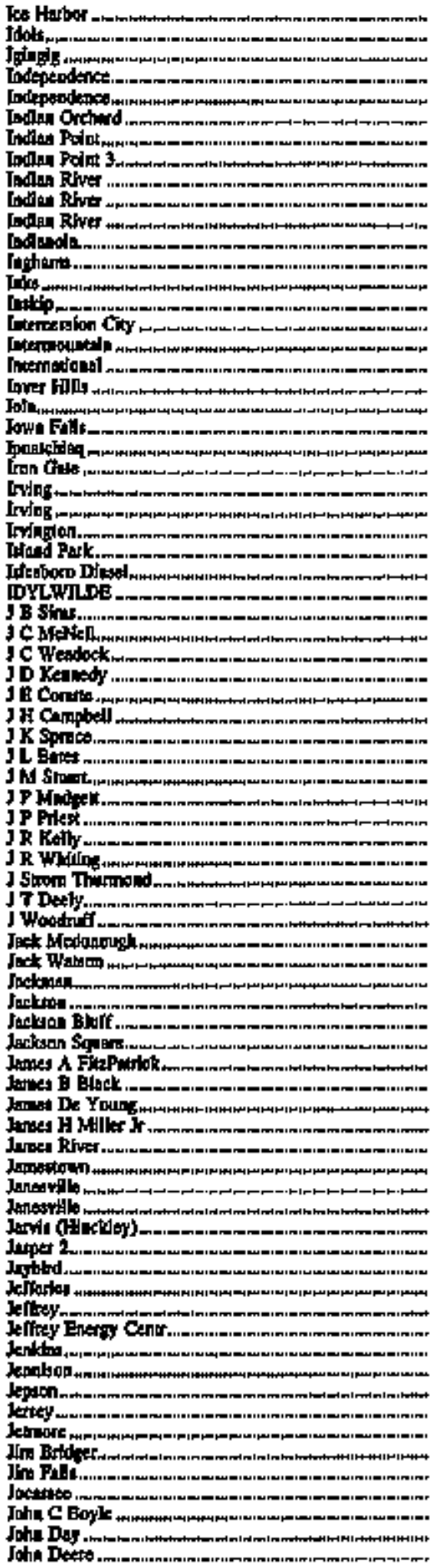 & 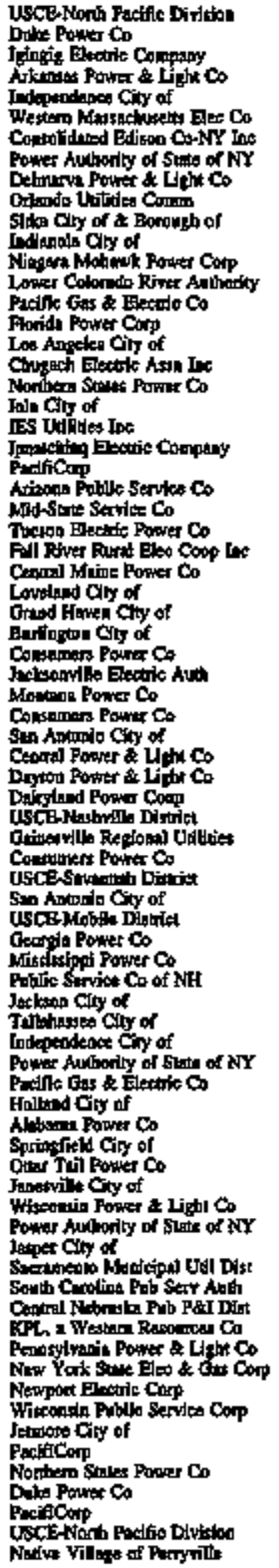 & 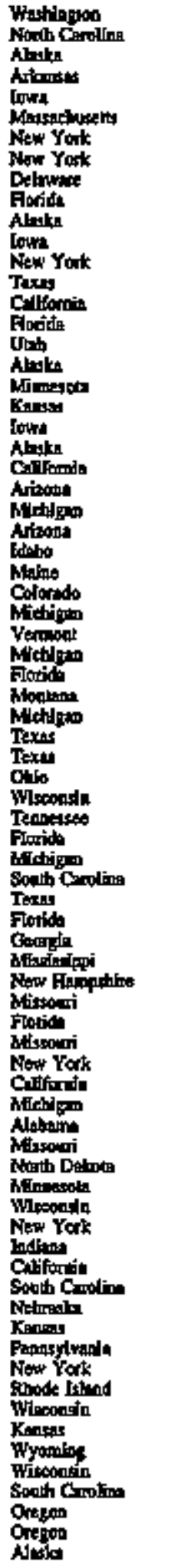 \\
\hline
\end{tabular}

Sec footenote at ead of table. 
Table D1. U.S. ERectric Utillty Plants, as of January 1, 1995 (Contioued)

\begin{tabular}{|c|c|c|}
\hline Pthent Nant & 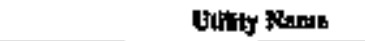 & Sinto \\
\hline 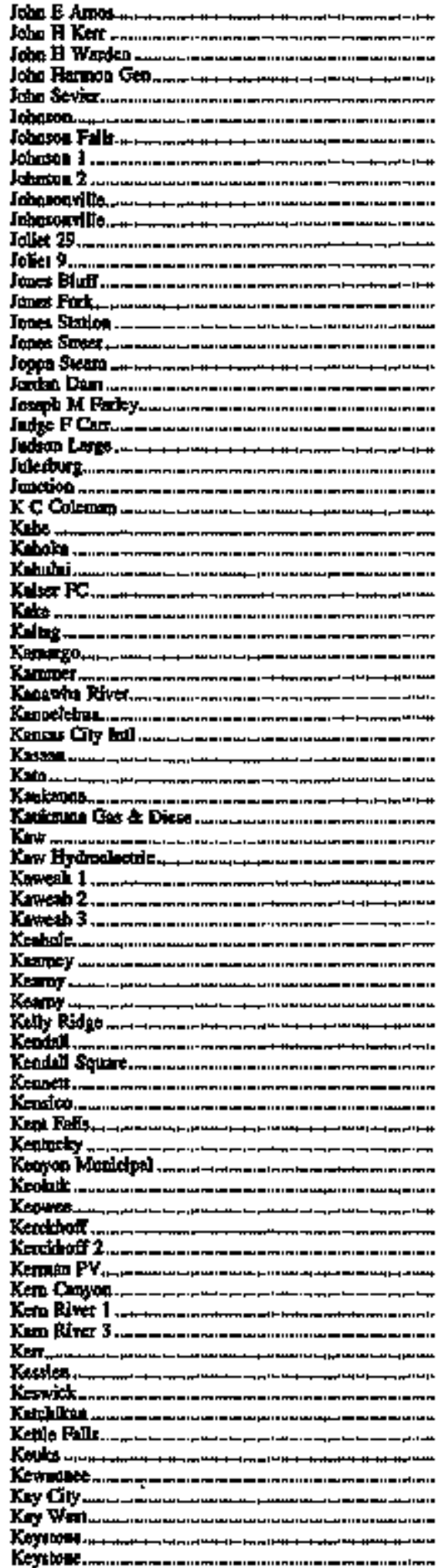 & 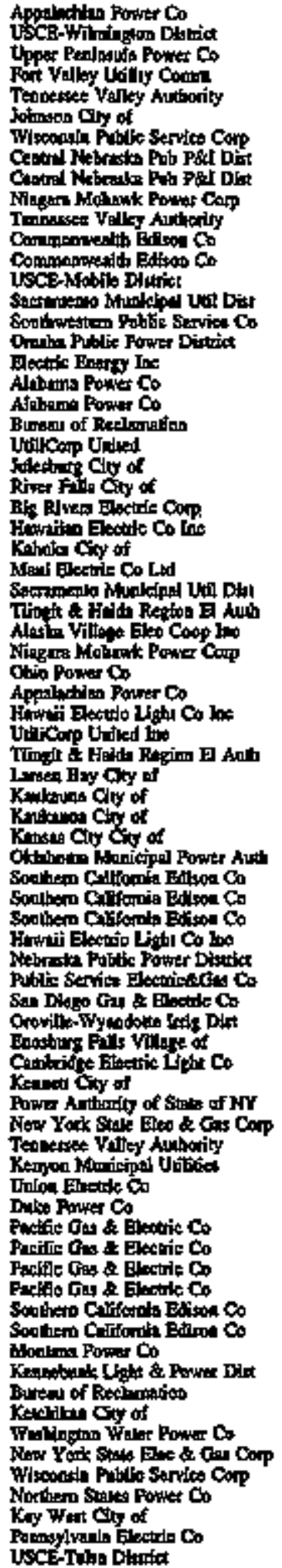 & 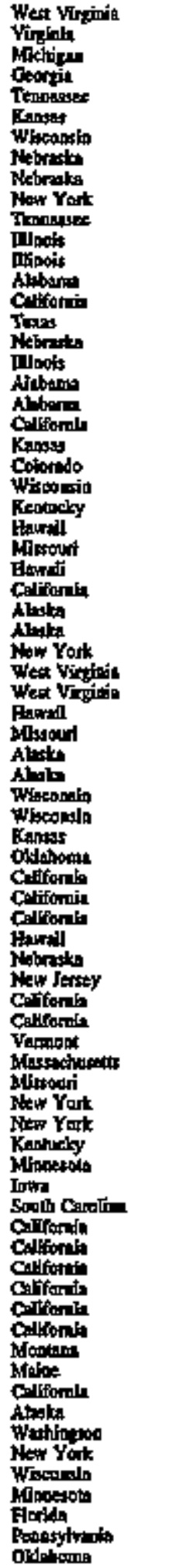 \\
\hline
\end{tabular}

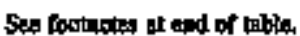


Table D1. U.S. Edectric Uthity Plants, as of January 1, 1995 (Contimued)

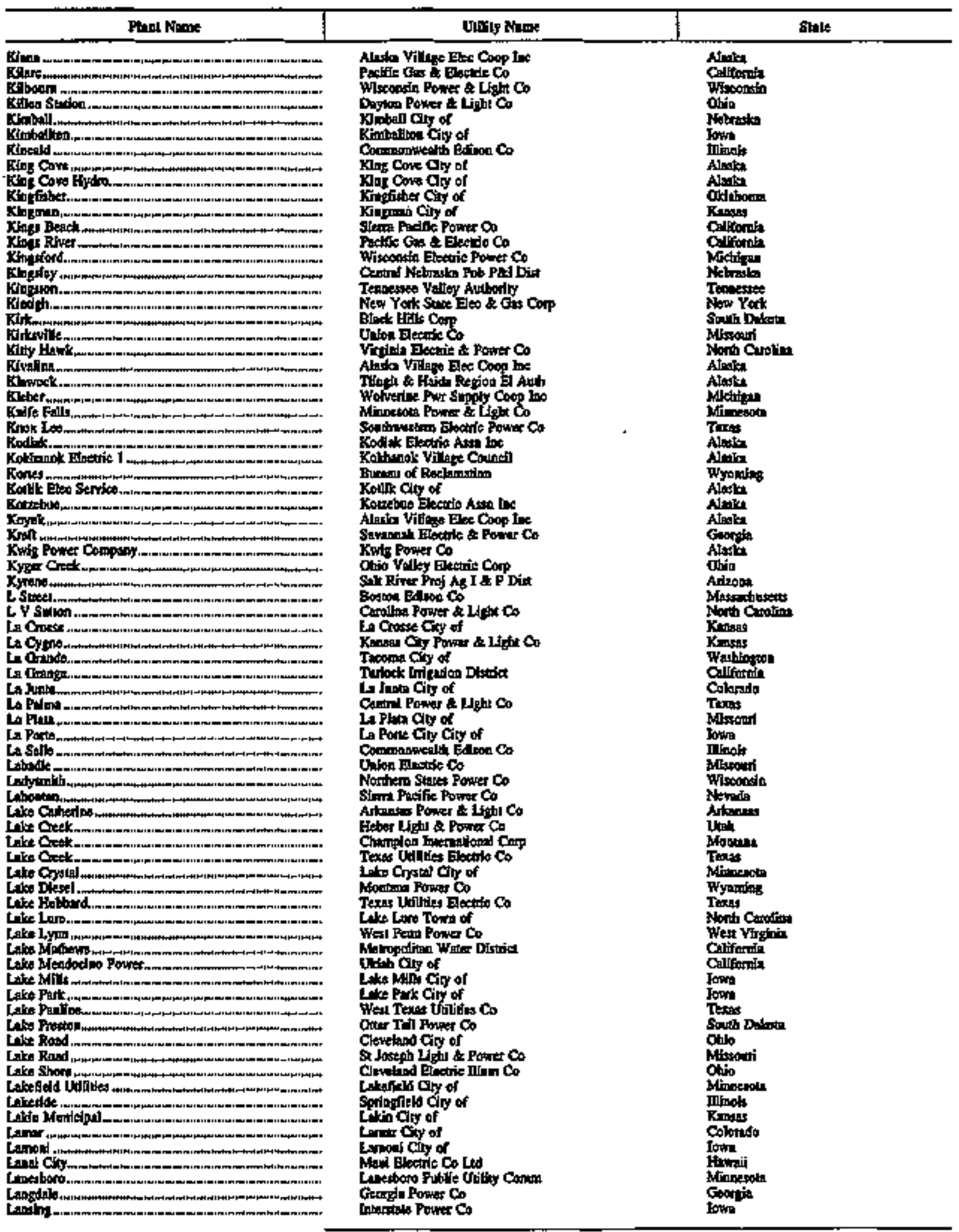

Seo soonobs at end of ublt. 
Tabile D1. U.S. Flectric Utility Planks, as of Jamary 1, 1995 (Continued)

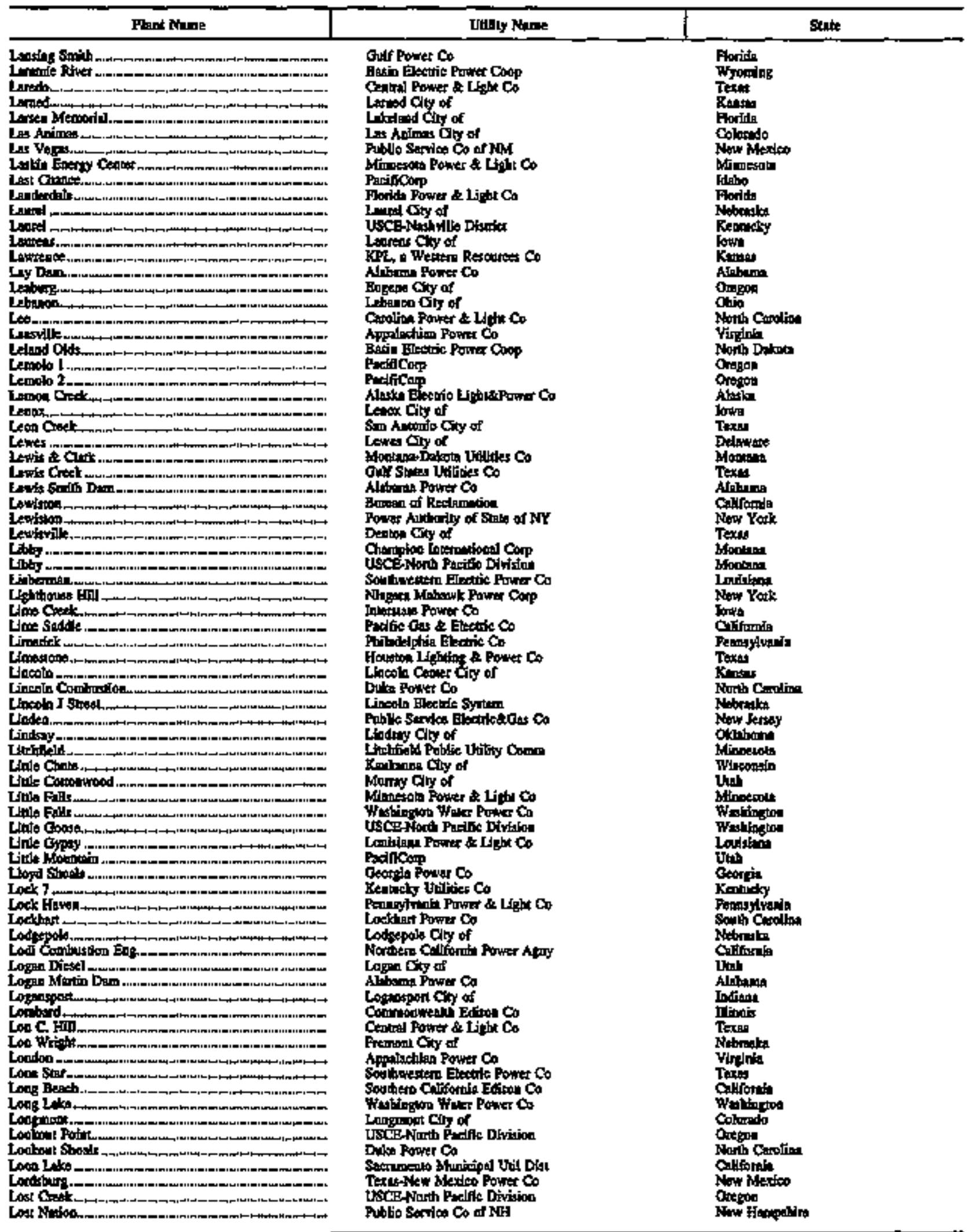

Sed foomolas at eod of whla 


\begin{tabular}{|c|c|c|}
\hline P'un kane & Dedlfy Nang & stat \\
\hline 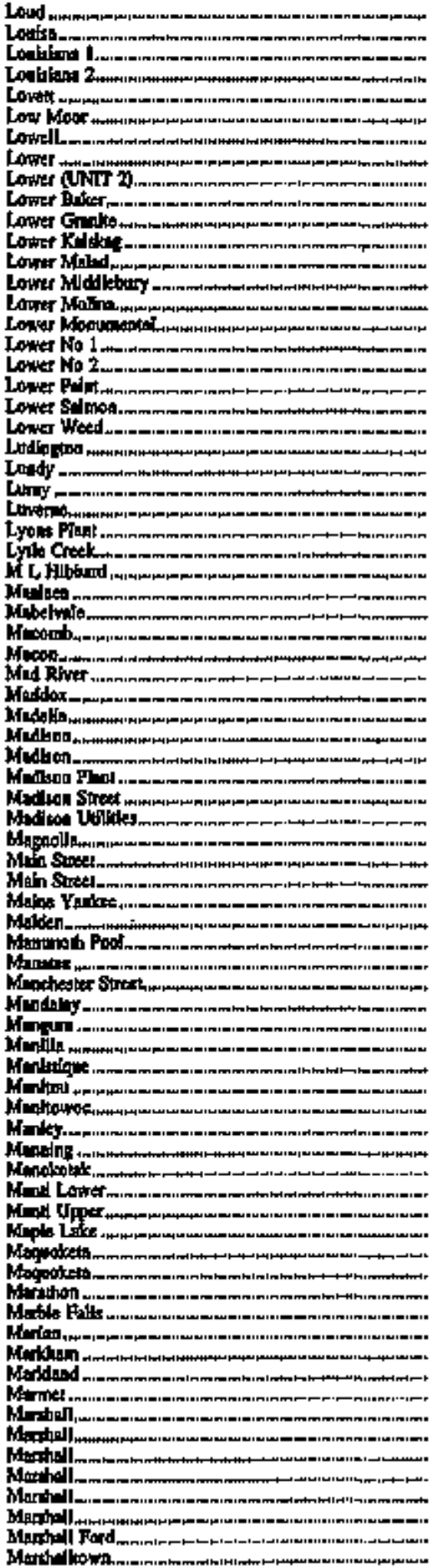 & 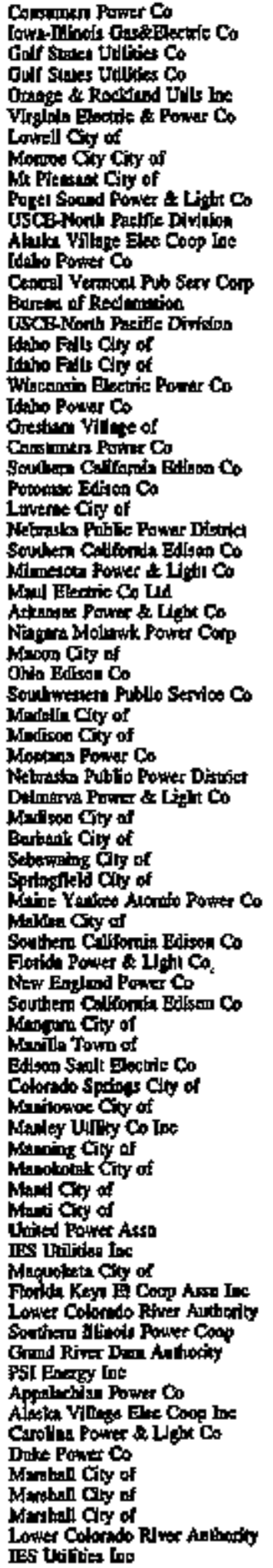 & 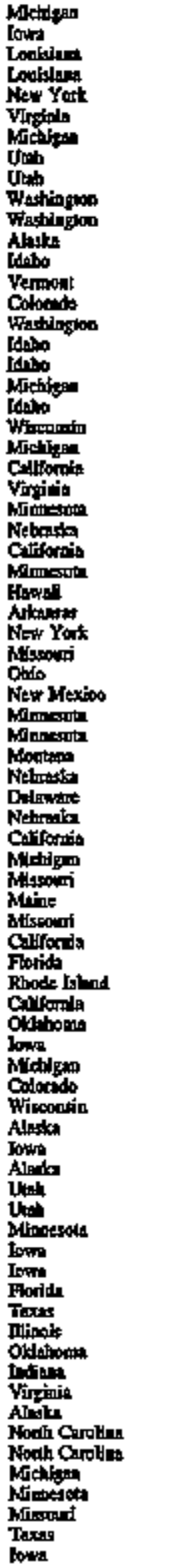 \\
\hline
\end{tabular}

Sos cootiontes an end of thbte. 
Table D1. U.S. Electric Utility Plants, as of Jamuary 1, 1995 (Continued)

\begin{tabular}{|c|c|c|}
\hline Plent Narit: & tkllty Nane & Stale \\
\hline 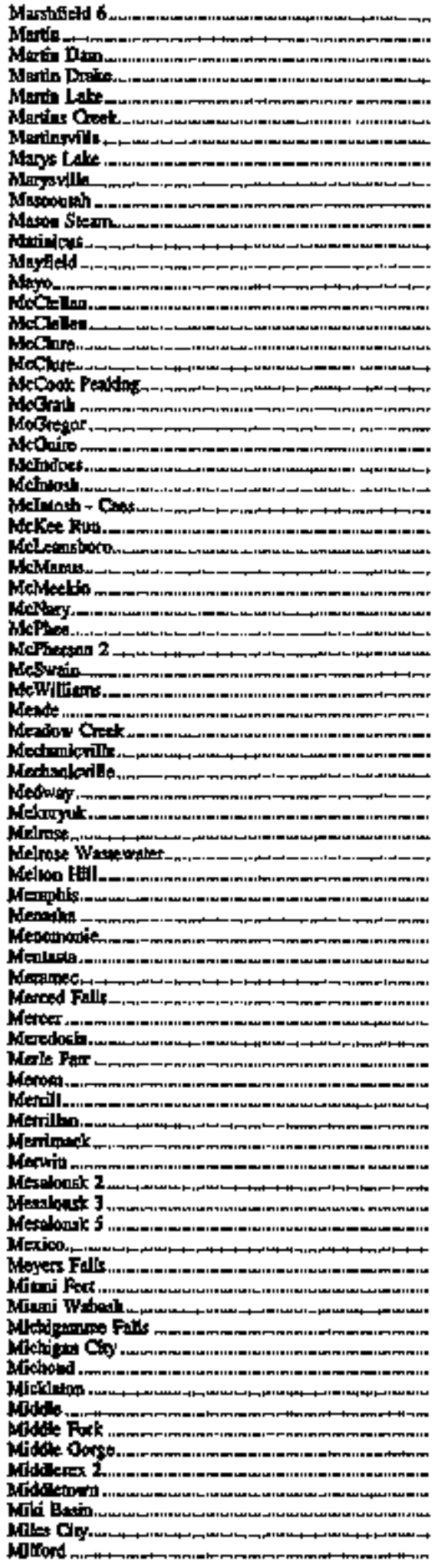 & 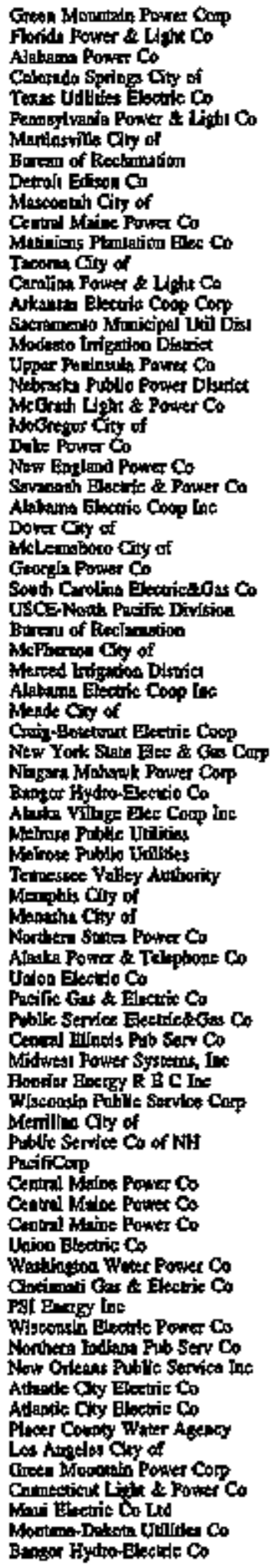 & 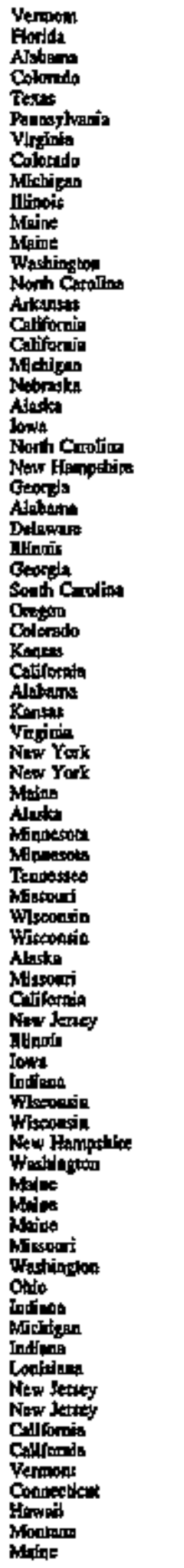 \\
\hline
\end{tabular}

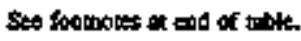


TabIc D1. U.S. Electric Utility Plants, as of January 1, 1995 (Continued)

\begin{tabular}{|c|c|c|}
\hline Plant Nhm: & U山l' Nom: & Elrite \\
\hline 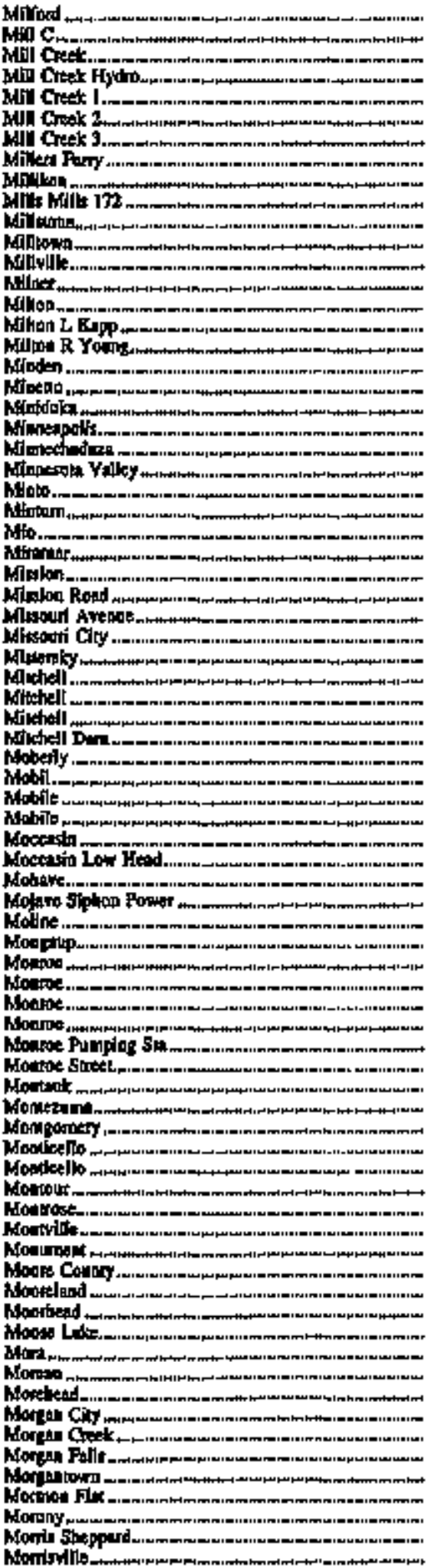 & 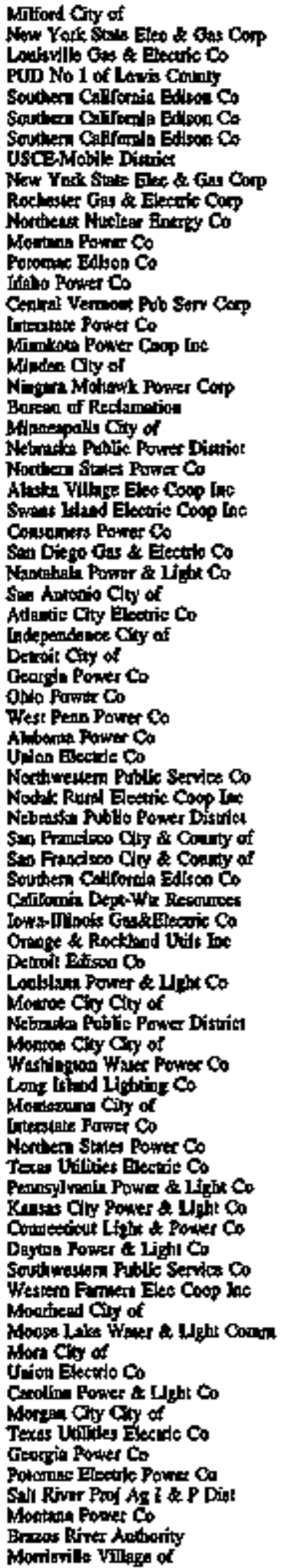 & 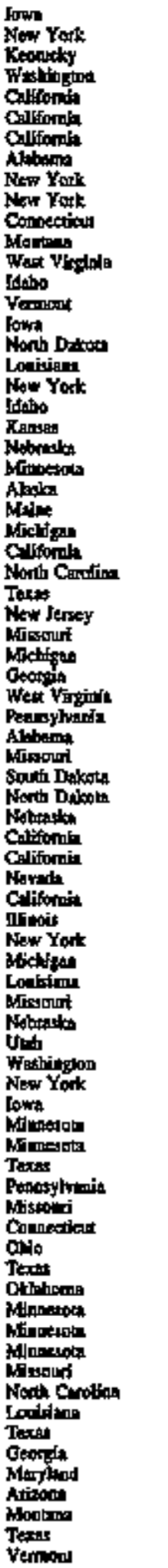 \\
\hline
\end{tabular}

See fochones at ead of uble. 
Table D1. U.S, Electric Utllity Plants, as of January 1, 1995 (Continued)

\begin{tabular}{|c|c|c|}
\hline Fant Nama & 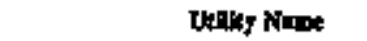 & Slinte \\
\hline 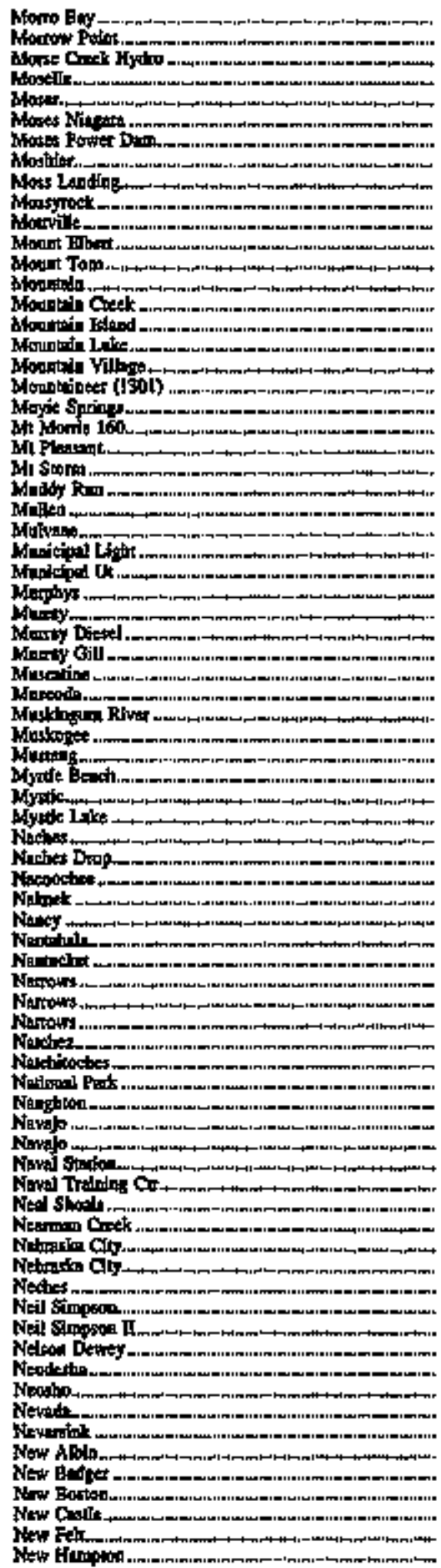 & 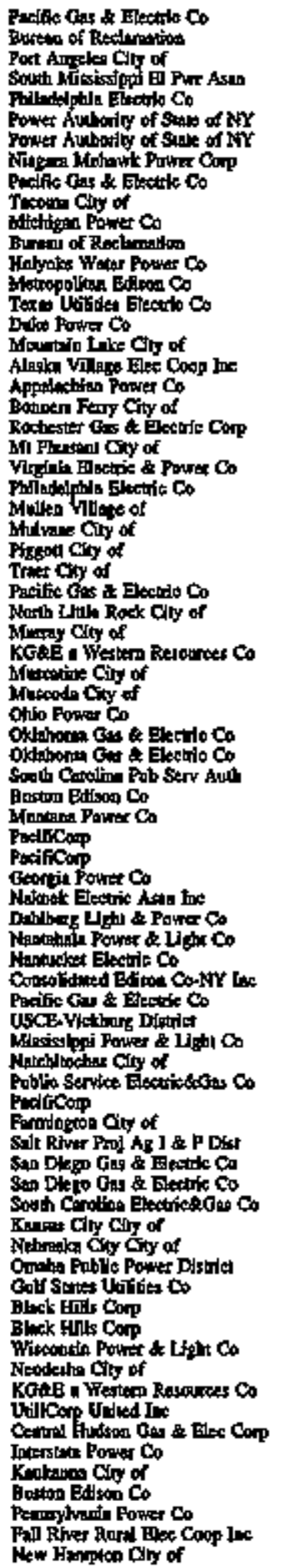 & 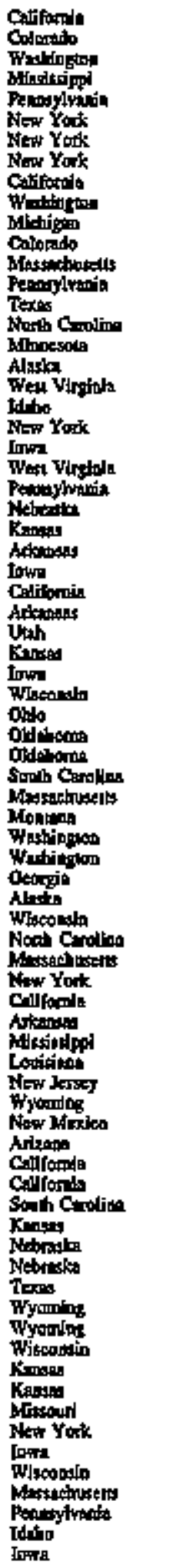 \\
\hline
\end{tabular}

Sen fortunates at end of table 
Table D1. U.S. Electric Utility Plants, as of January 1, 1995 (Continued)

\begin{tabular}{|c|c|c|}
\hline PHane Stame & Collty Nam & stalt: \\
\hline 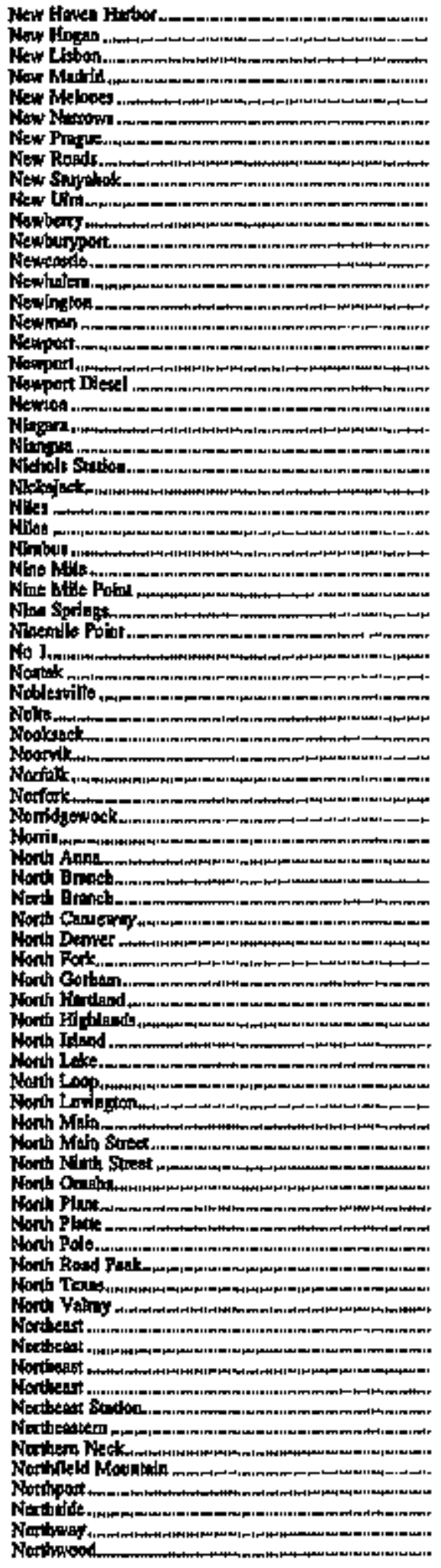 & 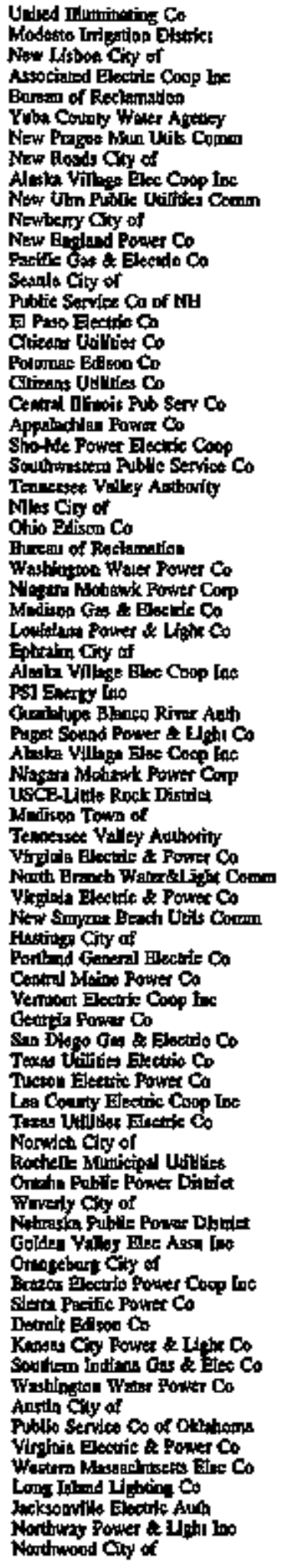 & 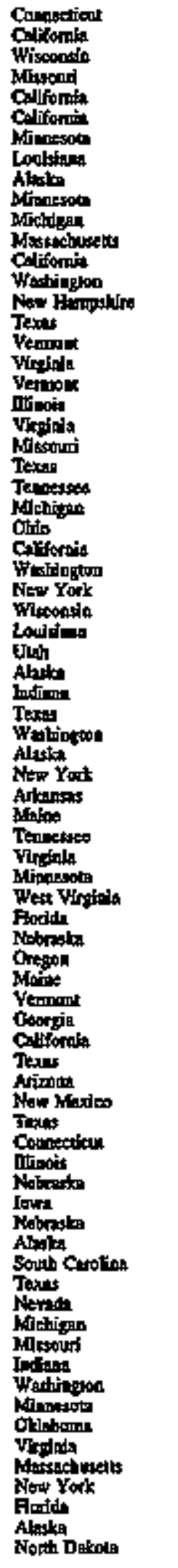 \\
\hline
\end{tabular}

Ses footurtios at and of able. 
Tabłe D1. U.S. Electric Utitity Phonts, es of January 1, 1995 (Continued)

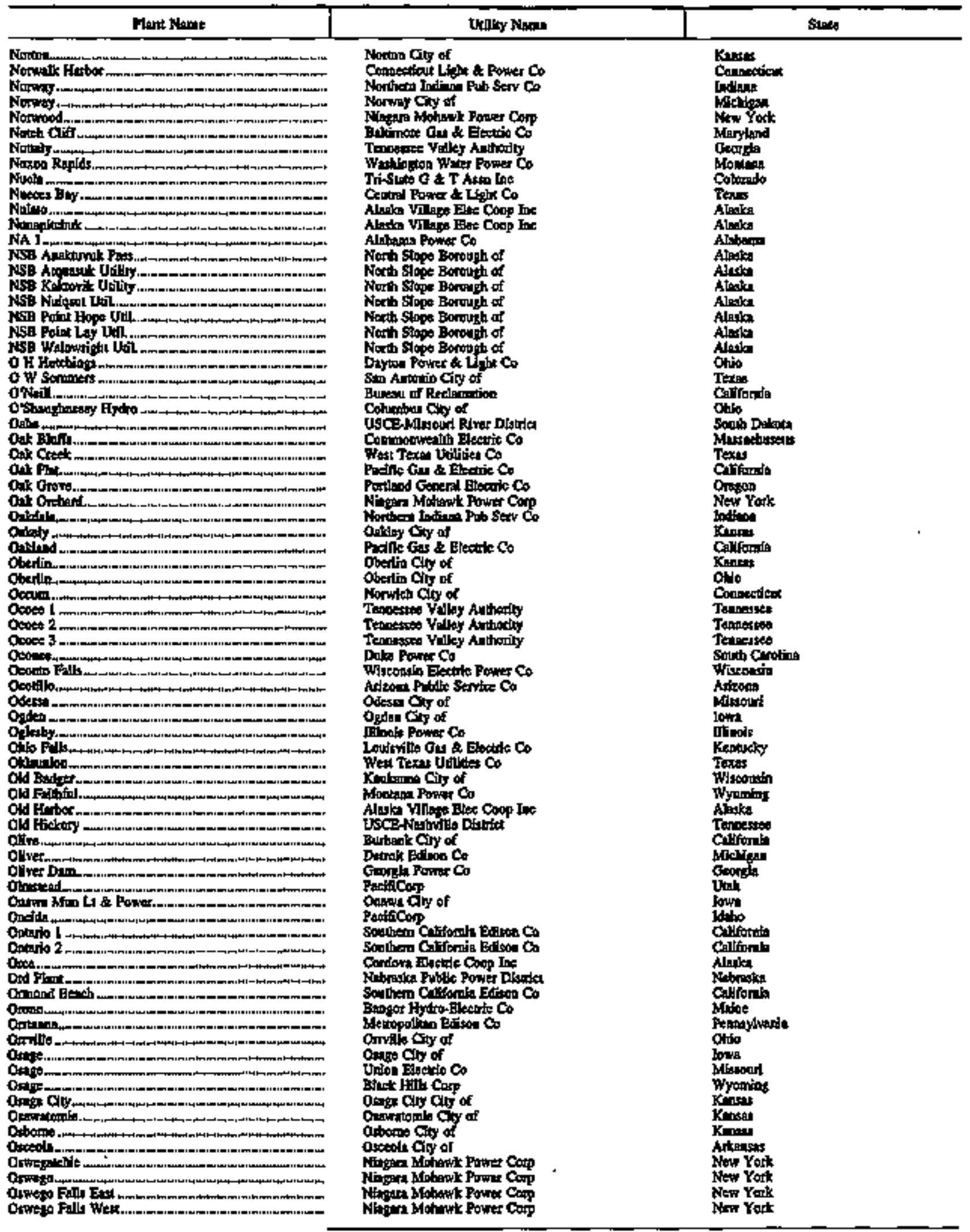

Son foomoles at end of who. 
Tahle D1. U.S. Electric Utility Plants, as of January 1, 1995 (Continued)

\begin{tabular}{|c|c|c|}
\hline Pant Nont & Uathy Nom & Sente \\
\hline 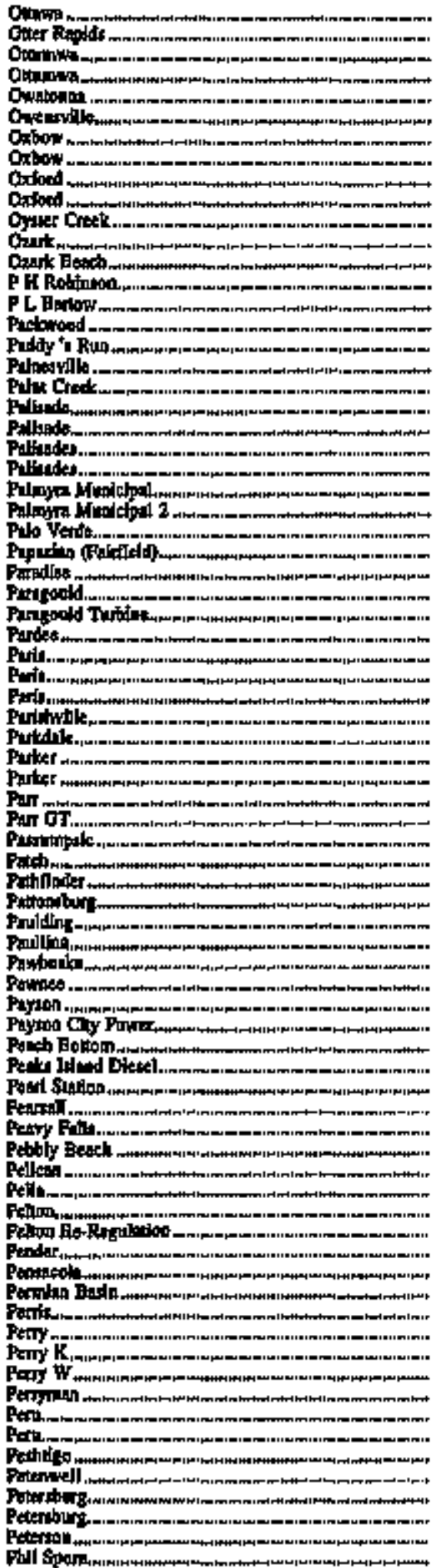 & 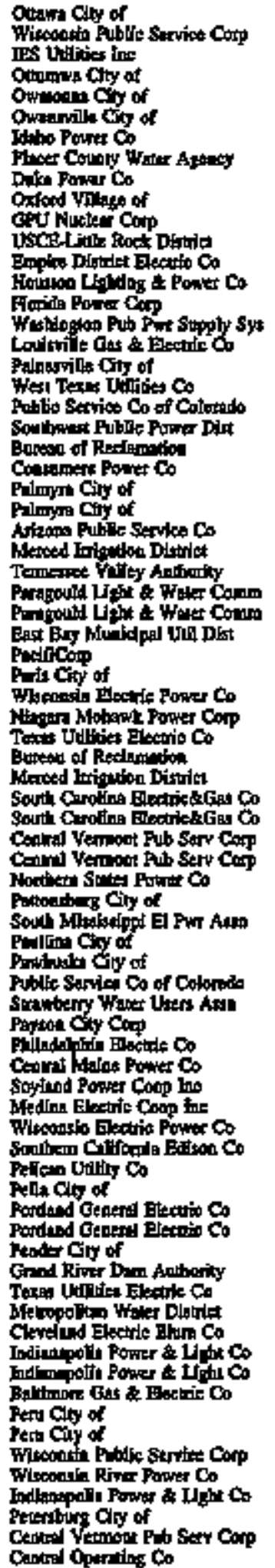 & 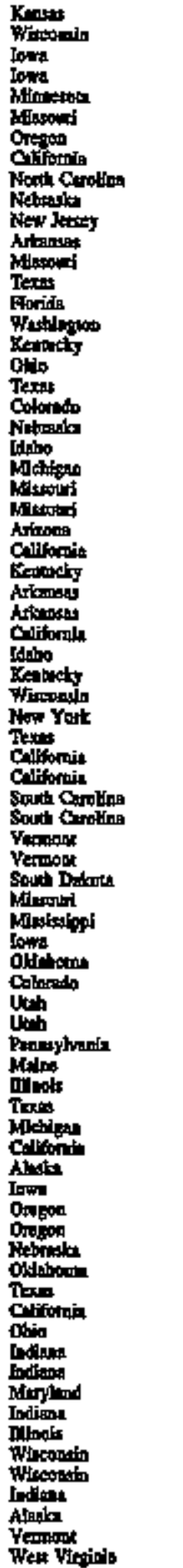 \\
\hline
\end{tabular}

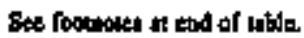


Table D1. U.S. Flectric Uttility Plants, as of January 1, 1995 (Continued)

\begin{tabular}{|c|c|c|}
\hline Plant Nhore & Dowlicy fabet & Stote \\
\hline 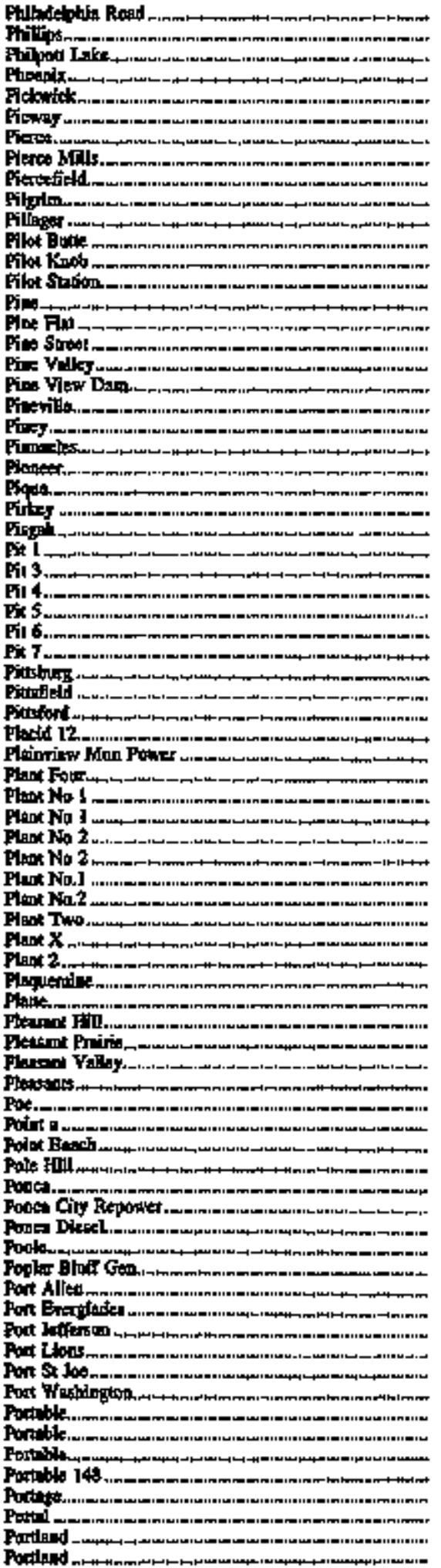 & 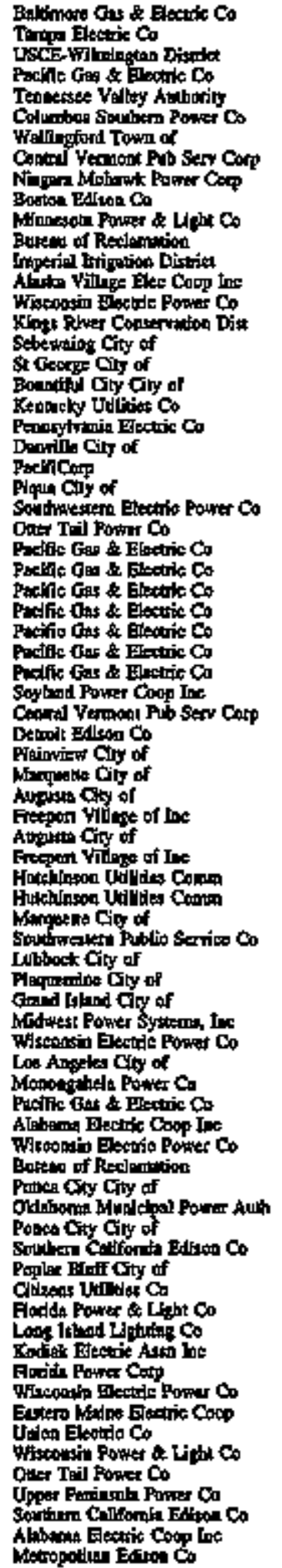 & 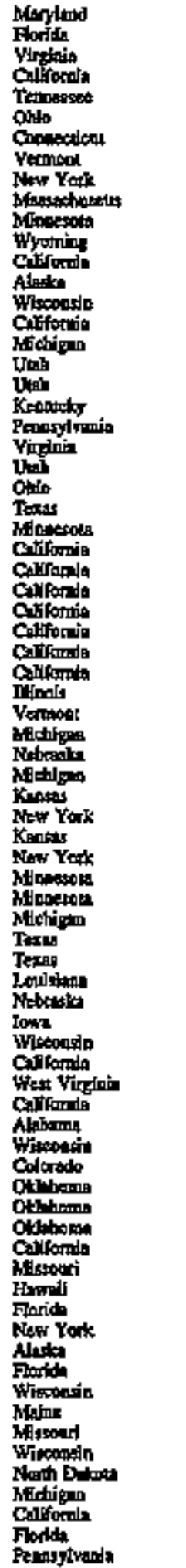 \\
\hline
\end{tabular}

see footnous at end of wble. 


\begin{tabular}{|c|c|c|}
\hline Fined Nime & Ullits xitut & States \\
\hline 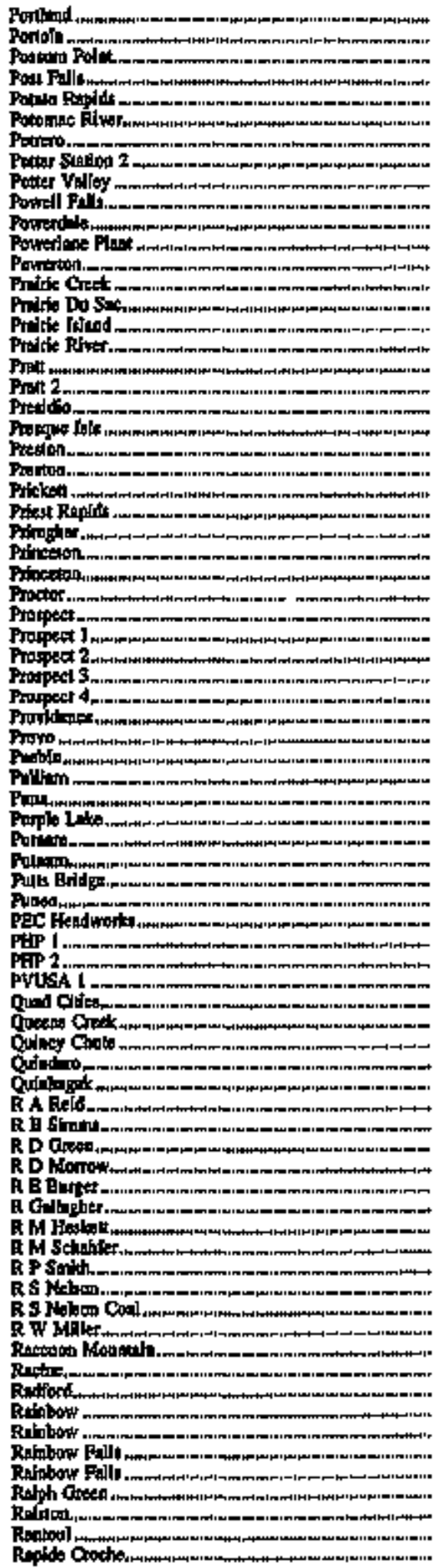 & 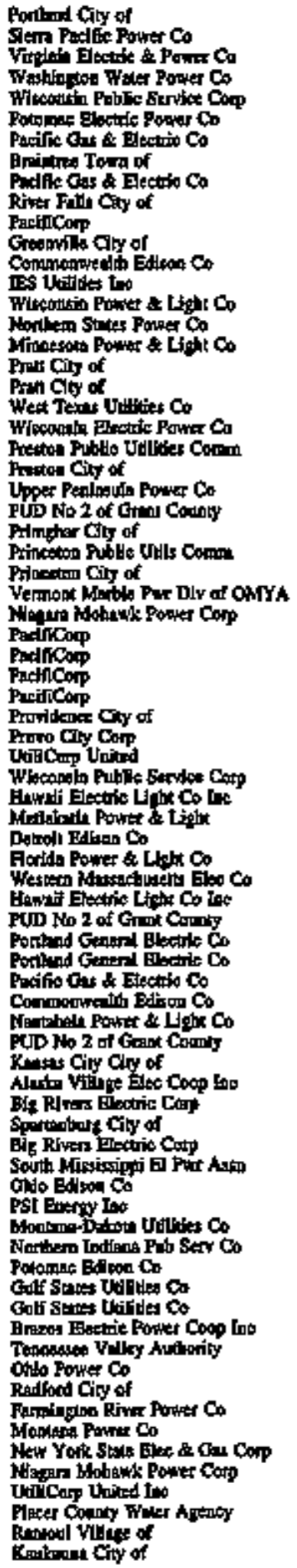 & 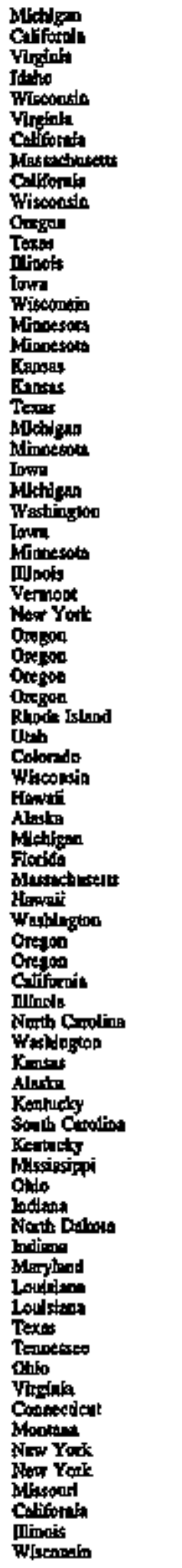 \\
\hline
\end{tabular}

Sat boototer at esd of mble. 
Table D1. U.S. Electrk Utility Ptants, as of January 1, 1995 (Contimued)

\begin{tabular}{|c|c|c|}
\hline Plant Thot & turly thant & Stre \\
\hline 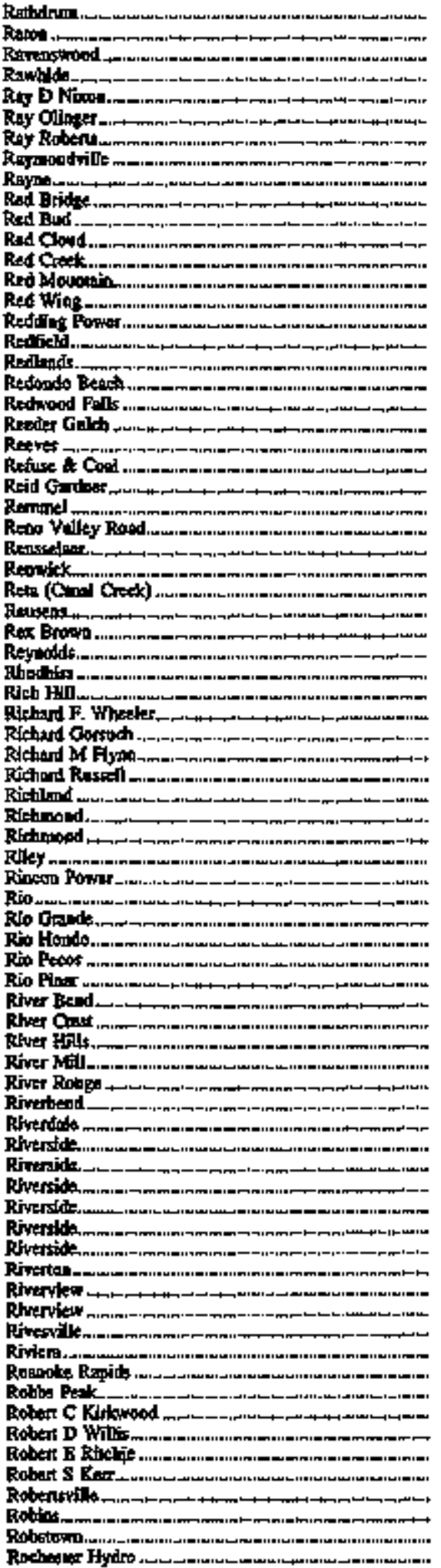 & 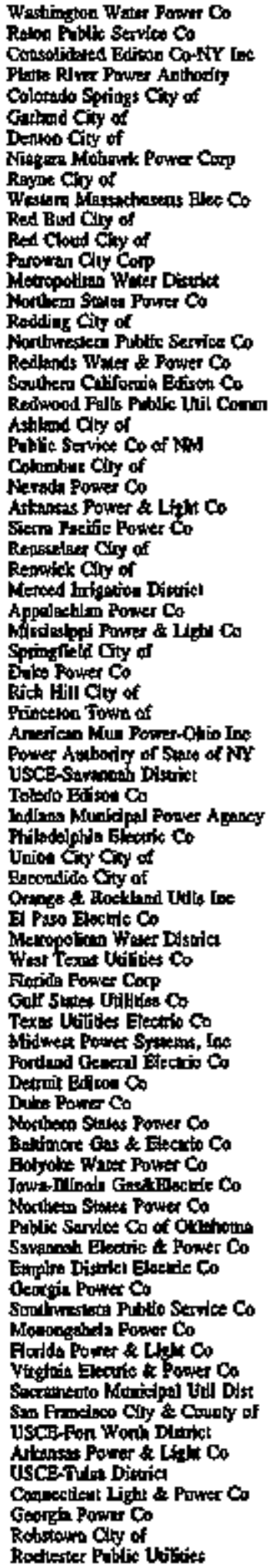 & 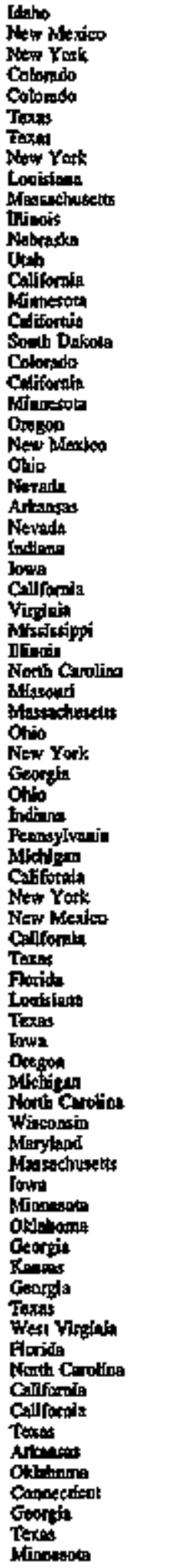 \\
\hline
\end{tabular}

Fee footuntes at end of thble. 
Tahle D1. U.S. Electric Utlity Planis, as of January 1, 1995 (Contimmed)

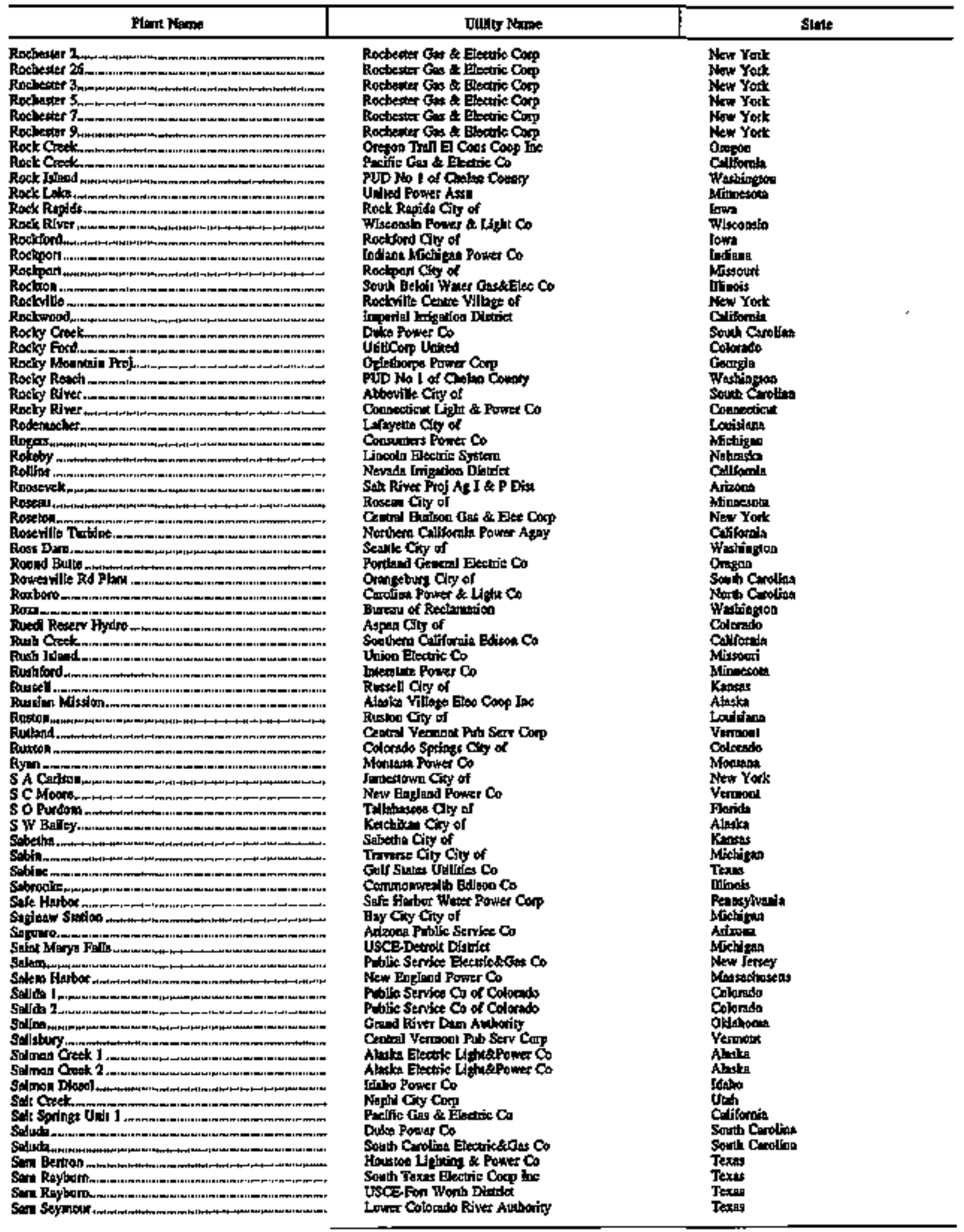

Set foolngtes in and of ablo. 


\begin{tabular}{|c|c|c|}
\hline Paint Nome & 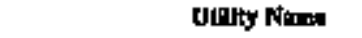 & SIda \\
\hline 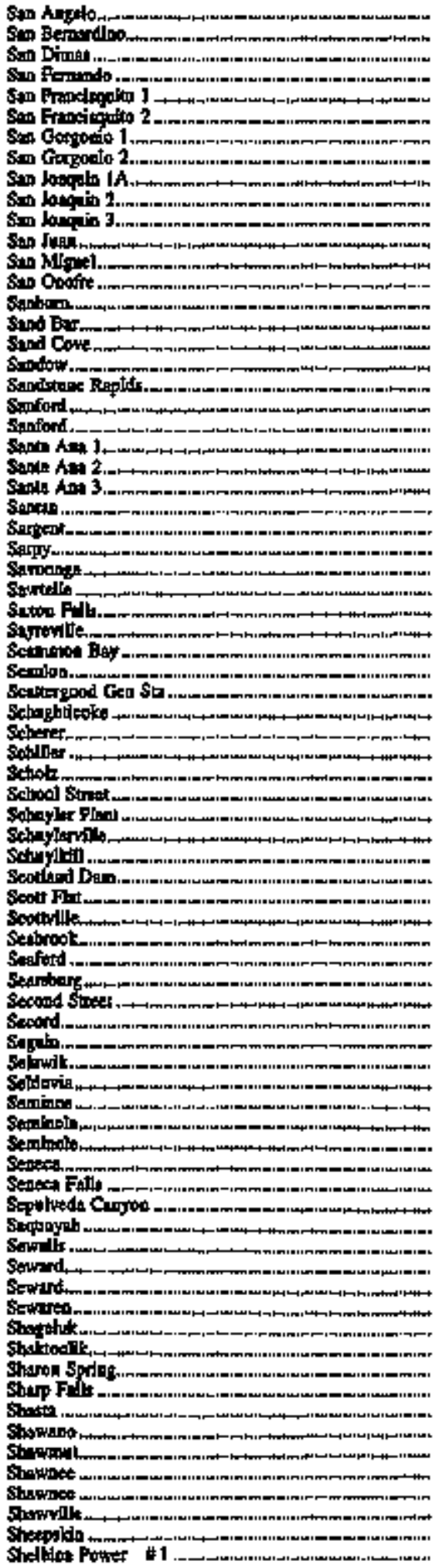 & 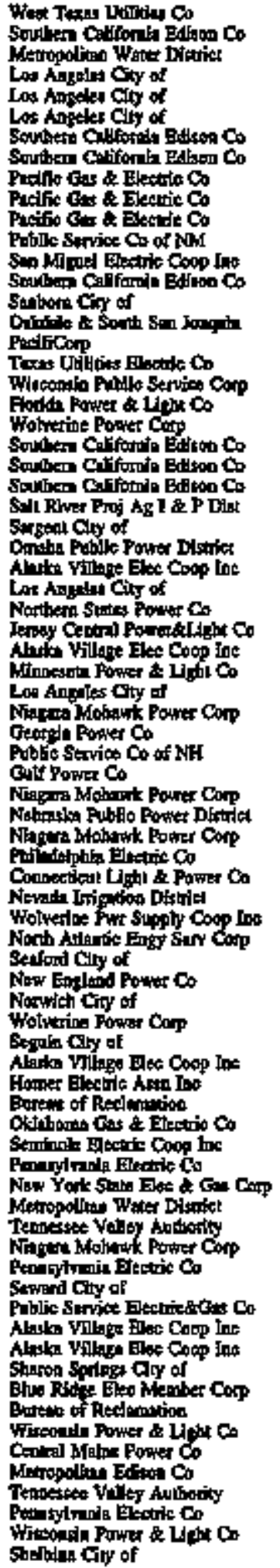 & 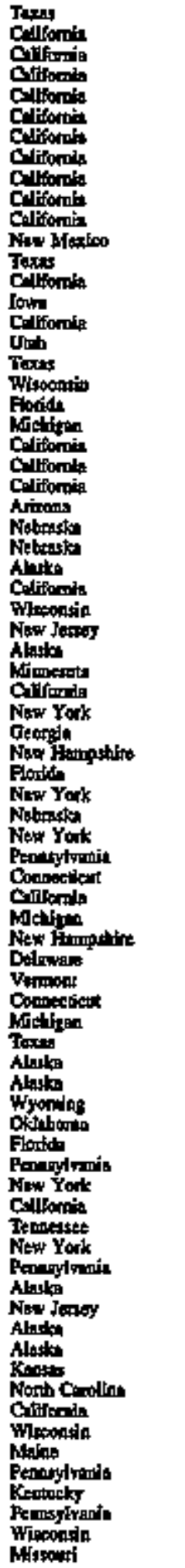 \\
\hline
\end{tabular}

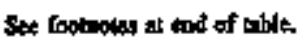


Table D1. U.S. Electrie Utiliky Phants, as of January 1, 1995 (Continued)

\begin{tabular}{|c|c|c|}
\hline Plante Nande & Ditilyy Nhon: & Strofte \\
\hline 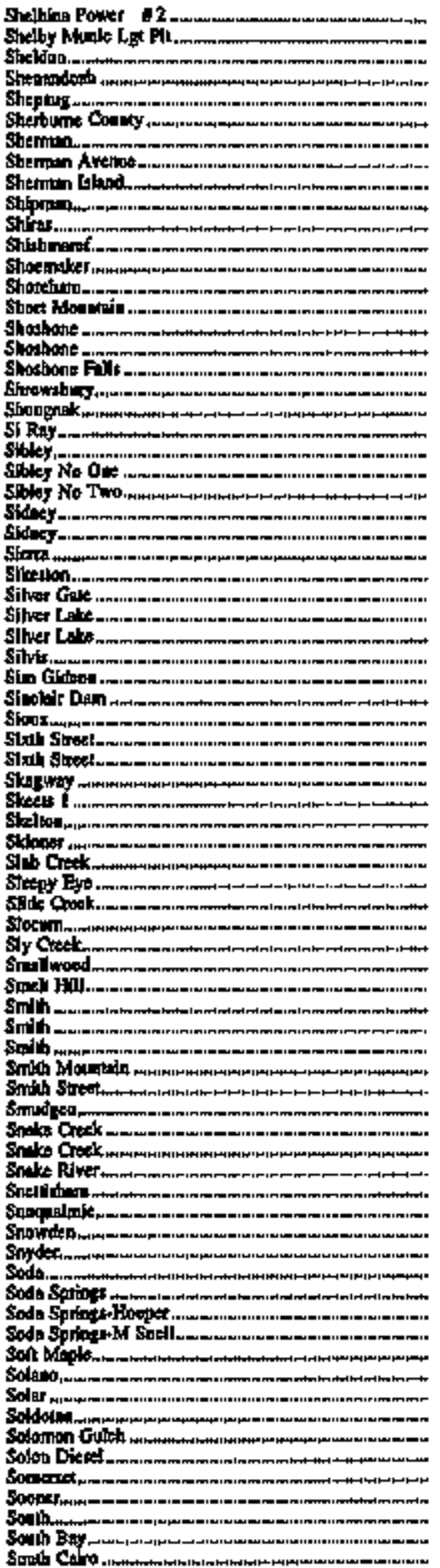 & 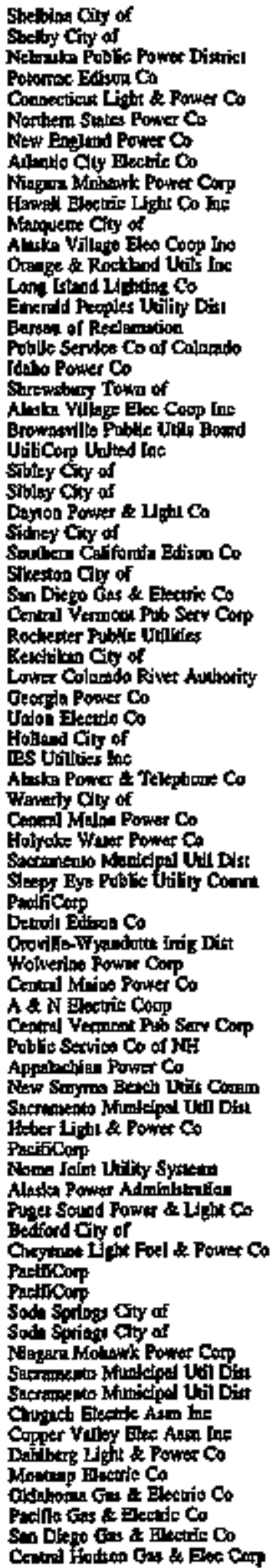 & 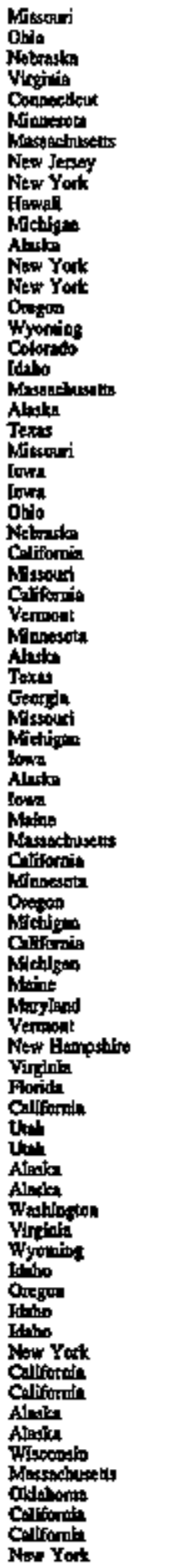 \\
\hline
\end{tabular}

Sen footingtes ate end of cubte. 
Tabje DL. U.S. Electric Utility Flauts, as of January 1, 1995 (Continned)

\begin{tabular}{|c|c|c|}
\hline Plant Nane & Vlaity Mhene & slite \\
\hline 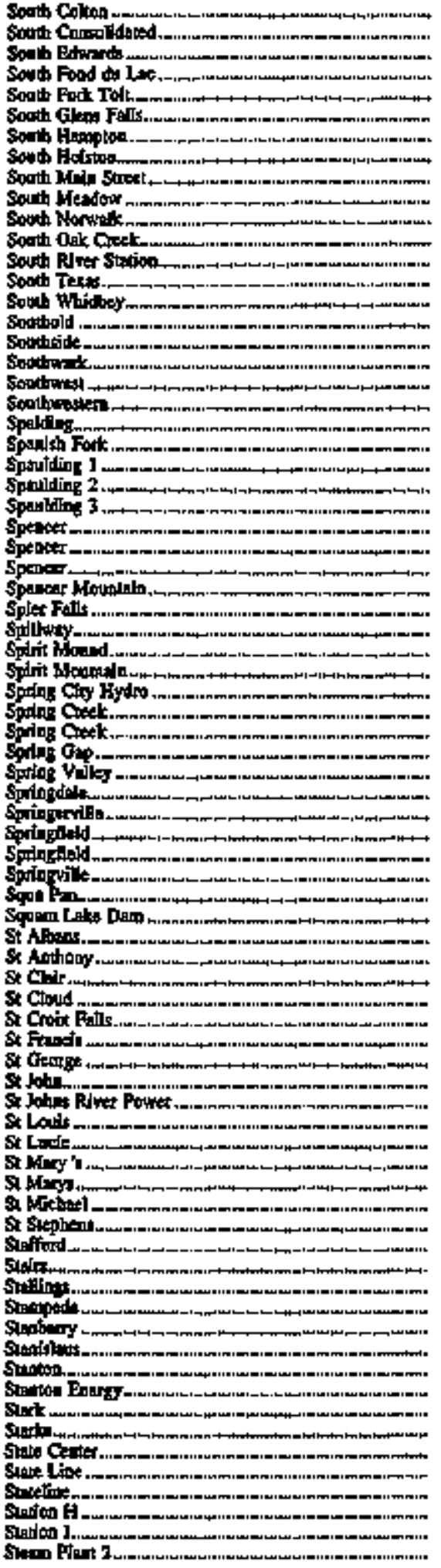 & 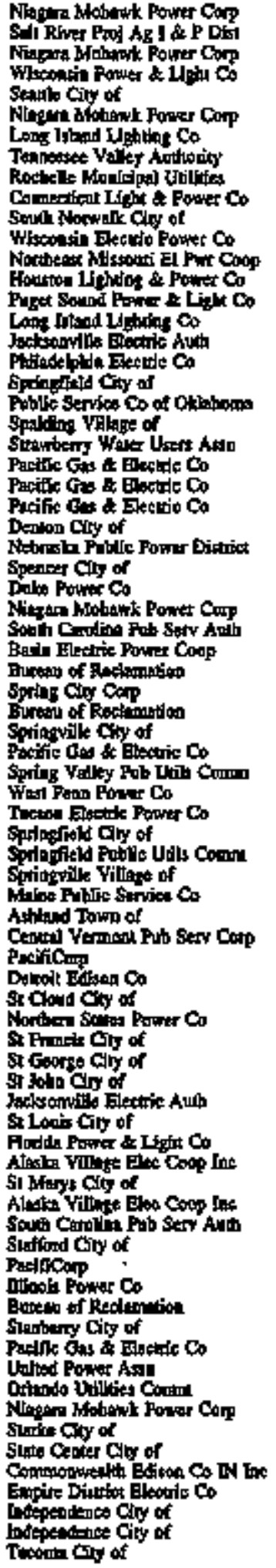 & 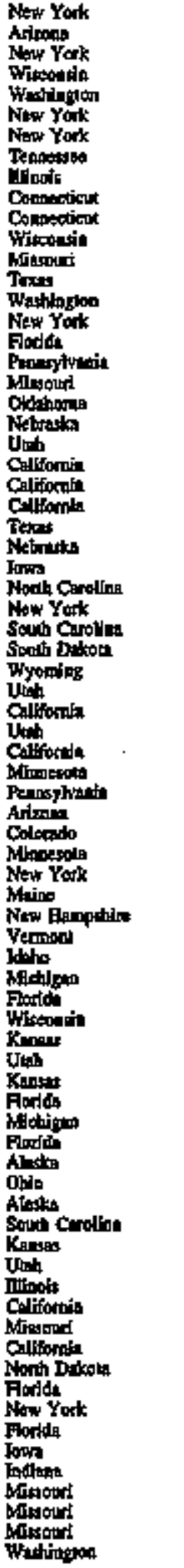 \\
\hline
\end{tabular}

Ser toonoxs of end of toble 
Table D1. U.S. Electric Utility Plants, as of January 1, 199 (Continued)

\begin{tabular}{|c|c|c|}
\hline Plint Nand & Denly Ntan. & Stanle \\
\hline 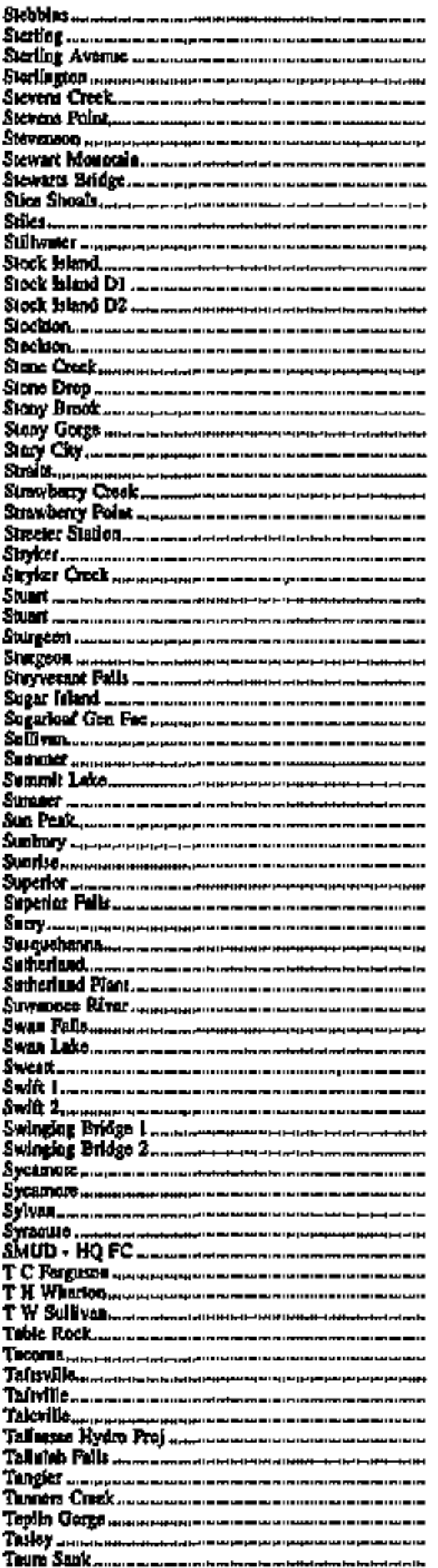 & 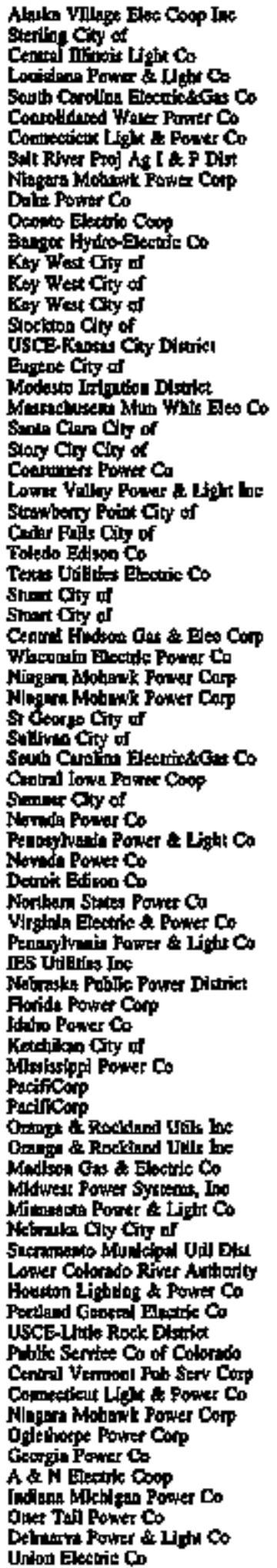 & 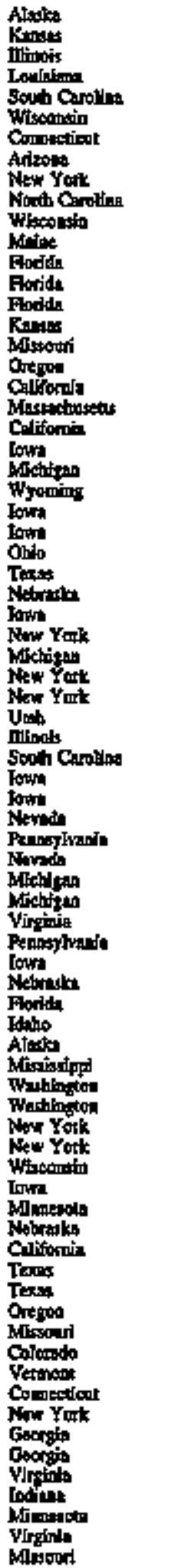 \\
\hline
\end{tabular}

Sec footinget th the of tible. 
Table D1. U.S. Electric Utlity Plants, as of Jamuary 1, 1995 (Conthmed)

\begin{tabular}{|c|c|c|}
\hline Plont Noma & Utaly Kame & Stante \\
\hline 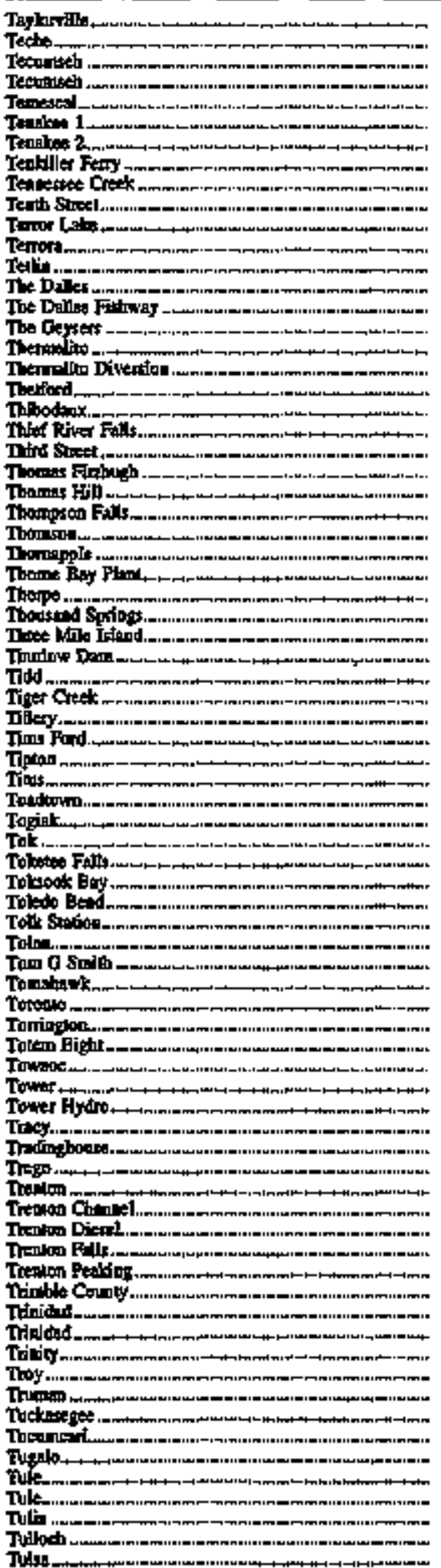 & 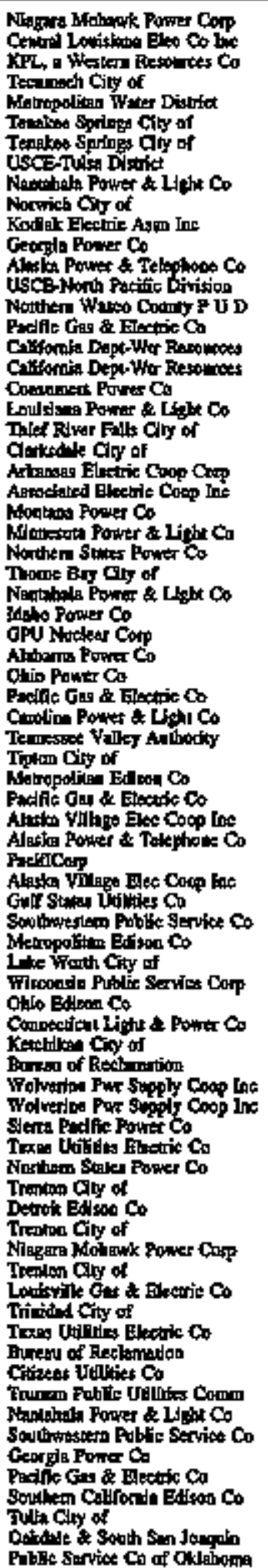 & 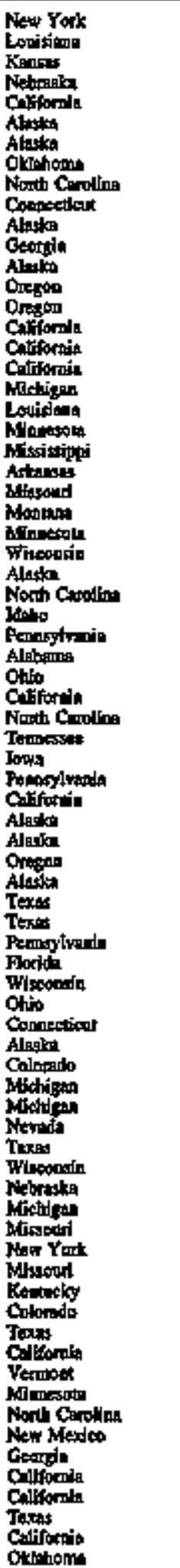 \\
\hline
\end{tabular}

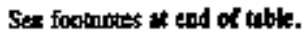


Table D1. U.8. Electrte Utility Plants, as of January 1, 1995 (Conttmued)

\begin{tabular}{|c|c|c|}
\hline Pind Name & Dills Name & Stets \\
\hline 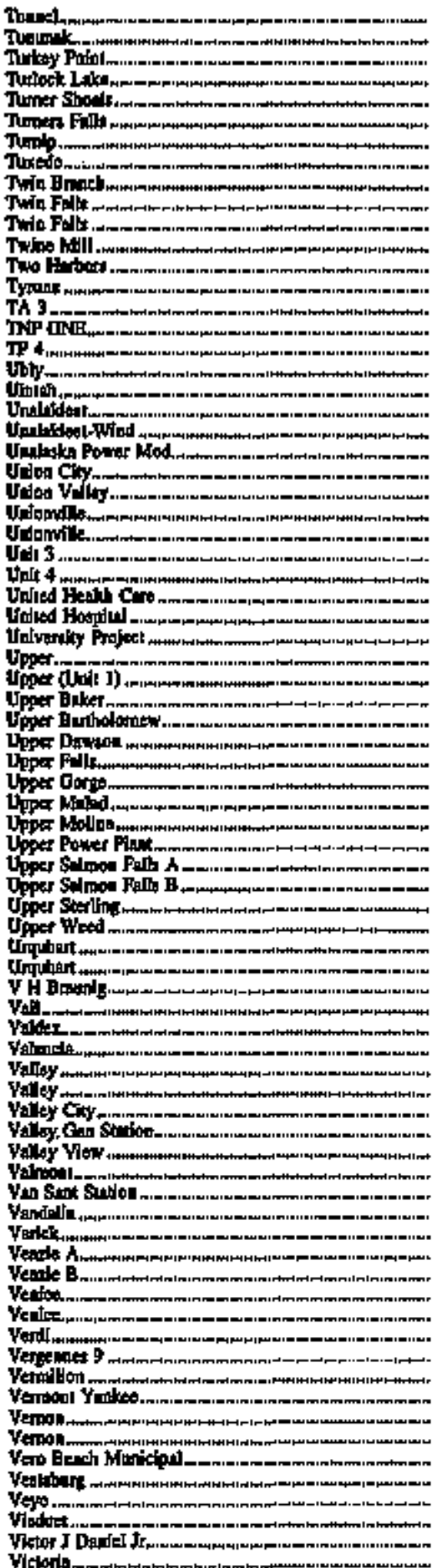 & 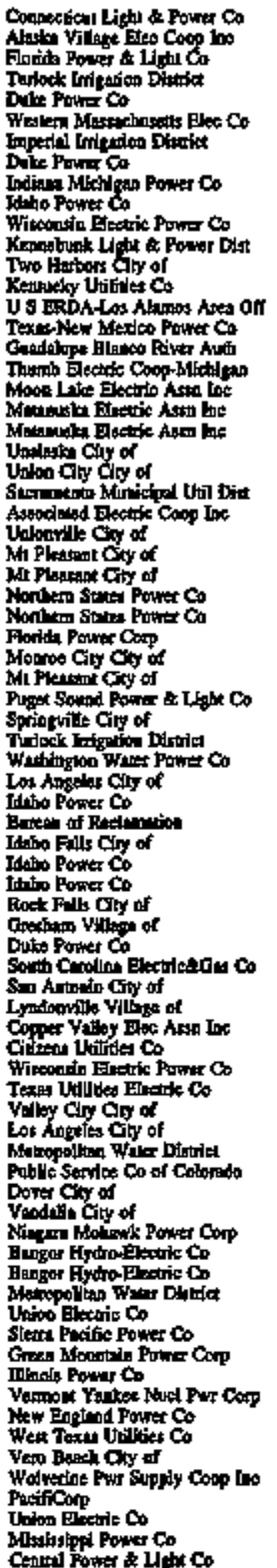 & 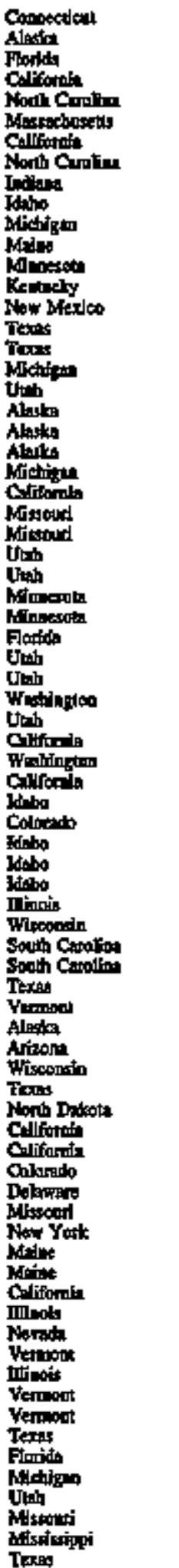 \\
\hline
\end{tabular}

Sos footrotes al and of toblt. 
Table D1. D.S. Electric Utility Plants, as of Jannary 1, 1995 (Continued)

\begin{tabular}{|c|c|c|}
\hline 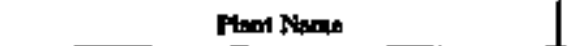 & Lathy Namo & Stote \\
\hline 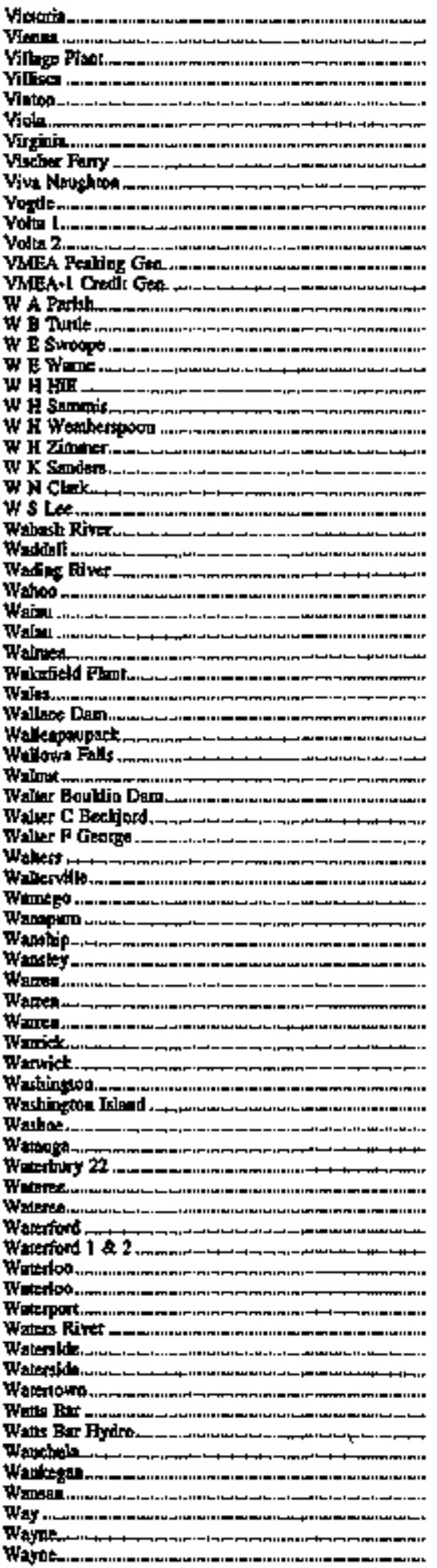 & 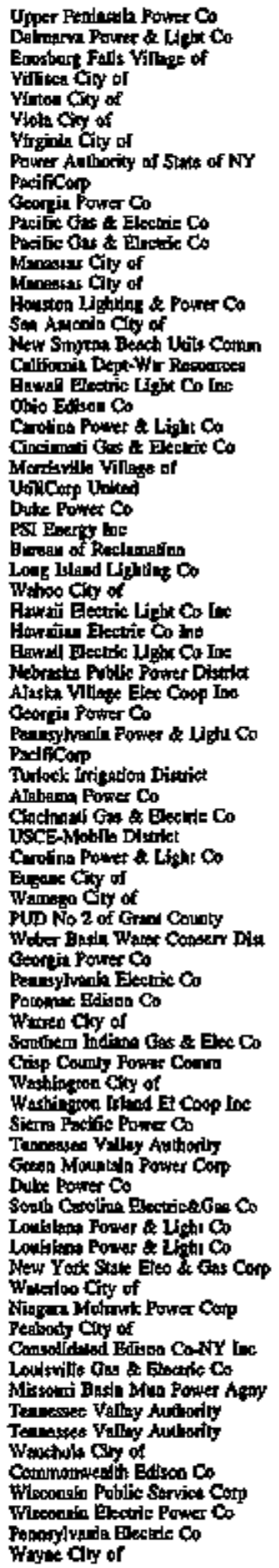 & 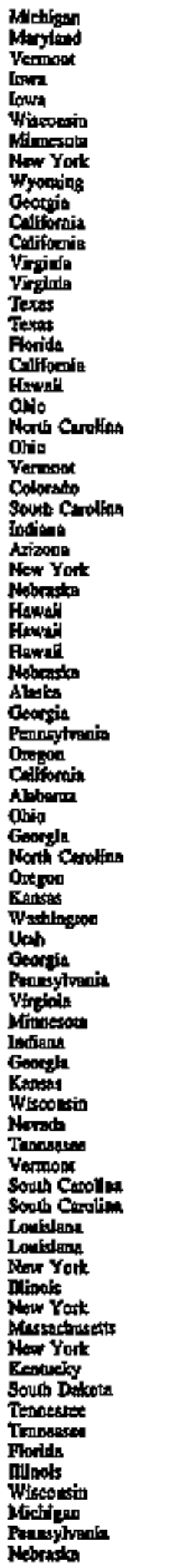 \\
\hline
\end{tabular}

See footnotes at exd of table. 
Table D1. U.S. Electric Utility Plants, as of Jamaary 1, 1995 (Continued)

\begin{tabular}{|c|c|c|}
\hline Plent None & UH'ty None & Slote \\
\hline 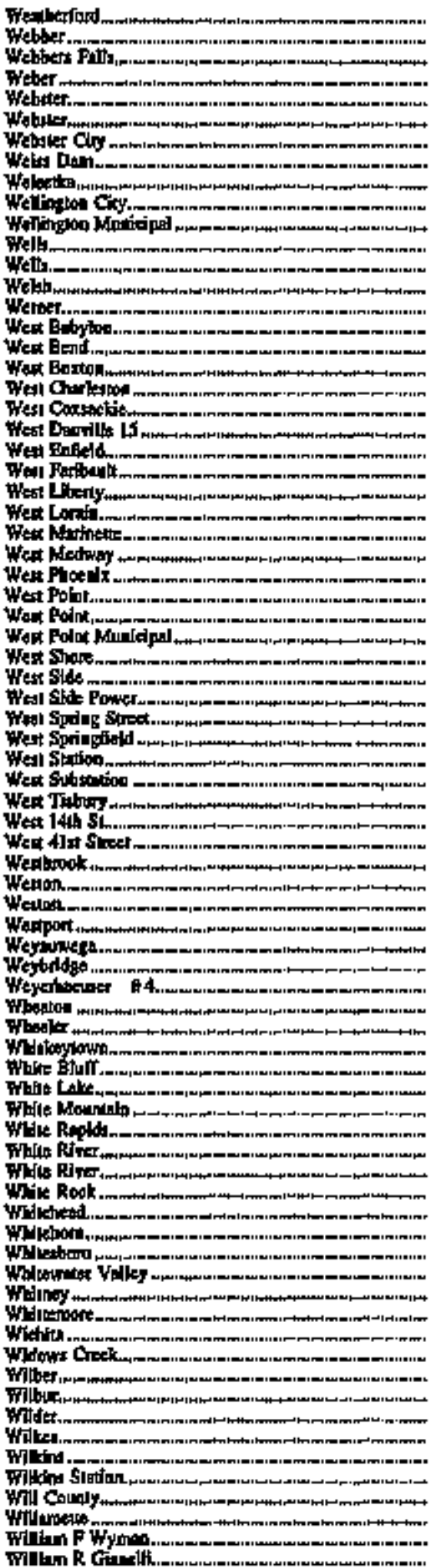 & 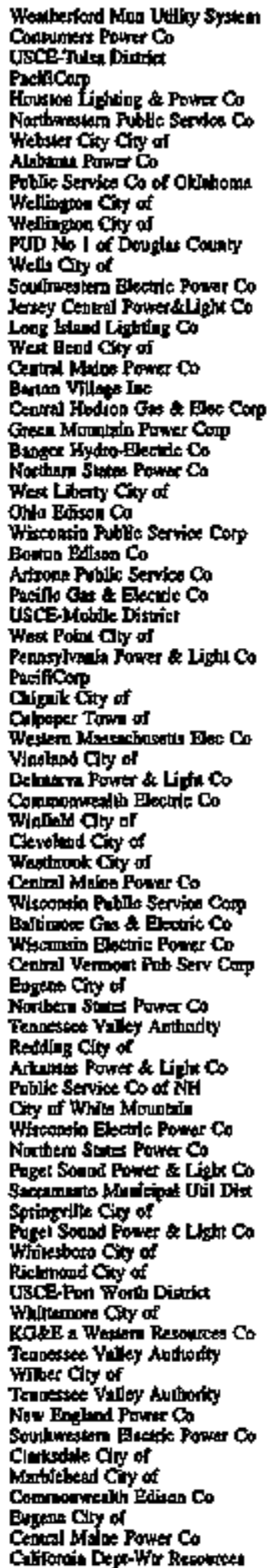 & 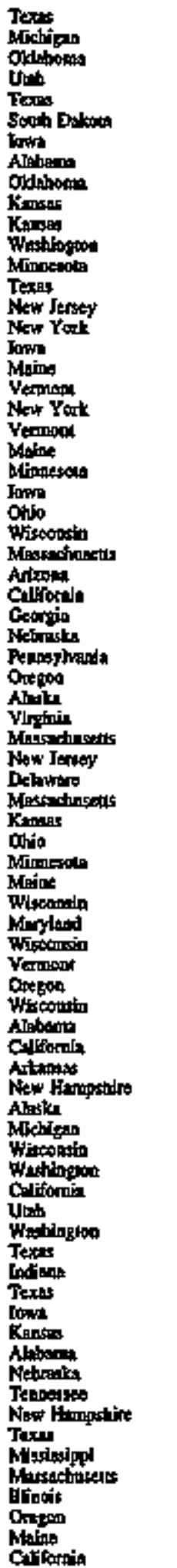 \\
\hline
\end{tabular}

see rootsougn ar and of bable. 
Tabłe DL. U.S. Electric Utility Pants, as of Janmary 1, 1995 (Continned)

\begin{tabular}{|c|c|c|}
\hline Pleal Nemo & thlikg Nomb & Stoto \\
\hline 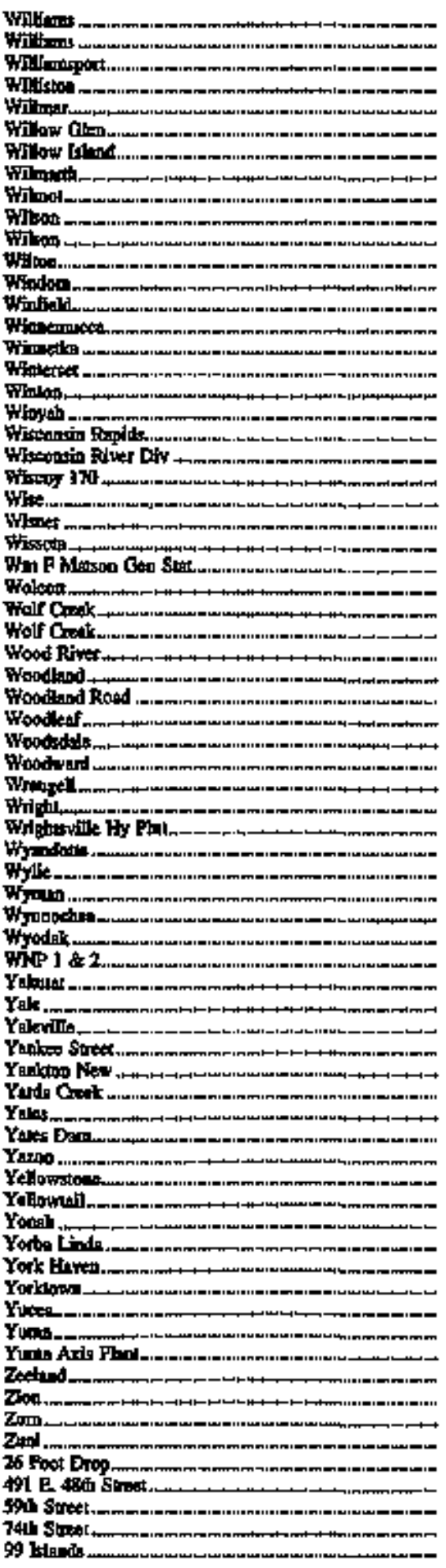 & 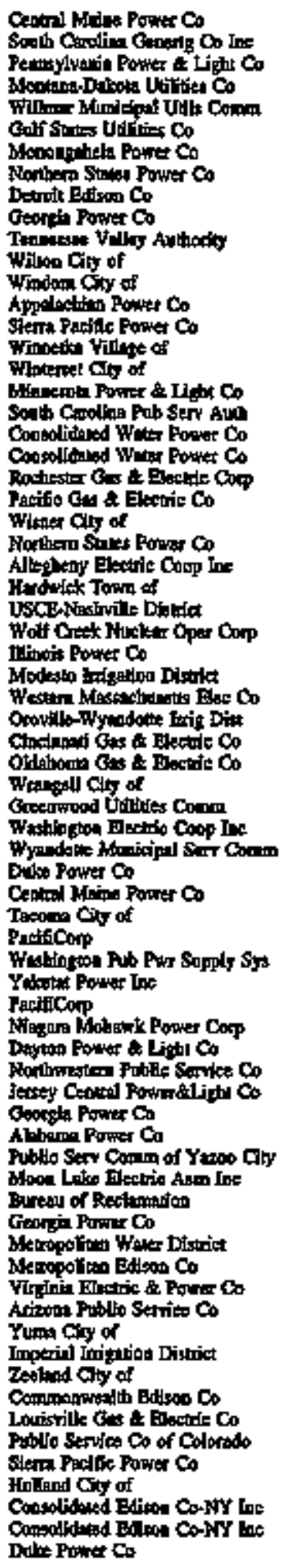 & 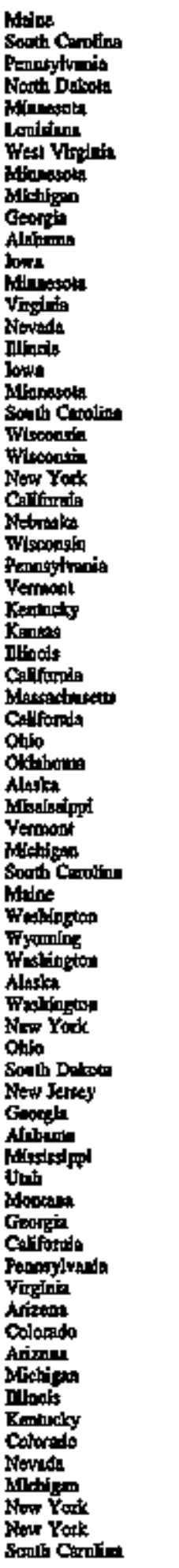 \\
\hline
\end{tabular}

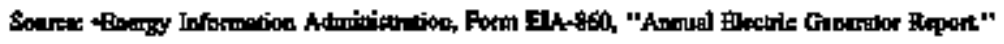


Tahle D2. U.S. Eectric Utility Plants by State, 1995

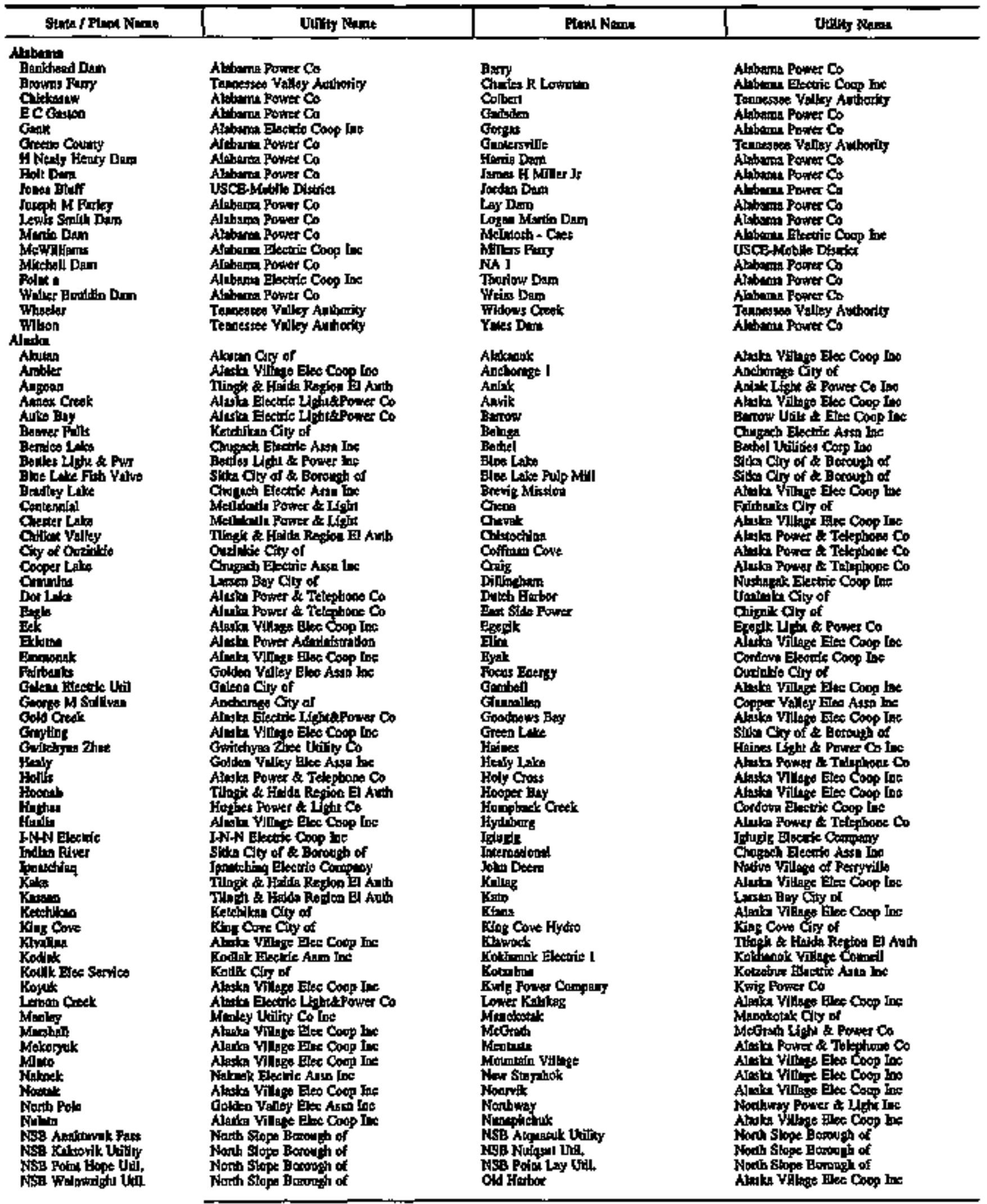

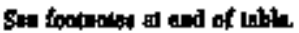


Tahle D2. U.S. Ełectric Utility Plants by State, 1995 (Continued)

\begin{tabular}{|c|c|c|c|}
\hline Slete / Fien Nene & Utity Name & Phant Nome & unty Nante \\
\hline 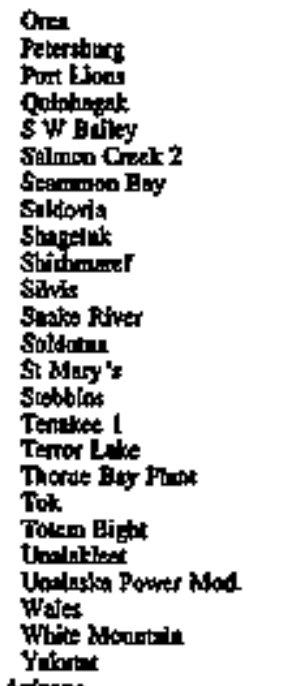 & 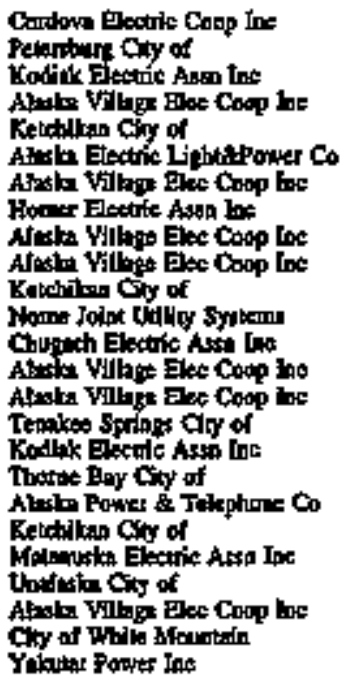 & 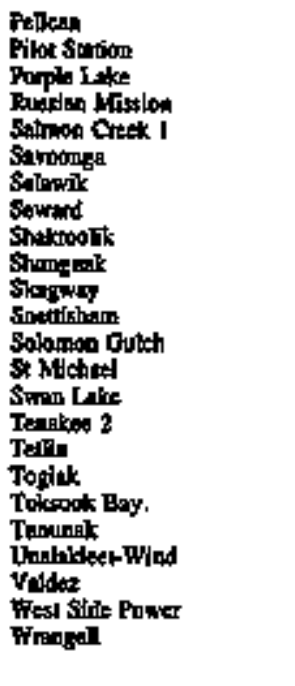 & 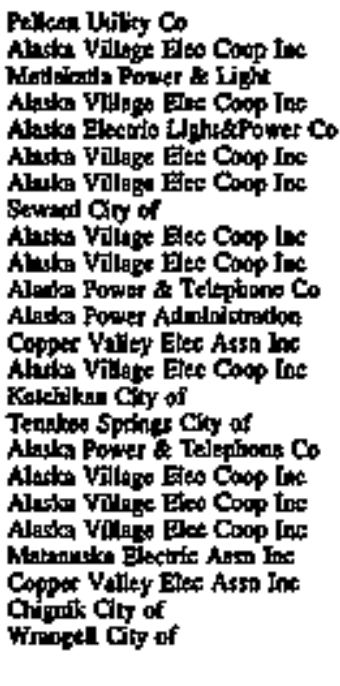 \\
\hline 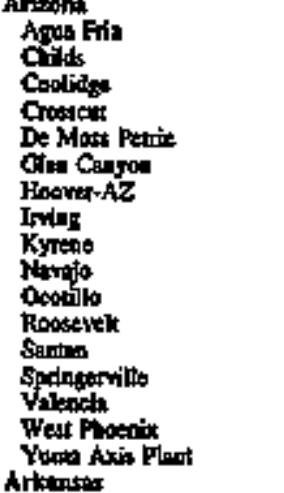 & 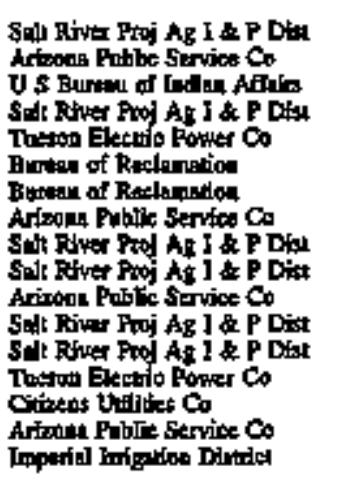 & 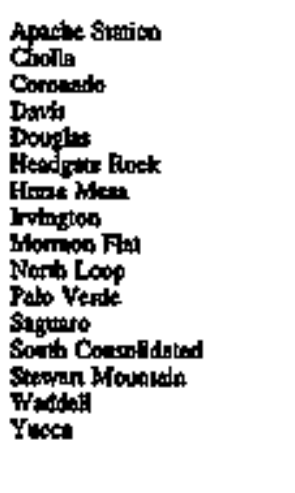 & 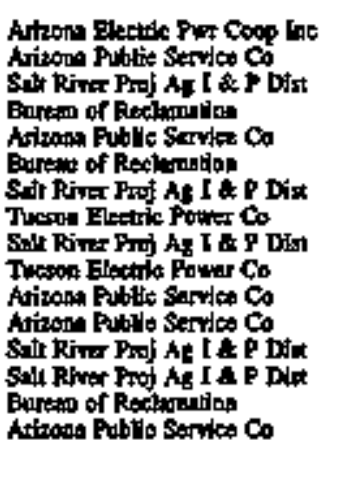 \\
\hline 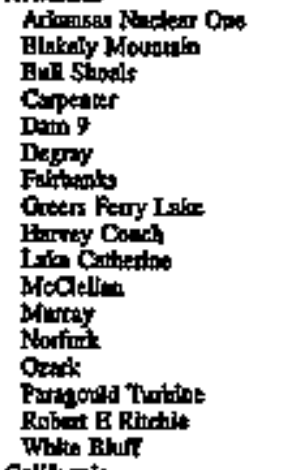 & 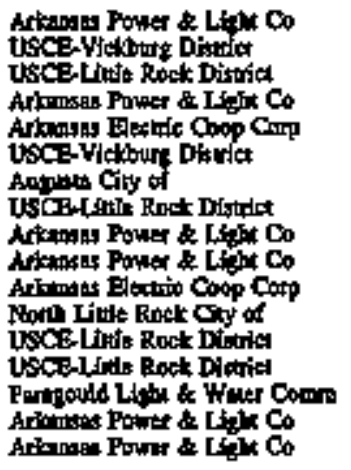 & 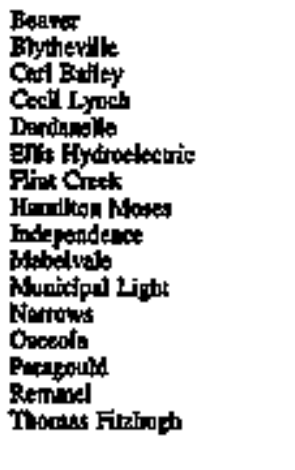 & 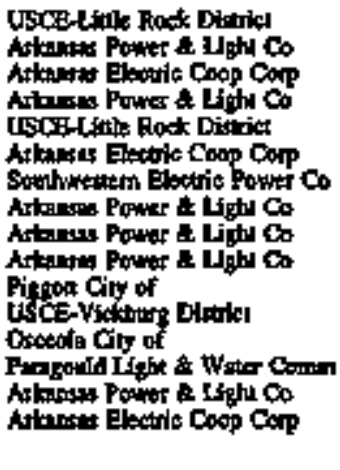 \\
\hline 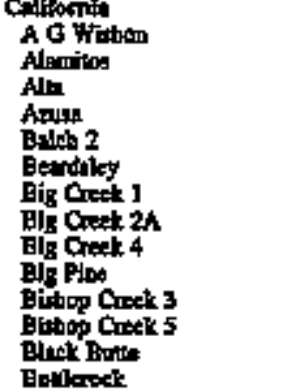 & 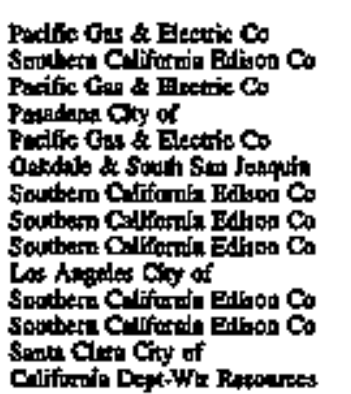 & 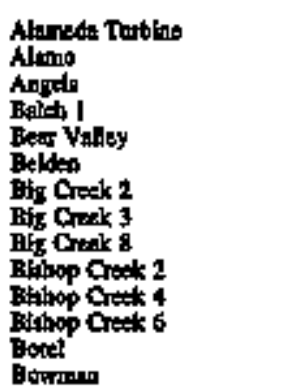 & 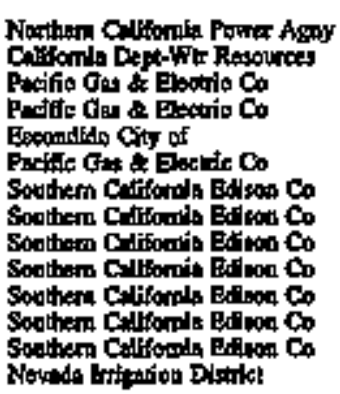 \\
\hline
\end{tabular}

Ses foomots to end of noblo. 
Table D2. U.S. Electric Utility PJants by State, 1995 (Continued)

\begin{tabular}{|c|c|c|c|}
\hline flede / Ptat Nown & Utiley Nome & Phut Nethe & untily Namt \\
\hline 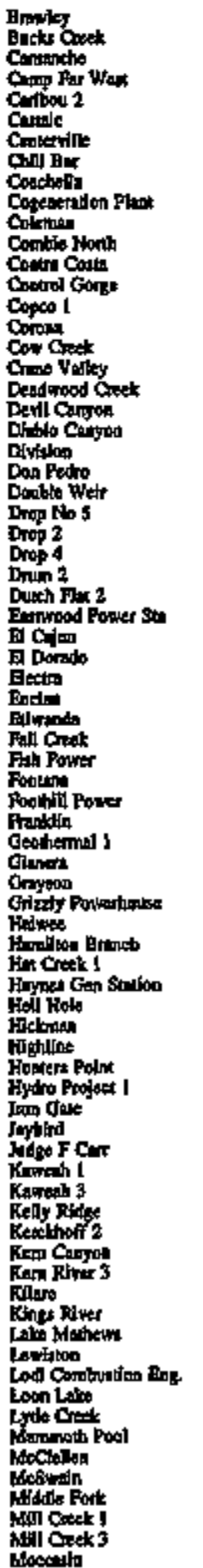 & 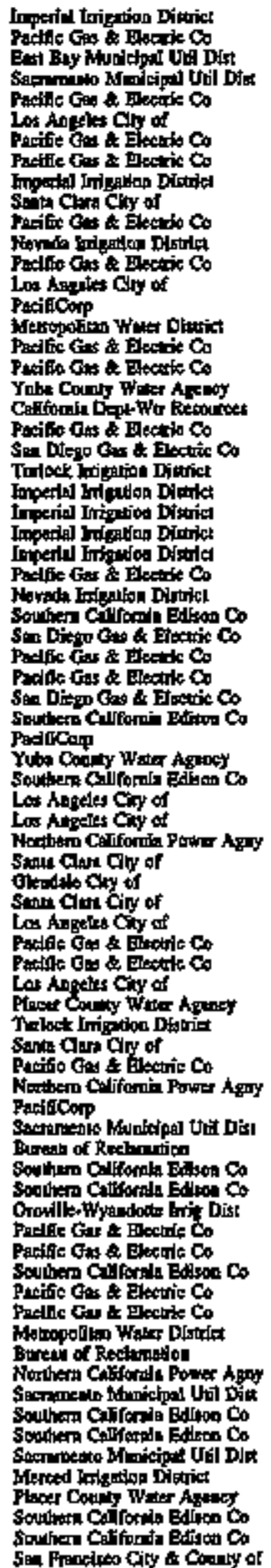 & 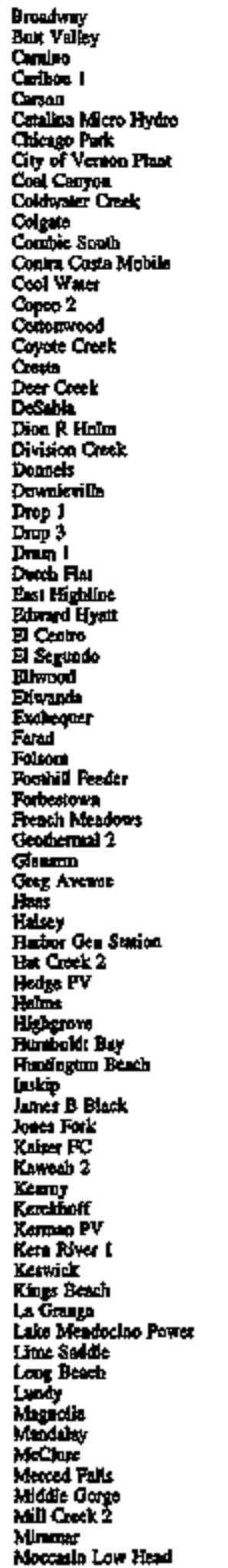 & 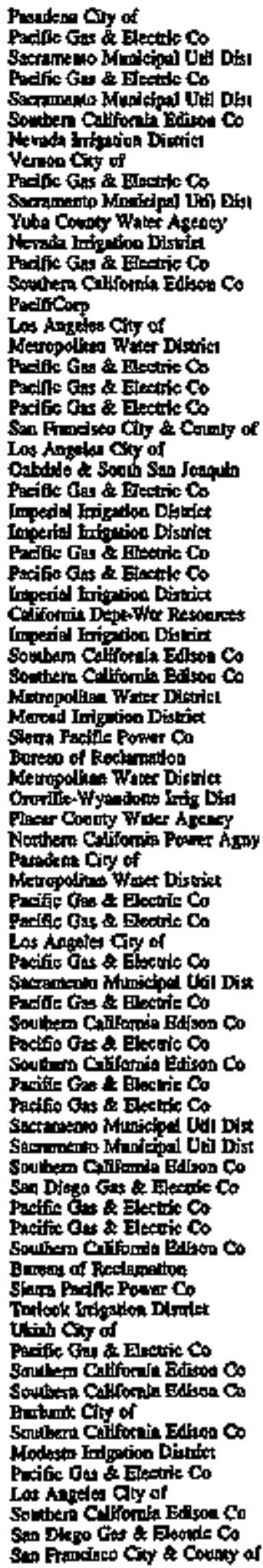 \\
\hline
\end{tabular}

See foolmolat al tod of labte 
Tahle D2. U.S. Electric Utility Plants by State, 1995 (Continued)

\begin{tabular}{|c|c|c|c|}
\hline Sirle / Pint Nome & 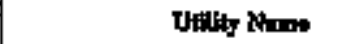 & Plant None & Cullty Kane \\
\hline 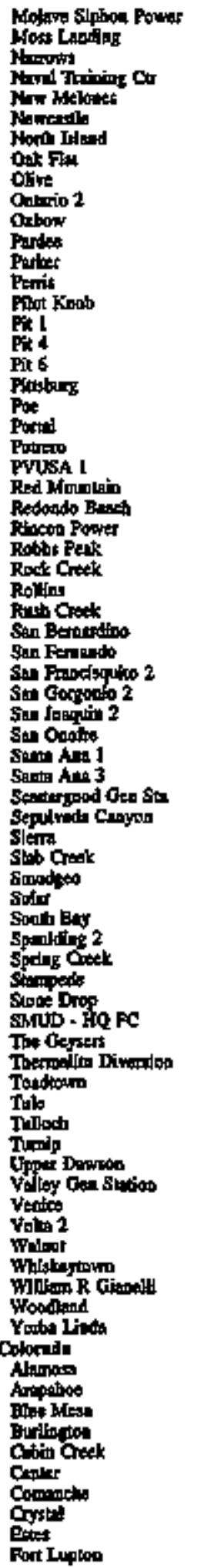 & 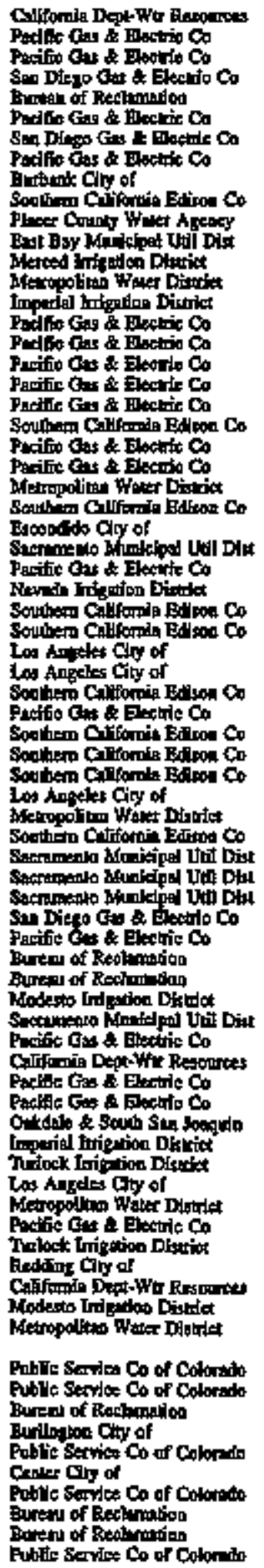 & 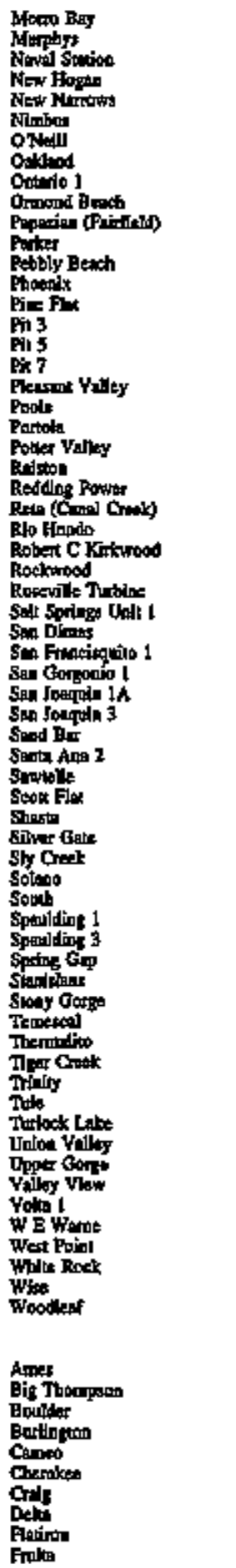 & 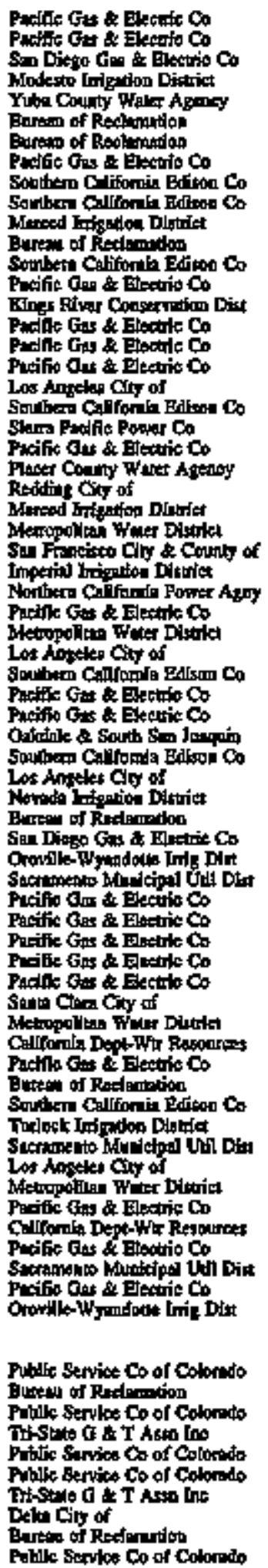 \\
\hline
\end{tabular}

$S=5$ toolooses at ead of tablot 
Table D2. U.S. Electric Utility Plants by State, 1995 (Continued)

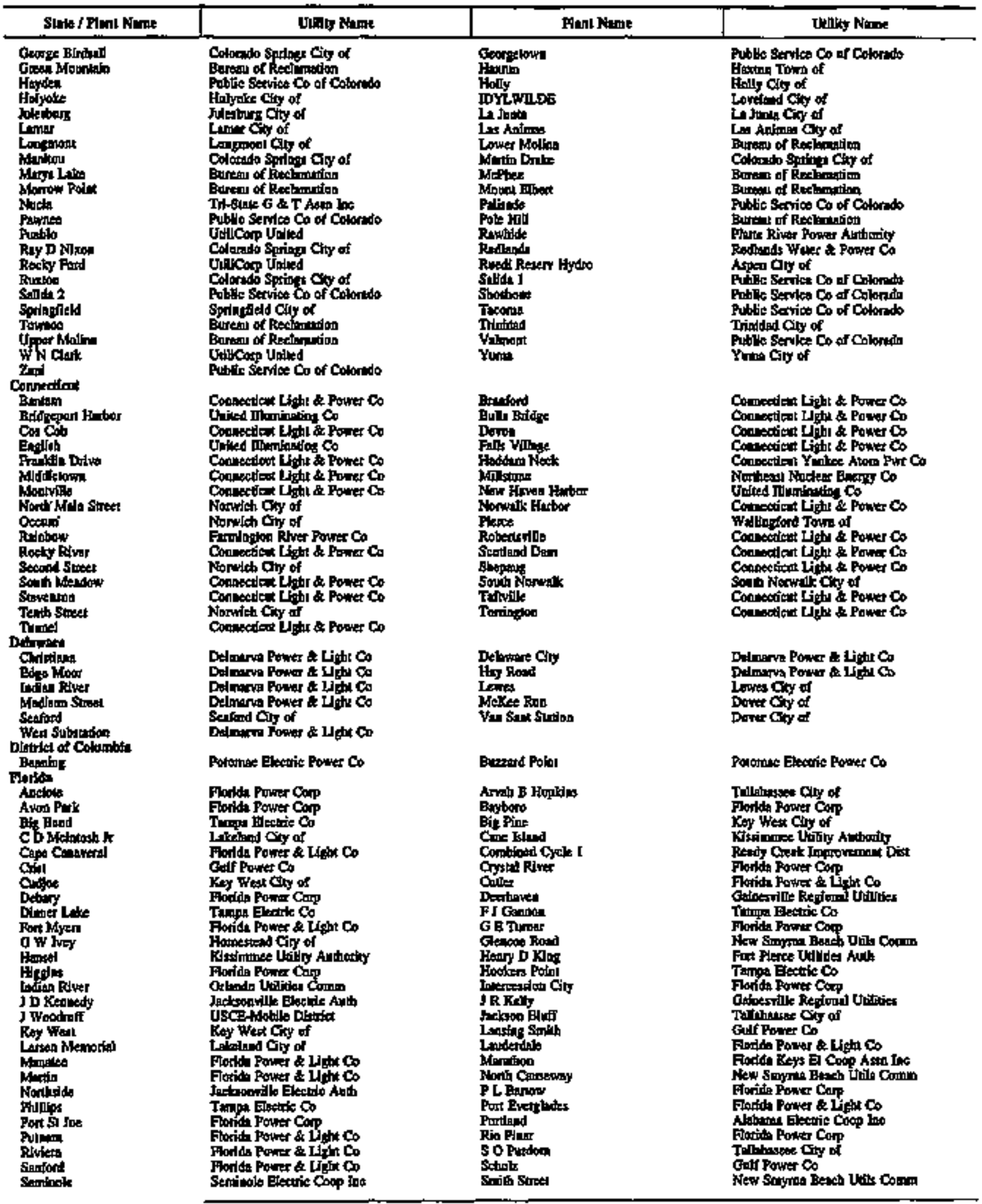

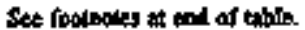


Tahle D2. U.S. Electric Utillty Plants by State, 1995 (Continued)

\begin{tabular}{|c|c|c|c|}
\hline Slate / Plool Nane & Ututity None & Plapt flanpe & Leqky Nan \\
\hline 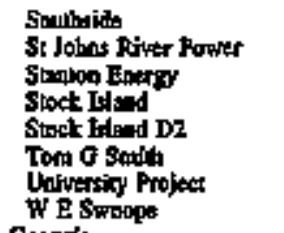 & 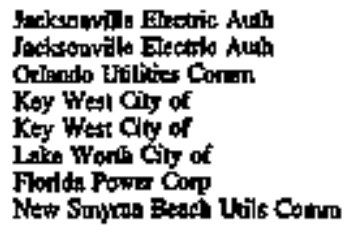 & 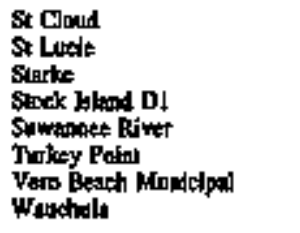 & 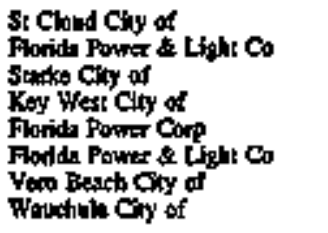 \\
\hline 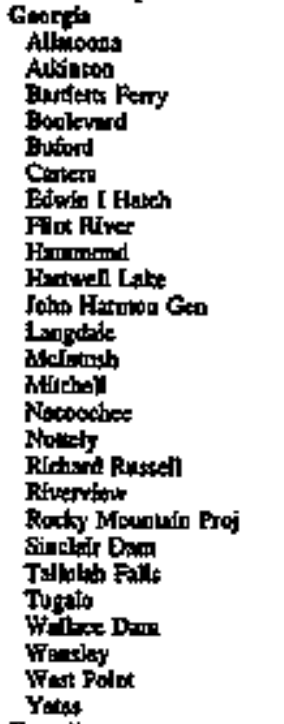 & 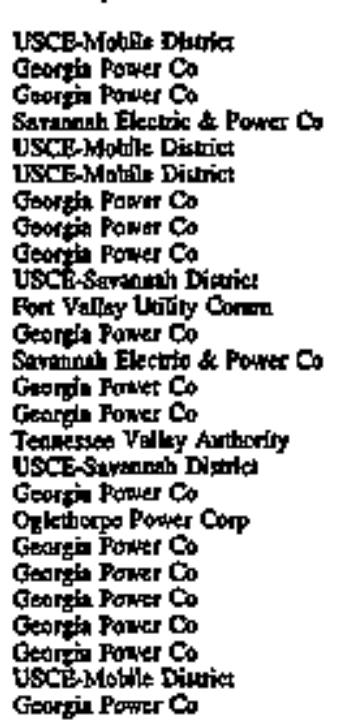 & 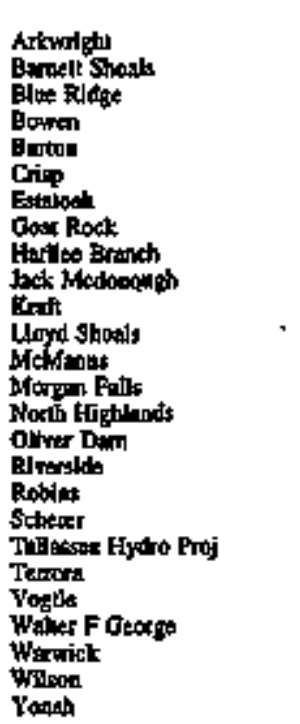 & 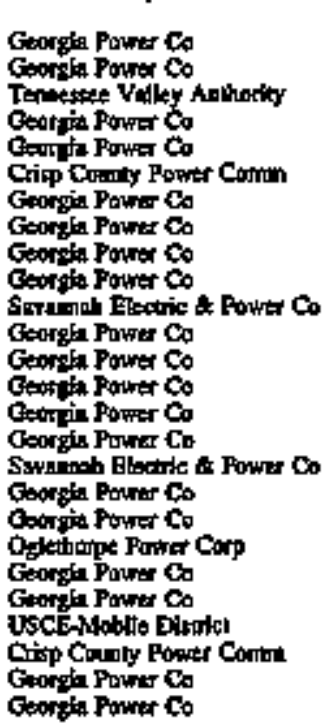 \\
\hline 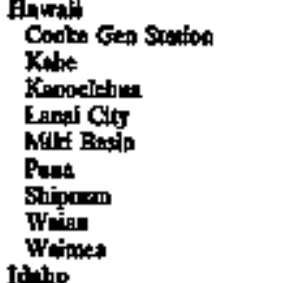 & 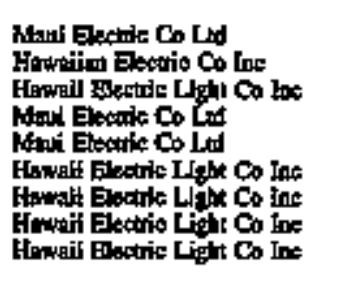 & 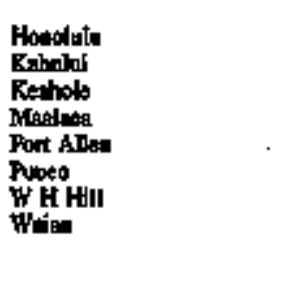 & 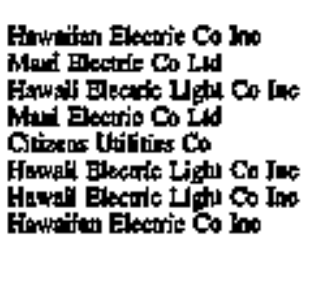 \\
\hline 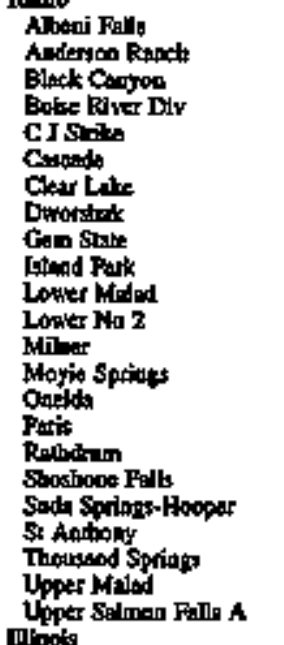 & 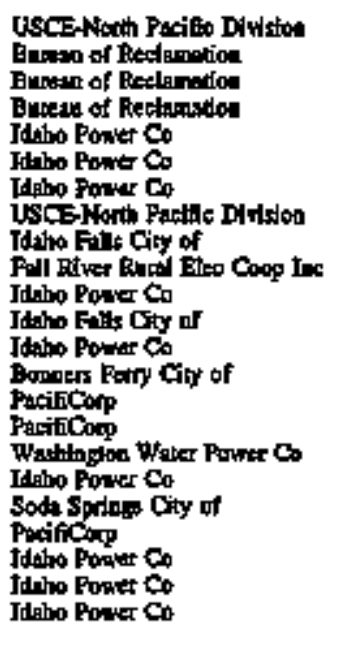 & 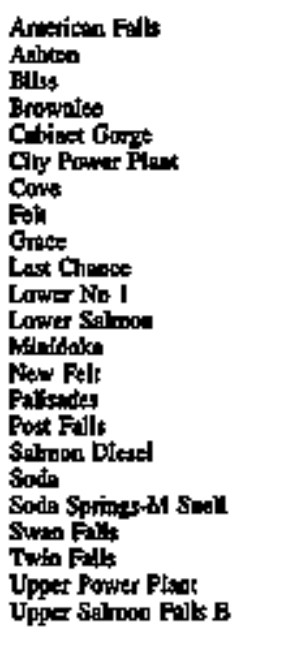 & 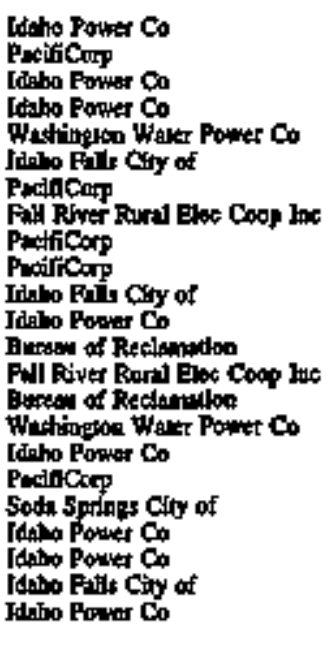 \\
\hline 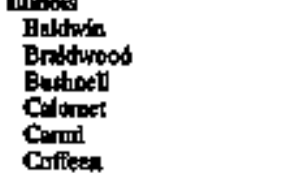 & 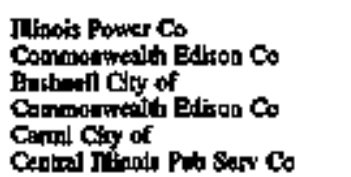 & 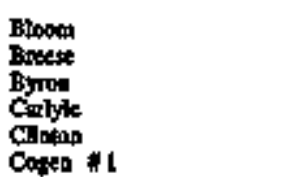 & 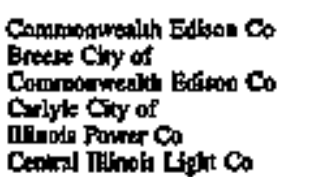 \\
\hline
\end{tabular}

See frotudes at and of thele. 
Table D2. U.S. Electric Utility Plants by State, 1993 (Continued)

\begin{tabular}{|c|c|c|c|}
\hline state / Phat Mand & thath Nothe & Phnt Nama & eanly Naphe \\
\hline 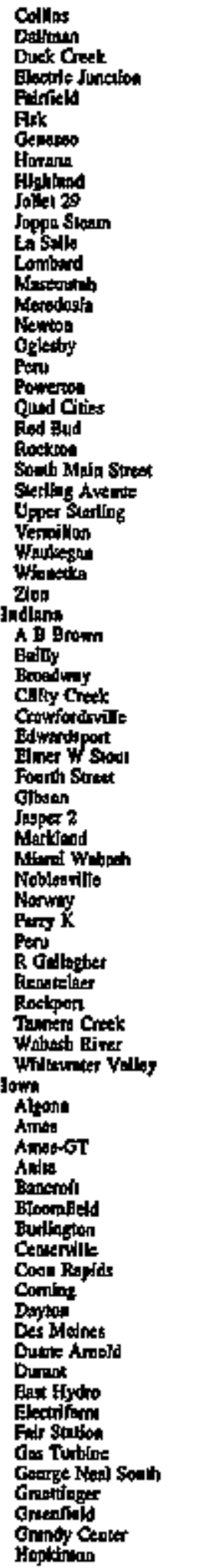 & 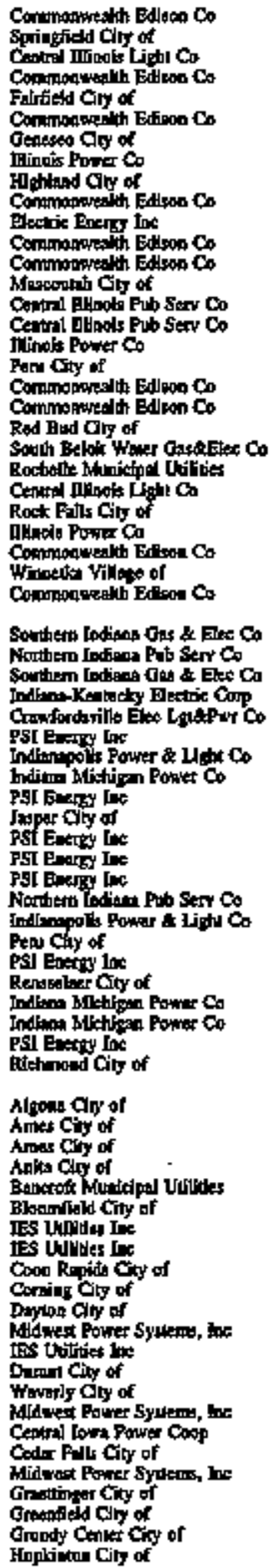 & 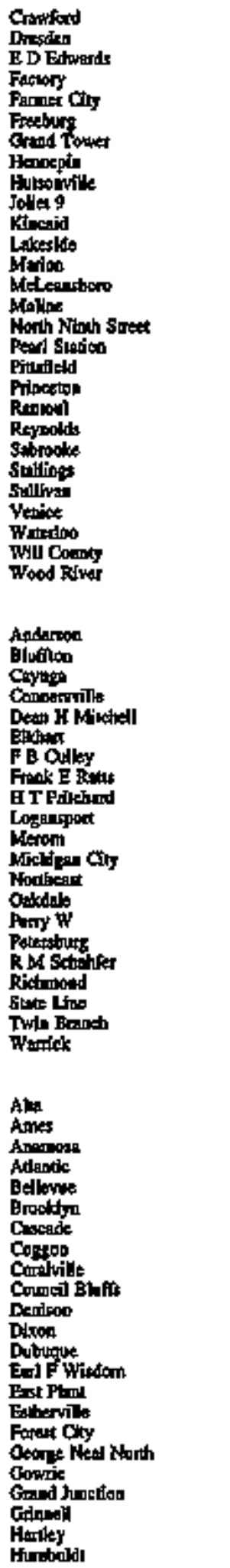 & 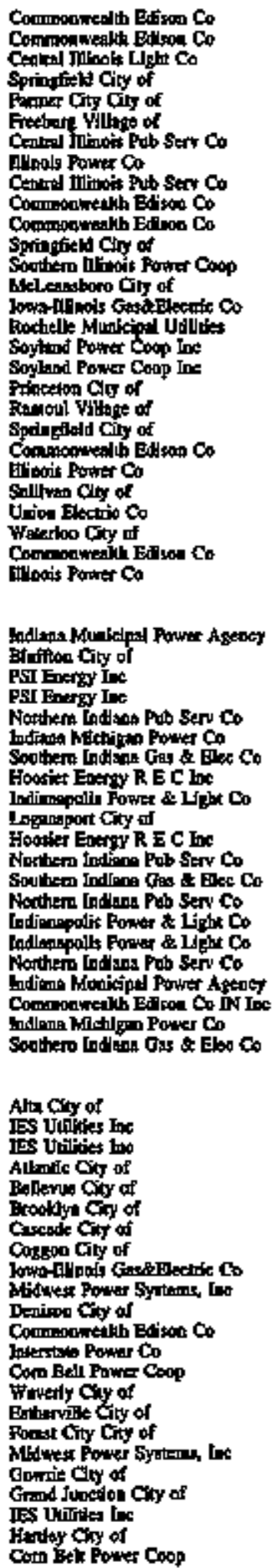 \\
\hline
\end{tabular}

Sen foolpows the tod of teble. 
Table D2. U.S. Glectric Utility Plants by State, 1995 (Contimaed)

\begin{tabular}{|c|c|c|c|}
\hline Stale / Pant Nane & Jollity lane & Plane Nate & thith Now \\
\hline 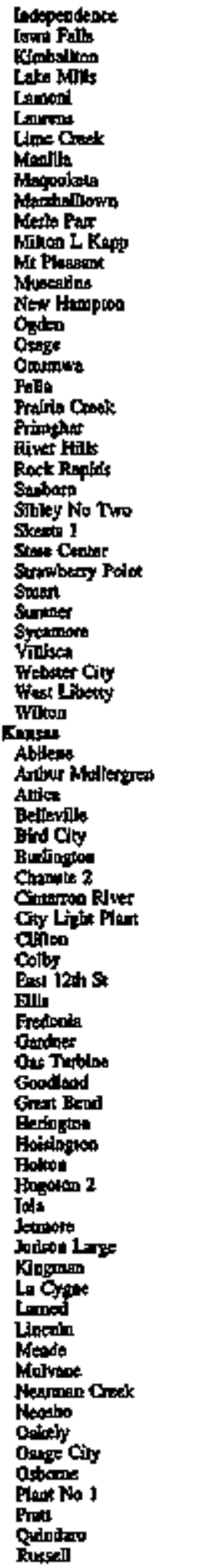 & 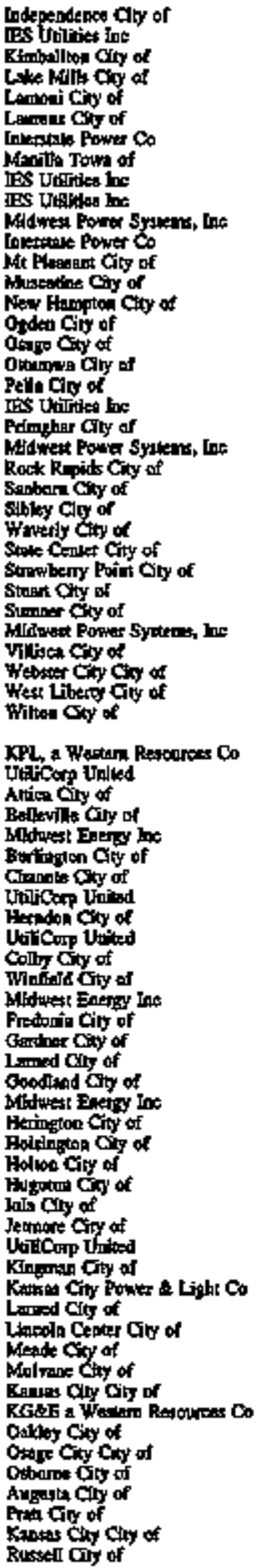 & 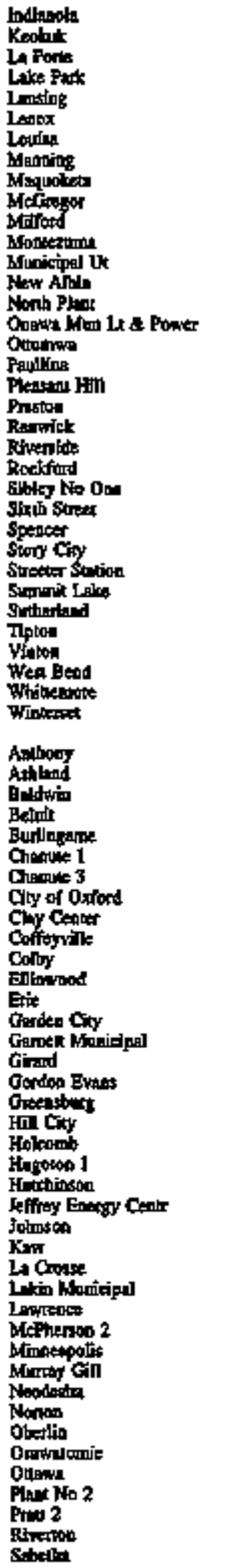 & 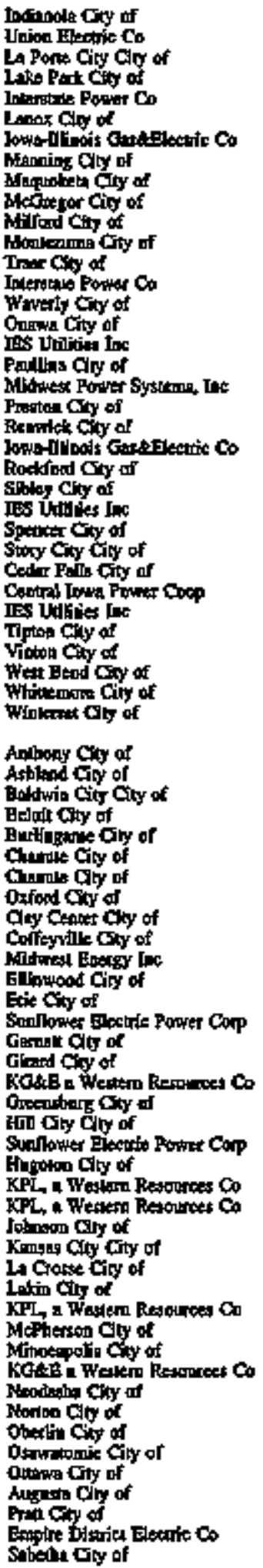 \\
\hline
\end{tabular}

Seet fooknotes at ard of tabk 
Table D2. U.S. Electric Utility Plants by State, 1995 (Continued)

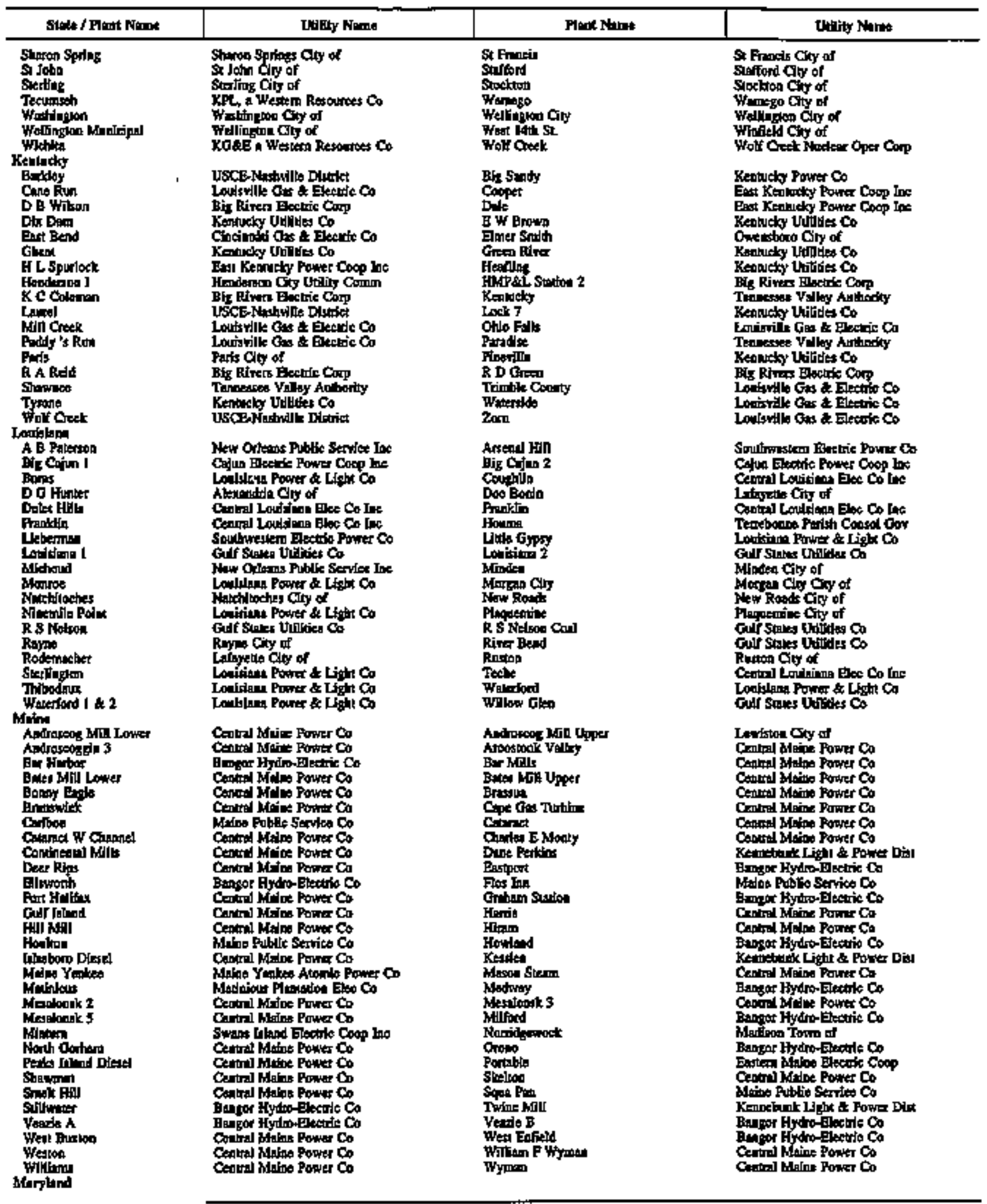

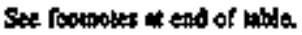


Table D2. U.S. Elestric Utility Plants by Stato, 1995 (Contioued)

\begin{tabular}{|c|c|c|c|}
\hline Staln / Phnt Nane & 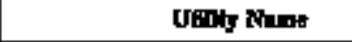 & Ptanl Ninge & Jolity Nimat: \\
\hline 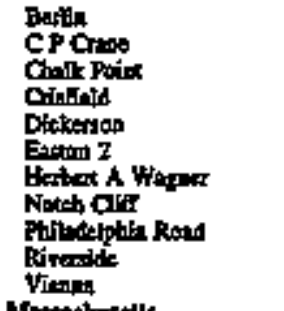 & 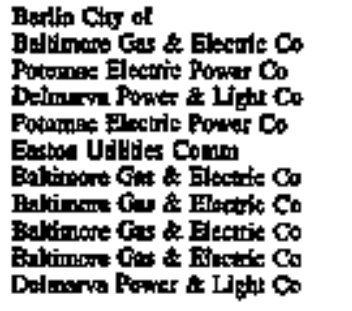 & 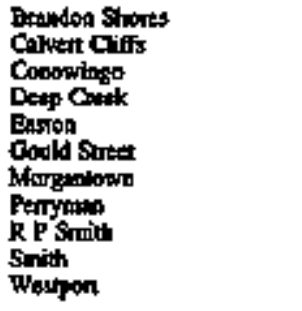 & 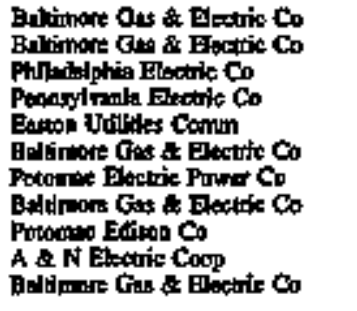 \\
\hline 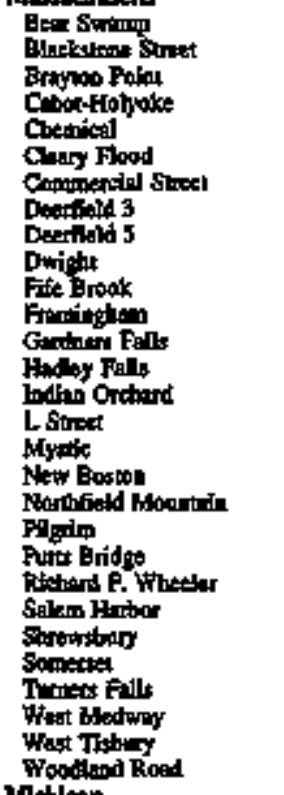 & 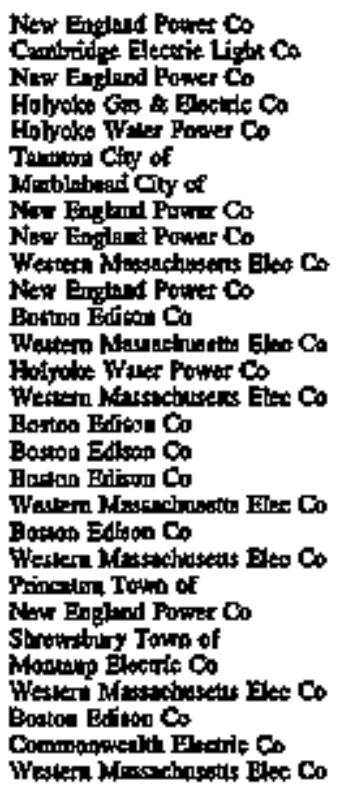 & 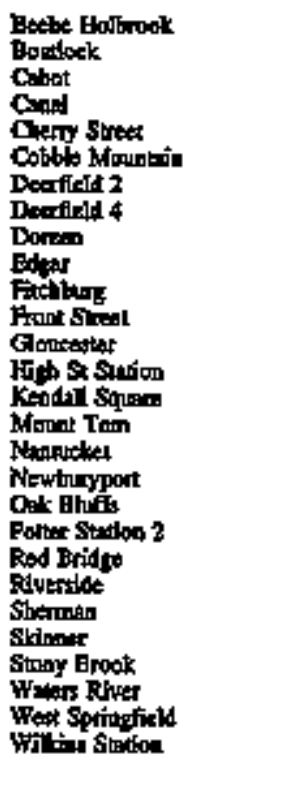 & 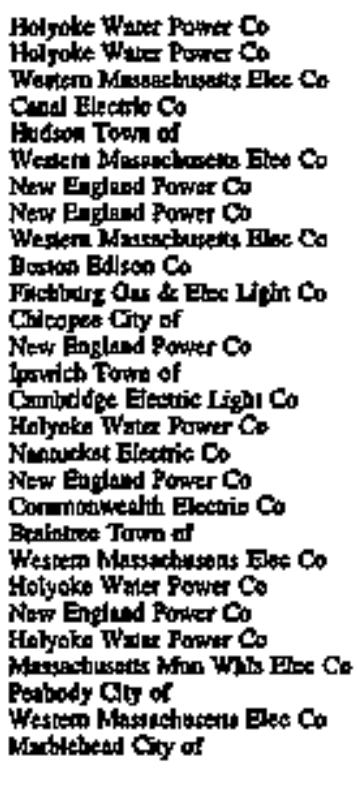 \\
\hline 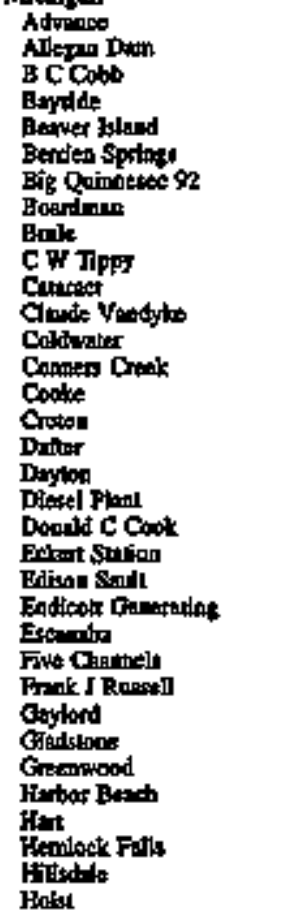 & 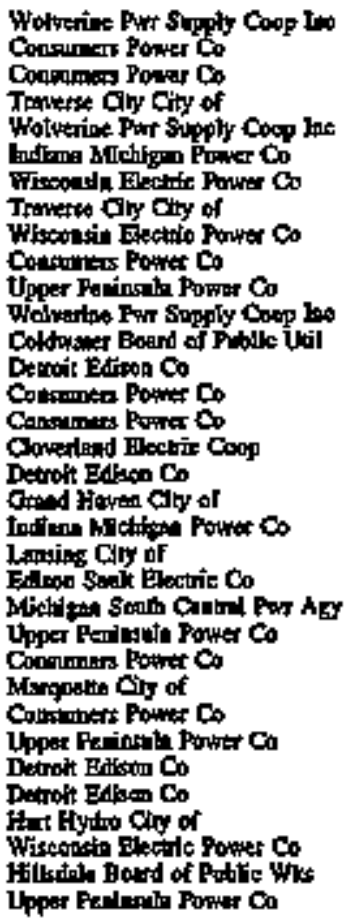 & 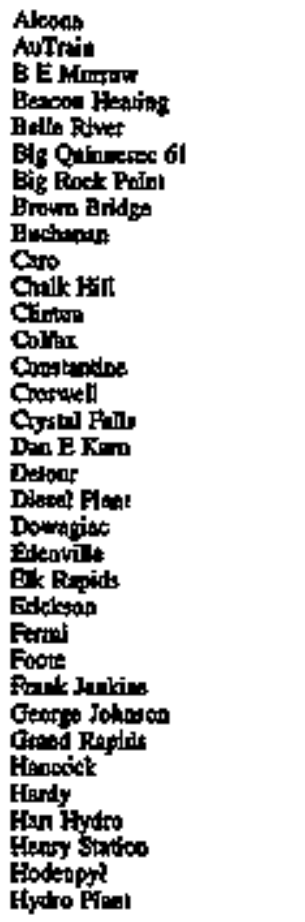 & 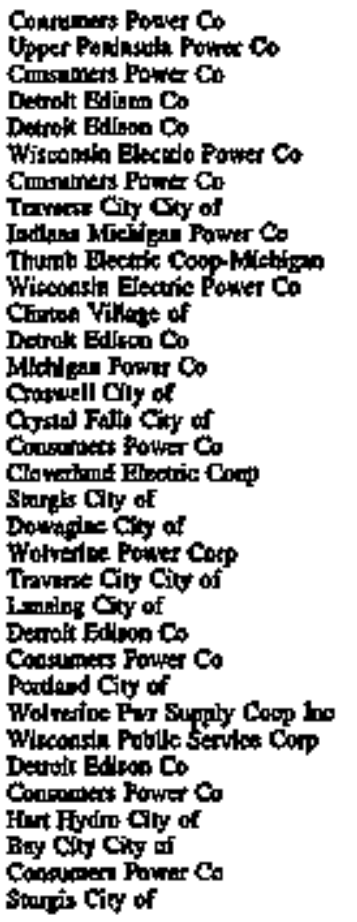 \\
\hline
\end{tabular}

Sec footnoles at End of trble. 
Table D2. U.8. Electric Utility Plants by State, 1995 (Continued)

\begin{tabular}{|c|c|c|c|}
\hline Btato / Fhane Nan & Litrity Name & Pland Nabe & Otwity Numer \\
\hline 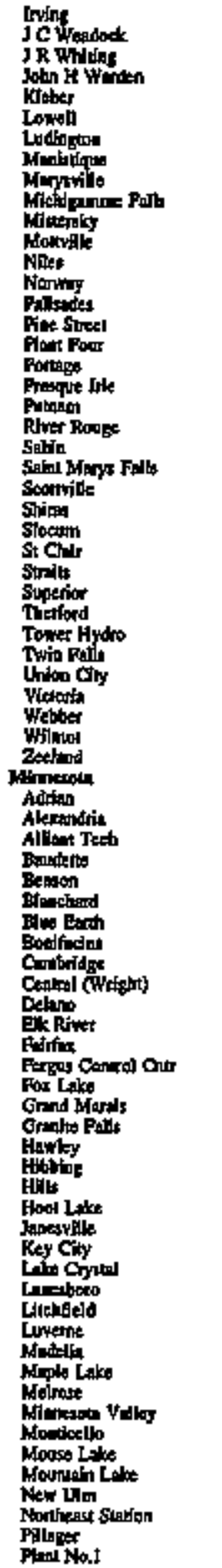 & 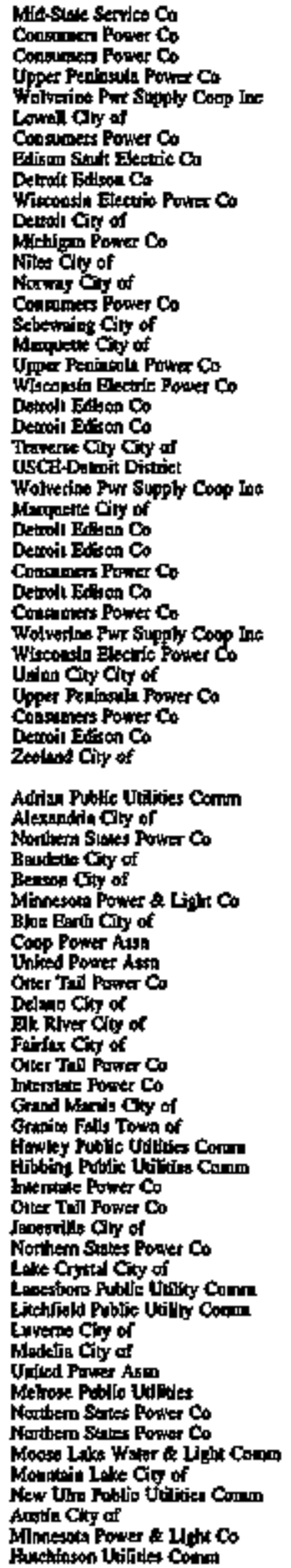 & 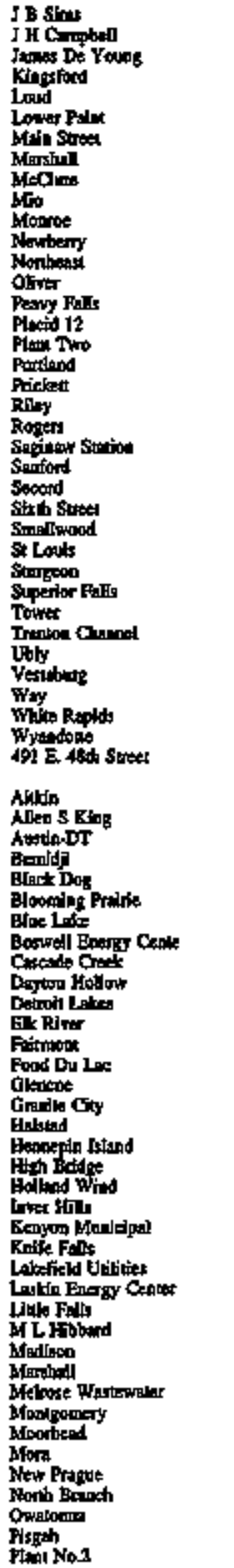 & 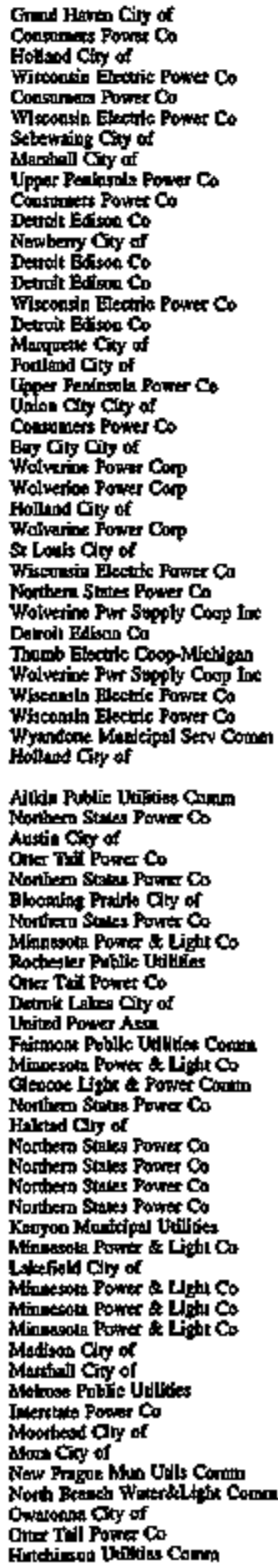 \\
\hline
\end{tabular}

See frodeoly at apd of thile. 
Table D2, U.S. Electric Utility Plants by State, 1995 (Cantimued)

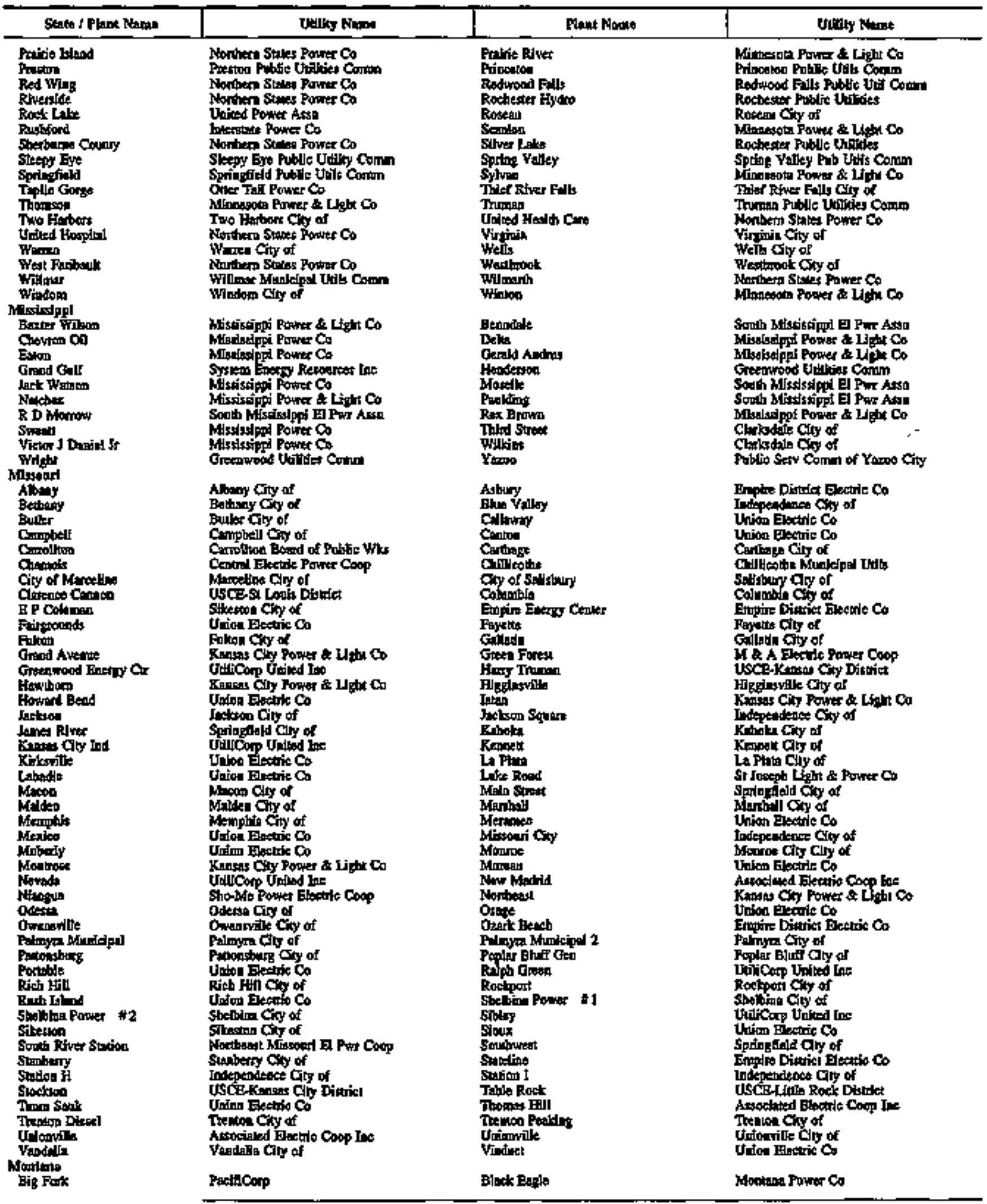

Ses loothotes at end of thble. 
Table D2. U.S. Electric Utility Plants by State, 1995 (Continued)

\begin{tabular}{|c|c|c|c|}
\hline Stacte f Thane Itange & Woilny Nome & Flu: haoe & Uum, Noms: \\
\hline 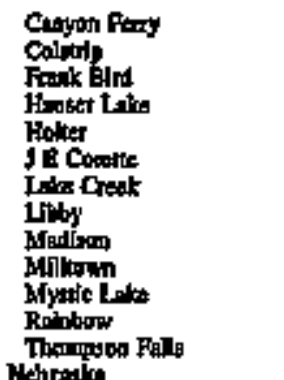 & 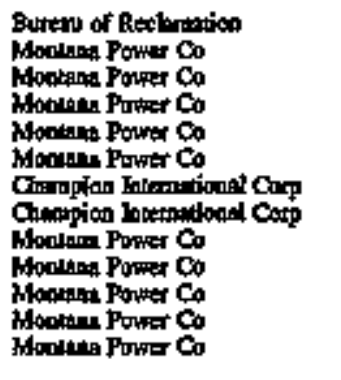 & 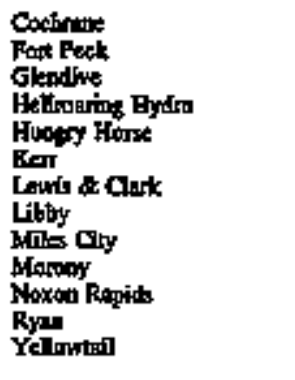 & 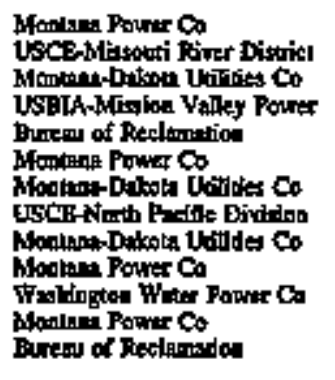 \\
\hline 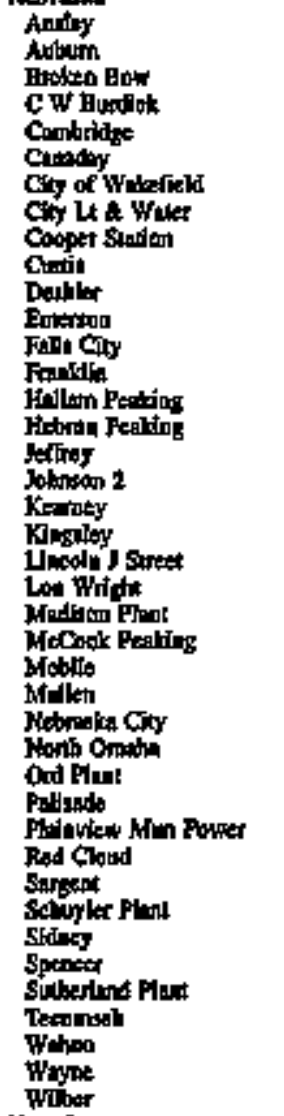 & 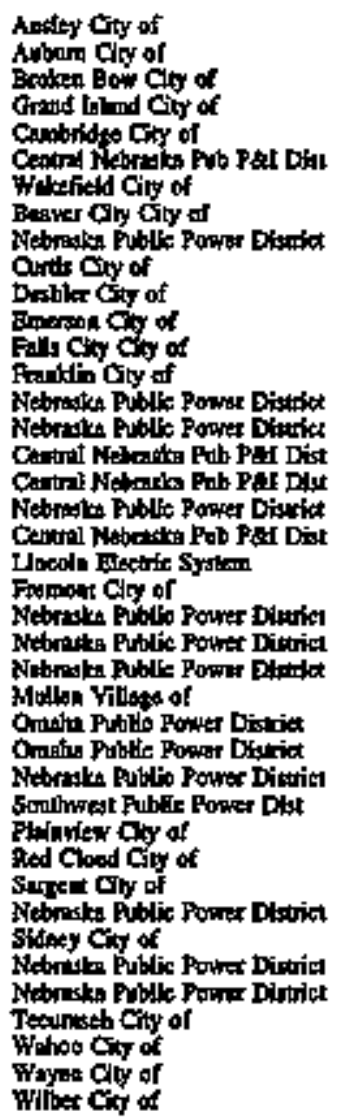 & 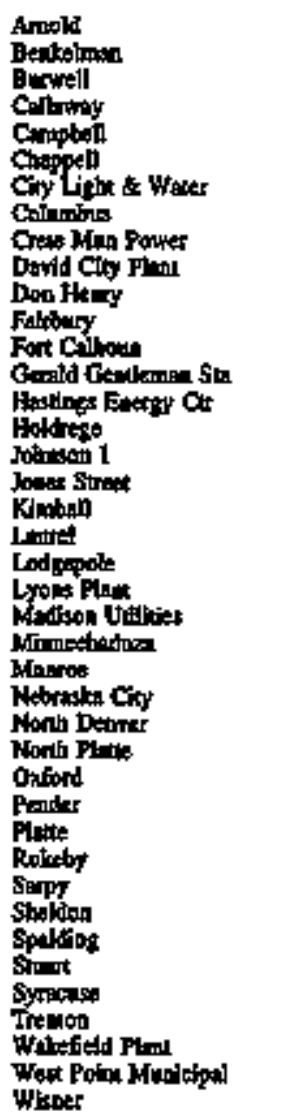 & 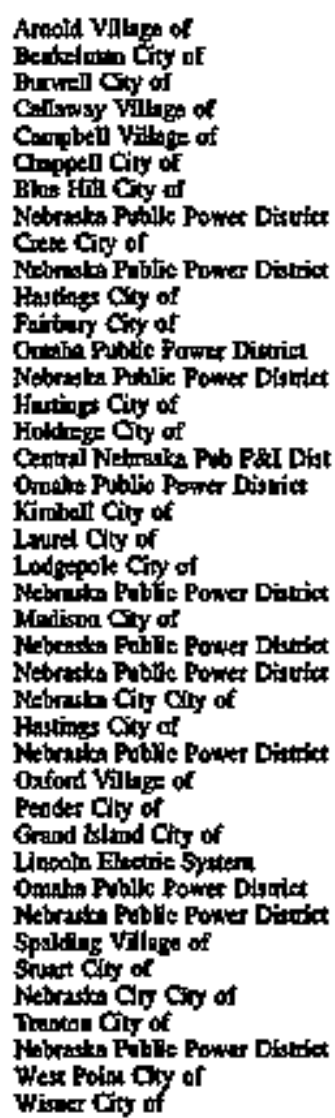 \\
\hline 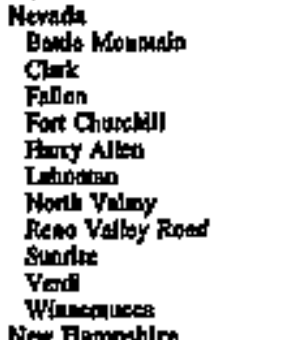 & 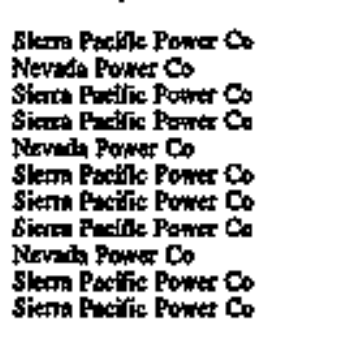 & 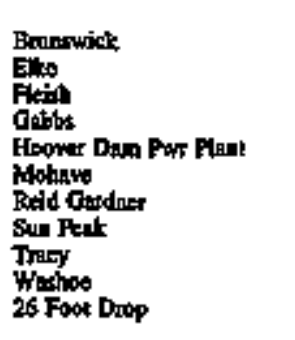 & 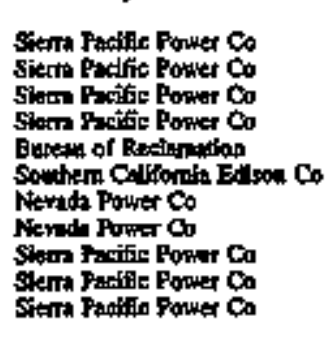 \\
\hline 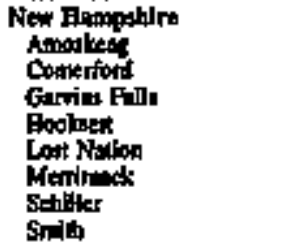 & 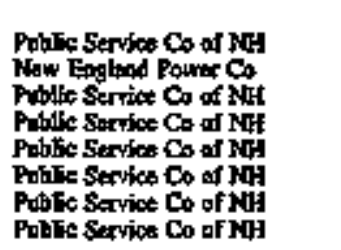 & 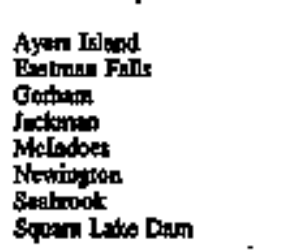 & 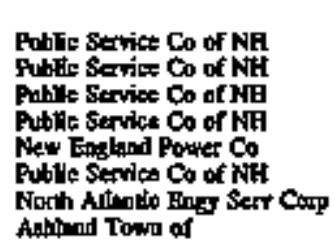 \\
\hline
\end{tabular}

Sor fortholes al ead of labje. 
Table D2. U.S. Blectric Utility Flants by State, 1995 (Continued)

\begin{tabular}{|c|c|c|c|}
\hline Stoth / Ptand Nowt & Cillats Nam: & Ftoml Nane & 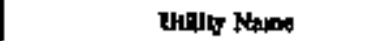 \\
\hline 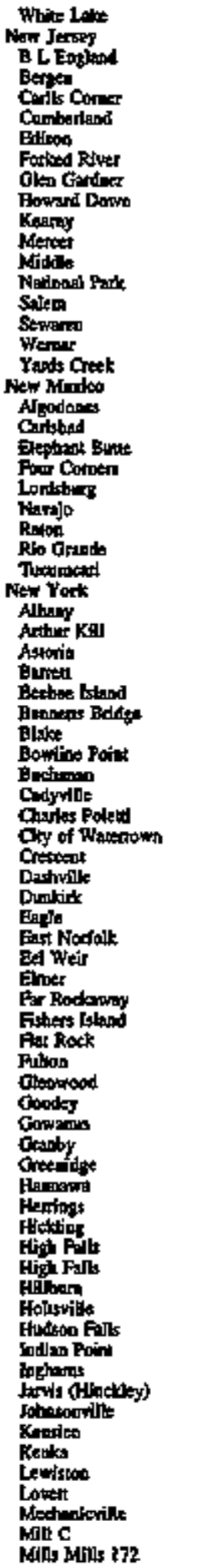 & 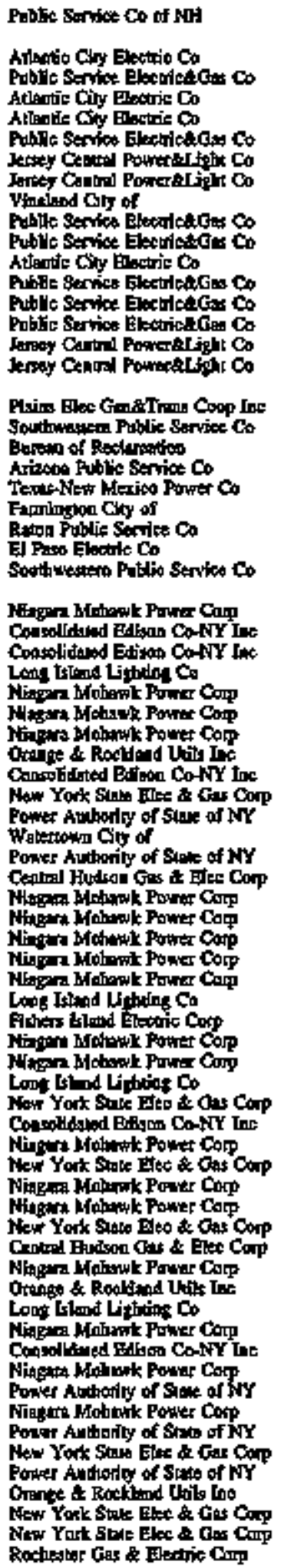 & 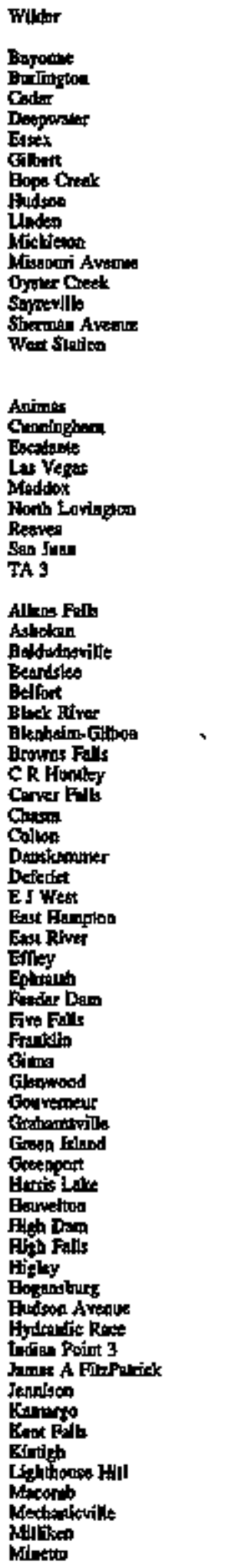 & 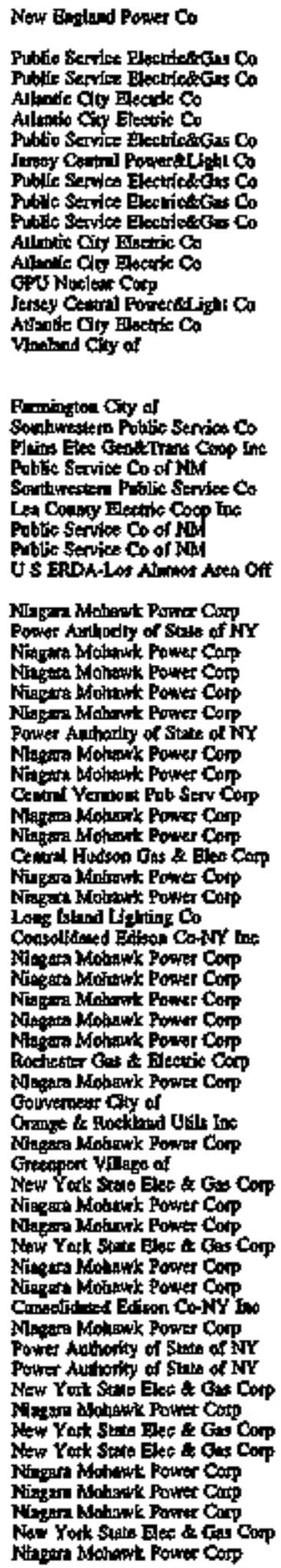 \\
\hline
\end{tabular}

Sex forbatis at und of toble. 
Tabje D2. U.S. Electric Utifty PJarts by State, 1995 (Continued)

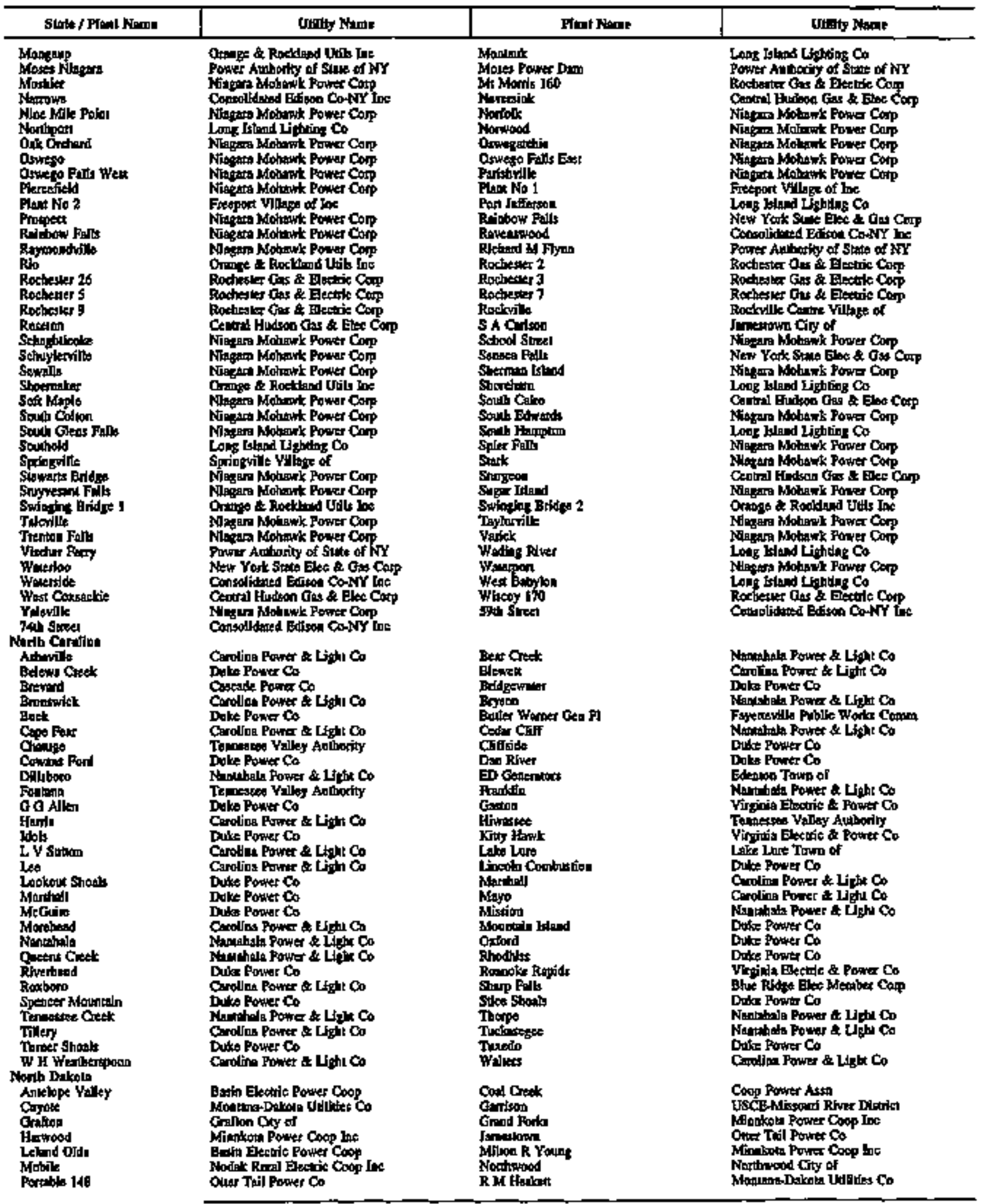

Ses foodooug at and of table. 
Tabje D2. U.S. Electric Utillity Plants by State, 1995 (Continued)

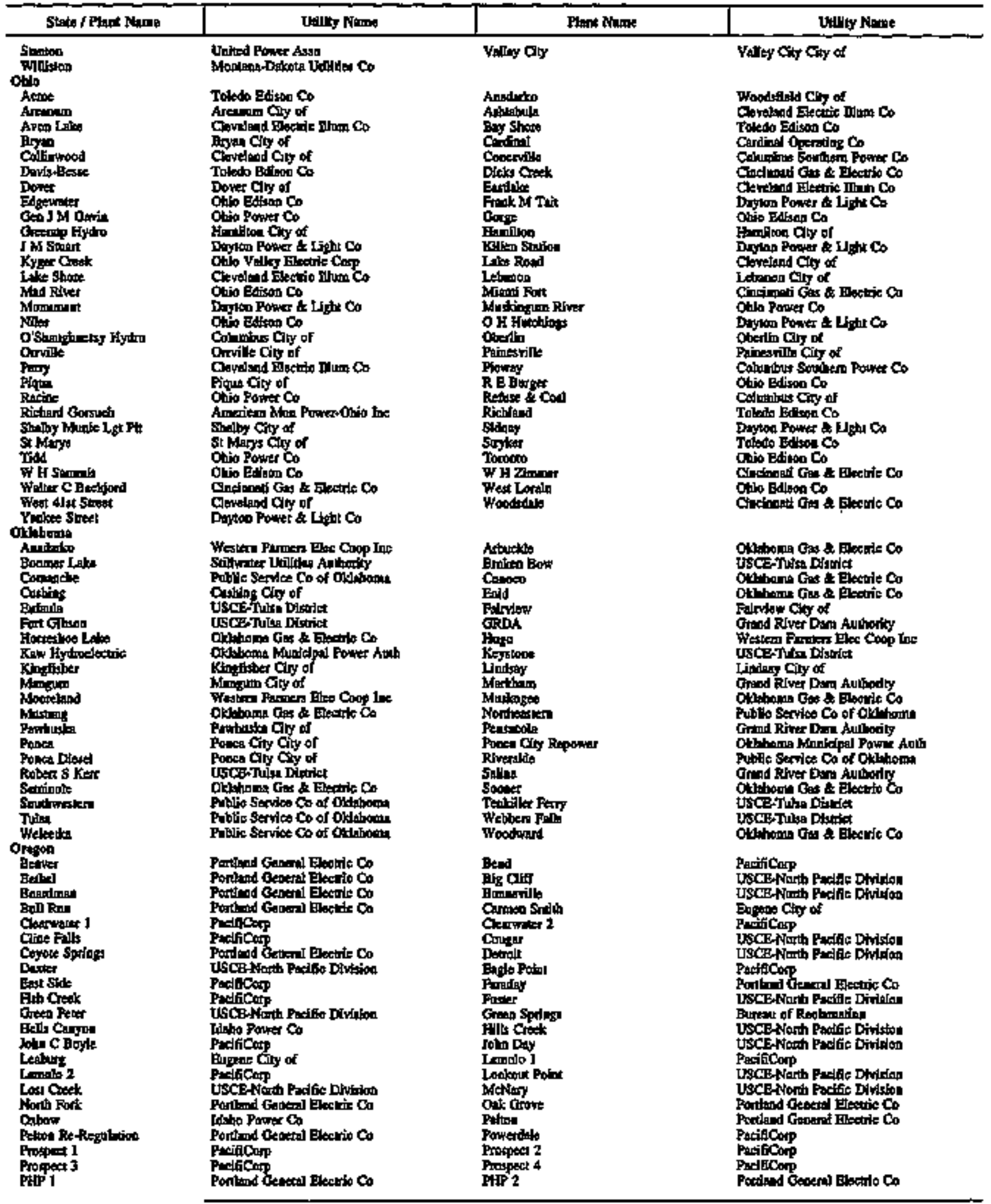

Ses poctoones ar and of table. 
Table D2. U.S. Electric Utility Plants by State, 1995 (Continued)

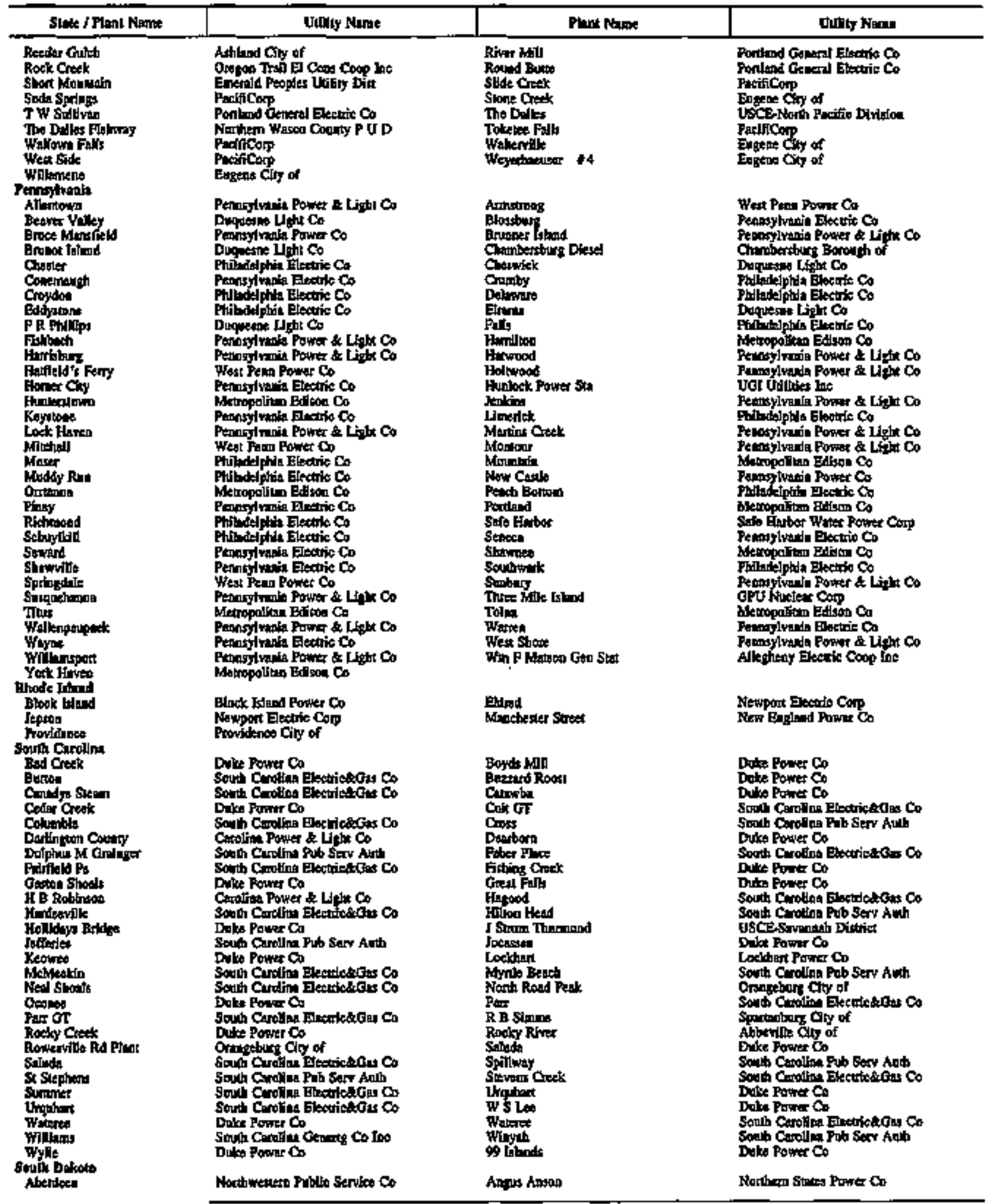

Ses foonow al end of labte. 
Table D2. U.S. Electric Uttility Plants by State, 1995 (Continued)

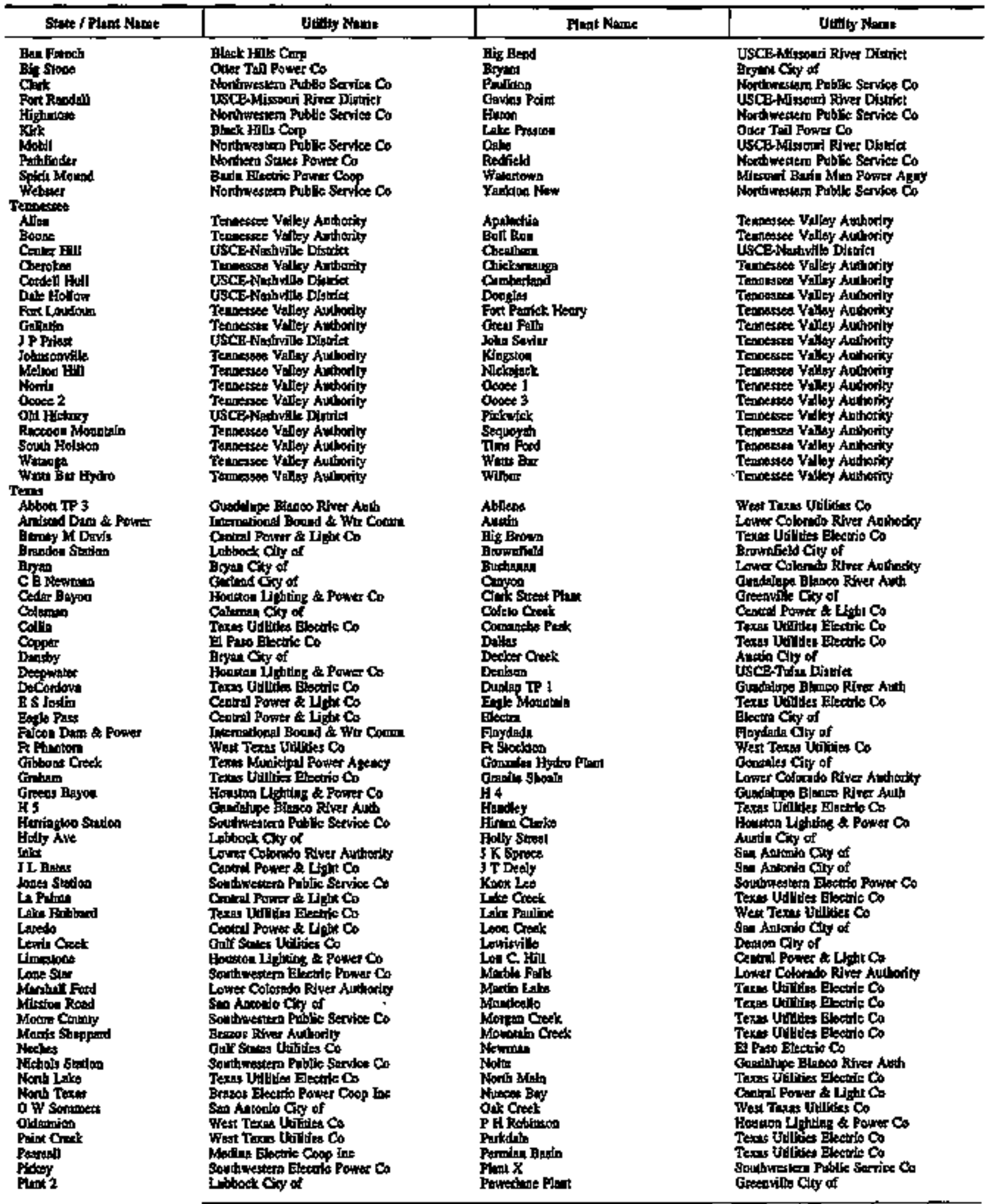

Ste foosotes al ead of tabk. 
Tahle D2. U.S. Electric Ufflity Pants by State, 1995 (Contimmed)

\begin{tabular}{|c|c|c|c|}
\hline Sick / Finnt Name & Utilty Nane & Pant Nure & 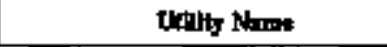 \\
\hline 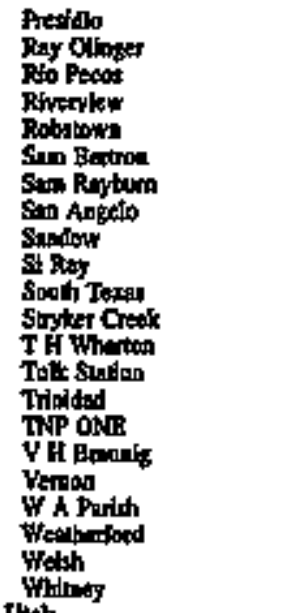 & 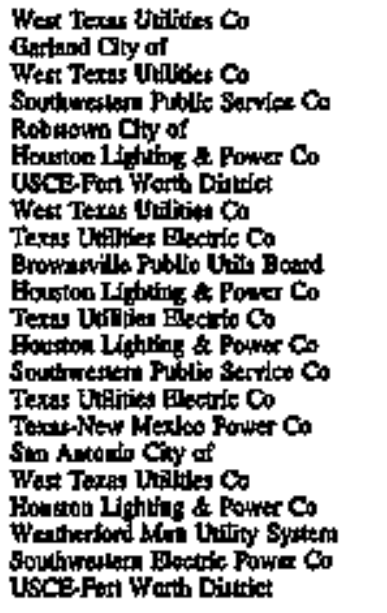 & 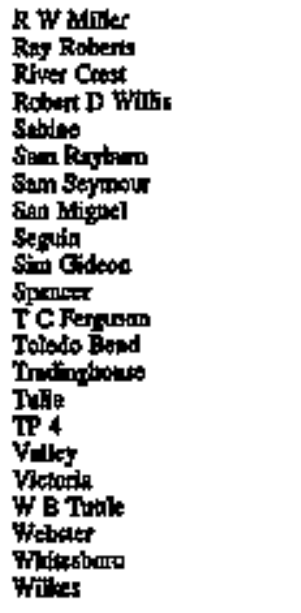 & 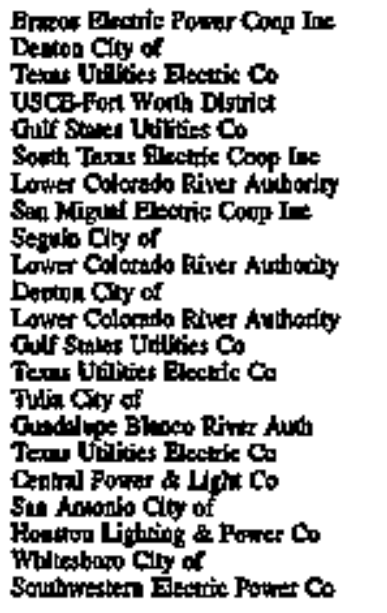 \\
\hline 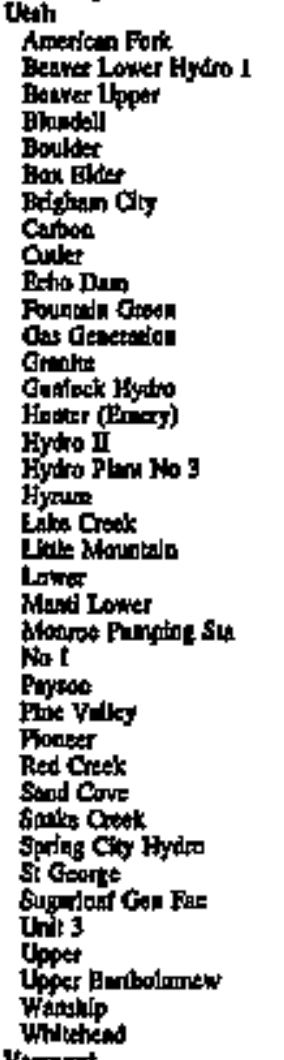 & 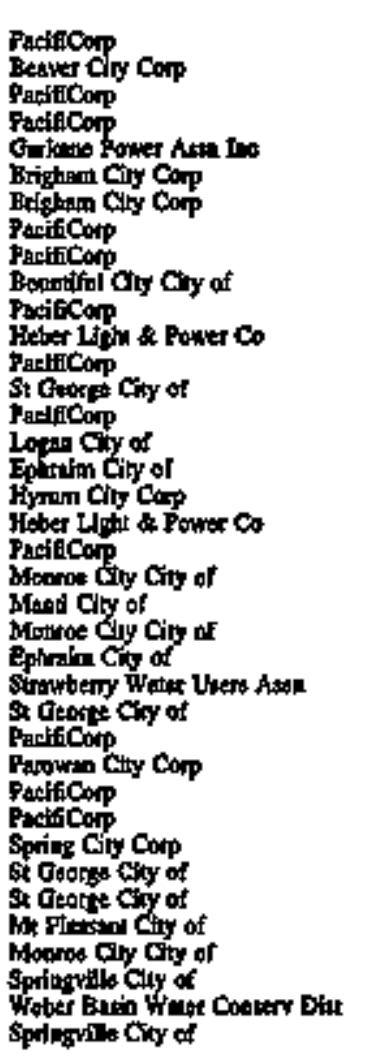 & 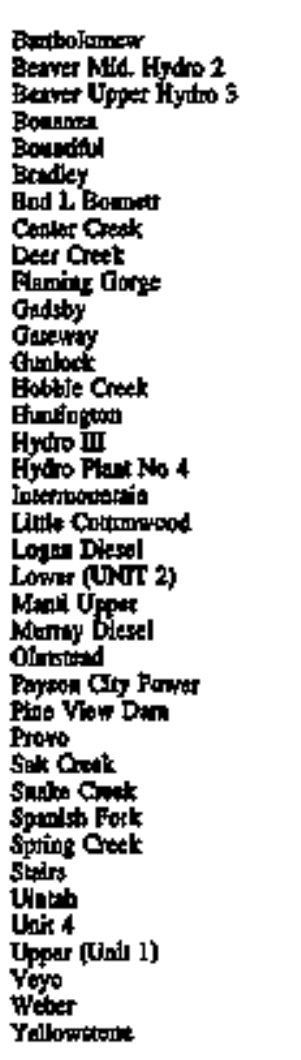 & 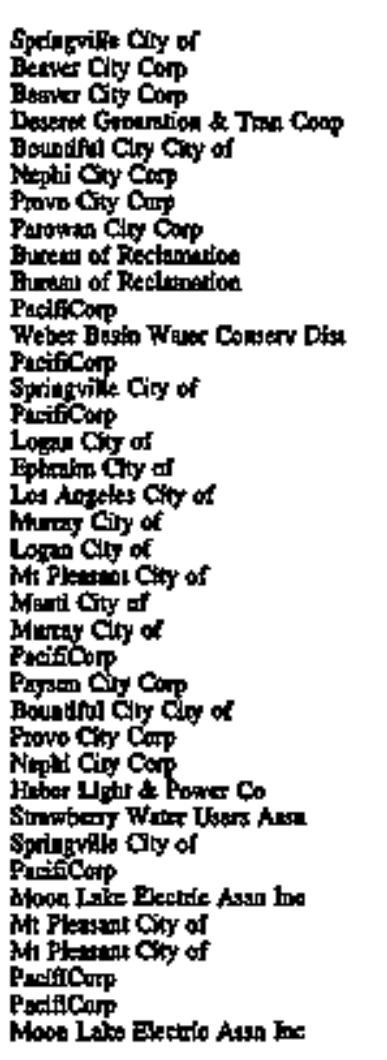 \\
\hline 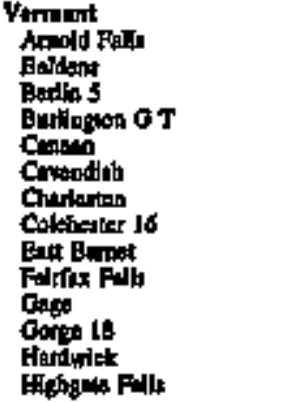 & 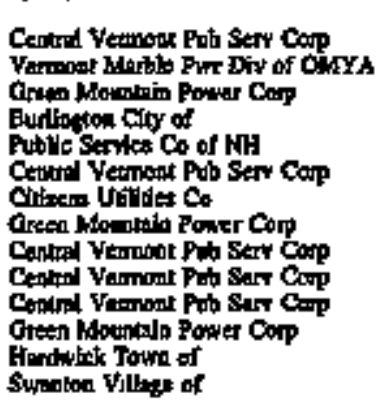 & 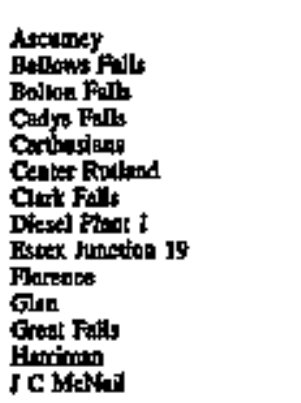 & 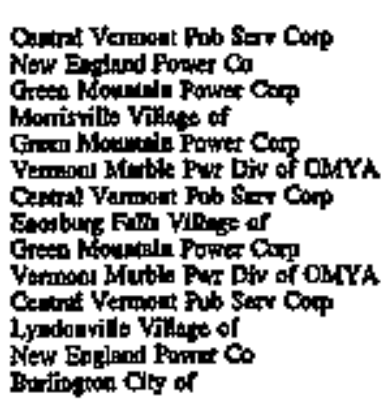 \\
\hline
\end{tabular}

Sea footnifue ul eod of ubla. 
Table D2. U.S. Electric Utility Plants by State, 1995 (Continued)

\begin{tabular}{|c|c|c|c|}
\hline Stalt I Phat Nane & Dulthy Nome & Plank Phan & Utrity Neme \\
\hline 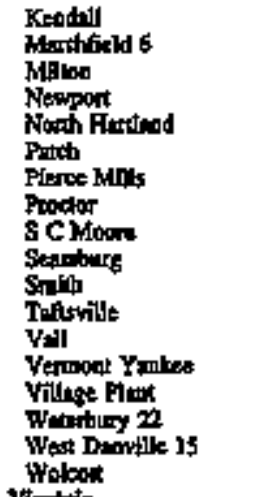 & 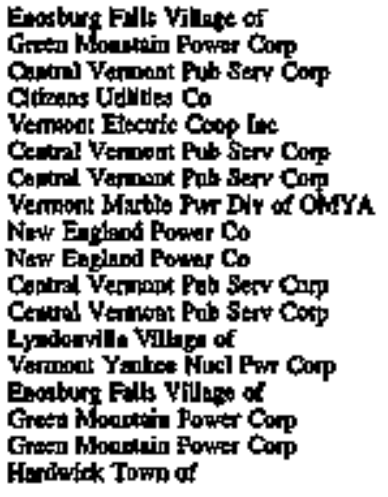 & 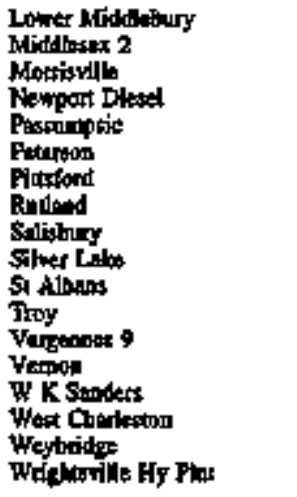 & 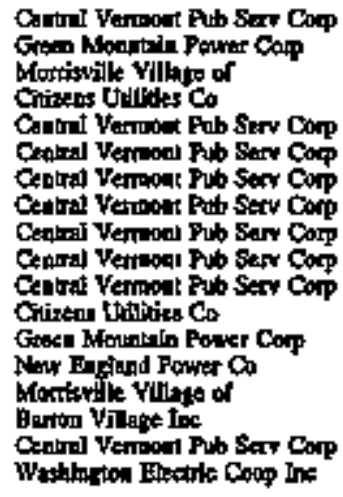 \\
\hline 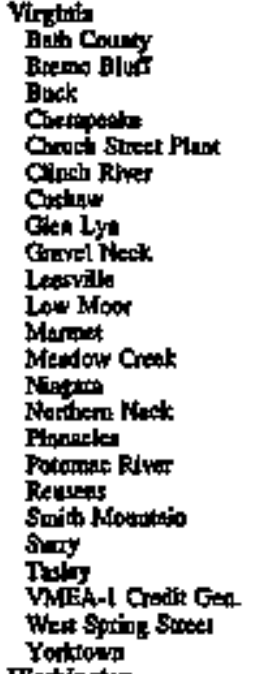 & 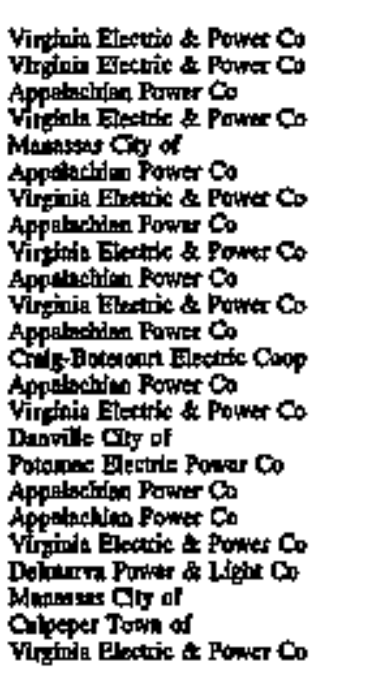 & 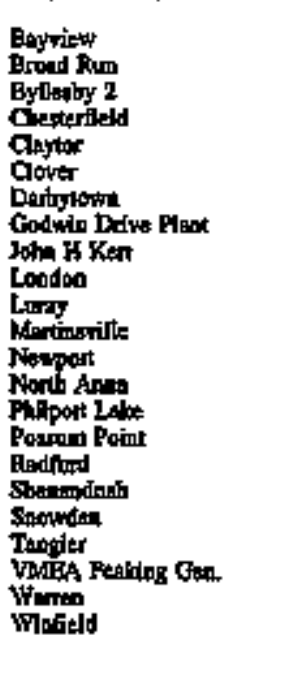 & 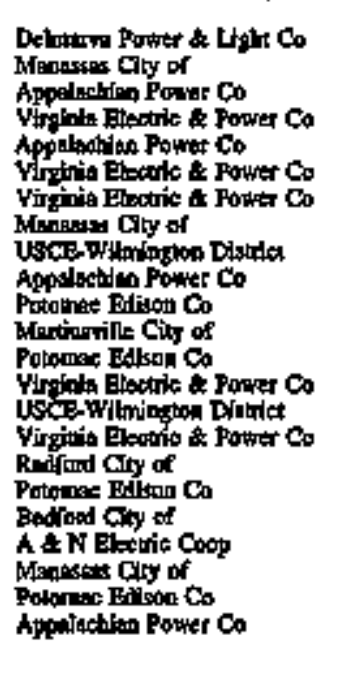 \\
\hline 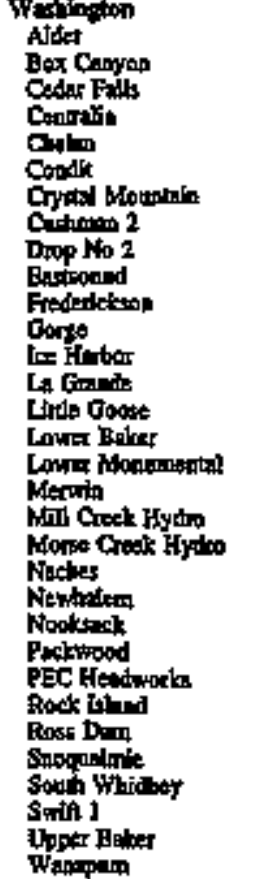 & 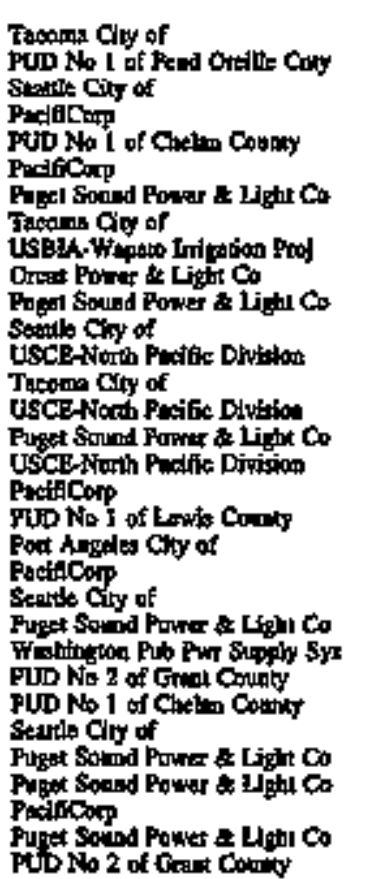 & 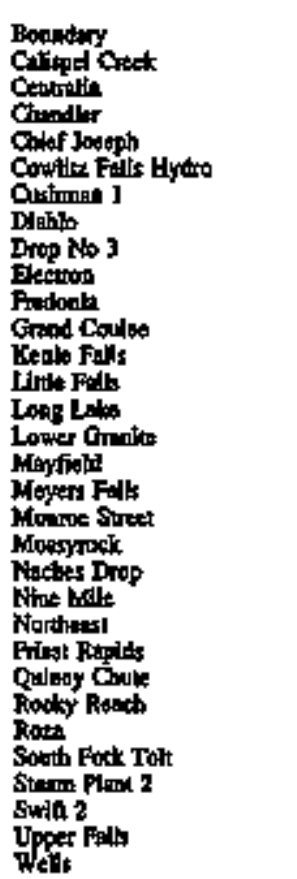 & 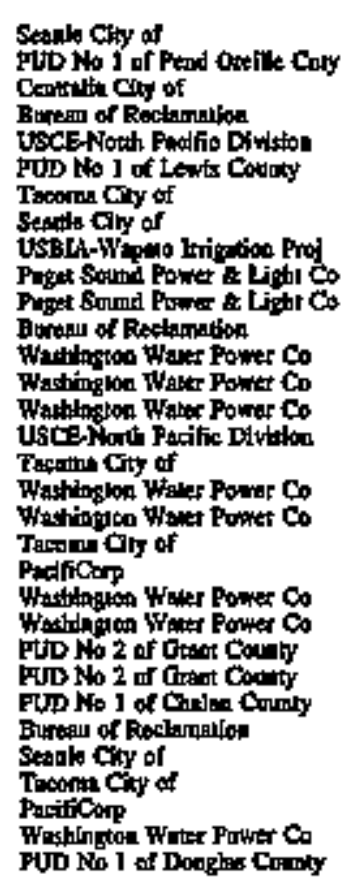 \\
\hline
\end{tabular}

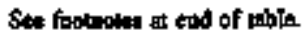


Tahle D2. U.S. Electric Utility Flants by State, 1998 (Continued)

\begin{tabular}{|c|c|c|c|}
\hline Sleta / Ptork Nime & Lutly Noter & Pland the & Unthts \\
\hline 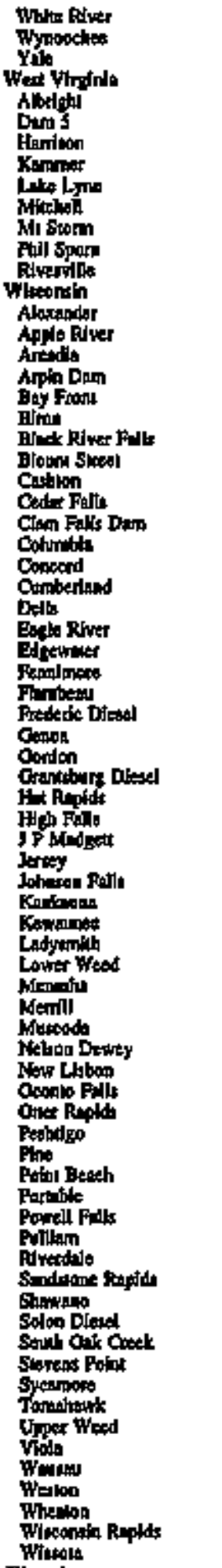 & 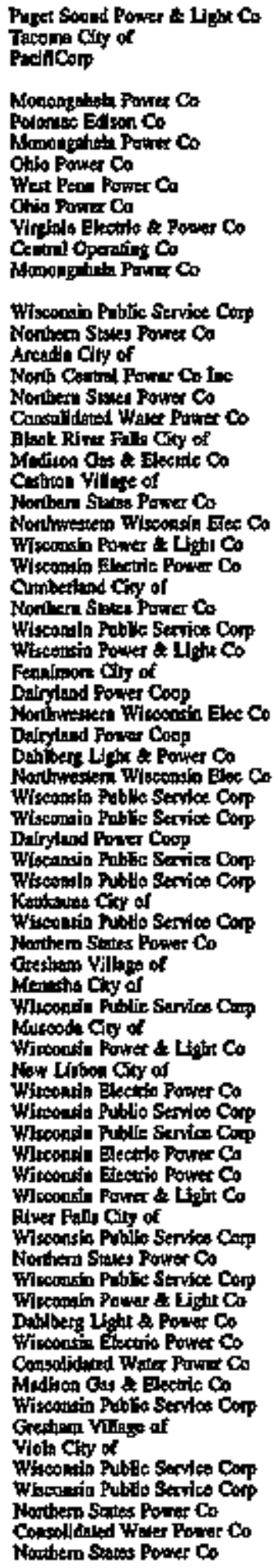 & 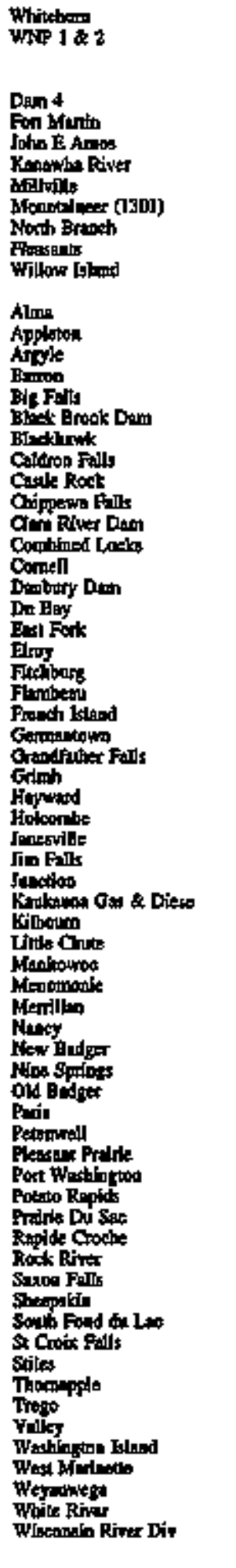 & 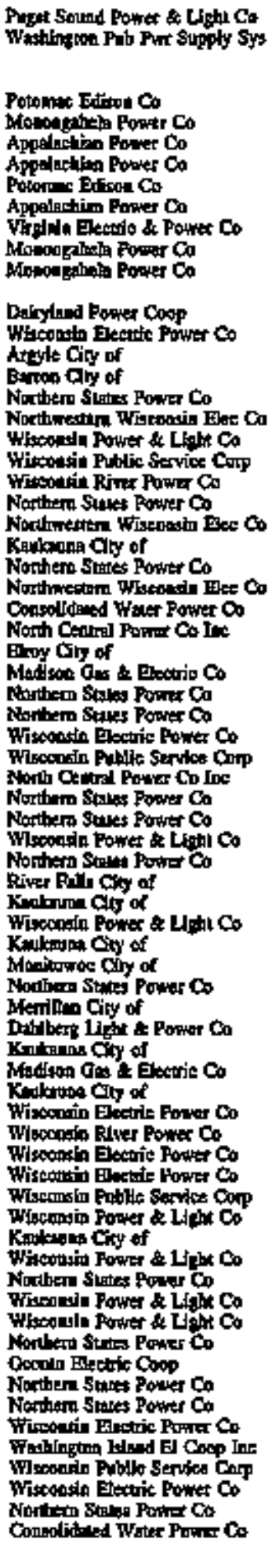 \\
\hline & Euram of Rrellat ation & Bo,titon & Buten of Reclarstion \\
\hline
\end{tabular}

See foonones al end of able. 
Table D2. U.S. Electric Utility Plants by State, 1995 (Continued)

\begin{tabular}{|c|c|c|c|}
\hline 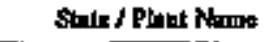 & Uutlky Nome & Pland Name & Dulfy Nom \\
\hline 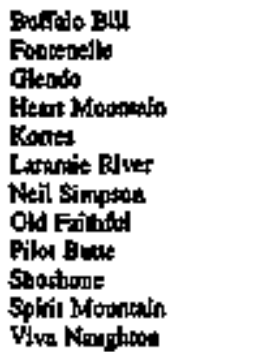 & 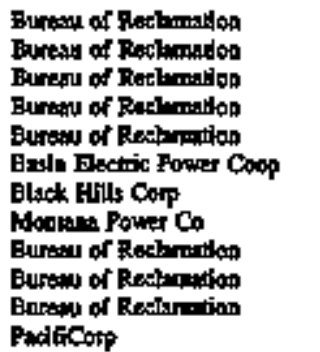 & 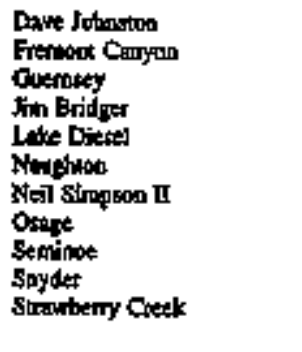 & 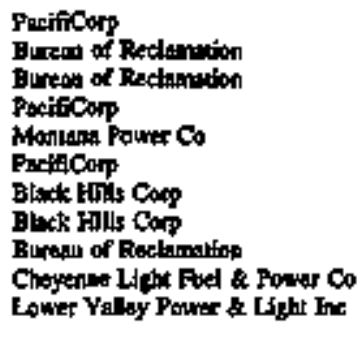 \\
\hline
\end{tabular}

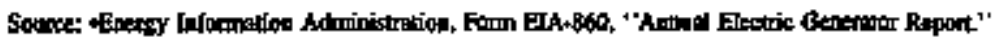


Table DS. US. Electric Ctallity Plants by Utility, 1995

\begin{tabular}{|c|c|c|c|}
\hline Usilky / Pant Nome & Slote & Utilly / Fisol Nant & stote \\
\hline 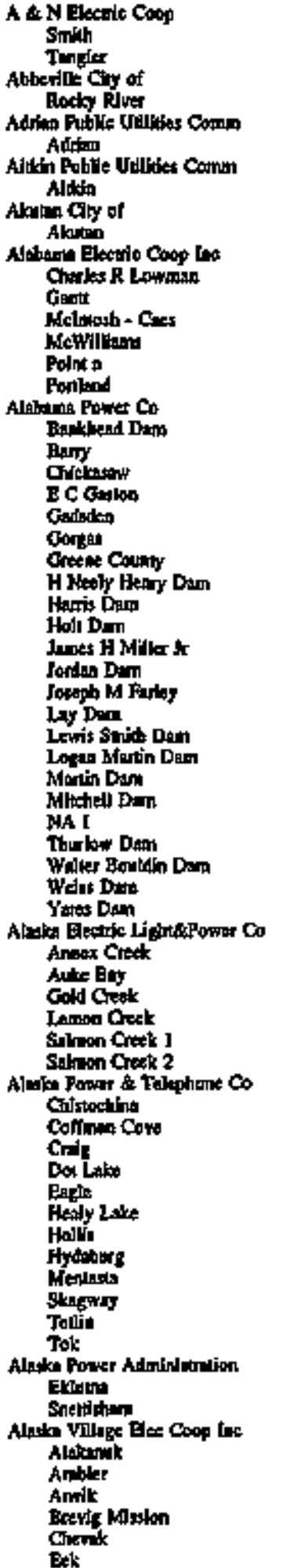 & 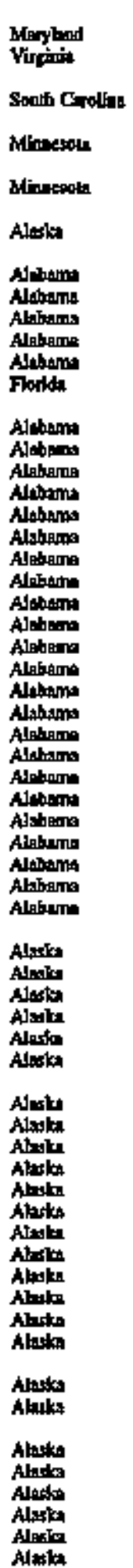 & 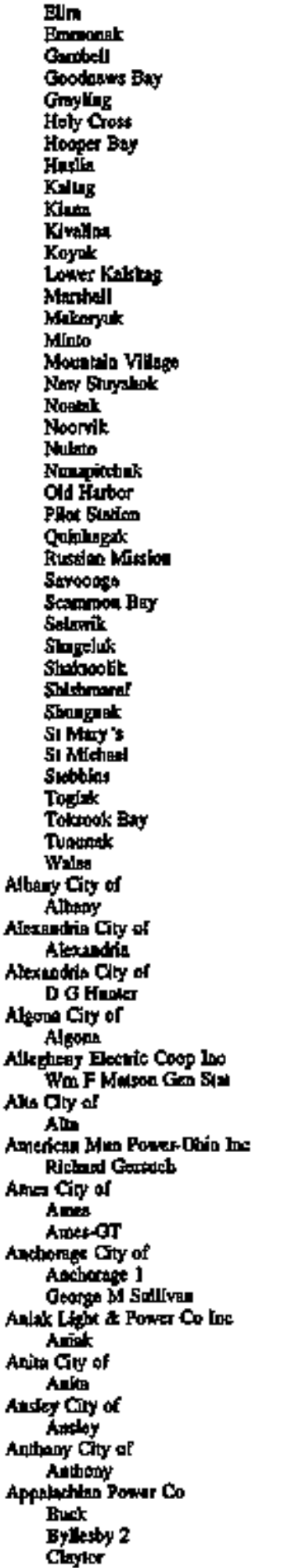 & 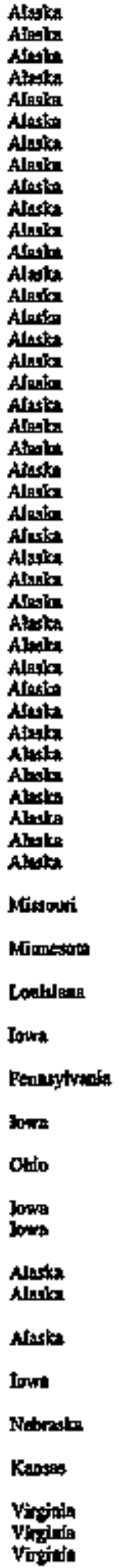 \\
\hline
\end{tabular}

Sec footeolan at ind of thats. 
Tahle D3. U.S. Electrtc Utillty Plants by Utulfty, 1995 (Continued)

\begin{tabular}{|c|c|c|c|}
\hline 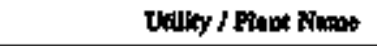 & Sonto & 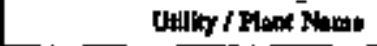 & Siote \\
\hline 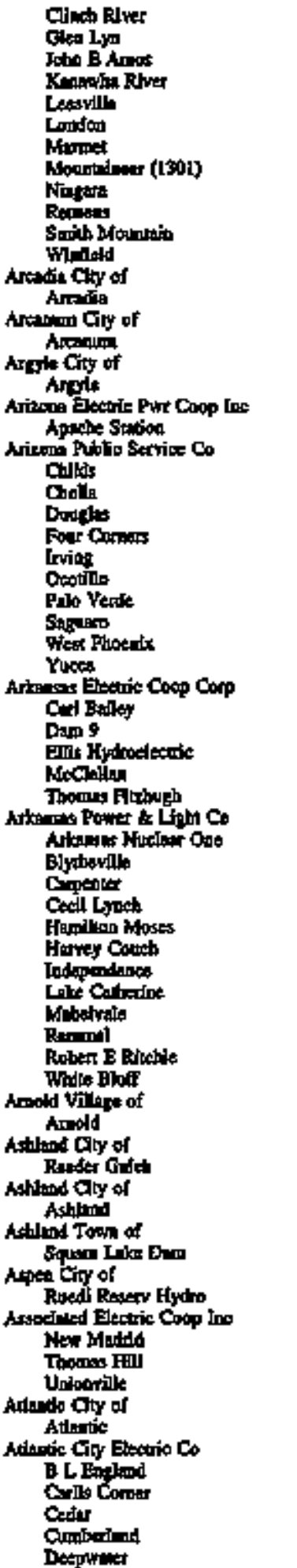 & 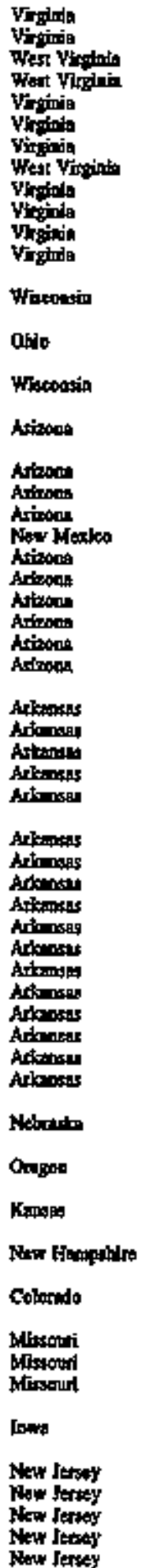 & 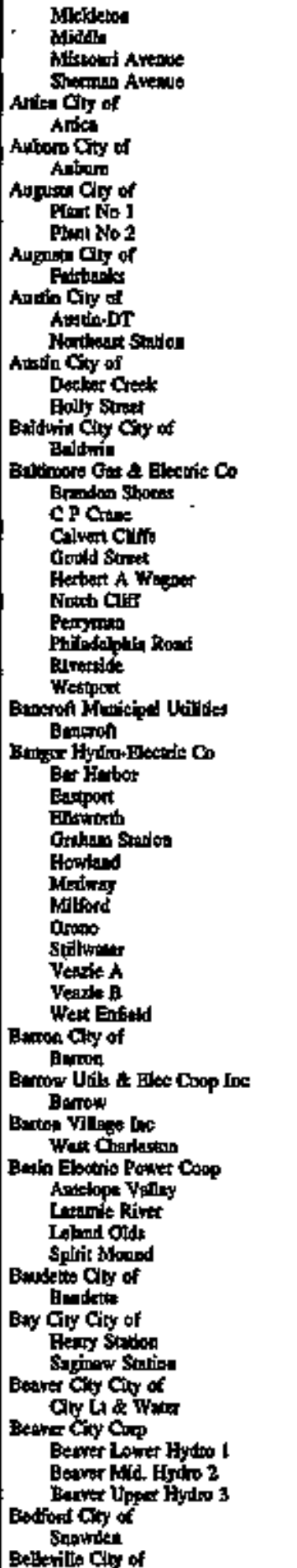 & 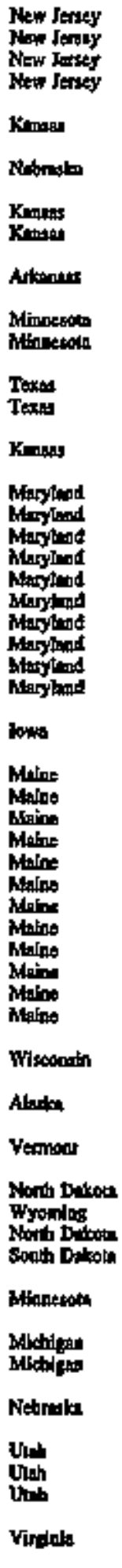 \\
\hline
\end{tabular}

Ses fooknow at ond of tholle 
Table D3. U.S. Electric Utility Plants by Utlity, 1995 (Continued)

\begin{tabular}{|c|c|c|c|}
\hline Udulky / Pant Mamt & Slote & Ul'tib I Pant Nathe & Slot: \\
\hline 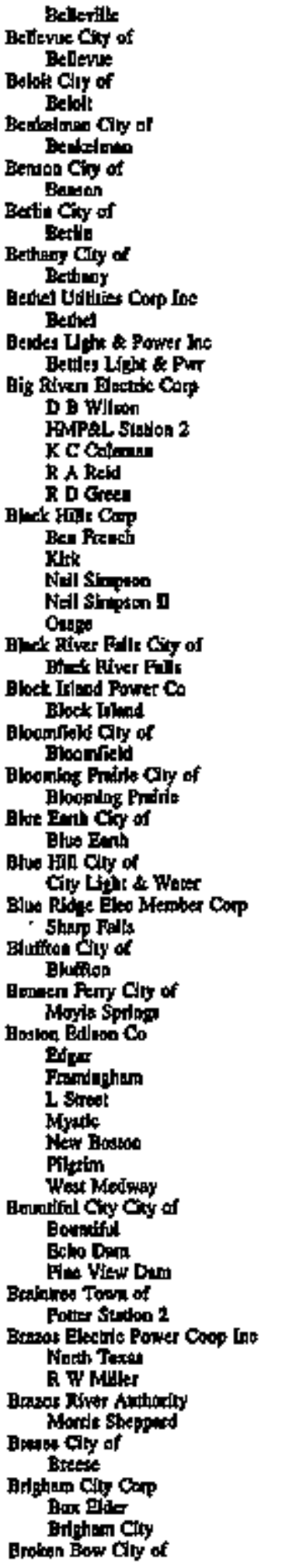 & 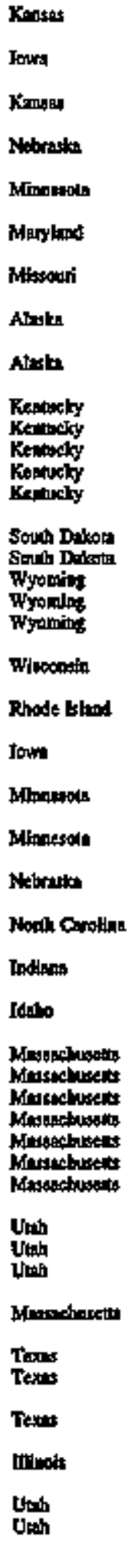 & 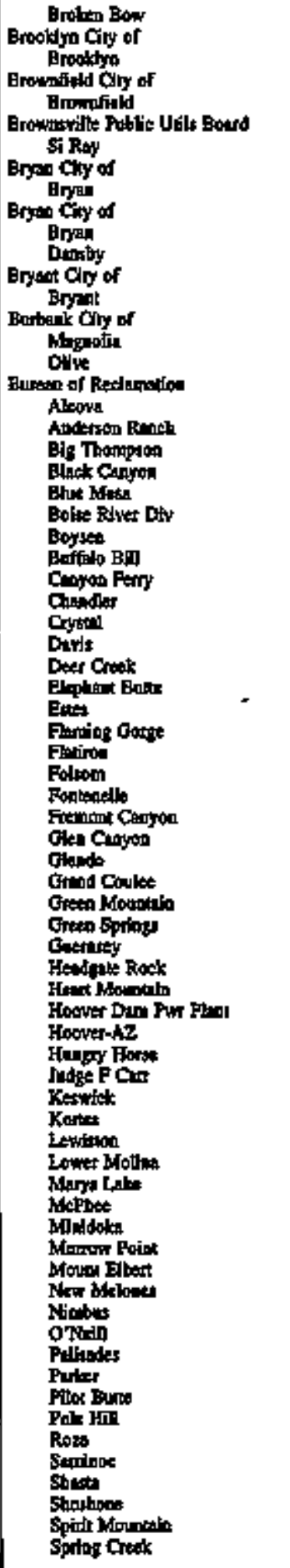 & 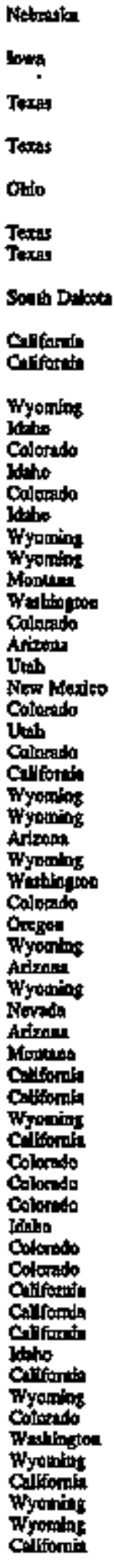 \\
\hline
\end{tabular}

Set frotholed al end of tablo. 
Table D3. U.S. Electric Utlity Plants by Utllty, 1995 (Continued)

\begin{tabular}{|c|c|c|c|}
\hline tullky / Mant Nhme & Btats & Lutiley / Ptant Name & Sinte \\
\hline 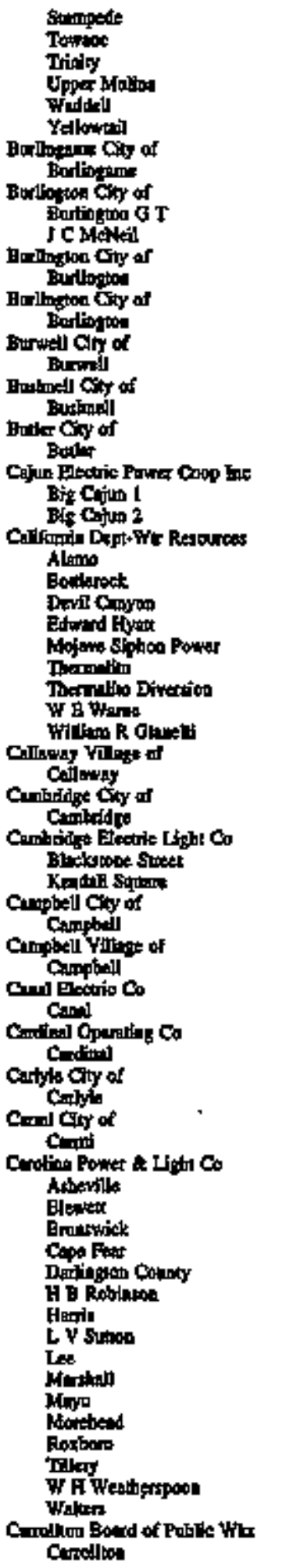 & 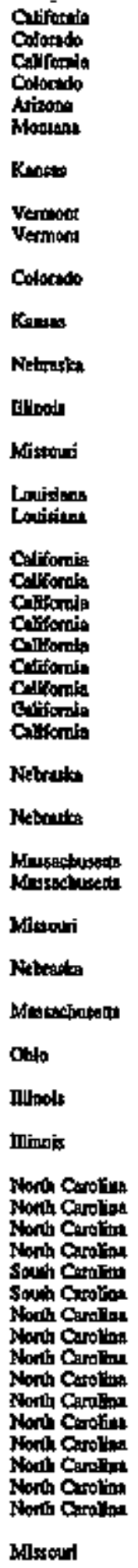 & 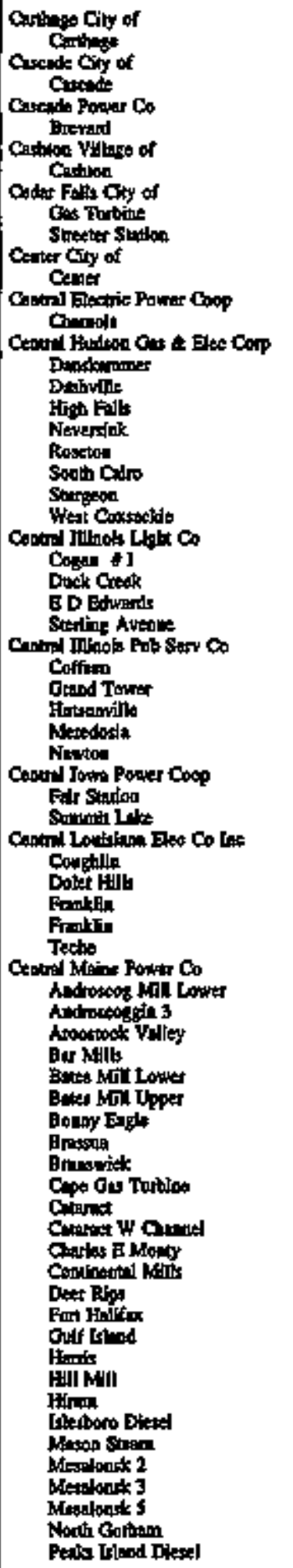 & 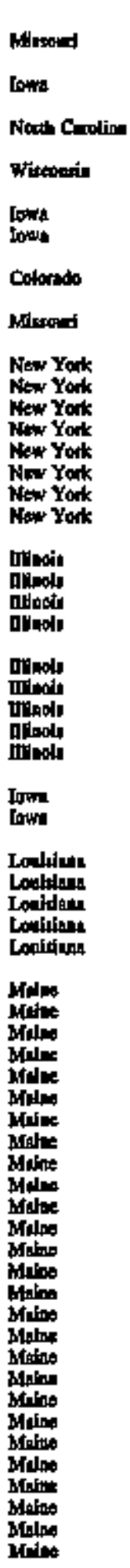 \\
\hline
\end{tabular}

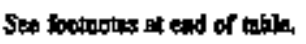


Table D3. U.S. Eledtric Utility Plants by Utility, 1995 (Contisued)

\begin{tabular}{|c|c|c|c|}
\hline Dally / Plane Xtane & Stet: & Utlity I Plant Nima & Soit \\
\hline 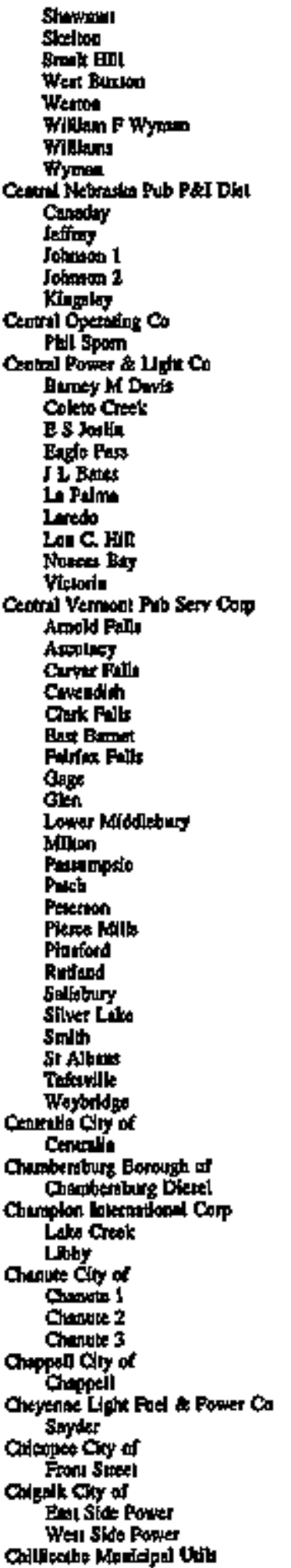 & 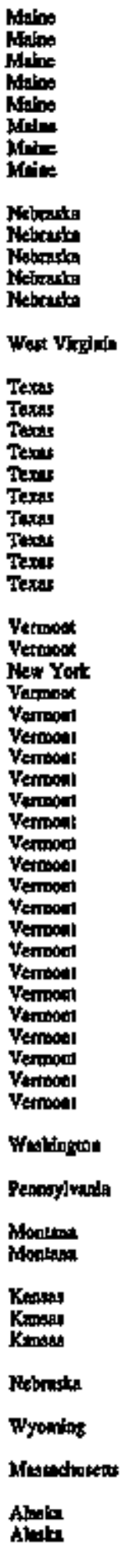 & 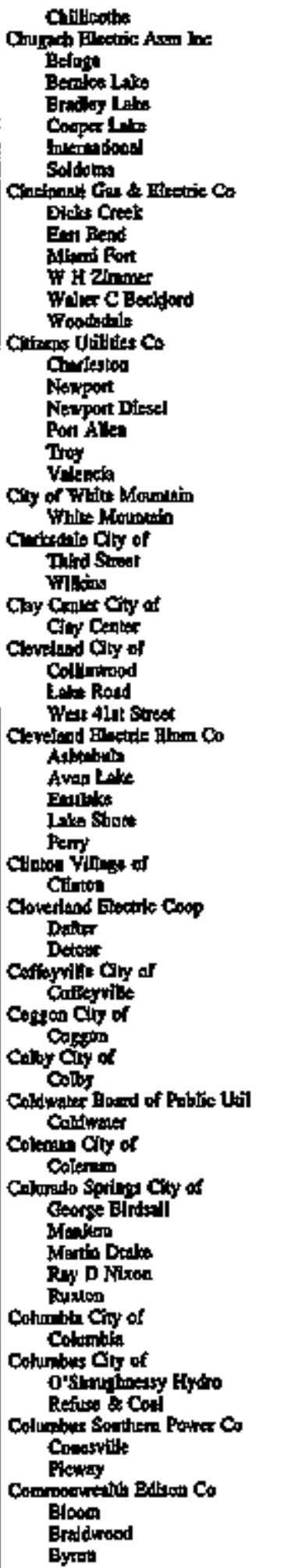 & 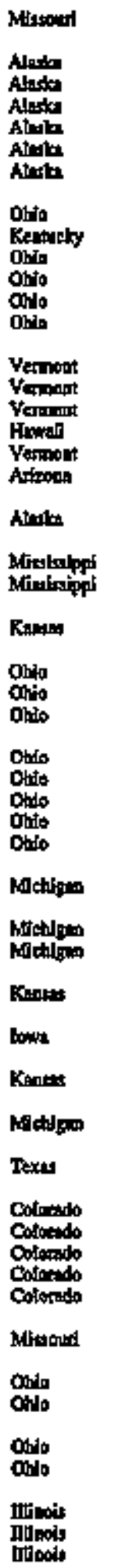 \\
\hline
\end{tabular}

Sec foctnoliet at end of tobla. 
Tohle D5. U.S. Electric Utility Pints by Utility, 1995 (Confinued)

\begin{tabular}{|c|c|c|c|}
\hline Uutis / Flant None & flente & Dithy / Ptunt Nomo & State \\
\hline 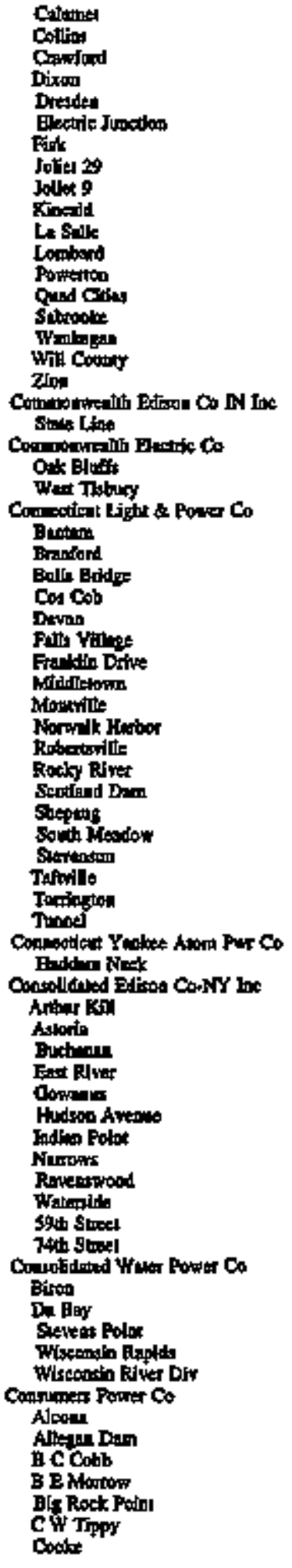 & 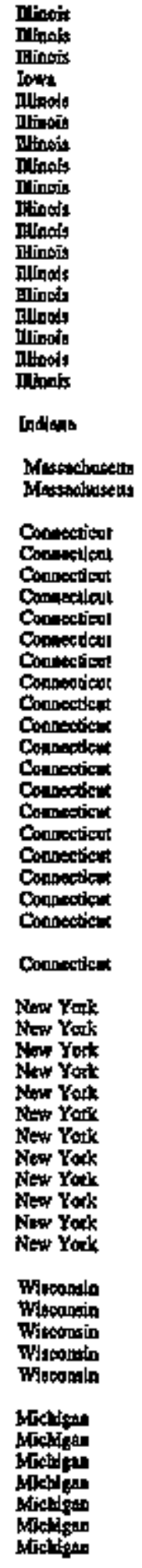 & 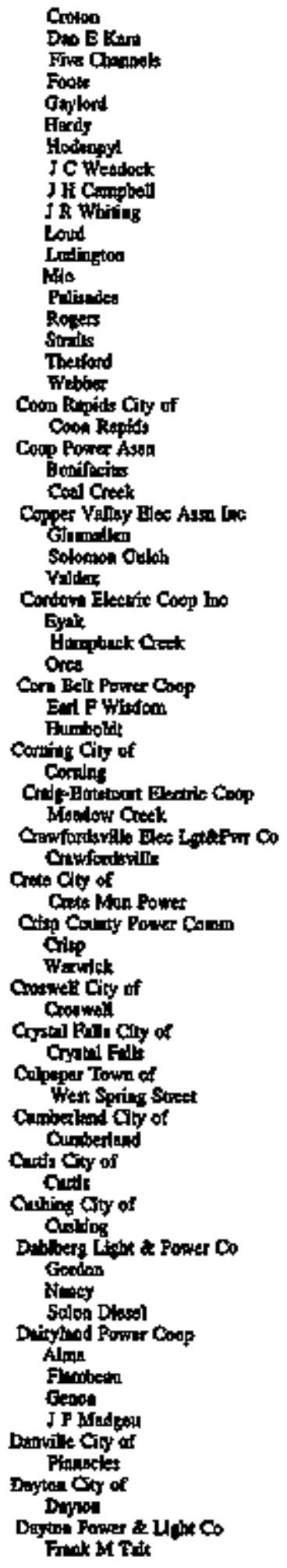 & 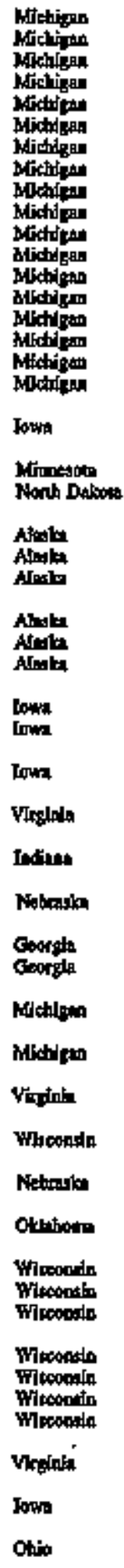 \\
\hline
\end{tabular}

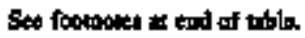


Table D3. U.S. Electric Utillty Plants by Utillty, 1995 (Conlinned)

\begin{tabular}{|c|c|c|c|}
\hline Udil's / Pant Mas & Sonte & Utiliky / Plonk Nobt & Staten \\
\hline 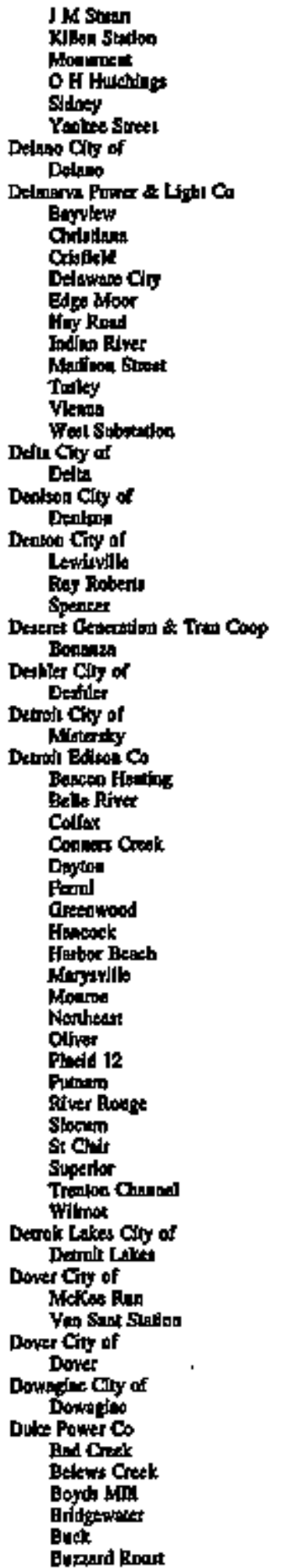 & 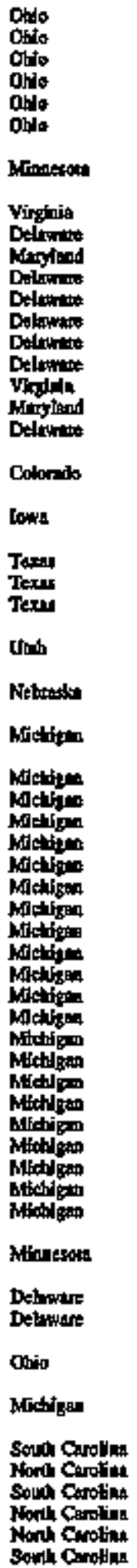 & 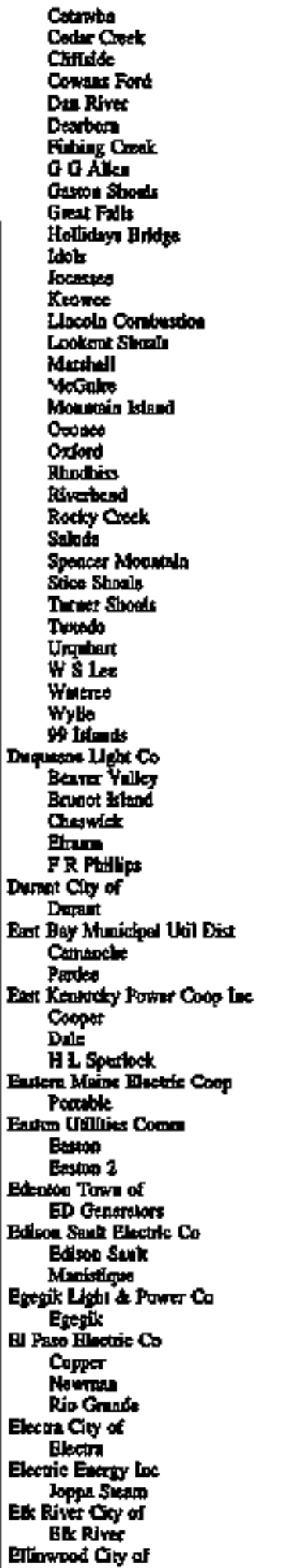 & 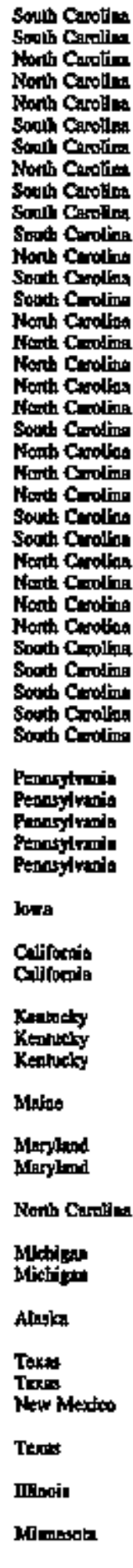 \\
\hline
\end{tabular}

Ses foodpontat ar end of whlo 
Table D3. US. Flectric Utility Plants by Utility, 1995 (Continued)

\begin{tabular}{|c|c|c|c|}
\hline Dently / Frent Nome & 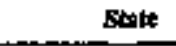 & Lorify / Fant tous & storts \\
\hline 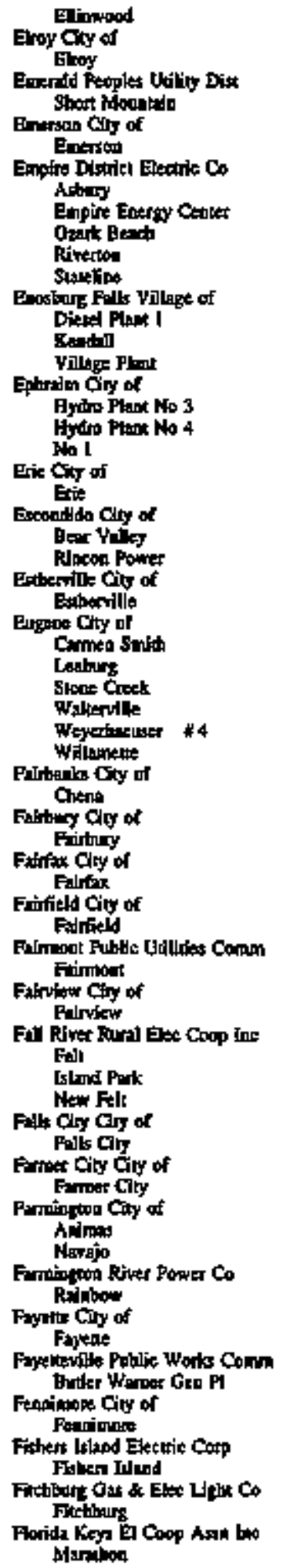 & 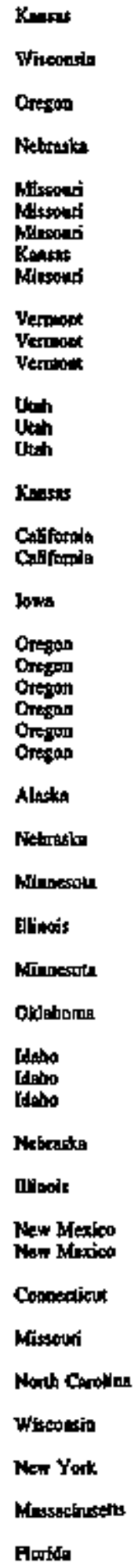 & 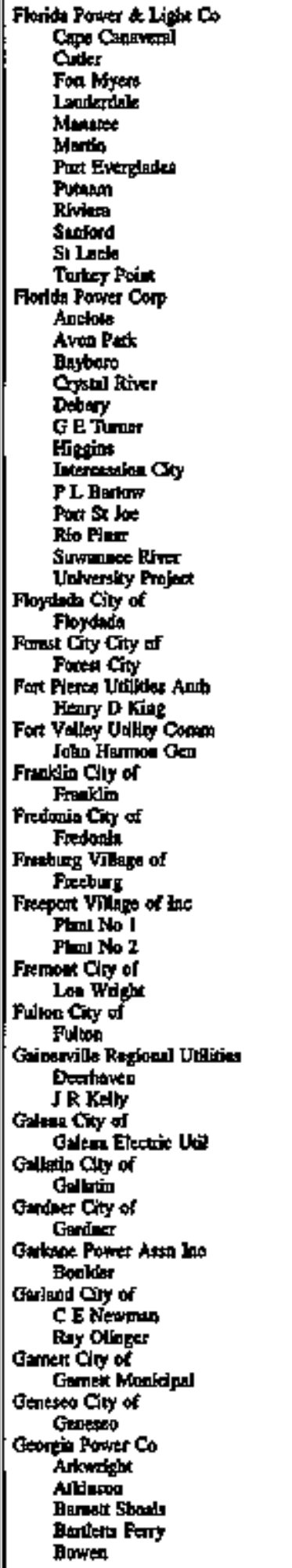 & 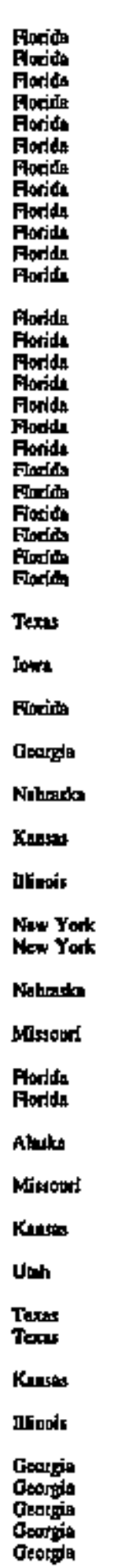 \\
\hline
\end{tabular}

Sed foowones at and of labk. 
Table D3, U.S. Electric Utility Plants by Utility, 1995 (Continued)

\begin{tabular}{|c|c|c|c|}
\hline Wully f Pland Nant & Sinte & 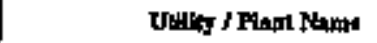 & Sieve \\
\hline 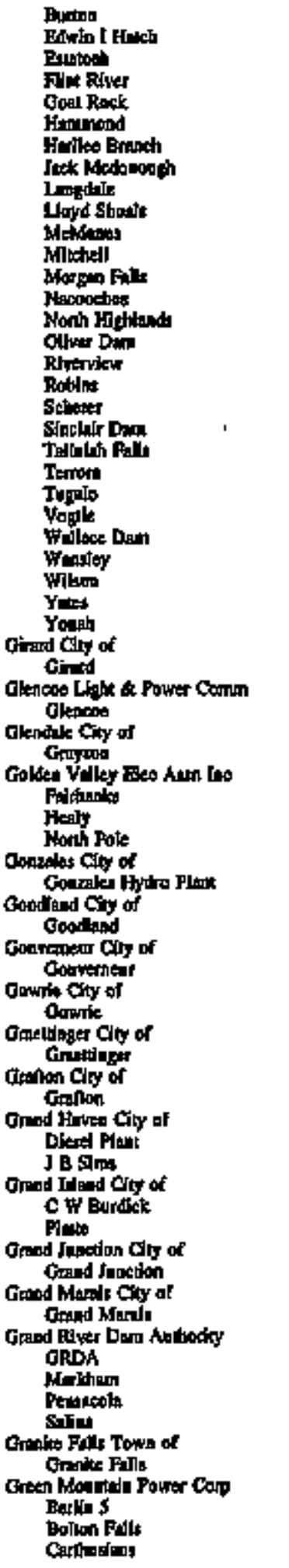 & 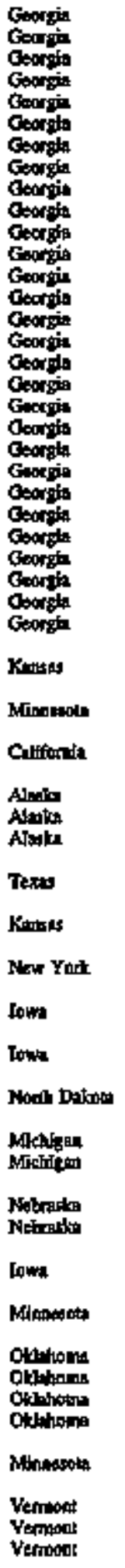 & 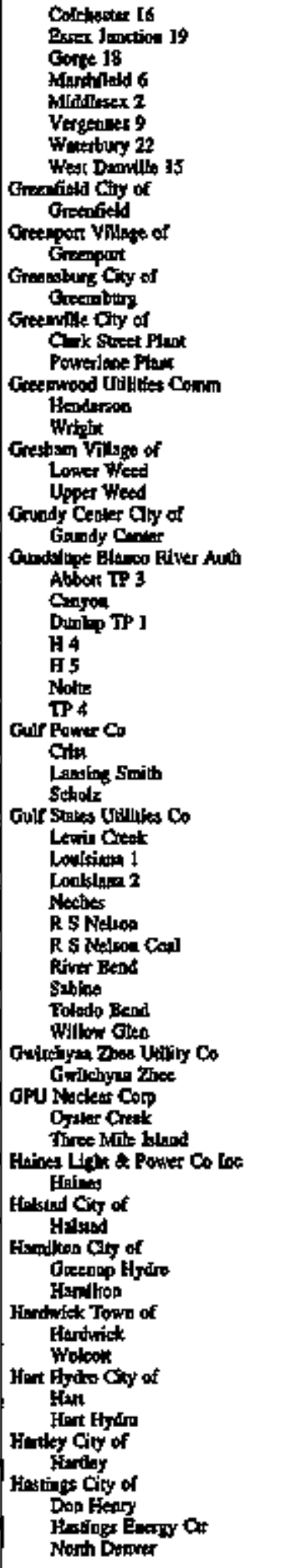 & 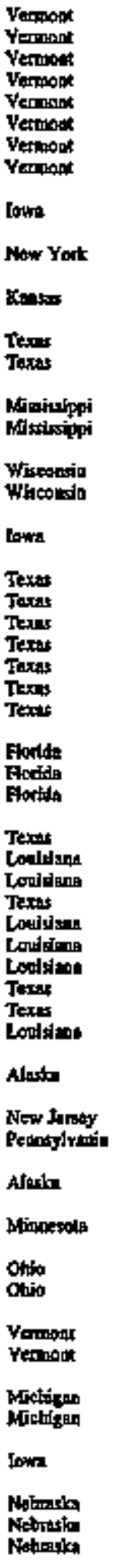 \\
\hline
\end{tabular}

Set froolnows nt and of thots. 
Table D3. U.S. Electric Utility Plants by Utitity, 1995 (Contimeed)

\begin{tabular}{|c|c|c|c|}
\hline Udilty / Ftonl Ntame & Stele & Uallity / Ftant Klane & since \\
\hline 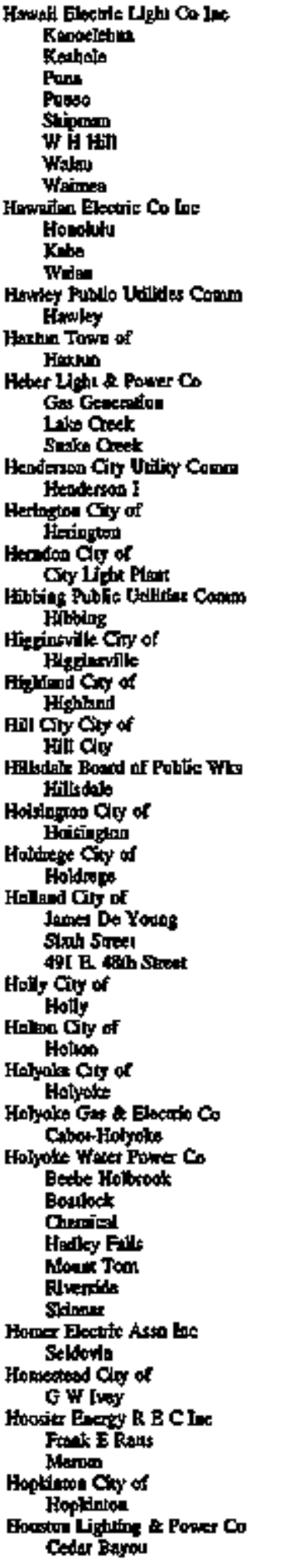 & 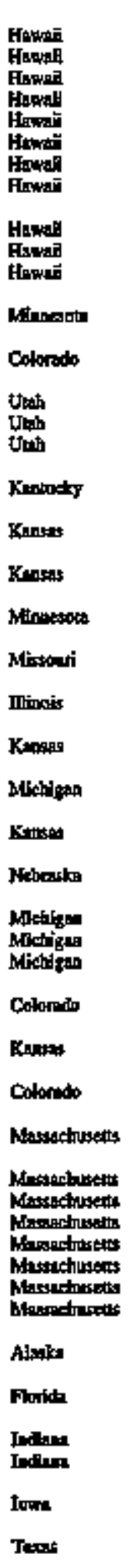 & 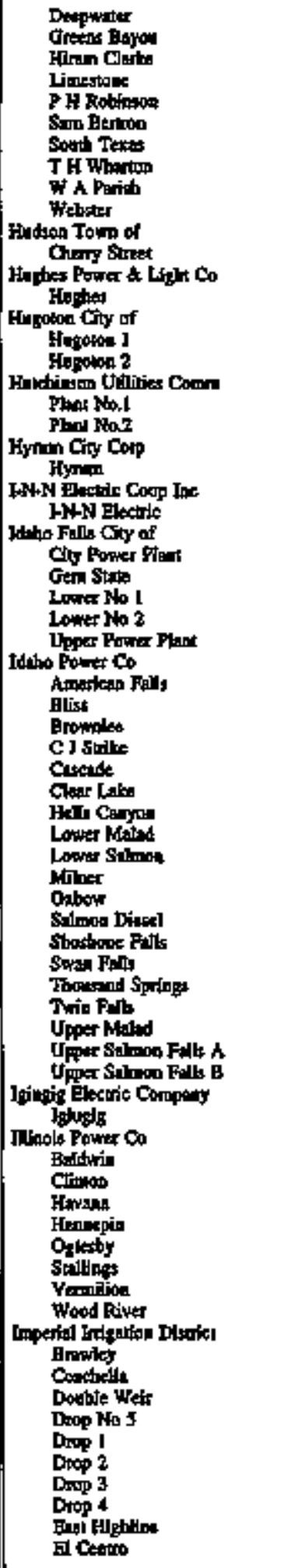 & 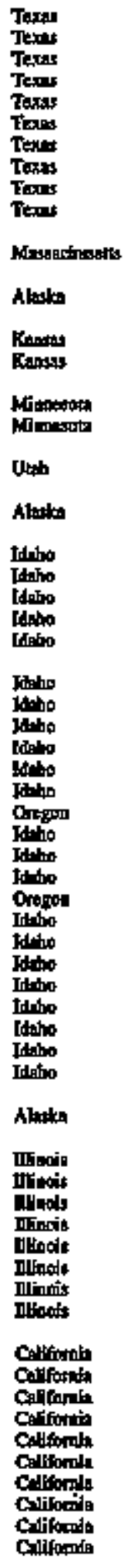 \\
\hline
\end{tabular}

Ses footoots al and of table 
Table D9. U.S. Electric Utility Planass by Utilfty, 1995 (Contioued)

\begin{tabular}{|c|c|c|c|}
\hline Uallby / Pions Nane & Slove & Utilig / Pant Nane & Stote \\
\hline 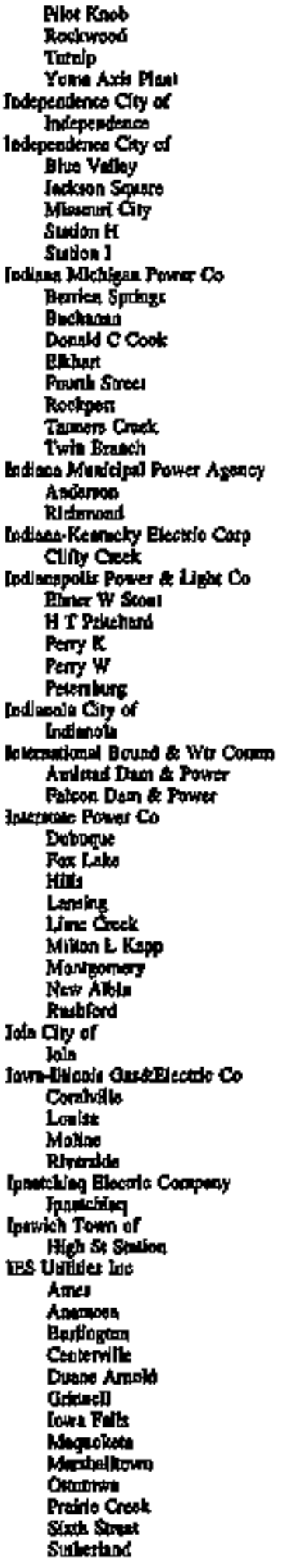 & 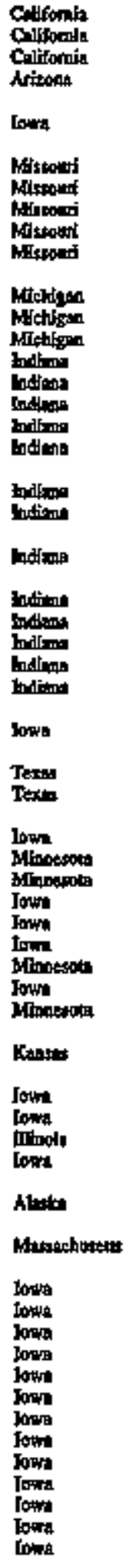 & 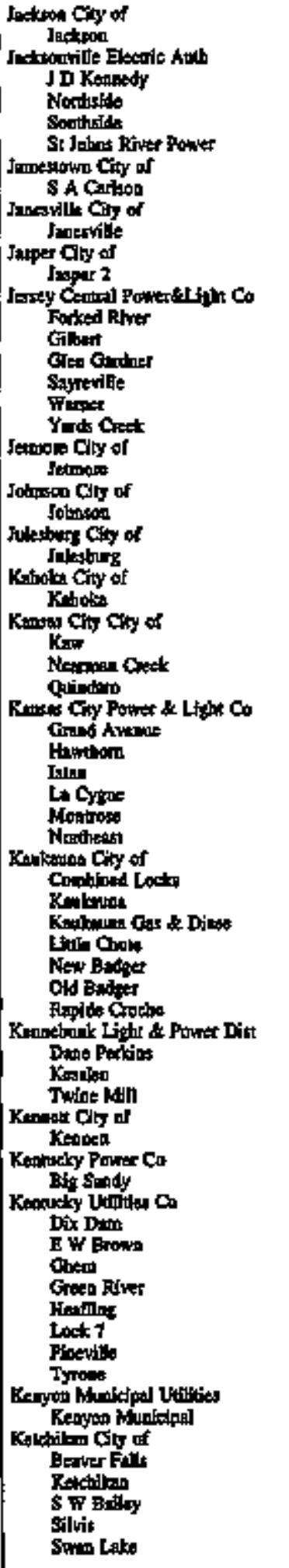 & 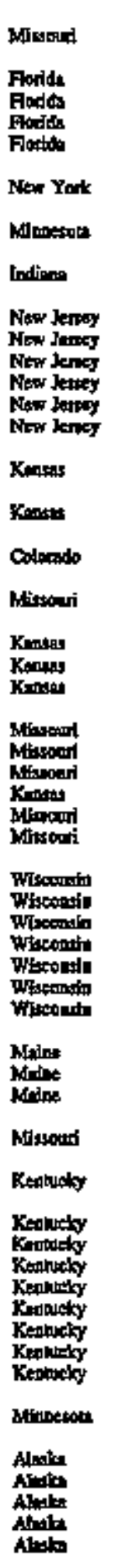 \\
\hline
\end{tabular}

Sed footmolat at end of ullthe 
Table D3. U.S. Electric Utility Flants by Utility, 1995 (Continued)

\begin{tabular}{|c|c|c|c|}
\hline Ddliby / Plant Nhe & State & Ulitiky / Ptapt Nomo & Shatp \\
\hline 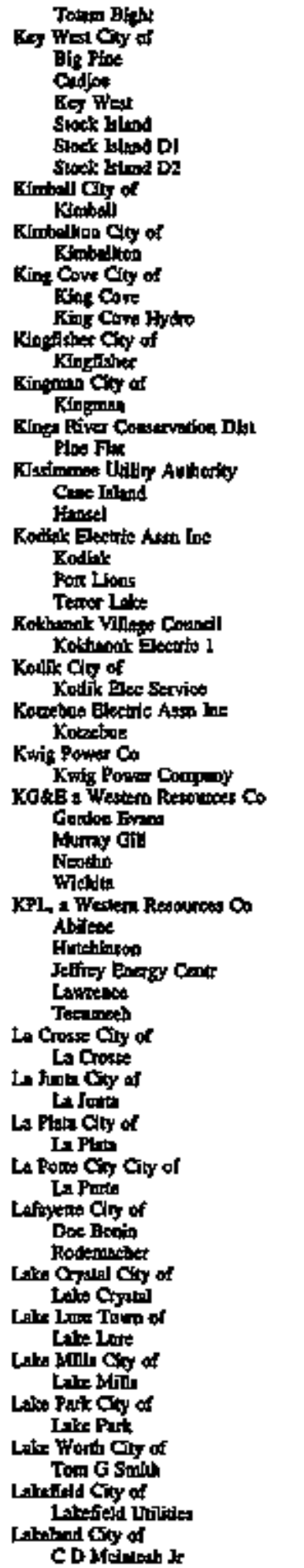 & 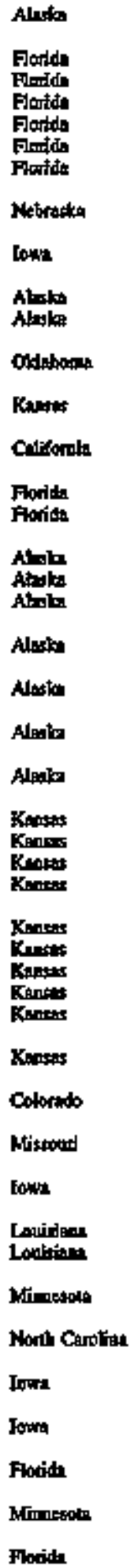 & 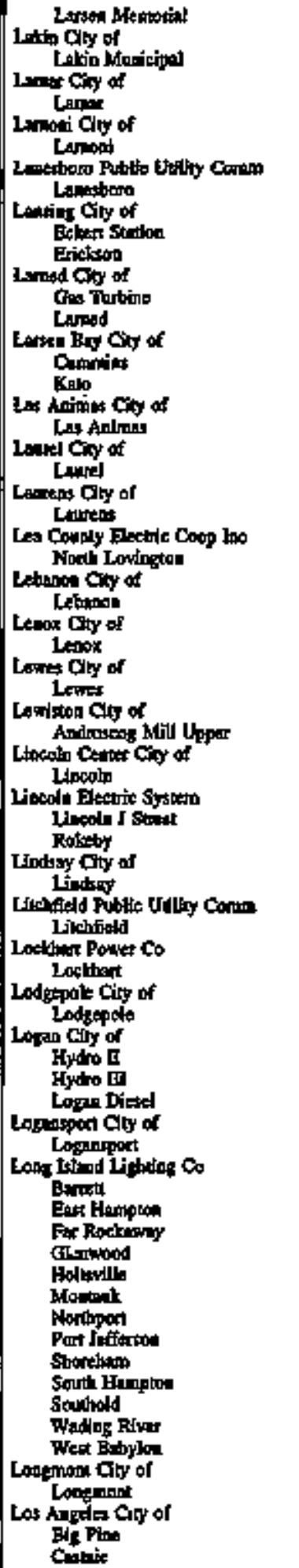 & 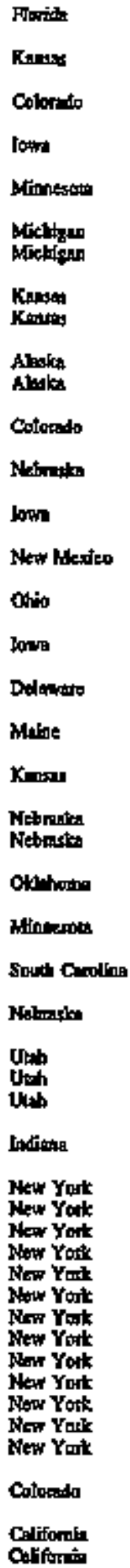 \\
\hline
\end{tabular}

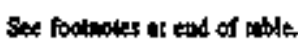


Tahle D3. US. Electric Ut"lity Plants by Utlity, 1995 (Continued)

\begin{tabular}{|c|c|c|c|}
\hline Oultity / Fisad None & Stante & UIW's I Fant Name & stale \\
\hline 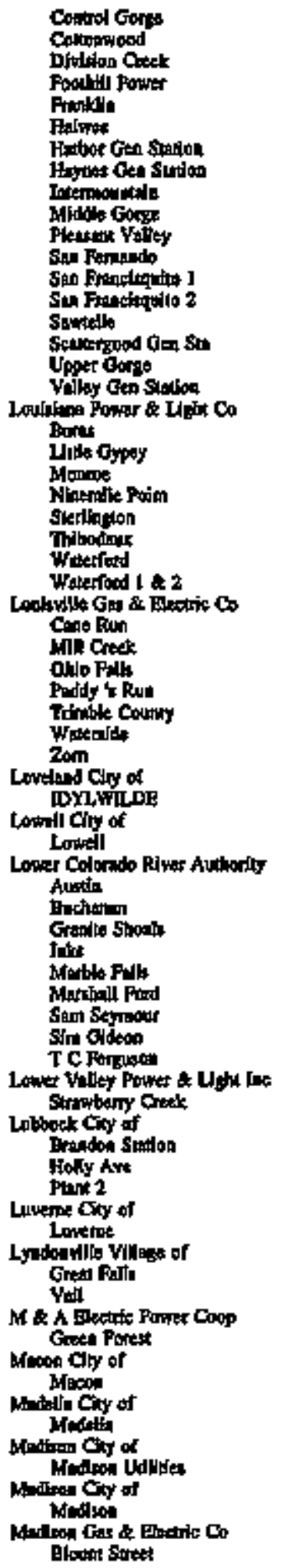 & 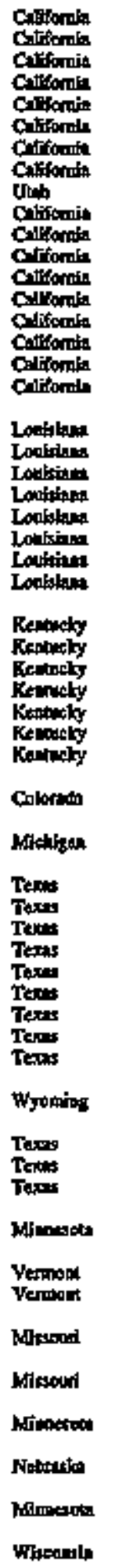 & 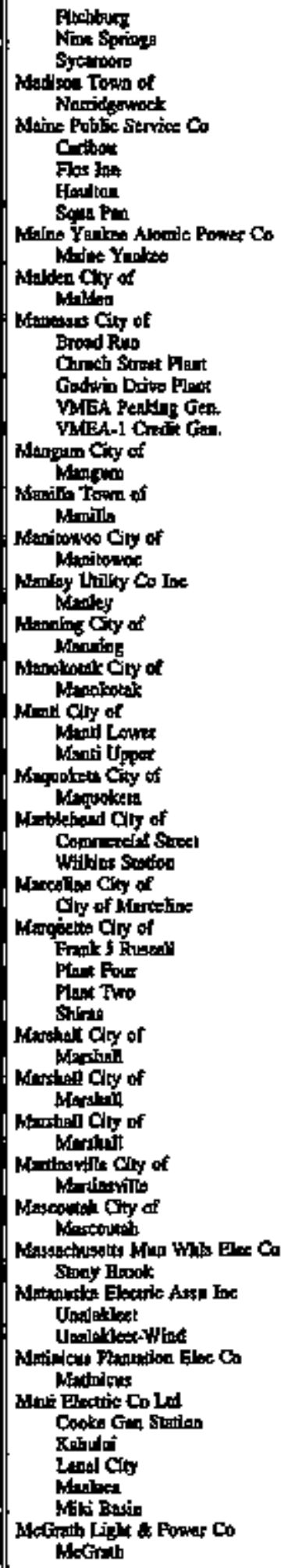 & 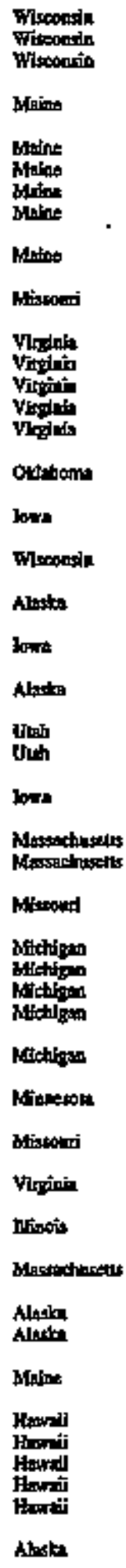 \\
\hline
\end{tabular}

Seo foconotes an end of lable. 
Talne D3. U.S. Electric Utility Plants by Utilty, 1995 (Contimued)

\begin{tabular}{|c|c|c|c|}
\hline 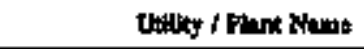 & state & 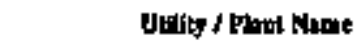 & Fale \\
\hline 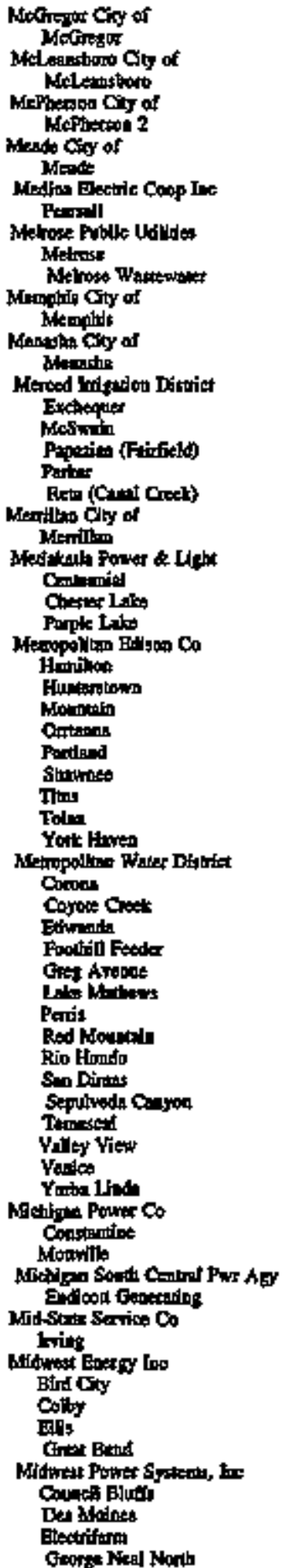 & 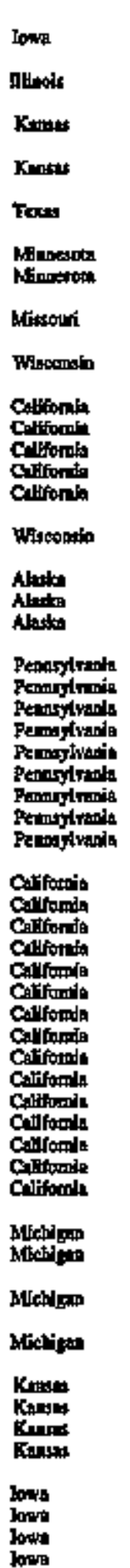 & 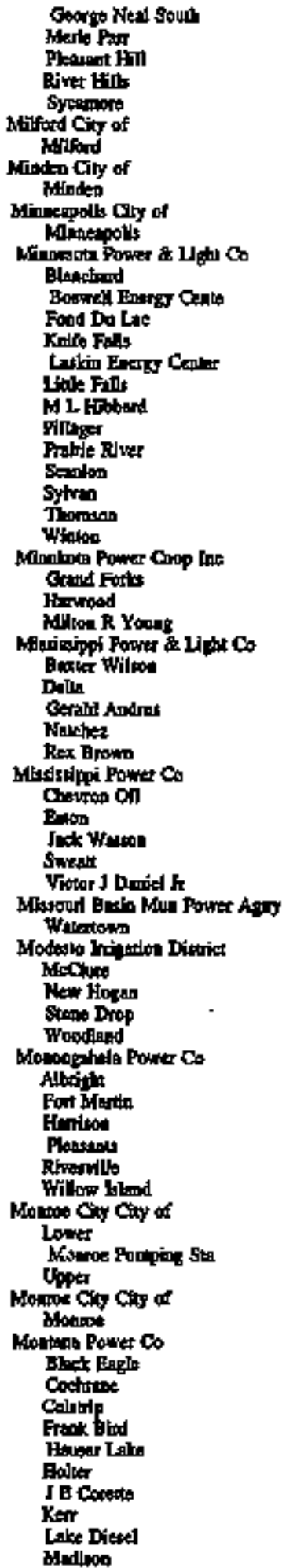 & 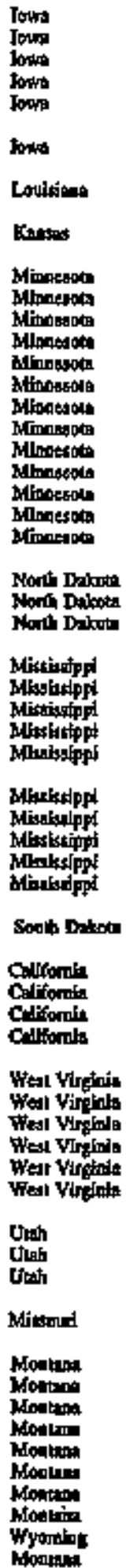 \\
\hline
\end{tabular}

Set footnoles at tend of thele. 
Table D3. DS. Flectric Utillity Plants by Utillty, 1995 (Contimued)

\begin{tabular}{|c|c|c|c|c|}
\hline Datlly / Flapt Neme & Senle & Dully f Plart ktanit & State & \\
\hline 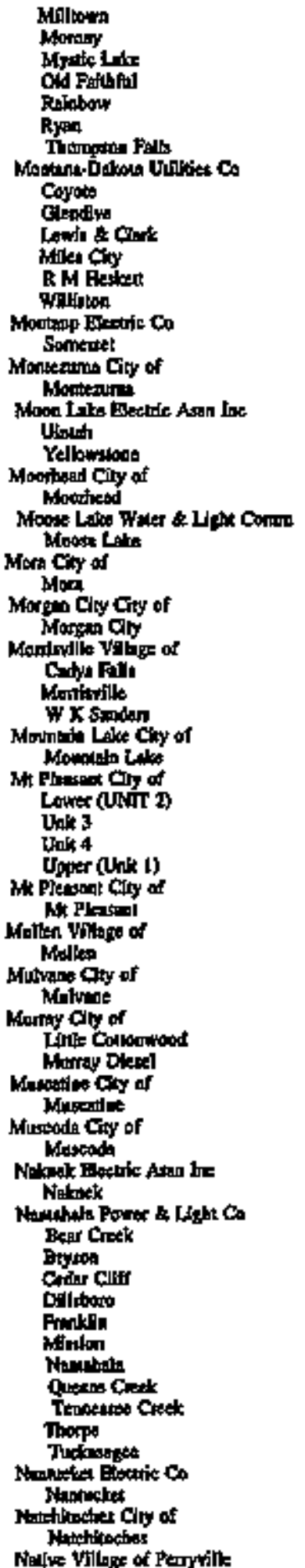 & 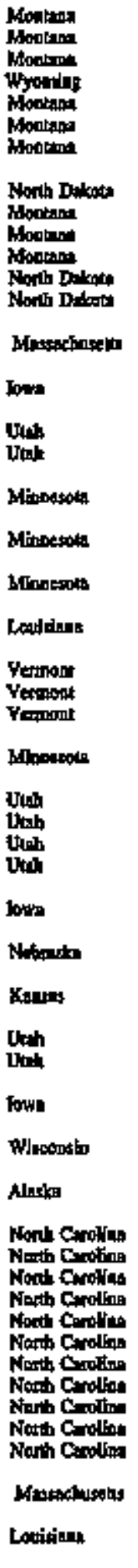 & 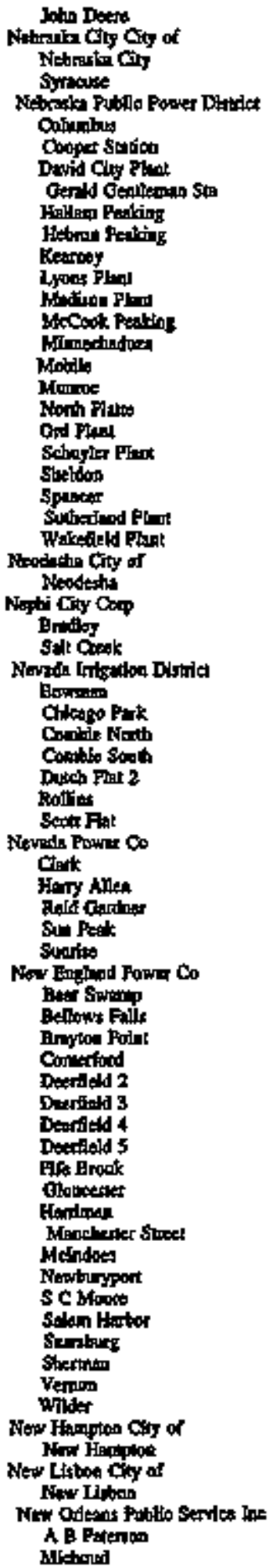 & 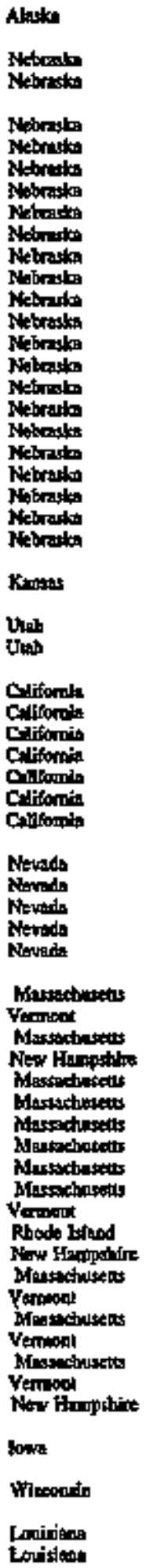 & \\
\hline
\end{tabular}

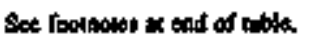


Tabie D3. U.S. Electric Uthity Plants by Utitity, 1995 (Contmned)

\begin{tabular}{|c|c|c|c|}
\hline 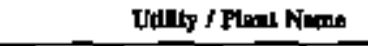 & Stah & Dlathy f Than thes & State \\
\hline 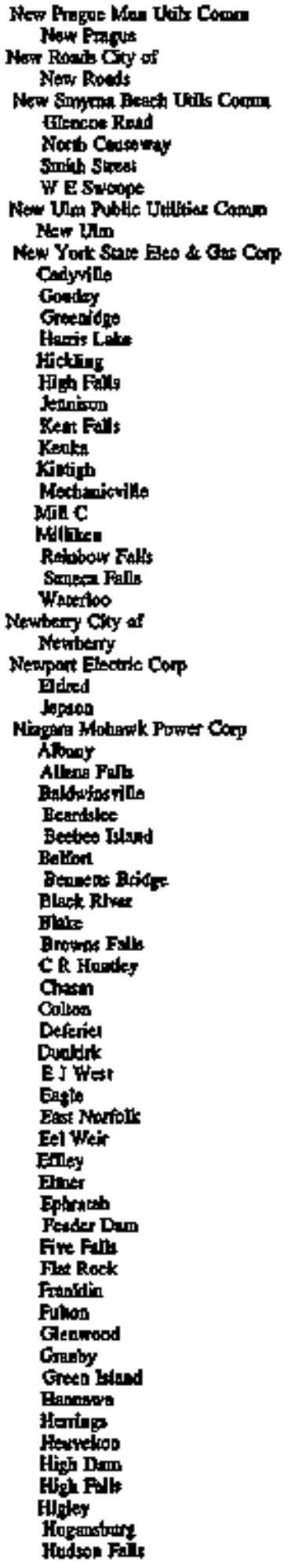 & 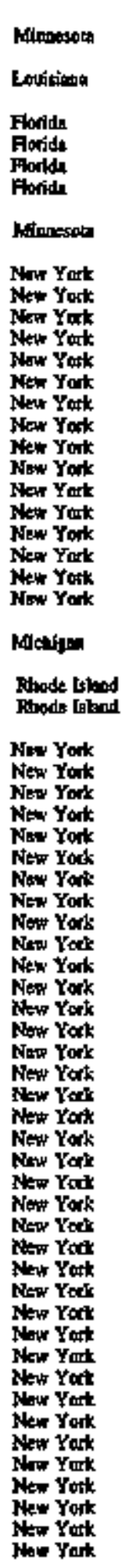 & 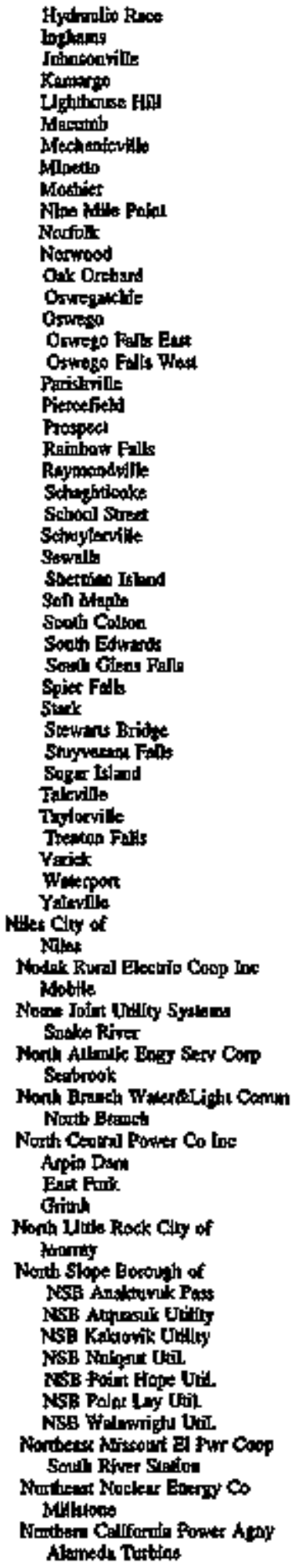 & 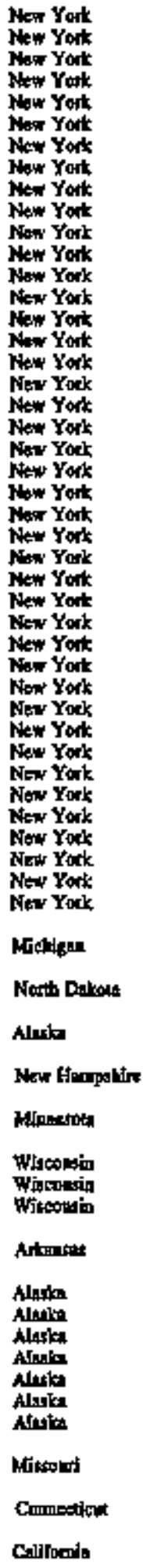 \\
\hline
\end{tabular}

Sen tootnotss at end of teble. 
Table D3. U.S. Electric Utility Plants by Utility, 1995 (Continued)

\begin{tabular}{|c|c|c|c|}
\hline 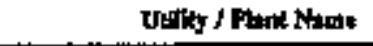 & 8tais & Whis / Plant Nase & stote \\
\hline 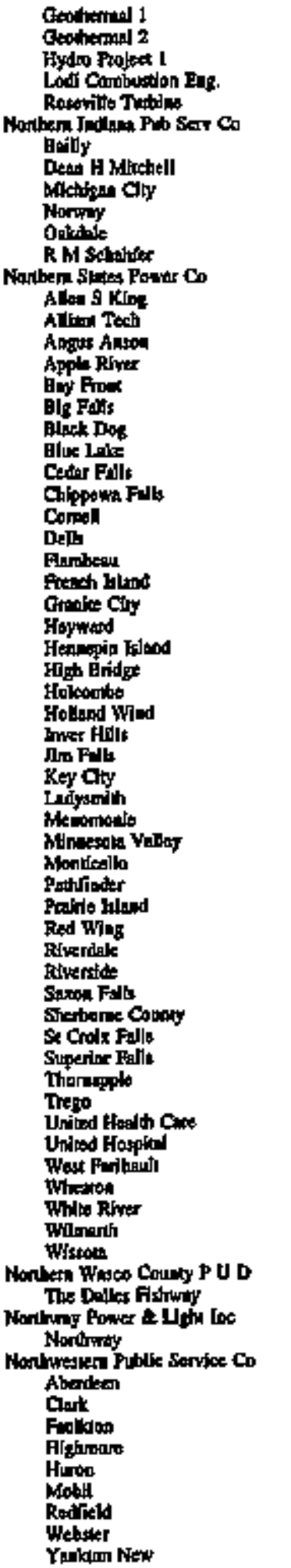 & 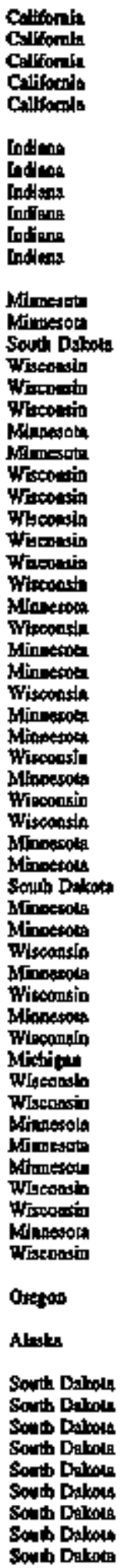 & 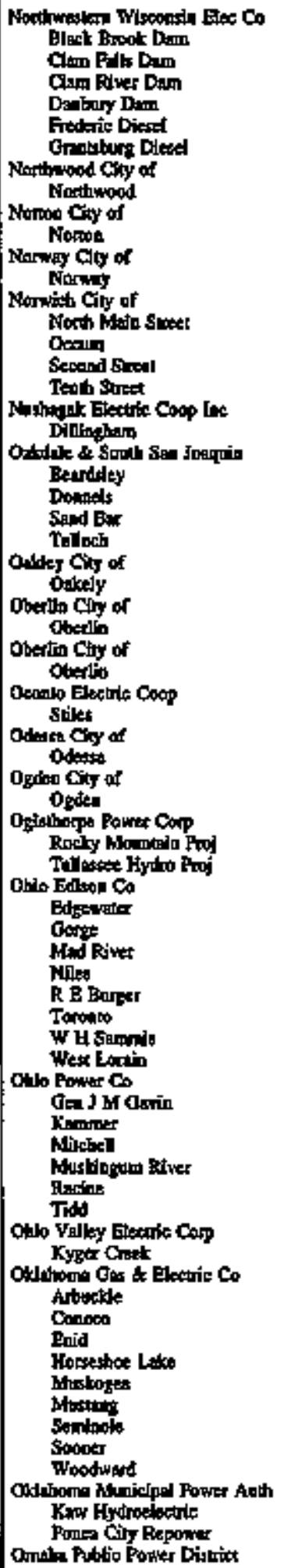 & 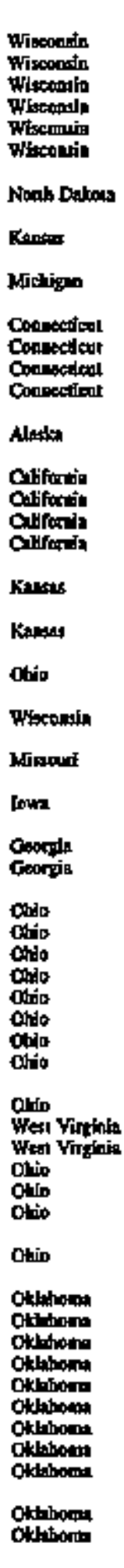 \\
\hline
\end{tabular}

Sex Footnotes to tad of toble. 
Table D3. U.S. Electric Utility PHants by Utility, 1995 (Continued)

\begin{tabular}{|c|c|c|c|}
\hline Jutbly / Pinat Nant & Siocte & Jitis f Phit Name & Stok \\
\hline 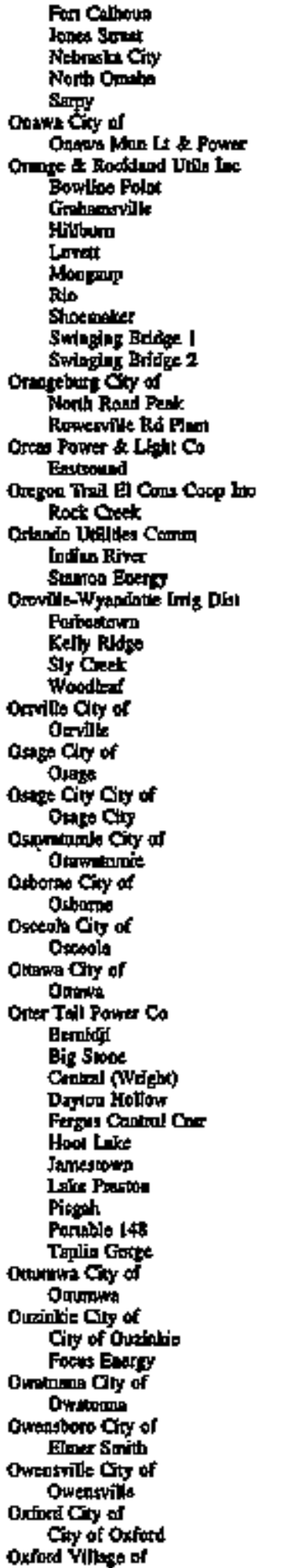 & 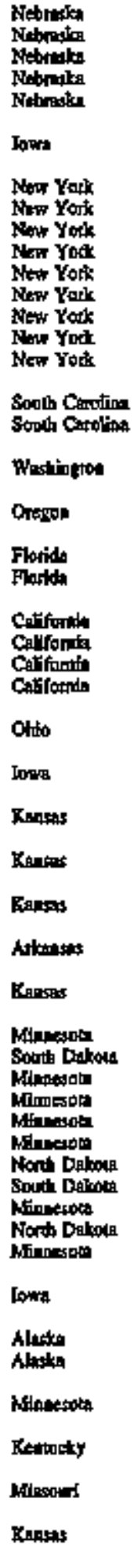 & 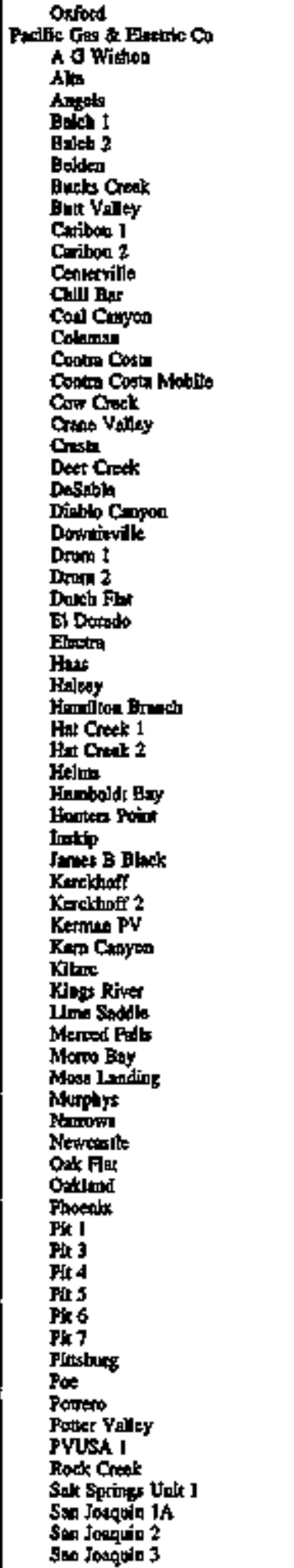 & 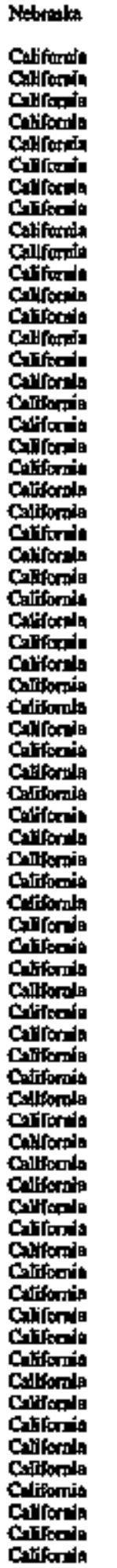 \\
\hline
\end{tabular}

Ses footonga al ced of lable. 
Table D3. U.S. Electrie Utillty Plants by Utillty, 1995 (Continued)

\begin{tabular}{|c|c|c|c|}
\hline UU!ty / Final Namo & Slate & tulth / Ptond Nine & Sole \\
\hline 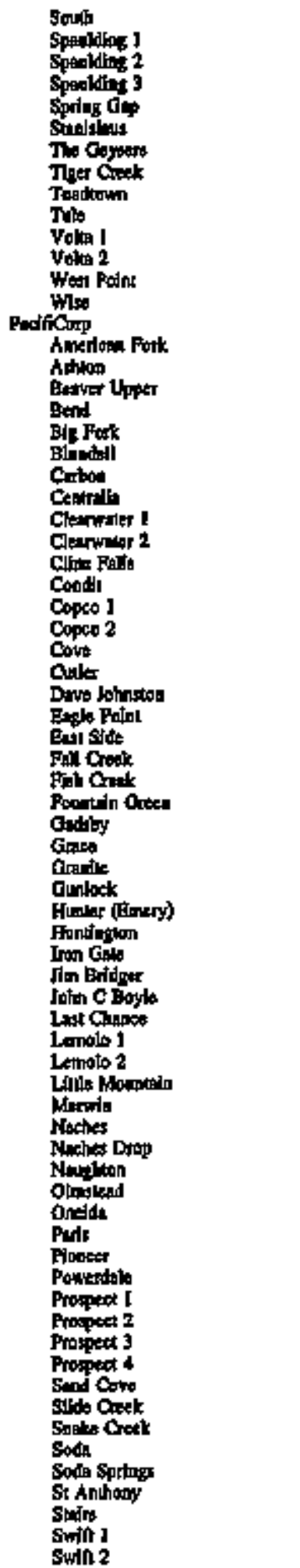 & 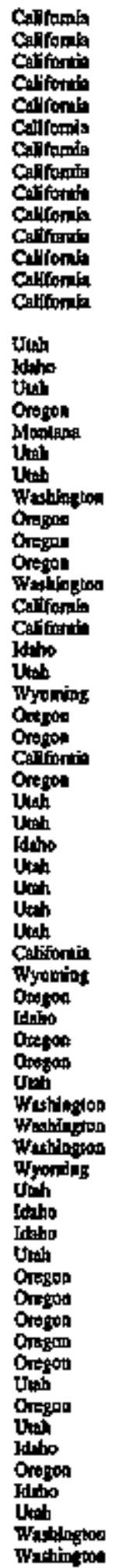 & 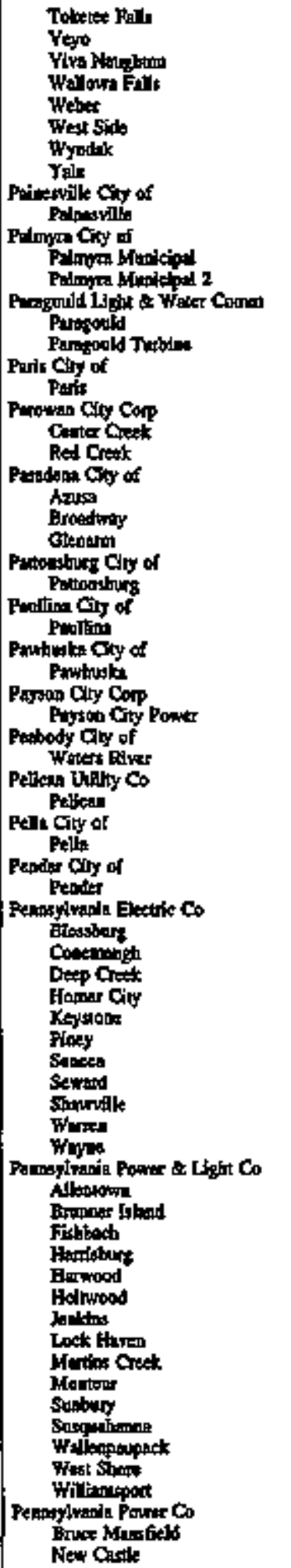 & 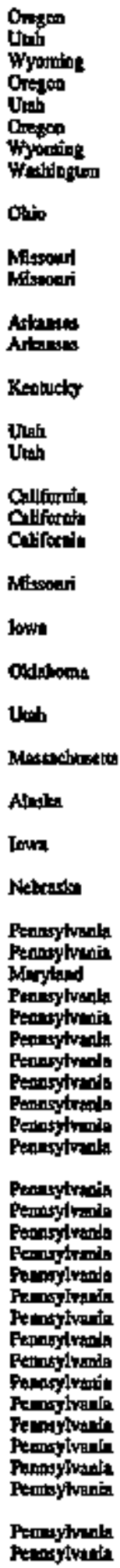 \\
\hline
\end{tabular}

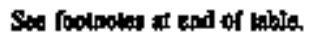


Table D3. U.S. Electric Utility Plants by Utlity, 1995 (Contimued)

\begin{tabular}{|c|c|c|c|}
\hline Ulethy f Phnt Namb & Silote & Ulily / Flank Name & State \\
\hline 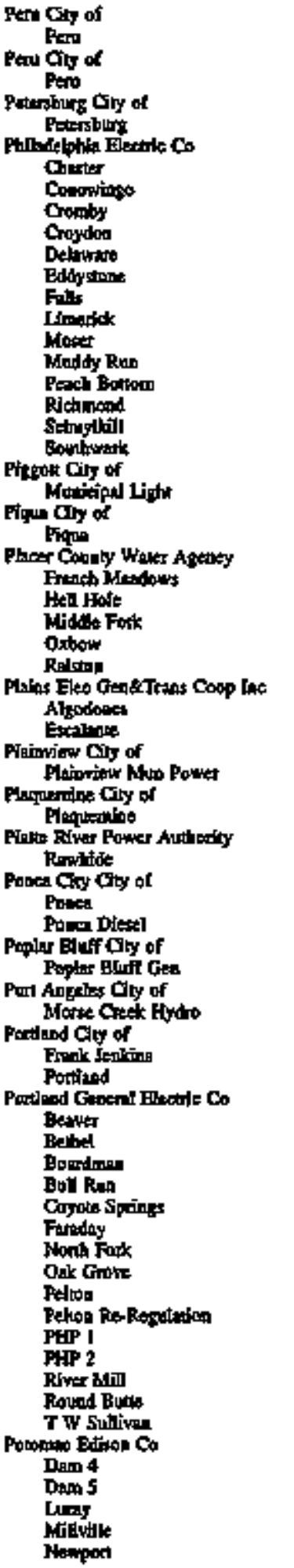 & 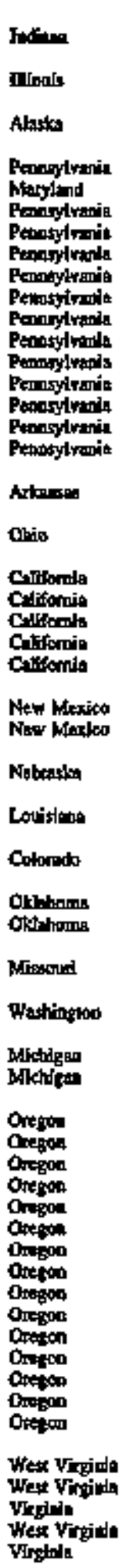 & 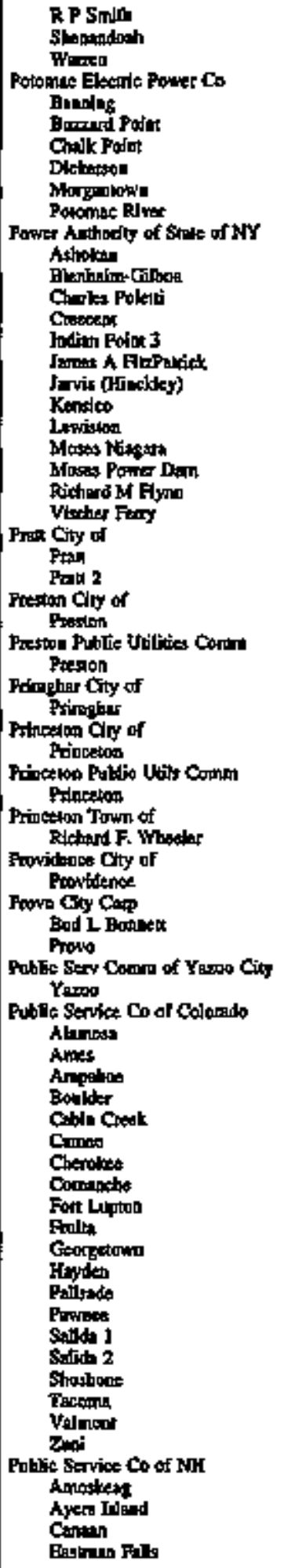 & 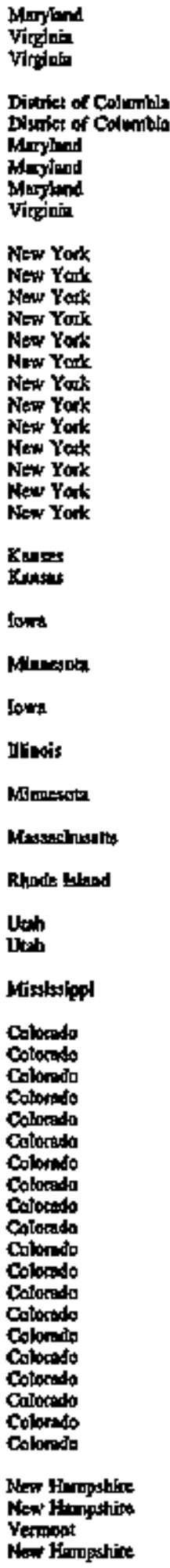 \\
\hline
\end{tabular}

Set fortmotrs of end of lable. 
Table D3. U.S. Electric Utillity Plants by Utility, 1995 (Continued)

\begin{tabular}{|c|c|c|c|}
\hline Uunly f Plate Nane & $\sin 0$ & Uinity f Plant Nonse & stalt \\
\hline 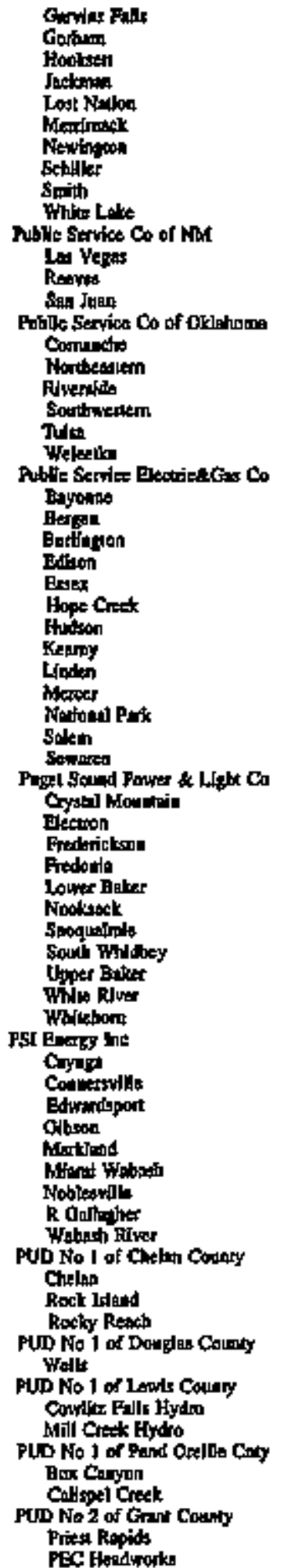 & 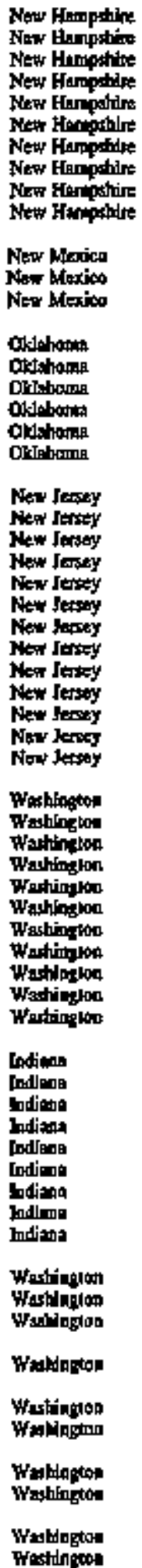 & 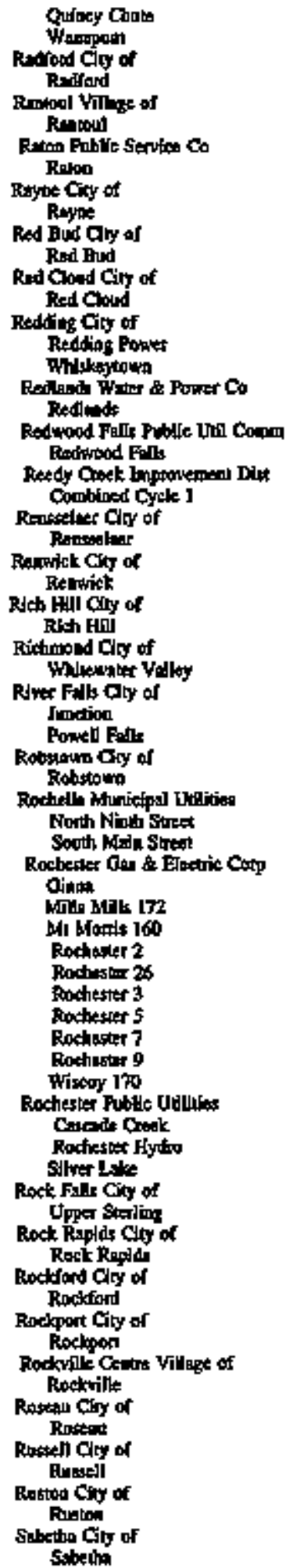 & 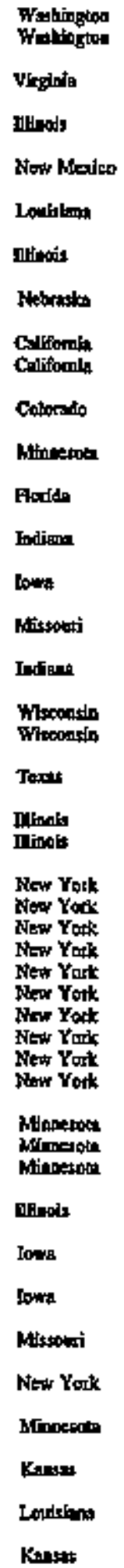 \\
\hline
\end{tabular}

See tootnotes all end of Iobke. 
Tahle D3. U.8. Electric Uthlity Plants by Utality, 1995 (Continned)

\begin{tabular}{|c|c|c|c|}
\hline Vinlty f Flant Nann & Slate & 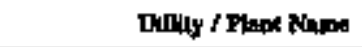 & Stove \\
\hline 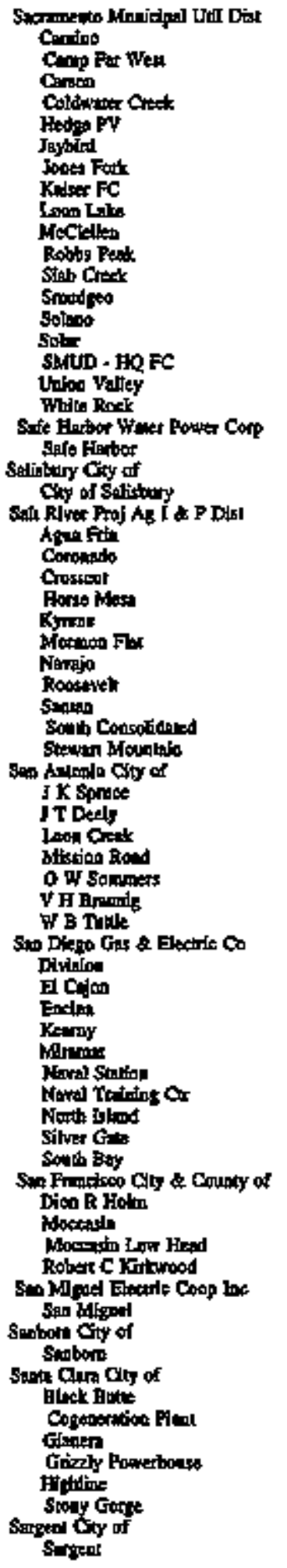 & 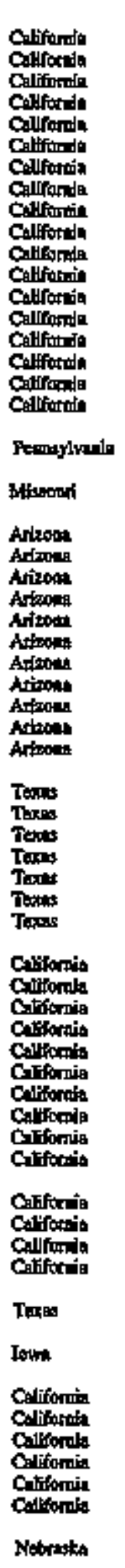 & 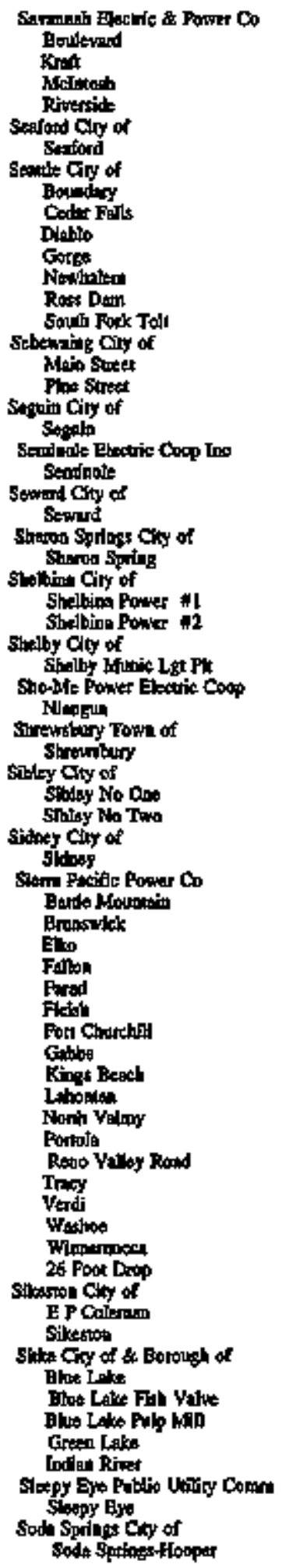 & 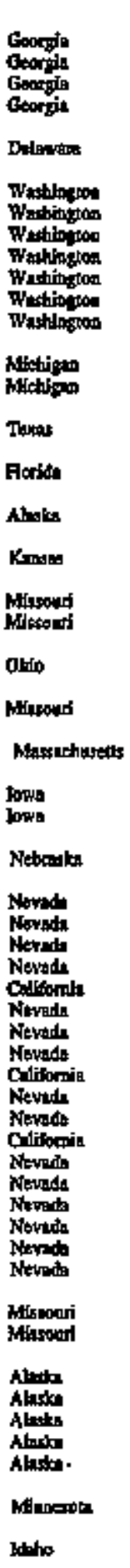 \\
\hline
\end{tabular}

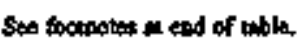


Tabje D3. U.S. Electrle Utility Plants by Utility, 1995 (Conturued)

\begin{tabular}{|c|c|c|c|}
\hline Dully / Ptont None & Slote & 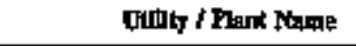 & Siste \\
\hline 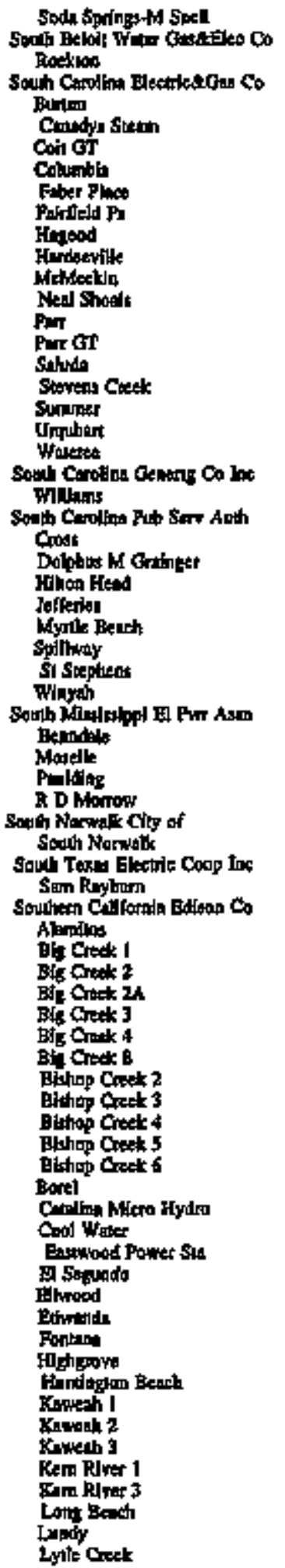 & 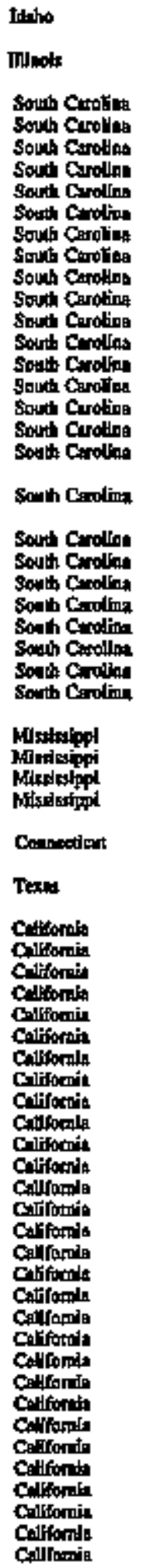 & 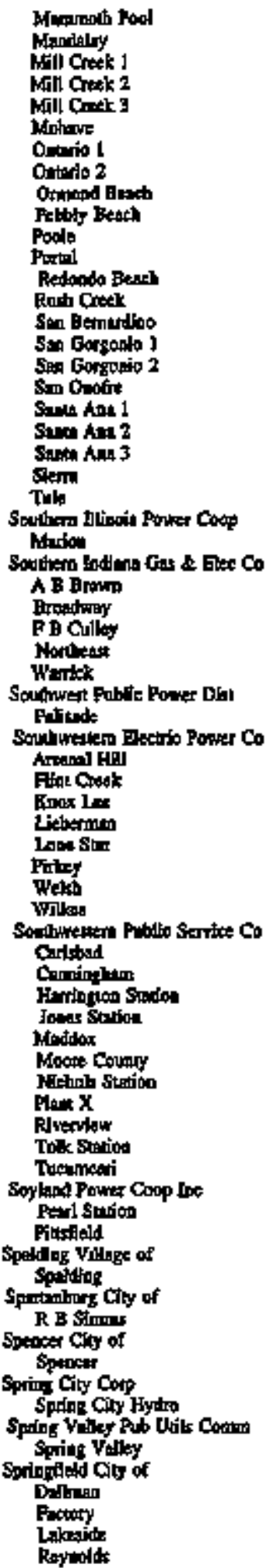 & 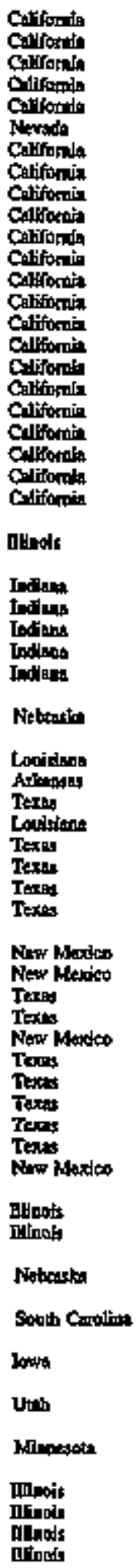 \\
\hline
\end{tabular}

Sect footwons al end of tobla, 
Tahle D3. U.S. Electrie Utility Plants by Utittty, 1995 (Continued)

\begin{tabular}{|c|c|c|c|}
\hline Uilly / Pisal Nmo & Seate & UAll'y / Fisal Nume & Elate \\
\hline 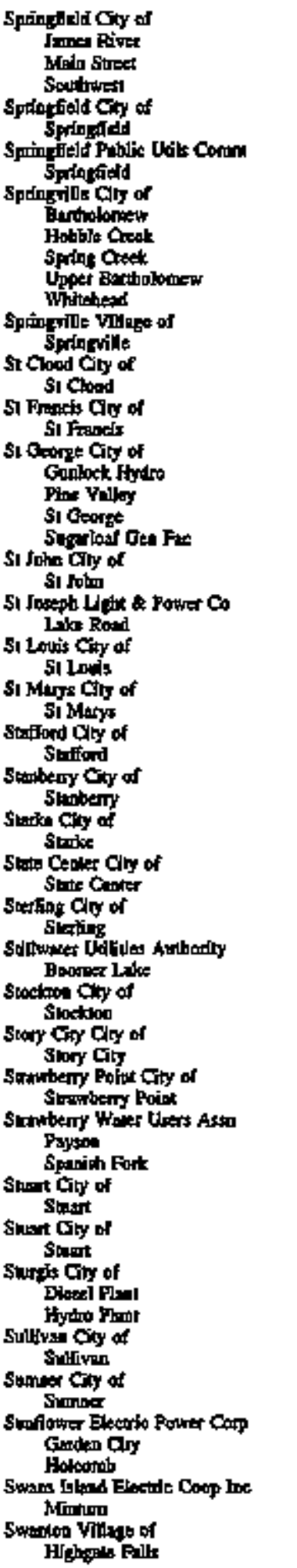 & 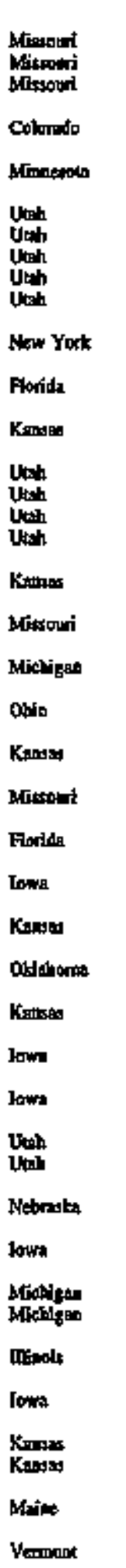 & 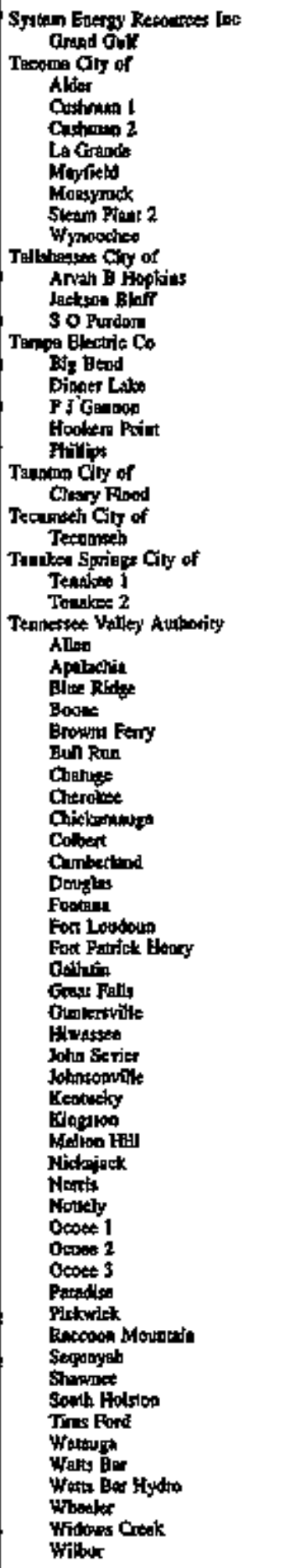 & 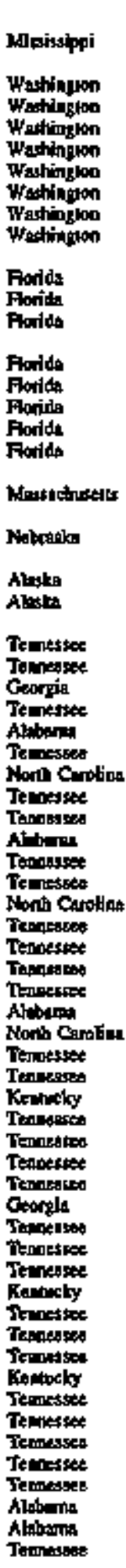 \\
\hline
\end{tabular}

Ses footonted al ead of thble. 
Table D3. U.S. Electric Utility Plants by Utiltty, 1995 (Continued)

\begin{tabular}{|c|c|c|c|}
\hline Cofliky f Ptink Nams & Stale & Dd:Ity / Fle:ul Hane & 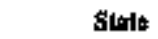 \\
\hline 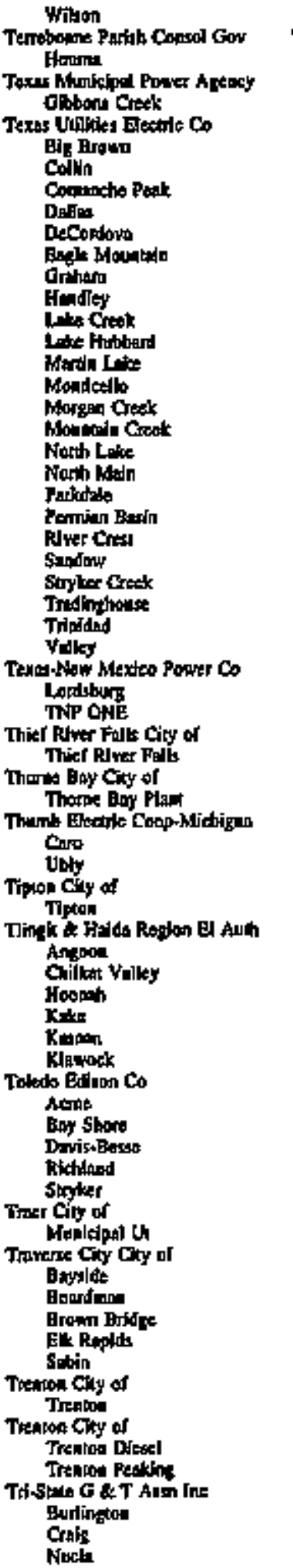 & 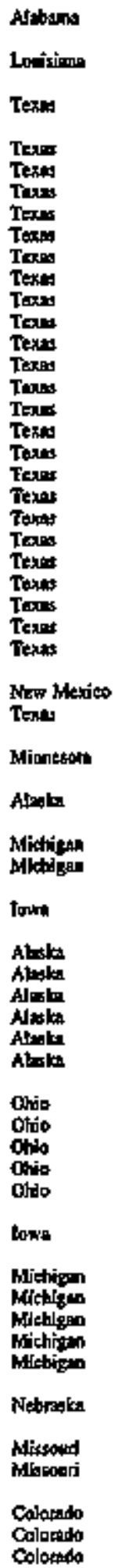 & 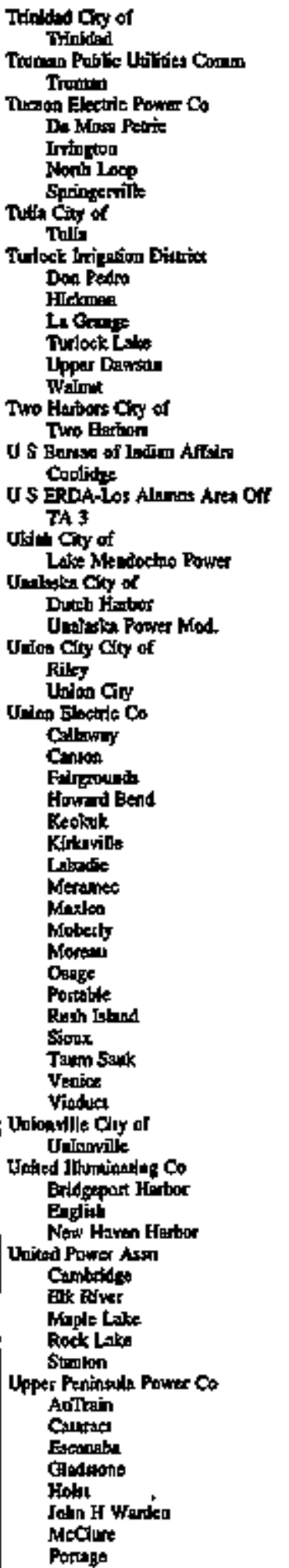 & 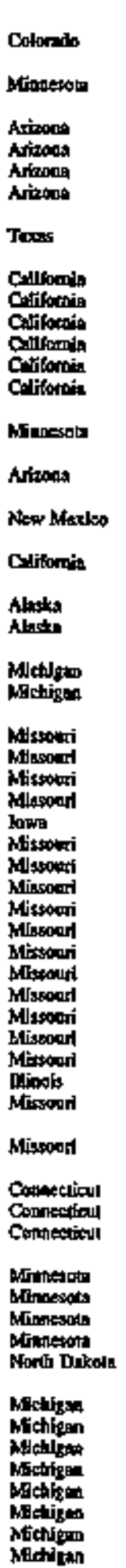 \\
\hline
\end{tabular}

Ses footnotes a end of trible. 
Table D3. U.S. Electric Utility Flants by Utiltty, 1995 (Continued)

\begin{tabular}{|c|c|c|c|}
\hline Wivly f Flend Ninde & Sitile & Thilly / Fiar lane & Slakt \\
\hline 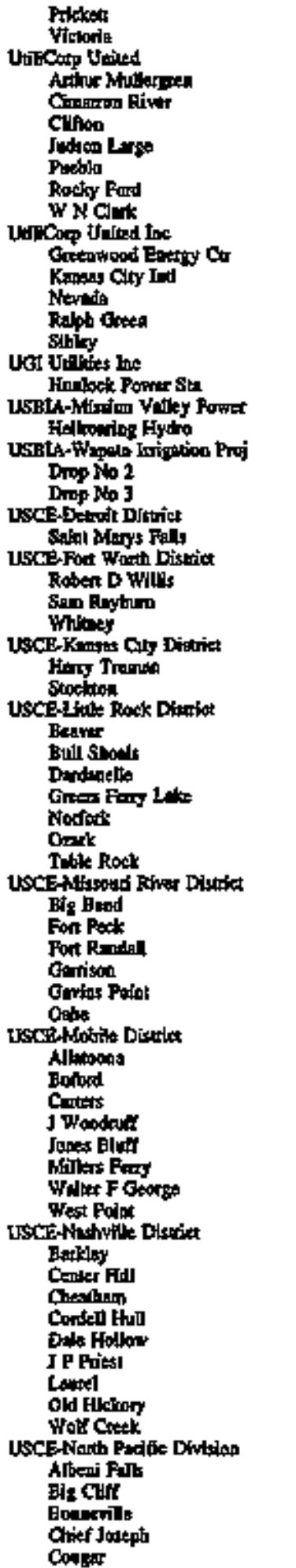 & 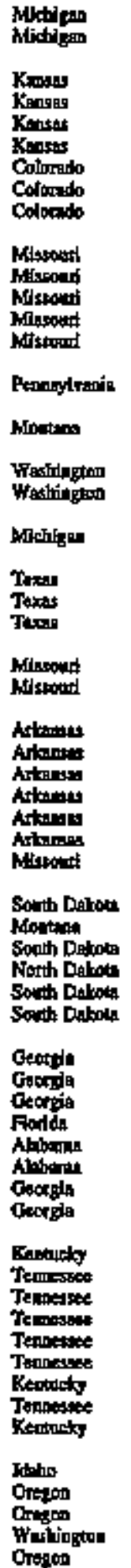 & 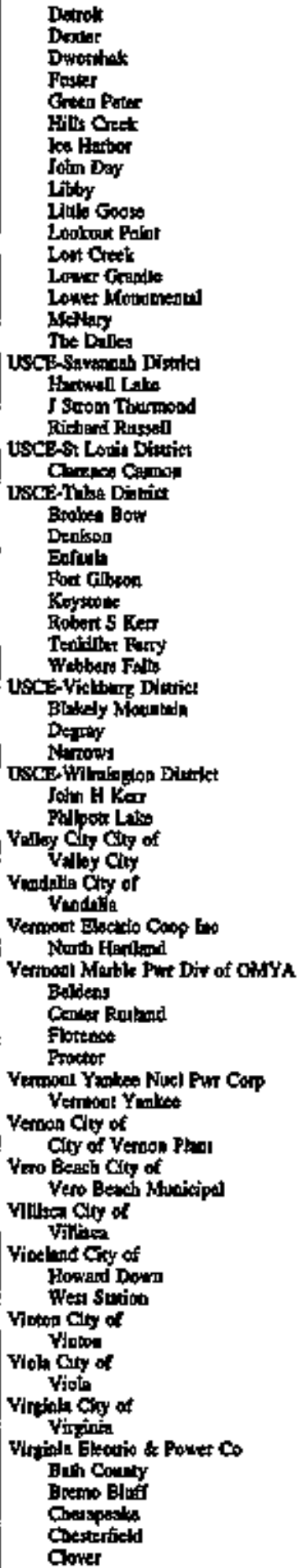 & 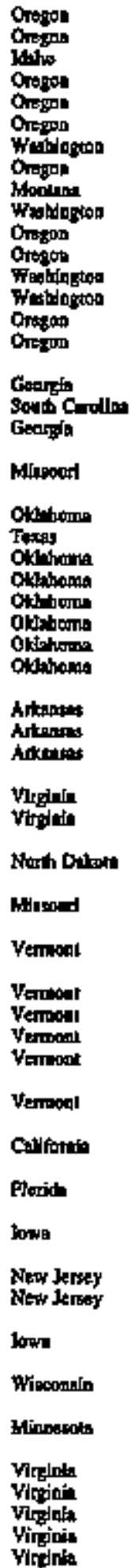 \\
\hline
\end{tabular}

Seo fontungtes al end of lable. 
Tabje D3. U.S. Electric Uthity Piants by Utility, 1995 (Contiaued)

\begin{tabular}{|c|c|c|c|}
\hline Utilly I Plant Kant & State & 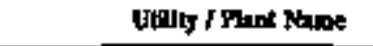 & Stet \\
\hline 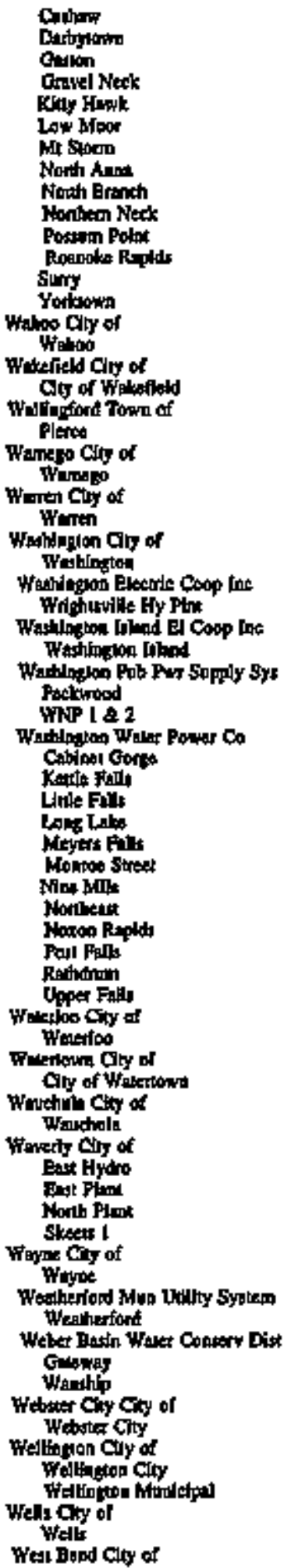 & 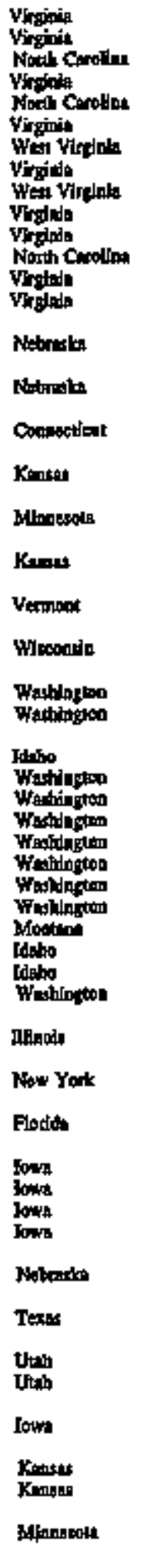 & 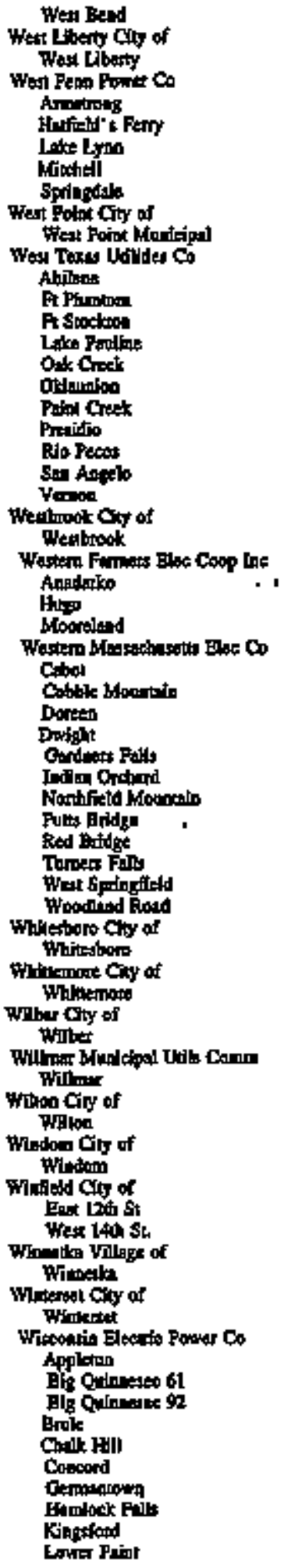 & 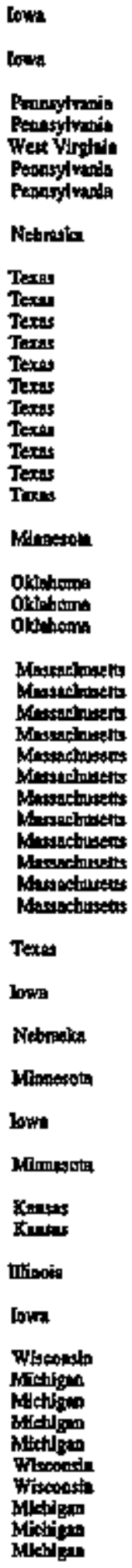 \\
\hline
\end{tabular}

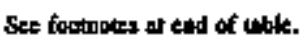


Tabłe D3. U.S. Electric Utility Phants by Uttity, 1995 (Continued)

\begin{tabular}{|c|c|c|c|}
\hline Uiflty / Fient Mome & 8lat: & Wirty / Punt Rase & Stent \\
\hline 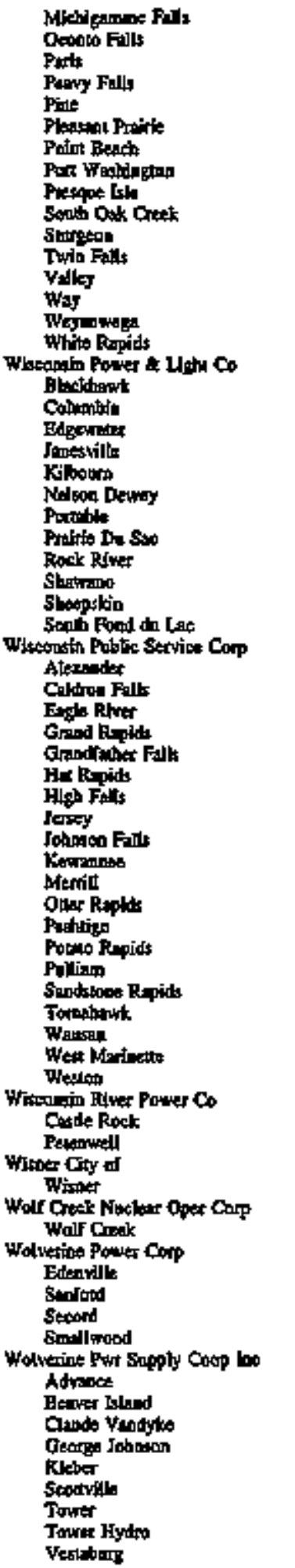 & 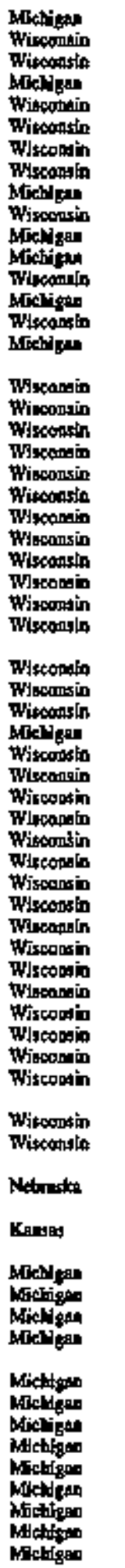 & 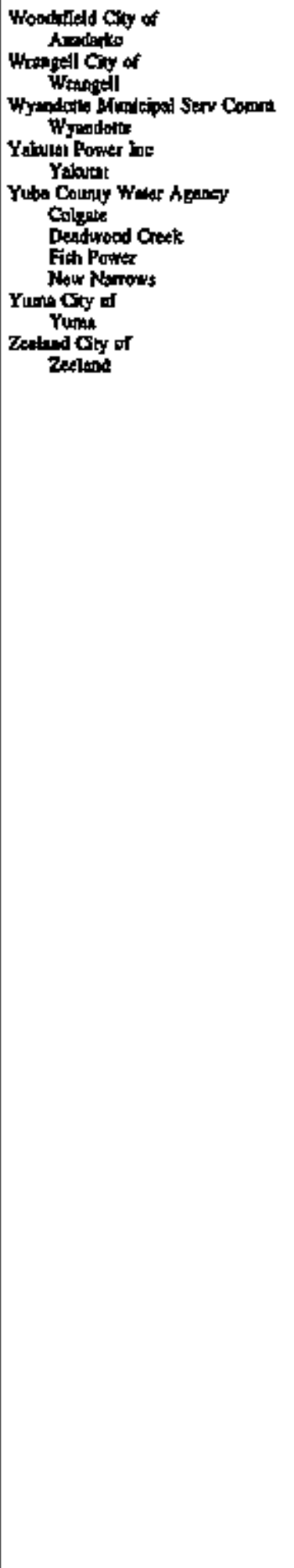 & 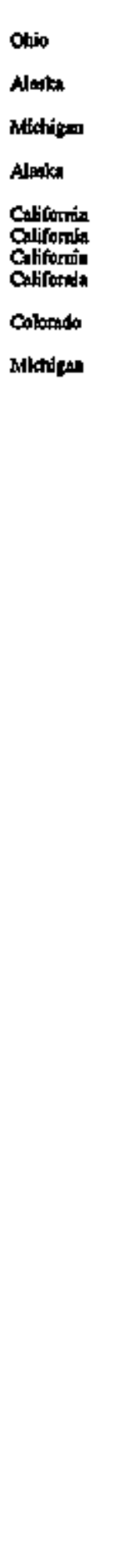 \\
\hline
\end{tabular}

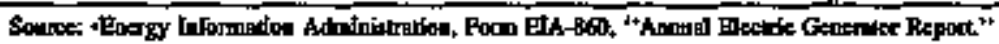




\title{
Appendix E
}

\author{
Plant-Level \\ Statistics for \\ U.S. Electric \\ Utilities
}





\section{Appendix $E$}

\section{Plant-Level Statistlcs for U.S. Electric Utilities}

Table E1. Number of Plants at U.S. Electric Utilities by Censtis Division and State, as of January 1, 1996

\begin{tabular}{|c|c|c|c|}
\hline $\begin{array}{c}\text { Census Divtsion } \\
\text { gth }\end{array}$ & $\begin{array}{c}\text { Numbirl } \\
\text { of } \\
\text { Plants }\end{array}$ & $\begin{array}{c}\text { Cerous Dolvition } \\
\text { Shale }\end{array}$ & Nhminerl \\
\hline 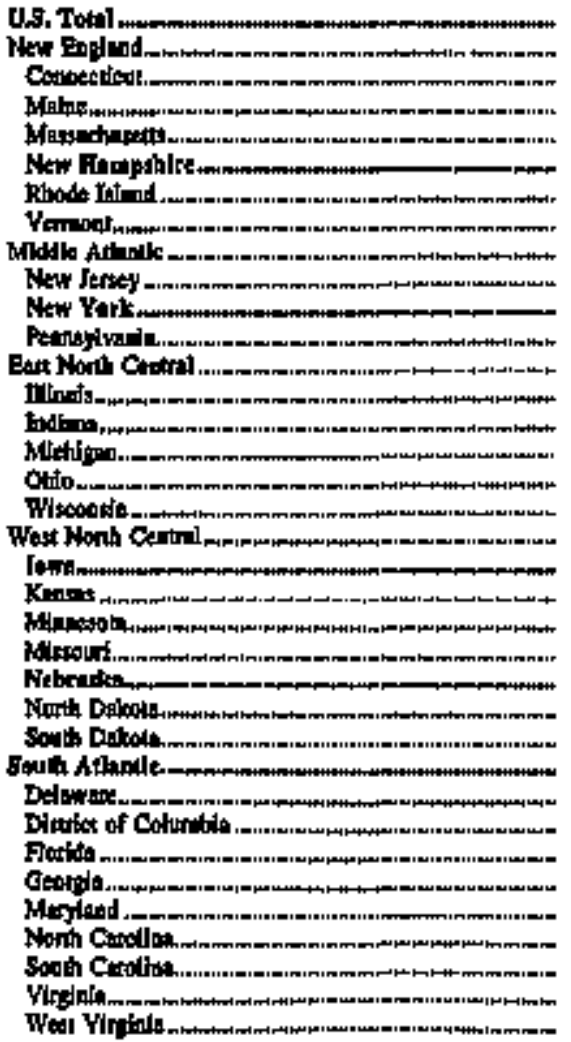 & 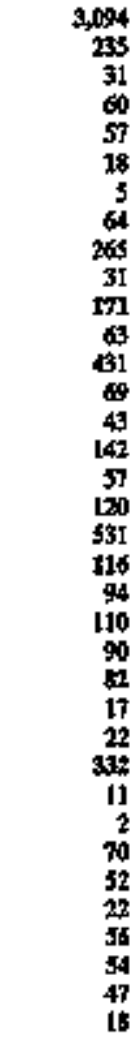 & 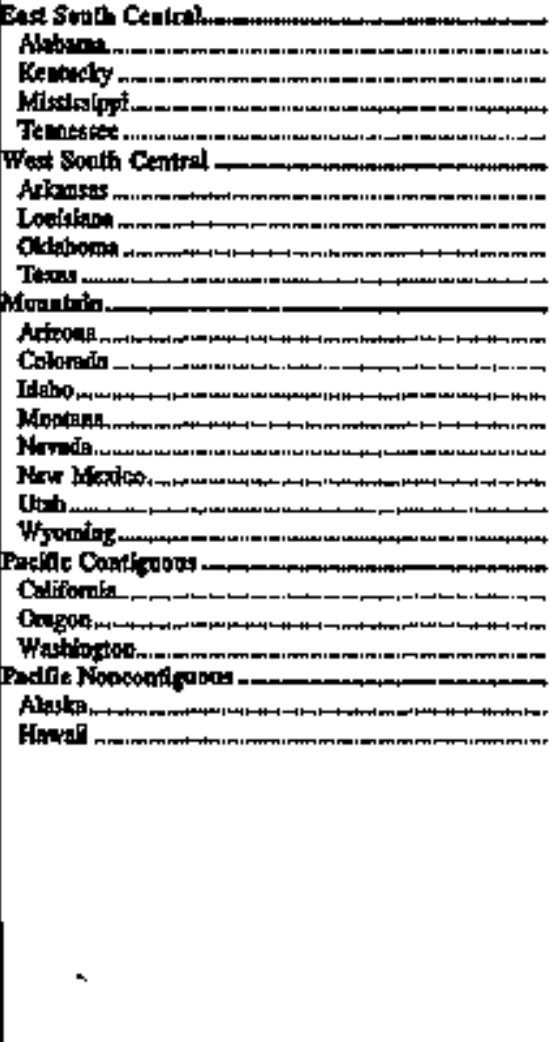 & $\begin{array}{r}126 \\
36 \\
34 \\
20 \\
36 \\
245 \\
33 \\
36 \\
40 \\
136 \\
312 \\
35 \\
63 \\
46 \\
28 \\
22 \\
18 \\
76 \\
26 \\
49 \\
309 \\
61 \\
69 \\
178 \\
161 \\
17\end{array}$ \\
\hline
\end{tabular}

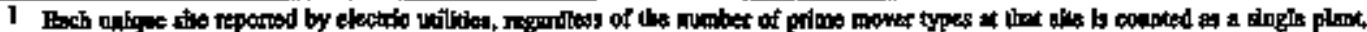

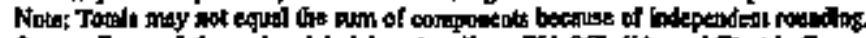

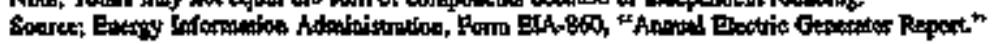


Table E2. Existing Capacity at U.S. Electric Utilttles by Census Division, State, and Prime Mover, as of January 1, 1996

\begin{tabular}{|c|c|c|c|c|c|c|}
\hline \multirow[b]{2}{*}{$\begin{array}{c}\text { Centind Dhrisiont } \\
\text { Stutc }\end{array}$} & \multicolumn{2}{|c|}{ Fand skent } & \multicolumn{2}{|c|}{ Nueleor } & \multicolumn{2}{|c|}{ Byilroaitedrk } \\
\hline & $\begin{array}{c}\text { Nowber } \\
\text { of } \\
\text { ronts }\end{array}$ & 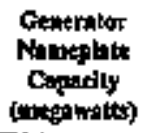 & $\begin{array}{c}\text { Nomber? } \\
\text { of } \\
\text { Findu }\end{array}$ & 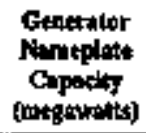 & $\begin{array}{c}\text { Nerober } \\
\text { of } \\
\text { Pintls }\end{array}$ & 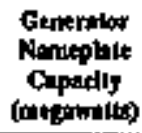 \\
\hline 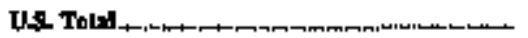 & $\mathbf{8 3}$ & 476,153 & 69 & this & $1,2 s$ & 91,114 \\
\hline 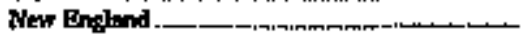 & $\mathbf{3}$ & $11+00$ & 6 & 6 gath & 146 & 2793 \\
\hline 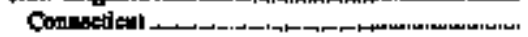 & 9 & 3,091 & 2 & 3,425 & 14 & 138 \\
\hline 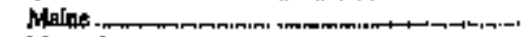 & $\mathbf{s}$ & 1,090 & $\mathbf{I}$ & 920 & 45 & 386 \\
\hline 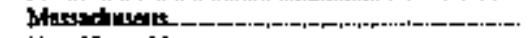 & 14 & 6,157 & $\mathbf{I}$ & 65 & $\mathbf{z 3}$ & 1,647 \\
\hline Niew Happthipt, & 3 & $1, \mathbf{0 0 3}$ & $\mathbf{I}$ & $1,2+2$ & 12 & 254 \\
\hline Fhodd lifand & J & 489 & - & - & $\mathbf{1}$ & 2 \\
\hline 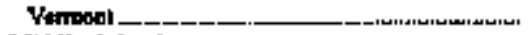 & $\mathbf{1}$ & 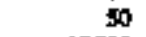 & I & 563 & 51 & 371 \\
\hline 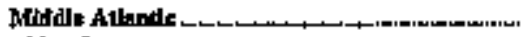 & 73 & 45,710 & $\mathbf{L}$ & 19,411 & 21 & 7,406 \\
\hline New Jessey & 13 & 5,342 & 3 & 4,151 & I & 387 \\
\hline NGW York & 32 & 17,365 & $\mathbf{s}$ & 5,624 & 112 & 5,162 \\
\hline 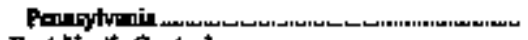 & $\mathbf{2 8}$ & 23,002 & 5 & 9,636 & 8 & 1,BSE \\
\hline 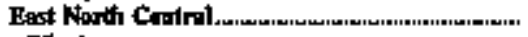 & 146 & 90,60 & Ig & 21,821 & 18 & 2,94 \\
\hline 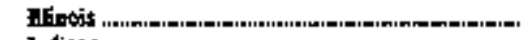 & 32 & 2099 & 7 & 13,134 & 3 & 1 \\
\hline 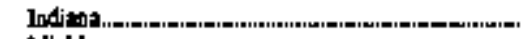 & 29 & 21,460 & 一 & - & $\mathbf{5}$ & 89 \\
\hline 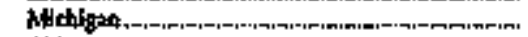 & 29 & 15,626 & 4 & 4,326 & 6 & 2,330 \\
\hline 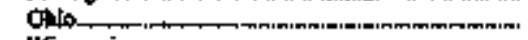 & 36 & 25,168 & 2 & $2[78$ & 3 & 12 \\
\hline 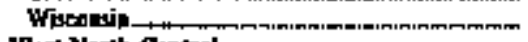 & 20 & 7,326 & 2 & Ists & 75 & 445 \\
\hline 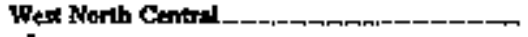 & 124 & 39,7n & 7 & 6,161 & Gs & 3,006 \\
\hline 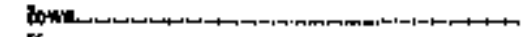 & 24 & 6,397 & I & 397 & $\tau$ & 134 \\
\hline 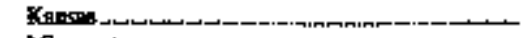 & $\mathbf{2 7}$ & 7,457 & I & 1,236 & - & - \\
\hline 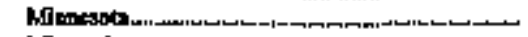 & 28 & 5,975 & 2 & 1,755 & 2 & 142 \\
\hline 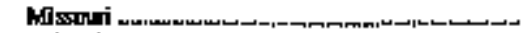 & 27 & 11.923 & $\mathbf{I}$ & 1,236 & 8 & 1,100 \\
\hline 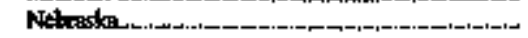 & $\overline{12}$ & 3.440 & $\mathbf{2}$ & 1,738 & $\mathbf{1 1}$ & 189 \\
\hline 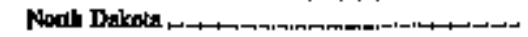 & 7 & 4,009 & - & - & $\mathbf{l}$ & $\$ 17$ \\
\hline 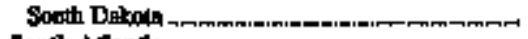 & 4 & 573 & - & - & 4 & 1,731 \\
\hline Soulb Alande & 125 & 91090 & 15 & 25,460 & 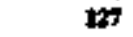 & 11,904 \\
\hline Dollawares & 4 & $1+791$ & - & - & - & - \\
\hline 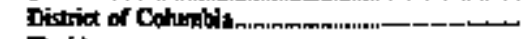 & $\mathbf{j}$ & 49:0 & 一 & - & - & - \\
\hline 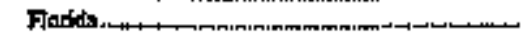 & 46 & 26,683 & 3 & $4,1,10$ & 2 & 42 \\
\hline 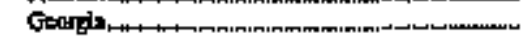 & 15 & 15,064 & 2 & 3,090 & 3t & 3,273 \\
\hline Maryleod & 10 & $7+146$ & I & $t, 849$ & $\mathbf{2}$ & 494 \\
\hline Nonth Canolins & 15 & 12,597 & 3 & 5,125 & 34 & 1,506 \\
\hline Sodth Cooding & 11 & 6,01) & 4 & 6,799 & 29 & 3,449 \\
\hline 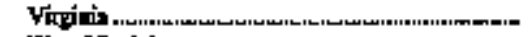 & 9 & 6,875 & $\mathbf{z}$ & $\mathbf{3 , 6 5 5}$ & 25 & 3,103 \\
\hline 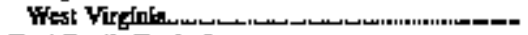 & 14 & 15,038 & 一 & - & 4 & 57 \\
\hline 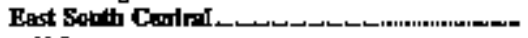 & 57 & 44,3112 & 4 & 9,016 & $\mathbf{5}$ & 7,268 \\
\hline 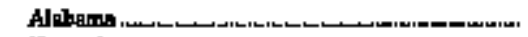 & I1 & 32,672 & 2 & 5,203 & 21 & 2,868 \\
\hline Kexhnty & 22 & 16,181 & - & - & $\mathbf{7}$ & 748 \\
\hline 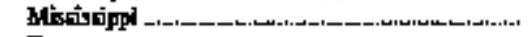 & 16 & 5,365 & $\mathbf{I}$ & 1,373 & - & - \\
\hline 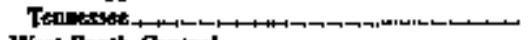 & 8 & 9,895 & 1 & 2,41 & 27 & 3,647 \\
\hline Wer Sanlh Central _. & $\mathbf{1 \$ 0}$ & 9.102 & $\boldsymbol{5}$ & 9204 & G1 & 2,67 \\
\hline 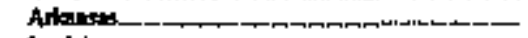 & 11 & 6,475 & I & E.\$AS & 14 & 1,196 \\
\hline 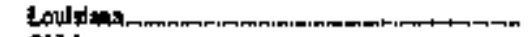 & 30 & 15,762 & 2 & 2736 & - & - \\
\hline Okjboms & 17 & 11,599 & - & - & 1t & 1,052 \\
\hline 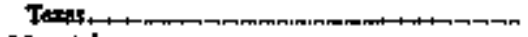 & 92 & 58,187 & 2 & 5,139 & 26 & 50 \\
\hline 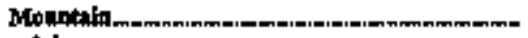 & 71 & 34 spes & $\mathbf{I}$ & 4,210 & 190 & 10,790 \\
\hline 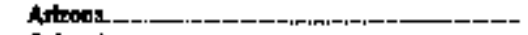 & 13 & 3.367 & 1 & 4,210 & 14 & 2,885 \\
\hline תolotsion. & 18 & 5,300 & 一 & - & 29 & 1,006 \\
\hline 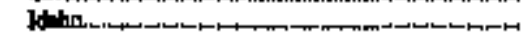 & - & - & - & 一 & 44 & 2,200 \\
\hline 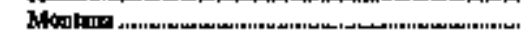 & $\mathbf{s}$ & 2,595 & 一 & $m$ & $\mathbf{2 1}$ & 2,137 \\
\hline 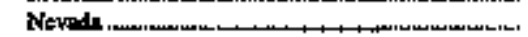 & 7 & 3,674 & - & - & 6 & 1,046 \\
\hline 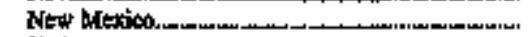 & 13 & 5,200 & - & - & 3 & 58 \\
\hline 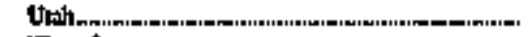 & 7 & 4,720 & ع & - & 58 & 27! \\
\hline 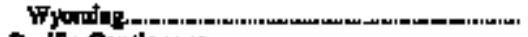 & $\mathbf{B}$ & 5,975 & - & - & 15 & 294 \\
\hline 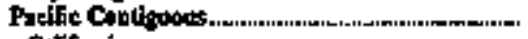 & 41 & 23,728 & 3 & 5,753 & 345 & 41,100 \\
\hline 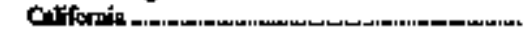 & 33 & 21,206 & 2 & 4,555 & 234 & 12,061 \\
\hline 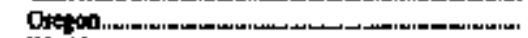 & 5 & 9601 & - & - & 54 & 5,154 \\
\hline Yothipgoth & $\mathbf{3}$ & 1,561 & $\mathbf{I}$ & 1,200 & 58 & 20,386 \\
\hline 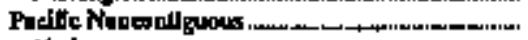 & 13 & 135 & - & - & 30 & 36 \\
\hline Altitis & 4 & 155 & 一 & 一 & 28 & 359 \\
\hline 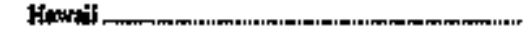 & 9 & 1,219 & 一 & 一 & 2 & 3 \\
\hline
\end{tabular}

Sectoctiotes at ead of abl. 
Table E2. Existing Capacity at U.S. Electric Utilities by Census Division, State, and Prime Mover, as of January 1, 1996 (Continured)

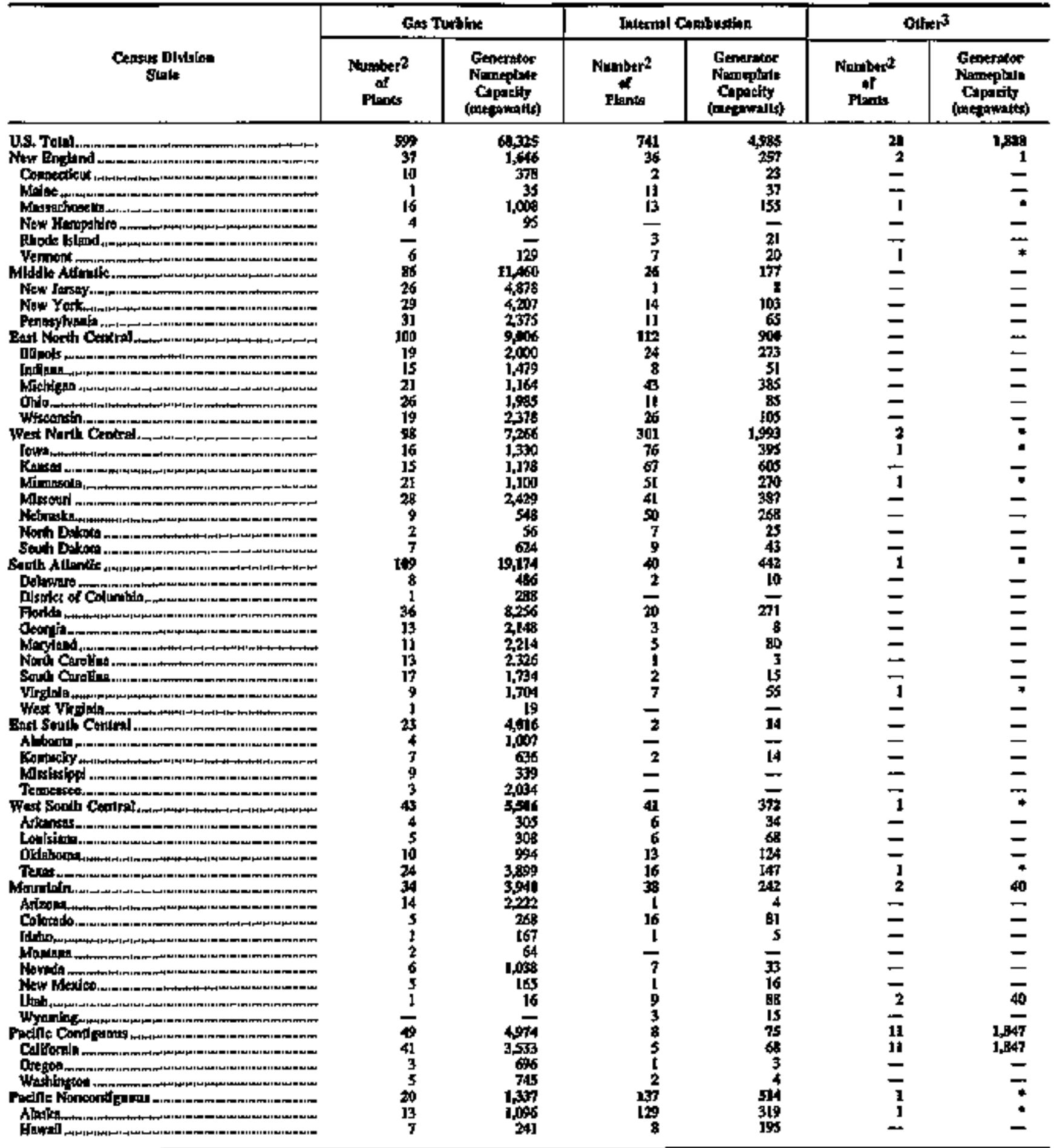

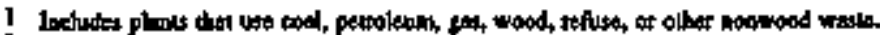

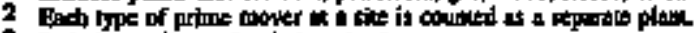

3 Inchadas genthermil, wied, and sake

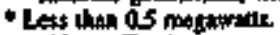

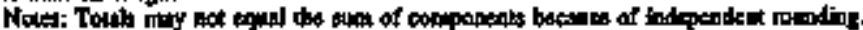

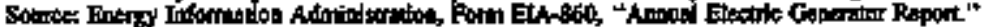


Table E3. Existing Capacity at US. Electric Utilities by Class of Ownership, Census Division, and State, as of December 31, 1995

(Megawatts)

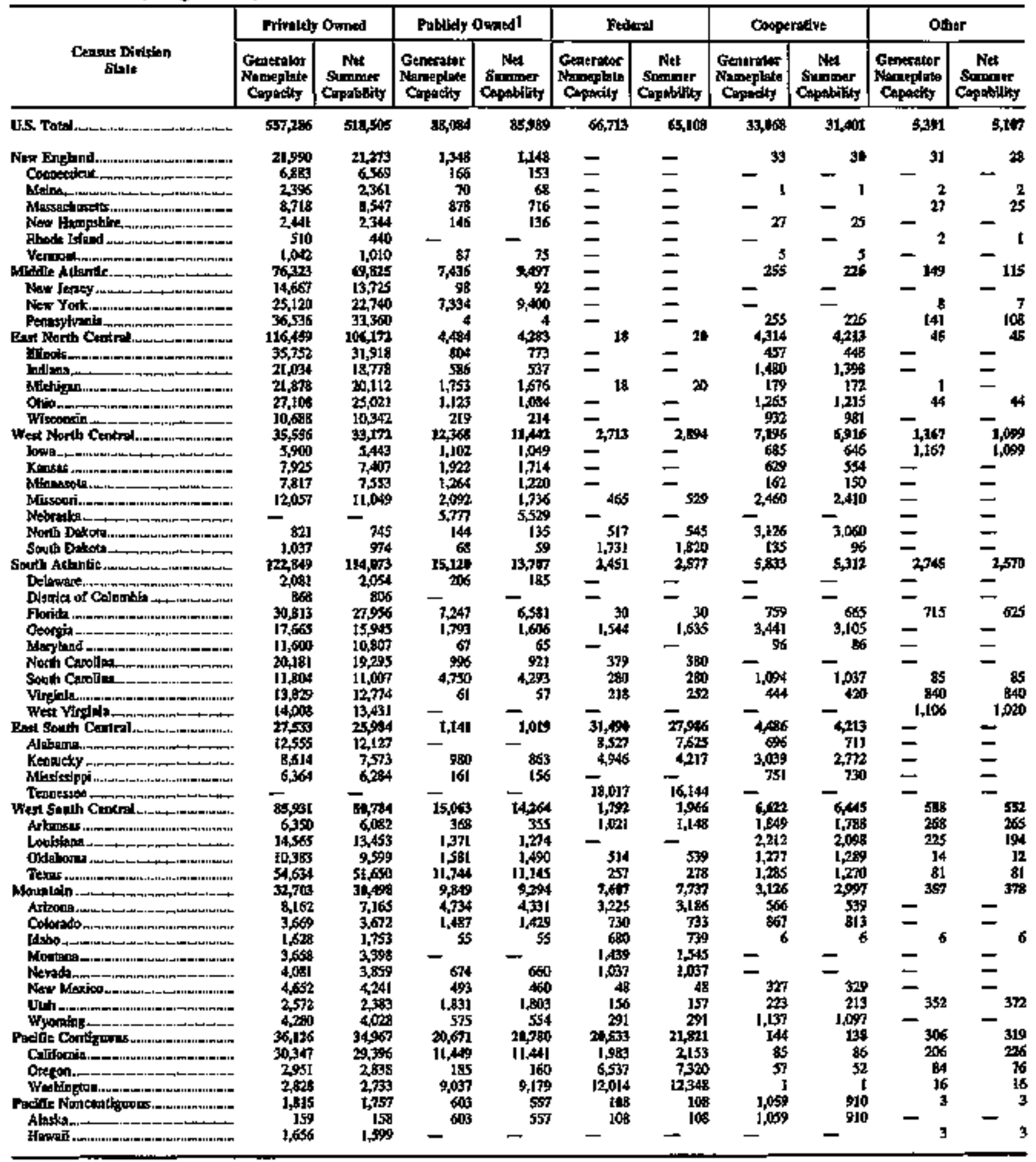

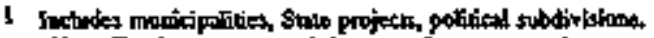

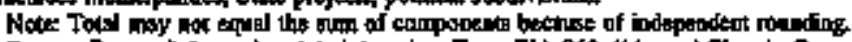

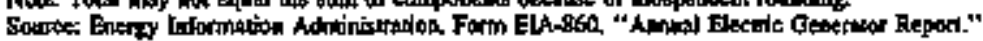


Appendix F

Maps 


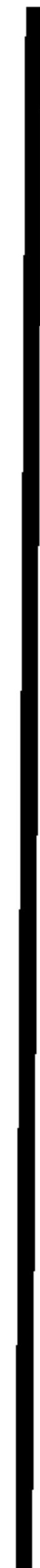




\section{Appendix F}

\section{Maps}

Figure F1. North American Electric Reliabillty Council Regions for the Contlguous United States and Alaska

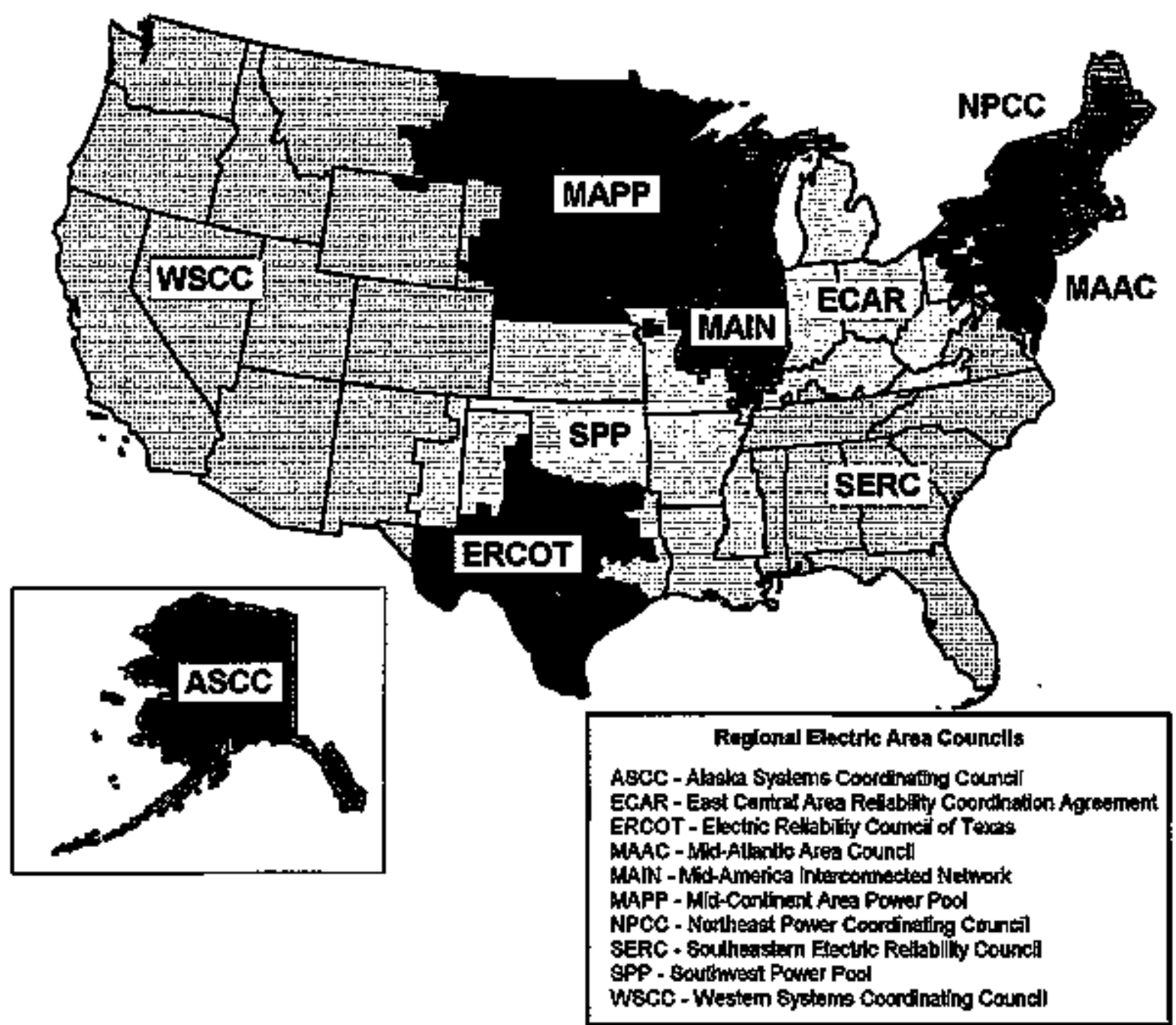

Source: Energy Information Admixigtration, Office of Coat, Nuclear, Electric and Alternate Furels. 
Flgure F2. U.S. Federal Regions

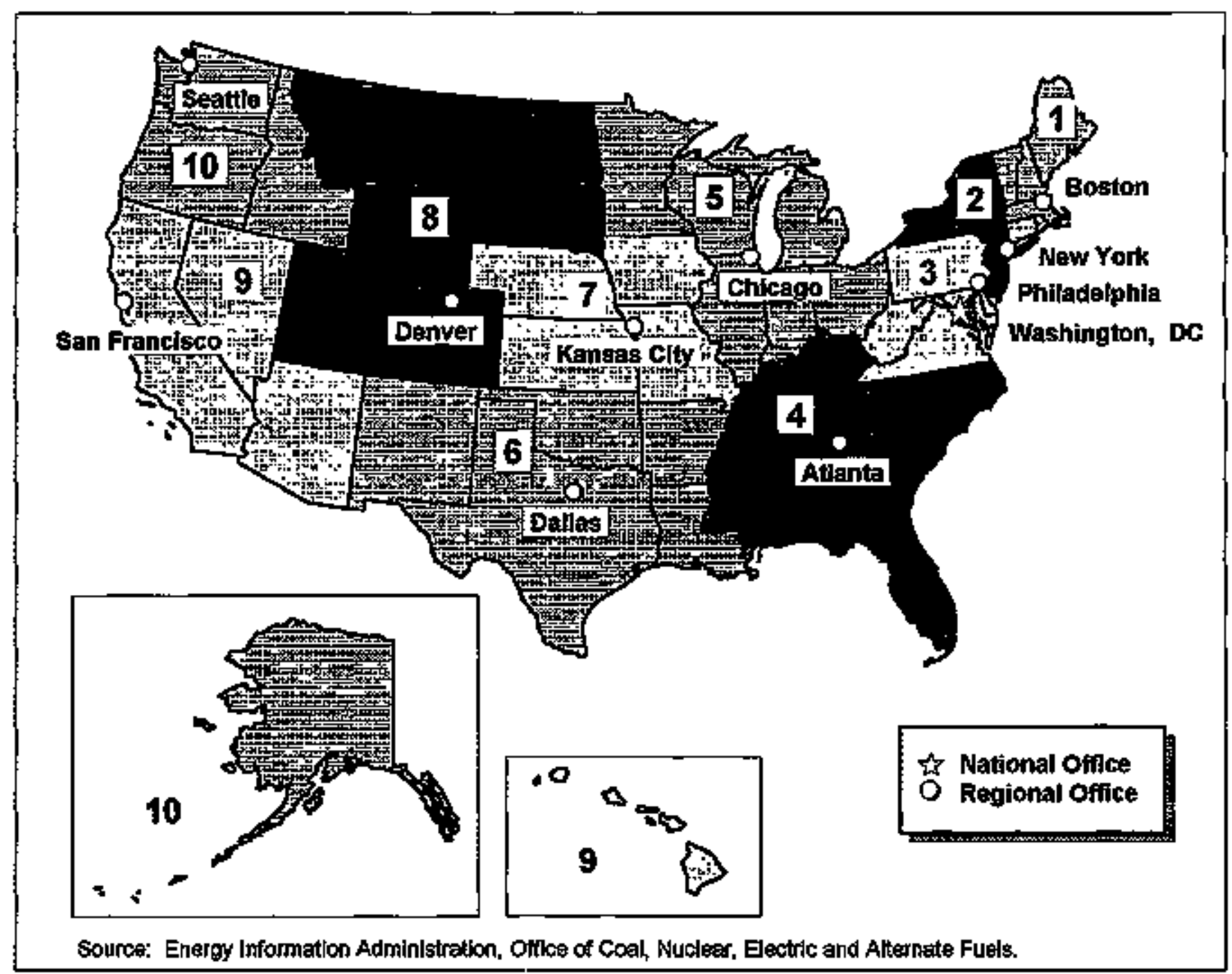

400 Energy Information Administration/Inventory of Power Plants in the United States as of January 1, 1996 


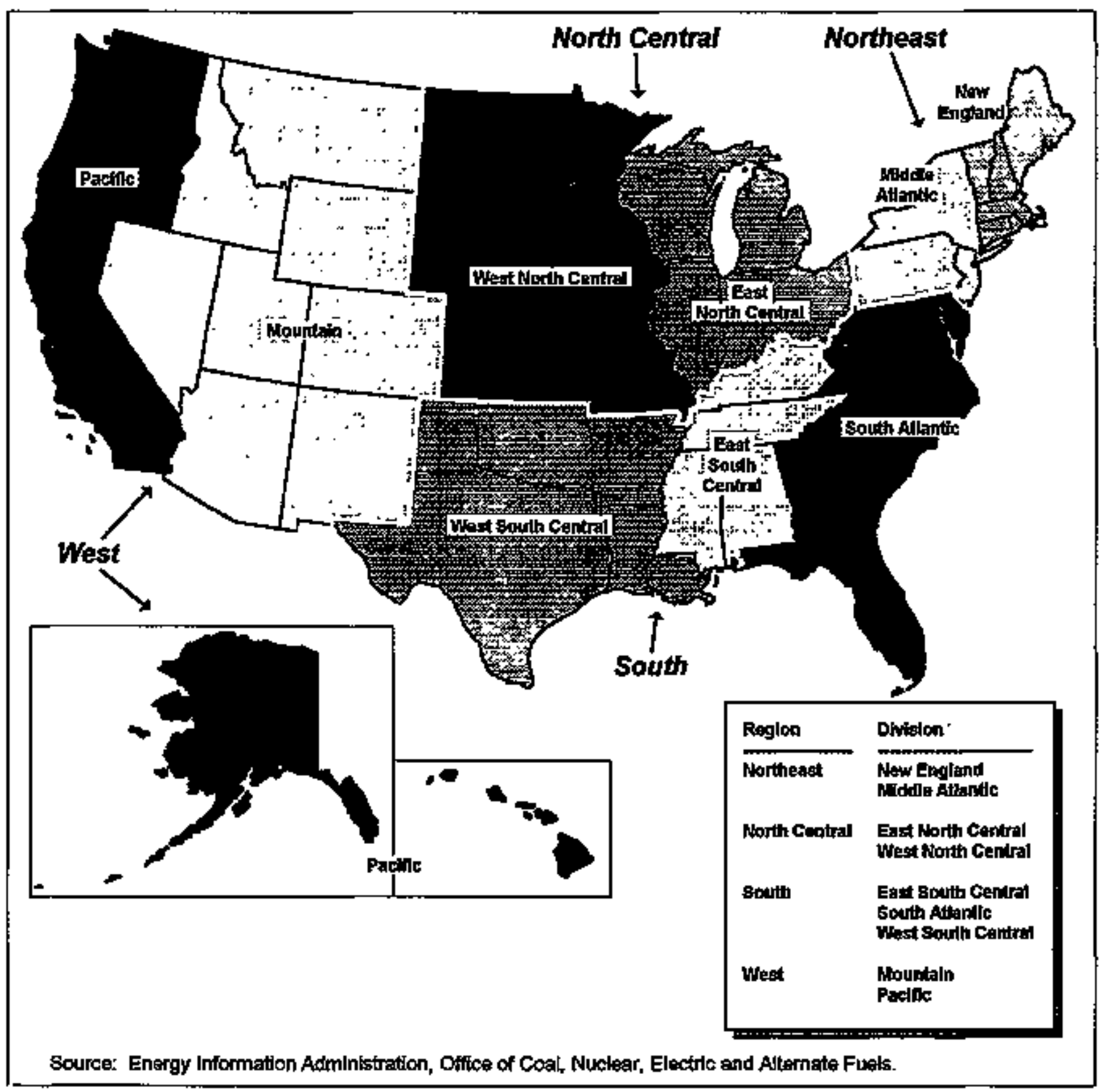

Energy Inforthation Administration/Inventory of Power Plants in the United States as of January 1, 1996401 
S

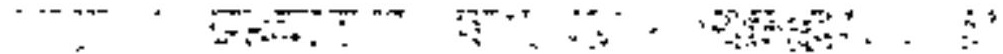




\section{Glossary}

Ampere: The unit of measurement of electrical corrent produced in a circuit by 1 volt acting through a resistance of 1 ohen. (See Current, Obm, Volt.)

Anthracite: Anthracite, or hard coal, is the highest rank of economically useable coal. It is jet black with a high luster. The moisture content generally is less than 15 percent. Anthracite contains approximately 22 to 28 mitlion Btu per ton as received and averages about $\mathbf{2 5}$ million Btu per ton. Its ignition temperature is approximately 925 to 970 degrees Fabrenhejt. Virtually all of the anthracite mined is from northeastern Pennsylvania. It is used mostly for space heating and generating electricity.

Barrel: A volametric unit of mcasure for crude oil and petroleum products equivalent to 42 U.S. gallons.

Baseload: The minimum amount of electric power delivered or required over a given perjod of time at a steady state. (See Baseload Plant.)

Baseload Capaclty: The generating equipment nortmally operated to serve loads on a round-the-clock basis. (See Baseload, Baseload Plant.)

Baseload Plant: A plant, usually bousing higheffictency steam-electric units, which is normally operated to take all or part of the minimum load of a system, and which consequently produces electricity at an essentially constant rate and runs coptinuously. These units are operated to maximize system mechanical and thermal efficiency and minimize system operating costs. (See Baseload.)

Biomass: Organic materials used as a source of energy. (See Other Generation.)

Bituminons Cosl: Bituminous coal, or soft cosl, is the most common coal. It is dense, black, often with well-defined bands of bright and dull material. Its moisture content usually is less than 20 percent. The beating value ranges from 19 to 30 million Btu per ton as received and averages about 24 million Btu per ton. The jgation temperature ranges from about 700 to almost 900 degrees Fahrenheit. Bituminons col is mined chiefly in the Appalachian and Interior coal ftelds. It is used for generating electricity, making coke, and space heating.

Blast Furnace: A furnace is which soltd fuel (coke) is burned with an air blast to smelt iron ore.

Hoilling-Water Reactor (BWR): A light-water reactor in which water, used as both coolant and moderator, is allowed to boil in the core. The resulting steam can be used ditectly to drive a turbine.
Btu (Brftish Thermal Unit): A standard unit for measuring the quantity of heat energy equal to the quantity of heat reguired to raise the temperature of 1 pound of water by 1 degree Fahregheit.

Capability: The maximum load that a generating unit, generating station, or other electrical apparatus can carry under specified conditions for a given period of time without exceedisg approved limits of temperature and stress.

Capacity: The amount of electric power delivered or required for which a gentrator, turbine, transformer, transmission circuit, station, or system is rated by the manufacturer. (See Genenator Nameplate Capacity.)

Capacity Factor: The ratio of the average load on the plant(s) for the period of time considered to the aggregate capacity of all the generating equipment installed in the plant(s).

Census Divisions: The tine geographic divistons of the United States established by the Bureau of the Census, U.S. Departmeat of Commerce for statistical analysis. The boundaries of Census divisions coincide with State boundaties. In some cases, the Pacific Division is subdivided into the Pacific Contiguous and Pacific Noncontiguous aress.

Coal: A black or browntsh-black solid combustible substance formed by the partial decomposition of veg. etable matter without access to ait. The rank of coal, whjch jncludes anthracite, bituminous coal, subbituminous coel, and lignite, is basted on fixed carbon, volatile matter, and beating value. Coal rank indicates the progressive alteration from jignite to anthracite. Lignite contains approximately 9 to 17 million Btu per ton. The contents of subbituminous and bituminous coal range from 16 to 24 million Btu per ton and from 19 to 30 million Bto per ton, respectively. Anthracite contains approximately 22 to 28 million Btu per ton.

Cogeneration: The seguential or simultaneous process in which useful heat/steam is generated, used in a variety of process applications, and then directed into a turbine to generate eleetricity and/or mechanical work frotn the useful thermat energy still available for use. (See Geperation, Energy.)

Coke: In general, a product made from bituminous coal and crude oil from which the volatile constituents have been driven off by beat, so that fixed carbon and ash aze fused together. Coke, being Jargely carbon, is hard and porous, and is a desirable fuel in certain metallurgical industries. 
Combined Cycle: A cogeneration technology in which additional electricity is produced sequentially from the otherwise lost waste heat exiting from one or more gas-fited toubines. The exiting hemat flow is routed to a exhaust-fired conventional boiler or to a heat recovery stearn generator for utilization by a steam turbine in the production of electricity. This process increases the efficiency of an electric generating system by turining the rejected heat into thermal steam rather than discharging it toto the atmosphere. (See Cogeneration, Turbine.)

Combined Hydroelectric Plant: A bydroelectric plant that uses both pumped water and natural streanflow for the production of power.

Combined Pumped-Storage Plant: A punpedstorage hydroelectric power plant that uses both pumped water and aatural streariflow to produce electricity.

Commerctal Operation: A generating unit is said to be in commercial operation when control of the foading of the unit is turned over to the system dispatcher.

Consumption (Fuel): The amount of fuel used for gross generation, providing standby service and start-up and/or flame stabilization. (See Fuel.)

Conrentional Hydroelectric Plant: A plant in which all of the power is produced from natural streamflow as regulated by available storage.

Crude ofl (including Lease Condensate): A mixture of hydrocerbons that existed in liquid plase in underground reservoirs and that remains liquid at atmospheric pressure after passing through surface separating faciljtits. Included are lease condensate and liquid hydrocarbons prodaced from tar sands, gilsonite, and shale oil. Drip gases are atso included, but topped crude oil (residual oil) and other unftuished ojls are excluded. Liguids produced at natural gas processing plants and mixed with crude oil are likewise excluded where identiftable. (See Petroleum.)

Corrent: A flow of electrons in an electrical conductor. The stsength or rate of movement of the electricity is measured in amperes. (See Ampere, Ohm, Volt.)

Demand: The rate at which electric energy is detivered to or by a system, part of a system, or piece of equipment, at a given instant or averaged over any designated period of time.

Design Electrical Rating (Capacity), Net: The nominal net electrical output of a nuclear unit, as specified by the utility for the purpose of ptant design.

Distillate Fuel Oil: A general classification for one of the petroleum fractions produced in conventional distillation operations. It is used prinarily for space heatíng, on-and-off-highway diesel engine fued (including raikroad engine fuel and fuel for agriculture machinery), and electric power geveration. Included are products known as No. 1, No. 2, and No. 4 fuel oils; No. I, No. 2, and No. 4 diesel fuels.

Electric Plant: A station containing prime movers, electic generalors, and auxiliary equipment for converting mechanical, chemical, and/or fission energy into electric energy.

Electric Power Industry: The public, private, and cooperative electric utility systems of the United States taken as a whole. This includes all electric systems serving the pubjic: regulated investor-owned electric utility companies; Federal power projects; State, municipal, and other government-owned systems, including electric public utility districts; electric cooperatives, including Generation and Transmission entities (" $G$ and $T^{\prime \prime} S^{n}$ ); jointly owned electric utility facilities, and electric utility facilities owned by a lessos and leased to an electric utilfty. Excluded from this list are the special purpose electric facilities or systems that do not offer servite to the public.

Electric Power System: An individual electric power entity-a company, an electric cooperative, a public electric supply corporation like the Tennessee Valley Authority, a similar Federal department or agency like the Bonneville Power Adminjstration, the Bureau of Reclamation or the Coxps of Engtneers, a municipally owned, electric department offoring service to the public, or an electric public utility district (a "PUD"); also a jointly owned electric supply project such as the Keystone.

Electric Utulity: A corporation, persod, agency, authority, or other legal entity or instrumentality that owis and/or operates facilsties within the United States, its territories, or Puerto Rico for the generation, transmission, distribution, or sale of electric energy, primarily for use by the public. An entity that solely operates qualifying facilities under the Public Utility Regulatory Policies Act of 1978 is not considered an electric utility.

Energy: The capacity for doing work as measured by the capability of doing work (potential energy) or the conversion of this capability to motion (kinetic energy). Energy has several forms, some of which are easily convertible and can be changed to another form useful for work. Most of the world's convertibje energy comes from fossil fuels that are burned to produce heat that is then used as a transfer mediutm to mechanical or other means in otder to accomplish tasks. Electrical energy is usually measured in kilowathours, while heat entergy is usually measured in British thermal units. (Sec Energy Source.)

Energy Source: The pritary source that provides the power that is converted to electricity through chemical, mechanical, or other means. Energy sources include coat, petroleum and petroleum products, gas, water, urapiom, wind, sunlight, geothermal, and other sources.

Fahrenheit: A temperature scale on which the boiling print of water is at 212 degrees above zero on the scale and the freezing point is at 32 degrees above zero at standard atmospheric pressurc. 
Federal Region: In a Presidential directive issued in 1969, various Federal agencies (among them the currently designated Department of Heaith and Human Services, the Department of Labor, the Office of Economic Opportunity, and the Small Business Administration) were instructed to adopt a uniform freld system of I0 geographic regions with common boundaries and headquarters cities. The action was taken to cortect the evolution of fragmented Federal field organization structures that each agency or component croated independently, usually with little reference to other agencies' arrangements. Most Federal domestic agencies or their components have completed realignments and relocations to conform to the Standard Federal Administration Regions (SFAR's) shown on the map at the end of this pablication.

Forced Outage: The shutơown of a generating unit, tcansutission line or other facility, for emergency reasons or a condition in which the generatsig equipment is unavailable for load due to unanticipated breakdown. (See Outage.)

Fossil Foel: Any naturally occurring organic foel, such as coal, crude oil, and patural gas.

Fossil Fuel Plant: A plant using cosi, petroleum, or gas as its source of energy.

Fuel: Any substance that can be burned to prodace heat; also, materials that can be ftssioned in a chain reaction to produce heat.

Fitel Cell: A device that produces electrical energy directly from the controlled electrochemical oxidation of the fuel. It does not contain on intermediate heat cycle, as do most other electrical generation techniques.

Ges: Includes natural gas, colse-oven gas, blastfurnace gas, and refinery gas. Manufactured gas is reported as natural gas on FERC Form 423. (See Natural Gas.)

Gas-Turbine Plant: A plant in which the prime mover is a gas turbine. A gas turbine consists typjcally of an axial-flow air compressor, one or more combustion chambers where liquid or gaseous fuel is burned and the hot gases are passed to the turbine; where the hot gases expand to drive the generator and then are used to run the compressor.

Generating Unit: Ap olectric generator together with its prime mover.

Generation: The process of producing electric energy by transforming other forms of energy; also, the atrount of electrie entergy produced, expressed in kilowatthours. (See Electric Plant, Energy.)

Generator: A machine that converts mechanical energy into clectrical energy.

Generator Nameplate Capacity: The full-load continvous rating of a generator, prime mover, or other electrical equipment under spectifted conditions as designated by the manufacturer. Generator nameplate capacity is usually indicated on a nameplate attacbed physically to the equipment. Installed station capacity does not include auxiliary or house units.

Geothermal Energy: Energy from the internal heat of the earth may be residuai beat, friction heat, or a result of radioactive decay. The heat is found in rocks and flujds at various depths and can be extracted by drilling andfor pomping.

Geothermal Plant: A plant in which the prime mover is a steam turbine. The turbine is driven either by steam produced from hoc water or by natural steam that derives its energy from heat found in rocks or fluids at various depths beneath the surface of the earth. The energy is extracted by drilling and/or pumping.

Gigaratt (GW): One billion watts. (See Watt.)

Gigawatthour (GWh): One billion wathours. (See Watthour.)

Grid: The layout of an electrical distribution system.

Gross Generation: The total anount of electric energy produced by a generating station or stations, messured at the generator terminals. (See Geperation, Electric Plant,)

Heat Rate: A measure of generating station thergual efficiency, generally expressed in Btu per net kilowatthour. It is comptsted by dividing the total Btw content of fuel barned for electric generation by the resulting net kilowatthour generation. (Sec Btu, Britisb Thermal Unit.)

Heary Oil: The fuel otis remaining after the lighter oils bave been distilled off during the refining process. Except for start-up and flame stabilization, virtually all petroleum used in steam plants is heavy oil.

Horsepower: A unit for measuring the rate of work (or power) equivalent to 33,000 foot-pounds per minute or 746 watts. (See Watt)

Hydroelectric Energy: The production of electricity from kitetic energy in flowing water. (See Energy.)

Hydroelectric Plant: A plant in which the turbine genetators are drivent by falling water.

Hydroelectric Power: The barnessing of flowing water to produce mechanical or electrical energy- (See HydroeIectric Energy, Hydroelectric Plant.)

Internal Combustion Plant: A plant in which the prime mover is an internal corrbustion engine. An intertal combustion engine has one or more cylinders in which the process of combustion takes place, converting energy released from the rapid burning of a fuel-air mixture jnto mechanical energy. Diesel or gas-fired engines are the principal types used in eloctric plants. The plant is ustually operated during periods of bigh demand for electricity. 
Kflowatt (kW): One thousand wats. (See Watt.)

Kilowatthour (kWh): One thousand watthours. (See Watthour.)

Life Extension: Investments made to majntain the operating status of an electric generating plant, into acceptable levels of availability and efficiency, beyond its originally anticipated retirement date.

Light Oil: Lighter fuel oils distilled off during the refining process. Virtually all petroleum used in internal combustion and gas-tarbine engines is light oil.

Light-Water Reactor (LWR): A puclear reactor that uses water as the primary coolant and moderator, with slightly enriched uranium as fuel. There are two types of commercial light-water reactor -- the boiling-water reactor (BWR) and the pressurized-water roactor (PWR).

Lignite: Lignite, the lowest rank of coal, is brownish black and has a bigh moisture contenc, sometimes as high as 45 percent. It tends to disintegrate when exposed to the weather. The heat content of ligpite ranges from 9 to 17 million Btu per ton as reteived and averages about 14 miltion Btu per ton. The ignition temperature is appsoximately 600 degrees Fabrenhekt. Lignite is mined in California, Lorisiana, Montana, North Dakota, and Toxas, and is used mainly to generate electricity in power plants that are relatively close to the mines.

Load (Electric): The anount of electric power delivered or required at any specific point or poínts on a system. The requirement originates at the energyconsuming equipment of the customers.

Load Management Technfque: Utility demand management practices directed at reducing the tnaximum bilowatt demand on an electric system, and/or modifying the coincident peak demand of one or more classers of service to better meet the utility systen capability for a given hour, day, week, season, or yoar. (See Demand, Losd (Electrie)).

Low-Power Testing: The period of time between a plant's initial fuel loading date and the issuance of its operating (Full Powm) ticense. The maximun level of operation during this period is 5 percent of the unit's design thermal rating.

Maximum Demand: The greatest of all demands of the load that has occurred within a specifled period of time.

Mct: One thousand cobic feet.

Megawatt (MW): One million watts. (See Watt.)

Megawatthour (MWh): One million watthours, (Soe Watthour.)

MMef: One million cubic feet.
Muniejpality: A city, county, irrigation district, draingge district, or a political subdivision or agency of a \$tate competent under the laws thereof to carry on the business of developing, transunitting, or distributing power.

Natural Gas: A naturally oecurring mixtsre of bydrocarbon and nonbydracartoon gases found in porous geological formations beneath the earth's surface, often in association with petrolevm. The principal constituent is methane.

Net Generation: Gross generation less plant use, measured at the high-Yoleage temrinals of the statson's step-up transformer. The energy required for pumping at pumped-storege plants is regarded as plant use and must be deducted from the gross generation. (See Generation, Electric Plant.)

Net Summer Capability: The steady hourly output which generating equipment is expected to supply 10 system load (exclusive of auxiliary) power as demonstrated by tosts at the time during summer peak demand.

Net Winter Capability: The steady hourly outpot which generating equipment is expected to supply to system load exclusive of auxiliary power as demonstrated by test at the time of winter peak demand.

North American Electrite Reliabillty Councit (NERC): A counctl formed in 1968 by the electric utisity industry to promote the reliability and adequacy of bulk power supply in the electric utility systems of North America. NERC consists of nipe Jegional reliability councils and encompasses essentially all the power systems of the contiguous United States, Cantada, and some in Mexico. The data summarized by NERC regions in this publication are limited to that portion applicable to the contiguous United States, thereby excluding that portion of NERC data applicable to Alaska, Hawaiti, Canada, and Mexico. The NERC Regions ar:

ECAR - East Central Area Reliability Coordination Agreement

ERCOT - Electric Reliability Council of Texas

MAIN - Mid-America Intereonnected Network

MAAC - Mid-Atlantic Area Council

MAPP - Mid-Continent Area Power Pool

NPCC - Northeast Power Coordinating Council

SERC - Southeastero Electric Reliability Counci]

SPP - Southwest Power Pool

WSCC - Western Systems Coordinating Counejl.

Nuclear Fuel: Fissionable materials that have been enriched to such a composition that when placed in a nuclear reactor will support a self-sustajuing fission chain reaction, producing heat in a coatrol]ed manner for process use.

Nuclear Power Plant: A plant in which the printe mover is a steam turbine. The steam used to drive the turbine is produced by a heat transfer from the reactor 
vessel during the period when the nuclear foel is undergoing fisston.

Nuclear Reactor: A device in whitch a fission chain reaction can be initiated, maintained, and controlled. Its essential components are a vessel containing a core with fissionable fuel, a moderator for the fission chain reaction, and a control system.

No. 1 Fuel Oil: A light distitlate fuel oil intended for use in vaporizing pot-type burnets. ASTM Specification D396 speciftes for this grade maximum distillation temperatures of 400 degrees $F$. at the 10-percent point and 550 degrees $F$. at the 90 -percent point, and kinematic viscosities between 1.4 and 2.2 centistokes at 100 degrees $F$.

No. 2 Fuel Oil: A distillate fuel oil for use in atomizing type burners for domestic heating or for moderate capacity commercial-industrial borner units. ASTM Specification D396 specifies for this grade distillation temperatures at the 90-percent point between 540 degrees and 640 degrees $F$., and kinematic viscosities between $\mathbf{2 , 0}$ and $\mathbf{3 . 6}$ centistokes at 100 degrees $\mathrm{F}$.

No, 1 and No. 2 Diesel Fuel Ods: Distillate fuel oils used in compression-ignition engines, as given by ASTM Spectfication D975:

No. 1-D - A volatile distillate fuel oil with a boiling range between 300-575 degrees $F$, and used in high-speed diesel engines generally operated under wide variations in speed and load. Includes type C-B diesel fuel used for city buses and stmilar operations. Properties are defined in ASTM Specifications D975.

No. 2-D - A gas-oil type of distillate of lower volatitity with distillation temperatures at the 90-percent point between 540-640 degrees $F$. for use in high-speed diesel engines generally operated under uniform speed and load conditions. Includes Type R-R diesel fuel used for rajlroad locomotive engines, and T.T for diasel-engine trucks. Properties are dofjped in ASTM Specification D975.

No. 4 Fuel Oil: A fuel oil for commercial burner installations not equipped with preheating facilities; used extensively in industrial plants. This grade is a blend of disti]late fuel oil and residual fuel otl stocks that conforn to ASTM Specification D396 or Federal Specifrestion VV-F-815C; its kinematic viscosity is between 5.8 and 26.4 centistokes at 100 degrees F. AIso included is No, 4-D, a fuel oil for tow- and medium-speed diesel engines that conforms to ASTM Specification D975.

Ohm: The unit of mteasurement of clectrical resistance. The resistance of a circtit in which a potential difference of 1 volt produces a current of 1 ampere. (Ste Ampere, Current, Volt.)

Oil: A mixture of hydrocarbons usually existitg in the liquid state in atural underground pools or reservoirs. Gas is often found in association with oil. (See Crude Oil (Including Lease condensate), Petroleam.)
Operable: A unit is operable when it is available to provide power to the grid. For a nuclear unit, this is when it receives its full power amendment to its operating license from the Nuclear Regulatory Commission.

Other Gas: Includes manufactured gas, coke-oven gas, blast-furnace gas, and refinery gas. Manufactured gas is obtained by distillation of coal, by the thermal decomposition of oil, or by the reaction of steam passing through a bed of heated coal or coke. (See Natural Gas)

Other Generation: Electricity originating from these sources: biomass, fuel cells, geothermal heat, solar power, waste, wind, and wood.

Ontage: The period during which a generating unit, transmission line, or other facility is out of service. (See Forced Outage, Scheduled Outage.)

Peak Load: The maximum load during a specified period of tirute.

Peak Load Plant: A plant usually housing old, lowefficiency steam units, gas turbines, djesets, or pumped-storage hydroelectric equipment normally used during the peak-load periods.

Peaking Capacity: Capacity of generating equtpment normally operated during the hours of highest daily, weekly, or seasonal loads. Some genterating equipment may be operated at certain times as peaking capacity and at other times to serve loads on a 'round-the-clock besis. (See Peak Load.)

Petroleum: A mixture of hydrocarbons existiog in the liguid state found in natural andergtound teservoirs, often associated with gas. Petroleum includes Fuel oil 2, 4, 5, 6, topped erude, kerosene, and jet fuet. (See Petroleum (Crade Oil.))

Petrolenm Coke: A residue, high in carbon content and low in hydrogen, that is the final product of thermal decomposition in the condensation process in cracking. This product is reported as marketable coke or catalyst coke. The converston factor is 5 bartels (of 42 U.S. gallons each) per short ton.

Petroleom (Crude Oil): A naturally occurring, oily, flammable liquid composed principally of hydrocarbons. Crude oil is occasionally found in springs or pools but usually is drilled from wells beneath the earth's surface.

Photoroltaic Cell: Device that produces electrical current by converting light or stmilar radiation. (See Other Generation.)

Plant: A station at which are located prime movers, electric genertators, and auxiliary equipment for converting mechanical, chemical, and/or nuclear energy into electric energy. A station may contain more then one type of prime mover. Electric utility plants exclude stations that satisfy the definition of qualsfying facility under the Public Utility Regulatory Poltcies Act of 1978 . 
Plant Use: The electric energy used in the operation of a plant. Included in this definition is the energy required for pumping at pusmp-storage plants.

Plant-Use Electricity: The electric energy used in the operation of a plant. This energy total is subtracted from the gross energy ptoduction of the plant; for reporting purposes the plant energy production is then reported as a net figure. The energy required for pumping at pumped-storage plants is by defintion subtracted, and the energy production for these plants is then reported as a net figure. (See Combined Pumped-Storage Plant, Pomped-Storage Hydroelectric Plant, Pure Pumped-Storage Hydroelectric PIant.)

Power: The rate at which energy is transferred, usualty measured in watts. Also used for a measurement of capacity. (See Capacity, Energy, Watt.)

Power (Electrical): An electric mexsurement unit of power called a voltampere is equal to the product of one volt and one ampere. This is equivalent 101 Watt for a direct curtent system and a unit of of apparent power is separated into real and reactive power. Real power is the work-producing part of apparent power that measures the rate of supply of energy and is denoted as kilowatts (KW). Reactsve power is the portion of apparent power that does no work and is referred to as kilovars; this type of power must be stapplied to most types of magnetic equipment, such as motors, and is supplied by generator or by electrostatic equipment. Vottamperes are usually divided by 1,000 and called kilovoltamperes (kVA). Energy is denoted by the product of reat powt and the length of time utilized; this prodact is expressed as kilowatthours.

Pressurized-Water Reactor (PWR): A quclear reactor in which heat is transferred from the cors to a heat exchanger via water kept under high pressure, so that bigh temperatures can be maintained in the primary system without boiling the water. Steam is generated in a secondary circuit.

Primo Mover: The engine, turbine, water whoel, or similar machine that drives an electric generator.

Privately Owned Electric Utility: A class of ownership found in the electric powor industry whete the utility is regulated and authorized to achieve an allowed rate of return. (See Electric Power Industry.)

Production (Electric): Act or process of producing electric energy from other forms of energy; also, the amount of electric energy expressed in watthours (Wh).

Pnblicly Owaed Electric Utility: A class of ownership found in the electric power industry. This group inclades those utilities operated by municipalities, and State and Federal power agencies.

Pnblic Utitity Regulatory Pollicies Act of 1978: One part of the Nattonal Energy Act, PURPA contains measures designed to encourage the conservation of energy, more efficient use of resources, and equitable rates. Principal among these were suggested retail rate reforms and new incentives for production of electricity by cogenerators and users of renewable resources. The Commission has primary authority for implementing several key PURPA programs.

Pumped-Storage Hydroelectric Plant: A plant that usuaily generates electric entrgy during peak-load periods by using water previously pumpeó into an ele. vated storage reservoir during off-ptak periods when excess generating capacity is available to do so. When additional generating capacily is needed, the water can be released from the reservoir through a conduit to turbine generators located in a power plant at a lower Ievel.

Pure Pumped-Storage Hydroelectric Plant: A plant that produces power only from water that has previously been pumped to an upper reservoir.

Renewable Energy Sourcet An energy source that is regenerative or virtually inexhaustible. Typical examples are wind, geothermal and water power. (See Other Generation.)

Repowering: Refurbishment of a plant by replace. ment of the combustion technology with a new combustion technology, tusually resultiog in better performance and greater capacity.

Residual Fuel Oil: The topped crude of refinery operation; includes No. 5 and No.6 fuel oils as defined in ASTM Specification D396 and Federal Specification VV-F-\$15C, Navy Special fuet oil as defined in Military Specification MIL-F-859E including Amendment 2 (NATO Symbol F-77), and Bunker $\mathrm{C}$ fuel oil. Residual fuel oil is used for the production of olectric power, space heating, vessel bunkering, and various industria] purposes. Imports of residual fuel oil include "Imported Crude Oil Burned as Fuet. "

Run-of-River Hydroelectric Plant: A low-head plant using the flow of a stream as it occurs, and having tittle ot no reservoir capacity for storage. (See Hydroelectric Power.)

Schednled Outaget The shutdown of a generating unit, transmission line, or other facility, for inspection or maintenance, in accordance with an advance schedule, (See Forced Oulage, Outage.)

Short Ton: A unjt of wejght equal to 2,000 pounds.

Solar Energy: Energy produced from the sun's radjation.

Standby Facllity: A facilicy that supports a utility sysiem and is generally running under no-joad. It is availabte to replace or supplement a facility normally in service. (See Standby Service, Outage.)

Standby Service: Support service that is available as needed to supplement a customer, a utility system, or to anther utility if a schedale or an agreenent authorizes the tranșaction. The service is not regularly used. (See Standby Facility, Ontago.) 
Station (Electrie): A plant containing prime movers, electric generators, and auxiltary equipment for converting mechanical, chenical, and/or nuclear energy into electric energy.

Storage Hydroetectric Plant: A hydroelectric plant with reservoir storage capacity for power use.

Subbltuminous Coal: Subbiluminous coal, or black lignite, is dutj black and genterally contains 20 to 30 percent moisture. The beat content of subbituminous coal ranges from 16 to 24 million Btu per ton as received and averages about 18 million Btu per ton. Subbituminous coal, mined in the western coal fields, is used for generating electricity and space heating-

System (Electric): Physically connected generation. transatission, and distribution facilities operated as an integrated unit under one central managemeat, or operating supervision.

Thermal: A term used to identify a type of electric generating station, capacity, capability, or output in which the source of energy for the prime mover is heat.

Turbjne: A machine for generating rotary mechan. ical power from the energy in a stream of fluid (such as water, steam, or hot gas). Turbines convert the kinetic energy of fluids to mexbanical energy through the principles of impulse and reaction, or a mixture of the two.

Uranlum: A heavy, naturally radjoactive, metallic element with atomic number 92 . The two isotopes that occur most frequently are Uranium-235 and Uranjum-238. Uranjum-235 is the only isotope existing in nature in any appreciable extent that is fissionable by thermal neutrons. Urabium is the batic raw material of nuclear energy. (See Nuclear Futel.)

Volt: The unit of measurement of voltage, electrical force, or pressure. The electrical force that, if steadily applied to a circuit with a rosistante of 1 ohm, will produce a current of 1 ampere. (See Ampere, Curreat, Ohm.)

Watt: The electrical unit of power. The rate of onergy transfer equivalent to 1 ampere flowing under a pressure of 1 volt at unity power factor.

Watthour (Wh): An electrical energy ubit of measure equal to 1 watt of power supplied to, or taken from, an electric circuit steadily for 1 howr.

Wind Energy: Energy produced by harnessing the force of the wind. In a wind energy conversion system such as a windmili, the energy of wind is used co turn the shaft of a generator, which in turn usually produces direct current. This direct current is usuatly converted to alternating current before being fed into a utility grid system. 